\title{
Synthesis of Graphene Using Plasma Etching and Atmospheric Pressure Annealing: Process and Sensor Development
}

\author{
Andrew Robert Graves \\ West Virginia University, agraves1@mix.wvu.edu
}

Follow this and additional works at: https://researchrepository.wvu.edu/etd

Part of the Condensed Matter Physics Commons, Electrical and Electronics Commons, Electronic Devices and Semiconductor Manufacturing Commons, Engineering Physics Commons, Engineering Science and Materials Commons, Materials Chemistry Commons, Nanotechnology Fabrication Commons, Other Chemical Engineering Commons, Semiconductor and Optical Materials Commons, and the Structures and Materials Commons

\section{Recommended Citation}

Graves, Andrew Robert, "Synthesis of Graphene Using Plasma Etching and Atmospheric Pressure Annealing: Process and Sensor Development" (2020). Graduate Theses, Dissertations, and Problem Reports. 7582.

https://researchrepository.wvu.edu/etd/7582

This Dissertation is protected by copyright and/or related rights. It has been brought to you by the The Research Repository @ WVU with permission from the rights-holder(s). You are free to use this Dissertation in any way that is permitted by the copyright and related rights legislation that applies to your use. For other uses you must obtain permission from the rights-holder(s) directly, unless additional rights are indicated by a Creative Commons license in the record and/ or on the work itself. This Dissertation has been accepted for inclusion in WVU Graduate Theses, Dissertations, and Problem Reports collection by an authorized administrator of The Research Repository @ WVU. For more information, please contact researchrepository@mail.wvu.edu. 
Synthesis of Graphene Using Plasma Etching and Atmospheric Pressure Annealing: Process and Sensor Development

Andrew Robert Graves

Follow this and additional works at: https://researchrepository.wvu.edu/etd

Part of the Condensed Matter Physics Commons, Electrical and Electronics Commons, Electronic Devices and Semiconductor Manufacturing Commons, Engineering Physics Commons, Engineering Science and Materials Commons, Materials Chemistry Commons, Nanotechnology Fabrication Commons, Other Chemical Engineering Commons, Semiconductor and Optical Materials Commons, and the Structures and Materials Commons 


\title{
Synthesis of Graphene Using Plasma Etching and Atmospheric Pressure Annealing: Process and Sensor Development
}

\author{
Andrew R. Graves \\ Dissertation submitted to the \\ Benjamin M. Statler College of Engineering and Mineral Resources \\ at West Virginia University
}

in partial fulfillment of the requirements for the degree of

Doctor of Philosophy

in

Chemical Engineering

Charter D. Stinespring, Ph.D., Chair

Robin S. Hissam, PhD

John W. Zondlo, PhD

Edward M. Sabolsky, PhD

Jeremy M. Dawson, PhD

Department of Chemical and Biomedical Engineering

Morgantown, West Virginia

2020

Keywords: Graphene, SiC, Buffer layer, ICP/RIE, Plasma Etching, Sensors, Gas Detection, Photo Detection, Schottky Diode, XPS, Raman, Electronic Devices

Copyright 2020 Andrew R. Graves 


\title{
Abstract
}

\section{Synthesis of Graphene Using Plasma Etching and Atmospheric Pressure Annealing: Process and Sensor Development}

\author{
Andrew R. Graves
}

Having been theorized in 1947, it was not until 2004 that graphene was first isolated. In the years since its isolation, graphene has been the subject of intense, world-wide study due to its incredibly diverse array of useful properties. Even though many billions of dollars have been spent on its development, graphene has yet to break out of the laboratory and penetrate mainstream industrial applications markets. This is because graphene faces a 'grand challenge.' Simply put, there is currently no method of manufacturing high-quality graphene on the industrial scale. This grand challenge looms particularly large for electronic applications where the synthesis process must be scaled to wafer dimensions and, for the most part, must utilize existing semiconductor processing tools. Two widely researched methods, selective thermal sublimation and chemical vapor deposition, have the potential to meet this grand challenge; yet despite a global development effort, each method continues to face significant challenges at this time.

The work detailed in this dissertation approaches the problem from a fundamentally different direction. To that end, a novel plasma assisted growth method for producing graphene on silicon carbide $(\mathrm{SiC})$ wafers has been investigated. This process has been under investigation in the Stinespring laboratory since 2012. In this two-step process, a halogen $\left(\mathrm{Cl}_{2}\right.$ or $\left.\mathrm{CF}_{4}\right)$ based inductively coupled plasma (ICP) combined with a reactive ion etch (RIE) was used to selectively etch silicon from the surface layers of a $\mathrm{SiC}$ wafer to produce a carbon rich film in the first step. In the second step, the film and substrate underwent a rapid thermal anneal (RTA) under ultrahigh vacuum (UHV) to crystalize the carbon film into graphene layers. The primary goals of the research described in this dissertation were to increase the manufacturability of the process, improve the quality of the graphene films and to develop and characterize simple sensors using these films.

To improve manufacturability of the graphene films, this work developed an atmospheric pressure (AP) RTA under an inert gas to replace the UHV process. Films produced by this APRTA method were characterized by x-ray photoelectron spectroscopy (XPS) and Raman spectroscopy. It was found that the standard process yielded good quality, two to three layer thick graphene films with low levels of oxygen defects. These analyses also provided strong evidence that the graphene films were tightly coupled to the $\mathrm{SiC}$ substrate by way of a buffer-layer. This buffer-layer was structurally and compositionally similar to graphene, but a substantial fraction of the carbon atoms were covalently tethered to silicon atoms of the $\mathrm{SiC}$ substrate surface. This result was confirmed by electrical characterization measurements of the films up to $800{ }^{\circ} \mathrm{C}$ in which a variety of electrical properties were measured. The temperature dependence of these electrical properties also yielded valuable insight into fundamental aspects of the films, such as the charge injection mechanisms, carrier scattering processes, and other phenomena which are only reveal at elevated temperatures.

To improve film quality, a parametric investigation was conducted to determine the influence of each processing parameter on the film properties. In total, nine different parameters (SiC polytype, crystal face, doping type, ICP power, RIE power, and plasma pressure as well as 
RTA temperature, heating rate and cooling rate) were investigated. While it was found that each parameter effected the film properties to varying degrees, the most important parameters were the RIE power, the anneal temperature, and the anneal time.

Despite optimizing theses key parameters, the process still produced a buffer-layer coupled film. To remedy this and enhance film quality, high temperature annealing of the graphene films in hydrogen was investigated. It was found that repeated high temperature thermal cycling of the samples in hydrogen resulted in the largest degree of buffer-layer decoupling. Ultimately, however, full film decoupling was not achieved. This indicates a fundamental difference between these buffer-layers and those produced by the thermal sublimation process.

In pursuit of the device development goal, simple chemoresistive gas sensors and photo detectors were fabricated and tested. The results of these studies both demonstrate the utility of the graphene films produced here and provide additional insight into the structural and electrical properties of the films.

The sensitivity of the chemoresistive sensors was investigated for a variety of gases including $\mathrm{H}_{2}, \mathrm{CO}$, and $\mathrm{CH}_{4}$ gas mixtures in $\mathrm{Ar}$ and $\mathrm{He}$ at temperatures ranging from $25^{\circ} \mathrm{C}$ to $>800^{\circ} \mathrm{C}$. The devices were found to function effectively as gas sensors by a thermal and chemical response mechanisms. With regard to photodetection, the devices were found to display a strong response to visible laser irradiation in both a power and unpowered state, including $650 \mathrm{~nm}$ (red or $461 \mathrm{THz}$ ), $532 \mathrm{~nm}$ (green or $564 \mathrm{THz}$ ) and 405nm (purple or $740 \mathrm{THz}$ ) wavelengths. Overall, these results demonstrate the utility of the films produced here in application areas including gas sensors, flow measurement, temperature measurement, and photodetection.

In conclusion, this work has significantly advanced the development of the plasma assisted graphene synthesis process by improving the manufacturability of the process and quality of the resulting films. Moreover, our scientific understanding of many fundamental aspects of the synthesis process, the film structure, and the electrical behavior of these tethered graphene films has been advanced in a significant way. These results, particularly as they relate to the electrical properties, charge injection and transport processes, will be of interest to researchers pursuing graphene growth by selective thermal sublimation and the related research community who must deal, in some cases, with tethered graphene films or who look to utilize tethered films for device applications. Finally, this work has uncovered a plethora of future research directions in both the synthesis and application arenas. 


\section{DEDICATION}

The entire body of work that this document represents is dedicated to my dad, Lenard Graves, who sparked my passion for science, my mom Stephanie Graves, who taught me to read, to my brother, Aaron Graves, who's been a constant source of motivation and to all the educators, from

grade school to graduate school, who have gotten me to this point. Without a doubt, without any of them, this work would not exist. 


\section{ACKNOWLEDGMENTS}

I'm a pretty nostalgic person by nature so, as I wrote this dissertation, I've been thinking a lot about everyone who has helped me get to the point where this work was possible. I've been incredibly lucky throughout my life to have been surrounded by many friends, colleagues, teachers and mentors who have all helped me get here. And although I would really like to mention everyone, frankly, there are just far too many people and I know I would end up leaving many out (I realize that's rather a cliché thing to say in an acknowledgement, but even so, is completely true in this case!). However, there are a few people who have had such a profound impact, I must thank them directly. To everyone I have not mentioned, please know that I am incredibly thankful, nevertheless.

First, I want to thank my research advisor and mentor for this work, Charter Stinespring. Charter has, for years, gone above and beyond what anyone has a right to expect from an advisor. He always shows an incredible amount of patience with me and is always willing to take the time to discuss any scientific or professional topic. I have learned an incredible amount from the many hours we have spent discussing various scientific principles in his office (many times, long into the evening) and I've always valued greatly his career advice. It has been an absolute pleasure working with him on this research, and I've thoroughly enjoyed every minute. Although, that's not to say there have not been many points of frustration! Experiments not performing as expected, or the answers to some observed phenomena remaining frustratingly elusive; Charter was always there to provide prospective and encouragement and has always remained relentlessly positive.

Very importantly, I'd like to thank Robin Hissam. I owe everything I've accomplished in my graduate career to her. Not only did she give me my first undergraduate research opportunity, she provided me a path into graduate school; something that, at the time, I had not seriously considered because I thought it was out of reach. Robin gave me the opportunity to pursue a career in science on a level that I never thought would be possible, and for that, I will always be grateful. She was also instrumental in the transition from my Master's research work in her laboratory, into this PhD work. She has been a constant source of encouragement and I have considered myself very fortunate to have her as a mentor. 
I would be remiss if I did not thank the other members of my research committee; John Zondlo, Edward Sabolsky and Jeremy Dawson. They have dedicated a large amount of their time to reviewing this work and each has provided valuable and constructive feedback.

In a way, there's a certain finality to all this. This work feels like the culmination of all my schooling and, I suppose, it marks the end of my academic career. Because of that, I want to thank some truly fantastic educators I've been privileged to have had; David Hubbs and Gary Seldomridge, who were the best math teachers I've ever had; Donald Wagner and Paula Waggy, both fantastic science teachers. And most importantly, I want to especially thank my special education teachers, Debbie Thompson and Juanita Murphy, without them, I unquestionably would not have been able to accomplish any of this.

Lastly, I'd like to thank my friends and colleagues who have put up with me while I've been working on this. I've been essentially (and quite selfishly) incommunicado while writing this dissertation, except when I needed a section proofread, someone to bounce ideas off of or simply just to take an evening off and relax. You all have helped more than you can know, and I'll always remember that.

As a final note, although I am privileged to have had many people help me greatly with this work in one form or another, any and all mistakes contained here are fully and completely my own.

Andrew R. Graves West Virginia University February 2020 


\section{TABLE OF CONTENTS}

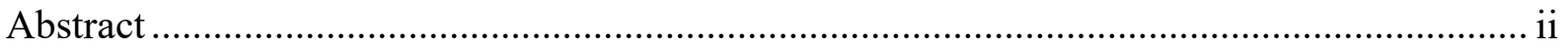

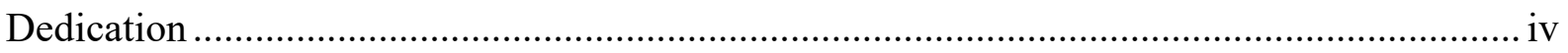

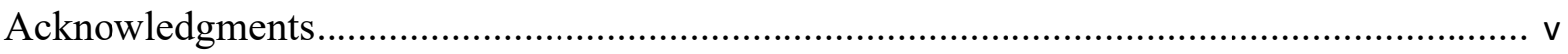

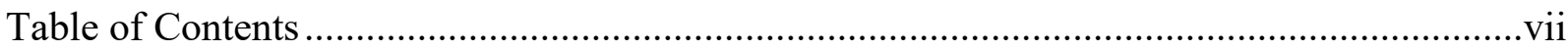

List of Tables ............................................................................................................ xi

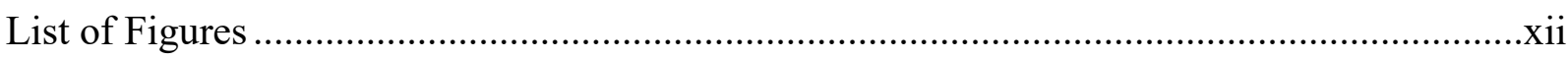

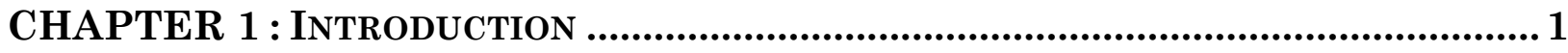

$\S 1.1$ Graphene and Its Properties: The Fundamentals....................................................... 1

$\S 1.2$ General Applications of Graphene: Graphene's Place in the World................................ 7

$\S 1.3$ Summary and Grand Challenges: Initial Problem Statement......................................... 14

\section{CHAPTER 2 : BRIEF REVIEW OF RELEVANT LITERATURE AND STATEMENT OF}

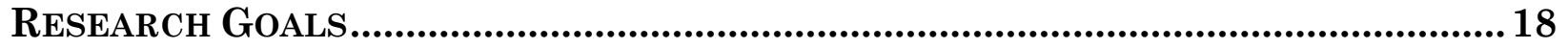

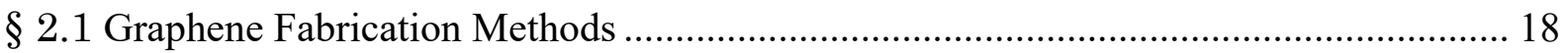

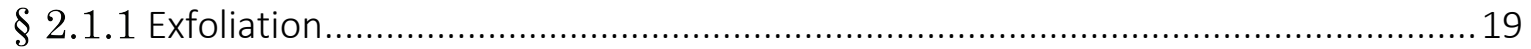

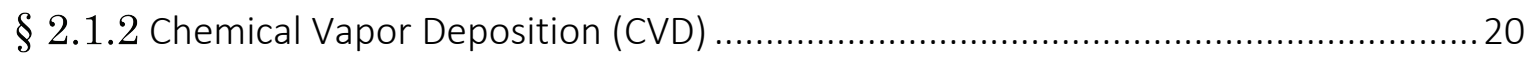

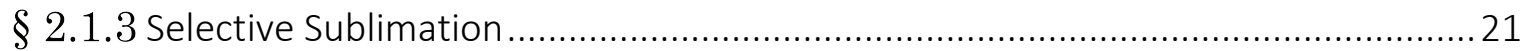

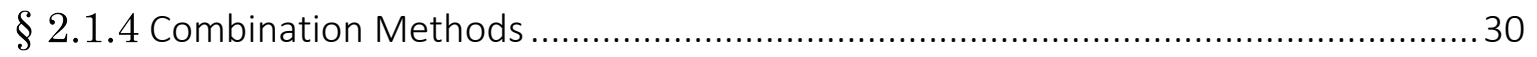

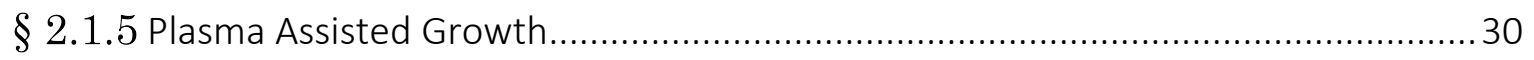

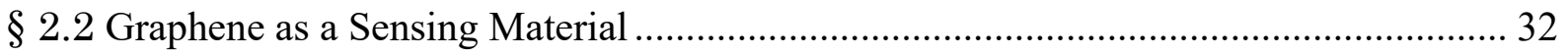

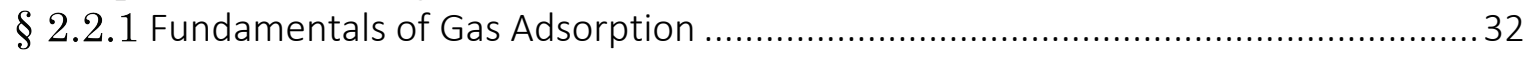

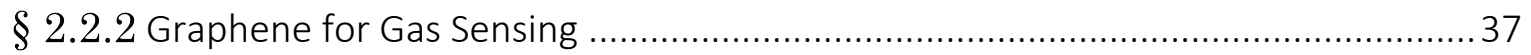

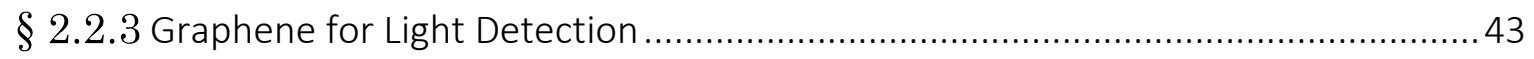

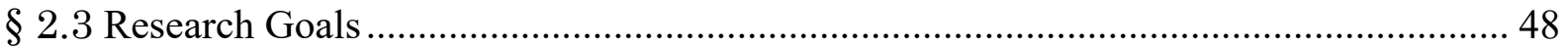

$\S 2.3 .1$ Goal 1: Investigation of the plasma assisted method of graphene production .....50

$\S 2.3 .2$ Goal 2: Application development of graphene produced via the plasma assisted

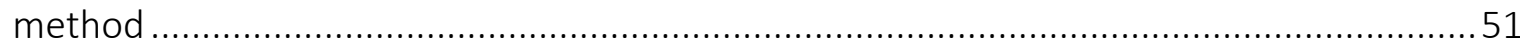

CHAPTER 3 : EXPERIMENTAL DESIGNS AND CHARACTERIZATION SETUPS ............53

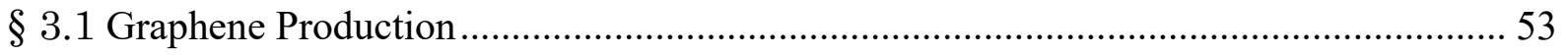

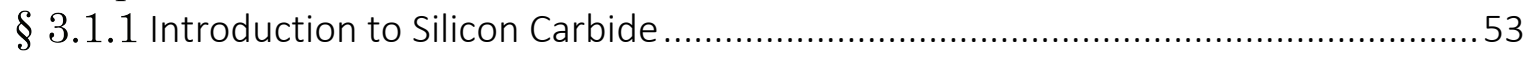

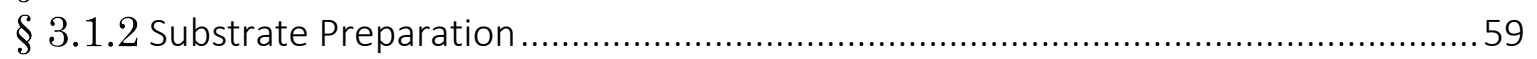

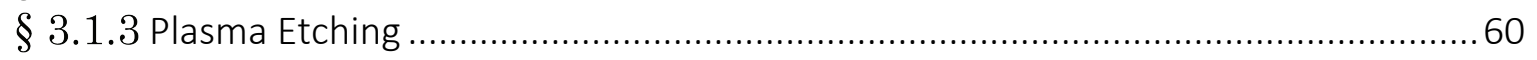

$\S$ 3.1.4 Atmospheric Pressure Rapid Thermal Annealing (AP-RTA)................................ 70

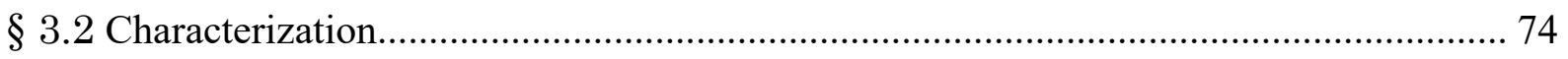

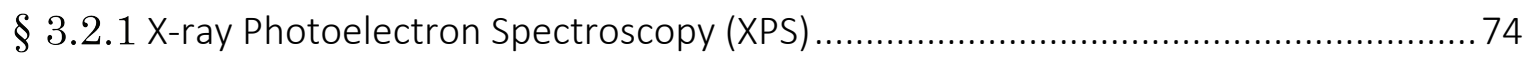

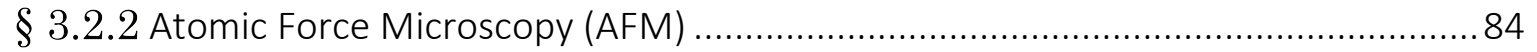

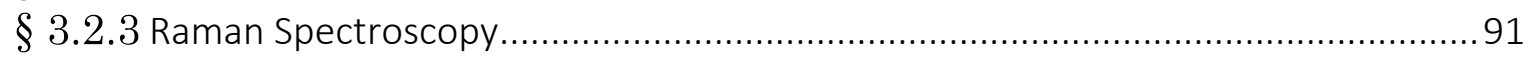

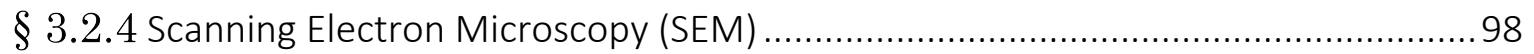

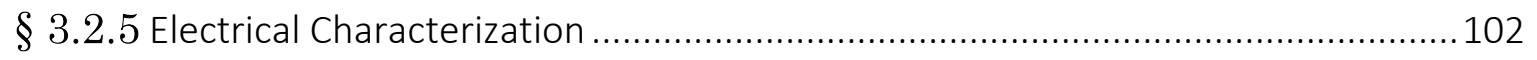

$\S 3.3$ Sensor Assembly and Packaging.......................................................................... 112 


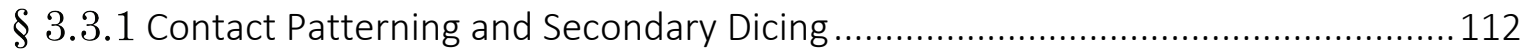

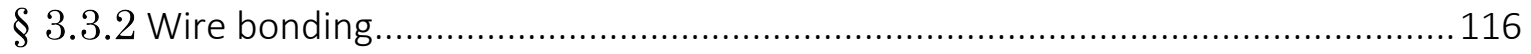

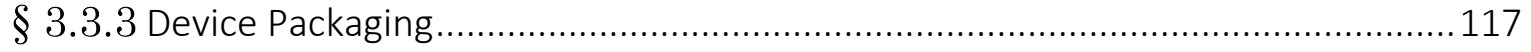

$\S 3.4$ Experimental Setup for Gas Sensing ............................................................. 125

$\S$ 3.4.1 Large Test Bed Setup .............................................................................. 126

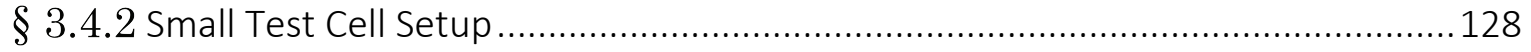

$\S 3.5$ Experimental Setup for Photo Detection............................................................. 131

CHAPTER 4 : Results AND Discussion - Graphene Synthesis, PARAMETRIC INVESTIGATION AND HYDROGEN TREATMENT ...................................................... 133

$\S 4.1$ Graphene Growth Using Standard Operating Procedure ............................................. 133

$\S 4.1 .1$ Characterization by AFM ...........................................................................137

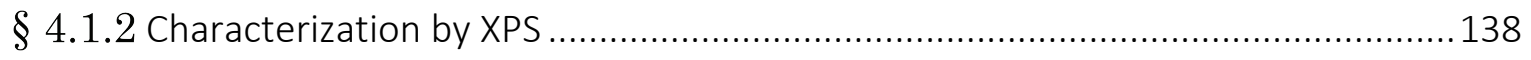

$\S 4.1 .3$ Characterization by Raman Spectroscopy …………………………………... 151

$\S$ 4.1.4 Summary of the Characterization Results from Graphene Films Produced by the

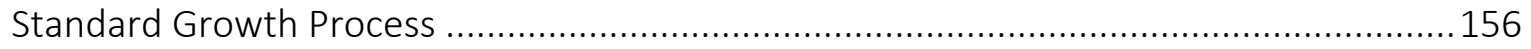

$\S 4.2$ Graphene Synthesis Parametric Investigation ........................................................... 160

$\S$ 4.2.1 Substrate Selection- Effect of SiC Polytype, Crystal Face and Doping ................. 160

$\S 4.2 .2 \mathrm{ICP} /$ RIE Etching-Effect of Plasma Power .......................................................... 167

$\S 4.2 .3 \mathrm{ICP} /$ RIE Etching-Effect of Plasma Chamber Pressure.......................................... 178

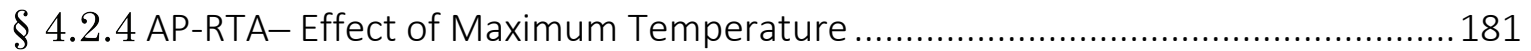

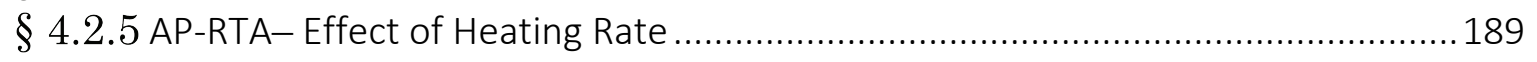

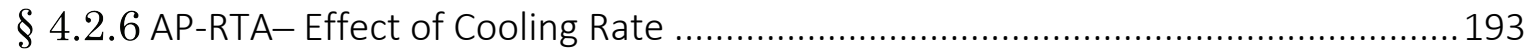

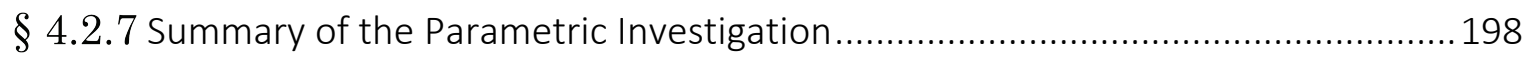

$\S 4.3$ Post Synthesis- Effect of High Temperature Hydrogen Treatment............................. 198

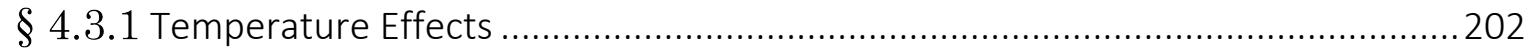

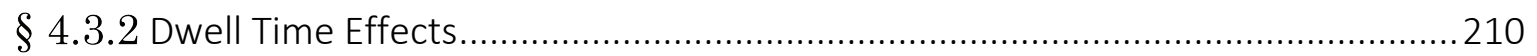

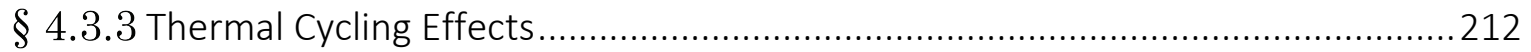

$\S$ 4.3.4 Summary of High Temperature Hydrogen Film Treatment ...............................2 214

CHAPTER 5 : RESUlTS AND DisCusSiON - EleCTRICAL CHARACTERIZATION, Temperature Behavior, Gas Testing AND Light TeSTING..............................215

$\S 5.1$ Room Temperature Electrical Characterization of Graphene-SiC Devices ................. 216

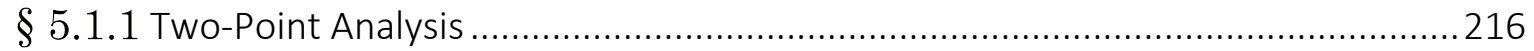

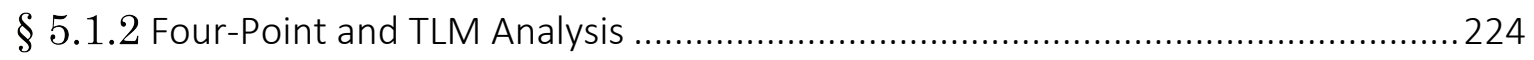

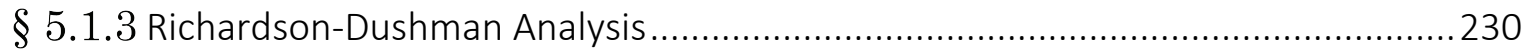

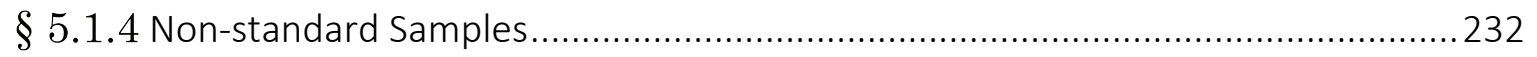

$\S$ 5.1.5 Summary of Room Temperature Electrical Characterization …….......................233

$\S 5.2$ Temperature Dependence of Device Properties ..................................................... 235

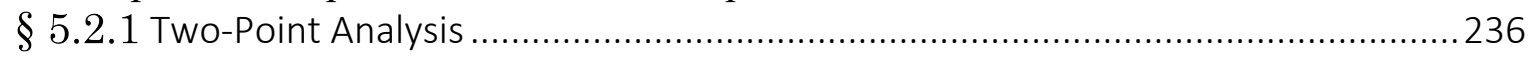

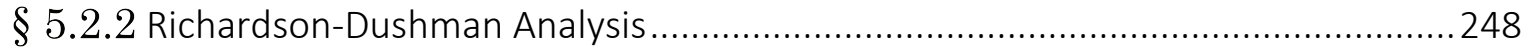

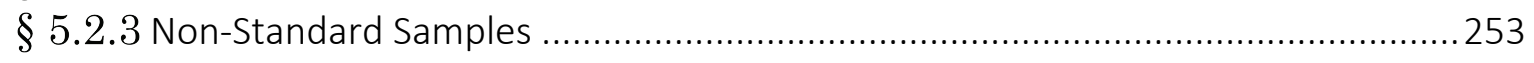

$\S 5.2 .4$ Summary of Temperature Dependent Device Properties ..................................263 


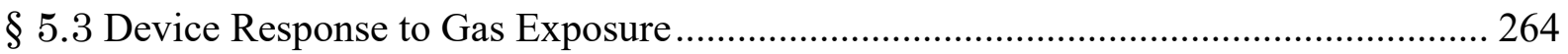

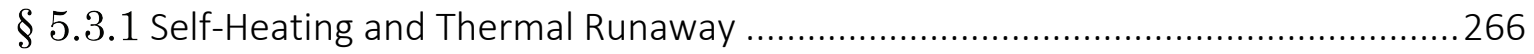

$\S 5.3 .2$ Startup Effects: Thermal and Electrical Device Equilibration.............................. 271

$\S$ 5.3.3 Inert Gas Exposure: Effect of Flow Rate, Physical Properties and Establishment of

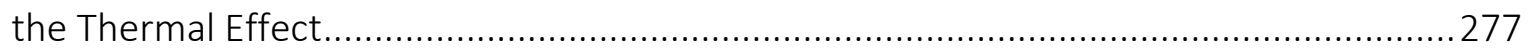

$\S 5.3 .4$ Restometric Sensing of Hydrogen Gas: Thermal and Chemical Response ...........284

$\S$ 5.3.5 Restometric Sensing of Methane and Carbon Monoxide Gas: Thermal Response

.

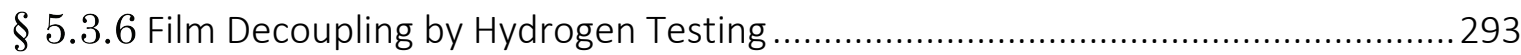

$\S 5.3 .7$ Gas Sensing Mechanism and Future Directions ................................................298

$\S 5.3 .8$ Summary of Device Performance in Gas Sensing Applications.............................304

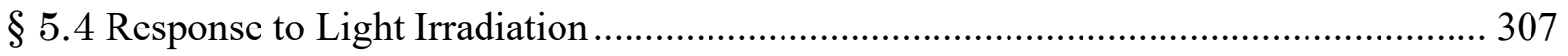

$\S 5.4 .1$ Positional Sensitivity ......................................................................................

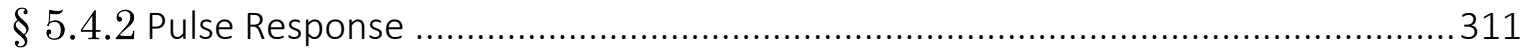

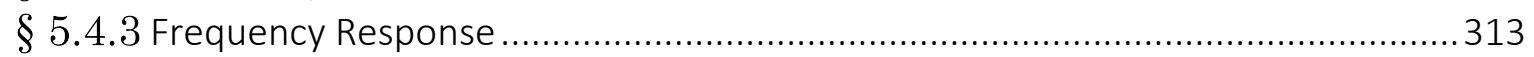

$\S 5.4 .4$ Powered Response......................................................................................

$\S 5.4 .5$ Sensing Mechanism and Future Directions ........................................................ 318

$\S 5.4 .6$ Summary of Device Performance in Photo Detection Applications ......................328

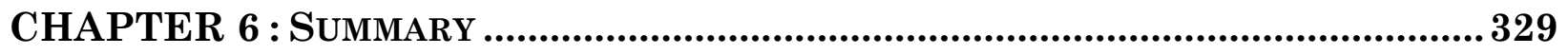

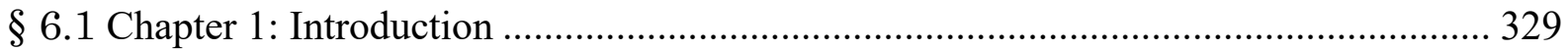

$\S 6.2$ Chapter 2: Brief Review of the Relevant Literature and Statement of Research Goals330

$\S 6.3$ Chapter 3: Experimental Designs and Characterization Setups .................................. 333

$\S 6.4$ Chapter 4: Results and Discussion - Graphene Synthesis, Parametric Investigation and

Hydrogen Treatment ......................................................................................................... 333

$\S 6.5$ Chapter 5: Results and Discussion - Electrical Characterization, Temperature Behavior,

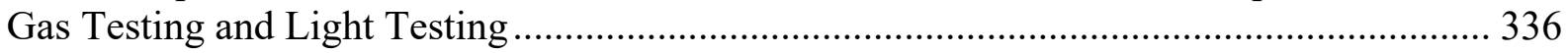

CHAPTER 7 : CONCLUSIONS AND FUTURE DIRECTIONS ..........................................

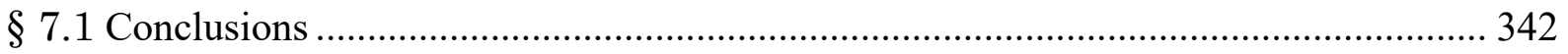

$\S$ 7.1.1 Goal 1: Process Development Conclusions .........................................................343

$\S 7.1 .2$ Goal 2: Application Development Conclusions .................................................345

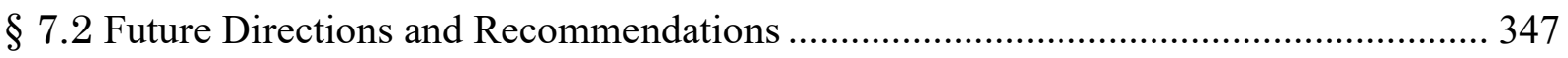

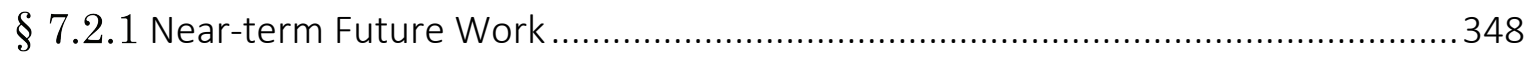

§ 7.2.2 Long-term Future Work .......................................................................... 349

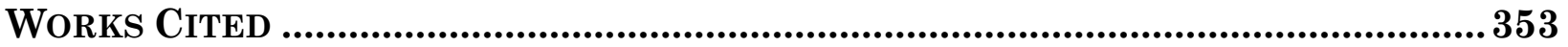

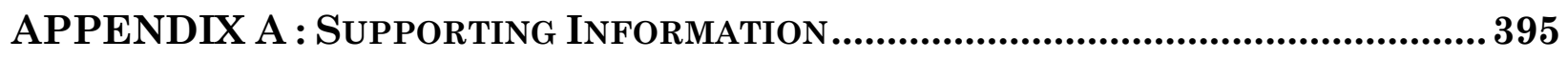

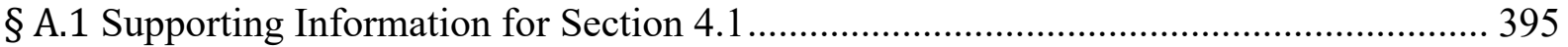

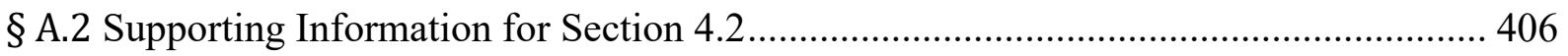

§ A.3 Supporting Information for Section 4.3 ................................................................. 416

§ A.4 Supporting Information for Section 5.3 ……….................................................. 417

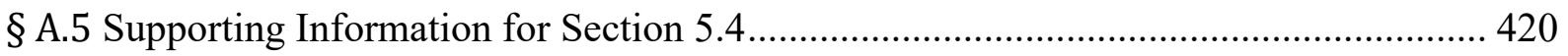




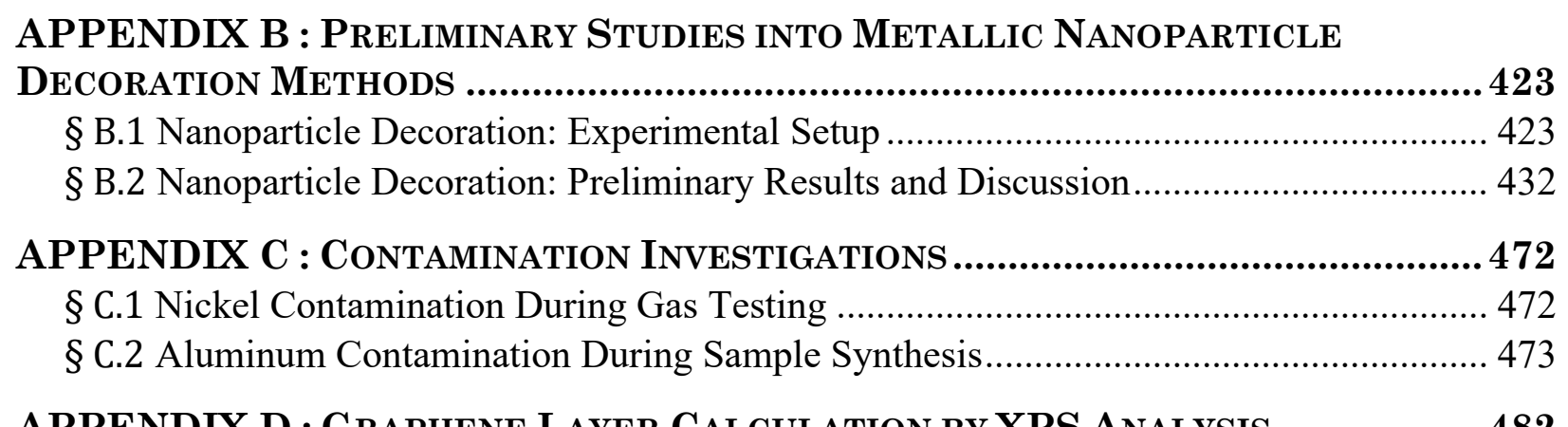

APPENDIX D : GRAPHENE LAYER CALCULATION BY XPS ANALYSIS....................482 


\section{LIST OF TABLES}

Table 1-1: Categorical list of select graphene applications.

Table 2-1: Select parameters for graphene growth on $\mathrm{SiC}$ and buffer-layer removal by hydrogen

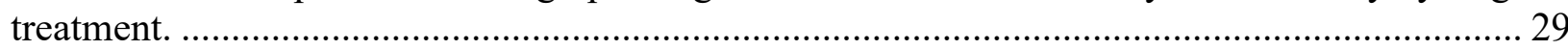

Table 2-2: Goal 1 and the related objectives. ...................................................................... 51

Table 2-3: Goal 2 and the related objectives. ................................................................ 52

Table 3-1: Select properties of silicon carbide and silicon ................................................... 55

Table 3-2: Comparison of 6H-SiC and 4H-SiC properties. ${ }^{(435)}(418)(443)(444)$.............................. 58

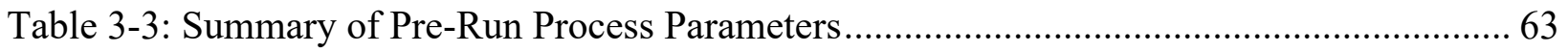

Table 3-4: The primary Raman peaks encountered in this work........................................... 96

Table 3-5: List of film parameters and related information gained from the TLM method...... 108

Table 3-6: Summary of wire bonding parameters ........................................................... 117

Table 4-1: Standard etch parameters................................................................................... 134

Table 4-2: Fitting criteria used for the $\mathrm{C} 1$ s fitting. ............................................................. 148

Table 4-3: The experimental matrix used to investigate the effects of hydrogen exposure on the

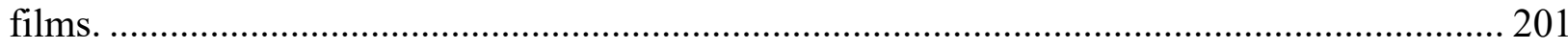

Table 4-4: Literature reports on the C1s graphene peak shifting after buffer-layer decoupling. 206 Table 5-1: Electrical parameters from a typical device constructed from the Graphene/SiC

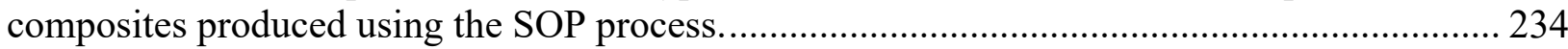

Table 5-2: Specific heat (CP) and thermal conductivity $(\mathrm{k})$ of the gases used in this study (data

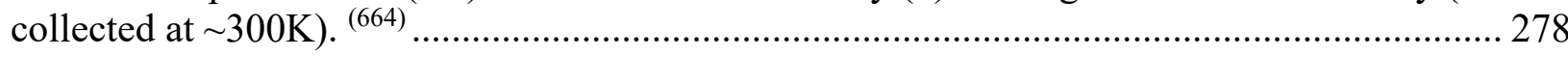

Table 5-3: Change in device parameters after high temperature hydrogen exposure................ 296

Table 7-1: Goal 1 and the related objectives. .................................................................... 343

Table 7-2: Goal 1 objectives and resulting conclusions. ................................................... 344

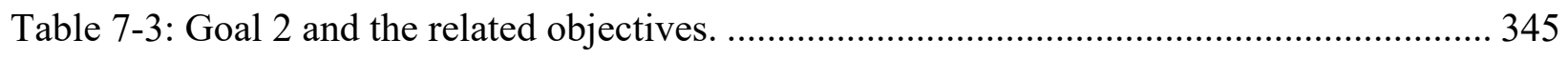

Table 7-4: Goal 2 objectives and resulting conclusions. ...................................................... 346

Table A-1: Literature explanations for the observed Graphene-SiC peak separation after buffer

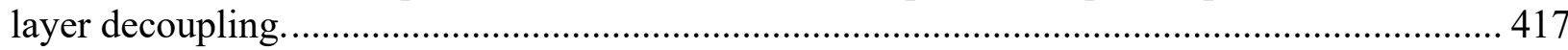

Table B-1: Optimum conditions found for the photo-nucleation of silver nanoparticles.......... 461

Table B-2: Partial sample list, arranged by nanoparticle coverage area................................ 469 


\section{LIST OF FIGURES}

Figure 1.1: The evolution of a carbon atom as it transitions from its ground state to an excited state, then finally to a sp ${ }^{2}$ hybridized state. Shown on the left are the electron spin configurations and on the right, the orbital configurations in 3D space. 2

Figure 1.2: Simple schematic of the carbon electron orbitals and how the $\mathrm{sp}^{2}$ hybridized orbitales give rise to $\sigma$ the bonds in graphene. Also illustrated is the how the lone $p_{z}$ orbital $\pi$ bonds and

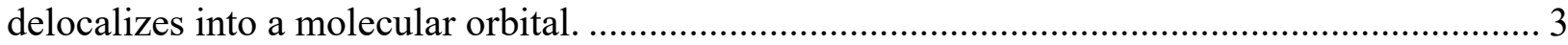

Figure 1.3: (A) The 2D crystal structure of graphene with the triangular unit cell, consisting of two inequivalent carbon atoms. The unit cell $\left(a_{\mathrm{n}}\right)$ and nearest-neighbor $\left(\delta_{\mathrm{n}}\right)$ vectors are given. (B) An illustration for the various pore sizes calculated for the graphene ring: $d_{1}$ using the $\mathrm{C}-\mathrm{C}$ bond length, $\mathrm{d}_{2}$ using a hard sphere model and $\mathrm{d}_{3}$ using the upper Van der Waals diameter. (C) Bond lengths and angles of a graphene ring as measured from the atom centers. (The widely used nomenclature can become somewhat confusing, note that the $a$ in the vector notation refers to the

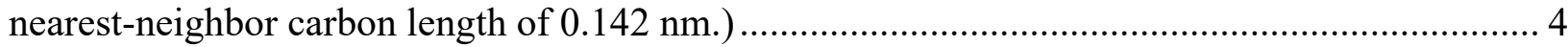

Figure 1.4: While various databases and reports provide slightly different numbers the trend is clear. The world's interest in graphene continues to accelerate. Note that only partial data for 2019 is available at the time of this writing; the dotted line indicates a projection value. 8

Figure 1.5: Tree map of graphene publications broken down by country of origin (tile area is proportional to the number of peer-reviewed publications with 'graphene' in the title.)............. 9

Figure 1.6: Tree map of graphene publications broken down by field of study (tile area is proportional to the number of peer-reviewed publications with 'graphene' in the title.)............. 9

Figure 1.7: Just some of the graphene-centric companies founded over the past few years. While their degree of success remains to be seen, this does indicate there is money to be made in graphene technology...... 11

Figure 2.1: Flow chart showing the relationships between the source of carbon and the graphene production method. The plasma assisted method has been developed and the method used in this research. Although most comparable to the sublimation method, the plasma assisted method is inherently different. 19

Figure 2.2: A schematic showing the transition from $\mathrm{SiC}$ to graphene. Initial Si sublimation forms a carbon rich buffer overlayer. Further sublimation causes the formation of a second carbon bufferlayer. The formation of the second buffer-layer allows the previous layer to relax into graphene. The simplest case for the interface between the buffer-layer and $\mathrm{SiC}$ substrate is shown, although more complex bonding arrangements have been predicted. 24

Figure 2.3: (A) Lateral schematic view of the interaction of the buffer-layer to the SiC substrate. (B) Top down view of the buffer-layer and $\mathrm{SiC}$ unit cells superimposed, illistrating the $\sim 30 \%$ missmatch and $\sim 30^{\circ}$ rotational miss match of the unit cells. The $\mathrm{SiC}$ and buffer-layer undergo AB stacking. Anotated values are take from references ${ }^{(212)}{ }^{(217)},{ }^{(218)}$ and ${ }^{(221)}{ }^{\ldots . . . . . . . . . . . . . . . . . . . . . . . . . ~} 25$

Figure 2.4: Schematic showing the use of hydrogen to passivate the SiC substrate bonds, free the buffer-layer and allow for its transition into a proper graphene layer. ................................... 27

Figure 2.5: (A) Lennard-Jones potential for physisorption and chemisorption, illustrating the difference in binding energy and binding distance and the gain in potential energy by adsorbing. (B) Simple cartoon illustrating the key difference between physisorption (weak, long range 
bonding involving intermolecular forces) and chemisorption (strong, short range bonding involving molecular orbitals). The interplay between potential plots showing the transition from physisorption to chemisorption with an energy barrier (C) and without (D). 35

Figure 2.6: The six types of adsorption isotherms as classified by IUPAC. Type I, the Langmuir isotherm, is the simplest case and is the most applicable to this work. Types II-IV more accurately describe adsorption on structural complex surfaces composed of various micro- and mesopores and their effects on adsorption. The following IUPAC citation is an excellent reference for classifying adsorption behavior and determining the underlaying phenomena at play. (293)...... 36

Figure 2.7: Cartoon depicting the fingerprinting of gasses using different sized arrays and operating in a simple trinary response mode (positive, negative or no change).......................... 43

Figure 2.8: The electronic band diagram of a metal and an n-type semiconductor (A) before contact and (B) after intimate electrical contact is achieved and electronic equilibration. The Schottky barrier presents itself as a barrier to charge injection. (C) The three mechanisms for passing the Schottky barrier. All three occur, but which mechanism dominates depends on the carrier concentration $\left(\mathrm{N}_{\mathrm{d}}\right)$ and the absolute temperature $(\mathrm{T})$, for a given system. (e is the charge of the carrier, the dielectric constant of the semiconductor $\left(\varepsilon_{s}\right), \mathrm{k}$ is the Boltzmann constant and $\mathrm{m}^{*}$ is the

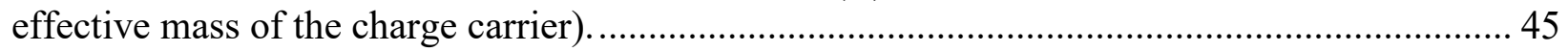

Figure 2.9: Band diagram and resulting carrier generation when (A) photons of energies between

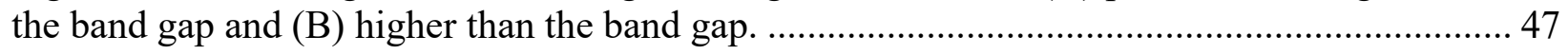

Figure 2.10: The overarching hierarchical view of this research.............................................. 49

Figure 3.1: The $6 \mathrm{H}-\mathrm{SiC}$ and $4 \mathrm{H}-\mathrm{SiC}$ crystal structure. Two unit cells are shown for each type. For $6 \mathrm{H}-\mathrm{SiC}$ the unit cell stacking sequence is $\mathrm{ABCACB}$ and is $\sim 15.119 \AA$ along the $\mathrm{c}$ axis. For $4 \mathrm{H}-$ $\mathrm{SiC}$ the unit cell stacking sequence is $\mathrm{ABCB}$ and is $\sim 10.085 \AA$ in height. Shown in the inset is the tetrahedral bonding geometry due to $s p^{3}$ hybridized bonding, the hexagonal unit cell of the SiC used in this work and the relative positions of the three possible bilayer stacking arrangments. 57 Figure 3.2: Photograph of one of the 3 inch diameter $6 \mathrm{H}-\mathrm{SiC}$ wafer used in this work, before

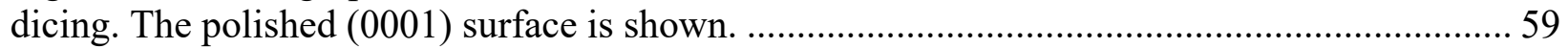

Figure 3.3: (A) The DAD3240 Disco automatic dicing tool. (B) The dicing arm with hub style diamond grit blade installed. Three water jet lines are visible. (C) A SiC wafer mounted onto adhesive tape and vacuum metal ring. (D) A $6 \mathrm{H}-\mathrm{SiC}$ wafer diced into $1 \mathrm{~cm}^{2}$ squares; after graphitization these were layer diced into 16 smaller $2.5 \mathrm{~mm}^{2}$ samples using the same dicer shown here. (E) An example of a slightly different dicing pattern. Shown is a $6 \mathrm{H}-\mathrm{SiC}$ wafer diced into samples measuring $10 \mathrm{~mm} \times 2.5 \mathrm{~mm}$. 60

Figure 3.4: (A) The Trion Minilock-Phantom iii ICP/RIE system to perform the etching step. (B) A view of the interior or the main process chamber where the plasma is formed......................... 62

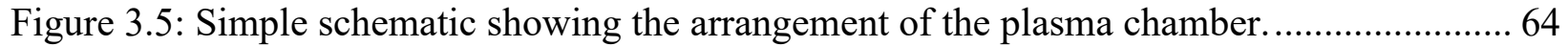

Figure 3.6: Schematic of the plasma system used in this work.................................................. 67

Figure 3.7: (A) Schematic of the chamber and the potential of the sample/substrate. The RIE RF field is offset by the DC self-bias as it develops. (B) The plasma potential along the $\mathrm{Z}$ direction (vertical axis) of the chamber, from the shower head to the sample chuck. (C) The plasma potential

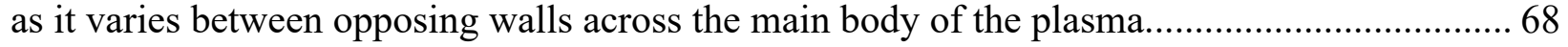

Figure 3.8: Views looking into the main chamber as seen through the side viewport, taken at different steps during the process. (A) The $\mathrm{O}_{2}$ cleaning step, used to clean the chamber of material from previous runs. (B) The $\mathrm{CF}_{4}$ conditioning step, used to bring the chamber behavior into a 
pseudo-steady state. (C) The etch step. Several samples can be seen undergoing the etch. (D) Close-up of the etch step, showing the samples, the main plasma and the ion sheath (dark band), due to the DC bias. 69

Figure 3.9: (A) Schematic of a typical AP-RTA tube, used to house the sample during the APRTA step. (B) Real world picture of a tube used in this work... 70

Figure 3.10: (A) The Thermo Scientific Lindberg Blue M furnace shown with quartz RTA tube (supported by a lab jack). This furnace was used for some sub $1000^{\circ} \mathrm{C}$ experiments. (B) The high temperature $\mathrm{C} \& \mathrm{M}$ furnace with alumina $\left(\mathrm{Al}_{2} \mathrm{O}_{3}\right)$ refractory tube used for a plurality of sub $1000^{\circ} \mathrm{C}$ experiments and all experiment which required temperatures $>1000^{\circ} \mathrm{C}$. Pictured is the furnace at $\sim 1500^{\circ} \mathrm{C}$. .71

Figure 3.11: (A) A simple schematic of the setup for hydrogen annealing. (B) A picture of a sample loaded into the quartz injector tube. (C) A picture of the gas inlet and outlet plumbing designed for the total containment of hydrogen annealing gas. (D) A picture showing the geometry of the injector-sleeve arrangement and a sample being loaded into the injector. 73

Figure 3.12: The solid-state interpretation of the origins of the XPS spectra and a graphical representation of the energy balance preformed to calculate the binding energy (BE). The equation is solved in terms of the kinetic energy, which in practices is the measured quantity. The $\Phi_{\text {Spec. and }}$ $\mathrm{h} v$ are known and are constant. Rearrangement of the equation show the simple calculation of the binding energy: $\mathrm{BE}=\mathrm{h} v-\mathrm{E}_{\mathrm{K}}-\Phi_{\text {Spec. }}$. Electrons are counted by the spectrometer by what is esentualy an amp meter. Electrons lost by the sample are made up by ground electrons........... 75 Figure 3.13: Simplified schematic of an XPS setup illustrating the relative arrangement of the photon source, sample and the hemispherical analyzer. The filtering of photoelectrons of the hemispherical analyzer by kinetic energy $\left(E_{K}\right)$ is also shown. 77 Figure 3.14: (A) The generic arrangement of XPS. The large volume of material driven into photoelectron excitation is shown. The large protuberance is due to scattering of X-ray within the sample. The comparatively small effective analysis volume is also shown; the surface is sampled to a greater extent than the bulk. (B) The surface sensitive nature of low angle detection is illustrated- small takeoff angles collect photoelectrons from a shallow escape depth. This effect is derived from (C) which shows the attenuation of photoelectrons as a function of distance traveled in terms of the number of IMFPs, (a decrease of 1/e per $\lambda$ ). (D) How angle resolved measurements are achieved in the system used in this work. High angle measurements collected both surface and bulk photoelectrons, while low angle measurements collected primarily surface photoelectrons. The sample is tilted, changing the angle to the detector, while the angle between the detector and

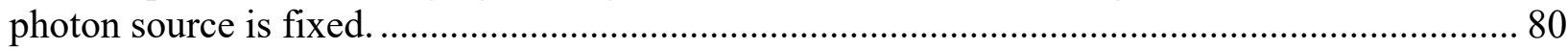

Figure 3.15: The universal curve for the inelastic mean free path $(\lambda)$ as a function of photoelectron energy. The data points correspond to various scattering media. Note that most information gained from XPS is generated from photoelectrons with energies above $10 \mathrm{eV}$, thus XPS is highly surface sensitive (i.e. IMFP $<10 \mathrm{~nm}$ ). This chart has been reproduced using data from reference (731). 82 Figure 3.16: A picture of the PHI 5700 VersaProbe XPS analysis tool used in this work. The main components have been labeled. Blowout: a view through the sample inspection window showing the X-ray source and detector arrangement. The relative position of the articulating sample stage can be manipulated to collect data at various takeoff angels 83

Figure 3.17: A qualitative view of some of the primary forces involved in AFM. At sufficiently large distances, effectively no atomic forces are felt. As the distance is decreased the effective forces felt are indicated by the blue curve labeled total force (Lennard-Jones potential). Points A 
and $\mathrm{B}$ on the total force curve are not optimum operational positions because the detected forces can be interpreted in multiple ways. Point " $C$ " is ideal due to the large forces involved and simple interpretation of the data. 85

Figure 3.18: (A) Top down view of the typical left-to-right and top-to-bottom raster pattern the tip takes across the sample surface. (B) Side view of the AFM tip showing the chip body, cantilever and tip..... 86

Figure 3.19: A simple illustration of how a surface feature deflection is detected and converted into an image: The tip encounters a feature, which causes a deflection of the tip due to the atomic forces, resulting in a deflection of the reflected laser beam position onto the photodetector. The photodetectors signal is then amplified, digitized and processed resulting in a $2 \mathrm{D}$ false color pixilated image. 87

Figure 3.20: (A) When the angle between the tip edge and feature edge is large the tip can accurately track across fine surface features. (B) When a tip with a large radius of curvature is used, or high aspect ratio features are mapped, artificial tip induced broadening effects can occur. 88

Figure 3.21: (A) The principle of phase images. The attractive or repulsive forces felt between the tip and the sample will drive the actualy occilation of he tip off of the drive signal. The diffrence between the two is the phase angle shift. This parameter is used to generate the phase image. (B) The principal of amplitude images. Here, the amplitude dampening is used to generate the image. The tip is kept at a consistent height, therefore when the tip encounters a feature the amplitude of the tip occilation is dampened. A complementary mode of opearation is posible where the amplitude is set and the height of the tip is continuously modulated to maintain a consistent amplitude (this is not illistrated here). 90

Figure 3.22: (A) The Agilent 5500 SPM AFM. (B) The AFM assembly. (C) The underside of the AFM showing the scanner head. The laser light reflecting from the AFM tip can be seen. (D) An AFM chip mounted in the AFM head. The tip can be seen in the zoomed in insert. 91

Figure 3.23: A schematic depicting Rayleigh scattering (I). The excitation laser light excites the system into a virtual state (II). The system then collapses back into the ground state (III) which spontaneously causes an emission of a photon (IV) with an energy equal to the energy difference between ground and virtual states. 92

Figure 3.24: Schematic of three distinct types of scattering processes. Anti-Stokes, where the incident laser excites a pre-excited system resulting in a higher frequency photon emission. Rayleigh, the most common scattering process, where the emitted photon is equal frequency to the excitation laser. Stokes, where the system does not fully collapse into the ground state resulting in a lower frequency photon. 93

Figure 3.25: Illustration of the type of qualitative data gained from a simple analysis of a Raman peak. 95

Figure 3.26: Real-space atom vibrations which result in the D, 2D and G peaks. The D and 2D always occur, however the D peak does not appear in the Raman spectra unless the ring is near a defect site or graphene edge. 96

Figure 3.27: A picture of the Renishaw micro-Raman inVia spectrometer during operation...... 98

Figure 3.28: A simple schematic of the generic principle of SEM opeation. 99 
Figure 3.29: (A) The HitachiS-4700 SEM and (B) the JEOL LSM 7600F SEM. The JEOL SEM was located in a Class 10,000 cleanrooom enviroment and housed inside a mechanl isolation chamber for vibration reduction. 101

Figure 3.30: The so-called Ohm's Wheel, showing the various relationships between current, voltage, power and resistance, as described by Ohm's law..... 103

Figure 3.31: Conceptual schematic for the (A) two-point probe arrangement and (B) the four-point probe arrangement used in this work. (C) The actual probe and SMU arrangement used in this work using 3 SMUs and a dedicated ground terminal. The four-point probe arrangements use of a high impedance voltage measurement device means that many elements of the circuit can be

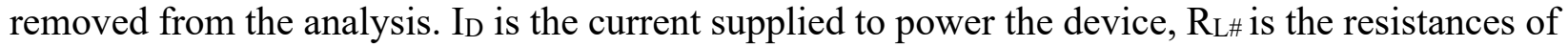
the test leads and $\mathrm{R}_{\mathrm{C} \#}$ are the contact resistances. Note that SMU1 acts as the current source, and SMU2 and SMU3 act as the HIDs to measure voltage. In reality, the setup is slightly more complex with several more unions and contact points. For clarity, these are not shown and can be simply lumped in with the terms shown. 105

Figure 3.32: (A) A TLM arrangement. The distance between pads $\left(\ell_{n}\right)$ and the pad width (W) are indicated. Current is forced across the pattern and the voltage is measured between two inner pads. (B) Graphical method for determining sheet resistance; resistance is plotted against pad spacing and a liner line is struck. The slope divided by the contact width is equal to the sheet resistance in $\Omega / \square$. 106

Figure 3.33: (A) The more complete TLM arrangement, which allows for the extraction of contact parameters as shown in (B). Namely, the contact resistance $\left(\mathrm{L}_{T}\right)$ and the transmission length $\left(\mathrm{L}_{T}\right)$. 107

Figure 3.34: Two pitfalls of the TLM. (A) A pattern not sized to the dimensions of the sample resulting in the current path length not fully accounted for, leading to artificially low resistivity values. (B) An illustration of the impact of a substrate which is not fully electrically insulating. This also leads to artificially low resistivity values. 109

Figure 3.35: Graphical interpretation of the modified Richardson-Dushman analysis employed in the analysis of the graphene devices. From this analysis the Schottky barrier height $\left(\Phi_{\mathrm{B}}\right)$ and ionized donor density or charge carrier concentration $\left(\mathrm{N}_{\mathrm{d}}\right)$..... 111

Figure 3.36: (A) The KJL e-beam evaporator used in this work. (B) The view of the main chamber, as seen through the view port during a deposition. In this specific run, five samples were concurrently processed. 112

Figure 3.37: (A) Example of one of the shadow masks used for the $1 \mathrm{~cm}^{2}$ samples. (B) A graphinated sample with deposited bimetallic electrical contacts ready for secondary dicing. (C) A diced $1 \mathrm{~cm}^{2}$ sample. Each square was then packaged into a sensing platform. 113

Figure 3.38: (A) SEM image of $2.5 \mathrm{~mm}^{2}$ with complete parallel contact pattern. The bright areas correspond to the metal contacts. (B) 3D projection of the same sample (arbitrary $\mathrm{Z}$ value). (C) Higher magnification of the center set of parallel contacts. (D) False color 3D projection of the same image. The substrate contrast seen in $(A, B)$ is due to a legacy processing step (pattered plasma oxidation step) which was not used extensively in this work. 114 Figure 3.40: (A) SEM image of $2.5 \mathrm{~mm}^{2}$ with complete interdigitated contact pattern. The bright areas correspond to the metal contacts. (B) 3D projection of the same sample (arbitrary $\mathrm{Z}$ value). (C) Higher magnification of the center set of the interdigitated contacts. (D) False color 3D projection of the same image. 115 
Figure 3.39: Magnified image of a sample used for TLM testing. 115

Figure 3.41: (A) The West Bond 74776E ultrasonic wire bonder wire. (B) A close up view of the tool head assembly; also shown is a gold thread, bonded to a sample and despoiling, in preparation for the crescent termination bond and completion of the jumper connection 117

Figure 3.42: (A) The MK I high temperature sensor package used in large test bed experimental setup. The graphinated sample and wire bonds can be seen. (B) The sensor package was mounted to the end of a two-hole ceramic rod. Electrical contact was made from the package to the testing equipment via platinum wires housed within the rod. The platinum wires from the package were braided to the platinum wires from the rod to ensure intimate electrical contact. NOTE: For scale, the sample is $2.5 \mathrm{~mm}^{2}$. 118

Figure 3.43: (A) A close up view of the MK I high temperature packaging showing the wire bonds from the sample to the package. In this instance, the bonds were made to $\mathrm{Ni} / \mathrm{Au}$ coated stainless steel washers. (B) A schematic showing how the coated washers were held in place and how the package was anchored to the ceramic rod. NOTE: For scale, the sample is $2.5 \mathrm{~mm}^{2}$. 119

Figure 3.44: (A) A back-to-back duel sensor arrangement version of the MK II design. (B) A magnified view of two sensors mounted on the same package for simultaneous testing. (C) A magnified view showing the bonding between the sensor and the flattened and coated package wires. NOTE: For scale, the sample is $2.5 \mathrm{~mm}^{2}$. 120

Figure 3.45: (A) A MK II package design which had only one sample. This particular package uses borosilicate glass. (B) The effect of high temperature exposure on the glass; this led to warping of the substrate and bond failure (arrow). (C) A failed MK II package design using quartz; the high temperature cement bonded poorly to the quartz leading ultimately to package failure. NOTE: For scale, the sample is $2.5 \mathrm{~mm}^{2}$. 120

Figure 3.46: (A) A version of the MK III design. (B) Due to poor bonding to the flattened wire, detachment of the ultrasonic bonds can be seen (arrow). (C) The connection of the package to the ceramic rod is shown. The prongs from the rod are inserted into the holes of the package which contain the flattened package wires. In this way, electrical contact is achieved. NOTE: For scale, the sample is $2.5 \mathrm{~mm}^{2}$. 121

Figure 3.47: (A) The modified MK III package design with improved electrical connection. (B) A magnified view of the connection between the Ni package wire and the gold thread. (C) Magnified view of the sample and hook connections. The sample is mounted with high temperature cement to the flattened side of the ceramic rod. NOTE: For scale, the sample is $2.5 \mathrm{~mm}^{2}$. 122 Figure 3.48: (A) The CD200SPM Micro Spot Resistance Welder used to spot weld the RTD and heater to the TO pins. (B) The TO body with RTD and heater element attached. (C) Magnified view of a completed device package. 123

Figure 3.49: (A) Thermal stability test of a completed device for use in the small gas test cell setup. This device is pictured operating at $\sim 800^{\circ} \mathrm{C}$. (B) The same device from a different angle, showing the wire bonds remain intact at high temperature. (C) Magnified view of the device showing the graphinated sample. Both the wire bonds and the deposited Ti/Au contacts can be seen......... 124

Figure 3.50: The MK0* package design. This is a simplified version of the MK 0 design and does not included the heating element and RTD. This design used in the small test cell and was useful for the rapid fabrication of devices for simple testing. 125 
Figure 3.51: A simplified schematic of the gas flow system. Both the large test bed and the small test cell were part of the same system. Other aspects of the system involving the RTA system and alternative arrangements of the large test bed are not shown. 125

Figure 3.52: A) A simple schematic of the large test bed setup. This design preheats the incoming gasses and maintains the entire test device at the desired temperature. B) The sensor probe and gas inlet assembly prior to insertion. (C) The complete sensor probe assembly fully inserted into the furnace at temperature. The cooling line is not assembled in this picture. 126

Figure 3.53: (A) Increase in the series resistance of the MK II design as a function of temperature. (B) Simple schematic of the circuit. (C) Effective resistance as a result of the various contributors to the series resistance in the MK I - MK III designs. Note that this does not including the actual device package, wire bonds, or $\mathrm{Ti} / \mathrm{Au}$ contact trace. These unaccounted-for contributions are estimated to be on the order of $\sim 1 \Omega$ at room temperature. 128

Figure 3.54: The small test cell, as indicated by the arrow and circle annotation, with an installed sensor device package. The friction fitted saber type connectors can be seen at the top of the test cell. In the background, some of the plumbing and mass flow controls can be seen. 129

Figure 3.55: A closeup of the small test cell with accompanying exploded view schematic, showing the gas flow pattern and how the device is installed within the cell. Note that the $1 / 4$ inch line protrudes past the exhaust port so that incoming gas mixes and impinges direction on to the sensor before it is exhausted. 130

Figure 3.56: (A) IV curve collected from the MK0 design, not including the film. (B) Simple schematic of the circuit. (C) Effective resistance as a result of the various contributors to the series resistance in the MK I - MK III designs. 131

Figure 3.57: (A) The photo detection setup. (B) The laser spot can be seen on an empty sample package. (C) A simplified schematic of the laser setup. 132

Figure 4.1: AFM analysis on an as-received $6 \mathrm{H}-\mathrm{SiC}$ wafer. A) The atomic steps can clearly be seen. The red dashed line shows the locations of a line scan across severial atomic steps. A step edge protrusion, marked by the blue arrow, can be seen. B) Height profile for the line scan shown in (A) with plane-leveled 3D projection of (A). C) The same data, but facit-leveld. Using the line scans, a miscut angle of $\sim 0.33^{\circ}$ is calculated. 133

Figure 4.2: The -DC bias developed on the sample chuck during a standard etch. A log best fit is shown to aid the eye. 135

Figure 4.3: Step produced using a partial shadow mask and standard etch parameters. Based on this profile and the powered etch time of 12 minutes, The $6 \mathrm{H}-\mathrm{SiC}(0001)$ etch rate is $\sim 233 \mathrm{~nm} / \mathrm{min}$ for the standard etch parameters. 136

Figure 4.4: Representative heating profile for the standard AP-RTA process. Heater temperature and sample temperature are both shown. Sample heating rate was $\sim 200{ }^{\circ} \mathrm{C} / \mathrm{min}$, cooling rate was $\sim 75^{\circ} \mathrm{C} / \mathrm{min}$ and max sample temperature was $990^{\circ} \mathrm{C} \pm 2 \%$. 137

Figure 4.5: (A) The as-received $6 \mathrm{H}-\mathrm{SiC}(0001)$ surface showing a clean, atomically stepped surface. (B) The same surface after undergoing the standard CF4 etching treatment. (C) The same surface after the AP-RTA process. Each image is $5 \mu \mathrm{m}$ X $5 \mu \mathrm{m}$. Scale bars are $1 \mu \mathrm{m}$................ 137 Figure 4.6: (A) Survey spectra collected from the same sample at various stages in the synthesis process (as-received $6 \mathrm{H}-\mathrm{SiC}$, post $\mathrm{CF}_{4}$ etch and post AP-RTA). All major peaks have been identified. Unlabeled features are due to various energy loss processes associated with the labeled photoelectron emission lines. (B) Atomic concentrations calculated from the survey spectra. (C) 
High resolution scans of the C1s peak at each stage in the synthesis process. The C-Si reference peak at $282.5 \mathrm{eV}$ is marked to guide the eye. 139

Figure 4.7: Deconvoluted C1s XPS spectra obtained using (A) the bulk and (B) the surface analysis modes. (C) Peak parameters for each fit and the percent area change of each peak as the technique transitions from a bulk sensitive mode to a surface sensitive mode; a decrease in peak area indicates a more sub-surface species and an increase indicates a more terminal-surface species. 141

Figure 4.8: The range of binding energy values reported for the S1, S2 and Graphene peaks. The red dashed arrows indicated where the values assigned in Figure 4.7 (A) for this work fall relative to what others have reported for the thermally driven selective sublimation process for graphene growth which results in buffer-layer formation. Although on the high side, values for this work fall within the range of reported values. (Note that the as-reported values have all be universally shifted to what is shown such that the $\mathrm{SiC}$ peak appears at a binding energy of $282.5 \mathrm{eV}$, so that they are directly comparable between each other and to this work.). 143

Figure 4.9: An idealized view of the carbon film with respect to the origins of the XPS S1, S2 and $\mathrm{G}$ (graphene) peaks. The atomic spacings have been labeled consistent with the literature review in Chapter 1 and 2. Atom sizes are to scale; bond distances are approximately to scale. 144

Figure 4.10: A plot showing the number of carbon overlayers vs the $\mathrm{IG}_{\mathrm{G}} / \mathrm{Isic}_{\mathrm{sic}}$ ration. The highlighted data point corresponds to the calculated value obtained by analysis of the data shown in Figure 4.7(A). Details of this analysis and attenuation model are given in APPENDIX D. 147

Figure 4.11: Parameters extracted from the analysis of survey (SUR) spectra and the C1s region from 23 randomly selected film produced using the standard synthesis process. (A) Violin and box plot key. (B) The number of carbon layers as calculated using the method depicted in Figure 4.10. (C) The percentage of carbon atoms within the film in a chemically defective state, assuming S2 is a pure buffer-layer peak, on a per layer basis (total percent defects divided by the calculated number of carbon layers). (D) The percent of carbon atoms in the carbon overlayer which are in a buffer-layer state $\left(\mathrm{S} 1+\mathrm{S} 2 / \mathrm{G}+\mathrm{S}_{1}+\mathrm{S}_{2}+\mathrm{D}_{3}+\mathrm{D}_{4}+\mathrm{D}_{5}\right)$. (E) The distributions binding energies for each peak within the fitted $\mathrm{C} 1 \mathrm{~s}$ region. The data points within the same dataset are linked; no correlation is observed. (F) Distribution of the atomic concentration as calculated from the survey data $(1000 \mathrm{eV}-0 \mathrm{eV})$. Data points within the same dataset are linked. Inset, corollary plot showing that lower oxygen concentrations correlated to higher carbon concentrations. 149

Figure 4.12: (A) The raw Raman signal from a graphinated sample produced using the standard process (TOP), Raman spectra from a as-received $6 \mathrm{H}-\mathrm{SiC}$ sample (MIDDLE) and the result of the scaled subtraction of the two (BOTTOM) revealing the contributions of the carbon overlayers to the raw signal from the graphinated sample. (B) The fitting results from fitting the subtracted spectrum with a 6-peak fit.

Figure 4.13: Statistical analysis of the fitted Raman data from 16 randomly selected samples produced using the standard method. Datapoints within the same dataset are linked; no correlation was observed between any measured parameter. (A) Range of center peak values for each fitted peak. (B) Peak areas for each fitted peak. (C) FWHM values for each fitted peak. (D) The D/G $[\mathrm{D} /(\mathrm{G} 1+\mathrm{G} 2)$ in this case $]$ ratio and the $\mathrm{G} 1 / \mathrm{G} 2$ ratios. 155

Figure 4.14: (A) Simple reaction coordinate diagram showing the generic steps in the standard synthesis process. (B) Schematic showing the planarization of the atomically stepped surface due to the aggressive etching action of the plasma.. 156

Figure 4.15: (A) Raman spectra collected from films grown from both polar surfaces on three different substrate types, all using the Standard Synthesis parameters. The spectra are largely 
indistinguishable indicating a strong coupling of the vDOS across all samples, regardless of substrate choice. (B) The $\mathrm{D} /\left(\mathrm{G}_{1+2}\right)$ ratios. Regardless of substrate, the silicon face appears to produce films with a slightly higher $\mathrm{D} / \mathrm{G}_{1+2}$ ratio, indicating these films contain slightly more defect. However, all points fall largely within the statistical bounds calculated from the Standard Synthesis. The black colored data (top) labeled $6 \mathrm{H}-\mathrm{SiC}(\mathrm{Si}-)$ in $(\mathrm{A}, \mathrm{B})$ was collected from a Standard Sample process around the same time as the other samples included in the figure. ... 161 Figure 4.16: The C1s XPS spectral region, collected from films produced using the Standard Process but grown from various $\mathrm{SiC}$ polytype $(6 \mathrm{H}-, 4 \mathrm{H}-)$, crystal faces $(\mathrm{C}-, \mathrm{Si}-)$ and doping (ntype, semi-insulating). Note that the $\mathrm{SOP}$ process uses $6 \mathrm{H}-\mathrm{SiC}$ Si-face and is naturally n-type doped. 163

Figure 4.17: Parameters calculated from XPS analysis. (A) The number of carbon layers. (B) The percentage of carbon atoms involved in a chemical defect on a per layer basis. (C) The percentage of carbon atoms participating in the buffer-layer. 164

Figure 4.18: The relationship between ICP and RIE (read) powers to the DC bias developed on the sample chuck, as read by the tool. The diamond data point corresponds to the parameters used during SOP process (i.e., samples produced here are SOP samples). Higher RIE powers lead to larger DC biases. Contour lines appear at every $100 \mathrm{~V}$ increment. The area between points has been spline interpolated. The black regions correspond to ICP/RIE combinations where a plasma could not be ignited. Not all points which were ignitable resulted in a stable plasma. 169

Figure 4.19: (A) The variation in the number of carbon layers is seen as a function of ICP power and the DC self-bias. (B) The percentage of carbon atoms in the film which are participating in the buffer-layer. 170

Figure 4.20: The relationship between the applied RIE power and the developed -DC self-bias. The dashed line is a simple polynomial best fit line to guide the eye. The error bars represent the bound of values the DC bias fluctuates through for a given run. 172

Figure 4.21: (A) Stacked XPS of the C1s region collected at different RIE powers (and similarly, -DC biases). All spectra have been normalized to the $\mathrm{SiC}$ peak intensity. The arrow indicates the direction of increasing RIE power / -DC bias. The black spectrum corresponds to a sample produced using the standard process parameters. (B) Stacked Raman spectra collected from the same samples. (C) Plot showing how the number of carbon layers and percent carbon participant in the buffer-layer changes a function of -DC bias. The distinct zones are discussed: (I) where chemical etching dominates (II) the synergistic region and (III) where non-discriminant physical etching dominates. (D) Plot showing how the D/G ratio and the percent carbon involved in a chemical defect state vary as a function of -DC bias. Arrows indicated the corresponding axis. Error bars in (C) and (D) are the standard deviation value of the DC bias for each run........... 173

Figure 4.22: (A) Plot showing the growth in the FWHM value of the D peak, while the magnitude remains constant. (B) Plot of the D peak location show a shift to higher wavenumbers with increasing -DC bias. Error bars are the standard deviations values of the DC bias for each run. 176

Figure 4.23: The relationship between the chamber pressure and the developed-DC self-bias. Note that the ICP and RIE power setpoint is $600 \mathrm{~W}$ ICP and $300 \mathrm{~W}$ RIE (standard process powers) in all cases. The data point corresponding to the standard process chamber pressure is diamond shaped and highlighted. A best simple 3rd order polynomial best fit line has been added to guide the eye. Pressure about $125 \mathrm{mT}$ and below $10 \mathrm{mT}$ could not be maintained. Error bars are the standard deviation values of the DC bias for each run. 179 
Figure 4.24: The effect of chamber pressure on the number of carbon layers produced and the percentage of carbon in the film participating in buffer-layer bonding. Error bars (small and largely obscured by the data points) are the standard deviation value for the pressure reading for each run.

Figure 4.25: (A) Real data point temperature profiles. The furnace setpoint was $1400^{\circ} \mathrm{C}$. The samples were withdrawn from the furnace once the desired temperature was reached. (B) Equations which approximately describe the heating and cooling rates for all samples where TSAMPLE is the temperature of the sample, TMAX is the maximum temperature seen by the sample and $t$ is time. Time is in seconds and temperature is in ${ }^{\circ} \mathrm{C}$. (C) The maximum temperature seen by each sample in (A) and the approximant time taken to reach that temperature. 182

Figure 4.26: (A) Stacked XPS spectra of the C1s region. The arrow indicates increasing AP-RTA temperature. (B) The number of carbon layers and the fraction of carbon in the layers associated with the buffer-layer are plotted. At very high temperatures, almost all the carbon is removed from the surface and that which remains is in the form of a buffer-layer. Silicon sublimation may also be partially responsible for the sharp increase in buffer-layer carbon after above $1200^{\circ} \mathrm{C}$. (C) The atomic concentration of fluorine and oxygen. Above $\sim 850^{\circ} \mathrm{C}$, all detectable fluorine remaining from the etch is removed. (D) The fraction of carbon in the film in a chemical defect state. The line shape appears to be a confluence of the lines in (C), indicating the defects to be primally fluorine based at low temperatures which transition to oxygen at high temperatures. (B, C, D) Arbitrary best fit lines are included as the dashed lines to glide the eye.............................. 184

Figure 4.27: (A) Normalized and stacked Raman spectra. The arrow indicates increasing annealing temperature. Samples exposed to $1400^{\circ} \mathrm{C}$ do not generate a meaningful Raman signal. (B) The D and $\mathrm{G}$ peak centers as a function of temperature. (C) The full width at half max (FWHM) of the $\mathrm{D}, \mathrm{G} 1+\mathrm{G} 2$ peak with the $2 \mathrm{D}$ peak area as a function of annealing temperature. Arbitrary best fit lines are included as dashed lines to guide the eye. 186

Figure 4.28: The area ratio of the D peak area to the sum of the G1 and G2 peak areas. The decrease is indicative of a films wich have fewer structural defects and therefore a higher crystilinity. The arbitrary best fit line is included as the dashed line to guide the eye. 188

Figure 4.29: (A) A semi- $\log _{10}$ plot of the three different heating rates used in this study. The natural log equations which describe the heating rates are shown next to their curves, where ' $t$ ' is the time in seconds. The time to $1000^{\circ} \mathrm{C}$ is annotated as well. Inset, the first 60 seconds of the same data but in a standard plot. (B) A representative cooling rate for all samples. This is the standard synthesis cooling rate. The cooling time to $\sim 25^{\circ} \mathrm{C}$ is annotated as well. 189

Figure 4.30: (A) XPS of the C1s region and (B) Raman spectra of three samples, each heating at a different rate. All other synthesis parameters were identical. (C-F) Parameters extracted from (A,B) plotted against the sample heating duration in a $\log _{10}$ scale. (C) Plot of the atomic concentration of carbon and oxygen. (D) Plots of the number of carbon layers and the percentage of carbon in the film associated with the buffer-layer. (E) The $\mathrm{D} / \mathrm{G}_{1+2}$ ratio is shown to decrease (improve) with extended annealing time. (F) The full width at half maximum (FWHM) of the D and $\mathrm{G}$ peaks. The narrowing of the $\mathrm{D}$ peak indicates a narrowing of the distribution of states responsible for the D peak. Likewise, the $G$ peak widening indicates additional $G$ peak vibrational modes. 191

Figure 4.31: The various cooling rates explored in this study. Heating rates were all identical. (A) Plots of the cooling rates, time shifted to the onset of the cooling phase. Curves are labeled with the exponential factors which govern the cooling rate. Inset, the same data in a semi-log 10 plot. (B) 
The equations used to generate the curves in (A) which approximate the cooling rate where ' $\mathrm{T}$ ' is the temperature of the sample in ${ }^{\circ} \mathrm{C}$ and ' $\mathrm{t}$ ' is the time is seconds. Equations are color coded to the curves in (A). Also tabulated is the amount of time the samples took to cool down from $\sim 1000^{\circ} \mathrm{C}$ to approximately room temperature.

Figure 4.32: (A) XPS of the C1s region and (B) Raman spectra of three samples, each cooled at a different rate. All other synthesis parameters were identical. (C-F) Parameters extracted from $(A, B)$ plotted against the sample cooling duration. (C) Plot of the atomic concentration of carbon and oxygen. (D) Plots of the number of carbon layers and the percentage of carbon in the film associated with the buffer-layer. (E) The $\mathrm{D} / \mathrm{G}_{1+2}$ ratio and (F) the fraction of carbons in each layer involved in a chemical defect state. The complexities of the curves of (E) and (F) indicated competing process may be at play. Arbitrary best fit lines are included as the dashed lines to guide the eye. 196 Figure 4.33: (A) The normalized and stacked Raman spectra grouped by temperature and stacked by increasing dwell time: $0 \mathrm{sec}, 900 \mathrm{sec}$ and $3600 \mathrm{sec}$. Shown for comparison is the spectrum for samples which have not seen any elevated temperature or exposure to hydrogen. (B) Raman spectra from samples produced using the standard AP-RTA in argon, cooled and then reheated in hydrogen and allowed to dwell for $0 \mathrm{sec}, 900 \mathrm{sec}$ or $3600 \mathrm{sec}$. Shown for comparison is the spectrum of the sample produced at the same time using the Standard Synthesis process and has not been exposed to hydrogen. 202

Figure 4.34: The shaded band bounded by the dashed lines represents the middle $75 \%$ of the distribution for the SOP samples. The solid line marks the average value recorded for the SOP samples. The box plot for the corresponding parameter is to the right of each plot along with the average value. The whiskers represent the middle $90 \%$ of values. (A-C) The samples which were etched only, then exposed to hydrogen. (D-F) Samples were etched, then AP-RTAed in argon, then exposed to hydrogen. 205

Figure 4.35: Stacked C1s spectra spectrum for etched only (A) and etched and AP-RTAed under argon (B) then exposed to hydrogen for a 0 second dwell from $500^{\circ} \mathrm{C}$ to $1000^{\circ} \mathrm{C}$. A slight shift to higher binding energies for the film related peaks is observed. Spectrum are normalized to the Si$\mathrm{C}$ reference peak. (C) The number of carbon layers produced in each case. (D) The atomic oxygen concentration. (E) Atomic fluorine concentration. The box and whisker plots show the range of values collected from SOP samples. Data outside this range is significant. 208

Figure 4.36: The effect of dwell time at $1000^{\circ} \mathrm{C}$ under hydrogen. (A) Stacked C1s spectra, the steady transformation of the spectra with increasing dwell time is seen. (B) Similarly, a refinement of the Raman spectra is also seen. (C) The number of graphene layers. (D) The $\mathrm{D} / \mathrm{G}_{1+2}$ ratio. (E) The Raman $\mathrm{G}_{1+2}$ and $\mathrm{D}$ peak full width at half maximum (FWHM). The box and whisker plots show the range of values collected from SOP samples. Data outside this range is significant. 210 Figure 4.37: XPS (A) and Raman (B) analysis of a standardly synthesized sample thermally cycled between room temperature and $1000^{\circ} \mathrm{C}$ in hydrogen 10 times. The dashed lines show the location of the graphene peak and Raman spectra line for a Standard Sample (no hydrogen exposure) for comparison. $\chi^{2}$ is the fitting parameter as determined by the fitting program (lower is better). 212 Figure 5.1: A schematic of a generic device. The specifics of the contact pattern, electrode setup and sample packaging varied, but the basic design was the same. The 'device' consists of the $\mathrm{SiC}$ substrate, the graphene film, Au/Ti contacts. The device 'package' consisted of the mounting hardware and related components as well as the electrical leads and connections. The image is not to scale. 215 
Figure 5.2: (A) IV plots of a representative graphene/SiC device and a reference $\mathrm{SiC}$ sample. The graphene/SiC device shows higher conductivity and exhibits a back-to-back Schottky behavior. Parameters of the graphene film, as determined by XPS and Raman analysis, are included in the upper inset. Lower inset, a schematic showing the sample contact pattern and electrode arrangement. Data was collected in 0.001A steps.

Figure 5.3: (A) A simple schematic of the physical interpretation of the potential electrical conduction paths in the graphene/SiC devices. The IV results indicated that the majority of current flows through the graphene layer(s). (B) Equivalent circuit diagram of this physical interpretation. The metal-graphene interface is represented by Schottky diode. Each film layer is discussed in detail within the text.. 218

Figure 5.4: An approximation of the electronic band structure of a back-to-back Schottky device under an external bias which shoes current injection by thermionic emission. There has been some debate over the exact shape of the Fermi level within the space change region; Rhoderick makes the case that it is flat. (745) This is the interpretation shown.... 221

Figure 5.5: (A) The positive region of the IV curve shown in Figure 5.2. (B) Plot of the $\ln (\mathrm{I})$ of the data in (A) against the voltage drop across the contact/film interface. Data with $(\circ)$ and without $(\diamond)$ the series resistance (Rs, packaging + film) removed (i.e. $\diamond$ 's takes Rs to be zero). The linear fit is used to calculate the ideality factor, $\eta$. 223

Figure 5.6: (A) IV curves collected from contact pads with various spacings. Dashed lines correspond to power law best-fit lines for each data set. (B) The corresponding TLM plot. the dashed line and accompanying equations represent the linear best-fit to the data. The slope of the linear fit is equal to the sheet resistance of the film $\left(\mathrm{R}_{\mathrm{S}}\right)$, the Y-intercept is twice the contact resistance $\left(2 R_{C}\right)$, the $\mathrm{X}$-intercept times the contact width $(\mathrm{W})$ is equal to twice the transfer length $(\mathrm{L} T)$ 225

Figure 5.7: (A) Force current plotted as a function of the voltage drop between two inner sense pads. The results of six different pad spacings are shown. The contact width was $0.233 \mathrm{~cm}$. (B) The resistance of the film as calculated by the voltage drop, plotted as a function of the aspect ratio of the film between the sense pads. 227

Figure 5.8: (A) The IV curve with a best-fit line shown. The film parameters contact pattern design, and sample packaging of the device are listed. (B) The Richardson-Dushman analysis of the data shown in (A). A best-fit linear line is shown. 230

Figure 5.9: Summary data for samples AP-RTA'ed to different temperatures. (A) Data points are two-point IV measurements from films AP-RTAed to various temperatures. The annotated numbers are the power law exponents for the best-fit power law lines shown. (B)Richardson-Dushman analysis plot for the data in (A). (C) The calculated Schottky Barrier height and carrier concentrations. (D) Schottky diode ideality factors for the data set. 232 Figure 5.10: (A) Stacked IV plots, collected from $25^{\circ} \mathrm{C}$ to $200^{\circ} \mathrm{C}$, showing the smooth transition from back-to-back Schottky to almost pure ohmic behavior. (B) Similar data from a different device across a larger temperature range. The transition to ohmic behavior is observed around $300^{\circ} \mathrm{C}$. Conductivity increases up to $\sim 550^{\circ} \mathrm{C}$ after which a small decline is seen up to $810^{\circ} \mathrm{C}$ (maximum temperature range of the RTD). 236

Figure 5.11: The Maxwell-Boltzmann distribution at three generic temperatures. The fraction of carriers with an energy greater than the Schottky barrier increases with increasing temperature. 
Figure 5.12: (A) IV plot, collected at $205.2^{\circ} \mathrm{C}$, of a device (only a single polarity is shown). There are two distinct regions separated by an inflection point. (B) Proposed contributors to the IV curve; a combination of thermionic conduction and charge trap hopping conduction. The solid black line is empirical data from (A). 239

Figure 5.13: (A) IV plot of a device (single polarity) at 54 different temperatures, collected sequentially, in $\sim 10^{\circ} \mathrm{C} \pm 7^{\circ} \mathrm{C}$ increments. (B) Calculated slope of the curves shown in (A). The red line marks the minima of each curve. Inset: zoomed in region of the same plot, as indicated by the arrow. (Note that the curve termination is due to the $10 \mathrm{~mA}$ current limit.) 241

Figure 5.14: (A) Semi-Log10 plot of the roll-off voltage (B) and semi-log 10 plot of the corresponding current. The best-fit lines fit well to an exponential curve, indicating that the charge traps are distributed exponentially across energy levels.

243

Figure 5.15: Electronic band diagram of the metal/graphene interface, illustrating the various mechanisms by which charge carriers are injected into the film........................................ 244

Figure 5.16: A non-zero crossing of the IV curves is observed for devices operating at very high temperatures. This is not seen at low temperatures.

245

Figure 5.17: (A) Two-point resistance of a device from $23^{\circ} \mathrm{C}$ to $800^{\circ} \mathrm{C}$ at $10 \mathrm{~mA}$ and (B) at $1 \mathrm{~mA}$ across the same temperature range. 246

Figure 5.18: Room temperature IV curves before and after a thermal cycle up to $700^{\circ} \mathrm{C}$ under argon over 4 hrs. Illustrated here is the improved conductivity via the contact bake-in or curing effect. After the initial bake-in, device IVs are essentially unchanging with further thermal cycles.... 247

Figure 5.19: (A) IV as $f(\mathrm{~T})$ plot and (B) corresponding Richardson-Dushman plots. The arrows indicate in what direction the curves increase in temperature.

Figure 5.20: (A)The estimated Schottky barrier height $\left(\Phi_{B}\right)$ and ideality factor $(\eta)$ as a function of temperature. (B) The mobility $(\mu)$ and carrier concentration $\left(N_{D}\right)$. The confidence of each point is related to the $\mathrm{R}^{2}$ plot above each graph and corresponds to the linear fit of the data from which the data are calculated; ( $\circ$ ) for $\Phi_{B}, \mu, N_{D}$ and ( $\square$ ) for $\eta$. (C) and (D) show a more detailed view of the results between $23^{\circ} \mathrm{C}-200^{\circ} \mathrm{C}$ of $(\mathrm{A})$ and $(\mathrm{B})$, respectively. 250

Figure 5.21: (A) The data from Figure 5.19(D) plotted in $\log _{10}-\log _{10}$. The dashed lines are the contributions of various scattering mechanisms as proposed by Sillman, et al. For comparison, (B) the data from Sillman, et al. (data points) with the scattering mechanisms (dashed lines) described by Fletcher, et al. This figure has been adapted from Fletcher, et al. (626) 253 Figure 5.22: Two-point IV as $f(\mathrm{~T})$ plots collected from a $4 \mathrm{H}-\mathrm{SiC}[\mathrm{SI}] /$ graphene composite sample produced using the SOP graphenization process described in Chapter 4. Arrows indicate the

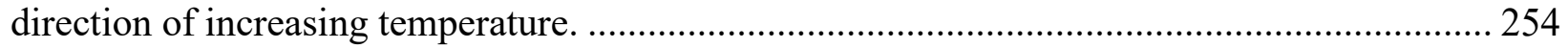
Figure 5.23: Two-point IV as $f(\mathrm{~T})$ plots collected from a 4H-SiC/graphene composite sample produced using the SOP graphenization process described in Chapter 4. Arrows indicate the direction of increasing temperature. ................................................................................ 256 Figure 5.24: (A) IV plot of a $4 \mathrm{H}-\mathrm{SiC} / \mathrm{Graphene}$ sample collected at $450^{\circ} \mathrm{C}$. Two regions, charge trap conduction and thermionic conduction are clearly apparent. The independent contributions of each mechanism are shown by the dashed lines. (B) Approximant roll-off voltage of charge trapping and $(\mathrm{C})$ corresponding current level. 257

Figure 5.25: Qualitative illustration of the resonant tunneling process responsible for the peak in current flow. Resonance in the electron wave function with the quantum wells formed by successive charge traps leads to a higher tunneling probability for those electrons. This process is 
carried out through the entire trap hopping circuit. (Note: In reality, the wave packet is perturbed by the barrier more than what is illustrated here, and the reflections are substantially more complex. Shown here is a simple cartoon to help qualitatively illustrate the general principle.) ............. 259

Figure 5.26: Energy diagram illustrated at several points in the IV curve of a resonant tunneling device. A description of the phenomena at each point is given in the text..... 260

Figure 5.27: The curves have been extended outside of the current and voltage limit range by using an ad hoc French curve fitting technique to estimate the peak to valley current ratio (PVCR), as indicated by the respective dashes lines. 261

Figure 5.28: Generic illustration of the fundamental heat transfer processes which dictate the device temperature. Electrical energy is converted into heat energy in the device by Ohmic heating. Heat is removed from the device by the gas stream via forced convective heat transfer. The difference in the rates between these two processes determines the steady state balance of heat energy residing in the device which dictates the device temperature. 267 Figure 5.29: Illustration of the thermal equilibration process from device startup to the first gas pulse. 268

Figure 5.30: (A) An example of a thermal runaway event for a device operating in constant voltage mode $(0.350 \mathrm{~V})$. The plot shows the first 10 seconds after power up of the device, while under a flow of 500 SCCM of UHP Ar. The specific conditions which led to a runaway event varied from sample to sample. This device used a TLM pattern and two-point probe data was recorded between two closely spaced pads. (B) The thermal runaway cycle: Ohmic heating leads to a temperature rise, which leads to a decrease in resistance, which leads to an increase in the current, which leads to an increase in power, which rises the temperature; the cycle repeats. 269 Figure 5.31: (A) Photo of the device tested. (B) Equivalent circuit diagram showing the various measurement points. (C) How the various resistances were calculated, using the measurement points as indicated in $(\mathrm{B})$. 271

Figure 5.32: (A) A two-point IV curve collected at room temperature. 12 points of constant current operation are indicated. (B) The absolute resistance recorded over the first 80 seconds after the power is supplied to the device. Note that the vertical axis is $\log _{10}$ scale and increases in the downward direction. 272

Figure 5.33: Device resistance as calculated from two-point measurements. (A) The absolute change (from $t=0$ ) in the resistance of the device at 12 different current setpoints. (B)The same data but viewed as the percent change in resistance. 273

Figure 5.34: Film resistance as calculated from four-point measurements. (A) The absolute change (from $\mathrm{t}=0$ ) in the resistance of the device at 12 different current setpoints. (B)The same data but viewed as the percent change in resistance... 274

Figure 5.35: The device behavior in response to cycling between two inert gases, argon and helium, with different physical properties. (A) The response taken as the change in resistance and (B) the same response calculated as a percentage change in resistance. The setpoint was $60 \mathrm{~mA}$....... 279

Figure 5.36: The device response due to changes in gas flowrate. The device was allowed to come to a full thermal equilibrium under a flow of 10 SCCM of argon. The flow was then stepwise increased to 700 SCCM for 200 seconds, then reduced back to 10 SCCM. The device response to a purely thermal (cooling) effect is thus illustrated. (A) The response as an absolute change in resistance and (B) a percentage change in resistance. The current setpoint was $100 \mathrm{~mA}$. The setpoint was $100 \mathrm{~mA}$. 280 
Figure 5.37: The startup behavior and subsequent response to reducing the Ar gas flowrate from 500 SCCM to 0 SCCM, effectively heating the device, performed at six different constant voltage setpoints. (A) $0.100 \mathrm{~V}$, (B) $0.500 \mathrm{~V}$, (C) $1.00 \mathrm{~V}$, (D) $1.50 \mathrm{~V}$, (E) $2.00 \mathrm{~V}$, (F) $2.30 \mathrm{~V} \ldots \ldots \ldots \ldots \ldots \ldots . . . . . . .281$

Figure 5.38: By modeling the test setup as a PFR in series with a CSTR, the concentration in the test chamber can be qualitatively calculated. (A) A generic schematic of the testing system. (B) The gas pulse shape at various points in the test system. The mixing is enhanced at higher temperatures. 283

Figure 5.39: (A) IV curve of the device under test. As expected, the device transitions from backto-back Schottky behavior at room temperature to apparent Ohmic behavior at high temperature. (B) The positive quadrant, spanning a reduced voltage range. Six different operation points are indicated by the crossed circles $(\square)$. (C) The two-point resistance measured at $0.100 \mathrm{~V}$ as the device is heated from room temperature to $810^{\circ} \mathrm{C}$. The three test temperatures are indicated by the diamonds $(\diamond)$. Inset: an expanded view of the plot around the resistance reversal point. Note the transition occurs at $296^{\circ} \mathrm{C}$. 285

Figure 5.40: The results of a hydrogen pulse test from the same device at (A) $0.100 \mathrm{~V}$ and (B) $2.00 \mathrm{~V}$. Both tests were carried out at $23^{\circ} \mathrm{C}$. The red line indicted the applied hydrogen pulse. 286 Figure 5.41: The results of a hydrogen pulse test from the same device at $0.100 \mathrm{~V}$ and $0.900 \mathrm{~V}$. Both tests were carried out at $230^{\circ} \mathrm{C}$. (A) Contrary to what would be expected for a thermal effect, the resistance decreases on hydrogen exposure, indicating this is a chemically derived effect when the device is operated at a low setpoint. (B) During high setpoint operation, only a thermal effect is observable. 287

Figure 5.42: The results of a hydrogen concentration study for a device operated in the chemically responsive regime and thermally responsive regime. (A) When operating the device at $0.200 \mathrm{~V}$, a chemical response is seen. (B) When operated at $1.40 \mathrm{~V}$, a thermal response is seen. The device is at the same temperature, $230^{\circ} \mathrm{C}$, in both cases. The hydrogen/argon pulse mixtures are indicated by the red bars and correspond to a 5\%,25\%,50\%,75\% and 100\% hydrogen concentrations. 289 Figure 5.43: (A) The results of the hydrogen concentration study showing the thermal and chemical response on the same scale. The red line indicates the hydrogen mix pulses. Sequentially, they are $5 \%, 25 \%, 50 \%, 75 \%$ and $100 \%$ hydrogen in argon. (B) A comparison of the device response curves to a $300 \mathrm{sec}$ pulse of $100 \% \mathrm{H}_{2}$ when the device is at a low setpoint (chemical effects dominant) and a high setpoint (thermal effects dominate). 290

Figure 5.44: The results of a hydrogen pulse test $\left(100 \% \mathrm{H}_{2}\right.$ for $300 \mathrm{sec}, 100 \%$ Ar for $\left.600 \mathrm{sec}\right)$ from the same device at (A) $0.100 \mathrm{~V}$ and (B) $0.900 \mathrm{~V}$. Both tests were carried out at $409^{\circ} \mathrm{C} \ldots \ldots \ldots \ldots . . . .291$ Figure 5.45: Four 300-second-long pulses of pure $\mathrm{CH}_{4}$ gas in a carrier stream of pure Ar. (A) Device response at $10 \mathrm{~mA}$ and at (B) $100 \mathrm{~mA}$. Both tests were conducted at room temperature in the small test cell..... 292

Figure 5.46: The device response to $\mathrm{H}_{2}$ and $\mathrm{CO}$ is shown at both a low set point (A) $0.650 \mathrm{~V}$ and at a higher setpoint (B) $1.00 \mathrm{~V}$. Both tests were conducted at room temperature in the small gas test cell. 293

Figure 5.47: An example of the irreversible and large change in device resistance occasionally observed in devices exposed to hydrogen at high temperatures. 294

Figure 5.48: The IV curve collected before $(\diamond)$ and after $(\circ)$ high temperature gas testing with hydrogen. Both curves were collected under the same conditions: $23^{\circ} \mathrm{C}$ and $20 \mathrm{SCCM}$ of UHP argon. 295 
Figure 5.49: (A) Top, the C1s spectra collected from the film after high temperature hydrogen gas testing. Bottom, the $\mathrm{C} 1 \mathrm{~s}$ spectra before gas testing. Inset are the calculated film parameters from the deconvolution of the data. (B) The corresponding deconvolution results. 296

Figure 5.50: Raman spectra collected from the film after high temperature hydrogen gas testing. The $\mathrm{D} / \mathrm{G}$ ratio $(\sim 1.31)$, narrowing of all peaks and slight growth of the two-phonon peaks are all indicative of the buffer layer decoupling process. 297

Figure 5.51: The IV plots collected at various temperatures in (A) a pure argon environment and in (B) a pure hydrogen environment. A substantial splash current is seen in the pure hydrogen environment. 303

Figure 5.52: Illustration of the two laser scan paths. Shown is a false color, 3D projection of a SEM image of a $2.5 \mathrm{~mm}^{2}$ sample with the parallel contact pattern design. The path of the laser spot across the sample is shown. The approximant laser spot size is shown to scale. 308

Figure 5.53: Photocurrent generated as a function of position by irradiation with red laser light. (A) Photocurrent as the laser is first scanned onto the sample and then across the length of a Ti/Au contact. (B) Photocurrent as the laser is scanned across the width of the same contact. The relative position is correlated with the photo response, as indicated by the false color images and the dashed drop lines. The laser spot size is drawn to relative scale. Note that the inset contact schematic has been rotated $90^{\circ}$ from (A) to (B) for clarity.... 309

Figure 5.54: The photocurrent circuit produced by irradiating at two different positions. 310 Figure 5.55: (A) Photovoltage due to five pulses of red laser light. (B) Higher resolution plot of one pulse, illustrating the fast response time. (C) Photocurrent due to four pulses of red laser light. (D) Higher resolution plot of one pulse, illustrating the, comparatively, slower response time. 312 Figure 5.56: (A) The photocurrent generated in response to five pulses of laser light. Three different laser wavelengths were tested. The inspection box to the right shows leading edge of a pulse, highlighting the difference in rise time. Higher resolution plots of the first two pulse are shown in (B) red laser light, (C) green laser light and (D) purple laser light. 314 Figure 5.57: (A) Photocurrent as a function of laser wavelength and (B) as a function of laser energy. The dashed lines correspond to the best fit power law line for each dataset. 315 Figure 5.58: The powered photo response. Both thermal effects and photo effects can be seen. 316

Figure 5.59: Photocurrent recorded due to irradiation with red laser light as a function of applied voltage across the device. Error bars are calculated from the minimum and maximum amplitude of the photo response to five pulses of laser light in each case. 317

Figure 5.60: Various charge carrier processes which occur when the device is irradiated. (A) Irradiation can lead to promotion of charge carriers from the valence band (VB) or from charge traps into the conduction band (CB); or carriers already in the VB may be promoted to even higher levels. (B) The carrier recombination and energy thermalization process. (C) The carrier multiplication process mediated by carrier-carrier interactions; in this case, rather than energy going into thermalization process, the energy causes additional e-h pairs to be generated. (D) The overall electron flow in a device due to the injection of photonic energy. The direction of charge pumping can reverse, if the opposite contact is irradiated. 321

Figure 5.61: The basic idea behind a light antenna constructed from nanoparticles. High frequency electromagnetic radiation drives the plasmon shell surrounding a nanoparticle into oscillation. A 
high-density array of nanoparticles can lead to a constructive interaction between theses oscillations of neighboring particles, leading to plasmonic resonance....................................... 324 Figure 5.62: The nominal metal work function as a function of atomic number. The metals used for the electrical contacts for the devices studied here are highlighted. These data are averaged from the tabulated data found in the most recently available edition of the CRC Handbook of Chemistry and Physics. (688) 326

Figure 5.63: Different geometrical designs for the metal contacts and the resulting depletion region. (A) The contact design used in this work. (B) A toothed design. (C) A comb design. (D) Starburst design. (E) An array of starbursts. (F) A larger array of the starburst contact design and

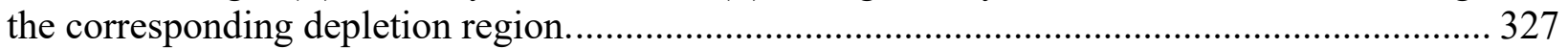

Figure 7.1: Suggested technological roadmap for the plasma assisted graphene growth process. Dashed lines represent future work. 349

Figure A.1: The wafer manufacturing process and the origin of the atomically stepped surface. (A) Ideal fully epitaxial crystal growth. (B) Actual growth occurs slightly off axis. The wafer dicing from the master ingot is also slightly off axis. The chemo-mechanical polishing step flattens the wafers such they the wafer as a whole is geometrically uniform. (C) Measurements of the wafer miss cut angle. All angles marked $\theta$ are equivalent. 396

Figure A.2: AFM images collected from six randomly chosen locations across the wafer surface. The atomic steps are clearly seen, as well as polishing debris. 397

Figure A.3: AFM images showing an area containing a high concentration of crystal edge defects. As indicated by the red arrows, the blades contain pores at least 0.4-0.6 nm deep. However, these are not fully resolved by the $10 \mathrm{~nm}$ AFM tip. 397

Figure A.4: AFM showing the atomically stepped surface. (B) Line profile corresponding to the red line in (A). (C) High magnification AFM image of the surface. (D) Line profile corresponding to the red line in $(\mathrm{C})$. 398

Figure A.5: XPS spectra from a sample produced using the standard process but with no rest step during the ICP/RIE. This yielded good films, but not significantly better than films which incorporated a rest step during the etch. 399

Figure A.6: The C1s region of a sample where no helium flow was used (top) and a sample where the standard helium flow of 1.0 SCCM was used. Running the etch process without helium flow resulted in more carbon layers, will lower defect less and less nitrogen contamination. For simplicity, the blue curve combines the S1 and S2 peaks. 400

Figure A.7: The effect of consecutive ICP/RIE etch runs (no $\mathrm{O}_{2}$ cleaning or $\mathrm{CF}_{4}$ conditioning steps between runs) on the amount of defective carbon and the number of graphene layers, as determined by XPS analysis of the C1s region. A clear trend is seen in that more defects and less graphene is formed as the number of successive runs increases. Including an $\mathrm{O} 2$ clean and one $\mathrm{CF}_{4}$ run (with not sample) was determined to be the best balance between a steady DC bias and chamber performance. This corresponds to operating the chamber with a sample loaded at the run $\# 2$ point in the figure, which yielded the most layers of defect free graphene. 401

Figure A.8: (A) XPS data collected from the material which builds up on the ICP/RIE chamber walls. The presence of fluorine indicated the material buildup is related to the $\mathrm{CF}_{4}$ etching. (B) Picture of the upper lid of the process chamber showing the showerhead (i) and upper chamber walls (ii). The material build-up is clear. This material was removed and analyzed give the spectra 
shown in (A). XPS results and literature reports indicated the material is most likely a combination of a fluorocarbon polymer film and aluminum fluoride polymer film. (689) (690)................. 403

Figure A.9: The fitting results using a single G peak fit. Substantial errors result and a poor fit is

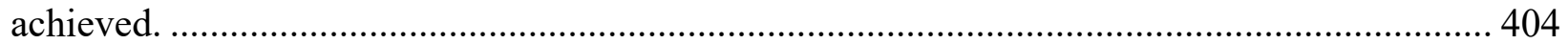

Figure A.10: Raman spectra collected for a sample which has been etched using the standard

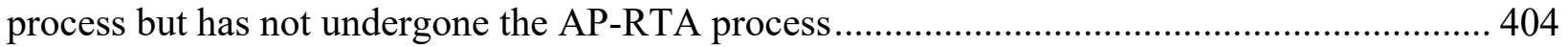

Figure A.11: XPS spectra of three different silicon test samples. They are: the as-received silicon, silicon which has undergone the SOP process using $\mathrm{Cl}_{2}$ gas as the halogen plasma source and the standard SOP process using $\mathrm{CF}_{4}$ gas as the halogen plasma source. The relative atomic concentrations are annotated. Also shown is a more detailed view of the same data. The ratio between the $\mathrm{C} 1 \mathrm{~s}$ and $\mathrm{Si} 2 \mathrm{p}$ peaks are annotated. ............................................................. 406

Figure A.12: The variation of the -DC bias can be seen a function of etch time. .................... 407

Figure A.13: Heat maps of the number of carbon layers and the percent carbon which is participating in the buffer layer across the rang of ICP and RIE powers. ............................. 407

Figure A.14: Plots of the D/G peak area ratio, \% carbon as buffer layer, D peak position and D peak FWHM plotted as a function of the number of carbon layers. No correlation was found. 408 Figure A.15: There is no correlation between the number of carbon layers and the chemical defect density of each layer. This indicates that all layers are equally defective (i.e. the $2^{\text {nd }}$ layer has as

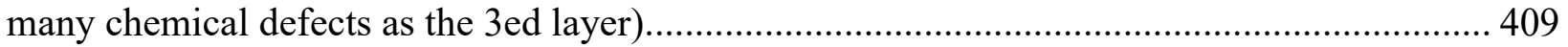

Figure A.16: Tabulated and plotted area under the curve for each temperature. Obviously, but importantly, a linear incase in the maximum temperature leads to an exponential increase in the time at a sample is exposed to elevated temperature. 410

Figure A.17: Plots of various film parameters, taken as a function of the annealing amount (area under the RTA curve). 411

Figure A.18: (A) The number of buffer layer carbon atoms per graphene carbon layer atoms as a function of AP-RTA temperature (ratio of buffer carbon to graphene carbon atoms). It is clear that for the majority of temperatures, the buffer layer is largely independent of the number of layers. Given the nature of the buffer layer, this observation agrees with intuition. At high temperatures $\left(>1100^{\circ} \mathrm{C}\right)$ the carbon in the uppermost graphene layers are consumed by trace oxygen. Leaving films which are mostly buffer layer. (B) The same data, but plotted against the amount of annealing the samples experience (the integrated are under the annealing curve). It is unclear whether the large uptick in buffer layer (indicating the graphene layers has been consumed or burnt off) is due to the high temperature (A) or the extended time spent at temperature (B). Results from the dwell time studies indicated that it may be the latter. 411

Figure A.19: The elemental atomic concentration across various AP-RTA temperatures. The increase in carbon concentration is due to the removal of fluorine species, as well as a densification of the carbon film leading to an attenuation of the silicon signal from the bulk. At temperatures above $1000^{\circ} \mathrm{C}$ as sharp increase in oxygen content is observed, coinciding with a decrease in carbon, indicating the carbon film is being consumed by oxygen...................................... 412

Figure A.20: (A) The approximant heating and cooling profile for the thermally cycled sample. (B) XPS analysis of the C1s region. The dominant peaks are labeled, and the peak parameters are tabulated below the figure. Inset also are the atomic concentration data collected from higher resolution scans of each element. (C) Raman analysis of the same sample. The primary peaks are labeled, and the peak parameters are tabulated below the figure. 
Figure A.21: (A) The approximant heating and cooling profile showing the extended anneal dwell time. (B) XPS analysis of the C1s region. The dominant peaks are labeled, and the peak parameters are tabulated below the figure. Inset also are the atomic concentration data collected from higher resolution scans of each element. (C) Raman analysis of the same sample. The primary peaks are labeled, and the peak parameters are tabulated below the figure. …………………………...... 415 Figure A.22: The D/G ratio as a function of increasing AP-RTA temperature......................... 416 Figure A.23: (A) The spectral radiance calculated at for different temperatures. (B) The same data on a simi-log $\log _{10}$ scale. (C) The visible spectral region of the same data. The arrow indicates the wavelength of light the devices have shown photosensitivity to............................................ 419 Figure A.24: The relationship between a photons energy and its wavelength, as given by the Planck-Einstein relation. 421

Figure A.25: (A) The current recorded from two contacts as one is irradiated with a purple laser. (B) Similar data collected when a red laser is scanned across one of the contacts. 422

Figure B.1: (A) The equipment setup for nucleation experiments using NaBH4. (B) Close up view illustrating the drip path of the burette solution into the flask solution with stir bar.................. 424

Figure B.2: (A) The XL-1500 UV crosslinker used to irradate the graphenenated samples. (B) A beaker of $\mathrm{AgNO}_{3}$ in a water-ethanol mixture with a graphenated sample under UV light......... 426 Figure B.3: A schematic of the photo-nucleation process. Depicted is the proposed mechanism for silver nucleation. Light irradiation of the semiconductor surface promotes electrons into the conduction band while ethanol scavenges holes left in the valence band, leading to a net negative charge accumulation on the surface. This charge accumulation acts on ions within the solution and

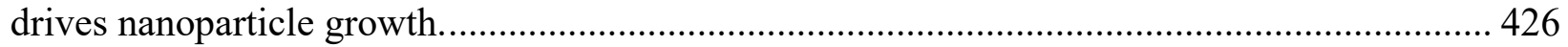

Figure B.4: (A) Magnified view of the macro set of elecro-nucleation experimental setup. (B) Higher magnification view of the experimental setup showing the positive electrode, solution aliquot and the ultra-sonic wire bonds used to make electrical contact with the sample. (C) Simple conceptual schematic of the experimental setup.................................................................... 428

Figure B.5: (A) The experimental setup for the micro set of elecro-nucleation experiments. (B) Magnified view showing the positioning of the positive electrode to the substrate. (C) Simple conceptual schematic of the experimental setup. (D) Magnified view of the positive electrode and substrate with aliquot of precursor solution. Note that in this set of experiments, the positive electrode was submerged in the liquid. 429

Figure B.6: (A) The AFM scanner head with insulated scanner body, AFM tip and Pt electrode. (B) Simple conceptual schematic of the experimental setup. (C) Magnified view of the liquid cell holding a test substrate and a liquid salt solution. (D) View of the sample stage with liquid test cell installed. The surface meniscus of the liquid salt solution within the cell can be seen as well.. 430 Figure B.7: (A) Generic example of the nanoparticle deposition power profile with each phase labeled. (B) Real world picture of the interior of the process chamber. The pertinent components are label. Note that the substrate shutter is shown in the OPEN position and the source shutter is shown in the CLOSED position. 431

Figure B.8: Bubble formation on a graphinated sample at 5, 10 and 15 minutes in a solution of $2.71 \mathrm{mM}$. NaBH 4 . At 15 min the sample has begone to become buoyant and has stood up on end. Shortly thereafter the sample floated to the surface. 434

Figure B.9: Both beakers contain the same mixture: $47 \mathrm{~mL}$ of $2.25 \mathrm{mM} \mathrm{AgNO}_{3}$ and $10 \mathrm{~mL}$ of $12.95 \mathrm{mM} \mathrm{NaBH}_{4}$. (A) A stable colloidal solution of silver particles was formed by using dropwise 
mixing. (B, C) Large particles have formed which precipitate out of the liquid as a result of the

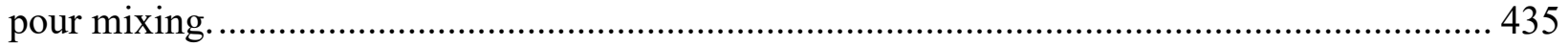

Figure B.10:(A) The result of dropwise mixing of dilute $\mathrm{NaBH}_{4}$ into a concentrated $\mathrm{AgNO}_{3}$ salt solution. Large particles have formed and have begun to precepted out of solution. (B) The result of dropwise mixing of dilute $\mathrm{AgNO}_{3}$ salt solution into concentrated $\mathrm{NaBH}_{4}$; a stable colloidal solution has formed. 435

Figure B.11: Evidence of nucleation runaway based on the observed structures. Two samples, treated under the same conditions, showing varying degrees of nucleation runaway. The key features in both are the large particles surrounded by a growing nest of smaller nanoparticles. The false color bar ranges have been truncated to more fully resolve the feature of both images. Also shown are false colored 3D renders of select nucleation sites at different stages of runaway... 437 Figure B.12: (A) The modified equipment setup for nucleation experiments using $\mathrm{NaBH}_{4}$. (B) Close up view illustrating the drip path of the burette solution into the flask solution with stir bar. 438

Figure B.13: (A) AFM image showing nanoparticles. (B) Histogram analysis showing the height and diameter distribution of the nanoparticles in (A), using a combination height and curvature thresholding detection method; inset in (B) are the detection maps. (C) 3-D false colored image of (A). (D) XPS results showing the elemental distribution and a section of the survey scan showing the presence of silver. 440

Figure B.14: (A) AFM image showing nanoparticles. (B) Histogram analysis showing the height and diameter distribution of the nanoparticles in (A), using a combination height and curvature thresholding detection method; inset in (B) are the detection maps. (C) 3-D false colored image of (A). (D) XPS results showing the elemental distribution and a section of the survey scan showing the presence of gold. 441

Figure B.15: (A) AFM image showing nanoparticles. (B) Histogram analysis showing the height and diameter distribution of the nanoparticles in (A), using a combination height and curvature thresholding detection method; inset in (B) are the detection maps. (C) 3-D false colored image of (A). (D) XPS results showing the elemental distribution and a section of the survey scan showing the presence of platinum. 442

Figure B.16: (A) AFM image showing nanoparticles. (B) Histogram analysis showing the height and diameter distribution of the nanoparticles in (A), using a combination height and curvature thresholding detection method; inset in (B) are the detection maps. (C) 3-D false colored image of (A). (D) XPS results showing the elemental distribution and a section of the survey scan covering the Ir4f emission range. (E) Phase image on the same sample, more clearly revealing the donutlike structure of the nanoparticles. (F) Zoomed in region of (C) showing the donut-like structure. (G) Phase image of $(F)$. 443

Figure B.17: SEM images of a $2.5 \mathrm{~mm}$ square SiC-Graphene sample after Au nucleation using $\mathrm{NaBH} 4$. Note the degradation of the Ti/Au contacts (bright areas of the image). 445

Figure B.18: (A) Schematic of the DC method. (B) IV plot showing the slight change in electrical character of the device after deposition. (C) The current flow resulting from a 1.0V bias applied across the device during the 600 seconds of deposition. Inset within is the first 20 seconds of deposition; the large drop indicates that the majority of the deposition occurs within the first 10 seconds. (D) SEM of a ball wire bond made to a deposited Ti/Au contact on the device. (E) Magnified view of the inspection box shown in (D), showing the tree-like growth, emanating out from the current injection site. (F) SEM image take approximately at the midpoint between two 
contacts, revealing the presence of nanoparticles. (G) SEM taken adjacent to another current injection site (shown in the inset) illustrating a layer by layer growth. (H) SEM of a Ti/Au contact with particles deposited on-top. Note the particle alignment, as illustrated by the dotted lines. 448 Figure B.19: (A) Schematic of the EC setup. (B) Real-world picture of the setup. The spacing between the probe and sample was estimated to be $\sim 5 \mathrm{~mm}$. (C) IV data collected from the sample before, after precursor addition and after deposition. (D-E) SEM images of the sample showing the extensive needle-like Ag growth. The arrows in (F) show the two types of Ag structures: thin,

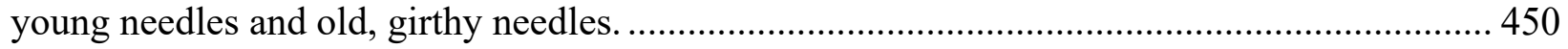

Figure B.20: (A, B) Drying effects, as a result of a decreasing droplet size, can be seen. (C) Ag crystals, as a result of the concentration of $\mathrm{AgNO}_{3}$ increasing to beyond the solubility point, crashing out of solution and crystalizing (magnified insets). ........................................... 451

Figure B.21: Simple schematic showing the modified EC deposition setup.......................... 451

Figure B.22: (A) Growth occurred relatively uniformly and appears not to be strongly influenced by the $\mathrm{Ti} / \mathrm{Au}$ contacts. The location of contacts has been annotated. (B) There are two regions of growth: a dense filed of growth, annotated "I" and a sparse field of growth, annotated "II". (C) SEM showing indiscriminate growth on the graphenated surface, Ti/Au contact and on an Au wire wedge bond. (D) Magnified view of the area 'I' annotated in (B). (E) Magnified view of the area 'II' annotated in (B). (F) Close up view of the primary growth feature ................................ 452

Figure B.23: Large area Au deposition of globular like structures........................................ 453

Figure B.24: Large area Ir deposition of globular like structures........................................ 453

Figure B.25: SEM analysis revealing the growth structures. (A) The large area deposition can be seen. (B) The delaminated sheets of material; also visible is growth on a gold wire bond wire. (C) Magnified view of growth on a wire bond wire; the nodule growth is easily seen. $(A, D, G, H)$ show successive magnified views revealing the interconnected bladed-like structure of the underlying sheets. (D,E,F) show successive magnified views showing a slightly different morphology to the underlying sheets. 454

Figure B.26: (A) The resulting Ag structure grown between the sample and the probe after the application of a $10 \mathrm{~V}$ bias for 120 seconds. (B) Magnified view of (C) showing the Ag structure after the probe has been removed. The structure is still attached to the substrate. 455 Figure B.27: (A) The probe and substrate after 30 seconds of $5 \mathrm{~V}$ biasing. No large structures are observed. (B) AFM image of the nanoscale dendritic structures formed beneath the biasing probe. (C) False colored 3D image of the large structure seen in (B). 456

Figure B.28:(A) AFM of the substrate in precursor liquid before the applied voltage. (B) AFM of the same area immediately after the nucleation experiment showing the growth of a single spherical particle exactly where the tip had been during the experiment. (C) Additional higher magnification of the same particle. (D) False colored 3D representation of the particle. (E) Perpendicular line profiles of the particle showing a rough symmetry, a particle height of $\sim 24 \mathrm{~nm}$ and radius of $\sim 50$ nm. 458

Figure B.29: The resulting growth when electrical contact being made between the AFM tip and the sample. (A) A view of the electrochemical test cell with sample. Electrical contact was made when an Ag wire grew between the AFM tip and sample. (B) Magnified view of (A) with arrow indicating the Ag wire. (C) A view through the built-in optical microscope of the AFM showing the dendritic growth which occurs when the AFM tip is in physical contact and a bias is applied. (D) AFM image of a different sample showing a similar effect. 459 
Figure B.30: (A) $5 \mu \mathrm{m} \times 5 \mu \mathrm{m}$ AFM image of a photo nucleated sample produced under the optimum conditions described in Table B-1. The XPS derived atomic concentrations and particle statistics are included below. (B) Histogram plots for particle diameter and height extracted from the analysis of $(\mathrm{A})$... 462

Figure B.31: Particle detection maps from two $5 \mu \mathrm{m}$ X $5 \mu \mathrm{m}$ AFM scans on the same sample. The effect of surface oxide on nucleation is illustrated. (A) The plain graphene surface sees moderate particle coverage. (B) An area of the same sample but exposed to a light oxygen plasm. A higher surface oxide concentration results in more nucleation as evident from the higher particle cover and count. The nucleation reaction was carried out in a quartz beaker containing $50 \mathrm{~mL}$ of a $3 \mathrm{mM}$ $\mathrm{AgNO}_{3}$ aqueous solution of $8 \%$ ethanol and was irradiated with $265 \mathrm{~nm}$ light for 30 minutes. 464

Figure B.32: Secondary X-ray emission intensity (SXI) map of two different areas of the same sample. The Ti/Au show up as bright areas in the image and correspond to areas of high electron emission. The blue plus marks the center of the collection area for the spectra shown in each panel. The red line represents the $\sim 100 \mu \mathrm{m}$ spot size of the XPS. (A) The SXI map showing the area sampled for the spectrum in (B). (B) The Ag3d region collected from a Ti/Au metal contact pad. Very high levels of silver are detected. (C) Corresponding SXI map and spectrum for the graphene surface of the same sample. The sample edges are also marked. Very low levels of silver are detected. This show the preferential silver deposition onto the metal contacts. 466

Figure B.33: Phase image of a silicon wafer decorated with gold nanoparticles. Excellent coverage and distribution are demonstrated. 468

Figure B.34: Particle detection maps corresponding top the samples listed in Table B-2, excluding sample L which did not have nanoparticles. All maps correspond to a $5 \mu \mathrm{m} \times 5 \mu \mathrm{m}\left(25 \mu \mathrm{m}^{2}\right)$ area, excluding B' which corresponds to a 90 X $90 \mu \mathrm{m}\left(1800 \mu \mathrm{m}^{2}\right)$ area. 470

Figure C.1: (A) An example of a device for reference before testing (B) After high temperature testing with carbon monoxide. The light-colored nickel film is clearly seen. The compostion of the film was determinond by XPS. 472

Figure C.2: (A) XPS survey data from a contaminated sample. Inset are the atomic concentrations. The presence of aluminum always correlates to high levels of oxygen. (B) The Al2p region showing the unambiguous presence of aluminum. (C) Representative AFM image collected from a contaminated sample. The surfaces are textured and covered with what appear to be nanoparticles. (D) Higher magnification AFM image from a different contaminated sample. (E) Phase image of (D). The phase shift indicates the particles to be of a different material than the background. 474

Figure C.3: Flow diagram summarizing the major experiments conducted to determine the source of aluminum contamination. 475

Figure C.4: The primary contamination source: (A) The anodized aluminum centering ring. This was found to be the primary source of contamination. The speckling of lighter color is where the anodization has broken through and the bare aluminum is exposed. (B) A magnified view of the inner portion of the ring showing excessive etching of the unprotected aluminum has occurred, producing pitting. The secondary contamination source: (C) The electrostatic chuck. (D) Zoomed in view of the edge of the electrostatic chuck, the light speckled area along the edge is bare aluminum exposed where the anodization has broken through. This is due to plasma undercutting occurring between the carrier wafer and the chuck. (E) The backside of a used carrier wafer, showing discoloration, indicating plasma undercutting has occurred. 477 
Figure C.5: Schematic of the proposed Al contamination mechanism. A) Cross-sectional view of the chamber interior with key components labeled. B) Depiction of the etching and sputtering process which damages the anodization and exposes the underlying bare aluminum. The arrows indicating the path of the $\mathrm{CF}_{\mathrm{x}}$ and aluminum. $\mathrm{C}$ ) Depiction showing the impregnation of the $\mathrm{SiC}$ by the aluminum. The size discrepancy between silicon atoms and aluminum has been exaggerated for clarity. 478

Figure C.6: Proposed aluminum concentration depth profiles which explain the bi-conditional process requirement for detectable aluminum contamination. XPSSD is the sampling depth of the XPS. XPS $S_{L D L}$ is the lower detection limit of XPS and [Al $]_{0}$ corresponds to the zero aluminum. For XPS analysis to detect aluminum, it must be at concentrations about the XPS LDL and within the XPSSD. A graphical representation of when these conditions are satisfied is labeled as the XPS detection regime.

Figure C.7: Atomic concentrations of aluminum and oxygen across the parameter space investigated during the ICP/RIE power study. 480

Figure D.1: Schematic of a three-layer (two graphene + one buffer) film on a $6 \mathrm{H}-\mathrm{SiC}(0001)$ substrate surface. This is an idealized model and does not attempt to account for complex interactions between the buffer layer and substrate. 483

Figure D.2: Plot of number of graphene layers $(\mathrm{N})$ as a function of intensity ratio $\left(\mathrm{IG}_{\mathrm{G}} / \mathrm{Isic}^{\mathrm{Sic}}\right)$. The dashed line corresponds to the pseudo-spline interpretation used to estimate the fractional coverage. 483 


\section{CHAPTER 1: INTRODUCTION}

The amount of literature available on graphene is staggering, with more being produced every year. To place this material in context and provide a basis for the subsequent discussion, this introduction presents a brief atomistic description of graphene (Section \$1.1), its current position in the world as a disruptive material as well as some general applications (Section $§ 1.2$ ), and concludes with a discussion some of the border challenges the material currently faces (Section $\$ 1.3)$.

Since graphene was theorized in 1947, it was suspected to have many remarkable properties across almost every conceivable metric. ${ }^{(1)}$ Finally isolated in 2004 (almost 60 years later), graphene attracted a veritable 'gold rush' of research attention. ${ }^{(2)}$ Initial efforts were aimed at testing and characterizing graphene to determine if it's theoretical properties would carry over into the real-world. In many cases they did, and in some cases, they even surpassed the predicted values. Over the past decade, the bulk research effort has shifted towards investigation into production methods of graphene and its applications. The work presented here focuses on both.

Graphene is classified as a "low dimensional" (low-D) material. A low-D material is a material where at least one of the dimensions is considerably smaller than the rest- to a point where the small dimension contributes little to the nature of the material and the remaining dimensions govern the material behavior.

It is worth noting that graphene is not the sole material to occupy this category. Quantum dots (0-D), carbon nanotubes (1-D), hexagonal boron nitride (2-D) are other examples of low-D materials. Graphene is itself a 2-D material, in that the two lateral dimensions are orders of magnitude larger than its depth, which is only one carbon atom in thickness. To a degree, all lowD materials share similar properties (specifically those properties which are derived from the inherent nanostructure of the materials). While graphene does share many properties with other low-D materials, graphene remains utterly unique, even among all other low-D materials. Of particular interest are graphene's unparalleled mechanical and electrical properties.

\section{\$1.1 Graphene and Its Properties: The Fundamentals}

Graphene has been thoroughly described numerous times in literature and so its structure is almost common knowledge in the scientific community, but it bares description once more here. 
All properties inherent to graphene are ultimately due to the bonding between the atoms which makeup graphene and the specific arrangement of the electron orbitals. Because of this, an understanding of graphene on an atomistic level is crucial for both the development of new graphene production techniques as well as for the intelligent design of sensing platforms based on graphene.

Graphene consists of a single atomic layer of $\mathrm{sp}^{2}$ hybridized carbon atoms arranged in a hexagonal pattern. In order to appreciate how the carbon atoms come to be in this type of arrangement, consider the electronic structure of atomic carbon in its ground state as depicted in Figure 1.1. Atomic carbon possesses a full 1s orbital, containing two tightly bound electrons that do not participate in any bonding events. In addition, there is a full 2 s orbital containing two electrons and two half-filled p orbitals; containing one electron each that are available for interaction with neighboring orbitals. In order for $\mathrm{sp}^{2}$ hybridization to occur, the carbon atom must enter an excited state where one electron from the $2 \mathrm{~s}$ orbital is promoted to a higher energy state as a third half-filled p- orbital. These orbitals then quantum mechanically mix, from a linear

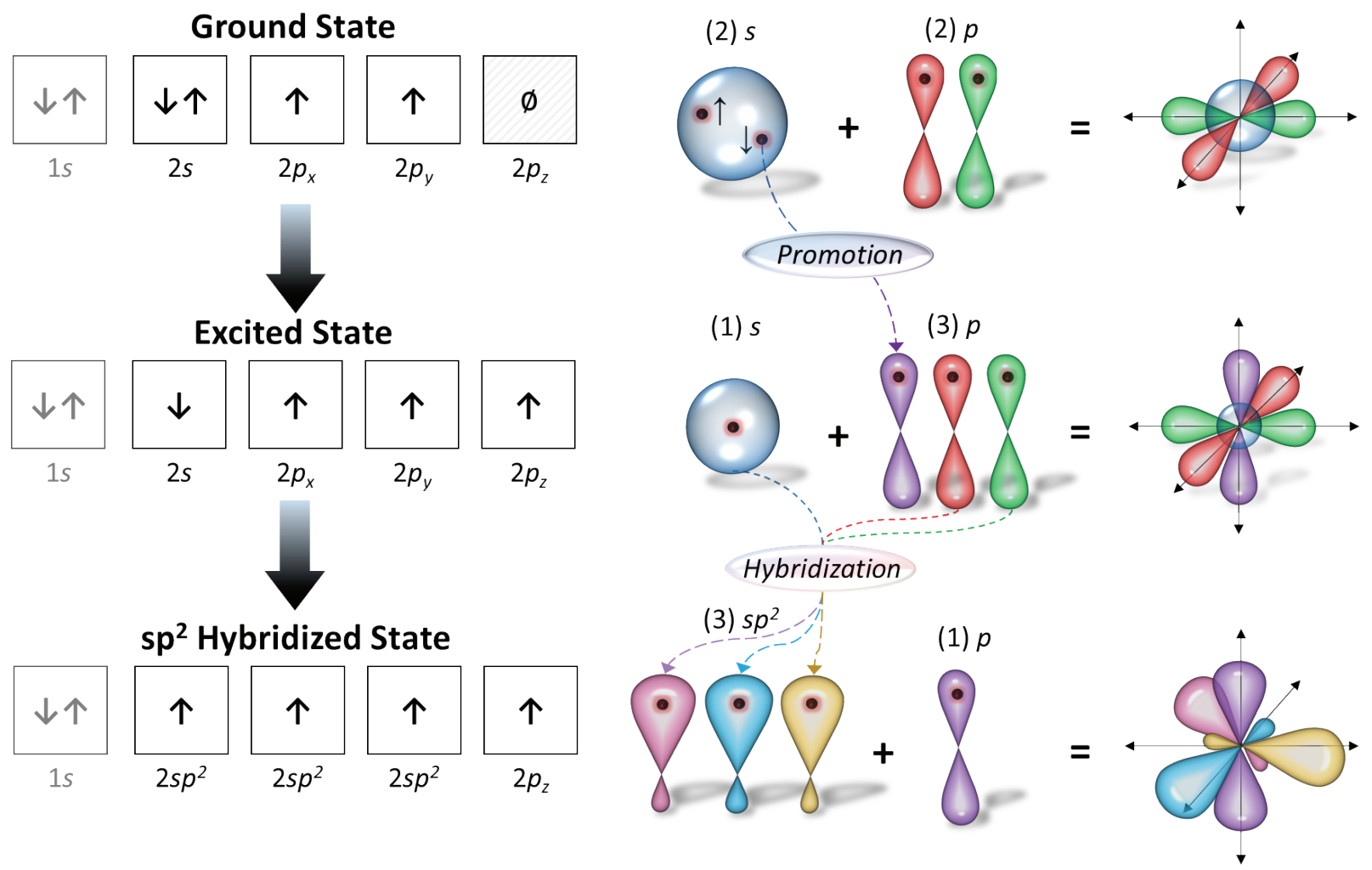

Figure 1.1: The evolution of a carbon atom as it transitions from its ground state to an excited state, then finally to a sp $\mathrm{s}^{2}$ hybridized state. Shown on the left are the electron spin configurations and on the right, the orbital configurations in $3 \mathrm{D}$ space. 
combination of states, to form the prototypical $\mathrm{sp}^{2}$ hybridized carbon atoms (recall that electron orbitales are simply a graphical representation of the probability distribution for the location of an electron, constructed from the solutions to the spherical harmonic equations).

A covalent bond between adjacent $\mathrm{C}$ atoms is formed when the $\mathrm{sp}^{2}$ orbitals from neighboring $\mathrm{C}$ atoms interact and from a $\sigma$ bond. This is illustrated in Figure 1.2. Each $\mathrm{C}$ atom forms three $\sigma$ bonds with three neighboring $\mathrm{C}$ atoms, which forces the atomic arrangement into a trigonal planar formation and is ultimately responsible for the 2-D nature of graphene. Each C-C bond has a high binding energy of $4.9 \mathrm{eV}$ and each $\mathrm{C}$ forms three of these bounds with its neighbors. ${ }^{(3)}$ The high bond strength and the resulting interlocking triangular arrangement is the basis for many of the robust physical properties of graphene. The remaining half-filled and unhybridized p- orbital orients itself perpendicular to the plane of the graphene sheet. Each of these $p_{z}$ orbitals overlap with their two nearest-neighbors and from a $\pi$ bond. The $\pi$ bonds then delocalize across the newly formed graphene ring then across the entire graphene sheet, forming a molecular orbital. It is this delocalization of $\pi$ orbitals and the high symmetry of the bonding which give rise to the unique electronic properties of graphene. ${ }^{(4)}$

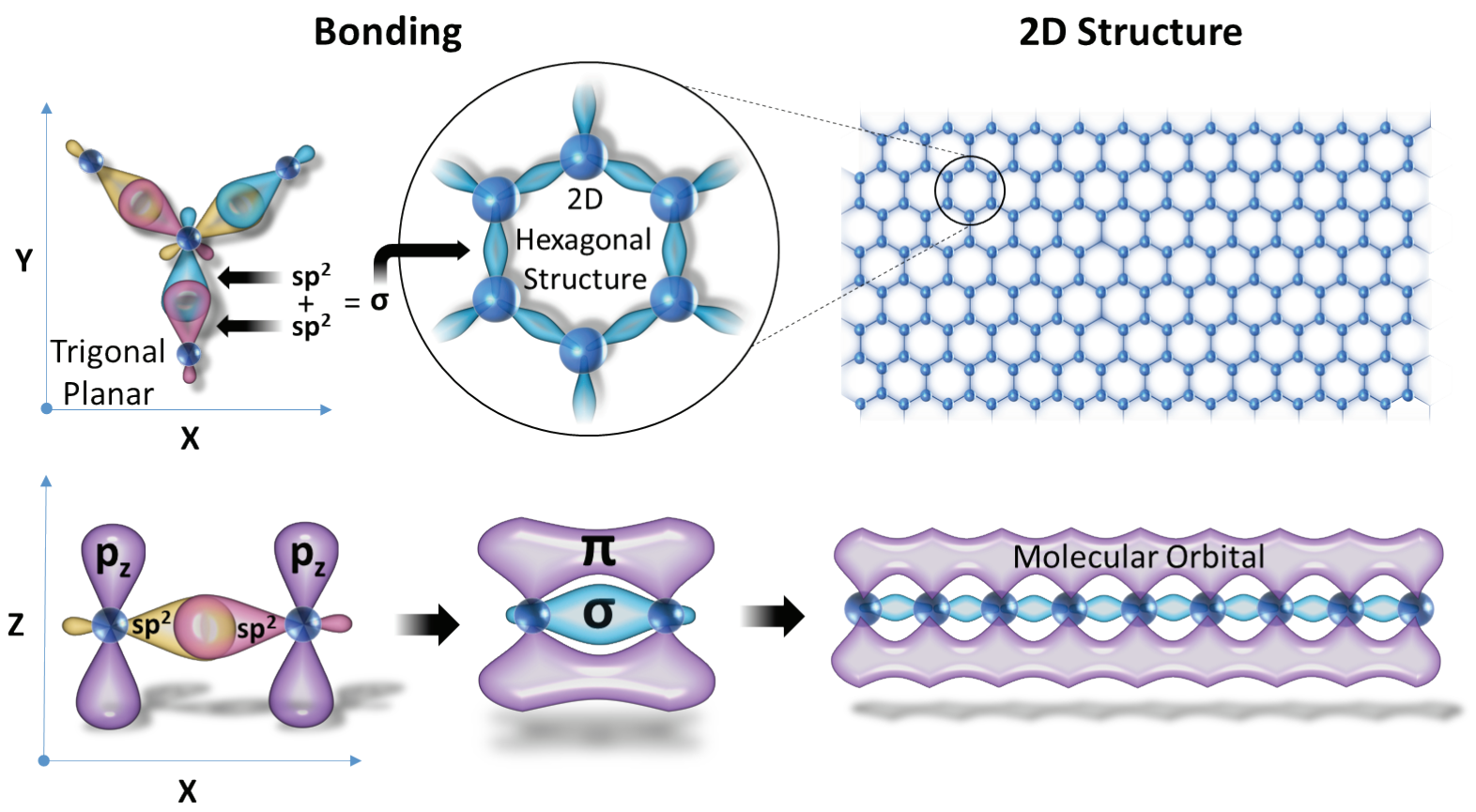

Figure 1.2: Simple schematic of the carbon electron orbitals and how the $\mathrm{sp}^{2}$ hybridized orbitales give rise to $\sigma$ the bonds in graphene. Also illustrated is the how the lone $\mathrm{p}_{z}$ orbital $\pi$ bonds and delocalizes into a molecular orbital.

Figure 1.3 shows the $2 \mathrm{D}$ crystal structure of graphene where $\mathrm{A}$ and $\mathrm{B}$ are inequivalent carbon atoms. The real space unit cell is triangular having unit vectors $\boldsymbol{a}_{1}$ and $\boldsymbol{a}_{2}$ and nearest- 
neighbor vectors $\boldsymbol{\delta}_{1}, \boldsymbol{\delta}_{2}$ and $\boldsymbol{\delta}_{3}$ which make the overall hexagonal pattern. ${ }^{(4)}$ The $\sigma$ bond formation results in a C-C bond length of approximately $1.42 \AA$ and a lattice constant of $2.45 \AA$ in graphene. (4) (5) This results in approximately $3.82 \times 10^{15}$ carbon atoms per centimeter (carbon atom areal density). ${ }^{(6)}$
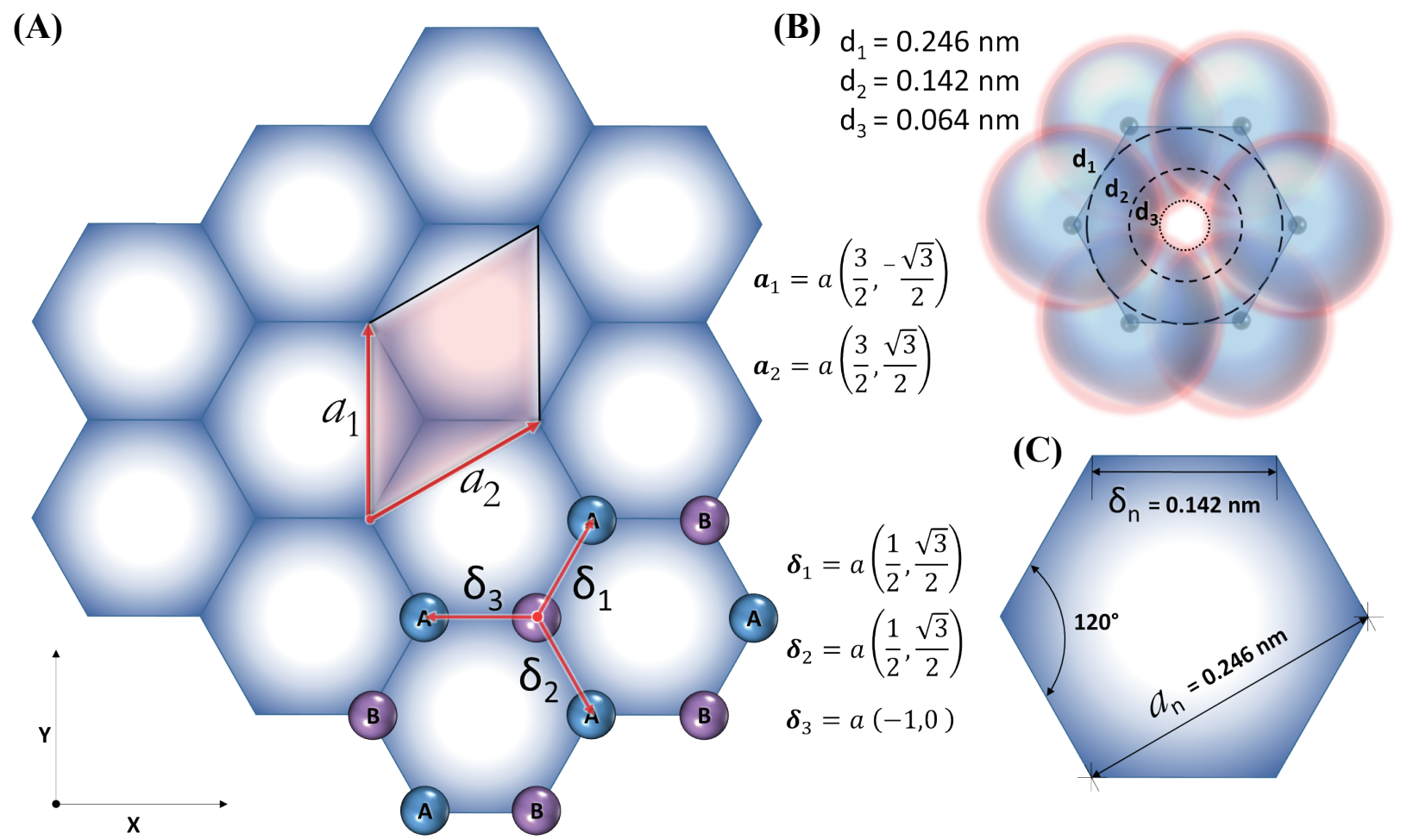

(C)

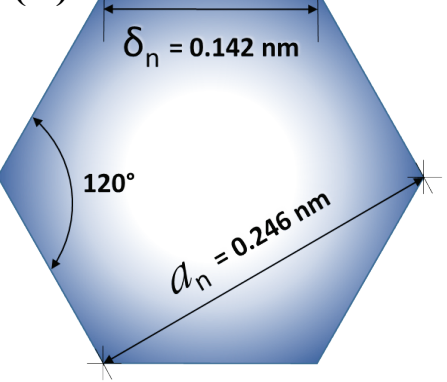

Figure 1.3: (A) The 2D crystal structure of graphene with the triangular unit cell, consisting of two inequivalent carbon atoms. The unit cell $\left(a_{\mathrm{n}}\right)$ and nearest-neighbor $\left(\delta_{\mathrm{n}}\right)$ vectors are given. (B) An illustration for the various pore sizes calculated for the graphene ring: $d_{1}$ using the C-C bond length, $d_{2}$ using a hard sphere model and $\mathrm{d}_{3}$ using the upper Van der Waals diameter. (C) Bond lengths and angles of a graphene ring as measured from the atom centers. (The widely used nomenclature can become somewhat confusing, note that the $a$ in the vector notation refers to the nearest-neighbor carbon length of $0.142 \mathrm{~nm}$.)

There has been some debate about the precise pore size (hole diameter) of the graphene ring, mostly resulting from the variations in definitions. This parameter plays a significant role on gas adsorption and intercalation, which are discussed later in this work. If only the C-C bond length (measured from carbon nuclei to carbon nuclei) is considered, a pore size of $0.246 \mathrm{~nm}$ is calculated. Of course, this does not consider the electron cloud (which is over three orders of magnitude bigger than the nucleus) and is therefore probably the least accurate. If a hard-sphere geometric model is used, a pore size of $0.142 \mathrm{~nm}$ is calculated. Using the carbon Van der Waals diameter, which has actual physical significance, would seem to most accurately reflect reality. However, the reported values for the Van der Waals diameter vary substantially. Values ranging from $0.300 \mathrm{~nm}$ to 0.388 
nm have been reported, depending on the calculation method. ${ }^{(7)}$ These are obviously too large for the $\mathrm{C}-\mathrm{C}$ spacing $(0.142 \mathrm{~nm})$ for carbon bound in a hexagonal pattern, in that the electron clouds would overlap the neighboring nuclei. Therefore, using an effective Van der Waals diameter of $0.220 \mathrm{~nm}$, the value at which the carbon spheres just touch the nearest-neighbor nuclei, a pore size of $0.064 \mathrm{~nm}$ is calculated. Thus, an upper bound of $0.220 \mathrm{~nm}$ for the carbon diameter is shown in Figure 1.3(B), which is consistent with the value used for graphene permeability studies. ${ }^{(3)}$

Regardless of the carbon values used, the sphere model lacks fidelity because, as was discussed earlier, the electron clouds around the carbon atoms are highly distorted to from the covalent $\sigma$ bonds and the dangling $\pi$ bonds and are thus not spherically distributed around each carbon nucleus. This is not to mention the thermally induced bond stretching, twisting and bending which is always occurring. However, the idealized model depicted in Figure 1.3(B) provides as a very useful conceptual viewpoint.

Graphene, having a second order elastic stiffness of $340 \mathrm{~N} \cdot \mathrm{m}^{-1}$ and a breaking strength of $42 \mathrm{~N} \cdot \mathrm{m}^{-1}$, is the strongest material ever tested. ${ }^{\left({ }^{(8)}\right.}{ }^{(9)}$ It has been noted that while the graphene bulk is incredibly robust, the graphene edges are fragile; this explains why graphene is "nanoscopically strong but is macroscopically weak". ${ }^{(10)}$ Graphene is one of the most thermally conductive of all carbon allotropes. ${ }^{(11)}$

Graphene has been observed displaying super-thermal-conductivity, as high as 5500 $\mathrm{W} / \mathrm{m} \cdot \mathrm{K}$, as well as many other unusual thermal characteristics. ${ }^{(12)}$ (13) These unique thermal characteristics are derived from the unique nature of the 2-D phonons which are the primarily heat carriers in graphene. ${ }^{(14)(15)}$ Graphene has many other interesting properties, though not explored here, they are still an active area of research. ${ }^{(16)}$

The attributes of graphene which have attracted the most attention are its superb electrical properties. Pristine graphene is a zero-band gap semiconductor. Therefore, any energetic or electronic perturbation to the system can easily promote charge carriers from the valence band into the conduction band, effecting graphene's conductivity. Due to the absence of an energetic band gap between the fully filled valence band and the empty conduction band, the zero-band gap nature of graphene means that essentially no (or very little) energy is required to do this. This makes pristine graphene very sensitive to its environment. Because conductivity is a function of both carrier concentration and carrier mobility, the interplay between these two factors can become 
complex when viewed as a function of temperature (this becomes relevant to this work in Section $\$ 5.2)$.

In general, an increase in temperature tends to increase the population of the conduction band, leading to an increase in charge carrier concentration and thus conductivity. Simultaneously, a decrease in conductivity occurs due to the generation of phonons in the graphene lattice. These phonons interact with and scatter the conduction elections (thereby effectively decreasing the mean free path of the charge carriers). This decreases charge carrier mobility and thus conductivity. ${ }^{(17)}$ Thermally induced rippling and buckling of graphene sheets has been shown to increase the electrical resistance as well. (18) (19) (20) (21) (22) These competing effects are always present and determining which effect is dominant is dependent on the specifics of the graphene system in question; the complexity of the system is further complicated by doping from the electrical contacts and other environmental effects, such as various substrate interactions. At low temperatures, suspended single layer graphene has been shown to display extremely high charge carrier mobilities and near ballistic transport has been observed. (23) (24) In single layer graphene, electron mobility as high as 200,000 to $250,000 \mathrm{~cm}^{2} /(\mathrm{V} \cdot \mathrm{s})$; even higher values, have been reported in two and three-layer graphene. (25) (26) (27) (28) (29) This high mobility arises from the lack of scattering sites in pristine graphene and occur at low carrier concentrations.

Graphene can be modified (doped) so that it possesses a nonzero band gap. In this case it exhibits a behavior more closely resembling that of a semiconductor. This is important for electronic device applications of graphene where the conductivity of graphene needs to be turned 'on' or 'off', the principle role of a semiconducting component in a device. There are many ways that a band gap may be created in graphene. Chemical modification, molecular doping, quantumwell formation, substrate selection and doping with other Group IV elements all induce an energy band gap. (30) (31) (32) (33) (34) Even by modifying the stacking order in few-layer graphene, a band gap can be opened. ${ }^{(35)(36)(37)}$

While the intrinsic conductivity of modified graphene is lower, an increase in conductivity with increasing temperature has be seen over certain regimes. (38) (36) (39) (40) Higher temperatures result in higher conductivity by providing the necessary energy (thermal) to promote electrons from the valence band across the band gap to the conduction band. Conductivity continues to increase until the competing effects of scattering (thermally induced phonons, crowding of the conduction band, etc.) become more pronounced. ${ }^{(41)}$ The temperature at which this occurs is 
dependent on the band gap width, the amount and type of impurities and by what mechanisms the band gap was induced into the material. ${ }^{(42)}$ Others have shown that through careful control of these parameters the band gap can be selectively tuned. Values as large as $\sim 50 \mathrm{meV}$ to $130 \mathrm{meV}$ have been reported. ${ }^{(43)(44)}$

It is worth noting that many properties of graphene can be tailored to a specific application by functionalization of the surface. This involves the addition of impurities or defects and other chemical modifications. ${ }^{(9)(45)(46)(44)(47)(48)(49)}$ This further speaks to the incredible versatility of graphene. Many of the typical properties reported for graphene are for pristine, ultraclean, suspended graphene flakes. However, for many foreseeable applications graphene will not be freestanding but instead attached to a substrate. This can complicate the characterization of the graphene system as the type of substrate and the degree of interaction greatly affect the electrical and thermal properties of the graphene. ${ }^{(50)(51)(52)(53)(54)}$

In 2014 (the 10-year anniversary of the discovery of graphene), there were 44 companies actively producing graphene and graphene-based materials. ${ }^{(55)}$ As of mid-2019, there are over 200. ${ }^{(56)}$ It is clear that graphene is on the rise.

\section{\$1.2 General Applications of Graphene: Graphene's Place in the World}

This section will first discuss graphene's status around the globe before highlighting just some of the applications of graphene. It will conclude with an argument for why the field of sensors was chosen as a testing ground for the newly developed plasma assisted method for graphene synthesis which has been pioneered by the Stinespring lab group.

Given the large number of fantastic graphene properties, it comes as no surprise that the range of proposed applications is similarly huge. The broad (and justifiable) enthusiasm for graphene has led to many exciting avenues of research and proposed applications; some more ambitious than others. They include everything from smart contact lenses and hair dye to space elevators. (57) (58) (59) Such a bold approach is healthy for the rapid development of a nascent technology. Furthermore, there are many intangible benefits to the aggressive and public pursuit of graphene. The hype surrounding this "super-material" has not only served as a source of inspiration for future scientist but has ignited the imagination of the general public. A simple google search for 'Graphene' returns over 41 million results. Beyond this, such a groundbreaking 
material demands an ambitious approach. Given its potential, it would be irresponsible to approach it any other way.

As can be seen in Figure 1.4, every year since its isolation, there has been a year-over-year increase in both the number of peer reviewed graphene publications and in the number of patents awarded. This indicates that not only is graphene more relevant than ever, but that the world's interest in graphene is accelerating. Furthermore, the fact that the number of patents is growing at an ever-increasing rate indicates that graphene is rapidly finding real-world applications.

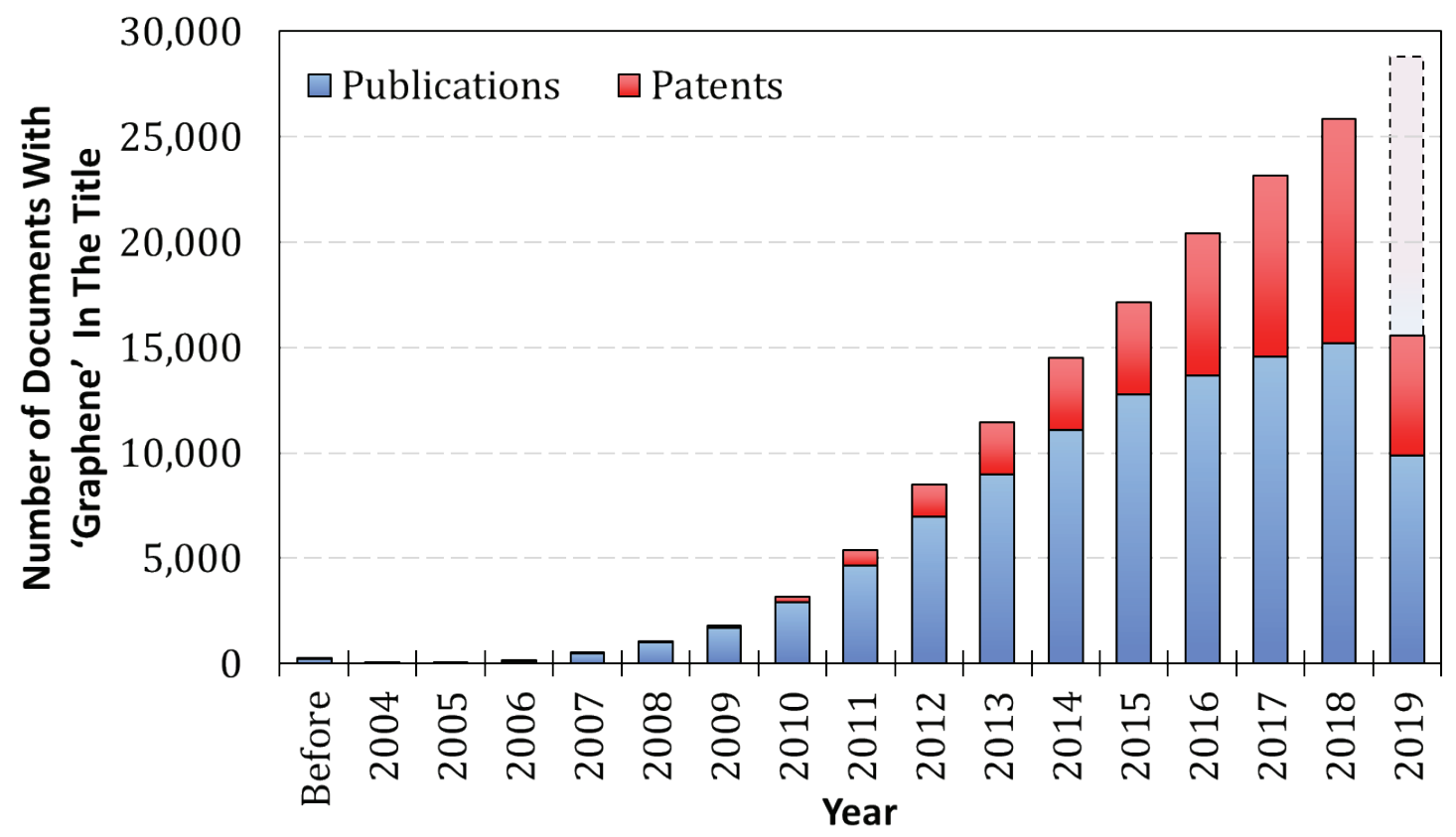

Figure 1.4: While various databases and reports provide slightly different numbers the trend is clear. The world's interest in graphene continues to accelerate. Note that only partial data for 2019 is available at the time of this writing; the dotted line indicates a projection value.

Figure 1.5 gives further context for the publication data shown in Figure 1.4. Figure 1.5 shows the breakdown of the $\sim 105,000$ graphene peer reviewed publications between 2004 to mid2019, by county. Clearly, graphene has attracted worldwide interest. In terms of the amount of published research (and as will be discussed next, funding moneys), graphene research is dominated by Asia-Pacific region, which is led by the People's Republic of China. Globally, the United States and, then the collective countries of the European Union, follow in sequence. Data was collected from the Web of Knowledge database. ${ }^{(60)}$ 


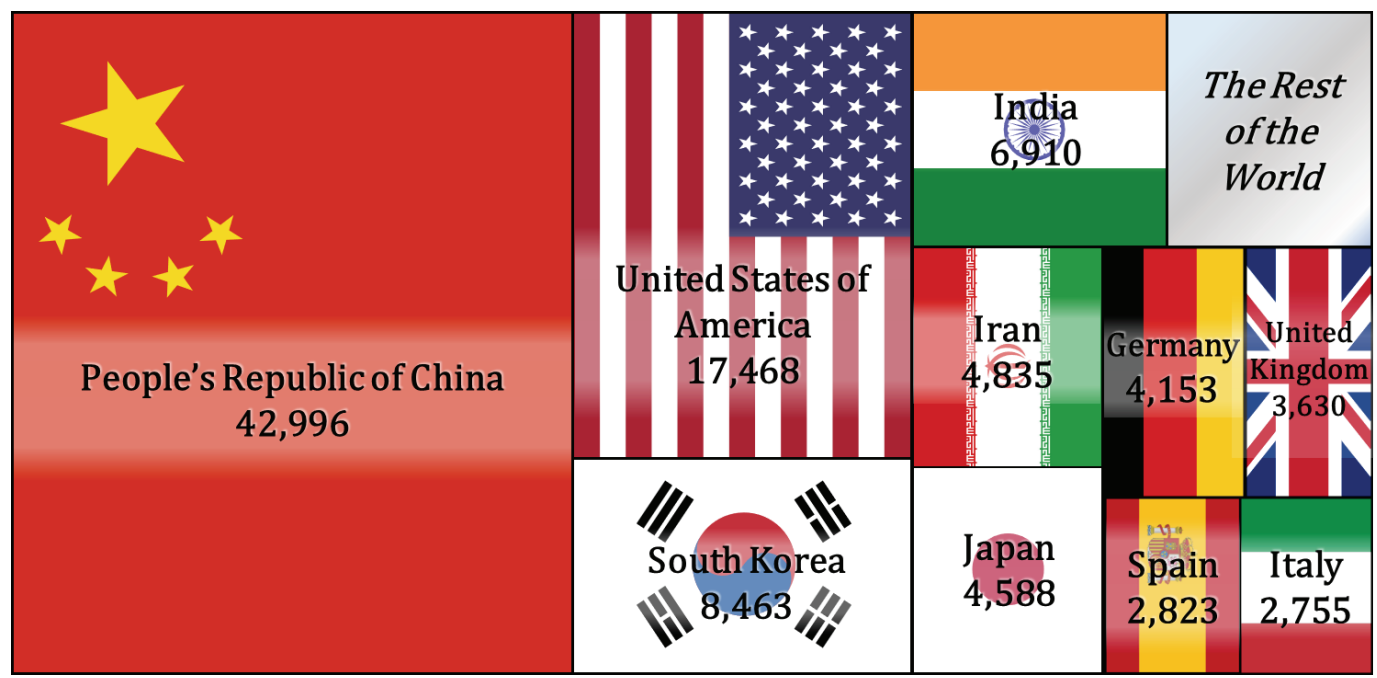

Figure 1.5: Tree map of graphene publications broken down by country of origin (tile area is proportional to the number of peer-reviewed publications with 'graphene' in the title.).

Figure 1.6 gives the same data, broken down by field of study. Note there is a high degree of crossover between research areas (a single paper may fit multiple categories). The wide range of research areas speaks to the fundamental versatility of graphene.

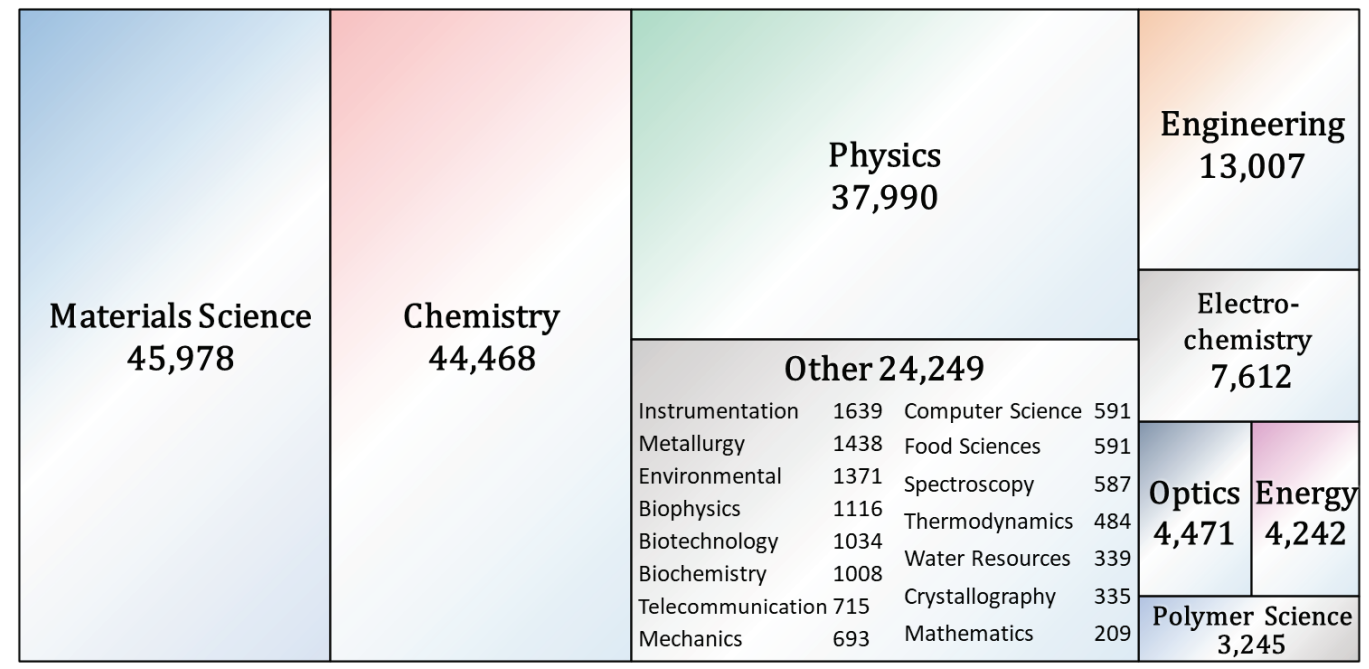

Figure 1.6: Tree map of graphene publications broken down by field of study (tile area is proportional to the number of peer-reviewed publications with 'graphene' in the title.).

A breakthrough in any one area of graphene development will have profound implications to both the scientific community and the world. Because of this, both corporations and world governments have invested heavily into the development of graphene. Because it is not known from which direction a breakthrough may occur, many governments have taken an 'all-of-theabove' approach to graphene funding. One of the more notable examples of this is the European Unions (EU) founding of the Graphene Flagship. Founded in 2013 with a 10-year operational 
budget of $€ 1.38$ billion euros ( 1.55 billion USD), it consists of over 150 academic and industrial research groups. ${ }^{(61)}$ The National Graphene Institute, a part of the University of Manchester (Manchester, UK) where graphene was first isolated in 2004 and funded jointly by the UK and EU, was opened in 2015 for a cost of $€ 53.3$ million euros (\$60 million USD). ${ }^{(62)}$ There are similar efforts all over the world. ${ }^{(63)}$ In the Asia-Pacific region, the Chinese government has heavily invested in graphene through both the support of state-owned enterprises as well as the creation of the $¥ 10$ billion yuan ( $\$ 1.57$ billion USD) Datong Graphene and Green Technology Industrial Park, and in 2018 with the founding of the Beijing Graphene Research Institute for an unspecified amount. ${ }^{(64)}{ }^{(65)}$ Private sector entities, such as Samsung Inc. and LG Electronics, lead South Koran efforts. In the U.S., graphene research occurs mainly within academic institutes supported by individual government agencies (National Science Foundation, Department of Energy, Department of Defense and National Institute of Standards and Technology, and others) but there is currently no high-level U.S. policy for building out the U.S. graphene industry. ${ }^{(66)}$

It has been predicted by both U.S. and European experts that there is a coming 'space-race' in development of graphene because of the significant national security implications. There have been several hearings held by the U.S. Congress with testimony by various field experts making the case for graphene and its strategic importance. ${ }^{(66)(67)(68)(69)}$ In 2017, the European Defense Agency (EDA) commission a study to investigate graphene's potential for defense applications. (70) (71) The full report is expected to be released at the end of 2019, however the pre-release executive summery states that graphene shows great potential in a range of defense applications, including sensors and optoelectronics. ${ }^{(72)}$

The development of graphene has not occurred solely from government and academic efforts. The private sector has been actively pursuing graphene as well. In fact, while $36 \%$ of graphene related intellectual property (IP) is owned by academic and government entities, the plurality, at $41 \%$, is owned by corporations (the balance being owned by private/public partnerships) as of 2018. ${ }^{(73)}$ This private sector interest in graphene has resulted in over 200 graphene startups being founded in the past few years, many as spinoffs from academic institutions. Some of these are shown in Figure 1.7. ${ }^{(74)}$ 


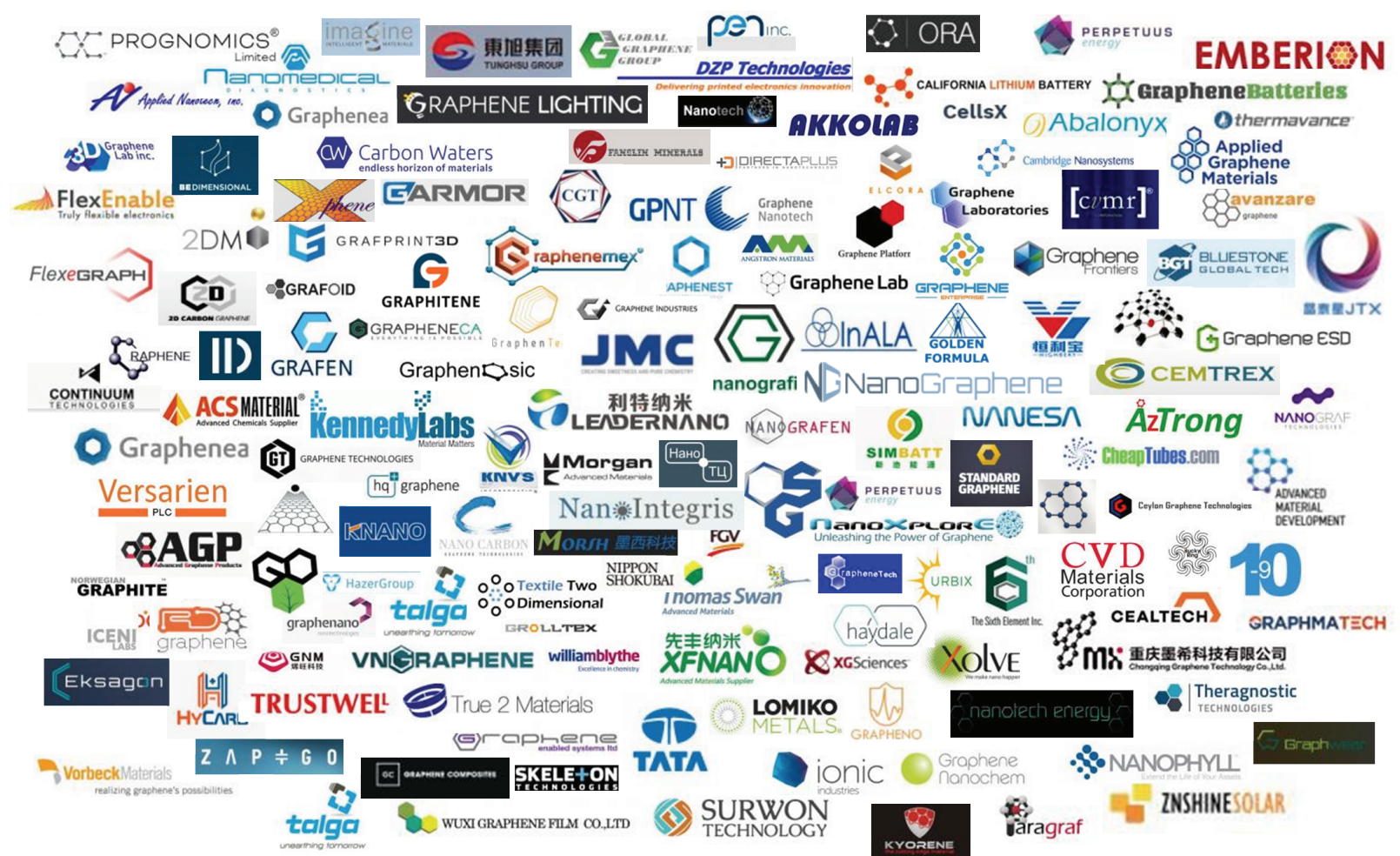

Figure 1.7: Just some of the graphene-centric companies founded over the past few years. While their degree of success remains to be seen, this does indicate there is money to be made in graphene technology.

While most of the companies shown in Figure 1.7 are privately held, there are many that are publicly traded. As of July 2019, the top 13 graphene focused companies have a total market capitalization of over $\$ 754.74$ million USD. ${ }^{(75)}$ While this is relatively small, it does reflect the general public's willingness to bet on graphene. Perhaps more notable is the fact that many large, well-established companies have also invested heavily into graphene research. Samsung Inc. is, by far, the largest single holder of graphene IP. (76) (77) Other well-known companies also hold substantial graphene IP including IBM, Intel Co., Sony, Lockheed-Marten, Boeing Co. and many others. (73) (78) (79) (80) The competition for graphene IP is occurring on a global scale. As of 2018 , of the top 30 companies (as ranked by number of secured graphene patents), 23 are based in China. (81) At 32,142 China holds $\sim 57 \%$ of all global graphene patens (South Korea is second with $\sim 7,000$

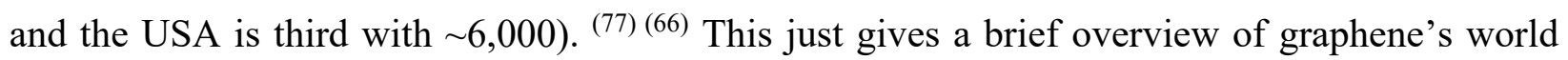
position at the time of this writing. However, there are more detailed reports published daily and, given the rapid pace of graphene advancement, more up-to-date information can easily be found.

As previously stated, there are a huge number of graphene applications. There are many popular, highly in-depth review articles which cover the topic. ${ }^{(82)(83)(84)}$ Provided in Table 1-1 is a list of some of the broad application categories and is by no means an exhaustive list. However, 
it provides a snapshot of the wide range of applications of graphene. The citations include recent review articles and highly referenced research articles which detail each category more thoroughly and represent a starting point for the interested reader.

Table 1-1: Categorical list of select graphene applications.

\begin{tabular}{|c|c|}
\hline \multicolumn{2}{|l|}{ Physical } \\
\hline Composites & \\
\hline Mixes & $(85)(86)(87)$ \\
\hline Coatings & $(88)(89)$ \\
\hline Other & \\
\hline Membranes & $(90)(91)(92)$ \\
\hline Actuators & (93) (94) (95) \\
\hline \multicolumn{2}{|l|}{ Electrical } \\
\hline \multicolumn{2}{|l|}{ Sensors } \\
\hline Electrochemical (gas, vapor, liquid) & (96) (97) (98) \\
\hline Photonic & (99) (100) \\
\hline \multicolumn{2}{|l|}{ Electronics } \\
\hline $\begin{array}{l}\text { Discrete Components } \\
\text { (Supercapacitors, high-speed electronic, etc.) }\end{array}$ & $(101)(102)(103)(104)$ \\
\hline Antennas & $(105)(106)(107)(108)$ \\
\hline Transparent and flexible films & $(109)(110)(111)$ \\
\hline \multicolumn{2}{|l|}{ Energy } \\
\hline Energy production (solar cells, fuel cells, etc.) & $(112)(113)(114)$ \\
\hline Energy storage & $(115)(116)(117)$ \\
\hline Photonics and Optoelectronics & $(118)(119)(120)(121)$ \\
\hline \multicolumn{2}{|l|}{ Chemistry } \\
\hline Biomedical Technologies & $(122)(123)(124)$ \\
\hline Catalysis & $(125)(126)$ \\
\hline Other & (127) \\
\hline
\end{tabular}

Table 1-1 has been broken down into three broad categories where each application category capitalizes or exploits a particular feature of graphene. The 'Physical' category applications take advantage of graphene's robust and rugged mechanical properties and/or its nanoscopic nature (incredibly high breaking strength, hardness, thermal conductivity, subnanometer pore size, etc.). The 'Electrical' category takes advantage of the unique electrical properties of graphene (high carrier mobility, low carrier count, zero band gap, plasmonic behavior, etc.). The 'Chemistry' category encompasses applications which utilize graphene as a 
tunable nanoscale substrate. In these applications, graphene is modified through the addition of irregularities in the structure (defects sites, functional groups, adatoms, etc.).

Of course, many applications fit multiple categories and utilize multiple aspects of graphene simultaneously. For example, conductive high strength composites seek to capture both the robust mechanical properties of graphene and its high thermal and electrical conductivity. ${ }^{(128)}$ (129) The field of Sensors is another area where multiple aspects of graphene are harnessed. For example, high temperature gas sensors would utilize the thermodynamic stability of graphene, its unique electrical properties and nanoscale nature.

As will be discussed in the following section, this work explores a newly developed plasma assisted method of graphene synthesis, as well as potential applications for the graphene it produces. In 2018 it was reported that the largest single technological application of all granted graphene patents (at 34\%) were for electronic applications. ${ }^{(73)}$ Clearly, the industry sees the electronic applications of graphene to be particularly promising. Of these electronic applications, sensors are an attractive candidate specifically for the evaluation of graphene produced through new synthesis methods.

There are four primary reasons for this. First, sensors construction is relatively simple since it requires few processing steps. In this work, for example, only three steps were required; contact deposition, wire bonding and package assembly. Second, sensor testing likewise straightforward. Third, the performance of these devices provides valuable insight into the relationship between material properties and the synthesis technique. And forth, there is widespread interest for new sensing devices. Therefore, to better understand the nature of this new synthesis method and to evaluate its potential in a real-world application, the field of sensors was chosen as a demonstration platform. A simple sensor design was adapted for both gas sensing and photo detection.

The plasma assisted method of graphene synthesis developed here is of interest because it addresses what is perhaps the single greatest challenge graphene faced by graphene: scalable graphene production. This is particularly important for electronic applications. This is achieved by providing an alternative method for the growth of graphene on a robust electrically insulating, thermally conductive semiconductor, using established semiconductor processing tools and techniques. 


\section{\$1.3 Summary and Grand Challenges: Initial Problem Statement}

In the realm of disruptive technologies, graphene is often compared to silicon or aluminum. Aluminum, first isolated in 1825 (Hans Christian Ørsted), only found widespread use as we know it today due to the importance of the airplane in World War I. Silicon, isolated in 1825 (Jöns Jacob Berzelius), is of course responsible for the semiconductor revolution. Both took over 100 years between their discovery and their appearance on the world stage. As to where graphene is in its technological development, only time will tell. However, using history as a guide, graphene is still in its infancy (or is at least a teenager, at 15 years old). As such, graphene still faces significant technological hurdles.

There is widespread agreement within the scientific community that graphene has the potential to be a revolutionary material. There are even whispers that graphene will become its own named era, much like the bronze or iron ages. However, for this to happen, the single greatest obstacle to the proliferation of graphene technology must be solved: producing graphene on a truly industry scale. Put simply, for graphene to ever leave the laboratory, methods must be developed for the mass production of high-quality graphene. This is especially true for high-performance electronic applications of graphene. Furthermore, the difficulty in producing quality graphene slows the advancement of the material by limiting the research of graphene devices to those entities who can produce a good quality graphene sample, a feat unto itself. If provided a reliable source of good quality graphene, the free market will solve many of the challenges graphene faces, while finding the best and most creative applications.

Many truly amazing devices have been fabricated from graphene. However, they are all 'one-off' research grade devices whose production cannot be easily scaled. As an example, the famous $300 \mathrm{GHz}$ transistor was constructed from a single graphene flake, mechanically exfoliated from a graphite block. ${ }^{(104)}$ The often-cited case of the $200,000 \mathrm{~cm}^{2} /(\mathrm{V} \cdot \mathrm{s})$ career mobility study was carried out by first “...optically locating a single-layer mechanically exfoliated graphene flake on top of a silicon [oxide] substrate..." and then building a device on top of it. ${ }^{(26)}$ These, and similar examples, are all truly amazing feats and represent important milestones in the maturation of graphene technology. They simultaneously showcase the fantastic potential of graphene and also highlight the need for a synthesis method which is scalable and manufacturable.

Chapter 2 will discuss various methods of producing graphene but in short, graphene can currently be produced in either, A) large amounts of poor-quality graphene (e.g. by chemical 
exfoliation) or B) very small quantities of pristine graphene (e.g. by mechanical exfoliation), but not both at the same time. Clearly, manufacturability of quality graphene is a critical issue. The global semiconductor industry, from the supply chain to fabrication tools, has evolved around and been built on the processing of material wafers. Even today, all integrated circuit and most electronic devices started out in one form or another as a wafer of semiconductor material. For the rapid adoption of graphene technology into the electronics industry, synthesis methods need to be compatible with today's semiconductor wafer processing pipeline. For this to happen, high-quality graphene crystal sheets would need to be synthesized at predefined locations across an entire wafer surface- potentially at multiple steps in the processes.

Furthermore, the recent move towards 3D integrated circuits further necessitates the ability to grow graphene in situ. This trend of expanding in the vertical dimension is occurring both within discreet component (for example the 3D Tri-Gate FET, FinFET and other multi-gate devices ${ }^{1}$ ), as well as in the bundling and incorporation of multiple complex components on a single chip. ${ }^{(130)}$ (131) (132) In fact, many next-generation sensors build the sensor material on the same chip as the related circuitry. ${ }^{(133)}$ Looking further into the future, there has been discussion of what will become the successor to the FinFET. Stacked 'nanosheet devices' promise to improve on all fronts; device density, power consumption and switching speeds. ${ }^{(134)}$ However, the nanosheet designs add further manufacturing complexity to their fabrication. The need for an integratable graphene growth process is clear.

A perfect solution to the graphene production problem for electronic devices would involve a synthesis method which grows graphene direction on an electrically insulating (and semiconducting), thermally conductive wafer, using a process which can scale with the wafer dimension and produces uniform graphene coverage and quality. Such a solution should be easily incorporated into the currently deployed fab technology pipeline and would pave the way for its rapid adoption without adding process complexity or substantially decreasing unit yields.

Chemical vapor deposition (CVD) and the selective sublimation methods of graphene growth both show promise as wafer scale technologies, however each possess their own

\footnotetext{
${ }^{1}$ Fin Field Effect Transistor (FinFET) first successfully commercialized by the Intel Corp. in 2012 using a 22nm process (using the 3-D Tri-Gate transistors variation), and then in 2016 by AMD using a $14 \mathrm{~nm}$ process. The FinFET is now the de facto industry standard for the transistor gate designs used in mainstream CPU microarchitectures. (723) (724) Base on widely available news reports, the current commercially available state-of-the-art, at the time of this writing (4Q2019), is AMD's 7nm process which uses 300mm wafers, produced by TSMC (Taiwan Semiconductor Manufacturing Company). (725) (726)
} 
technological difficulties. The CVD method requires removal of the graphene from the catalytic substrate and the selective sublimation results in inconsistent growth and pit formation. This work explores a plasma assisted growth method that, although not without its own challenges, presents an alternative approach to growing graphene films directly from a semiconducting substrate.

\section{\$1.4 Document Layout}

This document consists of seven chapters, each broken down into sections and subsections. Chapter 1 introduced graphene as a material (Section \$1.1), gave a broad overview of the global research efforts (Section \$1.2) and highlighted the basic motivation of this work (Section $\$ 1.3$ ). Chapter 2 expands on this by providing an overview of various graphene synthesis methods (Section \$2.1), including the novel method used in this work (Section \$2.1.5), and selected electronic sensing applications (Section $\$ 2.2$ ). This provides context for the goals of this work, which are explicitly stated at the end of the chapter (Section $\$ 2.3$ ).

Chapter 3 describes how these goals were approached by describing the experimental setups, materials and methods used. Each step of the graphene synthesis process is detailed (Section \$3.1). This is followed by a description of the various characterization techniques used as well as an overview of the fundamental principles of operation underpinning each technique, so that the data presented later may fully appreciated (Section \$3.2). The electronic device design and assembly process is detailed (Section \$3.3). Lastly, the experimental testing apparatus for gas sensing (Section \$3.4) and photo detection (Section \$3.5) are described.

Chapter 4 details the results and discussion pertaining primarily to research Goal 1 . This starts with characterization of the basic graphene synthesis process (Section $§ 4.1$ ), followed by a parametric investigation (Section \$4.2) and the effects of post synthesis high temperature $\mathrm{H}_{2}$ treatments (Section \$4.3). Chapter 5 details the results and discussion pertaining primarily to research Goal 2. The electrical characterization results are presented and discussed at the start of the chapter (Section \$5.1). The temperature dependences of the electrical device parameters are discussed next (Section §5.2). This is followed by an investigation into the device response to gas exposure (gas testing) (Section §5.3). The response to light irradiation (photo detection) concludes the chapter (Section §5.4). 
Chapter 6 provides a summary of each chapter (Sections $\$ 6.1$ - \$6.5). Chapter 7 presents the overarching conclusions and contextualizes them in the frame of the larger research landscape (Section §7.1). Finally, suggested future directions are provided (Section §7.2).

An extensive appendices section is included at the end of this document which is referenced throughout the main text. APPENDIX A provides supplemental information and data. APPENDIX B details preliminary studies into four different metallic nanoparticle decoration methods. APPENDIX $\mathbf{C}$ details the investigation on the origins and mechanisms of two metallic contamination phenomena encountered during this work. APPENDIX D details the methodology of the layer calculation as determined by XPS analysis. 


\section{CHAPTER 2: BRIEF REVIEW OF RELEVANT LiterATURE AND STATEMENT OF RESEARCH GOALS}

This chapter begins with a discussion of the most common methods for currently employed for synthesizing graphene (Section $\$ 2.1$ ), followed by its utilization in select sensing applications (Section $\$ 2.2$ ) and finally, the specific goals of this research work (Section $\$ 2.3$ ). No attempt has been made to provide a comprehensive review- merely a relevant synopsis. There are entire book volumes written on the subject which go into greater breadth and depth. ${ }^{(135)(136)}$ The information provided here has been chosen so as to provide a framework and context by which the research presented here may be evaluated.

\section{§2.1 Graphene Fabrication Methods}

A large segment of past graphene research has been dedicated towards discovering new methods for its production, with the ultimate goal of large area, high quality and low-cost graphene. Because of this, there are now a wide array of production methods from which to choose, each offers its own advantages and some methods are more suited towards specific applications than others. There are many review articles comparing the different methods, and they can be found in the references here. ${ }^{(17)(137)(138)}$ A summary of the most popular production methods is discussed next to provide context for the novel plasma assisted graphene growth method described in this 
section. A flow chart can be found in Figure 2.1 illustrating the relationships between the different methods in the context of the carbon source used to produce the graphene films.

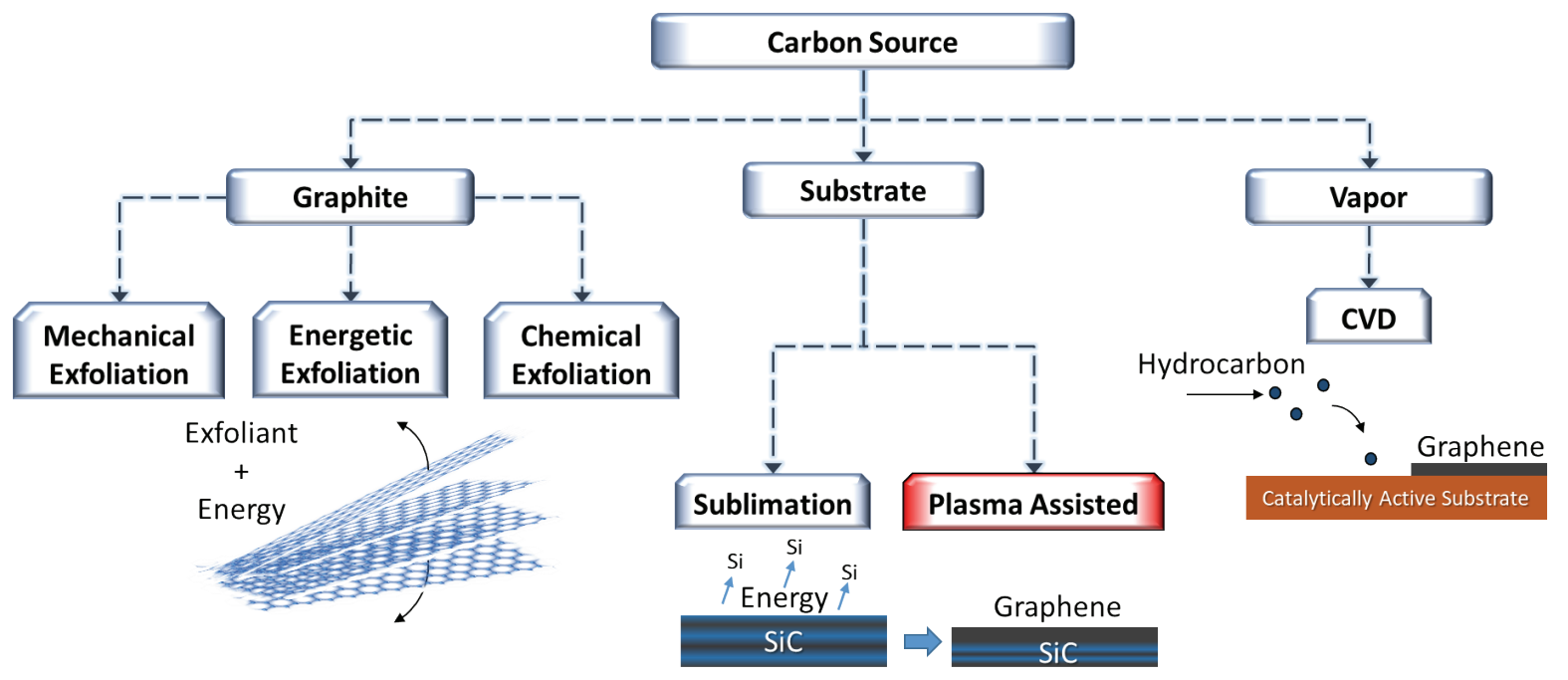

Figure 2.1: Flow chart showing the relationships between the source of carbon and the graphene production method. The plasma assisted method has been developed and the method used in this research. Although most comparable to the sublimation method, the plasma assisted method is inherently different.

\section{§2.1.1 Exfoliation}

Perhaps the most well-known method for graphene production, mechanical exfoliation, was used to produce isolated graphene for the first time in $2004 .{ }^{(2)}$ Due to the small inter-planar binding energy of $\sim 25 \mathrm{meV}$ per atom between graphene sheets (corresponding to a force of $\sim 300$ $\mathrm{nN} / \mu^{2}{ }^{2}$ ) mechanical delamination of carbon layers is easily achieved. ${ }^{(139)(137)}$ Using adhesive tape, a thin layer of graphite is removed from a sample of highly ordered pyrolytic graphite (HOPG). The graphite is then laboriously whittled down layer by layer with additional adhesive tape until 1 to 3 layers are left (termed "few-layer graphene"). The graphene is then transferred to a more suitable substrate. Because this method is known to produce the highest quality graphene (most of the exceptional properties commonly cited for graphene were measured using graphene produced with this method) there has been a concerted effort to improve this method and significant progress has been made; there are now many different methods used to mechanically exfoliate graphene. ${ }^{(140)}$ However, these methods are still inherently labor and time intensive, very difficult to scale up and unreliable. This severely impedes its use in applications other than lab scale experiments.

In an attempt to overcome the limitations of the original tape exfoliation method, many types of chemical exfoliation were developed. These methods use a variety of chemical, electronic 
and ultrasonic techniques to delaminate the carbon layers in HOPG to produce graphene. ${ }^{(141)(142)}$ (143) (144) While these new methods dramatically increase the yield, the product is of lower quality. In general, due to the relatively harsh environments and high shearing forces required to first reach and then chemically degrade the van der Waals forces between the stacked carbon layers in HOPG, these methods damage the graphene layers. This degrades their unique properties and typically results in some form of graphene oxide. ${ }^{(145)(146)(147)}$ Graphene produced via these methods is more suited for applications where the basic mechanical properties of graphene are of interest (rather than the electrical properties) and high quantities are sought over high quality. For example, in polymer mixes and other bulk applications, where 10's to 1000's of kilograms of graphene are required, as compared to electronic applications where atomically thin-films of high-quality graphene are required. (148) (149) (150) (17) These bulk production methods tend to be carried out in the liquid phase. This is done to either facilitate the dispersion of a chemical surfactant and/or exfoliation agent or, to mediate the dispersal of added energy to assisted in the exfoliation (i.e. ultrasonic power or the bulk application of shear stresses by mechanical mixing). ${ }^{(51)}$ Because of this, graphene produced by these methods is well fitted for use as an additive in various mixes, coatings, simple printable circuits or other applications where it is a prerequisite the graphene be homogeneously dispersed in a liquid. ${ }^{(152)}$

\section{\$2.1.2 Chemical Vapor Deposition (CVD)}

Commonly characterized as a 'Top Down' method of graphene production, chemical vapor deposition (CVD) can produce large scale, wafer size, areas of graphene. The generic approach to the CVD of graphene involves two key ingredients: a clean carbon source and a clean, lightly catalytically active substrate. ${ }^{(153)}$ The surface chemistry of the substrate, the physical conditions of the growth chamber and the type of carbon source are selected so that thermodynamic forces drive the dissociation of carbon from their host molecules in the vapor phase to chemisorb as a monolayer of carbon on the substrate. ${ }^{(154)(155)}$ Low order hydrocarbons are typically used as the carbon source (e.g. $\left.\mathrm{CH}_{4}, \mathrm{C}_{2} \mathrm{H}_{2}\right)$. ${ }^{(156)}$ Growth temperatures and pressure can vary but typically center around $1000^{\circ} \mathrm{C}$ and between medium-vacuum to $1 \mathrm{~atm}$, depending on the substrate of choice. ${ }^{(157)}$ Nickle substrates, for example, can lower growth temperatures to as low as $600^{\circ} \mathrm{C} .{ }^{(158)}$ The substrate plays an important role in lowering the energy barrier for the diffusion of carbon across the substrate surface and for the healing of defects in the carbon layer, both of which allow higher quality graphene films to form. ${ }^{(159)(160)}$ Transition metals such as ruthenium, iridium, nickel 
and copper are common choices for substrates. ${ }^{(161)}$ Copper is attractive due to its low cost, is widely available as ultra-clean foil and reliably produces large area, high quality graphene. ${ }^{(162)}$ (163) (164) (165) Higher process pressures, which increase growth rate, can be used because the chemisorption process is self-limiting, restricted by the low solubility of carbon on copper. ${ }^{(161)}$ (166)

Because transition metals are ill-suited for most electronic applications of graphene, a large research effort has been spent on methods for the transfer of CVD graphene to more suitable substrates. ${ }^{(167)(168)}$ Again, copper is a leader as a growth substrate in this regard due to the very low adhesion forces between graphene and copper of $\sim 0.40 \mathrm{eV}$ per atom which is $\sim 60 \%$ less than with other CVD substrates. ${ }^{(169)}$ Compared to the high binding energy between carboncarbon atoms of $\sim 7.972 \mathrm{eV}$ in graphene, delamination of intact graphene sheets from copper foil substrates is possible. ${ }^{(170)}$ There has been progress made in producing graphene via CVD without a substrate, though the resulting graphene is multi-layer, aggregated and crumpled. ${ }^{(171)}$

\section{\$2.1.3 Selective Sublimation}

Also first reported in 2004 by Berger, et al. (only a few months after the now famous paper by K.S. Novoselov and A.K. Geim on mechanical exfoliation), is the selective sublimation of silicon carbide ( $\mathrm{SiC}$ ), which has been used to grow graphene films. ${ }^{(172)}$ This method is also referred to as, 'thermal decomposition' of $\mathrm{SiC}$. This approach is commonly characterized as a 'Bottom Up' method of graphene production and is a promising route for growing wafer-scale graphene films. As such, it has become an area of high research activity and there are several review papers summarizing the area. ${ }^{(173)(174)(175)(176)}$ Although this method most commonly utilized polymorphs of $\mathrm{SiC}$ with hexagonal unit cell arrangements, growth from cubic $\mathrm{SiC}$ has been extensively investigated as well. ${ }^{(175)}$

The selective removal of $\mathrm{Si}$ atoms from a $\mathrm{SiC}$ crystal matrix is achieved by exploiting the vapor pressure differences between $\mathrm{Si}$ and $\mathrm{C}$ in the crystal matrix. ${ }^{(177)}$ The sublimation method is of particular interest for electronic applications because the end result is a graphene layer which lies on top of a semiconducting $\mathrm{SiC}$ substrate crystal. Because of its wide bandgap, the $\mathrm{SiC}$ substrate can serve as an electrically insulating and thermally conductive substrate. Thus, the basic structure of many electrical devices (a thin conducing channel on an insulating layer) is fabricated in a single step. The high thermal conductivity of $\mathrm{SiC}$ is beneficial for the transport of waste heat away from active components. $\mathrm{SiC}$ is a high performance semiconducting material and has itself 
attracted much research attention over the past several decades. ${ }^{(178)(179)(180)(181)} \mathrm{SiC}$ has widely been considered as an extremely promising material for future electronic applications, practically for high power and high temperature applications, and possibly even the successor to $\mathrm{Si}^{(182)}\left({ }^{(183)}\right.$ (184) (185)

The arrangement of a conductor-semiconductor stack is the basic backbone for many solidstate electronics and the heterostructure of composed of a Graphene-on-SiC stack is particularly promising. ${ }^{(186)(174)}$ Because of this, unlike with the exfoliation or CVD methods, there is no need for a tedious 'peal and stick' step to transfer the graphene to an appropriate substrate. As the sublimation technique has matured, the parametric window for successful growth has expanded. However, this process still requires extreme conditions such as high temperatures and very low pressures. ${ }^{(173)}$

Given the proper thermodynamic conditions, initial $\mathrm{Si}$ sublimation can start as low as $1100^{\circ} \mathrm{C} .{ }^{(187)}$ At this temperature, a carbon rich layer is formed containing the $\sigma$-bonds characteristic of graphene. Due to the tight association this layer has with the SiC bulk, it lacks the corresponding $\pi$-bonds. It has been reported that the temperature required for good quality graphene is between $1400^{\circ} \mathrm{C}$ to $1850^{\circ} \mathrm{C}$, although the exact temperature is highly dependent on many other parameters including growth time, background gas pressure and heating rate. ${ }^{(173)(188)}$ If growth is allowed to continue after formation of the first carbon layer, a second carbon lay is formed beneath the first initial layer. With the formation of this second layer, the covalent bonds between the initial layer and the substrate are broken. This allows the initial layer to relax and transform into a quasi-free-standing graphene layer. The new layer is covalently bound to the substrate and shields the graphene overlayer from substrate driven effects. ${ }^{(189)}$ Because of this, the interfacial carbon rich layer is called a buffer-layer (occasionally in the literature also as $0^{\text {th }}$ layer graphene) and is the reconstructed carbon surface of the $\mathrm{SiC}(0001)$ crystal with $(6 \sqrt{3} \times 6 \sqrt{3}) \mathrm{R} 30^{\circ}$ periodicity. The buffer-layer and its relevance to this work are discussed in detail at the end of this subsection.

Depending on the exact crystal structure of the $\mathrm{SiC}$ wafer (3C-SiC, $6 \mathrm{H}-\mathrm{SiC}$, etc), orientation (Si-face or C-face) and the level of vacuum (which effects the sublimation temperature) it is not uncommon for the temperature required for this growth process to range from anywhere between $1125^{\circ} \mathrm{C}$ to $2000^{\circ} \mathrm{C} .{ }^{(190)(191)(192)}$ Not only do the substrate properties influence the required temperature of graphene formation but can strongly effect the properties of the produced graphene. 
Others have found a correlation between increasing degrees of wafer miss-cut (relative to the (0001) face) and an increase in carrier concentration coupled with a corresponding decrease in carrier mobility. ${ }^{(193)(194)}$ Due to the geometry that the multi-faceted surface of a miss-cut wafer presents, sublimation across the wafer is not uniform and occurs more rapidly on areas whose acute angles decrease the energy barrier for sublimation and provide more area over which sublimation may occur. This leads to the preferential formation of graphene along the step edges and pit formation. (195) (196) (197) (198) (193) (199) (197) (198) The degree of atomic step bunching has also been shown to influence graphene growth. ${ }^{(200)}$ In summary, the specifics of the starting substrate strongly influence the buffer-layer growth and graphene growth process.

It is worth noting that graphene formed at relatively low temperatures under ultra-high vacuum (UHV) are of lower quality than graphene grown at higher temperatures and higher pressures. Higher quality films can be grown by increasing the pressure in the reactor by injecting an inert gas, which allows a higher temperature to be reached before sublimation begins. (201) Higher temperatures increase the degree of annealing and higher pressures slow down the $\mathrm{Si}$ sublimation rate. By more slowly removing the Si from the crystal structure, while at the same time providing more thermal energy for the remaining atoms to rearrange, the carbon layer can more adequately self-heal. This benefit is not realized when the process is carried out at low temperatures. Low temperature synthesis generally produces graphene with high levels of structural defects. ${ }^{(177)(202)}$

The interactions between graphene and its substrate greatly influence the properties of the supported graphene. The spacing between graphene and the substrate ranges from $2.05 \AA$ to 3.41 $\AA$, depending on the substrate. ${ }^{(169)}$ The more tightly associated the graphene is to the substrate and the greater the lattice mismatch, the more pronounced the substrate-driven effects are. ${ }^{\text {(203) (204) As }}$ was previously stated, for multilayer graphene grown directly from a $\mathrm{SiC}$ substrate, there exists a carbon buffer-layer. However, even with the presence of a buffer-layer, graphene has been shown to not fully relax and is under compressive stress due to the lattice mismatch between the underlying SiC, buffer-layer and graphene overlayer. ${ }^{(205)}$ These substrate interactions can either enhance or limit the utility of the graphene, depending on the application. Substrate effects such as distortion of the graphene electron cloud, rippling or buckling of the graphene layer and charge transfers can all affect the properties of the supported graphene. (206) (207) Additionally, in some instances charge impurities from the substrate can act as scattering sites which will reduce the 
conductivity in otherwise pristine graphene. ${ }^{(208)}$ Others have shown that a band gap of $\sim 0.26 \mathrm{eV}$ can be generated exclusively from the interactions between single layer graphene and a $\mathrm{SiC}$ substrate. ${ }^{(50)}$ The bandgap does decrease to zero as the number of graphene layers grows to greater than four layers. The amount of charge transfer between the $\mathrm{SiC}$ substrate and graphene overlayers has been found to be dependent on the interface geometry and influenced by the buffer-layer. ${ }^{(209)}$ Furthermore, it has been shown that the reactivity of graphene can be greatly enhanced or muted based on the substrate. This is thought to be due to perturbation of the electron cloud distribution of the graphene, induced by charges from substrate. ${ }^{(210)}$

As mentioned earlier, the selective sublimation process results in the formation of a bufferlayer as part of the graphene growth process. The buffer-layer decouples and frees the carbon layer from the bulk SiC. The buffer-layers remain tightly associated with the substrate and experiences a mix of $\mathrm{sp}^{2}$ and $\mathrm{sp}^{3}$ hybridized bonding. ${ }^{(211)}$ As successive graphene overlayers are grown, the first buffer-layer transforms into a quasi-freestanding graphene sheet as a new buffer-layer is formed beneath. A simple illustration of this process is shown in Figure 2.2. The buffer-layer has been predicted computationally and has been detected experimentally in low energy electron diffraction (LEED) data and both Raman and x-ray photoelectron spectroscopy (XPS) spectra. ${ }^{\text {(212) }}$ (202) (211) Figure 2.2 depicts the simplest bonding interface between the buffer-layer and SiC substrate. This arrangement is what is most comely cited in the literature and assumes atomically ideal atom positions and stacking. However, much more complex bonding arrangements have been predicted. ${ }^{(213)}$

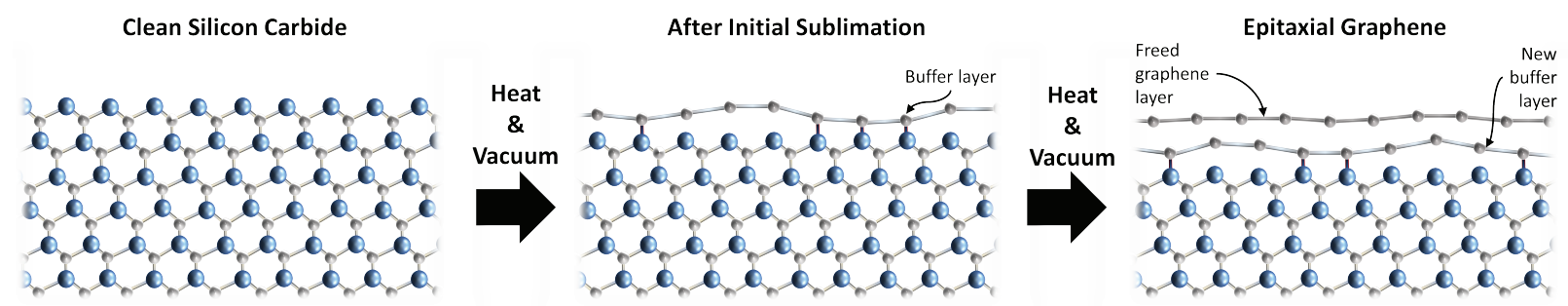

Figure 2.2: A schematic showing the transition from $\mathrm{SiC}$ to graphene. Initial Si sublimation forms a carbon rich buffer overlayer. Further sublimation causes the formation of a second carbon buffer-layer. The formation of the second buffer-layer allows the previous layer to relax into graphene. The simplest case for the interface between the buffer-layer and SiC substrate is shown, although more complex bonding arrangements have been predicted.

Many of the finer details of the buffer-layer are not well understood at this time, but a periodicity of $6 \sqrt{3} \times 6 \sqrt{3} \mathrm{R} 30^{\circ}$ has been computationally predicted as the most stable and this is the ordering that is most commonly cited in the literature (where they have shown this to be the 
case explicitly or not). ${ }^{(214)}$ However, there have been several other buffer-layer arraignments produced. For example, He, et al. produced buffer-layer with a periodicity of $6 \sqrt{43} \times 6 \sqrt{43}$ R7.6 (215)

Due to the partial covalent binding with the $\mathrm{SiC}$ substrate and the disparity in unit cell spacings between the bulk and buffer-layer, the buffer-layer undergoes strain relief in the form of buckling. (214)(216) (211) Additionally, the buffer-layer unit cell experiences a $30^{\circ}$ rotation relative to the bulk SiC. ${ }^{(212)}$ In the areas where the buffer-layer is pinned to the SiC substrate by covalent bonding, the SiC-buffer-layer spacing has been reported to be $1.90 \AA$ and $2.80 \AA$ in the unpinned regions. (217) (218) However, measured values for this spacing vary (to as high as $3.24 \pm 0.20 \AA$ ) again indicating that not all buffer layers are the same. ${ }^{(219)}$ A schematic of the buffer-layer interactions with the $\mathrm{SiC}$ substrate can be found in Figure 2.3. It has been reported that $a$ lattice parameter is $2.460 \AA \pm 0.001$ (corresponding to a C-C bond length of 1.420) in a decoupled graphene bilayer and $2.467 \AA \pm 0.001$ in the buffer-layer (corresponding to a $\mathrm{C}$-C bond length of

(A)

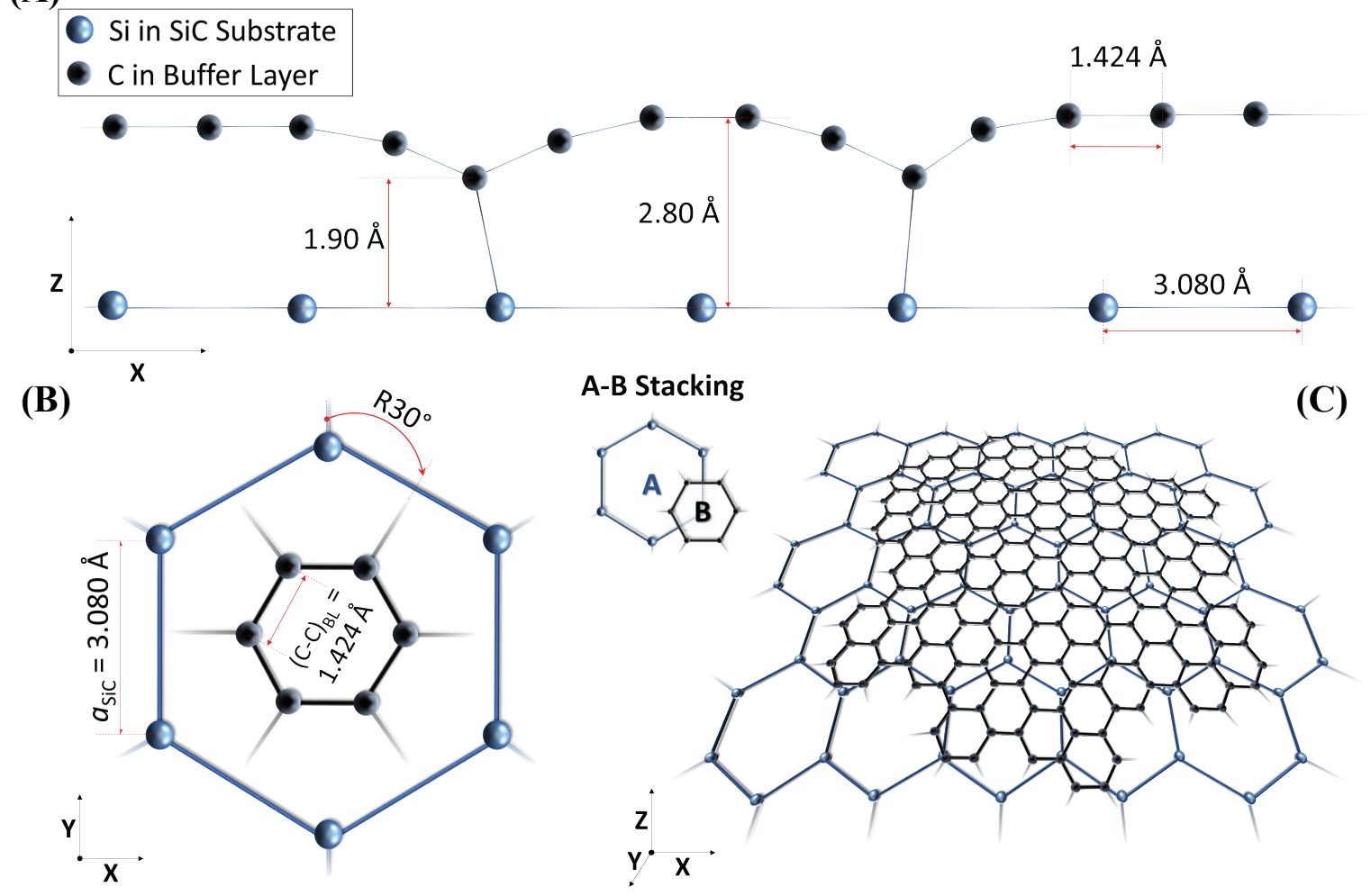

Figure 2.3: (A) Lateral schematic view of the interaction of the buffer-layer to the SiC substrate. (B) Top down view of the buffer-layer and $\mathrm{SiC}$ unit cells superimposed, illistrating the $\sim 30 \%$ missmatch and $\sim 30^{\circ}$ rotational miss match of the unit cells. The $\mathrm{SiC}$ and buffer-layer undergo A-B stacking. Anotated values are take from references (212), (217), (218) and (221). 
1.424). ${ }^{(220)}$ This indicates that the buffer-layer is under slight tensile stress (compared to the relaxed decoupled state) due to strong coupling to the $\mathrm{SiC}$ substrate, which has a larger lattice spacing.

The decoupling effect caused by the buffer-layer allows for the unperturbed formation of the free dangling $\pi$-bonds in the carbon overlayer and allows the carbon overlayer to relax into the natural unit cell spacing of graphene, which is different from that of the SiC bulk. ${ }^{(212)(220)(221)}$ The exact nature of the buffer-layer and its interactions with both the SiC bulk and graphene layer remains an area of intense research. It has been reported that approximately 1 in 3 carbon atoms in the buffer-layer are covalently bound to $\mathrm{Si}$ in the substrate. ${ }^{(22)}$ However, ratios as high as 1 in 10 (bound to unbound) have also been reported. ${ }^{(189)}$ Undoubtedly, each buffer-layer/substrate system is unique.

Because the buffer-layer does not behave the same as a graphene layer, there has been a concerted research effort to decouple this graphene layer form the bulk. By decoupling, the detrimental influences of the substrate on the carbon layer can be largely eliminated and allows for true single layer graphene-on-SiC structures to be realized. There have been several successful methods reported for decoupling of the buffer-layer ${ }^{2}$, including selective oxidation of the substrate, thermal shock and intercalation of other adatoms. (223) (224) (225) (224) (226) (227) (228) (229)

However, the most widely used method is hydrogenation of the substrate. It has been shown that on exposure to hydrogen gas at elevated temperatures, hydrogen can intercalate into the graphene/buffer-layer/SiC stack and decouple the buffer-layer from the substrate. Hydrogen first breaks the covalent bonds between carbons in the buffer-layer and silicon atoms in the substrate. By hydrogen bonding to the silicon, buffer-layer carbon atoms convent from an $\mathrm{sp}^{3}$ back to an $\mathrm{sp}^{2}$ conformation which allows these carbons to relax and participate in the prototypical graphene bonding scheme. The now decoupled buffer-layer, now a quasi-free graphene sheet, 'floats' on the bed of hydrogen atoms which now make up the terminal layer of the substrate.

\footnotetext{
${ }^{2}$ In the literature, "decoupling the buffer-layer" is used interchangeably with "removing the buffer-layer". However, note that the buffer-layer is not removed per se, but is rather freed and allowed to transform into a quasi-freestanding graphene layer.
} 
In this way, the hydrogen acts as a pseudo buffer-layer by increasing the atomic spacing between layers and passivating the covalent bonding between the carbon buffer-layer and $\mathrm{SiC}$ substrate. (230) (231) This allows the carbon buffer-layer to relax into a graphene layer and vastly improves carrier mobility in the layer. ${ }^{(232)}$ A schematic of this is illustrated in Figure 2.4. This has been shown computationally as well. ${ }^{(233)}$ The structural relaxation of the buffer-layer into a graphene sheet occurs simultaneously with a restoration of the electrical properties of the layer to those of graphene. ${ }^{(234)}$ Hydrogen treatment of graphene field effect transistors (GFET) has been shown to vastly improve device performance due to buffer-layer elimination effect; among other improvements, the carrier mobility in the GFET was found to increase from 800 to over 2000 $\mathrm{cm}^{2} \cdot(\mathrm{V} \cdot \mathrm{s})^{-1} \cdot(235)$
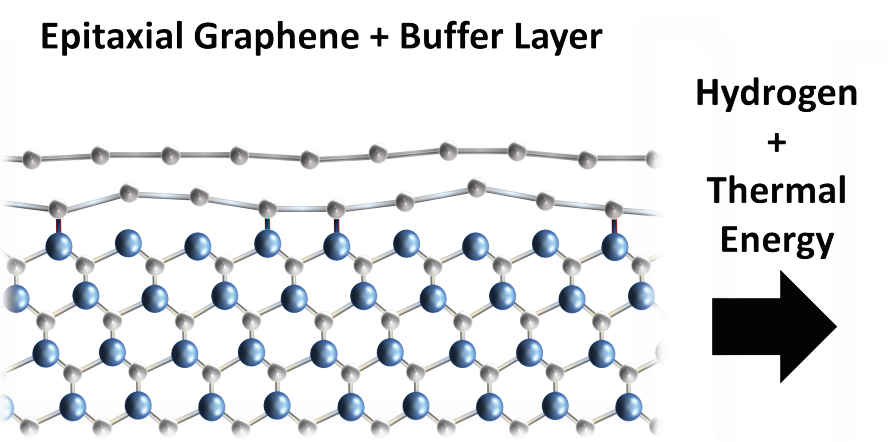

\section{Bi-layer Graphene}

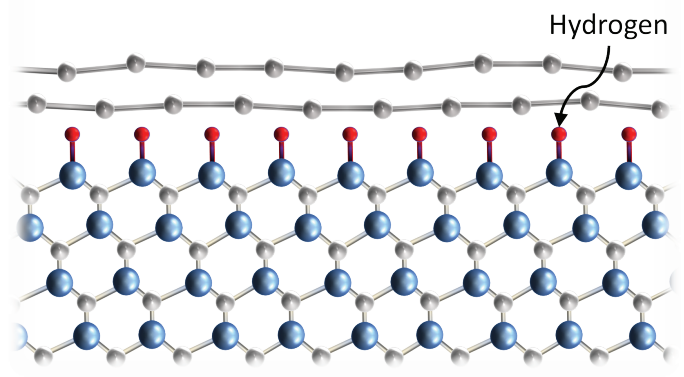

Figure 2.4: Schematic showing the use of hydrogen to passivate the SiC substrate bonds, free the bufferlayer and allow for its transition into a proper graphene layer.

It should be noted that this is an idealized view of the system and the specific mechanism of hydrogen transport and reaction with the interface is not completely understood at this time. There are two generic path types for hydrogen transport from the bulk, though the graphene overlayers and the buffer-layer to the interface. The first being a circuitous route where hydrogen would travel between graphene sheets, using defect sites and grain boundaries as transit points to diffused between layers and eventually reach the interface. Thermally induced, long range flexural phonons (out of plain vibrations) could help in the transport of hydrogen between sheets, enhancing the probability of finding an inter-sheet transit point beyond what would be expected from simple Brownian motion between two inflexible plains. ${ }^{(236)}$

The second path type would be a direct diffusion though graphene sheets. Graphene has been touted as being impermeable to gasses as small as helium (which actually has a smaller Van

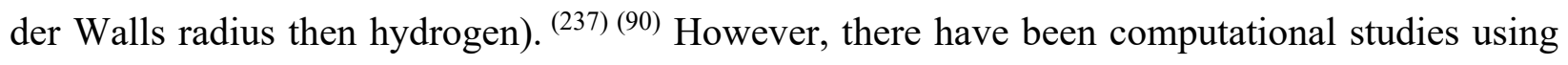
first principles and harmonic transition state theory indicating it is possible for a hydrogen atom to 
transit through the center of a graphene ring (the process having an energy barrier of only 2.86 $\mathrm{eV})$. Additionally, hydrogen atoms can chemisorb onto a carbon atom and then 'flip' to the opposing side of the graphene sheet (the energy barrier for this is slightly higher at $\sim 4.5 \mathrm{eV}$ ). Defect sites can drastically increase the probability of graphene penetration. ${ }^{(238)}$ (239)

A short list of the reported conditions for the removal of the buffer-layer via hydrogenation are given in Table 2-1 (all values, units and wordings are listed as they are presented in the corresponding references). An important note is that the optimum conditions for buffer-layer decoupling varies widely between studies and both atomic and molecular hydrogen have been used successfully. This gives further indications that the precise nature of the buffer-layer is not universal and varies from situation to situation. There does not appear to by any correlation between the conditions required for decoupling and the allotrope of $\mathrm{SiC}$ or crystal face. This indicates that, even given the same substrate and growth conditions, there are variations in the details of the buffer-layer - substrate coupling. In addition to high temperature hydrogen treatment, exposure to a weak hydrogen plasma has been shown to successfully decouple the buffer-layer. (240) 
Table 2-1: Select parameters for graphene growth on $\mathrm{SiC}$ and buffer-layer removal by hydrogen treatment.

\begin{tabular}{|c|c|c|c|c|c|c|c|c|c|}
\hline \multirow{2}{*}{ Year } & \multirow{2}{*}{ Substrate } & \multicolumn{3}{|c|}{ Initial Growth Conditions } & \multicolumn{4}{|c|}{ Hydrogen Treatment } & \multirow{2}{*}{ Ref \# } \\
\hline & & Time & Pressure & Temperature & Time & Pressure & Temperature & Hydrogen Description & \\
\hline 2014 & $4 \mathrm{H}-\mathrm{SiC}(0001)$ & $10 \mathrm{~min}$ & 800 mbar & $1550^{\circ} \mathrm{C}$ & $?$ & $?$ & $820^{\circ} \mathrm{C}$ & "100\% hydrogen" & $(241)$ \\
\hline 2010 & $4 \mathrm{H} \& 6 \mathrm{H}-\mathrm{SiC}(0001)$ & $?$ & UHV & \begin{tabular}{|c|}
$1100-1150$, \\
$1200,1350^{\circ} \mathrm{C}$ \\
\end{tabular} & $10 \min$ & 950 mbar & $600-1000{ }^{\circ} \mathrm{C}$ & Molecular Hydrogen & $(242)$ \\
\hline 2017 & $6 \mathrm{H}-\mathrm{SiC}(001)$ & $?$ & $0.1 \mathrm{Mpa}$ in $\mathrm{Ar}$ & $1450^{\circ} \mathrm{C}$ & $\begin{array}{c}30,240,480,900 \\
\text { sec }\end{array}$ & $?$ & $300 \mathrm{~K}$ & Atomic Hydrogen & $(243)$ \\
\hline 2018 & $4 \mathrm{H}-\mathrm{SiC}$ & $5 \min$ & $\begin{array}{c}1000 \text { mbar under } \\
30 \mathrm{SLPH} \mathrm{Ar}\end{array}$ & $\begin{array}{l}1600-1670{ }^{\circ} \mathrm{C} \\
1530-1570^{\circ} \mathrm{C} \\
\end{array}$ & $\begin{array}{c}8,18,22,24,27 \\
29,40 \mathrm{~min} \\
\end{array}$ & $\begin{array}{l}1000 \mathrm{mbar} \\
@ 10 \mathrm{SLPH} \\
\end{array}$ & \begin{tabular}{|c|}
$790,810,950,1050,1090$ \\
$1190,1260,1510^{\circ} \mathrm{C}$ \\
\end{tabular} & $\begin{array}{c}10 \mathrm{SLPH} \\
\text { Hydrogen }\end{array}$ & $(244)$ \\
\hline 2016 & $4 \mathrm{H}-\mathrm{SiC}(0001)$ & $\mathrm{N} / \mathrm{A}$ & $1 \times 10^{-5} \mathrm{mbar}$ & $\begin{array}{c}\text { e-beam } \\
\text { Irradiation }\end{array}$ & $30 \mathrm{~min}$ & ? & $900^{\circ} \mathrm{C}$ & $\begin{array}{c}100 \text { SCCM Molecular } \\
\text { Hydrogen }\end{array}$ & $(245)$ \\
\hline 2017 & $4 \mathrm{H}-\mathrm{SiC}(0001)$ & $2 \mathrm{hrs}$ & 1 bar & $1600^{\circ} \mathrm{C}$ & $2 \mathrm{hr}$ & 800 mbar & $700,900,1100,1200^{\circ} \mathrm{C}$ & "hydrogen atmosphere" & (246) \\
\hline 2014 & $4 \mathrm{H} \& 6 \mathrm{H} \mathrm{SiC}(0001)$ & $?$ & \begin{tabular}{|c|}
600 Torr in \\
background of $\mathrm{Ar}$
\end{tabular} & $1650^{\circ} \mathrm{C}$ & $1 \mathrm{hr}$ & $1 \mathrm{~atm}$ & $600,700,800,950^{\circ} \mathrm{C}$ & Molecular Hydrogen & (247) \\
\hline 2009 & $6 \mathrm{H}-\mathrm{SiC}(0001)$ & $?$ & $?$ & $?$ & $600 \mathrm{sec}$ & $0.26 \mathrm{mPa}$ & $200,377,600,800^{\circ} \mathrm{C}$ & Atomic Hydrogen & (248) \\
\hline 2011 & $\mathrm{SiC}(0001)$ & $15 \mathrm{~min}$ & 1 torr & $1625^{\circ} \mathrm{C}$ & $30-120 \min$ & 600 torr & $600-1200^{\circ} \mathrm{C}$ & Molecular Hydrogen & $(235)$ \\
\hline 2014 & $6 \mathrm{H}-\mathrm{SiC}(0001)$ & $0.42 \mathrm{hr}$ & $\begin{array}{c}100 \text { mbar in } \\
\text { background of } \mathrm{Ar}\end{array}$ & $1540^{\circ} \mathrm{C}$ & $0.5 \mathrm{hr}$ & 900 mbar & $1050^{\circ} \mathrm{C}$ & "Pd-purified H2" & (249) \\
\hline 2010 & $4 \mathrm{H} \& 6 \mathrm{H}-\mathrm{SiC}$ & $?$ & Various & Various & $10 \min$ & $1 \mathrm{~atm}$ & $600,730,800,830,1060^{\circ} \mathrm{C}$ & Molecular Hydrogen & $(250)$ \\
\hline 2011 & $6 \mathrm{H}-\mathrm{SiC}$ & $?$ & $1 \mathrm{~atm}$ of $\mathrm{Ar}$ & $2000^{\circ} \mathrm{C}$ & $15 \mathrm{~min}$ & $\begin{array}{c}1 \times 10^{-6} \\
\mathrm{mbar}\end{array}$ & $700^{\circ} \mathrm{C}$ & Atomic Hydrogen & (251) \\
\hline 2012 & $4 \mathrm{H}-\mathrm{SiC}(0001)$ & $50 \mathrm{~min}$ & $5 \times 10^{-6} \mathrm{mbar}$ & $1500^{\circ} \mathrm{C}$ & $40 \mathrm{~min}$ & 500 mbar & $700^{\circ} \mathrm{C}$ & "H" & $(232)$ \\
\hline 2013 & $4 \mathrm{H}-\mathrm{SiC}(0001)$ & $?$ & \begin{tabular}{|c|}
600 Torr in \\
background of $\mathrm{Ar}$
\end{tabular} & $1650^{\circ} \mathrm{C}$ & $?$ & $1 \mathrm{~atm}$ & $800,1000^{\circ} \mathrm{C}$ & Molecular Hydrogen & $(252)$ \\
\hline 2010 & $4 \mathrm{H} \&$ 6H-SiC (0001) & $?$ & Various & Various & $10 \min$ & $1 \mathrm{~atm}$ & $600-1000^{\circ} \mathrm{C}$ & Molecular Hydrogen & $(253)$ \\
\hline 2014 & $6 \mathrm{H}-\mathrm{SiC}(0001)$ & $15 \mathrm{~min}$ & UHV & $1050^{\circ} \mathrm{C}$ & $40 \mathrm{~min}$ & $?$ & $700^{\circ} \mathrm{C}$ & Atomic Hydrogen & $(231)$ \\
\hline 2012 & $4 \mathrm{H}-\mathrm{SiC}(0001)$ & $?$ & 100 Torr in of $\mathrm{Ar}$ & $1800^{\circ} \mathrm{C}$ & $?$ & 25 Torr & $1250^{\circ} \mathrm{C}$ & "H" & $(254)$ \\
\hline 2014 & $6 \mathrm{H}-\mathrm{SiC}(0001)$ & $?$ & UHV & $1300^{\circ} \mathrm{C}$ & $30 \mathrm{~min}$ & $?$ & $800^{\circ} \mathrm{C}$ & "hydrogen atmosphere" & $(255)$ \\
\hline 2009 & 4H \& 6H-SiC(0001) & $?$ & Various & Various & $?$ & $?$ & $600-1000^{\circ} \mathrm{C}$ & "Molecular Hydrogen" & $(256)$ \\
\hline 2019 & $4 \mathrm{H}-\mathrm{SiC}$ & ? & ? & $?$ & $60 \mathrm{~min}, 30 \mathrm{~min}$ & 200 mbar & $900,1100^{\circ} \mathrm{C}$ & "H $\mathrm{H}_{2}$ & $(257)$ \\
\hline
\end{tabular}




\section{§2.1.4 Combination Methods}

More recently there has been an effort to combine different methods for producing graphene in an attempt to capitalize on the advantages each individual method offers. One such example is the combination of the CVD and selective sublimation methods whereby sublimation of $\mathrm{SiC}$ at $1600^{\circ} \mathrm{C}$ starts initial graphene growth and exposes nucleation sites; the CVD process is run simultaneously with a gas mixture contacting $\mathrm{CH}_{4}$ which continues and enhances graphene growth. ${ }^{(258)}$ Another method combines a hydrogen plasma to assist in the CVD growth of graphene on copper. ${ }^{(259)}$

Other creative methods include the synthesis of graphene nano-flakes by injecting $\mathrm{CH}_{4}$ directly into an ICP Ar- $\mathrm{H}_{2}$ plasma. ${ }^{(260)}$ Others have also reported on the cracking of $\mathrm{CH}_{4}$ to produce nano-flake graphene. ${ }^{(261)}$ Because the process occurs in the gas/plasma phase, the graphene is initially an aerosol, which is then collected as it falls out of suspension. This presents a unique opportunity for various method for coatings (for example, electrostatically directed depositions of the nano-flakes). Given the huge interest in graphene, the modification of existing methods and the search for novel method will continue for the foreseeable future.

\section{\$2.1.5 Plasma Assisted Growth}

A novel method of producing graphene has been in development for several years by the Stinespring laboratory group at West Virginia University, Morgantown WV. ${ }^{(262)}{ }^{(263)}$ This is the method by which all the graphene used in the work presented here was produced. The specifics of this method are expanded on in detail in Chapter 3 and the effects of various processing parameters are presented in Chapter 4. In many ways, this method is similar to the selective sublimation method for producing graphene. As with the sublimation method, the starting material is a crystallin $\mathrm{SiC}$ wafer. The method described here primarily uses $6 \mathrm{H}-\mathrm{SiC}$.

Rather than relying on thermal energy to selectively remove $\mathrm{Si}$ from the $\mathrm{SiC}$ matrix, this method uses a plasma-chemically assisted route to selectively remove $\mathrm{Si}$. An inductively coupled $\mathrm{CF}_{4}$ or $\mathrm{Cl}_{2}$ plasma (ICP), in combination with a reactive ion etch (RIE), is used to chemically and physically etch a $6 \mathrm{H}-\mathrm{SiC}$ wafer. Under the appropriate conditions, $\mathrm{Si}$ is etched at a faster rate than the $\mathrm{C}$, leading to a $\mathrm{SiC}$ wafer with an amorphous carbon rich layer on its surface. This carbon layer is impregnated with halogenated species leftover from the plasma etch. The wafer is then thermally 
annealed in an ultrahigh vacuum environment at $970{ }^{\circ} \mathrm{C}$. However, in this work, the annealing step was modified to an atmospheric pressure rapid thermal anneal.

This annealing step provides the necessary thermal energy to desorb the volatile halogenated species and rearrange the amorphous carbon to form epitaxial graphene. The end result is a highly coupled buffer-layer, covered by small domain graphene, containing a low level of oxygen-based defects, laying on top of a robust semiconductor $(\mathrm{SiC})$. It is worth noting that a small number of oxygen defects are desirable for the applications pursued here. Oxygen defects can promote nanoparticle nucleation, can impart a small bandgap to the graphene and act as reactive sites for gas adsorption. Furthermore, a key parameter in any graphene synthesis process is the number of graphene layers produced. With this plasma assisted method, the number of layers is controlled to an extent by the ICP and RIE powers during the plasma etch step.

The plasma assisted method shares many of the benefits of the selective sublimation method (i.e. large area growth on an idea substrate, no required transfer step, compatibility with current wafer fabrication technology, etc.). Due to the aggressive nature of the plasma etch and the resulting planarization of the surface, this method may not be as strongly templated by the substrate. Phenomena specific to the sublimation process, such as rapid growth at step edges and pit formation, may not occur with the plasma assisted method. ${ }^{(195)(196)(197)(198)(193)(199)}$ The plasma assisted method eliminates the need for the ultrahigh temperatures and high vacuum environment required for sublimation. Furthermore, because the plasma assisted method only results in graphene growth on areas of the substrate which have been exposed to the etch, this method allows for the potential patterning of circuitry and micro device structures on the wafer scale. This process uses off-the-shelf industrial standard plasma etch processes commonly in use in today's mature semiconductor industry and can potential be scaled to the diameter of commercial SiC wafers.

However, unlike the selective synthesis method, which has been in development by a number of research groups since 2004, many fundamental aspects of the plasma assisted method remain a mystery. As such, questions regarding many synthesis parameters and structural aspects of the resulting material have yet to be answered. This work aims to elucidate the process as a whole and fill in some of the knowledge gaps. By investigating the effects of various synthesis parameters and testing the as-synthesized material for use in real world applications, a deeper understanding of the underlying science can be achieved. 
Just prior to the beginning of the present work, it was discovered that the ultrahigh vacuum anneal (UHVA) could be replaced with a rapid thermal anneal in a flow of atmospheric pressure argon. ${ }^{(264)}$ The work presented here focuses exclusively on graphene films formed using the new atmospheric pressure rapid thermal anneal (AP-RTA) process.

\section{§2.2 Graphene as a Sensing Material}

The excellent physical, mechanical and electrical properties of graphene are well known, and over recent years there have been many attempts to utilize these properties. These have been met with varying degrees of success, and the field of sensors a prime example. ${ }^{98)}$ (265) (266) (267) (268) Graphene based gas sensing has been of particular interest, and ${ }^{(97)(269)(270)(271)}$ more recently, so too have graphene based light sensors. ${ }^{(99)}(272)$ This section begins with a short review on the fundamentals of gas adsorption as a primer for the use of graphene as a gas sensor. This section concludes with a short review of graphene based used light sensors.

\section{\$2.2.1 Fundamentals of Gas Adsorption}

Adsorption phenomena has been a field of study since the 1880's when the term 'adsorption' was first coined by Du Bois-Reymond. ${ }^{(273)}$ In 1916, Irving Langmuir formulated a general theory for adsorption (resulting in most notably, the Langmuir isotherm); work for which he was awarded the 1932 Nobel Prize in Chemistry. ${ }^{3}(274)$ Soon thereafter, the mathematical description of two body interaction at atomic distances, proposed by Lennard-Jones in 1924 (the famous Lennard-Jones potential, discussed later), was a significate step towards the understanding of adsorption on an atomistic level. ${ }^{(275)}$ Since then, the field has matured significantly and there are now many models describing adsorption. ${ }^{(276)}$ The early 1950's saw the first use of digital computing to assist in adsorption modeling. ${ }^{(277)}$ With the introduction of complex density functional theory (DFT), ab initio calculations, Monte-Carlo (and many others) combined with supercomputing in the 1990's-2010's, high fidelity molecular simulations of various adsorption events have been modeled. (278) (279) (280) (281) More recently, artificial neural networks, genetic algorithms and other machine learning techniques have been applied to adsorption phenomena. (282) (283) (284) (285) (286) Accordingly, there is extensive literature available on the topic. There are

\footnotetext{
31932 Nobel Prize in Chemistry, Irving Langmuir, "for his discoveries and investigations in surface chemistry." (727)
} 
many highly regarded textbooks on the subject, therefor only a brief primer is included here and only specify as it pertains to the work detailed later. ${ }^{(273)}$ (287) (288)

Consider a material, of arbitrary size and shape, in a gaseous environment. Through Brownian motion, a fraction of molecules which make up the gas environment will eventually interact with the surface of the material. When a gas molecule approaches the surface, the molecule will interact and either collided elastically (e.g. ricochet off the surface and rejoin the main body of gas) or will interact in a manner which results in the molecule not leaving the surface. The latter is an adsorption event. If, after adsorption, the gas molecule then leaves the surface, this is called a desorption event. Clearly, not all molecule/surface interactions will lead to an adsorption event. The fraction that do adsorb to the surface verses those which interact but do not adsorb (i.e. ricochet off) is the sticking coefficient; a sticking coefficient of 0.20 , for example, indicates that for every five molecules that interact with the surface, four bounce off and one remains, or "sticks", to the surface. The sticking coefficient is fundamentally a result of the energy loss a molecule can achieve by attaching to the surface verses remaining in the main body of gas; the more energetically favorable it is for a gas to remain bound to the surface, the higher the sticking coefficient. Because of this, the sticking coefficient is a function of many things; including temperature, pressure, surface coverage and notably, the chemistry that occurs between the gas and surface. This is specific to each gas-surface combination.

In most cases there are a finite number of sites available for adsorption (ignoring for the moment surface stacking of adsorbed molecules). Surface coverage defines the percentage of available sites that are occupied by an adsorbed molecule; a surface cover of $100 \%$ indicates that all available sites are occupied.

Adsorption events are generically classified as either physisorption or chemisorption; although the separation between the two is not always clearly defined. In fact, the classification criteria are rather loose and there many conflicting definitions can be found in the literature. ${ }^{4}$ However, framing adsorption events in this context is useful in the interpretation of the phenomena and presents a convenient basis for describing what would otherwise be complex situations. By classifying an adsorption event, which may not be well understood, as either physisorption of

\footnotetext{
${ }^{4}$ Hydrogen bonding and electrostatic interactions have been attributed to both physisorption and chemisorption, thus illustrating the overlap between the two sorption nomenclatures.
} 
chemisorption, assumptions can be made about the system based on what has been determined from other, more well understood, systems which fall into the same category.

Physisorption events involve weak, long range bonding and rely on van-der-Waals interactions (such as London dispersion forces and dipole-dipole interactions) and other intermolecular forces. In the classic description of physisorption, these bonding events do not require specific features of the surface and occur at large distances. ${ }^{5}$ The adsorbent 'sees' a smeared surface adsorption potential representing the attractive forces averaged over a large area of the surface. Because physisorbed species are not tightly bound to one specific binding site, these species tend to have high surface mobility. Because of this high mobility, physisorbed species can be viewed as a 2D fluid on the surface. ${ }^{(273)}$ Some finite amount of physisorption will always occur. ${ }^{(289)}$ Due to their small binding energy, physisorbed species are easily desorbed.

Chemisorption events involve strong, short range bonding and rely on strong interactions between molecular orbitals between the adsorbent and the surface (covalent bonding, ionic bonding, etc.). These interactions are highly specific to the adsorption site and the adsorbent. Due to the tight binding, chemisorbed species are relatively immobile, although site hopping can occur if given sufficient energy and site density. (290) (291) (292)

The Lennard-Jones potential is the resulting effect of van-der-Waals attractive forces combined with Pauli-repulsion, plotted as a function of the distance between the molecule and the surface. There are other potential functions which describe two body behavior, such as the Morse potential, but are qualitatively very similar. These types of potential plots are useful for conceptualizing adsorption behavior. In Figure 2.5(A), the Lennard-Jones potential is shown qualitatively for physisorption and chemisorption. Several defining aspects of each adsorption are evident; the relative difference in magnitude of the potential energy change between chemisorption $\left(-\Delta \mathrm{E}_{\text {phys }}\right)$ and chemisorption ( $\left.-\Delta \mathrm{E}_{\mathrm{phem}}\right)$, as well as the equilibrium bonding distance of each binding type $\left(Z_{\text {che-eq }}\right.$ and $Z_{\text {phys-eq, }}$ respectively). Figure $2.5(B)$ shows a simplified carton of each adsorption

\footnotetext{
${ }^{5}$ Surface irregularities (which perturb the surface energy distribution) can enhance the propensity for a molecule to adsorb. But this is still a physorption event because the bonding occurs at long range, do not involve intimate electron orbital interactions (i.e. no chemistry) and results from various in the physical surfaces structure and not variations in the local chemical environment).
} 
case. Note the long range, lightly interacting and largely site independent nature of physisorption verses the short range, highly interacting and site-specific bonding for chemisorption.
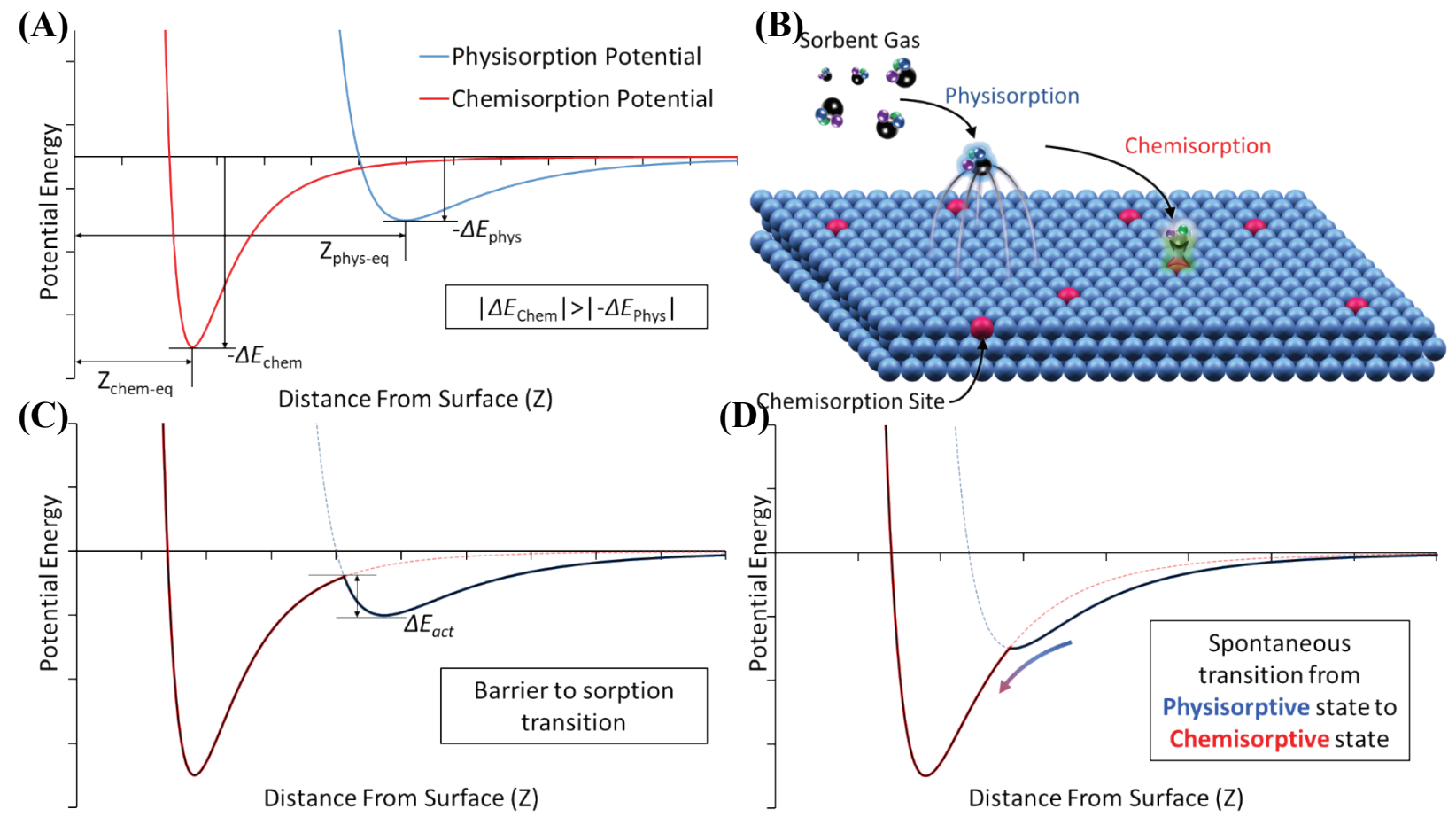

Figure 2.5: (A) Lennard-Jones potential for physisorption and chemisorption, illustrating the difference in binding energy and binding distance and the gain in potential energy by adsorbing. (B) Simple cartoon illustrating the key difference between physisorption (weak, long range bonding involving intermolecular forces) and chemisorption (strong, short range bonding involving molecular orbitals). The interplay between potential plots showing the transition from physisorption to chemisorption with an energy barrier (C) and without (D).

Both physisorption and chemisorption can occur simultaneously on a surface and adsorbed species can transition from a physisorbed state to a chemisorbed state. The ease in which this occurs depend on the interplay between the adsorption potential for each case. There can be a significant energy barrier to the transition $\left(\Delta \mathrm{E}_{\mathrm{act}}\right)$, as is shown in Figure 2.5(C), or a negligible barrier, as shown in Figure 2.5(D). In the latter case, the transition from a physisorbed to chemisorbed state can occur spontaneously.

This treatment describes simple adsorption, where the properties of the surface and the adspecies are independent of one another. However, there are scenario where what would otherwise be a weak interaction, but due to a specific substrate-adspecies combination, results in a very strong interaction. For example, a random dipole field may exist at the surface due to the nature of the substrate. A charged adspecies can approach the surface and cause the dipole fields to align, producing a local site-specific field which can result in a strong physiorption. 
The potential plots shown in Figure 2.5 for physisorption are a simplified case and do not show other adsorption effects which do occur (time dependent effects, diffusion effects, capillary condensation, nucleation, molecular dissociation and other surface reaction events, etc.). In reality, adsorption is a chaotic and dynamic process. The complex adsorption behavior is reviled in isotherm diagrams. Such diagrams plot the amount of adsorbed gas against the relative pressure of the adsorbent, at a constant temperature. The International Union of Pure and Applied Chemistry (IUPAC) have officially classified the various adsorption behaviors into six categories, as described by their isotherm adsorption diagrams. ${ }^{(293)}$ Their generic shapes are shown in Figure 2.6.

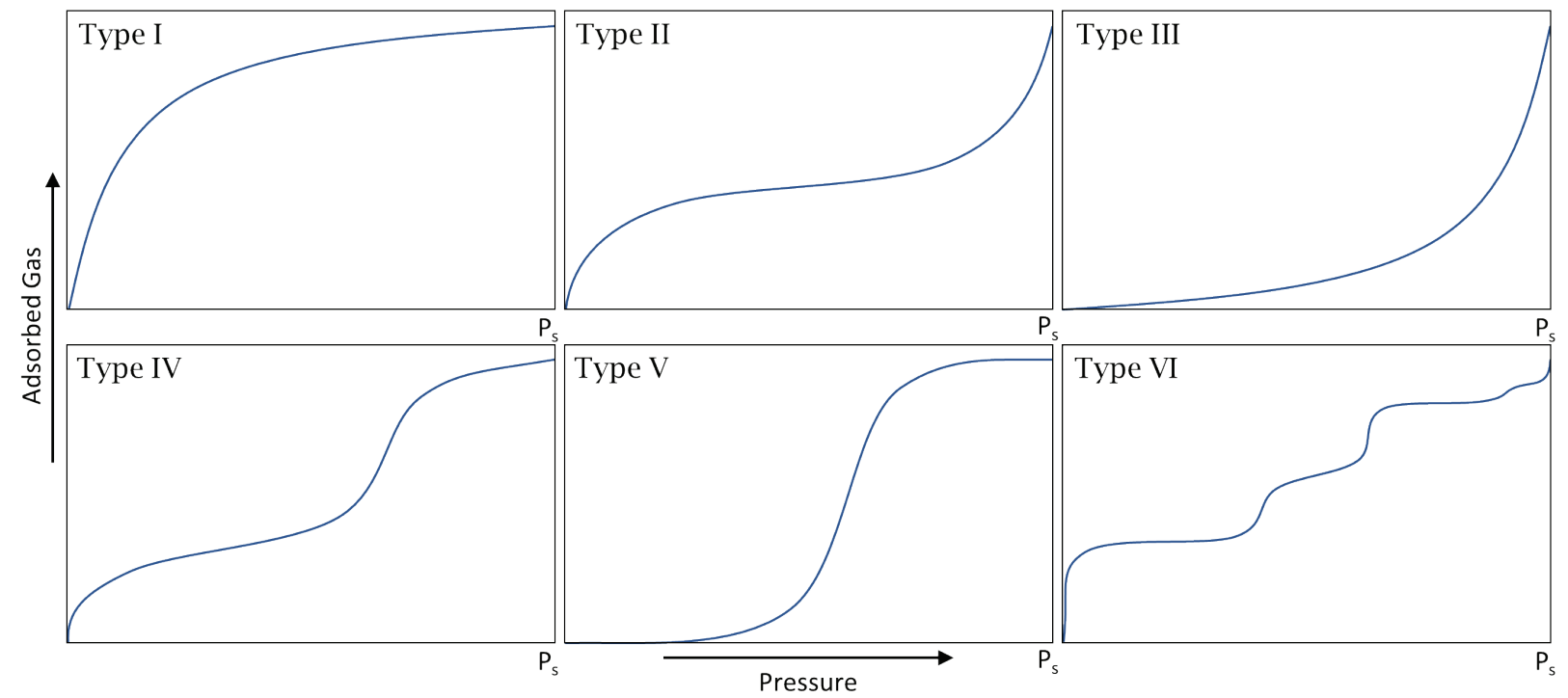

Figure 2.6: The six types of adsorption isotherms as classified by IUPAC. Type I, the Langmuir isotherm, is the simplest case and is the most applicable to this work. Types II-IV more accurately describe adsorption on structural complex surfaces composed of various micro- and mesopores and their effects on adsorption. The following IUPAC citation is an excellent reference for classifying adsorption behavior and determining the underlaying phenomena at play. (293)

Type I is the classic Langmuir adsorption case and shows the simple development of a monolayer. Types II-VI are cases where various other effects dominate, the criteria of which have been official determined by IUPAC. ${ }^{293)}$ These curves describe adsorption onto structurally complex surfaces which have various combinations of micro- and mesopores. The resulting combinations lead to complex adsorption rates governed by various mass transport effects and other phenomena, like pore condensation. There are analogous desorption plots as well. ${ }^{(293)}$

Because chemisorption involves interaction with the electron orbitals of the surface atoms, chemisorption is primarily limited to a maximum of a single monolayer (i.e. no stacking). ${ }^{(273)}$ After a mono-layer has developed, chemisorbed molecules shield the surface orbitals of the 
chemisorption sites from further interactions. ${ }^{(294)}$ For physisorption however, the long-range nature of the binding means that adsorbent molecules can stack on one another (newly arriving molecules can 'see though' the already physiosorbed species to the surface) or physisorbed molecules can at as physisorption sties themselves; the latter is described by Brunauer-EmmettTeller (BET) theory. Thus, physisorption can lead to greater the $100 \%$ surface coverage.

The plots of Figure 2.6 are useful in a gas sensing context for two key reasons. First, an increase in the concentration of an analyte gas in a carrier stream will relate monotonicity to the partial pressure of the analyte gas. Second, sensor response is often directly related to the amount of adsorbed gas. Therefore, the overall character of these plots can be used to predict sensor response. Or alternatively, a sensor response can be retroactively compared to these plots, thereby providing insight into the specific adsorption process which drove the response. Although the plots of Figure 2.6 describe physisorption only, they can be used as a starting point for understanding the much more complex chemisorption processes.

\section{\$2.2.2 Graphene for Gas Sensing}

In the most basic sensing scenario, sensing is achieved by monitoring for a change in the value of some arbitrary property of a material as it is exposed to a stimulus; for example, observing the material's change in electrical resistance. The magnitude of the response is proportional to the amount of material the stimulus effects. In general, a stimulus interacts primarily with the surface of a material. But the bulk, which is shielded from interaction with the environment by the surface layer, does not 'see' the stimulus and remains unaffected. Therefore, while a large response may be elicited from a material, because interactions only occur at the surface, little or no response is detected.

For example, consider a best-case scenario for gas sensing where $100 \%$ surface coverage is achieved. If the sensing material is a $1 \mathrm{~cm}^{3}$ cube of graphite, only $0.0000007 \%$ of all atoms which make up the cube would be directly affected. ${ }^{6}$ Because a materials properties are a confluence of both the bulk and surface contributions, the change in any particular property of this hypothetical cube would be proportionally minute. Therefore, to more easily detect surface-environment interactions, it is advantageous to minimize the fraction of the material that is the inactive bulk and maximize the exposed surface area (i.e. maximize the ratio of surface area to internal volume).

${ }^{6}$ This calculation assumes a graphite density of $2.26 \mathrm{~g} / \mathrm{cm}^{3}$, an atomistic surface density of $7.63 \times 10^{-8} \mathrm{~g} / \mathrm{cm}^{2}$ and the molecular weight of carbon to be $12.011 \mathrm{~g} / \mathrm{mol}$. 
In this way, the response is not swamped out from the nonresponsive bulk fraction of the sensing material. This is the basic motivation for nanomaterial-based gas sensing which, over recent years, has become an intense are of research. ${ }^{(295)}$ Generally, this leads to a better signal to noise ratio and an increased detection ability. This is why low-D materials are such attractive sensing materials. Graphene, having no internal volume and existing as a pure surface (every atom is a surface atom), is an ideal candidate for gas sensing applications for this very reason. Furthermore, the unique electrical properties make graphene ideal as well. In particular, the combination of highly mobile charge carriers with an overall low carrier concentration could lead to both a rapid sensor response and a low threshold for detection.

There have been may mechanisms reported to explain gas sensitivity in research devices. (296) (297) The more complex the sensing device, the more complex the mechanisms tend to be. Some complex devices required external illumination or involve spectroscopic analysis, for example. In contrast, most graphene-based gas sensors tend to be of simple construction and consist of only a few components. They are:

1) The sensing area, composed of some form of graphene, which can range from a single graphene sheet to a mosaic of drop-cast graphene oxide sheets.

2) Metalized contact pads to electrical contact between the electronics and the sensing area.

3) A substrate on which the device is built.

For this class of devices, the basic sensing mechanisms revolves around the adsorption of gas molecules onto graphene sensing area, which will then disrupt the delicate electrical structure device. This disruption can then be electrically detected. This usually takes the form of monitoring the conductivity or resistance of the sensing film. ${ }^{(97)}$ Although other electrical parameters can be used as detection schemes, such as the electrical impedance or capacitance of a device. (298) (299) (300) However, only the former will be considered here. Sensors operating under this detection scheme are broadly referred to as a chemiresistor sensors.

The conductivity of a material is a function of the number of charge carriers and their mobility. Adsorbed gases can alter the carrier concentration of the adsorbent by donate holes/electrons. A slightly different mechanism can come into play where gas adsorption leads to charge trapping or acts as charge scattering sites, effecting both carrier mobility and concentration, and thereby altering the resistivity. Additionally, intricate situations can arise from complex 
interactions between the graphene sensing material and other components of the sensor, like the substrate or the metal contacts. For example, the formation of Schottky junctions at the metalgraphene interface results in a charge depletion region formed in the graphene and a built-in electric field. Such a situation can provide additional opportunities for complex gas/material interactions which ultimately lead to a change in the effective resistance of the device. ${ }^{\text {(301) (302) (303) }}$

Classic solid-state gas detection systems operate under a similar principle (typically charge transfer events or an adsorbed surface reaction) but suffer from a relatively high noise level, arising from defects in the crystal structure and erratic charge carrier motion. ${ }^{(304)(305)}$ Both problems are strongly temperature dependent and common to a wide range of materials. ${ }^{(306)}$ Furthermore, relativity few carriers, as compared to the bulk population, are involved and thus much of the sensor is inert.

Again, graphene arises as an ideal candidate in that it may overcome these problems while simultaneously achieving much greater sensitivity due to its low defect level, high mobility and low carrier density. The low number of highly mobile charge carriers, combined with the low level of defects in graphene, lead to a large mean free path for charge carriers with no charge trapping. This results in ultra-fast response and a low noise level because the charge carriers can move freely and unimpeded in the 2-D gas of massless Dirac fermions on the surface of graphene. ${ }^{7(307)}$ Because of this, ultra-high sensitivity can be achieved.

However, it should be noted that the graphene used to build devices need not be pristine. Eftekhari, et al. makes the important point that defects in the graphene structure play an important role in many applications of graphene. ${ }^{(308)}$ This is true in particular for sensing applications where defects serve as active sites available for interaction with the analyte. Highly defect graphene oxide (GO) and reduced graphene oxide (rGO) have been shown to perform quite well as gas sensors. (309) (310) (311) (312) The decrease in crystal quality is compensated for by the much more chemically active surfaces that GO and rGO present for gas interaction. ${ }^{(145)}$ (313) Furthermore, functionalize of the sensing surface has become a common method for increasing the performance of carbonbased material gas sensing. ${ }^{(314)}$

\footnotetext{
${ }^{7}$ Stated another way, the $\pi$ orbitals of the graphene sheet (which are delocalized and form a molecular orbital that spans the entire graphene surface) is presented to the environment for interaction. This molecular orbital is a 'sea' in which carriers can move freely and travel long distances before encountering a scattering site. The adsorption of gas onto this sea disrupts this situation by adding or removing carriers (charge transfer) or impede their movement (charge trapping or screening) or by decreasing the distance carriers can travel before encountering an obstacle (charge scattering site); all of which leads to a change in resistance (the detectable device response).
} 
There are many reports in the literature that show that the response of graphene devices is not only sensitive but also selective to the target gas species. ${ }^{(315)}$ In these cases, the ability to differentiate gases is derived from the direction of charge transfer between the graphene and target gas. The direction of charge transfer depends on whether the starting graphene is n-type or p-type doped and if the adsorbed molecule donates or accepts electrons. ${ }^{(316)(317)(318)(319)(320)}$

The addition of metallic nanoparticles to graphene devices has been shown to increase the sensitivity of the graphene devices. ${ }^{(321)}$ (322) The exact mechanism is unclear but is thought to be a combination of the additional gas interaction sites provided by the particle, electron/hole doping effects of the graphene-metal heterostructure or surface reactions induced by the catalytic nature of the metals. ${ }^{(323)(324)} \mathrm{Pd}, \mathrm{Pt}, \mathrm{Ag}, \mathrm{Au}$ are common metals that have shown to increase sensitivity. (325) (326) (327) (328) (329) (330) (331) (332) It has been computationally predicted that the addition of adatoms can increase binding energies of a target gas and increase sensitivity and stability. ${ }^{(333)}$

Regardless of the mechanism, for gas detection to occur, gases must interact with the sensing surface; the stronger the interaction the stronger the response. As discussed in Section $\$ 2.2 .1$, gas interactions with a graphene surface can be broken down into two sub categories: physisorption and chemisorption. However, it is not always clear which is occurring in any particular case and the distinction between the two is not absolute.

In general, physisorption events rely on dipole interactions and Van der Waals forces which result in weak interactions between molecules and the graphene surface. ${ }^{(334)}$ While this can cause physical bending or buckling of the graphene surface, resulting in a change in resistance of the graphene (due to perturbation of the 2-D gas of charge carriers on its surface), the changes are relatively small. ${ }^{(335)(336)(337)}$ Due to the exothermic nature of physisorption, high temperatures lead to fewer adsorption events which would tend to decrease sensor response at elevated temperatures. ${ }^{(338)}$ Additionally, thermally induced bending and buckling of the graphene films can obscure the signal; deconvolution the signal due to physisorption events and thermal effects could prove challenging. ${ }^{(20)(22)}$

Chemisorption events are more energetic. Strong chemisorption interactions can rehybridization molecular orbitals; this can have a larger impact on the electrical properties as compared to physisorption. But chemisorption events generally have higher activation energy requirements as compared to physisorption, and therefore occur less frequently. Following first principles one suspects that, due to the pristine nature of the graphene surface, chemisorption 
events on the basal plane are rarer than the more energetically active edges sites. ${ }^{(339)(340)}$ Oxygen terminated graphene edges are preferential sites for gas adsorption. Binding energies are in the 100-200 meV range. ${ }^{(341)}$ Adsorption on the graphene surface have been calculated to be an order of magnitude lower. ${ }^{(317)}$ Cleaved $\sigma$ bonds are reactive and the asymmetry of the graphene edge prevents full delocalization of the $\pi$ bonds, presenting energetically active sites for interaction. ${ }^{(342)}$ (343) The specific chemistry of the edges is complex and depend on the edge geometry (the socalled arm-chair vs. zig-zag arrangement). (344) (345) However, chemisorption can occur on the pristine surface of graphene as well. It has been reported that adsorption of large aromatic molecules on the surface of graphene sheets (via $\pi-\pi$ orbital interaction) leads to charge transfer and doping of the graphene. ${ }^{(346)}$

Due to their higher binding energy, chemisorbed molecules require more energy to drive their desorption, this can lead to sensor saturation of chemisorbed molecules. ${ }^{(347)}$ This has been observed experimentally, but it was found that a simple bakeoff step can recover the device with no loss of performance. ${ }^{(348)}$

Due to the greater level of interaction, chemisorption events can result in a higher magnitude of device response as compared to physisorption. However, chemisorption requires active chemisorption sites. By adding defects into the otherwise pristine graphene surface, the energy barrier to chemisorption events can be lowered thus increasing their frequency. By introducing defect sites into the graphene surface, local energy disruption occurs. This leads to energetically active sites which are available for complex interactions with the target molecules and can increase the frequency of chemisorption events. ${ }^{(349)}$ These defects can be structural in nature like vacancies or Stone - Wales like crystal defects or metallic atoms added into the graphene lattice or the presence of attached functional groups. ${ }^{(350)(351)(352)(353)}$ Even bending of the graphene film has been computationally shown to influence both chemisorption and physisorption probabilities. ${ }^{(354)}$

It has been reported that, due to changes in the local carrier concentration by chemical doping from the analytic gas, the detection of single gas molecules is possible. ${ }^{(348)}$ Single molecule gas detection has also been reported by means of Coulomb scattering of charge impurities resulting from adsorbed molecules. ${ }^{(355)}$ It has also been reported that adsorption of gas molecules modifies graphene's band gap; an easily detectable change useful as a gas detection mechanism. (32) (33) Based on the many different detection mechanics of graphene gas detectors already reported, and regardless of the type of sorption event, graphene is a good choice as a backbone on which a gas 
detection device may be built on. This is due both to the dimensionality and unique electronic structure of graphene.

Vapor sensing is occasionally discussed separately from gas sensing however, in the strict sense, vapor sensing is fundamentally the same as gas sensing. A brief discussion on the progress of graphene-based vapor sensing applications is presented here to complete the review of relevant sensing literature in this area. There is much overlap between the vapor and gas sensing categories; however, 'vapor sensing' tends to refer to the detection of gas or vapor volatilized from the surface of a liquid. Therefore, the attraction of graphene-based vapor sensing is much the same as graphene-based gas sensors. The main difference being that the detection of vapor typically involves larger molecules such as volatile organic compounds (VOCs) like alcohols, acetic acid,

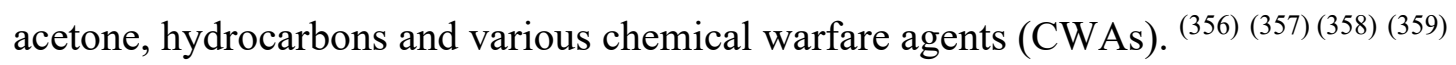

Because the detection mechanism for vapor sensing is similar to gas sensing, vapor sensors operate as chemiresistors as well, whereby detection is achieved through monitoring changes in resistance/conductance of the graphene. ${ }^{(358)(360)}$ A primary difference is in the transport of the target species to and from the graphene surface, with the larger molecules adsorption/desorption kinetics being slower and showing lower rates of intra-surface diffusion after adsorption. This is evident when comparing $\mathrm{H}_{2}$ and $\mathrm{NH}_{3}$ for example. ${ }^{(361)}$

An effect of the decrease in mobility for these heavier molecules are slower signal response and sensor saturation. This leads to a slow recovery and decrease in response over time. Though this could be avoided by periodic bake-off steps where short pulses of current are passed through the device to drive off adsorbed species via the ohmic heating of the graphene surface. It has been shown that this is less of a problem at higher temperatures due to higher (less negative) $\Delta H_{\text {ads }}$ values, indicating that an adsorbed state is less desirable at higher temperatures. ${ }^{(362)(363)}$ This is due to an increase in desorption coefficient as a result of the elevated temperatures.

As is the case with gas sensors, there are reports of increased response to select vapors with the addition of metallic nanoparticles to the sensing system or doping the graphene lattice with metal atoms. ${ }^{(364)(365)(366)}$ Molecular simulations have also shown preferential adsorption to defects sites. ${ }^{(367)}$ Multilayer graphene has been shown to have greater interaction with target molecules, probably due to the role of step edges. ${ }^{368)(369)}$

In summary, it is easy to see how a practical, chemiresistive gas or vapor sensing system could be constructed. Chemiresistor detection is attractive due to the simple nature of operation, 
no requirement for specific temperature ranges (which would require external heating) and low power requirements leading to intrinsically safe designs. All of these advantages increase the potential deployment scenarios of these sensors. Graphene is highly attractive as a gas sensing material for may reason previously discussed. In particular, due to its extreme ruggedness, a niche exists for graphene's use in harsh environments. Previously, ceramic gas sensors have attempted to fill this niche. And although graphene must face its own challenges, others have stated that that the need to miniaturize and transition to thin-film applications present substantial technological changes to ceramic sensors- two challenges graphene excels at. ${ }^{(370)}$

By adding nanoparticles to the surface of the graphene or controlling the level of defects in the graphene, the sensors can be tuned to a particular type of gas. By combining different sensors on the same platform, each tuned to a different type of gas, a typical fingerprinting methodology can be employed. Each sensor can be electrically monitored independently while the platform is exposed to a gas mixture. The larger the sensor array, the more specific the fingerprint. A simple cartoon illustrating the basic concept of gas fingerprint using a sensor array is shown in Figure 2.7 .

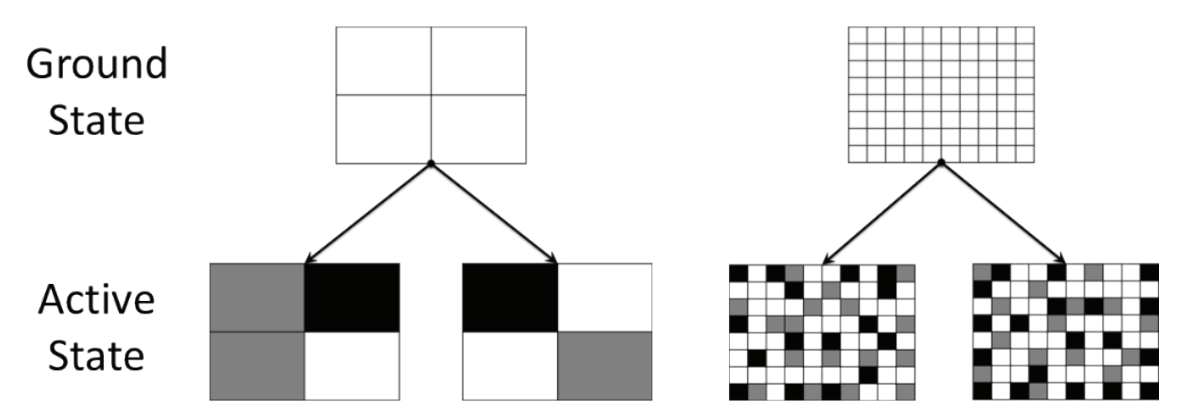

Figure 2.7: Cartoon depicting the fingerprinting of gasses using different sized arrays and operating in a simple trinary response mode (positive, negative or no change).

This illustration shows a sensors' array operating in a chemiresistive mode were the sensors respond in one of three different ways: an increase, decreases or no change in resistance (trinary response mode) as compared to a reference state. By simply monitoring the resistance of an array of nanoparticle-decorated sensors, the detection of a complex range of gases could be achieved.

\section{§2.2.3 Graphene for Light Detection}

The use of graphene as a light sensing platform is a relatively new area of research interest. Due to its relatively low absorbance over the entire visible range, with a maximum of only $\sim 2.3 \%$, 
graphene was initially ignored for uses in optoelectronics. ${ }^{8}{ }^{(371)}$ Considering the atomically thin nature of graphene, this absorbance value is quite large; however, many other materials used in optoelectrical applications easily exceed this value.

Recently this has begun to change, and graphene is beginning to attract a lot of research attention for optoelectronic applications outside of utilizing its transparency. ${ }^{(119)(372)(373)}$ Studies have suggested that due to the tight confinement and long life (orders of magnitude longer than in conventional materials) of induced surface plasmons, graphene is a good candidate for strong lightmatter interactions. ${ }^{(374)}$

Virtually all optoelectronic applications of graphene involve the deposition of metallic pads to make electrical contact to the device. The interface between doped graphene and a metal contact has been known to generate a Schottky junction. ${ }^{(375)(376)(377)}$ This type of interaction between a metal and semiconductor is well understood and is thoroughly described in many textbooks. ${ }^{(378)}$ ${ }^{(379)}$ Additionally, there are several review articles describing the generation of Schottky junctions in graphene-based devices and the resulting photo detection phenomena. ${ }^{(380)}(112)$

Briefly, as it applies to graphene devices, a graphene Schottky junction involves the generation of a charge depletion region within the graphene layer. This is due to a miss alignment of the energy bands between the metal and graphene. Once intimate contact occurs between the metal and graphene, the Fermi levels align to reach an electronic equilibrium. To do this, there is charge transfer between the two materials. Due to the wide conduction band in the metal, there exist an almost unlimited number of unoccupied low energy states. In the graphene (taken to be ntype doped in this example), immediately adjacent to the interface, mobile higher energy charge carriers in graphene spontaneously jump the interface and fall into the lower energy states which are available in the metal. The electronic band diagram of the interface region before and after contact is shown in Figure 2.8(A,B), respectively, based on Bartolomeo's interpretation. ${ }^{(380)}$

\footnotetext{
${ }^{8}$ In fact, its primary use was considered to be as a transparent conductive electrode for solar cell, touch screen and organic light-emitting diodes (OLEDs) applications (although this remains an intense research application of graphene). (729) (109) (728)
} 
(A)

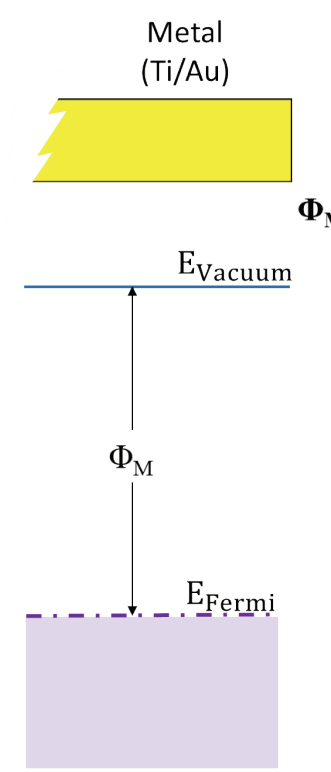

(B)
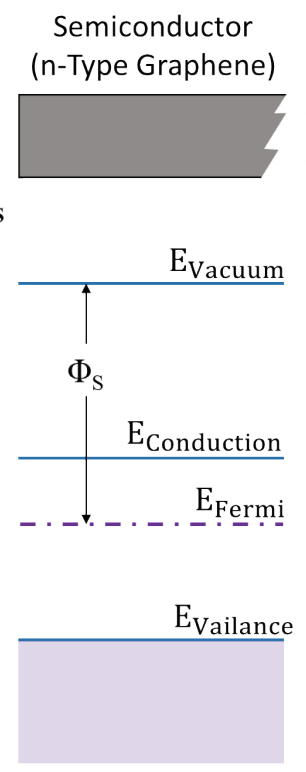

(C)

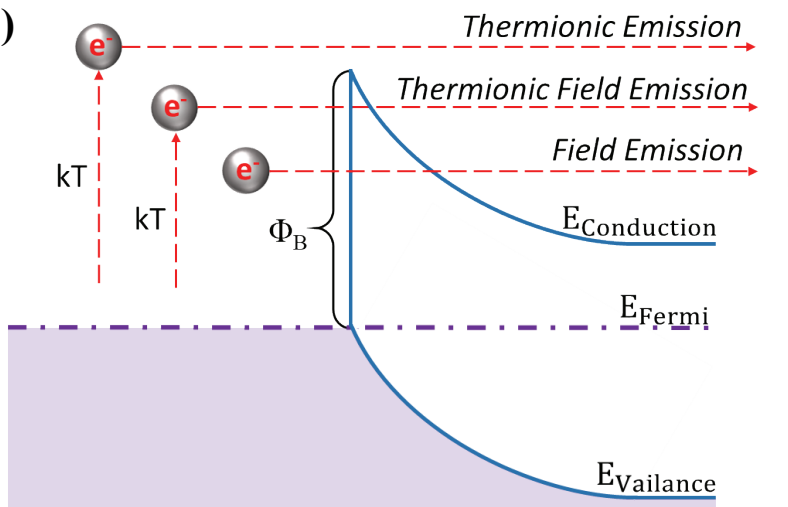

Thermionic Emission

\begin{tabular}{|c|c|c|}
\hline \multirow{2}{*}{$\left(\frac{e h}{4 \pi} \sqrt{\frac{N_{d}}{\varepsilon_{s} m^{*}}}\right)$} & $\leq 0.2$ & Thermionic \\
\cline { 2 - 3 } & 0.2 to 5 & Thermionic Field \\
\cline { 2 - 3 } & $>5$ & Field \\
\hline
\end{tabular}

Figure 2.8: The electronic band diagram of a metal and an n-type semiconductor (A) before contact and (B) after intimate electrical contact is achieved and electronic equilibration. The Schottky barrier presents itself as a barrier to charge injection. (C) The three mechanisms for passing the Schottky barrier. All three occur, but which mechanism dominates depends on the carrier concentration $\left(\mathrm{N}_{\mathrm{d}}\right)$ and the absolute temperature (T), for a given system. (e is the charge of the carrier, the dielectric constant of the semiconductor $\left(\varepsilon_{\mathrm{s}}\right)$, $\mathrm{k}$ is the Boltzmann constant and $\mathrm{m}^{*}$ is the effective mass of the charge carrier).

The area that these charge carriers are pulled from becomes the charge depletion region (or the "space charge region" as it is sometimes referred to in the literature). Depending on the degree of mismatch between the electronic band structures and the mobility of the charge carriers in the graphene, the charge depletion layer can extend far into the graphene layer, on the order of several hundred $\mu \mathrm{m}$. Due to asymmetrical carrier distribution around the junction, this region is under the influence of a built-in electric field. The built-in electric field is seen as a barrier to charge injection from the contact to the graphene. This field is responsible for the non-linear IV behavior of Schottky devices, in that a threshold voltage is required to overcome the energetic barrier to charge 
injection. Given the distribution of energies of the charge carrier population to be injected, the threshold is not a step function but rather is continuous and produces a smooth curve.

There are three mechanisms by which carriers can pass the Schottky barrier, each are illustrated in Figure 2.8(C). In thermionic emission, carriers of low energy are thermally promoted to higher energy levels above the barrier height. The barrier is always present but, at sufficiently high temperatures, the energy of all carriers is higher than the barrier and barrier effect is not seen. Lacking sufficient temperature, quantum effects can dominate. Carriers have a non-zero probability of tunneling through the barrier. Given the statistical nature of these events, this process, called field emission, dominates when the number of carriers is high (i.e. a large population increases the absolution number of carriers which tunnel). The third mechanism, thermionic field emission, is a combination effect. Thermal energy (kT), promotes carriers to higher energy levels which, while not higher than the barrier, increase the likelihood of tunneling though the barrier which is energetically thin at higher energy levels (thermal assisted tunneling). A.D. Bartolomeo gives the equation shown in Figure 2.8(C) to determine generically which mechanism dominates. ${ }^{(380)}$

The built-in field of the Schottky junction due to the space charge region is significant to the field of photodetectors. Although the built-in field is relatively weak, charge mobility becomes high in this region due to the lack of carrier-carrier interactions. When this region is irradiated with photons, one of two processes may occur; both are shown in Figure 2.9. ${ }^{9}$ If the photons are of an energy greater than the Schottky barrier $\left(\Phi_{\mathrm{B}}\right)$, but less than the band gap of the semiconductor (EBandGap), charge carriers may be photonically promoted over the barrier and contribute to the current (Figure 2.9(A)). If the photon energies are greater than the bandgap of the material (EBandGap), electron-hole pairs are generated within the space charge region. Due to the built-in field and the near high mobility of charge carriers in this region, the electron-hole pairs are quickly accelerated away from each other before they can recombine and thermalize their energy into the graphene lattice. Therefore, the carrier lifetime is high in this region compared to carriers generated outside this region. The continued generation of electron-hole pairs results in a photo-current

\footnotetext{
${ }^{9}$ It is important to note that the exact carrier motion depends on the details of the band alignment. Promoted carriers will drift in a direction which lowers their energy. This may be from the metal to the semiconductor or vice versa, it depends on the relative alignment of the energy bands, which can be affected by external biasing. Figure 2.9 shows one possibility. The diagrams presented in the literate vary depend on if the graphene is taken to behave as a metal or as a semiconductor and as a result, the band alignment changes correspondingly.
} 
(Figure 2.9(B)). Most graphene photodetectors reported in the literature attribute the device response to one of these two effects. ${ }^{(380)}$ This has become the basis for many optoelectronic device applications of graphene. ${ }^{(118)(381)(382)}$

Photodetection devices, using graphene based Schottky contacts, have been shown to far

(A)

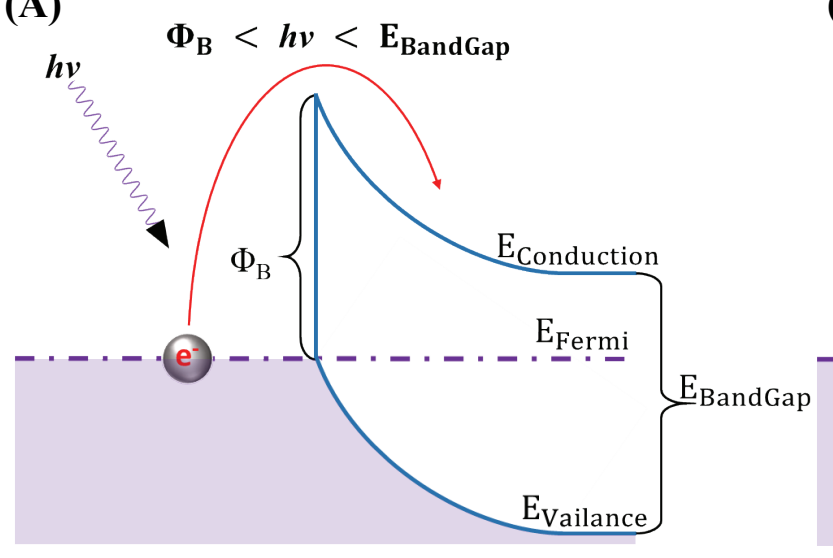

(B)

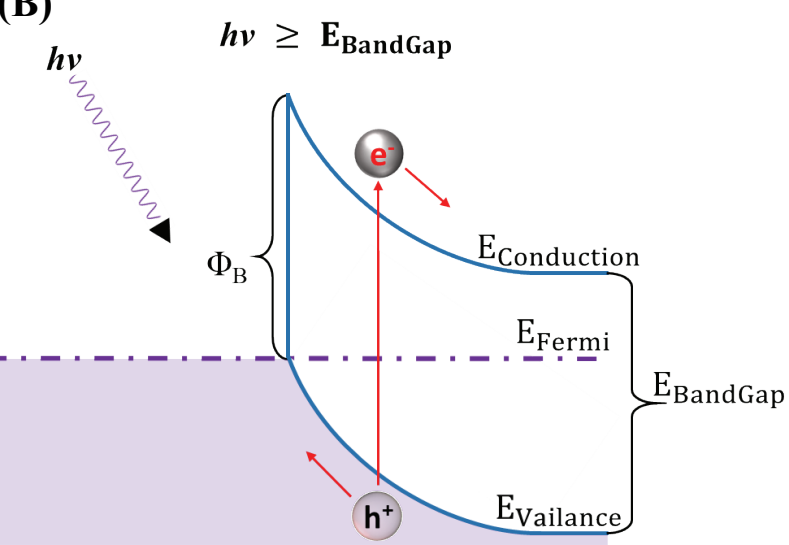

Figure 2.9: Band diagram and resulting carrier generation when (A) photons of energies between the band gap and (B) higher than the band gap.

outperform identical reference devices that do not utilize graphene in the design. ${ }^{(383)}$ A 4-fold increase in light energy conversion has also been reported. ${ }^{(384)}$ This suggests graphene could play a key role in high-performance photo detectors. In addition to light detection, Schottky junctions are also the basis for graphene based solar cells which has recently begun to receive intense research attention. ${ }^{(385)}$

Ultrafast graphene photodetectors have been reported that show a high internal quantum efficiency across a wide bandwidth of visible light. ${ }^{(386)}$ This translates to a high photo-response, spanning the $200 \mathrm{~nm}-1000 \mathrm{~nm}$ range have been reported. ${ }^{(387)}$ It has also been reported that the addition of metallic nanoparticles ( $\mathrm{such}$ as $\mathrm{TiO}_{2}, \mathrm{Ag}, \mathrm{Au}$ ) to the surface of graphene can act as sensitizers and can enhance the interaction between graphene and light. (388) (389) (390) (391) (392) By combining graphene Schottky contact with a random array of deposited nanoparticles, an ultrafast high performance photodetector has been constructed. ${ }^{(393)}$ However, by the controlled deposition of nanoparticles, plasmonic structures can be generated. These plasmonic structures can greatly enhance the interaction between light and matter.

Plasmonic structures are constructed from nano-scale elements which display free surface electrons that are free to oscillate in a coherent manner (such as is the case for metallic nanoparticles). Plasmonic resonant structures arise from precisely arranged arrays of plasmonic 
elements such that their plasmon oscillations constructively interact with one another, increasing the overall effect (although this interaction can be complex). ${ }^{(394)(395)}$ The collective plasmon resonance of the plasmonic array can then couple with the surface plasmons of the graphene sheet. (396) (397) (398) Thus, plasmonic structures built on a graphene surface can act as an impedance matching device between light and graphene, greatly increasing energy transfer between the two. (399) (400)

Due to the selective nature of plasmonic resonance, appropriately constructed plasmonic structures can ensure that such interactions are highly discriminatory (e.g. specific to a narrow bandwidth of light). ${ }^{(401)}$ Coupling of plasmonic resonant structures (essentially light antennas) with surface plasmons of the graphene sheet and has been found to be effective even with micro sized resonant structures. ${ }^{(402)}$ Utilizing these types of arrays and depositing similar plasmonic structures of metallic nanoparticles onto graphene devices, the internal quantum efficiency of a graphene device has been markedly increased at selected wavelengths. ${ }^{(403)}$ Advantageous in this approach is the ability to tune the sensitivity of such devices by changing the geometric layout and sizes of the plasmonic structures. ${ }^{(404)}$ (405) (381) (406) (407) Nano rods, acting as resonant dipole antennas, have also been shown to strongly couple with surface plasmons. ${ }^{(408)}$

Graphene has a uniquely flat absorption spectrum, virtually independent of frequency from the IR through the visible range. ${ }^{(409)}$ Such "blank-slate" behavior makes graphene an ideal starting material upon which to build a tuned photodetector via plasmonic coupling engineering. By tuning plasmonic structures to resonate at desired frequencies, the sensitivity of the devices can be enhanced to more than 20 times that that of undecorated devices at the tuned frequency. ${ }^{(399)}$

Furthermore, graphene devices built on $\mathrm{SiC}$ substrates are uniquely suited for optoelectronic applicants due to the synergistic effect the $\mathrm{SiC}$ substrate has been suspected to pay. ${ }^{(410)}$ Complex substrate effects (gating effects and capacitive coupling) have been shown to dramatically enhance device sensitivity. ${ }^{(411)}$ Graphene photo detectors could operate by monitoring for changes in resistance on exposure to light (resistometer) or by monitoring for induced photo currents (photometer).

\section{§2.3 Research Goals}

To address the production and manufacturability hurdles facing graphene (referred to in this work as the 'grand challenge', and discussed in Section \$1.3) this research focuses on the 
novel plasma assisted growth process, which has been in development for several years by the Stinespring laboratory group at West Virginia University, Morgantown WV (described in Section \$2.1.5). (262) (263) This method provides an alternative process for the growth of high-quality graphene thin-films on the wafer scale. However, although this method shows great potential, it is not in an as developed state as many of the more widely used methods. Fundamentally, this work aims to investigate and mature this process.

A simple contextualized hierarchical of view of this research is shown in Figure 2.10. The principal motivation for this work starts with the grand challenge facing graphene. This work postulates that part of the solution to this challenge lies with the development of the novel plasma assisted graphene growth process. To that end, Goal 1 and Goal 2 aim to improve the current understanding of this process. A multifaceted approach was taken, with Goal 1 focusing on the direct development of the synthesis process and Goal 2 focusing on the application of the films produced by the process.

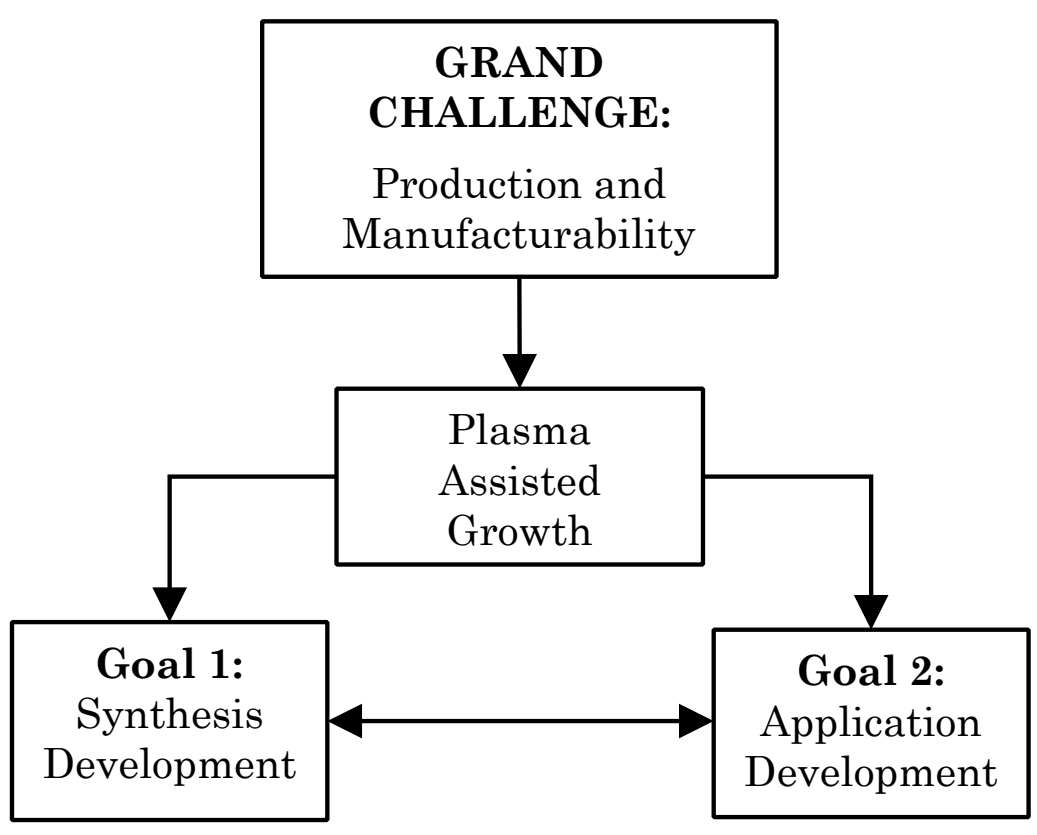

Figure 2.10: The overarching hierarchical view of this research.

There are two primary goals of this research: process development and application development. These goals were chosen for their synergistic relationship; insight gained from one area can be applied to the other. Stinespring, et.al have shown that the UHV plasma assisted method is a viable means by which to produce high-quality graphene films. ${ }^{(262)(263)(264)}$ The work presented here investigates the graphene films produced using a modification of this process where an atmospheric pressure, rather than an ultra-high vacuum, rapid thermal anneal is utilized. This 
has the benefit of greatly reduced the complexity of the annealing equipment while simplifying the process as a whole. Additionally, the effects of the processing parameters and the degree of their impact on the films produced will be investigated. Post-synthesis treatment of the films, aimed at further improving the film quality, will also be investigated.

The fabrication of simple electronic devices using the films produced by the plasma assisted method can provide additional insight into the films produced. Using these simple devices, the electrical properties of the films can be determined. Investing how these properties change as a function of temperature can provide yet another method to probe the fundamental aspects of the films and their interplay with the $\mathrm{SiC}$ substrate.

Clearly, others have shown the utility of graphene as a sensing material. However, the graphene produced by the recently developed plasma assisted method has yet to be investigated for its sensing capabilities. There is reason to believe that graphene produced via the plasma assisted method is different from graphene produced using traditional means. Therefore, an investigation into the sensing applications of graphene produced using this method is called for. In addition to the practical knowledge gained by fabricating and testing these devices, their performance can provide unique insight into the underlying phenomena at play which govern the device behavior. Additionally, this assists in further understanding the nature of the films.

Using the groundwork laid here, future work can build on the knowledge gained by this research to further develop the plasma assisted growth method. Using the various data acquired from the simple devices fabricated here as a guide, more complex and better preforming devices can also be designed. Based on these considerations, the following goals for this research were established.

\section{§2.3.1 Goal 1: Investigation of the plasma assisted method of graphene production}

Goal 1 is to develop the plasma assisted method of graphene growth. Just prior to the beginning of the present work, it was discovered that the ultrahigh vacuum anneal (UHVA) could be replaced with a rapid thermal anneal in a flow of atmospheric pressure argon. ${ }^{(264)}$ Therefore, the first objective of Goal 1 was the characterization of the graphene films produced by the plasma assisted method using the modified atmospheric pressure RTA. The results of this objective are detailed in Section $\$ 4$.1. The effects of the various process parameters on the films produced was also investigated, which constitutes the second objective of Goal 1. The results of this parametric 
investigation are detailed in Section $\$$ 4.2. Additionally, to further improve the films, effects of post-synthesis high temperature $\mathrm{H}_{2}$ treatments were investigated. This objective was motivated by conclusions drawn during the pursuit of the first objective for Goal 1. The results of this are detailed in Section $\$$ 4.3. The overarching conclusions related to Goal 1 are given in Section \$7.1.1. Table 2-2 lists the objectives of Goal 1 and the sections within this document where the related results are discussed.

Table 2-2: Goal 1 and the related objectives.

\begin{tabular}{|c|c|}
\hline \multicolumn{2}{|c|}{ Goal 1: Development of the novel plasma assisted graphene growth method. } \\
(Chapter 4) & Section $\S 4.1$ \\
\hline Objective 1A: Characterize the SOP process. & Section $\S 4.3$ \\
\hline Objective 1B: Probe the impact of processing parameters on the films produced. & Section $\S 4.2$ \\
\hline Objective 1C: Decouple the SOP films by hydrogen intercalation. &
\end{tabular}

\section{§2.3.2 Goal 2: Application development of graphene produced via the plasma assisted method}

Goal 2 is an exploratory study on the utilization of the plasma assisted graphene for sensing applications. The main objective of Goal 2 is to electrically characterize the films and investigate the sensing performance of the $\mathrm{SiC} /$ graphene composites produced by the plasma assisted method. Therefore, the first objective of Goal 2 was the electrical characterization of the simple electronic devices fabricated. The results of this objective are detailed in Section \$5.1. Investigating how the electrical properties changed as a function of temperature was the second objective of Goal 2 . This is detailed in Section \$5.2. The third objective was to probe the gas sensitivity of the devices. This is detailed in Section $\$$ 5.3. The last objective was to investigate the photo sensitivity of the devices. This is detailed in Section $\$$ 5.4. The overarching conclusions related to Goal 2 are given in Section \$7.1.2. Table 2-3 lists the objectives of Goal 2 and the relevant sections where their results are discussed. 
Table 2-3: Goal 2 and the related objectives.

Goal 2: Development of simple electronic devices using graphene produced by the plasma assisted method. (Chapter 5)

Objective 2A: Electrically characterizes the simple electronic devices. Section $\$ 5.1$

Objective 2B: Investigate the temperature dependence of the device electrical properties.

Section $\$ 5.2$

Objective 2C: Investigate the gas sensing performance of the devices. Section $\$ 5.3$

Objective 2D: Investigate the photo diction capability of the devices.

Section $\$ 5.4$




\section{CHAPTER 3: EXPERIMENTAL DESIGNS AND CHARACTERIZATION SETUPS}

In this chapter, the experimental setup for graphene production, materials characterization and sample testing will be detailed. The basic theories which underpin the graphene production method will be explored as well. This is done here because the motivation behind the experimental design necessitates a discussion of the underlying theory. The relevant operational principles of the characterization techniques will also be explored. This is done here to set the stage for the discussion of the results within Chapter 4 and Chapter 5.

\section{§3.1 Graphene Production}

Beginning with an introduction to silicon carbide $(\mathrm{SiC})$, this section will describe the experimental method used in this work to synthesize graphene form a $\mathrm{SiC}$ substrate. Each processing step and the relevant characterization equipment are covered here, complete with a discussion of the underlying theory as it relates to this work.

Developed by the Stinespring Laboratory, the graphene production method used in this work is a modification of previously published methods. ${ }^{(262)(263)}$ A detailed investigation of the process parameters has been conducted, which is covered in Chapter 4. The overall process consisted of three primary steps. First, was a sample preparation step where a silicon carbide wafer was diced into several small rectangular pieces which were then cleaned (detailed in Section \$3.1.2). Second, was the plasma etching step in which $\mathrm{SiC}$ was dry etched under conditions which lead to the selective removal of silicon from the surface layer of the $\mathrm{SiC}$ (detailed in Section \$3.1.3). Third, was a rapid thermal annealing step where the etched $\mathrm{SiC}$ was rapidly thermally annealed under atmospheric pressure gas, leading to the formation of graphene (detailed in Section \$3.1.4).

\section{§3.1.1 Introduction to Silicon Carbide}

Silicon carbide (abbreviated $\mathrm{SiC}$, historically named carborundum) was first synthetically produced by E.G. Acheson in 1893 where it found its primary use first as an abrasive and then later as a refractory material. ${ }^{(412)}$ In Germany $1934, \mathrm{SiC}$ began to be used in electronic applications such as photodetectors and rectifiers. ${ }^{(413)}$ Later in the United States, $\mathrm{SiC}$ was studied for its use as an inert electrode for electrochemical reactions (late 1930's) and as the basis for non-ohmic resistor 
elements (early 1940's). ${ }^{(414)(415)}$ The recent (2018) review of SiC growth technology by Willmann provides an overview of the various methods of $\mathrm{SiC}$ growth in use today. ${ }^{(416)}$

Even before high-quality $\mathrm{SiC}$ wafers became commercially available in 1991 (by Cree, Inc.), $\mathrm{SiC}$ has long been touted as heralding in a new wave of high performance electronic devices. (417) (183) The main attraction was the promise that devices based on SiC would be extremely mechanically rugged and electrically robust. This is due in part to SiC's high temperature stability, hardness, large electrical bandgap, high break down voltage and large thermal conductivity. ${ }^{(183)}$ The high temperature stability of $\mathrm{SiC}$ is a result of the large $\mathrm{Si}-\mathrm{C}$ bond strengths and incredibly low dopant diffusion coefficients. ${ }^{(418)}$ Additionally, with such a long list of $\mathrm{SiC}$ polymorphs from which to choose, scientists could choose a precise $\mathrm{SiC}$ polymorph for the specific application.

For example, the electronic bandgap (the single most critical parameter for any semiconducting material) of $\mathrm{SiC}$ can range from as low as $\sim 2 \mathrm{eV}$ to as high as $\sim 7 \mathrm{eV}$, depending on the polymorph. ${ }^{(183)}$ For comparison, silicon has a bandgap of around $1.11 \mathrm{eV}$ at room temperature. ${ }^{(419)}$ While a higher bandgap is not necessarily 'better', the ability of a single material type to have a wide range of bandgaps from which to choose, is ideal from a design prospective.

This wide bandgap, combined with a high breakdown voltage $(2.4 \mathrm{MV} / \mathrm{cm})$, very high thermal conductivity $(4.9 \mathrm{~W} / \mathrm{cm} \cdot \mathrm{K})$ and high saturation velocities $\left(2.0 \times 10^{7} \mathrm{~cm} / \mathrm{s}\right)$ position $\mathrm{SiC}$ as an ideal material for high temperature and high power electronic applications. ${ }^{(182)}$ The general consensus is that the outlook for the future of $\mathrm{SiC}$ remains bright. Particularly for use in high performance electronic devices, power devices, microelectromechanical systems (MEMS), rugged sensors and many other micro and nano-scale applications. ${ }^{(420)(421)(422)(423)(424)(425)}$ For example, it has been stated that the adaptation of high performance $\mathrm{SiC}$ based electronics (in particular high power, high speed metal-oxide semiconductor field-effect transistor (MOSFETs) and junction gate field-effect transistor (JFETs)) is poised to disrupt the photovoltaic industry. ${ }^{(426)}$

Select properties of $\mathrm{SiC}$ are tabulated in Table 3-1, along with the corresponding properties of silicon for the sake of comparison. Although there are some exotic, large bandgap or high temperature semiconducting materials (diamond, $\mathrm{CsK}_{2} \mathrm{Sb}, \mathrm{GaN}, \mathrm{GaAs}, \mathrm{B}_{4} \mathrm{C}, \mathrm{Ru}_{2} \mathrm{Si}_{3}, \mathrm{BaTiO}_{3}$ and many others) which have properties that approach or even surpass those of $\mathrm{SiC}$, they each possess their own challenges (such as large scale production limitations and the rare or toxic compounds involved) and are not yet mature materials. On the other hand, silicon carbides' technological 
maturity has been steadily progressing over the past three decades, and there now exists a wide verity of production routes for the growth of high quality $\mathrm{SiC}$ on the industrial scale. (427) (428) (429) (430)

Table 3-1: Select properties of silicon carbide and silicon

\begin{tabular}{|c|c|c|}
\hline Parameter & $\begin{array}{l}\text { Silicon Carbide } \\
\text { (various polytypes) }\end{array}$ & Silicon \\
\hline Hardness (Vickers Hardness) & $20.0 \mathrm{GN} / \mathrm{m}^{2}(419)$ & $11.0 \mathrm{GN} / \mathrm{m}^{2(431)}$ \\
\hline Electronic Bandgap & $2-7 \mathrm{eV}^{(183)}$ & $1.11 \mathrm{eV}^{(419)}$ \\
\hline Thermal Conductivity & $4.90 \mathrm{~W} / \mathrm{cm} \cdot \mathrm{K}^{(182)}$ & $1.48 \mathrm{~W} / \mathrm{cm} \mathrm{cm} \cdot \mathrm{K}^{(432)}$ \\
\hline Breakdown Voltage & $1.8-3.8 \mathrm{MV} / \mathrm{cm}^{(421)}$ & $0.3 \mathrm{MV} / \mathrm{cm}^{(421)}$ \\
\hline Melting Point (approx.) & $2,830^{\circ} \mathrm{C}^{(432)}$ & $1,414^{\circ} \mathrm{C}^{(432)}$ \\
\hline
\end{tabular}

And while $\mathrm{SiC}$ has slowly been adopted by the electronics industry, outside of extreme applications, silicon still reigns supreme in this space. ${ }^{(182)}$ However, through the union of SiC with graphene many of the shortcomings of both materials could potentially be solved. As an example, $\mathrm{SiC}$ has a room temperature electron mobility of only $100-980 \mathrm{~cm}^{2} / \mathrm{V} \cdot \mathrm{s}$ compared to $1350 \mathrm{~cm}^{2} / \mathrm{V} \cdot \mathrm{s}$ for $\mathrm{Si}^{(421)(419)}$ Graphene on the other hand has been reported to have mobilities well over 200,000 $\mathrm{cm}^{2} / \mathrm{V} \cdot \mathrm{s}$. ${ }^{(26)}$ The fact that graphene can be grown from $\mathrm{SiC}$, a process which produces an ideal semiconducting-conducing heterostructure, makes the combination a natural fit for electronic applications. There are several review articles devoted to illustrating the potential of this specific SiC-graphene heterostructure. ${ }^{(186)(174)(173)}$

In general, $\mathrm{SiC}$ consists of approximately equal parts silicon and carbon; a 1 to 1 stoichiometric ratio (this is not always the case and nonstoichiometric $\mathrm{SiC}$ is an emerging research area). ${ }^{(43)}$ However, the number of ways in which these atoms can arrange in is astounding. In fact, with more than 250 polymorphs of $\mathrm{SiC}$, there are more known polymorphs of $\mathrm{SiC}$ than almost any other material. (434) Each polymorph (differences in crystal morphology such as cubic, hexagonal, etc.) and polytype (difference is bilayer stacking such as $4 \mathrm{H}, 6 \mathrm{H}$, etc.) has a unique set of electronic properties; these properties can be further modified by doping with adatoms.

Being a semiconductor, the conductivity of $\mathrm{SiC}$ can be tuned by doping the crystal structure with adatoms or inducing vacancy sites. This results in a net number of positive charge carriers (resulting in p-type doping) or a net negative number of charge carriers (resulting in n-type doping). Most commercially available $\mathrm{SiC}$ substrates are naturally n-doped with nitrogen. The $6 \mathrm{H}-$ $\mathrm{SiC}$ and $4 \mathrm{H}-\mathrm{SiC}$ substrates used here are $\mathrm{n}$-doped with nitrogen and have a manufacturer specified 
resistivity of $0.020-0.200 \Omega \cdot \mathrm{cm}$ and have a nitrogen n-type doping density of $9 \times 10^{14}-9 \times 10^{19} \mathrm{~cm}^{-}$ ${ }^{3} \pm 25 \%$ on the silicon face and $1 \times 10^{16}-1 \times 10^{19} \mathrm{~cm}^{-3}$ on the carbon face. ${ }^{(435)}$

Alternatively, semi-insulating $\mathrm{SiC}$ (generically classified as such when the $\mathrm{SiC}$ resistivity is over $\sim 1 \times 10^{5} \Omega \cdot \mathrm{cm}$ ) can be created by doping with vanadium or by growing high purity crystals at high temperature resulting in atomic vacancies (carbon vacancies). ${ }^{(436)}$ Vanadium is an amphoteric dopant (it can lead to n-type or p-type depending on the Fermi level). To from semiinsulating $\mathrm{SiC}$ using vanadium, vanadium acts as a p-type counter-dopant to compensate for natural occurring n-type nitrogen dopants. ${ }^{(437)}$ Alternatively, doping by using growth conditions which impart intentional carbon vacancies (which act as electron acceptor sites resulting in p-type doping) can also be used to produce semi-insulting wafer, sometimes referred to as high purity semi insulating (HPSI) wafers. ${ }^{(438)}{ }^{(437)}$ Data from the manufacture indicate the 4H-SiC[SI] substrates were grown using this method (nitrogen compensated by carbon vacancies) and has a manufacturer specified resistivity of $>1 \times 10^{5} \Omega \cdot \mathrm{cm} .{ }^{(435)}$ However, analysis performed by others has shown even high purity semi-insulting wafers have contaminations, such as $\mathrm{B}, \mathrm{N}, \mathrm{Na}, \mathrm{Al}, \mathrm{V}, \mathrm{Cr}$, $\mathrm{Fe}, \mathrm{P}, \mathrm{Ni}, \mathrm{F}, \mathrm{Cu}$ and $\mathrm{As}$ in the $10^{13} \mathrm{~cm}^{-3}$ to $10^{15} \mathrm{~cm}^{-3}$ range, depending on the specific contaminant. (439) (440) (441) (416) The precise contamination level is difficult to quantify given that the levels are close to or at the detection limit of the analysis technique (SIMS is commonly employed).

In this work, three types of $\mathrm{SiC}$ were used. They were: $6 \mathrm{H}-\mathrm{SiC}, 4 \mathrm{H}-\mathrm{SiC}$ and semi-insulting $4 \mathrm{H}-\mathrm{SiC}$. The vast majority of samples made and tested used $6 \mathrm{H}-\mathrm{SiC}$. The $6 \mathrm{H}-$ and $4 \mathrm{H}-$ (excluding the semi-insulting $4 \mathrm{H}$ ) were doped with nitrogen resulting in n-type doping. Simple schematics of the unit cells of $6 \mathrm{H}$ and $4 \mathrm{H}$ polytypes can be seen in Figure 3.1. Each stacking layer consists of an $s p^{3}$ bond silicon and carbon bilayer resulting in a tetrahedral arrangement. The specific stacking arrangement and crystal lattice system differentiates one polytype from another. An unofficial but widely used nomenclature follows that the first number in the sequence indicates the number of Si-C bilayers which make up the unit cell $(4,15,75,256$, etc.) and the subsequent letter indicates the crystal family ( $\mathrm{H}$ for hexagonal, $\mathrm{C}$ for cubic, $\mathrm{R}$ for rhombohedral, etc.). For $6 \mathrm{H}-\mathrm{SiC}$ the unit cell stacking sequence is $\mathrm{ABCACB}$ and is $\sim 15.119 \AA$ along the $\mathrm{c}$ axis. For $4 \mathrm{H}-\mathrm{SiC}$ the unit cell stacking sequence is $\mathrm{ABCB}$ and is $\sim 10.085 \AA$ on the $\mathrm{c}$ axis. Two unit cells are depicted for each polytype in Figure 3.1 . 


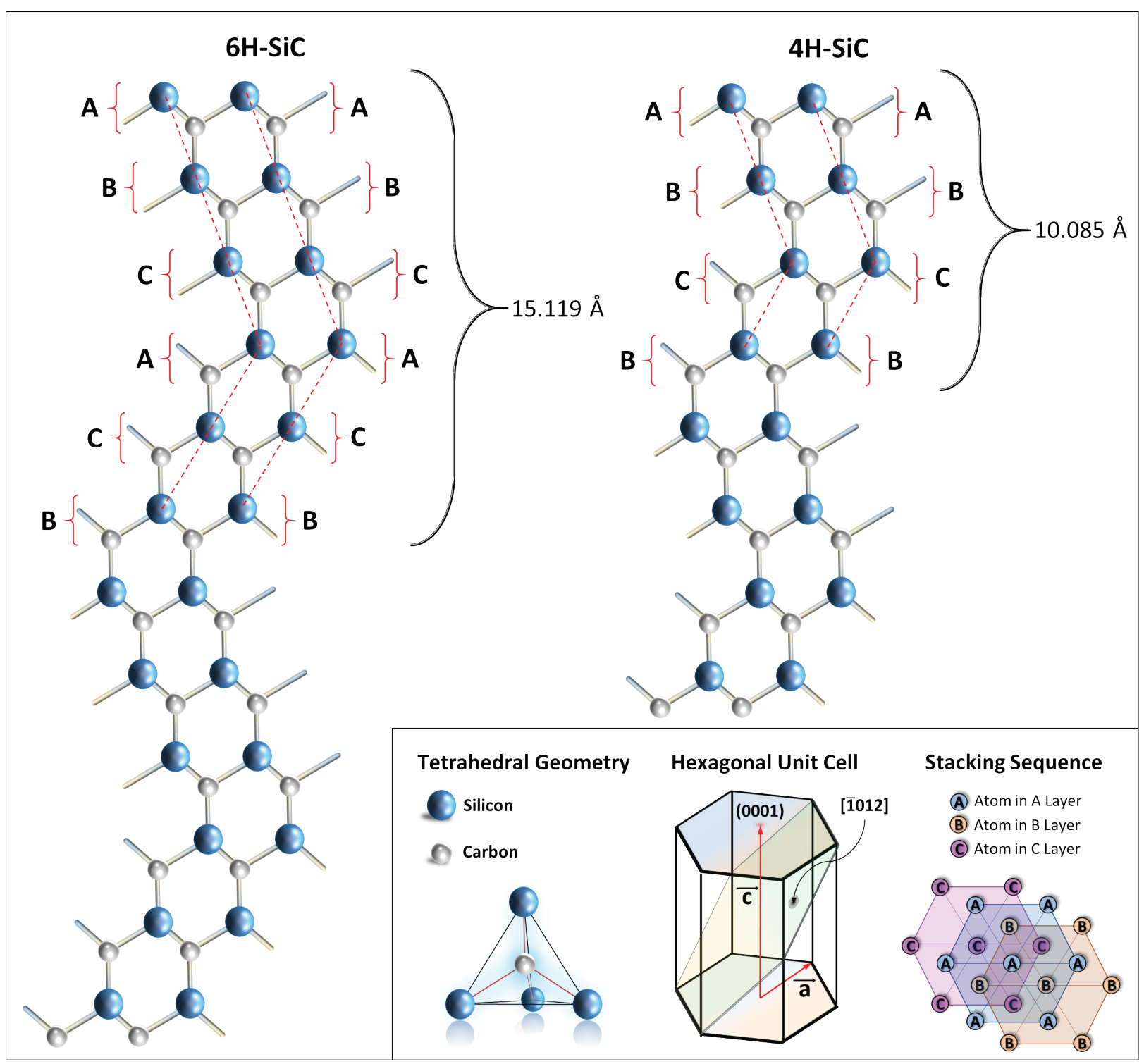

Figure 3.1: The 6H-SiC and 4H-SiC crystal structure. Two unit cells are shown for each type. For $6 \mathrm{H}-\mathrm{SiC}$ the unit cell stacking sequence is ABCACB and is $\sim 15.119 \AA$ along the $\mathrm{c}$ axis. For $4 \mathrm{H}$-SiC the unit cell stacking sequence is ABCB and is $\sim 10.085 \AA$ in height. Shown in the inset is the tetrahedral bonding geometry due to $s p^{3}$ hybridized bonding, the hexagonal unit cell of the $\mathrm{SiC}$ used in this work and the relative positions of the three possible bilayer stacking arrangments. 
Despite the only structural difference between $6 \mathrm{H}-\mathrm{SiC}$ and $4 \mathrm{H}-\mathrm{SiC}$ being the lack of two additional bilayers in the latter, the material properties of the two polytypes are different. Many of the optical properties are quite dissimilar, for example. ${ }^{(442)}$ A comparison of some properties of $6 \mathrm{H}-\mathrm{SiC}$ and $4 \mathrm{H}-\mathrm{SiC}$ can be found in Table 3-2. The values tabulated here are nominal. The literature reports a range of values for each parameter; this is reflective of the slight variability encountered with periodic materials (crystals, for example) whose properties can be strongly influenced by defects.

Table 3-2: Comparison of $6 \mathrm{H}-\mathrm{SiC}$ and $4 \mathrm{H}-\mathrm{SiC}$ properties. ${ }^{(435)}(418)(443)(444)$

\begin{tabular}{|l|l|l|}
\hline \multicolumn{1}{|c|}{ Property } & \multicolumn{1}{c|}{ 6H-SiC } & \multicolumn{1}{c|}{ 4H-SiC } \\
\hline Bandgap (eV) & 3.03 & 3.26 \\
\hline Thermal Conductivity $(\mathrm{W} / \mathrm{cm} \cdot \mathrm{K}){ }^{@ 298 K}$ & $3.0-3.8$ & $3.0-3.8$ \\
\hline Intrinsic Carrier Concentration $\left(\mathrm{cm}^{-3}\right)$ & $\sim 10^{-5}$ & $\sim 10^{-7}$ \\
\hline Saturated Electron Velocity $\left(10^{7} \mathrm{~cm} / \mathrm{sec}\right)$ & 2 & 2 \\
\hline Intrinsic Electron Mobility $\left(\mathrm{cm}^{2} / \mathrm{V} \cdot \mathrm{s}\right)$ & 380 & 947 \\
\hline Intrinsic Hole Mobility $\left(\mathrm{cm}^{2} / \mathrm{V} \cdot \mathrm{s}\right)$ & 115 & 95 \\
\hline Thermal Conductivity $(\mathrm{W} / \mathrm{cm} \cdot \mathrm{K})$ & 5 & 5 \\
\hline Electron Affinity $(\mathrm{V})$ & 3.7 & 3.3 \\
\hline Mohs Hardness & $\sim 9$ & $\sim 9$ \\
\hline $\begin{array}{l}\text { Lattice Parameters (manufacture } \\
\text { specified) }\end{array}$ & $\begin{array}{l}\mathrm{a}=3.081 \AA \\
\mathrm{c}=15.117 \AA\end{array}$ & $\begin{array}{l}\mathrm{a}=3.073 \AA \\
\mathrm{c}=10.053 \AA\end{array}$ \\
\hline
\end{tabular}

A final aspect of $\mathrm{SiC}$ crystals important to this work is the crystal face experimented on. The two crystal faces discussed here are either the carbon face or the silicon face; two inequivalent planes. This simply indicates whether a silicon layer (the (0001) direction using the Miller indices) or a carbon layer is presented (the $(000 \overline{1})$ direction using the Miller indices) on the surface. Due to the bilayer nature of $\mathrm{SiC}$, this also indicates from which direction the crystal is approached. Referring to Figure 3.1, approaching either crystal from the bottom up will encounter a carbon layer. If a bilayer is removed, again a carbon layer is encountered; proceeding up through the crystal uncovers a reverse of the stacking sequence (for example in $4 \mathrm{H}-\mathrm{SiC}$, BCBA rather than $\mathrm{ABCB})$. The same logic holds true when approaching the crystal from the top down in Figure 3.1. This is of particular importance in an epitaxial context; for example, the selective sublimation method of graphene growth on the Si face results in markedly different graphene growth than on the $\mathrm{C}$ face. Both the amount of graphene grown, the rate and the temperature dependence are affected (however, the morphology of the graphene appears to be unaffected). ${ }^{(445)}$ 


\section{§3.1.2 Substrate Preparation}

The first step in the substrate preparation is the dicing of the as-received 4-inch whole wafers into smaller pieces for individual processing. SiC wafers, purchased from University Wafer, Inc., were diced using a DAD3240 automatic dicing saw from Disco, Inc. to produce 10 full $1 \mathrm{~cm}^{2}$ samples and 14 smaller samples of varying sizes. A hub style, diamond grit ZH05 series dicing blade operated at a cutting speed of 30,000 RPM and with a feed speed of $1 \mathrm{~mm} / \mathrm{sec}$ was used to dice the $\mathrm{SiC}$ wafer. Two different polytypes of hexagonal $\mathrm{SiC}$ were used; $6 \mathrm{H}$ and $4 \mathrm{H}$, both n-doped. 4H-SiC semi-insulating silicon carbide was also briefly investigated. $6 \mathrm{H}$-SiC was used for the vast majority of the experimentation. A photograph of a 3 inch $6 \mathrm{H}-\mathrm{SiC}$ is shown in

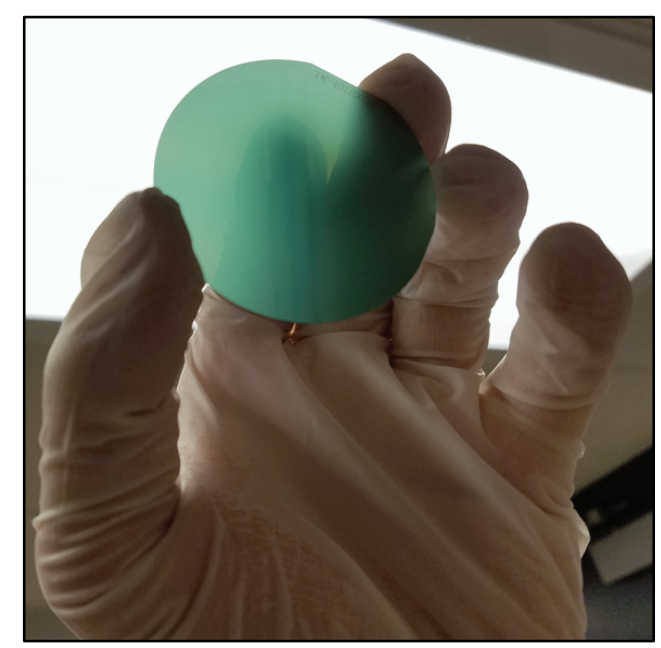

Figure 3.2: Photograph of one of the 3 inch diameter $6 \mathrm{H}-\mathrm{SiC}$ wafer used in this work, before dicing. The polished (ooo1) surface is shown.

A photograph of the Disco dicer is shown in Figure 3.3. The smaller samples were used for a variety of tests and experiments while the full squares are processed as device substrates. The following cleaning and plasma etching steps are conducted entirely within a cleanroom to prevent sample contamination. All chemicals used were semiconductor grade. 


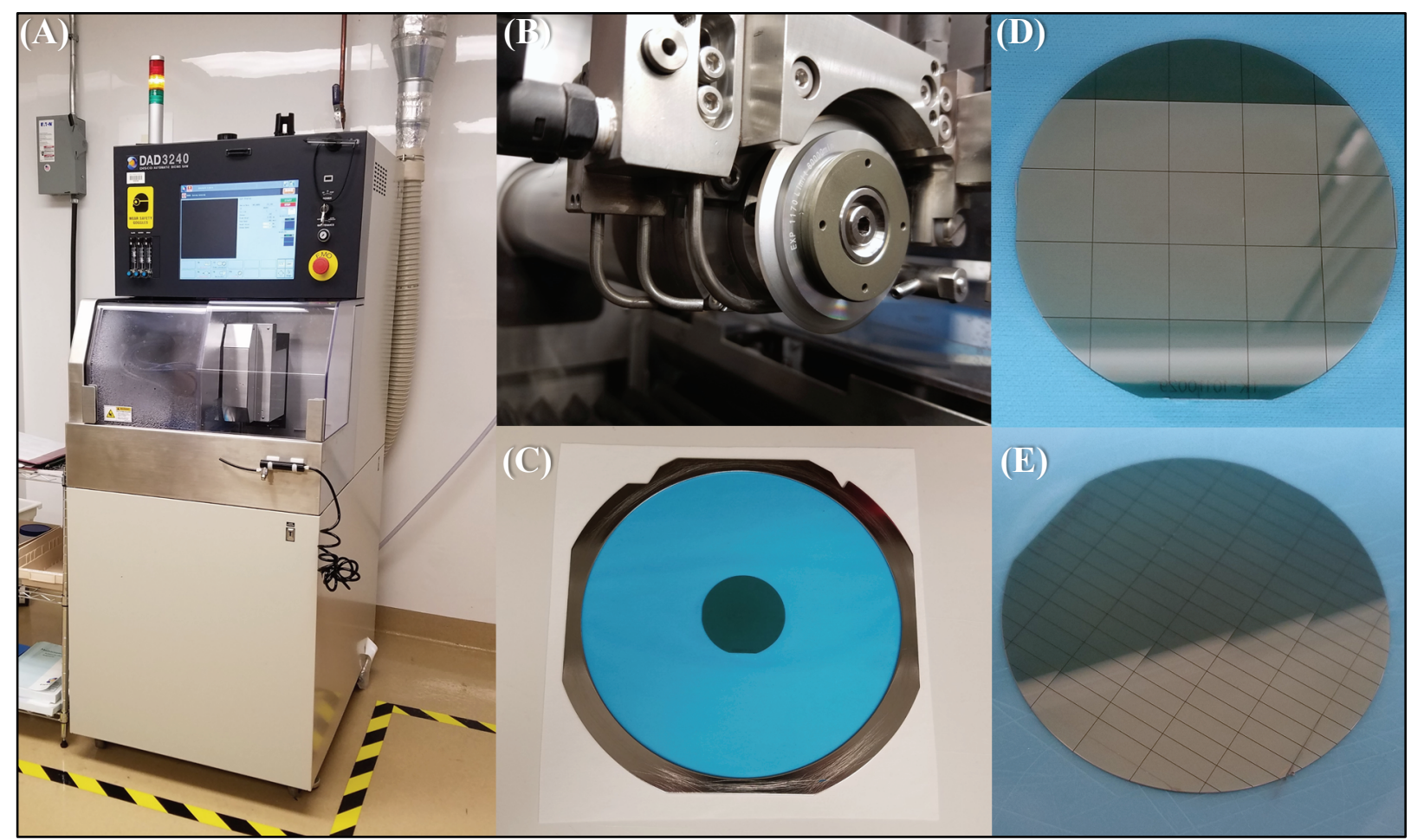

Figure 3.3: (A) The DAD3240 Disco automatic dicing tool. (B) The dicing arm with hub style diamond grit blade installed. Three water jet lines are visible. (C) A SiC wafer mounted onto adhesive tape and vacuum metal ring. (D) A $6 \mathrm{H}-\mathrm{SiC}$ wafer diced into $1 \mathrm{~cm}^{2}$ squares; after graphitization these were layer diced into 16 smaller $2.5 \mathrm{~mm}^{2}$ samples using the same dicer shown here. (E) An example of a slightly different dicing pattern. Shown is a $6 \mathrm{H}-\mathrm{SiC}$ wafer diced into samples measuring $10 \mathrm{~mm} \times 2.5 \mathrm{~mm}$.

The diced SiC underwent a 10-minute sequential soak of ethanol, then deionized (DI) ultrapure water and then acetone to degrease the surface. Next, the samples were sonicated in a DI water bath for 15 mins to further clean the surface. The samples were then soaked for 15 mins in glacial acetic acid. This was done to remove any remaining particles due to electrostatic charging effects. The samples were then rinsed with DI water and then acetone. Samples were then blow dried with dry house nitrogen.

\section{§3.1.3 Plasma Etching}

This section will describe both the plasma etching step as it was performed in practice, as well as touch on the relevant plasma theory. Plasma etching is commonly referred to as a 'dry etch'. This is as opposed to a 'wet etch' where chemically reactive liquids are used to remove material. In a dry etch, a plasma is used to remove (etch) the material. Because a plasma is essentially an ionized gas, it is highly chemically active. The reaction kinetics of a dry etch are generally much faster than those of a wet etch and the chemistry can be complex. Because the 
plasma is electrically generated, it can be remotely controlled. This allows for a high degree of real-time control over the plasmas. This makes it a versatile and highly tunable processing technique to a degree that other processing techniques cannot achieve.

For this work, plasma conditions were chosen that produced a plasma which shows a greater reactivity to silicon over carbon. This selectively etched silicon out of the $\mathrm{SiC}$ crystal matrix of the substrate. Both carbon and silicon are removed in this process, however the rate of silicon removal is higher than the rate of carbon removal. This results in a carbon rich surface that is quickly established on the surface of the SiC substrate at the start of the etch. This balance is maintained as the etch proceeds down through the $\mathrm{SiC}$ crystal stack. Allowing the process to proceed beyond the initial establishment of the carbon rich surface ensures that the final film is constructed from fresh $\mathrm{SiC}$ which, to this point, has never been exposed. Doing so allows the etch to penetrate down through any surface contamination and crystal damage remaining from wafer polishing and handling. On the completion of the etch, the result is a $\mathrm{SiC}$ substrate with a carbon rich surface. This process is discussed in Section $\$ 4.1$.

The plasma used here is a radio frequency (RF) glow discharge plasma and is classified as a low temperature, weakly ionized plasma (around 1 ion is generated for every 10,000 neutrals). (446) (447) Specifically, an inductively coupled plasma (ICP) combined with a reactive ion etch (RIE) was utilized. The main advantage of an ICP/RIE is the ability to independently control the plasma density and species distribution (by manipulating the ICP power) and the impact energy of ions on the sample surface (by manipulating the RIE power). This is accomplished by using two independently controlled power supplies and impedance matching networks for the ICP and RIE. By combining an ICP with an RIE, a synergistic effect is achieved which results in higher etch rates that either method used individually. ${ }^{(448)}$ 
A Trion Technologies Minilock-Phantom iii inductively coupled plasma with a reactive ion etch (ICP-RIE) system was used in this work. ${ }^{(449)}$ A photograph of the etching system is shown in Figure 3.4. The system is equipped with a load lock and a robotic transfer arm. The system vacuum is maintained by a mechanical roughing pump staged with a turbomolecular pump. The ICP power supply can be varied between $0 \mathrm{~W}-1000 \mathrm{~W}$. The RIE power supply can be varied between $0 \mathrm{~W}-600 \mathrm{~W}$. The system is equipped with an automatic matching network (a Trion "PhaseMag” network) for both the ICP and RIE. During operation the process chamber pressure can be varied from 0 mTorr to 125 mTorr. The process gas flowrates can be varied from 0 SCCM to 100 SCCM. However, it is noted that a stable plasma may not be achieved for all parameter combinations.

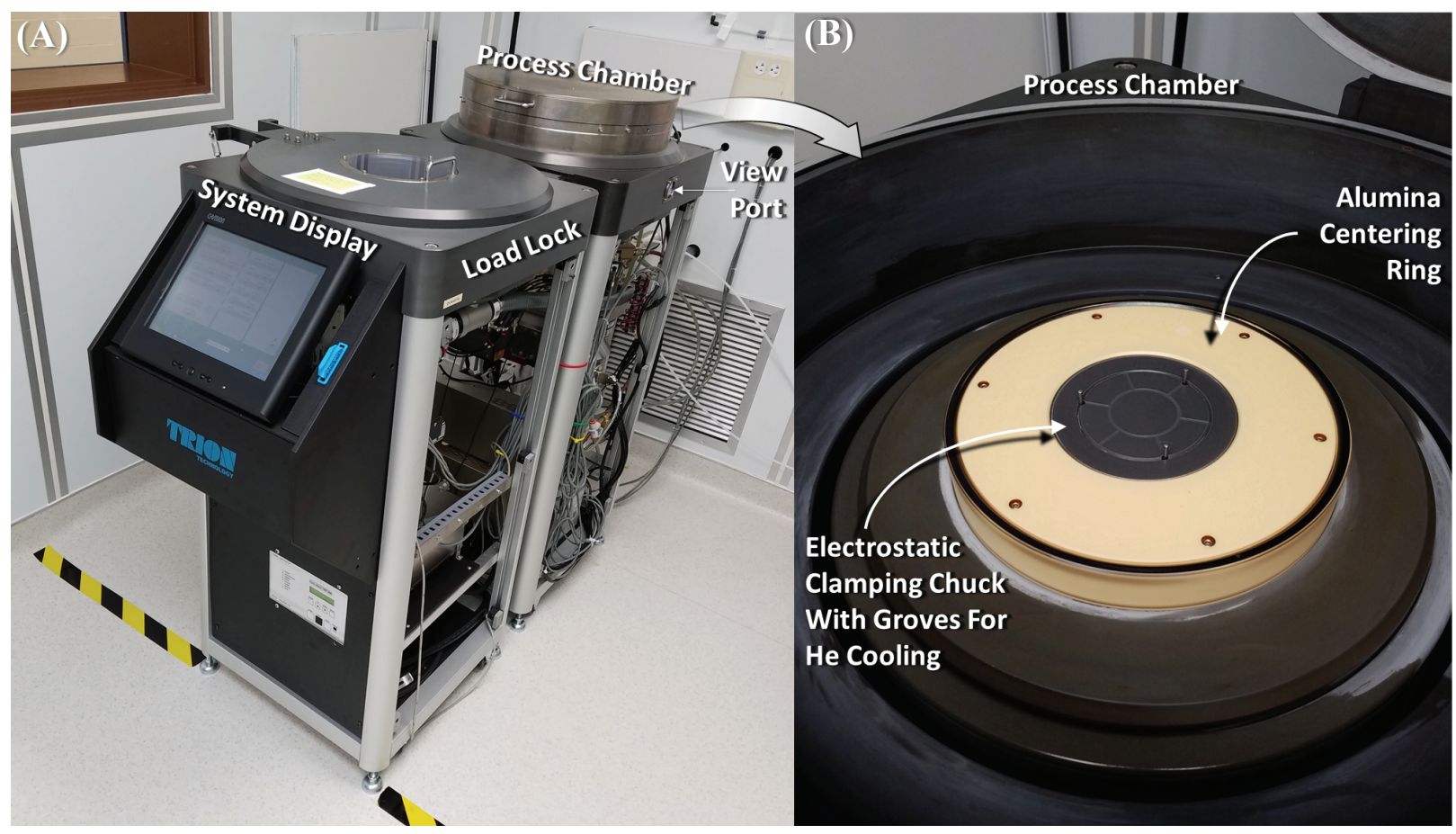

Figure 3.4: (A) The Trion Minilock-Phantom iii ICP/RIE system to perform the etching step. (B) A view of the interior or the main process chamber where the plasma is formed.

The system is also equipped with an anodized aluminum electrostatic clamping chuck, used to electrostatically clamp the carrier wafer to the bottom electrode during etching. The chuck is grooved so that a helium backing flow can be used to cool the back side of the carrier wafer. A large centering ring surrounds the chuck. For part of this work, this ring was constructed from anodized aluminum. This was later replaced with an alumina $\left(\mathrm{Al}_{2} \mathrm{O}_{3}\right)$ chuck for issues relating to contamination described in Appendix $\$$ C.2. 
A $100 \mathrm{~mm}$ silicon carrier wafer was always used when the system is operated. This wafer serves two purpose. First, this wafer is the transport substrate for the transition of samples from the load lock to the main chamber. Second, the wafer provides physical protection of the electrostatic chuck from the reactive plasma.

Before the primary sample etching step, the etching system itself underwent a two-part process preformed to bring the chamber into a clean and stable starting status before sample processing. First, an oxygen-based cleaning recipe was run. Second, a $\mathrm{CF}_{4}$ chamber conditioning recipe was run. The parameters for each are listed in Table 3-3. The cleaning step utilized a highly reactive oxygen plasma to consume contaminants remaining in the chamber from previous runs. Using a new carrier wafer, so as to prevent oxygen-based cross contamination, the $\mathrm{CF}_{4}$ chamber conditioning step was performed. This was done to expose the chamber to a plasma environment similar to would be seen by the chamber during the primary run where a $\mathrm{SiC}$ sample was to be processed. More information regarding this step is detailed in Appendix $\$ \mathbf{A}$.1.4.

Table 3-3: Summary of Pre-Run Process Parameters

\begin{tabular}{|l|c|c|}
\hline \multicolumn{1}{|c|}{ Parameter } & Oxygen Plasma Cleaning Recipe & Chamber Conditioning Recipe \\
\hline Time & $1200 \mathrm{sec}$ & $(120 \mathrm{sec}$ ON, 60 sec OFF $)$ X 4 \\
\hline ICP Power & $600 \mathrm{Watts}$ & $600 \mathrm{Watts}$ \\
\hline RIE Power & $80 \mathrm{Watts}$ & $300 \mathrm{Watts}$ \\
\hline Gas Used & $\mathrm{O}_{2}$ & $\mathrm{CF}_{4}$ \\
\hline Flow Rate & $98 \mathrm{SCCM}$ & $20 \mathrm{SCCM}$ \\
\hline Chamber Pressure & $50 \mathrm{mTorr}$ & $25 \mathrm{mTorr}$ \\
\hline
\end{tabular}

Others have shown a $\mathrm{CF}_{4}$ inductively coupled plasmas will deposit an insulating material onto the chamber walls. ${ }^{(450)}$ This detunes the electrical characteristics of the system, resulting in parameter drift as a function of usage. This is in agreement with observations recorded during this work; see Appendix $\boldsymbol{\$} \boldsymbol{A}$.1.4. By performing the conditioning step, the chamber properties reach a pseudo steady-state before the primary sample run. It is noted that this machine is part of a multiuser facility; otherwise the precursor steps would be different (i.e. these steps are independent of the fundamental science of the process and are instead preformed as part of equipment maintenance). This is typical for the type of plasma chamber design used in this work. ${ }^{(447)}$

During these steps, no samples were in the chamber. Immediately following these two steps, the primary sample etching step was performed. This step used the same carrier wafer as the chamber conditioning step. Using this wafer during the chamber conditioning step ensured the 
wafer experienced the same conditioning environment as the process chamber. The aggressive etch of the conditioning step exposes a fresh, clean silicon surface on the carrier wafer which further reduces possible contamination routes.

Once cleaned, the SiC substrates were mounted onto the silicon carrier wafer with a small dab of boron nitride Cool-Grease (CGR7016) from AI Technology, Inc. This paste is electrically insulating and thermally conductive. It is used in this work primarily to mechanically adhere the sample to the carrier wafer to prevent movement during the etch. The typical use for this paste is to enhance the thermal contact between the sample and the carrier wafer and reduce sample heating due to the plasma. The samples were then etched under a variety of conditions. The effect of each of these parameters in discussed in Section $\$ 4.2$. The total etch time remained constant at 12 mins for all experiments.

A generic description of the primary etch follows. High purity tetrafluoromethane $(99.7 \%$ $\mathrm{CF}_{4}$ ) was injected at a rate of $20 \mathrm{SCCM}$ into the etching chamber through a gas distributer head located at the top of the chamber. A schematic of the chamber can be seen in Figure 3.5. The chamber was automatically maintained at a predefined pressure (typically this was 25 mTorr) during the entire process by dynamically controlling a throttle exhaust valve between the process chamber and the turbomolecular pump.

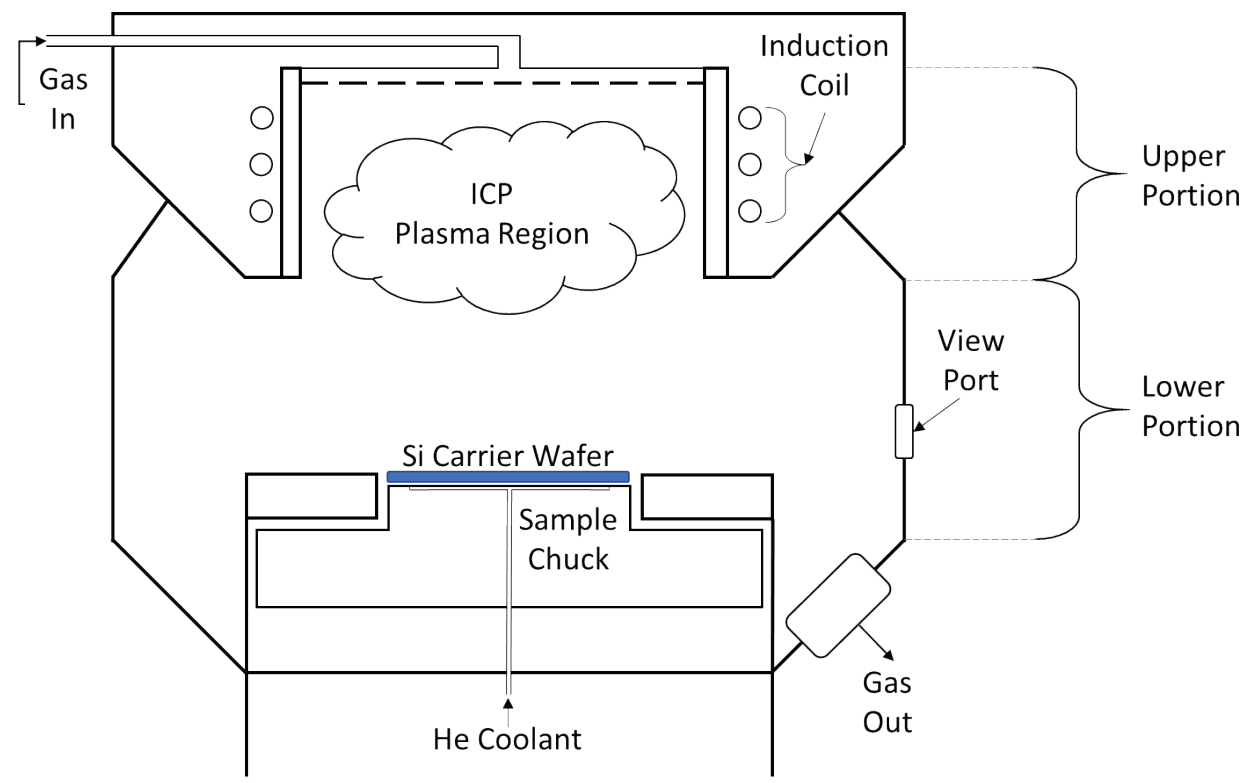

Figure 3.5: Simple schematic showing the arrangement of the plasma chamber. 
This geometry of the chamber allows for the generation of a so-called 'remote' inductively coupled plasma. The ICP plasma generated in the upper portion of the chamber is coupled to the induction coils by transformer action. This confines the high-density plasma primarily to the upper portion of the chamber. However, by design, some plasma is generated in the main process area (lower portion). This separation of the ICP from the sample electrode limits the influence of the ICP on the sample and allows for greater control by the reactive ion etch portion of the process (governed by the powered bottom electrode). In this way, greater independence (and thus control) can be achieved between the primary ICP effects (the species distribution and ion/radical amounts) and the RIE effects (ion-bombardment energy). ${ }^{(447)}$

The primary etching process was broken into four cycles. Each cycle consisted of a powered etch step and an unpowered rest step. The etch step ran for 180 seconds and both the ICP and RIE power were set to the full set-point power. The rest period ran for 60 second during which the gas flowrate and chamber pressures were maintained at the set values, but no power was supplied to the chamber.

During the etch step the gas in the chamber is subjected to a radio frequency (RF) electromagnetic field operating at $13.56 \mathrm{MHz}$ via a water cooled 3-turn copper induction coil which encompasses the upper portion of the chamber. ${ }^{10}$ This generated a relatively low-density plasma consisting of ions, radicals and electrons. The coil was driven by an RF generator, regulated by an impedance matching network. This network continuously matched the generator output impedance (at $50 \Omega$ ) to the coil impedance so that the appropriate set point power was dissipated into the plasma. This minimized power losses resulting from impedance mismatch; the plasma induction coils' impedance can vary greatly as the electrical permeability (effective dielectric strength) of the gas/plasma mixture within the coil changes due to the dynamic nature of the process and pressure fluctuations. ${ }^{(451)}$ Under some conditions, the network cannot match with the coil. This leads to cases where the amount of power actually dissipated into the plasma is much less than the set point power and the plasma because unstable. This was encountered during the parametric investigation, discussed in Section $\$ 4.2 .2$ and Section $\$ 4.2 .3$.

\footnotetext{
${ }^{10}$ There is no inherent scientific reason for the specific frequency of $13.56 \mathrm{MHz}$; this is simply the center frequency in the ISM band (industrial, scientific and medical) reserved by the FCC (federal communications commission) and ITU (international telecommunications union) for scientific and medical equipment. (734) (735)
} 
Once power is applied to the ICP coil, electrons are stripped from a small number of gas molecules by the strong RF field. These electrons are caught in the oscillating RF field and collide with other gas molecules in the chamber. Due to the mass and charge difference between the heavy ions and the light electrons $\left(9.1 \times 10^{-31} \mathrm{~kg}\right.$ for an $\mathrm{e}^{-} \mathrm{vs} .1 .5 \times 10^{-25} \mathrm{~kg}$ for $\mathrm{CF}_{4}$, a difference of 6 orders of magnitude), the electrons are rapidly oscillated back and forth (at velocities on the order of $1 \times 10^{6} \mathrm{~m} / \mathrm{s}$ and change directions $13,560,000$ times a second), in sync with the RF field, while the ions lag behind. ${ }^{(452)}$ This results in a difference in oscillation periodicity and dramatically increases the collision frequency between electrons and ions. These collisions are highly energetic and generates ions, radicals and more electrons (as well as broad spectrum light via photoemission from electron energy transition events which produces the characteristic plasma glow). The secondarily generated electrons go on to collide with more gas molecules which create more ions, radicals and electrons. This results in an electron-ion cascade process which leads to the ignition of a stable plasma. This is how the RF electric field becomes inductively coupled with the etchant gas in the chamber and gives rise to the inductively coupled plasma (ICP). ${ }^{(453)}$

Although the majority of the gas in the chamber remains as $\mathrm{CF}_{4}$, an appreciable fraction devolves into a plasma consisting of electrons and all manner of $\mathrm{CF}_{\mathrm{x}}$ ions and radicals. Others have shown that the majority of the charged particles exist as $\mathrm{CF}_{2}{ }^{2+}$ ions. ${ }^{(454)}{ }^{(455)}$ However, the exact species distribution is complex and is a function of the power, pressure and location within the chamber. The $\mathrm{CF}_{\mathrm{x}}$ radicals constantly recombine into higher order ions and are subsequently ripped apart by the strong RF field and re-ionized by free electrons, thus creating the dynamic plasma. ${ }^{(456)}$

For the RIE component, a secondary RF signal (also at $13.56 \mathrm{MHz}$ ) is applied to the sample chuck, which sets at the bottom of the chamber directly below the induction coil. A circuit is created consisting of the RF generator, matching network, sample plate, plasma and chamber walls. Because the electrons are light and fast moving, some escape the main plasma region. These electrons strike the chamber walls, which are grounded and remain neutral as the electrons are bled to ground. This makes the plasma slightly electron deficient, and as a whole has a slight positive bias.

In the lower region of the chamber, near the RIE electrode, electrons also escape the plasma and strike the sample chuck. However, this plate is not grounded but instead is capacitively coupled to the RIE RF generator. By coupling in this manner, this allows for the accumulation of negative 
charges on the chamber side of the capacitive plate. Negative charges are not bled off to ground due to the isolating nature of the blocking capacitors in the matching network to DC flow (a capacitor appears as an open circuit to DC flow). A schematic of the ICP and RIE circuit networks is shown in Figure 3.6. ${ }^{(451)}$

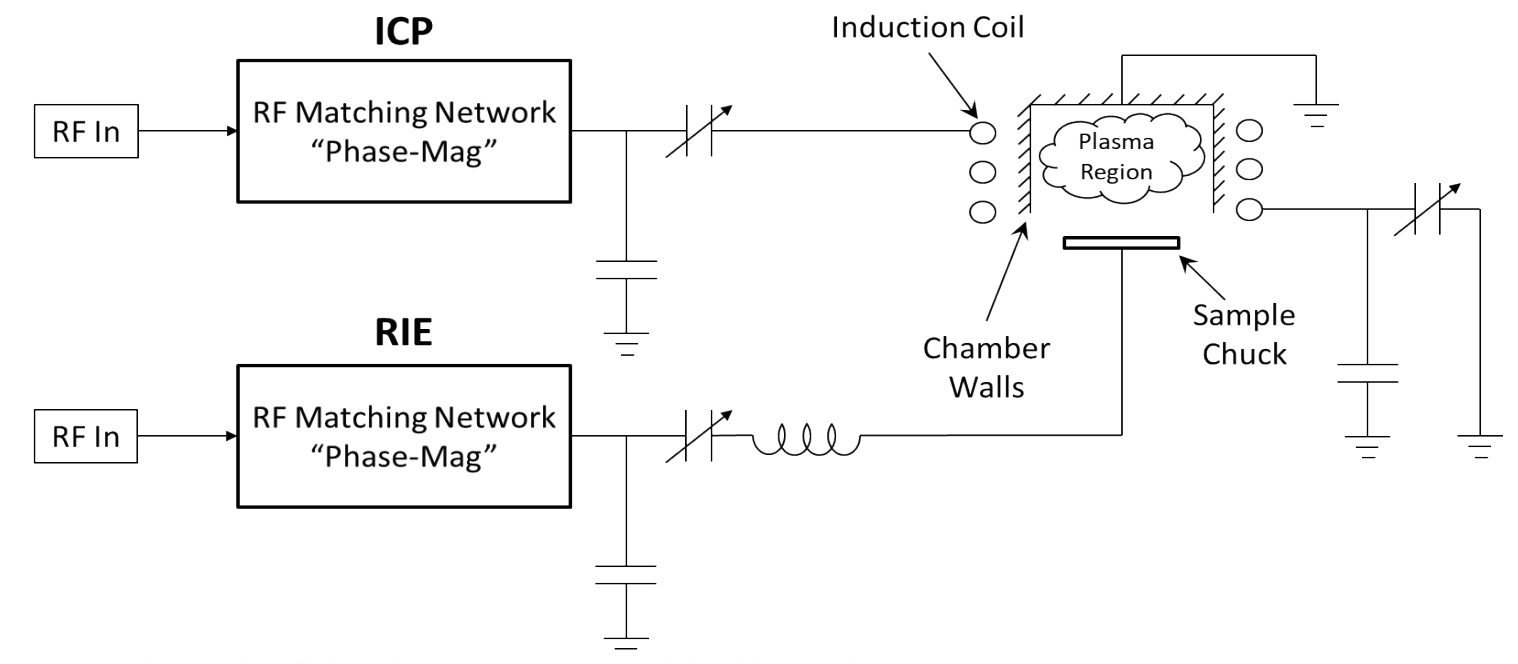

Figure 3.6: Schematic of the plasma system used in this work.

This arrangement allows a large DC biases ( $\left.\mathrm{VDC}_{\mathrm{DC}}\right)$ of several hundred volts to develop on the chuck; this is called a 'self-bias'. The VDC is an important 'side-effect' of the RIE (in that it is not a directly controlled parameter and is a function of the applied RIE power). ${ }^{(446)}$ The bias is negative (relative to the plasma) and acts on the positivity charged ions in the plasma, attracting them to the sample surface and repelling the free electrons. This effect is confined to a narrow band immediately adjacent to the electrode, called the ion sheath. The high frequency RIE RF signal is seen on the chamber side of the capacitive plate (a capacitor appears as a closed circuit to AC flow- in this case the RF signal). The RF signal is negatively offset by the DC self-bias. The physics which govern the effects of the secondary RF field on the plasma are complex, but the result is highly energetic positive ions accelerated to the sample surface. ${ }^{11(457)}$

The time resolved charge on the substrate is shown in Figure 3.7(A). The $13.56 \mathrm{MHz}$ signal is offset by the DC bias as a result of charge build up from electrons captured from the plasma. Figure 3.7 $(B, C)$ shows the plasma potential as a function of vertical ( $\mathrm{Z}$ direction) and horizontal (X direction) positions within the chamber. However, these are idealized view of the plasm

\footnotetext{
${ }^{11}$ For an in-depth discussion of the field theory as it relates to the wafer RF field, the reader is directed to Chapters 11 and 12 of Principles of Plasma Discharges and Materials Processing. (447) The following references also provide a more complete look into the plasma theory. (732) (733)
} 
potential. In reality, the spatial fluctuations in plasma potential can be substantial and complex. (447) The main plasma area is slightly positive, due to a loss of electrons to the walls (ground and perpetually at $0 \mathrm{~V}$ ) and bottom electrode (sample chuck). The capacitively isolated sample chuck builds a large negative change due to the accumulation of electrons from the main plasma region.

(A)

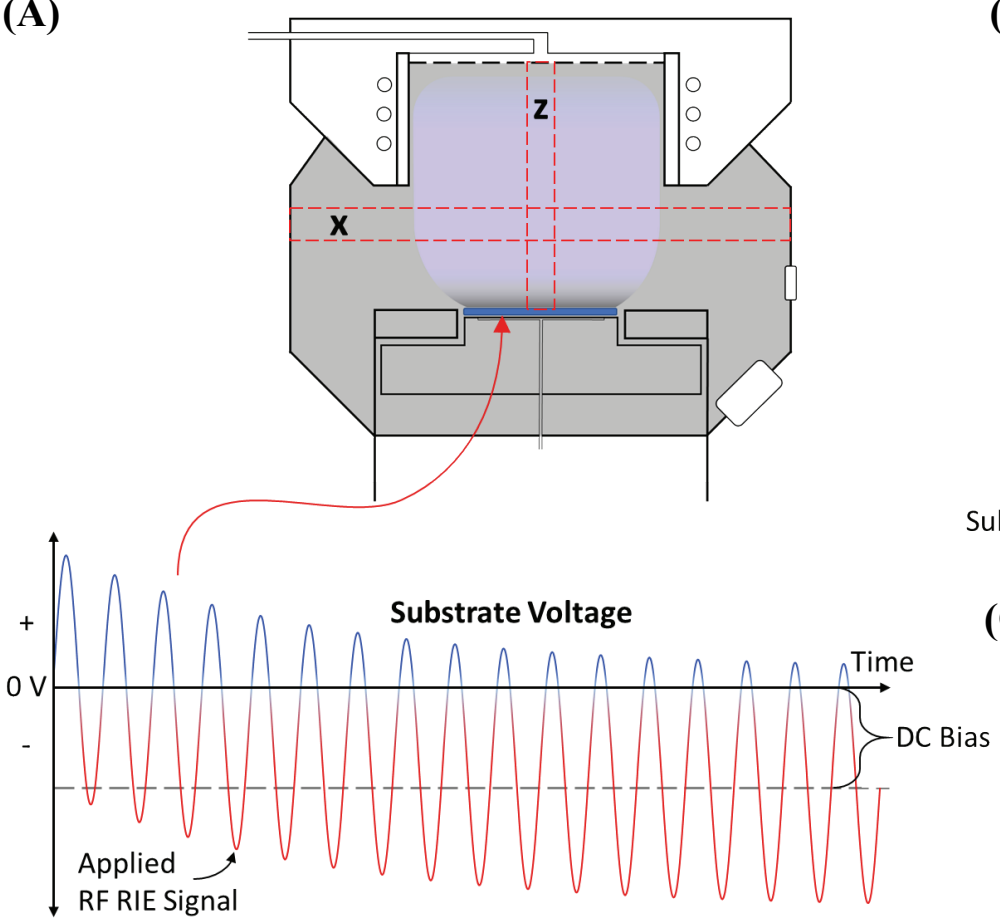

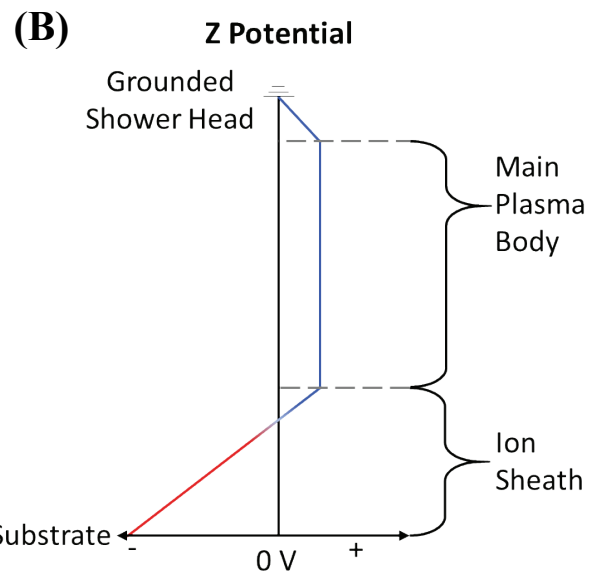

(C)

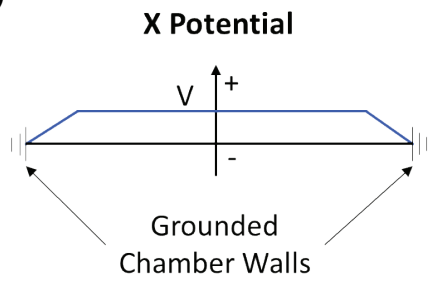

Figure 3.7: (A) Schematic of the chamber and the potential of the sample/substrate. The RIE RF field is offset by the DC self-bias as it develops. (B) The plasma potential along the $\mathrm{Z}$ direction (vertical axis) of the chamber, from the shower head to the sample chuck. (C) The plasma potential as it varies between opposing walls across the main body of the plasma.

The combination of the DC bias and the RF field have two effects. First, the negative field repels the free electrons in the plasma near the sample electrode. This prevents non-discriminant electron radiation damage to the sample surface and limits substrate heating. The area of the plasma that is electron free is the ion sheath. Because there are no photons emitted in this region, it can be seen by the naked eye and is also referred to as the "dark band" in the plasma. The larger the DC self-bias and RIE power, the larger the dark band becomes. ${ }^{(452)}$

The second and more important effect is the aggressive directional etching due to ion bombardment. The negative $\mathrm{DC}$ field acts on the positively charged $\mathrm{CF}_{\mathrm{x}}$ ions within the dark band. Ions are accelerated by the electric field towards the sample electrode. The ions impact the sample with an energy proportional to the ion size (mass), the ion change $(+1,+2,+3, \ldots$, etc.), the sum of the potential gradient generated by the -DC bias and the slight positive charge of the main body 
of the plasma. This results in a non-discriminant physical etch (i.e. both carbon and silicon are etched). ${ }^{(447)}$

This non-discriminant physical etch works synergistically with the selective chemical etch, which occurs simultaneously. The chemical etch results from the specific chemistry occurring between the $\mathrm{CF}_{\mathrm{x}}$ ions and $\mathrm{SiC}$ substrate. The $\mathrm{CF}_{\mathrm{x}}$ ions attack the $\mathrm{SiC}$ bonds, damaged by the physical etching and resulting heating, and selectively binds to the Si to form volatile compounds. (458) (459) The chemistry is complex, but the primary reaction is a surface decomposing reaction to form $\mathrm{SiF}_{\mathrm{x}}$ compounds. These $\mathrm{SiF}_{\mathrm{x}}$ compounds are quickly volatilized from the sample surface due their low evaporation energy and the elevated sample temperature and low chamber pressure. ${ }^{12}$ This leads to rapid and preferential removal of silicon from the $\mathrm{SiC}$ crystal matrix. Some of the volatile compounds re-enter the plasma field and are re-ionized but most are exhausted from the chamber. ${ }^{(447)}$ Figure 3.8 shows photographs of the various plasmas used in the graphene synthesis. The images are taken though the side view port (the view port is annotated in Figure 3.4). The ICP

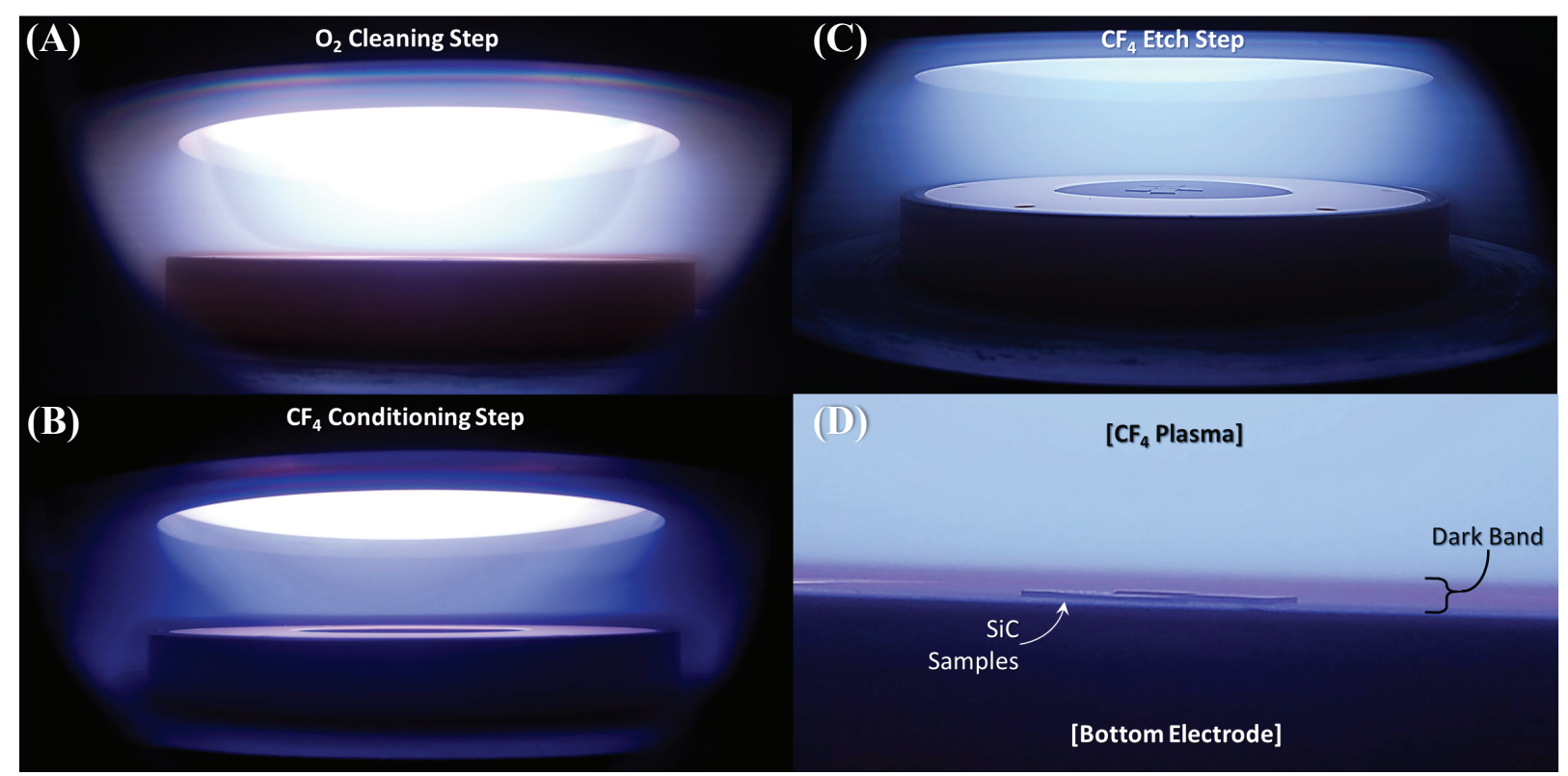

Figure 3.8: Views looking into the main chamber as seen through the side viewport, taken at different steps during the process. (A) The $\mathrm{O}_{2}$ cleaning step, used to clean the chamber of material from previous runs. (B) The $\mathrm{CF}_{4}$ conditioning step, used to bring the chamber behavior into a pseudo-steady state. (C) The etch step. Several samples can be seen undergoing the etch. (D) Close-up of the etch step, showing the samples, the main plasma and the ion sheath (dark band), due to the DC bias.

\footnotetext{
${ }^{12} \mathrm{As}$ an example, $\mathrm{SiF}_{4}$ (silicon teterafluoride), the most abundantly produced product by the reaction between a $\mathrm{CF}_{4}$ plasma reaction with a $\mathrm{Si}$ substrate, has a vaporizes point of $-86.2^{\circ} \mathrm{C}$ at $1 \mathrm{~atm}$. (447) (739)
} 
is generated in the upper area of the chamber. The large object at the bottom of the chamber is the bottom electrode to which the RIE power is applied.

\section{§3.1.4 Atmospheric Pressure Rapid Thermal Annealing (AP-RTA)}

This section will describe the experimental procedure used to perform the AP-RTA step. The AP-RTA step accomplishes two things: it provides the thermal energy need for the carbon atoms to rearrange into the thermodynamically favorable graphene orientation and removes the volatile F containing species. The impact of this step and a parametric study is discussed in Section $\$ 4.2 .4-\$ 4.2 .6$.

The atmospheric pressure rapid thermal annealing (AP-RTA) step is a substantial deviation from the previously reported methods for plasmas assisted growth of graphene. ${ }^{(264)}$ The previous method performed the rapid thermal annealing step under an ultra-high vacuum. For this work, the rapid thermal anneal was performed under atmospheric pressure ultra-pure argon or ultra-pure hydrogen. To accomplish this, several custom recurved quartz tubes were fabricated. A schematic accompanied by a real-world photograph can be seen in Figure 3.9 for AP-RTAs using argon. Several tubes were fabricated, but the basic design remained the same.

(A)
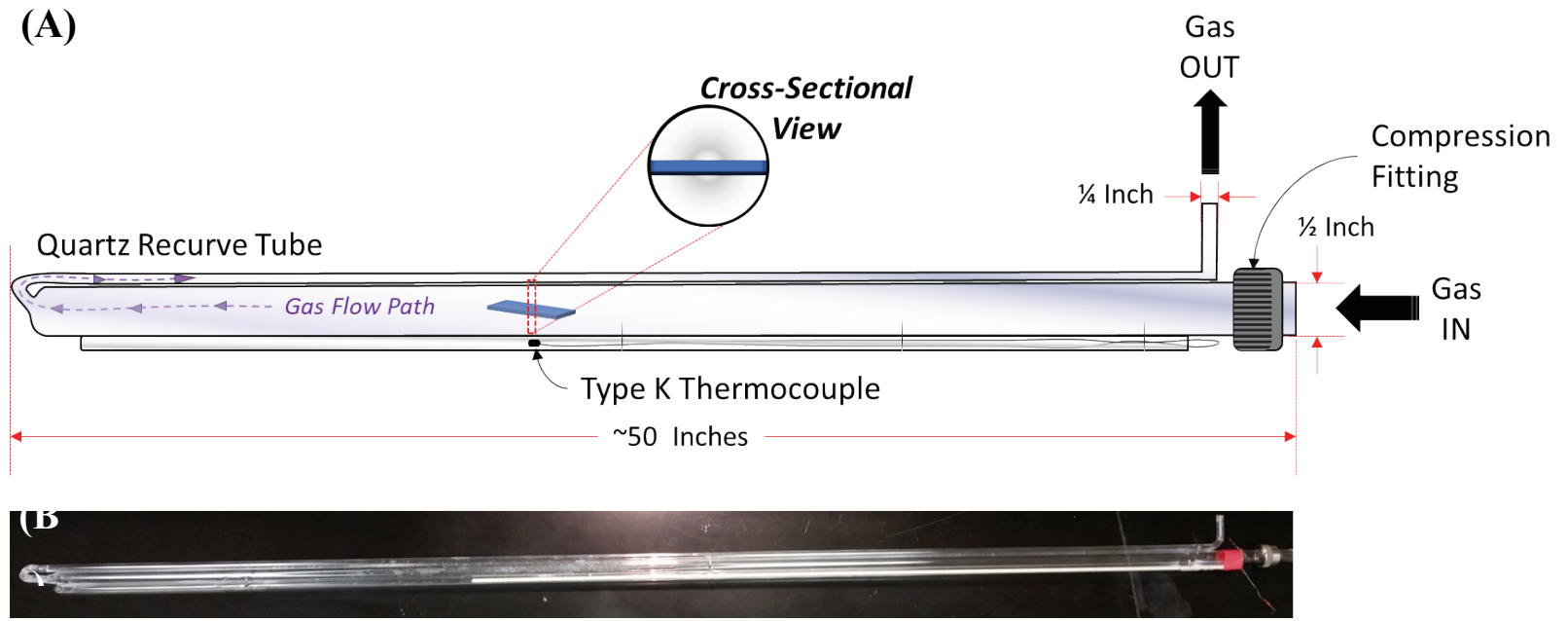

Figure 3.9: (A) Schematic of a typical AP-RTA tube, used to house the sample during the AP-RTA step. (B) Real world picture of a tube used in this work.

Semiconductor grade, ultra-pure argon (99.99999\% Ar, obtained from Airgas) was flown down the tube at a rate of $100 \mathrm{SCCM}$. Argon was used to protect the sample from oxidation during the AP-RTA step. The tube was designed with a recurve to minimize backflow of atmosphere from the open exhaust of the tube. The high temperatures encountered necessitated the use of quartz. A compression fitting was used to seal and mate the tube to the argon gas line. A flexible gas line 
was employed to allow for the freedom of movement of the tube to be rapidly inserted and extracted from the heater. A small quartz line was tacked to the side of the tube to house a type K thermocouple. The thermocouple wires were threaded through a two-hole alumina $\left(\mathrm{Al}_{2} \mathrm{O}_{3}\right)$ tube to protect and insulate them from one another. The thermocouple was attached to an Omega DP462 temperature readout which allowed for the real time monitoring of the temperature in the vicinity of the sample.

Three different heaters were used in this work. A series 1600 C\&M High Temperature Furnace was the primary heater used in a plurality of the $<1000^{\circ} \mathrm{C}$ anneals and in all anneals requiring temperatures $>1000^{\circ} \mathrm{C}$. An FD 303A Hoskins Electric Furnace from Hoskns Mfg. Co. and a Lindberg Blue $\mathrm{M}$ from Thermo Scientific were also used for some annealing experiments below $1000^{\circ} \mathrm{C}$. The C\&M and Thermo Scientific furnaces are shown in Figure 3.10.

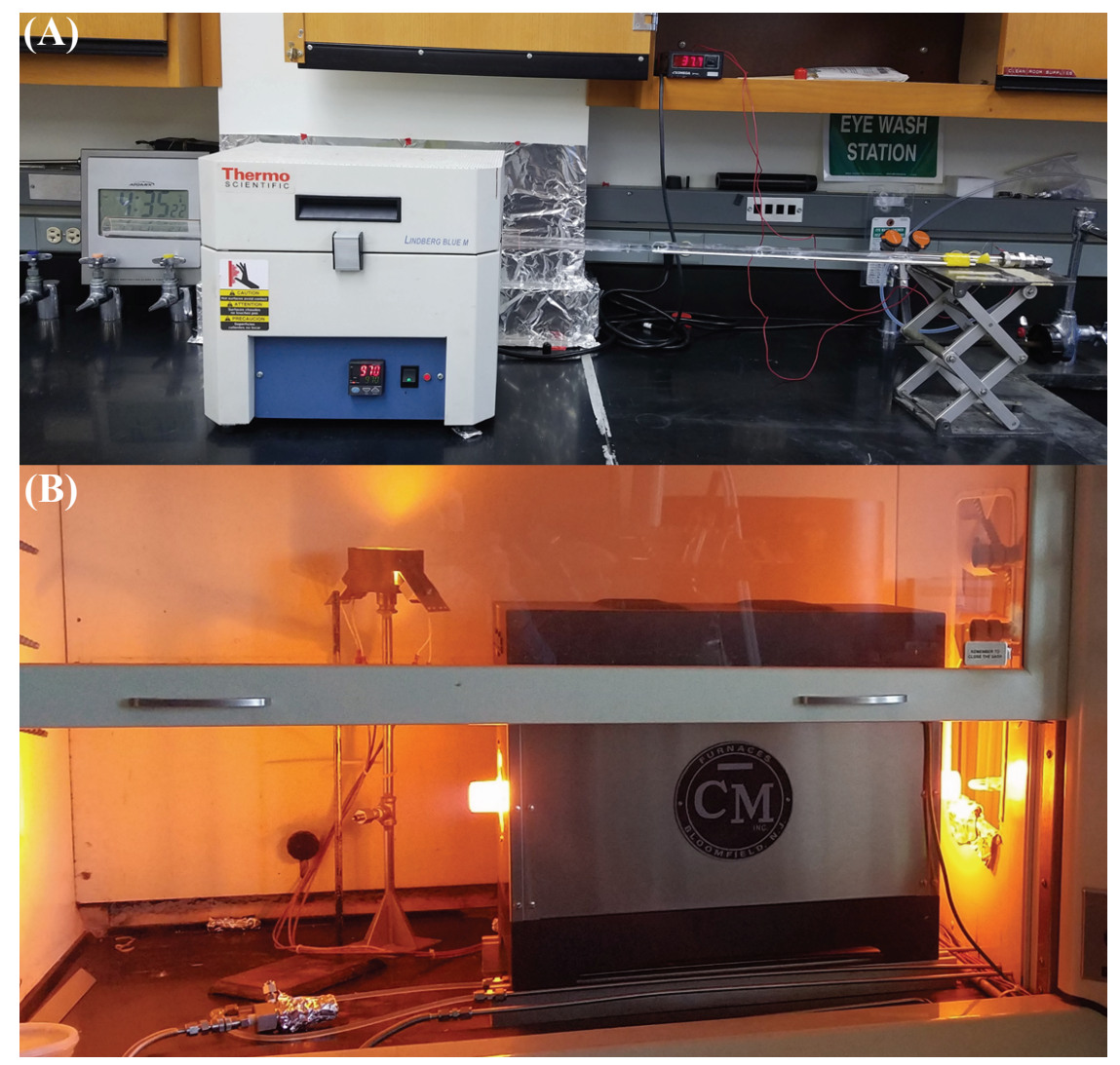

Figure 3.10: (A) The Thermo Scientific Lindberg Blue M furnace shown with quartz RTA tube (supported by a lab jack). This furnace was used for some sub $1000^{\circ} \mathrm{C}$ experiments. (B) The high temperature C\&M furnace with alumina $\left(\mathrm{Al}_{2} \mathrm{O}_{3}\right)$ refractory tube used for a plurality of sub $1000^{\circ} \mathrm{C}$ experiments and all experiment which required temperatures $>1000^{\circ} \mathrm{C}$. Pictured is the furnace at $\sim 1500^{\circ} \mathrm{C}$.

The quartz tube was loaded into the heater during the heater heat up time and continuously purged with argon at $100 \mathrm{SCCM}$. After the four hours heat up stage, the tube was allowed to bake 
for an additional 15 mins at the heater setup point (typically $1000^{\circ} \mathrm{C}$ for most RTAs). This prebake of the tube was done to desorb atmospheric containments, such as water, from the inner tube walls prior to sample loading. The tube was removed from the heater and allowed to cool to room temperature. The etched substrates were then loaded into the quartz tube and positioned axially so that the sample rested next to the thermocouple (approximately $3 / 4$ down the length of the tube). The tube geometry ensured that the sample sits directly on the center diameter chord of the tube, exposing both surfaces to the Ar flow. The tube was purged with ultra-pure Ar for a period of at least one hour at a rate between 100 - 500 SCCM to ensure that the gas in the tube was exchanged several times.

Once the heater had reached the set temperature and the tube had been thoroughly purged, the AP-RTA step was performed. During the AP-RTA the Ar gas flow was maintained at 100 SCCM for all experiments. The tube was quickly inserted into the heater cavity and both the temperature and time were monitored. Once the desired set point was reached (either a specific time or specific temperature) the tube was quickly withdrawn from the heater and allowed to rapidly and naturally cool to room temperature. The argon flow was maintained at 100 SCCM during the cool down period. Once the sample had cooled to room temperature, the gas flow was terminated, and the samples extracted from the tube for analysis.

For thermal treatment of samples under hydrogen gas, the series 1600 C\&M High Temperature Furnace was used exclusively. Due to the hazardous nature of hydrogen gas at high temperature, a different design of the quartz containment tube was used. This can be seen in Figure 3.11. The design used a sealed $1 / 2$ inch quartz containment sheath with a $1 / 4$ inch quartz injector. This design allowed for the controlled containment of the gas as it flowed through the tube. The $\mathrm{H}_{2}$ gas was exhausted from the tube into a $1 / 4$ flex line. This line was plumbed directly into the exhaust of the fume hood where the gas stream was diluted by laboratory air down to $<0.002 \% \mathrm{H}_{2}$ (well below the flammability limit of hydrogen). There was dead space gas between the end of the injector and the end of the sealed sleeve tube. This volume was not in-line with the gas flow path and consequently was not fully swept out by the gas stream. Samples were loaded in the injector line ensured that the samples were constantly exposed to fresh gas only. 
(A)
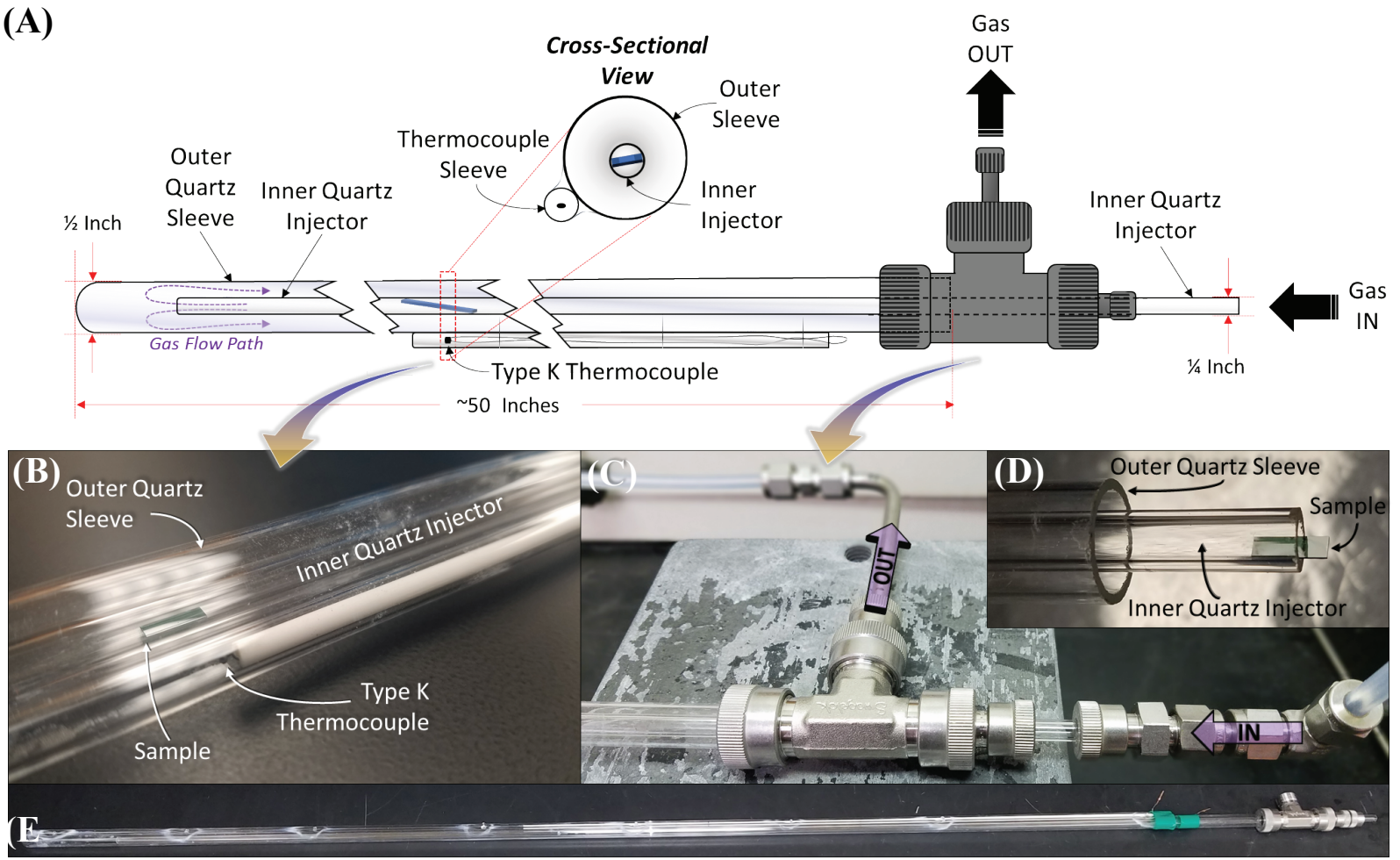

Figure 3.11: (A) A simple schematic of the setup for hydrogen annealing. (B) A picture of a sample loaded into the quartz injector tube. (C) A picture of the gas inlet and outlet plumbing designed for the total containment of hydrogen annealing gas. (D) A picture showing the geometry of the injector-sleeve arrangement and a sample being loaded into the injector.

Samples were loaded into the injector tube and the tubes sealed via the fitting assemblies. The tube was then purged with ultra-high purity (UHP) argon at a rate of 700 SCCM for approximately 5 mins to sweep the tube of laboratory air. All purge times were calculated based on the volume of the system and flow rate such that the system was swept out multiple times by the purge gas. The tube was then purged with UHP hydrogen at a rate of 700 SCCM for approximately five mins to sweep the tube of argon. The hydrogen flow rate was decreased to 100 SCCM (this matched the flowrate of argon during the RTA step of previous experiments). The tube assembly was then inserted into the heater and then withdrawn after the prescribed annealing time or temperature was reached. The tube was allowed to cool down naturally under a flow of UHP hydrogen. Once the thermocouple reading dropped below $100^{\circ} \mathrm{C}$ the gas flow was switched to 700 SCCM of argon for approximately five mins to fully purge the tube. The tube assembly was then disassembled, and the samples extracted for analysis. 


\section{§3.2 Characterization}

A variety of analysis techniques were used to characterize the materials produced in this work. A thorough analysis of the principle material was conducted at each processing step as the material transitions from $\mathrm{SiC}$ to the graphene films. This section will give a brief introduction to the techniques which were used the most extensively. This will touch on both the underlying theoretical principles involved and on their application. Particular attention is directed towards the theory and application as they pertain to this work specifically, so that the results presented in Chapter 3 may be properly interpreted and given context.

\section{§3.2.1 X-ray Photoelectron Spectroscopy (XPS)}

XPS analysis is a surface sensitive characterization technique that has been widely used since its inception in the early 1960s. ${ }^{(460)(461)}$ For his work on what eventually became the basic physics behind XPS analysis, Kai M. Siegbahn was awarded one half of the 1981 Nobel Prize in physics. ${ }^{13(462)}$ A rudimentary setup resembling an XPS apparatus was constructed in 1907 by P.D. Innes. In the 1950's, Siegbahn and others improved on Innes setup. His work with Hewlett-Packard Co. led to the first commercial XPS instrument in 1969. ${ }^{(463)}$

When irradiated with photons, a material may first adsorb and then re-emit the energy through a variety of mechanisms. Often, adsorbed photonic energy is simply thermalized into vibrations within the material. However, given photons of sufficient energy, the material may reemit the photonic energy as ejected electrons through the photoelectric effect. Electrons emitted in this fashion are commonly termed photoelectrons.

The amount of energy required to produce a photoelectron is call the binding energy (BE) (referenced to the Fermi level). This quantity provides valuable insight into the material which produced the photoelectrons. Most importantly, information such as the atomic composition of the material (what elements are present) and the chemical environment of the elements (bonding states) can be collected. ${ }^{(464)}$

\footnotetext{
${ }^{13}$ The other half of the 1981 Nobel Prize in physics was awarded to Nicolass Bloembergen and Arthur Schawlow for their contributions to the development of laser spectroscopy. (462)
} 
Due to the quantum nature of photons and electron energy states, the energies of the emitted photoelectrons are independent of the intensity of the excitation photon source. Therefore, by preforming a simple conservation of energy calculation, the BE is easily determined. Only three parameters are need. ${ }^{(465)}$ They are the energy of the excitation source (hv; plank's constant times the frequency of the photon), the kinetic energy of the emitted photoelectrons ( $\left.E_{k}\right)$ and the work function of the spectrometer/detector ( $\Phi_{\text {Spec. }}$ ). The calculation is depicted graphically in Figure 3.12 .

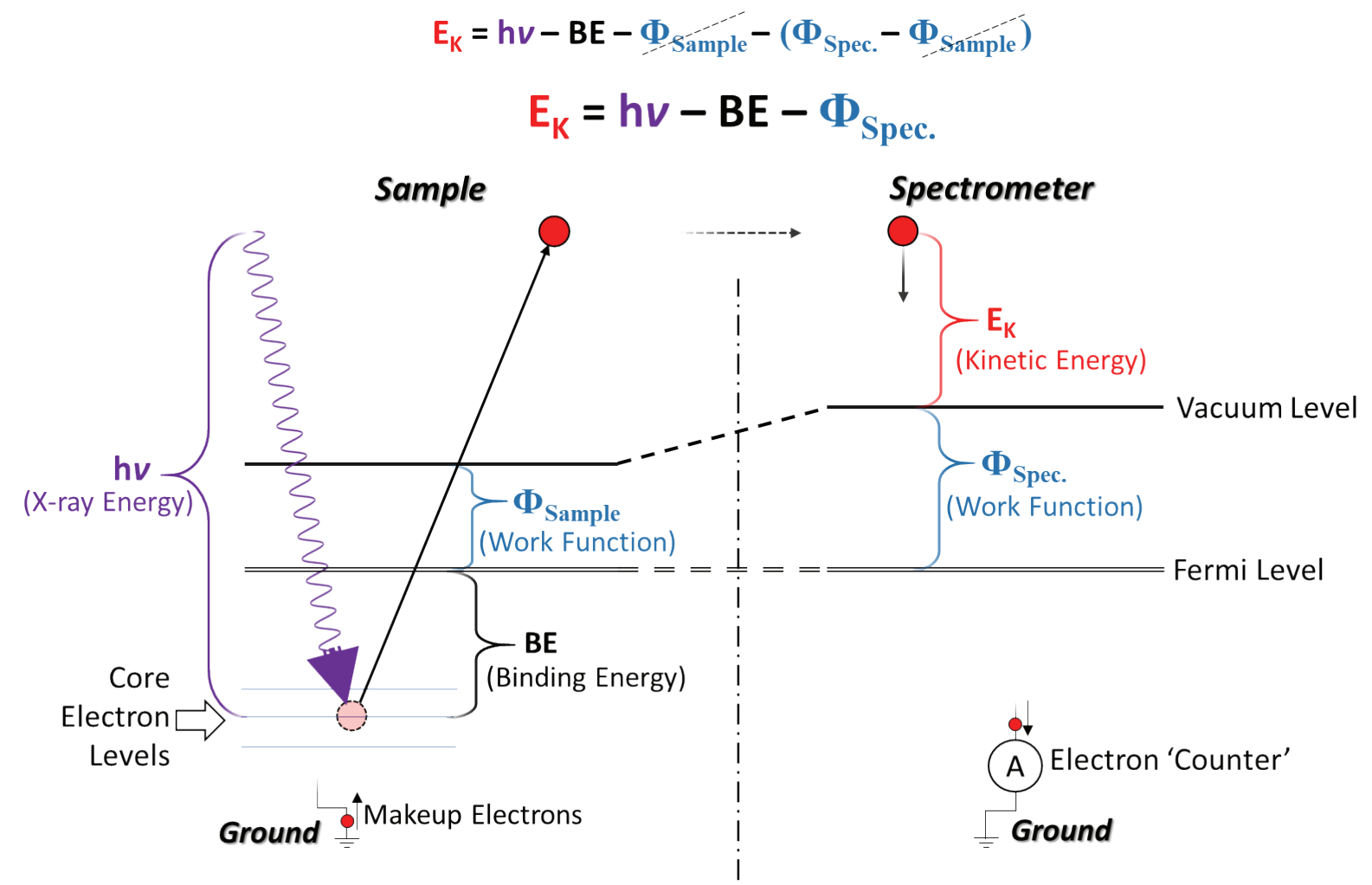

Figure 3.12: The solid-state interpretation of the origins of the XPS spectra and a graphical representation of the energy balance preformed to calculate the binding energy (BE). The equation is solved in terms of the kinetic energy, which in practices is the measured quantity. The $\Phi_{\text {Spec. }}$ and $h v$ are known and are constant. Rearrangement of the equation show the simple calculation of the binding energy: $\mathrm{BE}=\mathrm{h} v-\mathrm{E}_{\mathrm{K}}$ - $\Phi_{\text {Spec. }}$ Electrons are counted by the spectrometer by what is esentualy an amp meter. Electrons lost by the sample are made up by ground electrons.

The photon energy $(\mathrm{h} v)$ is known and is constant. In this work, $\mathrm{Al} \mathrm{k} \alpha \mathrm{X}$-rays were used as the photon source. X-rays are produced in a manner such that they have a narrow, well characterized energy. This is done by heating a filament, acting as an anode, which produces electrons by thermionic emission. The electrons are accelerated towards the cathode by an electric field. The electrons strike the cathode, which produces photoelectrons by the photoelectric effect. This leaves a vacancy in the electron levels of the cathode material, into which electrons from a 
higher energy orbital fall into (in this case, the primary transition event is from the $L$-shell to the $K$-shell). The difference in electron energy levels is quantized and, by conservation of energy, this energy is emitted as photons. The photon energy is equal to the difference in electron energy levels and thus, have a narrow energy distribution. The beam of photons is directed towards a quartz crystal which, acting as a Bragg reflector, monochromats the beam. This further narrows the energy distribution of the X-ray beam. In this work, an aluminum cathode is used. The produces an X-ray beam with a spectrum that peaks at the $\mathrm{K} \alpha$ line at $1486.60 \mathrm{eV}$. The monochromator refines this line to a width of $\sim 0.85 \mathrm{eV}$ as cited by the manufacture.

The spectrometer work function $\left(\Phi_{\text {Spec. }}\right)$ is an equipment parameter of the spectrometer $\left(\Phi_{\text {Spec }}=4.86 \mathrm{eV}\right.$ for the equipment used in this work $)$. Physically, it is the amount of energy required to bring an electron from the edge of the Fermi level (what would be the highest filled energy level at $0 \mathrm{~K}$ ) to the vacuum level (the theoretical energy level at which an electron is no longer bound to the solid and is stationary, in a vacuum). The work function of the sample, which is different for every sample, does not need to be known. This is because the work function of the sample cancels out in the energy balance calculation (Figure 3.12), due to the alignment of the Fermi levels caused by keeping the sample and spectrometer at the same electrical potential (intimate electrical contact).

The kinetic energy $\left(E_{k}\right)$ is the energy of the photoelectrons as measured at the detector. Photoelectrons strike the detector which produces a current, which is recorded as an arbitrary intensity or as photoelectron counts. The detector cannot differentiate between photoelectrons of different kinetic energies and thus all photoelectrons appear identical to the detector. To measure the photoelectron $E_{k}$, a hemispherical analyzer is used to selectively filter photoelectrons of only a narrow range of energies by electrostatic gating.

To do this, an electrostatic field is applied to the photoelectrons which directs them into a semi-circular path within the analyzer. The field strength is tuned such that only photoelectrons of a narrow band of energies can complete the half circle turn and strike the detector. Energies higher than the tuned band of allowable energies will not be deflected enough and will strike the outer wall of the hemispherical analyzer and never reach the detector. Photoelectrons of too low an energy will be deflected too far by the field and will follow a tighter path, striking the inner wall of the analyzer. The analyzer sweeps the allowable band through a wide energy range at a set speed 
and recorders the generated current, which is proportional to the number of photoelectron strikes at the detector. This process is illustrating in Figure 3.13.

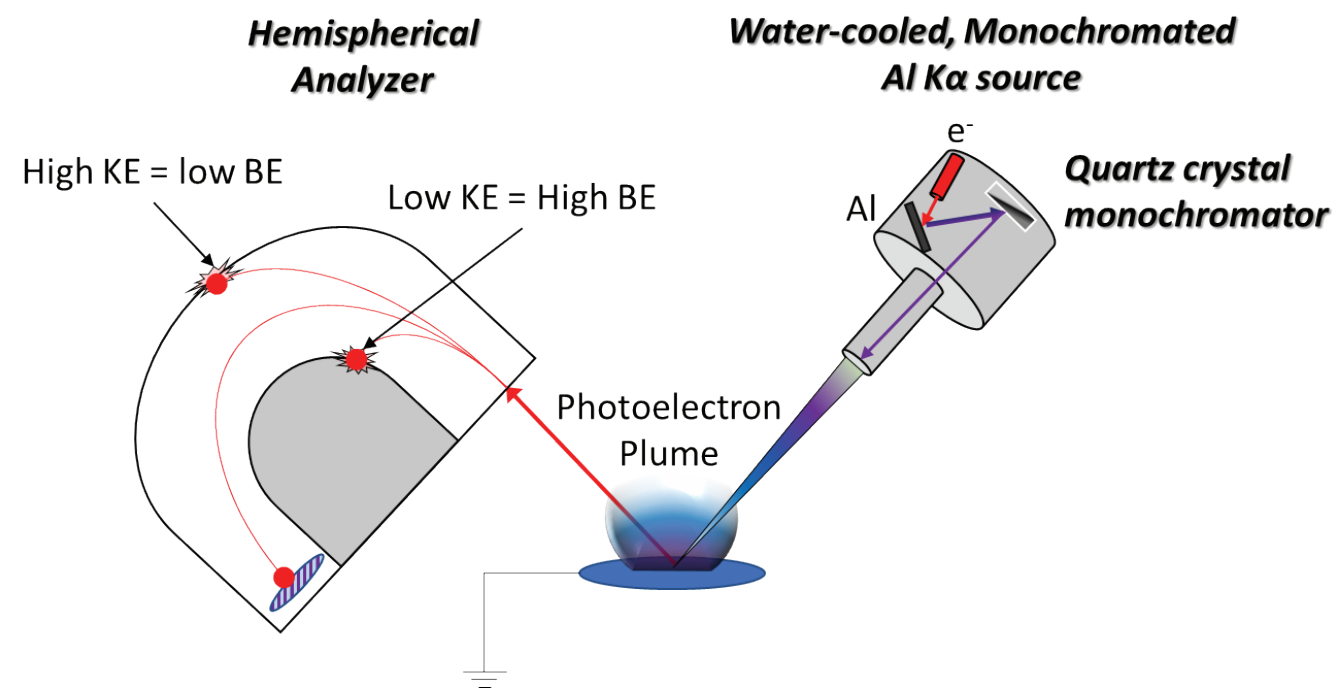

Figure 3.13: Simplified schematic of an XPS setup illustrating the relative arrangement of the photon source, sample and the hemispherical analyzer. The filtering of photoelectrons of the hemispherical analyzer by kinetic energy (Ек) is also shown.

The range of energies allowed to hit the detector by the analyzer at any one time is the band pass parameter (in eV), the increment that the band pass is stepped is the step size (in $\mathrm{eV}$ ) and the time the detector is counting photoelectron strikes over a particular range is the integration time (in ms). It is common to collect multiple sweeps across the desired energy range and average the spectra to increase the signal to noise ratio; this parameter is the sweep count. The entire process is carried out in an ultra-high vacuum (UHV, $\sim 10^{-8} \mathrm{~Pa}$ ) environment to decrease the number of collisions with gas molecules and thereby increase the number of photoelectrons which reach the detector and increase the signal.

In the end, this produces a spectrum whose $\mathrm{X}$-axis corresponds to the kinetic energy of the photoelectrons and whose $\mathrm{Y}$-axis corresponds to the number of photoelectrons collected (plotted as an arbitrary intensity or as photoelectron 'counts'). It is common, however, to convert from kinetic energy to the binding energy (BE) as this is the more meaningful value, although they are directly and fundamentally related.

A material will produce many photoelectron peaks over a wide range of binding energies. Because the precise electronic state of the electrons within a material are unique to the elements which make up the material, the binding energies of the photoelectrons are likewise unique. Thus, each spectrum produced from a pure material is unique to the elements which make up that 
material. This provides a spectral 'fingerprint' unique to each element. Therefore, by comparing the spectrum produced from a sample (of unknown composition) to a database (which identifies each element by their characteristic fingerprint), XPS can be used to identify the elemental composition of a sample. ${ }^{(466)}$ Excluding hydrogen and helium, the two elements not detectable by XPS. ${ }^{14}$ Additionally, the relative atomic concentration of elements in a heterogeneous material can be determined by comparing the relative intensities (peak areas) of the characteristic emission line for each element detected. ${ }^{15}$

A similar methodology is employed for the identification of the chemical state of each element. The chemical environment of an atom will influence the electronic state of the electrons in that atom. This modification of the electronic state is reflected as a shift of the photoelectron BEs from host atom. The shifts in the photoelectron BE peaks (as compared to the BEs of a pure substance) is used to identify the chemical state of the element. Information such as whether a carbon atom is bound to a silicon atom or to an oxygen atom can be determined. For example, an oxygen contacting compound will reveal itself at a higher binding energy due to the increased electron affinity, as compared to the pure material. ${ }^{(467)}$ Finer details can also be determined, such as whether an oxygen is bound to a carbon atom as part of an epoxide group or a carboxyl group. These determinations are made by comparisons to various chemical shift databases or to literature reports where spectra have been collected from known materials. ${ }^{(468)}$

Elemental identification is accomplished by collecting a survey scan. In a survey scan, data is collected at a low (spectral) resolution but over an energy range broad enough to capture sufficient data for the fingerprinting of the elements suspected to make up the sample. Once an element has been identified, a particular photoelectron peak is chosen. Generally, this is the line with the highest intensity, and which does not overlap with any other element peak. Data is collected over only this range but at a high resolution (by using a smaller step sizes and longer

\footnotetext{
${ }^{14}$ This is due to the extremely low ionization cross sections of the lone H1s orbital $(5,000 \mathrm{x}$ less than $\mathrm{C} 1 \mathrm{~s})$ and the fact that it does not possess core electrons. Its lone election must participate in bonding within the solid and thus only appears in the spectra in the broad valence band region where it is convoluted with all other valence elections. Likewise, He is practically imposable to detect for the same reasons. (736)

${ }^{15}$ Parameters other than intensity must also be taken into consideration, such as the ionization cross section of each element, line widths, etc. and is specific to each emission line for an element. This is combined into a correction factor, termed the Relative Sensitivity Factor (RSF). (730) This term is typically lumped into the Corrected Relative Sensitivity Factor (CRSF), which includes many equipment specific factors such as the type of photoelectron analyzer used, the focus lensing used, the collection angle, etc. (466)
} 
integration times, but at the cost of a longer collection time overall). These are commonly referred to as high resolution scans.

Individual XPS peaks are assigned designations corresponding to the atomic symbol of the element and electron orbital responsible for the peak. For example, a "C1s" peak consists of photoelectrons excited from the 1 s electron orbital of all the carbon atoms within the sampling depth. For a sample which is chemically heterogeneous, a high-resolution scan can reveal that the primary peak is actually composed of several peaks. Each peak corresponds to a unique chemical state of that element.

To gain useful information from such as spectra, first a determination of an appropriate baseline is required. For this work an iterative Shirley baseline estimation was used. This takes into account the systematic rise in baseline resulting from various energy loss processes and is the generally accepted best practice for baseline determination. ${ }^{(469)}$ Gaussian-Lorentzian peaks (also known as the Voigt function) were used to fit the component peaks. This method is consistent with the generally accepted practice because integration always result in finite areas (as opposed to other line fits which can lead to mischaracterizations of the data derived from purely mathematical idiosyncrasies). ${ }^{(470)(471)}$ Further detail on the specific peak fitting methodology and reasoning is given in Section $\$$ 4.1.2. The program MultiPak Spectrum Version 9.9.0.8 (ULVUAC-PHI, Inc.) was used to fit and analyze all XPS data.

For this work, monochromated Al Ka X-rays were used as the photonic excitation source. This type of soft X-ray can penetrate a solid sample up to $\sim 10 \mu \mathrm{m}$ deep. ${ }^{(466)}$ The incident X-ray excites all material within the penetration depth into photoelectron emission. This depth is called the excitation depth. However, due to the short inelastic mean free path of photoelectrons in a solid (orders of magnitude shorter than the X-ray photons), only the photoelectrons emitted from the uppermost surface of the material (the top 10 - $30 \AA$ ) can escape and are detectable.

A sampling depth of 3 times the inelastic mean free path (IMFP or $\lambda$ ) is commonly cited. ${ }^{16}$ This means that only $<5 \%$ of the signal comes from photoelectrons traveling greater than $3 \lambda$. ${ }^{(472)}$ This is illustrated in Figure 3.14(A,B). Therefore, most of the photoelectrons generated in the

\footnotetext{
${ }^{16}$ The IMFP is the average distance a particle can travel before it undergoes a collision. For a collection of radiative particles, $\lambda$ is described mathematically leaving $1 / \mathrm{e}$ of the population unaffected, meaning that over a distance of $1 \lambda$ approximately $63 \%$ will have undergone a collision event, leaving $\sim 37 \%$ unaffected; at a distance of $3 \lambda, \sim 95 \%$ of particles have experienced an inelastic collision event. (731)
} 
excitation depth never exit the material. The depth from within the material that photoelectrons can escape from is called the sampling depth. Because of this, XPS is an excellent surface characterization tool due to its high degree of surface sensitivity. Photoelectrons which do escape but are deep within the sample are strongly attenuated and contribute to the spectra background. This is the cause of the stepped-like character to the spectra corresponding to the higher binding energy side adjacent to intense peaks.

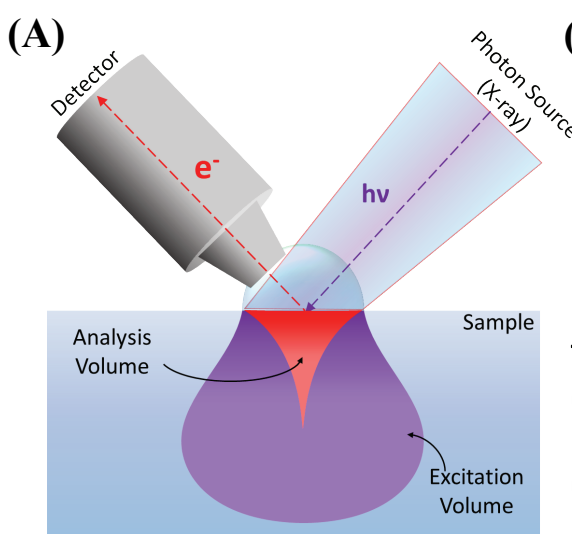

(D)
(B)

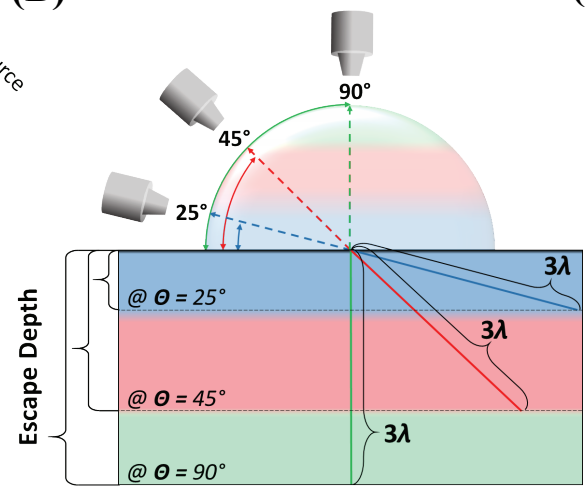

(C)

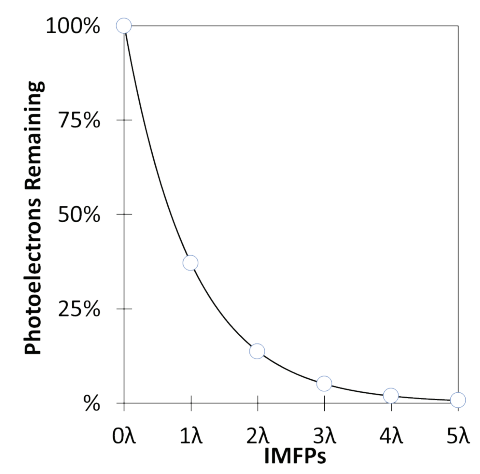

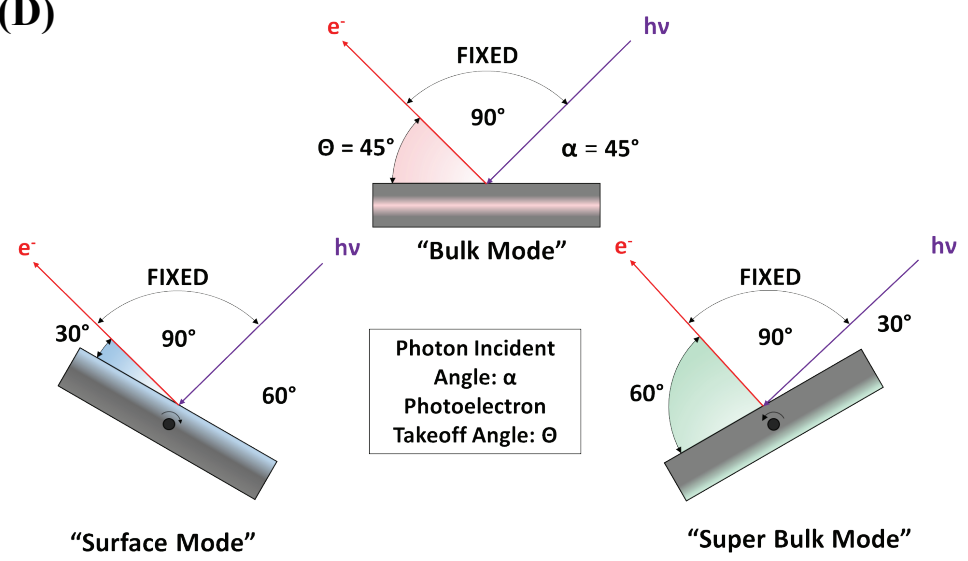

Figure 3.14: (A) The generic arrangement of XPS. The large volume of material driven into photoelectron excitation is shown. The large protuberance is due to scattering of X-ray within the sample. The comparatively small effective analysis volume is also shown; the surface is sampled to a greater extent than the bulk. (B) The surface sensitive nature of low angle detection is illustrated- small takeoff angles collect photoelectrons from a shallow escape depth. This effect is derived from (C) which shows the attenuation of photoelectrons as a function of distance traveled in terms of the number of IMFPs, (a decrease of 1/e per $\lambda$ ). (D) How angle resolved measurements are achieved in the system used in this work. High angle measurements collected both surface and bulk photoelectrons, while low angle measurements collected primarily surface photoelectrons. The sample is tilted, changing the angle to the detector, while the angle between the detector and photon source is fixed.

Another beneficial consequence of the short attenuation length of photoelectrons is that the depth sensitivity of XPS can be tuned (i.e. become more or less surface sensitive). This is done by sampling the photoelectron plume from different angles. A larger fraction of photoelectrons which 
exit the sample at shallow angles originate from surface layers, as compared to those which exit more normal to the surface. Therefore, by collecting photoelectrons which have a shallow takeoff angle, the resulting spectra is composed of a larger fraction of photoelectrons which have originated from surface layers.

For the equipment used in this work, this is accomplished by tilting the sample, relative to the detector (Figure 3.14(D)). In this work, the takeoff angle is defined as the angle between the sample and the detector axis. ${ }^{17}$ The angle between the detector and X-ray source is fixed. This is illustrated in (Figure 3.14(D)). This arrangement does have the disadvantage of also changing the angle of incidences of the $\mathrm{x}$-ray beam and the resulting spot size and shape. However, this is largely inconsequential for the analysis preformed in this work.

In this work, low angle measurements $\left(<45^{\circ}\right)$ are referred to as surface sensitive modes. Data collected at $\sim 45^{\circ}$ is referred to here as bulk mode, as comprise the vast majority of the data collected for this work. Note that regardless of the angle, XPS is always highly surface sensitive; the terms bulk modes and surface sensitive modes are simply relative terms used to differentiate between the two.

The exact length of one IMFP $(\lambda)$ for a photoelectron is a function of the photoelectron energy and the composition of the material in which the photoelectron is scattered. The IMFP is most strongly a function of the photoelectron energy. This relationship is captured in the so-called "universal curve" for photoemission. This curve is "universal' in the sense that its shape is independent of the composition of the scattering medium. The material composition simply shifts the curve globally to higher or lower values of $\lambda$. This is shown in Figure 3.15.

\footnotetext{
${ }^{17}$ This definition was chosen for the pertinent angle because it is how the manufacture of the XPS used in this study defines the relative detector angle. Note that it is also common in the literature to cite the pertinent angle as the angle made between the axis of the detector and a line normal to the surface of the sample; as long as the appropriate math is used, it is inconsequential.
} 


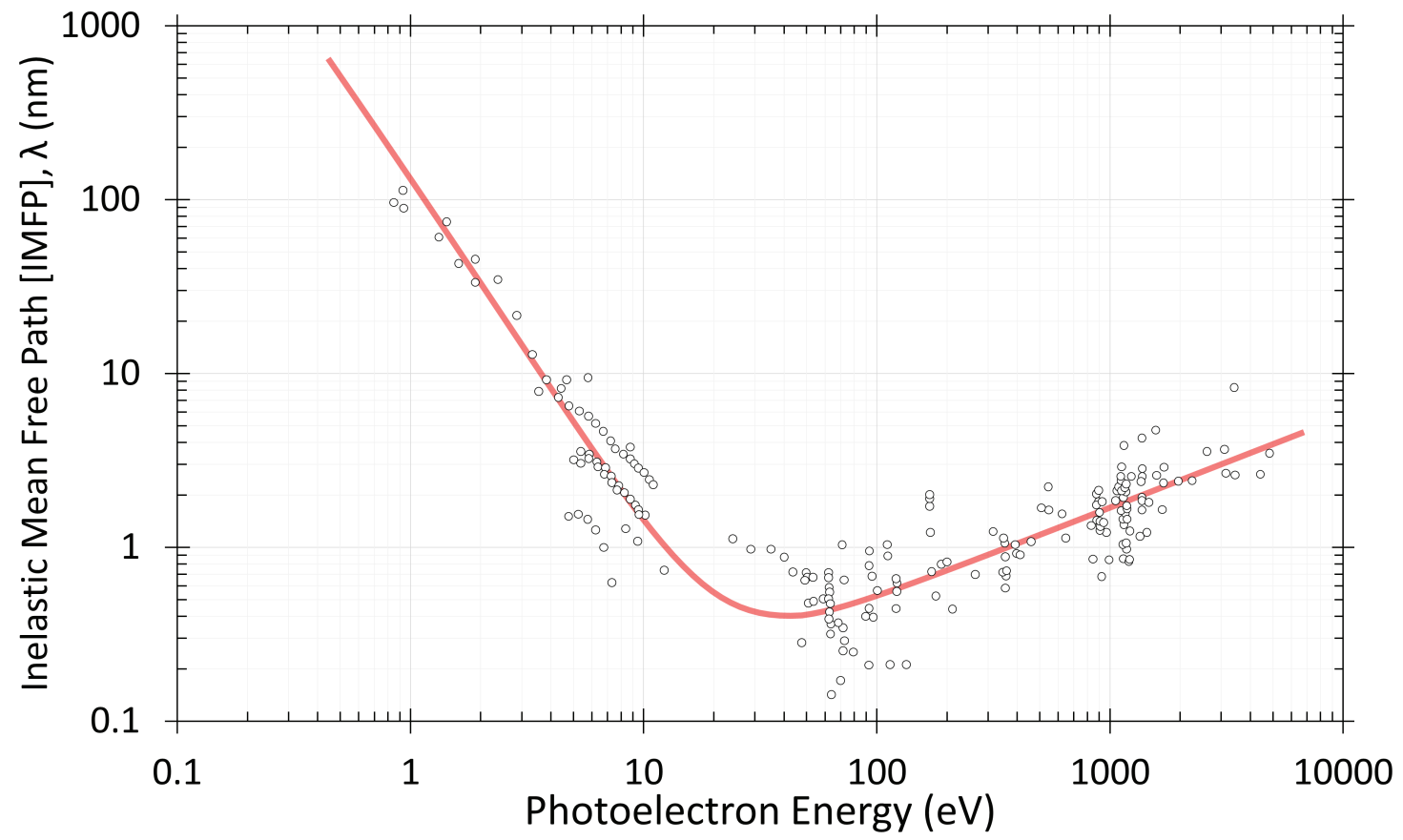

Figure 3.15: The universal curve for the inelastic mean free path $(\lambda)$ as a function of photoelectron energy. The data points correspond to various scattering media. Note that most information gained from XPS is generated from photoelectrons with energies above $10 \mathrm{eV}$, thus XPS is highly surface sensitive (i.e. IMFP $<$ 10nm). This chart has been reproduced using data from reference (731).

The non-linear nature of the universal curve is due to the various scattering mechanism which dominate at different energies. In particular, the quantized nature of the interaction of fundamental particles. For example, the sharp uptick in $\lambda$ at low photoelectron energies is due to these electrons not being energetic enough to undergo some of the mechanisms responsible for inelastic scattering, thus there $\lambda$ is increased. There have been numerous empirical and theoretical studies which have validated this.

Another consequence of a short attenuation length and the resulting depth sensitivity of the photoelectron single is that the thickness of the carbon films produce in this work can be calculated. This takes advantage of a uniform, chemically pure and 'infinitely thick' substrate overlaid with a thin film. This is discussed at the end of this section.

A frequently encountered problem of XPS is sample charging. Electrons lost by the sample are made up by ground electrons. This requires that the sample be conductive and in perfect electrical contact to ground (or electron source). However, this is rarely the case. This results in the sample building a small positive charge due to sample becoming slightly electron deficient. The positive change increases the electron affinity of the sample and makes the photoelectrons 
appear to have a higher BE (i.e. it requires more energy to produce photoelectrons). This can be compensated for by electrically basing the sample stage or bombardment of the sample by negatively charge ions. Alternatively, the data can be simply shifted such that a characteristic peak appeared at a known (uncharged) reference value. In this work, the Si-C peak appearing at 282.5 $\mathrm{eV}$ was used as the reference peak for charge compensation.

It is noted that there are many other considerations in both the theory and operation of XPS that, while important, are not discussed here because they have little impact on this work (such as Auger emission, spin orbital splitting, shake off peaks, plasmon loss peaks, ghost peaks, etc.). ${ }^{(466)}$

For this work, XPS data were collected using a Physical Electronics PHI 5700 VersaProbe (Figure 3.16) with an Omni Focus V lens and an aluminum monochromated (via quartz crystal) $\mathrm{k} \alpha$ X-ray source with a beam energy of $1486.60 \mathrm{eV}(359.5 \mathrm{PHz}$ in the frequency domain) generating an on-sample beam diameter of $\sim 100 \mu \mathrm{m}$ at $\theta=45^{\circ}$. The setup used in this work can be seen in Figure 3.16. with the pertinent parts labeled.

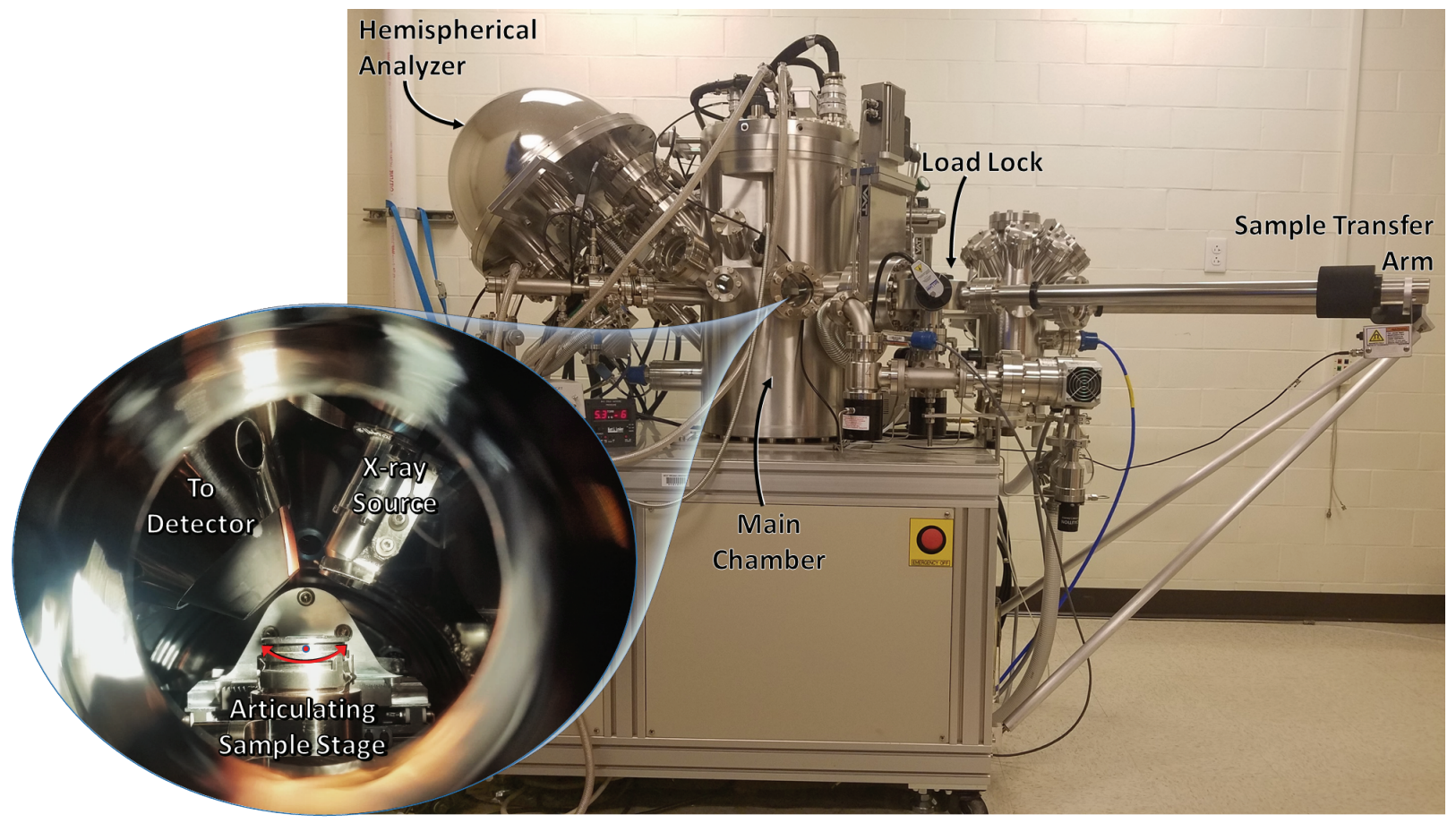

Figure 3.16: A picture of the PHI 5700 VersaProbe XPS analysis tool used in this work. The main components have been labeled. Blowout: a view through the sample inspection window showing the X-ray source and detector arrangement. The relative position of the articulating sample stage can be manipulated to collect data at various takeoff angels.

Unless otherwise noted, the takeoff angle used was $45^{\circ}$. For conducting samples, no neutralization of any type was used. For survey spectra, a pass energy of $117.4 \mathrm{eV}$, a $0.500 \mathrm{eV}$ step 
size and a time per step of $25 \mathrm{~ms}$ was used. For detailed scans a pass energy of $23.5 \mathrm{eV}$, a step size of $0.025 \mathrm{eV}$ and a time per step of $50 \mathrm{~ms}$ was used. Sample charging was compensated for by a simple mathematical shift of the spectra using the $\mathrm{SiC}$ peak at $282.50 \mathrm{eV}$ in the $\mathrm{C} 1 \mathrm{~s}$ region as the reference peak. ${ }^{(473)}$ The instrument was calibrated using the $\mathrm{Au}_{4} \mathrm{f}_{7 / 2}$ reference line from a $\mathrm{Au}$ standard sample.

Finally, XPS analysis was also used to determine the number of graphene layers on the samples produce throughout this work. This calculation is based on the attenuation ratio of the photoelectron signal produce from carbon in the bulk to the signal produce by the carbon in the graphene overlayer. The method is discussed in depth within APPENDIX D.

\section{§3.2.2 Atomic Force Microscopy (AFM)}

Atomic Force Microscopy (AFM), a surface characterization technique, was used in this work primarily to characterize the surface morphology of synthesized samples. The basic idea underpinning AFM is straightforward. Atomic forces are detected at discrete points across a sample's surface. Each data point corresponds to a real-world position on the sample. These data points are then mapped 2-dimensionally to generate a representation of the surface based on the detected atomic forces. The way in which the atomic forces are detected, the type of atomic force and how the forces are interpreted constitute the rest of the field of AFM. For this work, with respect to utilizing the AFM as a characterization tool, the AFM was operated in a typical and standard fashion. However, as is discussed in the Appendix $\S B$.1.3 and $\S$ B.2.2, the AFM was also utilized in a novel method for the controlled deposition of metallic nanoparticles. This will not be discussed here.

AFM was developed in 1986 by Gerd Binning, Calvin Quate and Christoph Gerber, partially as a result of Binnings' and Heinrich Rohrers previous work on scanning tunneling microscopy (STM), for which they later each received a $1 / 4$ share of the 1986 Nobel prize in physics. ${ }^{18}{ }^{(474)}$ In 2016 Binning, Quate and Gerber received the $\$ 1,000,000$ Kavli prize for their development of the AFM, specifically. ${ }^{(475)}$ AFM was first reported in 1987; while many improvements have been made over the preceding three decades, the basic operation remains the same. ${ }^{(476)}$

\footnotetext{
${ }^{18}$ The other $1 / 2$ of the 1986 Nobel Prize in Physics was awarded to Ernst Ruska for his "fundamental work in electron optics, and for the design of the first electron microscope".
} 
In the context of AFM, detected atomic forces are either attractive or repulsive in nature. The distance from the sample surface will determine if attractive or repulsive atomic forces are felt. This function is well known and is illustrated by the Lennard-Jones potential (Figure 3.17), which is a simple summation of all forces acting between two bodies at atomic scale distances where atomic forces dominate.

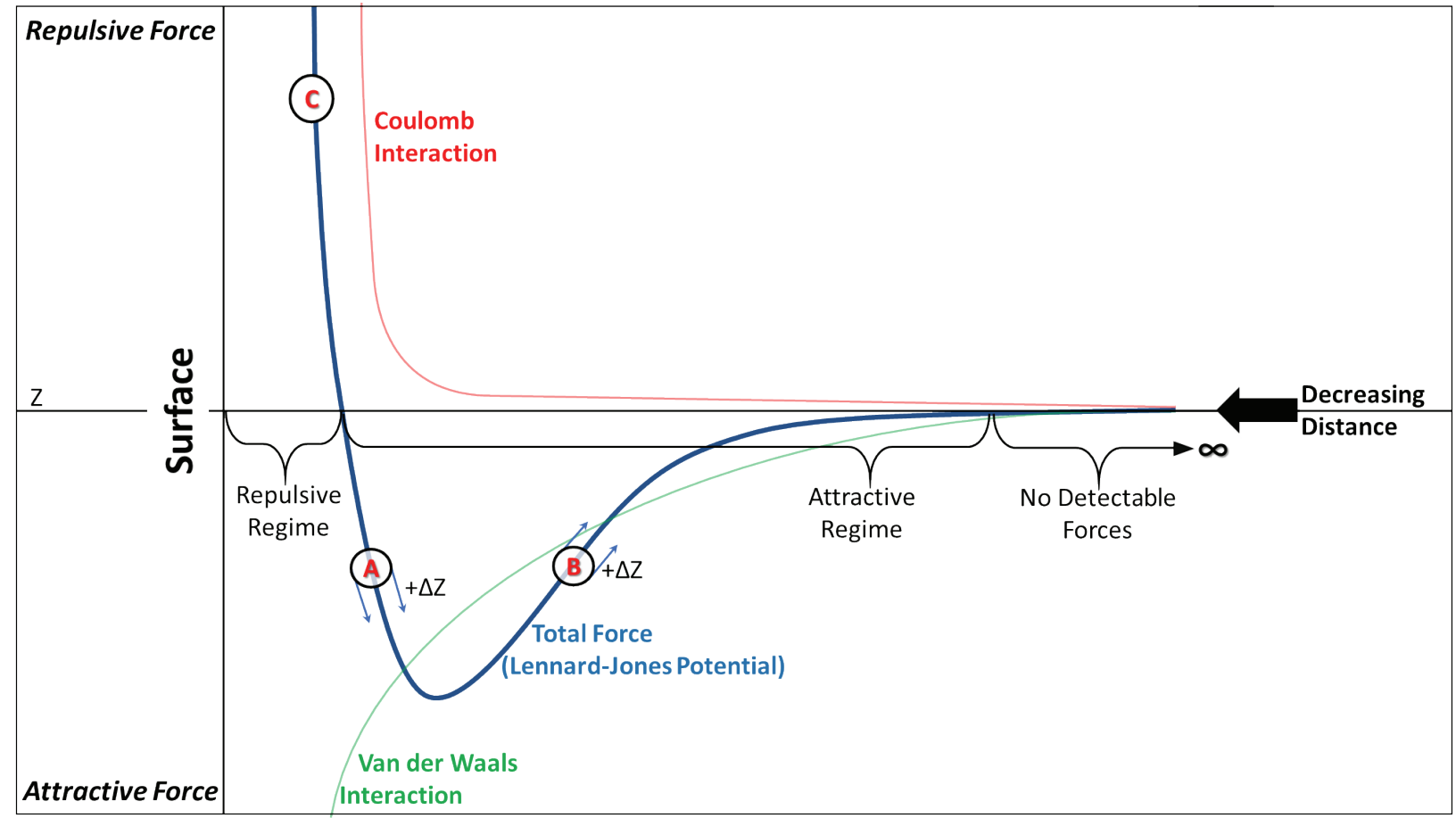

Figure 3.17: A qualitative view of some of the primary forces involved in AFM. At sufficiently large distances, effectively no atomic forces are felt. As the distance is decreased the effective forces felt are indicated by the blue curve labeled total force (Lennard-Jones potential). Points A and B on the total force curve are not optimum operational positions because the detected forces can be interpreted in multiple ways. Point "C" is ideal due to the large forces involved and simple interpretation of the data.

As the distance between two bodies is decreased, the sum of the forces felt between the bodies are first attractive (where van der Waals forces dominate) and then repulsive at very small distances (where short-range Coulomb interactions dominate). ${ }^{(47)}$ The distance above the sample, and thus where on the Lennard-Jones potential curve the AFM is operated at, is a user defined parameter. This is determined indirectly by choosing an approach distance. While this is an important parameter consideration, for this work, the primary concern was to ensure the AFM was not within the transient region as this would lead to ambiguous, poor quality images. For example, if the AFM were operating at position "A" on the Lennard-Jones curve in Figure 3.17 and a protrusion on the sample surface were encountered, the AFM would detect this as an increase in attractive forces (increase $\Delta \mathrm{Z}$ and moving right along the Lennard-Jones curve). However, if 
operating at position "B" and the same protrusion were encountered, the AFM would detect a decrease in the attractive forces. Obviously, operating in the transient regime around positions " $\mathrm{A}$ " and "B" can lead to ambiguous results. Therefore position "C", fully in the repulsive regime, was chosen as the preferred point of operation with respect to vertical positioning (additionally, this regime results in larger forces and thus a better signal-to-noise can be achieved). AFM operation in this regime has been shown to result in clearer images. ${ }^{(478)}$

The component used to detect the atomic forces consists of three elements: a chip body, cantilever and tip. The chip body is used to handle and mount the component in the AFM. The cantilever extends off the end of the chip body and is free to flex. At the end of the underside of the cantilever is an ultra-sharp protrusion simply called the 'tip'. It should be noted that the entire component is often referred to as a 'tip'. Figure 3.18 shows a simple illustration of a tip.

(A)

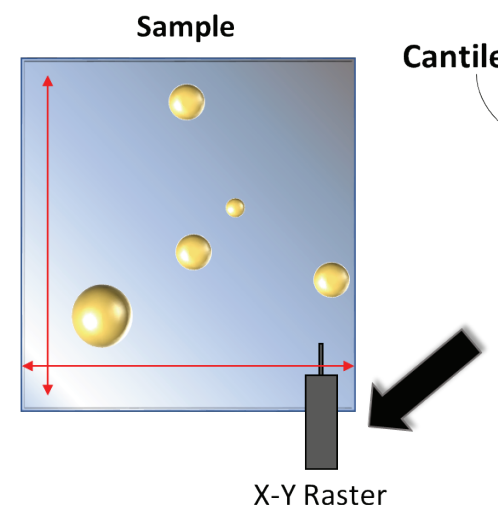

(B)

ever

Figure 3.18: (A) Top down view of the typical left-to-right and top-to-bottom raster pattern the tip takes across the sample surface. (B) Side view of the AFM tip showing the chip body, cantilever and tip.

The tip is mounted on to a scanner head. Within the scanner head are high precision actuators (piezoelectric, in this work) used to precisely position the tip above the sample. The tip is brought into contact or near contact with the surface of the sample. The actuators then raster the tip across the sample. The tip traces over the topological features causing the cantilever to flex in response. The cantilever/tip deflection is monitored by reflecting a small, tightly focused laser onto the back of the cantilever. The laser is reflected off of the cantilever and strikes a quadrated photodetector. The geometry of this arrangement means that small deflections of the cantilever (in the nanometer range) are amplified by orders of magnitude when translated to the deflection of the reflected laser beam onto the photodetector. The photodetector outputs an analog voltage, the sign 
and amplitude of which are proportional to the position of the laser spot. This signal is first amplified/digitized and then processed. The processed data is then rendered as a false color 2dimensional image which corresponds to the deflection of the cantilever and ultimately, to what drove the deflection: atomic forces as felt by the tip; hence, atomic force microscopy.

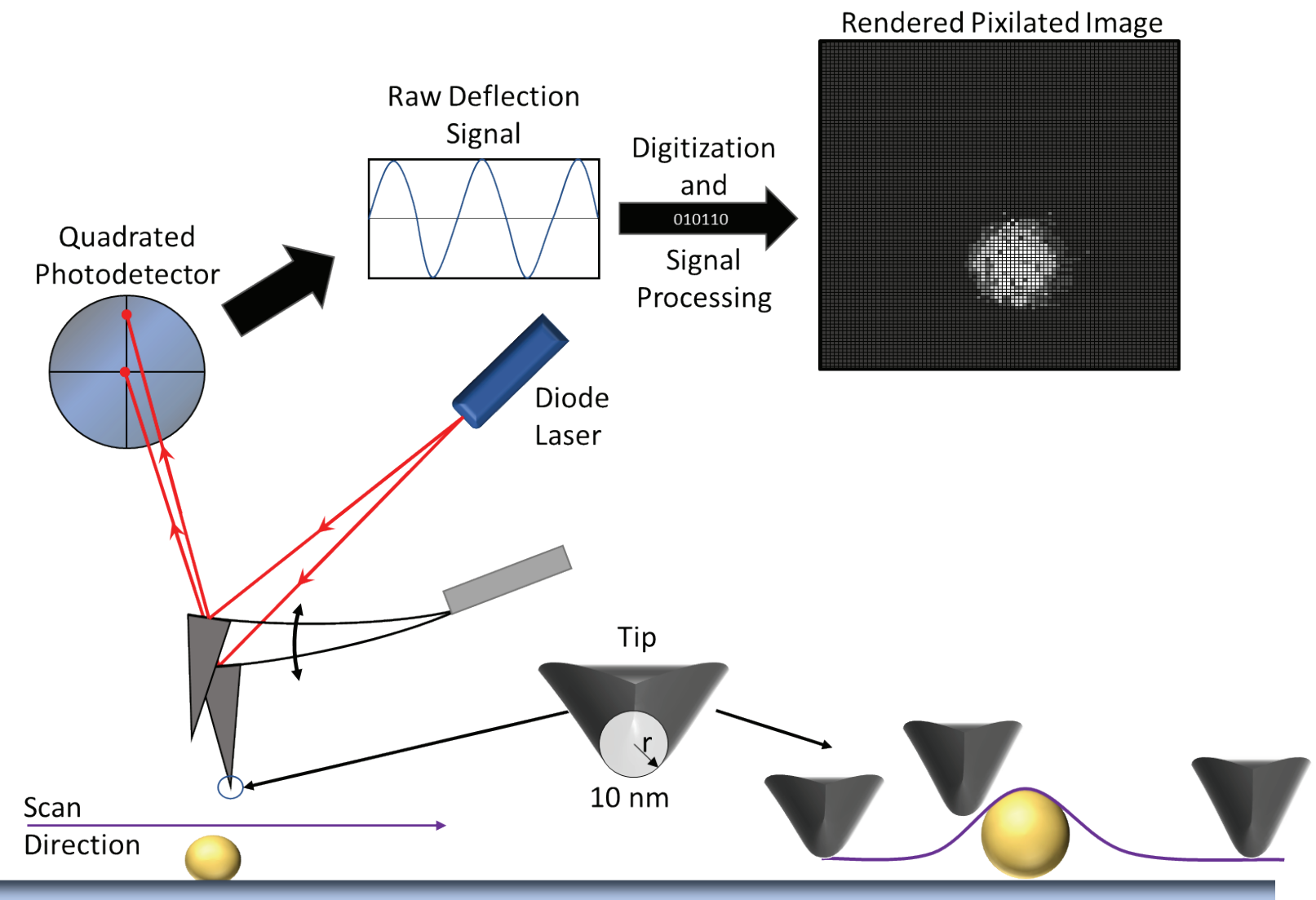

Figure 3.19: A simple illustration of how a surface feature deflection is detected and converted into an image: The tip encounters a feature, which causes a deflection of the tip due to the atomic forces, resulting in a deflection of the reflected laser beam position onto the photodetector. The photodetectors signal is then amplified, digitized and processed resulting in a 2D false color pixilated image.

Tips can be made from a variety of materials and with a wide variety of geometries and sizes. For this work, silicon tips were used. Tips were backside coated with either a reflective Au or Al film to enhance laser reflection. An important tip parameter is the tip size, characterized by the radius of curvature of the end of the tip. The tip size has a direct impact on the resolution of the collected image; smaller tips can yield both higher image resolution (higher pixel count) and higher surface resolution (resolve smaller surface features). Small surface features, which are on the same size scale as the tip, will result in the rendered image of the surface feature appearing artificially large. This is a tip broadening effect resulting from the geometry of the pyramidal or conical shape of the tip. As the tip rasters across the surface, the side of the tip can make contact 
with the feature before the actual apex of the tip does. This is illustrated in Figure 3.20. Tip broadening effects only result in artificial enlargement in the lateral (X-Y) dimensions; the vertical (Z) direction is unaffected. Note that tip broadening occurs to features of all sizes, however, is only an important consideration when the broadening effect is an appreciable fraction of the feature size. This effect applies to this work specifically in characterization of the deposited nanoparticles, which are close in size to the tip radius. The amount of tip broadening that occurs is a function of both the slope of the surface feature and the slope of the side tip. Tip broadening is minimized when the slope of a feature is small, and the tip has a high aspect ratio (high edge slope). The tip broadening effect is illustrated in Figure 3.20. Therefore, tip compensation is done on a case by case basis. The situation is further complicated by the tip ageing effects, where tip geometry changes as the tip is worn down or fractures with use.

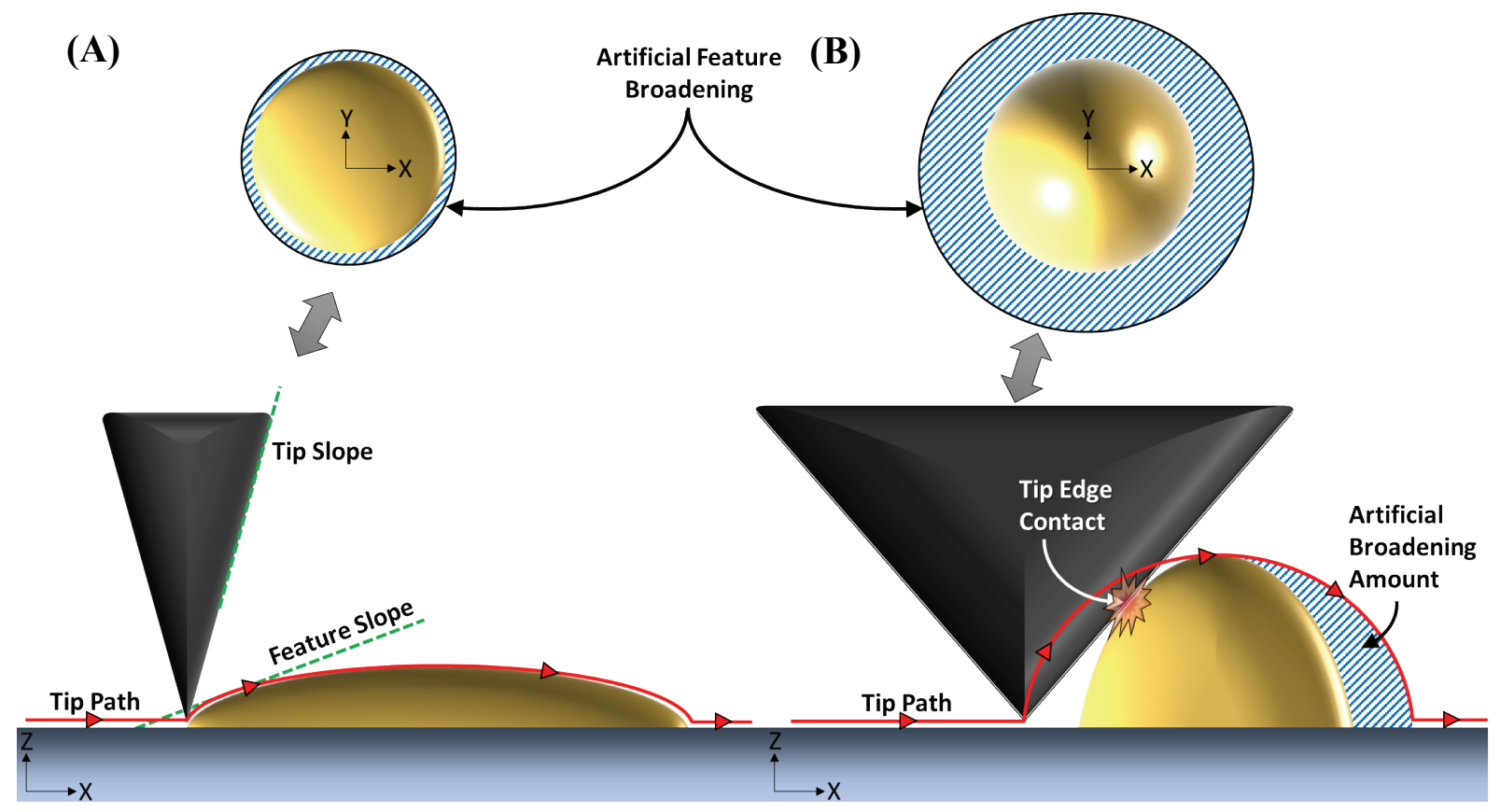

Figure 3.20: (A) When the angle between the tip edge and feature edge is large the tip can accurately track across fine surface features. (B) When a tip with a large radius of curvature is used, or high aspect ratio features are mapped, artificial tip induced broadening effects can occur.

For this work, the AFM was operated in tapping mode (also called 'AC mode'). In this mode, the cantilever is driven into oscillation at or near its resonance frequency (which is a function of the tip materials and geometry of the cantilever). The tip is rastered across the surface and both the frequency and amplitude of the oscillating tip are recorded. Under no external influence, the tip will oscillate at the drive frequency (a user defined parameter and typically a value near the natural resonant frequency of the tip is chosen) and with a large amplitude. This is 
called the free tip oscillation. When the tip is brought close to a sample surface, the oscillation amplitude will become dampened as repulsive atomic forces are felt by the tip. This dampening is used to determine that sufficient contact with the surface has been achieved. For this work contact was determine once the oscillation had been dampened to between $88 \%$ to $95 \%$ of the free tip osculation (i.e. a 5-12\% reduction in oscillation amplitude).

As the tip is rastered across the sample surface, changes in surface topology will further dampen or free the tip amplitude of oscillation. This amplitude modulation is mapped and a 2dimensional image, called an amplitude image, is generated. This process is illustrated in Figure 3.21 (B). Based on calibration and tooling factors, the amplitude image is converted into a topography image and is an accurate representation of the true surface from which quantitative information can be extracted.

Operating in tapping mode allows for a second type of image to be generated called a phase image. Here, the difference between the drive frequency and the actual oscillation frequency of the tip is recorded. This difference is recorded as a phase shift and when mapped, generates the phase image. A phase shift is caused when there are changes in the attractive/repulsive forces, including those caused by height $(Z)$ effects, between the tip and sample. These types of forces will cause the real tip oscillation to either lag or lead the drive single. Changes in chemical makeup, crystal domain or material phase (physical phase, not to be confused with the angular phase of the signal) can all cause a phase shift. Attractive forces result in a negative phase shift where repulsive forces result in a positive phase shift. ${ }^{(479)}$ An illustration of this principle is shown in Figure 3.21 (A). Additional references regarding AFM. ${ }^{(480)(481)}$ 
(A)

Phase Shift

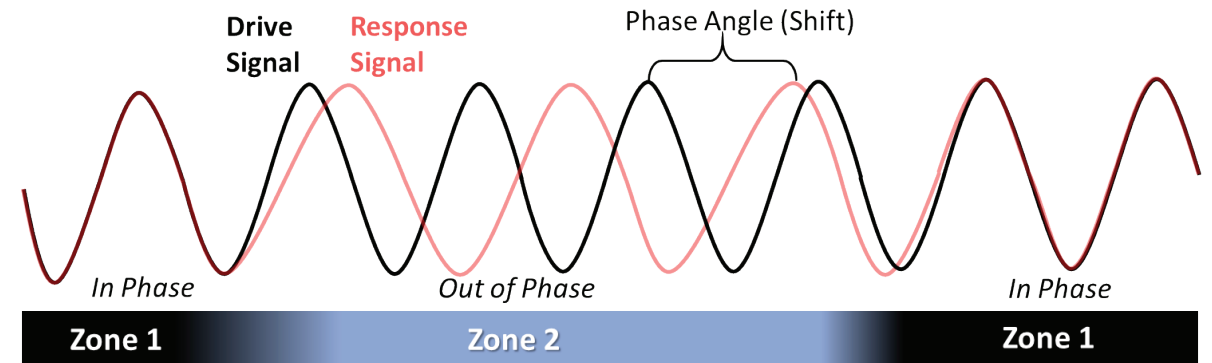

(B)

Amplitude Change

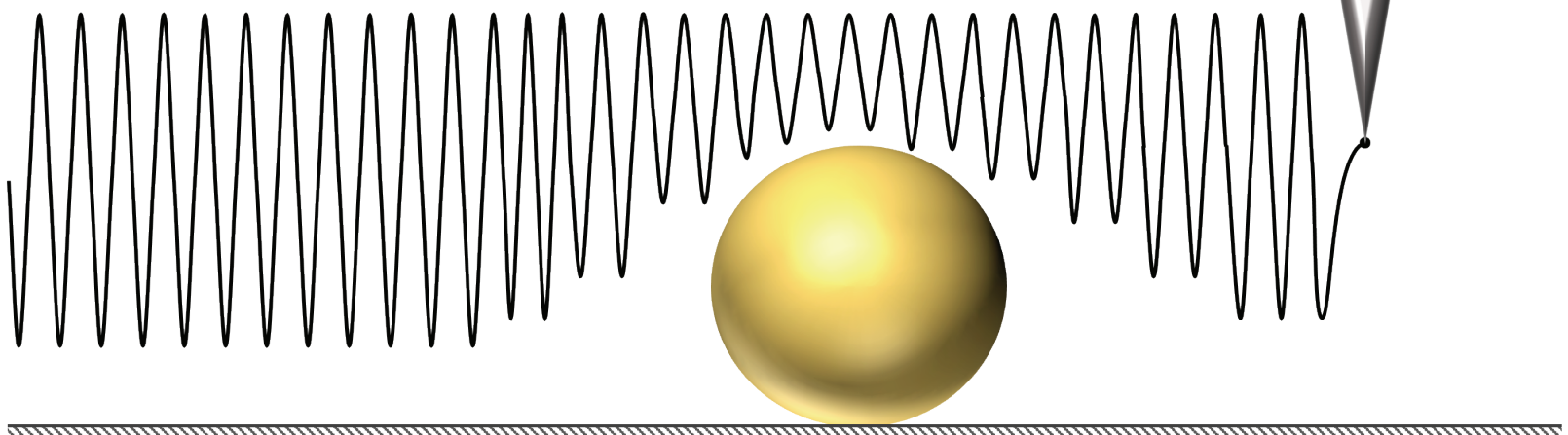

Figure 3.21: (A) The principle of phase images. The attractive or repulsive forces felt between the tip and the sample will drive the actualy occilation of he tip off of the drive signal. The diffrence between the two is the phase angle shift. This parameter is used to generate the phase image. (B) The principal of amplitude images. Here, the amplitude dampening is used to generate the image. The tip is kept at a consistent height, therefore when the tip encounters a feature the amplitude of the tip occilation is dampened. A complementary mode of opearation is posible where the amplitude is set and the height of the tip is continuously modulated to maintain a consistent amplitude (this is not illistrated here).

Due to the largely featureless nature of the graphene-SiC composites of this work, AFM was primarily used in the characterization of deposited nanoparticles through various methods (discussed extensively in APPENDIX B). An Agilent 5500 SPM AFM was used in this work; a photograph of which can be found in Figure 3.22. Tapping mode (AC Mode) AFM was used exclusively. The AFM was housed in a Herzan acoustic isolation chamber and rested on an isolation mount. The AFM was equipped with a video camera and optical microscope. A variety of tips were used, all with a tip radius of $<10 \mathrm{~nm}$. Specific parameters such as $\mathrm{P}$ and I gains, scan speed and sample approach distance were optimized on a case by case basis. P and I gains used were between 5\% and 90\%. Tips were driven slightly off of the resonate frequency. Tip approach was stopped when the tip oscillation was dampened to between $88 \%$ to $95 \%$ of the free air oscillation. Scan speed was typically between $0.5-2.0$ lines/sec. Scan resolution was between 512 X 512 data points for low resolution images and as high as 2048 X 2048 data points; most images 
used a 1024 X 1024 data point resolution. Sample approach was typically between 89\% - 95\% dampening of free top oscillation. Images were analyzed using the open source program Gwyddion (V2.51). ${ }^{(482)}$

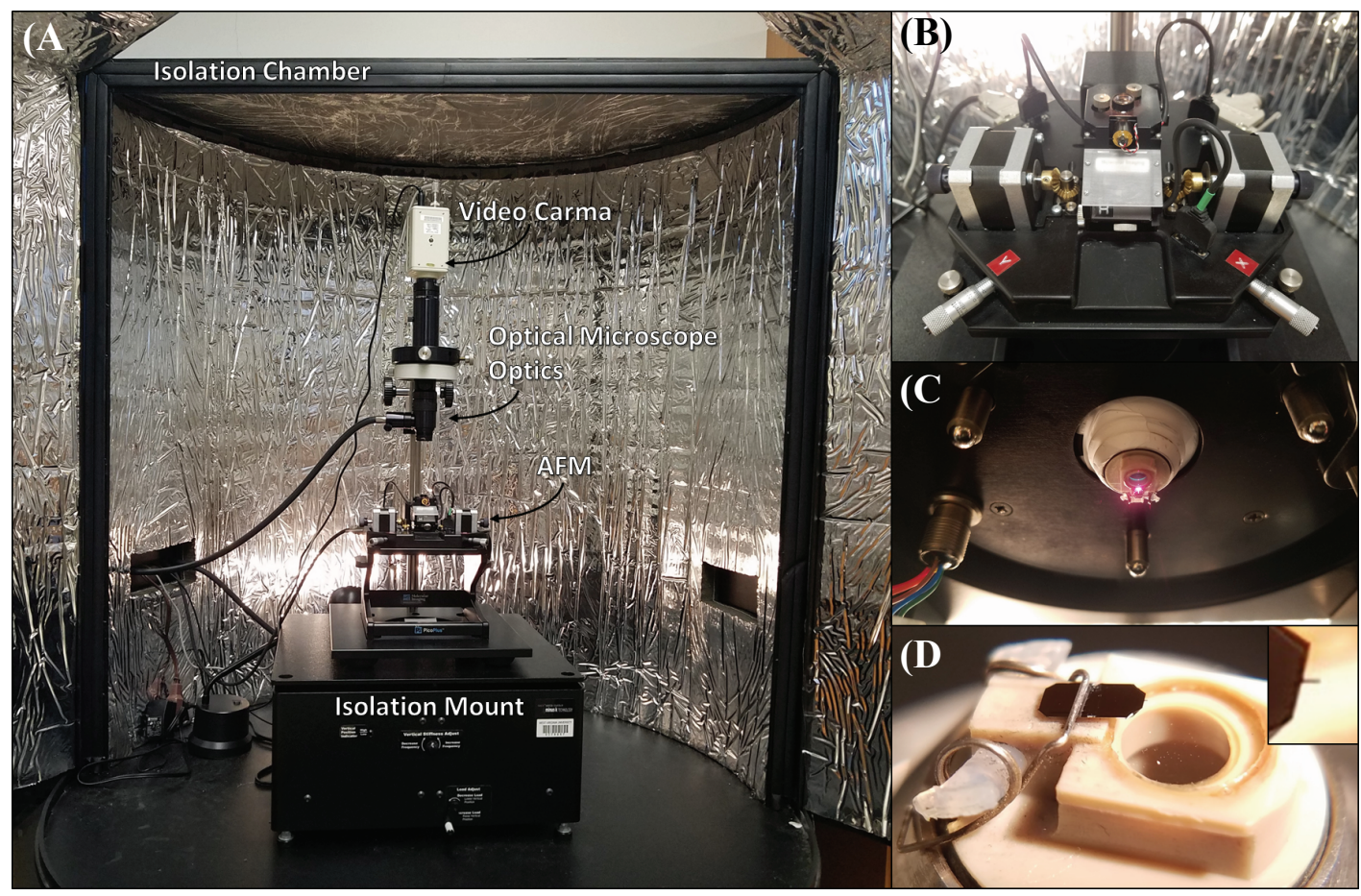

Figure 3.22: (A) The Agilent 5500 SPM AFM. (B) The AFM assembly. (C) The underside of the AFM showing the scanner head. The laser light reflecting from the AFM tip can be seen. (D) An AFM chip mounted in the AFM head. The tip can be seen in the zoomed in insert.

\section{§3.2.3 Raman Spectroscopy}

Raman spectroscopy utilizes the complex interaction of light with matter. Light can interact with matter in one of four ways: reflection, transmission, absorption or scattering. Raman spectroscopy relies on the scattering of light by matter. In 1928, Sir Chandrasekhara Venkata Raman discovered the underlying optical principal this technique is based on and for which he was the sole recipient of the 1930 Nobel Prize in Physics. (483) (484) (485) Both the technique and the underlying phenomena are named after him.

There are three mechanisms by which the scattering of light (sometimes referred to as diffuse reflections) may occur. They are Rayleigh Scattering, Stokes and Anti-Stokes scattering. The vast majority of scattered light (99.999\% to $99.999999 \%)$ under goes Rayleigh scattering, where the scattered light is at the same frequency of the incident light (i.e. it undergoes no spectral shift). ${ }^{(486)(487)}$ 
A schematic of the Rayleigh scattering process is depicted in Figure 3.23. Rayleigh scattering is a four-step process. First (Figure 3.23: I), incident light (monochromated visible laser light in the case of Raman spectroscopy) is absorbed by the sample. The sample internalizes this energy in step two (Figure 3.23:II); the incident light excites the system from a ground vibrational state into a higher energy virtual vibrational state (this state does not actually exist but is useful for illustrative purposes; the energy of the virtual state is simply equal to the incident light energy). The system then collapses back to its ground state (obeying the second law of thermodynamics with respect to blackbody radiation) and a photon is emitted with an energy equal to the difference between the virtual state and the ground state (Figure 3.23:III, IV).

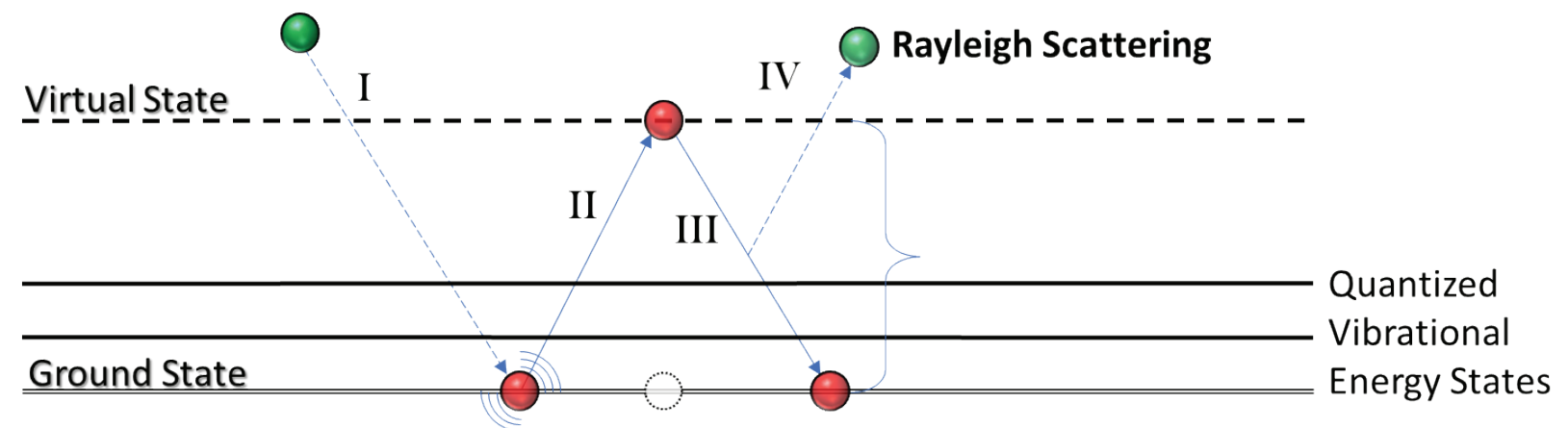

Figure 3.23: A schematic depicting Rayleigh scattering (I). The excitation laser light excites the system into a virtual state (II). The system then collapses back into the ground state (III) which spontaneously causes an emission of a photon (IV) with an energy equal to the energy difference between ground and virtual states.

Stokes scattering occurs when the system collapses to an energy state higher than the ground state. This process is depicted in Figure 3.24. Again, a photon is emitted in this process but because the system did not undergo the full energy transition back to the ground state the emitted photon energy is consequently lower. The difference between the energies of the Rayleigh photon and the Stokes photon is the Raman shift. Because all photons move at the same speed (the speed of light) and have the same mass (somewhat paradoxically, this is zero), the manner by which a photon can express an energy differential is by frequency shift; higher frequencies correlated to photons with higher energies; conversely, lower frequencies correspond to lower energy photons. Therefore, in practice it is the lower frequency shift that differentiates a Stokes photon from a Rayleigh photon. For Raman spectroscopy, it is the absolute difference in frequency 
between the Stokes photons and Rayleigh photons which are plotted as a wavenumber in $\mathrm{cm}^{-1} .{ }^{19}$ This gives rise to the Raman shift. This is advantageous because the frequency of the excitation laser is largely independent of the Raman shift and the data analysis is less cumbersome. ${ }^{20}$

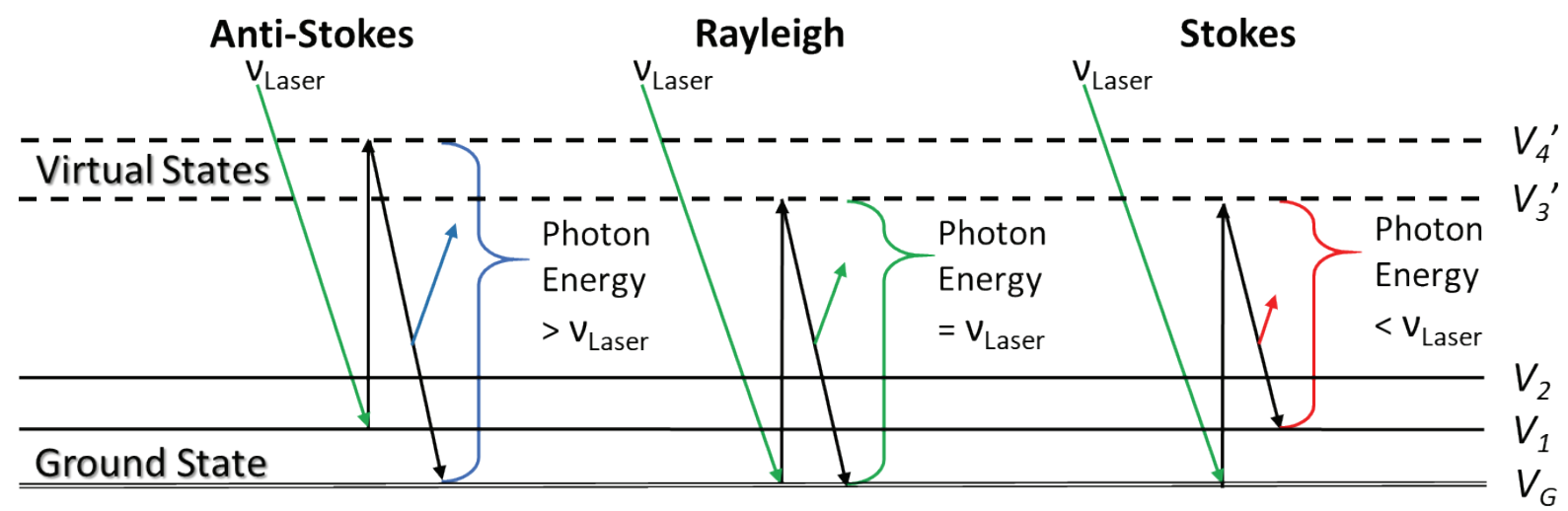

Figure 3.24: Schematic of three distinct types of scattering processes. Anti-Stokes, where the incident laser excites a pre-excited system resulting in a higher frequency photon emission. Rayleigh, the most common scattering process, where the emitted photon is equal frequency to the excitation laser. Stokes, where the system does not fully collapse into the ground state resulting in a lower frequency photon.

A discussing of Raman scattering is not complete without mentioning Anti-Stokes scattering. This process is also depicted in Figure 3.24. Anti-Stokes scattering occurs when the excitation light excites a system, which is already at an excited vibrational state, up to a virtual state. This leads to the system occupying a virtual state which is higher than either the Rayleigh or Stokes virtual states. When the system collapses into the ground state the emitted photon has a higher energy and is therefore at a higher frequency as compared to the excitation light. This type of scattering, which always occurs with Stokes scattering, is rarely used in Raman spectroscopy except in a few specific circumstances (e.g. if a sample shows excessive florescence). Anti-Stokes scattering is always lower in intensity than Stokes scattering, because it occurs less frequently. This is due to the thermal energy requirement needed to put the system into a pre-excited state (a prerequisite for Anti-Stokes scattering). The probability of this occurring at any finite temperature is a function of the wavenumber (larger Raman shifted wavenumbers require higher energy) and

\footnotetext{
${ }^{19}$ The wavenumber, in $\mathrm{cm}^{-1}$, is the number of wavelengths which fit within $1 \mathrm{~cm}$ of real space. It is the inverse of the wavelength and thus directly relates to frequency. Recall that wavelength is equal to the speed of light (c) divided by frequency $(v)$.

${ }^{20}$ In practice, different excitation sources will produce slightly different Raman shifts. This is true specifically in carbon films. Therefore, the source should be kept in mind when comparing spectra collected using different excitation sources. (494) (507) (737) (738)
} 
follows the Boltzmann distribution. This, among other considerations, limits the utility of analysis involving Anti-Stokes scattering. ${ }^{(488)}$

For Raman scattering to occur, the electric filed component of the incident light must cause a change in polarizability of the system. This is a result of coherent molecular vibrations which distort the electron cloud (resulting in an induced dipole). The vibrational frequency is highly dependent on the atom type and bonding within the material. This specificity gives rise to the characteristic Raman spectra for a given system. There are several vast databases which have been compiled of the characteristic Raman fingerprints of various molecular groups and systems. ${ }^{(489)}$ The comparison of empirical data to these types of databases can give insight on the system studied.

To collect spectra Raman data, the incident laser frequency is compared to that of the scattered laser light. In practice, this is done with a series of optics and a diffraction grating. The diffraction grating causes reflected light from its surface to be distributed by wavelength as a function of the reflection angle. Diffractions gratings with more gratings give higher spectral resolution. By changing the geometry between the diffraction grating and the detector, only a narrow bandwidth of light is allowed to strike the detector at any one time. By sweeping through an angular range, the light frequency exposed to the detector is likewise swept through a range. If any of the incident laser light has been Raman shifted into this range the detector can record this. This is seen as an intensity (in 'counts' or arbitrary units) and plotted against the difference between the frequency of the collected light and the frequency of the excitation light as the Raman shift in wavenumbers $\left(\mathrm{cm}^{-1}\right)$.

Although the science behind Raman spectroscopy can be complex, the border details of extracted data is relatively straightforward to analyze. In general, and as it pertains to this work, the analysis of a given peak is illustrated in Figure 3.25 and is as follows: peak position gives the identity of the molecular structure responsible for the peak; the height of the peak is directly proportional to the amount or concentration of the molecular structure of the peak; any shift of the peak position indicates stress on the structure (relative to the reference material and data), with shifts to higher wavenumbers indicating compressive stress and shifts towards lower wavenumbers indicating tensile stress; the width of the peak indicate the crystallinity of the specific molecular structure within the material- a narrower peak is due to a smaller distribution of photon energies and thus a more crystalline material. ${ }^{(488)}$ 


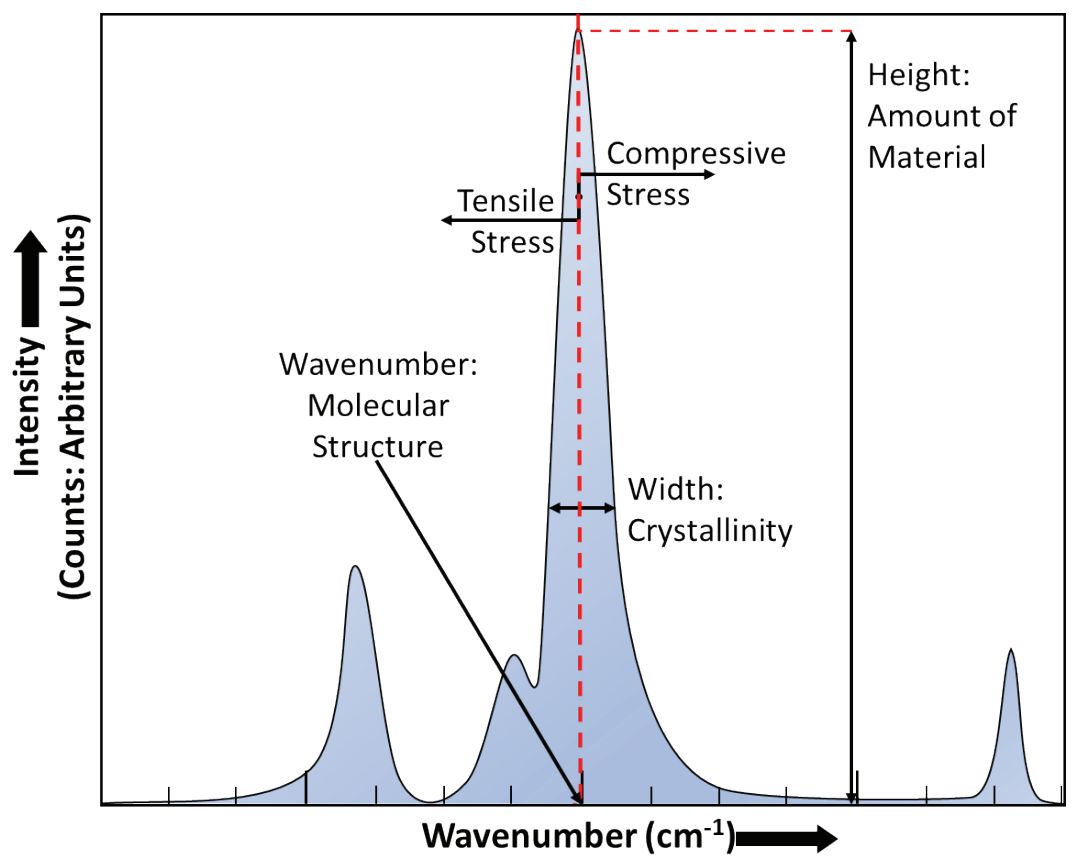

Figure 3.25: Illustration of the type of qualitative data gained from a simple analysis of a Raman peak.

For graphene research, Raman can be a rich analytical tool. Even before the rise of graphene, Raman spectroscopy has long been a powerful analytical tool for the characterization of carbon materials. ${ }^{(490)}$ Over the past decade there has been a large volume of work published on the characterization of graphene by Raman spectroscopy. In particular, the work conducted by A. C. Ferrari, et. al. has laid the foundation for the current understanding of the fundamental processes involved and the interpretation of the empirical data. (491) (492) (493) Additionally, there are many review articles which are particularly elucidating. ${ }^{(494)(495)(496)(497)}$ However, there still remains a debate as to the solid-state phenomena responsible for the more complex phonon processes. ${ }^{(491)}$

In this work, spectra were collected from around $1000 \mathrm{~cm}^{-1}$ to $3500 \mathrm{~cm}^{-1}$. This range captures many of the Raman peaks characteristic of graphene. This spectral range can contain up to 16 additional graphene peaks which if present, are each indicative of a particular characteristic of graphene (such as rotational disorder, for example). ${ }^{(498)}$ However, in this work, most of these peaks are not seen. The primary peaks are the D peak, the G peak, the 2D peak, the D+D' peak and the 2D' peak; these have been tabulated in Table 3-4. An in-depth explanation on the phonon interactions and resonance processes which lead to these peaks can be found in the references. ${ }^{(496)}$ 
Table 3-4: The primary Raman peaks encountered in this work.

\begin{tabular}{|c|c|c|}
\hline Peak ID & $\begin{array}{c}\text { Approximant } \\
\text { Location }\end{array}$ & Information \\
\hline D & $\sim 1350 \mathrm{~cm}^{-1}$ & $\begin{array}{l}\text { Requires aromatic carbon rings near defect sites or graphene edges } \\
\text { (broken symmetry), ring "breathing mode" (Raman inactive if no } \\
\text { defects). }\end{array}$ \\
\hline G & $\sim 1600 \mathrm{~cm}^{-1}$ & $\begin{array}{l}\text { Bond stretching of C-C pairs in the graphene aromatic carbon ring, } \\
\text { sensitive to strain. }\end{array}$ \\
\hline 2D (G') & $\sim 2670 \mathrm{c}$ & $\begin{array}{l}\text { Requiring two phonons with opposite momentum, is a double } \\
\text { resonance process between phonons and electrons, second order of } \\
\text { the D peak, but does not require the presence of the D peak, requires } \\
\text { large range order of aromatic carbon rings; will split into multiple } \\
\text { peaks for multilayer graphene; the quintessential graphene peak. }\end{array}$ \\
\hline D+D' & $\sim 2910$ & Combination of phonons with different momenta, a defect peak. \\
\hline 2D'(G”) & $\sim 3160 \mathrm{~cm}^{-1}$ & An overtone of the D' (The D' peak is not seen in this work) \\
\hline
\end{tabular}

The real space vibrations responsible for the $\mathrm{D}$ and $\mathrm{G}$ peaks are the most easily visualized. A schematic of these vibrations within the graphene sheet are shown in Figure 3.26. The ratio of the $D$ to $G$ peak intensity $\left(\mathrm{I}_{\mathrm{D}} / \mathrm{I}_{\mathrm{G}}\right)$ is commonly cited as a figure of merit for graphene, with a higher ratio indicating less defective graphene. However, it has been shown that the relationship between the $\mathrm{ID}_{\mathrm{D}} / \mathrm{IG}_{\mathrm{G}}$ ratio and the actual amount of graphene defects is not linear. In fact, there is a maximum $\mathrm{ID}_{\mathrm{D}} / \mathrm{IG}_{\mathrm{G}}$ ratio that occurs once there are $\sim 5 \mathrm{~nm}$ between defect sites. Higher defect densities $(<5 \mathrm{~nm}$ between defect sites) show a decrease in the ID/IG ratio. ${ }^{(499)(500)}$

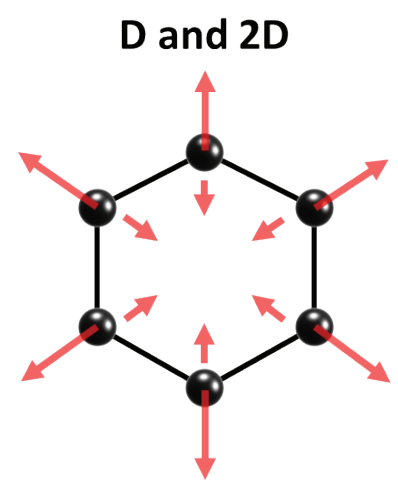

Breathing mode of the aromatic carbon ring

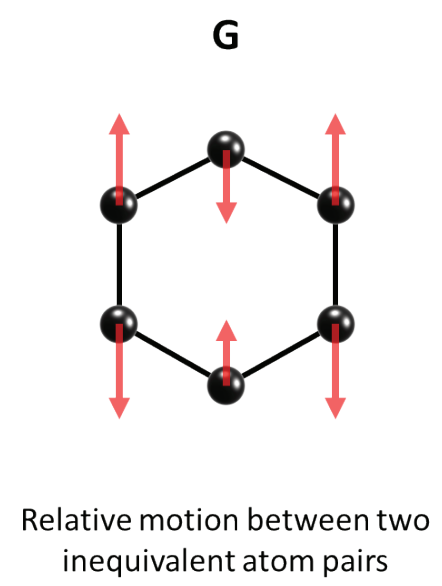

Figure 3.26: Real-space atom vibrations which result in the $\mathrm{D}, 2 \mathrm{D}$ and $\mathrm{G}$ peaks. The $\mathrm{D}$ and $2 \mathrm{D}$ always occur, however the D peak does not appear in the Raman spectra unless the ring is near a defect site or graphene edge.

For composite systems, the Raman spectra will show contributions from all the material excited by the laser that are Raman active. For SiC samples irritated with a $500 \mathrm{~nm}$ Raman laser, the penetration depth of the Raman laser is several millimeters. ${ }^{(501)}$ If the characteristic peaks do 
not overlap (i.e. appear at sufficiently different wavenumbers), the analysis is straightforward. However, if there are spectra overlap of the materials, steps must be taken to extract the pertinent data.

For this work, samples investigated consisted of a graphene/SiC stacked composite system. The characteristic peaks of both the $\mathrm{SiC}$ and the graphene appear over the same range; in particular the spectral range between $1000 \mathrm{~cm}^{-1}$ to $2000 \mathrm{~cm}^{-1}$ contains both the $\mathrm{D}$ and $\mathrm{G}$ peaks associated with graphene, as well as the second order carbon related vibrational peaks in SiC. ${ }^{(502)}$ The highly ordered SiC displays strong Raman activity and thus, the graphene component of the Raman signal is swamped by the SiC signal. To obtain meaningful information on the graphene overlayer only, a reference $\mathrm{SiC}$ spectrum is collected from a clean, as-received $\mathrm{SiC}$ wafer. This $\mathrm{SiC}$ spectrum is then subtracted from the graphene/SiC spectrum. The difference between the two spectra show the graphene contribution only. This is consistent with analysis techniques reported in the literature. (503) (504)

The intensity of the $\mathrm{SiC}$ reference spectra and the graphene/SiC spectra must be the same such that the $\mathrm{SiC}$ signal is appropriately subtracted out. To ensure an accurate subtraction, the $\mathrm{SiC}$ reference spectra was scaled to the graphene/SiC spectra by matching the spectra intensity of the two spectra at wavenumbers where only $\mathrm{SiC}$ peaks were present (i.e. no graphene component). The SiC shelf between $1800 \mathrm{~cm}^{-1}$ and $1900 \mathrm{~cm}^{-1}$ was found to give consistent results and thus was used as the scaling point. This peak is an overtone of a LO (longitudinal optical) phonon at 964 $\mathrm{cm}^{-1}$ in $\mathrm{SiC}$ and is unaffected by doping. ${ }^{(502)(505)}$ This, combined with the large spectra range the peak presents for matching, make it an ideal reference peak for intensity scaling. All spectra were leveled using the simplest mathematical baselining function which gave good results and was chosen on a case by case basis. This part of the data analysis was performed using the open-source program, "Spectragryph" version 1.2.10. The graphene Raman peaks were fitted using Lorentzian peak shapes, consistent with what has widely been reported in the literature for similar materials. (506) (507) The open-source program, "Fityk" version 1.3.1 was used in the fitting of the data. ${ }^{(508)}$ 
For this work a Renishaw micro-Raman inVia spectrometer equipped with a $532 \mathrm{~nm}$ green laser excitation source was used. The on-sample diameter was $\sim 5 \mu \mathrm{m}$ and a 50X objective was used. The laser was operated at $100 \%$ power as this was found to give the best results and no deleterious heating effects were observed. A diffraction grating with 1800 lines per mm was used. The exposure time was varied on a case by case basis but was always between 30 seconds and 180 seconds. The goal was to maximize the relatively weak contribution of the graphene to the total spectra without saturating the detector with the SiC peaks. An alternative method of increasing the spectral intensity is to add together several repeated spectral scans across the same range. However, to minimize hysteresis effects involving the electro-mechanical motion associated with the laser optics and diffraction grating, only a single spectral accumulation (spectra rescan) was used. The spectrometer was calibrated before each session using a single silicon crystal standard and the characteristic peak which appears around $521 \mathrm{~cm}^{-1}$. A photograph of the setup can be seen in Figure 3.27.

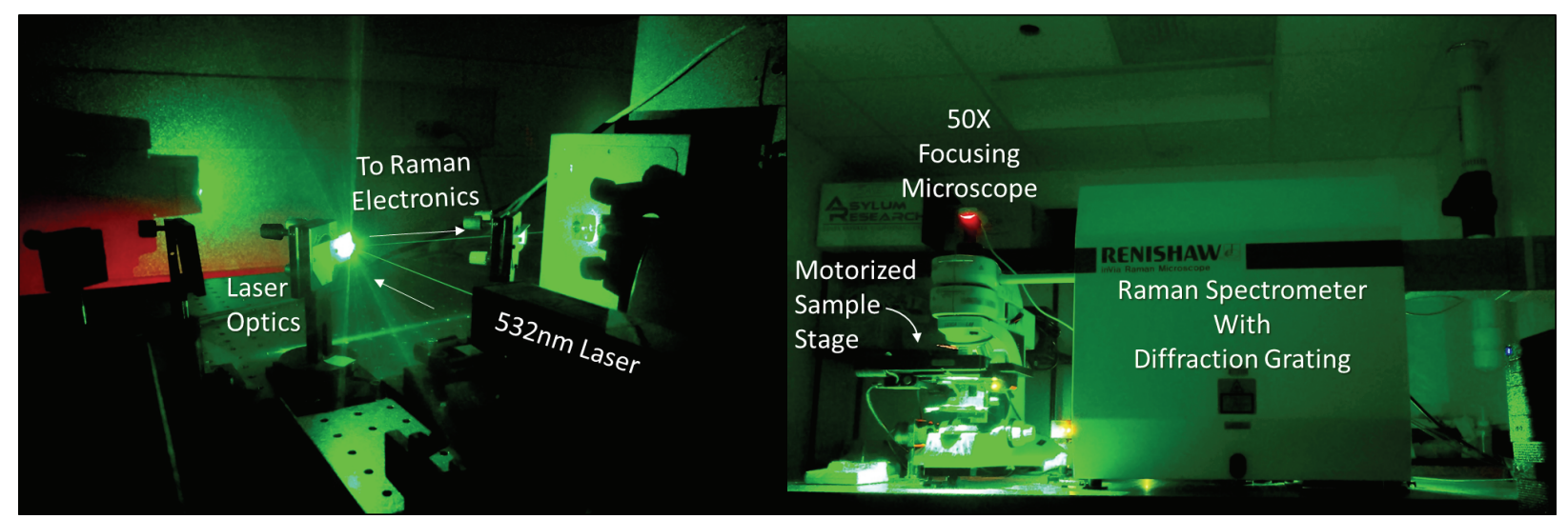

Figure 3.27: A picture of the Renishaw micro-Raman inVia spectrometer during operation.

\section{§3.2.4 Scanning Electron Microscopy (SEM)}

Scanning electron microscopy (SEM) is a surface imaging technique in wide use today. The first commercial SEMs were made available for sale by the Cambridge Instrument Company (UK) and JEOL JSM-1 (Japan) in 1965. ${ }^{(509)}$ However, the SEM can trace its origins all the way back to 1933 when Ernst Ruska was the first to image a surface with an electron beam. Ruska later won one-half of the 1986 Nobel Prize in Physics for his work on electron optics which led to the invention of the scanning electron microscope. ${ }^{21}(510)$

\footnotetext{
${ }^{21}$ The other half of the 1986 Nobel Prize in Physics was shared by Gerd Binning and Heinrich Rohrer for their work on the design of the scanning tunneling microscope (STM).
} 
The most basic advantage of SEM imaging over traditional optical imaging is the far greater magnification that can be achieved. Visible light imaging is limited to resolutions on the order of magnitude of the wavelength of light used to illuminate the sample. SEM on the other hand, utilizes a highly focused beam of electrons to extract surface information and therefor can resolve much finer details.

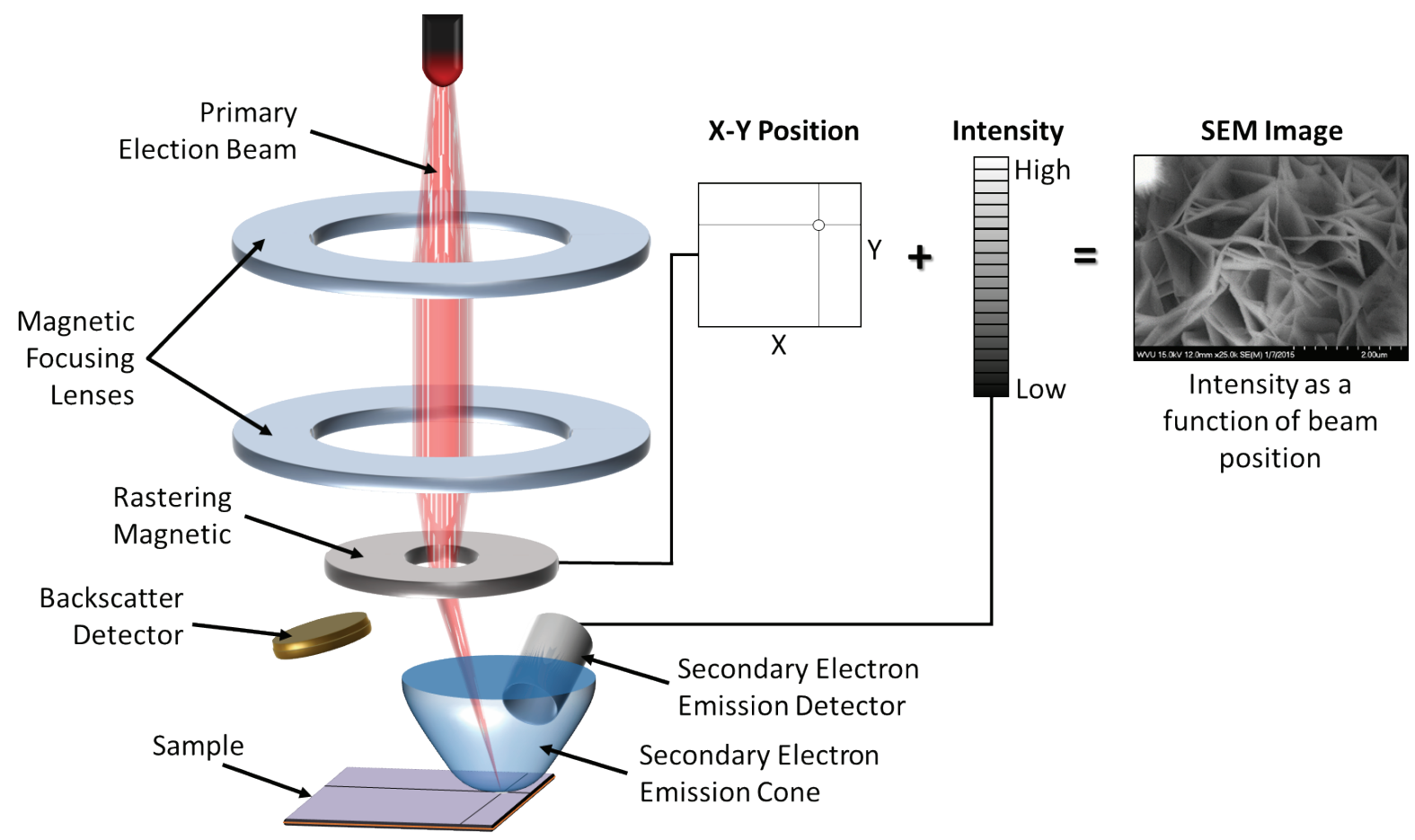

Figure 3.28: A simple schematic of the generic principle of SEM opeation.

A SEM operates under high vacuum conditions. In typical operation, an electron beam is generated and is focused using a series of electromagnetic lenses. The beam is rastered over the sample being imaged. Once the beam of electrons strikes the sample, several physical phenomena occur. As it pertains to SEM, the two most important events are the emission of secondary electrons and back scattered electrons. Both types of electrons are detected by electron detectors positioned within the SEM. By combining the known position of the electron beam on the sample surface (electronically controlled by the SEM software) and on the intensity of the secondary and backscattered electrons striking the detectors, a high-resolution gray-scale image can be constructed. The value of the gray scale is proportional to the amount of detected electrons corresponding to the position of the primary beam at the time. This creates the contrast seen in SEM images. A simple schematic illustrating this process can be seen in Figure 3.28. 
Secondary electron emission occurs when a material is irradiated with a primary radiation source (in this case, the electron beam) with an energy greater than the ionization potential of the material. When this occurs, the material under irradiation absorbs the beam energy and, in order to return to a thermodynamic/electrodynamic equilibrium, an electron is emitted. This emitted electron is the secondary electron. Secondary electrons have a low kinetic energy. This, combined with the small inelastic mean free path of electrons in solids, mean that only secondary electrons emitted at or near the surface of the material will make their way to the detectors. This is adventitious as it adds contrast to topologically complex surfaces. Surface features which have a large aspect ratio or acute angles (i.e. sharp edges) will have a greater fraction of produced secondary electrons escape and reach the detectors. This higher electron flux is rendered as a bright area in SEM images. ${ }^{(511)}$ Therefore, SEM is primarily a surface characterization technique. However, geometry is not the only factor in determining if a secondary electron emission event occurs. The $\mathrm{Z}$ number of the atoms in the sample, among other properties, also influence secondary electron emission and therefore the rendered image. ${ }^{(512)}$

In an ideal scenario, the sample is conductive and electrically grounded. In such a case, a ground electron is pulled to neutralize the ion produced by the primary electron beam. The process then repeats and thus a continuous stream of secondary electrons is emitted. If the sample is nonconductive or not properly grounded, the material will not re-neutralize, and the sample will quickly become electrostatically charged. Sample charging will drastically reduce the emission of secondary electrons and will deflect the electron beam, reflecting primary electrons back to the detector. The SEM cannot differentiate these reflected primary electrons from electrons originated from the sample. This leads to a rendered image with a large bright spot or cloud masking the areas of the sample experiencing charging effects. ${ }^{(513)}$ This can be avoided by coating the sample with a conductive material, but this can mask small features. Due to the highly conductive nature of the materials imaged in this work, charging was rarely an issue and no sample coating was used.

The back scattered electron emission process occurs when an incident electron is deflected by the nucleus of an atom in the sample. The propensity for a back-scatter event to occur is proportional to the cross-sectional area of interaction of the atom, which itself is a function of the size of the nucleus ( $\mathrm{Z}$ number) and electron cloud screening effects of the atom. Thus, backscattered electrons can give image contrast based on the element type in a sample; the so 
called, "Z-contrast" image. The back scattered electron process is also affected by sample charging due to the primary beam deflection.

By using separate detectors and taking advantage of the differences in the electron energies of the back scatter and secondary emission processes, images can be produced using electrons from either process or from a blend of both processes. The images collected in this work used a blend. The SEMs used in this work have multiple detectors within the chambers positioned at different locations relative to the sample. The images used in this work were generated with data collected from both secondarily emitted electrons and from back scattered elections.

Part of the energy of the incident beam is thermalized and the sample undergoes electron beam heating. This heating must be taken into consideration when operating at high magnification (i.e. using a tightly focused electron beam) or when using long collection times (resulting in long primary beam irradiation time). In this work, this was rarely a problem due to the thermal stability of the materials imaged. Many other interactions do occur which are useful (such as secondary Xray generation, Auger electrons, etc.) but do not pertain to the work detailed here.

In this work two SEMs were used. They were a Hitachi S-4700 SEM and a JEOL LSM $7600 \mathrm{~F}$ SEM and. These SEMs can be seen in Figure 3.29. No special preparation was done to the samples imaged. The working distance, acceleration voltage and other parameters were all chosen on a case by case basis with a goal of achieving good image quality.

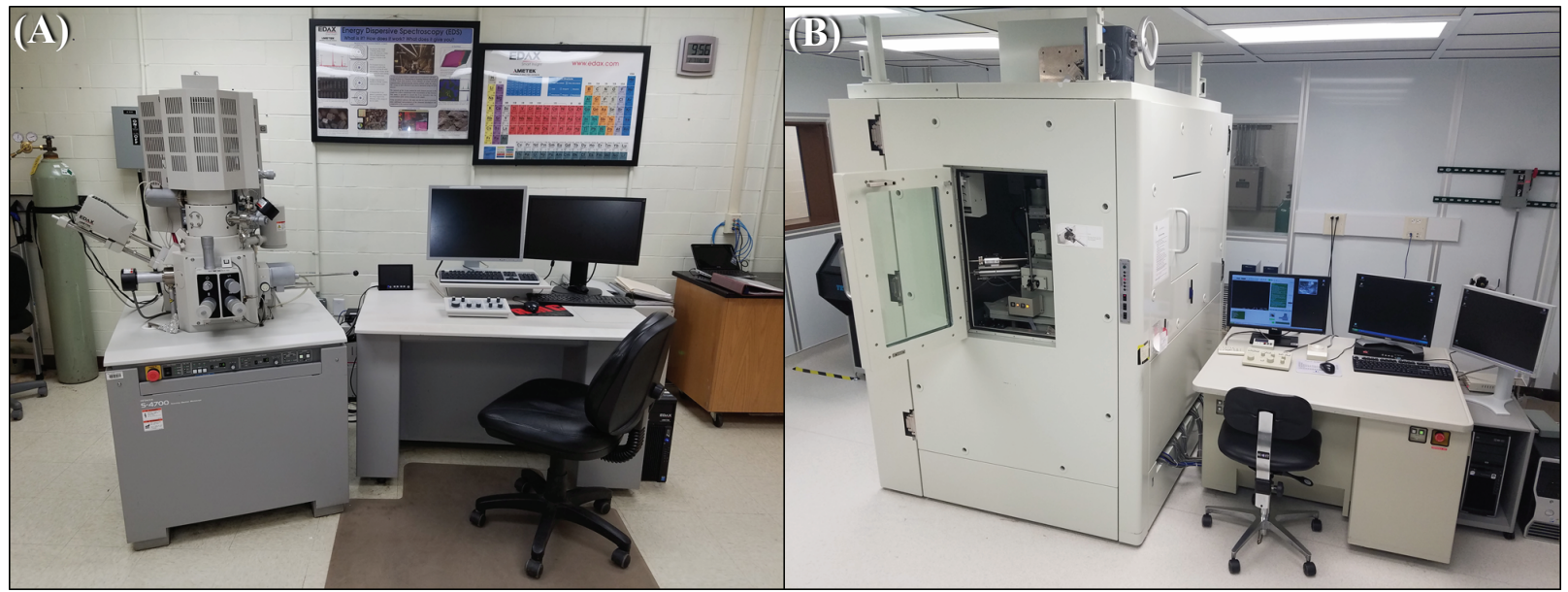

Figure 3.29: (A) The HitachiS-4700 SEM and (B) the JEOL LSM 7600F SEM. The JEOL SEM was located in a Class 10,000 cleanrooom enviroment and housed inside a mechanl isolation chamber for vibration reduction.

SEM was used primarily to characterize the nucleation methods, the deposited contacts and the masked etching experiments. Due to the atomically flat nature of the graphenated samples and 
the homogeneity of the sample surface, SEM saw limited use in this work for the characterization of the produced graphene films. Samples were mounted to the SEM stage using either double sided copper tape or carbon tape to mechanically adhere the sample and electrically ground the sample.

\section{§3.2.5 Electrical Characterization}

In order to electrically characterize the films produced in this work, reliable electrical contact to the films was required. This was accomplished by depositing bi-metallic contacts through an e-beam evaporation technique (detailed in Section \$3.3.1), ultrasonic wirebonding (detailed in Section \$3.3.2) and custom sample packing (detailed in Section \$3.3.3). The electrical characterization following the assembly is detailed in this section.

The materials fabricated in this work were electrically characterized with a Keithley 4200 Semiconductor Characterization System (4200-SCS). The unit used in this work had three DC source measurement units (SMU) capable of acting as independent power supplies as well as high impedance voltage measurement units and current measurement units. Each unit could be operated in one of four modes: constant voltage, constant current, voltage sweep or current sweep mode. Each SMU had a voltage range of $-20 \mathrm{~V}$ to $+20 \mathrm{~V}$ and a current range of $-100 \mathrm{~mA}$ to $+100 \mathrm{~mA}$. This setup allows for a wide variety of tests to be conducted. The center lead of a triaxial cable was used to make electrical contact from the SMU to the device package. The outer sheading was grounded to reduce noise in the signal. The combined circuit resistance of two triaxial cables was $\sim 1.4 \Omega$.

Much of the electrical characterization uses a simple application of Ohm's law to relate the current, voltage, power and resistance. ${ }^{22}$ From this simple relationship, a high level of insight into the device is gained. This is one of the reasons the simple device design and operational mode were chosen. Although simple, the famous relationships described by Ohm's law are an incredibly useful tool for determining various electrical parameters in a circuit. In fact, it has recently been reported by Weber, et al. that Ohm's law remains valid down to the atomic limit. ${ }^{(514)}$ For these reasons, it bears repeating here. Ohm's Law is most commonly given as

$$
I=\frac{V}{R}
$$

\footnotetext{
${ }^{22}$ Described by George Ohm in 1827 in his book, "Die galvanische Kette, mathematisch bearbeitet" (English: The
} Galvanic Circuit Investigated Mathematically). 
where I is the current (in amperes, A), V is the potential voltage across the material (in volts, V) and $\mathrm{R}$ is the resistance (in ohms, $\Omega$ ). Given the fundamental nature of Ohm's law, and its relationship to electrical power, it can be described in many forms. There are many iterations of Ohm's law and, when combined with the Joule-Lenz power law, they are summarized in the socalled Ohm's wheel shown in Figure 3.30.

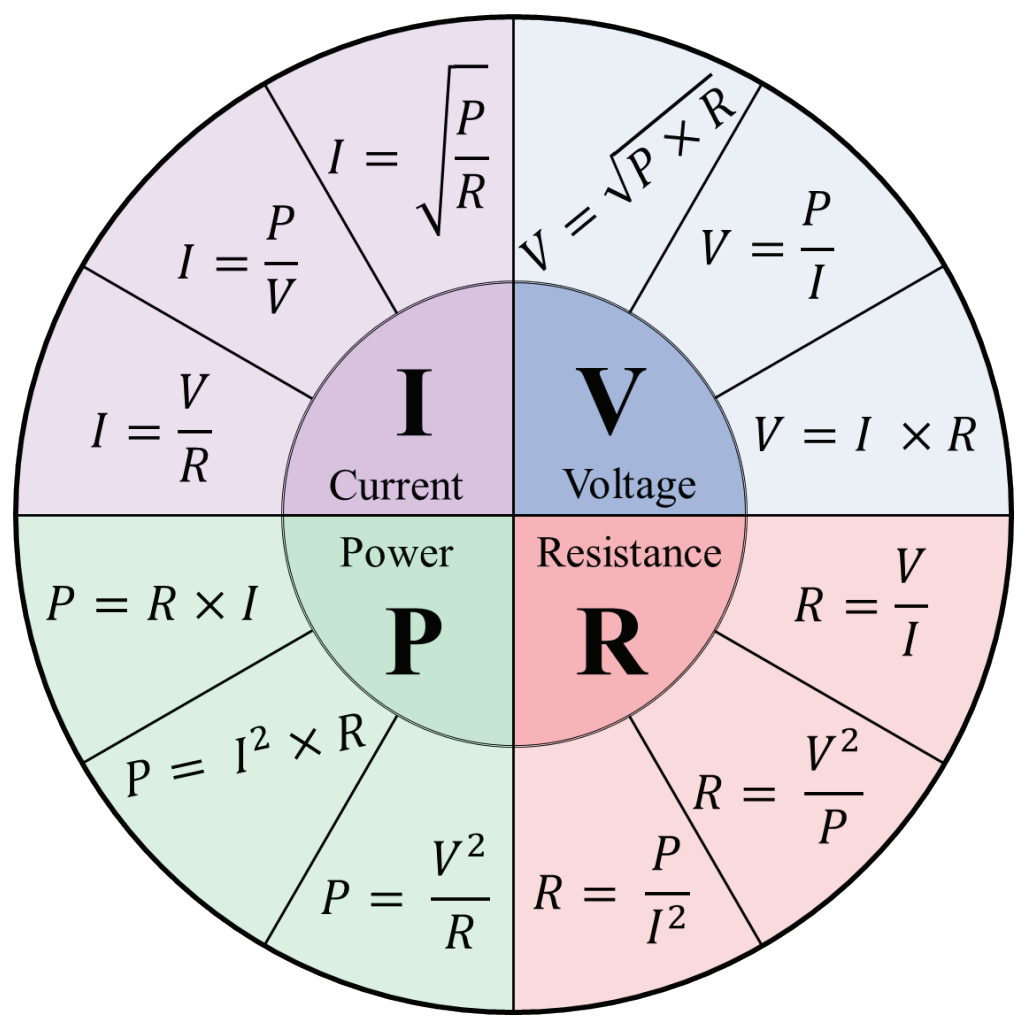

Figure 3.30: The so-called Ohm's Wheel, showing the various relationships between current, voltage, power and resistance, as described by Ohm's law.

For this work, the electrical measurements can be categorized into two types. The first type, termed IV measurements, were used for basic electrical characterization. These measurements plotted the current passing through the device against the voltage applied across the device. These tests were conducted in one of two ways: 1) sweeping the applied voltage through a predefined range and measuring the corresponding current or 2) by sweeping the desired current through a range and recording the voltage required to pass the desired current. These types of testes were used to capture a 'snap-shot' of the electrical properties of the device at a single instance in time.

The second type was used for device performance testing (gas sensing, photo detection, etc.) where the devices were electrically monitored as a function of time. These testes were also conducted in one of two ways: 1) by applying a predefined voltage and recording the current as a 
function of time (constant voltage mode) or 2) selecting a predefined current and modulating the voltage required (constant current mode).

From these simple tests, fundamental electrical properties of a material can be determined. Furthermore, both types of tests are subcategorized based on the electrical probe arrangement. They are a simple 2-point arrangement or a more complex 4-point arrangement. The electrode nomenclature used in this work is as follows: If a probe is suppling power or is grounded, it is referred to as a force probe. If a probe is connected to a high impedance voltage measurement device (such that a negligible amount of current flows through the sensing circuit) the electrode is referred to as a sense probe.

The two-point probe arrangement is one of the simplest methods employed for the electrical characterization of a material. In this arraignment, only two electrodes (force probes) are used; a source and a ground. If the instrument is operating in a constant voltage mode, the source probe supplies the voltage and the ground (always $\triangleq 0 \mathrm{~V}$ ) serves as the charge return path (current sink). If the instrument is operating in a constant current mode, the configuration is the same; however, the voltage is modulated to maintain the user defined current flow. Although the simplicity of the two-point probe arrangement is attractive, the primary drawback is that the resulting measurements captures and convolutes the electrical behavior of every element in the circuit. This includes effects like the lead wire resistance/impedance, the contact resistance of each connection in the circuit, etc. For the resistance parameter, the measured resistance is simply the sum of the individual resistances of each discreet component and union.

A typical four-probe arrangement used in this work is as follows; a source and ground probe are arranged in the same manner as in the two-point arrangement, but two additional probes are added. The additional probes act as sense probes and make contact in-between the outer two force probes. A voltage is applied across the force probes producing a current. The voltage is measured (with respect to ground) at the two sense probe locations. A schematic of a generic twoand four-point probe measurement is shown in Figure 3.31 $(A, B)$. The actual four-point probe arrangement using all three SMUs and a dedicated ground sink are shown in Figure 3.31(C). By using dedicated SMUs for the voltage measurements (rather than a single SMU measuring the $\mathrm{V}_{\text {drop }}$ across the device) and a dedicated ground terminal, the current interlock on the 4200-SCS could be increased from $10 \mathrm{~mA}$ to $100 \mathrm{~mA}$, as well as added flexibility to the setup. 
(A)

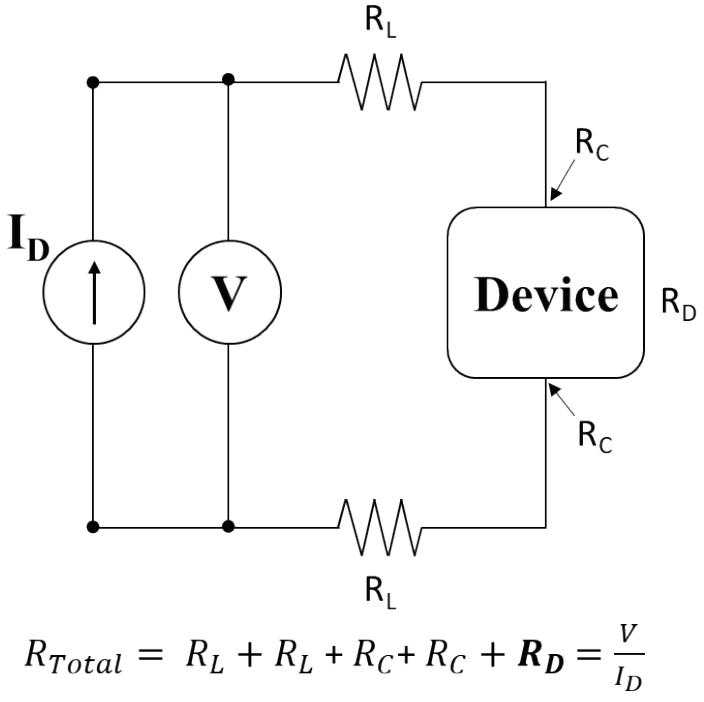

(B)

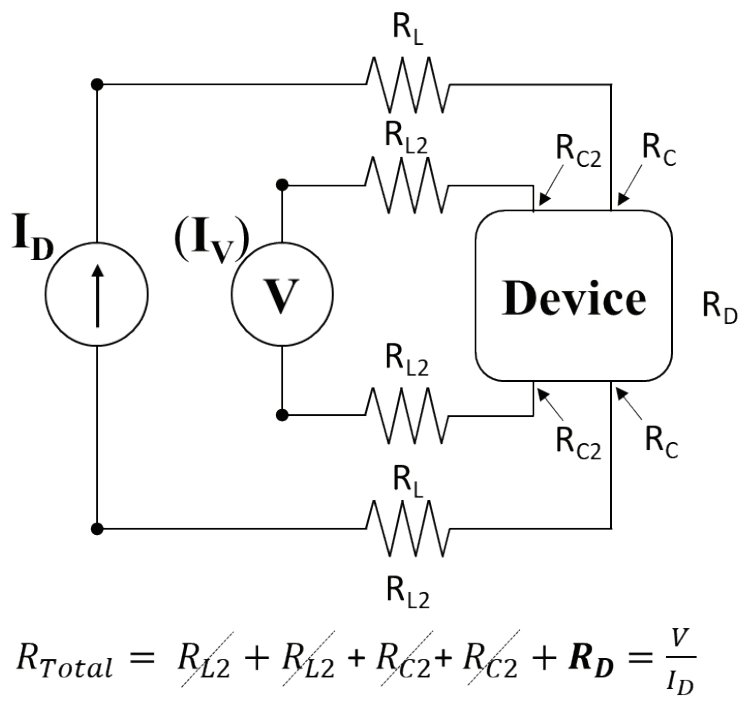

(C)

SMU1

SMU2

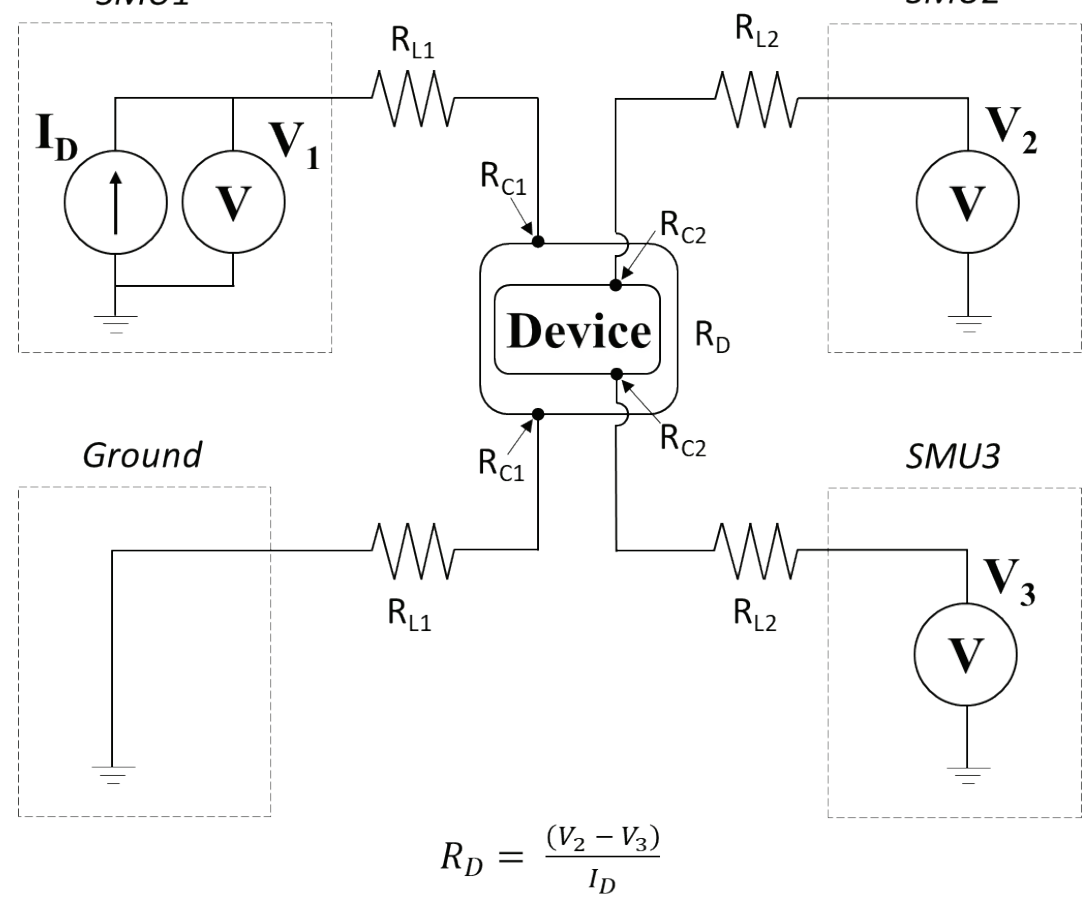

Figure 3.31: Conceptual schematic for the (A) two-point probe arrangement and (B) the four-point probe arrangement used in this work. (C) The actual probe and SMU arrangement used in this work using 3 SMUs and a dedicated ground terminal. The four-point probe arrangements use of a high impedance voltage measurement device means that many elements of the circuit can be removed from the analysis. $\mathrm{I}_{\mathrm{D}}$ is the current supplied to power the device, $\mathrm{R}_{\mathrm{L} \#}$ is the resistances of the test leads and $\mathrm{R}_{\mathrm{C} \#}$ are the contact resistances. Note that SMU1 acts as the current source, and SMU2 and SMU3 act as the HIDs to measure voltage. In reality, the setup is slightly more complex with several more unions and contact points. For clarity, these are not shown and can be simply lumped in with the terms shown.

While the four-point probe is a more complex arrangement (necessitating additional test 
leads and contact points), the inherent advantage of this method is that these additional components can be ignored in the analysis ( $\mathrm{R}_{\mathrm{L} 2}$ and $\mathrm{R}_{\mathrm{C} 2}$ in Figure 3.31). This is accomplished by the use of a high impedance $\left(>10^{12} \Omega\right)$ voltage measurement device (HID). Such a device allows only a minute amount of current to flow. This current is required to measure a voltage (labeled Iv in Figure $3.31(B))$ and is incredibly small, on the order of $\sim 10 \times 10^{-15}$ amps for the HIDs used in this work.

Because the parasitic voltage drop (and other deleterious effects) is proportional to the current flow, this arrangement allows the additional components of the HID (lead wires, contacts, etc.) to be ignored in the analysis. In this way, by using the voltage measured by the HIDs (SMU2 and SMU3 in Figure 3.31(C)) and the known current passing though the device (as supplied by SMU1 in Figure 3.31(C)), the resistance of the device only can be calculated $\left(\mathrm{R}_{\mathrm{D}}\right) .{ }^{\left({ }^{(15)}\right.}$ Figure $3.32(A)$ shows a typical probe arrangement used in this work for four-point analysis.
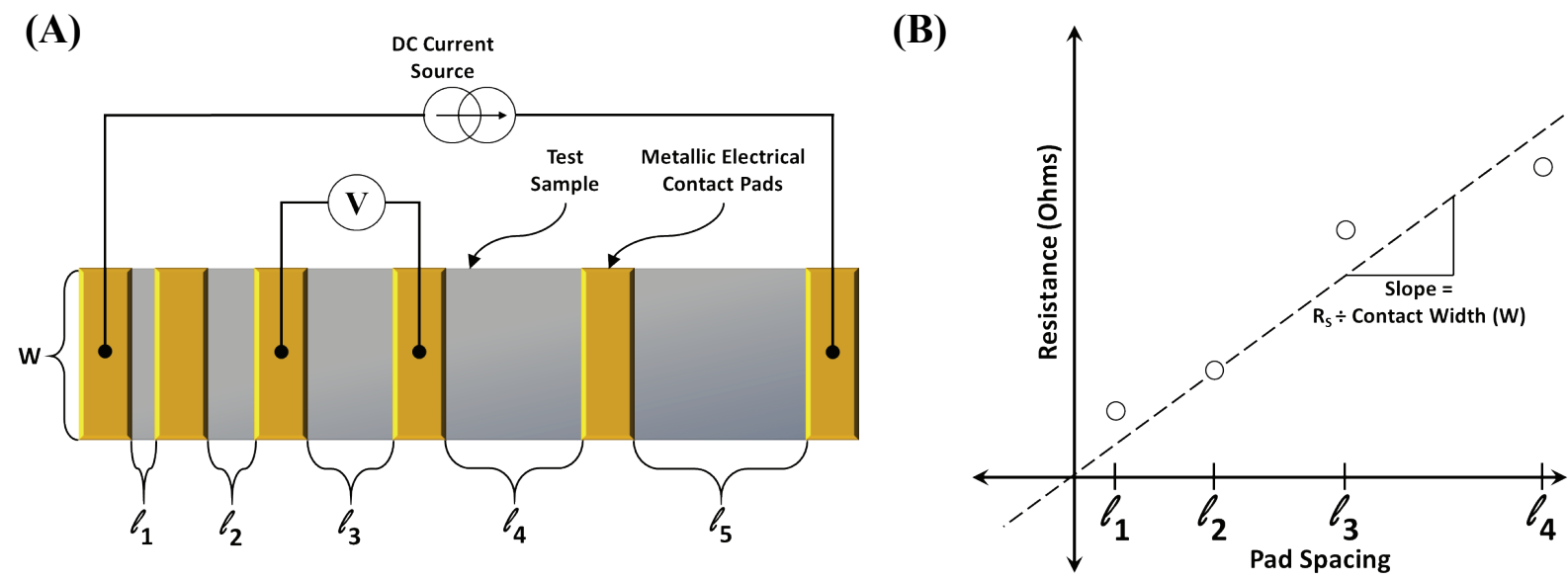

Figure 3.32: (A) A TLM arrangement. The distance between pads ( $\left.\ell_{n}\right)$ and the pad width (W) are indicated. Current is forced across the pattern and the voltage is measured between two inner pads. (B) Graphical method for determining sheet resistance; resistance is plotted against pad spacing and a liner line is struck. The slope divided by the contact width is equal to the sheet resistance in $\Omega / \square$.

The four-probe measurement allows for a simple transmission line model (TLM) analysis method to be employed (also referred to as the transfer length method). This method is commonly used to extract electrical properties of thin films. This analysis is based on the fact that the resistance of a thin film will vary linearly with distance (i.e. if the length of the film is doubled, the resistance will also double) and assumes that the current flow is strictly 2-dimentianal. The TLM method, originally proposed in 1964, utilizes an electrical test pattern consisting of a series of irregularly spaced, linearly arranged, identical electrical contacts pads. ${ }^{(516)}$ This probe arrangement is depicted in Figure 3.32(A). 
A current is forced across the test pattern between two force probes (an SMU and a dedicated ground, in this work). The voltage (relative to ground) is then measured by two sense probes at two discreet points in the pattern. The voltage difference between the points is calculated as a voltage drop ( $\left.\mathrm{V}_{\text {drop }}\right)$. Based on the $\mathrm{V}_{\text {drop }}$ and the known current flow (which is the same at all points in a series circuit), the resistance of the film between the two sense probes is calculated via Ohm's law; this value is then plotted against the spacing between the pads. From this, the film resistance can be calculated. Resistance measurements are taken between several pairs of contact pads, each with a different spacing, and plotted as a function of spacing. From this locus of points, a best fit linear line is struck as shown in Figure 3.32(B). If the slope of the line is divided by the contact width, the resistance of the film can be quantified in units of $\Omega / \square$ (ohms per square). This unusual unit has the benefit of being independent of film size and quantifies the resistance on a per square basis (i.e. a $1 \mathrm{~cm} \times 1 \mathrm{~cm}$ film would have the same sheet resistance as a $10 \mathrm{~cm} \times 10 \mathrm{~cm}$ identical film). This allows of the direct comparison between films of different sizes and shapes, as long as the restively is expressed in terms of $\Omega / \square$.

If the voltage is measured on the same contact pads as the force probes, the contributions of the contacts through which current is inject and removed are lumped into the measurements. This probe arrangement is shown in Figure 3.33. The film resistance is offset by the contact resistance; the locus of points is shifted up by an amount equal to twice the additional contact resistance (the effects of both the source and drain contacts are additive in the series circuit). Therefore, the contact resistance can be taken as $1 / 2$ the $Y$ - intercept. The length of the contact utilized to inject current (and overcome the contact resistance) is called the transmission length
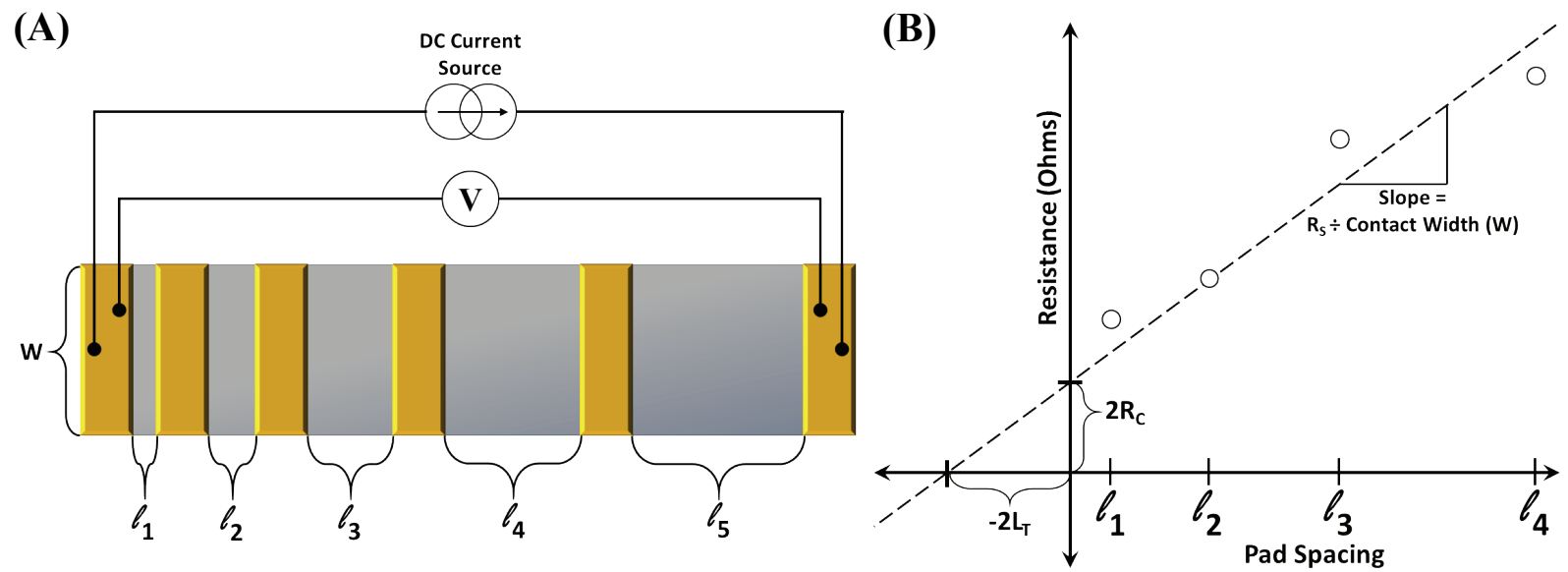

Figure 3.33: (A) The more complete TLM arrangement, which allows for the extraction of contact parameters as shown in (B). Namely, the contact resistance $\left(\mathrm{L}_{\mathrm{T}}\right)$ and the transmission length $\left(\mathrm{L}_{\mathrm{T}}\right)$. 
$\left(\mathrm{L}_{\mathrm{T}}\right)$ and is found by taking $-1 / 2$ of the $\mathrm{X}$-axis intercept. The disadvantage to this method is complex contact/film interactions are also lumped into the measurements. As will be discussed later, the devices fabricated in this work produced Schottky contacts. This makes the resistance measurements of the film complicated when the sense probes (voltage measurements) are collected on the same contacts as the force probes (current injection).

A summary of the properties derived from a classic TLM test are provided in Table 3-5. (517) Many other parameters can be determined with simple modifications to the classic TLM test. (518) (515) The specific contact resistance is a useful term for comparisons between devices of differing size or geometry. Depending on how the current flow path between the contact and the film interface is defined, the specific contact resistance can be characterized by the contact width as, $\rho_{C}(\mathrm{~W})=\mathrm{R}_{\mathrm{C}} \cdot \mathrm{W}$ or by the contact area used for current injection as $\rho_{\mathrm{C}(\mathrm{A})}=\mathrm{R}_{\mathrm{C}} \cdot \mathrm{W} \cdot \mathrm{L}_{\mathrm{T}}{ }^{(519)}$

Table 3-5: List of film parameters and related information gained from the TLM method.

\begin{tabular}{|c|c|c|c|c|}
\hline \multicolumn{2}{|l|}{ Properties } & Derived From & Units & Physical Meaning \\
\hline $\begin{array}{c}\text { Contact } \\
\text { Resistance }^{\dagger}\end{array}$ & $\mathrm{R}_{\mathrm{C}}$ & $1 / 2$ of $Y$-intercept & $\Omega$ & $\begin{array}{l}\text { Resistance due to the contact } \\
\text { pad and material interface }\end{array}$ \\
\hline $\begin{array}{c}\text { Sheet Resistance } \\
\text { (Film } \\
\text { Resistance) }\end{array}$ & Rs & (Slope) $*$ (Contact Width) & $(\Omega / \square)^{\dagger \dagger}$ & $\begin{array}{l}\text { Resistance of the thin film } \\
\text { sheet independent of size and } \\
\text { geometry }\end{array}$ \\
\hline Transfer Length ${ }^{\dagger}$ & $\mathrm{L}_{\mathrm{T}}$ & $-1 / 2 * X$-intercept & $\mathrm{cm}$ & $\begin{array}{l}\text { Length of the electrical } \\
\text { contact utilized for current } \\
\text { flow (1/e of the current is } \\
\text { dissipated over this length) }\end{array}$ \\
\hline $\begin{array}{l}\text { Width Specific } \\
\text { Contact } \\
\text { Resistance }^{\dagger} \\
\end{array}$ & $\rho c(W)$ & $\left(\mathrm{RC}_{\mathrm{C}}\right) *($ Contact Width $)$ & $\Omega \cdot \mathrm{cm}$ & $\begin{array}{l}\text { Contact Resistance based on } \\
\text { the contact edge }\end{array}$ \\
\hline $\begin{array}{l}\text { Area Specific } \\
\text { Contact } \\
\text { Resistance }^{\dagger}\end{array}$ & $\rho C(A)$ & $\begin{array}{c}\left(\mathrm{R}_{\mathrm{C}}\right) *\left(\mathrm{~L}_{\mathrm{T}}\right) *(\text { Contact } \\
\text { Width })\end{array}$ & $\Omega \cdot \mathrm{cm}^{2}$ & $\begin{array}{l}\text { Contact Resistance on the } \\
\text { contact area used for current } \\
\text { injection }\end{array}$ \\
\hline
\end{tabular}

As an alternative to plotting the resistances as a function of contact spacing and then compensating for the contact width in the calculation of the sheet resistance and contact resistance, the resistances can be plotted as a function of the aspect ratio of the film between the contacts. The data is usually not presented in this way in the literature, but the determination of the sheet resistance is more easily intuited by the reader from the plot. This is the way the data are presented in Chapter 5. 
There are several requirements a TLM setup must meet in order for the assumptions made in the derivation of the method to hold true. They are discussed in the literature and are mainly concerned with the geometry and spacing of the pattern. ${ }^{(520)}$ There are two presupposition of this technique that are pertinent to this work.

First, is that the current flux lines are entirely confined within the known bounds of the test pattern. This requires that the area around the pattern be electrically insulating or the pads extend to the physical edge of the sample. If this is not the case, then the current flux lines, emanating from the force pads, extend into the expanse of the material beyond the dimensions of the patterned area. This leads to additional film area participating in the conduction, more than what is accounted for by considering the area of the test pattern only. This results in the recorded current not corresponding to the actual current passing between the sensing pads where the voltage is read from. Thus, the measured voltage drop is incorrectly associated with the total current flux, rather than that which is actually flowing between the sense pads. This is illustrated in Figure 3.34(A).
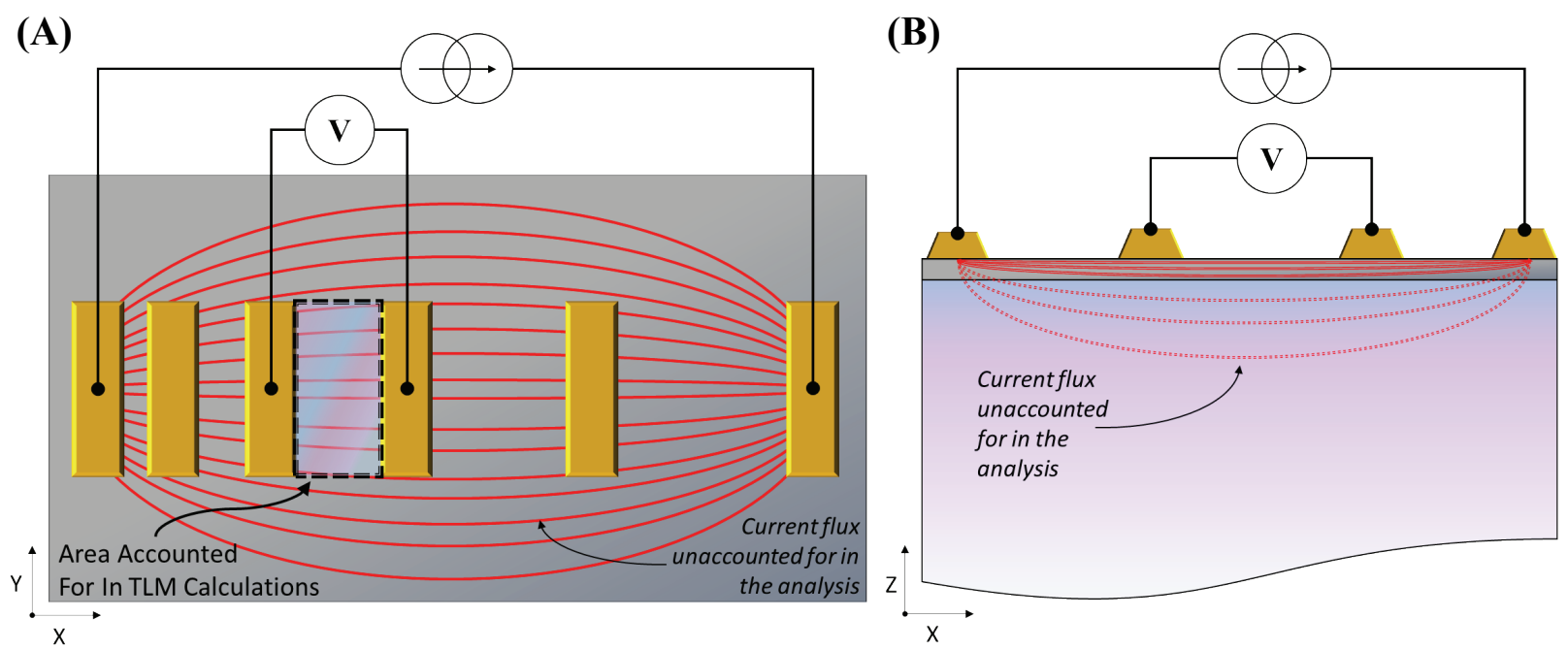

Figure 3.34: Two pitfalls of the TLM. (A) A pattern not sized to the dimensions of the sample resulting in the current path length not fully accounted for, leading to artificially low resistivity values. (B) An illustration of the impact of a substrate which is not fully electrically insulating. This also leads to artificially low resistivity values.

The second presupposition is that the substrate on which the thin film is on is electrical insulating. The TLM method was developed for the electrical characterization of thin films, and thus a key assumption is that there is no conduction in the depth of the substrate (i.e. a conductive film on a perfectly insulating substrate). Therefore, the second presupposition of the TLM technique is that the substrate does not participate in conduction. The impact of this is illustrated 
in Figure 3.34(B). Current penetrates into the substrate, utilizing additional conduction paths not accounted for in the analysis, leading to artificially low resistivity values.

To gain further insight into the nature of the metal-graphene interface, the nonlinear I-V behavior was analyzed using Anwar's modification of the Richardson-Dushman equation for a two-dimensional semiconductor. ${ }^{(521)}$ This yields the equation:

$$
I=\left(\frac{q W \sqrt{8 \pi m^{*}}}{h^{2}}\right)(k T)^{3 / 2} e^{\left(\frac{-q \Phi_{B}}{k T}\right)} e^{\left(\frac{q \Delta \Phi_{B}}{k T}\right)}
$$

Here, I is the current, $\mathrm{q}$ is the carrier charge, $\mathrm{W}$ is the contact width, $\mathrm{m} *$ is the charge carrier's effective mass, $\mathrm{T}$ is the film temperature, $\mathrm{h}$ is Planck's constant $\left(6.6260 \times 10^{-34} \mathrm{~J} \cdot \mathrm{Sec}\right), \mathrm{k}$ is Boltzmann's constant $\left(8.6173 \times 10^{-5} \mathrm{eV} / \mathrm{k}\right), \Phi_{\mathrm{B}}$ is the Schottky barrier height, and $\Delta \Phi_{\mathrm{B}}$ is the barrier lowering due to the applied electric field.

Following the treatment given by $\mathrm{Wu}$ and co-workers, $\Delta \Phi_{\mathrm{B}}$ can be written,

$$
\Delta \Phi_{B} \approx\left(\frac{q^{3} N_{d} V}{8 \pi^{2}\left(\varepsilon^{\prime}\right)^{2} \varepsilon}\right)^{1 / 4}
$$

where $\varepsilon^{\prime}$ and $\varepsilon$ are the high frequency and static permittivity of the film, respectively, and $\mathrm{Nd}$ is the carrier density. ${ }^{(522)}$ Substituting this expression into Equation 3-2 and rearranging yields:

$$
\ln \left(\frac{I}{\frac{q W \sqrt{8 \pi\left(k_{B} T\right)^{3} m^{*}}}{h^{2}}}\right)=-\frac{q \Phi_{B}}{k_{B} T}+\left(\frac{q^{7} N_{d}}{8 \pi^{2}\left(\varepsilon^{\prime}\right)^{2} \varepsilon\left(k_{B} T\right)^{4}}\right)^{1 / 4}\left(V^{1 / 4}\right) \quad \text { Equation 3-4 }
$$

The term on the left-hand side of this equation represents the natural log of a reduced current which, when plotted as a function of $\mathrm{V}^{1 / 4}$, should yield a straight line (if current injection is by thermionic emission). From the slope and intercept of this line, the carrier density $\left(\mathrm{N}_{\mathrm{d}}\right)$ and Schottky barrier height $\left(\Phi_{\mathrm{B}}\right)$, respectively, may be calculated. This is illustrated graphicly in Figure 3.35. 


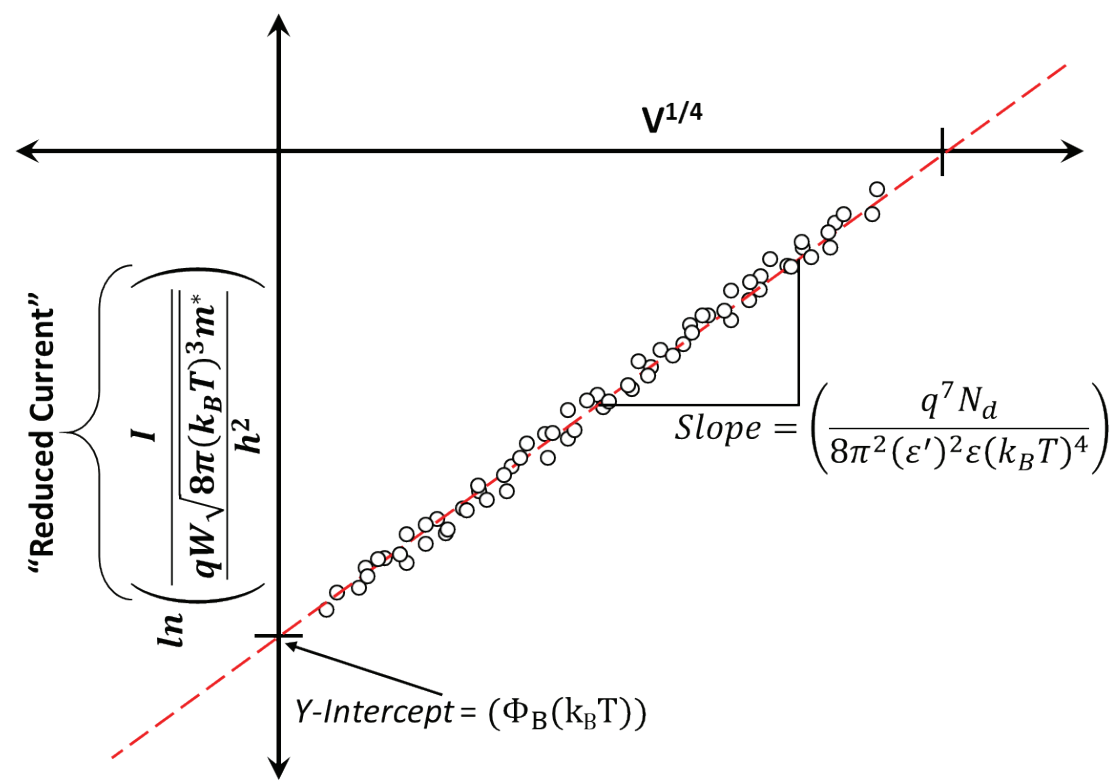

Figure 3.35: Graphical interpretation of the modified Richardson-Dushman analysis employed in the analysis of the graphene devices. From this analysis the Schottky barrier height $\left(\Phi_{\mathrm{B}}\right)$ and ionized donor density or charge carrier concentration $\left(\mathrm{N}_{\mathrm{d}}\right)$.

In the calculations of the reduced current preformed in this work, it was assumed that the charge carriers were electrons, so q was taken as the electron charge $\left(1.6022 \times 10^{-19} \mathrm{C}\right)$. Following $\mathrm{Wu}$ and coworkers, ${ }^{(522)}$ it was further assumed that $\mathrm{m}^{*}$ was numerically equal to the mass of a free electron $\left(9.1093 \times 10^{-31} \mathrm{~kg}\right) .{ }^{(522)}$ As they point out, the calculations are not particularly sensitive to changes in this value since variations of two orders of magnitude for $\mathrm{m}^{*}$ produce less than a $10 \%$ in the calculated value of the Schottky barrier height. Based on these values, and the extracted intercept from the plot, the Schottky barrier height was determined.

Although the Schottky barrier height is not particularly sensitive to $\mathrm{m}^{*}$, the value calculated for $\mathrm{Nd}$ is strongly dependent on the values used for $\varepsilon$ and $\varepsilon^{\prime}$. Wu and coworkers used approximate values based on the average for the $\mathrm{SiC}$ substrate and the air above the graphene film. ${ }^{(522)}$ Since those studies, however, Huang, et al. have observed values of $\varepsilon^{\prime} / \varepsilon_{0} \sim 10,000$ and $\varepsilon^{\prime} / \varepsilon_{0} \sim 2$ for GO (where, $\varepsilon_{0}=8.854 \times 10^{-12} \mathrm{~J} \cdot \mathrm{m} / \mathrm{C}^{2}$ is the permittivity of a vacuum) and, because the graphene films produced here have oxygen-based defect levels on the order of $\sim 20 \%$, these values were used in the present calculations. ${ }^{(523)}$

Additionally, by using the relationship between conductivity (as determined by TLM measurements), carrier mobility (as determined by the previously discussed Richardson-Dushman analysis) and the charge of a carrier (again, taken to be an electron in this case) the carrier mobility 
of the film may be calculated. When this value is divided by the number of graphene layers (as determined by XPS analysis detailed in APPENDIX D), the nominal mobility is calculated.

\section{§3.3 Sensor Assembly and Packaging}

This section will start with a description of the manufacturing steps used to fabricate devices from the SiC-graphene composites synthesized by the plasma assisted process. This includes the deposition of electrical contacts by e-beam evaporation followed by thermosonic wirebonding to the device package. This section will conclude with a brief description of the various device packaging used to mount and house the devices during and electrical characterization and gas/light testing.

\section{§3.3.1 Contact Patterning and Secondary Dicing}

Bimetallic contacts were deposited onto the graphenated substrates by an e-beam evaporation process. The contacts, consisting of $30 \mathrm{~nm}$ of titanium and $300 \mathrm{~nm}$ of gold, were deposited by a custom Kurt J. Lesker E-Beam Evaporator e-beam evaporator. This can be seen in Figure 3.36. E-beam evaporation is a common technique for the deposition of electrical contacts. Under high vacuum, an electron beam is directed onto metal ingots housed within a crucible. Under electron beam irradiation the ingots liquefy and produce a melt. Material then evaporates from the

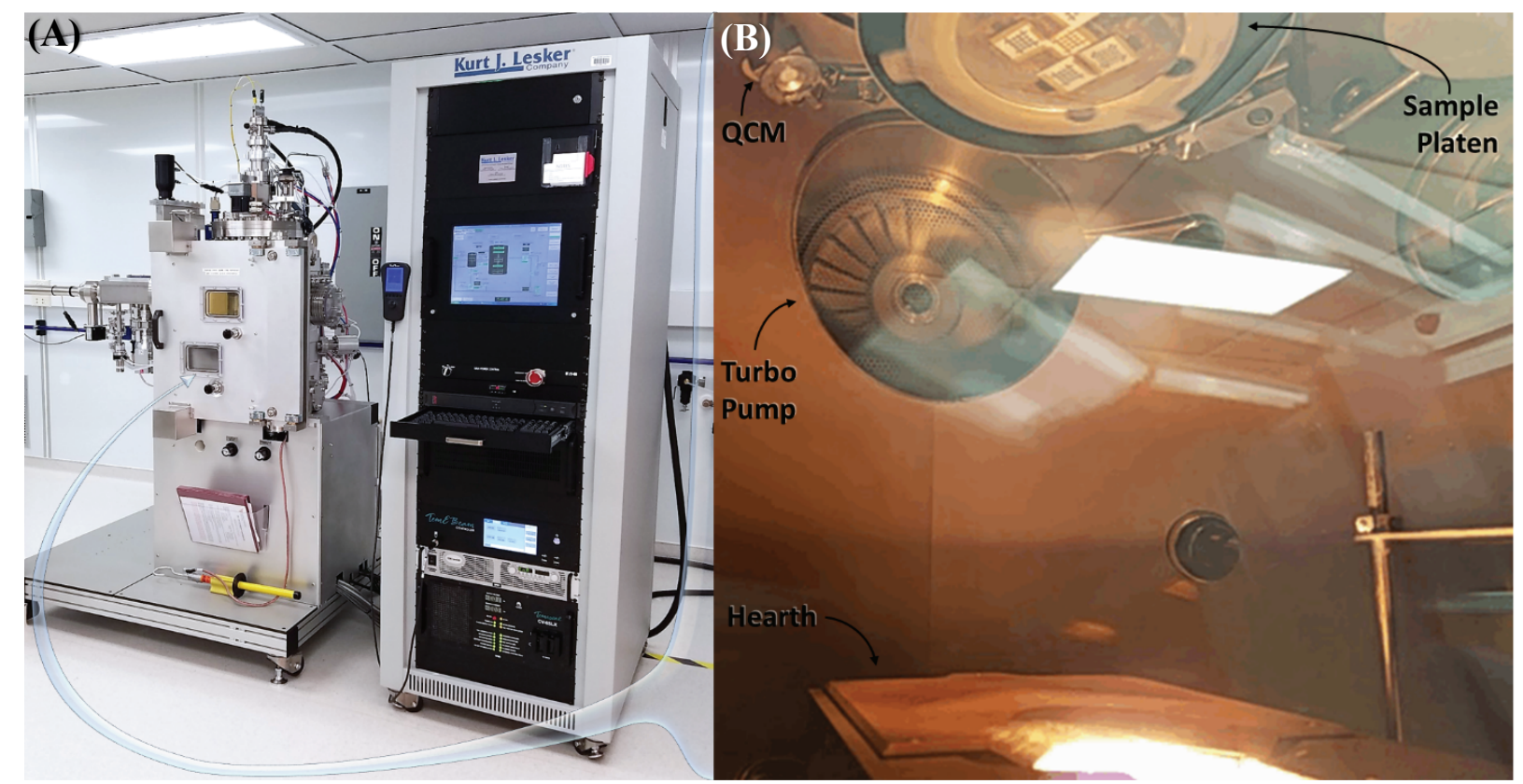

Figure 3.36: (A) The KJL e-beam evaporator used in this work. (B) The view of the main chamber, as seen through the view port during a deposition. In this specific run, five samples were concurrently processed. 
crucible where it coats the interior of the vacuum chamber. The rate of evaporation can be controlled by modifying the power of the electron beam.

The deposition is monitored by a quartz crystal microbalance. These types of high accuracy balances use a well characterized quartz crystal driven into oscillation; as material is deposited onto the crystal, the resonate frequency is shifted. Based on the density of the evaporated material and the shift in resonance frequency of the crystal, the amount of material deposited onto the crystal is then calculated.

Titanium was deposited at a rate of $0.5 \AA / \mathrm{sec}$. Gold was deposited at a rate of $2.0 \AA / \mathrm{sec}$. Titanium was deposited first to act as a mediation layer between the substrate and gold layer. This increased the pad adhesion and mechanical integrity of the contacts. Gold was deposited as the second layer to serve as the contact surface for the wirebonding step.

For the graphenated $1 \mathrm{~cm}^{2}$ squares, metal contacts were deposited through a custom designed shadow mask containing both an interdigitated pattern and a parallel contact pattern, which can be seen in Figure 3.37(A). The metal shadow masks were purchased from Photo Sciences, Inc. From this point, these samples proceeded to the secondary dicing step. For the $2.5 \mathrm{~mm}$ X $10 \mathrm{~mm}$ graphenated samples, handmade shadow masks were used for the deposition of parallel contacts for electrical TLM tests. These shadow masks used Kapton tape as the masking material (tacky side up). These samples proceeded directly to the mounting and wire bonding step.
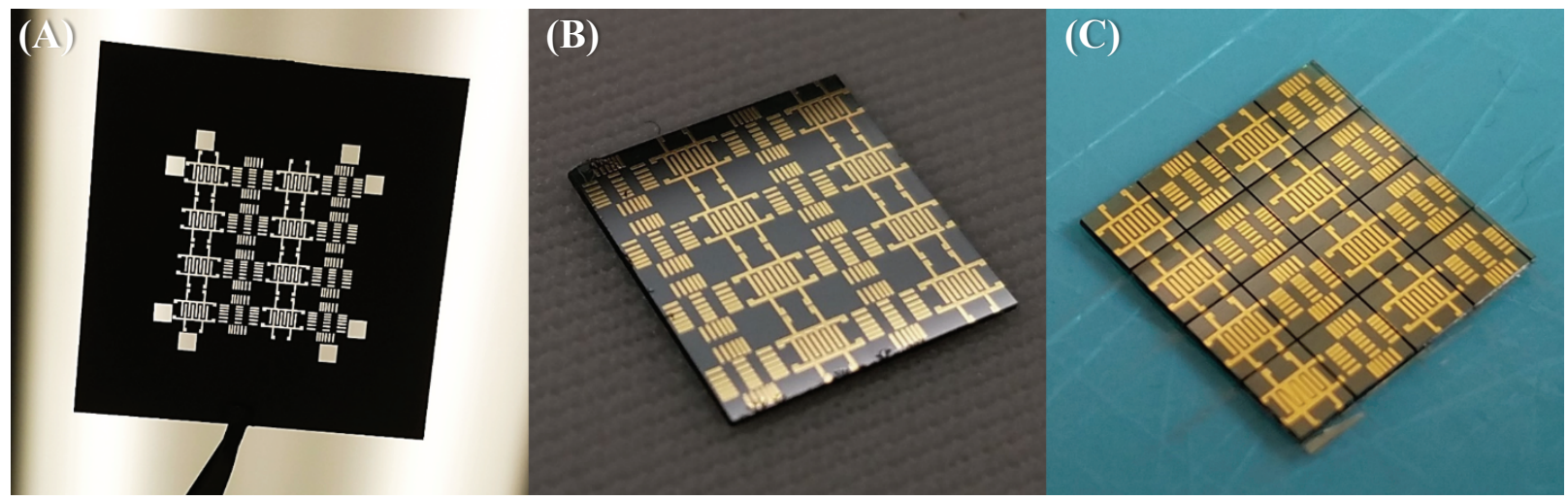

Figure 3.37: (A) Example of one of the shadow masks used for the $1 \mathrm{~cm}^{2}$ samples. (B) A graphinated sample with deposited bimetallic electrical contacts ready for secondary dicing. (C) A diced $1 \mathrm{~cm}^{2}$ sample. Each square was then packaged into a sensing platform. 
The $1 \mathrm{~cm}^{2}$ graphinated samples (now with metal contacts) were then diced into 16 equal pieces approximately $2 \mathrm{~mm}^{2}$, each containing a complete metal pattern. The DAD3240 Disco dicer used in this step was the same tool used in the primary dicing step. An example of a $1 \mathrm{~cm}^{2}$ graphenated sample diced into 16 smaller samples can be found in Figure 3.37(B,C). One of the 2 $\mathrm{mm}^{2}$ squares was then mounted to a sensor package; exactly how and to what type of sensor package depended on the type of testing to be conducted. To illustrate the size and spacing of the electrical contacts, an SEM image of a $2 \mathrm{~mm}^{2}$ sample with a complete interdigitated pattern contact pattern can be seen in Figure 3.38. Also shown is a close up of a single set of parallel contacts.

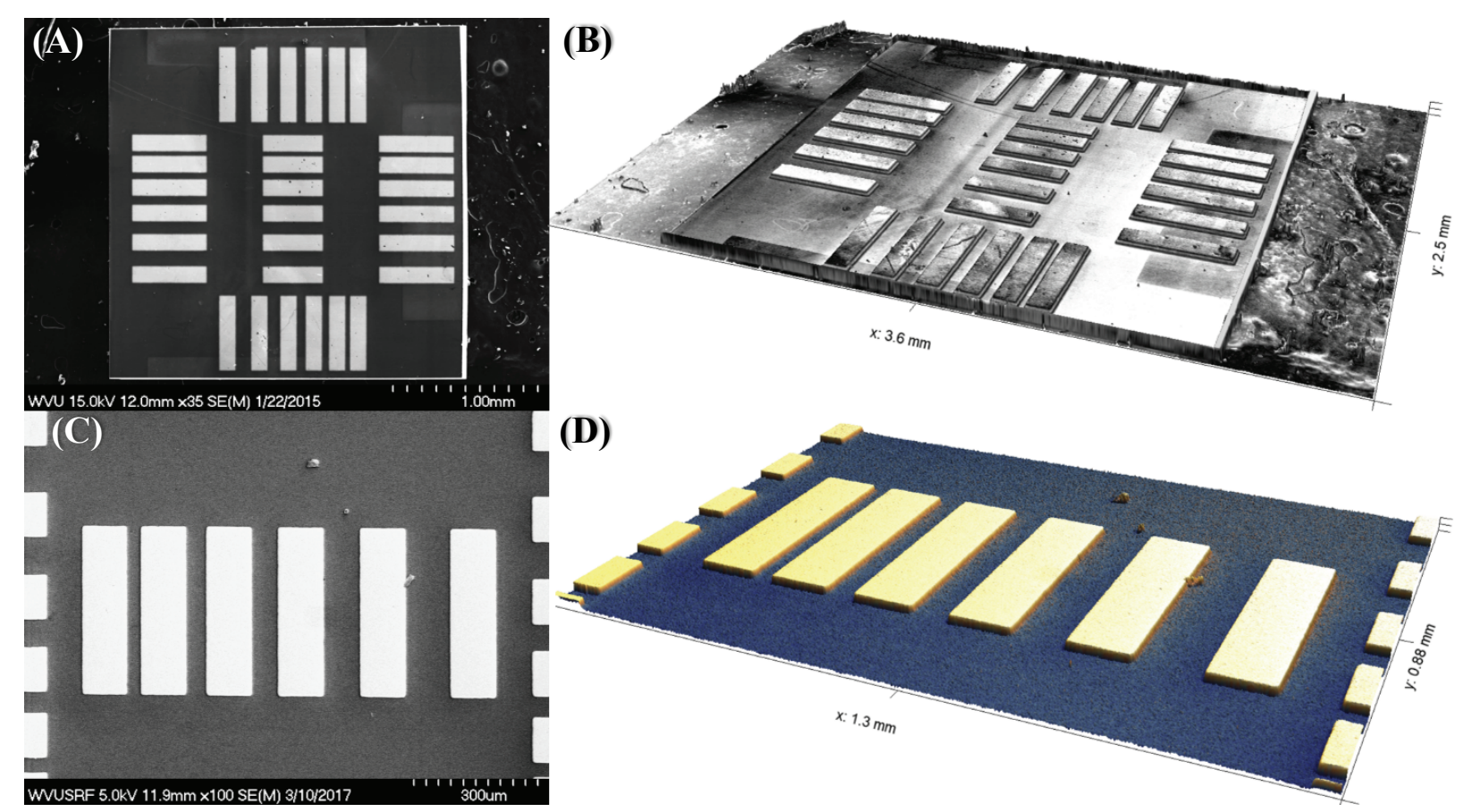

Figure 3.38: (A) SEM image of $2.5 \mathrm{~mm}^{2}$ with complete parallel contact pattern. The bright areas correspond to the metal contacts. (B) 3D projection of the same sample (arbitrary Z value). (C) Higher magnification of the center set of parallel contacts. (D) False color $3 \mathrm{D}$ projection of the same image. The substrate contrast seen in $(A, B)$ is due to a legacy processing step (pattered plasma oxidation step) which was not used extensively in this work.

Note that the contrast difference seen on the substrate in Figure 3.38 is due to an oxygen plasma etch, used to increase the adhesion between the Ti/Au contacts and the substrate. This was a technique carried over from previous work. By modifying the wire bonding parameters, this step was found to be unnecessary and other than a few samples (one of which is shown in Figure $3.38(A))$ was ultimately not used extensively in this work. In Figure 3.39 SEM images of the interdigitated pattern can be seen. 

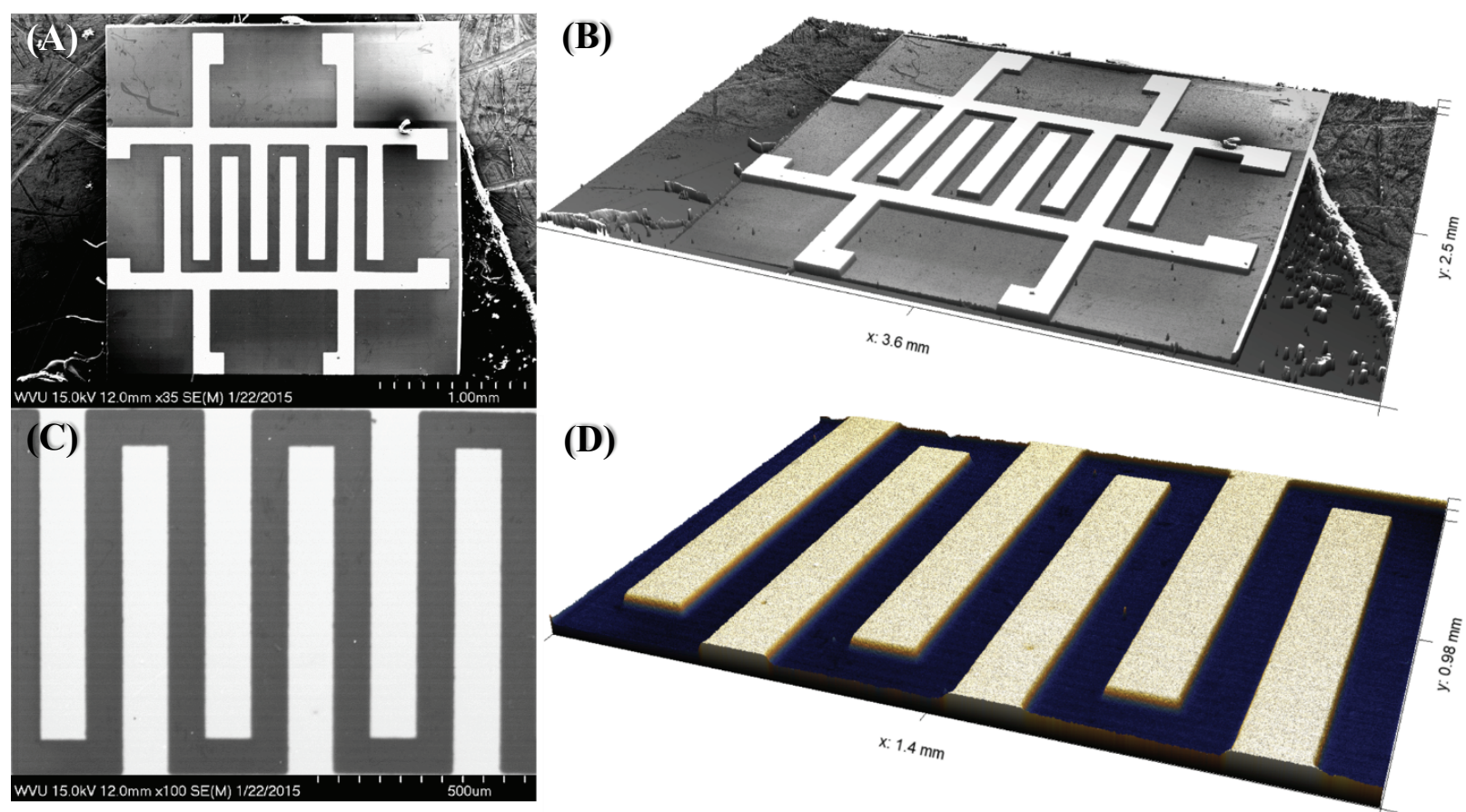

Figure 3.39: (A) SEM image of $2.5 \mathrm{~mm}^{2}$ with complete interdigitated contact pattern. The bright areas correspond to the metal contacts. (B) 3D projection of the same sample (arbitrary Z value). (C) Higher magnification of the center set of the interdigitated contacts. (D) False color $3 \mathrm{D}$ projection of the same image.

Figure 3.40 shows an optical image of a $2.5 \mathrm{~mm}$ X $10 \mathrm{~mm}$ sample with contacts for TLM measurements. Strips of Kapton tape were lain across the sample, with the adhesive side up, such that the majority of the same was protected. The exposed area between strips received the metal flux during e-beam deposition. Because the mask was applied by hand, the spacing between the contacts varied from sample to sample. Therefore, the spacing between the metal contacts was measured for each sample after deposition. This measured value was then used in the TLM calculations. Any non-parallelism between adjacent contacts was accounted for by calculating the average spacing between the two contacts.

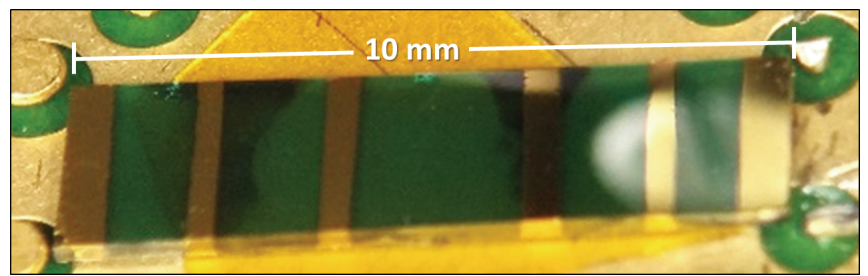

Figure 3.40: Magnified image of a sample used for TLM testing. 


\section{§3.3.2 Wire bonding}

Wire bonding (or wirebonding) is a commonly used technique for making electrical interconnects in micro devices. There are three main types of wire bonding: thermosonic bonding, thermocompression bonding and ultrasonic bonding. This work used thermosonic bonding. A combination of heat and ultrasonic power is used to fuse a gold wire with a gold substrate. Both the substrate and the gold wire are heated.

To start the bonding process, a high voltage arc is formed between the end of the gold wire and the electronic flame off wand (EFO wand). This melts the tip of the gold wire and through surface tension, a small gold ball is formed. On contact with a clean gold surface, heat and ultrasonic power is applied to the ball. The ultrasonic power scrubs the surfaces together. This fuses the ball to the substrate and a ball bond is formed. With the ball anchored to the surface, the tool is retracted from the surface and gold wire is allowed to unspool. The tool is then brought back down to the surface and again heat and ultrasonic power are applied. This shears the wire from the tool head at the same time fusing the wire to the substrate resulting in a crescent bond. The ball bond area is now jumpered to the crescent bond area and are electrically connected though the gold wire.

In this work, a West Bond 74776E wire bonder was used to make electrical contact from the sample to the sensor package. A photograph of this tool can be seen in Figure 3.41. The wire bonder used a $90^{\circ}$ tooling head also shown in Figure 3.41 (B). The wire used was $\sim 25.5 \mu \mathrm{m}$ in diameter and was $99.99 \%$ annealed gold with $3-10 \mathrm{ppm}$ of beryllium to enhance ball bond formation and $50 \mathrm{ppm}$ of total impurities of $\mathrm{Cu}, \mathrm{Ag}, \mathrm{Fe}$ and $\mathrm{Mg}$. The electrical resistivity was reported to be $\sim 2.3 \mu \Omega \cdot \mathrm{cm}$ with a thermal conductivity of $\sim 315 \mathrm{~W} / \mathrm{m} \cdot \mathrm{K}$ and a melting point of $1063{ }^{\circ} \mathrm{C} .{ }^{(524)}$ 


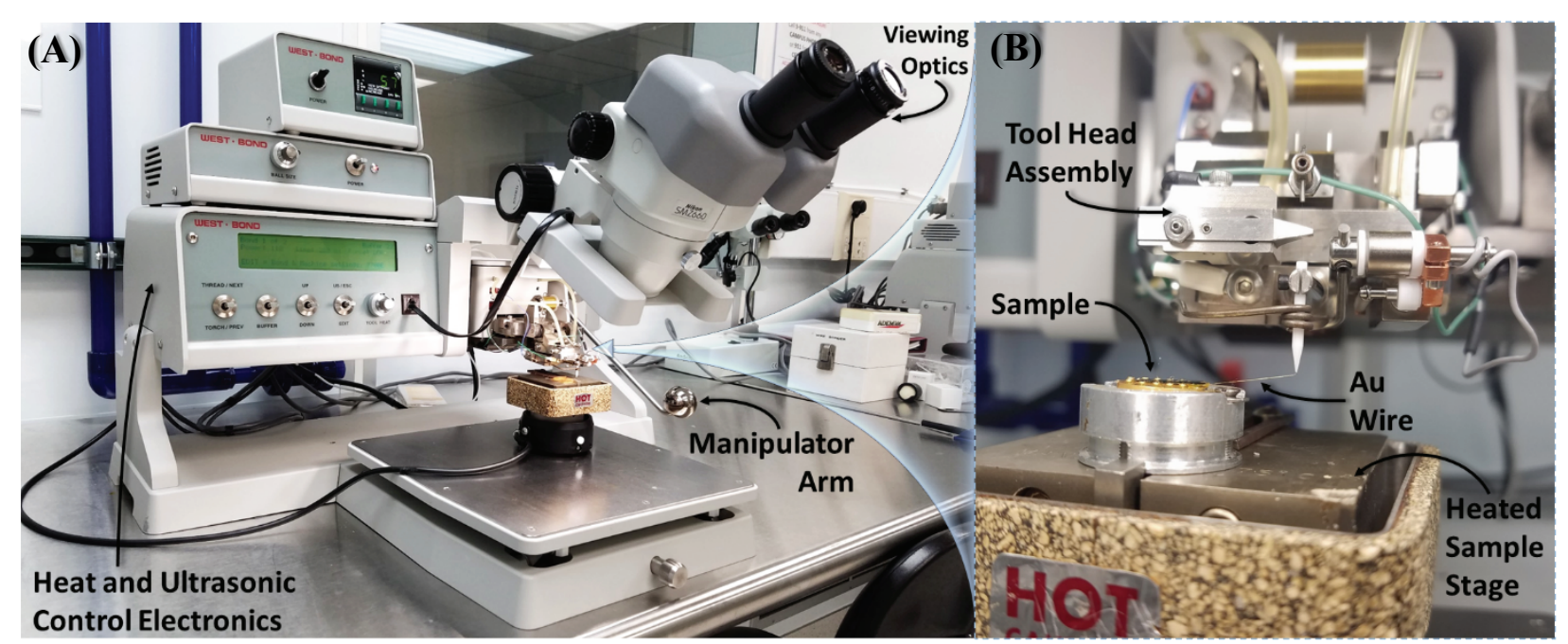

Figure 3.41: (A) The West Bond 74776E ultrasonic wire bonder wire. (B) A close up view of the tool head assembly; also shown is a gold thread, bonded to a sample and despoiling, in preparation for the crescent termination bond and completion of the jumper connection.

The stage heat, ultrasonic power and ultrasonic duration are all user-controlled parameters that can be modified to achieve the desired bond. These parameters varied greatly over the course of this work and were chosen on a case by case basis. The range of values used are tabulated in Table 3-6.

Table 3-6: Summary of wire bonding parameters

\begin{tabular}{|c|l|l|}
\hline \multicolumn{2}{|c|}{ Parameter } & \multicolumn{1}{c|}{ Value } \\
\hline \multicolumn{2}{|c|}{ Stage Temperature } & $105^{\circ} \mathrm{C}-125^{\circ} \mathrm{C}$ \\
\hline \multirow{3}{*}{ Ball Bond } & Ultrasonic Power & $30 \mu \mathrm{W}-200 \mu \mathrm{W}$ \\
\cline { 2 - 3 } & Pulse Duration & $20 \mathrm{~ms}-200 \mathrm{~ms}$ \\
\hline \multirow{2}{*}{ Wedge Bond } & Ultrasonic Power & $50 \mu \mathrm{W}-200 \mu \mathrm{W}$ \\
\cline { 2 - 3 } & Pulse Duration & $20 \mathrm{~ms}-50 \mathrm{~ms}$ \\
\hline
\end{tabular}

\section{§3.3.3 Device Packaging}

Several device packages were used in this work. These were designed on an as needed, ad hoc basis. Although ancillary to the main thrust of this work, these package designs were a key component of the experimental setup. This section will give a brief overview of the most used designs and touch on the reasoning behind each package choice. The sensor package used in the small test cell (discussed in Section \$3.4.1) included an integrated heater and temperature monitoring element imbedded within the device package. This design is inherently different and is discussed at the end of this section. 
To ruggedize the graphenated samples against handling and testing, the samples were anchored to a sensor package platform. Electrical contact was made from the sample to the package via ultrasonic wire bonding (described in Section \$3.3.2). Electrical contact was then made from the sample package to the electrical monitoring equipment (a Keithley 4200 SCS) via triaxial cables. This was done for practical purposes and was a necessary step to both mechanically isolate the sample and to reliably mount the sample within the testing platforms.

Figure 3.42 shows one of the first high temperature sensor packages used in the large test bed (the large test bed is detailed in Section $\$ 3.4 .1$ ) termed here as the MK I design. Two pyrolytic boron nitride (PBN, a high temperature ceramic) wafers with dimensions of $12.0 \mathrm{~mm} \times 15.0 \mathrm{~mm}$ x $0.50 \mathrm{~mm}$ had four $1.60 \mathrm{~mm}$ ID holes machined at each corner, $2.40 \mathrm{~mm}$ from the edges of the wafer. The pair of PBN wafers were sandwiched around the ceramic rod that housed the electrical leads (Figure 3.42 A, B).

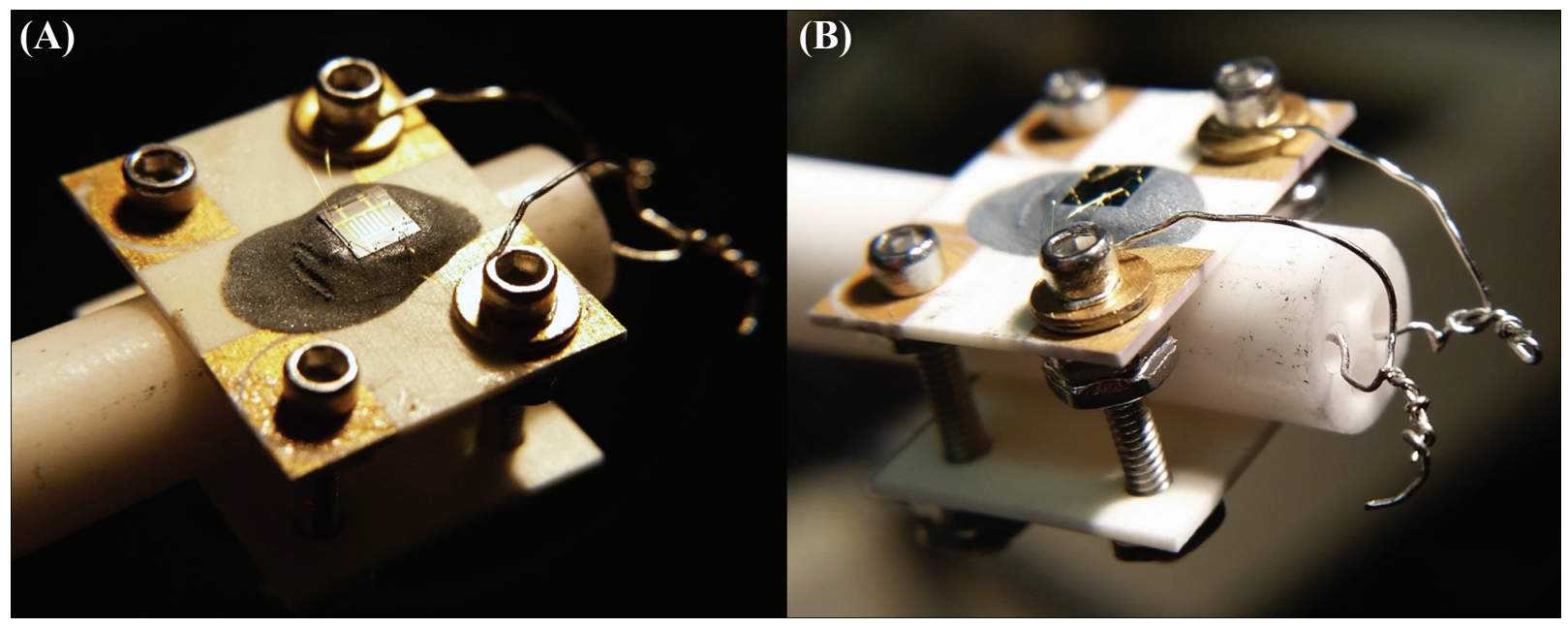

Figure 3.42: (A) The MK I high temperature sensor package used in large test bed experimental setup. The graphinated sample and wire bonds can be seen. (B) The sensor package was mounted to the end of a twohole ceramic rod. Electrical contact was made from the package to the testing equipment via platinum wires housed within the rod. The platinum wires from the package were braided to the platinum wires from the rod to ensure intimate electrical contact. NOTE: For scale, the sample is $2.5 \mathrm{~mm}^{2}$.

A bolt-washer-nut-nut assembly was mounted through two adjacent holes (seen in technical detail in Figure 3.43 D). The double nut arrangement was used to prevent loosening due to the cyclical thermal expansion experienced by the components over the course of testing. The washers were coated with $200 \mathrm{~nm}$ of nickel and $600 \mathrm{~nm}$ of gold via e-beam evaporation. A platinum wire was sandwiched between the bolt head and the washer. The sensors were cemented to the PBN using Caramabond 890 (Aremco Products, Inc.), a high temperature cement. Using ultrasonic bonding, gold wire bonds were jumpered between the sensor's contacts and the Ni-Au 
coated washers (Figure 3.43(A)). Alternatively, the gold wire bonds were jumpered between the sensors contacts and to Ti-Au pads, which were evaporated onto the PBN wafer (as seen in Figure 3.42). The Pt wires from the sensor platform were braided with the Pt wires from the end of the ceramic rod (Figure 3.43 (B)). For size scale reference, the graphene samples in Figure 3.42 and Figure 3.43 are $2.5 \mathrm{~mm}^{2}$. The assembly was tedious and the wire bond to the platform was mechanically unreliable. Additionally, the stainless-steel components were not chemically stable at elevated temperatures. The effect of this was a redeposition of material evolved from the stainless-steel components onto the sample. For these reasons, this design was not used for testing above $\sim 500^{\circ} \mathrm{C}$.

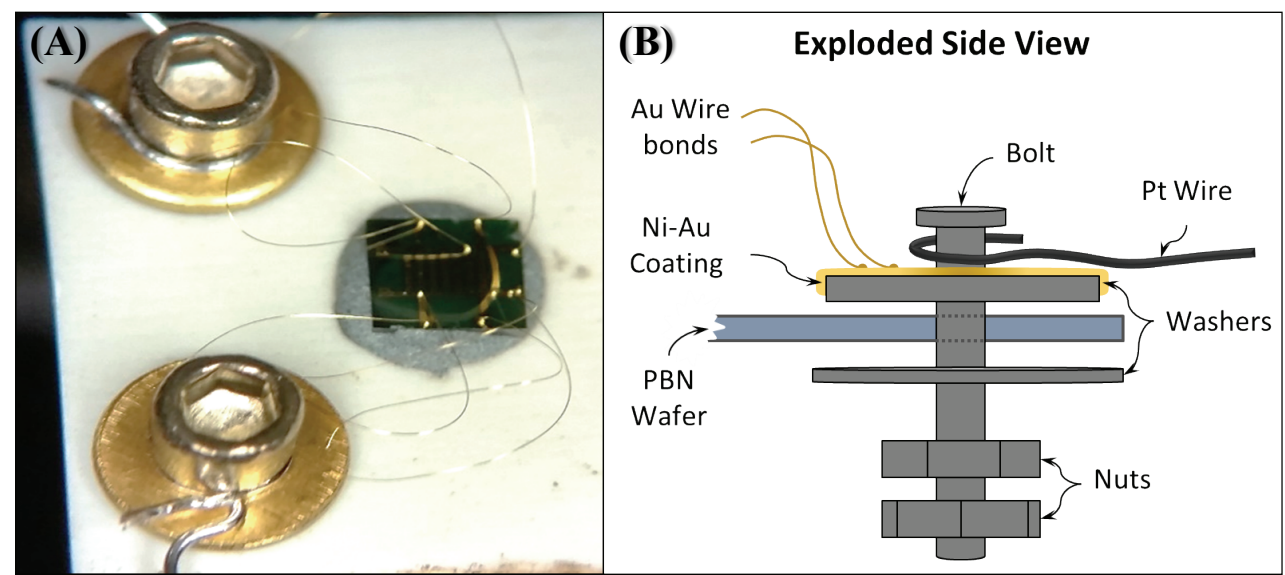

Figure 3.43: (A) A close up view of the MK I high temperature packaging showing the wire bonds from the sample to the package. In this instance, the bonds were made to Ni/Au coated stainless steel washers. (B) A schematic showing how the coated washers were held in place and how the package was anchored to the ceramic rod. NOTE: For scale, the sample is $2.5 \mathrm{~mm}^{2}$.

The next iteration of the high temperature sensor package, the MK II design, can be seen in Figure 3.45. The MK II design eliminated the costly PBN and the use of stainless steel. Both the wires from the sensor package and the wires within the ceramic rod were upgraded to Ni wires due to hydrogen embrittlement of the Pt wires, which was observed to occur at high temperature. To compensate for the higher resistivity of Ni compared to $\mathrm{Pt}$, the wire diameter was tripled. When used in conjunction with a four holed ceramic rod, the MK II design could accommodate two samples at a time in back-to-back arrangement. This can be seen in Figure 3.45(A,B). Platinum wires were flattened on one end in a hydraulic press to provide a smooth and flat surface for wire bonding. The partly flattened wires were then coated with a bilayer of Ti-Au via e-beam evaporation. The wire was then sandwiched between two differently sized insulating substrates and bonded together using high temperature cement. This arrangement provided mechanical 
isolation between the un-flattened ends (which were wound around the electrical leads from the ceramic rod) and the flattened ends. Ultrasonic wire bonds were made between the sample and the flattened and coated wires (seen in Figure 3.45(C))

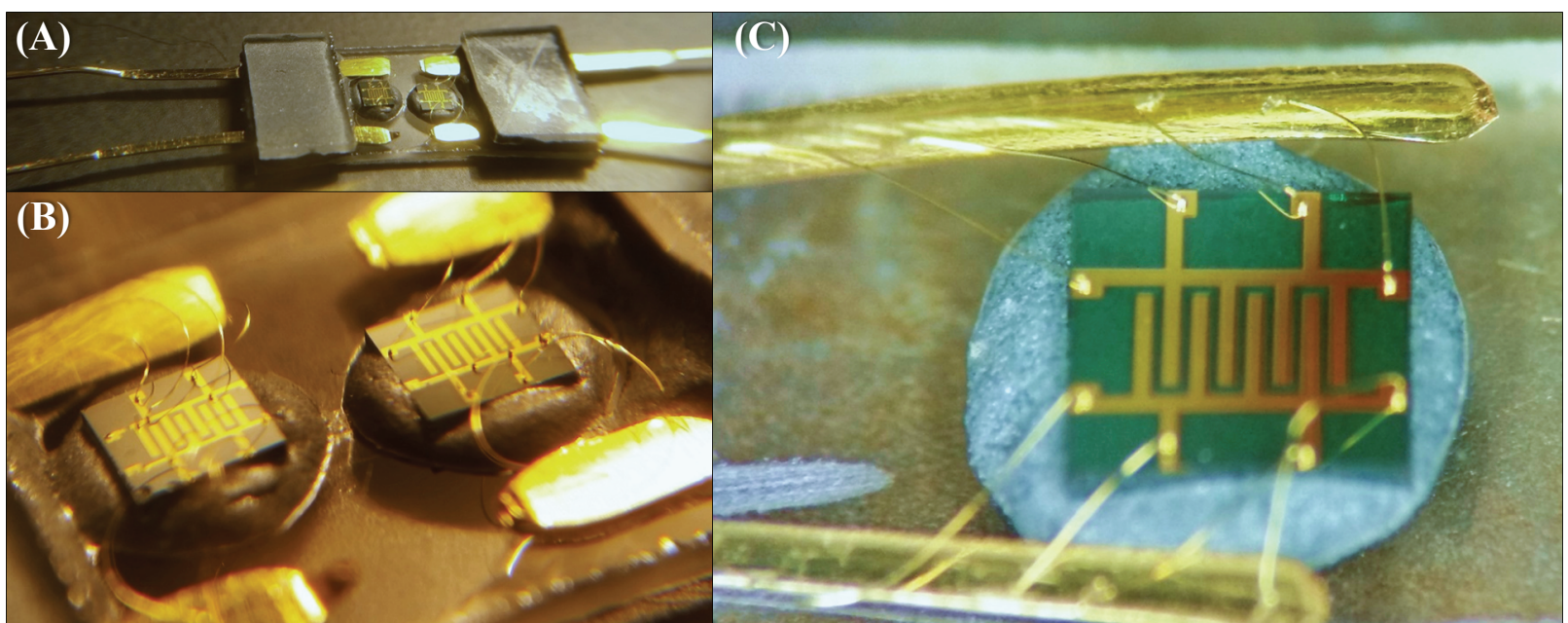

Figure 3.44: (A) A back-to-back duel sensor arrangement version of the MK II design. (B) A magnified view of two sensors mounted on the same package for simultaneous testing. (C) A magnified view showing the bonding between the sensor and the flattened and coated package wires. NOTE: For scale, the sample is $2.5 \mathrm{~mm}^{2}$.

Initially, cost-effective borosilicate glass was used as a substrate. This was later upgraded to quartz due to borosilicate's comparatively low softening temperature which resulted in package degradation at high temperature. High temperatures caused warping of the substrate, placing stress
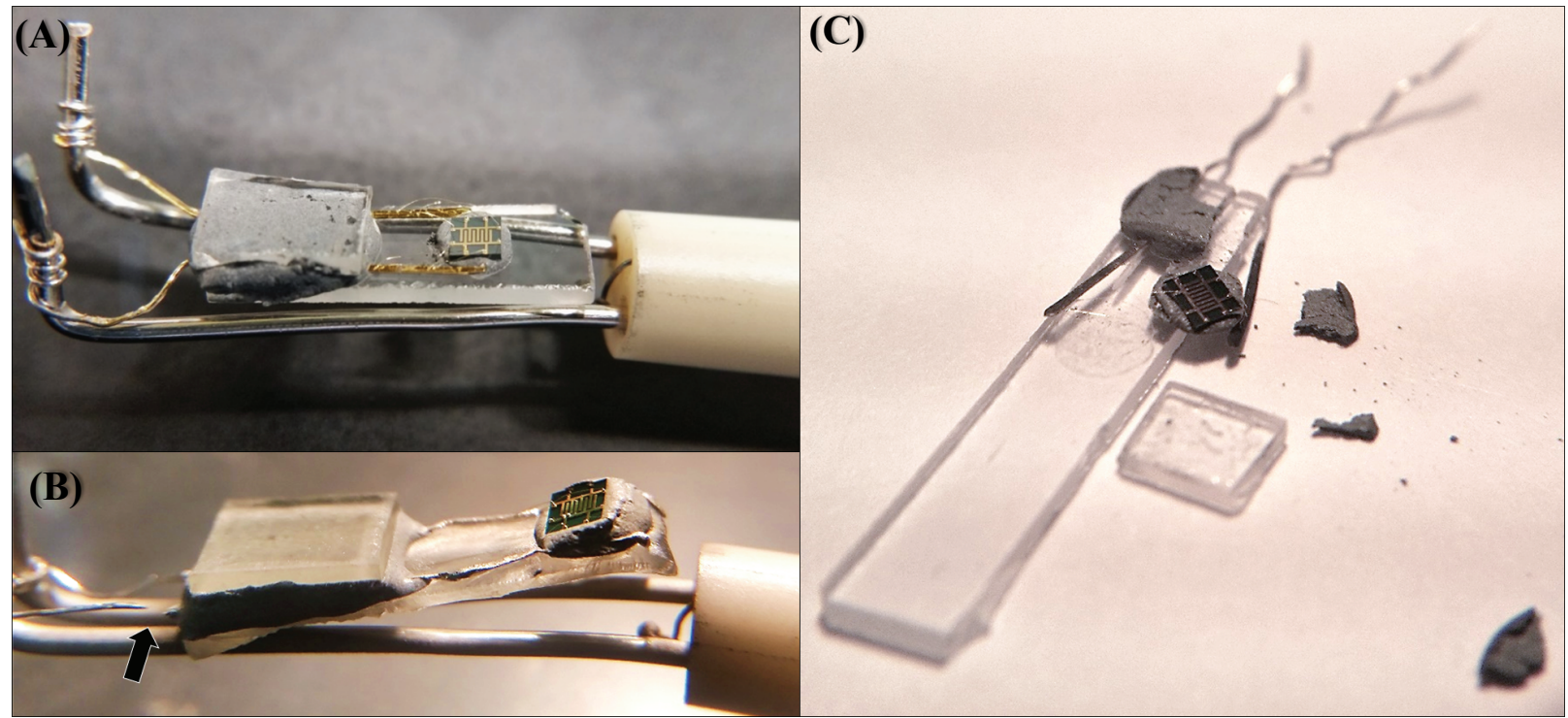

Figure 3.45: (A) A MK II package design which had only one sample. This particular package uses borosilicate glass. (B) The effect of high temperature exposure on the glass; this led to warping of the substrate and bond failure (arrow). (C) A failed MK II package design using quartz; the high temperature cement bonded poorly to the quartz leading ultimately to package failure. NOTE: For scale, the sample is $2.5 \mathrm{~mm}^{2}$. 
on the fragile gold wire bonds and Pt leads which then failed. This can be seen in Figure 3.44. However, the use of the quartz substrate resulted in poor adhesion of the high temperature cement to the quartz substrate, leading to package failure at high temperature. This can be seen in Figure $3.44(C)$.

A version of the last design, the MK III, can be seen in Figure 3.46. The MK III used a short section of a four-hole alumina $\left(\mathrm{Al}_{2} \mathrm{O}_{3}\right)$ rod as the substrate. Two sides of the rod were filed down so that a flat surface was presented. Gold coated, flattened Ni wires were fed through the holes of the ceramic and bent back $180^{\circ}$ and made to lay on the filed flat surface of the rod. The sample was mounted with high temperature cement to the flat area of the rod. Ultrasonic wire bonds were made from the sample to the coated, flattened wires. The holes were pumped full of cement and cured. This mechanically isolated and stabilized the wires and prevented the wires from rotating within the holes (which would cause the wire bonds to detach). The sensor package (consisting of ceramic rod and Au coated flattened wire) were joined to the ceramic rod which housed the Ni wires which lead to the testing equipment. This was done by fitting the Ni wire prongs from the rod into the holes of the rod section which made up the sensor package. Electrical contact was made through the friction-fit formed within the hole between the Ni wires from the rod and the Ni wires from the sensor package, as seen in (Figure $3.46(C)$ ).

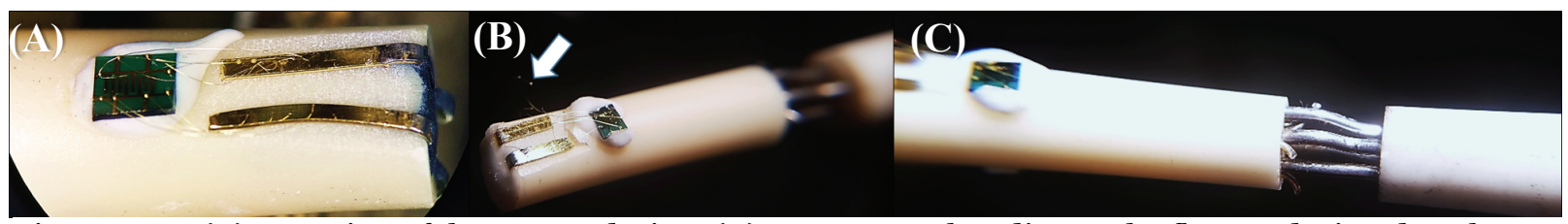

Figure 3.46: (A) A version of the MK III design. (B) Due to poor bonding to the flattened wire, detachment of the ultrasonic bonds can be seen (arrow). (C) The connection of the package to the ceramic rod is shown. The prongs from the rod are inserted into the holes of the package which contain the flattened package wires. In this way, electrical contact is achieved. NOTE: For scale, the sample is $2.5 \mathrm{~mm}^{2}$.

The first version of the MK III design had the flattened and coated Ni wire acting as the bonding surface through which electrical contact between the sensor and the package was made. However, this did not prove to be a reliable method as can be seen from the detached wire bonds in Figure 3.46(B). A unique method was devised for making electrical contact between the sensor and the package. First, the package wire was bent back into a hook shape rather than flattened and coated. Then an ultrasonic wire ball bond was formed on the sample. The gold thread was allowed to unspool as it was wrapped around the Ni wire hook. After several loops around the Ni hook, the 
gold thread was brought back to the sample and terminated as a crescent bond on the sample surface. In this way a rugged and redundant link was made. This can be seen in Figure 3.47.

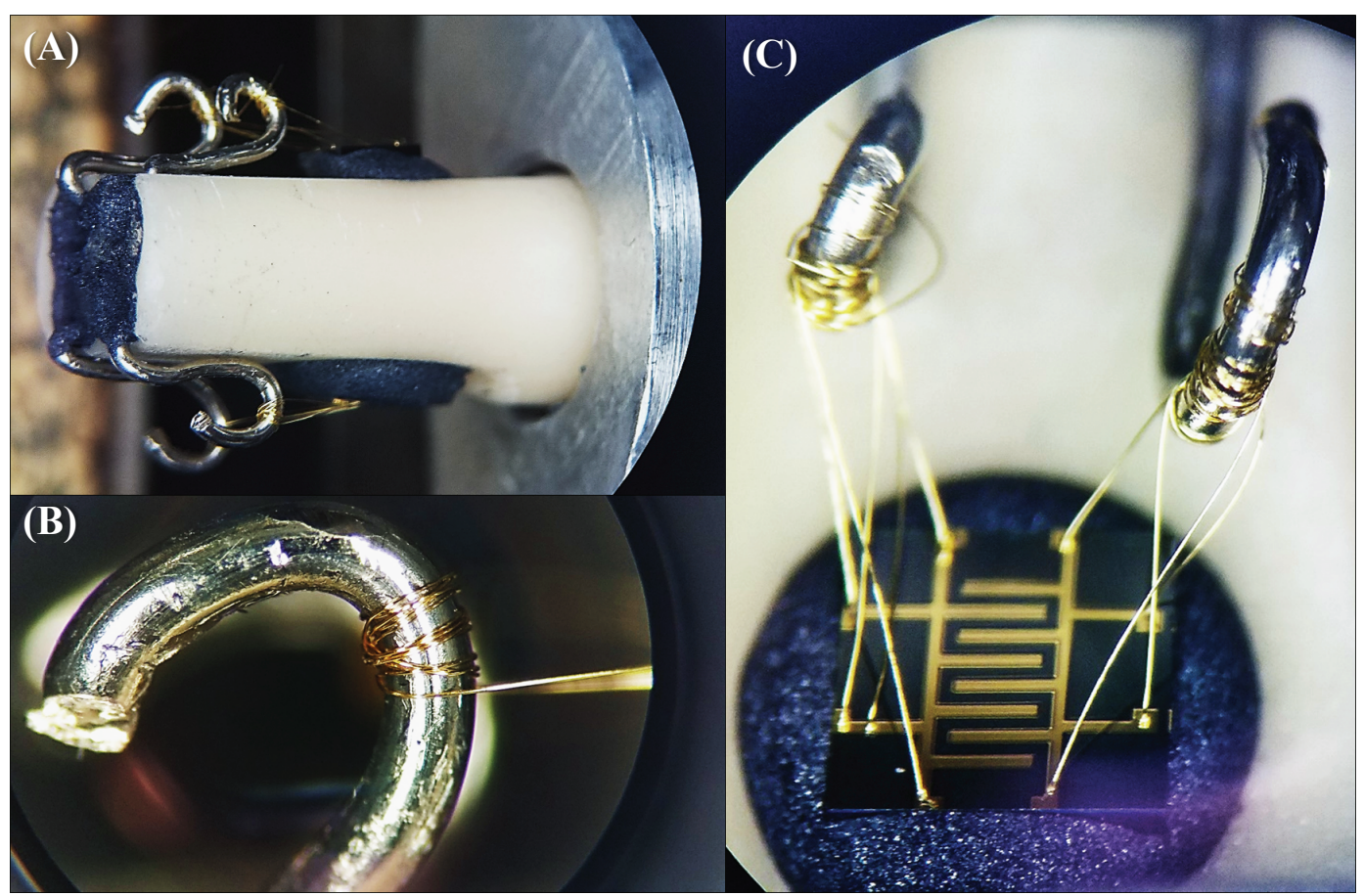

Figure 3.47: (A) The modified MK III package design with improved electrical connection. (B) A magnified view of the connection between the Ni package wire and the gold thread. (C) Magnified view of the sample and hook connections. The sample is mounted with high temperature cement to the flattened side of the ceramic rod. NOTE: For scale, the sample is $2.5 \mathrm{~mm}^{2}$.

The main drawback to the method was the lack of slack in the wire bond connection, making the bond more susceptible to breakage due to movement at the Ni wire. Although more challenging than the MK II design, the newer design still allows for the mounting of two samples, as seen in Figure 3.47 (A).

Gas testing in the small test cell setup used a sample packaging that was inherently different from the design used in the large test bed setup. This is referred to as the MK0 design. A complete device can be seen in Figure 3.48. 


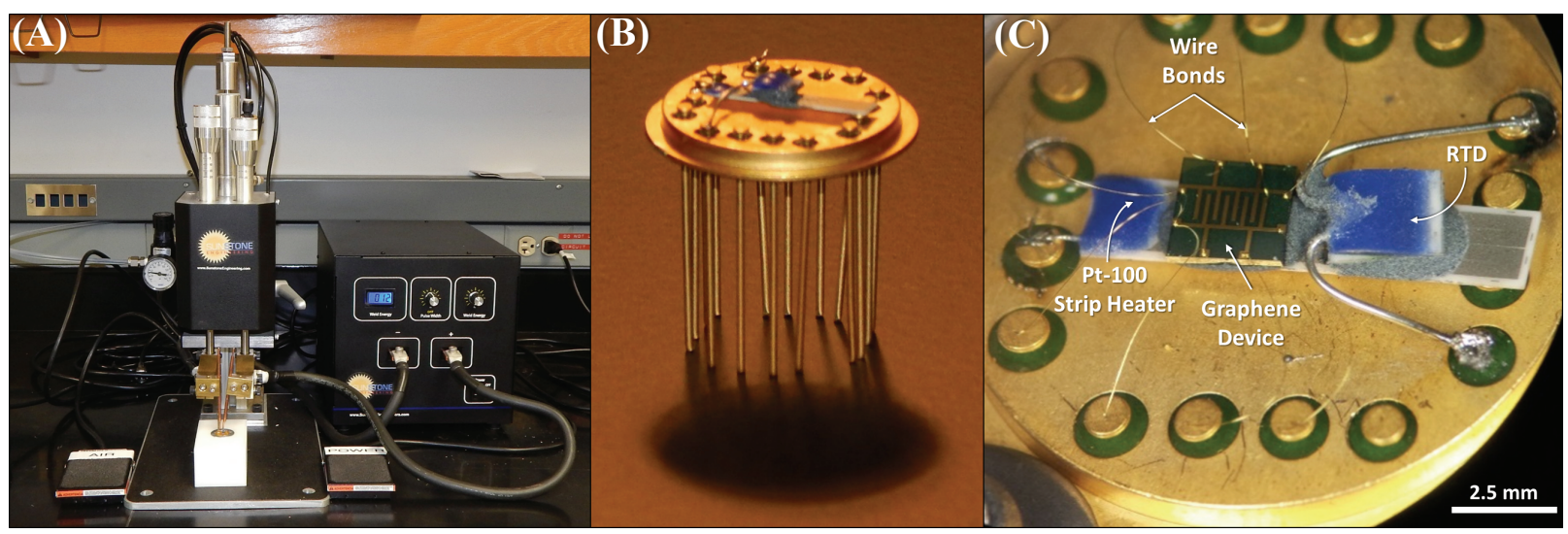

Figure 3.48: (A) The CD20oSPM Micro Spot Resistance Welder used to spot weld the RTD and heater to the TO pins. (B) The TO body with RTD and heater element attached. (C) Magnified view of a completed device package.

The devices used in the small test cell had a heat source and temperature monitoring element integrated into the sample packaging. The temperature of the test device was controlled with a M1020 platinum strip heater and monitored by a M222 resistance temperature detector (RTD) both provided by Harlem Sensor Technology USA. Using a CD200SPM Micro Spot Resistance Welder from Sunstone Engineering, these package elements were spot welded to a small ( 0.6" diameter) transistor outline (TO) header (HDR00823) provided by Spectrum Semiconductor Materials. The RTD and graphinated sample was attached to the heater using high temperature cement (Caramabond 890 from Aremco Products, Inc.). Electrical contact to the sensor was made through wire bonds from the device to the output pins of the TO header. The heater was driven by a manually controlled, variable DC power supply, while the RTD was read using an Omega Engineering RDXL4SD digital thermometer. 
Figure 3.49 shows one of the first device package designs and was used in initial open-air testing of the graphenated samples. Figure 3.49 shows a device in open air and operating at $\sim 800^{\circ} \mathrm{C}$. The device and packaging maintain integrity for extended time at high temperature in an open-air environment. These images show what the package would look like once inside the small test cell with the heater powered up.

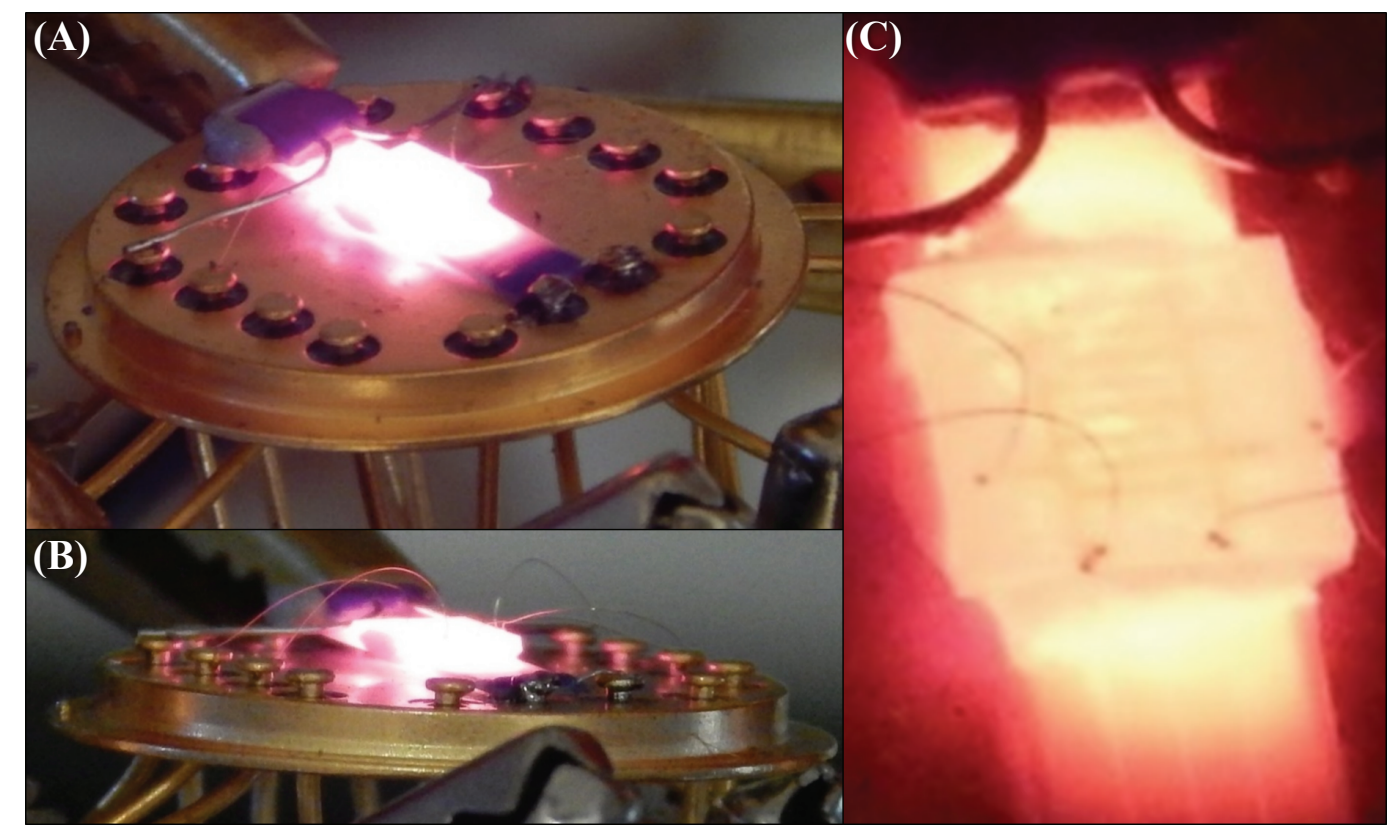

Figure 3.49: (A) Thermal stability test of a completed device for use in the small gas test cell setup. This device is pictured operating at $\sim 800{ }^{\circ} \mathrm{C}$. (B) The same device from a different angle, showing the wire bonds remain intact at high temperature. (C) Magnified view of the device showing the graphinated sample. Both the wire bonds and the deposited Ti/Au contacts can be seen.

For room temperature gas testing in the small test cell, a simplified version of this packaging design was used. The simplified version did not have a heating element or RTD; the graphinated sample was mounted to the TO header via double sided Kapton tape. Both versions were used in the small gas test cell. This package design is referred to as MK0* design and is shown in Figure 3.50 . 


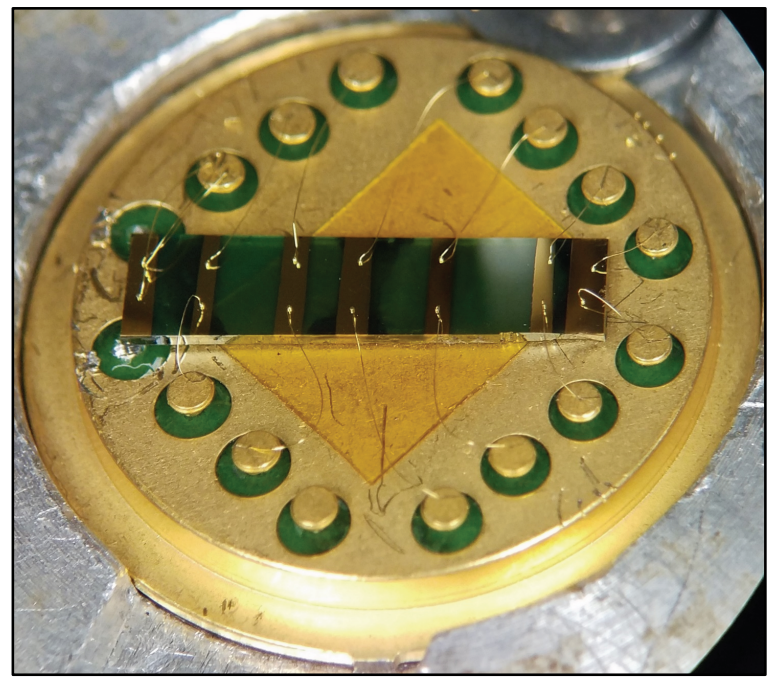

Figure 3.50: The MKo* package design. This is a simplified version of the MKo design and does not included the heating element and RTD. This design used in the small test cell and was useful for the rapid fabrication of devices for simple testing.

\section{§3.4 Experimental Setup for Gas Sensing}

There were two gas sensing test setups: a self-contained, "Small Test Cell" and a larger, "Large Test Bed." This section will describe the experimental setup of both. The sensor packaging is discussed in Section $\$ 3.3$. Both setups were part of the same gas flow system, as shown in the simplified schematic in Figure 3.51. The system used 1/4 inch 316 stainless steel lines and a combination of Swagelok ferruled fittings and Ultra-Torr compression fittings for all connections.

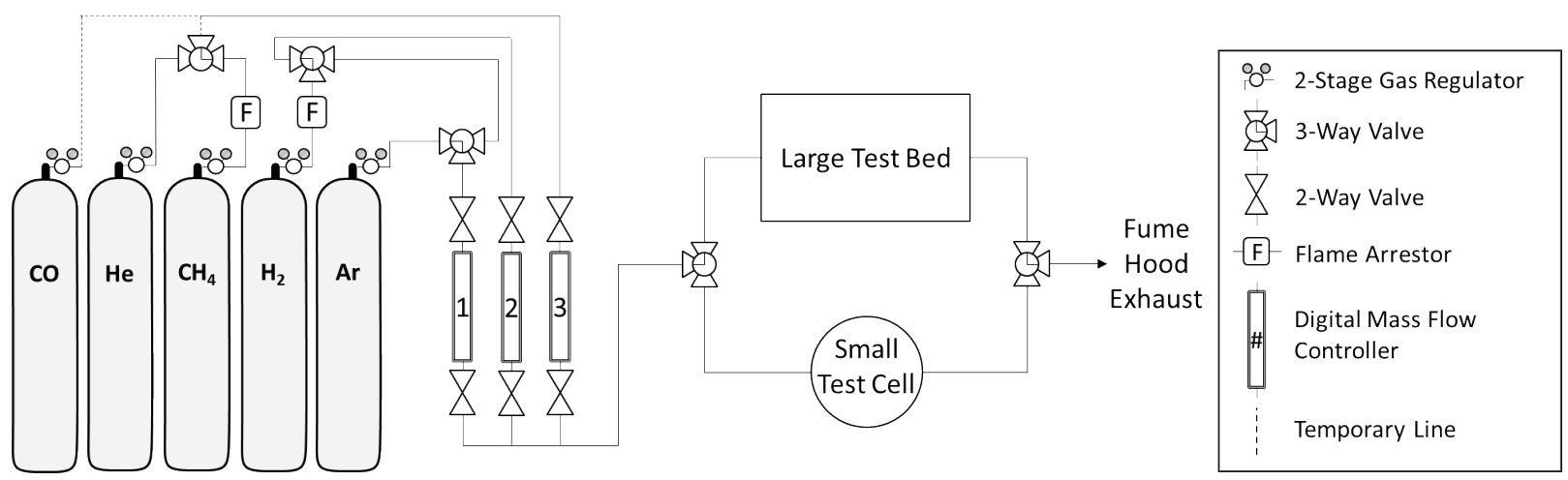

Figure 3.51: A simplified schematic of the gas flow system. Both the large test bed and the small test cell were part of the same system. Other aspects of the system involving the RTA system and alternative arrangements of the large test bed are not shown.

The gas flow system pictured was the most commonly used arrangement. However, the actual plumbing was considerably more complex as to allow for two separate RTA systems, which 
were also part of the same flow system. Additionally, several different gas flow arrangements were used with the large test bed but are not depicted here.

For gas testing a three-line gas manifold was used to control the gas flow to test setup. The gas flows were controlled by an MKS model 247C digital flow controller which allowed independent control over each gas line. The three mass flow controllers used had capacities of 500 SCCM, 20 SCCM and 10 SCCM. Throughout this work several gases were used; argon (Ar), helium $(\mathrm{He})$, carbon monoxide $(\mathrm{CO})$, hydrogen $\left(\mathrm{H}_{2}\right)$ and methane $\left(\mathrm{CH}_{4}\right)$. However, only three were used at any one time. Electrical readings from the sensor were monitored by a Keithley 4200 semiconductor characterization system (SCS). For both setups, gas tests consisted of varying the gas composition and flowrates while simultaneously monitoring the electrical characteristics of the installed device.

\section{§3.4.1 Large Test Bed Setup}

The large test bed, shown schematically in Figure 3.52(A), consisted of a bench top hightemperature furnace (a $1600 \mathrm{C} \& \mathrm{M}$ High Temperature Furnace) with a 1.5-inch quartz liner that served as the gas test chamber inside the furnace. The sensor package was mounted on the end of
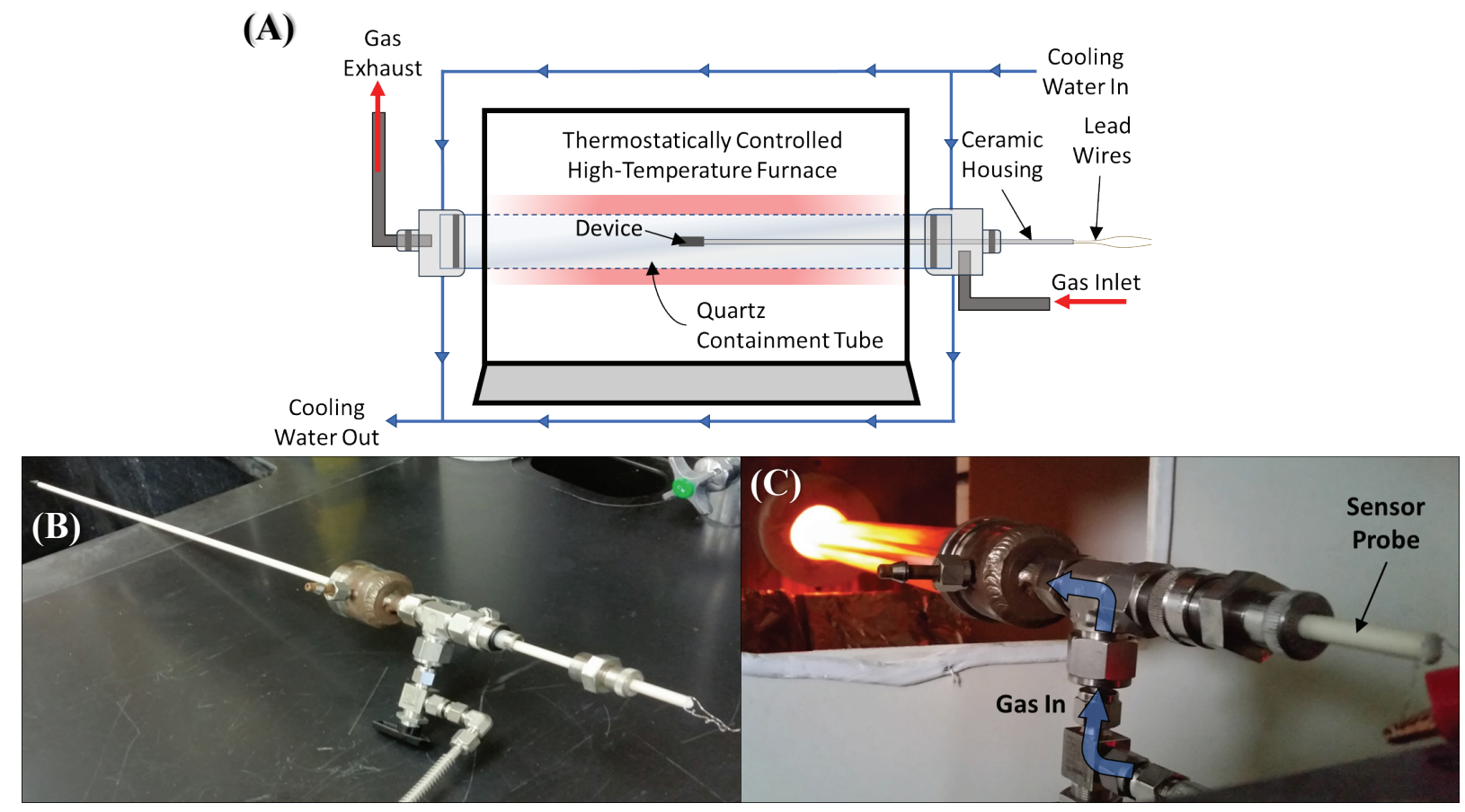

Figure 3.52: A) A simple schematic of the large test bed setup. This design preheats the incoming gasses and maintains the entire test device at the desired temperature. B) The sensor probe and gas inlet assembly prior to insertion. (C) The complete sensor probe assembly fully inserted into the furnace at temperature. The cooling line is not assembled in this picture. 
a 36 inch four-hole alumina $\left(\mathrm{Al}_{2} \mathrm{O}_{3}\right)$ rod seen in Figure 3.52(A,B). The rod was fitted through two legs of a $1 / 2$ inch Swagelok union tee. On the tube side, the fitting was sealed with a custom welded, water jacketed, 1.5-inch Ultra-Torr Swagelok compression fitting to $1 / 2$ inch Swagelok fitting. On the lab side, the rod was sealed with a modified $1 / 2$ inch to $1 / 4$ inch Ultra-Torr Swagelok compression fitting to seal against the laboratory air.

The rod was positioned within the fitting such that the sensor package was placed at the center of the furnace hot zone. The ceramic rod housed two or four Pt or Ni test leads, or two thermocouple wires. The rod provided physical protection and electrical isolation of the leads. The rod holes were filled with Varian Torr Seal (\#9530001) two-part epoxy to provide a gas tight seal between the laboratory and the test chamber. On the furnace-side of the rod, the leads made electrical contact to the sensor package and on the lab-side, the leads wires were connected to Keithley 4200 semiconductor characterization system (SCS). For experiments which used only two wires for electrical contact to the sample, a chromel-alumel type- $K$ thermocouple was fabricated and installed in the rod to monitor the temperature at the sensor. The exhaust side of the tube was sealed with a second custom welded, water jacketed, 1.5-inch Ultra-Torr Swagelok compression fitting to $1 / 2$ inch Swagelok fitting.

For simple two-point electrical measurements, each test setup (sample package and experimental setup) adds a series resistance to the circuit, which is convoluted into the device data. The triaxial test leads, which connects the experimental setup to the SCS-4200 electrical measurement equipment, is common to all designs and contribute approximately $1.4 \Omega$ to the total circuit resistance. This contribution is constant for all package designs and for all device temperatures. The wire leads contained within the ceramic rod used in the large test bet setup (and used the MK I, MK II and MK III package designs) are exposed to the heater. Therefore, their contribution to the circuit resistance changes as a function of temperature due to their ohmic behavior. Figure 3.53(A) plots the resistance of the circuit (without a device or sample packaging) of the large test bed setup as a function of temperature. A simple schematic of the setup in shown in Figure 3.53(B). However, in general the contributions are small compared to the device resistance, which dominates. 
(A)

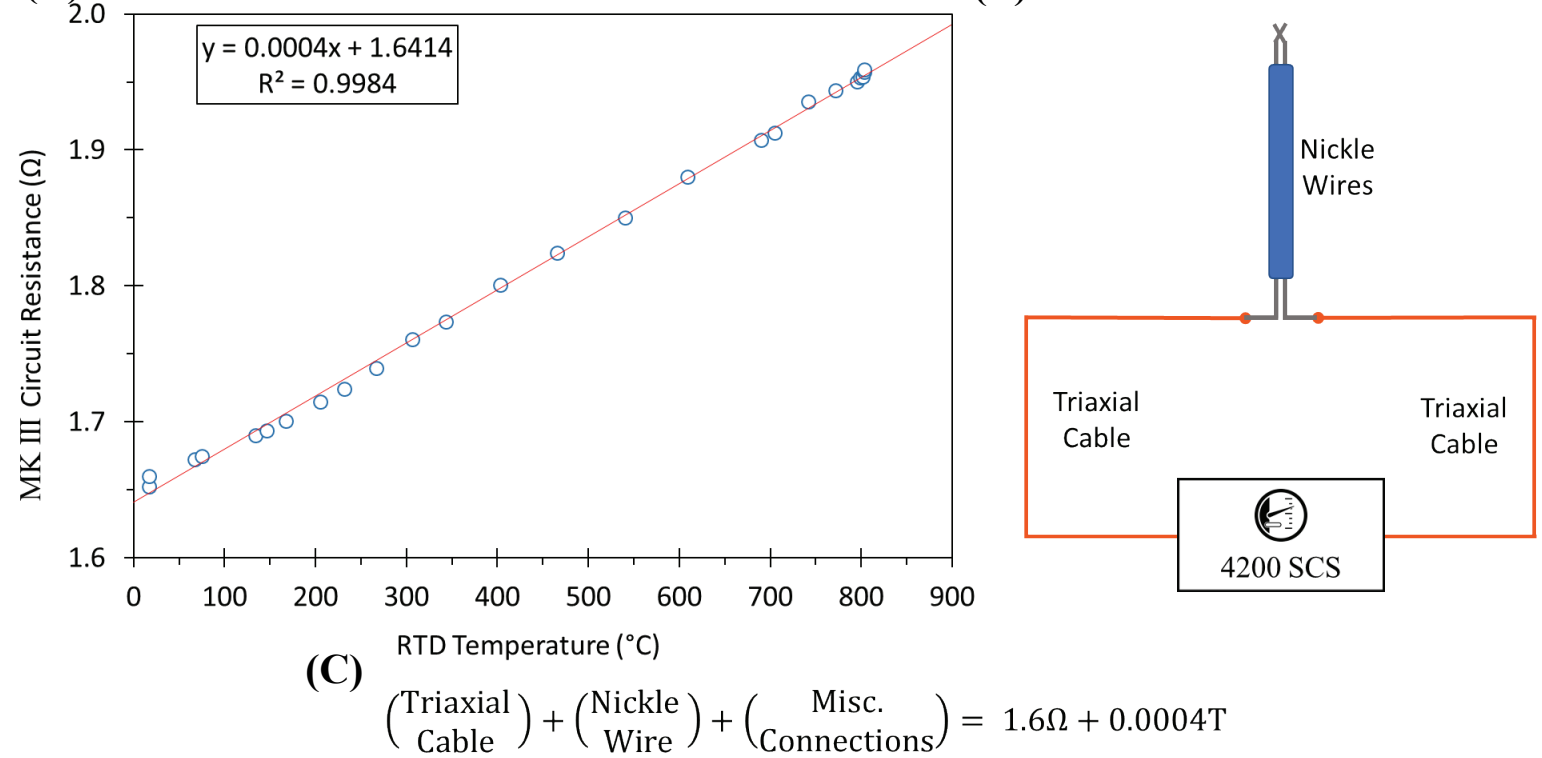

Figure 3.53: (A) Increase in the series resistance of the MK II design as a function of temperature. (B) Simple schematic of the circuit. (C) Effective resistance as a result of the various contributors to the series resistance in the MK I - MK III designs. Note that this does not including the actual device package, wire bonds, or $\mathrm{Ti} / \mathrm{Au}$ contact trace. These unaccounted-for contributions are estimated to be on the order of $\sim 1 \Omega$ at room temperature.

\section{§3.4.2 Small Test Cell Setup}

The small test cell, seen in Figure 3.54, was designed for device testing from room temperature up to $\sim 700^{\circ} \mathrm{C}$. The cell's small volume allowed for quick gas turnover times and rapid gas pulsing. The cell itself was a 0.5 " Swagelok union Tee fitting mounted in line with the gas flow system. From the gas manifold assembly, the test gases were introduced by way of the bottom leg of the tee, where the gas line expanded into the 0.5 " Swagelok Tee fitting. 


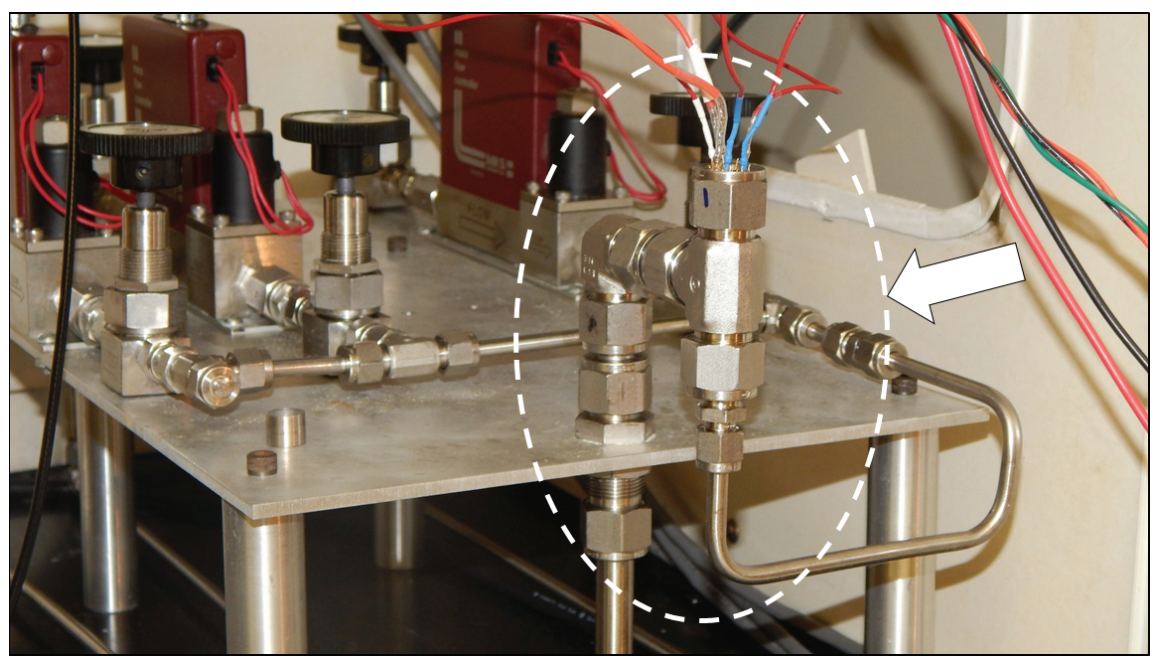

Figure 3.54: The small test cell, as indicated by the arrow and circle annotation, with an installed sensor device package. The friction fitted saber type connectors can be seen at the top of the test cell. In the background, some of the plumbing and mass flow controls can be seen.

The packaged device was mounted on the top leg of the tee and sealed against the laboratory air via a modified Swagelok ferrule fitting when mated against the surface of the TO header lip. The plumbing was designed such that gas flowed into the tee and impinged directly onto the device, and then is exhausted out of the remaining leg. A close-up view of the small test cell and illustration of an installed device is shown in Figure 3.55. Friction fitted saber type connections were made to the TO heater pins, external to the test chamber. From these test leads, contact was made with the Keithley 4200 test unit.

Although the small test cell allowed for rapid sample testing and a simple sensor assembly, there were several limitations inherent in the design. Constant temperature control was difficult to maintain due to limitations of the small strip heater and the inability to rapidly track the changes in thermal characteristics of the test environment during gas pulse tests. Rapid changes in gas composition and flowrate overwhelmed the heater and resulted in large temperature fluctuations of the test chamber. However, the small test cell was ideal for room temperature tests and moderately high temperature tests. The large test bed filled the equipment gap for very high temperature tests. 

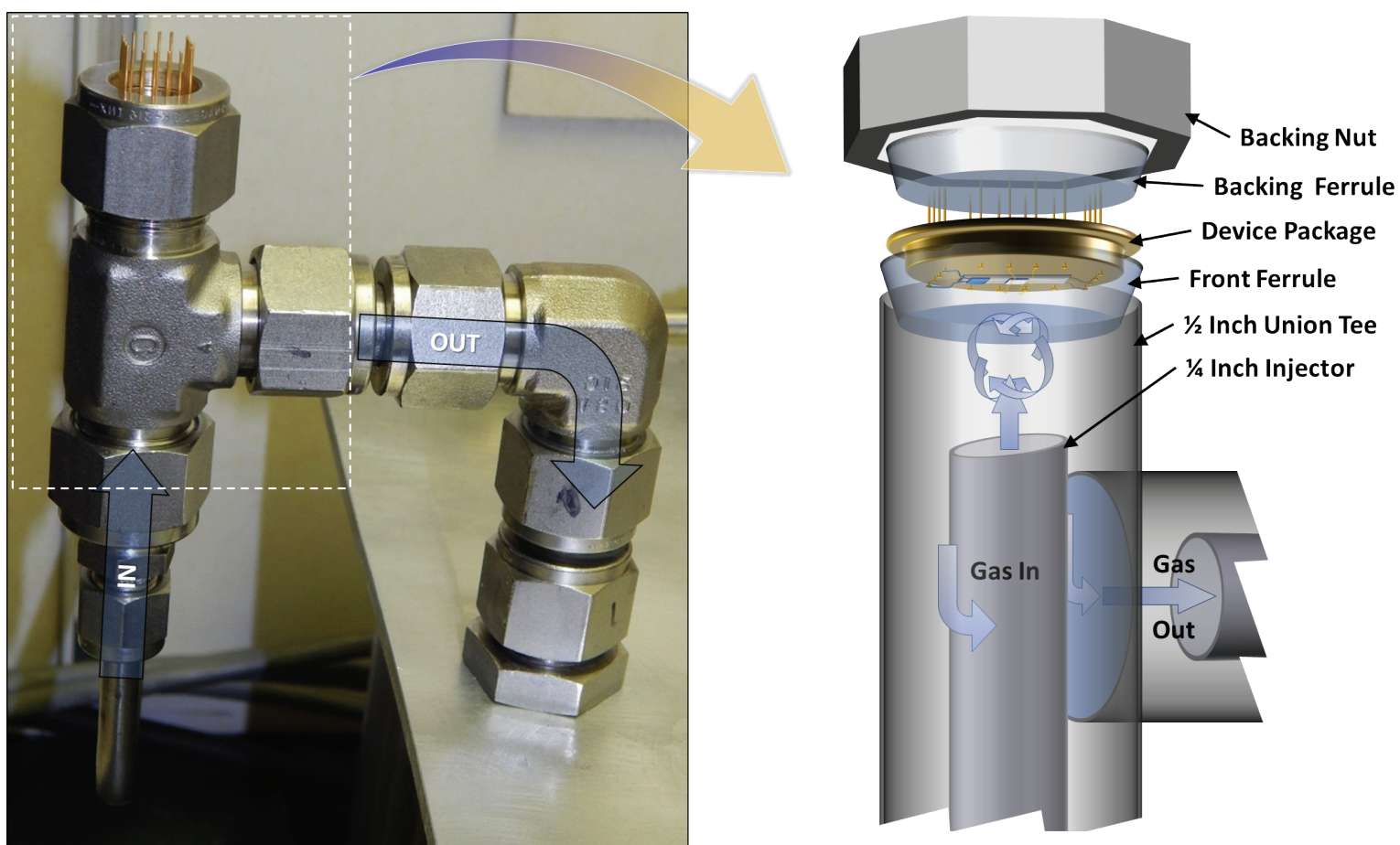

Figure 3.55: A closeup of the small test cell with accompanying exploded view schematic, showing the gas flow pattern and how the device is installed within the cell. Note that the $1 / 4$ inch line protrudes past the exhaust port so that incoming gas mixes and impinges direction on to the sensor before it is exhausted.

As with the large test bed setup (using MKI-III package designs), the device packaging of the MK0 design also contributes to the series resistance of two-point measurements. However, unlike the previous designs, the localized heating of the MK0 design limits effect of temperature on these contributions. Figure 3.56 shows the contributions of the sample packaging of the MK0 design to the overall resistance of the device. This data convolutes the contributions of the triaxial cables, TO post feed throughs, gold wire bonds, Ti/Au contact pads and all interconnects. At only $2.1 \Omega$, the contribution of the MK0 packaging in the small test cell is small. Although this value is large than the series resistance given for the large test bed, note that unlike the data shown in Figure 3.53, this value includes the Ti/Au contact trace and wire bonds. Overall, the small test cell a smaller series resistance than does the large test bed. 


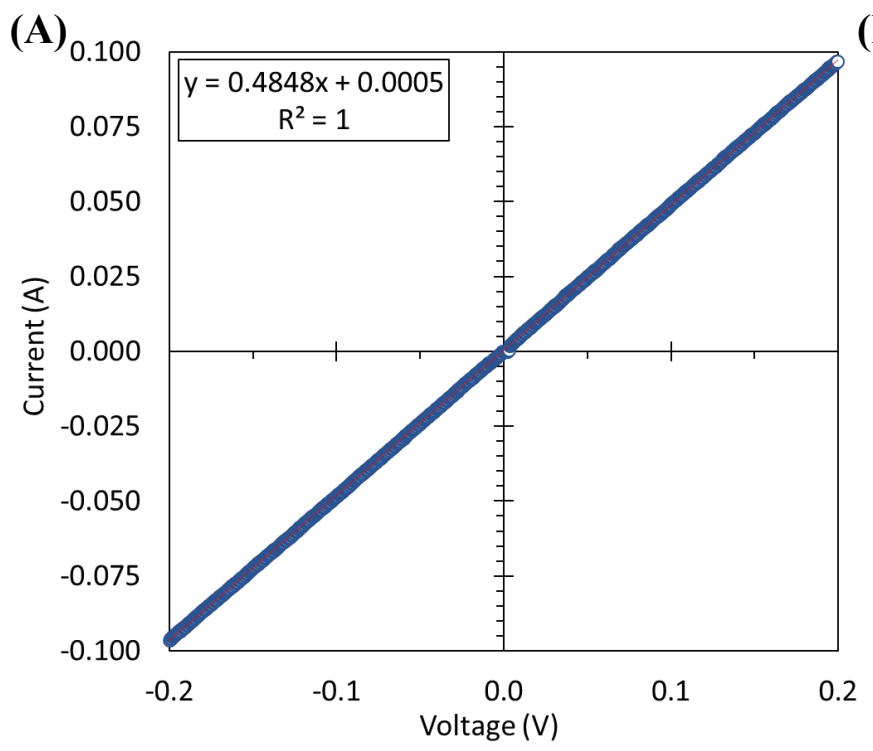

(B)

(C)

$$
\left(\begin{array}{c}
\text { Triaxial } \\
\text { Cable }
\end{array}\right)+\left(\begin{array}{c}
\text { TO Post } \\
\text { Feedthrough }
\end{array}\right)+\left(\begin{array}{c}
\text { Au Wire } \\
\& \text { Bonds }
\end{array}\right)+\left(\begin{array}{c}
\text { Ti/Au } \\
\text { Trace }
\end{array}\right)+\left(\begin{array}{c}
\text { Misc. } \\
\text { Connections }
\end{array}\right)=\sim 2.06 \Omega
$$

Figure 3.56: (A) IV curve collected from the MKo design, not including the film. (B) Simple schematic of the circuit. (C) Effective resistance as a result of the various contributors to the series resistance in the MK I - MK III designs.

The Ti/Au contacts and the wire bonds are heated during high temperature device testing in all test setups. Resistance data collected using the setup shown Figure 3.56(B), indicates that the series resistance contribution change due to heating of the $\mathrm{Au}$ wire bond and $\mathrm{Ti} / \mathrm{Au}$ trace is approximately $10 \%$ over a $650^{\circ} \mathrm{C}$ temperature increase. Because this is due to the Ti/Au contacts and wire bonds, this contribution would be common to all test setups (small test cell, large test bed and all package designs).

\section{§3.5 Experimental Setup for Photo Detection}

The light sensitive setup was a simple scaffold arrangement to control the focus and position of a low power laser spot on the graphinated samples. The sample package used for these tests were the simplified version of the design used in the small gas test cell using the TO header which did not have the integrated heater and RTD. All tests were done in the open laboratory and at room temperature. Figure 3.57 shows the experimental setup and a simplified schematic. 

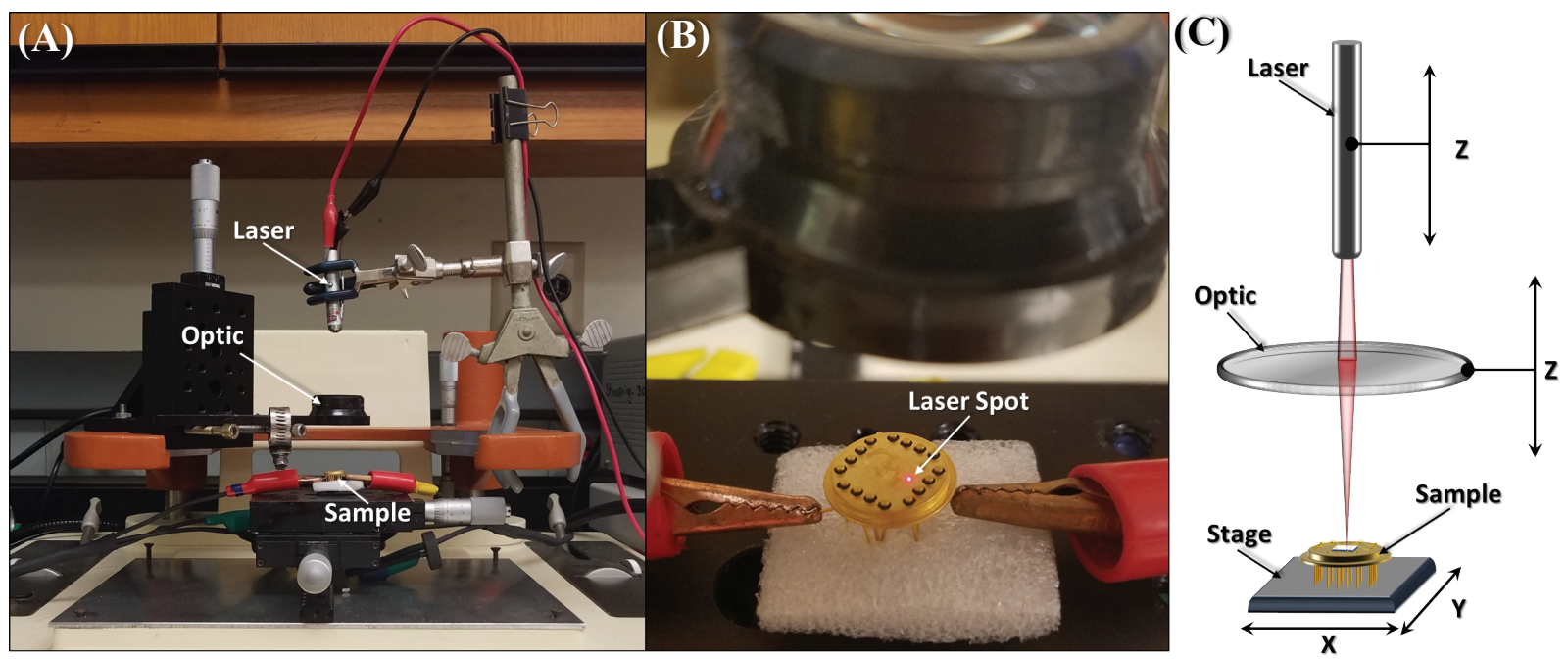

Figure 3.57: (A) The photo detection setup. (B) The laser spot can be seen on an empty sample package. (C) A simplified schematic of the laser setup.

Three consumer-grade, low power $(<5 \mathrm{~mW})$ diode lasers were used: red $(650 \mathrm{~nm} \pm 10)$, green $(532 \mathrm{~nm} \pm 10)$ and purple ( $405 \mathrm{~nm} \pm 10)$. The lasers were modified from their original battery powered design to instead operate on a DC laboratory power supply (a GW Instek GPS-3030DD from Good Will Instrument Co.). A 1.5-inch optic was placed in the beam path to decrease the onsample spot size from $4.5 \mathrm{~mm}$ to $\sim 200 \mu \mathrm{m} \pm 25 \%$. Both the laser and the optic were independently mounted. The elevation (Z-axis) of both could be changed on the fly to maintain a tight focus of the laser spot on the sample at all times. The laser was mounted approximately 8-12 inches from the sample surface. Simple on/off pulse tests were conducted by either energizing/deenergizing the laser or by alternatingly placing an opaque material in/out of the beam path. The latter method did reduce the lifetime of the laser used but did eliminate emission power fluctuation due to the diode laser warm up on each on pulse, which was detectable by the graphenated sensors. The sample was mounted on a manual X-Y stage which allowed for the precise positioning of the sample within the laser beam path. This allowed for an additional type of test where the sensor response was measured as a function of the laser spot position on the sample surface. Response was measured by the same Keithley 4200 electrical characterization equipment as was used in all other studies. 


\section{CHAPTER 4: RESUlts AND Discussion - GRAPHENE SYNTHESIS, PARAMETRIC INVESTIGATION AND HYDROGEN TREATMENT}

The experimental methods and procedure are described in Chapter 3. In Chapter 4, the results for graphene synthesis studies are presented and discussed. Over 300 graphene/SiC samples were produced and their surfaces individual analyzed using AFM, XPS and Raman spectroscopic

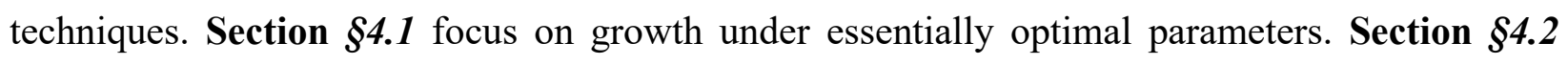
discusses the parametric optimization studies. Section $\$ 4.3$ discusses the effects of post-synthesis high temperature hydrogen treatments on the films.

\section{\$4.1 Graphene Growth Using Standard Operating Procedure}

This section, as noted, presents the results of growth studies at optimal or near optimal conditions. These process parameters were based on previous work by Chaudhari, et al. but have been modified. ${ }^{(525)(263)}$ Films produced in this manner are referred to as standard films produced using the standard operating procedures (SOP). These films constitute the base case. Studies on the effects of various synthesis parameters (detailed in Section $\$ 4.2$ and Section $\$ 4.3$ ) use these standard films as a convenient reference point.
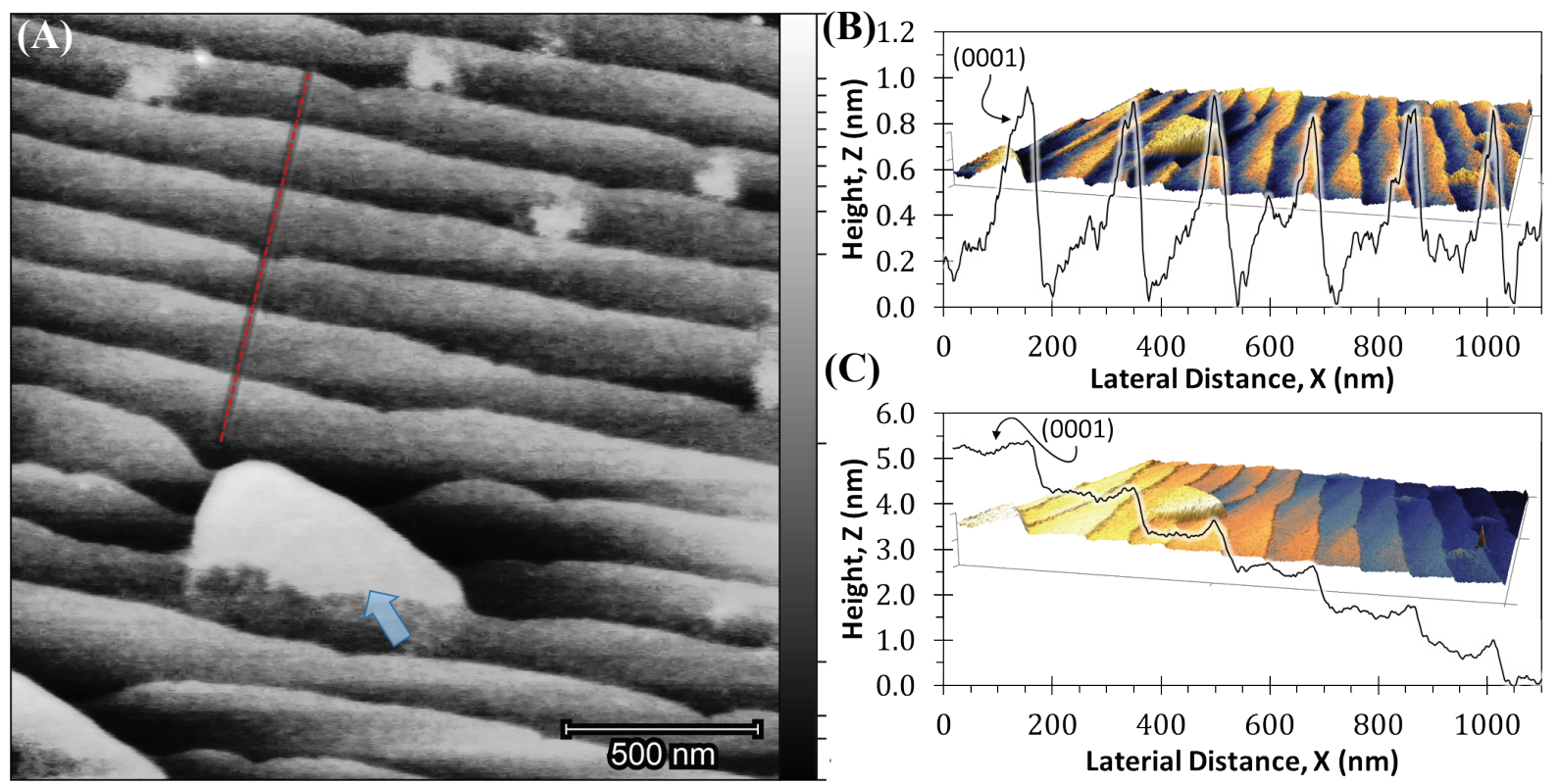

Figure 4.1: AFM analysis on an as-received 6H-SiC wafer. A) The atomic steps can clearly be seen. The red dashed line shows the locations of a line scan across severial atomic steps. A step edge protrusion, marked by the blue arrow, can be seen. B) Height profile for the line scan shown in (A) with plane-leveled 3D projection of (A). C) The same data, but facit-leveld. Using the line scans, a miscut angle of $\sim 0.33^{\circ}$ is calculated. 
For standard films, the graphitization process was performed on the Si-face (0001) of a substrate cut from a chemical/mechanical polished on-axis $6 \mathrm{H}-\mathrm{SiC}(0001) \mathrm{n}$-doped wafer. AFM of the as-received material is shown in Figure 4.1 (A). Analysis shows a clean surface with the atomic steps of the $6 \mathrm{H}-\mathrm{SiC}$ clearly visible. Additionally, the presence of a crystal edge defects is revealed, as indicated by the arrow. Figure 4.1(B,C) plane-leveled and facet-leveled height profiles corresponding to the line scan shown in Figure 4.1 (A) with correspondingly leveled 3D renders.

The analysis shows a step height of $\sim 0.85 \mathrm{~nm}$ indicating some step bunching is occurring (the unit cell height of $6 \mathrm{H}-\mathrm{SiC}$ is $\sim 0.15 \mathrm{~nm}$ ). The measurement shows an off-axis angle of $\sim 0.33^{\circ}$ in this case, which is consistent with the manufacturer reported value. A broader AFM survey of the wafer morphology and defects sites can be found in Appendix $\boldsymbol{\$}$ A.1.1. The step height, terrace width and defect density were found to vary across the surface of the wafer slightly. This is consistent with others have observed in the characterization of both $6 \mathrm{H}-$ and $4 \mathrm{H}-\mathrm{SiC}$ wafers. ${ }^{(526)}$ The as-received 2-inch 6H-SiC wafers were diced and cleaned (as detailed in Section §3.1.2).

Following the standard multistep chamber cleaning and conditioning process (detailed in Section \$3.1.3), the samples were etched using the standard etching parameters detailed in Table 4-1. The effects of varying these parameters are detailed in Section \$4.2. The programed 60 second unpowered rest portion of the etch was included in the recipe for practical purpose. This decrease the duty cycle of the ICP/RIE power supplies and decreased the workload on the chamber and pumping system (i.e., there was no scientific reason specific to the graphitization process). Films produced using an etch recipe without rests steps did not result in markedly different films (see Appendix $\$$ A.1.2 for supporting information).

Table 4-1: Standard etch parameters.

\begin{tabular}{|l|l|}
\hline \multicolumn{1}{|c|}{ Parameter } & \multicolumn{1}{c|}{ Value } \\
\hline Chamber Pressure & $25 \mathrm{mTorr}$ \\
\hline $\mathrm{CF}_{4}$ Flowrate & $20 \mathrm{SCCM}$ \\
\hline ICP Power & $600 \mathrm{~W}$ \\
\hline RIE Power & $300 \mathrm{~W}$ \\
\hline He Cooling Flowrate & $0.0 \mathrm{SCCM}$ \\
\hline Power Cycle & ON: $180 \mathrm{Sec}$ \\
Duration $(4$ cycles) & OFF: $60 \mathrm{Sec}$ \\
\hline Total Etch Time & $12 \mathrm{~min}$ total \\
\hline
\end{tabular}

It was observed that by not using a cooling helium backing flow on the carrier wafer during the etch step (counter to the typical standard practice for ICP/RIE etching), higher quality films 
were produced. In general, this resulted in a thicker graphene film, fewer defects per layer and a lower elemental nitrogen contamination (see Appendix $\boldsymbol{\$} \boldsymbol{A}$.1.3 for supporting information).

The DC bias developed on the sample chuck varied slightly from run to run and was a function of the chamber history (what processes were run and how many) and the elapsed time from the previous manual cleaning of the chamber (where the chamber was physically opened and cleaned by hand). Additionally, as components in the chamber were replaced over time (the gas distribution head, electrostatic chuck and centering ring in particular) the DC bias was affected. Nonetheless, for each run, the DC bias provided a useful measurement of the energy of the incident ions impinging onto the $\mathrm{SiC}$ surface.

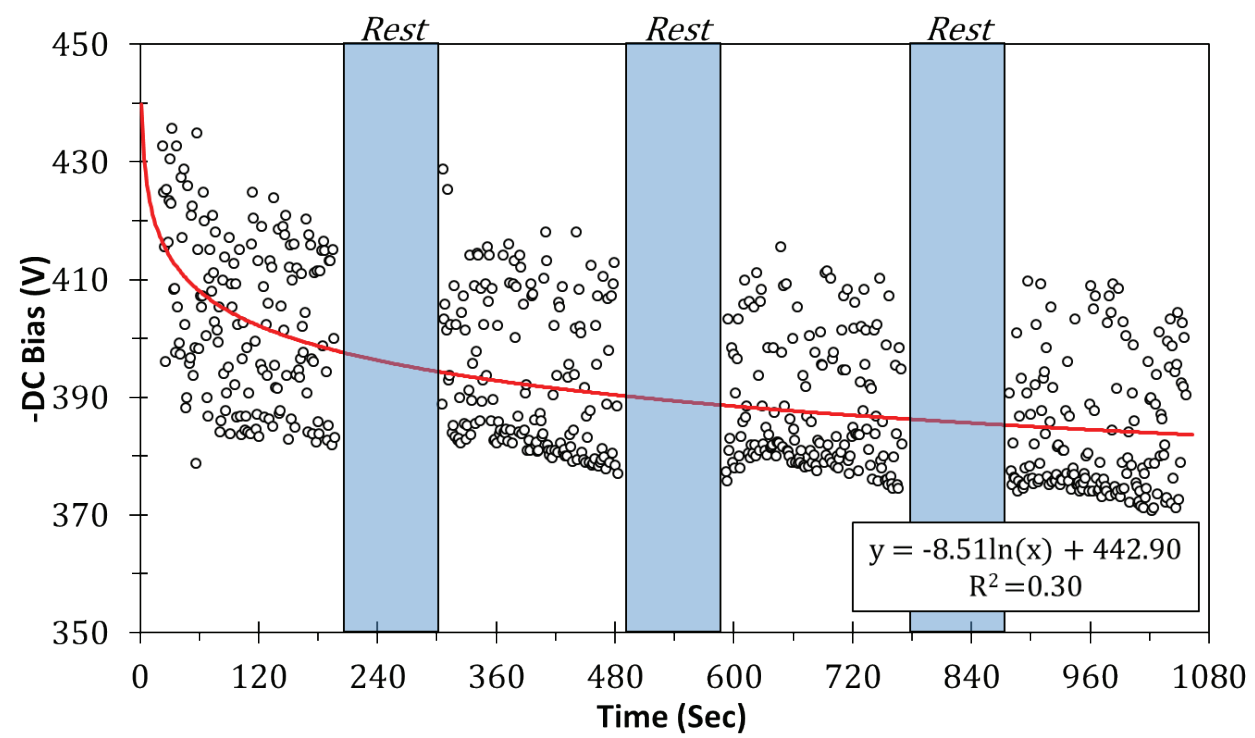

Figure 4.2: The -DC bias developed on the sample chuck during a standard etch. A log best fit is shown to aid the eye.

A DC bias plot vs. time is shown in Figure 4.2 and is representative of the typical DC bias developed during the standard etch process. Note that with power supply ramp up/down times, the actual duration over which power is supplied is approximately 108 seconds. The rapid DC bias fluctuations are a result of the Phase-Mag network rapidly tuning to match the output impedances of the power supplies to match with those of the induction coil and sample chuck in real-time due to plasma fluctuations. The steady downward drift in DC bias is a result of the chamber conditions approaching a pseudo-steady state. This effect is minimized by running the pre-etch conditioning step. A single chamber $\mathrm{O}_{2}$ clean and a $\mathrm{CF}_{4}$ conditioning step were found to strike the best balance between good steady state performance during the etch step and the electrodynamic detuning of the chamber from material buildup (Appendix $\boldsymbol{\$} \boldsymbol{A . 1 . 4}$ ). 
To determine etch rate, a $6 \mathrm{H}-\mathrm{SiC}$ sample was partially shadow masked using a silicon wafer segment before the etch step. This protected the covered area form the plasma during the etch. After the etch, the mask was removed, and the resulting step height was measured. Stylus profilometry results for a sample masked and etched using the standard etch parameters is shown in Figure 4.3 and reveals a total etch depth of approximately $2.8 \mu \mathrm{m}$. This corresponds to a H$\mathrm{SiC}(0001)$ etch rate of $\sim 233 \mathrm{~nm} / \mathrm{min}$ for the $12 \mathrm{~min}$ powered etch. Using the $6 \mathrm{H}-\mathrm{SiC}$ lattice parameters, this corresponds to a removal of approximately $\sim 11,100 \mathrm{Si}-\mathrm{C}$ bilayers.

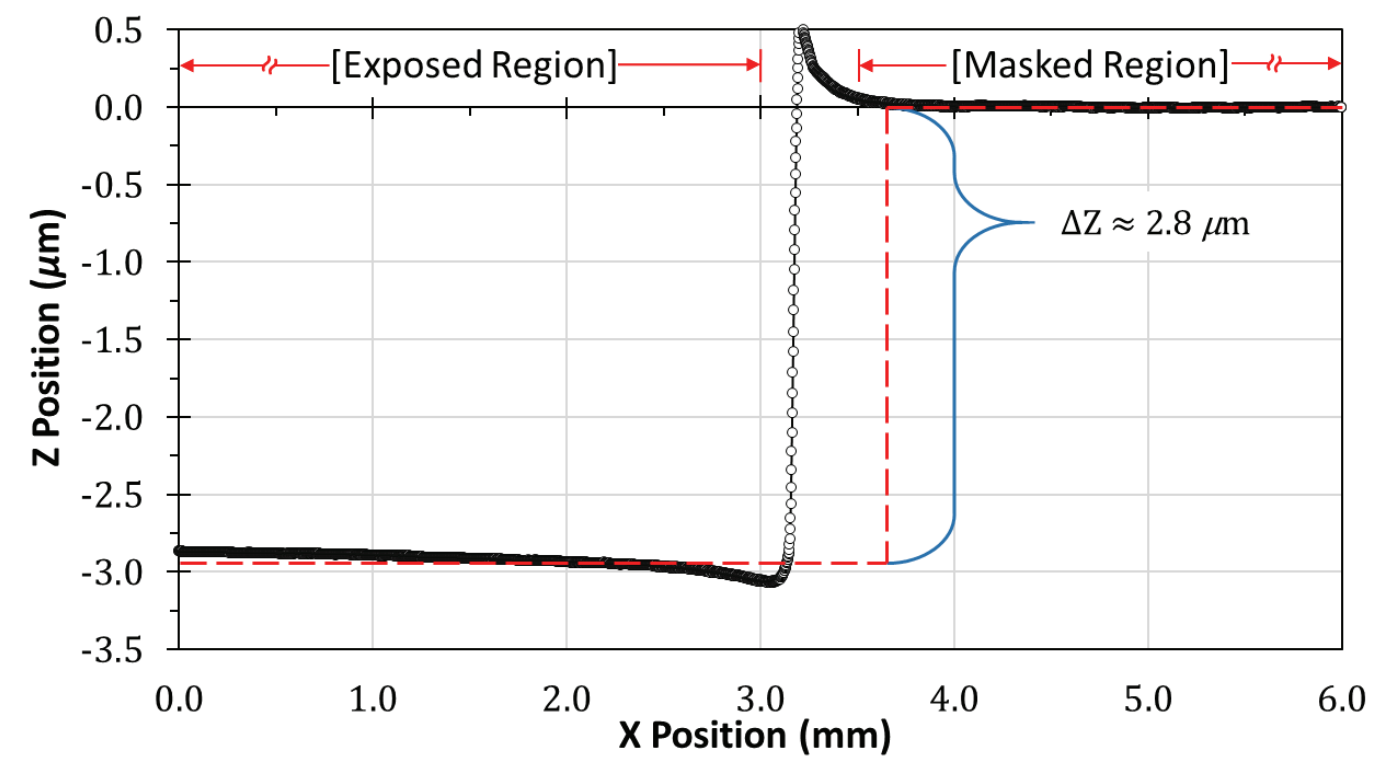

Figure 4.3: Step produced using a partial shadow mask and standard etch parameters. Based on this profile and the powered etch time of 12 minutes, The $6 \mathrm{H}-\mathrm{SiC}(0001)$ etch rate is $\sim 233 \mathrm{~nm} / \mathrm{min}$ for the standard etch parameters.

Following the etch step, the sample was transported in air to the atmospheric pressure rapid thermal annealing (AP-RTA) setup. The standard AP-RTA process was performed at atmospheric pressure under a flow of 100 SCCM of $99.99999 \%$ pure Ar. The samples were rapidly heated to approximately $990^{\circ} \mathrm{C} \pm 2 \%$, then withdrawn from the furnace and allowed to naturally cool to room temperature. The heating rate was dictated by the furnace setpoint temperature, which was $1000^{\circ} \mathrm{C}$. This resulted in an average heating rate of $\sim 200{ }^{\circ} \mathrm{C} / \mathrm{min}$ from $25^{\circ} \mathrm{C}$ to $990^{\circ} \mathrm{C} \pm 2 \%$. The cooling rate was $\sim 75^{\circ} \mathrm{C} / \mathrm{min}$ from $990^{\circ} \mathrm{C} \pm 2 \%$ to $\sim 25^{\circ} \mathrm{C}$ (laboratory ambient temperature) and was dictated by the open-air laboratory conditions. The finer details of the heating and cooling rates, and their effects on the film growth, are expanded on in Section $\$ 4.2 .5$ and Section \$4.2.6. A representative heating profile for the standard AP-RTA process is shown in Figure 4.4 and shows both the heater 
temperature and the sample temperature. Note that the nominal accuracy limits for the Type K thermocouples used in this work are $\sim 0.75 \%$ (or $\pm 7.5^{\circ} \mathrm{C}$ at $1000^{\circ} \mathrm{C}$ ). ${ }^{(527)}$

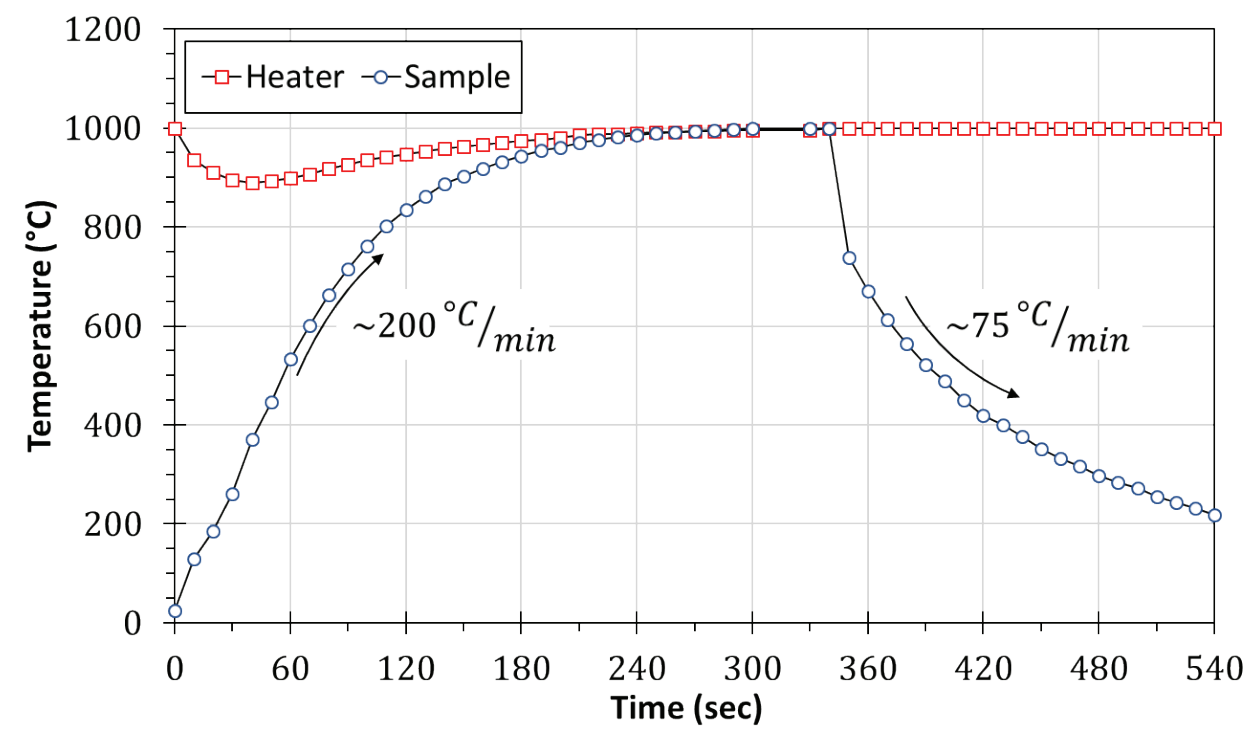

Figure 4.4: Representative heating profile for the standard AP-RTA process. Heater temperature and sample temperature are both shown. Sample heating rate was $\sim 200{ }^{\circ} \mathrm{C} / \mathrm{min}$, cooling rate was $\sim 75^{\circ} \mathrm{C} / \mathrm{min}$ and max sample temperature was $990^{\circ} \mathrm{C} \pm 2 \%$.

\section{\$4.1.1 Characterization by AFM}

Figure 4.5(A) shows the characteristic step and terrace morphology previously discussed.

Figure $4.5(B, C)$ shows the escalating planarized surface produced by etching and annealing, respectively. The difference between pre and post process surfaces is remarkable. It should also be recalled that nearly $3 \mu \mathrm{m}$ of material has been etched away during the process $(\sim 11,100 \mathrm{Si}-\mathrm{C}$ bilayers). Consequently, the surfaces should be free of any surface defects or contaminations associated with the pre-processed surface (polishing damage, handling contaminations, etc.).

\begin{tabular}{|c|c|c|c|c|c|c|c|c|}
\hline \multicolumn{3}{|c|}{ As Received 6H-SiC } & \multicolumn{3}{|c|}{ Post $\mathrm{CF}_{4}$ Etch } & \multicolumn{3}{|c|}{ Post AP-RTA } \\
\hline RMS Roughness & $148.0 \mathrm{pm}$ & & RMS Roughness & $138.0 \mathrm{pm}$ & & RMS Roughness & $136.0 \mathrm{pm}$ & \\
\hline Mean Roughness & $121.9 \mathrm{pm}$ & & Mean Roughness & $109.8 \mathrm{pm}$ & & Roughness & 107 & \\
\hline (a) & 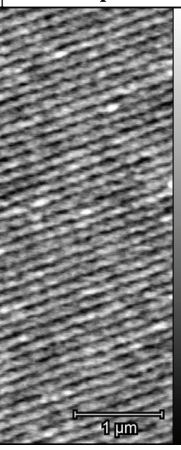 & $\begin{array}{l}0.953 \mathrm{~nm} \\
0.900 \\
0.800 \\
-0.700 \\
-0.600 \\
-0.500 \\
0.400 \\
0.300\end{array}$ & Shen $\times$ & 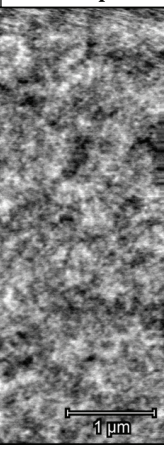 & $\begin{array}{l}1.123 \mathrm{~nm} \\
1.000 \\
0.900 \\
0.800 \\
0.700 \\
0.600 \\
0.500\end{array}$ & & & \begin{tabular}{|l|}
$1.035 \mathrm{~nm}$ \\
0.900 \\
0.800 \\
0.700 \\
0.600 \\
0.500 \\
0.400 \\
0.215 \\
\end{tabular} \\
\hline
\end{tabular}

Figure 4.5: (A) The as-received 6H-SiC(0oo1) surface showing a clean, atomically stepped surface. (B) The same surface after undergoing the standard CF4 etching treatment. (C) The same surface after the AP-RTA process. Each image is $5 \mu \mathrm{m} X 5 \mu \mathrm{m}$. Scale bars are $1 \mu \mathrm{m}$. 
In the subsequent discussion, the morphology of the post etched and annealed surface (i.e., the graphene film) is referred to as rolling hillocks or low laying hillocks. Here the variations in height ( $\min$ to $\max$ ) is $0.8 \mathrm{~nm}$ over the distance on the order of lum (1000nm). This height represents about three $\mathrm{C}-\mathrm{Si}$ bilayers, or about one-half the unit cell height for $6 \mathrm{H}-\mathrm{SiC}$.

\section{\$4.1.2 Characterization by XPS}

XPS analysis of the pre and post processed surface reveals the change in both the elemental make-up and chemical composition of the surface film as the material proceeds through the SOP process. In Figure 4.6(A) survey spectra for the as-received $6 \mathrm{H}-\mathrm{SiC}$, post $\mathrm{CF}_{4}$ etched and post APRTA surface. The corresponding atomic concentrations as calculated from these spectra are given in Figure 4.6(B). Spectrum for the starting 6H-SiC material reveals the presence of silicon and carbon, in what is approximately a stochiometric ratio ${ }^{23}$, as well as the presence of oxygen. Inspection of the high resolution $\mathrm{O} 1 \mathrm{~s}$ and $\mathrm{Si} 2 \mathrm{p}$ spectral regions suggest the oxygen fraction exists as various silicon suboxides ( $\mathrm{Si}-\mathrm{O}-\mathrm{Si})$ or oxycarbide (Si-O-C). ${ }^{(528)}{ }^{(529)}$ Inspection of the highresolution spectra of the $\mathrm{O} 1 \mathrm{~s}$ peak are consistent with this interpretation in that essentially only a single peak (oxygen species) is present.

\footnotetext{
${ }^{23}$ Note that the $\mathrm{SiC}$ is, in fact, stoichiometric. The apparent discrepancy is due to the specific sensitivity factors used by the instrumentation in calculating the concentration. Sensitivity factors vary slightly between manufactures, depending on the particular database the values are pulled from.
} 


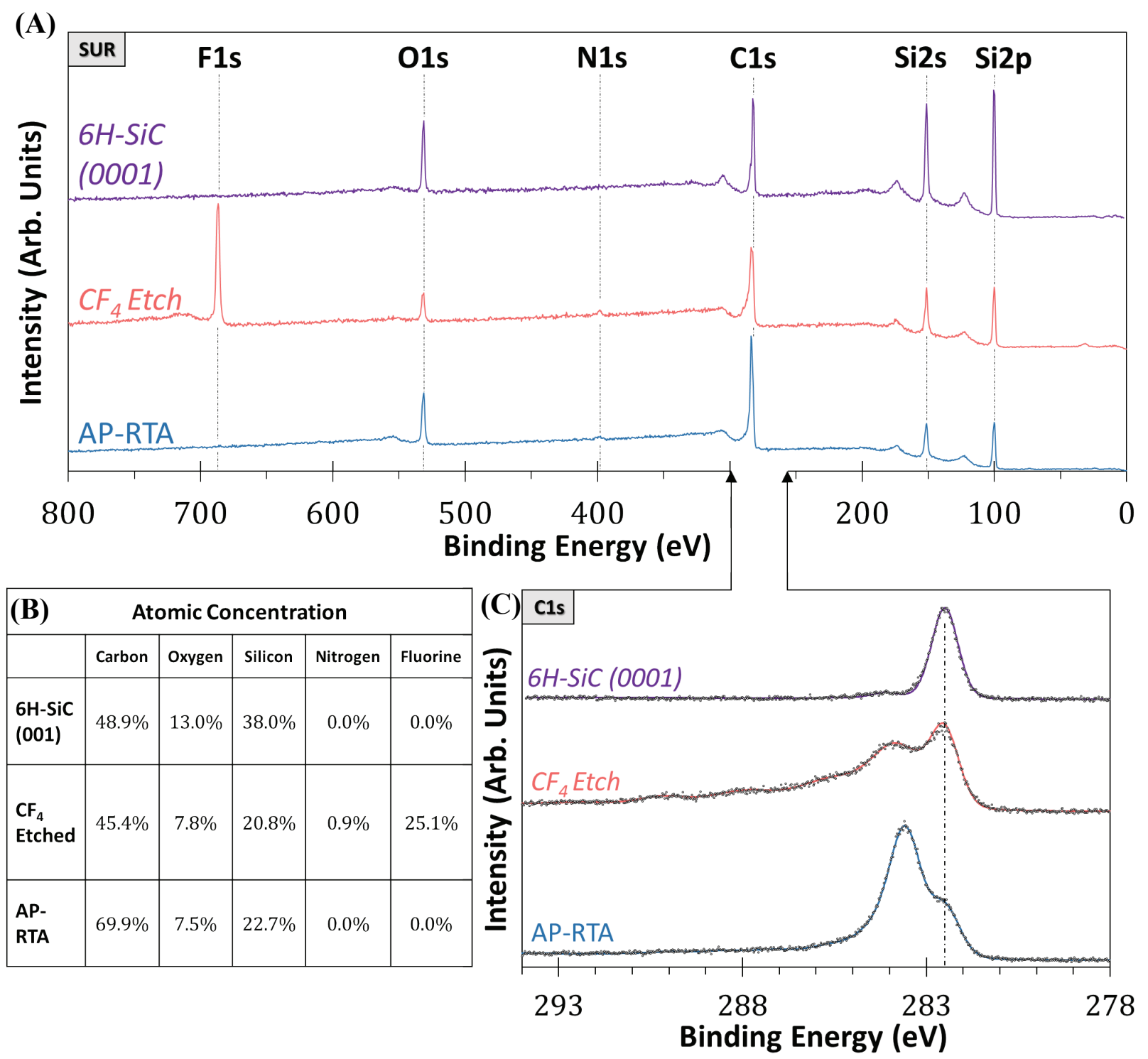

Figure 4.6: (A) Survey spectra collected from the same sample at various stages in the synthesis process (as-received $6 \mathrm{H}-\mathrm{SiC}$, post $\mathrm{CF}_{4}$ etch and post AP-RTA). All major peaks have been identified. Unlabeled features are due to various energy loss processes associated with the labeled photoelectron emission lines. (B) Atomic concentrations calculated from the survey spectra. (C) High resolution scans of the C1s peak at each stage in the synthesis process. The C-Si reference peak at $282.5 \mathrm{eV}$ is marked to guide the eye.

After ICP-RIE etching, there is a slight increase in intensity of the C1s relative to the Si2s peak as well as a broadening of the C1s peak. In addition, an O1s, F1s and a very low level N1s peak can be observed. The F1s is clearly associated with the etch process. The O1s and N1s are primarily associated with air exposure as samples were transferred from the ICP-RIE system to the XPS system; however, contributions from low level oxygen contaminants in the etching gas cannot be excluded. 
Following the anneal, there is a substantial increase in the intensity of the $\mathrm{C} 1 \mathrm{~s}$ peak relative to the Si2s peak. As discussed later, this enhancement of the C1s peak is due to the formation of multi-layer graphene film and buffer-layer. All fluorinated species appear to be desorbed during the AP-RTA. The O1s peak intensity remains essentially unchanged suggesting little if any additional contribution from oxygen-based contaminants in the $99.99999 \%$ pure (Research Plus purity) Ar purge gas during the anneal or from the atmosphere during transit to the XPS. This is consistent with the analysis of the high resolution Si2s peak. The absence of the N1s peak also suggests the annealed surface is more resistant to adsorption of gases from the atmosphere.

The high-resolution spectra of the $\mathrm{C} 1 \mathrm{~s}$ region at each step in the synthesis process are shown in Figure 4.6(C). At all three stages the peak at $282.5 \mathrm{eV}$ is associated with carbon in the $\mathrm{SiC}$ bulk. ${ }^{(473)}$ This peak is used to compensate for changing which occurs during the XPS analysis process. In addition, the as-received $6 \mathrm{H}-\mathrm{SiC}$ shows a small adventitious carbon surface contaminate appearing on the higher binding energy side of the SiC-C peak $(\sim 284.5 \mathrm{eV})$, likely atmospheric in origin. This is consistent with reports in the literature. High resolution spectra of the same spectral region on the post etched surface reveal a much more complex signal. The multiple broad peaks with components out to $290 \mathrm{eV}$ indicate a wide distribution of carbon surface species in various chemical states. Undoubtedly many of these peaks are fluorinated carbon states remaining from the etching process. For the post AP-RTA surface, the $\mathrm{C} 1 \mathrm{~s}$ region is narrower and indicates a smaller distribution of chemical states. Additional insight into the chemistry of the etched and annealed surface can be obtained from the deconvolution of the high-resolution C1s spectra taken for a representative sample, as shown in Figure 4.7. 
(A)

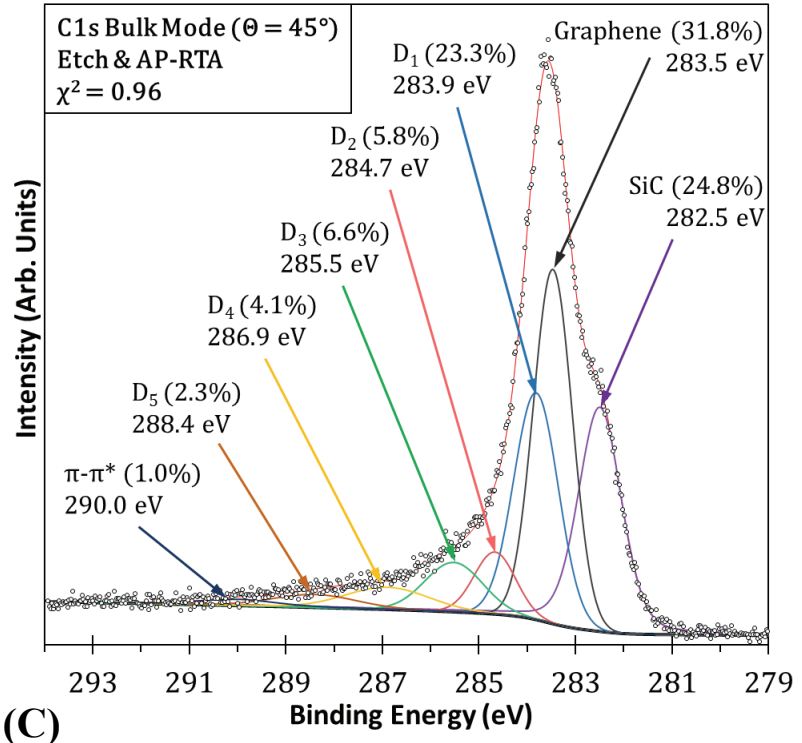

(B)

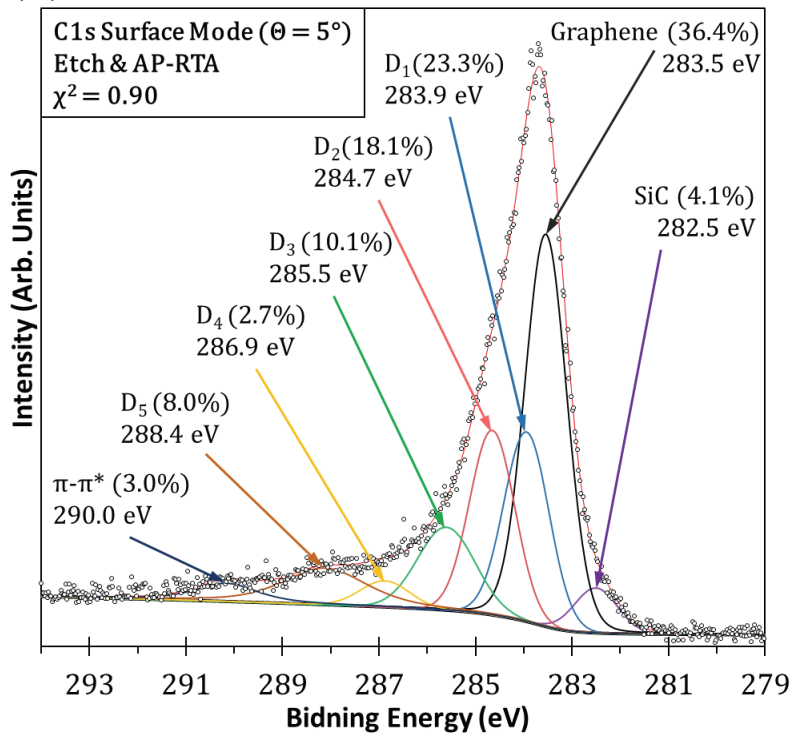

\begin{tabular}{|c|c|c|c|c|c|c|c|c|c|c|c|}
\hline \multicolumn{5}{|c|}{ Bulk Sensitive $\left(\theta=45^{\circ}\right)$} & \multicolumn{2}{|c|}{$\begin{array}{l}\text { Change in Peak Area } \\
\text { (Bulk to Surface Mode) }\end{array}$} & \multicolumn{5}{|c|}{ Surface Sensitive $\left(\Theta=5^{\circ}\right)$} \\
\hline Band ID & Position & Separation & FWHM & \%Area & As \% & Ratio $\left(R_{x}\right)$ & Band ID & Position & Separation & FWHM & \%Area \\
\hline SiC & 282.5 & 0.0 & 1.1 & 24.8 & $-84 \%$ & 0.16 & $\mathrm{SiC}$ & 282.5 & 0.0 & 1.1 & 4.1 \\
\hline Graphene & 283.5 & 1.0 & 1.0 & 31.8 & $+14 \%$ & 1.14 & Graphene & 283.5 & 1.0 & 1.0 & 36.4 \\
\hline D1: S1 & 283.9 & 1.3 & 1.1 & 23.3 & $-22 \%$ & 0.78 & D1: S1 & 283.9 & 1.4 & 1.1 & 18.1 \\
\hline $\mathrm{D} 2: \mathrm{S} 2, \mathrm{C}-\mathrm{OH}$ & 284.7 & 2.2 & 1.0 & 5.8 & $+202 \%$ & 3.02 & $\mathrm{D} 2: \mathrm{S} 2, \mathrm{C}-\mathrm{OH}$ & 284.7 & 2.2 & 1.1 & 17.6 \\
\hline D3: C-O-C & 285.5 & 3.0 & 1.4 & 6.6 & $+55 \%$ & 1.55 & D3: C-O-C & 285.6 & 3.1 & 1.4 & 10.1 \\
\hline D4: $C=0$ & 286.9 & 4.4 & 2.0 & 4.1 & $-34 \%$ & 0.66 & D4: $C=0$ & 286.9 & 4.4 & 1.2 & 2.7 \\
\hline D5: $\mathrm{C}=\mathrm{O}-\mathrm{OH}$ & 288.4 & 5.9 & 2.0 & 2.3 & $+243 \%$ & 3.43 & D5: $\mathrm{C}=\mathrm{O}-\mathrm{OH}$ & 288.1 & 5.6 & 2.1 & 8.0 \\
\hline Pi-Pi* & 290.0 & 7.5 & 1.9 & 1.3 & $+133 \%$ & 2.33 & $\mathrm{Pi}-\mathrm{Pi}^{*}$ & 290.2 & 7.7 & 1.5 & 3.0 \\
\hline
\end{tabular}

Figure 4.7: Deconvoluted C1s XPS spectra obtained using (A) the bulk and (B) the surface analysis modes. (C) Peak parameters for each fit and the percent area change of each peak as the technique transitions from a bulk sensitive mode to a surface sensitive mode; a decrease in peak area indicates a more subsurface species and an increase indicates a more terminal-surface species.

Due to the lack of overall character in the raw $\mathrm{C} 1$ s spectra and the many peak assignments which are possible, the deconvolution process must be approached with a critical eye. Additionally, it is reasonable to assume that the surface film is not strictly homogeneous in structure over the XPS analysis spot size area, which is roughly circular with a diameter of $\sim 100$ $\mu \mathrm{m}$. Thus, the spectra will be a convolution of all the variations in film composition and film structure experienced by the film over the $7854 \mu \mathrm{m}^{2}$ analysis area. The important note here is that the spectra represent an average of the film, collected over a relativity large area. While it is tempting to continue to add peaks to a spectrum to aggressively minimize the fitting parameter $\left(\chi^{2}\right.$ in this case) it is important that care is taken to ensure that the deconvoluted spectra comports with reality; or at least as close as can be intuited based on first principles and remaining consistent with what others have reported in the literature. To that end, a description follows detailing the generic fitting methodology and motivation used in this work to deconvolute the C1s spectra. 
Figure 4.7(A) shows the C1s peak obtained using the bulk analysis mode. This spectrum has been resolved into eight different components. The peak at $282.5 \mathrm{eV}$ is associated with carbon in the substrate (SiC-C). This assignment is self-evident; it lays far enough away from the other component peaks that its position and low binding energy tail is clearly resolved in the raw data. Additionally, XPS analysis of the as-received SiC gives confirmation of this assignment. Because the $\mathrm{SiC}-\mathrm{C}$ peak location is unambiguous, its position is used to determine the charging occurring in each spectrum. Therefore, by design, this peak appears at $282.50 \mathrm{eV}$ in all spectra presented here (i.e., all spectra are individually shifted such that this specific peak lies at $282.50 \mathrm{eV}$ ). The precise binding energy location of this peak was determined from previous work conducted by Stinespring, et al. ${ }^{(473)}$

In Figure 4.7, the peak at $283.5 \mathrm{eV}$ is associated with $\mathrm{sp}^{2}$-bound carbon $\left(\mathrm{sp}^{2}-\mathrm{C}\right)$ in the graphene film. The identification of this peak is likewise relatively unambiguous due to its amplitude which is partially resolved in the raw data. Additionally, the shift in position of the graphene peak relative to the $\mathrm{SiC}-\mathrm{C}$ peak $(\Delta \mathrm{SiC} \sim 1.0 \mathrm{eV})$ is in excellent agreement with the observations made by Oliveira, et al. and Langer, et al. using XPS systems similar to that used here. ${ }^{(226)(530)}$

In Figure 4.7, the peaks designated D1 at $283.9 \mathrm{eV}(\Delta \mathrm{SiC} \sim 1.4 \mathrm{eV})$ and D2 at $284.7 \mathrm{eV}$ $(\Delta \mathrm{SiC} \sim 2.2 \mathrm{eV})$ are associated with the buffer-layer between the graphene and the SiC substrate. These peaks are frequently referred to as $S_{1}$ and $S_{2}$ in the literature. Their position, or more precisely their shift relative to the SiC-C peak, has been established based on the results of Oliveira, et al. and Langer, et al. who used XPS equipment comparable to that used here. (226) (530) These shifts also are in good agreement with those observed in spectra obtained using a variety of synchrotron light sources. (240) (531) (532) The D2 peak, as discussed later, has also been associated $\mathrm{C}-\mathrm{OH}$ type defects in the graphene film. Furthermore, this assignment of the D1 and D2 peaks as buffer-layer related peaks fall well within the range of values reported by others in the literature. Figure 4.8 lists and plots many of the reported binding energy values for the $\mathrm{S}_{1}, \mathrm{~S}_{2}$ and graphene peaks. As indicated by the red dashed arrows in this figure, the values observed in this work fall within the range of reported values. 


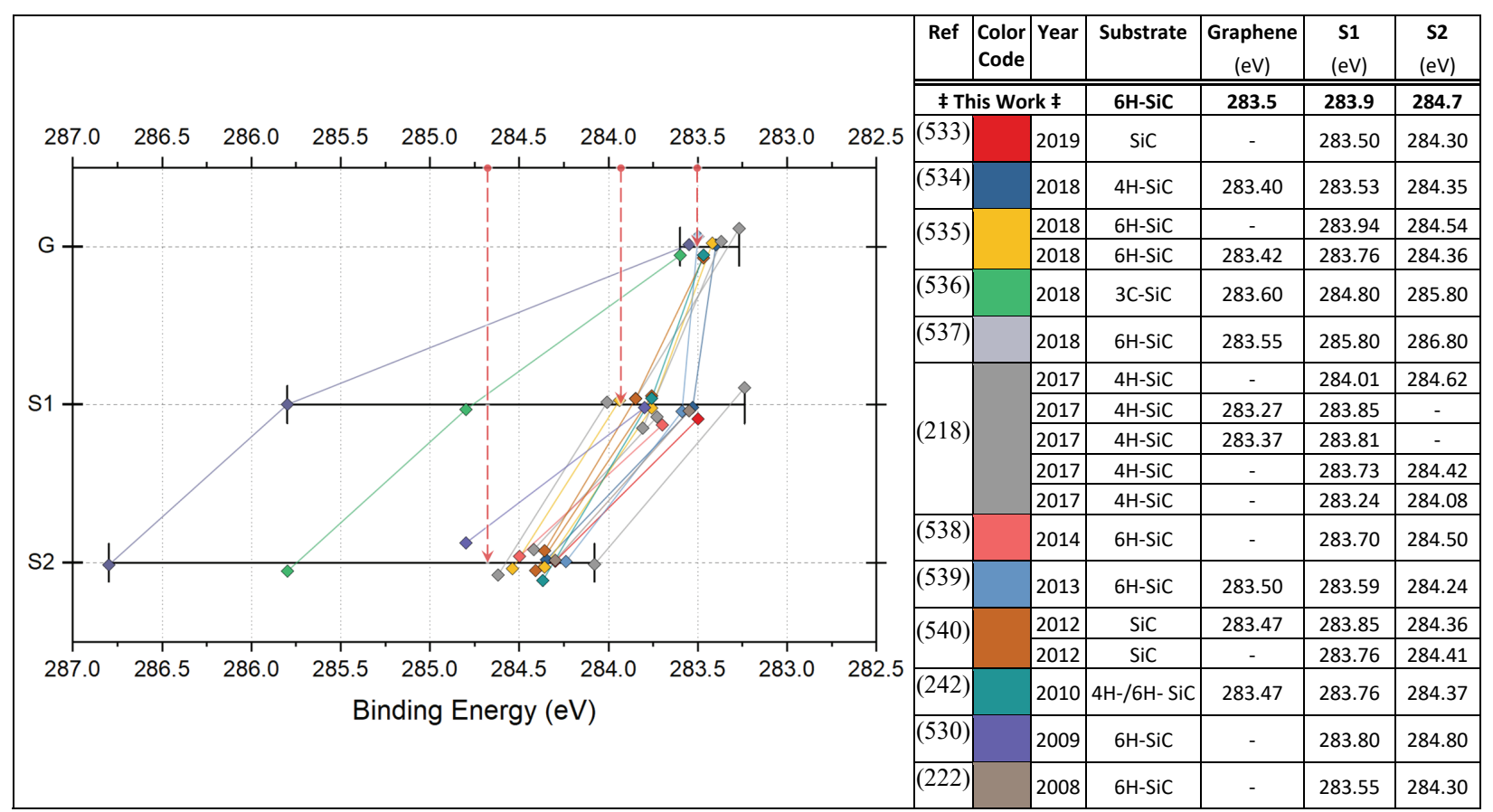

Figure 4.8: The range of binding energy values reported for the S1, S2 and Graphene peaks. The red dashed arrows indicated where the values assigned in Figure 4.7 (A) for this work fall relative to what others have reported for the thermally driven selective sublimation process for graphene growth which results in buffer-layer formation. Although on the high side, values for this work fall within the range of reported values. (Note that the as-reported values have all be universally shifted to what is shown such that the $\mathrm{SiC}$ peak appears at a binding energy of $282.5 \mathrm{eV}$, so that they are directly comparable between each other and to this work.)

With regard to the buffer-layer species, the details vary somewhat, but it is generally thought that the lower binding energy peak (D1 or $\left.\mathrm{S}_{1}\right)$ is associated with carbon atoms in the bufferlayer which are tethered to silicon atoms in the $\mathrm{SiC}$ substrate in a $\mathrm{sp}^{3}$ like state. In contrast, the higher binding energy peak (D2 or $\mathrm{S}_{2}$ ) is associated with $\mathrm{sp}^{2}-\mathrm{C}$ in the buffer-layer which must interact with the tethered carbon atoms. In this case, the $\mathrm{C}-\mathrm{C}$ bonds for these carbon atoms must be highly strained. It is worth noting that there has been some disagreement within the scientific community on the physical interpretation of the $\mathrm{S}_{1}$ and $\mathrm{S}_{2}$ components of the spectrum. Lima, et al. have stated that the $\mathrm{S}_{2}$ peak is due to carbon covalently bound to the terminal silicon in the substrate (tethered), while more recent work by Conrad, et al. have assigned $\mathrm{S}_{1}$ to be due to carbon covalently bound to the terminal silicon in the substrate (tethered). ${ }^{(538)(218)}$ The later view $\left(\mathrm{S}_{1}\right.$ as the covalent bound carbon) is the more generally excepted interpretation and this is the interpretation adopted here. Based on this, it is evident that, given the D1/D2 $\left(\mathrm{S}_{1} / \mathrm{S}_{2}\right)$ ratio observed here ( $\sim 3$ based on peak areas), the buffer-layer for this film is highly tethered. 
Although there is not yet consensus in the scientific community on the details of the bufferlayer, Figure 4.9 shows an idealized schematic illustration of the generic arrangement of the carbon and silicon atoms of the interface (additional information on the buffer-layer formed during the selective sublimation method, as reported by others, is given in Section $\$ 2.1 .3$ ). The various binding states detected by XPS are shown and the peak assignments are annotated.

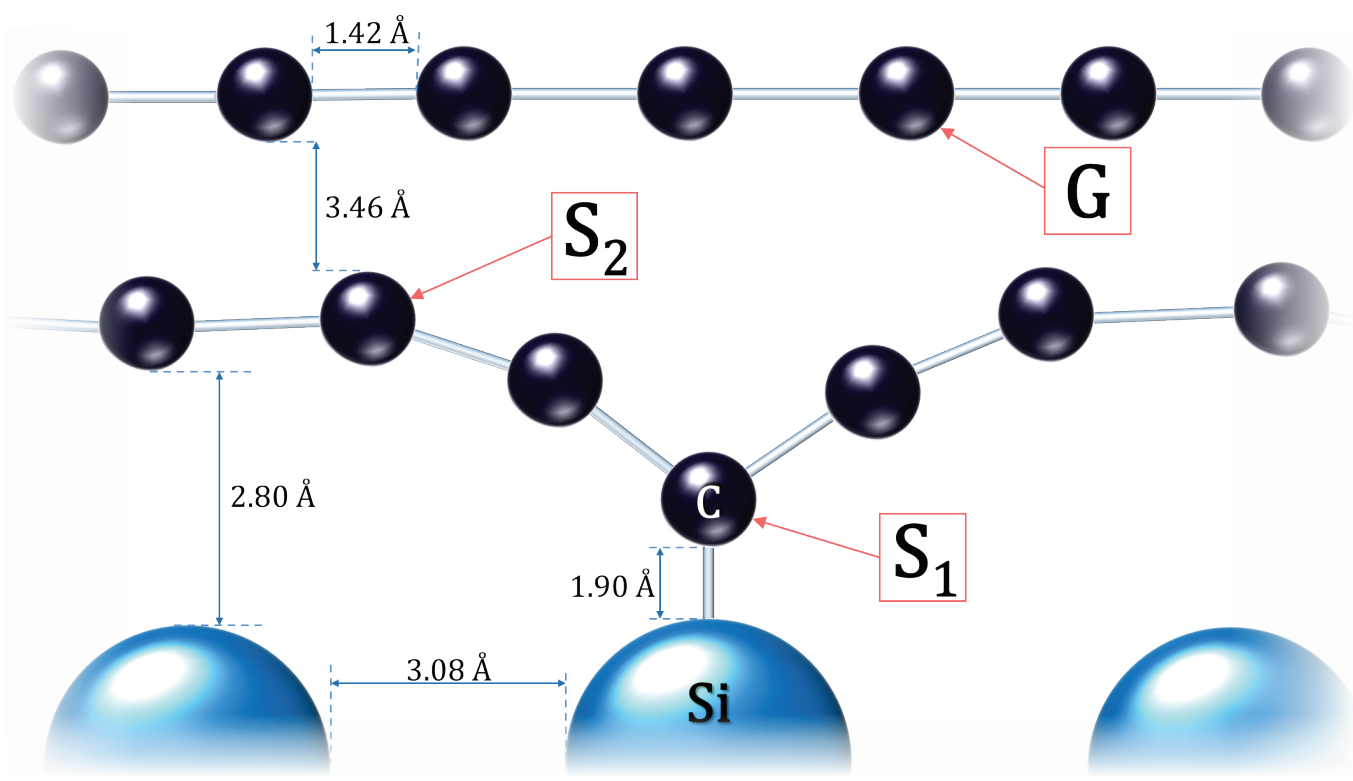

Figure 4.9: An idealized view of the carbon film with respect to the origins of the XPS S1, S2 and G (graphene) peaks. The atomic spacings have been labeled consistent with the literature review in Chapter 1 and 2. Atom sizes are to scale; bond distances are approximately to scale.

In reality the interfacing mechanics, and more broadly the film itself, are certainly more chaotically ordered that what is depicted in Figure 4.9. Computational studies have revealed the interface can be substantial more complex. ${ }^{(213)}$ It is again reiterated here that this interpretation and physical model is based on the corpus of work others have produced and is based on their analysis, which used x-ray diffraction techniques and XPS analysis using synchrotron radiation (advanced analysis techniques not available here). Therefore, while their interpretation may be a reasonable approximation of the physical reality of films produced using the selective sublimation growth technique, the buffer-layer and interfacing mechanicals of the films produced here may be substantially different. This stands to reason base on a conceptual bias, given the inherently different film formation mechanics, as well as the results presented in Section $\$ 4.3$, which indicates, at the very least, a substantial difference in the buffer-layer adhesion to the substrate. These disclaimers notwithstanding, the following interpretation has been developed based on what is detectable by the XPS analysis and takes advantage of the large about of work others have 
compiled over recent years on what is the most analogous structure (i.e., films produced using selective sublimation and analyzed using advanced techniques). It is the most mature depiction of the situation yet established for films grown using the novel plasma assisted growth method.

In Figure 4.7, along with the SiC-C, graphene, D1 and D2 peaks, three additional peaks are also identified in the spectrum; D3 at $285.5 \mathrm{eV}, \mathrm{D} 4$ at $286.9 \mathrm{eV}$ and D5 at $288.4 \mathrm{eV}$. The position, intensity, and full width at half max (FWHM) of D3, D4 and D5 were allowed to vary within a specified reasonable range (discussed later) in order to minimize the $\chi^{2}$ fitting parameter while maintaining the position and FWHM of the other peaks. The D3, D4 and D5 peaks are associated with oxygen-based defects typically found in graphene oxide (GO) and reduced graphene oxide (rGO). When discussing the peaks, it is convenient to speak in terms of binding energy shifts relative to the graphene peak $(\Delta \mathrm{G})$ since a $\mathrm{SiC}-\mathrm{C}$ reference peak is not present in $\mathrm{GO}$ and rGO materials. In the case of $\mathrm{D} 3, \Delta \mathrm{G} \sim 2.0 \mathrm{eV}$ corresponds reasonably well with the value reported for epoxide (C-O-C) defects. ${ }^{(541)}$ The defect species identified as D4 with $\Delta \mathrm{G} \sim 3.4 \mathrm{eV}$ has

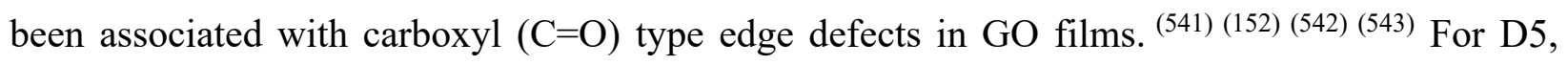
$\Delta \mathrm{G} \sim 4.2 \mathrm{eV}$ corresponds to the value reported for carboxyl $(\mathrm{O}=\mathrm{C}-\mathrm{OH})$ defects found at the sheet edge. (541) (152) (542) (543) As observed above, the $\mathrm{S} 2$ peak $(\Delta \mathrm{G} \sim 1.2 \mathrm{eV})$ which is attributed to the buffer-layer also overlaps (and is most likely due in part) to $\mathrm{C}-\mathrm{OH}$ defect species formed on the graphene surface. Lastly, the peak seen at $290.0 \mathrm{eV}$ is due to the $\pi-\pi^{*}$ transition. ${ }^{(530)(544)}$ This peak is shifted by $\sim 6.5 \mathrm{eV}$ relative to the graphene peak and is associated with an excitation (shake up) of an electron from the $\pi$-bonding to $\pi$-antibonding $\left(\pi^{*}\right)$ orbital.

Figure 4.7(B) shows the C1s spectrum corresponding to that shown in Figure 4.7(A) but obtained using the surface sensitive analysis mode (a larger fraction of the total photoelectron signal is collected from the surface, as compared to the bulk analysis mode). By determining which peaks grow/shrink in intensity, a comparison between spectra collected from bulk and surface modes give an indication as to which species are more strongly associated with the buke verses the surface. This spectrum has been resolved into the same peaks identified in Figure 4.7(A) as $\mathrm{SiC}$, graphene and the states D1, D2, D3, D4, D5 and the $\pi-\pi^{*}$ transition. In this spectrum, the SiC peak is strongly attenuated relative to the graphene and defect peaks. Quantitatively, the percentage change and ratios $(\mathrm{Rx})$ of the surface to bulk peak areas (normalized) for each species $(\mathrm{X})$ are given in Figure $4.7(C)$. The ratio $\mathrm{R}_{\mathrm{SiC}}$ of $\sim 0.16$ (corresponding to signal decrease of $-84 \%$ ) clearly 
suggests that the SiC-C peak is associated with the underlying substrate, while $\mathrm{R}_{\text {Graphene }} \sim 1.14$ (corresponding to signal increase of $+14 \%$ ) indicates that the graphene peak is associated with the surface film. The ratio $\mathrm{R}_{\mathrm{D} 1} \sim 0.78$ (signal decrease of $-22 \%$ ) is intermediate between what is observed for the substrate (SiC-C) and the surface film (graphene). This is consistent with its identification as a buffer-layer species. Given this it is reasonable to expect the value of $\mathrm{R}_{\mathrm{D} 2}$ to be $\sim 0.9$; however, this ratio is found to be twice that value. This is may be due to the fact that the D2 peak has contributions from both subsurface $(\mathrm{S} 2)$ and surface $(\mathrm{C}-\mathrm{OH})$ species. The ratios for D3 and D5 are consistent with what are expected for surface species.

Based on the spectra shown in Figure $4.7(A)$, the graphene peak represents $\sim 75 \%$ of the surface film species, while the total oxygen-based defects, D2, D3, D4 and D5, represent $\sim 25 \%$. Given the number of layers (discussed later) this corresponds to a per layer defect concentration of $\sim 9 \%$. Note that D2 is included here as an oxygen-based defect to obtain the maximum defect concentration. If the entirety of D2 is taken to be all S2 (buffer-layer), with no oxide component, the total defect level is $\sim 18 \%$ or $\sim 6 \%$ per layer. Although these levels may vary from run to run, they are typical of all the films produced in these studies. Moreover, they are very comparable to the defect levels observed in prior work where a UHV anneal rather that an atmospheric pressure RTA was used. In the case of the UHV anneal the defects were due to a combination of oxygenand fluorine-based defects. ${ }^{(263)}$ These defect levels stand in marked contrasted to the oxygen-based defect levels of $\sim 60 \%$ for GO and $35 \%-43 \%$ for RGO. ${ }^{(542)}$

As described in APPENDIX D, analysis of the ratio of peak areas within the C1s region can give an indication of the average number of graphene layers in the film over the XPS analysis area ( 100 $\mu \mathrm{m}$ spot size). ${ }^{(545)(546)(547)(263)}$ Specifically, this is done by first calculating the total intensity (area) of all graphene associated peaks (i.e., the main graphene peak, plus all of the defect peaks, not including the $\pi-\pi^{*}$ peak) and dividing it by the area of the C-Si peak (carbon signal from the bulk). In this way, attenuation ratio of the photoelectron signal produce from carbon in the bulk to the signal produce by the carbon in the graphene overlayer is quantified. This ratio denotated $\mathrm{I}_{\mathrm{G}} / \mathrm{ISiC}_{\mathrm{SiC}}$ is then used in the computational model described in APPENDIX $\boldsymbol{D}$ to determine the number of carbon overlayers. This number includes the buffer-layer between the outer graphene layer and the $\mathrm{SiC}$ substrate. Thus, a two-layer film represents one buffer-layer and one graphene layer. This technique presents a convenient and consistent method for the comparison of the 
amount of ordered carbon between samples and gives a good approximation for the number of equivalent carbon layers.

Figure 4.10 shows an example of the analysis based on the previously described computational model. The attenuation ratio $\left(\mathrm{I}_{\mathrm{G}} / \mathrm{ISiC}_{\mathrm{SiC}}\right)$, corresponding to the $\mathrm{C} 1 \mathrm{~s}$ data shown in Figure 4.7(A), is indicated on the master curve. This analysis shows the buffer-layer and graphene overlayer has an average combined thickness of $1.0 \pm 0.1 \mathrm{~nm}$ over the XPS analysis area. For multilayer epitaxial graphene films formed by sublimation of $\mathrm{Si}$ from the $6 \mathrm{H}-\mathrm{SiC}(0001)$ surface, the average graphene monolayer (ML) thickness is $0.346 \mathrm{~nm}$ as reported by Weng, et al. ${ }^{(548)}$ Assuming this is also representative of the buffer-layer thickness, the overlayer in this case has a thickness of 2.8 monolayers (ML) of carbon or approximately $1 \mathrm{ML}$ due to the buffer-layer (BL) and $2 \mathrm{ML}$ due to graphene (i.e., approx. 1 ML of buffer-layer carbon + 2 ML of graphene, or 3 carbon layers in total). This calculation is shown in Figure 4.10. A simple $3^{\text {rd }}$ order polynomial fit is used to calculate the approximant number of carbon layers for a given ratio, valid over the range shown (a new fit polynomial is calculated to accurately capture data points which fall outside of this range).

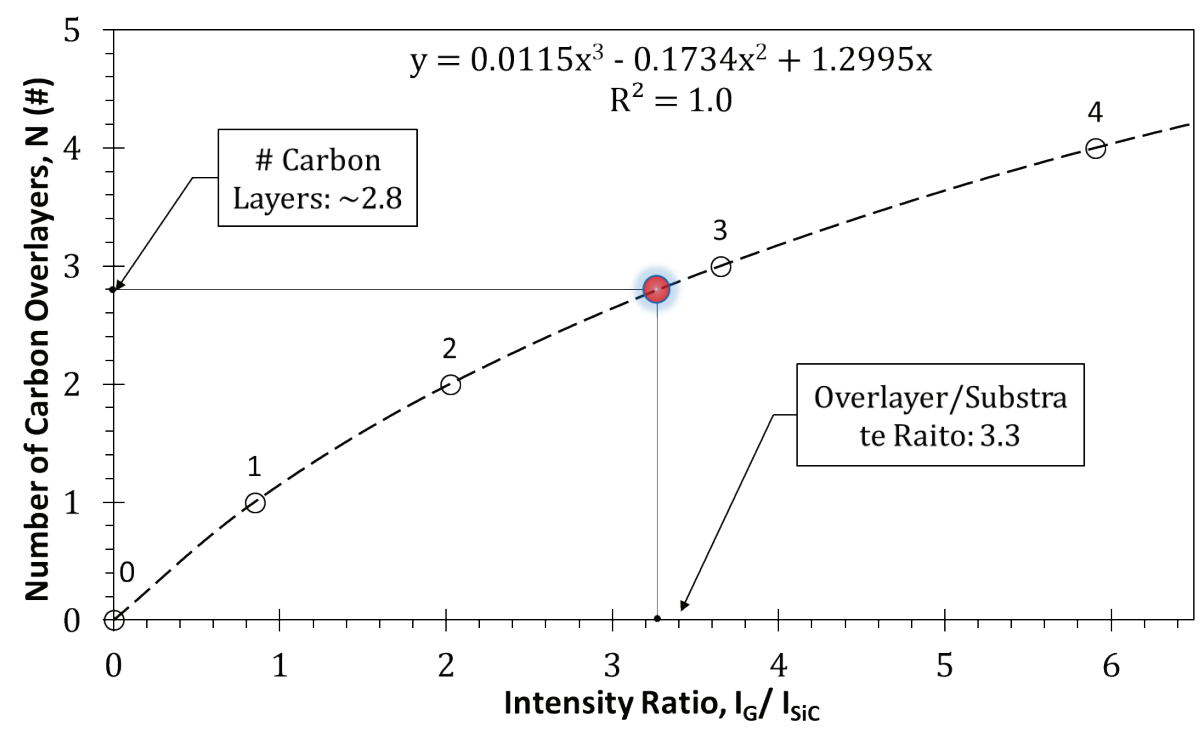

Figure 4.10: A plot showing the number of carbon overlayers vs the $\mathrm{I}_{\mathrm{G}} / \mathrm{I}_{\mathrm{Sic}}$ ration. The highlighted data point corresponds to the calculated value obtained by analysis of the data shown in Figure 4. 7(A). Details of this analysis and attenuation model are given in APPENDIX D.

The following XPS analysis paradigm was used as the basis for all films produced in this work. The program MultiPak Spectrum Version 9.9.0.8 (ULVUAC-PHI, Inc.) was used to fit the XPS data. To ensure that the fitting model was not overly rigid and was flexible enough to accurately represent the variations which occur between samples, the fitting criteria listed in Table 
4-2 were used. By design, the $\mathrm{SiC}$ peak appears at $282.5 \mathrm{eV}$ highlighted by the shaded box. Combination Gaussian-Lorentz line shapes were used for all peaks.

Table 4-2: Fitting criteria used for the C1s fitting.

\begin{tabular}{|c|c|c|c|c|c|c|c|c|}
\hline $\begin{array}{c}\text { Band } \\
\text { Parameter }\end{array}$ & SiC & Graphene & $\begin{array}{c}\text { D1 } \\
\text { (S1) }\end{array}$ & $\begin{array}{c}\text { D2 } \\
\text { (S2, C-OH) }\end{array}$ & $\begin{array}{c}\text { D3 } \\
\text { (C-O-C) }\end{array}$ & $\begin{array}{c}\text { D4 } \\
\text { (C=O) }\end{array}$ & $\begin{array}{c}\text { D5 } \\
\text { (C=O-OH) }\end{array}$ & Pi-Pi* \\
\hline \multirow{2}{*}{ Position (eV) } & \multirow{2}{*}{282.50} & $283.55-$ & $284.00-$ & $284.80-$ & $285.80-$ & $286.90-$ & $288.40-$ & $290.40-$ \\
& & 283.30 & 283.80 & 284.60 & 285.60 & 286.70 & 288.20 & 290.20 \\
\hline \multirow{2}{*}{ FWHM (eV) } & $1.50-$ & $1.20-$ & $1.50-$ & $1.50-$ & $1.80-$ & $2.00-$ & $2.00-$ & $2.50-$ \\
& 0.80 & 0.90 & 0.90 & 0.90 & 0.90 & 0.90 & 0.90 & 1.00 \\
\hline \multirow{2}{*}{$\%$ Gaussian } & $100-$ & $100-$ & $100-$ & $100-$ & $100-$ & $100-$ & $100-$ & $100-$ \\
& 50 & 50 & 50 & 50 & 50 & 50 & 50 & 50 \\
\hline
\end{tabular}

Using the fitting criteria from Table 4-2, the C1s region were fitted for 23 randomly selected samples produced over a span of 35 months using the SOP process. The statistical distribution of select key parameters extracted from each fit are shown in the box and violin plots of Figure 4.11. Over this time span, several different furnaces were used for the AP-RTA step (which slightly effected the heating and cooling rates) and the conditions in the ICP/RIE system changed considerably (chamber ageing, components were replaced and upgraded, etc.). These factors undoubtedly contributed to the spread in the data. 
(A)

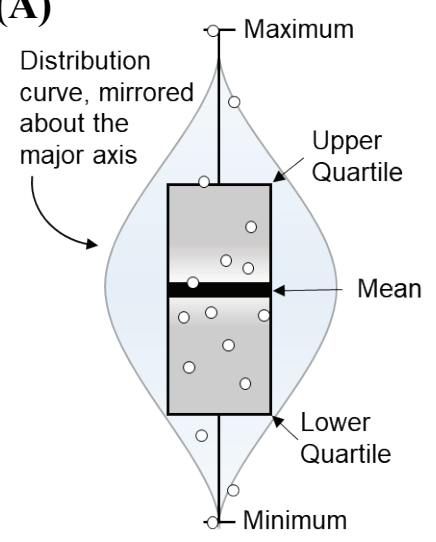

(B)

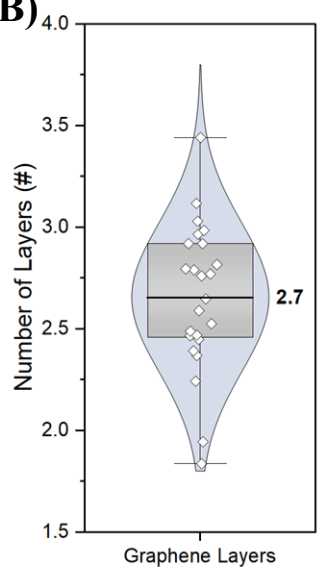

(C)

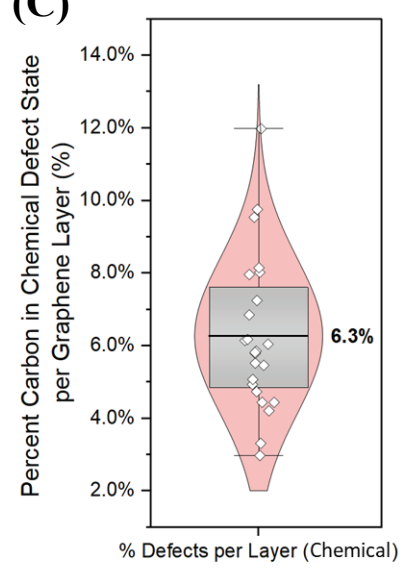

(D)

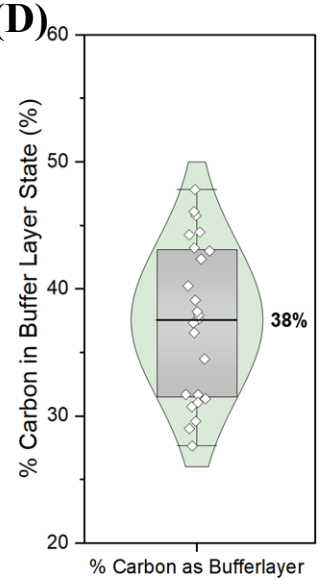

(E)

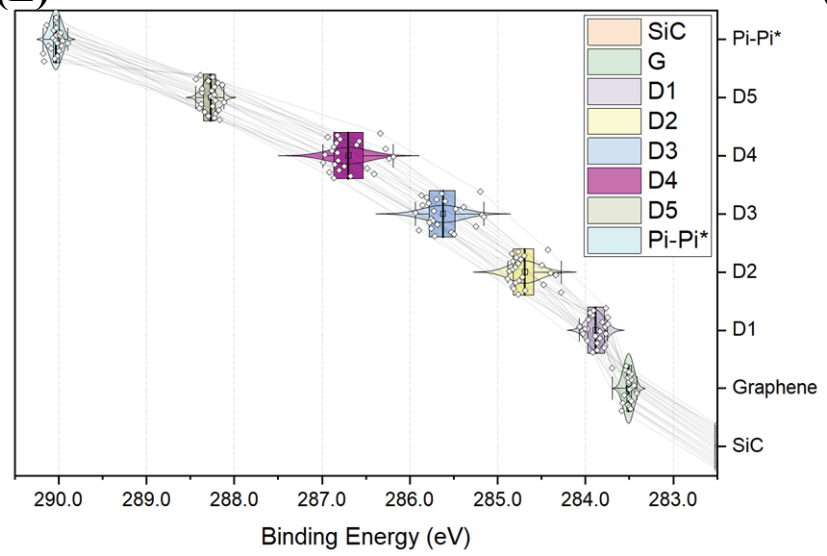

(F)

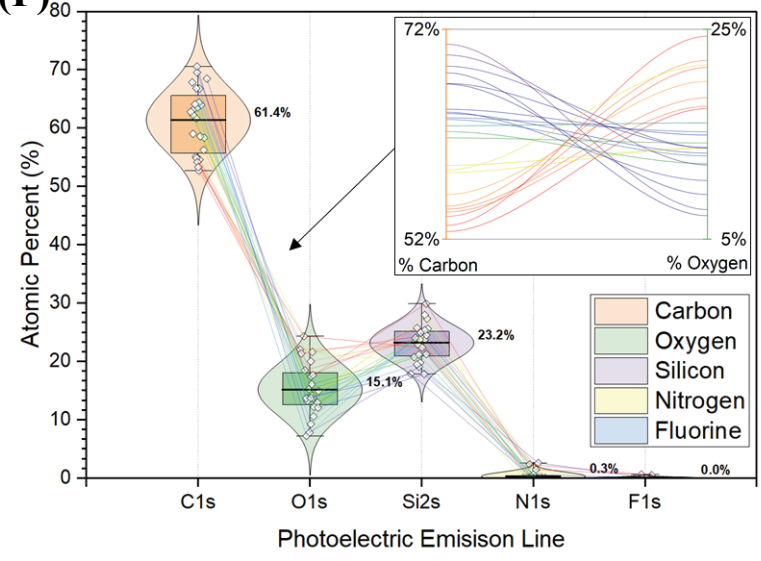

Figure 4.11: Parameters extracted from the analysis of survey (SUR) spectra and the C1s region from 23 randomly selected film produced using the standard synthesis process. (A) Violin and box plot key. (B) The number of carbon layers as calculated using the method depicted in Figure 4.10. (C) The percentage of carbon atoms within the film in a chemically defective state, assuming S2 is a pure buffer-layer peak, on a per layer basis (total percent defects divided by the calculated number of carbon layers). (D) The percent of carbon atoms in the carbon overlayer which are in a buffer-layer state $\left(\mathrm{S} 1+\mathrm{S} 2 / \mathrm{G}+\mathrm{S}_{1}+\mathrm{S}_{2}+\mathrm{D}_{3}+\mathrm{D}_{4}+\mathrm{D}_{5}\right)$. (E) The distributions binding energies for each peak within the fitted C1s region. The data points within the same dataset are linked; no correlation is observed. (F) Distribution of the atomic concentration as calculated from the survey data $(1000 \mathrm{eV}-\mathrm{oeV})$. Data points within the same dataset are linked. Inset, corollary plot showing that lower oxygen concentrations correlated to higher carbon concentrations.

The SOP process leads to an average of $\sim 2.7$ carbon layers (Figure 4.11(B)). Approximately $17 \%$ of all carbon atoms in the film participates in a defect bond. This corresponds to a lower limit of $\sim 6.3 \%$ of the carbons in each layer, when the number of layers in each case are considered (Figure 4.11(C))). This assumes that S2 is purely a buffer-layer peak and is not included as an oxide species in this calculation. Comparison of the bulk/surface spectra in Figure 4.7(B) indicated that the defects are preferentially segregated to the surface layers. However, by taking the defect percentage on a per layer basis, a more direct comparison can be drawn between the SOP films and films where both the number of carbon layers and total defect percentages vary 
greatly (as is the case for films produced during the parametric investigation studies, presented in Section $\$ 4.2)$.

Figure 4.11(D) shows that the SOP process results in $\sim 38 \%$ of the carbon atoms are associated with the buffer-layer. This was calculated by taking buffer-layer peak areas, $\left[\mathrm{S}_{1}+\mathrm{S}_{2}\right]$, as a percentage of all carbon overlayer related peak areas, $\left[\mathrm{G}+\mathrm{S}_{1}+\mathrm{S}_{2}+\mathrm{D}_{3}+\mathrm{D}_{4}+\mathrm{D}_{5}\right]$ (this counts all oxygen defects as residing in the graphene overlayer. This is a reasonable assumption based on the conclusions drawn in Sections $\$$ 4.2.4 - \$4.2.6). Figure 4.11(E) shows the distribution of binding energies of each peak used to fit the data for each sample. Data points from the same spectra (dataset) are linked. This shows no correlation between the relative binding energy locations of each peak within a dataset.

Lastly, Figure 4.11(F) shows the atomic concentrations as calculated from the survey spectra $(1000 \mathrm{eV}$ to $0 \mathrm{eV})$. Data points from the same sample (dataset) are linked. The inset corollary plot shows a generic correlation between low oxygen concentrations and high carbon concentrations and vice versa. 


\section{\$4.1.3 Characterization by Raman Spectroscopy}

Raman analysis of a representative set of SOP samples is shown in Figure 4.12. The spectrum for the graphinated sample is from a film shown to have a combined buffer-layer and graphene thickness of $0.7 \pm 0.1 \mathrm{~nm}$ ( $\sim 1 \mathrm{ML}$ of buffer-layer $+1 \mathrm{ML}$ of graphene, or $\sim 2$ complete carbon layers in total) as determined from XPS analysis. Contributions from the $\mathrm{SiC}$ substrate are clearly evident in this spectrum. The difference spectrum is obtained by scaling and subtracting the graphinated spectra from the reference spectra. The resulting spectrum is representative of the carbon overlayers only. This spectrum is shown in Figure 4.12(A). Close analysis of the subtracted spectra shows the presence of peaks identified as the $\mathrm{D}, \mathrm{G}, 2 \mathrm{D}, \mathrm{D}+\mathrm{D}^{\prime}$ and 2D'. These peaks are routinely seen in graphene. ${ }^{(549)}$ It is clear that the $G$ peak cannot be adequately fitted to a single peak (see Appendix $\boldsymbol{\S} \boldsymbol{A . 1 . 5}$ for supporting information) and is revealed to be bimodal.

(A)

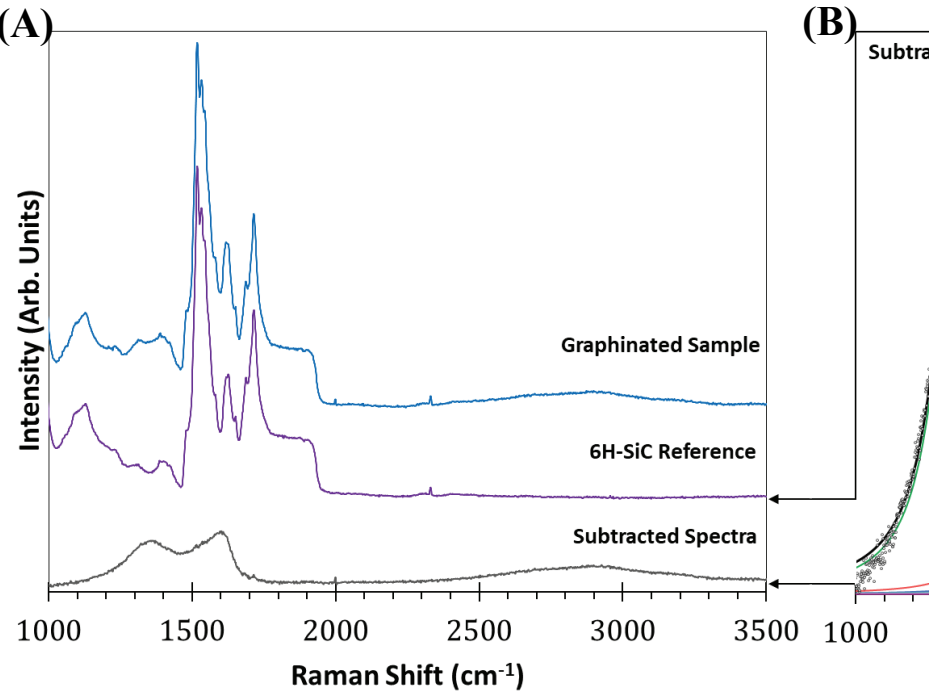

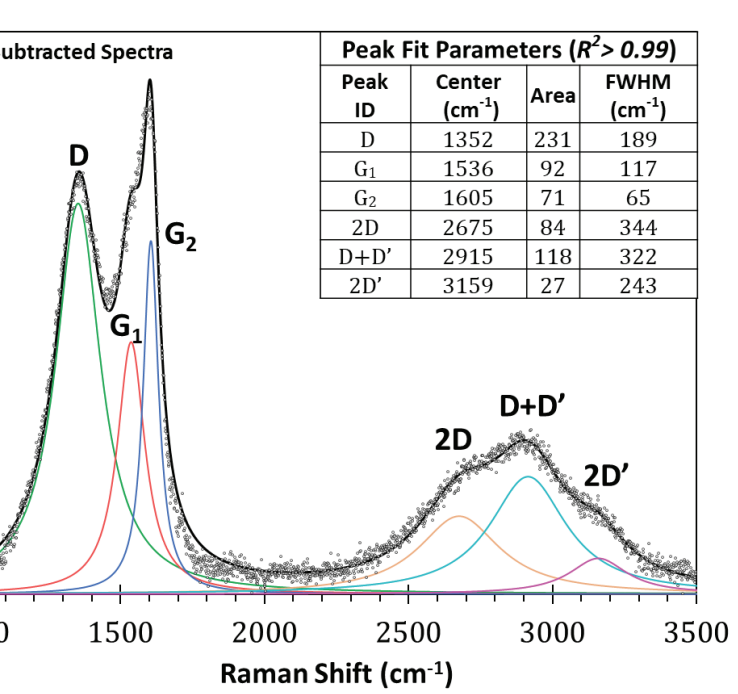

Figure 4.12: (A) The raw Raman signal from a graphinated sample produced using the standard process (TOP), Raman spectra from a as-received 6H-SiC sample (MIDDLE) and the result of the scaled subtraction of the two (BOTTOM) revealing the contributions of the carbon overlayers to the raw signal from the graphinated sample. (B) The fitting results from fitting the subtracted spectrum with a 6-peak fit.

As indicated in Figure 4.12(B), the dominant peaks associated with the graphene film are located at $1352 \mathrm{~cm}^{-1}\left[189 \mathrm{~cm}^{-1}\right], 1566 \mathrm{~cm}^{-1}$ (the weighted average of peaks at $1538 \mathrm{~cm}^{-1}\left[188 \mathrm{~cm}^{-}\right.$ $\left.{ }^{1}\right]$ and $\left.1603 \mathrm{~cm}^{-1}\left[70 \mathrm{~cm}^{-1}\right]\right), 2667 \mathrm{~cm}^{-1}\left[313 \mathrm{~cm}^{-1}\right], 2917 \mathrm{~cm}^{-1}\left[296 \mathrm{~cm}^{-1}\right]$, and $3150 \mathrm{~cm}^{-1}\left[187 \mathrm{~cm}^{-}\right.$

${ }^{1}$ ]. Here, the numbers in brackets are the corresponding FWHM values for each component peak of the spectrum. Although it is important to note that these Raman peak positions may be shifted due to doping and strain, they are in generally good agreement with the $\mathrm{D}$, the $\mathrm{G}$, the $2 \mathrm{D}$ (also called G'), the D+D' (also called D+G), and the 2G' (also called G+D') peaks reported by Cancado et al. for exfoliated graphene. ${ }^{(550)(494)(551)}$ There are four possible physical interpretations for the 
overall character of the Raman spectra that are presented in this work. What follows is a discussion and evaluation of each case.

In their studies, Cancado, et al. investigated the effects of crystal defects produced by Ar+ ion bombardment of exfoliated graphene. ${ }^{(494)}$ Starting with largely defect free single layer (1 ML) graphene which exhibited the usual well defined G and 2D peaks, they observed the emergence of defect related peaks including the $\mathrm{D}, \mathrm{D}+\mathrm{D}^{\prime}$ and G+D'(2D'). These peaks, as noted above can be associated with the corresponding spectral features observed here. In addition, as the defect density increased, significant peak broadening was observed. Specifically, as the defect separation decreased from $24 \mathrm{~nm}$ to $2 \mathrm{~nm}$, the FWHM increased from $20 \mathrm{~cm}^{-1}$ to $75 \mathrm{~cm}^{-1}$ for the D peak, 10 $\mathrm{cm}^{-1}$ to $40 \mathrm{~cm}^{-1}$ for the $\mathrm{G}$ peak, and $25 \mathrm{~cm}^{-1}$ to $140 \mathrm{~cm}^{-1} 2 \mathrm{D}$ peak. As seen in Figure 4.12(B), the broadening observed here is typically a factor of two greater. Based on these observations, defect levels do not appear to provide an obvious explanation of the peak broadening.

The situation is complicated, however, by the fact that based on similar studies, Ferreira, et al. report line broadening comparable to the levels reported here. ${ }^{(551)}$ Consequently, based solely on the Raman spectra, crystal defects cannot be ruled out as the source of the observed line broadening. It is important to note, however, that the Raman spectra for the graphene produced here using the atmospheric pressure RTA processes are in every way comparable to those for graphene produced using the UHV annealing process. Moreover, the UHV annealed films yielded well defined reflection high-energy electron diffraction (RHEED) patterns which indicate a high level of crystal perfection. Based on this, lattice defects as a source of the observed line broadening are not likely. ${ }^{(263)}$

A second potential explanation for the observed broadening of the Raman peaks is the presence of oxygen-based defects on the films. In fact, broadening of the type seen here is found in the Raman spectra for GO and RGO. (543) (552) (553) Based on XPS analyses, the oxygen-based defect levels for RGO and GO are on the order of $40 \%$ to $60 \%$, respectively. ${ }^{(542)}$ This is well above the most aggressive calculation of the upper bound of oxygen-based defect levels found for these films $(<25 \%)$ as discussed previously. Moreover, for these materials, and particularly for RGO, the ratio of $\mathrm{D} / \mathrm{G}$ are much higher then what are observed here (although the splitting of the $\mathrm{G}$ peak, and the various method for calculating, by area or intensity, makes a direct comparison of the D/G ratios complicated). Further, the two phonon components, if present, are less significant than in 
the present case. Consequently, oxygen-based defects do not appear to be a strong candidate for the broadening seen here.

The third, and most plausible, explanation for the observed peak broadening is related to the presence of the buffer-layer. The XPS results presented above show a clear indication of a buffer-layer between the graphene and the SiC substrate. Several groups have investigated the evolution of the Raman spectrum during the formation of graphene by sublimation of Si from SiC. (220) (189) (212) (554) Both Schumann, et al. as well as Strupinski, et al. report Raman spectra for the buffer-layer with supposedly no graphene overlayer. ${ }^{(220)(189)}$ These spectra are essentially replicas of that shown in Figure 4.12(B) with respect to both peak broadening and relative intensities for the D, G, and two phonon region. In contrast, however, Fromm, et al. observe the D and G peak broadening equivalent to that reported here, but they do not observe any peaks in the two phonon region. (212) As pointed out by Strupinski, et al, this discrepancy may be partially due the discontinuous nature of the graphene films formed by Si sublimation from SiC and the fact that the laser spot covers both buffer and graphene areas at the same time in some cases. ${ }^{(189)}$

Fromm, et al. make the crucial observation and demonstrate that the Raman spectra for the buffer-layer represent a mapping of the vibrational density of states (vDOS) of the buffer-layer. ${ }^{(212)}$ Further, they show that the vDOS continues to contribute to the graphene spectrum to a smaller and smaller extent as the graphene is increasingly decoupled from the buffer-layer and the D, G, and 2D peaks emerge. Consequently, the XPS and Raman results presented here suggest that the graphene films produced by the RTA process have not been fully released from the buffer-layer. As discussed later (Section $\$ 5.1$ ), this conclusion is supported by the results of the electrical measurements presented here.

A fourth possible explanation, which is not entirely consistent with the XPS and electrical measurements but cannot be ruled out, is that the graphene films are nanocrystalline. A discussion of this possibility is included here for completeness. Robinson, et al. studied the nucleation of graphene on $\mathrm{SiC}$ (0001) surfaces using the sublimation process. ${ }^{(555)}$ They observed graphene nucleation at step edges on the surface and, using $488 \mathrm{~nm}$ radiation. Moreover, they found that the $\mathrm{D} / \mathrm{G}$ (peak area) ratio varied from $0.05 / 1$ to $0.73 / 1$ as the annealing temperature decreased from $1425^{\circ} \mathrm{C}$ to $1225^{\circ} \mathrm{C}$. They attributed this variation to temperature dependent changes in grain size. Similarly, Cancado, et al. have also studied the effect of grain size on the ratio of the D to G peak 
intensities for exfoliated graphene and have developed an empirical equation which related grain size to the $\mathrm{D} / \mathrm{G}$ ratio. ${ }^{(556)}$ More recently, Daas, et al. have found that growth on non-polar SiC produces nanocrystalline graphene, which had D/G ratios similar this work in many cases. ${ }^{(557)}$ In these studies, the crystallite size (La) was determined using the equation

$$
L_{a}=\frac{560 \mathrm{~nm} \cdot e V^{4}}{E_{l}^{4}} \times\left(\frac{I_{D}}{I_{G}}\right)^{-1}
$$

Here $\mathrm{E}_{1}$ is the laser energy in $\mathrm{eV}(532 \mathrm{~nm}=2.33 \mathrm{eV})$ and $\mathrm{I}_{D}$ and $\mathrm{I}_{\mathrm{G}}$ are integrated intensities (areas) of the respective peaks. Based on the various $\mathrm{D} / \mathrm{G}$ ratio calculated for samples produced using the SOP process, the estimated size of the graphene nanocrystallites formed by the two-step process described here are on the order of 10-50 nm (via (4-1).

It is possible that the last two scenarios are occurring simultaneously. The weight of evidence of XPS, Raman, and electrical analysis suggests that the intensity and line broadening observed here are primarily due to coupling of the vibrational density of states (vDOS) of the buffer-layer. However, the graphene overlayers (the quasi-freestanding graphene layers on top of the buffer-layer) may also exist as nanocrystalline graphene (nano-sized domain sizes). However, determining how much of the Raman character is due to the coupled vDOS and how much is due to the nanocrystallinity is difficult to determine. Therefore, the nanocrystalline aspect of the films are not considered explicitly in this work. Furthermore, if both scenarios are operating, it is likely that (4-1 is no longer valid and would lead to a under estimate of the domain size (due to the as yet unaccounted for effect of the buffer-layer on the Raman spectrum). The conclusion reached here is that the films most certainly have a buffer-layer and additionally, the graphene overlayer may also have an element of nanocrystallinity.

The preceding Raman analysis paradigm was used as the basis for all films produced in this work. The statistical distribution of select key parameters extracted from the fit of the Raman spectrum of 15 randomly selected SOP samples are shown in the box and violin plots of Figure 4.13. The broad nature of the peaks contributes to the range of peak locations seen in Figure 4.13(A). The range of peak areas and FWHM values encountered with films produced using the standard method are shown in Figure 4.13(B,C), respectively. The $\mathrm{D} /\left(\mathrm{G}_{1}+\mathrm{G}_{2}\right)$ ratio, commonly cited as a figure of merit for Raman analysis of graphene, is shown in Figure 4.13(D). From their Ar+ bombardment studies, Cancado, et al. have determined a relationship between the D/G ratio 
and the distance between defect sites. Applying their empirically derived curves to the average $\mathrm{D} / \mathrm{G}_{1+2}$ ratio of the standardly produced samples here indicated an average defect spacing of either $\sim 2 \mathrm{~nm}$ or $\sim 9 \mathrm{~nm}$, depending on which side of the curve the data fall. ${ }^{(494)}{ }^{(549)}$ This is consistent with theoretical work conducted by A.C. Ferrari, et al. ${ }^{(500)}$
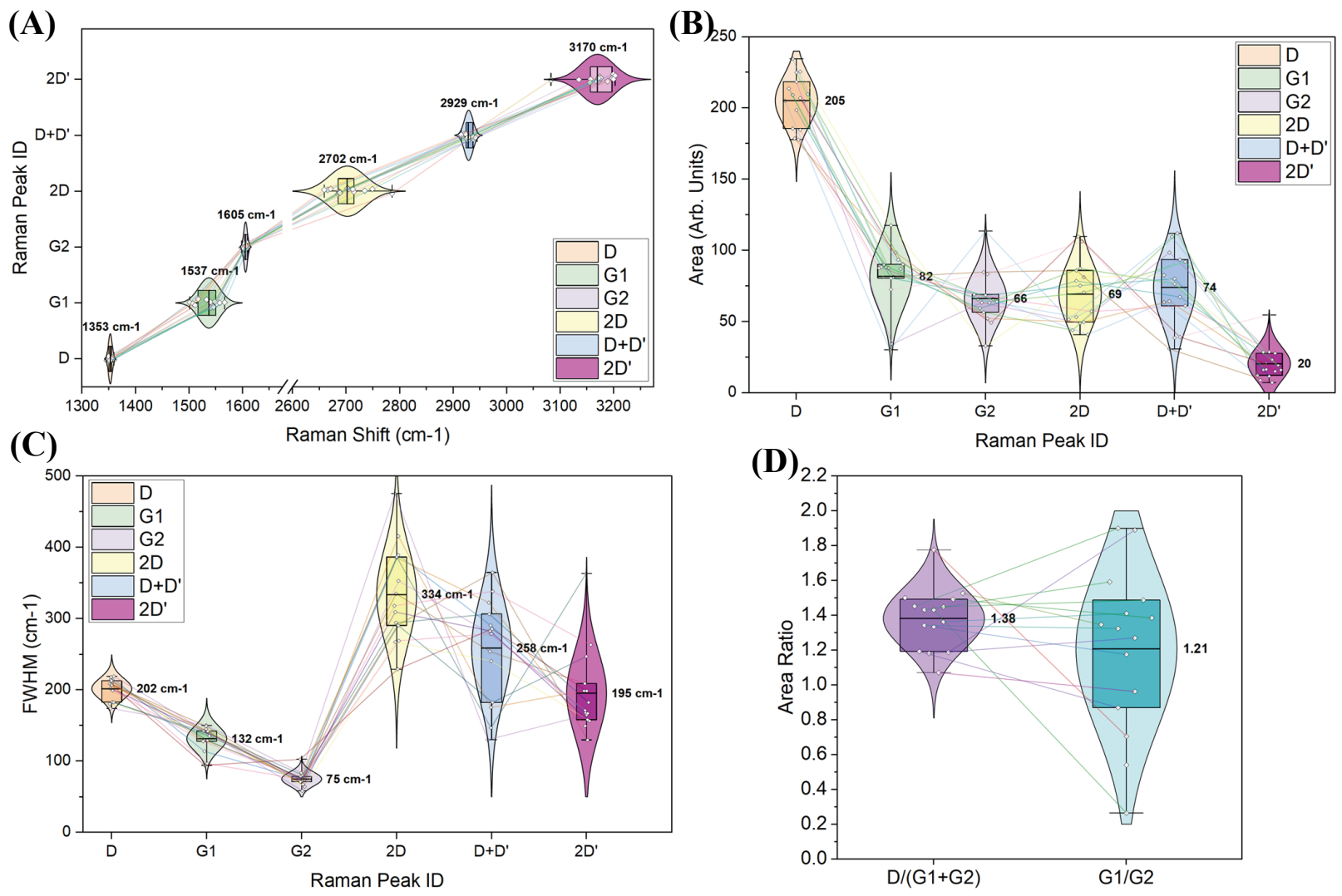

Figure 4.13: Statistical analysis of the fitted Raman data from 16 randomly selected samples produced using the standard method. Datapoints within the same dataset are linked; no correlation was observed between any measured parameter. (A) Range of center peak values for each fitted peak. (B) Peak areas for each fitted peak. (C) FWHM values for each fitted peak. (D) The D/G $[D /(G 1+G 2)$ in this case] ratio and the $\mathrm{G} 1 / \mathrm{G} 2$ ratios.

Also seen in Figure 4.13(D) is the $\mathrm{G}_{1} / \mathrm{G}_{2}$ ratio. The precise nature of the $\mathrm{G}$ peaks is difficult to determine. This is because strong overtones of the longitudinal and transverse optical modes (TO and LO peaks) from the $\mathrm{SiC}$ substrate lie directly over the same range as the $\mathrm{G}_{1}$ peak (these are seen in the spectrum from a $6 \mathrm{H}-\mathrm{SiC}$ reference sample in Figure 4.12(A)). This results in noise around the $1600 \mathrm{~cm}^{-1}$ region in the subtracted spectrum, masking the finer details of the data and is unavoidable as others have noted. ${ }^{(212)}$ Consequently, this leads to a degree of uncertainty in the details of peaks. This is particular true for deconvoluting the G peaks. At a minimum, however, it is clear there are two G peaks (see Appendix $\boldsymbol{\S} \boldsymbol{A}$.1.5 for supporting information). 


\section{\$4.1.4 Summary of the Characterization Results from Graphene Films Produced by the Standard Growth Process}

In summary, the standard film synthesis is a two-step process. The first step is a combined inductively coupled plasma and reactive ion etch (ICP-RIE) etching process and the second is an atmospheric pressure rapid thermal anneal (AP-RTA) annealing process. The overall process is illustrated in a thermodynamic context in Figure 4.14(A). The etch step was observed to have a smoothing effect on the SiC surface. The crystal step edges, as well as surface crystal defects (step edge protrusions from the $\mathrm{SiC}$ surface), were etched away. This is not unexpected as the planarization effect of $\mathrm{CF}_{4}$ plasma etch on $\mathrm{SiC}$ has been reported in the literature. ${ }^{(558)}$ The etching process is a combination of chemical etching (due to reactions that from volatile species) and physical etching (due to energy transfer from energies ions like $\mathrm{CF}_{\mathrm{x}}^{+}$). Although there may be some preferential (high rate) crystallographic directions, these are overwhelmed by the much higher vertical (normal to the surface) physical rate associated with the plasma ions. This is increasingly true for as the RIE power (effecting bias voltage) increases. Consequently, the ICPRIE process produces a planarizing etch. An illustration of the planarization process is shown schematically in Figure 4.14(B).

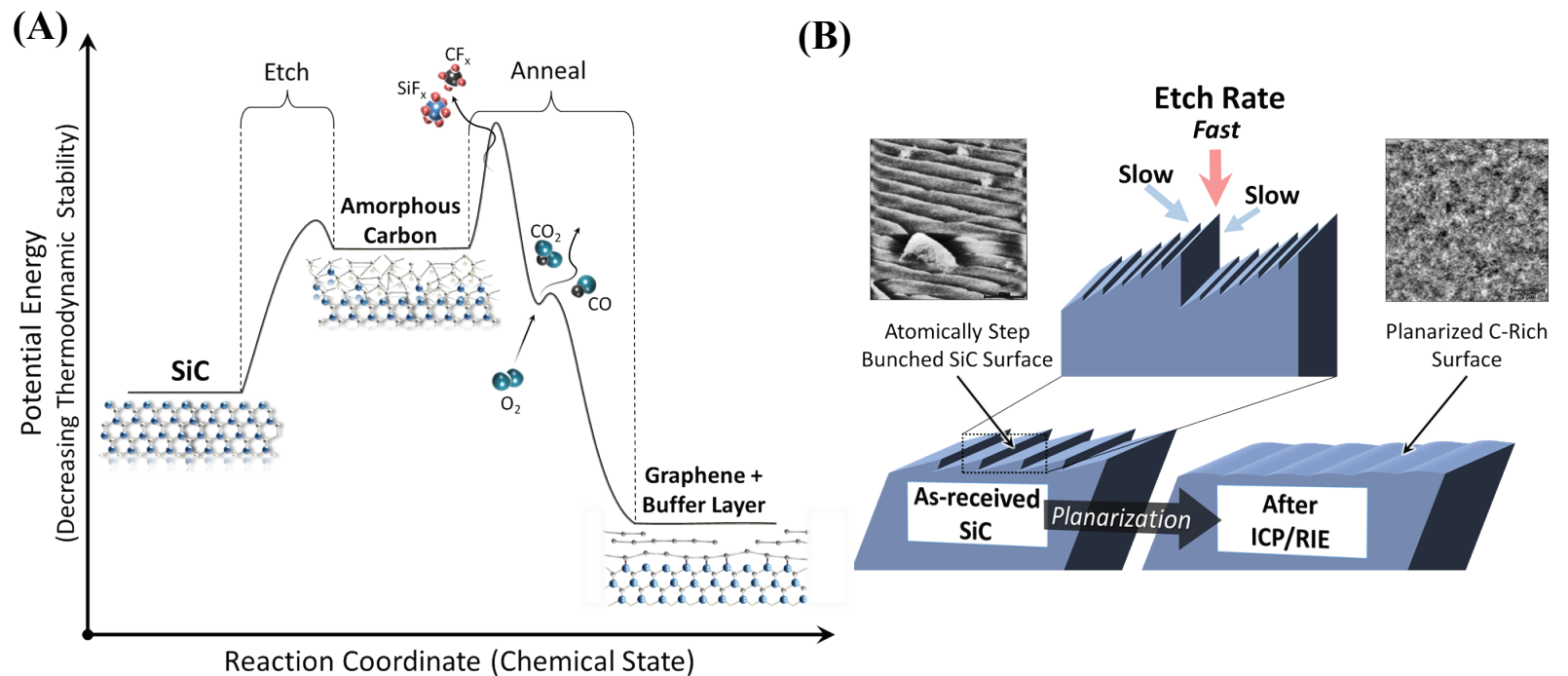

Figure 4.14: (A) Simple reaction coordinate diagram showing the generic steps in the standard synthesis process. (B) Schematic showing the planarization of the atomically stepped surface due to the aggressive etching action of the plasma.

XPS analysis of the post-etched material shows the surface to be largely amorphous (as determined from the wide distribution of carbon related chemical species observed in the $\mathrm{C} 1 \mathrm{~s}$ region of XPS and the weak and poorly defined Raman spectra see for Appendix $\boldsymbol{\$ A}$ A.1.6 
supporting information). More importantly this surface is carbon rich, due to the selective removal of $\mathrm{Si}$ from the $\mathrm{SiC}$ matrix by the $\mathrm{CF}_{4}$ plasma. Additionally, XPS analysis of a pure silicon sample exposed to the standard process (a $\mathrm{CF}_{4}$ ICP/RIE etch and AP-RTA anneal) do not show the formation of surface carbon. Furthermore, etching of $\mathrm{SiC}$ with alternative carbon free halogen gases, $\mathrm{Cl}_{2}$, results in a similarly rich carbon surface. These two observations indicated that the carbon atoms of the graphene overlayers originate from $\mathrm{SiC}$ substrate carbon and not the carbon component of the etchant gas. This has also been demonstrated in previously reported work using this method. ${ }^{(262)}$

As depicted schematically in Figure 4.14(A), the AP-RTA step adds thermal energy to the carbon overlayer and allows the system to leave the local energy minima and fall down the thermodynamic potential energy hill into a more stable and more crystalline form. Higher fluorinated silicon $\left(\mathrm{SiF}_{\mathrm{x}}\right)$ and carbon $\left(\mathrm{CF}_{\mathrm{x}}\right)$ species will also be desorbed. Oxygen present during the AP-RTA (either as a surface contaminant remaining from the etching step or newly introduced during the AP-RTA step) will scavenge carbon from the surface (by as a simple combustion reaction) reducing the overall number of carbon layers, and contributing to chemical defect states in the remaining carbon. Thus, the observed correlation between higher oxygen content and fewer carbon layers as shown in Figure 4.11(F).

This smooth and featureless carbon rich SiC surface presents an inherently different surface from which to grow graphene, as compared to the highly crystalline atomically stepped SiC surface used with the selective sublimation methods. Many of the complexities involved with the selective sublimation method, which stem from various structural irregularities of the starting surface (such as crystal face, atomic step bunching, preferential growth on step edges, pit formation, etc., discussed in Section $\$ 2$.1.3), may be diminished with this plasma assisted method. However, many aspects of the plasma assisted method remain to be investigated.

After the AP-RTA step, the samples were analyzed with XPS and Raman spectroscopy. The analysis shows a complete removal of fluorine related species remaining from the etch step. A densification of the surface film, as a result of the increase in crystallinity of the surface, is also observed.

As will be discussed later in more detail, what others have reported on similar epitaxial $\mathrm{SiC} /$ graphene systems has been used as a starting framework to interpret the characterization 
results (even though the synthesis methods differ substantially). This has led to the following conclusion: The plasma assisted method reported here results in a strongly coupled buffer-layer, along with a fractional-layer to few-layer graphene overlayer. There is a strong coupling of the vibrational density of states (vDOS) via the covalent bonding of the buffer-layer to the $\mathrm{SiC}$ substrate. While the fine details of this system are not completely understood at this time, this current interpretation is reasonable and correlates generically with what others have reported.

There remain, however, some idiosyncrasies with this analysis that are worth noting for a comprehensive summary. For example, XPS analysis occasionally indicates that there is slightly more than one layer's worth of carbon participating in the buffer-layer $\left(\mathrm{S}_{1}+\mathrm{S}_{2}\right)$, which does not strictly comport with the current description of the system. This could be explained by a number of factors, with the last (\#5) being the most likely:

1) Simple peak fitting errors (over contribution of $S_{1}$ and $S_{2}$ ).

2) Errors caused by the overlap between the $\mathrm{S}_{2}$ and $\mathrm{C}-\mathrm{OH}$ peaks; $\mathrm{C}-\mathrm{OH}$ defect species formed on the graphene surface may incorrectly be attributed to the $\mathrm{S}_{2}$ peak and erroneously contribute to the buffer-layer carbon count when they should be counted with the graphene overlayer.

3) Unaccounted for differences in the mean free path (MFP) of photoelectrons transiting though the buffer-layer and the graphene layer (the layer model assumes the MFP is uniform throughout the entirety of the carbon film).

4) Corrugations in the buffer-layer (which others have observed and as is detailed in Section \$2.1.3) leading to differences in the areal carbon densities between the buffer-layer and graphene layer; the layer model used here assumes they are identical. (This would result in differences in MFPs)

5) The current physical description of the buffer-layer may be incomplete, in that the interface between the substrate and the buffer-layer is more complex than what is depicted in Figure 4.9. Computational studies have shown there are geometric arrangements of the interface more carbons than would normally exist in a one atom thick mono layer of material could participate in a buffer-layer bonding, particular in the intermediate stages of buffer-layer formation. ${ }^{(213)}$

Because of these irregularities, throughout the rest of this work, the amount of carbon atoms in the film assigned as buffer-layer-related are taken as a percentage of the total carbon atoms (\%) of the film, rather than as a number of buffer-layers (\#). This avoids scenarios were more than a one-layer thick buffer-layer is present, which would be misleading and most likely 
would not be reflective of the reality. This is because what constitutes the buffer-layer is dependent on the understanding or model of the system, which may be incomplete. However, the fraction of carbon atoms which participate in the buffer-layer (peaks identified as $S_{1} \& S_{2}$ ) is a direct measurement taken from the deconvolution of the $\mathrm{C} 1 \mathrm{~s}$ spectrum. In this way, a meaningful comparison in the amount of buffer-layer carbons can be made between samples, without a need to generate a new physical model in each case (for example, see Section $\$ 4.2$ ).

Due to the tight association the buffer-layer has to the substrate, and the fact that the bufferlayer itself is grown directly epitaxially from the substrate, it stands to reason that the buffer-layer is strongly templated by the substrate. Thus, differences in substrates may explain some of the buffer-layer variations found in the literature reports for the selective sublimation method. Overall, this is indicative of complexities of the SiC-buffer-layer-graphene composite system. More importantly, it also indicates that not all buffer-layers are the same. However, even allowing for these variations, the buffer-layer produced here by the plasma assisted method stands apart from those produced using the selective sublimation method. As will be discussed later, the buffer-layer produced here appears to be more highly tethered, and there appears to be a larger fraction of carbon participating in buffer-layer bonding events.

Because the plasma assisted method reported here is inherently different than the selective sublimation method, there is even more reason to suspect that the buffer-layers generated in this work are substantially different from what others have reported. Given the very different way in which silicon is removed from the $\mathrm{SiC}$ substrate, the fact that the buffer-layers are different between the selective sublimation method and the plasma assisted method is not surprising. In the selective sublimation method, thermally energy is used to effectively 'vibrate out' silicon atoms from the substrate (sublimation). In the plasma assisted method, the $\mathrm{SiC}$ surface is impacted with energetic and chemically reactive plasma species which physically and chemically etch the surface. These different methods undoubtedly have different effects on the resulting interface between the carbon overlayers and the substrate. The room temperature electrical behavior of these SOP films are discussed in Section $\$ 5.1$ and, as a function of temperature, in Section \$5.2.

In summary, the plasma assisted method is significantly different from any other $\mathrm{SiC}$ derived graphene syntheses method. Section $\$ 4.2$ begins to explore some of the key synthesis 
parameters and their effect on the resulting material. As will become evident, however, these studies only scratch the surface.

\section{\$4.2 Graphene Synthesis Parametric Investigation}

This section will explore the effects of various processing parameters on the graphene films. These parameters include substrate selection as well as plasma and annealing conditions. Films were characterized using primarily Raman spectroscopy and XPS. Electrical characterization of devise fabricated using select non-SOP conditions are discussed in Section $\$ 5.1 .4$.

As discussed in the previous section and as detailed in Appendix $\boldsymbol{\$} \boldsymbol{A . 1 . 4}$, some of the variability observed in the standard films results from gradual changes in the processing equipment which occur over time. Therefore, when possible, an SOP sample is produced alongside the test samples as a reference. In this way, comparisons can be made to both a reference sample processed using equipment in a relatively similar condition as the test samples, as well as to the range of values typical for the SOP samples.

\section{\$4.2.1 Substrate Selection- Effect of SiC Polytype, Crystal Face and Doping}

This section explores the effects of the $\mathrm{SiC}$ polytype $(6 \mathrm{H}$ and $4 \mathrm{H})$ as well as orientation and doping. Recall, the SOP process utilizes the $6 \mathrm{H}-\mathrm{SiC}(0001)$ polytype. As noted previously, the $6 \mathrm{H}-\mathrm{SiC}$ polytype has six Si-C bilayers stacked in the sequence ABCACB to form the unit cell (diagrams of the various $\mathrm{SiC}$ crystal structures and other parameters are given in Section \$3.1.1). The (0001) surface is the Si-terminated basal (hexagonal) plane frequently referred to as the Siface. The $(000 \overline{1})$ surface orientation is $\mathrm{C}$ - terminated and is referred to as the $\mathrm{C}$-face. The $4 \mathrm{H}-\mathrm{SiC}$ polytype also has both $\mathrm{Si}$ - and $\mathrm{C}$ - face. The difference is that the unite cell for $4 \mathrm{H}-\mathrm{SiC}$ consist of four Si-C bilayers with a stacking sequence of $\mathrm{ABCB}$. An addition differentiator is the doping in each substrate. The SOP 6H-SiC(0001) substrate used have n-type electrical conductivity (doping) exclusively. The $4 \mathrm{H}-\mathrm{SiC}$, on the other hand, have either n-type or are semi-insulating (SI). Thus, for the $4 \mathrm{H}-\mathrm{SiC}$ orientations, additional variations of conductivity type are possible. In summary, the $6 \mathrm{H}-\mathrm{SiC}(0001)$ n-type represents the SOP while $6 \mathrm{H}-\mathrm{SiC}(000 \overline{1})$ n-type, $4 \mathrm{H}-\mathrm{SiC}(0001)$ n-type,

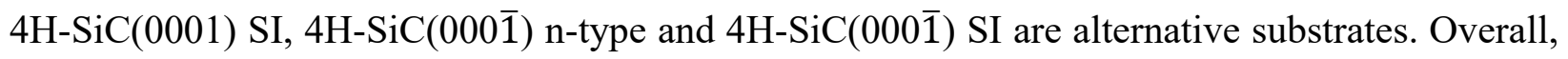
this represents five alternative (to the SOP) structures, orientations and conductivities. 
Raman spectra of the surface film resulting from the ICP/RIE etch and AP-RTA steps preformed on various substrates are shown in Figure 4.15(A). While there are some subtle differences between the spectra, these differences fall well within the range of variability observed for the SOP process carried out on the Si-face of a $6 \mathrm{H}-\mathrm{SiC}$ substrate. Analysis of the deconvoluted data bears this out as well. Peak locations, FHWM and peak areas are similarly within the range of values seen from the standard process conducted on Si-terminated face of a $6 \mathrm{H}-\mathrm{SiC}$ wafer.
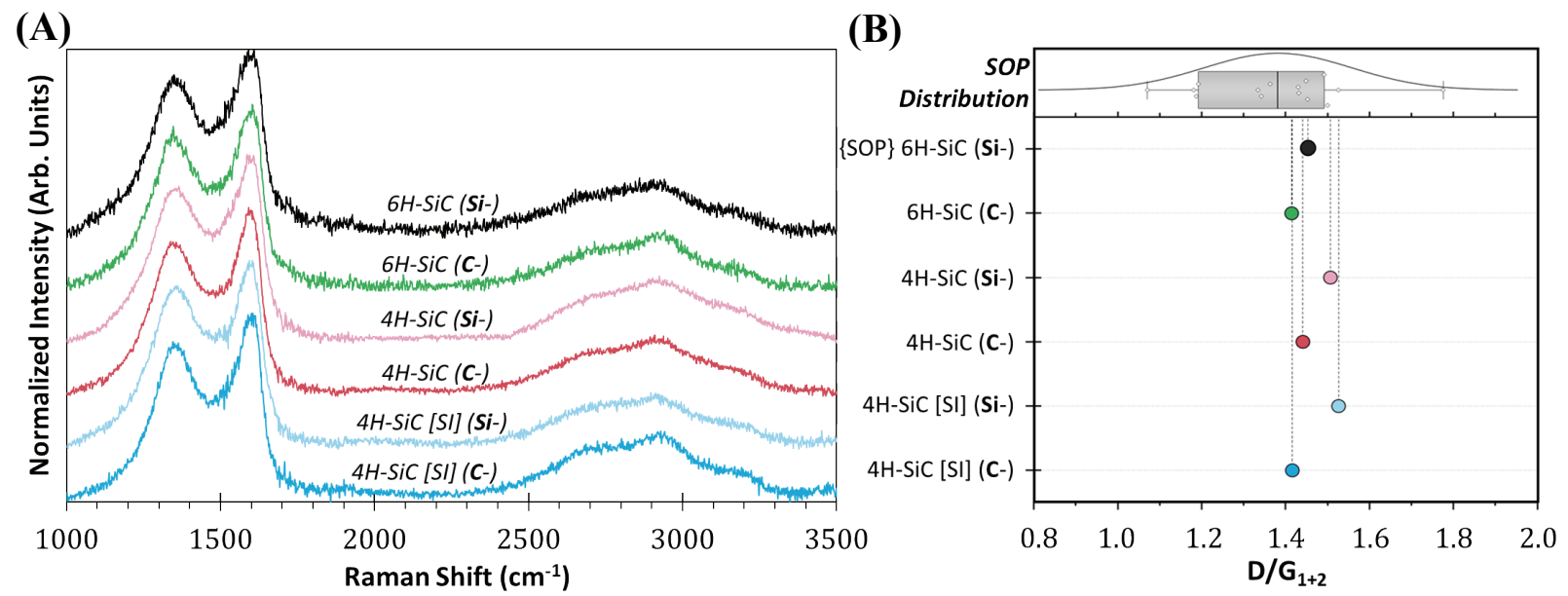

Figure 4.15: (A) Raman spectra collected from films grown from both polar surfaces on three different substrate types, all using the Standard Synthesis parameters. The spectra are largely indistinguishable indicating a strong coupling of the vDOS across all samples, regardless of substrate choice. (B) The $\mathrm{D} /\left(\mathrm{G}_{1+2}\right)$ ratios. Regardless of substrate, the silicon face appears to produce films with a slightly higher $\mathrm{D} / \mathrm{G}_{1+2}$ ratio, indicating these films contain slightly more defect. However, all points fall largely within the statistical bounds calculated from the Standard Synthesis. The black colored data (top) labeled 6H-SiC $(\mathrm{Si}-)$ in $(\mathrm{A}, \mathrm{B})$ was collected from a Standard Sample process around the same time as the other samples included in the figure.

Shown in Figure 4.15(B) are the $\mathrm{D}_{/ \mathrm{G}_{1+2}}$ ratios (the $\mathrm{G}$ peak is taken as the sum of $\mathrm{G}_{1}$ and $\mathrm{G}_{2}$ ) for each substrate choice. As can be seen, all data points fall within the bounds of values collected from SOP samples. Nonetheless, it appears that etching the silicon face, regardless of the substrate, consistently results in films with a slightly higher $\mathrm{D} / \mathrm{G}_{1+2}$ ratio. This would seem to indicate that these films have a slightly higher defect level. Given the spread in the data observed for the standard process, further study is required to determine if this trend holds.

XPS analysis were also performed on the films. The C1s spectral regions are shown in Figure 4.16. Annotated in each panel are the number of carbon layers calculated, the percentage of surface carbon assigned as buffer-layer related, the percent of surface carbons involved in a chemical defect state and the elemental atomic concentrations. The table included in Figure 4.16 tabulates the peak areas in each case. 
The most readily apparent observation is that for the $4 \mathrm{H}$ polytype, a larger fraction of the $\mathrm{C} 1$ s region is assigned as buffer-layer related $(\mathrm{S} 1+\mathrm{S} 2$ peaks) and with less participating in the graphene overlayer ( $\mathrm{G}$ peak, $\mathrm{sp}^{2}$ hybridized carbon), while the total number of carbon layers is similar. In other words, the total amount of carbon is approximately the same on $6 \mathrm{H}$ and $4 \mathrm{H}$ polytypes, but a larger fraction is devoted to buffer-layer carbon in the $4 \mathrm{H}$ samples. This appears constant across crystal faces (both $\mathrm{Si}$ - and $\mathrm{C}$-). 

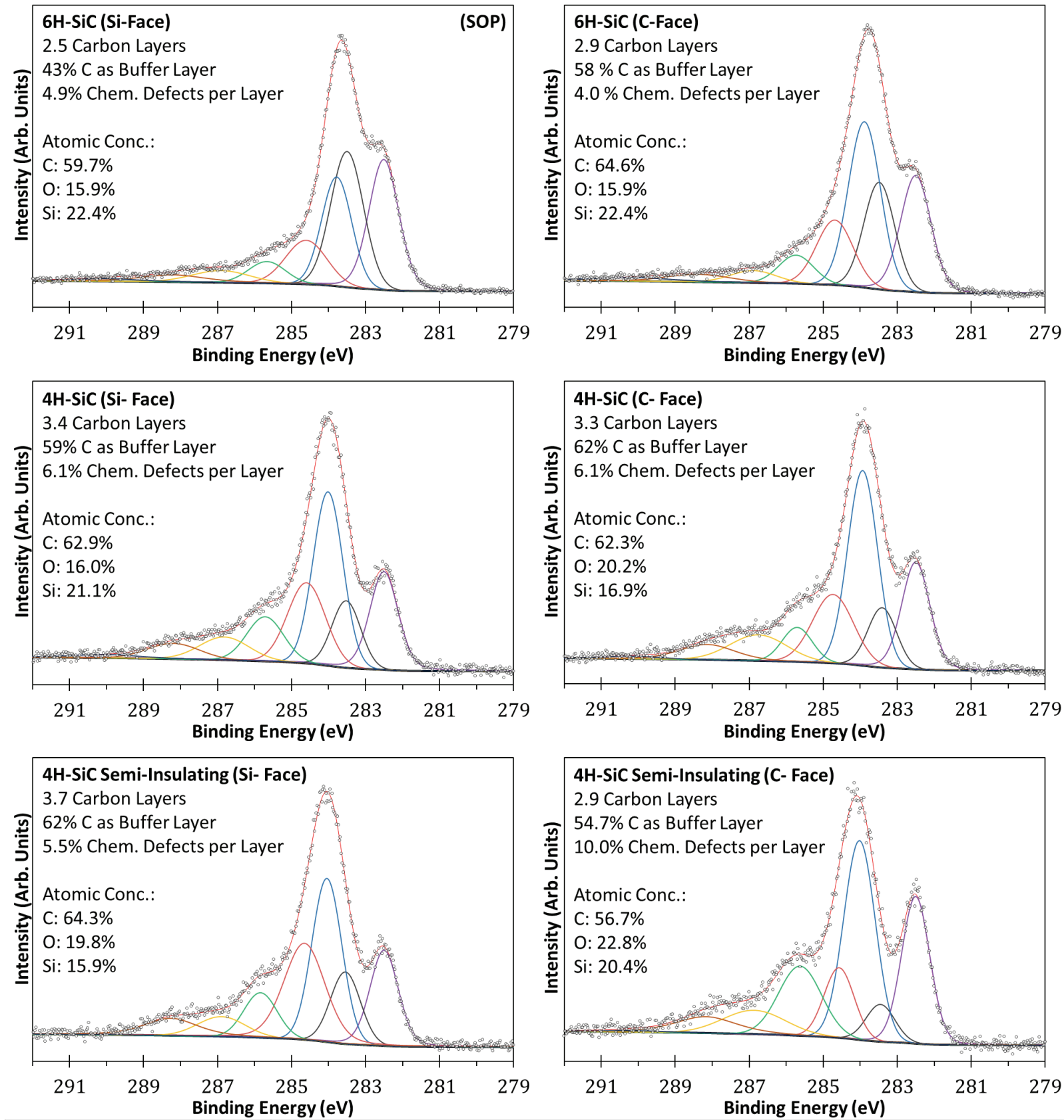

\begin{tabular}{|c|c|c|c|c|c|c|c|c|}
\hline \multirow[b]{2}{*}{ Polytype } & \multicolumn{8}{|c|}{ C1s Peak Areas } \\
\hline & SiC & $\begin{array}{c}G \\
\left(s p^{2}\right)\end{array}$ & $\begin{array}{c}\text { D1 } \\
\left(S_{1}\right)\end{array}$ & $\begin{array}{c}\text { D2 } \\
\left(\mathrm{S}_{2}, \mathrm{C}-\mathrm{OH}\right)\end{array}$ & $\begin{array}{c}\text { D3 } \\
(\mathrm{C}-\mathrm{O}-\mathrm{C})\end{array}$ & $\begin{array}{c}D 4 \\
(C=0)\end{array}$ & $\begin{array}{c}\text { D5 } \\
(\mathrm{C}=\mathrm{O}-\mathrm{OH})\end{array}$ & $\pi-\pi^{*}$ \\
\hline $6 \mathrm{H}-\mathrm{SiC}$ (Si-Face) & $26 \%$ & $28 \%$ & $21 \%$ & $11 \%$ & $5 \%$ & $4 \%$ & $3 \%$ & $1 \%$ \\
\hline $6 \mathrm{H}-\mathrm{SiC}$ (C-Face) & $22 \%$ & $19 \%$ & $31 \%$ & $14 \%$ & $6 \%$ & $3 \%$ & $3 \%$ & $2 \%$ \\
\hline $4 \mathrm{H}-\mathrm{SiC}$ (Si- Face) & $18 \%$ & $11 \%$ & $31 \%$ & $18 \%$ & $10 \%$ & $7 \%$ & $5 \%$ & $1 \%$ \\
\hline $4 \mathrm{H}-\mathrm{SiC}$ (C- Face) & $19 \%$ & $10 \%$ & $34 \%$ & $16 \%$ & $6 \%$ & $9 \%$ & $5 \%$ & $1 \%$ \\
\hline 4H-SiC Semi-Insulating (Si- Face) & $16 \%$ & $12 \%$ & $28 \%$ & $24 \%$ & $8 \%$ & $5 \%$ & $6 \%$ & $0 \%$ \\
\hline 4H-SiC Semi-Insulating (C- Face) & $22 \%$ & $5 \%$ & $32 \%$ & $11 \%$ & $15 \%$ & $8 \%$ & $7 \%$ & $1 \%$ \\
\hline
\end{tabular}

Figure 4.16: The C1s XPS spectral region, collected from films produced using the Standard Process but grown from various $\mathrm{SiC}$ polytype $(6 \mathrm{H}-, 4 \mathrm{H}-)$, crystal faces (C-, Si-) and doping (n-type, semi-insulating). Note that the SOP process uses $6 \mathrm{H}-\mathrm{SiC}$ Si-face and is naturally n-type doped. 
Figure 4.17 compares the number of layers, the percent defects per layer and the percent carbon in the film associated with the buffer-layer for each sample to the SOP samples (which use $6 \mathrm{H}-\mathrm{SiC} \mathrm{Si}$-face). Although there is a spread in the values, the number of carbon layers there does not appear to be a consistent relationship between the substrate type, crystal face or doping.

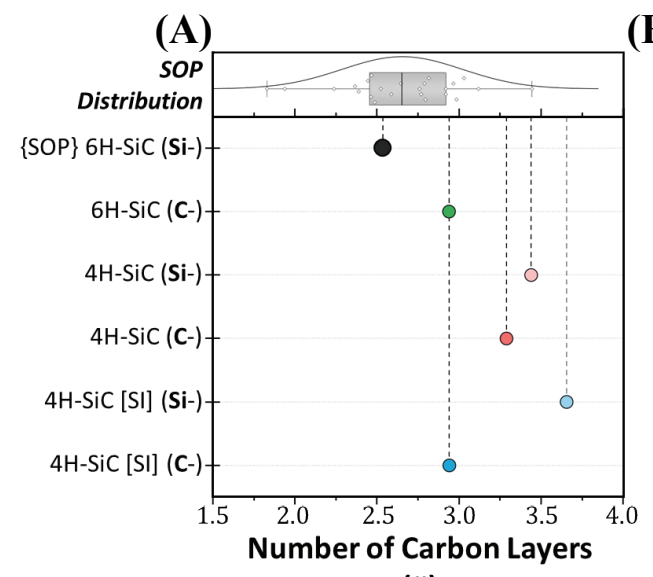

(\#)
(B)

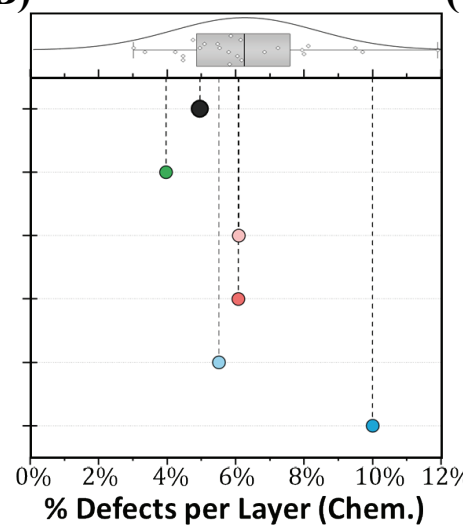

(\%)
(C)

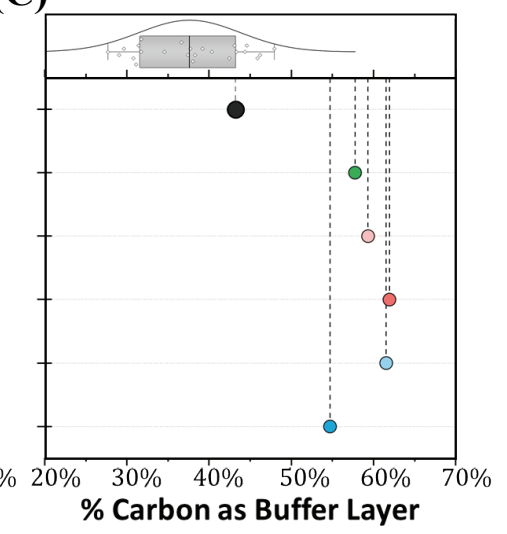

(\%)

Figure 4.17: Parameters calculated from XPS analysis. (A) The number of carbon layers. (B) The percentage of carbon atoms involved in a chemical defect on a per layer basis. (C) The percentage of carbon atoms participating in the buffer-layer.

These results further illustrate an impact of the substantial differences between this plasma assisted method and the selective sublimation method. The plasma assisted method, when carried out on the C-face surface, does not produce thicker films as compared to when it is carried out on the Si-face. This is counter to what has been widely reported for selective sublimation (where thicker films are almost always produced when the sublimation is carried out on the C-face). Considering the inherent differences in the Si removal process (between the plasma assisted method reported here and the selective sublimation) this is not suppressing. As will be discussed later, a key fundamental difference between the two methods is the effective penetration depth of each method. In the sublimation method, only a few atomic layers are affected by the process. Thus, if the terminal layer is composed of carbon (rather than silicon) the material used in the reconstruction process will contain more carbon which, when fully reconstructed into graphene layers, produces thicker films. Stated another way, the initial carbon layer makes up a substantial fraction of the surface atoms used to form the film. On the other hand, for the plasma assisted method, large amounts of both $\mathrm{Si}$ and $\mathrm{C}$ are removed. As previously stated, for the $6 \mathrm{H}-\mathrm{SiC}$ variant at least 11,100 Si-C bilayers are removed (likely comparable amounts are removed for the $4 \mathrm{H}$ 
variant). Thus, the initial carbon layer has little to no effect on the final thickness (it accounts for only $\sim 1 / 22,200$ of the total layers affected by the process).

Figure 4.17(B) compares the per layer chemical defect concentration for each substrate. It is shown to be likewise unaffected by the substrate, other than for the $4 \mathrm{H}-\mathrm{SiC}$ semi-insulating $\mathrm{C}$ face sample. Most other samples cluster around the average defect concentration value observed in SOP samples. Although it still falls within the range of values collected from SOP samples, the 4H-SiC semi-insulating C-face data point is most likely an outlier. This is reasonable because there is no physical basis to explain why a film grown from this specific surface would produce a film with a disproportionately high defect concentration.

Figure $4.17(C)$ plots the percent of carbon atoms in the film participating in the bufferlayer. The $4 \mathrm{H}-\mathrm{SiC}$ substrates appear to consistently produce films with a larger percent of carbon involved in the buffer-layer, specifically in the coupled state (the S1 peak). This observation can be interpreted in several ways. First, the peak assignment could be incorrect as a result of various substrate-induced electronic doping effects, which could shift the film peak positions outside of the bounds allowed for by the fitting criteria (the fitting bounds are given in Table 4-2). Unadulterated graphene/buffer-layer films grown on $4 \mathrm{H}-\mathrm{SiC}$ substrates show peak separation between the $\mathrm{SiC}$ and graphene components $\left(\Delta_{\mathrm{SiC}-\mathrm{G}}\right)$ of $\sim 1.00-0.80 \mathrm{eV}$, similar to the $\Delta_{\mathrm{SiC}-\mathrm{G}}$ on $6 \mathrm{H}$ of $\sim 1 \mathrm{eV}$. (534) (218) (242) Therefore, the fitting model used for $6 \mathrm{H}$ substrates is still valid for $4 \mathrm{H}$ substrates (i.e. the graphene peak does not have an intrinsically higher binding energy on $4 \mathrm{H}$ substrates). Oxygen induced buffer-layer decoupling has been shown to increase the $\Delta$ SiC-G from $1.0 \mathrm{eV}$ to $1.9 \mathrm{eV}{ }^{(559)}$ However, there is no reason to expect a spontaneous buffer-layer decoupling due to the use of $4 \mathrm{H}$ substrates. Further, the Raman spectra show no evidence of decoupling. Consequently, spontaneous decoupling has most likely not occurred.

The second possibility, and the most likely, is that the as-grown films have a greater propensity to remain coupled to a $4 \mathrm{H}$ substrate than to a $6 \mathrm{H}$ substrate. This may be due to the buffer-layer maintaining a more complex interfacing scheme with the $4 \mathrm{H}$ substrates. Molecular dynamic computational studies on the buffer-layer formation process predicted complex interfacing structures are possible. ${ }^{(213)}$ The fact that these complex interfacing schemes would result in a higher aerial atomic density of the buffer film may explain the apparent overcount in the amount of carbon in the buffer-layer (i.e. in cases where more than one layer's worth of carbon 
is calculated for the buffer-layer, which should only ever be, at most, one layer). However, this cannot be confirmed using the available analysis techniques and thus this scenario is larger speculative (this presents another opportunity for the utilization of more complex analysis techniques in future work). If this scenario is occurring, it is unclear what may drive the different interfacing mechanics. The answer most likely lies in differences in the surface plasma chemistry during the etch step.

The $\mathrm{C}$-face of the $6 \mathrm{H}-\mathrm{SiC}$ sample also shows a disproportionately large fraction of carbon assigned to the buffer-layer. However, this is more likely a function of the roughness of the starting surface. Recall that this was the only surface studied which was not chemo-mechanically polished to mirror finish by the manufacturer. Although the etch step does has a planarization effect on nano-scale features and the fact that 1000's of nm of material are removed, aspects of the large micron-scale roughness of the original unpolished surface are stenciled down though the crystal during the etch. The roughness is not fully removed on this surface as evident by an examination of the surface by the naked eye. Therefore, given the roughness of the surface, a possible explain for the large buffer-layer content may lie in a decreased carbon mobility. The remaining roughness of the surface may not allow for the carbon layer to gain enough mobility during the AP-RTA step to form contiguous areas of graphene overlayer growth over the buffer-layer. Others have shown that more highly polished surfaces result in better graphene which supports this conclusion. ${ }^{(560)} \mathrm{A}$ further complicating factor is that the rougher surface may also cause a slight overcount in the number of carbon layers. The layer model loses fidelity when the surface is not atomically flat due to surface scattering effects which tend to enhance surface species signals.

In summary, none of the substrates used produced radically different films. In fact, the films are largely similar. While there are some small differences between films, the tight coupling between vDOS (leading to a reduction and blending of vibrational modes) masks any finer details of the system and results in almost identical Raman spectra. The only difference between the films seen by XPS analysis is the higher buffer-layer content of films grown on $4 \mathrm{H}$ substrates. Therefore, based on this evidence it appears that the SOP process leads to roughly the same tightly coupled carbon overlayer (along with a fractional graphene overlayer) regardless of the crystal face ( $\mathrm{Si}$ - or $\mathrm{C}-)$, the polytype $(6 \mathrm{H}$ or $4 \mathrm{H})$ or the doping (n-doped or semi-insulating) of the starting material. While there are studies in the literature which show various substrate driven effects (such as growth 
rates, crystal quality and thickness), absent additional characterization, the plasma assisted process appears to be mostly insensitive to substrate type. ${ }^{(445)(561)}$

The primary reason for this could lie in the aggressive nature of the ICP/RIE etch step. Due to the large amount of material removed during the etching process (several 1000's of nm) the specific crystal face presented to the plasma at the start of the etching process and the order that the crystal bilayers are uncovered, is of little consequence. However, while the polytype does not appear to affect the fundamental nature of the film, it does affect the amount of coupling between the carbon overlayer to the substrate, with the $4 \mathrm{H}$ polytype leading to a higher degree of coupling.

Although there are undoubtedly differences between the films produced here that are not captured by the Raman and XPS characterization, this does illustrate one of the primary differences between this plasma assisted method and the selective sublimation methods. Specifically, the penetration depth of the silicon removal process. Due to the nature of the silicon removal process of the plasma assisted method, many atomic layers are affected at any one time in the etch process. The effect of the RIE on the crystal structure and its composition, is felt many atomic layers deep into the substrate (this is part of the reason for the planarization effect), and thus the specifics of the substrate are less important (the templating effect of the substrate is reduced). This is in contrast to the much more layer-by-layer process of the surface sublimation of $\mathrm{Si}$ atoms in the selective sublimation methods were only the top few atomic layers are affected in total.

Other than the amount of substrate coupling, the SOP process does not appear to be affected by the substrate choice based on Raman and XPS analysis. However, as will be discussed in Section $\$ 5.1 .4$ and $\$ 5.2 .3$, the electrical properties are substantially different.

\section{\$4.2.2 ICP/RIE Etching- Effect of Plasma Power}

The study detailed in this section explores the effect of the ICP power, RIE power and indirectly, the DC self-bias. The DC self-bias is an important 'side-effect' of the applied RIE power, but it is not a directly controllable parameter.

The ICP power is of interest because it affects the plasma density and species distribution. Higher ICP powers lead to a denser plasma. A larger fraction of the feed gas is ionized and that which is ionized, is ionized to a greater extent. This results in a species distribution skewed towards smaller and more reactive particles and higher electron temperature. 
The RIE power is of interest because it effects the DC self-bias, which is negative with respect to the main plasma cloud. The DC bias, which sets the electric field strength through which the positive ions are accelerated. Consequently, the DC bias effects the directionality and kinetic energy of the ions generated by the ICP. Higher RIE powers correspond to higher ion kinetic energy. The polarity of the DC-bias also sets the direction of the electric field and thus the direction of the ion motion. In this work, the plasma ions are most likely primely $\mathrm{CF}_{\mathrm{x}}^{+}(\mathrm{x}=1,2,3)$. As a result, the negative bias observed here caseous the positive ions to be directed towards the substrate.

In practice, the DC bias is influenced by a wide array of factors but is mostly a strong function of the RIE power. Figure 4.18 shows the relationship between ICP, RIE and the DC bias. The data points correspond to the parametric location of the samples used in this study and the average DC bias recorded during the etch. As was shown to be the case for the standard process (Figure 4.2), the DC bias varies over the duration of the etch in most cases. Plots of the DC bias as a function of etch time can be found in Appendix $\boldsymbol{\$}$ A.2.1). The black region is the parametric space where a plasma could not be ignited. Some combinations of programed ICP/RIE powers resulted in highly unstable plasmas. Therefore, to accurately reflect the conditions in the chamber, all plots used the read values for the ICP/RIE powers rather than the programed values. 
From Figure 4.18, it is clear that the DC self-bias is most strongly a function of the applied RIE power. Higher RIE powers result in higher -DC biases. The applied ICP power also has some effect, but to a lesser extent. Due to the low number of positive ions available to neutralize the sample plate at low ICP powers, low ICP powers result in high -DC biases.
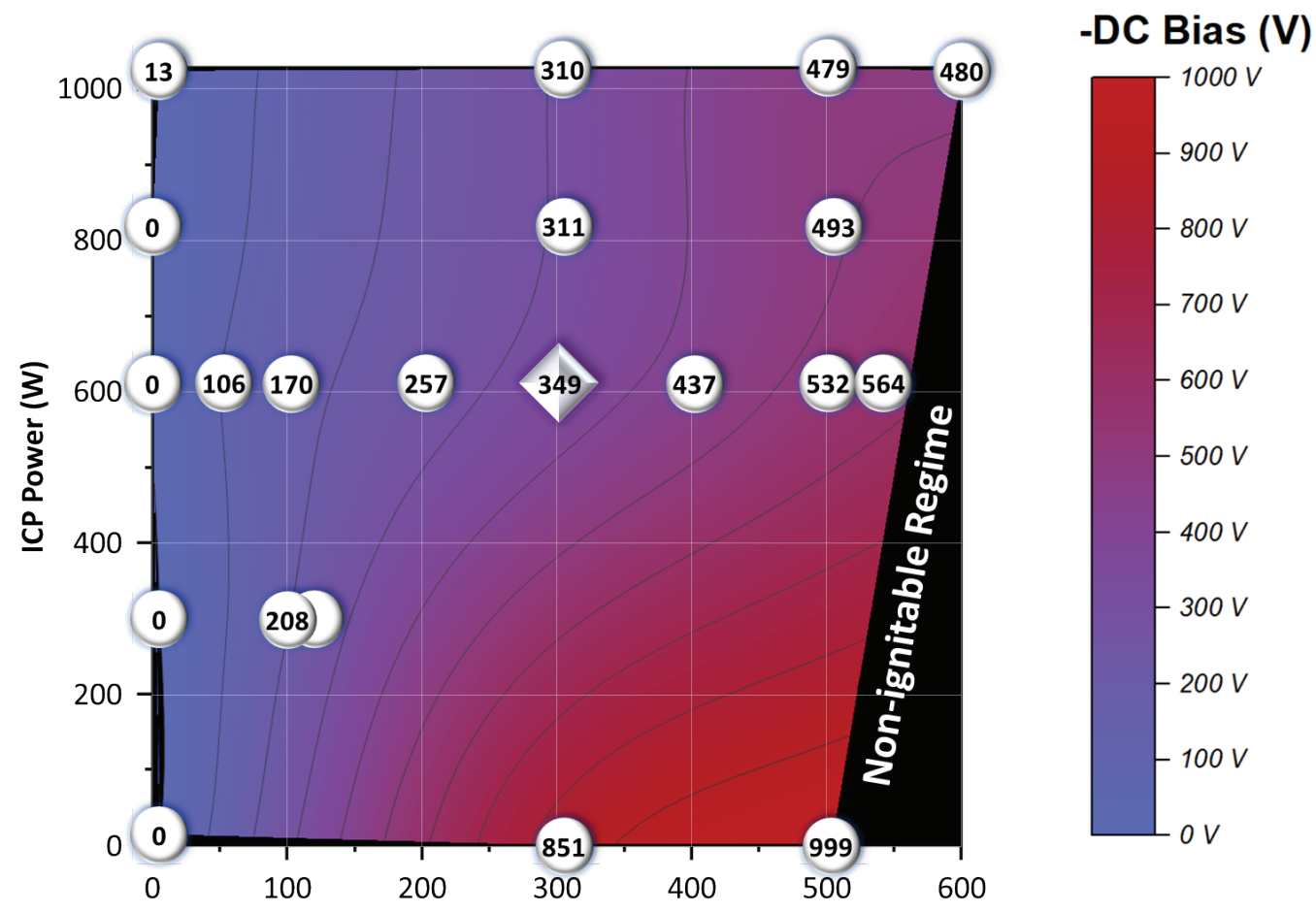

Figure 4.18: The relationship between ICP and RIE (read) powers to the DC bias developed on the sample chuck, as read by the tool. The diamond data point corresponds to the parameters used during SOP process (i.e., samples produced here are SOP samples). Higher RIE powers lead to larger DC biases. Contour lines appear at every $100 \mathrm{~V}$ increment. The area between points has been spline interpolated. The black regions correspond to ICP/RIE combinations where a plasma could not be ignited. Not all points which were ignitable resulted in a stable plasma.

It should also be noted that parameters other than the ICP and RIE powers effect the DC bias. Variations in the electrodynamics of the process chamber also effect the DC bias. These variations are primarily due to material build up on the chamber walls and the slow etching of some internal components (these effects are discussed in Appendix $\$ \boldsymbol{A . 1 . 4}$ and the etching of components in Appendix $\$ \boldsymbol{C}$.2). These effects contribute to the variations in the exact DC bias developed for a given ICP/RIE power combination at any given time. 
Although the RIE power is the independently varied parameter, it is the resulting DC bias which is most strongly correlated with the resulting film parameters. Furthermore, variations in electrodynamics of the chamber are captured in the DC bias value. Therefore, the same RIE power do not always result in the same DC bias. As such, it is convenient to plot the ICP power vs. -DC bias to extract generic trends (plots of ICP power vs. RIE power for the number of carbon layers and the percent carbon as buffer layer are included in Appendix $\$$ A.2.2). Figure 4.19(A) shows a heat map distribution in the number of carbon layers across the parameter space. Figure 4.19(B) shows the percent of carbon in the film which is associated with the buffer-layer. Analysis of these plots allow for some general conclusions to be made about the process, discussed next.
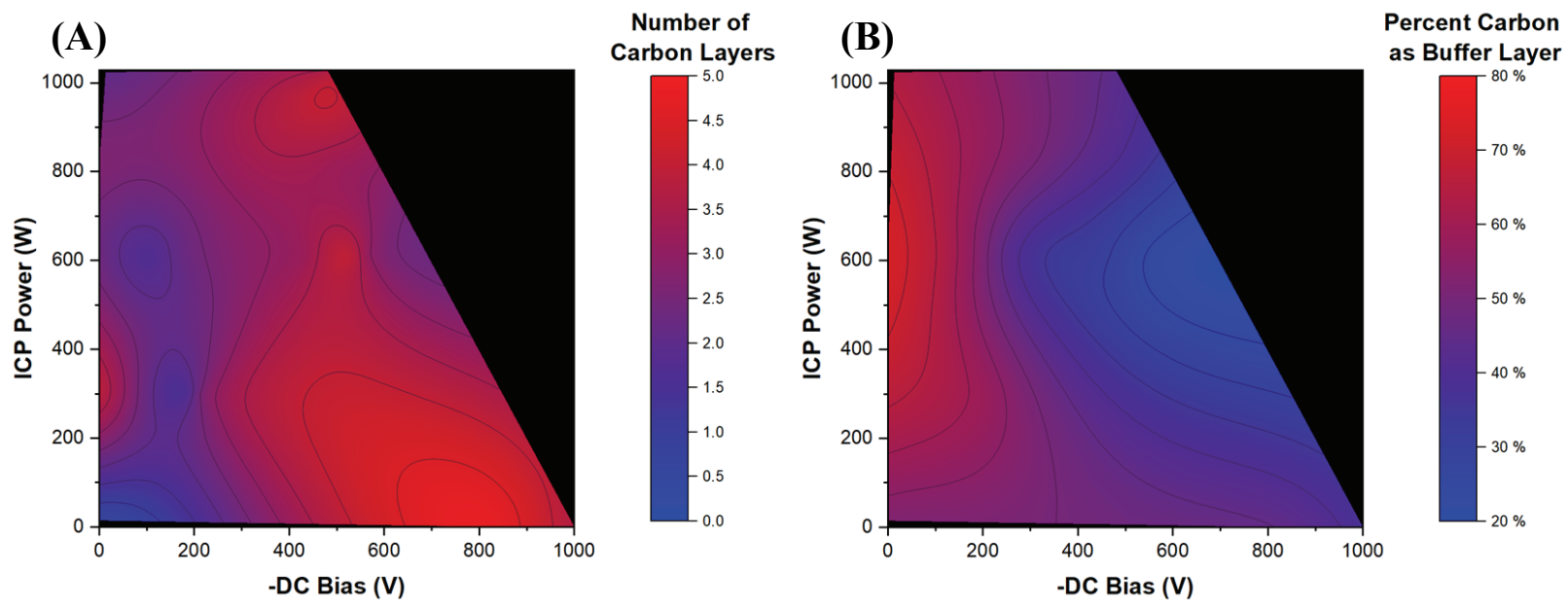

Figure 4.19: (A) The variation in the number of carbon layers is seen as a function of ICP power and the DC self-bias. (B) The percentage of carbon atoms in the film which are participating in the buffer-layer.

The ICP-RIE etching process is a combination of physical etching (due to sputtering caused energy and momentum transfer) as well as chemical etching (due to reactions between the surface and reactive plasma species and the subsequent formation and removal of volatile chemical species). All process parameters influence film thickness. However, the DC bias (the electrical potential which develops between the plasma and substrate), plays a particularly useful and intuitive role. A more negative DC bias increases the kinetic energy and momentum transfer of positive ions impinging on the $\mathrm{SiC}$ surface result in thicker films (more layers) as seen Figure 4.5(A). The enhanced chemistry which occurs at higher ICP powers (due to the denser and more reactive plasma) also enhances the chemical etching process and results in more layers. These chemical enhancements are generally less than the energy and momentum driven enhancement derived from higher RIE powers (higher DC bias) over much of the parameter space. However, 
this picture becomes more complicated at the edges of the parameter space as will be discussed in the latter half of this section.

While the exact relationship varies over the range of ICP powers, the percentage of carbon atoms in the film participating in the buffer-layer is a function of the DC bias, Figure 4.19(B). By the nature of the calculation, this percentage is affected by the number of layers (more layers will reduce the percentage of buffer-layer carbon, even if the absolute amount is the same). But even when this is accounted for, low DC bias result in a disproportionately high amount of buffer-layer carbon in the final films. This is discussed in more detail in the latter half of this section.

Extracting finer detail conclusions over this parameter space is challenging for several reasons. Heat maps of various film parameters can be generated but their analysis is complicated by the intermittent appearance of aluminum contamination which skews the results (in particular, the defect related calculations are particularly sensitive to aluminum contamination). The aluminum contamination originates from the electrostatic chuck which is etched due to plasma undercutting beneath the carrier wafer (the investigation on the aluminum contamination is explored in detailed in the Appendix $\$$ C.2). While largely random, some combinations of ICP/RIE powers tend to lead to an aluminum contamination. As such, it is difficult to draw conclusions from films produced under these conditions due to the influence the deleterious aluminum has on the film formation. Also, variations in plasma stability over the range of ICP/RIE combinations, can result in large swings in the DC bias which also complicate the issue. Plasma stability may be improved in future work by a precise manual tuning of the capacitive matching network at each processing point. However, the chamber design (electrode area and spacing, coil diameter, etc.) also affect the plasma characteristics.

As a result, some ICP/RIE combinations may never generate a stable plasma in the current system. Clearly, such a large parameter space would benefit from data coverage at a higher resolution so that more detailed conclusions could be drawn. This should be part of future work. However, the area corresponding to the constant ICP line at $600 \mathrm{~W}$ resulted in no aluminum contamination, fairly stable plasmas and a largely liner relationship between RIE power and the developed DC self-bias. Figure 4.20 shows this relationship. 


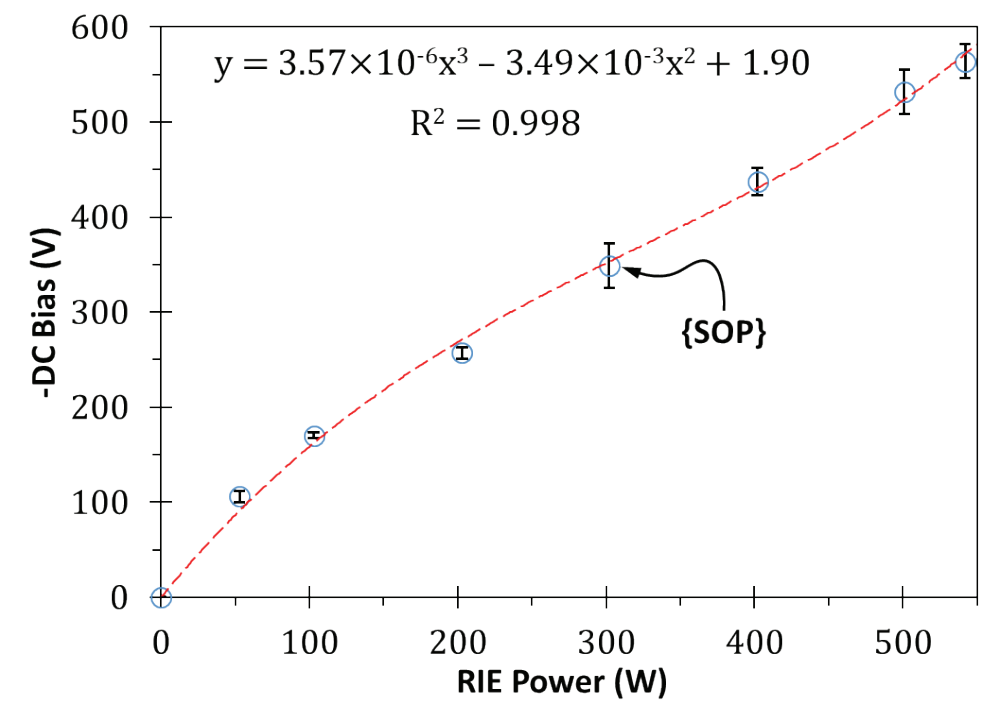

Figure 4.20: The relationship between the applied RIE power and the developed-DC self-bias. The dashed line is a simple polynomial best fit line to guide the eye. The error bars represent the bound of values the DC bias fluctuates through for a given run.

To this point the impact of plasma parameters on the number of graphene layers has been considered. Valuable insight into the impact of these parameters on characteristics of these films can be obtained from both XPS and Ramana spectroscopic analysis of films formed under various etch conditions.

The C1s region of the XPS spectra and the Raman spectra are shown stacked in Figure 4.21 $(A, B)$, respectively. The locations of the primary peaks have been indicated. The arrows indicated the direction of increasing -DC bias. Recall that in the XPS spectra the $\mathrm{S}_{1}$ and $\mathrm{S}_{2}$ peaks have been identified as buffer-layer related peaks, $\mathrm{G}$ is the graphene peak and the $\mathrm{SiC}$ peak is used as a charge reference. The region labeled $D_{x}$ corresponds to the various oxide states. In the Raman spectra the D peak (indicative of the amount of structural defects) and the $G$ peaks (indicative of the amount of pristine graphene) are labeled. The two phonon region peaks are labeled as well. The black colored spectrum lines correspond to the sample produced during the course of this study using the SOP parameters (600W ICP and 300W RIE). 


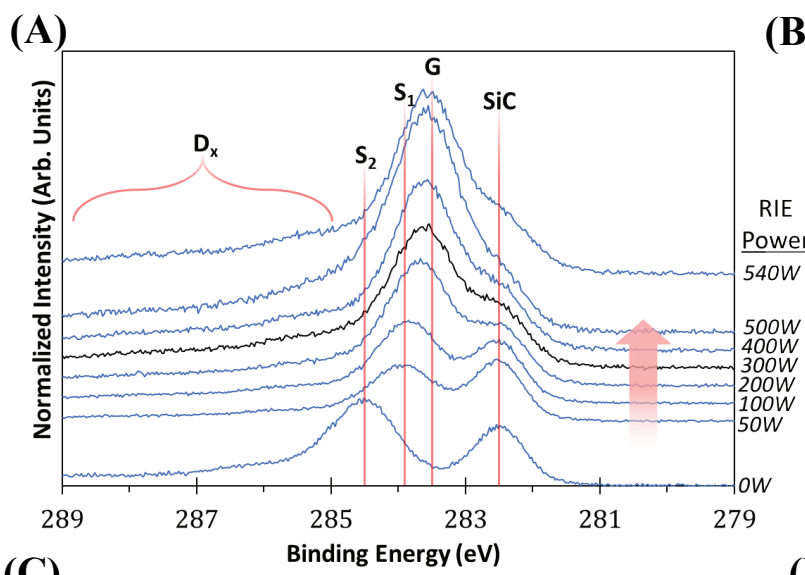

(B)
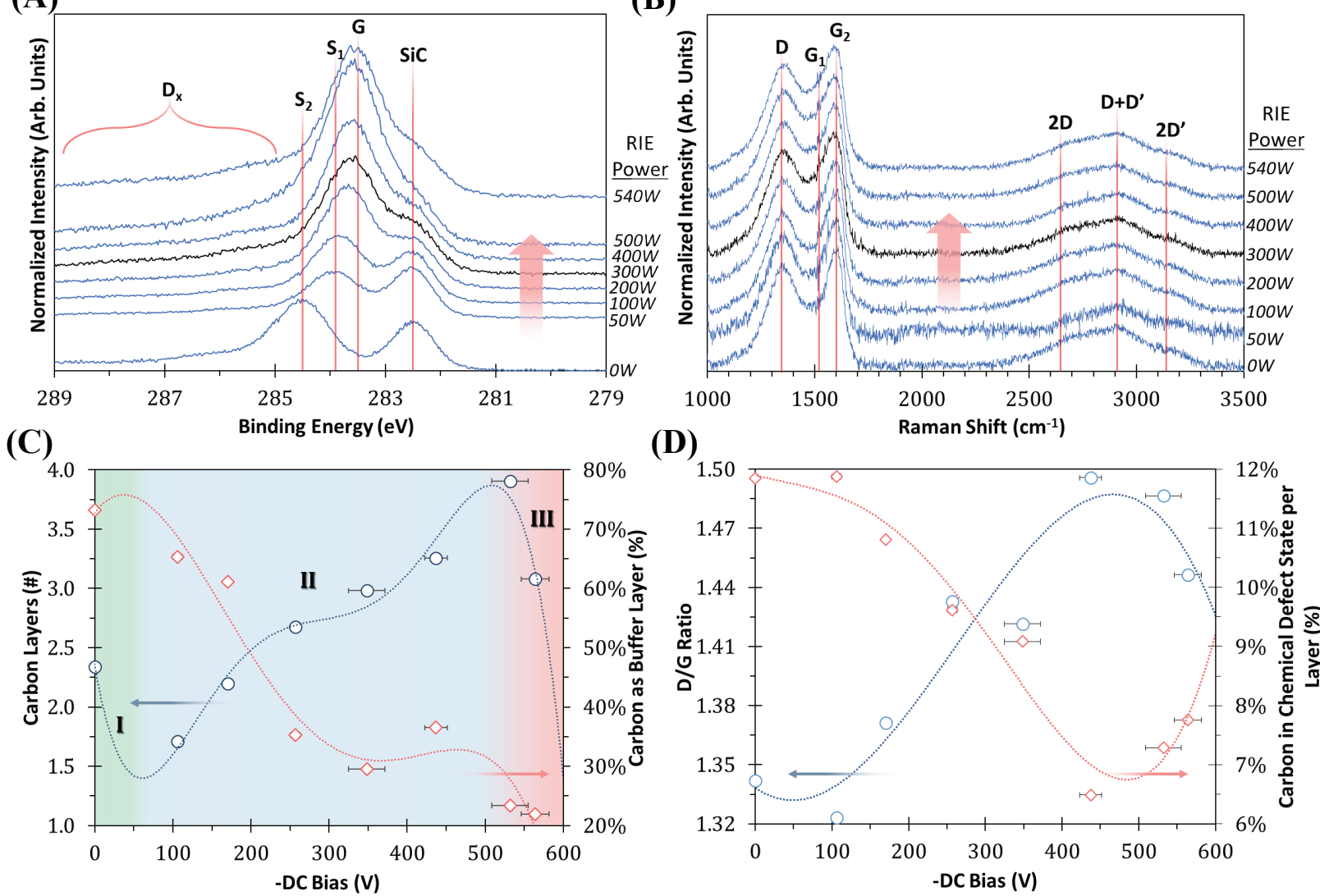

(D)

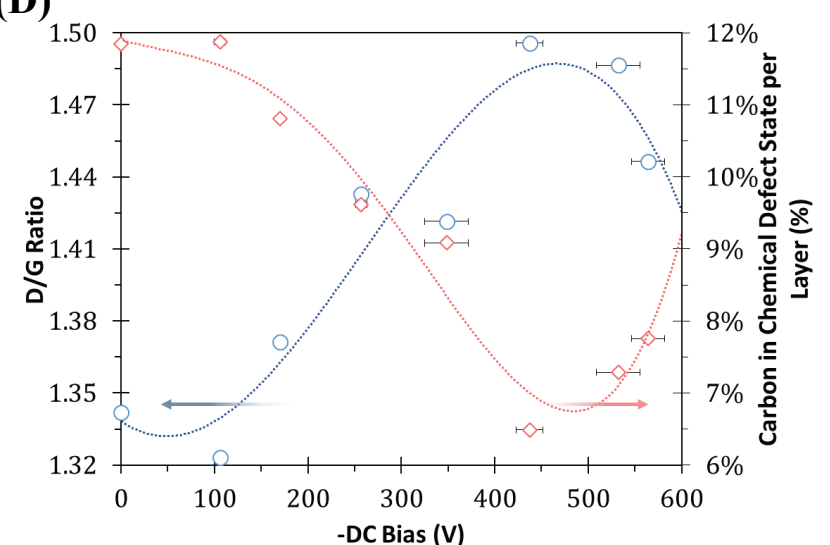

Figure 4.21: (A) Stacked XPS of the C1s region collected at different RIE powers (and similarly, -DC biases). All spectra have been normalized to the $\mathrm{SiC}$ peak intensity. The arrow indicates the direction of increasing RIE power / -DC bias. The black spectrum corresponds to a sample produced using the standard process parameters. (B) Stacked Raman spectra collected from the same samples. (C) Plot showing how the number of carbon layers and percent carbon participant in the buffer-layer changes a function of -DC bias. The distinct zones are discussed: (I) where chemical etching dominates (II) the synergistic region and (III) where non-discriminant physical etching dominates. (D) Plot showing how the D/G ratio and the percent carbon involved in a chemical defect state vary as a function of -DC bias. Arrows indicated the corresponding axis. Error bars in (C) and (D) are the standard deviation value of the DC bias for each run.

Figure 4.21(C) plots the number of carbon layers and the percentage of carbon in the film associated with the buffer-layer against the -DC bias. The broader trend is that higher -DC biases lead to more carbon layers (thicker films). However, close analysis reveals that this trend is not maintained at very low or very high -DC bias. This is explained by the complex interplay of the ICP with the RIE etching. Essentially, the curve is a result of competing effects between the selective chemical etch due to the ICP and the non-discriminant etch of the RIE.

Recall that the selective nature of the etch is derived from the chemistry of the $\mathrm{CF}_{4}$ plasma, as generated by the ICP (CFx ions prefer to react with silicon atoms rather than carbon atoms). The RIE power effectively dictates the directionality and impact energy of the reactive ions in the ICP plasma. The kinetic energy associated with the ion impact results in a non-discriminant etch 
of the surface. In the region labeled (I) in Figure 4.21(C), at very low -DC biases there is effectively no ion impact energy and thus no non-discriminant etching. This allows for the selective chemical etch of the ICP to dominate, resulting in a net balance of carbon atoms on the surface (the reactive ions arrive at the sample surface through randomly driven Brownian motion and various field effects of the ICP coil and chamber). Once a bias is developed on the sample, the sample surface is impacted by ions and the non-discriminant etch begins. This initially reduces the balance of carbon on the surface.

In the region labeled II in Figure 4.21(C), a synergistic effect is achieved between the chemistry due to the ICP and the arrival of reactive ions to the sample surface due to the RIE. This synergy is derived from the enhancement in interaction between the reactive ions and the surface of the sample. A further increase in ion impact energy, while causing a more aggressive nondiscriminant etch, allows for the ions to implant deeper into the $\mathrm{SiC}$ stack and react with a greater fraction of silicon atoms (the chemistry of the ICP is 'felt' deeper into the SiC stack). Thus, the effectiveness of the ICP is enhanced and a larger balance of carbon is left on the surface at the end of the etch. However, in the region labeled III in Figure 4.21(C), the ion impact energy is so high that the non-discriminant etch dominates, and the excess carbon generated on the surface by the ICP chemistry is etch away at a rate approaching its production.

With respect to the amount of buffer-layer carbon on the surface, the trend is more straightforward. Larger -DC biases result in less carbon in the film participating in buffer-layer bonding. Because this metric is taken as a percentage, it is influenced by the number of carbon layers by the nature of its calculation. However, even when this is accounted for (by taking the amount of buffer-layer carbon on a per layer basis, for example) the trend remains the same. Furthermore, there was no correlation observed between the number of layers and the amount of buffer-layer carbon in the standardly synthetized samples (i.e., all things being equal, except for variations in the number of layers which did not correlate to variations in the amount of bufferlayer carbon; plots of this data can be found in Appendix $\boldsymbol{\$} \boldsymbol{A . 2 . 3}$ ).

Therefore, it can be concluded that the decrease in the amount of buffer-layer carbon seen with increasing -DC biases (via increasing RIE energy) is due to the increase in ion impact energy. This analysis is supported by examination of data points which resulted in approximately the same number of layers but lie at different -DC biases. For example, the amount of carbon on the surface 
is the roughly the same at $0 \mathrm{~V}$ bias and $\sim 180 \mathrm{~V}-\mathrm{DC}$ bias. The film produced at $0 \mathrm{~V}$ consists of substantially more buffer-layer carbon. Similarly, at $\sim 450 \mathrm{~V}$-DC bias and $\sim 550 \mathrm{~V}$-DC bias an approximately 3-layer film is produced. Yet, the film produced at the lower-DC bias (and was thus exposed to a less energetic ion flux) results in more buffer-layer associated carbon. This indicates that ion impact energy plays a role in decoupling of the buffer-layer, perhaps by inducing damage to the film/substrate interface.

Previous experiments using a $\mathrm{Cl}_{2}$ based plasma, which were shown to result in much lower etch rates overall, do no show the uptick in film thickness at low -DC biases. ${ }^{(263)}$ This supports the hypothesis presented here, in that the less chemically active $\mathrm{Cl}_{2}$ plasma requires the $\mathrm{DC}$ bias assist to achieve any meaningful selectivity. Thus, the high selectivity of the $\mathrm{CF}_{4}$ plasma is responsible for the uptick seen in this work at low -DC biases.

Raman analysis tells a similarly complex story. Recall that the $\mathrm{D} / \mathrm{G}$ ratio is indicative of the amount of damage in the film (a large $\mathrm{D} / \mathrm{G}$ ratio correlate to a more damaged film). The blue circles and trend line in Figure 4.21(D) show how the D/G ratio changes as a function of -DC bias. All other Raman parameters are essentially unchanged as a function of RIE power (peak locations, areas, FWHM, height and ratios). Higher -DC bias values lead to a higher $\mathrm{D} / \mathrm{G}$ ratio. This would seem to indicate that higher -DC biases, and thus higher ion impact energies, lead to higher defect levels in the final films. This intuitively makes sense, and undoubtedly contributes in part to the higher $\mathrm{D} / \mathrm{G}$ ratio. However, close analysis reveals a more complex picture.

The red triangles and trend line in Figure 4.21(D) show the percentage of carbon in a chemical defect state taken on a per layer basis, as determined from XPS analysis. Clearly, increasing the -DC bias actually decreases the chemical defect concentration. Although the $\mathrm{D} / \mathrm{G}$ ratio and the chemical defect states are measures of inherently different aspects of the system (ratios of vibrational modes vs. photoelectron binding energies) they are related, and it would be expected that they would correlate with each other. However, this is obviously not the case. 
A possible explanation for this behavior lies in why the $D / G$ area ratio increases. The $D$ peak area increases, while the $\mathrm{G}$ peak area $\left(\mathrm{G}_{1}+\mathrm{G}_{2}\right)$ remains essentially constant. Recall from Section $\$ 3.2 .3$ that the magnitude of a Raman peak is proportional to the amount of material generating the signal. The breadth of the peak is indicative of the distribution of stress/strain states the structure is under as well as the coupling of vibrational modes. Also, shifts to lower wavenumbers correspond to an increase in the tensile stress. Figure 4.22(A) plots the full width at half maximum (FWHM) and the normalized peak intensity against the -DC bias. It is clear that it is the broadening of the $\mathrm{D}$ peak that is responsible for the area increase (not an increase in amplitude). If the increase in area was due to an increase in the concentration of defect sites, one would expect the magnitude of the D peak to increase. However, this is not the case and the D peak intensity remains constant. Additionally, from Figure 4.22(B), the D peak center shifts to substantial higher wavenumbers. Therefore, the Raman data is not showing more defective films at higher DC bias, but rather, something else.

(A)

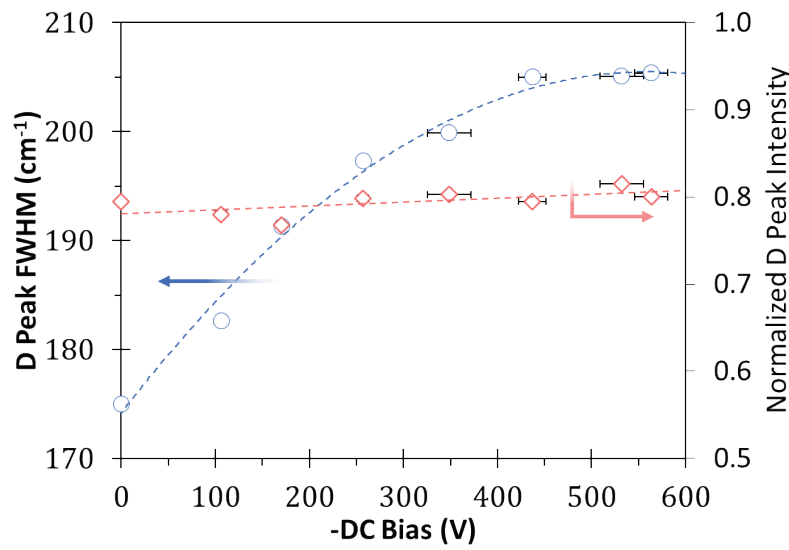

(B)

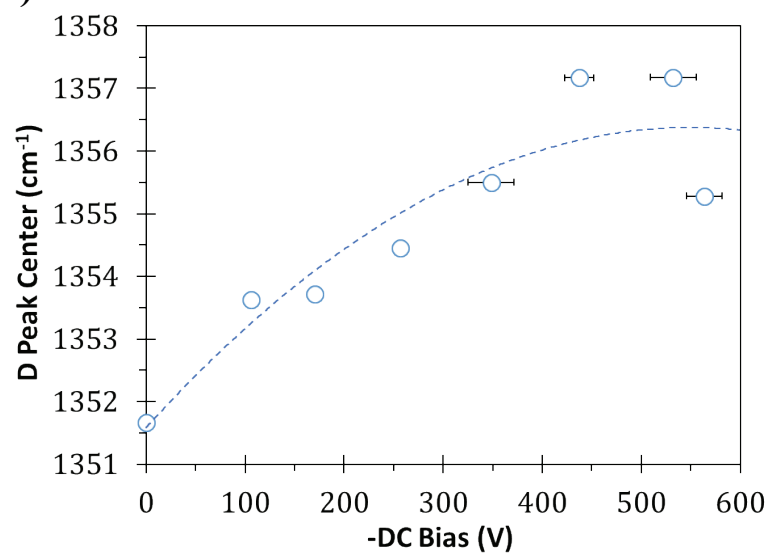

Figure 4.22: (A) Plot showing the growth in the FWHM value of the D peak, while the magnitude remains constant. (B) Plot of the D peak location show a shift to higher wavenumbers with increasing -DC bias. Error bars are the standard deviations values of the DC bias for each run.

What these results may be illustrating is the beginning emergence of a second D vibrational mode; one which is shifting towards higher wavenumbers, resulting from the more relaxed films that are produced at higher -DC biases. State another way, the spectra may be the superposition of signals from a highly coupled film (the major peaks) plus the spectra from part of the film which is beginning to become partially decoupled (the second D peak, which appears as a high wavenumber shoulder on the primary $\mathrm{D}$ peak). Whether this relaxation is the result of a decrease in buffer-layer content (due to ion bombardment) or simply by the fact that increasing the number 
of layers would naturally result in those layers being more relaxed (as a result of being more distant from substrate and its effects), is not clear.

Examination of samples produced using the SOP parameters which all experience similar ion impact energies (Appendix $\boldsymbol{\$} \boldsymbol{A}$.2.3), show no correlation between the number of layers and the $\mathrm{D} / \mathrm{G}$ ratio, indicating it is the increase in ion impact energy (and the subsequent reduction in buffer-layer) that is responsible for the relaxation (rather than an effect of increasing layers). Because the standard process does not produce the number of layers that the very high -DC biased experiment did, it is also possible these results are a confluence of both effects.

In summary, the generic relationship between the applied ICP power and RIE power effects on the -DC self-bias developed on the sample were determined. While the ICP power does have some influence, it is primarily the RIE power which determine the -DC self-bias in the $\mathrm{CF}_{4}$ based plasmas. Higher -DC biases lead to more carbon layers and a smaller fraction of the layer participating in buffer-layer bonding. Practical considerations (such as matching network tuning issues leading in part to plasma instability and aluminum contaminations) make drawing more detailed conclusions across the entirety of the parameter space explored in this study difficult.

By examining the subsection of the parameter space at a constant $600 \mathrm{~W}$ RIE power, where stable plasmas are generated, substantial insight is gained on the interplay between ICP power, DC bias and the selective etching of the $6 \mathrm{H}-\mathrm{SiC}$ substrates. At $0 \mathrm{~V}-\mathrm{DC}$ bias, the selective chemical etch dominates, resulting in a net positive balance of carbon on the surface. On the application of RIE power a -DC bias develops, which leads to a non-discriminant etch of the surface. This initially leads to a reduction in the balance of carbon on the surface, but at higher -DC biases, the synergistic combination of an aggressive ion bombardment and a selective chemical etch result in a large balance of carbon on the surface, and ultimately more carbon layers. At very high -DC biases, the aggressive etch dominates and the steady state balance of carbon is reduced. Due to the increased ion bombardment energies, a substantial reduction in the amount of buffer-layer content is observed with increasing -DC bias. Furthermore, higher -DC biases lead to less chemically defective films as determined by XPS analysis, but the relaxation of these layers leads to an artificial increase in the calculated defect density as determined by Raman analysis. This may be due to the emergence of a secondary D vibrational mode resulting from a fraction of the new layers produced at high -DC biases experiencing less strain. However, because the larger character of the 
overall Raman spectrum remains, there is still a substantial coupling of vDOS across all films produced.

Regardless of the specific etching mechanism, physical or chemical, the overarching picture of the etching process under the conditions used here is that the SiC surface is rapidly etched at tens to hundreds of nanometers per minute. When the etch process begins, selective etching of the Si relative to the $\mathrm{C}$ atoms rapidly establishes steady state $\mathrm{C}$-enriched layer which is maintained as the etch progresses into the substrate. Increasing the RIE power / bias voltage increase the penetration depth of etching species into the $\mathrm{SiC}$ matrix (as well as the overall etch rate), and thus, increases the number of C-rich layers. This, in turn, increase the number of graphene layers that can be formed during the RTA.

\section{\$4.2.3 ICP/RIE Etching- Effect of Plasma Chamber Pressure}

Increasing the chamber pressure increases the gas density within the chamber. This affects both the mean free path of all particles (electrons, ions and radicals) and the gas power density (the amount of power dissipated per mole of gas). This which in turn effects everything that the ICP and RIE powers do, such as the DC bias, ion kinetic energy, etc. A detailed discussion of the ICP/RIE process is included in Section $\$ 3.1 .3$.

The chamber pressure of the etching system can be set by controlling either the inlet gas flow rate or the chamber exhaust valve position. In these studies, the $\mathrm{CF}_{4}$ flowrate was held constant at the standard processing value of 20 SCCM. The pressure was automatically set by dynamically controlling the chamber exhaust value position during the process. Increasing the pressure results in more process gas residing in the chamber at any one time, resulting in a higher gas density.

For plasmas, the precise relationship between pressure and density can be complex. Plasmas do not necessarily follow the same relationships as do gases (this is part of the reason

plasmas are occasionally referred to as the $4^{\text {th }}$ state of matter). This is due to the complex electric field effects on the plasma species and their variety of charge states, as well as the many intramolecular forces felt between the particles (electrons, ions, neutrals). Therefore, the precise 
relationship between pressure and density (and thus their downstream effects) are difficult to predict, a priori. However, by taking a simplistic approach, some can be made. ${ }^{24}$

First, by changing the chamber pressure, the distribution of plasma species and their degree of ionization will be changed. This is because, for the same applied power, the same amount of energy will be dissipated into more material (gas) if the pressure is raised, effectively decreasing the power density (watts per mol gas). The converse is also true. A lower pressure means more energy is applied to the gas on a per mol basis, which increases the degree of ionization. This also affects the plasma potential. Second, the increase in gas density decreases the mean free path of all plasma components and increases collision frequency. This affects the amount of gas converted into plasma and further effect the distribution of plasma species.

By observation, the overall impact of these various effects can be seen. Ostensibly, increasing the chamber pressure leads to an increase in the -DC bias as can be seen in Figure 4.23 which plots the -DC bias a function of gas pressure. Note that set ICP and RIE powers are constant at $600 \mathrm{~W}$ and $300 \mathrm{~W}$, respectively. However, due to the change in the electrical impedance of the system, the actual dissipated ICP and RIE powers are decreased by as much as $10 \%$. Based on this alone, increasing the pressure which would tend to decrease the DC bias. Counter to this however, the -DC bias is observed to drastically increase with pressure.

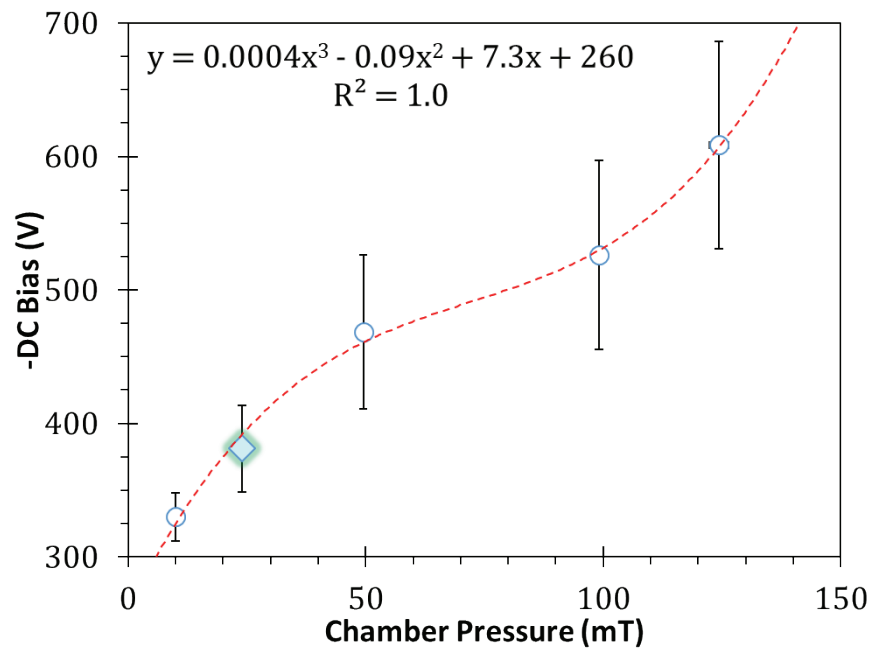

Figure 4.23: The relationship between the chamber pressure and the developed -DC self-bias. Note that the ICP and RIE power setpoint is 600W ICP and 300W RIE (standard process powers) in all cases. The data point corresponding to the standard process chamber pressure is diamond shaped and highlighted. A best simple 3rd order polynomial best fit line has been added to guide the eye. Pressure about $125 \mathrm{mT}$ and below $10 \mathrm{mT}$ could not be maintained. Error bars are the standard deviation values of the DC bias for each run.

${ }^{24}$ This simple approach assumes that, for the same gas flowrate, ICP and RIE powers, a higher pressure necessarily means more gas resides in the chamber at any one time, resulting in a higher partial density. 
Given the fact that the DC bias changes so drastically with pressure, and that the DC bias has been shown to drastically impact the film properties, separating out the purely pressure driven effects is difficult. The number of carbon layers and the percent of carbon bound in the bufferlayer is plotted against chamber pressure Figure 4.24. Best fit lines have been added to guide the eye, but they are a poor fit to the data. Generically however, a higher chamber pressure leads to fewer carbon layers with a larger fraction participating in the buffer-layer.

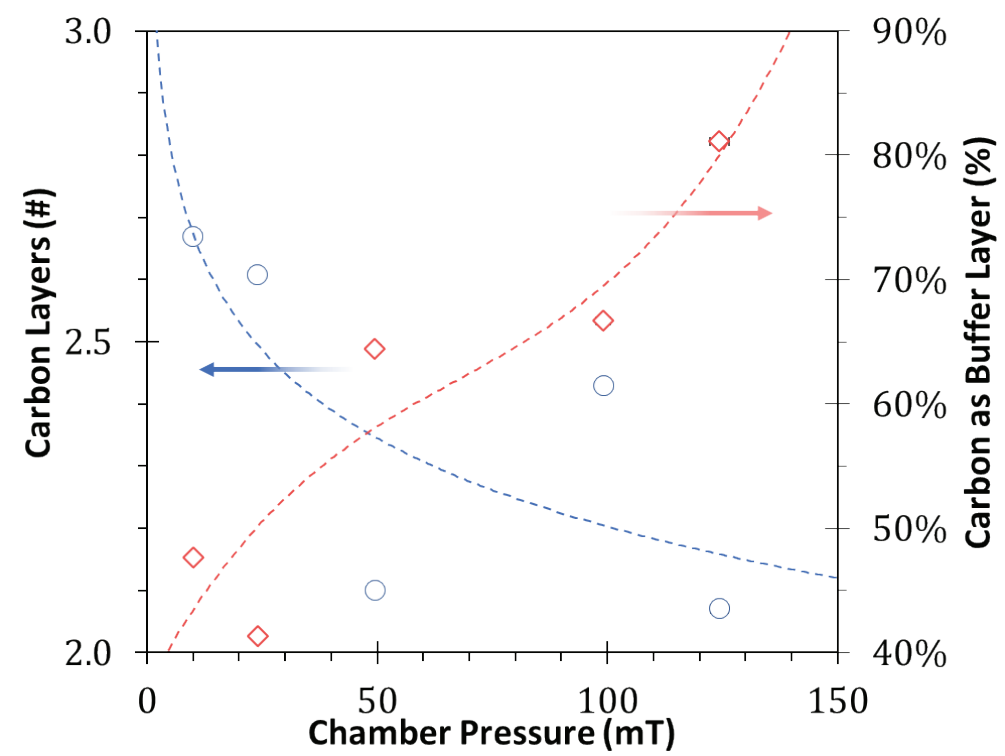

Figure 4.24: The effect of chamber pressure on the number of carbon layers produced and the percentage of carbon in the film participating in buffer-layer bonding. Error bars (small and largely obscured by the data points) are the standard deviation value for the pressure reading for each run.

Note that although the chamber pressure increases the -DC bias, the increased -DC biases is itself not responsible for the change in properties. For example, a DC bias of $-500 \mathrm{~V}$ generated at $100 \mathrm{mT}$ produced a nominally $\sim 2.5$ layer thick film, with approximant $68 \%$ of the carbon participating in buffer-layer bonding (from Figure 4.23). However, a similar DC bias of -500V generated at $25 \mathrm{mT}$ produces a nominally $\sim 3.7$-layer film with approximant $33 \%$ carbon participating in buffer-layer bonding (from Figure 4.21(C)). This indicates the impact of chamber pressure is multifaceted. However, the kinetic motion of the particles which make up the plasma may explain the observations. When the pressure goes up, the mean free path of the plasm particles goes down (due to the increased particle density). This drastically increase the number of random scattering events resulting in less ion acceleration into the substrate, effectively reducing the effects of the larger -DC bias. The energy and momentum transfer are reduced at higher pressure, leading to less ion penetration producing less effective etching, leading to less carbon and ultimately, thinner films. 
In summary, the fact that higher pressures lead to fewer layers, while simultaneously much higher -DC bias, indicated that evidently the increased scattering effects of a denser plasma dominated over the increase in ion impact energy. The increase in buffer-layer carbon further points to a decrease in etching effectiveness. The decrease in etching effectiveness may be due to the increase in collision frequency, which hinders mass transport to and from the sample surface. Furthermore, the larger amount of inactive gas (gas which has not been ionized into a plasma state) may effectively shield the $\mathrm{SiC}$ surface from the barrage of reactive ions by screening and scattering reducing their kinetic energy by the time they reach the surface.

\section{\$4.2.4 AP-RTA- Effect of Maximum Temperature}

This section will explore the atmospheric pressure rapid thermal anneal (AP-RTA) step of the two-step synthesis process. Specifically, the effect of the maximum temperature seen by the sample during the AP-RTA. The etch parameters for all samples in this study followed the SOP parameters (600W ICP, 300W RIE, etc.). Effects of the heating rate, cooling rate and dwell time of the AP-RTA will be examined in the next sections. For this set of experiments, the series 1600 C\&M High Temperature Furnace was held at a set point of $1400^{\circ} \mathrm{C}$. The integrated thermocouple of the RTA tube was used to determine the approximate temperature of the sample. Detailed information on the heater and RTA tubes can be found in Section \$3.1.4. The RTA tube, containing the sample, was rapidly inserted into the tube furnaced and the thermocouple readout was monitored. Once the desired temperature was reached, the RTA tube was rapidly withdrawn and suspended in the forced air flow of the containment hood. The lag time of the thermocouple read out lead to an over or undershoot of the desired temperature for samples which had very short RTA durations. All the temperatures cited in this study are the actual maximum temperatures as read by the thermocouple. 
Room temperature research plus purity argon (99.9999\%) was flown through the RTA tube before, during and after the RTA step, consistent with the SOP procedure. More detailed information on the RTA tube cleaning and degassing are included in Section \$3.1.4. The temperature profiles for several of the samples tested in this study are shown in Figure 4.25(A). To accurately represent the AP-RTA profiles, a logarithmic equation is used to describe the heating profile and exponential decay equation is used to describe the cooling profile.

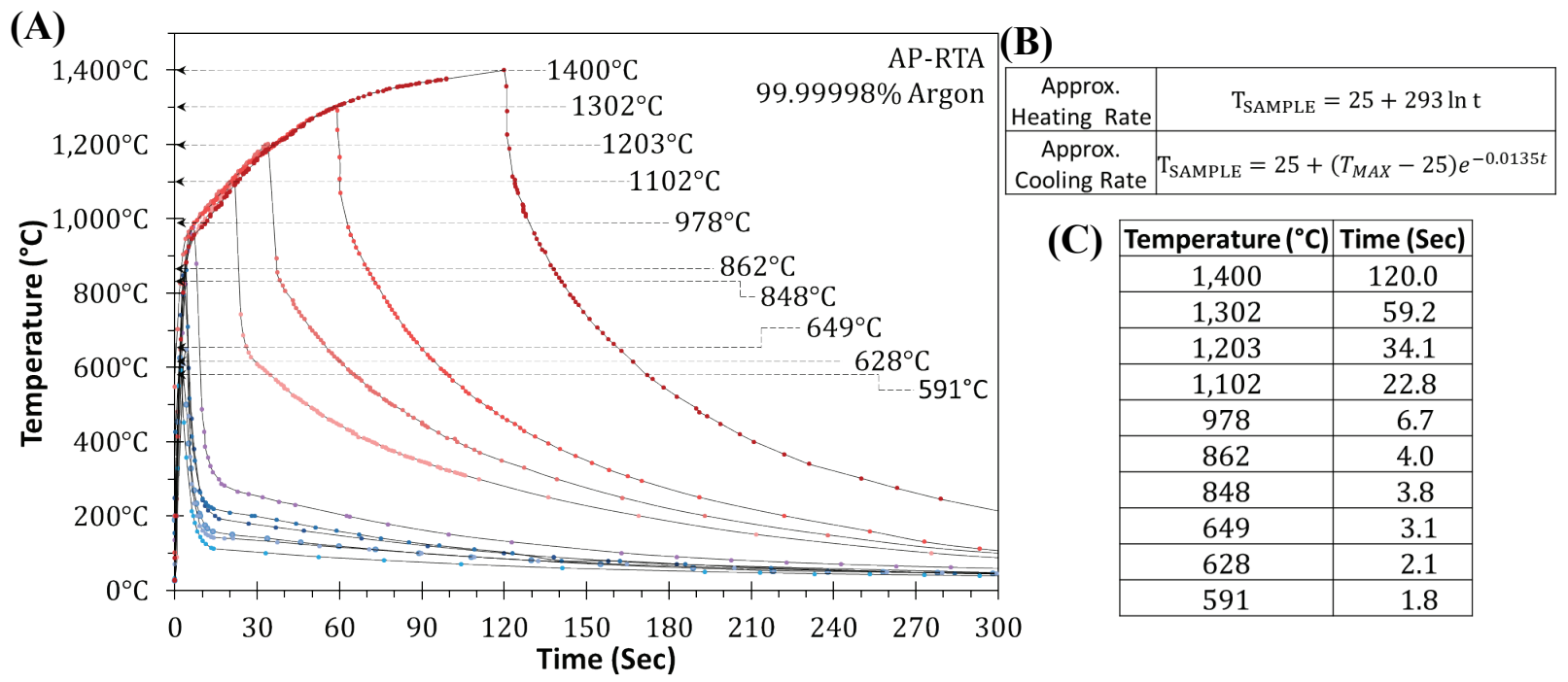

Figure 4.25: (A) Real data point temperature profiles. The furnace setpoint was $1400^{\circ} \mathrm{C}$. The samples were withdrawn from the furnace once the desired temperature was reached. (B) Equations which approximately describe the heating and cooling rates for all samples where $\mathrm{T}_{\text {SAMPLE }}$ is the temperature of the sample, TMAX is the maximum temperature seen by the sample and $t$ is time. Time is in seconds and temperature is in ${ }^{\circ} \mathrm{C}$. (C) The maximum temperature seen by each sample in (A) and the approximant time taken to reach that temperature.

The heating and cooling rates were largely governed by Newtons Law of Cooling. However, because the heaters thermal mass does not provide an infinite thermal reservoir, the temperature of the heater drops on the insertion of the RTA tube. This results in an initially very high heating rate (up to $\sim 700^{\circ} \mathrm{C}$ ) then the heating rate slows. Once the PID controller detects the furnace temperature drop, the heating elements are energized which leads to an increase in heating rate. Thus, all samples experience an overall accelerated heating rate that would normally be predicted. These effects are more pronounced at very short or very long RTA times. This leads to a standard deviation in heating rate of $\sim 10 \%$ for samples in this study. The cooling rates all follow Newtons Law of Cooling quite well and are governed by the maximum temperature seen during the RTA, the temperature of the laboratory air and the containment hood air flowrate. The laboratory is climate controlled at $\sim 25^{\circ} \mathrm{C}$ and the hood air flowrate is constant. Therefore, for this 
study it can be concluded that the only parameter which is changing any meaningful amount between samples is the maximum RTA temperature.

The XPS analysis of the samples can be seen in Figure 4.26. Figure 4.26(A) show the evolution of the stacked C1s spectra, which have all be normalized to the SiC peak intensity which appears at $282.50 \mathrm{eV}$ which is also used for a charging reference. All other analysis parameters are consistent with the rest of this work. The graphene peak (labeled G) and the buffer-layer related peaks (labeled $\mathrm{S}_{1}, \mathrm{~S}_{2}$ ) are also annotated. The spectra are labeled according to the maximum temperature the corresponding sample was exposed to. For the sample which did not undergo the AP-RTA process (i.e., was etched only), the maximum temperature is taken to be $25^{\circ} \mathrm{C}$ (room temperature). However, it is worth noting that the maximum temperature that sample would have seen would have occurred during the etching process and was undoubtedly higher than $25^{\circ} \mathrm{C}$. This is especially true given that not cooling helium backing flow was used. Recall that the samples reach temperatures high enough to discolor and bake the high temperature coolgrease used on the back on the samples (more information on this can be found in Appendix $\$$ A.1.3). An additional test was performed where a sample was thermally cycled between $1200^{\circ} \mathrm{C}$ and $500^{\circ} \mathrm{C}$ five times. This data is included in Appendix $\boldsymbol{\$} \boldsymbol{A}$.2.7. It was found that this was largely deleterious, resulting in only a fractional carbon layer remained; the rest having, presumably, been consumed by trace oxygen in a simple combustion reaction. ${ }^{25}$

\footnotetext{
${ }^{25}$ An additional note on this parameter study: Although a rigorous attempt was made to limit the number of dependent variables, by the natural fact that the heating and cooling rates are constant between samples, samples exposed to higher temperatures also experienced more time at elevated temperature. This leads to the natural question whether the observed trends are a function of the exposure to high temperature or on the extended time at high temperature. This aspect is explored in detail in Appendix § A.2.5. It was found that, in most cases, the most likely primary driving force for the trends observed in this study are due to the increasingly high temperature as seen by the samples and not the additional time samples spend at the higher temperatures.
} 
(A)

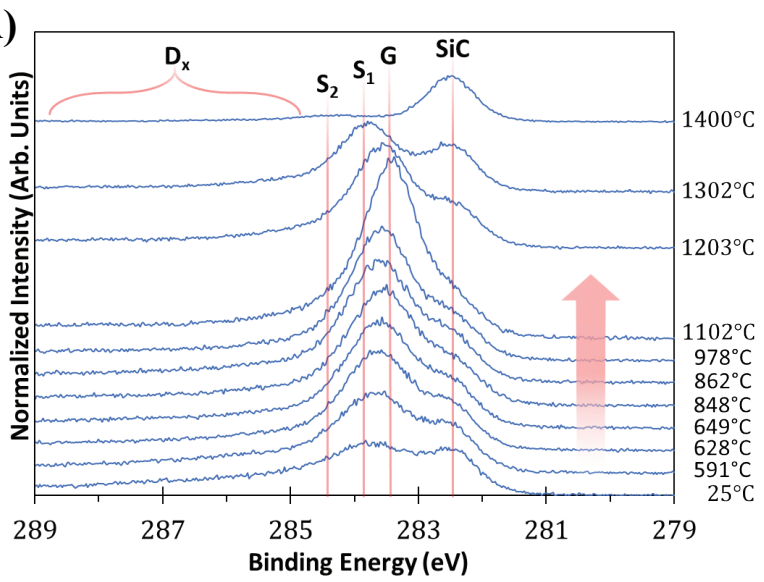

(C)

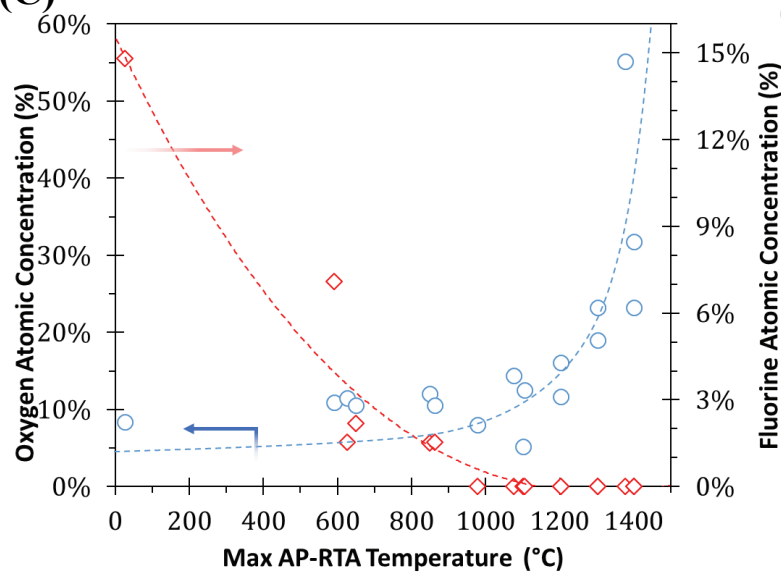

(B)

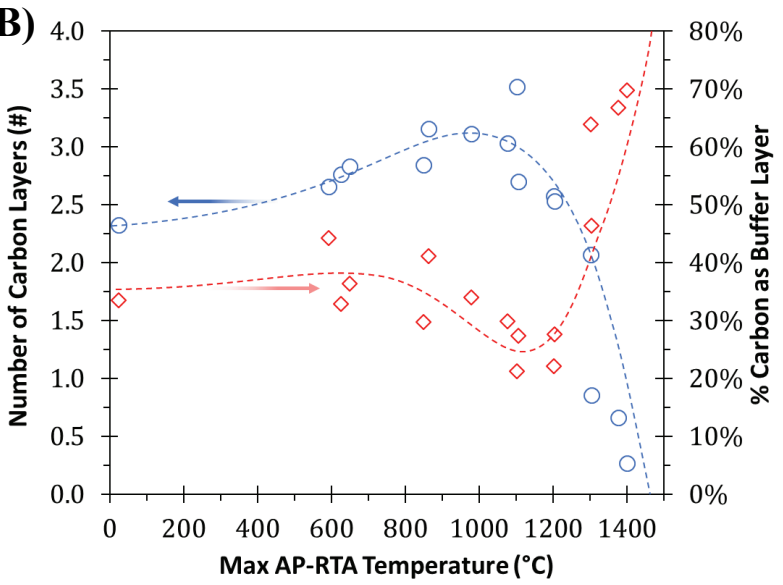

(D)

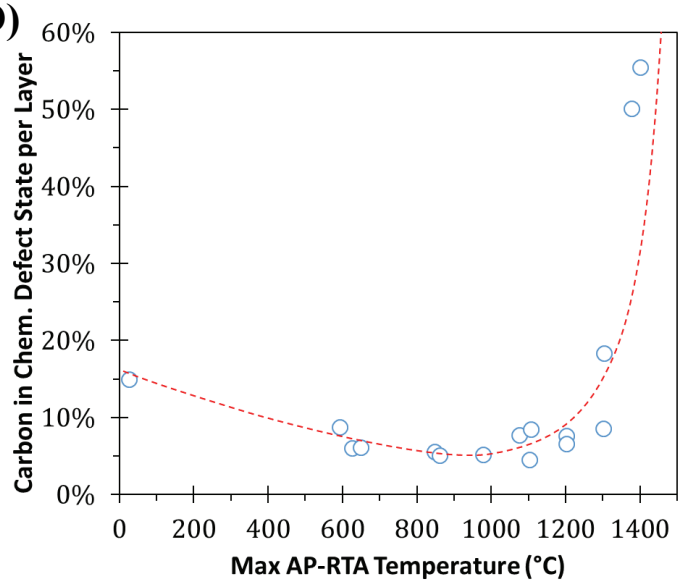

Figure 4.26: (A) Stacked XPS spectra of the C1s region. The arrow indicates increasing AP-RTA temperature. (B) The number of carbon layers and the fraction of carbon in the layers associated with the buffer-layer are plotted. At very high temperatures, almost all the carbon is removed from the surface and that which remains is in the form of a buffer-layer. Silicon sublimation may also be partially responsible for the sharp increase in buffer-layer carbon after above $1200^{\circ} \mathrm{C}$. (C) The atomic concentration of fluorine and oxygen. Above $\sim 850^{\circ} \mathrm{C}$, all detectable fluorine remaining from the etch is removed. (D) The fraction of carbon in the film in a chemical defect state. The line shape appears to be a confluence of the lines in (C), indicating the defects to be primally fluorine based at low temperatures which transition to oxygen at high temperatures. (B, C, D) Arbitrary best fit lines are included as the dashed lines to glide the eye.

The effect of annealing temperature on the number of layers and the amount of buffer-layer carbon is shown in Figure 4.26(B). Up to a temperature of $\sim 1100^{\circ} \mathrm{C}$, the number of carbon layers increases. Because the amount of carbon on the surface is set during the etch (which is the same for all samples in this study), the increase in the calculated number of layers must be due to a densification and coalescence of carbon species into the graphene film, causing a further attenuation in the $\mathrm{SiC}$ bulk signal. There is a corresponding decrease in the amount of buffer-layer carbon. Note that the absolute amount of buffer carbon is largely the same, but the fraction of buffer-layer carbon decreases due to the increase in the amount of calculated graphene carbon. This is clearly apparent when the total number of carbon layers are taken into account and 
the ratio of buffer-layer carbons to graphene layer carbons are calculated (this data can be found in Appendix $\$$ A.2.5).

At temperatures above $1100^{\circ} \mathrm{C}$, there is a sharp decrease in the number of carbon layers and an increase in the amount of carbon in the film associated with the buffer-layer. This also coincides with a large drop in the atomic concentration of carbon (atomic concentration as a function of annealing temperature for all elements can be found in Appendix $\boldsymbol{\$ A . 2 . 6}$ ). This is all indicative of the removal of the graphene overlayers. The removal of graphene is most likely a result of a surface reaction where volatile species are formed, and carbon atoms are scavenged from the surface. From the atomic concentration data shown in Figure 4.26(C), reactions with oxygen are probably responsible.

The uptick in buffer-layer carbon above $\sim 1100^{\circ} \mathrm{C}$ is due to two reasons. First, the elimination of the graphene overlayers leaves mostly buffer-layer carbon left. Second, the beginning stages of silicon sublimation occur at this temperature. As discussed in Section $\$ 2.1 .3$, the first stage of the selective sublimation process is the generate of a carbon buffer-layer. The increase in the amount of buffer-layer is clear from the large increase in the relative intensity of the buffer-layer peaks (S1 and S2) to the SiC peak, as can be seen in Figure 4.26(A). For example, the spectra collected at $1302^{\circ} \mathrm{C}$ shows a clear buffer-layer peak that accounts for the majority of carbon film states, more than at any other temperature. The only way for this to happen is if more buffer-layer carbon is generated. The additional buffer-layer carbon is more likely to originate from the substrate as opposed to a conversion of graphene layers to buffer-layer. This is not unexpected as the atmospheric pressure sublimation temperature of $\mathrm{SiC}$ is in this temperature range. The fact that there is almost no film carbon on the surface at $1400^{\circ} \mathrm{C}$ indicates that the oxygen-based carbon removal process occurs at a higher rate than the any silicon sublimation process which may be occurring simultaneously.

Figure 4.26(C) shows the atomic concentration of oxygen and fluorine components. Fluorine, remaining from the etching step, is observed to decrease with increasing temperature. Simultaneously, an increase in oxygen is observed. While some of the fluorine and oxygen are associated with the substrate, Figure 4.26(D) indicates the majority are in the film as defects. The data in Figure 4.26(D) is calculated from the XPS analysis of the $\mathrm{C} 1 \mathrm{~s}$ region, but roughly correlates to the sum of the fluorine and oxygen trendline shapes of Figure 4.26(C). This indicates that the 
defects are primally fluorine based at low temperatures, which are removed during the AP-RTA, but then oxygen defects are added at high AP-RTA temperatures.

In Figure 4.27(A), stacked Raman data collected at various AP-RTA temperatures are shown. Note that the samples exposed to $1400^{\circ} \mathrm{C}$ do not generate any meaningful Raman signal once the SiC contributions are subtracted out. This is consistent with the XPS analysis, which indicated there is little to no carbon film on samples exposed to $1400^{\circ} \mathrm{C}$. Again, the broad similarity in the overall character of the spectra indicate a substantial coupling of the vDOS continues to occur regardless of the annealing temperature. However, films exposed to higher AP-RTA

(A)

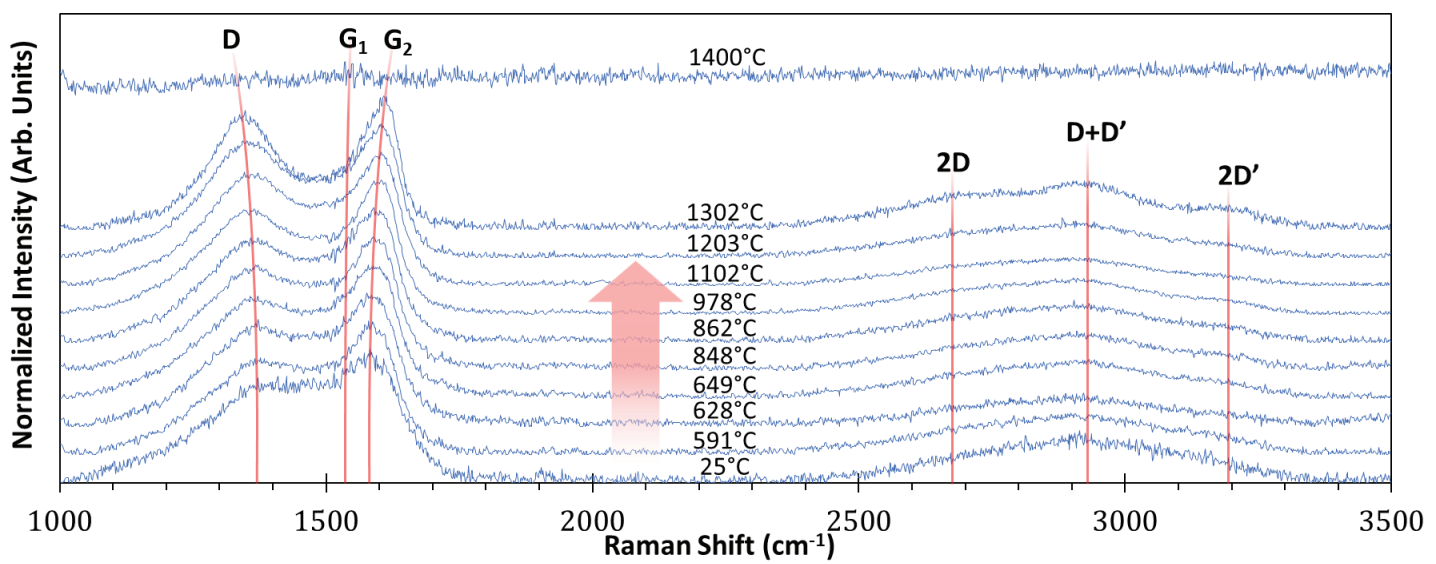

(B)

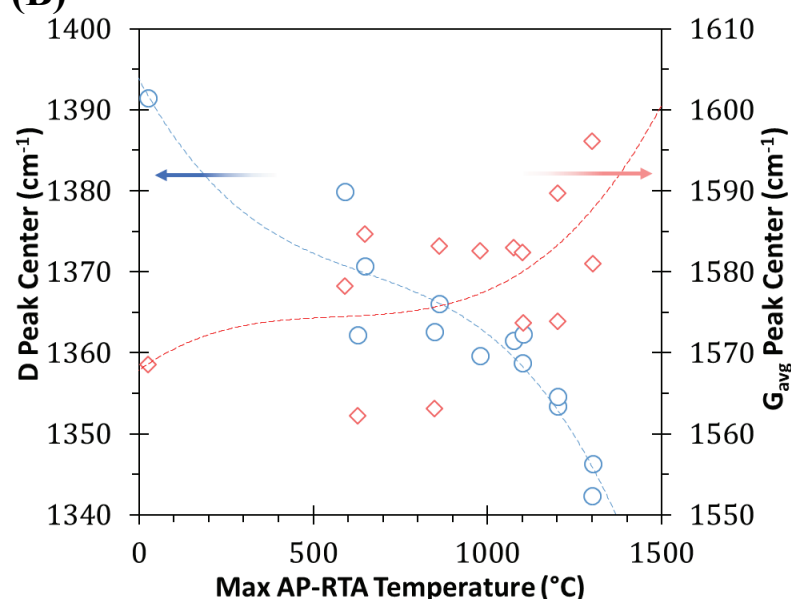

(C)

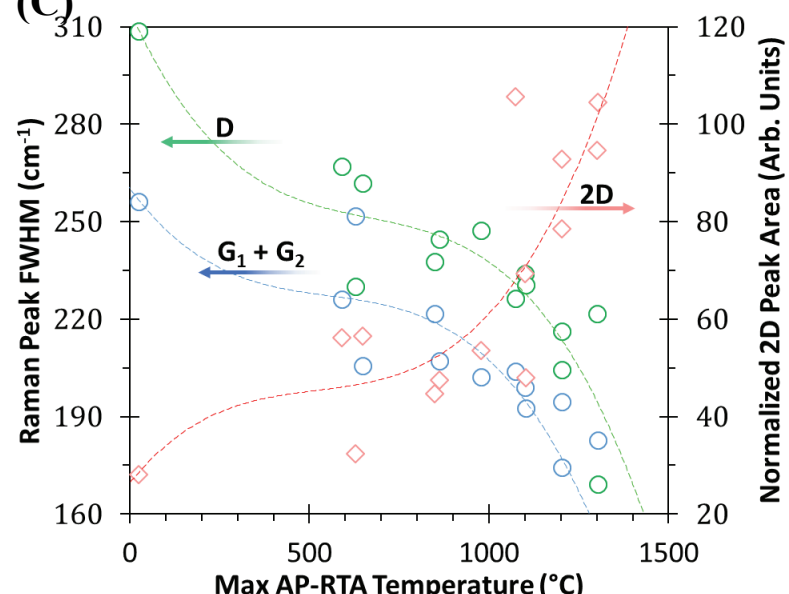

Figure 4.27: (A) Normalized and stacked Raman spectra. The arrow indicates increasing annealing temperature. Samples exposed to $1400^{\circ} \mathrm{C}$ do not generate a meaningful Raman signal. (B) The D and G peak centers as a function of temperature. (C) The full width at half max (FWHM) of the D, G1+G2 peak with the 2D peak area as a function of annealing temperature. Arbitrary best fit lines are included as dashed lines to guide the eye.

temperatures do generate clean and more well-defined spectra. Figure 4.27(B,C) illustrates the more subtle differences in the spectra. 
Here again the implications of the low character in the overall Raman spectra are apparent. Suffice it to say that the G peak is bimodal (requiring two peaks to achieve a reasonable fit), but beyond this the finer details are obscured. There are a vast number of unique combinations for the $\mathrm{G}_{1}$ and $\mathrm{G}_{2}$ precise locations, FWHM and intensities. Most combinations result in equivalently good fits and no particular fit presents itself as making more intuitive sense. None the less, by taking the combined FWHM and average centers of the G peaks, the spectrum range which covers the G peak region can be quantified. Following this logic, Figure 4.27(B) plots the peak centers of the D and the average of the $\mathrm{G}$ peaks as a function of temperature. Figure 4.27(C) plot the full width at half maximum of the $D$ and $G$ peaks (sum of $\mathrm{G}_{1}+\mathrm{G}_{2} \mathrm{FWHM}$ ), as well as the normalized $2 \mathrm{D}$ area.

Figure 4.27(B) shows that the D peak shifts to substantially lower wavenumbers while the G peaks shift to higher wavenumbers with increasing annealing temperature. This indicates an increase in film crystallinity, consistent with the reverse of the 'amorphization trajectory' predicted by Ferrari, et al. in his studies of carbon films. ${ }^{(500)(562)}$ The 2D, D+D' and 2D" peaks do not change position.

Figure 4.27(B) also shows a narrowing of the D and G peaks. The decrease in the FWHM of the $\mathrm{D}$ and $\mathrm{G}$ peaks indicate a decrease in the distribution of vDOS with increasing temperatures indicating a slight improvement in the film crystallinity. It has been shown that the buffer-layer alone does not generate a 2D peak. ${ }^{(212)}$ Therefore, its growth with increasing temperature further indicates an increase in film crystallinity or slight decoupling of the parts of the buffer-layer. The fact that the films which did not undergo the AP-RTA step, yet still produced a Raman signal which includes a small 2D peak, indicate that there is some degree of reordering of the carbon into aromatic rings (which are not part of the film bound to the substrate) occurring during the etching process. In fact, spectra very similar to this have been called a "low-sp" carbon film. ${ }^{341)}$ All things considered, the etched only films (the $25^{\circ} \mathrm{C}$ line in Figure $4.27(A)$ ) are surprisingly well ordered. 
Finally, Figure 4.28 shows the $\mathrm{D} / \mathrm{G}_{1+2}$ ratio as a function of annealing temperature. While both the $\mathrm{D}$ and $\mathrm{G}_{1+2}$ peaks narrow, the overall decrease of the $\mathrm{D} / \mathrm{G}_{1+2}$ ratio is indicative of fewer structural defects in the remaining films at higher temperatures. Note that there is a substantial reduction in the film thickness as the temperature increases as well, with the samples exposed to $1400^{\circ} \mathrm{C}$ showing no Raman signal and thus there is no $\mathrm{D} / \mathrm{G}_{1+2}$ data point for that temperature.

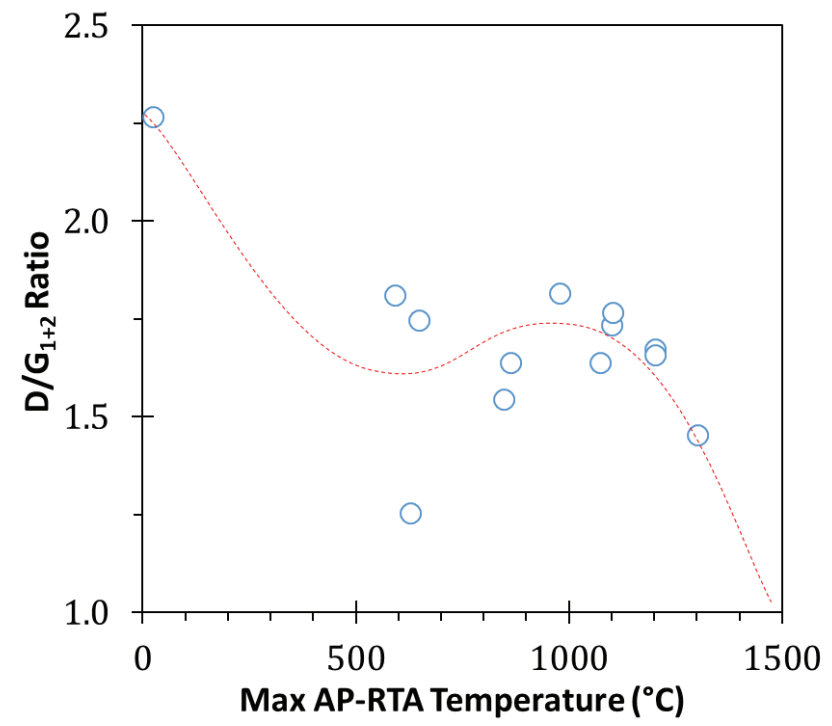

Figure 4.28: The area ratio of the D peak area to the sum of the G1 and G2 peak areas. The decrease is indicative of a films wich have fewer structural defects and therefore a higher crystilinity. The arbitrary best fit line is included as the dashed line to guide the eye.

In summary, increasing the annealing temperature has several effects on the film quality. By XPS analysis, the lowest defect concentration is seen at around $1000^{\circ} \mathrm{C}$. Around this temperature, all fluorine species have been removed from the film and a minimum is found in the oxygen-based film defects. At higher temperatures, reactions with oxygen begin to consume the carbon in the overlayer, reducing the amount of surface carbon and increasing the defect concentration of the remaining film. At very high temperatures $\left(>1200^{\circ} \mathrm{C}\right)$ films of mostly bufferlayer, with a fractional graphene overlayer, are left. The densification of the film (as determined from the increased attenuation of the $\mathrm{SiC}$ substrate signal) combined with the improvements in the Raman spectra indicate that higher temperatures lead to films with a greater degree of crystallinity. The overarching trend is that higher AP-RTA temperatures result in substantially thinner films that are slightly more crystallin. However, at no point do the films fully decouple from the substrate. This is clear from the overall character of the Raman spectra which still indicated a coupling of the vDOS and in the XPS where the $S_{1}$ and $S_{2}$ peaks remain present. 


\section{\$4.2.5 AP-RTA- Effect of Heating Rate}

This study investigated the effect of heating rate on the films after they underwent the standard ICP/RIE etching process. Samples were heated to $\sim 1000^{\circ} \mathrm{C} \pm 10 \%$ at three different rates. After the samples reached $1000^{\circ} \mathrm{C}$, the samples were withdrawn from the heater and allowed to cool natural in the laboratory air, resulting in the SOP cooling rate. Figure 4.29(A) show a semi$\log _{10}$ plot of the heating rate and a standard plot of the SOP cooling rate. Consistent with the previous section, to accurately represent the AP-RTA profiles, logarithmic equations are used to describe the heating profile and exponential decay equations are used to describe the cooling profiles.

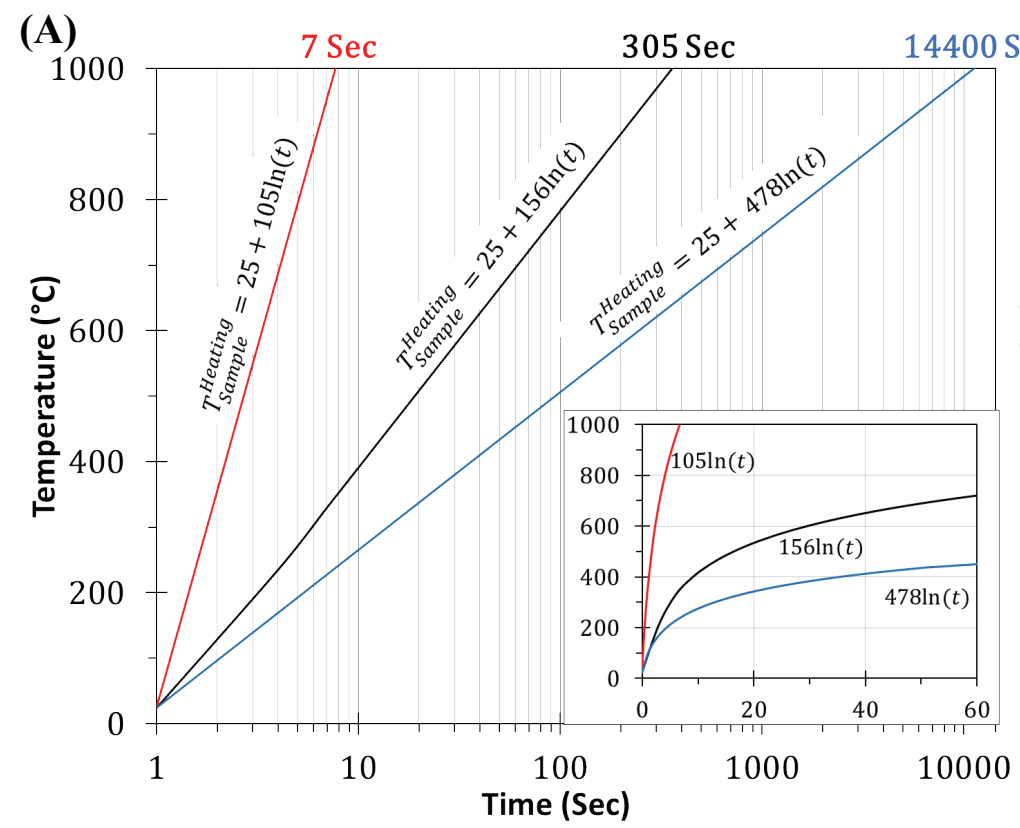

(B)

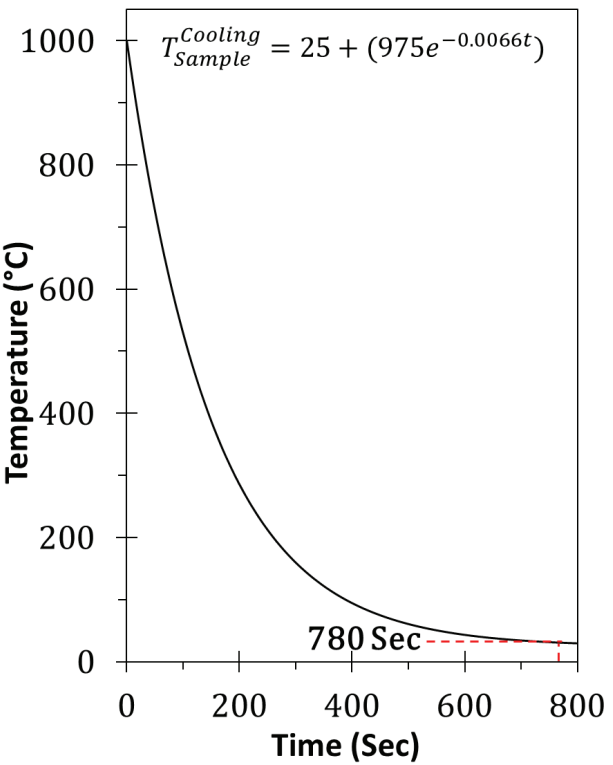

Figure 4.29: (A) A semi- $\log _{10}$ plot of the three different heating rates used in this study. The natural log equations which describe the heating rates are shown next to their curves, where ' $t$ ' is the time in seconds. The time to $1000^{\circ} \mathrm{C}$ is annotated as well. Inset, the first 60 seconds of the same data but in a standard plot. (B) A representative cooling rate for all samples. This is the standard synthesis cooling rate. The cooling time to $\sim 25^{\circ} \mathrm{C}$ is annotated as well.

The fastest heating rate was achieved by setting the heater to $1400^{\circ} \mathrm{C}$ and withdrawing the RTA tube when the tube thermocouple read $\sim 1000^{\circ} \mathrm{C}$. This resulted in a total heating time of 7 seconds from $\sim 25^{\circ} \mathrm{C}$ to $\sim 1000^{\circ} \mathrm{C}$ (a heating rate of $105 \ln (\mathrm{t})$ ). For the next fasted heating rate, the heater was set to $\sim 1000^{\circ} \mathrm{C}$ and the tube was withdrawn when the tube thermocouple read $\sim 1000^{\circ} \mathrm{C}$. This resulted in a total heating time of 305 seconds from $\sim 25^{\circ} \mathrm{C}$ to $\sim 1000^{\circ} \mathrm{C}$ (a heating rate of $156 \ln (\mathrm{t}))$. For the slowest heating rate, the RTA tube with sample was inserted into a cold heater before it was powered (i.e., it was at room temperature). The heater was then programed to a setpoint of $1000^{\circ} \mathrm{C}$. The heating rate of the tube was thus dictated by the furnaces slow ramp to 
the setpoint. Although the PID controller of the furnace leads to a complex heating profile, for simplicity it is described here using a logarithmic equation. The total heating time was 14400 seconds or 4 hours (a heating rate of $478 \ln (t))$.

Stacked XPS and Raman spectra are shown in Figure 4.30(A,B), respectively. The most readily apparent trend is that the slower the heating rate, the fewer number of carbon layers are produced. Clearly, the slowest rate (corresponding to the $478 \ln (\mathrm{t})$ heating rate) results in, on average, only a fractional carbon monolayer and is only weakly Raman active. For ease of viewing, the plots of Figure 4.30(C-F) are simi- $\log _{10}$ and plot various parameters extracted from the XPS and Raman spectra shown in Figure 4.30(A,B). 
(A)

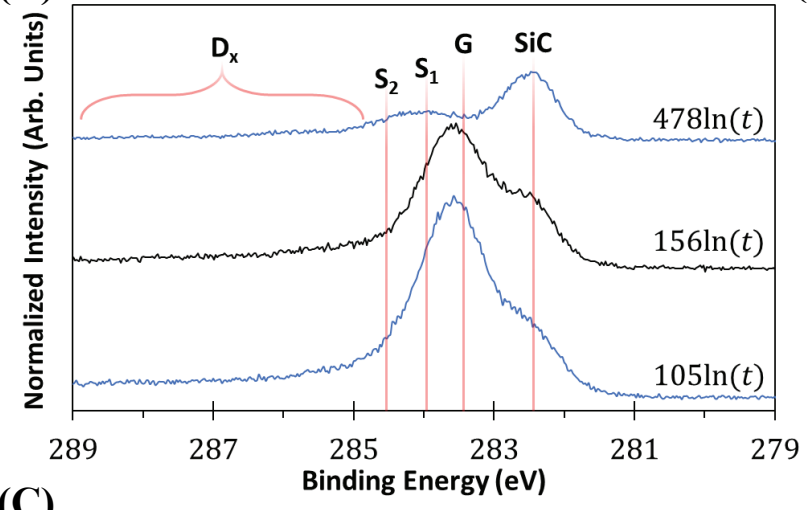

(C)
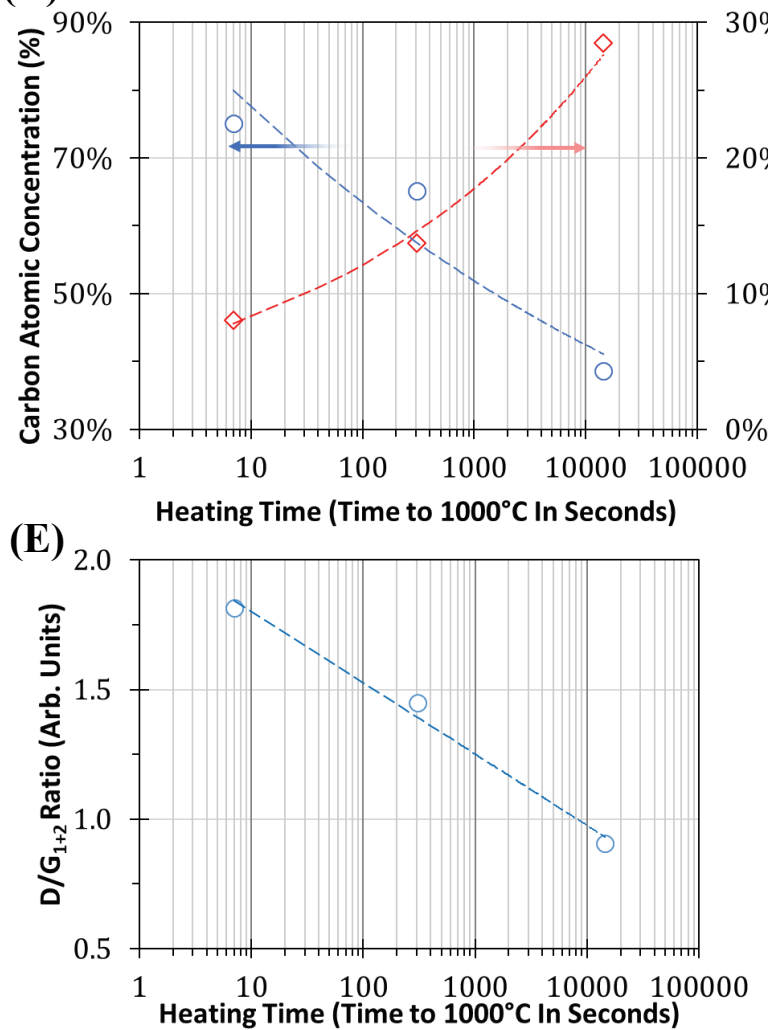

(B)

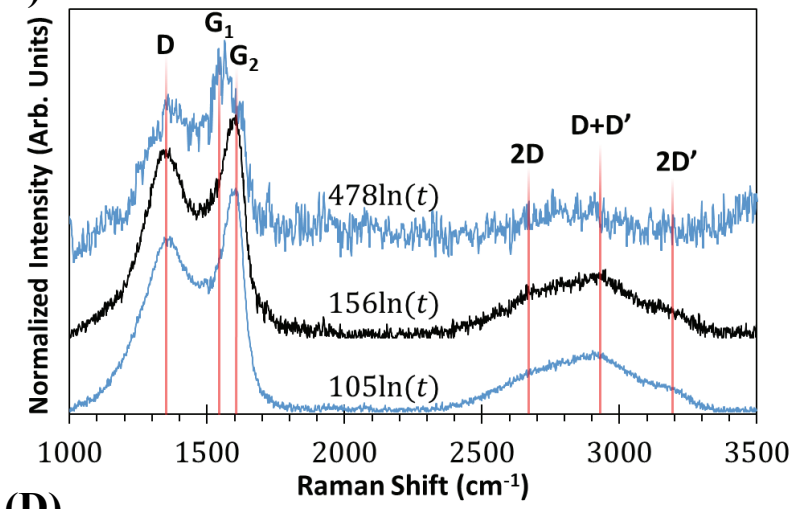

(D)

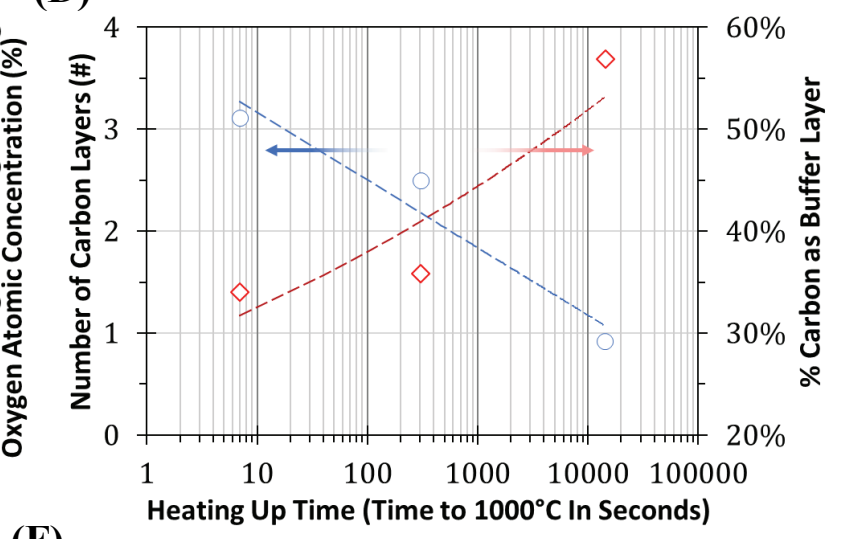

(F)

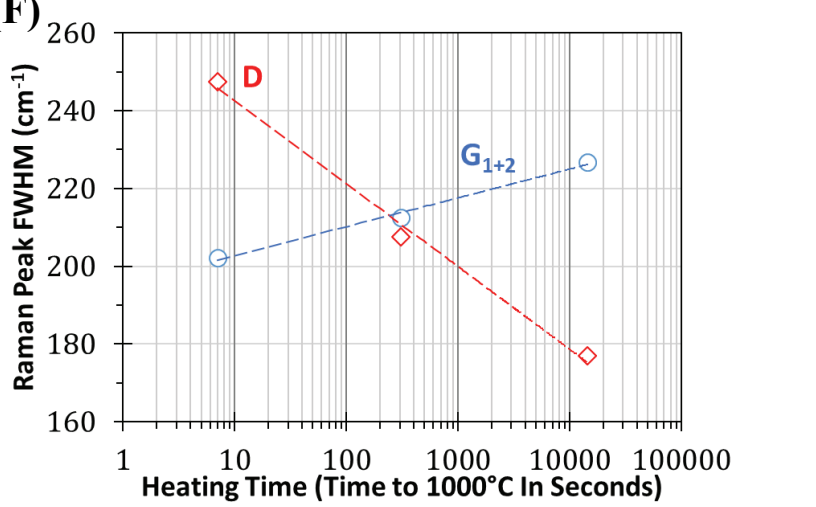

Figure 4.30: (A) XPS of the C1s region and (B) Raman spectra of three samples, each heating at a different rate. All other synthesis parameters were identical. (C-F) Parameters extracted from $(A, B)$ plotted against the sample heating duration in a $\log _{10}$ scale. (C) Plot of the atomic concentration of carbon and oxygen. (D) Plots of the number of carbon layers and the percentage of carbon in the film associated with the bufferlayer. (E) The $\mathrm{D} / \mathrm{G}_{1+2}$ ratio is shown to decrease (improve) with extended annealing time. (F) The full width at half maximum (FWHM) of the D and G peaks. The narrowing of the D peak indicates a narrowing of the distribution of states responsible for the $\mathrm{D}$ peak. Likewise, the $\mathrm{G}$ peak widening indicates additional $\mathrm{G}$ peak vibrational modes.

Figure 4.30(C) shows an exponential decrease in the atomic concentration of carbon with a simultaneous and exponential increase in the atomic concentration of oxygen. This is similar to what was observed in the study investigating the maximum AP-RTA temperature (Section $\$ 4.2 .4$ ) for temperatures above $1200^{\circ} \mathrm{C}$. In both cases, it appears that at the extremes of the study an 
oxidation of the film and removal of surface carbon occurs. Figure 4.30(D) shows the number of carbon layers and the fraction of carbon in the film associated with the buffer-layer. Slower ramp rates lead to fewer layers and leaving a larger fraction of buffer-layer carbon. Again, this is similar to the very high temperature experiments in the maximum AP-RTA study.

Figure 4.30(E,F) details aspects of the $\mathrm{D}$ and $\mathrm{G}_{1+2}$ peaks. Figure 4.30(E) shows that by slowing the heating rate, the $\mathrm{D} / \mathrm{G}_{1+2}$ area ratio is decreased. This is indicative of films with fewer defects. From Figure 4.30(F), the D peak is seen to narrow substantially while the G peak grows in width. Figure 4.30(E,F) taken together indicate that higher quality films are produced by slower heating rates. However, from Figure $4.30(C, D)$, we see that this comes at the cost of film oxidation and removal.

The simplest interpretation of Figure 4.30 is as follows. The decrease in the distribution of $\mathrm{D}$ states and the corresponding increase in the amount of $\mathrm{G}$ states, may be indicative of a film healing process. Carbon atoms initially in a defective state (contributing to the breadth of the $\mathrm{D}$ peak), rearrange to participate in a complete aromatic ring (contributing to the breadth of the $G$ peak). The peak intensities follow a similar trend, but the effect is more pronounced in the FWHM values. This fact indicates that while substantial film healing may be occurring, the distribution of states is still wide. The film is still coupled and is under a wide distribution of physical stress and strain states.

An alternant interpretation may be a 'film evolution' via a natural selection process, rather than a film healing process. In this case, broken aromatic carbon rings (and stressed rings near defect sites) which contribute to the D peak, would be more susceptible to the oxygen decomposition reaction which removes part of the film. In this way, carbon atoms that are in a defective state are consumed first, leaving only the atoms that are in a more structurally perfect state (and less vulnerable) in place. This would have the same effect of decreasing the $\mathrm{D} / \mathrm{G}_{1+2}$ ratio by selectively removing defective carbon. Figure $4.30(C, D)$ clearly indicates a carbon removal process is occurring. Figure $4.30(E, F)$ clearly indicates that the carbons that remains is of higher crystallinity. Most likely, some degree of both film healing and the film evolution process are both occurring.

In summary, this study investigated the effects of the heating duration across three orders of magnitude (a 4-fold increasing the heating rate). While this study ostensibly studied the heating 
rate, the main driving force for the observed film effects is most likely the effective time at elevated temperature the samples are exposed to. For example, an impact of a slow heating rate is that more time is spent by the sample at elevated temperature during the slow approach to the temperature end point $\left(1000^{\circ} \mathrm{C}\right.$ in this case). The effect on the films is two-fold. First, by spending more time at an elevated temperature, the reaction which consumes carbon from the sample surface is allowed to continue longer, leading to thinner films. Second, slower heating rates also result in higher quality films.

Extended anneal times at lower temperatures will be a subject of future work. Depending on the temperature sensitivity of the kinetics and on the activation energy of the carbon removal process, as compared to the carbon rearrangement process, there may be temperatures were the removal process is disproportionately limited. Therefore, by annealing for longer times at lower temperatures, the advantages of the film healing process may be realized, and the disadvantages of the film removal process could be reduced or eliminated.

\section{\$4.2.6 AP-RTA- Effect of Cooling Rate}

This study investigated the effect of the cooling rate on the films after they underwent the standard ICP/RIE etching process. All samples were etched and at the same time and then annealed sequentially, except for the sample exposed to liquid nitrogen (discussed later) which was processed at a later date. Samples were heated to $\sim 1000^{\circ} \mathrm{C}$ at the standard rate by inserting the RTA tube containing the sample into the tube furnace which was set to $1000^{\circ} \mathrm{C}$. Consistent with the previous sections, to accurately represent the AP-RTA profiles, logarithmic equations are used to describe the heating profile and exponential decay equations are used to describe the cooling profiles. Figure 4.31(A) show the various cooling profiles labeled with the pre-exponential factor which approximates the cooling rate. Figure $4.31(B)$ is color coded and tabulates the heating and cooling equations. The black lines correspond to the standard heating and cooling rates. The various cooling rates were achieved by slowly withdrawing the RTA tube from the FD 303A Hoskins Electric Furnace (Hoskns Mfg. Co.) and while manually monitoring the thermocouple readout so that the desired rate was achieved. Therefore, the cooling rate equations in Figure $4.31(B)$ are approximant. As with the vast majority of AP-RTAs, the samples were under a flow of 100 SCCM of room temperature research plus purity argon (99.9999\%) before, during and after the heating and cooling. 
To achieve the most rapid cooling rate, the RTA tube was decoupled from the flow system once the thermocouple read $1000^{\circ} \mathrm{C}$. The tube was upended so that the etched sample slid out into a modified dewar containing $500 \mathrm{~mL}$ of liquid nitrogen. The tube motion was such that the open end of the tube was never exposed to the laboratory air, taking advance of the nitrogen blanket covering the dewar. The whole process was completed in less than two seconds. Given the thermal mass of the RTA tube which kept the sample hot during the 2 second transit and the small mass of the sample $(<<1 \mathrm{~g})$ and the comparatively large reservoir of liquid nitrogen, it is estimated that the sample cooled from $1000^{\circ} \mathrm{C}$ to $-196^{\circ} \mathrm{C}$ in less than one second. This is consistent with visual observations. A dewar geometry was chosen such that the sample would fall and tumble through $\sim 15 \mathrm{~cm}$ of liquid before it reached the bottom of the vessel rather than simply set stationary during the cooling process. This turbulence further enhanced the heat transfer by reducing film boiling effects by sweeping the surface of nitrogen bubbles during the samples fall to the bottom of the dewar. All of this was done to achieve the maximum cooling rate possible.

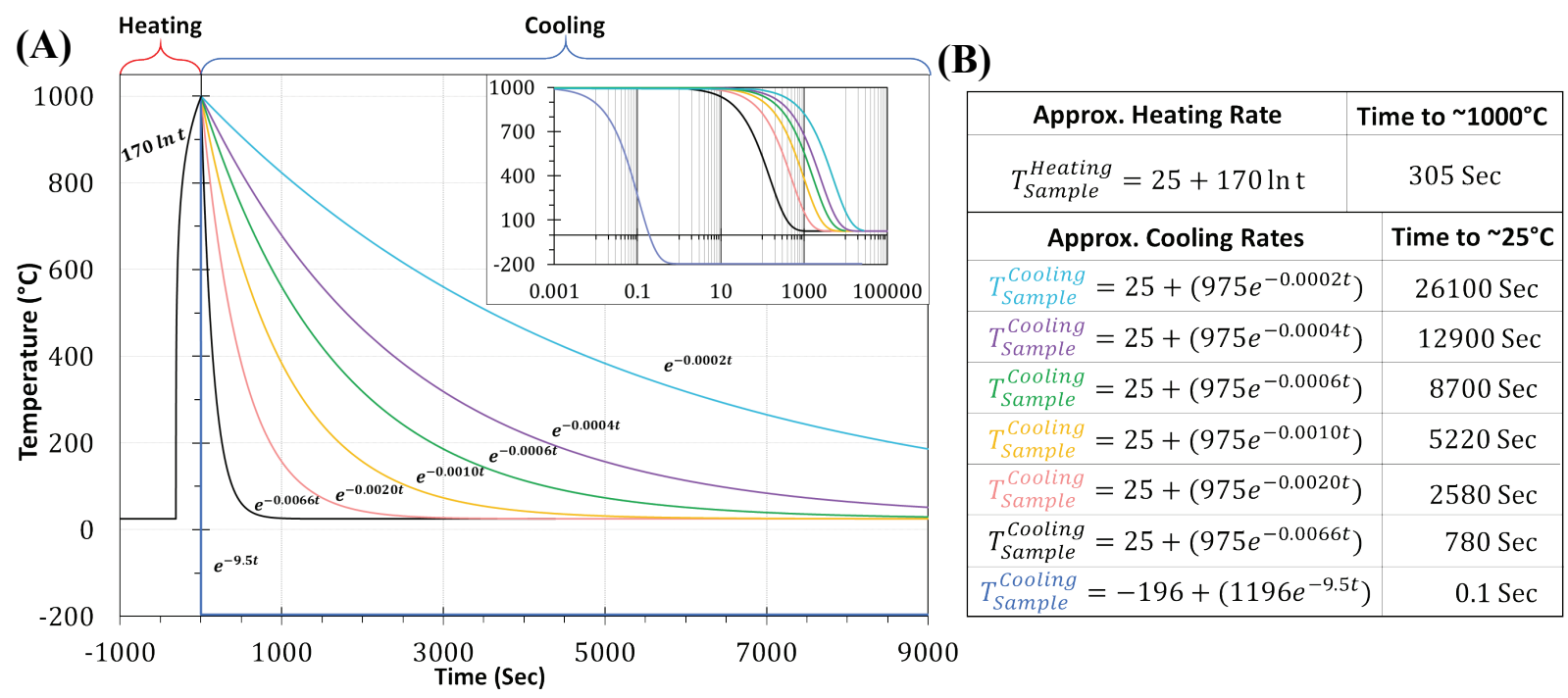

Figure 4.31: The various cooling rates explored in this study. Heating rates were all identical. (A) Plots of the cooling rates, time shifted to the onset of the cooling phase. Curves are labeled with the exponential factors which govern the cooling rate. Inset, the same data in a semi- $\log _{10}$ plot. (B) The equations used to generate the curves in (A) which approximate the cooling rate where ' $\mathrm{T}$ ' is the temperature of the sample in ${ }^{\circ} \mathrm{C}$ and ' $\mathrm{t}$ ' is the time is seconds. Equations are color coded to the curves in (A). Also tabulated is the amount of time the samples took to cool down from $\sim 1000^{\circ} \mathrm{C}$ to approximately room temperature.

Figure 4.32(A,B) show stacked XPS and Raman spectra across the various cooling rates. The black line corresponds to the standard cooling rate. Figure 4.32(C-F) plot the various extracted parameters from the spectra against the time it took for each sample to cool from $1000^{\circ} \mathrm{C}$ to $\sim 25^{\circ} \mathrm{C}$. A similar trend to the heating rate study is seen with extended cooling times. Figure 4.32(C) show 
the atomic concentrations of carbon and oxygen. In general, longer cool down times result in less carbon and more oxygen on the surface. Figure 4.32(D) plots the number of carbon layers and the percentage of carbon associated with the buffer-layer against the cooling time. Again, Figure 4.32(D,C) are internally consistent and the trends are similar to the heating study. 
(A)

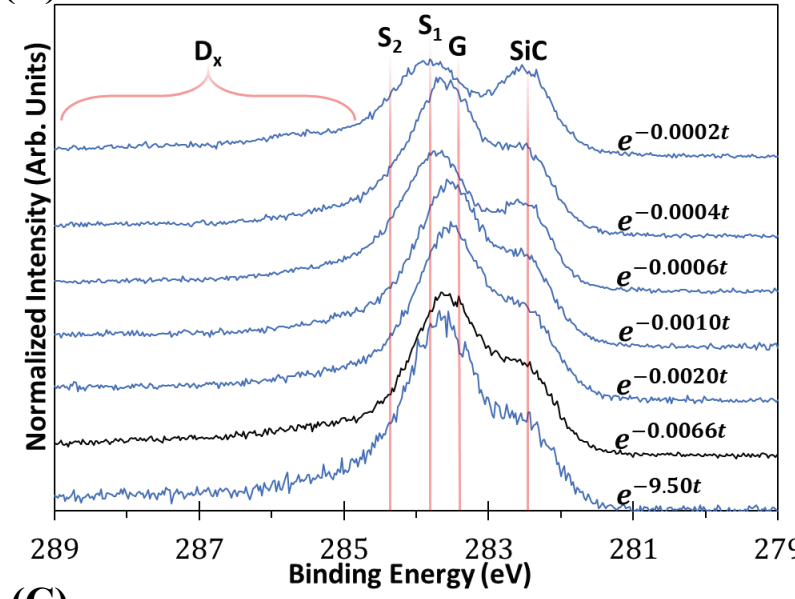

(C)

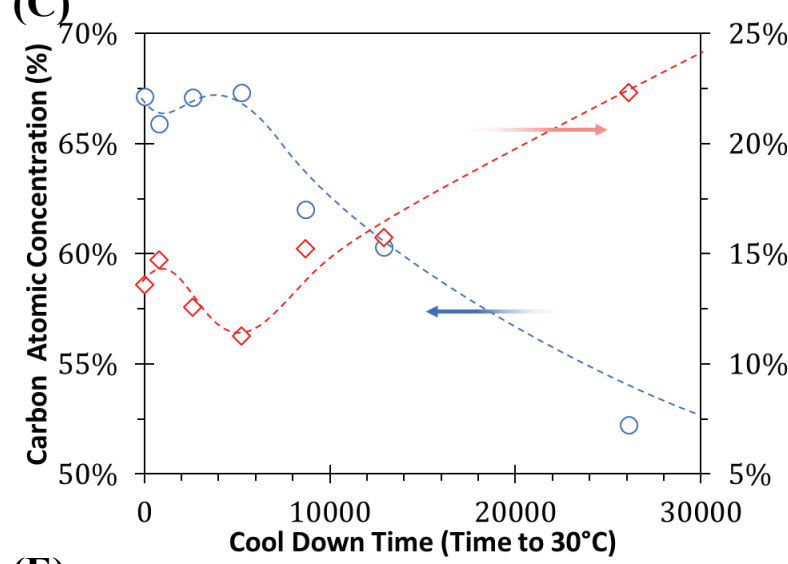

(E)

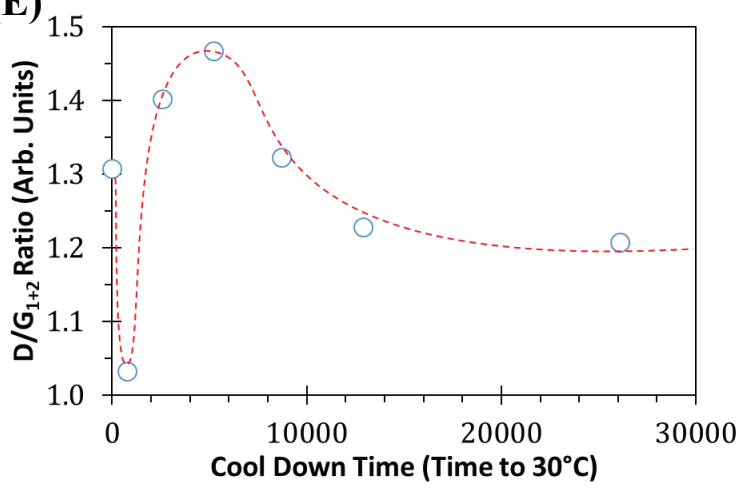

(B)

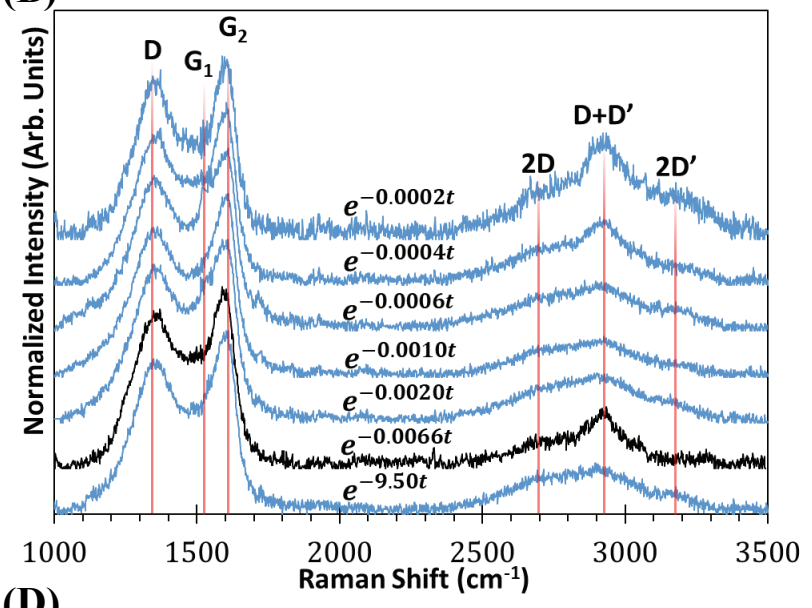

(D)
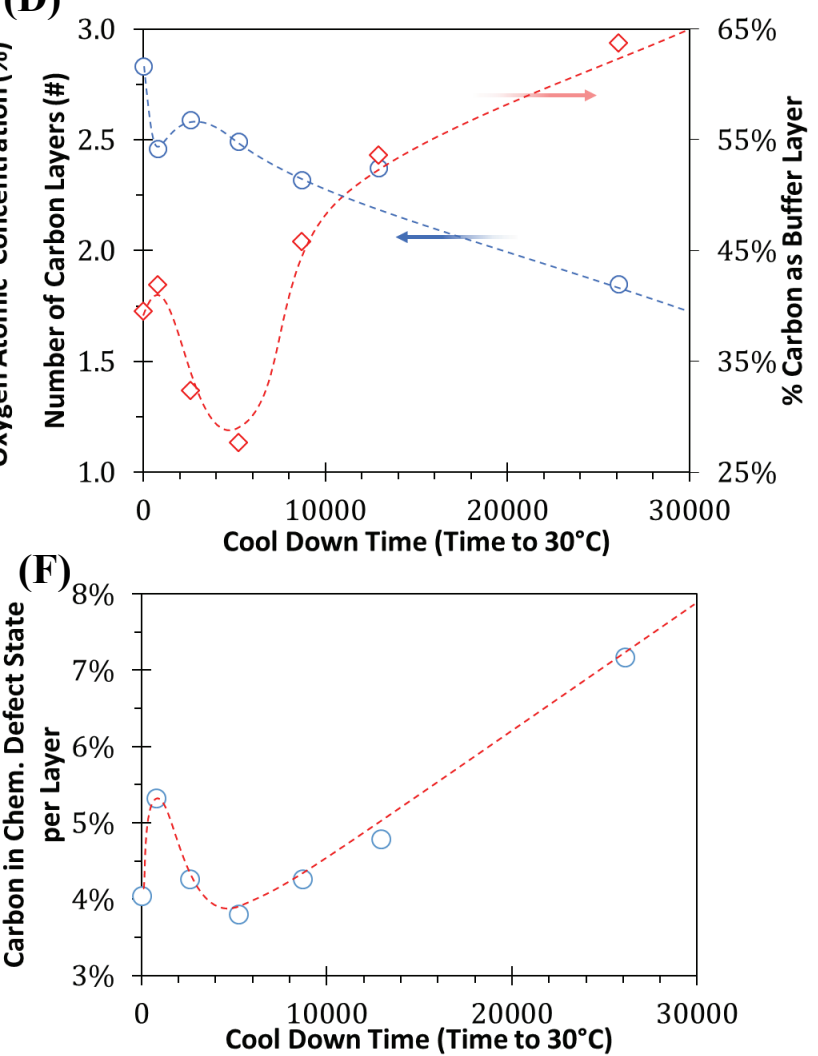

Figure 4.32: (A) XPS of the C1s region and (B) Raman spectra of three samples, each cooled at a different rate. All other synthesis parameters were identical. (C-F) Parameters extracted from (A,B) plotted against the sample cooling duration. (C) Plot of the atomic concentration of carbon and oxygen. (D) Plots of the number of carbon layers and the percentage of carbon in the film associated with the buffer-layer. (E) The $\mathrm{D} / \mathrm{G}_{1+2}$ ratio and $(\mathrm{F})$ the fraction of carbons in each layer involved in a chemical defect state. The complexities of the curves of (E) and (F) indicated competing process may be at play. Arbitrary best fit lines are included as the dashed lines to guide the eye.

Figure 4.32(E,F) show the trends in crystallinity and chemical defects respectively. The complexities of the curves indicate that there are several competing factors at work during the cool down stage. From theory, a slow cooling period would allow for the decreasingly mobile carbon 
atoms to settle into their thermodynamically favorable position; in this case, the aromatic graphene ring. This is evident by the decrease in the $\mathrm{D} / \mathrm{G}_{1+2}$ ratio (Figure 4.32(E)). However, the extended time also exposes the vulnerable carbon atoms (atoms not yet incorporated into a large graphene domain) to oxidation. For cooling periods longer than $\sim 600 \mathrm{sec}$, the oxidation process appears to dominate (Figure 4.32(F)) and a point of diminishing returns is reached with respect to the crystallinity (Figure 4.32(E)).

The jitter between the first three data points in Figure 4.32(C-F) may be simple 'noise' in line with what could be expected due to sample-to-sample variability. However, if this is not the case, the sample which was cooled very rapidly with liquid nitrogen may provide additional insight into the annealing process. Considering the ultra-fast cooling rate, film atoms are quickly locked into their positions as they fall into local energy minimum (as opposed to a border global minimum that a slower cooling rate would theoretically allow for). Thus, this sample provides a unique 'snap-shot' of the film frozen in the initial stages of the reordering process that the annealing step provides. The comparatively thick film and low chemical defect density indicated that the carbon consuming oxidation reaction has not proceeded far. To the extent that it has proceeded has most likely occurred during the heating ramp phase, as indicated by the heating rate study detailed in Section $\$$ 4.2.5. Compared to its neighboring data point, this film has a high $\mathrm{D} / \mathrm{G}_{1+2}$ ratio which would be consistent with a film which has not undergone extensive reordering.

To investigate the effect of an extended annealing time, a sample was allowed to rest at $1000^{\circ} \mathrm{C}$ for 15 minutes under a flow of $100 \mathrm{SCCM}$ following the SOP heating rate. The sample was then withdrawn from the furnace and allowed to rapidly and naturally cool down following the SOP cooling rate. The results are included in Appendix $\$ \mathbf{A . 2 . 8}$. The overall conclusion is that extended anneal times longer than standard anneal leads to deleterious effects very similar to what was observed in this study. Atomic Force Microscopy (AFM) analysis was also conducted on all samples in this study. However, each surface is practically indistinguishable from one another and are identical to the samples produced using the Standard Process.

In summary, the cooling rate has a similar effect on the films as the heating rate does. The etched carbon film reorganization process is undoubtedly complex; however, the general trends are consistent with classic annealing processes. Longer anneal times lead to more crystallin films, 
but this film improvement is hampered by the pervasive film oxidation process. The tradeoff between the two processes indicate that the SOP parameters are near optimum.

\section{\$4.2.7 Summary of the Parametric Investigation}

This section detailed the effect of various processing parameters on the films produced using the plasma assisted method. Each of the parameters investigated effected the film. The SiC polytype, crystal face and doping were minimal. This is in contrast to the selective sublimation method, which is strongly dependent on the specific of the starting substrate. The difference between this plasma assisted method and the sublimation method arises due to the fundamental difference between the silicon removal processes. The sublimation process is a much slower process, is more selective towards Si removal and the reorganization of the carbon film is highly templated by the immediate underlying substrate.

The plasma powers were found to be the most important parameters of the synthesis process. The interplay between the ICP power (which determines the chemistry of the plasma) and RIE power (which sets the DC bias, which determines the ion energies) control the number and quality of the graphene layers produced. At low RIE power, the chemistry of the ICP plasma dominates, lending to a highly selective etch and a multi-layered film produced. Increasing the ICP power increases the physical etching, which decrease the balance of carbon on the surface established by the chemical etch. Increasing the ICP power further leads to a synergistic effect between the chemical and physical etching, resulting in a sharp increase in the number of carbon layers produced. At the highest ICP powers tested, the trend revers due to the dominance of the physical etch, which reduces the amount of carbon on the surface.

Variation in the AP-RTA parameters can be summarized in the fact that higher temperatures and longer times produced more crystallin films, but at the cost of an increased oxygen defect concentration. It was found that a temperature of $\sim 800^{\circ} \mathrm{C}$ was required to fully desorb the fluorine component in the film remining from the etching process.

\section{§4.3 Post Synthesis- Effect of High Temperature Hydrogen Treatment}

As discussed in Section \$2.1.3, it is desirable to decouple the buffer-layer from the substrate to improve potential device performance. In short, by physically freeing the buffer-layer from the substrate, both the electronic states and the vibrational states between the two can be 
decoupled. On freeing, the buffer-layer can relax into the natural conformation of graphene and the advantages of a quasi-freestanding low-D material can then be fully realized.

The most commonly cited method in the literature is through high temperature exposure to hydrogen. Common parameters and the relevant mechanisms are discussed in Section $\$ 2.1 .3$. There are two distinct steps to the hydrogen induced decoupling process:

I) Mass transport of hydrogen to the buffer-layer/substrate bonding sites.

II) Reaction with, and passivation of, the substrate bonding sites.

Briefly, hydrogen intercalates into the stacked structure, where it reacts with the covalent bond between the $\mathrm{sp}^{3}$ hybridized silicon atoms of the substrate and the carbon atoms of the buffer-layer. Hydrogen then bonds to the silicon atoms and passivates the otherwise reactive sites. This leads to a decoupling of the buffer-layer allowing it to relax and transition into a quasi-freestanding graphene sheet. This is discussed extensively in Section $\$ 2.1 .3$.

As will be discussed, the Raman and XPS spectra are expected to change once decoupling has occurred. It is these changes (if they occur) that are of interest in this study. This section will present evidence that, although full buffer-layer decoupling has not occurred, changes in the Raman and XPS spectra indicate the initial stages of decoupling have started. It is noted that, as discussed in Section \$3.2.1, hydrogen itself cannot be practically detected using XPS. Furthermore, without the use of surface-enhanced Raman spectroscopy (SERS), the Si-H stretching mode is difficult to detect and the $\mathrm{C}-\mathrm{H}$ bending and stretching modes are covered by the $\mathrm{D}$ and D+D' peaks. (563) (562) (564) Therefore, the presence of hydrogen is not easily directly observed; however, the indirect effects of the buffer-layer release can be observed in subtle chemical shifts in the C1s region of XPS spectra and more obviously in the overall character of the Raman spectra.

Because the decoupling would most readily become apparent in the Raman spectra, this study primarily used Raman analysis to investigate the effects. Select samples were analyzed using XPS as well, but the analysis is complicated for reasons discussed later. Nevertheless XPS, in conjunction with Raman spectroscopy, gives additional insight. A broad survey of the literature indicates that decoupling of the buffer-layer is evident in the Raman spectra by: initially a small decrease in the $\mathrm{D} / \mathrm{G}$ ratio, followed by a narrowing of all Raman peaks and finally, substantial 
growth of the 2D peak. This which indicate a complete decoupling of the buffer-layer into a qusifree standing graphene layer (QFSG).

The decrease in $\mathrm{D} / \mathrm{G}$ is due to the removal of $\mathrm{C}$-Si bonds which act as defect sites and contribute, in part, to the $\mathrm{D}$ peak. The narrowing of peaks is a direct result of a decoupling of the vibrational states between the carbon layers and the substrate (similar to coupled oscillators with different periodicities versus the free resonance of a single oscillator at its natural resonance) and an improvement in crystallinity (analogous to vibrating a bowl of jelly versus a tuning fork). And Finally, the unambiguous growth of the 2D peak, the quintessential graphene peak, indicates a full decoupling (note that the buffer-layer has no 2D peak and in decoupled graphene, the 2D peak is approximately $4 \mathrm{x}$ the D peak intensity). ${ }^{(212)(494)(492)}$

To investigate the effects of high temperature exposure to hydrogen, a wide range of experiments were conducted. They are summarized in Table 4-3. The effect of dwell time and maximum annealing temperature were studied on two categories of samples. In the first category, are the samples which had undergone the full SOP process (etched and annealed under argon at $1000^{\circ} \mathrm{C}$ ), and in the second, the samples had experienced the $\mathrm{CF}_{4}$ etch only (i.e., no annealing, had not been exposed to high temperature in any environment).

The same apparatus was used for both the AP-RTA step and the high temperature hydrogen exposure experiments. The samples which were AP-RTAed in argon and then heated in hydrogen were not exposed to air between the steps. The samples remained in the RTA tube and once the samples reached room temperature, the gas was switched from argon to hydrogen. The tube was purged at a flow rate and for a duration such that the gas was exchanged many times over. This study follows the same procedures as described in Section $\$ 3.1 .4$ for the AP-RTA. 
Table 4-3: The experimental matrix used to investigate the effects of hydrogen exposure on the films.

\begin{tabular}{|c|c|c|c|c|c|c|c|c|c|c|c|c|c|}
\hline & \multicolumn{12}{|c|}{ Maximum Annealing Temperature Under Pure Hydrogen } \\
\hline & & \multicolumn{2}{|c|}{$500^{\circ} \mathrm{C}$} & \multicolumn{2}{|c|}{$600^{\circ} \mathrm{C}$} & \multicolumn{2}{|c|}{$700^{\circ} \mathrm{C}$} & \multicolumn{2}{|c|}{$800^{\circ} \mathrm{C}$} & \multicolumn{2}{|c|}{$900^{\circ} \mathrm{C}$} & \multicolumn{2}{|c|}{$1000^{\circ} \mathrm{C}$} \\
\hline \multirow{4}{*}{ 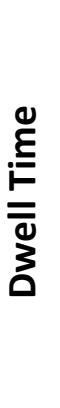 } & $\begin{array}{c}0 \\
\min \end{array}$ & $\begin{array}{c}\text { Etched } \\
\text { Only }\end{array}$ & SOP & $\begin{array}{c}\text { Etched } \\
\text { Only }\end{array}$ & SOP & $\begin{array}{c}\text { Etched } \\
\text { Only }\end{array}$ & SOP & $\begin{array}{c}\text { Etched } \\
\text { Only }\end{array}$ & SOP & $\begin{array}{c}\text { Etched } \\
\text { Only }\end{array}$ & SOP & $\begin{array}{l}\text { Etched } \\
\text { Only }\end{array}$ & SOP \\
\hline & $\begin{array}{c}15 \\
\min \end{array}$ & $\begin{array}{c}\text { Etched } \\
\text { Only }\end{array}$ & SOP & $\begin{array}{c}\text { Etched } \\
\text { Only }\end{array}$ & SOP & $\begin{array}{c}\text { Etched } \\
\text { Only }\end{array}$ & SOP & $\begin{array}{c}\text { Etched } \\
\text { Only }\end{array}$ & SOP & $\begin{array}{c}\text { Etched } \\
\text { Only }\end{array}$ & SOP & $\begin{array}{c}\text { Etched } \\
\text { Only }\end{array}$ & SOP \\
\hline & $\begin{array}{c}60 \\
\mathrm{~min}\end{array}$ & $\begin{array}{c}\text { Etched } \\
\text { Only }\end{array}$ & SOP & \begin{tabular}{|c|} 
Etched \\
Only
\end{tabular} & SOP & $\begin{array}{c}\text { Etched } \\
\text { Only }\end{array}$ & SOP & $\begin{array}{c}\text { Etched } \\
\text { Only }\end{array}$ & SOP & $\begin{array}{c}\text { Etched } \\
\text { Only }\end{array}$ & SOP & $\begin{array}{c}\text { Etched } \\
\text { Only }\end{array}$ & SOP \\
\hline & $\begin{array}{l}120 \\
\text { min } \\
\end{array}$ & & & & & & & & & & & & SOP \\
\hline$\frac{0}{v}$ & \begin{tabular}{|c}
5 \\
$\min$ \\
$\times 10$
\end{tabular} & & & & & & & & & & & & SOP \\
\hline
\end{tabular}

These three parameters each provide insight into various aspects of the decoupling process. Variations of the maximum annealing temperature can reveal information on the bonding strength between the buffer-layer and substrate. For instance, if decoupling occurred only after a critical temperature threshold were reached, this would give insight into the energy required for the cleavage of the $\mathrm{Si}-\mathrm{C}$ bond (specifically, by the hydrogenation reaction on the $\mathrm{Si}$ atom site).

Variations in dwell time can provide insight to the kinetics of the process. Given that the path of a hydrogen atom to the substrate-buffer-layer interface is circuitous (and thus a diffusion limited process), dwell time variations speak more to the diffusion kinetics rather than the reaction kinetics (which would be comparatively much faster).

Lastly, by conducting the same sets of experiments on the two types of samples (SOP samples and etched-only samples) the role that hydrogen plays (if any) during the film reordering process can be studied. Additionally, the diffusion of hydrogen through the more ordered film produced by the AP-RTA samples is expected to be different then through the partially amorphous film of the unannealed samples.

For samples which were not allowed to dwell at temperature (i.e. a dwell time of $0 \mathrm{~min}$ ), the furnace was set to $1000^{\circ} \mathrm{C}$ and the samples were withdrawn once the thermocouple read the desired temperature, (similar to the maximum AP-RTA temperature in Section \$4.2.4). Therefore, the heating rates of these samples are similar. For all other samples, the heater was set to the desired temperature and the dwell time clock was started once the sample temperatures reached within 5\% 
of the furnace setpoint. Thus, the heating rates are all different. Similarly, the cooling rates are different in that they are dictated by the maximum temperature.

\section{\$4.3.1 Temperature Effects}

Figure 4.33 shows the stacked Raman spectra of samples which were etched only (no APRTA in argon) and then annealed in hydrogen at various temperatures $\left(500^{\circ} \mathrm{C}-1000^{\circ} \mathrm{C}\right)$ for three different dwell durations: $0 \mathrm{sec}, 900 \mathrm{sec}(15 \mathrm{~min})$ and $3600 \mathrm{sec}(1 \mathrm{hr})$. The relevant films for comparison are shown as well. For the 0 second dwell time, the samples were allowed to reach the desired temperature and were then immediately removed to cool. A qualitative analysis of Figure 4.33 indicates four key points.

\section{(A)}

Dwell

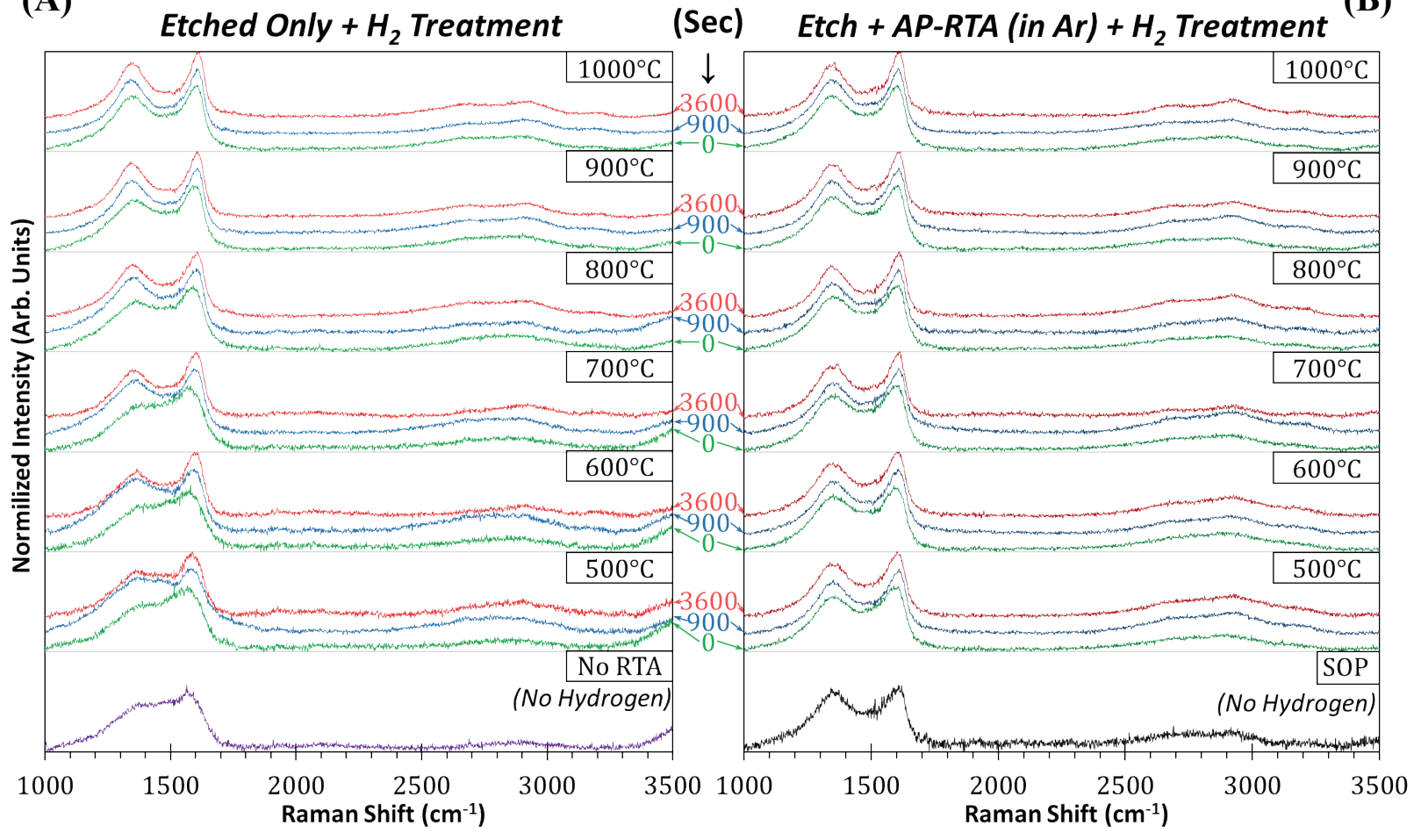

Figure 4.33: (A) The normalized and stacked Raman spectra grouped by temperature and stacked by increasing dwell time: o sec, 900 sec and 3600 sec. Shown for comparison is the spectrum for samples which have not seen any elevated temperature or exposure to hydrogen. (B) Raman spectra from samples produced using the standard AP-RTA in argon, cooled and then reheated in hydrogen and allowed to dwell for o sec, $900 \mathrm{sec}$ or $3600 \mathrm{sec}$. Shown for comparison is the spectrum of the sample produced at the same time using the Standard Synthesis process and has not been exposed to hydrogen.

First, films appear to be more stable in hydrogen for extended dwell times then they are in argon. Recall that extended annealing at high temperature in argon produced much thinner and more oxidized films (Section $\$ 4.2 .5$ and $\$ 4.2 .6$ and Appendix $\$$ A.2.8). As will be discussed in the 
XPS analysis, this may be due to a lower level of oxygen-based contaminants in the hydrogen stock or to a competition for free oxygen between the film carbon and gaseous hydrogen.

Second, hydrogen exposure does not have a drastic effect on the Raman spectrum. As evidenced by the absence of growth in 2D peak or drastic narrowing of the $\mathrm{D}$ and $\mathrm{G}$ peaks, the films appear to remain largely coupled to the substrate. Note that the times and temperatures used in this study cover the majority of what has been reported in the literature for successful decoupling (see Table 2-1). However, as discussed later, close analysis indicates the films do show signs of the beginning stages of decoupling. This speaks to the unique nature of these films and the strong bonding of the buffer-layer to the substrate.

Thirdly, there are some overt changes that can be seen in Raman spectra from the etchedonly samples (no AP-RTA in Ar) with increasing temperature (Figure 4.33(A)). Note the continued refinement of the spectra over the transition from $500^{\circ} \mathrm{C}$ to $1000^{\circ} \mathrm{C}$ in Figure $4.33(\mathrm{~A})$. Much of this is undoubtedly due to the film annealing, similar to the results of Section \$4.2.4. In this case, the simple fact that the samples are exposed to an elevated temperature is the primary driving force for the evolution of the spectra, rather than a hydrogen induced effect. This is evident when comparing the $1000^{\circ} \mathrm{C}$ data sets from Figure $4.33(A, B)$, which at this point have each seen $1000^{\circ} \mathrm{C}$ temperatures under hydrogen. The only difference being at what point during the film reordering process they were exposed to hydrogen (during the annealing for Figure 4.33(A) or after an initial anneal under argon for Figure 4.33(B)).

It might be expected that, given the more ordered nature of the SOP films (Figure 4.33(B)), that hydrogen would more easily diffuse through the etched-only films (Figure 4.33(A)) to the interface, attack the anchoring bonding and result in a more decoupled film. However, the spectra are largely the same. This indicates that, with respect to the broader goal of buffer-layer decoupling, whether the samples are exposed to hydrogen before or after the reordering of the carbon film does not have a large effect, based on this analysis. Stated another way, exposing unannealed films to hydrogen did not increase hydrogen penetration to the interface as compared to the already annealed films. This may indicate that the covalent bonding between the bufferlayer and substrate is already established following the $\mathrm{CF}_{4}$ etch, before the anneal. This is supported by the (relatively) well-ordered Raman spectra of the etched-only samples, which itself is very similar to buffer-layer only spectra. ${ }^{(212)}$ An alternative explanation, however, may involve 
reactions with fluorine embedded within the film of the un-annealed samples. For example, available $\mathrm{H}_{2}$ might be the consumption to from HF from the fluorinated species on the surface. In this case, extended annealing times may resolve this.

Fourth, ignoring for the moment any hydrogen effects, the data sets in Figure 4.33(A) (the etched-only samples) give further insight into the annealing process. Specifically, the interplay between the annealing temperature and the dwell time. Note that for the 0 second dwell, a temperature of at least $900^{\circ} \mathrm{C}$ is required for the etched-only samples (Figure 4.33(A)) to produce a spectrum similar to the SOP films. However, by dwelling at $700^{\circ} \mathrm{C}$ for 900 seconds or at $600^{\circ} \mathrm{C}$ for 3600 seconds, produces films comparable to the SOP films. This shows that, to an extent, temperature can be exchanged for time with respect to the annealing process. By reproducing these experiments under argon and conducting them across a finer resolution of temperature steps and dwell time steps, a clearer picture of the influence of time and temperature could be constructed. Such a series of experiments is an attractive candidate for future work and would overlap well with the future work proposed in Section $\$ \mathbf{4 . 2}$ on the effects of extended dwell times at lower temperature. The improved stability of the films under hydrogen may allow for longer dwell times than would otherwise be achieved under argon.

Figure 4.34 plots the figures of merit extracted from the Raman spectra of Figure 4.33., the $\mathrm{D} / \mathrm{G}_{1+2}$ ratio and the $\mathrm{D}$ and $\mathrm{G}$ full width at half maximum (FWHM) values. The data are plotted against the maximum RTA temperature and each curve connects the data sets for each dwell duration. The shaded bands bounded by the dotted lines annotate the standard range of values recorded on the SOP samples ( $\mathrm{CF}_{4}$ etch, AP-RTA in argon and no hydrogen exposure) to provide context. 


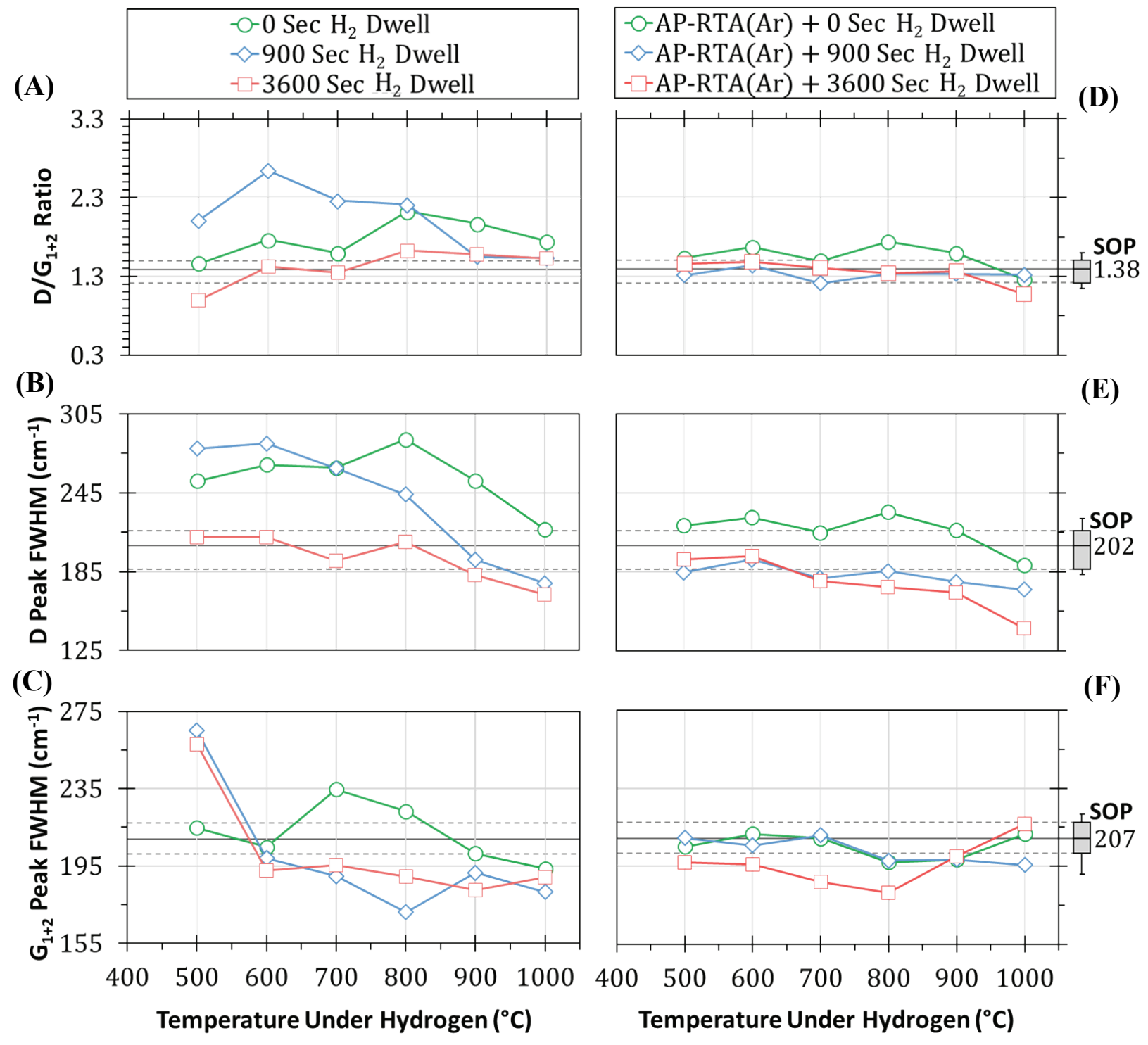

Figure 4.34: The shaded band bounded by the dashed lines represents the middle $75 \%$ of the distribution for the SOP samples. The solid line marks the average value recorded for the SOP samples. The box plot for the corresponding parameter is to the right of each plot along with the average value. The whiskers represent the middle $90 \%$ of values. (A-C) The samples which were etched only, then exposed to hydrogen. (D-F) Samples were etched, then AP-RTAed in argon, then exposed to hydrogen.

Not surprisingly, most data points in Figure 4.34(A-C) (etched-only) fall above the SOP values. This is typical of the non-annealed films, which are less crystallin. As well, the trend to higher crystallinity is seen with an increasing annealing temperature and, in general, with increasing the anneal time. The effect of hydrogen appears to be negligible, but if anything, results in a slight improvement in film quality. In Figure 4.34(D-F), films which had underwent the SOP AP-RTA and were then exposed to hydrogen did not undergo as drastic a change. This is consistent with the hypothesis that the substantial film reordering has already occurred, and therefore 
subsequent heating's have only a small effect on film quality. However, the long dwell time samples (3600 sec, red squares in Figure 4.33) show a significant improvement in the FWHM values of the D and G peaks. Because the broad FWHM of the D and G peaks is due to a blending of vibrational states (from the mixing of the phonon branches due to buffer-layer-substrate coupling, as described by F. Fromm, et al. ), a reduction in the FWHM indicates the start of the decoupling process. ${ }^{(212)}$ Although even the 'best' samples still show spectra that indicate a coupled buffer-layer.

The XPS analysis of a SiC/Buffer-layer/Graphene system becomes complicated due to various band bending and doping effects which shift the location of the graphene peak towards higher binding energies, relative to the $\mathrm{SiC}$ peak. Table 4-4 lists some of the relative graphene-SiC binding energy separation reported in the literature recorded before and after buffer-layer decoupling. The exact explanations cited in each source are quoted in Appendix $\$$ A.3.1; essentially, decoupling of the film decreases the sharing of electrons from the substrate to the film. This reduces the overall electron charge experienced by the graphene film and increases its electron affinity, resulting in a slightly higher photoelectron binding energy (i.e. the propensity of a photoelectron to remain in the film is increased).

Table 4-4: Literature reports on the C1s graphene peak shifting after buffer-layer decoupling.

\begin{tabular}{|c|c|c|c|}
\hline \multicolumn{3}{|c|}{ Separation Between Graphene and SiC Peaks in the } & \multirow{2}{*}{ Ref. } \\
\cline { 1 - 3 } Before Decoupling & After Decoupling & $\Delta_{\text {Before-After }}$ & \\
\hline $1.04 \mathrm{eV}$ & $1.42 \mathrm{eV}$ & $0.38 \mathrm{eV}$ & ${ }^{(537)}$ \\
\hline $1.00 \mathrm{eV}$ & $1.80 \mathrm{eV}$ & $0.80 \mathrm{eV}$ & ${ }^{(565)}$ \\
\hline $1.00 \mathrm{eV}$ & $1.77 \mathrm{eV}$ & $0.77 \mathrm{eV}$ & ${ }^{(559)}$ \\
\hline $0.90 \mathrm{eV}$ & $1.10 \mathrm{eV}$ & $0.20 \mathrm{eV}$ & \multirow{2}{(241)}{} \\
\hline $0.70 \mathrm{eV}$ & $1.47 \mathrm{eV}$ & $0.77 \mathrm{eV}$ & \multirow{2}{*}{$(225)$} \\
\hline $0.94 \mathrm{eV}$ & $1.38 \mathrm{eV}$ & $0.68 \mathrm{eV}$ & \\
\hline $0.94 \mathrm{eV}$ & $1.26 \mathrm{eV}$ & $0.40 \mathrm{eV}$ & \multirow{2}{(226)}{} \\
\hline $1.00 \mathrm{eV}$ & $1.16 \mathrm{eV}$ & $0.61 \mathrm{eV}$ & \multirow{2}{(256)}{} \\
\hline
\end{tabular}

The upshift of the graphene binding energy increases the overlap with the buffer-layer and oxide peaks. Therefore, it becomes difficult whether (and where) to assign the peaks between $283.5 \mathrm{eV}-285.0 \mathrm{eV}$ as buffer-layer peaks or to the now partially decoupled graphene layer. Again, this is a situation where high resolution spectroscopic would be useful. The decoupling induced 
upshift of the graphene peak is an exogenous event and therefore the fitting model used in the rest of this work cannot account for substantial shifts in the relative peak positions. This becomes particularly problematic for films frozen in the transition from highly coupled to partially decoupled.

Obviously, the spectra can be deconvoluted, but because there are no clear indicators for which fits accurately reflect the reality, the peaks could be assigned to fit any arbitrary narrative. As such, only the XPS derived parameters that are largely independent from peak shifts have been extracted from the data and plotted in Figure 4.35, but little attention is payed to the specific peak locations in these transitional films. The key observation is, however, that the boarder character of the spectra indicates that there are substantial shifts occurring within the spectral envelope, even if the specifics are obscured by the complexity. This is indicative of the beginning stages of film decoupling, as evident from the decoupling of electron states, which reveal itself in the spectra as reduced n-type substrate-induced doping effects resulting in an upshift to higher binding energies. More specifically, a reduction of substrate induced doping effects on the films. The effect of the annealing temperature in hydrogen are shown in Figure 4.35. Stacked C1s XPS spectra for the samples exposed to hydrogen from $500^{\circ} \mathrm{C}$ to $1000^{\circ} \mathrm{C}$ are shown in Figure $4.35(A, B)$. These samples constitute the 0 second dwell time samples.

The most apparent change in Figure 4.35(A) is the coalescence of chemical states into the dominant graphene peak with increasing temperature. As previously stated, this is due to the thermal effects of annealing and not to the presence of hydrogen (identical behavior was observed in Section $\$ 4.2 .4$ for annealing in argon). In Figure 4.35(B) the film reordering has already occurred, and therefore the spectra do not change much. There appears to be a slight shift of the dominant peak to higher binding energies at $900^{\circ} \mathrm{C}$ and $1000^{\circ} \mathrm{C}$ in both Figure 4.35 (A.B), as compared to the SOP spectrum. 
(A)

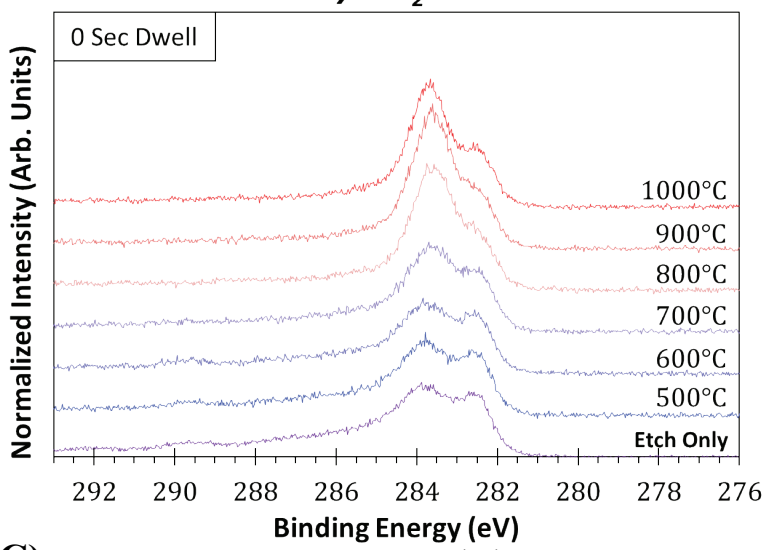

(C)

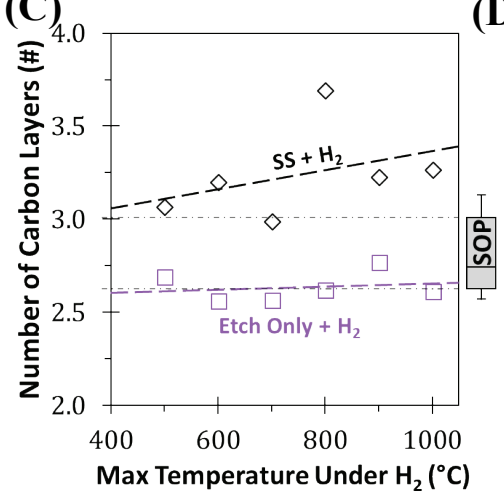

(D)
(B)

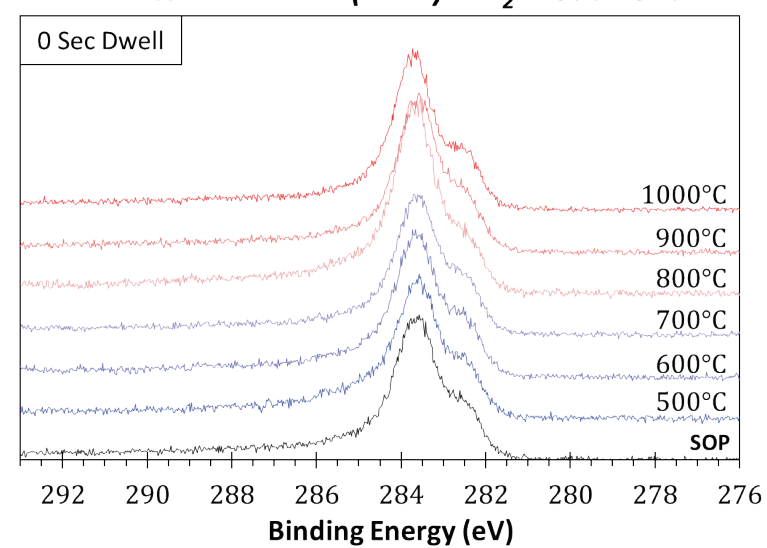

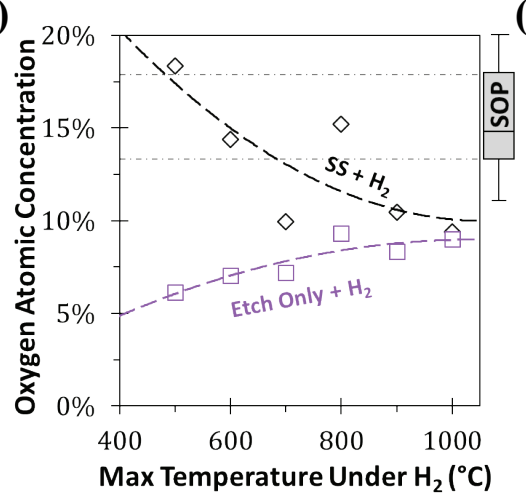

(E)

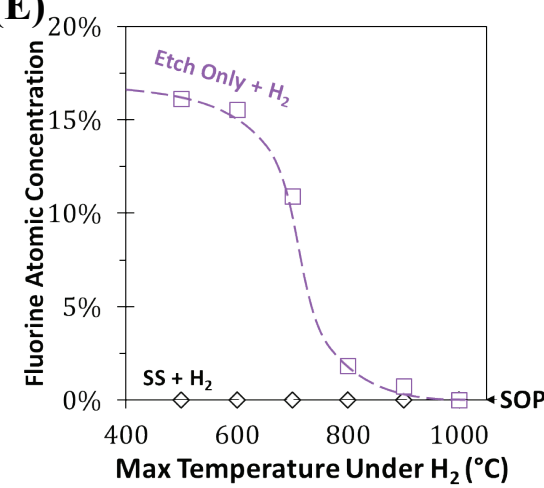

Figure 4.35: Stacked C1s spectra spectrum for etched only (A) and etched and AP-RTAed under argon (B) then exposed to hydrogen for a o second dwell from $500^{\circ} \mathrm{C}$ to $1000^{\circ} \mathrm{C}$. A slight shift to higher binding energies for the film related peaks is observed. Spectrum are normalized to the Si-C reference peak. (C) The number of carbon layers produced in each case. (D) The atomic oxygen concentration. (E) Atomic fluorine concentration. The box and whisker plots show the range of values collected from SOP samples. Data outside this range is significant.

Figure $4.35(C)$ plots the number of carbon layers against the hydrogen annealing temperature for both sets of samples. Interestingly, the samples which have undergone the SOP AP-RTA consistently produced more layers than did the samples which had only been exposed to hydrogen. Given that the films are shown to slowly oxidize in the $1000^{\circ} \mathrm{C}$ argon environment, it is interesting that these films are substantially thicker. In fact, they are thicker than the 2.7-layer average observed for SOP samples. Given that there is no reason to expect an increase in the amount of carbon on the surface (in fact, the atomic concentration of carbon is only slightly higher than the average for the SOP samples) the increase in thickness of the films after exposure to hydrogen may be explained by a densification of the carbon film following the partial decoupling of the buffer-layer. As the buffer-layer relaxes into the denser natural unit cell spacing of graphene, the $\mathrm{SiC}$ signal is more strongly attenuated (due to the increase in the areal density of the carbon overlayers), which is the basis for the thickness calculation. A detailed description of the layer 
calculation is included in APPENDIX $\boldsymbol{D}$ (recall the calculation assumes a constant areal density for each carbon layer) and a description of the atomic structure of the buffer-layer and graphene layer is given in Section $\$ 2.1 .3$.

In regard to the low thickness of etched-only films, recall that these films have a high concentration of fluorine remaining from the etching process. The high temperature reaction of gaseous hydrogen with embedded fluorine to produce a hydrofluorocarbon, which is then volatilized from the surface, may explain the carbon loss. Because all fluorine is removed during the AP-RTA process, which is carried out under argon, when these samples are exposed to hydrogen, there is no fluorine to react with. Instead, as shown in Figure 4.35(D) from the decrease in atomic oxygen, the hydrogen reaction with oxygen defects partially reducing the surface. Note that both curves approach the same values. This may be reflective of the base oxygen contamination level in the hydrogen gas used or on the effective oxygen contamination level (considering there may be competition between the film carbon and hydrogen gas for errant oxygen at the elevated temperatures used here which effectively reduces the concentration of oxygen). However, from Figure 4.35(E), there does not appear to be a correlation with the removal of fluorine with the reduction in carbon layers for the etched-only samples. Therefore, either the fluorine mediated carbon removal reaction is completed at temperatures below this or there are more complex processes at play. 


\section{\$4.3.2 Dwell Time Effects}

The effect of dwell time at $1000^{\circ} \mathrm{C}$ in hydrogen is illustrated in Figure 4.36. Figure 4.36(A) shows the stacked C1s XPS spectra and in Figure 4.36(B), the corresponding Raman spectra. The number of carbon layers, $\mathrm{D} / \mathrm{G}_{1+2}$ ratio and FWHM values of the Raman $\mathrm{D}$ and $\mathrm{G}$ peaks are shown in Figure 4.36(C-E). Note that the SOP reference spectra is included for comparison and was not exposed to hydrogen.

(A)
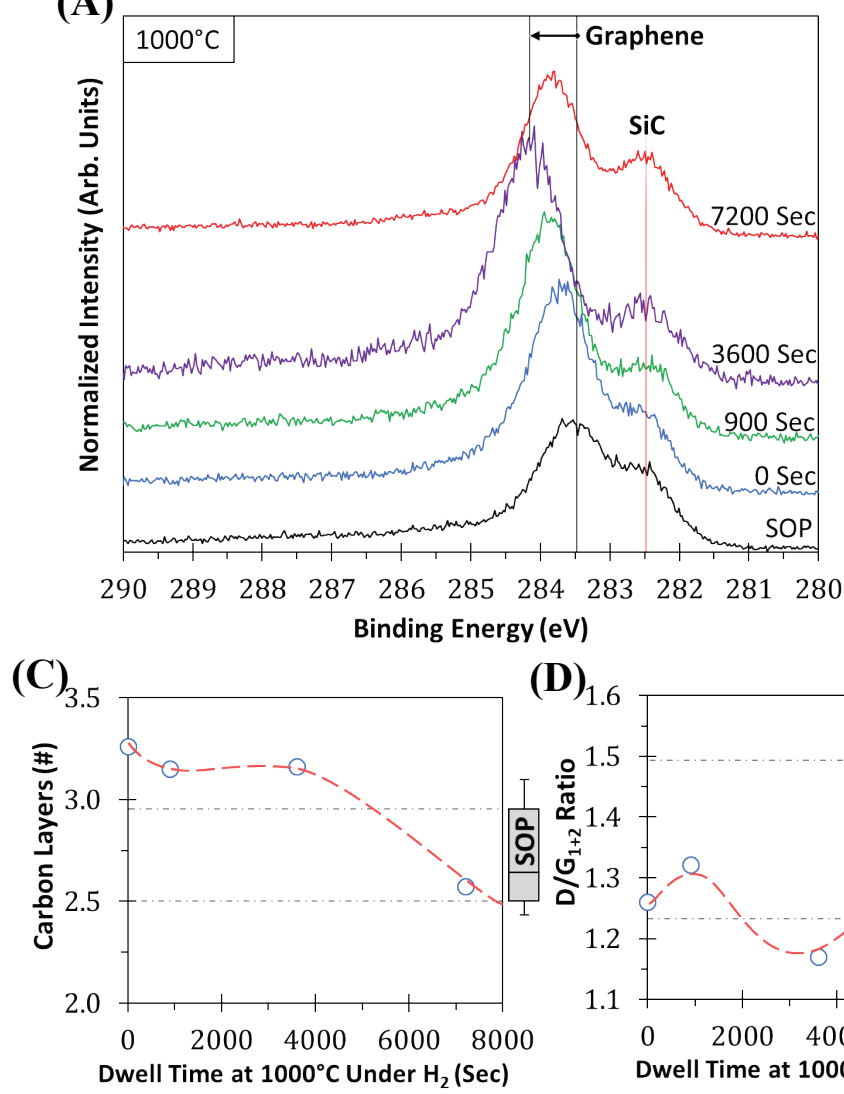

(B)

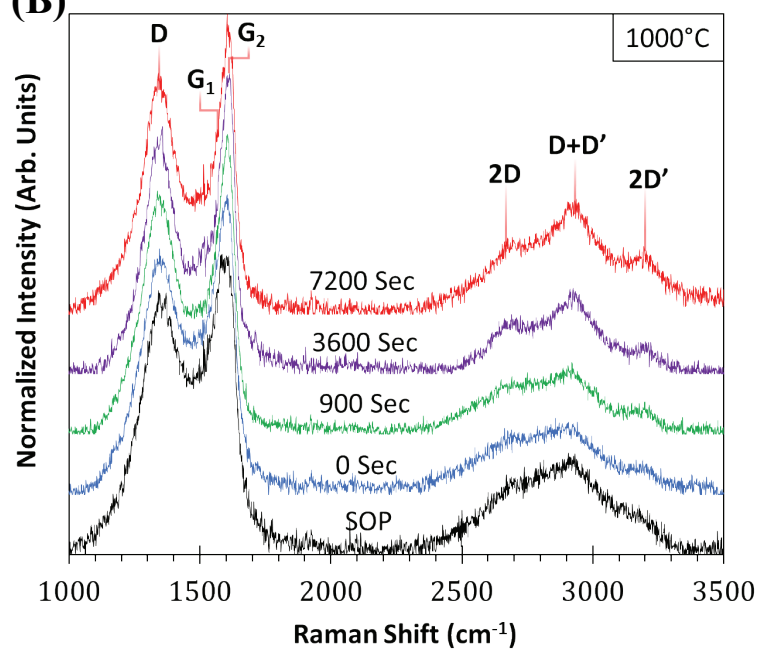

(E)
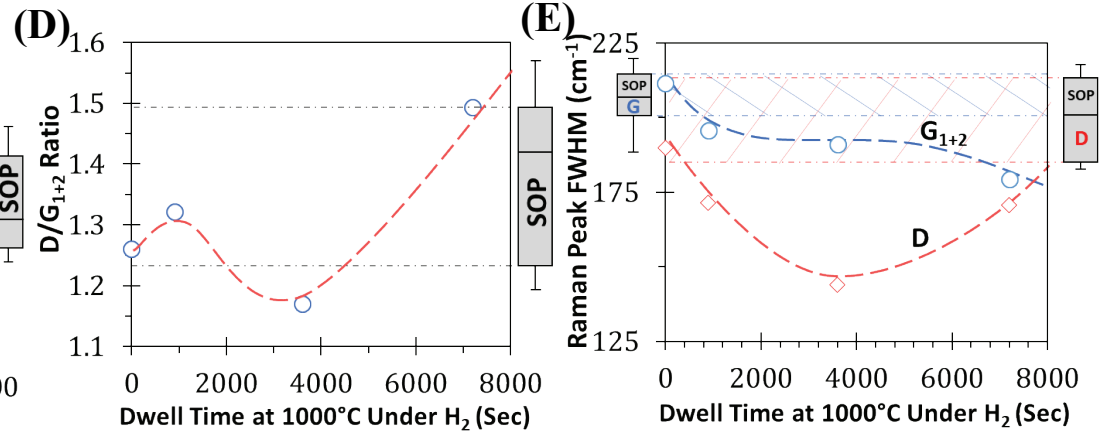

Figure 4.36: The effect of dwell time at $1000^{\circ} \mathrm{C}$ under hydrogen. (A) Stacked C1s spectra, the steady transformation of the spectra with increasing dwell time is seen. (B) Similarly, a refinement of the Raman spectra is also seen. (C) The number of graphene layers. (D) The $\mathrm{D} / \mathrm{G}_{1+2}$ ratio. (E) The Raman $\mathrm{G}_{1+2}$ and $\mathrm{D}$ peak full width at half maximum (FWHM). The box and whisker plots show the range of values collected from SOP samples. Data outside this range is significant.

An unambiguous shift of the peaks in the C1s region can clearly be seen in Figure 4.36(A). This transformation of the spectrum continues with increasing dwell time. The high intensity of the dominant feature, relative to the C-Si peak, precludes the possibility that these are solely bufferlayer peaks. Similarly, the low oxygen concentration indicates the peaks are likely not oxygen defect related. Thus, the transformation of the border character of the spectrum is most likely due to an up shift in the binding energy of the graphene peak due to the electronic effects of decoupling 
mentioned early and predicted by the literature. Furthermore, the graphene peak is the only peak in the $\mathrm{C} 1 \mathrm{~s}$ region with a large enough intensity to reshape the spectrum in this way. The shift continues until a dwell time of 3600 seconds, after which both the magnitude and the shift decrease.

Figure $4.36(B)$ show the stacked Raman results where again, a transformation in the broader character of the spectra is shown to continue with increasing dwell time under hydrogen at $1000^{\circ} \mathrm{C}$. The enhancement of the two-phonon region (2D, D+D' and 2D') is clearly observed. The enhancement takes the form of both an increase in intensity and a narrowing of the peaks, revealing the emergence of the individual peaks.

In addition to the uniform increase in the calculated number of carbon layers (for the reasons stated earlier), Figure $4.36(\mathrm{C})$, shows that the number of layers remains stable until dwell times exceed 3600 seconds, after which a substantial drop off is seen. This may be reflective of a similar, although much slower, process that is observed to be detrimental to the films and occur during extend dwell times in argon.

The $\mathrm{D} / \mathrm{G}_{1+2}$ ratio is plotted in Figure 4.36(D). As expected, the trend is initially towards lower ratios (better) with increasing dwell times. However, it is notable that there is a sharp increase in the ratio (worse) for the last data point (dwelling for 3600 seconds). This may indicate film decomposition is occurring, which is affecting the crystallinity. On the other hand, as seen in Figure 4.36(E) which plots the individual $\mathrm{D}_{\text {and }} \mathrm{G}_{1+2} \mathrm{FWHM}$ values, it is clear that the higher $\mathrm{D} / \mathrm{G}$ ratio is due to an increase in the FWHM value of the D peak. Note that the G peak continues to narrow, a sign of increasing decoupling. The increased breadth of the $\mathrm{D}$ peak may be due to the emergence of a new peak. A C-H bending mode has been shown to appear at $\sim 1450 \mathrm{~cm}^{-1}$ in graphene. ${ }^{(564)}$ This mode may be present here, but is eclipsed and lumped in with the D peak, contributing to its breadth and driving up the $\mathrm{D} / \mathrm{G}_{1+2}$ ratio.

While there are samples which show a large shift of the graphene peak (the $3600 \mathrm{sec}$ dwell sample in Figure 4.36(A) in particular), the breadth of the spectral envelope make the fitting of the graphene, S1, S2 and C-O-C peaks somewhat arbitrary. However, for films which were thermally cycled in a hydrogen environment, the XPS spectra is more easily deconvoluted. These films show much larger large shifts of the graphene peak, presumably as a result of a more decoupled film. The next section discussed these experiments. 


\section{\$4.3.3 Thermal Cycling Effects}

In an effort to 'exercise' and stress the buffer-layer-substrate interface, a sample was thermally cycled from $\sim 25^{\circ} \mathrm{C}$ to $1000^{\circ} \mathrm{C}$ ten times under hydrogen. The sample was allowed to bake at temperature for 300 seconds ( 5 minutes) in each cycle, for a total of 3000 seconds (50 minutes). The dashed line in Figure 4.37 $(A, B)$ is from the analysis of a SOP sample (labeled SOP in the figure) produced around the same time as the samples in this study and show the location of the graphene peak and the Raman spectral envelope from a typical SOP sample. The effects of thermal cycling in hydrogen are clearly illustrated.

(A)

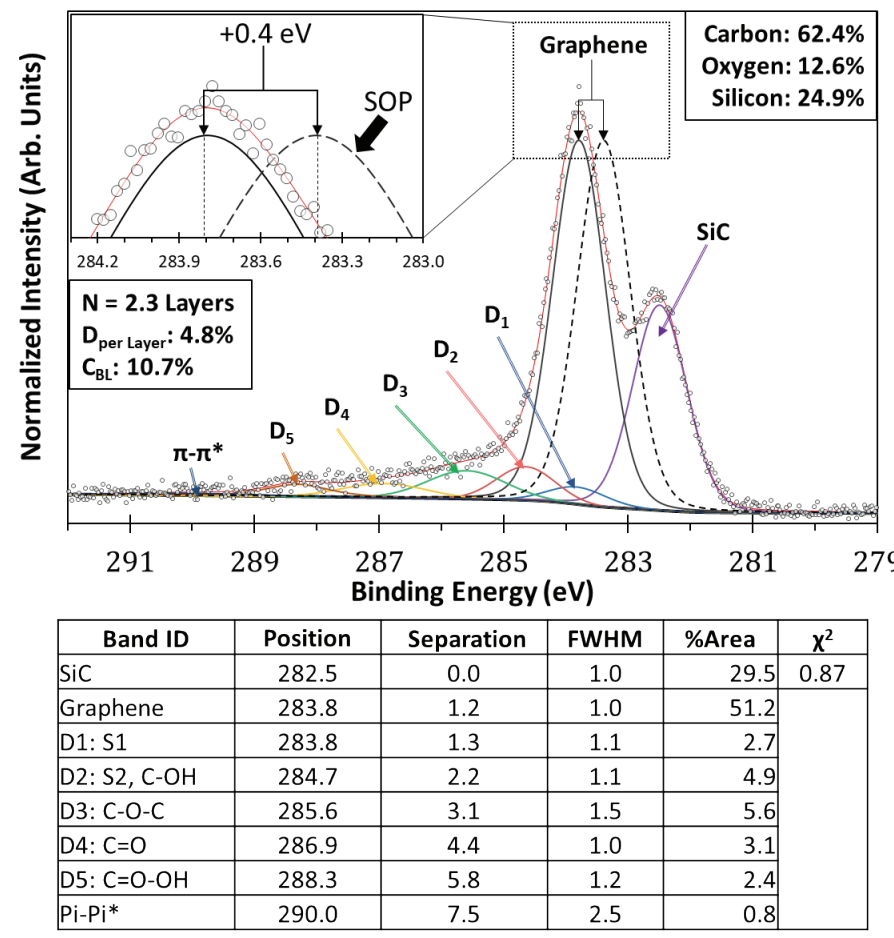

(B)

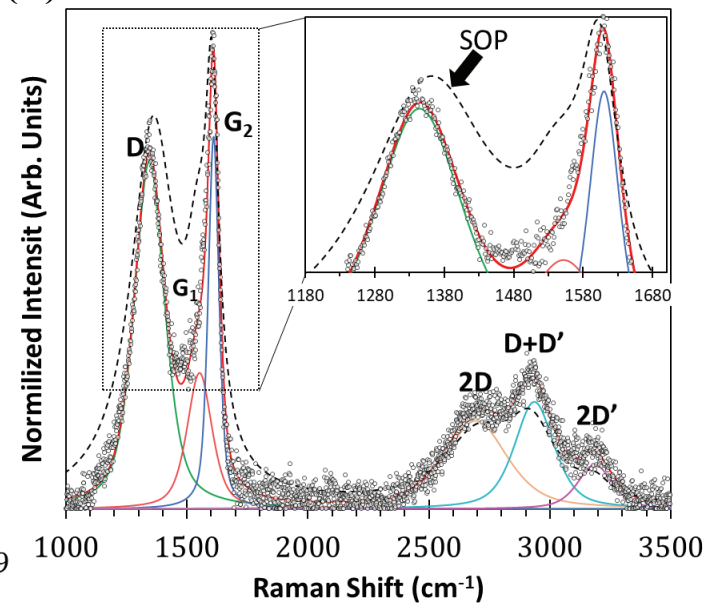

\begin{tabular}{|l|c|c|c|c|}
\hline Peak ID & $\begin{array}{c}\text { Center } \\
\left(\mathbf{c m}^{-\mathbf{1}}\right)\end{array}$ & $\begin{array}{c}\text { Area } \\
\text { (Arb. Units) }\end{array}$ & $\begin{array}{c}\text { Height } \\
\text { (Arb. Units) }\end{array}$ & $\begin{array}{c}\text { FWHM } \\
\left(\mathbf{c m}^{-\mathbf{1}} \mathbf{)}\right.\end{array}$ \\
\hline $\mathrm{D}$ & 1344 & 147.1 & 0.73 & 150 \\
\hline $\mathrm{G}_{1}$ & 1552 & 47.2 & 0.29 & 123 \\
\hline $\mathrm{G}_{2}$ & 1610 & 55.2 & 0.78 & 53 \\
\hline $2 \mathrm{D}$ & 2694 & 76.2 & 0.19 & 306 \\
\hline $\mathrm{D}+\mathrm{D}^{\prime}$ & 2936 & 59.1 & 0.23 & 195 \\
\hline 2D' & 3186 & 21.3 & 0.09 & 169 \\
\hline
\end{tabular}

Figure 4.37: XPS (A) and Raman (B) analysis of a standardly synthesized sample thermally cycled between room temperature and $1000^{\circ} \mathrm{C}$ in hydrogen 10 times. The dashed lines show the location of the graphene peak and Raman spectra line for a Standard Sample (no hydrogen exposure) for comparison. $\chi^{2}$ is the fitting parameter as determined by the fitting program (lower is better).

Figure 4.37(A) shows the C1s spectra. The peak parameters are tabulated below the plot. The dashed line shows the location of the graphene peak if it were located where it is normally observed in an SOP sample. The inset in Figure 4.37(A) clearly shows the graphene peak shift.

Figure 4.37(B) plots the corresponding Raman spectrum from the sample thermally cycled in hydrogen. The peak parameters are tabulated below the plot. The dashed line shows the spectra from a sample which was produced at the same time but has not been exposed to hydrogen (i.e., a 
SOP sample). The refinement and narrowing of the peaks are clear. Again, this is consistent with what would be expected for a film undergoing the initial stages of buffer-layer decoupling. As is the case with most samples examined in this study, while the $\mathrm{D} / \mathrm{G}_{1+2}$ ratio is only slightly lower (as compared to SOP samples), the most striking aspect of the Raman spectra is the reducing in the FWHM of all peaks. This seems to indicate that the hydrogen treatment is not healing structural defects, but rather, is reducing the coupling of the vibrational states of the film, which is evidence of decoupling.

In summary, this study shows the beginning stages of a hydrogen induced buffer-layer decoupling process. This is evident by XPS analysis that show a shift to higher binding energies of the graphene peak in the $\mathrm{C} 1 \mathrm{~s}$ region. Others have shown this to be due to a decreased in substrate induced doping effects. Additionally, a corresponding narrowing of all Raman peaks, coupled with a subtle growth in the two-phonon region, is clear evidence of initial decoupling. It is worth noting that an alternative explanation for the changes observed in the XPS spectra is the formation of graphane or graphane platelets. Graphane, proposed in 2007 and has since been produced, is essentially a graphene sheet whose dangling pi bonds have been hydrogenated. ${ }^{(566)}$ Graphane has been proposed as an intermediate step in the transport of hydrogen to the SiC-buffer-layer interface, particularly in relatively low temperature decoupling experiments $\left(500-700^{\circ} \mathrm{C}\right) .(231)$

In general, both longer dwell and higher temperatures show more decoupling occurring (greater graphene peak shifts in the XPS spectra and a greater degree of peak narrowing in the Raman spectra). Duration appears to have a slightly greater effect on decoupling. Surprisingly however, no combination of time or temperature lead to a complete decoupling of the film as many others have reported. The best films are produced around $800-900^{\circ} \mathrm{C}$ and dwell time of 3600 seconds. Thermally cycling the samples under hydrogen did not appear to show demonstrably greater decoupling occurring. This seems to indicate the buffer-layers produced here are different or are at least the interface is more resistant to hydrogenation, then those produced by the sublimation method. Again, this further speaks to the uniqueness of the plasma assisted graphene synthesis method.

Other than a global reduction in the number of layers produced, exposing the etched-only samples to hydrogen did not produce remarkably different films than the SOP samples that were exposed to hydrogen. The layer reduction may be due to reactions with the gaseous hydrogen and 
fluorine remaining in the film from the etch. The fact that hydrogen does not appear to diffuse to the film-substrate any differently between the two types of samples may indicate that the bufferlayer is largely already established by the end of the etch.

Note that for this decoupling to occur, hydrogen must penetrate the film and reach the tethering sites. Hydrogen transport though a graphene sheet has been calculated to be energetically expensive. Gupta, et al. calculate a transit energy of a hydrogen atom though a pristine graphene sheet to be $2.53 \mathrm{eV}$ and Miao, et al. calculated a similar value of $2.86 \mathrm{eV}$. (239) (238) Considering this, hydrogen transport most likely takes the more circuitous route to the interface by way of defect sites and grain boundaries. However, sheet edges of the areas are likely oxygen terminated which, due to their large electron cloud and screening effect, would hinder hydrogen transient. Clearly, the transport of hydrogen to the interface is not a trivial or simple process.

\section{\$4.3.4 Summary of High Temperature Hydrogen Film Treatment}

In summary, this study investigated the effect of high temperature hydrogen exposure on the decoupling of the films from the substrate. Of all the conditions tested, it was found that repeated thermal cycling of the SOP films in hydrogen resulted in the most decoupling. Although similar conditions were employed to what has been shown by others to fully decouple films produced by the sublimation process, only a partial decoupling was achieved here. The partial decoupling was observed by significant changes in the XPS and Raman spectra of the post-traded films. This indicates a fundamental difference in the buffer-layers produced by the plasma assisted method.

This study illustrates the fertile ground left for future work involving the role of hydrogen in the synthesis process. The impact of other exposure times and temperatures would be elucidating. Most intriguingly, however, is the potential incorporation of hydrogen gas during various ICP/RIE steps. Exposing the samples to a light hydrogen plasma before and/or after the etch could impact the system in very distinct ways. Additionally, the incorporation of a small fraction of hydrogen gas to the $\mathrm{CF}_{4}$ plasma during the ICP/RIE etching step would present a particularly fascinating investigation. 


\section{CHAPTER 5: RESUlts AND Discussion - ELECTRICAL CHARACTERIZATION, TEMPERATURE BEHAVIOR, GAS TESTING AND Light TESTING}

This chapter focuses on characterization of devices constructed using the graphene/SiC composites produced using the plasma assisted graphene growth process detailed in Chapter 4. Section $\$ 5.1$ presents and discusses the electrical characterization of the devices at room temperature in an inert environment. Section $\$ 5.2$ presents and discusses device behavior as a function of temperature in an inert environment. Section $\$ 5.3$ presents and discusses the device behavior under various gas environments across a wide temperature range. Section $\$ 5.4$ presents and discusses device behavior when irradiated with light at room temperature in air. Unless otherwise noted, all graphene used was produced using the SOP process (detailed in Section $\$ 2.1 .5$ and Section $\$ 4.1$ ).

The fabrication of the graphene-SiC devices and packaging are detailed in Section $\$ 3.3$. They are termed in this work as the MKI, MKII, MKIII, MK0 and MK0* designs. Briefly, the devices consisted of a $\mathrm{SiC}$ substrate from which a thin film of graphene was grown using the methods detailed in Section $\$ 4$.1. To these composites, Ti/Au contacts were then evaporated onto the graphene/SiC composites as described in Section $\$ 3.3 .1$. This collective assembly is referred

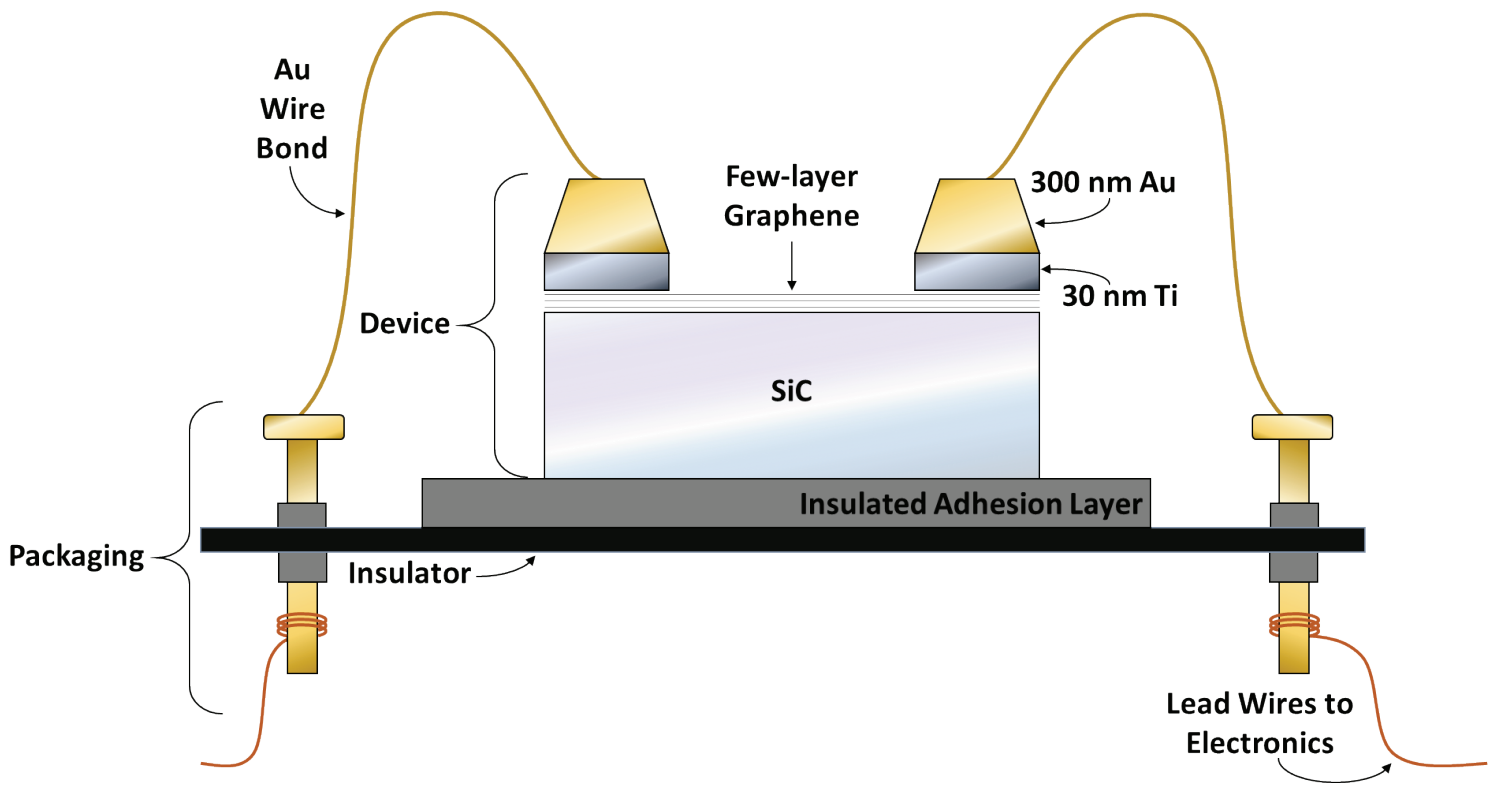

Figure 5.1: A schematic of a generic device. The specifics of the contact pattern, electrode setup and sample packaging varied, but the basic design was the same. The 'device' consists of the SiC substrate, the graphene film, $\mathrm{Au} / \mathrm{Ti}$ contacts. The device 'package' consisted of the mounting hardware and related components as well as the electrical leads and connections. The image is not to scale. 
to here as a 'device'. Gold wire bonds are made between the device and the sample packaging. In total, three different device designs were used in combination with five different package designs. A simple schematic of a generic device and packaging is shown in Figure 5.1; components have been enlarged and many of the complexities have been omitted for clarity.

\section{§5.1 Room Temperature Electrical Characterization of Graphene-SiC Devices}

The room temperature electrical properties of the graphene/ $\mathrm{SiC}$ composite devices are discussed in this section. Over 3,000 individual measurements were collected, discussed here are the nominal values and typical behaviors. Two-point analysis reveal that the devices exhibit classic back-to-back Schottky behavior with an ideality factor $(\eta)$ between 12-17. As discussed here, fourpoint and transition line model (TLM) analysis show the graphene films display a per-layer resistance of $0.3-0.9 \Omega / \square$. Richardson-Dushman analysis show that the devices possess a room temperature Schottky barrier height $\left(\Phi_{\mathrm{B}}\right)$ of $\sim 0.41$. The graphene films were found to have a room temperature carrier concentration $\left(\mathrm{N}_{\mathrm{d}}\right)$ of $2.4 \times 10^{14} \mathrm{~cm}^{-2}$ and a room temperature carrier mobility ( $\mu$ ) of $7,086 \mathrm{~cm}^{2} \mathrm{~V}^{-1} \mathrm{~s}^{-1}$.

\section{§5.1.1 Two-Point Analysis}

Current readings were collected as a function of voltage to generate IV plots. These were obtained using the simple two-point measurement technique described in Section \$3.2.5. These two-point measurements require only two contact points. Therefore, two-point measurements make the package design, wirebonding, electrode setup and analysis much simpler as compared to four-point measurements, which are discussed in the next subsection. Two-point measurements captures and combines the effects of all elements in the electrical circuit. This includes the series resistance of the test leads and sample packaging jumpers and connections.

For the MKI, MKII and MKIII package designs (described in Section \$3.3.3), the contribution of the lumped series resistance is approximately on the order of $\sim 1.6 \Omega$ and increases slightly with increasing temperature. For the MK0 and MK0* designs, the contribution is approximately $2 \Omega$, and largely independent of temperature. The resistance of the Ti/Au contact and pattern trace on the devices was found to vary by only $\sim 10 \%$ over a $650^{\circ} \mathrm{C}$ temperature swing. Both series resistance values are small compared to the resistance of the film at room temperature. The series resistance contribution of the package design and test leads are discussed in greater 


\section{detail in Section $\$ 3.3 .3$.}

In this work, over 700 current versus voltage (IV) curves were collected from 124 devices. As described above, several different package designs and electrical contact arrangements were utilized in these measurements. Figure 5.2 shows a representative IV plot collected from a typical graphene/SiC device (blue circles). The data shown was collected from a device using the TLM pattern arrangement and the MK0* package design, as depicted in the inset. Note that throughout this work the AC source symbol is used to indicate the polarity swing (negative to positive) in the applied device voltage (i.e. one cycle) for all tests, even when only one polarity is shown in later figures (the symmetry of the device results in identical curves). All devices show the same generic IV behavior, with slight differences resulting from the various packaging deigns and contact arrangements used. ${ }^{26}$ For comparison, a SiC reference device (purple diamonds) is also shown. The graphene device was synthesized using the SOP process, with electrical contacts in the TLM

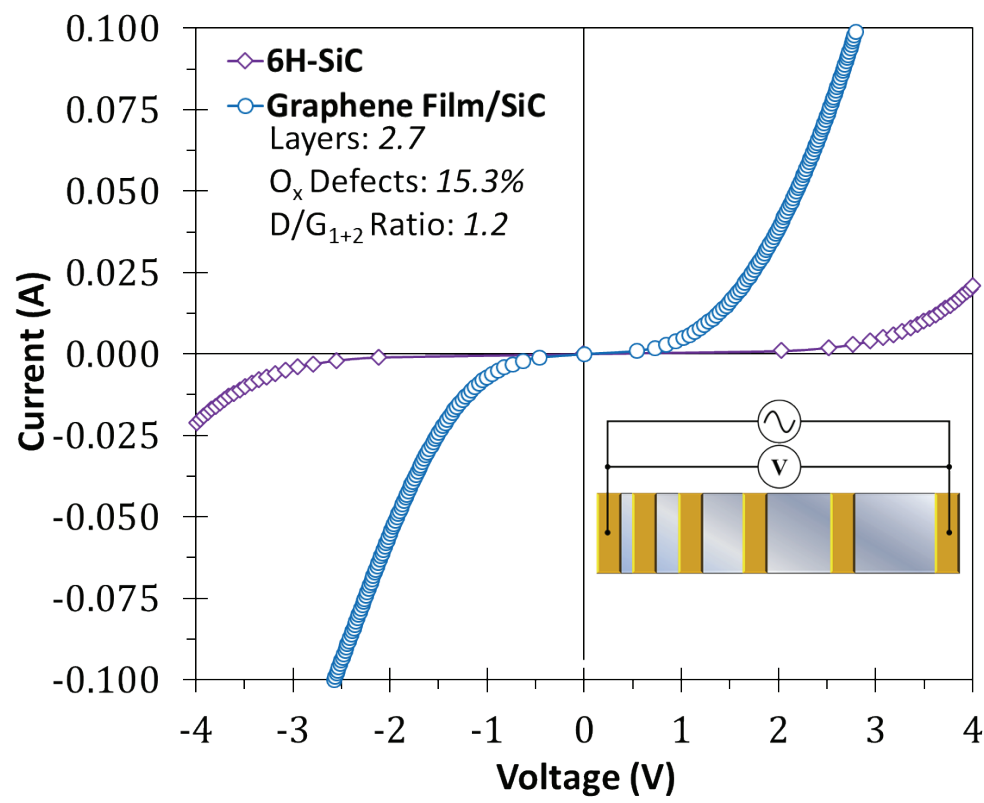

Figure 5.2: (A) IV plots of a representative graphene/SiC device and a reference SiC sample. The graphene/SiC device shows higher conductivity and exhibits a back-to-back Schottky behavior. Parameters of the graphene film, as determined by XPS and Raman analysis, are included in the upper inset. Lower inset, a schematic showing the sample contact pattern and electrode arrangement. Data was collected in 0.001A steps.

\footnotetext{
${ }^{26}$ For example, variations in the wirebonding technique used resulted in up to a $2 \%$ difference in the measured resistance. Furthermore, variations in the quality of the electrical connection made between the test setup/sample package and the measurement equipment (BNC triaxial leads via copper alligator clips) were found to have the largest effect and were the primary culprit for the differences in the series resistance contribution between otherwise identical setups. Furthermore, variations of the background pressure in the e-beam chamber have been shown to have a large impact on the quality of the electrical contact formed. (589) Therefore, the normal fluctuations in the nominal pressure of the e-beam chamber over the time span (years) the samples were made in this work has undoubtedly contributed, in part, to the slight variations observed in the IV plots from sample-to-sample.
} 
pattern and used the MK0* package design. Data was collected at room temperature, with the device under a steady state flow of 10 standard cubic centimeters per minute (SCCM) of ultra-high purity (UHP) argon. The test conditions and packaging were identical for the $\mathrm{SiC}$ reference sample.

There are two crucial points to be taken from Figure 5.2. First, is that the graphene/SiC device exhibits a much higher conductivity as compared to the $\mathrm{SiC}$ reference sample. The second point is that the devices exhibit back-to-back Schottky behavior. The first point indicates that, while the SiC substrate participates somewhat in conduction over this voltage range, the majority of current appears to flow through the graphene film. However, there are several additional possible contributors to the conduction path present in the graphene device which are not available in the virgin SiC. Figure 5.3 $(A, B)$ provides a conceptual diagram of these paths and an equivalent circuit diagram, respectively.

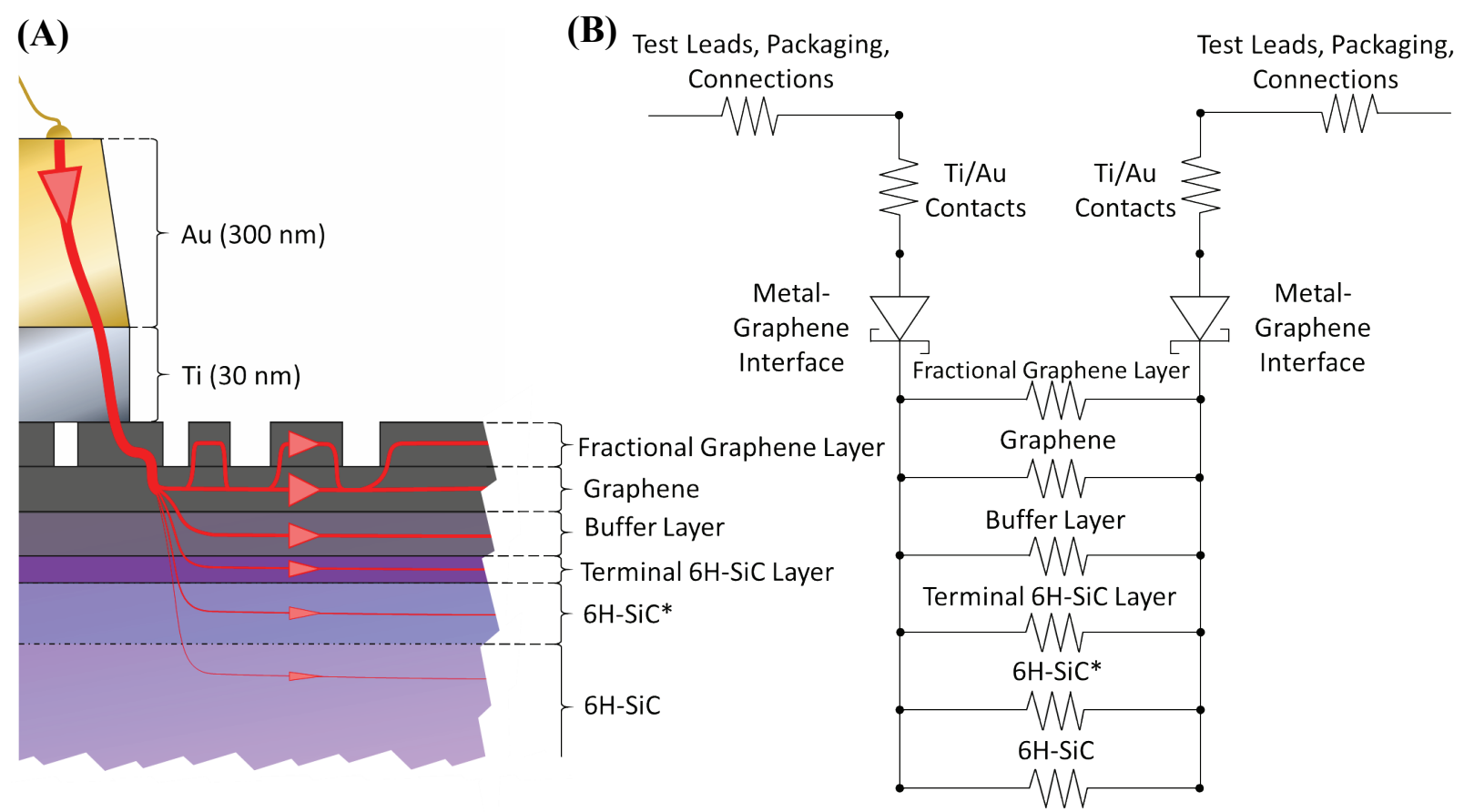

Figure 5.3: (A) A simple schematic of the physical interpretation of the potential electrical conduction paths in the graphene/SiC devices. The IV results indicated that the majority of current flows through the graphene layer(s). (B) Equivalent circuit diagram of this physical interpretation. The metal-graphene interface is represented by Schottky diode. Each film layer is discussed in detail within the text.

Conceptually, there are potentially six different parallel conduction paths between electrical contacts in the graphene/SiC devices. In this conceptual picture, the topmost layer would be a fractional graphene layer. This layer consists of the excess carbon atoms remaining from the fully formed graphene layers beneath but does not contain enough atoms to fully form a continuous layer. Given that this layer is the most terminal and likely contains many carbons in an exposed 
(chemically vulnerable) edge state, this carbon layer would most likely contain the plurality of the oxygen defects (oxygen defects are calculated by XPS analysis, Section \$4.1.2). The second layer(s) are the fully formed graphene layer(s). While there may be many crystal domains ( 10-50 $\mathrm{nm}$ in size by Raman analysis, Section $\$ 4$.1.3) these domains are contiguous (i.e. have touching boundaries) and would be the most conductive of all the layers.

The next layer down would be the buffer-layer. This layer is discussed extensively in Section $\$ 4.1 .4$, where Raman and XPS analysis strongly suggest its presence in these samples. In short, it is a graphene-like layer, with many of the carbon atoms participating in covalent bonding with silicon atoms in the terminal layer of the $\mathrm{SiC}$ substrate and whose orbitals exhibit some $\mathrm{sp}^{3}$ character. This causes a slight buckling of the layer. Additionally, this is the layer through which the differences in the crystal lattice sizes of the graphene layer and the $\mathrm{SiC}$ substrate are expressed. Thus, this layer is also under tensile stress.

The next layer down would be the terminal layer of the $6 \mathrm{H}-\mathrm{SiC}$ substrate. This is the analog to the buffer layer for the substrate. This layer contains the silicon atoms which are bound to the carbons in the buffer layer. Beneath this layer is the $6 \mathrm{H}-\mathrm{SiC}$ crystal. Given that this segment of the device starts at $\sim 4$ atomic layers down from the surface, the crystal structure and composition may be pristine. However, considering the aggressive nature of the etch, this portion of the $\mathrm{SiC}$ crystal may contain some structural damage and/or may not contain a stochiometric ratio of carbon to silicon. Furthermore, considering the kinetic energy of the fluorine etchant species, there may be some fluorine atoms contained within. Although XPS analysis does not show any fluorine present, it is important to note that effective doping levels for semiconductors are routinely in the parts per million range, which is well below the detection limit of XPS (parts per thousand range). It is unclear how thick this hypothetical horizontal slice of effected material would be, but a reasonable range would be in the $0.5-2.0 \mathrm{~nm}$ range.

It is possible that the annealing step may heal some of the structural defects in the $\mathrm{SiC}$, as well as remove the vast majority of fluorine atoms, however enough of either may remain to be sufficient in affect the conductivity. Given these considerations, it is reasonable to conclude that this area of the $\mathrm{SiC}$ crystal may be different, either structurally or compositionally, from the bulk $6 \mathrm{H}-\mathrm{SiC}$. Because of this, this section of the $\mathrm{SiC}$ crystal may be more (or less) conductive than the pristine $6 \mathrm{H}-\mathrm{SiC}$ crystal. It is labeled $6 \mathrm{H}-\mathrm{SiC}^{*}$ in Figure 5.3. Below this section, the last conduction path would be the unadulterated and lightly nitrogen doped (n-type) $6 \mathrm{H}-\mathrm{SiC}$ as-received crystal. 
This layer would be the least conducting and has the IV curve shown in Figure 5.2.

Each of the layers discussed would contribute to conduction (proportional to their resistance) and their contributions would be lumped together into the IV curve shown in Figure 5.2 (blue circles). However, considering the graphene layers are the most conductive, the IV curve is most representative of the carbon film. The other parallel layers are mentioned for the sake of presenting a complete picture of the system and may explain some of the observed behavior, discussed later (namely, the apparent high graphene layer conductivity).

The second point taken from Figure 5.2, is the back-to-back Schottky behavior (the origins of a Schottky contact are discussed in Section \$2.2.3). This indicates that the films are semiconducting. This back-to-back Schottky behavior is not unexpected, due to doping from oxygen based chemical defects (as indicated by XPS analysis, Section \$4.1.2) and interactions with the substrate. This doping and the substrate interactions shift the graphene films from a metallic behavior to a semiconducting behavior. As will be discussed in Section \$5.3.6, there is strong evidence that it is the interaction with the substrate which make these films have semiconducting characteristics. Furthermore, essentially identical Schottky junctions $\left(\Phi_{1} \approx \Phi_{2}\right)$ are formed at both contacts, and thus, the IV curves are (essentially) independent of the polarity of the applied bias and the curves show back-to-back reverse bias behavior; due to the symmetrical construction (metal-graphene-metal) there is an energy barrier to charge injection at both contacts and the device resists current flow (i.e. reverse bias condition) under both polarities. ${ }^{(567)}$ For clarity, analysis of the IV plots (discussed later) focus on only one quadrant of the full IV plot; the same analysis can be performed on the other quadrant and will produces similar results (regardless of which quadrant of the IV plot analyzed, the extracted parameters pertain to the source contact in each case).

A theoretical electronic band structure for a device produced here, showing back-to-back Schottky device under an external bias, is shown in Figure 5.4 and illustrates the underlying principle. Note that the specific details of how the band diagram is depicted (i.e. the shape of the Fermi level within the depletion region and the vacuum energy level across the device structure) varies in the literature; therefore, Figure 5.4 should be taken simply as a generic conceptual guide only. 


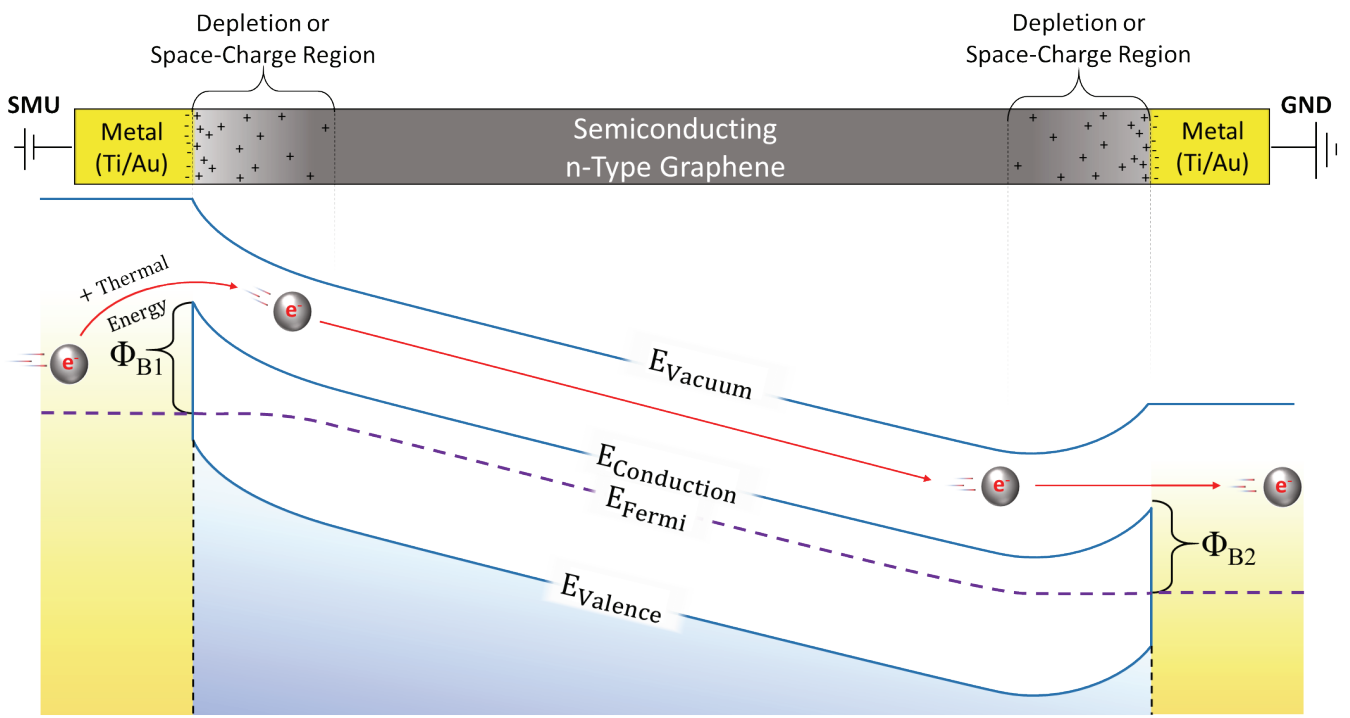

Figure 5.4: An approximation of the electronic band structure of a back-to-back Schottky device under an external bias which shoes current injection by thermionic emission. There has been some debate over the exact shape of the Fermi level within the space change region; Rhoderick makes the case that it is flat. (745) This is the interpretation shown.

The Schottky junction is discussed in a general theoretical sense in Section $\$ 2.2 .3$ and, in regard to the practical impact on these devices, in Section $\$ 5.2$. An excellent review of graphene Schottky junctions is given by Bartolomeo, which thoroughly explains the principle. (380) Occasionally, there is a slight asymmetry observed in the IV curves ( $<10 \%$ of samples tested). This is likely due to variations in the Schottky barrier height at each contact/film interface. This could be due to slight differences in the interfacing of the metal contact with the film.

There are many types of potential conduction mechanisms for the type of system illustrated here. ${ }^{(568)}$ Determining which mechanism is dominant can be challenging. For the purposes of these analysis, it is initially assumed that current injection is due to simple thermionic emission. However, observations collected during thermal response experiments (Section \$5.2) may indicate there are more complex mechanisms at play (namely, charge trap hopping). The conclusions drawn at the end of this section support this.

The analysis of the two-point measurements can give insight into the nature of the Schottky contact which is formed. Considered here is a model which assumes the dominant current flow from the contact to the film is by thermionic emission. The ideality factor $(\eta)$ is a correction factor required to fit experimental data to the standard thermionic emission model. Stated another way, the ideality factor is a measure of how close to an ideal diode (showing pure thermionic emission) a device is, with $\eta=1$ being a perfectly ideal thermionic diode. The deviation from ideal behavior is due to any number of real-world effects. Deviation can occur when current injection mechanisms 
other than purely thermionic (such as field emission or carrier generation and recombination within the depletion region, for example) contribute appreciably to the IV curve. ${ }^{(568)}$

Additionally, the thermionic model assumes a constant $\Phi_{\mathrm{B}}$. However, $\Phi_{\mathrm{B}}$ can vary as a function of applied voltage due to image force effects and self-heating. Image force effects occur when a charge carrier produces a virtual carrier, of opposite sign, when it approaches a barrier. This, in turn, affects the local electric field at the interface and thus the $\Phi_{\mathrm{B}}$. The overall effect is to lower $\Phi_{\text {в. }}{ }^{(567)}$ Because the effective $\Phi_{\mathrm{B}}$ is sensitive to temperature (this is discussed in Section \$5.2.2), the self-heating effects of the applied bias required to collect the IV curve will itself shift $\Phi_{\mathrm{B}}$ and cause a deviation from ideal behavior. Furthermore, interface irregularities at the electrical contacts (variations in the local $\Phi_{\mathrm{B}}$ due to interface inhomogeneities) will also increase $\eta{ }^{\text {. }}{ }^{(569)(570)}$ Along similar lines, the presence of an oxide (i.e. insulating) layer between the contact and the film will also increase $\eta{ }^{(571)}$

Following the 'standard forward-IV method' for devices with a small series resistance (Rs), as detailed by Aubry and Meyer, $\eta$, the ideality factor was calculated. ${ }^{27(572)}$ This is accomplished by plotting $\ln (\mathrm{I})$ versus the applied voltage. They show that, for small series resistance values, the series resistance can be removed from the analysis by taking the voltage across the diode to be,

$$
V_{\text {diode }}=V-R_{S} I \quad \text { Equation 5-1 }
$$

where $\mathrm{V}$ is the applied voltage, $\mathrm{R}_{\mathrm{S}}$ is series resistance (in this case, the estimated film and device packaging) and I is the current. ${ }^{28}$ This approach was originally proposed by Lien, et al. ${ }^{(573)}$ This is done so that the voltage used in the analysis is the voltage drop across the diode (contact-film interface), so as to remain consistent with the theory. Furthermore, Cibils, et al. showed that a more accurate value for $\eta$ can be determined by considering only the fully linear region of the $\ln (\mathrm{I})$ vs. V curve. ${ }^{(574)}$

\footnotetext{
${ }^{27}$ This has also been referred to as the Rhoderick method. (744) (571)

${ }^{28}$ Given that the series resistance of the test setup is small $(\sim 2 \Omega)$ and the bulk material is small (i.e. the graphene film, $\sim 3 \Omega$ for the specific device and electrode arrangement considered here) compared to the resistance of the whole device ( $30 \Omega-500 \Omega$ depending on the voltage) the standard method provides a reasonable approximation (for comparison, Aubry and Meyer consider modifications to this method for series resistance in the $60 \Omega-200 \Omega$ range). (572)
} 
Figure 5.5(A) shows the positive quadrant of the IV plot shown in Figure 5.2. Fitted to this is a best-fit power law equation. Because a thermionic emitter would show a power law IV, the goodness of the fit validates the diode behavior and this general approach. Note however, as will be discussed in Section $\$ \mathbf{5 . 2}$, this is true for room temperature measurements; at elevated temperature, other conduction mechanisms begin to contribute. Figure 5.5(B) shows a plot of $\ln (\mathrm{I})$ vs. $\mathrm{V}_{\text {diode. }} \mathrm{V}_{\text {didoe }}$ is the calculated voltage drop occurring across the diode, with the black diamond $(\diamond)$ data points taking Rs to be zero (i.e. the series resistance of the test setup is not removed) and the blue circles $(\circ)$ representing the voltage across the diode by removing the voltage drop due to the estimated series resistance (the series resistance + the resistance of the film between contacts, $\sim 6 \Omega)$. The portion of the curve fitted with a linear line is indicated by the red filled red circles $(\bullet)$.

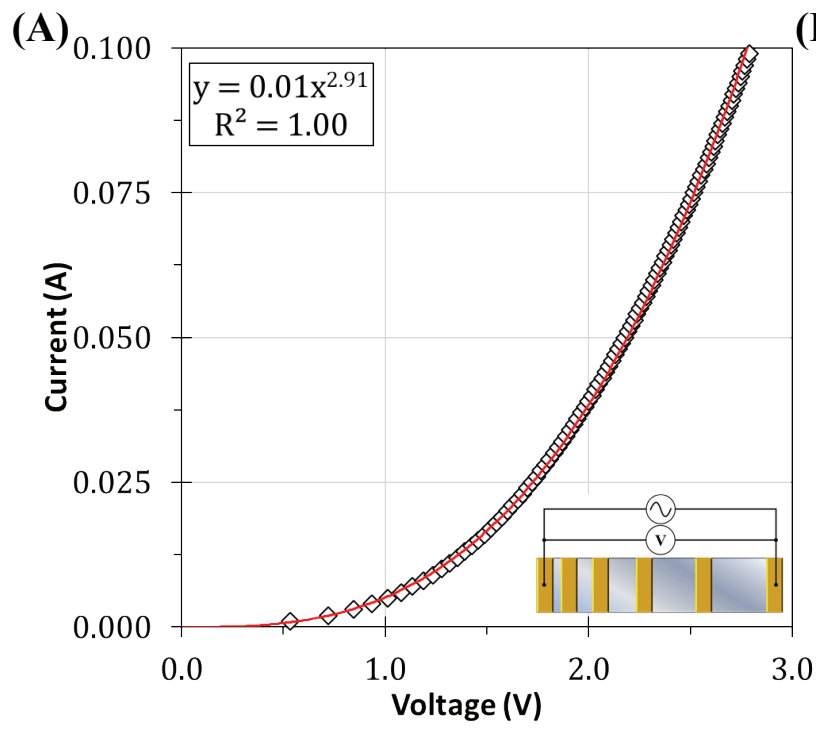

(B)

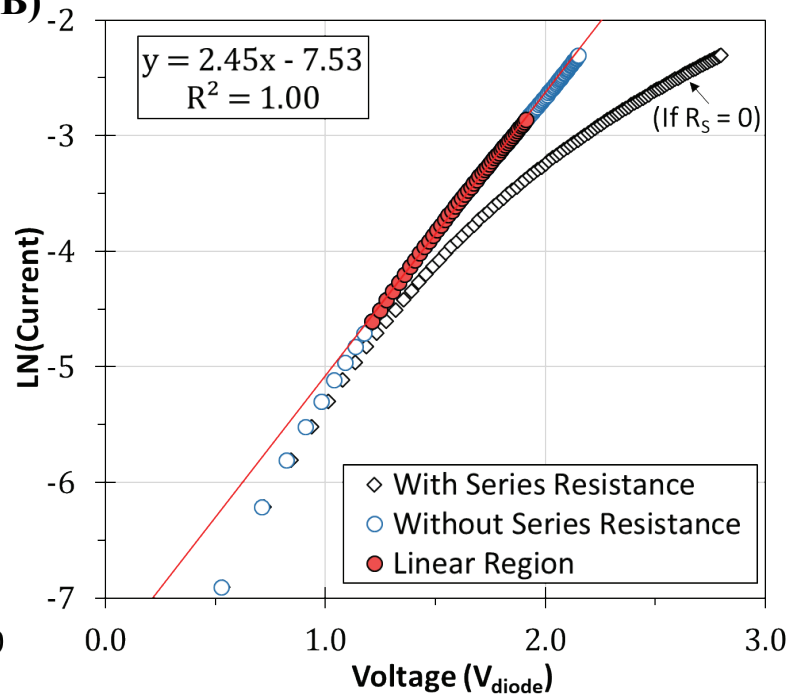

Figure 5.5: (A) The positive region of the IV curve shown in Figure 5.2. (B) Plot of the $\ln (\mathrm{I})$ of the data in (A) against the voltage drop across the contact/film interface. Data with ( $($ ) and without $(\diamond)$ the series resistance (Rs, packaging + film) removed (i.e. $\diamond$ 's takes Rs to be zero). The linear fit is used to calculate the ideality factor, $\eta$.

Based on this analysis, and using the slope of the linear region, the ideality factor is calculated by,

$$
\eta=\frac{q}{m k_{B} T}
$$

Equation 5-2

where $\mathrm{q}$ is the charge of an electron $\left(1.60 \times 10^{-19} \mathrm{C}\right), \mathrm{m}$ is the slope of the best-fit linear line in Figure 5.5(B), $\mathrm{k}_{\mathrm{B}}$ is the Boltzmann constant $\left(1.38 \times 10^{-23} \mathrm{~J} / \mathrm{K}\right)$ and $\mathrm{T}$ is the absolute temperature (taken to be $298 \mathrm{~K}$ ). Depending on the precise range of data included in the linear region, the value of $\eta \approx 15-17$ in this device, which used the TLM pattern and the MK0* package design. Samples 
which used other patterns, such as the interdigitated sensor pattern, show approximately $50 \%$ lower values of $\eta$. This may be due to both the large difference in the physical size of the samples (the interdigitated sensor patterned samples are $\sim 4 \mathrm{X}$ smaller) and the much larger effective contact length of the sensor pattern (the circuitous nature of the sensor pattern results in more contact 'shoreline' and film interfacing) as compared to the parallel contacts of the TLM pattern.

Alshaeer conducted a study on various calculation methods for determining $\eta$ and found that the standard method (Aubry and Meyer or Rhoderick method), while simple in its application, can lead to an overestimation of the $\eta$ by as much as $\sim 30 \%$. ${ }^{(571)}$ It was also found that the overestimation was greater for cases with low $\Phi_{\mathrm{B}}$ (as is the case here). Regardless of this, as compared to classic diodes, the value calculated here for $\eta$ is high. But this is not unusual for graphene devices. For devices consisting of a layered graphene heterostructures, very large values of $\eta$ have been calculated (as high as $\sim 34$ ). ${ }^{(575)} \mathrm{Hong}$, et al. attributed this to complex RC networks (resistance-capacitance networks) formed from the layered structure of the devices. ${ }^{29}$ (575) Considering the theoretical structure presented in Figure 5.4, this may be part of the case here. Other conduction mechanisms, mentioned earlier, may also contribute to the non-ideality. ${ }^{(568)}$

\section{\$5.1.2 Four-Point and TLM Analysis}

The four-point measurement method and the transmission line model (or transfer length method) are discussed in Section $\$$ 3.2.5. The results obtained by these methods to the devices produced here are discussed in this subsection. All results presented in this section were collected from samples using the TLM electrical contact design and the MK0* package design.

The nonlinear behavior of the IV data precludes the possibility of directly measuring the films true resistance from simple two-point measurements. However, Wu and coworkers, as well as Zhang, et al. , note that the measured voltage drop is across the Schottky barrier, the film, and the contacts, but in the high voltage ${ }^{30}$ portion of the curve (i.e., $\mathrm{V}>\Phi_{\mathrm{B}} / \mathrm{e}$ ), the voltage drop across the Schottky barrier becomes less significant. ${ }^{(522)(576)}$ Consequently, the resistance in this portion

\footnotetext{
${ }^{29}$ For simple cases where the layered structure adds only parallel conductance (no capacitive effects), the additional conduction paths can easily be taken into account in the analysis. However, the parallel conductance $\left(\mathrm{G}_{\mathrm{p}}\right)$ must be known. (751)

${ }^{30}$ Note that the 2013 U.S. department of energy technical standard defines high voltage as voltages greater than 600V $\mathrm{AC}$, the International Electrotechnical Commission (IEC), the National Electrical Code (NEC) and National Electrical Manufactures' Association (NEMA) cite varying, but similarly large values. (759) The terms 'high-', 'moderate-' and 'low-' voltages are used here in a colloquial sense only and simply refer to the relative voltage positions on the currentvoltage (IV) curve for the particular device being discussed.
} 
of the IV curve provides an upper limit on the combined film and contact resistance. Following their approach, IV measurements were made using contacts with varying spacings (d) and a common contact width (W). That is, for four different aspect ratios (d/W). Representative IV curves for each of these aspect ratios are shown in Figure 5.6(A). From the slope of these curves the resistance $\left(\mathrm{R}_{\mathrm{T}}\right)$ was determined at a voltage of $2.0 \mathrm{~V}$ (i.e., four times greater than the voltage needed to overcome the Schottky barrier in this case) for each aspect ratio. Neglecting any contribution from the Schottky barrier, $\mathrm{R}_{\mathrm{T}}$ should be given by,

$$
R_{T}=R_{S} \frac{d}{W}+2 R_{C}
$$

were $\mathrm{Rs}_{\mathrm{s}}$ is the sheet resistance (measured in $\Omega / \square$ ) and $\mathrm{R}_{\mathrm{C}}$ is the contact resistance $(\Omega)$. Figure 5.6(B) shows these resistance values plotted as a function of aspect ratio. This yields a near linear plot with a slope equal to $\mathrm{R}_{\mathrm{S}}$ and a $\mathrm{y}$-axis intercept equal to $2 \mathrm{R}_{\mathrm{c}}$. Following this general approach known as the transmission line method (TLM), it can be shown that the x-axis intercept times $\mathrm{W}$ equals twice the transfer length $\left(\mathrm{L}_{T}\right)$. The film resistivity $\left(\rho_{\mathrm{f}}\right)$ is given by the product $\mathrm{R}_{\mathrm{S}} \cdot \mathrm{t}$, where $\mathrm{t}$ is the film thickness. ${ }^{(577)(578)}$ This method is discussed more fully in Section $\$ 3.2 .5$.

(A)

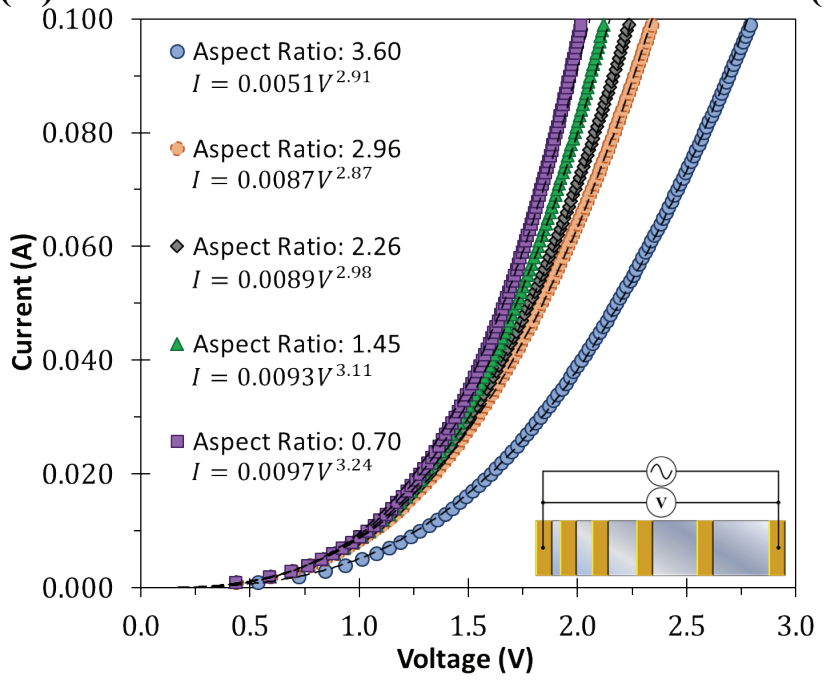

(B)

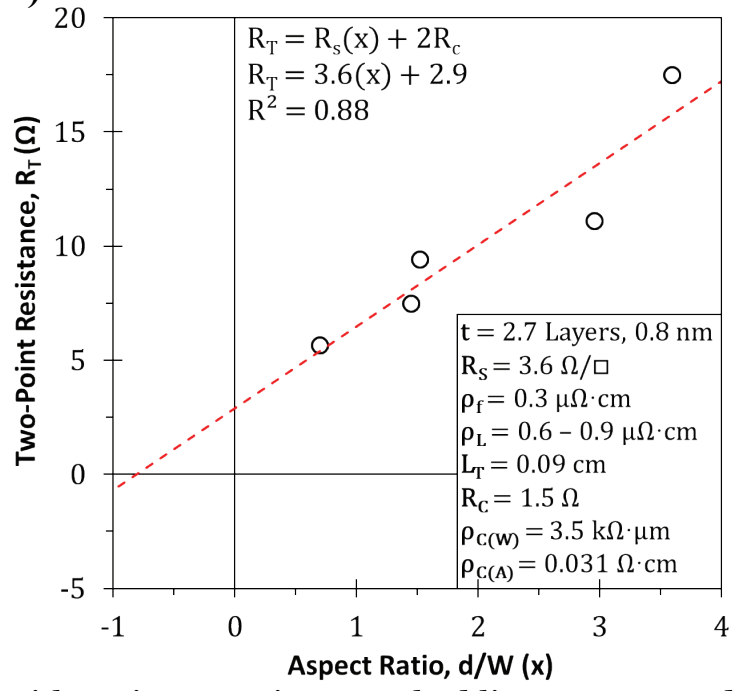

Figure 5.6: (A) IV curves collected from contact pads with various spacings. Dashed lines correspond to power law best-fit lines for each data set. (B) The corresponding TLM plot. the dashed line and accompanying equations represent the linear best-fit to the data. The slope of the linear fit is equal to the sheet resistance of the film $\left(\mathrm{R}_{\mathrm{S}}\right)$, the $\mathrm{Y}$-intercept is twice the contact resistance $\left(2 \mathrm{R}_{\mathrm{C}}\right)$, the $\mathrm{X}$-intercept times the contact width $(\mathrm{W})$ is equal to twice the transfer length $\left(\mathrm{L}_{\mathrm{T}}\right)$.

Based on the indicated linear fit to the TLM data shown in Figure 5.6(B), Rs $=3.6 \Omega / \square$, 
$\mathrm{R}_{\mathrm{C}}=(0.5) \times(2.9) \Omega=1.5 \Omega$, and $\mathrm{L}_{\mathrm{T}}=(0.5) \times(0.8) \times(0.233) \mathrm{cm}=0.09 \mathrm{~cm}^{31}$ This $\mathrm{L}_{\mathrm{T}}$ value is comparable to the contact length which indicates that the entire contact area is used in collecting the injected electrons. That is, there is no current crowding at the contacts. ${ }^{(579)}$ The value of $\mathrm{R}_{\mathrm{C}}$ leads to a contact resistivity, $\rho_{\mathrm{C}}$, which may be calculated as $\rho_{\mathrm{C}}=\mathrm{R}_{\mathrm{C}} \cdot \mathrm{W}=1.5 \Omega \times 0.233 \mathrm{~cm}=$ $0.35 \Omega \cdot \mathrm{cm}=3.5 \mathrm{k} \Omega \cdot \mu \mathrm{m}$. This is comparable to the lower $\rho \mathrm{c}$ values reported by Nagishino, et al. for Ti/Au contacts and Anteroinen, et al. for Ti contacts. ${ }^{(519)(577)}$

The specific contact resistance, $\rho_{\mathrm{C} \square}$, calculated as, $\rho_{\mathrm{C} \square}=\mathrm{R}_{\mathrm{C}} \cdot \mathrm{W} \cdot \mathrm{L}_{\mathrm{T}}=1.5 \Omega \times 0.233 \mathrm{~cm} \times$ $0.09 \mathrm{~cm}=0.031 \Omega \cdot \mathrm{cm}^{2}$. This is close to (slightly higher) the value reported by Robinson, et al. for an unannealed $\mathrm{Ti} / \mathrm{Au}$ contacts made to graphene grown from $6 \mathrm{H}-\mathrm{SiC}$ by selective sublimation. ${ }^{(580)}$ Further, using an average graphene layer thickness of $0.346 \mathrm{~nm}$ based on the measurements of Weng, et al. for multi-layer epitaxial graphene films on $6 \mathrm{H}-\mathrm{SiC}(0001)$, the calculated resistivity of the $0.8 \mathrm{~nm}\left(2.7\right.$ monolayers (ML)) thick film is $\rho_{\mathrm{f}}=\mathrm{R}_{\mathrm{S}} \cdot \mathrm{t}=3.6 \Omega / \square \times 0.8 \mathrm{~nm}=0.3 \mu \Omega \cdot \mathrm{cm}$. ${ }^{(548)}$

$\mathrm{Yu}$, et al. showed that the individual graphene layers in multilayer films and multilayer stacks can be treated as an equivalent set of parallel resistors representing the individual graphene layers. ${ }^{(581)}$ Assuming that the buffer and graphene layers contribute equally, the measured resistivity of the $\sim 2.7 \mathrm{ML}$ film represents a single layer resistivity approximately two to three times higher than the measured resistivity. This yields a single layer resistivity ( $\rho_{\mathrm{L}}$ ) of $\sim 0.6$ to $0.9 \mu \Omega \cdot \mathrm{cm}$ which is reasonably close to the nominal value of $1 \mu \Omega \cdot \mathrm{cm}$ frequently quoted for single layer exfoliated graphene and epitaxial graphene on $\mathrm{SiC}$. ${ }^{(582)(583)}$ This value is slightly lower than the single layer resistivity $(1.6 \mu \Omega-\mathrm{cm})$ observed for graphene produced by plasma etching and UHV annealing. ${ }^{(263)}$

Based on the observed film resistivity and carrier density, the carrier mobility within the film can be calculated as $\mu=\left(\mathrm{N}_{\mathrm{d}} \cdot \mathrm{q} \cdot \rho_{\mathrm{f}}\right)^{-1}$. Assuming once again that the carriers are electrons leads to a rather high mobility of $7,086 \mathrm{~cm}^{2} \mathrm{~V}^{-1} \mathrm{~s}^{-1}$. For graphene formed by thermal decomposition of $\mathrm{SiC}$, mobility values vary widely; however, representative values seem to range from 1,100 to $11,600 \mathrm{~cm}^{2} \mathrm{~V}^{-1} \mathrm{~s}^{-1}{ }^{(584)}$

\footnotetext{
${ }^{31}$ The unit " $\Omega / \square$ " is read as "Ohm per Square" or "Ohms Square"; and unit used solely for sheet resistance and disparate from $\Omega^{2}$. Treated dimensionally as Ohms. This unusual unit has the benefit of being independent of film size and quantifies the resistance on a per square basis (i.e. a $1 \mathrm{~cm} \times 1 \mathrm{~cm}$ film would have the same sheet resistance as a $10 \mathrm{~cm} \times 10 \mathrm{~cm}$ identical film). This allows of the direct comparison between films of different sizes and shapes, as long as the restively is expressed in terms of $\Omega / \square$.
} 
To further support the above results, four-point measurements were also performed to determine the sheet and film resistivity. The four-point analysis method is described in Section \$3.2.5. In short, a current is forced between two outer sets of contacts (force probes) and the voltage drop is measured between two inner contact points (sense probes). The voltage drop is monitored by a high impedance measurement device. This is done so the voltage may be 'sampled' without effecting the measurement. Due to the ultra-low current flow used to measure the voltage, there is minimal voltage drop across the contact and lead wires used to collect the measurement. Additionally, since the voltage is measured using a contact not injecting current, the effect of the Schottky contacts are not seen. In other words, the Schottky barrier has already been surmounted and current has already been injected into the film, therefore the voltage drop between the two inner contacts is due only to the resistance of the film. Thus, the four-point measurement examines the properties of the film only. The contributions of the contacts and the Schottky nature of the graphene-SiC interface are still present, but their effects do not appear in the measurement (as evident from the linear nature of the IV curves collected using this method).

In these measurements, a voltage was applied to the terminal contacts to force a current through the film. The voltage drop between two interior contacts, separated by a known spacing, was then measured. Figure 5.7(A) plots the voltage drop versus current obtained in this manner for the same $0.8 \mathrm{~nm}$ thick film represented in Figure 5.6, although different sets of contacts were

(A)

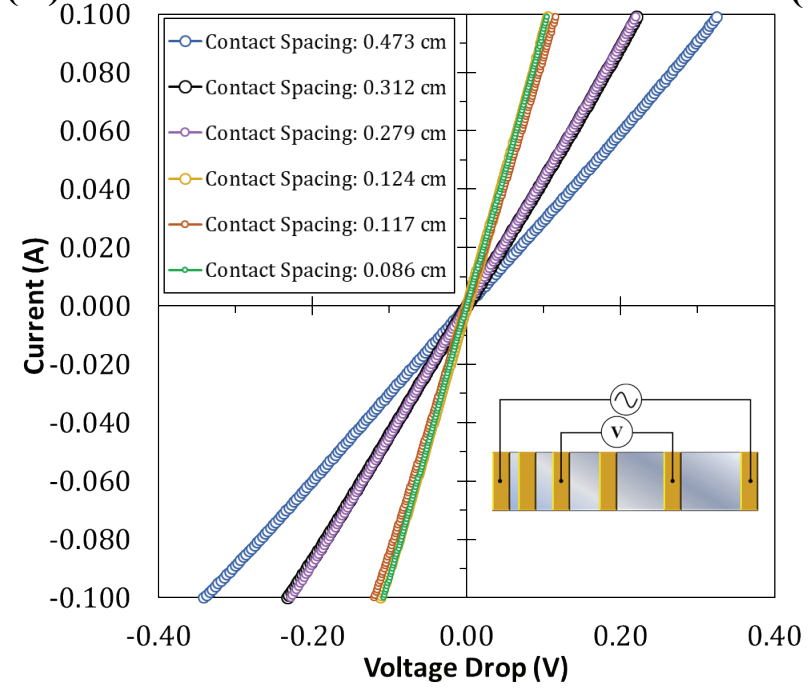

(B)

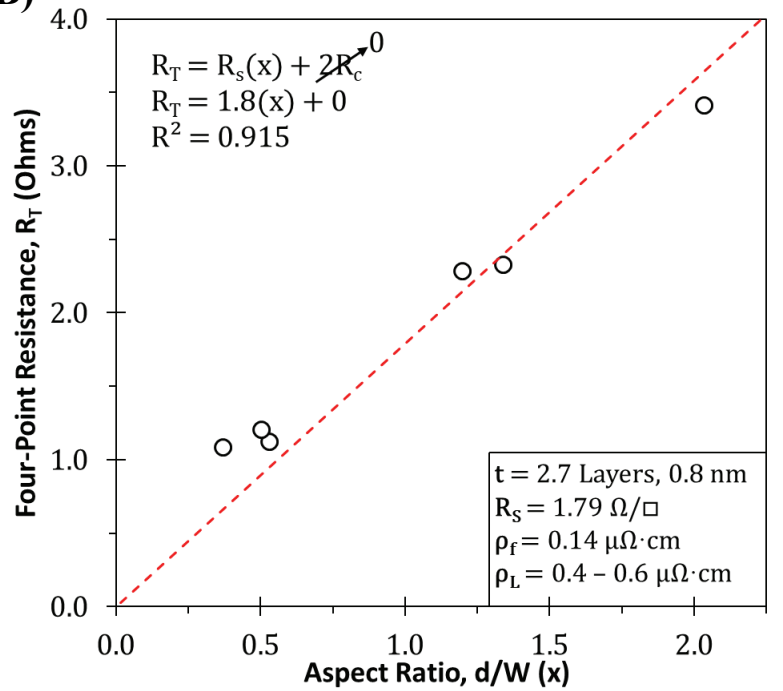

Figure 5.7: (A) Force current plotted as a function of the voltage drop between two inner sense pads. The results of six different pad spacings are shown. The contact width was $0.233 \mathrm{~cm}$. (B) The resistance of the film as calculated by the voltage drop, plotted as a function of the aspect ratio of the film between the sense pads. 
utilized in this test. Figure 5.7(B) plots the obtained resistance values as a function of the spacing between six different sets of sense pads. The intercept of the fit line is set to zero based on the fundamental principle that a film of zero length would have zero resistance (the contact pads would be touching) and that the four-point probe arrangement effectively removes the contribution of the contact resistance.

As expected, the voltage drop varies linearly with the current flow. From these measurements, the resistance of the expanse of film between the corresponding contacts is calculated using Ohm's law. Based on the slope and aspect ratio, the sheet resistance (Rs) of this film is $1.8 \Omega / \square$ and the corresponding film resistivity ( $\rho_{\mathrm{f}}$ ) is $0.14 \mu \Omega \cdot \mathrm{cm}$. Again, taking into account the fact that this is a $0.8 \mathrm{~nm}$ thick $(\sim 2.6 \mathrm{ML})$ film places the single layer resistivity at 0.4 to 0.6 $\mu \Omega \cdot \mathrm{cm}$. As expected, these values are comparable to, but slightly lower than, the values determined using TLM methods.

The lower than normal film resistivities observed here may be the result of several factors. The high carrier concentration, presumably due to oxygen doping, may play a role. In studies of doping effects on carrier mobility in few-layer graphene, Khan et al. demonstrated three to four fold increases in both electron and hole mobility which presumably leads to corresponding reductions in film resistivity. ${ }^{(585)}$ Moreover, the usual assumption is that charged defects (e.g. oxygen-based defects such as those observed here) serve as scattering centers. Work by Ponomarenko, et al. however, suggests Coulomb impurities on the graphene surface do not limit the carrier mean free path. ${ }^{(586)}$

The Ti/Au contacts, which cover a substantial fraction of the film, may also influence the conductivity measurements. The contacts will provide a parallel conduction path for the areas of the film over which they cover, leading to a smaller voltage drop across the film region overlaid with a contact than would otherwise occur. This would then lead to a lower calculated resistance for films which have metal contacts between the measurement electrodes. Additionally, metal contacts have been shown to lead to significant doping and other electronic modifications to the graphene beneath. ${ }^{(169)}{ }^{(587)} \mathrm{A}$ further possibility is that the buffer layer leads to a coupling of the electrons in the graphene film with electrons in the $\mathrm{SiC}$, resulting in a decrease in resistivity, which, as noted previously, would be consistent with the XPS and Raman data. This brings into question the role that the substrate (and related layers) play in the conduction, as illustrate in Figure 5.3. 
It is important to note that while both GO and RGO exhibit back-to-back Schottky behavior, their resistivity is orders of magnitude higher and the mobility is correspondingly lower than that reported for the films produced here. Specifically, the resistivity of GO was found to be $200-1000 \Omega-\mathrm{cm} .{ }^{(588)}$ After reduction in hydrazine for 24 hours to produce RGO, the resistivity decreased to $0.5-20 \Omega-\mathrm{cm}$. The hole mobility for RGO was less than $200 \mathrm{~cm}^{2} \mathrm{~V}^{-1} \mathrm{~s}^{-1}$, while the electron mobility was less than $30 \mathrm{~cm}^{2} \mathrm{~V}^{-1} \mathrm{~s}^{-1}$. Thus, the films produced here using the two-step process used in this work have as good or better electrical properties than the best RGO films.

In regard to the contact resistance, although not excessively high, there was no attempt made to aggressively minimize it. Based on literature reports, there are several methods which could be easily applied to the devices produced here to reduce the Rc. Russo, et al. showed a direct correlation between the contact resistance $\left(\mathrm{R}_{\mathrm{C}}\right)$ and the background pressure of the e-beam evaporator used to deposit the Ti/Au contacts onto graphene films, with higher pressures leading to higher contact resistances. ${ }^{(589)}$ The obvious mechanism here is that the larger number of contaminate molecules present at higher pressures contaminate the metal/graphene interface (undoubtedly atmospheric based contaminates like water, or hydrocarbon based from the vacuum pumping system). Therefore, a refinement of the contact deposition process could be an easy avenue for reducing the contact resistance (for example, increasing the pump down time to reach a lower base pressure). An additional method for reducing the contact resistance is patterning of the graphene film under the contact. Smith, et al. showed that by patterning the graphene under the contacts by adding cuts to the film (thereby increasing the amount of graphene edges) the contact resistance can be significantly reduced. ${ }^{(590)}$ 


\section{\$5.1.3 Richardson-Dushman Analysis}

Following the procedure as detailed in Section $\$ 3.2 .5$, the Schottky barrier height $\left(\Phi_{\mathrm{B}}\right)$, carrier concentration $\left(\mathrm{N}_{\mathrm{d}}\right)$ and carrier mobility $(\mu)$ were determined using the modified RichardsonDushman analysis. Figure 5.8(A) plots one quadrant of the IV curve of a device. Figure 5.8(B) plots the IV data as the natural log of the "reduce current" vs. the cube root of the voltage $\left(\mathrm{V}^{1 / 4}\right)$ (the reduced current is defined in Section $\$ 3.2 .5$ ).

(A)

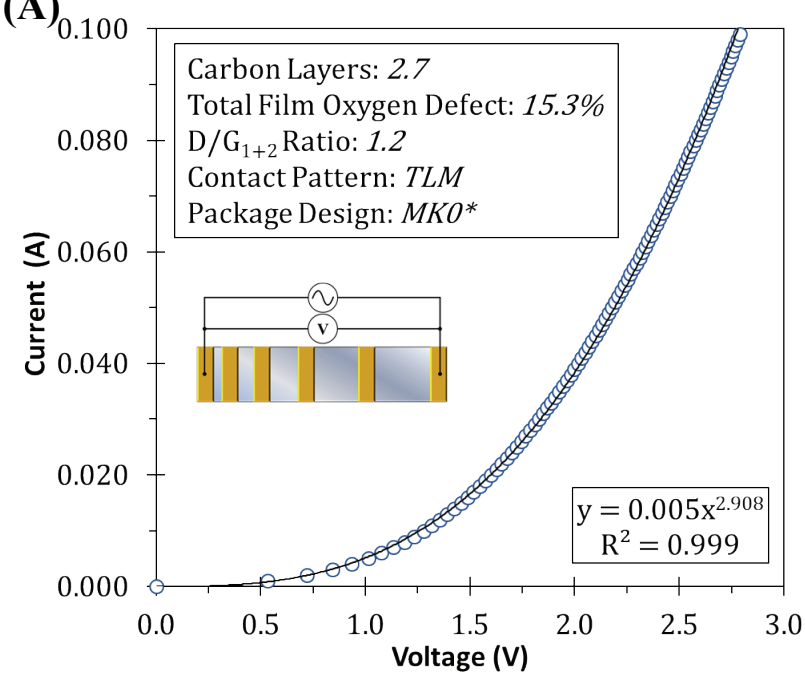

(B)

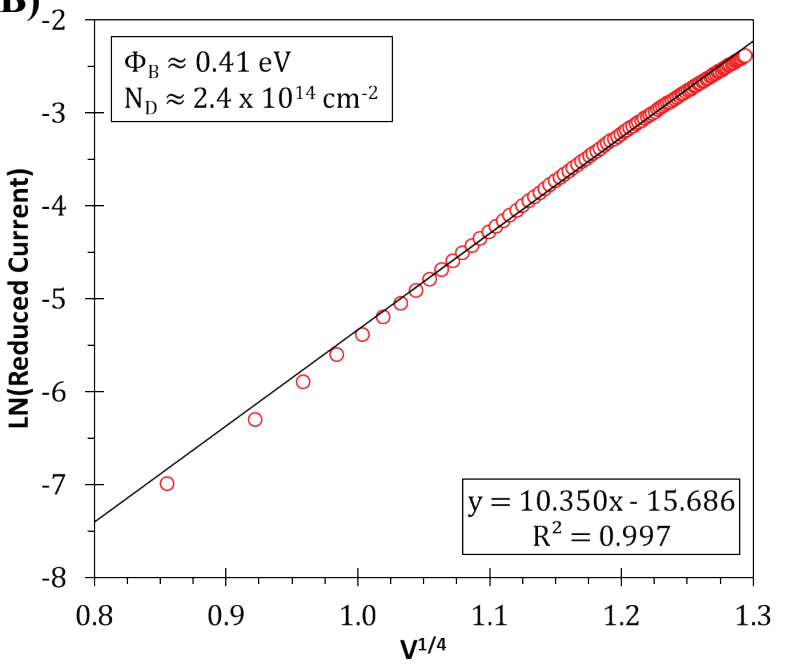

Figure 5.8: (A) The IV curve with a best-fit line shown. The film parameters contact pattern design, and sample packaging of the device are listed. (B) The Richardson-Dushman analysis of the data shown in (A). A best-fit linear line is shown.

Using this method, a Schottky barrier height $\left(\Phi_{\mathrm{B}}\right)$ of $\sim 0.41 \mathrm{eV}$ was calculated. This value is close to the values observed by $\mathrm{Wu}$ and coworkers who found Schottky barrier heights ranging from $0.45 \mathrm{eV}$ to $0.70 \mathrm{eV}$ for GO. ${ }^{(522)}$ Moreover, the $0.41 \mathrm{eV}$ value observed here is close to the range of values $(0.45-0.70 \mathrm{eV})$ observed for graphene films produced by plasma etching and UHV annealing. ${ }^{(523)}$ The recent review of graphene Schottky junctions by Bartolomeo shows that, for devices built from $\mathrm{SiC}$ (the $4 \mathrm{H}$ polymorph was used in all cases), values of the $\Phi_{\mathrm{B}}$ ranged from 0.36 to $1.15 \mathrm{eV} .{ }^{(380)}$ This puts this work on the low end of that range. It is interesting to note that the lowest value observed by Sonde and coworkers, albeit with devices using a significantly different design than what is used here, was from films which were grown epitaxially from the substrate (as opposed to experiments they performed with deposited graphene on $\mathrm{SiC}$, or graphene on non-SiC substrates). ${ }^{(591)}$ They suggest that the low $\Phi_{\mathrm{B}}$ value $(\sim 0.49 \mathrm{eV}$ lower than for their experiments using exfoliated graphene deposited onto $\mathrm{SiC}$ as opposed to an epitaxial growth method) is due to the presence of a buffer layer. 
Although this analysis does lump in the series resistance of the circuit, note that the carrier concentration only relies on the slope of the line in Figure 5.8(B) and is thus unaffected. Additionally, data presented by Aubry and Meyer indicated that a change in the value of the series resistance by an order of magnitude only effects the calculated barrier height (which uses the intercept of the line shown in Figure 5.8(B) and therefor is affected by the series resistance) by only $0.1 \mathrm{eV}$ when the plot of $\ln (\mathrm{I}) \mathrm{vs} . \mathrm{V}$ is linear, as is largely the case here. ${ }^{(572)}$

From Figure 5.8, the charge carrier density $\left(\mathrm{N}_{\mathrm{d}}\right)$ was found to be $\sim 2.4 \times 10^{14} \mathrm{~cm}^{-2}$. If an areal density of $3.8 \times 10^{15} \mathrm{~cm}^{-2}$ for carbon atoms is assumed for an ideal graphene film, this represents a doping density $\sim 6 \%$ which is significantly less than the level of oxygen-based defects in the film observed by XPS (nominally $\sim 17 \%$ for a 2.7 layer film). This may simply reflect the fact that not all defects are effective in creating charge carriers. Overall, these values for $\mathrm{N}_{\mathrm{d}}$ are comparable to those found for graphene films produced by plasma etching and UHV annealing (e.g., $5.6 \times 10^{14} \mathrm{~cm}^{-2}$ ) and they are approximately one to two orders of magnitude higher than those typically observed for epitaxial graphene formed by thermal annealing of $\mathrm{SiC}$. ${ }^{592)}$

A minor complication to this analysis is that the devices always experience some selfheating when power is applied (this is discussed in greater detail in Section \$5.3.1). The impact of this is that the devices heat up when a measurement is collected. Because the parameters extracted from these measurements are temperature sensitive, the details of how the data is collected impact the results. Depending on the parameters of the data acquisition (step size, setting time, etc.) an IV measurement can take from $2.25-33.57$ seconds to complete in the setup used here. Longer times lead to, presumably, higher sample temperatures. It was found that by varying the collection time, and therefore indirectly the sample temperature, $\Phi_{\mathrm{B}}$ value varied by $\pm 0.9 \%, \mathrm{~N}_{\mathrm{d}}$ by $\pm 4.3 \%$ and the $\mu \pm 3.8 \%$. The sensitivity of these parameters to an explicit temperature changes are detailed in Section $\$ 5.2$. 


\section{\$5.1.4 Non-standard Samples}

Due to the large number of samples produced, many of the non-standard samples were not electrically characterized. For those samples which were electrically characterized, further testing is required before clear conclusions can be made; however, some observations are worth reporting and are included here. This data, and analysis, is shown in Figure 5.9 and is discussed next briefly.

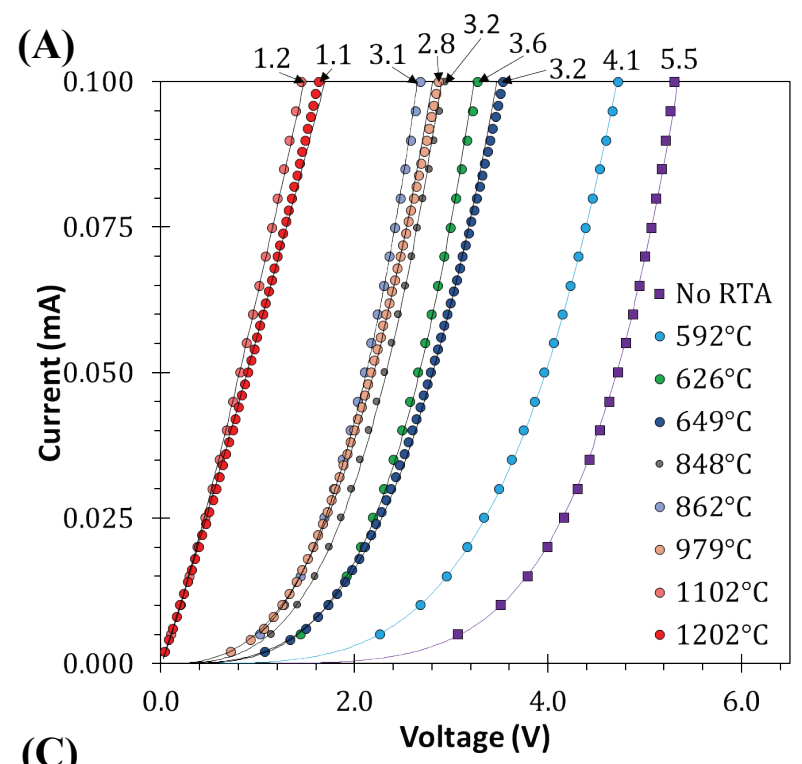

(B)
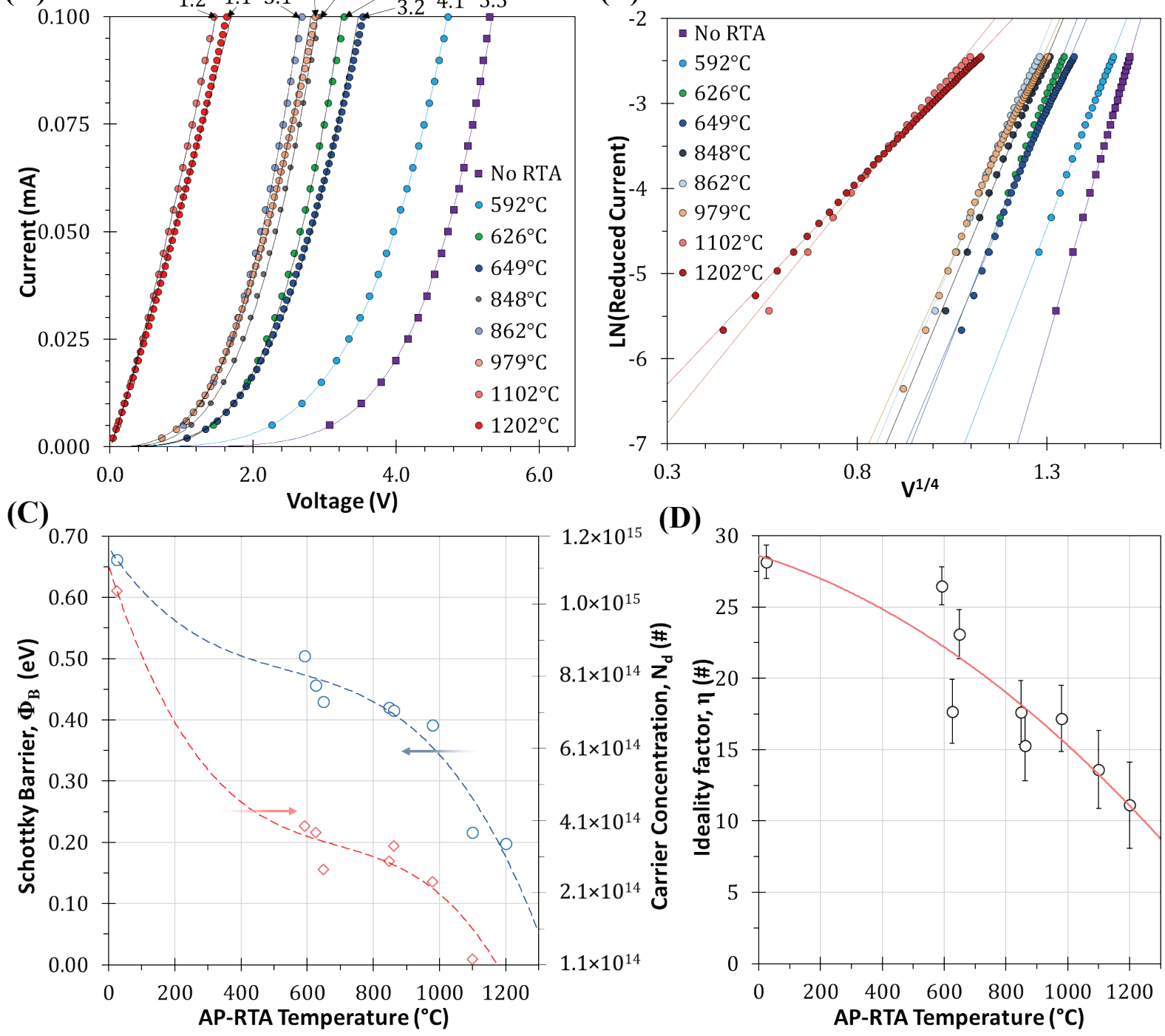

Figure 5.9: Summary data for samples AP-RTA'ed to different temperatures. (A) Data points are twopoint IV measurements from films AP-RTAed to various temperatures. The annotated numbers are the power law exponents for the best-fit power law lines shown. (B)Richardson--Dushman analysis plot for the data in (A). (C) The calculated Schottky Barrier height and carrier concentrations. (D) Schottky diode ideality factors for the data set.

Two-point electrical data (IV plots and TLM analysis) were collected at room temperature on devices made from the samples produced AP-RTA'ed up to different maximum temperatures (as detailed in Section $\$ 4.2 .4$ ). 
Current is proportional to the applied voltage. To quantify the curvature of the IV plot shown in Figure 5.9(A), the curves are be fitted to a power law equation. The proportionality thus becomes,

$$
I \propto V^{n}
$$

Equation 5-4

where $\mathrm{n}=1$ for an Ohmic device. The values of $\mathrm{n}$, annotated in Figure 5.9(A), show the clear trend that high annealing temperatures lead to values of $\mathrm{n}$ close to 1 (Ohmic behavior) and a low annealing temperature correlate to high values of $\mathrm{n}$ (Schottky behavior).

Figure 5.9(B) shows the Richardson-Dushman analysis of the data from Figure 5.9(A), which is then used to calculate the Schottky barrier height and carrier concentrations, plotted in Figure $5.9(\mathrm{C})$. The maximum temperature reached during the annealing obviously effects the electrical properties of the device. Recalling from Section \$4.2.4, variations in the AP-RTA temperature effect the films in several ways including thickness, oxygen content and crystallinity.

It is unclear which specific material property is responsible for the observed trends in the electrical properties. However, it is clear that the totality of these effects is that higher AP-RTA temperatures lead to devices with lower $\Phi_{\mathrm{B}}$ heights and lower charge carrier concentrations. Surprisingly, the per layer conductivity (as determined from TLM analysis) is not drastically affected by the AP-RTA temperature. Using these conductivity values to calculate the charge carrier mobility leads to unrealistically large values, particularly for samples annealed at temperatures above $1000^{\circ} \mathrm{C}$. This may indicate that, for these non-standard samples, the general approach breaks down and the physical reality of the situation is substantially different from our conceptual model of the system. Further electrical characterization and analysis is required for all non-standard samples. This presents a rich area for future work.

\section{§5.1.5 Summary of Room Temperature Electrical Characterization}

A summary of the device electrical parameters is given in Table 5-1. The results of the electrical analysis show that devices constructed from the graphene-SiC composites produced using the SOP process exhibit back-to-back Schottky behavior. The electrical analysis used a classic thermionic emitter model to calculate the diode parameters. The high ideality factor $(\eta)$ of these devices, while not unusual for graphene-based devices, indicate there are other mechanisms at play. These could include various non-thermionic current injection mechanisms (such as tunneling currents), Schottky barrier height variations (such as voltage dependent image force 
effects) or other complexities occurring at the contact/graphene interface (such as charge trapping or carrier recombination in the depletion region). ${ }^{(567)}$ To determine the precise current injection mechanism and the bulk film conduction mechanism will require further study and analysis.

Table 5-1: Electrical parameters from a typical device constructed from the Graphene/SiC composites produced using the SOP process.

\begin{tabular}{|r|l|}
\hline Device Behavior & Back-to-back Schottky \\
\hline Diode Ideality Factor $(\eta)$ & $\sim 15-17$ \\
\hline Schottky Barrier Height $\left(\Phi_{\mathrm{B}}\right)$ & $\sim 0.41 \mathrm{eV}$ \\
\hline Film Carrier Concentration $\left(\mathrm{N}_{\mathrm{D}}\right)$ & $\sim 2.4 \mathrm{x} 10^{14} \mathrm{~cm}^{-2}$ \\
\hline Film Sheet Resistance, 2.7 layer $\left(\mathrm{RS}_{\mathrm{S}}\right)$ & $\sim 1.8-3.6 \Omega / \square$ \\
\hline Per Layer Resistivity $\left(\rho_{\mathrm{L}}\right)$ & $\sim 0.14-0.9 \mu \Omega \cdot \mathrm{cm}$ \\
\hline Transfer Length $\left(\mathrm{LT}_{\mathrm{T}}\right)$ & $\sim 0.09 \mathrm{~cm}$ \\
\hline Contact Resistance $\left(\mathrm{RC}_{\mathrm{C}}\right)$ & $\sim 1.5 \Omega$ \\
\hline Specific Contact Resistance $\left(\rho_{\mathrm{C}}(\mathrm{W})\right)$ & $\sim 3.5 \mathrm{k} \Omega \cdot \mathrm{cm}$ \\
\hline Specific Contact Resistance $\left(\rho_{\mathrm{C}}(\mathrm{A})\right)$ & $\sim 0.031 \Omega \cdot \mathrm{cm}^{2}$ \\
\hline
\end{tabular}

Adding complexity to the system is the multilayered heterostructure of the graphene devices. This can present multiple parallel conduction paths for current flow. Given that these layers are not uniform in their electrical properties (conductivity, electrical permeability, etc.) and that the electrical communication between these dissimilar layers could be complex, their involvement in conduction process is likewise complex. This could include voltage dependent layer activation (i.e. all layers may not participate across all voltages) or capacitive effects occurring between layers.

In conclusion, while the finer details of the system require further study to unravel, the larger electrical picture generally matches with what would be expected from the materials characterization of the films as discussed in Chapter 4. Specifically, the films are highly doped with a large number of charge carriers. The carrier mobility is correspondingly low (relatively) as compared to literature reports on graphene of similar quality. The higher than expected film conductivity is explained by coupling with charge carriers in the substrate via the buffer layer and participation of the substrate in conduction. This adds further motivation for the decoupling of the films produced by this plasma assisted method (discussed in greater detail in Chapter 4). Decoupling could reduce the substrate induced doping/coupling thereby decreasing the charge carrier concentration, increasing carrier mobility and electrically isolating the film from the substrate. Dharmaraj, et al. have claimed a 30\% increase in carrier mobility and an order of magnitude decrease in the carrier concentration by hydrogen intercalation (for graphene films 
grown from $4 \mathrm{H}-\mathrm{SiC}(0001)$ by selective sublimation). ${ }^{(245)}$ Given the unusually high interaction between the buffer layer and the substrate for films produced using the plasma assisted method studied here, successful decoupling may produce even greater performance gains.

\section{\$5.2 Temperature Dependence of Device Properties}

This section will summarize the results and present representative data for over 1,800 tests collected from 120 devices in which the electrical properties of the graphene-SiC devices were observed as a function of temperature. The temperature response plays a convoluting role during both gas sensing (Section \$5.3) and photo detection (Section \$5.4). Therefore, a characterization of the device temperature response is key to interpreting the gas and photo responses. This section will discuss the device behavior due to an explicit temperature change. The device response due to temperature changes resulting from secondary effects (self-heating and physical environmental effects) are discussed in Section $\$ 5.3 .1$ - Section $\$ 5.3 .3$.

Measurements were conducted from room temperature (the laboratory temperature was measured to be $\sim 24^{\circ} \mathrm{C}$ with slight day-to-day variations of $\pm 4^{\circ} \mathrm{C}$ ) up to a maximum of $\sim 810^{\circ} \mathrm{C} .{ }^{32}$ All experiments detailed in this section were carried out under a flow of 500 SCCM of UHP argon. These experiments used both the large test bed (which heated the entire sample package and electrode leads, described in Section \$3.4.1) and small test cell (which directly heated the sample only and indirectly the sample packaging, described in Section \$3.4.2). For the large test bed setup, due to the harsh thermal environment, sample packaging was limited to the simpler designs which only allotted two of the four electrical feedthroughs for electrodes to make electrical contact on the sample. Therefore, only two-point measurements were collected as a function of temperature. The remaining two electrical feedthroughs were used in monitoring of the device temperature. A platinum resistance temperature device (RTD) was used for experiments in the small test cell and a type-K thermocouple (nickel-chromium/nickel-alumel) was used for experiments in the large test bed.

\footnotetext{
${ }^{32}$ The temperature limit in the small test cell $\left(\sim 800^{\circ} \mathrm{C}\right)$ is due to limitations of the RTD used to measure the sample temperature. The limit in the large test bed $\left(\sim 800^{\circ} \mathrm{C}\right)$ is due thermal degradation of the sample packaging. The device itself remains functional at temperatures well in excess of $800^{\circ} \mathrm{C}$. This speaks to the thermal stability of the graphene$\mathrm{SiC}$ composites.
} 


\section{§5.2.1 Two-Point Analysis}

The precise temperature sensitivity of the graphene/ $\mathrm{SiC}$ devices are difficult to quantify. This is because the temperature sensitivity is a function of both temperature and voltage. Furthermore, due to the self-heating effects experienced by the device (discussed in Section \$5.3.1), the interdependence of temperature and voltage become more complex. In order to fully capture the device behavior as a function temperature, IV plots were collected across a range of temperatures and stacked to form the 3D plot shown in Figure 5.10. Figure 5.10(A) shows a 3D surface plot (current, as a function of voltage, stacked against temperature) constructed from 29 IV plots, collected from $25^{\circ} \mathrm{C}$ to $200^{\circ} \mathrm{C}$ in $\sim 7^{\circ} \mathrm{C}$ steps. ${ }^{33}$ Both samples were $\sim 2.5 \mathrm{~mm}^{2}$ in size and utilized the sensor pattern design with the MK0 packaging, mounted in the small test cell. The two key observations are a gradual transition from back-to-back Schottky behavior to an almost fully Ohmic behaver, as well as a uniform increase in device conductivity across all voltage ranges.
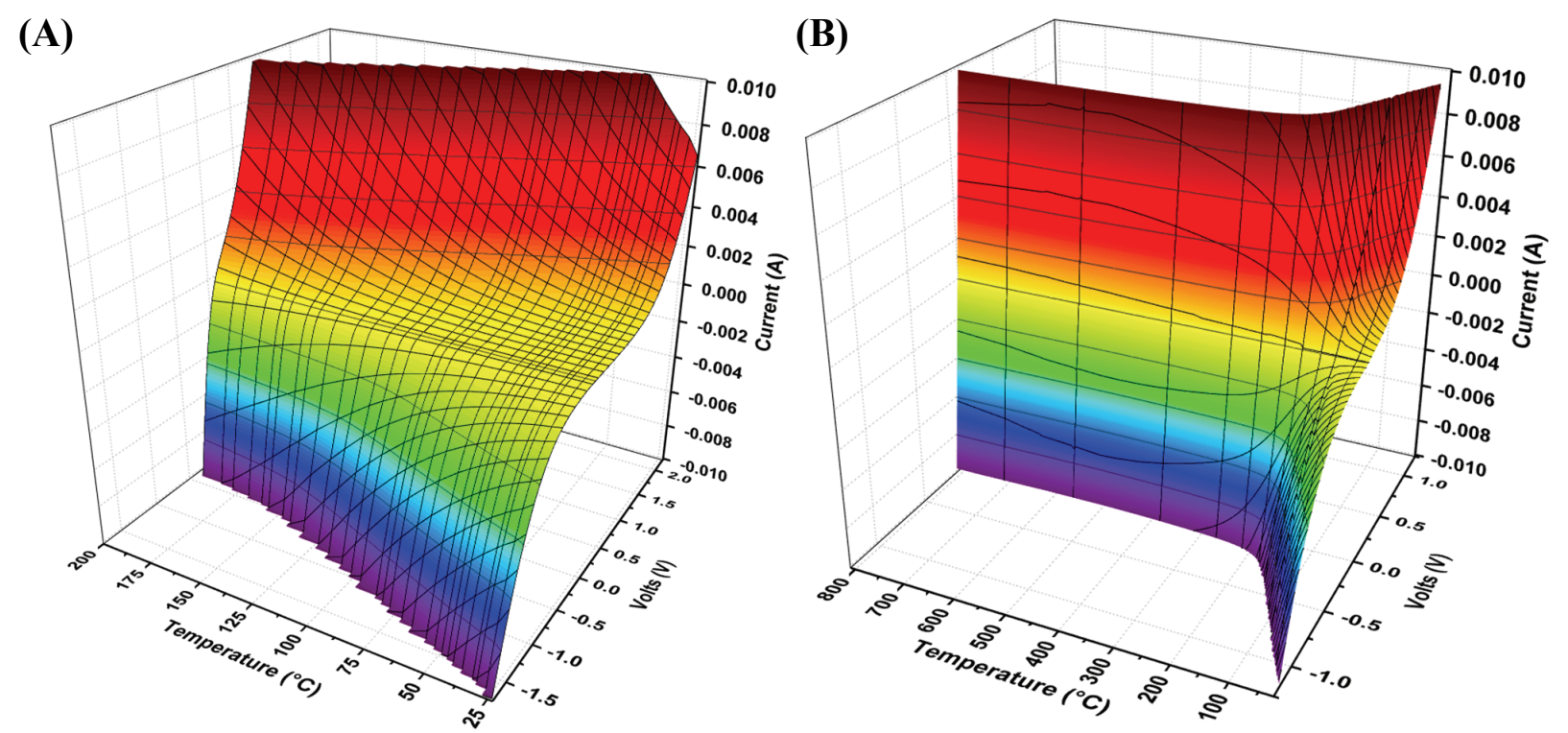

Figure 5.10: (A) Stacked IV plots, collected from $25^{\circ} \mathrm{C}$ to $200^{\circ} \mathrm{C}$, showing the smooth transition from backto-back Schottky to almost pure ohmic behavior. (B) Similar data from a different device across a larger temperature range. The transition to ohmic behavior is observed around $300^{\circ} \mathrm{C}$. Conductivity increases up to $\sim 550^{\circ} \mathrm{C}$ after which a small decline is seen up to $810^{\circ} \mathrm{C}$ (maximum temperature range of the RTD).

Figure 5.10(B) shows a similar surface plot (constructed of $71 \mathrm{IV}$ plots recorded from $25^{\circ} \mathrm{C}$ to $810^{\circ} \mathrm{C}$ in $\sim 10^{\circ} \mathrm{C}$ steps, collected from a separate, but otherwise identically fabricated sample) and shows the same trend across a larger temperature range. Here, a transition to what is essentially

\footnotetext{
${ }^{33}$ Note that at the time this data was collected, the experimental setup imposed a current limit of $10 \mathrm{~mA}$ for the SCS4200 power supplies. The setup was later modified to increase the current limit to $100 \mathrm{~mA}$. Both arrangements had a $\pm 20 \mathrm{~V}$ voltage limit.
} 
Ohmic behavior is observed around $\sim 300^{\circ} \mathrm{C}$. Device conductivity increases up to a temperature of $\sim 550^{\circ} \mathrm{C}$. Above this temperature, although the device remains Ohmic, the conductivity begins to decrease with further increasing temperature. This decrease in conductivity at very high temperature is typical of all devices measured, however, the precise temperature of this transition varies from sample to sample $\left( \pm 200^{\circ} \mathrm{C}\right)$ and depends on the device design and packaging.

The general behavior shown in Figure 5.10 is explained by classic thermionic emission of charge carriers over the Schottky barrier and into the film. ${ }^{(380)}$ As the temperature is increased, charge carriers in the metal contact are promoted to higher energy levels. There is a wide statistical distribution in the energies of these carriers which is directly related to the temperature (similar to a Maxwell-Boltzmann distribution but with quantized energy states). Recall that temperature is simply a measure of the characteristic energy of this distribution and is proportional through the Boltzmann constant. Therefore, as the temperature is increased, the fraction of charge carriers that

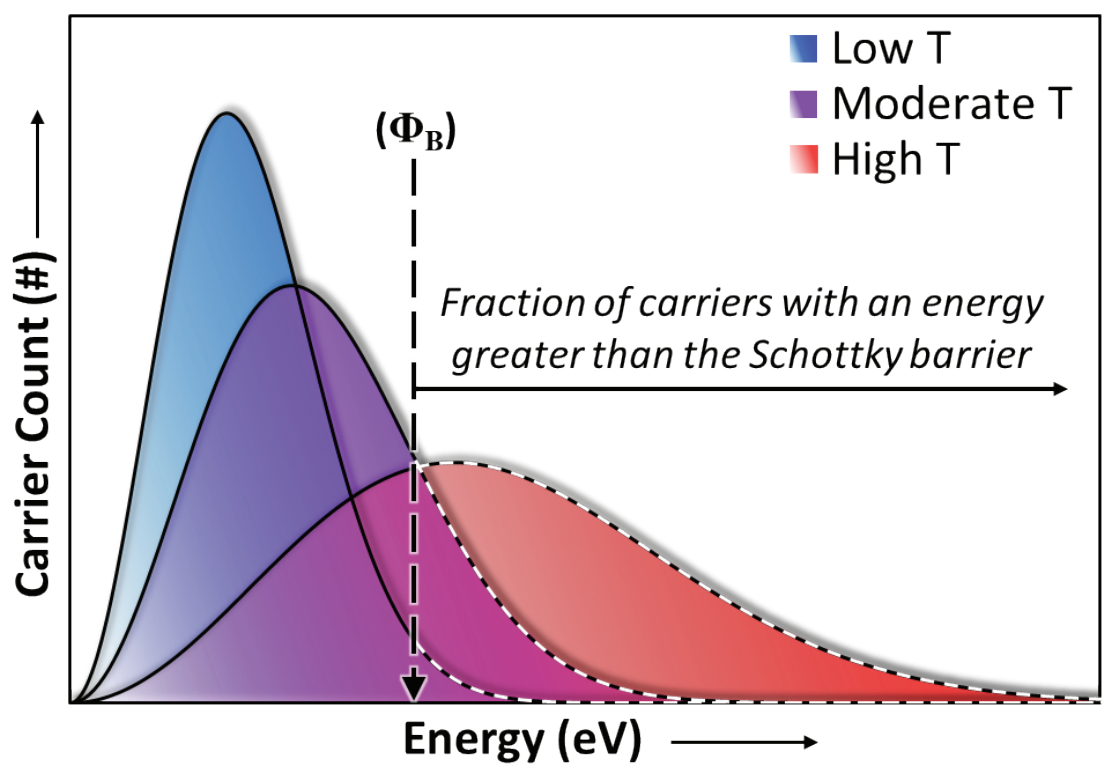

Figure 5.11: The Maxwell-Boltzmann distribution at three generic temperatures. The fraction of carriers with an energy greater than the Schottky barrier increases with increasing temperature.

approach the Schottky barrier with an energy higher than the barrier height is also increased. The relationship between the carrier distribution to the Schottky barrier, at various temperatures, is shown generically in Figure 5.11. To these high energy carriers, the Schottky barrier is not seen. Thus, the barrier-induced curvature of the IV plot is reduced by this high energy fraction of carriers and the device begins to conduct at lower voltages. As the number of carriers in this fraction grow (with increasing temperature), the curve becomes linear (and shifts to lower voltages) and only a 
small fraction of carriers do not have sufficient energy to surmount the barrier. It is important to note that, even though the IV plot is fully linear at high temperature, the Schottky barrier is still present (and in fact, as discussed in the next section, the measured barrier height increases with temperature).

There is a slight decrease in device conductivity at very high temperatures (observable most clearly in the constant voltage contour lines of Figure $5.10(\mathrm{~B})$ from $550^{\circ} \mathrm{C}$ through to $810^{\circ} \mathrm{C}$ ). This is most likely due to a combination of film effects and effects of the device packaging. At low temperature the contributions of the device packaging to the total circuit resistance is small. However, at high temperature, the resistance of the device decreases to a point where this may be no longer true (i.e. the packaging constitutes the majority of the measured circuit resistance). The Ohmic nature of these packaging components add a compounding effect as well, in that their absolute resistance increases with temperature (while the device resistance decreases with increasing temperature).

As for the film driven effects, an increase in scattering sites may be responsible for the observed increase in device resistance. It is likely that thermally generated phonons are a prime scattering element, which are known to increase the resistivity of graphene with increasing temperature. (593) (594) (595) (596) Phonons are discrete and quantized oscillations in the atoms which make up the crystal lattice. These localized perturbations are free to coherently move within the lattices and can be treated as quasiparticles. Phonons can interact with charge carriers, acting as scattering sites and reduce the carrier mean free path. This interaction negatively impacts carrier mobility and thus, conductivity.

The large number of mobile charge carriers in the film at high temperature may also contribute to a reduction in conductivity. The high carrier densities at elevated temperatures, due to thermally promoted carriers, may increase the number of carrier-carrier interactions (via Coulomb interactions). ${ }^{(597)(598)(599)}$ In such a case, the density of carriers increases to a point where the probability of carriers encountering each other becomes high and, because they are of like charge, repel each other. This can reduce carrier mobility and thus, conductivity. Contributing to the high number of carriers are both extrinsic carriers (i.e. carriers injected from the metal contact) as well as the generation of intrinsic carriers due to the promotion of carriers from the valence 
band to the conduction band in the film. Similarly, the generation of mobile carriers from the bulk $\mathrm{SiC}$ substrate (mediated via the buffer layer) may occur at very high temperatures as well.

Close inspection of the IV plots in the transitional region from Schottky to Ohmic behavior reveal subtle complexities in the curves. Figure 5.12(A) shows an IV plot collected at $\sim 205^{\circ} \mathrm{C}$. Two subtly distinct regions are marked by the dashed lines. They are largely linear but are separated by an inflection point. By way of an explanation for this behavior, the concept of interfacial charge trapping is introduced.

(A)

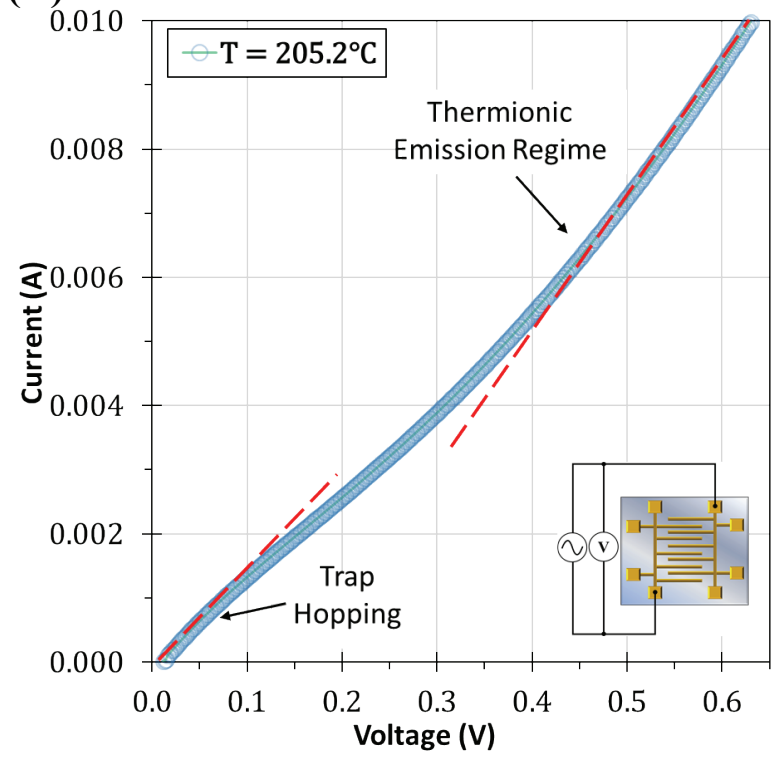

(B)

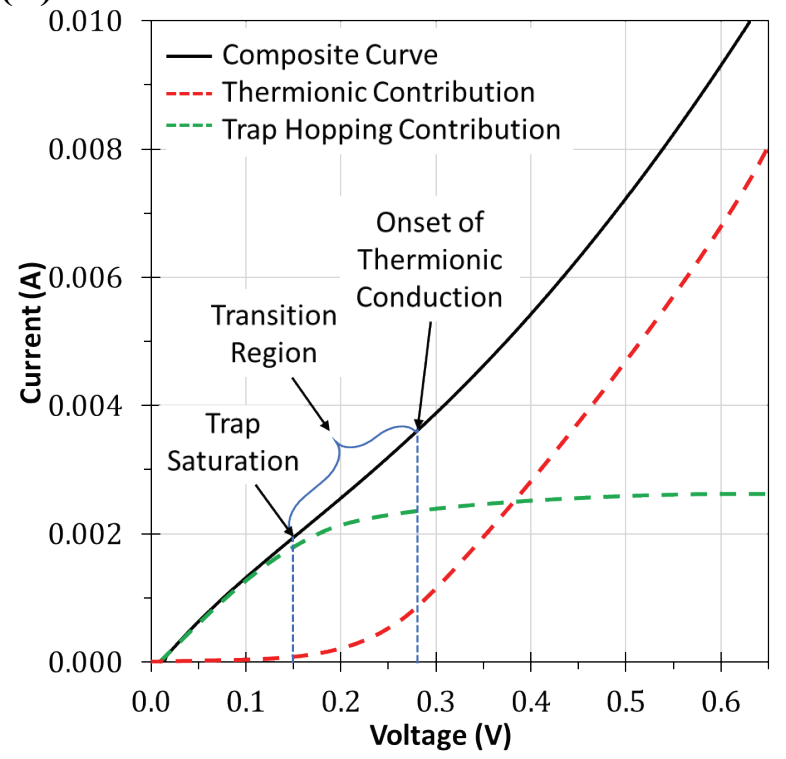

Figure 5.12: (A) IV plot, collected at $205.2^{\circ} \mathrm{C}$, of a device (only a single polarity is shown). There are two distinct regions separated by an inflection point. (B) Proposed contributors to the IV curve; a combination of thermionic conduction and charge trap hopping conduction. The solid black line is empirical data from (A).

Charge traps are energetically discreet, and physically isolated, energy states available within in the depletion region which carriers can occupy. Charge traps are due to physical inhomogeneities (structural irregularities, dangling bonds, etc.) within the physical space of the depletion region. In graphene grown epitaxially from $6 \mathrm{H}-\mathrm{SiC}$, traps have been associated with interactions between the film and substrate. ${ }^{(600)(601)}$ Charge traps are energetic wells in that they represent a local energetic minima for carriers. Because they reside within the depletion region, carriers must tunnel to them. This is driven by quantum effects (specifically, the wave-like nature of electrons in this case) $)^{34}$ and the potential energy gradient between the carrier and trap. ${ }^{(602)(603)}$

\footnotetext{
${ }^{34}$ In quantum mechanics the probabilistic location of a particle (in this case, an electron) is given by its wave function, $\Psi$, described by the Schrödinger equation. The component of the equation which describes the particle in onedimensional position-space as a function of time is qualitatively similar to a sine wave contained within a packet
} 
${ }^{(604)}$ Because the tunneling distance to a trap is shorter than the distance to transit the entire barrier, tunneling to traps occurs more frequently. A quantitative discussion on the kinetics of change trapping is given by Afanas. (605)

If the density of charge traps is high enough within the depletion region, carriers can traverse the barrier by sequentially tunneling (hoping) between traps until they exit the depletion region and enter the conduction band of the film. This is known as charge trap hopping and can contribute to the current flow across the interface. This is a thermally activated process for two reasons. First, carriers must exist at the trap energy level before they can tunnel, which may require thermal promotion if the traps are distributed across a range of energy levels. Stated another way, the electron and trap must be at approximately the same energy level to increase the likelihood of tunneling. Secondly, the probability for a carrier to hop between traps is increased at higher temperatures. Because traps are energetic wells, carriers do not require energy to fall into a trap. However, energy assists in the carrier exiting (tunnel out of) a trap, where it may then fall into the next trap. In this way, higher thermal energy correlates with a higher hopping frequency.

The externally applied bias increases the population of carriers on the current source side of the metal/graphene interface. Due to quantum mechanical effects, a finite fraction of this carrier population will tunnel into a charge trap pathway. Because there is no barrier to overcome, current through the barrier begins immediately (at any $\mathrm{V}>0$ ). Current flow increases linearly with increasing voltage as more carriers accumulate at the interface. The carrier population increases linearly with voltage and thus, the number of carriers in the fraction which tunnel to the trap pathway follows the same trend (linear). This continues until all the trap pathways through the barrier are saturated. At this point, the total current through the barrier levels off. At higher biases, the increased voltage 'tilts' the energy bands such that carriers 'spill over' the barrier (the voltage required for spill over is a function of temperature as determined by thermionic emission). At this

having a Gaussian distribution in amplitude. This is called the 'wave packet' and can move through space. The most statistically probable location of the particle corresponds to the maximum value of the square of the amplitude of $\Psi$ (following the interpretation of Born's Law, discussed later). When next to an energy barrier (forbidden zone), the wave packet wave function will penetrate into the barrier region. The amplitude of $|\psi|^{2}$ within the barrier decays exponentially with distance but if it does not fall to zero, it may exit the other side of the barrier and continue to propagate; albeit with a now reduced amplitude and thus, probability. Therefore, the particle will have a non-zero chance to pass through (i.e. tunnel through) the barrier, which is proportional to the barrier width; particles have a higher probability to tunnel through thin barriers. The tunneling probability is then multiplied to the entire carrier population (which is large) and so, even if the tunneling probability for one particle is low, there are an appreciable number of particles which can tunnel and thus contribute to tunneling current flow. 
point conduction increases rapidly again. The interplay between the roll-off in conduction (due to the trap saturation) and onset of thermionic conduction (due to carriers surmounting the Schottky barrier) determines the overall IV curve. In general, the density of traps determines the roll-off voltage. More traps result in more hopping pathways, which can accommodate more carriers which are made available at higher voltage. The Schottky barrier height determines the voltage required for classic thermionic diode conduction. This is illustrated schematically in Figure 5.12(B). The device temperature strongly influences both mechanisms.

The temperature-dependence interplay between the two mechanisms is evident in Figure 5.13. Figure 5.13(A) plots one quadrant of the IV curve at 54 different temperatures, collected in $\sim 10^{\circ} \mathrm{C} \pm 7^{\circ} \mathrm{C}$ steps. The transition from low conductive Schottky behavior to highly conductive Ohmic behavior is clear. The slope (first derivative) of these curves is plotted in Figure 5.13(B).
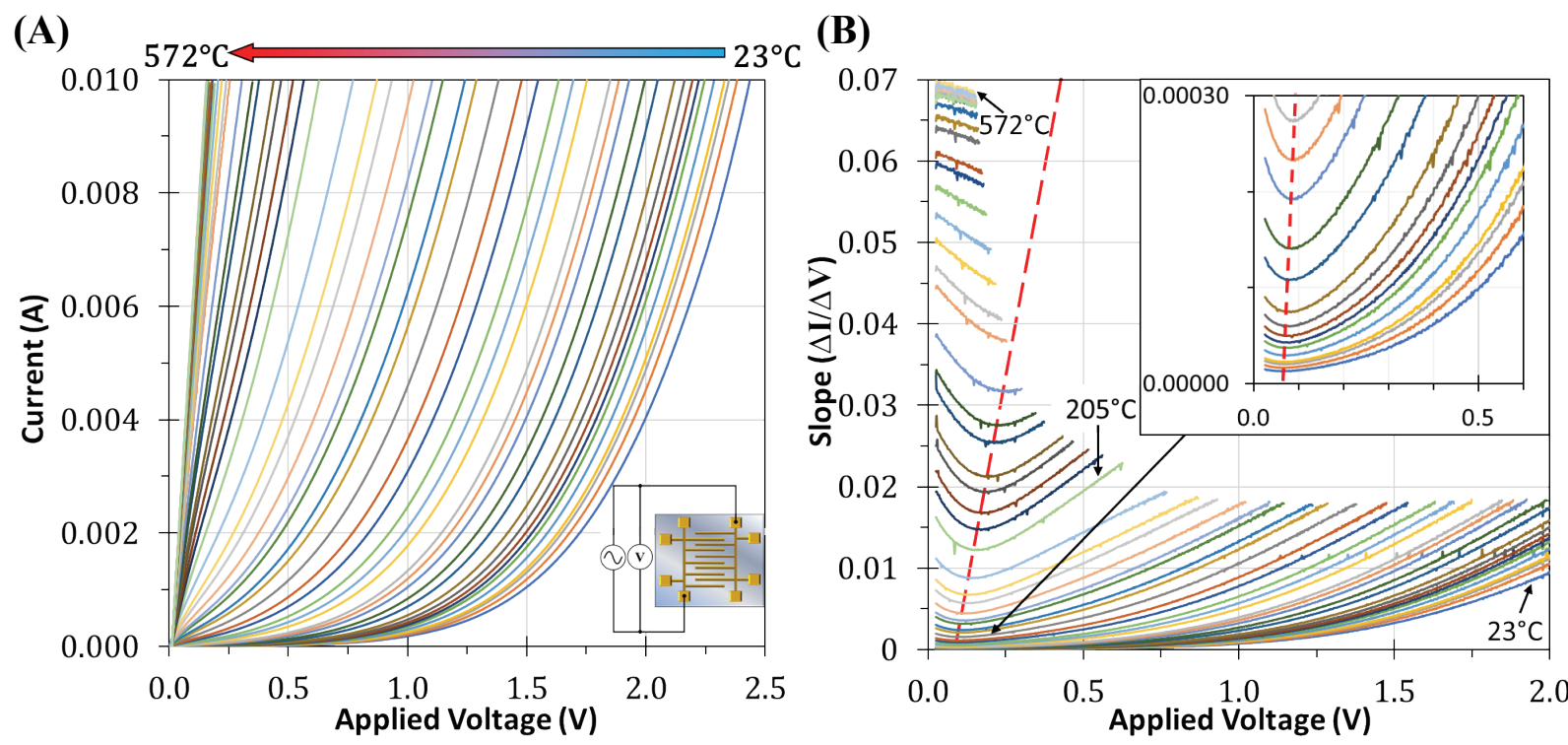

Figure 5.13: (A) IV plot of a device (single polarity) at 54 different temperatures, collected sequentially, in $\sim 10^{\circ} \mathrm{C} \pm 7^{\circ} \mathrm{C}$ increments. (B) Calculated slope of the curves shown in (A). The red line marks the minima of each curve. Inset: zoomed in region of the same plot, as indicated by the arrow. (Note that the curve termination is due to the $10 \mathrm{~mA}$ current limit.)

The dip in the slope curves is indicative of a transition between two regions of the parent IV curve. This corresponds to the transition from the charge trapping roll-off to the onset of the thermionic conduction mechanism. The red dashed line in Figure 5.13(B) maps the locus of points corresponding to the curve minima. The steady shift towards higher voltages shows that charge trap hopping contributes to conduction more at higher temperatures and is therefore a thermally activated process. This is expected if the traps exist across a range of energies, some of which are 
only accessible to high energy carriers. This observation is reflective of the physical reality that an increasingly large number of charge carriers are reaching the high energy traps via the increased thermal energy made available to the carriers at higher temperatures. The dashed line effectively maps this behavior.

Inset within Figure 5.13(B) is a zoomed in region of the same plot, showing the detail of the low temperature $\Delta \mathrm{I} / \Delta \mathrm{V}$ plots. From this, it is evident that a curve minima exists for all temperatures, and there is a small trap hopping current even at low temperatures. The charge hopping effect is reduced at these lower temperatures and the resulting contribution to current flow is small. This reflects the fact that few traps are utilized in conduction through the barrier at these reduced temperatures. This is because few carriers have the energy required to reach the traps (those that do are contained within the high energy tail of the population distribution, which accounts for only a small fraction of the total). An additional contributing factor may be that, although carriers have the energy to enter a trap pathway, they do not have sufficient energy to exit a trap within the pathway. In this case, carriers become literally trapped and do not contribute to conduction and freeze out the occupied (blocked) pathway from other more energetic carriers. Furthermore, the breadth of the Schottky barrier at the lower energy level requires more hops to traverse. Thus, the number that make the trip is small (this is similar to the reason thermionic-field emission occurs at higher temperatures, where the barrier is thinner).

By plotting the trap hopping roll-off voltage at each temperature, insight into the energy distribution of the charge traps can be gained. Figure 5.14(A,B) plots the voltage at which the trap hopping conduction rolls-off at each temperature where the behavior was clearly observable ${ }^{35}$ and the corresponding current at each critical voltage, respectively. Fitted to these data are exponential best-fit lines, which effectively map the saturation of the charge trap hopping pathways at each temperature (assuming that there are no other contributing factors). Because the temperature directly relates to the energy of the carriers, Figure 5.14(A) plots the distribution in energy of the

\footnotetext{
35 The roll-off voltage for temperatures above $\sim 300^{\circ} \mathrm{C}$ corresponded to currents $(>10 \mathrm{~mA})$ which were outside the current limit of the testing equipment and were therefore not recorded.
} 
charge traps, revealing them to be distributed exponentially towards higher energies. The impact of temperature on the frequency of trap hopping, is however a complicating factor. However, work reported by Joung, et al. showed a similar trend for the distribution of charge traps in reduced graphene oxide device (although only five temperatures were tested between $77 \mathrm{~K}-295 \mathrm{~K}$ ). ${ }^{(606)}$
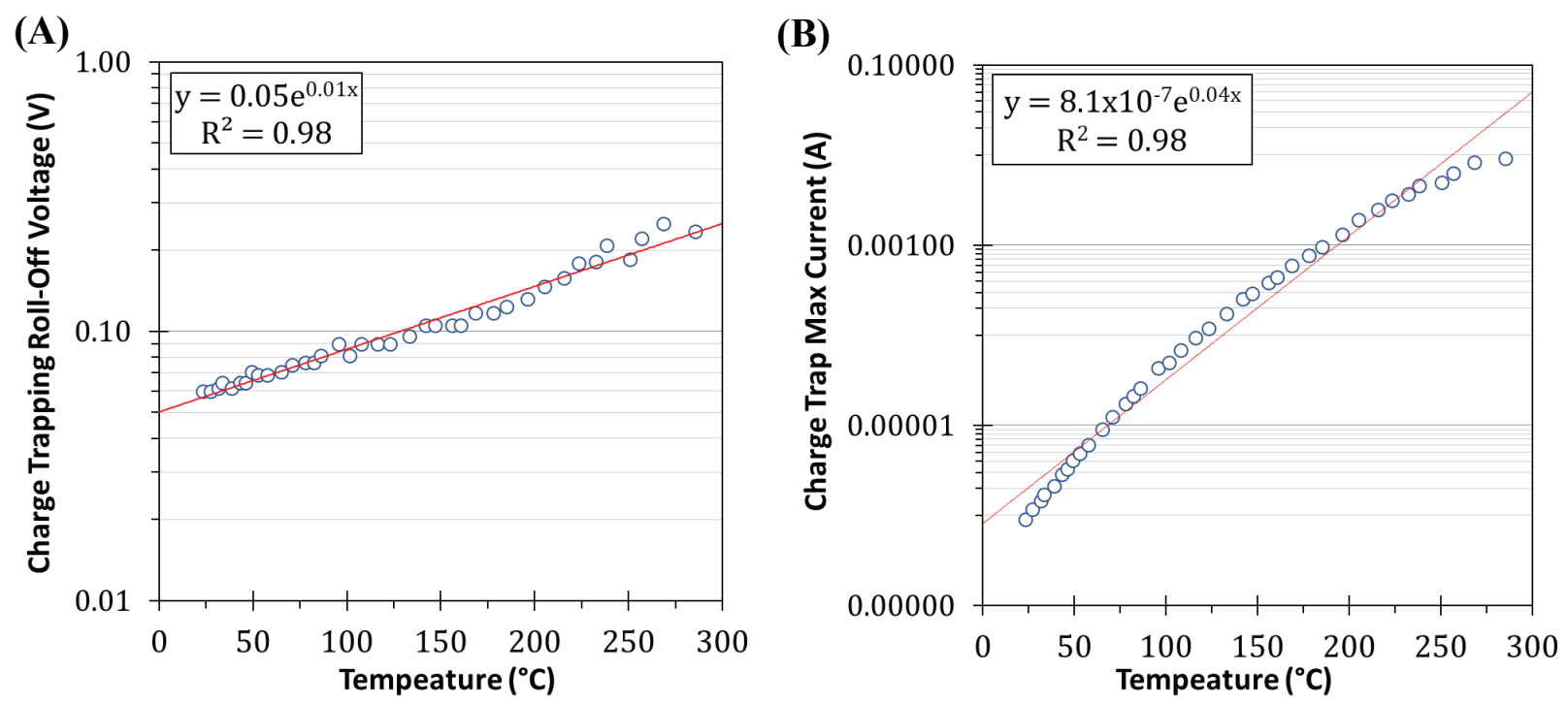

Figure 5.14: (A) Semi- $\log _{10}$ plot of the roll-off voltage (B) and semi-log ${ }_{10}$ plot of the corresponding current. The best-fit lines fit well to an exponential curve, indicating that the charge traps are distributed exponentially across energy levels.

Figure 5.14(B) plots the current at the trap roll-off voltage. This relates to the maximum current flow through the trap pathway, as a function of temperature. This shows that higher temperatures (where more trap pathways are accessible) effectively allows more current flow through the Schottky barrier (shorting it out).

While there are undoubtedly other effects at play, these two effects (charge trap hopping and thermionic emission) appear to be the primary mechanisms responsible for the overall device behavior as a function of temperature. ${ }^{(607)}$ Provided here is only an elementary description of the phenomena at work. Figure 5.15 illustrates the band diagram of the metal/graphene interface and the carrier injection mechanisms discussed. The Fermi level in the metal is assumed to be positioned well into the conduction band (i.e. plenty of electrons populate the metal conduction band) and the be within the band gap of the graphene film (i.e. the film is semiconducting with a small band gap). There have been more complex mechanisms described for current flow through 
the barrier, which may more accurately reflect the physical reality, but they are fundamentally charge trap effects combining with thermionic emission effects. ${ }^{(608)}$

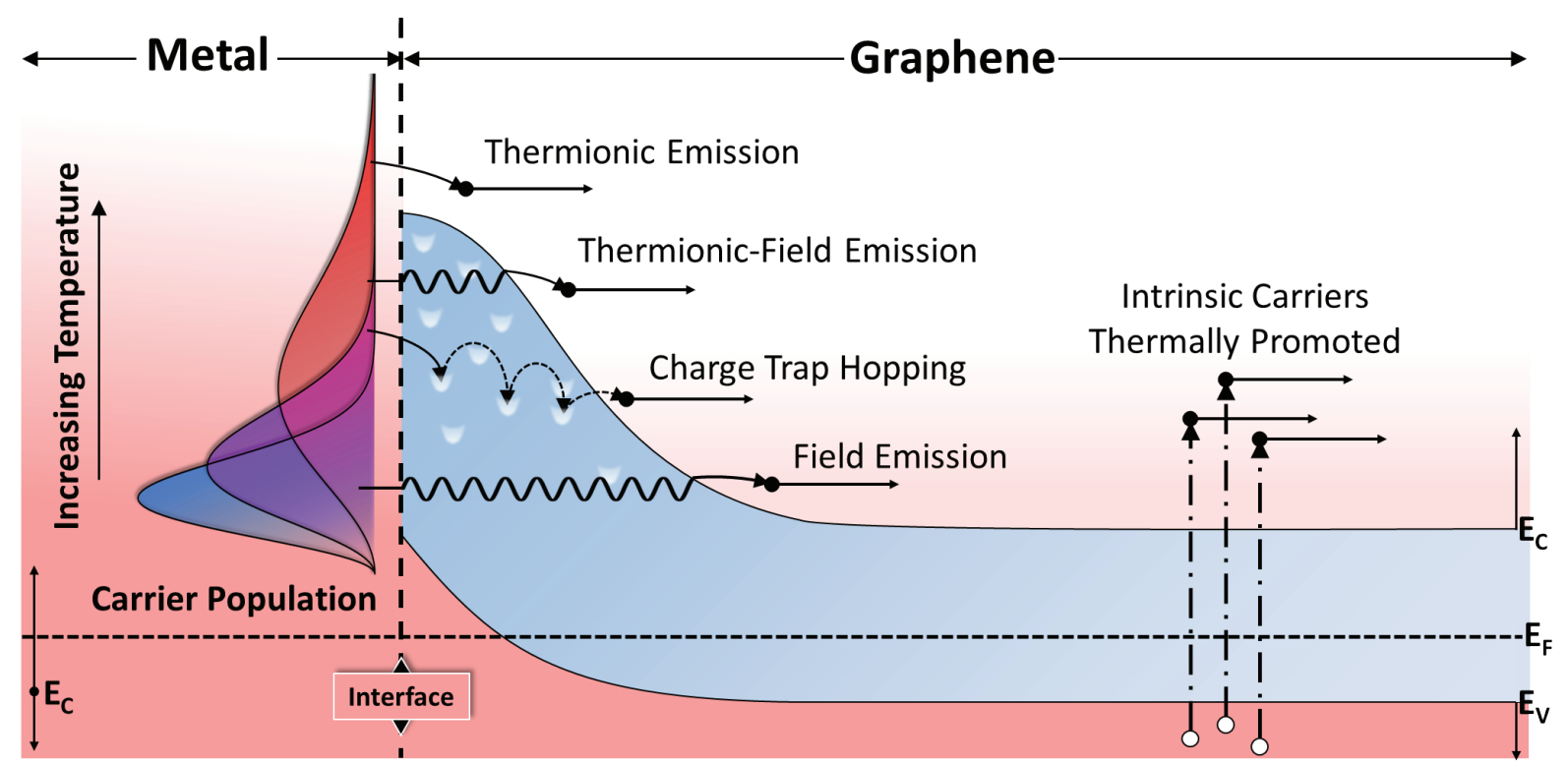

Figure 5.15: Electronic band diagram of the metal/graphene interface, illustrating the various mechanisms by which charge carriers are injected into the film.

A further observation may be noted in regard to the device behavior as a function of temperature. Close inspection of the data reveal that IV curves collected at very high temperatures display a non-zero axis crossing (there is current flow at $0 \mathrm{~V}$ ). This behavior is observed in this device when operated above $300^{\circ} \mathrm{C}$, as shown in Figure 5.16. Although there are few literature reports on solid state devices operating in this extreme temperature range, this phenomenon is most likely what has been termed a splash current ${ }^{36}$, which has been used in the context of hot-filament vacuum tube operation (which also utilize thermionic emission in their operation). ${ }^{(609)(610)}$ In those cases, hot electrons are thermionically emitted across a physical gap between electrodes which results in a small current flow, even under a $0 \mathrm{~V}$ bias condition. In the solid-state devices studied here, a similar process is likely at play. In this case, at very high temperature, charge carriers spontaneously surmount the Schottky barrier and fall into the lower energy states available in the metal contacts, which are electrically connected to the source measurement units (SMUs). This leads to a small trickle current in one direction at a $0 \mathrm{~V}$ externally applied bias (the energy responsible for this current is due to the external heating of the device and make up electrons are

\footnotetext{
${ }^{36}$ Splash Current; this is an antiquated term for the phenomena, but there is no contemporary term for this specific effect (other than generically, thermionic emission). It is not to be confused with splash electrons which is another outdated term in use around the same period (which are now commonly known as secondary electrons) and is unrelated. (754)
} 
supplied by the SMUs). This process likely occurs at both contacts, but due to a slight difference in barrier height, the contact with the lower barrier height dictates the direction of the overall thermally induced current flow.

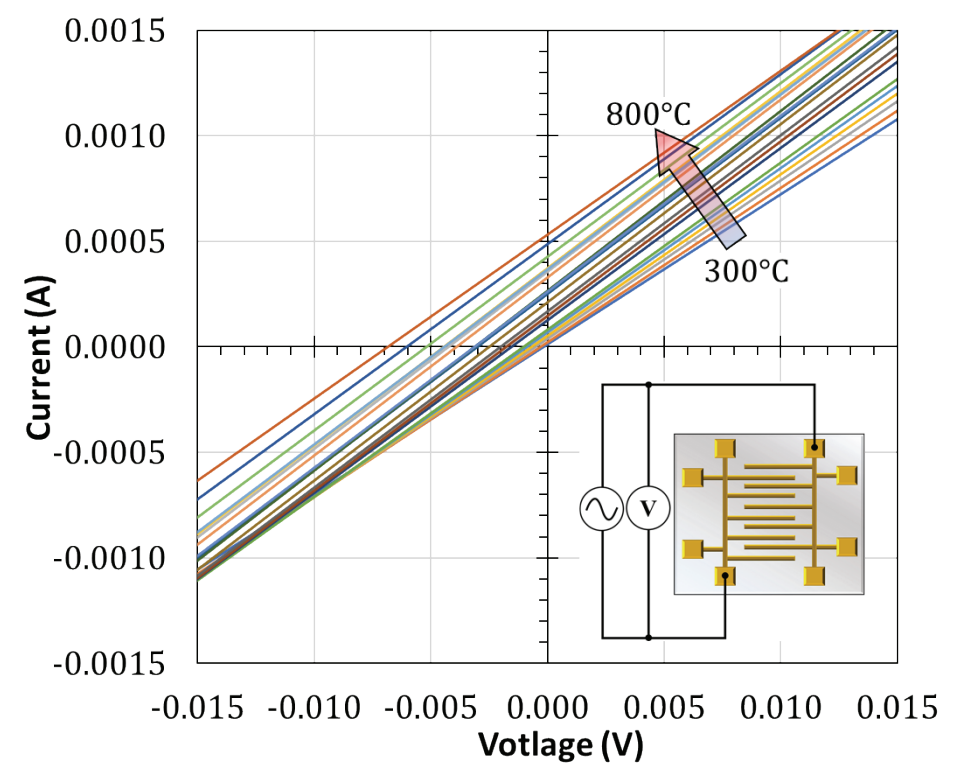

Figure 5.16: A non-zero crossing of the IV curves is observed for devices operating at very high temperatures. This is not seen at low temperatures.

An alternative explanation to splash current is the thermoelectric or photoelectric effects, both of which have been observed in graphene. ${ }^{(611)(612)(613)}$ However, there is no temperature gradient present, which is a necessity of the thermoelectric effect (the entire device is in approximant thermal equilibrium and the self-heating of the device is not sufficient to generate the large thermal gradient needed to drive the effect). Therefore, the thermoelectric effect it is not likely responsible for the results seen here. Likewise, the photoelectric effect is not the culprit because the device is shielded from all light sources. The device shown in Figure 5.16 was mounted in the small test cell and is thus not exposed to ambient light or the IR light from the heating elements (as would be the case in the large test bed). Therefore, a splash current is the most likely explanation and fits within the current understanding of the device operation.

Finally, to provide a more quantitative (and practical) view of the device resistance as a function of temperature Figure 5.17(A) plots the calculated two-point resistance of the device under a $10 \mathrm{~mA}$ current flow (corresponding to the lowest resistance calculated at each temperature for which data was available) and Figure 5.17(B) plots the same parameter calculated at $1 \mathrm{~mA}$ current flow (similar data, collected at a constant voltage and at a higher sampling rate, is provided in the course of the decision in Section $\$$ 5.3.4). Here, the broad trends previously discussed are 
apparent. The rapid reduction in resistance up to $\sim 200^{\circ} \mathrm{C}$ is seen at bout current levels. The suppression of current flow at (relatively) low temperatures is consistent with the thermionic emission model of current injection as described by Wu, et al. for graphene devices. ${ }^{(522)}$ Note that the largest temperature sensitivity is observed in the low current (and thus low voltage) regime. This behavior, combined with the fact that the effect of self-heating is most pronounced at high current levels, act synergistically and result in a device which is highly temperature sensitive across all of the operational parameter space. The specifics of the device self-heating effects are discussed in Section $\$ 5.3 .1$.

(A)

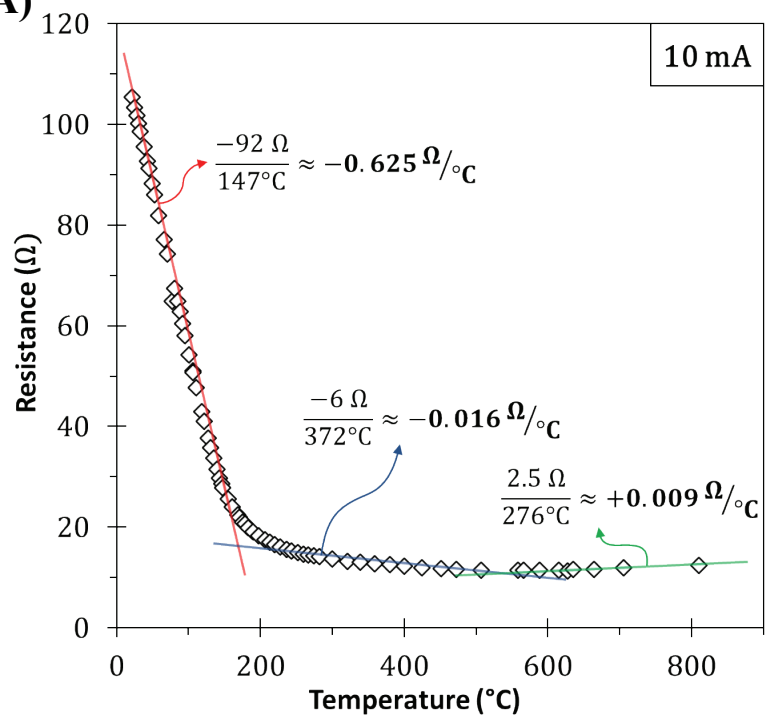

(B)

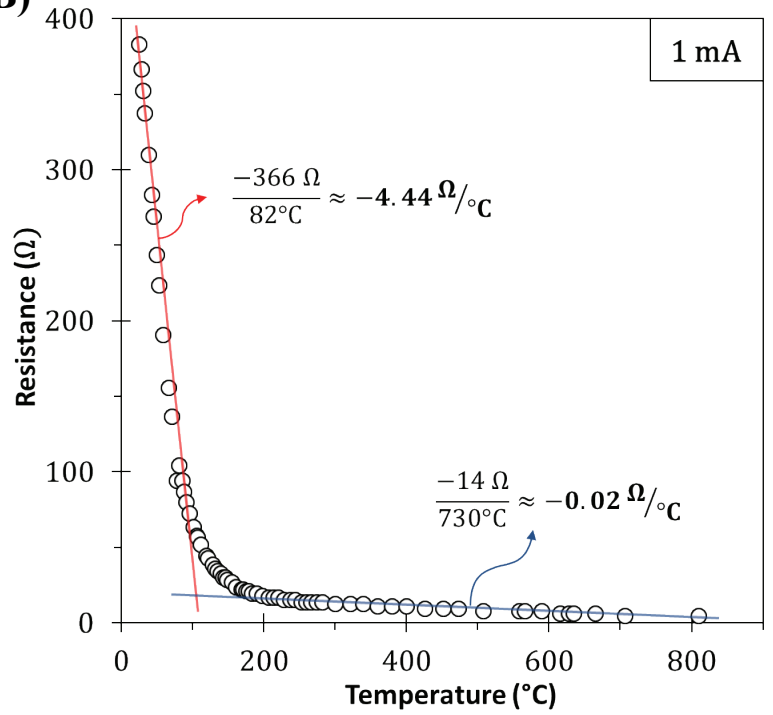

Figure 5.17: (A) Two-point resistance of a device from $23^{\circ} \mathrm{C}$ to $800^{\circ} \mathrm{C}$ at $10 \mathrm{~mA}$ and (B) at $1 \mathrm{~mA}$ across the same temperature range.

This section concludes with a brief discussion of the temporary hysteresis observed in the device IV curves after thermal cycling. Figure 5.18 shows the room temperature IV plot of a device before (blue $\circ$ ) and after (red $\diamond$ ) a gentle thermal cycle up to $700^{\circ} \mathrm{C}$ under a flow of argon over 4hrs (small test cell, sensor pattern, MK0 package design). Clearly, the thermal cycle improves the conductivity of the device across all voltages. The largest improvement in conductivity was observed after an initial soft bake (slow ramp to $200^{\circ} \mathrm{C}$, over $2 \mathrm{hrs}$ ). After a hard bake (ramp to $700^{\circ} \mathrm{C}$, over $4 \mathrm{hrs}$ ), the room temperature device conductivity fully stabilized and showed little or no change after additional thermal cycles. For example, after the hard bake the device from Figure 5.18 was backed again at $600^{\circ} \mathrm{C}(120 \mathrm{~min}$ dwell time $)$, and then again at $800^{\circ} \mathrm{C}(200 \mathrm{~min}$ dwell time). The room temperature IV showed little change after either case. 


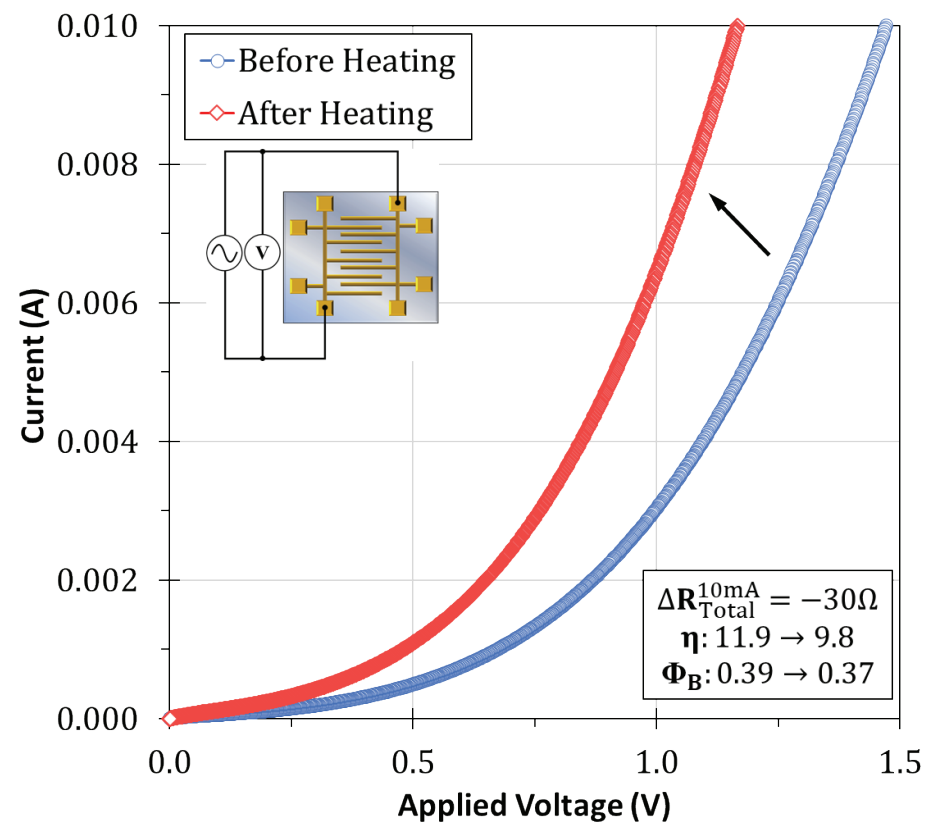

Figure 5.18: Room temperature IV curves before and after a thermal cycle up to $700^{\circ} \mathrm{C}$ under argon over $4 \mathrm{hrs}$. Illustrated here is the improved conductivity via the contact bake-in or curing effect. After the initial bake-in, device IVs are essentially unchanging with further thermal cycles.

The behavior observed here is most likely due to a 'bake-in' or contact curing effect of the $\mathrm{Ti} / \mathrm{Au}$ contacts (as opposed to an evolution in the properties of the film). This effect has been observed in graphene devices before. ${ }^{(614)}$ For example, Smith, et al. observed a $27 \%$ reduction in resistance after an anneal at $350^{\circ} \mathrm{C}$, Balci, et al. saw a reduction after a $10 \mathrm{x}$ thermal cycle to $300^{\circ} \mathrm{C}$ totaling $60 \mathrm{~min}$ and Robenson, et al. saw a significant resistance reduction after a 60 second bake at $400^{\circ} \mathrm{C}$. ${ }^{(590)(615)(580)}$ Considering these reports, the increase in device conductivity observed here is not surprising.

The improved conductivity observed here could be due to several simultaneous effects. For instance, contact baking has been shown to reduce the contact resistance $\left(\mathrm{Rc}_{\mathrm{c}}\right)$ contribution to the total circuit resistance. ${ }^{(615)}$ Although not directly measured here (this would require the application of the four-point method), a reduction in the $\mathrm{R}_{\mathrm{C}}$ which would lead to a decrease in the measured two-point resistance. (580) (590) (615) Application of the Richardson-Dushman analysis (detailed in Section $\$ 3.2 .5)$ analysis shows a lowering of the Schottky barrier height $\left(\Phi_{\mathrm{B}}\right)$ from $0.39 \mathrm{eV}$ to 0.37 $\mathrm{eV}$. This effect has been attributed to a 'blurring' of the physical interface between the metal contact and the graphene film. To this point, Leong, et al. observed a marked decrease in contact resistance after annealing at only $300^{\circ} \mathrm{C}$ for $2 \mathrm{hrs} .{ }^{(614)}$ They identify the effect as a result of a slight dissolution of carbon into the metal contact and chemisorption of metal to the dangling bonds of 
the carbon film. Such a scenario would explain the decrease in both the contact resistance and the reduction in barrier height. Furthermore, analysis of the data shown in Figure 5.18 reveal the ideality factor $(\eta)$ of the device decreases from 11.9 to 9.8 after heating. This could be explained by an increase in the uniformity of the contact/film interface from the bake-in process, thereby decreasing the degree of interfacial inhomogeneities and leading to better conductivity between the contact and film. (616) (569) (570) In their study of the effects of rapid thermal annealing of graphene-metal contacts, Osman, et al. conclude that fundamental effects (i.e. barrier reduction, doping, etc.) combined with process related effects (i.e. the quality of the metal graphene interface, which effects $\mathrm{R}_{\mathrm{C}}$ ) both lead to the improvement in conductivity.

In summary of the annealing effects, the device conductivity is markedly improved after the initial low temperature bake under inert gas. After a higher temperature bake-in, this improvement is locked in and maintained, even after additional thermal cycles. This is typical of all devices fabricated in this work. An important caveat to this is for devices exposed to hydrogen gas at very high temperatures. This, however, is most likely not related to contact baking, but rather film/substrate effects. This is discussed in Section $\$$ 5.3.6.

\section{§5.2.2 Richardson-Dushman Analysis}

This section describes the results of applying the modified Richardson-Dushman analysis (detailed in Section $\$ 5.1 .3$ ) to the two-point IV data collected as a function of temperature from an SOP device. These analyses give considerable insight into the film behavior. Importantly, these analyses reveal how the Schottky barrier $\left(\Phi_{\mathrm{B}}\right)$, mobility $(\mu)$ and carrier concentration $\left(\mathrm{N}_{\mathrm{D}}\right)$ change as a function of temperature. However, because the temperature data is collected using two-point measurements, some film parameters must be estimated. The room temperature film resistance was taken to be the same as the identically produced sample analyzed in Figure 5.8. The contact length was modified such that the $\Phi_{\mathrm{B}}$ calculated at room temperature was the same as that found for the identical sample analyzed in Figure 5.8. This compensates for the fact that the device studied here utilized the interdigitated sensor design of the electrical contacts and assumes all other device parameters are identical. Finally, for the calculation of mobility $(\mu)$, the change in the resistance of the film was assumed to be proportional to the overall change in the resistance of the device. Although not entirely accurate, these assumptions provide the best estimates of the unknown parameters. Because of these estimations, the absolute values calculated for $\Phi_{B}, N_{D}$ and 
$\mu$ should be taken as only approximations. The more important observation here are the overall trends.

Figure 5.19 plots the IV curves and corresponding Richardson-Dushman plots of a device heated from $23^{\circ} \mathrm{C}$ up to $810^{\circ} \mathrm{C}$. The turnabout in the trends indicated by the arrows corresponds to the reduction in device conductivity observed above $\sim 550^{\circ} \mathrm{C}$, which was described in the previous section (Section $\$ 5.2 .1)$.
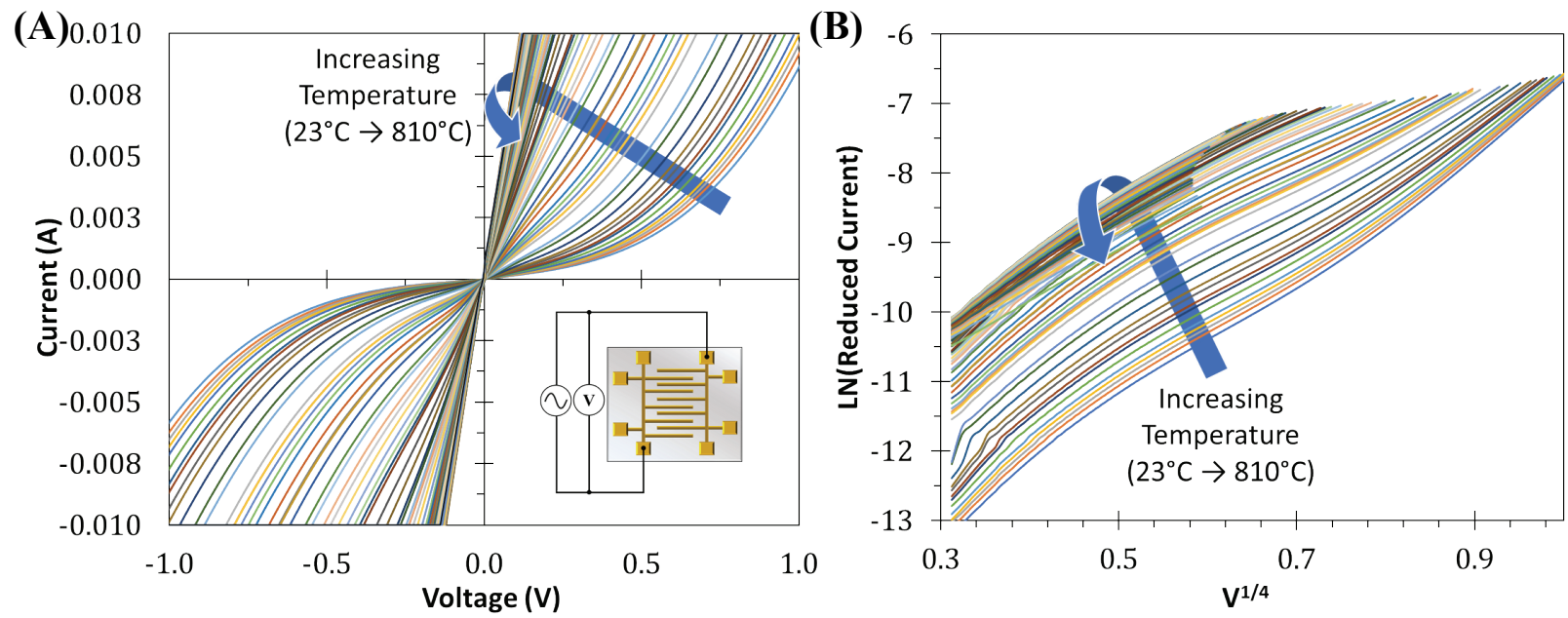

Figure 5.19: (A) IV as $f(\mathrm{~T})$ plot and (B) corresponding Richardson-Dushman plots. The arrows indicate in what direction the curves increase in temperature.

The parameters extracted from Figure 5.19 are plotted as a function of temperature in Figure 5.20. The confidence in the value of each point is related to the quality of the linear fit applied to the curves in Figure 5.20(B), (and the corresponding plots for the determination of $\eta$, which are not shown) from which the data are calculated. Above the graphs shown in Figure 5.20 are the corresponding goodness-of-fit values, $\mathrm{R}^{2}$ (the square of the Pearson correlation coefficient). It is interesting to note that the worst fits correlate to the abrupt change in the $\Phi_{B}, N_{D}, \mu$ and $\eta$ trends. This may indicate a crossover point between the various competing fundamental effects which, together, are responsible for the overall trends and a subtle breakdown in the models. 
(A)

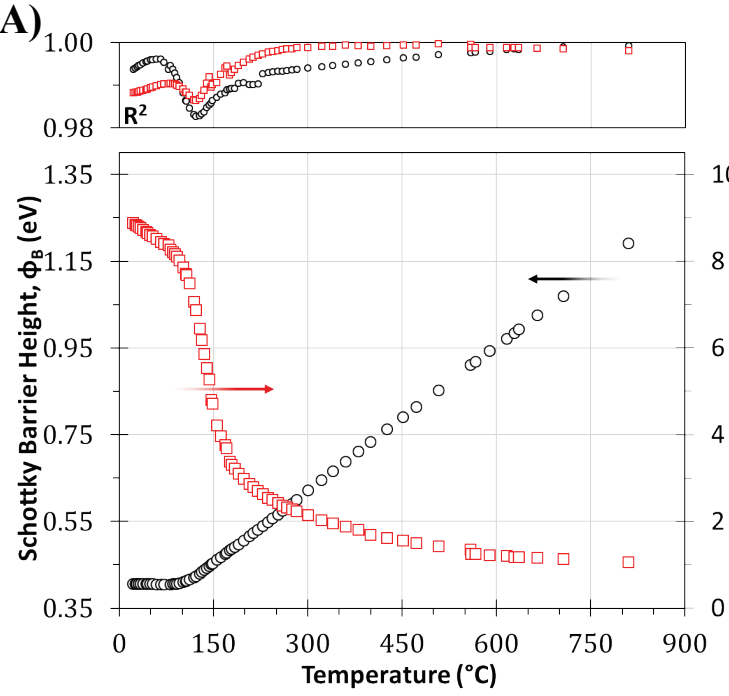

(C)

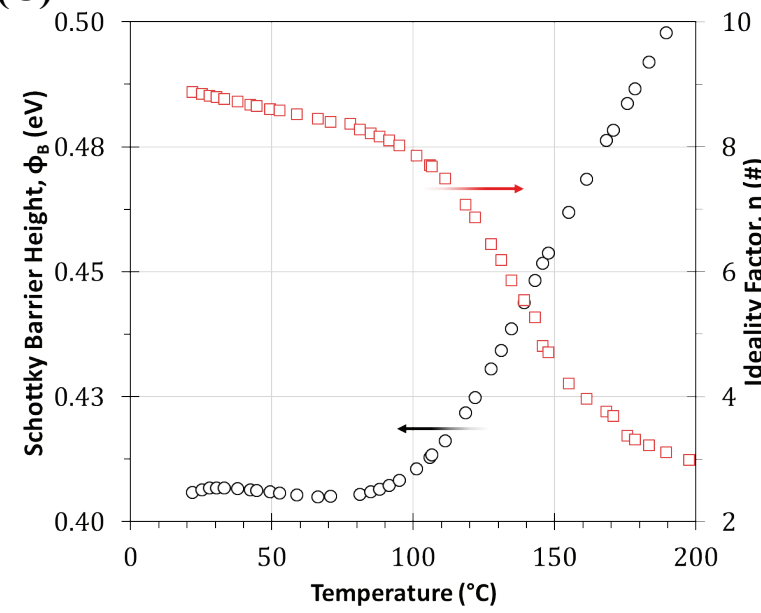

(B)

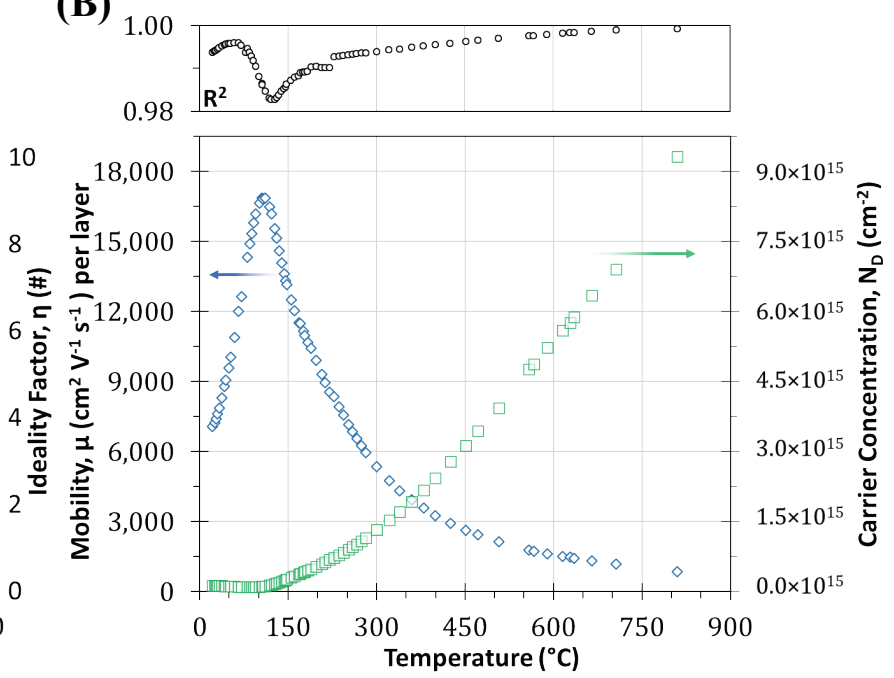

(D)

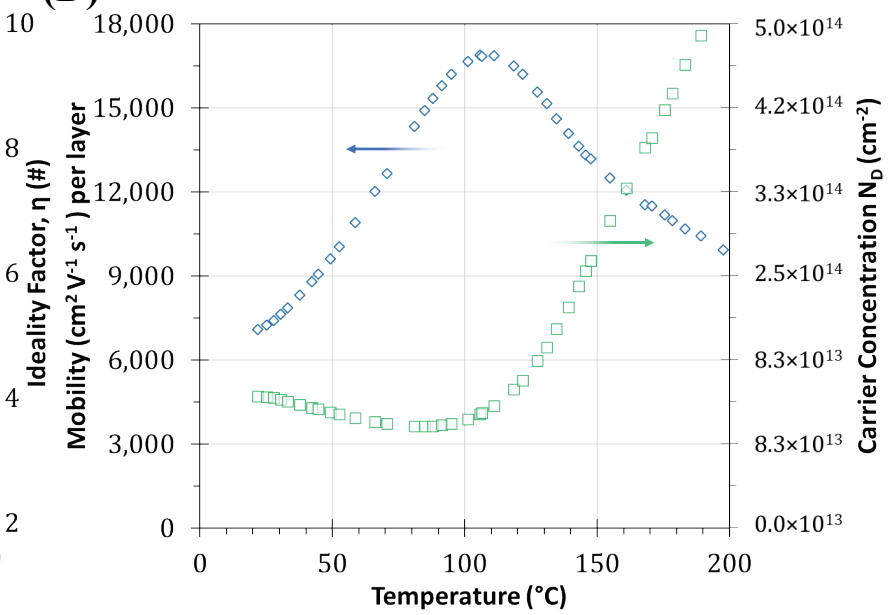

Figure 5.20: (A)The estimated Schottky barrier height $\left(\Phi_{\mathrm{B}}\right)$ and ideality factor $(\eta)$ as a function of temperature. (B) The mobility $(\mu)$ and carrier concentration $\left(N_{D}\right)$. The confidence of each point is related to the $\mathrm{R}^{2}$ plot above each graph and corresponds to the linear fit of the data from which the data are calculated; ( $(\circ)$ for $\Phi_{B}, \mu, N_{D}$ and ( $\square$ ) for $\eta$. (C) and (D) show a more detailed view of the results between $23^{\circ} \mathrm{C}-200^{\circ} \mathrm{C}$ of (A) and (B), respectively.

Figure 5.20(A,C) shows that, above $\sim 100^{\circ} \mathrm{C}$, the Schottky barrier $\left(\Phi_{\mathrm{B}}\right)$ increases linearly from $0.40 \mathrm{eV}$ at $23^{\circ} \mathrm{C}$ to $\sim 1.20 \mathrm{eV}$ at $810^{\circ} \mathrm{C}$. The ideality factor $(\eta)$ displays a slightly more complex behavior but ultimately decreases monotonically with increasing temperature. Although not tested in the same temperature range and using a different device design, similar trends have been observed in both graphene devices and non-graphene devices. ${ }^{(617)(608)(618)}$

One of the more thorough investigations on the apparent temperature dependence of $\Phi_{\mathrm{B}}$ and $\eta$ has been provided by Sullivan, et al. ${ }^{(619)}$ Although his investigations did not involve graphene devices, the general physics governing Schottky device operation are taken to be similar. The trends of $\Phi_{\mathrm{B}}$ as a function of temperature $(f(\mathrm{~T}))$ and $\eta$ as $f(\mathrm{~T})$ have both been attributed, by 
he and others, to barrier height inhomogeneities. (619) (569) (617) (620) Specifically, that $\Phi_{\mathrm{B}}$ varies between discreet points over interface area (i.e. local spatial variations in the Schottky interface). Consequently, the change in the extracted $\Phi_{\mathrm{B}}$ as $f(\mathrm{~T})$ is due to the interface regions with a high $\Phi_{\mathrm{B}}$ becoming activated (thermionically) at higher temperatures. The $\Phi_{\mathrm{B}}$ height calculations extract the parameters associated with the average $\Phi_{\mathrm{B}}$ height of the active regions of the interface. Thus, counter to the observed trend, the $\Phi_{\mathrm{B}}$ does not actually change with temperature. Merely, the apparent rise in $\Phi_{\mathrm{B}}$ is reflective of the thermally induced activation of interfacial regions with high $\Phi_{\mathrm{B}}$, which occurs at high temperatures. Based on this interpretation of the results shown in Figure $5.20(A, C)$, there are many high $\Phi_{\mathrm{B}}$ interfacial regions which are only activated above $\sim 100^{\circ} \mathrm{C}$.

Similar to $\Phi_{\mathrm{B}}, \eta$ should theoretically be independent of temperature. ${ }^{(619)}$ However, as observed here and elsewhere, the value of $\eta$ shows a strong temperature dependence, which has also been explained by inhomogeneities of the $\Phi_{\mathrm{B}}$ interface. Specifically, the origins of this temperature dependency have been ascribed to complex interactions between interfacial regions with different $\Phi_{\mathrm{B}}$. These interactions include so-called pinch-off effects of low $\Phi_{\mathrm{B}}$ regions surrounded by high $\Phi_{\mathrm{B}}$ regions (which results in saddle points in the voltage contour lines across the interface), voltage dependent $\Phi_{\mathrm{B}}$ heights and other intra-region effects. ${ }^{(619)(620)}$ However, as stated by Parui, et al. , other than this generic mechanism related to inhomogeneous at the interface, there has yet to be a clear explanation given for this behavior. ${ }^{(617)}$

Figure $5.20(B, D)$ plots the carrier mobility $(\mu)$ as a function of temperature. Maximum mobility is seen around $\sim 100^{\circ} \mathrm{C}$, above which an exponential decay is observed. The carrier concentration $\left(\mathrm{N}_{\mathrm{D}}\right)$ decreases slightly up to $\sim 100^{\circ} \mathrm{C}$, above which a linear increase is observed up to $810^{\circ} \mathrm{C}$. The observed increase in $\mathrm{N}_{\mathrm{D}}$ as $f(\mathrm{~T})$ is not surprising given the thermionic nature of the device and the presence of intrinsic carriers thermally promoted into the conduction band of the film. The reason for the initial decrease of $\mathrm{N}_{D}$ between $23-75^{\circ} \mathrm{C}$ is however, unclear.

The general behavior of $\mu$ as $f(\mathrm{~T})$ (notably the peak in $\mu$, as seen in Figure 5.20(D)), which has been observed in other devices, is commonly explained by a crossover between two dominant scattering mechanisms. (621) (622) (623) Specifically, the ionized impurity scattering, which is known to dominate at low temperatures, with thermal phonon (coherent quantized oscillations in the atoms which make up the lattice) induced scattering, which dominates at high temperatures. ${ }^{(624)}$ The ionized impurity scattering decreases with temperature because, at high temperature, carriers 
have higher velocities and spend less time under the influence of the Coulomb forces surrounding the ionized impurities and thus, their effect on scattering is reduced. The number of thermally produced phonons, on the other hand, scale monotonically with increasing temperature. The peak in the $\mu$ as $f(T)$ curve is explained by the crossover point in these two dominant scattering effects. In this case, ionized impurity scattering sites may be due to oxygen defects in the film (as observed in the XPS analysis detailed in Section \$4.1.2) or to covalent interactions with the substrate (which may perturb the local electron cloud distribution due to a rehybridization from $\mathrm{sp}^{2}$ to $\mathrm{sp}^{3}$ ).

It is routinely stated that ionized impurity scattering and phonon scattering scale proportionally to $(\mathrm{T})^{3 / 2}$ and to $(\mathrm{T})^{-3 / 2}$, respectively. ${ }^{(621)}$ Although the $\mu$ as $f(\mathrm{~T})$ data presented here follow these trends generically, there are subtle complexities in the curve which are not fully accounted for by the composite of these two proportionalities. To further elucidate on the specific behavior of $\mu$ as $f(\mathrm{~T})$, it is interesting to note that the mobility data shown here (Figure 5.20(D)) match remarkably well with Hall mobility data collected by Stillman et al. on n-GaAs films, although the temperature range covered by Stillman, et al. ( $77 \mathrm{~K}$ to $300 \mathrm{~K}$ ) was much smaller than what is covered here $\left(23^{\circ} \mathrm{C}\right.$ to $\left.810^{\circ} \mathrm{C}\right) .{ }^{(625)}$ Through numerical studies, Fletcher, et al. applied a convolution of five types of scattering mechanisms to Sillman, et al. results, which fit Fletcher, et al. data quite well (within 10\%). ${ }^{(626)}$ The five mechanisms considered were, neutral impurity scattering (non-Coulomb defect scattering), ionized impurity scattering (previously discussed), piezoelectric scattering (due to noncentrosymmetric crystals structure deformation; in this case, most likely due to local interactions with the substrate which perturb the symmetry of the graphene structure or with regions around oxygen defect sites which perturb both crystal symmetry and disrupts the local charge distribution), deformation potential scattering (a type of phonon scattering, previously discussed) and polar scattering (a type of phonon scattering, previously discussed). ${ }^{(621)(626)}$ Considering the similarities in Stillman, et al. data with the data reported here, it is reasonable to conclude similar scattering mechanisms (described by Fletcher, et al.) are at play here. To illustrate this, Fletcher, et al. scattering mechanisms are compared qualitatively to those produced here, as shown in Figure 5.21(A). For comparison, a figure from Fletcher, et al. has been 
adapted and is shown in Figure 5.21(B). The similarities in the two data sets (from this work and Sillman, et al. data) are apparent.
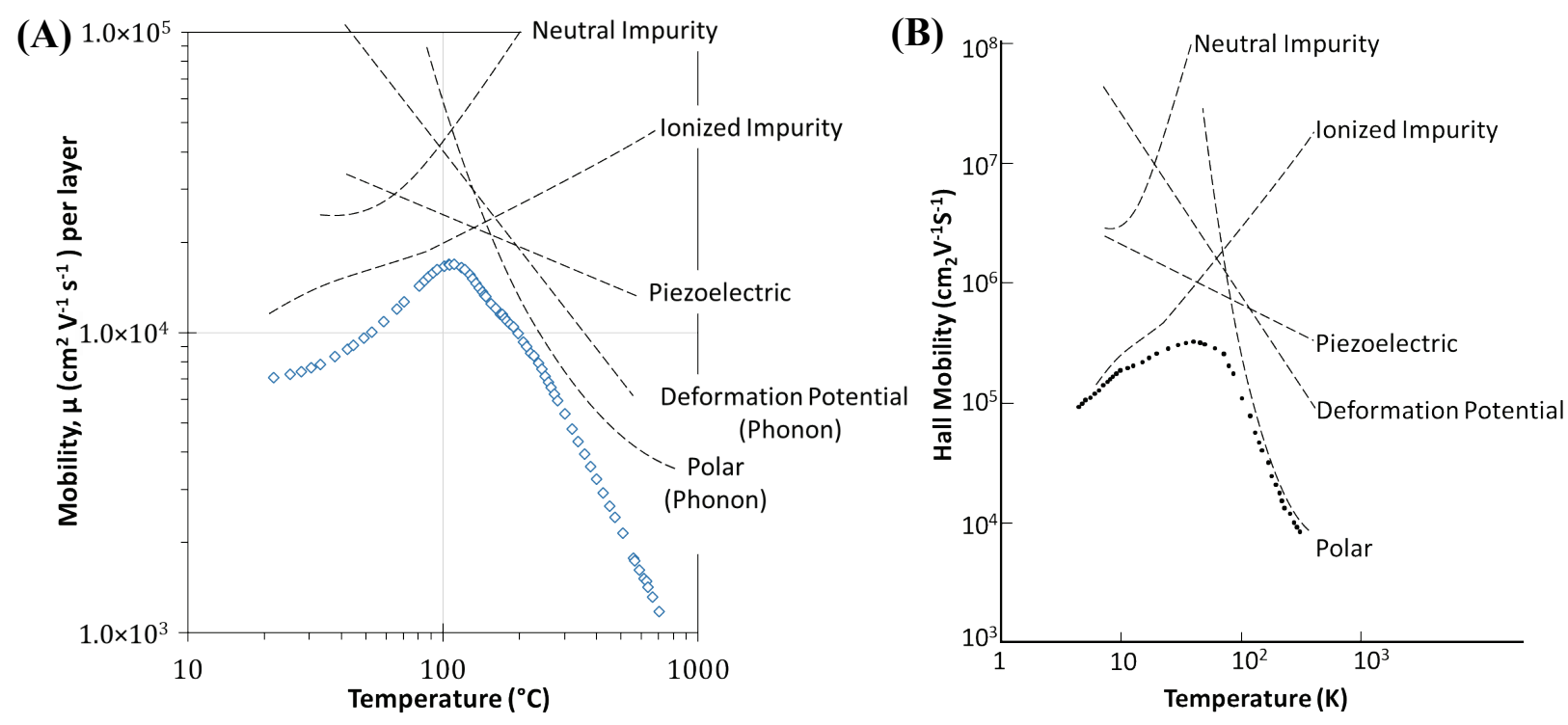

Figure 5.21: (A) The data from Figure 5.19(D) plotted in $\log _{10}-\log _{10}$. The dashed lines are the contributions of various scattering mechanisms as proposed by Sillman, et al. For comparison, (B) the data from Sillman, et al. (data points) with the scattering mechanisms (dashed lines) described by Fletcher, et al. This figure has been adapted from Fletcher, et al.(626)

\section{§5.2.3 Non-Standard Samples}

This section concludes with a brief discussion of the temperature behavior of two types of non-SOP samples. They are the samples produced by carrying out the SOP process on the $4 \mathrm{H}-\mathrm{SiC}$ and $4 \mathrm{H}-\mathrm{SiC}$ semi-insulating([SI]) polytype substrates. Both show a significant departure from any behavior observed for devices using the $6 \mathrm{H}-\mathrm{SiC}$ polytype.

Two-point IV curves, collected as a function of temperature, from the $4 \mathrm{H}-\mathrm{SiC}[\mathrm{SI}] /$ graphene variant are shown in Figure 5.22. Clearly, the 4H-SiC[SI]/graphene composite is significantly less conductive than the $6 \mathrm{H}-\mathrm{SiC} /$ graphene or $4 \mathrm{H}-\mathrm{SiC} /$ graphene counterpart. Further, the $4 \mathrm{H}-$ $\mathrm{SiC}[\mathrm{SI}] /$ graphene device shows strictly Ohmic behavior across all temperatures tested. These results, of both $4 \mathrm{H}-\mathrm{SiC}[\mathrm{SI}] /$ graphene and $4 \mathrm{H}-\mathrm{SiC} /$ graphene (discussed shortly), are particularly unexpected considering the similarities in both the XPS and Raman spectra to the SOP $6 \mathrm{H}-$ $\mathrm{SiC} /$ graphene composites. Fundamentally, this suggests there are subtle (but important) aspects of the $\mathrm{SiC} /$ graphene composites which are not detectable by the material characterization techniques at our immediate disposal. 


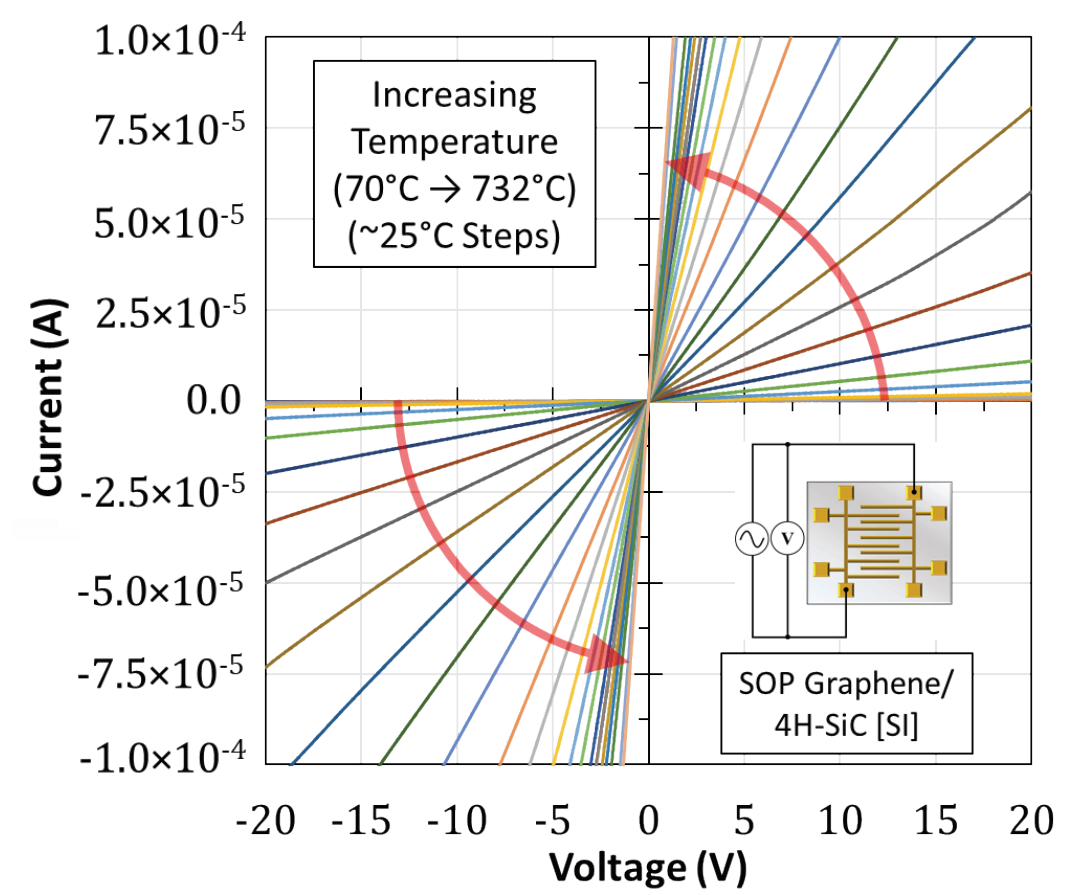

Figure 5.22: Two-point IV as $f(\mathrm{~T})$ plots collected from a $4 \mathrm{H}-\mathrm{SiC}[\mathrm{SI}] /$ graphene composite sample produced using the SOP graphenization process described in Chapter 4. Arrows indicate the direction of increasing temperature.

Although further study is required to determine the precise reason for these unexpected behaviors, two explanations for the results shown in Figure 5.22 are presented here. The low conductivity may be reflective of the fact that there is little or no participation of the substrate in conduction. Because the substrate is semi-insulating and thus cannot participate in conduction, the overall device is less conductive. However, this would then indicate that the substrate of the $6 \mathrm{H}-$ $\mathrm{SiC} /$ graphene devices contributes to conduction to a much greater extent than previously thought, based on the IV measurements. This will require further investigation to determine.

An alternant explanation involves a discussion of the $\mathrm{CF}_{4}$ etching process. Recall that the key aspect of the $\mathrm{CF}_{4}$ etch is the selectivity of the $\mathrm{CF}_{\mathrm{x}}$ ion towards silicon over carbon. Also recall (as discussed in Section \$3.1.1) that to produce the semi-insulating 4H-SiC substrates, the use of either counter-dopants (like vanadium) or intentional growth of vacancy states are employed. Both types of semi-insulating $\mathrm{SiC}$ are known to contain contaminating elements such as boron, nitrogen and aluminum, among others. ${ }^{(440)(441)}$ It is unknown what the selectivity of the etch is in this specific scenario between $\mathrm{C}, \mathrm{Si}$ and the contaminants/counter-dopants. If the selectivity is low, then the 12-minute ICP/RIE process will effectively concentrate these additives, which remain over from the volume of material removed during the etch $(\sim 2.8 \mu \mathrm{m})$, into the surface film. These foreign materials may then participate in the reorganization of the carbon film during the AP-RTA. 
While the contamination/counter-dopants concentration is low in the bulk $\mathrm{SiC}$, the effective concentration of these foreign materials into the final film may have a large impact on the electrical properties (even though the level is below the practical detection limit of XPS). This is a situation unique to the novel plasma assisted method for graphene growth employed in this work. In this method, over 11,100 Si-C bilayers are ultimately affected by the process (in the 12minute SOP etch). This is in stark contrast to the selective sublimation method, in which only the first few terminal layers of the $\mathrm{SiC}$ crystal are affected. Given the relative atomic densities of $\mathrm{SiC}$ and graphene, three Si-C bilayers contain enough carbon atoms to form one graphene layer. And although the selectivity of $\mathrm{Si}$ over $\mathrm{C}$ of the $\mathrm{CF}_{4}$ is not infinite (i.e. in practice, more than three $\mathrm{SiC}$ bilayers are needed to produce one graphene layer), the SOP etch of 11,100 layers is more than sufficient to produce a $\sim 3$-layer graphene film.

The advantage of the 12-minute etch is that fresh, unadulterated SiC crystal material, far removed from surface damage or contamination, is ultimately used in the final reconstruction of the graphene film. However, if further studies reveal that a side effect of this deep etch is the inadvertent concentration of dopants/contaminating elements into the reconstructed film, (following a study on the minimum etch depth required to form a quality film) the etch time can easily be shortened. 
The IV plots collected as a function of temperature of the $4 \mathrm{H}-\mathrm{SiC} /$ graphene composites also show a radical departure from the $6 \mathrm{H}-\mathrm{SiC} /$ graphene devices, as shown in Figure 5.23. In Figure 5.23 two distinct, but ultimately related, curve defining aspects can be observed. The first, seen in the positive quadrant of the IV plot, is an obvious evidence of charge trapping conduction. The second, seen in the reverse bias quadrant of the IV plot, is a peak in device conductivity, followed by a negative differential resistance (NDR, where current flow decreases with increasing voltage), will be discussed shortly.

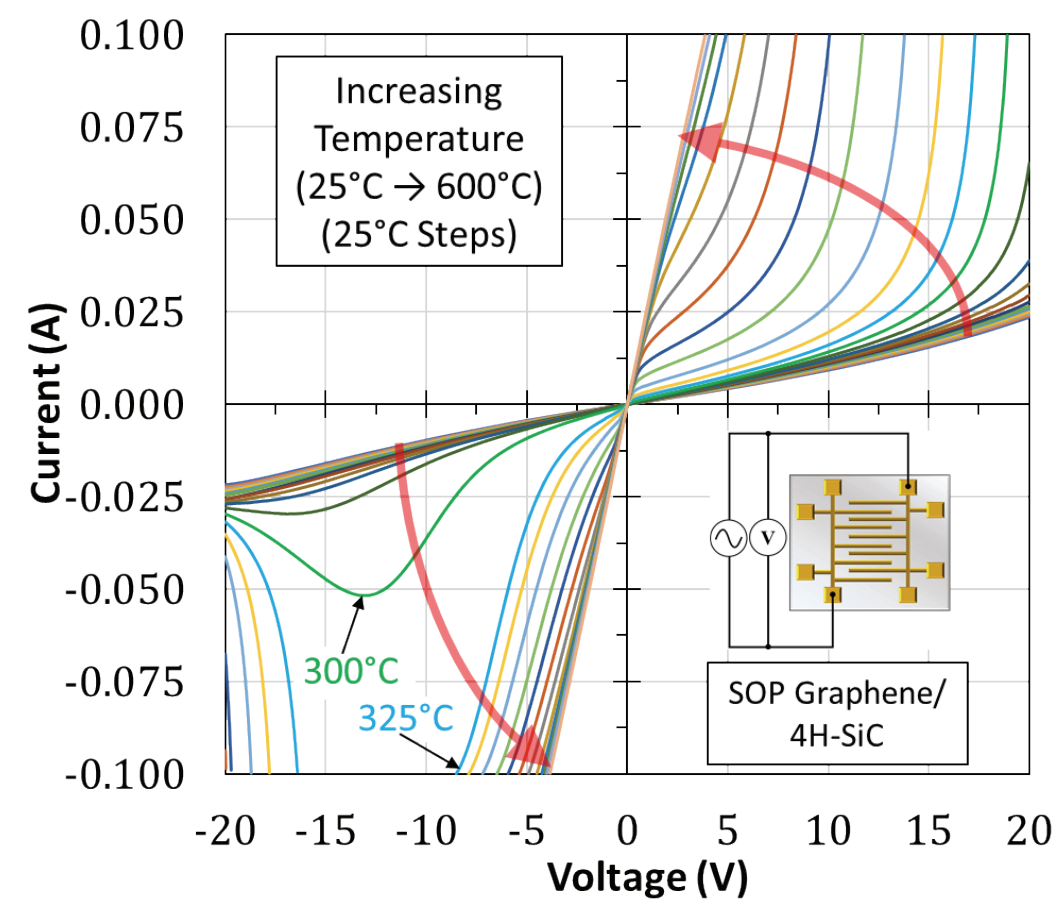

Figure 5.23: Two-point IV as $f(\mathrm{~T})$ plots collected from a $4 \mathrm{H}-\mathrm{SiC} /$ graphene composite sample produced using the SOP graphenization process described in Chapter 4. Arrows indicate the direction of increasing temperature.

To illustrate the first point (charge trapping conduction), the positive portion of the IV curve collected at $450^{\circ} \mathrm{C}$ is shown in Figure 5.24(A). The curve shape shows clear evidence of charge trap conduction and thermionic conduction, as indicated by the dashed lines. Following an analysis similar to what was described earlier (Figure 5.13), the roll-off voltage for trap conduction is shown to increase exponentially with temperature. This is shown in Figure 5.24(B) along with the corresponding current, shown in Figure 5.24(C). Similar to the 6H-SiC/graphene samples, the charge traps appear to be distributed exponentially in energy. The fact that charge trapping conducting accounts for a large portion of the IV curve (i.e. is sustained over a large voltage range) may indicate that there are many more charge traps present. Correspondingly, there are more trap 
pathways through the barrier which can then accommodate a large carrier flow which is made available to the system at higher voltages.

(A)

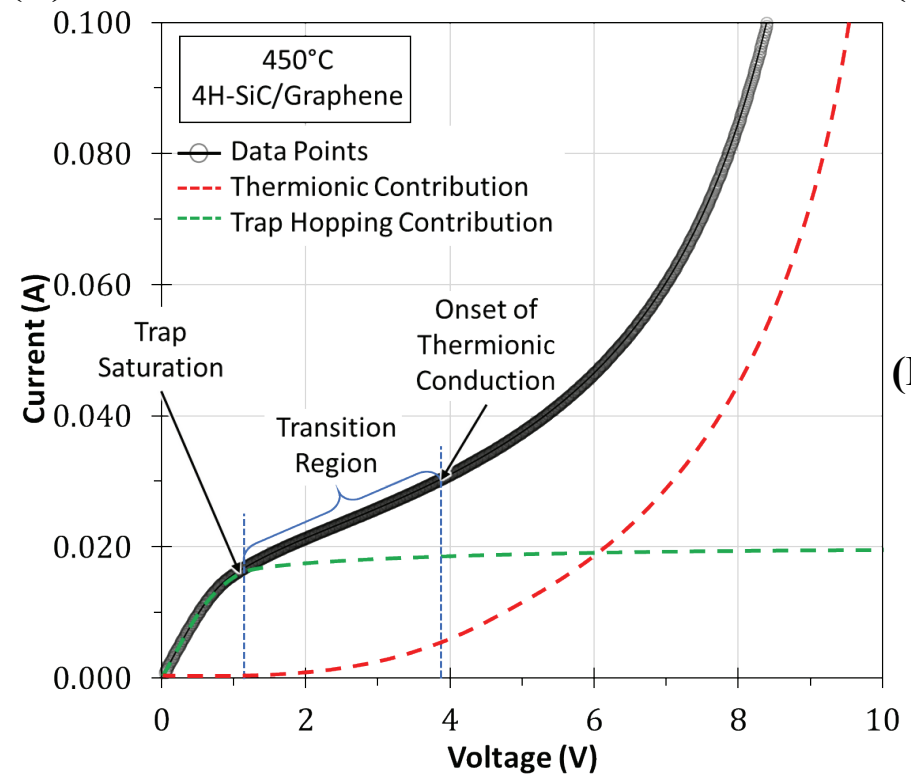

(B)
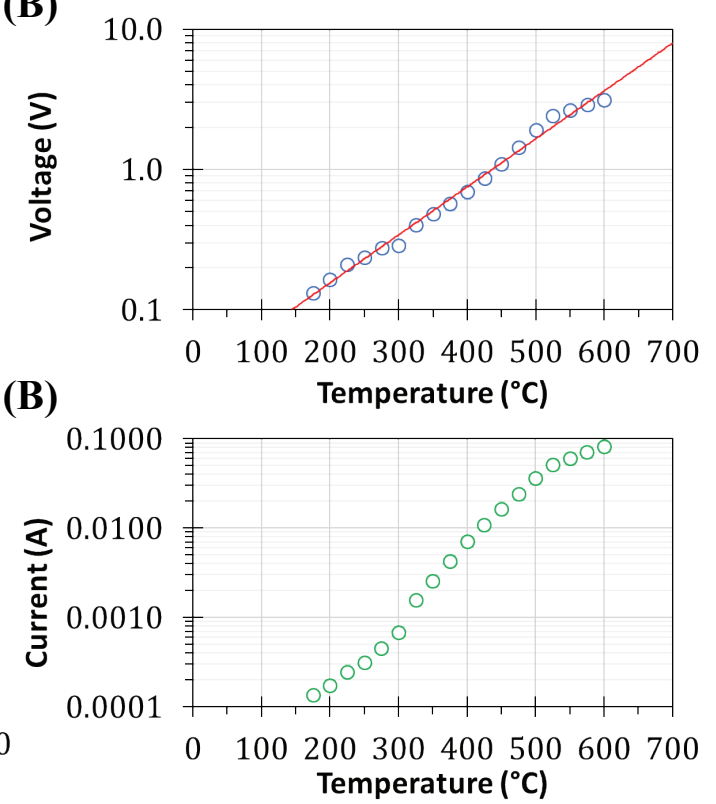

Figure 5.24: (A) IV plot of a $4 \mathrm{H}-\mathrm{SiC} / \mathrm{Graphene}$ sample collected at $450^{\circ} \mathrm{C}$. Two regions, charge trap conduction and thermionic conduction are clearly apparent. The independent contributions of each mechanism are shown by the dashed lines. (B) Approximant roll-off voltage of charge trapping and (C) corresponding current level.

The negative differential resistance (NDR) seen in the reverse bias quadrant of Figure 5.23 is characterized by a decrease in current flow as a function of increasing voltage. This NDR behavior is most commonly utilized by electronic devices called resonant tunneling diodes. Resonant tunneling diodes have been an intense area of research which is much too broad to summarize here (as of 2019 there are over 16,000 research articles published on resonant tunneling diodes). However, a brief description of their operating principle gives insight into what is occurring with the $4 \mathrm{H}-\mathrm{SiC} /$ graphene composites which produced the results shown in Figure 5.23. The references provide a more detailed discussion of the theory, operation and applications of resonant tunneling diodes. ${ }^{(627)(628)(629)(630)(631)}$

Simply stated, the peak in conductivity seen in Figure 5.23 is most likely due to resonance of the electron wave function (described by the Schrödinger equation) with a charge trap(s). ${ }^{(627)}$ (632) This type of resonant conduction is a purely quantum effect driven process (see Footnote 34) and is typically discussed in the context of two thin energy barriers in close proximity. The area between the barriers is then described as a quantum well. If the wave function of an electron 
matches with the dimension of the quantum well, such that a resonance in the wave function is achieved, the probability of tunneling into and then out of the well approaches unity (100\% transmission probability). A more detailed discussion follows. ${ }^{(633)(634)}$

Two events occur when a particle wave packet approaches an energy barrier. A fraction of the wavefunction may reflect back from the barrier and a fraction may penetrate into the barrier. (635) The wavefunction decays exponentially over the width of the barrier until it exits the other side. The probabilistic location of the particle is described by the square of the wavefunction (following Born's Law). ${ }^{37(636)}$ Therefore, if the barrier is thin (and thus the wavefunction has not decayed excessively by the time it exists the other side of the barrier) the particle has a non-zero statistical chance to tunnel through the barrier at zero energy cost.

The wavefunctions can be treated using classical mechanics via the superposition principle. (637) Therefore, they may interact with themselves and other wavefunctions, much like mechanical waves (i.e. constructively or destructively). A unique situation arises when two thin barriers in series are separated from each other by a distance on the order of the particle wavefunctions wavelength. In this case, the fraction of the wavefunction which penetrates the first barrier constructively interferes with the fraction which is reflected from the second barrier (and back to the inner wall of the first barrier, and so on). This is analogous to classic resonance inside an acoustic chamber. The result is a high amplitude of the wavefunction within the confines of the barrier. Therefore, the probability of the particle residing within the quantum well formed by the two barriers is high, but only for the particle frequency (energy) which leads to resonance, which is a function of the quantum well width.

Applying this concept to the experimental results observed here lead to the following interpretation: Conduction occurs readily through a charge trap (or a series of charge trap paths), whose spacing and energies couple with the energy of the electron, leading to a resonance of the charge carrier wavefunctions within the trap.

\footnotetext{
${ }^{37}$ This statistical interpretation of the wavefunction, originally postulated in 1926 by Max Born, resulted in Born being awarded 1/2 of the Nobel Prize in Physics in 1954. (756)
} 
A simple illustration of this resonant process is illustrated qualitatively in Figure $5.25 .{ }^{38} \mathrm{It}$ is unclear if a series of resonating quantum wells are formed or if a single well is responsible for the observed behavior. The probability of tunneling through a charge trap (or successive charge traps) is highly increased when the electron wavefunctions achieve resonance with the trap energies and spacing, which occurs for a narrow voltage range. This is observable in the IV curves as a voltage band showing unusually high conductivity.

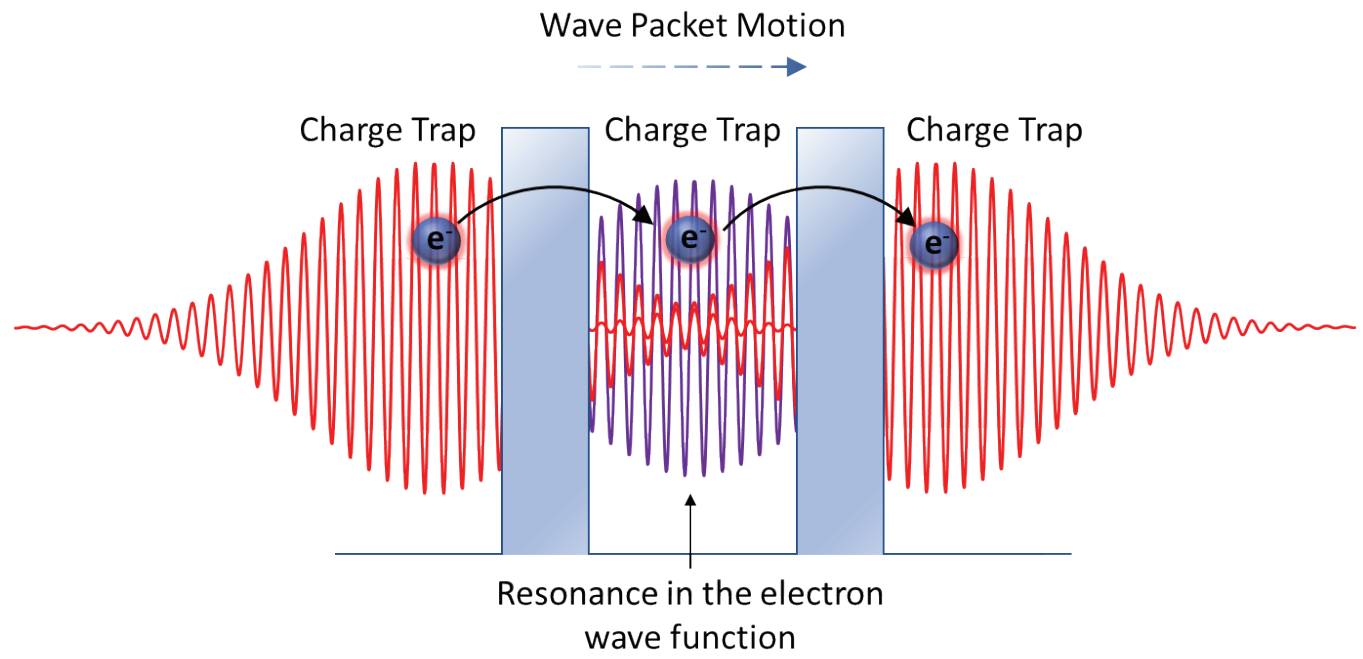

Figure 5.25: Qualitative illustration of the resonant tunneling process responsible for the peak in current flow. Resonance in the electron wave function with the quantum wells formed by successive charge traps leads to a higher tunneling probability for those electrons. This process is carried out through the entire trap hopping circuit. (Note: In reality, the wave packet is perturbed by the barrier more than what is illustrated here, and the reflections are substantially more complex. Shown here is a simple cartoon to help qualitatively illustrate the general principle.)

The operation of resonant tunneling diodes is also described in the context of an energy band diagram, which may be more intuitive for some readers. The narrow band of energy at which resonance occurs (a function of the physical dimensions of the quantum well) can be treated as a narrow band of allowable energy states through the well. This presents a 'shortcut', so to speak, for electrons to transits the well, but one which is only accessible to electrons with a specific energy.

The integrated area of the carrier distribution (recall that the carrier population is distributed across a range of energies via a pseudo Maxwell-Boltzmann function) that aligns with

\footnotetext{
${ }^{38}$ Note that the wavefunction has a dispersive character as it moves in position-space with time, not easily illustrated here. Furthermore, the real mathematical solution to the wavefunction interaction (reflection/transmittance) with the energy barriers are substantially more complex that what is shown. Particularly when the barrier is not static (i.e. varies with time in magnitude and position) which is a more accurate reflection of reality, particularly when the matter which generates the barrier is at elevated temperature. (750) (753)
} 
this narrow band (shortcut) are readily transmitted through the width of the well via the path provided by the narrow band. The alignment of this pathway with the carrier population can be modified by the voltage applied across the system. Additionally, the distribution of the carrier population envelope can be shifted in energy space by changing the temperature of the system. These two facts explain the peak in current which occurs at an intermediate voltage (the voltage at which the narrow band of allowable energy states aligns with the bulk of the carrier population) and the temperature activation (the carrier population envelope must be shifted to an energy level where an applied voltage can make the population accessible to the pathway through the well). The band diagram associated with each point in the IV curve is shown in Figure 5.26.

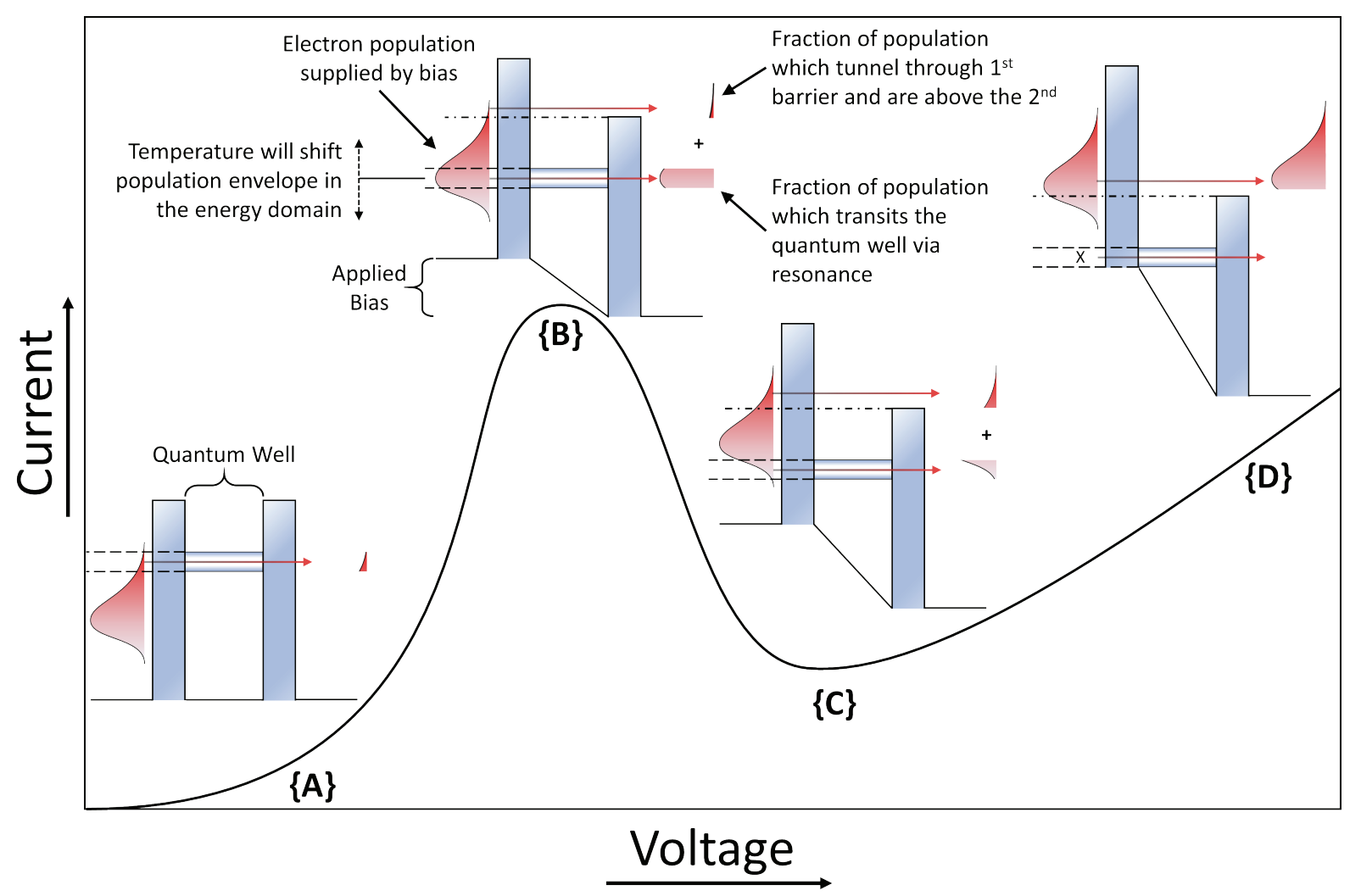

Figure 5.26: Energy diagram illustrated at several points in the IV curve of a resonant tunneling device. A description of the phenomena at each point is given in the text.

At position $\{\mathrm{A}\}$ in Figure 5.26, few carriers are at the resonate energy pathway level and thus the current is correspondingly low. At $\{\mathrm{B}\}$ the bias-induced tilt of the band diagram brings the bulk of the carrier distribution in line with the resonant pathway leading to a large current flow. At $\{C\}$ current drops (this is the NDR region) due to the higher bias shifting the resonate pathway below the bulk of the carrier distribution envelope. At $\{D\}$ the bias has shifted the energy of the second barrier such that it is below the energy of many of the carriers and thus, does not impede 
their flow. Current flow thus increases as now carriers need only to tunnel through the first barrier. In reality, the carrier distribution is much broader than what is shown, and all of the processes occur to a varying extent across the entire curve. Increasing the voltage bias also increases the total number of carriers in the population, which also impacts the current flow, but this is not the dominating effect. For simplicity, only the dominant processes are shown. Carriers which are not above the barriers, or are not at the resonant energy level, must tunnel the entire quantum well. These tunneling events have a low probability of occurring due to the large total width (first barrier width, plus the well spacing, plus the second barrier width).

To quantify the resonant behavior, IV plots collected at two different temperatures are examined in Figure 5.27. A French Curve ${ }^{39}$ fitting technique was used to give a reasonable approximation of the curve behavior outside of the current and voltage limits (represented by the dashed lines). At $300^{\circ} \mathrm{C}$ and $325^{\circ} \mathrm{C}$, reaching resonance with the charge traps increases the current

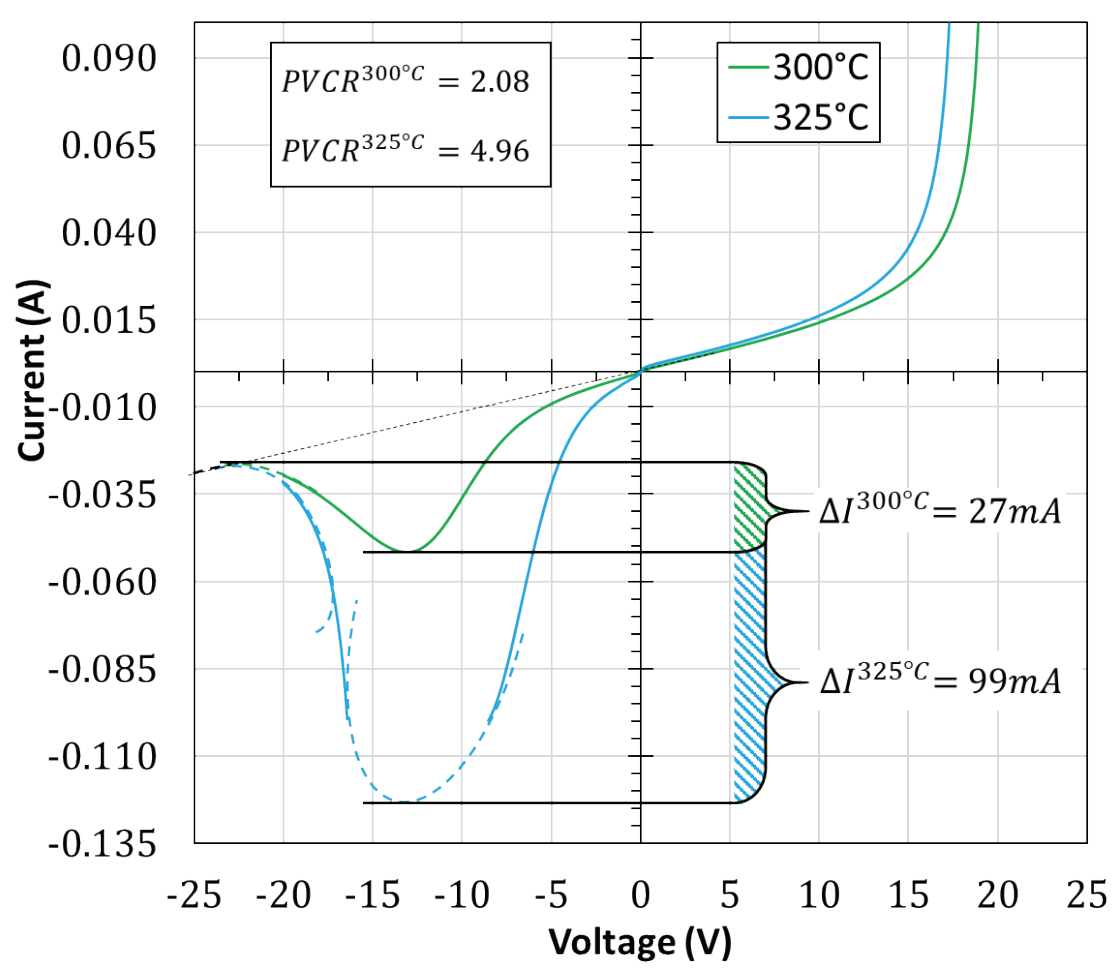

Figure 5.27: The curves have been extended outside of the current and voltage limit range by using an $a d$ hoc French curve fitting technique to estimate the peak to valley current ratio (PVCR), as indicated by the respective dashes lines.

\footnotetext{
${ }^{39}$ A French Curve fit uses segments of the Euler spiral to project and extend simple curve behavior. A French Curve fit was slightly modified to take into account the slopes of the known portions of the curve and is used here to interpolate between data points. This was done to give a conservative estimate for the apex value of the curve peak and trailing edge values. This fitting method is similar to spline interpolation but is a more constrained approach.
} 
flow $37 \mathrm{~mA}$ and $109 \mathrm{~mA}$, respectively (from what the current would otherwise be). These two IV curves represent the sharpest increase in resonate current, characterized by an increase of $72 \mathrm{~mA}$ for a $\Delta \mathrm{T}$ of $25^{\circ} \mathrm{C}$. Based on the current peak, the resonant voltage is approximately $13 \mathrm{~V}$. Based on the rate of change in the current peak across temperatures, the activation temperature lies between $300^{\circ} \mathrm{C}$ and $325^{\circ} \mathrm{C}$.

A common figure of merit for resonant tunneling diodes is the peak to valley current ratio (PVCR). This is the ratio of current flow between the peak current at resonance and the next subsequent current valley encountered in the direction of increasing voltage. The PVCR for the device at $300^{\circ} \mathrm{C}$ is 2.08 and at $325^{\circ} \mathrm{C}$ the PVCR is 4.96 . Above $325^{\circ} \mathrm{C}$, the PVCR appears to not substantially change, indicating that the temperature required to bring the carrier population to the energy level of the resonant pathway has been reached. The maximum PVCR displayed by the device here is $\sim 4.96$. This appears to compare well with the majority of reports on resonant tunneling diodes. There are, however, a few reports with exceptionally high PVCR values at roomtemperature. ${ }^{(638)(639)}$

A final comment on Figure 5.27 is the breadth of the resonate peak in the IV curve. The breadth is indicative of the energy width of the shortcut pathway. That is, the window of energies which leads to resonance. Because the resonance energy is a function of the physical dimensions of the quantum well, a wide range of resonant energies indicates a wide range in quantum well widths. Therefore, if the distribution of well widths can be reduced, the resonant energy range may be reduced (producing a sharper resonant peak in the IV curve).

The high temperature performance and stability of the device detailed here sets it apart from other resonant tunneling diodes. Many devices operate at room temperature or below, with higher temperatures generally reducing performance. (640) (641) (642) (639) Because most resonant tunneling diodes are constructed from precisely layered films, the temperature stability is an aspect of concern due to diffusion of the atoms between layers, which negatively effects performance. (643) (644) The devices reported on here were thermally cycled to $600^{\circ} \mathrm{C}$ four times and no loss in the resonant performance was observed.

The majority of resonant tunneling diodes are constructed from complex multilayered heterostructures using Group III - V compounds (specifically, Si, Ga, As, Sb and Al are common). There are a few reports on graphene based resonant tunneling diodes, however most are 
computational studies of theoretical devices. ${ }^{(645)(646)(647)}$ Overall, this appears to be an area of limited experimental research. For resonant tunneling diodes more broadly, there appears to be no literature available on the performance and operation of devices in the temperature regime covered in this work $\left(23^{\circ} \mathrm{C}-600^{\circ} \mathrm{C}\right)$. Clearly, this is an area of this work well suited for future studies.

\section{\$5.2.4 Summary of Temperature Dependent Device Properties}

As discussed in Section $\$ \mathbf{5 . 1 . 1}$, at room temperature the devices exhibit back-to-back Schottky behavior. On heating in an inert environment (ultra-pure argon), the device behavior transitions to Ohmic (linear IV) behavior. The exact temperature of transition from Schottky to Ohmic varies slightly from sample to sample and depends largely on the contact pattern design and the sample packaging. However, all devices display Schottky behavior at $\sim 25^{\circ} \mathrm{C}$ and in general, are fully Ohmic at temperatures $\geq 200-300^{\circ} \mathrm{C}$.

The $\mathrm{SiC} /$ graphene devices studied here show exceptional robustness to temperature and are capable of withstanding extended periods of time (hours) at high temperature (up to $\sim 800^{\circ} \mathrm{C}$ ). In fact, the device failure in all cases is due to destruction of the device packaging; a component easily improved. Overall, the SOP devices exhibit a high level of sensitivity to temperature. The sensitivity is primarily derived from the thermionic emission of charge carriers over the Schottky barrier, which is a highly temperature dependent phenomena which is easily observable as a change in device resistance. This presents an opportunity for their use as an on-chip, planar microthermometer circuit element or as discrete temperature sensing elements in high temperature environments. Further testing is required to establish a calibration curve and determine the limits of the temperature sensitivity, but given the results presented here detection of fractional degree changes should be easily achievable across a wide temperature range.

Richardson-Dushman analysis indicates there is a degree of contact inhomogeneity, as evident by the thermal activation of interfacial regions with a high Schottky barrier height. Analysis of the carrier mobility as a function of temperature indicate the dominate scattering mechanism at low temperature to be a combination of neutral and ionized impurity scattering. The dominate scattering mechanism at high temperature appears to be scattering by thermal phonons. The crossover of these two mechanisms results in a peak in carrier mobility around $100^{\circ} \mathrm{C}$.

The study of the $\mathrm{SiC} /$ graphene devices electrical behavior as a function of temperature have revealed several fascinating phenomena. This has provided additional insight into the films 
produced using the SOP method described in Section $\$ 4.1$. The observation of charge trapping current at high temperature indicates there are exponentially distributed high-level charge traps formed at the metal/film interface. It is unclear what feature of the film is responsible for these traps, but oxygen defects (acting as electron acceptors) and structural defects (leading to dangling bonds) seem likely. Trace amounts of fluorine (a highly electronegative element) or inadvertent aluminum contamination (forming $\mathrm{Al}_{\mathrm{x}} \mathrm{O}_{\mathrm{x}}$ ) may also play a role. Further study will be required to determine if the amount of charge trapping current scales with the Raman D peak (indicative of structural defects) or with the number of oxygen defects as detected by XPS analysis. Testing of non-SOP samples annealed at lower temperatures, where fluorine is definitively present, may also provide insight into the origins of the charge traps.

The preliminary testing of devices using the non-standard $4 \mathrm{H}-\mathrm{SiC}[\mathrm{SI}]$ and $4 \mathrm{H}-\mathrm{SiC}$ substrates have revealed several fascinating results. First, the $4 \mathrm{H}-\mathrm{SiC}[\mathrm{SI}]$ show Ohmic behavior across all temperatures tested. Although conductivity is increased with elevated temperature, the devices are several orders of magnitude lower in conductivity. This may be the result of an inadvertent concentration of intrinsic contaminations in the $\mathrm{SiC}$ crystal into the film by the selective etch process. Or, this may indicate that the substrate participates in conduction to a much greater extent than previously thought. Further testing will be required.

Preliminary testing of devices using the $4 \mathrm{H}-\mathrm{SiC}$ substrate variant show clear evidence of charge trapping and the unusual property of a negative differential resistance. This is observable in the IV curve at elevated temperature beginning around $250^{\circ} \mathrm{C}$ and is most extreme at temperatures above $300^{\circ} \mathrm{C}$. It is unclear why the $4 \mathrm{H}-\mathrm{SiC}$ substrate leads to this behavior but may be related to the specifics of the charge traps formed. Further testing will be required, but ultimately, the plasma assisted graphene growth method applied to the $4 \mathrm{H}-\mathrm{SiC}$ material is a promising avenue for fabrication of unique $2 \mathrm{D}$ circuit elements, such as planar resonant tunneling diodes. An unoptimized device has shown excellent temperature stability ( $4 \mathrm{x}$ thermal cycle to $600^{\circ} \mathrm{C}$ ), high temperature operation (above $300^{\circ} \mathrm{C}$ ) and good resonant tunneling diode performance (PVCR of 4.96).

\section{\$5.3 Device Response to Gas Exposure}

This section will present and discuss the test results for devices operating as gas sensors. Gas sensing was chosen because it is of interest to a wide range of funding agencies and it is one 
of the simplest applications for the SiC-Graphene composites produced in this work. The focused interest on high temperature gas sensors by the natural gas and petrochemical refining communities has motivated and funded this portion of the work. Of the numerous gas sensing device designs, resistometric detection is the simplest. ${ }^{(648)}$ In resistometric operation, the resistance of the device is simply monitored as a function of the environmental conditions (temperature, gas composition, etc.).

The graphene films used in these tests were produced using the "SOP" procedure. Over 1,500 tests were performed on $146 \mathrm{SiC}$-Graphene devices. The high temperature gas tests were restricted to simple two-point measurements, which is the de facto standard for this type of resistometric gas sensing (where the device resistance is the monitored metric). As will be discussed, the test results appear to be simple at first glance. However, careful examination of the results shows several complicating factors are at play. These will be discussed in the first subsections of this section. Many of these effects only became apparent as subtle patterns in device response emerged after extensive gas testing. Although it will require extensive modification to the testing apparatus, these observations show that four-point probe measurements will be key for future work involving these films. The rich data provided by such measurements will add to the understating of the devices, which has only begun to be revealed by the work presented here.

The experimental setup for the gas testing apparatus is detailed in Section $\$ 3.4$. In short, the devices act as a resistometer where response is evaluated by a change in the electrical resistance as aspects of the gas environment change. The device packaging used to house the SiC/Graphene composites is detailed in Section $\$$ 3.3.3. The analyte gases were ultra-high purity (UHP, $>99.9999 \%$ pure $)$ hydrogen $\left(\mathrm{H}_{2}\right)$, methane $\left(\mathrm{CH}_{4}\right)$ and carbon monoxide $(\mathrm{CO})$. Inert carrier gasses used were UHP argon (Ar) and helium (He). The analyte gases were chosen for their importance to the natural gas and energy industries. For these industries, and others, the ability to detect these gases at high temperatures is of interest. To this end, tests were carried out at temperatures ranging from room temperature to as high as $700^{\circ} \mathrm{C}$. As will be discussed, the device temperature response complicates the analysis and, in most cases, swamps out the response derived from chemical interactions with the analyte gases.

All devices showed a clear and unambiguous response to each analyte gas. However, the precise mechanism of this response depends on the testing regime. Broadly, the sensing mechanisms are divided into two categories. They are identified here as 'thermal effects or thermal 
response' and 'chemical effects or chemical response'. The former is due to thermally driven changes in the device properties and the latter are due to changes of the device properties that result from chemical interactions with the analyte gases.

Because the thermal effects are key to interpreting the device results, this section will begin with a discussion of the role that the thermal response plays in gas sensing. It should be noticed that the response data collected at high temperature are inherently noisy. This is most likely due to Johnson-Nyquist temperature dependent noise, which is particularly prevalent in graphene devices. ${ }^{(649)(650)}$ This noise is compounded for measurements collected at low setpoints (low voltages or low currents levels) which are also inherently noisy in and of themselves.

It should also be noted that for clarity all pulse data has been artificially time shifted to account for the plug flow lag of the feed system to the test chamber. Stated another way, the input analyte pulse data indicated in each graph was time-shifted such that the step increase in analyte concentration syncs with the onset of the device response.

\section{\$5.3.1 Self-Heating and Thermal Runaway}

During gas sensing, the thermal response of the device is a result of changes in the device temperature due to a shift in the thermal equilibrium of the device with its environment. As discussed in Section $\$ 5.2$, the devices exhibit a high degree of temperature sensitivity. The device temperature is determined by the balance between the heat generation and heat removal processes.

Heat is generated in the device by self-heating effects (also known as Joule or Ohmic heating) when the device is powered (i.e. when a voltage is applied across the device). Self-heating is described by the Joule-Lenz law,

$$
P=I^{2} \times R
$$

Equation 5-5

where $\mathrm{P}$ is the power generated (the rate of energy generated in $\mathrm{W}, \equiv \mathrm{J} / \mathrm{sec}$ ), $\mathrm{I}$ is the current (in amps, A) and $\mathrm{R}$ is the resistance (in Ohms, $\Omega$ ). The rate of heat transfer to or from the device and its environment is described by the following heat transfer equation,

$$
\dot{Q}=\frac{k A}{l}\left(T_{S}-T_{\infty}\right)
$$

where $\dot{Q}$ is the rate of heat transfer in (W or $\mathrm{J} / \mathrm{sec}), k$ is the thermal conductivity of the $\mathrm{gas}(\mathrm{W} / \mathrm{m} \cdot \mathrm{K})$, A is the surface area over which heat is removed $\left(\mathrm{m}^{2}\right), T_{s}$ is the temperature of the sample (in $\mathrm{K}$ or ${ }^{\circ} \mathrm{C}$ ), $T_{\infty}$ is the temperature of the gaseous environment (in $\mathrm{K}$ or ${ }^{\circ} \mathrm{C}$ ) and $l$ is the thermal boundary 
layer thickness $(\mathrm{m})$ over which the temperature changes from $T_{s}$ to $T_{\infty}$. The boundary layer thickness can be decreased (heat transfer increased) by increasing the gas flow rate. The combination of terms $\left(\frac{k A}{l}\right)$ determines the heat transfer rate for a given $T_{s}$ and $T_{\infty}$. A simplified generic view of the overall process is illustrated in Figure 5.28.

$$
\left(\begin{array}{c}
\text { Heat } \\
\text { Generation }
\end{array}\right)-\left(\begin{array}{c}
\text { Heat } \\
\text { Removal }
\end{array}\right)=\left(\begin{array}{c}
\text { Heat } \\
\text { Accumulation }
\end{array}\right) \propto\left(\begin{array}{c}
\text { Device } \\
\text { Temperature }
\end{array}\right)
$$

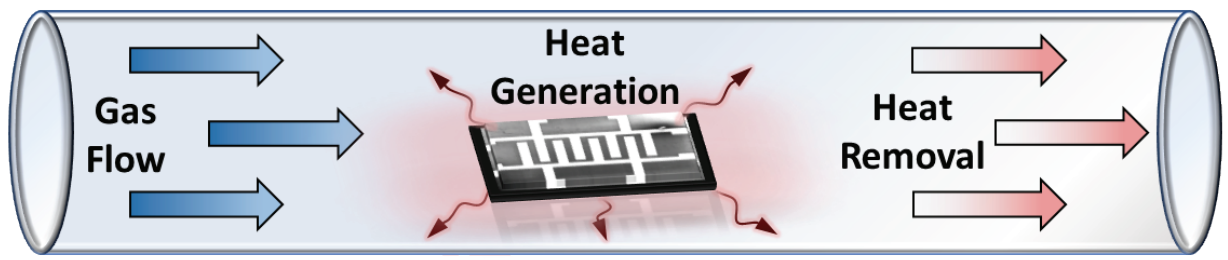

Figure 5.28: Generic illustration of the fundamental heat transfer processes which dictate the device temperature. Electrical energy is converted into heat energy in the device by Ohmic heating. Heat is removed from the device by the gas stream via forced convective heat transfer. The difference in the rates between these two processes determines the steady state balance of heat energy residing in the device which dictates the device temperature.

If these two effects, described by Equation 5-5 and Equation 5-6, are taken to be the dominant mechanisms which dictate the energy balance of the device, a typical scenario is as follows. A gas test begins with the device setting in a steady state flow of inert gas. The device is unpowered and is in thermal equilibrium with the environment. That is, the device is at the same temperature as the gas stream. At the start of the test $(\mathrm{t}=0)$, a voltage is applied across the device and data collection begins. This results in heat generation within the device, consistent with Equation 5-5. The temperature of the device rises, and a thermal gradient is established between the device and the gas stream. The temperature differential drives the heat removal process, consistent with Equation 5-6. After a brief equilibration time, the device reaches a new thermal equilibrium and is now at a new (elevated) temperature, determined by the operational power level, thermal conductivity of the gas and the thickness of the thermal boundary layer as established by the gas flowrate.

When the composition of the gas stream is changed, the process repeats. The change in the thermal conductivity of the gas mixture shifts the thermal equilibrium point by either increasing or decreasing the rate of the heat removal (by modifying $k$ in Equation 5-6). This in turn, changes the temperature of the device, which affects the resistance and thus the self-heating (via Equation 5-5), as well as the heat removal rate (by modifying the temperature differential quantity in Equation 5-6). This then affects the device temperature and so on. In this way, a new equilibrium is 
established, and the device sets at a new temperature and exhibits a new resistance value. This process is shown schematically in Figure 5.29.

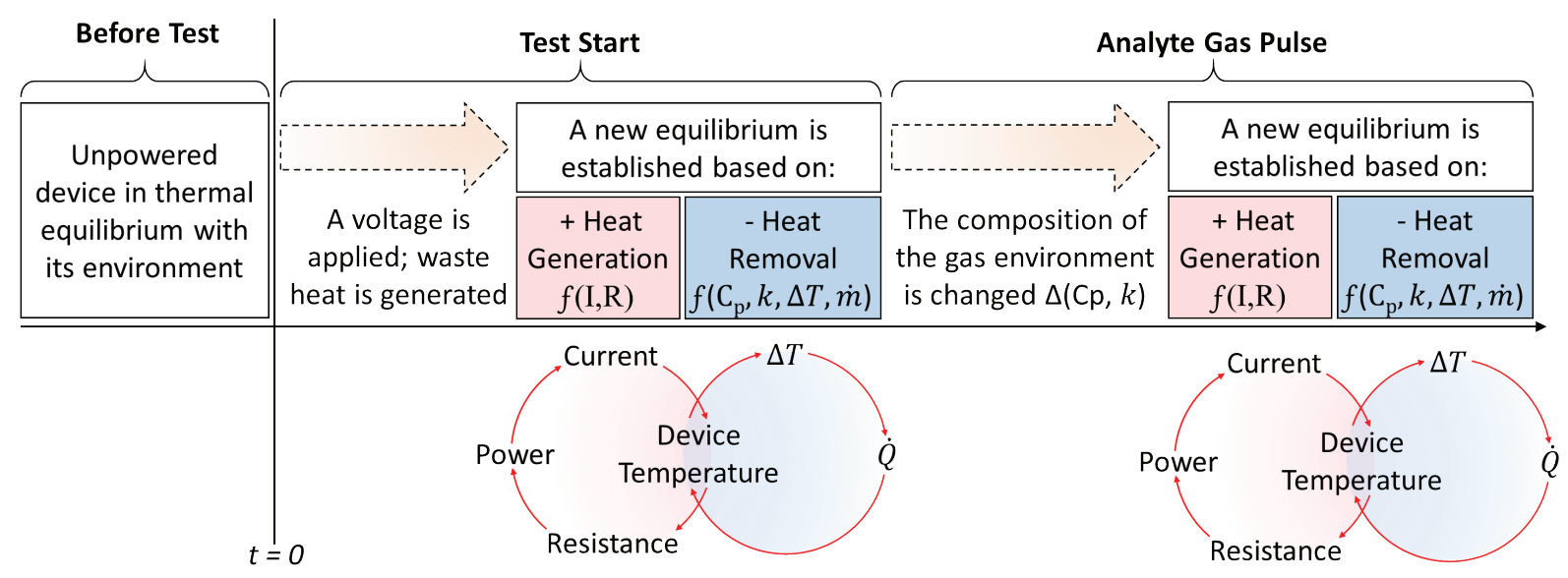

Figure 5.29: Illustration of the thermal equilibration process from device startup to the first gas pulse.

The equilibration time for device after initial startup (a bias is applied across the device) was observed to take as little as $\sim 5$ seconds up to as much as $\sim 500$ seconds. This wide range in the equilibration time is due to the several processes at play. For example, the amount of heat generated is, in part, a function of the resistance of the device. Additionally, the resistance of the device is a function of the temperature of the device. Furthermore, under constant voltage control, the thermally induced changes in resistance influences the level of current flow. In opposition to this is heat removal, $\dot{Q}$, which is proportional to the temperature gradient between the device and the gas environment $\left(\Delta \mathrm{T}_{\mathrm{S}-\infty}\right)$; and $\Delta \mathrm{T}_{\mathrm{S}-\infty}$ is proportional to the device power. The interplay rate between these effects determines the equilibration time.

There are scenarios where the first two effects dominate (Joule heating and the decrease in resistance) and lead to a thermal runaway event. In these cases, the feedback loop between temperature and resistance is such that an equilibrium between the device temperature and device electrical properties is never reached. Data for such an event is shown in Figure 5.30. In this case, Ohmic heating increases the temperature of the device, which decreases the device resistance. This allows more current to flow at the setpoint voltage. Following Equation 5-5, power is proportional to the square of the current, and thus the increase in the current dominates over the reduction in resistance. The 'vicious cycle', leading to a runaway, is illustrated in Figure 5.30(B). Current increases until the interlock limit of the testing equipment is reached $(100 \mathrm{~mA})$. 
(A)

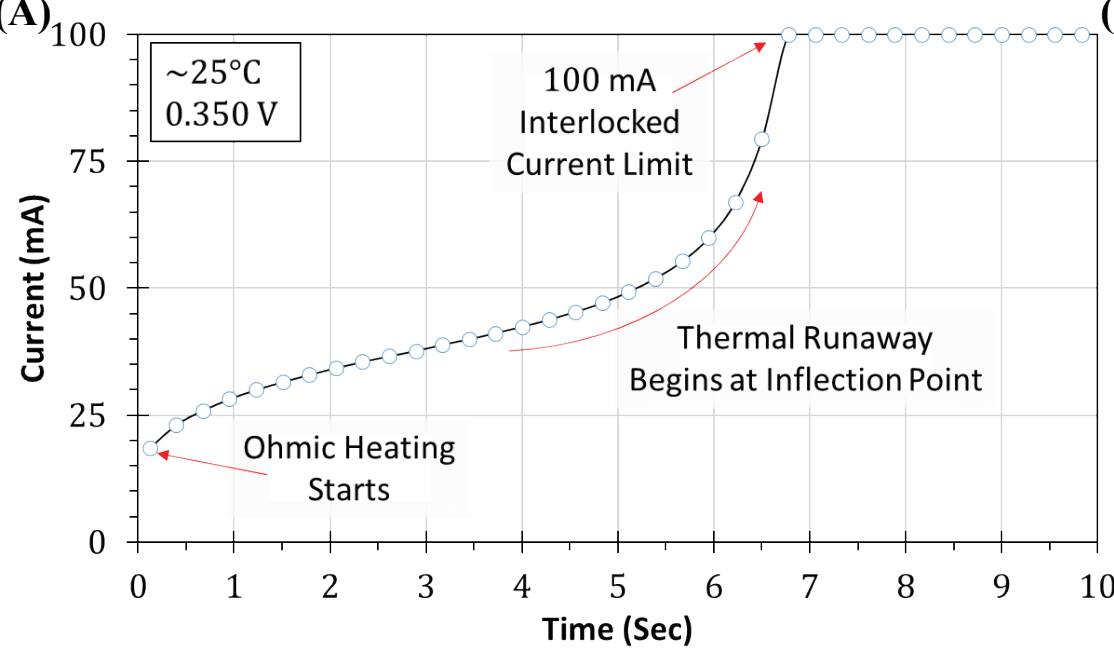

(B)

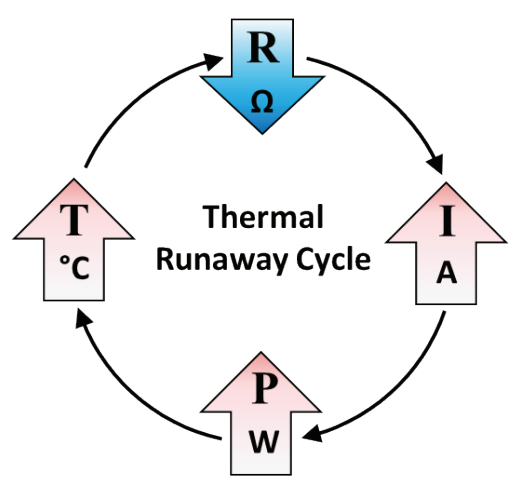

Figure 5.30: (A) An example of a thermal runaway event for a device operating in constant voltage mode $(0.350 \mathrm{~V})$. The plot shows the first 10 seconds after power up of the device, while under a flow of 500 SCCM of UHP Ar. The specific conditions which led to a runaway event varied from sample to sample. This device used a TLM pattern and two-point probe data was recorded between two closely spaced pads. (B) The thermal runaway cycle: Ohmic heating leads to a temperature rise, which leads to a decrease in resistance, which leads to an increase in the current, which leads to an increase in power, which rises the temperature; the cycle repeats.

The process shown in Figure 5.30 will not continue indefinitely. The resistance will decrease to a finite value in response to an increase in temperature and the device will eventually equilibrate. However, for the scenario shown in Figure 5.30(A), the equilibration point is outside the measurement window of the testing equipment (the 100mA current limit in this case). To avoid encountering these thermal runaway sceneries during testing, the devices are operated at either low voltages or in a constant current mode. By operating at low voltages, the current is reduced, which limits sample heating. However, very low voltage operation results in a poor signal-to-noise ratio. This is compounded for devices operating at high temperature. Devices operating at high temperature are inherently noisier due to Johnson-Nyquist temperature dependent noise, which is particularly prevalent in graphene devices. ${ }^{(649)(650)}$ Alternatively, by operating in constant current mode, the current is set and only the voltage is allowed to vary. Temperature effects are of course still present, but the comparatively larger parameter space of the $0 \mathrm{~V}-20 \mathrm{~V}$ voltage range mean that in practice, it is easier to stay within the operational space of the measurement equipment.

Although in principle, Equation 5-5 and Equation 5-6 seem simple to solve, the determination of the device temperature is difficult due to predict due to several real-world complicating factors. In particular, the heat sinking effects of the sample packaging must be included and the fact that the resistance of the device itself is temperature dependent. 
In summary, self-heating (also known as Joule heating or Ohmic heating) of graphene devices has been widely reported. ${ }^{(651)(652)(653)(525)}$ While reports vary, self-heating can lead to the graphene film being at a substantially higher temperature than the surroundings. Using empirical results with electrothermal simulations, Bae, et al. show areas of a transistor constructed from exfoliated graphene to be as high as $100^{\circ} \mathrm{C}$, while Islam, et al. calculate even higher temperatures. (654) (655) By comparing IR imaging techniques with Raman defect mapping of graphene films, Beechem, et al. conclusively show that structural defects result in areas of exceptionally high temperatures due to self-heating. ${ }^{(656)}$ In fact, the self-heating can be high enough to drive the film into photoemission. Riaz, et al. have recorded the photoemission spectrum for areas of high resistance in nanocrystalline graphene films. ${ }^{(657)}$ From the collected spectrum, they calculate the electron temperature in the film to be $\sim 1664 \mathrm{~K}$. For comparison, their device was $0.5 \mu \mathrm{m}$ wide by $1.0 \mu \mathrm{m}$ long and the applied voltage was $33 \mathrm{~V}$, producing a current flow of $150 \mu \mathrm{A}$.

The effect of self-heating of graphene devices has been shown to have complex effects on graphene device behavior. ${ }^{(655)(658)\left({ }^{659)}\right.}$ Peltier cooling at the metal contacts and localized heating around defect sites and multi-layer islands graphene have also been observed. ${ }^{(660)(661)}$ Charge trapping adds further complexity to the effect of self-heating on device response, effecting the time constant of the effect. ${ }^{(662)}$

Self-heating effects are clearly observable in the test data collected from the devices studied in this work. Obviously, the temperature of the film is a function of many factors, including the power dissipated by the device, the size of the device, how the device is physically supported and its thermal interaction with the environment. The latter being dependent on the thermal conductivity of the gas and the gas flowrate. The interplay between these two effects determines the equilibration temperature of the device. As will be discussed in Section \$5.3.2, in addition to a thermal equilibration, the devices also undergo an electrical equilibration observable on startup (i.e. when a voltage is first applied across the device). The gas tests are inherently transient in nature (i.e. they consist of pulses of an analyte gas) causing the thermal equilibrium point to be cyclically shifted. The effect of self-heating on the device resistance is most clearly observable when the device is shifted off thermal equilibrium, such as during device startup (initial application of voltage) or when the thermal properties of the environment are changed (gas pulse). 


\section{\$5.3.2 Startup Effects: Thermal and Electrical Device Equilibration}

In order to fully appreciate the device behavior, so that the gas response may be more fully understood, the device behavior immediately after startup was investigated. This section details this brief investigation. Additionally, the practical impact of operating the device at different current (or voltage) setpoints is illustrate. Note that the term 'startup' is used here generically to describe the behavior of the device immediately following the application of a voltage across the device. Also note that data collection begins immediately on startup. Therefore, both the application of voltage and the start of data collection begin at time equal to zero on all plots $(t=$ $0)$.

Adding to the complexity of the device response is the fact that the metal/graphene interface behaves differently after startup than does the graphene film. Two-point measurements record the convolution of the interface response and film response together. However, using fourpoint measurements which include only the film response, the interface response component of the two-point measurands can be identified and its implications understood. This becomes extremely important in interpreting the high temperature gas testing results which utilized twopoint measurements only.

A photo of the device under test and an equivalent schematic is shown in Figure 5.31 $(A, B)$. Testing was carried out in the small test cell, using the MK0* design, under a flow of 10 SCCM of UHP argon. As described in Section \$3.2.5, two-point resistance measurements capture the contributions of all circuit elements (the test leads $\&$ connections resistance + contact/graphene interface resistance + film resistance). Four-point resistance measurements, on the other hand, capture the resistance of only the film between voltage sensing contacts. The locations of where the voltage readings are collected and how they are used to calculated the two-point resistance

(A)

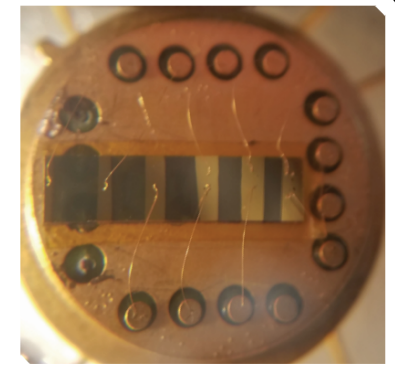

(B)

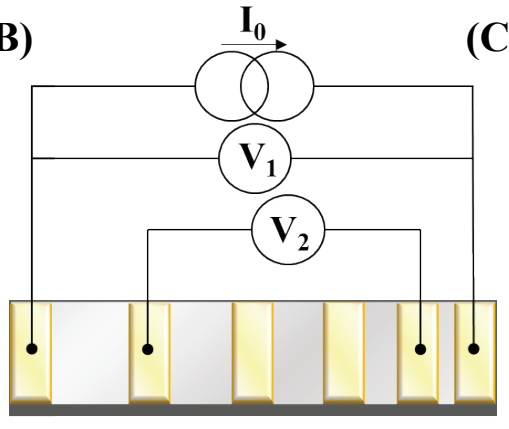

(C) 2-Point Resistance, (Total Resistance)

$$
\left(\frac{\mathrm{V}_{1}}{\mathrm{I}_{0}}\right)=\mathrm{R}_{2 \mathrm{pt}}
$$

4-Point Resistance, (Film Resistance)

$$
\left(\frac{\mathrm{V}_{2}}{\mathrm{I}_{0}}\right)=\mathrm{R}_{4 \mathrm{pt}}
$$

Figure 5.31: (A) Photo of the device tested. (B) Equivalent circuit diagram showing the various measurement points. (C) How the various resistances were calculated, using the measurement points as indicated in (B). 
$\left(\mathrm{R}^{(2 \mathrm{pt})}\right)$ ) the calculations are shown in Figure $5.31(B, C)$. The current $\left(\mathrm{I}_{0}\right)$ is the same in both calculations because all measurement points are colinear (i.e. a series circuit) and the current flow is the same at all points in the circuit. First, the device resistance after startup calculated using the two-point measurement will be discussed.

Figure 5.32(A) shows a two-point IV measurement, collected at room temperature. Due to the nonlinear nature of the device IV curve, operating the device at different voltages (or correspondingly, different current setpoints) results in a wide range of resistances for the device. In Figure 5.32(A), the blue dashed lines show 12 different operational setpoints for the device operating in constant current mode and their corresponding position on the IV curve. For each operational setpoints, Figure 5.32(B) shows the resistance of the device (on the $\log _{10}$ scale) recorded as a function of time (for clarity the $\mathrm{Y}$-axis increases in the downward direction). Clearly, a natural consequence of the nonlinear Schottky behavior is the disproportionately large resistances seen at low voltages/currents.

(A)

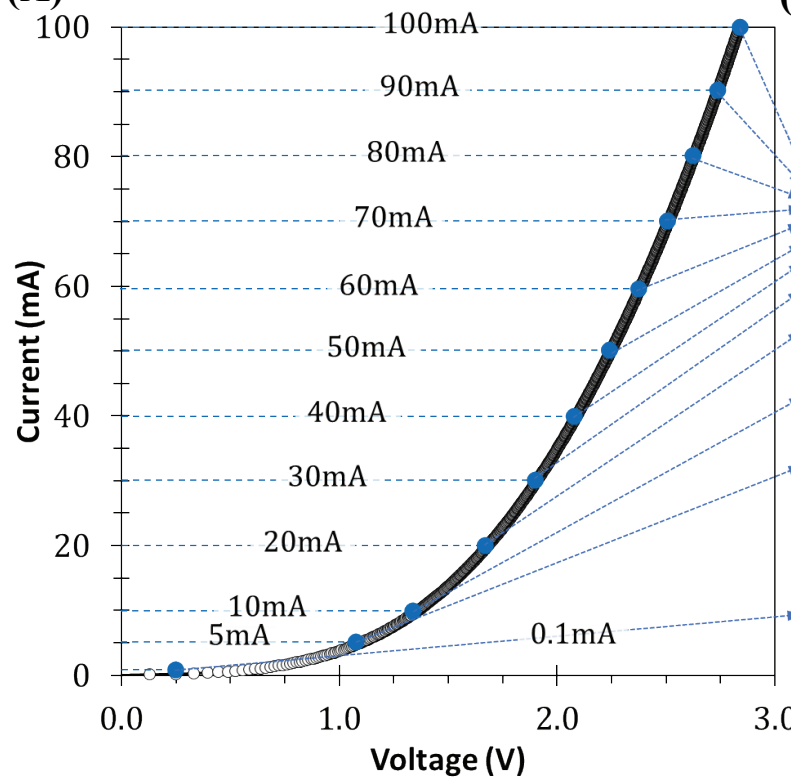

(B)

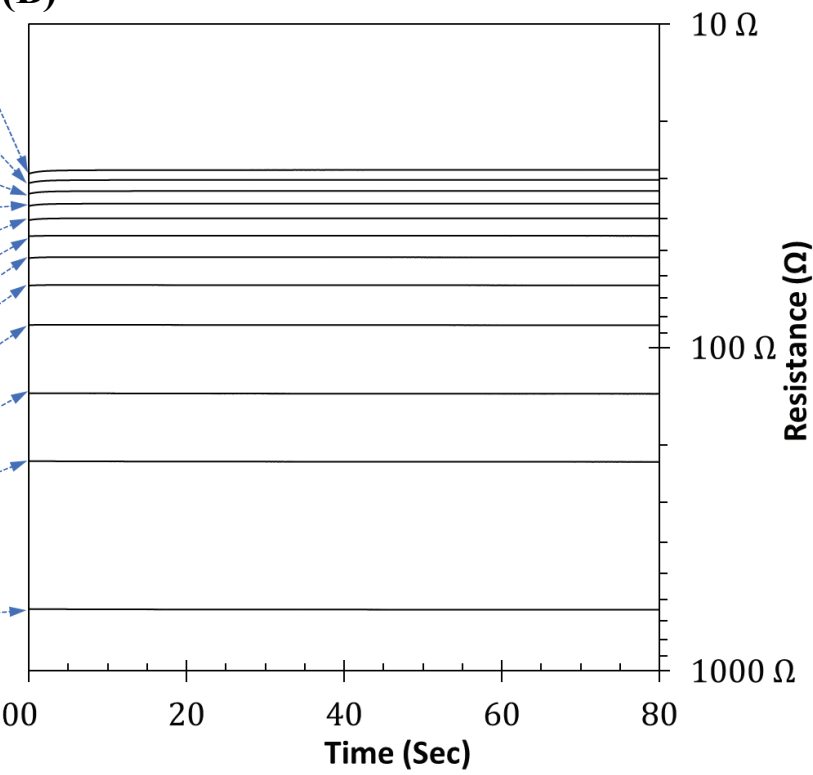

Figure 5.32: (A) A two-point IV curve collected at room temperature. 12 points of constant current operation are indicated. (B) The absolute resistance recorded over the first 80 seconds after the power is supplied to the device. Note that the vertical axis is $\log _{10}$ scale and increases in the downward direction.

Although Figure 5.32(B) illustrates how the resistance of a device can vary widely (by orders of magnitude) simply by operating at different setpoints, the important subtleties of how the device resistance changes after startup are obscured. More importantly, the different device behavior observed when operated at these different setpoints is also obscured. When the same data 
(Figure 5.32(B)) are viewed on a relative scale, however, the setpoint-dependent device behavior is clearly revealed, as shown in Figure 5.33. Figure 5.33(A) shows plots of the change in resistance $(\Delta \mathrm{R})$. Here, $\Delta \mathrm{R}$ is the change from the initial device resistance, defined as $\left(R_{t}-R_{0}\right)$, where $\mathrm{R}_{\mathrm{t}}$ is the resistance of the device at arbitrary time, t. Figure 5.33(B) shows the same data, but plotted as the percentage relative change in resistance, defined as $\left(\left(\frac{R_{t}-R_{0}}{R_{0}}\right) \times 100\right)$. It can be seen from Figure 5.33(A) that the low current setpoints lead to the largest change in device resistance. However, due to its Schottky nature, the device exhibits a very large resistance at these low setpoints. Thus, as seen from Figure 5.33(B), the percentage change in device resistance, relative to the total resistance, is small for the low setpoints.
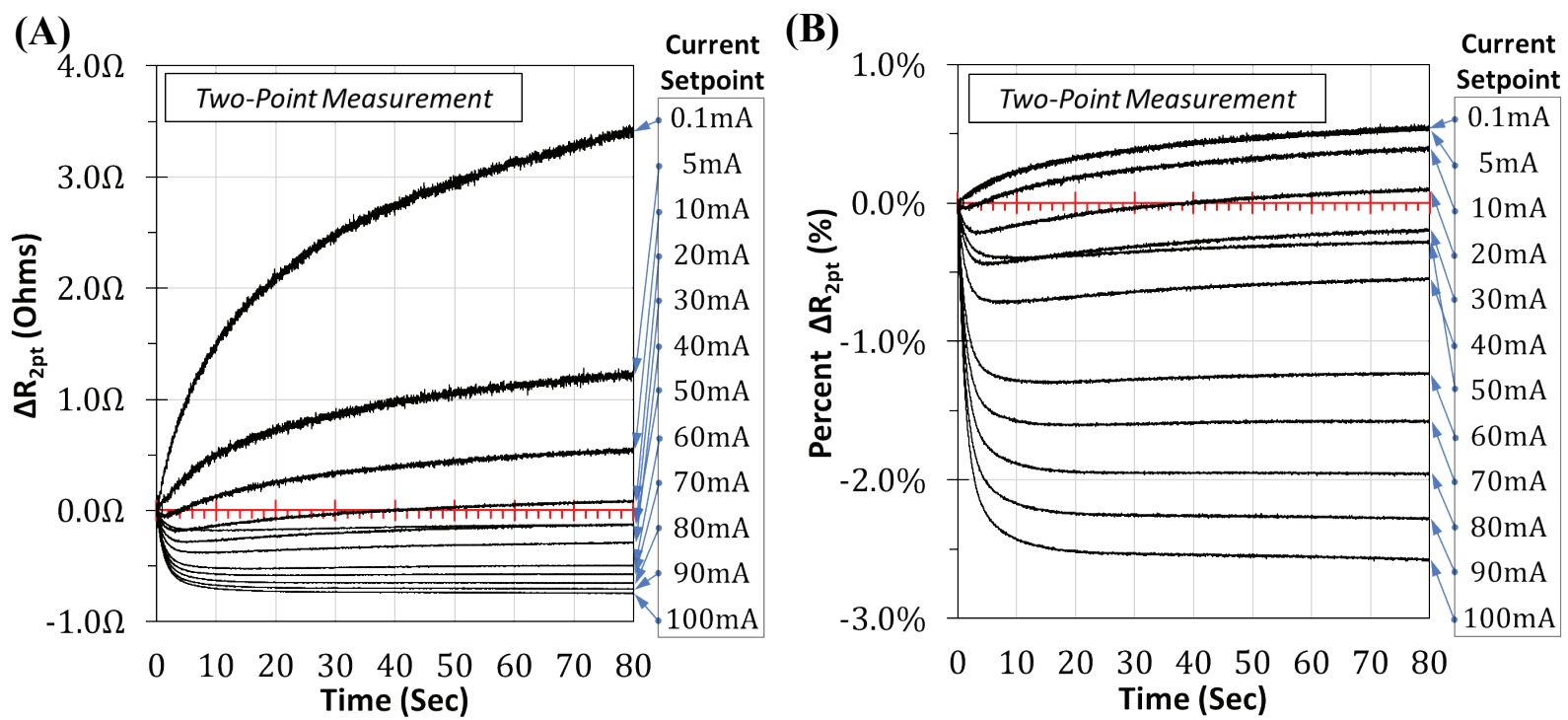

Figure 5.33: Device resistance as calculated from two-point measurements. (A) The absolute change (from $t=0$ ) in the resistance of the device at 12 different current setpoints. (B)The same data but viewed as the percent change in resistance.

The salient point to be taken from Figure 5.33 is that, at higher current levels $\left(\mathrm{I}_{0}>60 \mathrm{~mA}\right)$, the device resistance decreases monotonically. Presumably, this is due to the self-heating induced temperature rise and the resulting increase in thermionic conduction through the Schottky contact/interface. At low current levels $\left(\mathrm{I}_{0}<10 \mathrm{~mA}\right)$ the resistance increases monotonically. Intermediate current levels $\left(20 \mathrm{~mA} \geq \mathrm{I}_{0} \leq 50 \mathrm{~mA}\right)$ show a transitional behavior; first a drop, then rise in resistance (i.e. the resistance value changes non-monotonically). The data shown here is taken from a device operating in constant current mode. Identical behavior was observed for devices operating in constant voltage mode. It is unclear what the driving force is for the unexpected increase in device resistance observed at low setpoints. The following discussion on 
four-point measurements makes the case that this increase in resistance is exclusively due to effects of the metal/film interface. Possible mechanisms regarding an electronic equilibration process are discussed at the end of this section.

The analogous four-point measurements collected during the same test are shown in Figure 5.34. Again, both $\Delta \mathrm{R}$ and $\% \Delta \mathrm{R}$ are plotted at 12 different current setpoints, in Figure 5.34(A,B), respectively. The four-point method eliminates not only the effect of the leads and connections but also all contact effects. Consequently, these measurements capture the resistance behavior of the film exclusively (i.e. no metal-film effects).
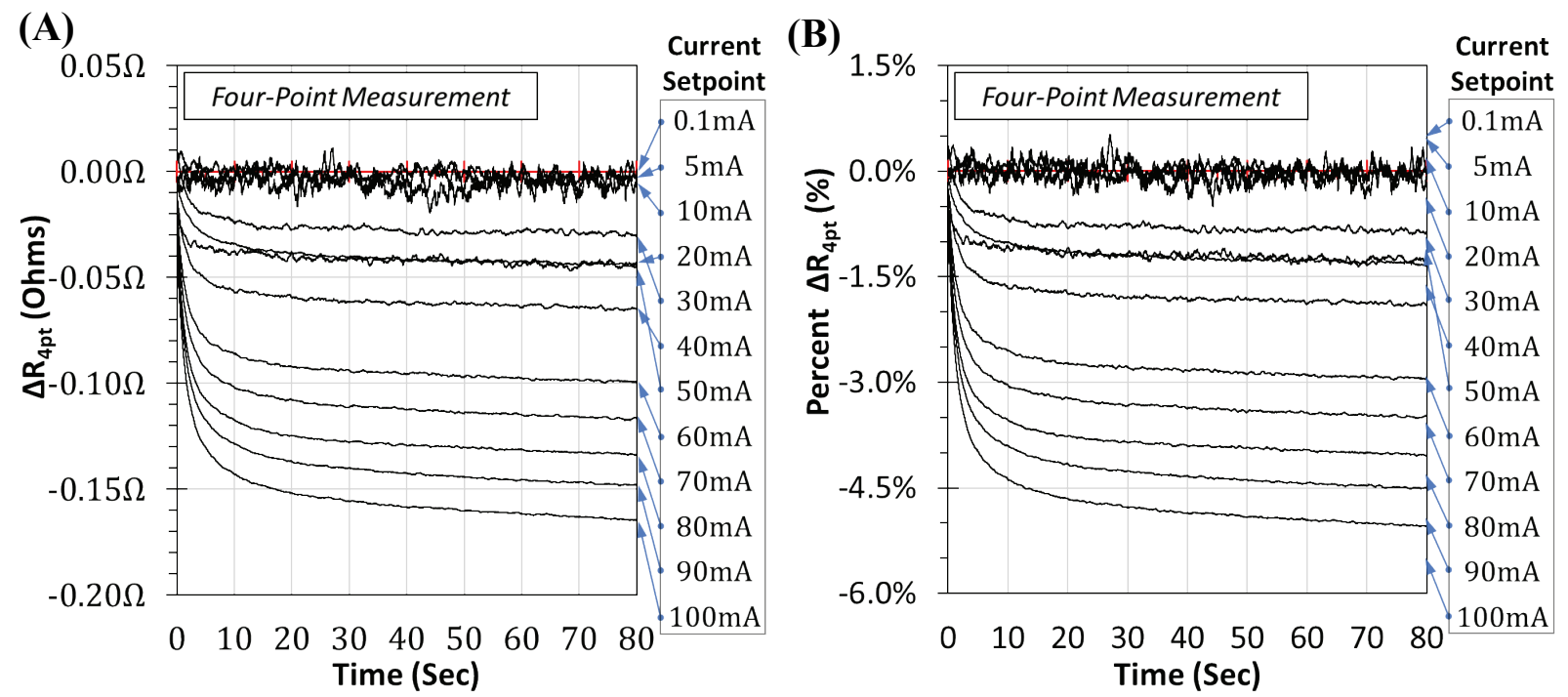

Figure 5.34: Film resistance as calculated from four-point measurements. (A) The absolute change (from $\mathrm{t}=\mathrm{o}$ ) in the resistance of the device at 12 different current setpoints. (B)The same data but viewed as the percent change in resistance.

The notable difference between Figure 5.33 (two-point measurement) and Figure 5.34 (four-point measurement) is that, while the two-point device resistance may either increase or decrease with time (depending on the setpoint), the four-point measurement show that the film resistance either remains constant for low setpoints $(<10 \mathrm{~mA})$ or decreases for higher setpoints. Therefore, by process of elimination, the increase in the device resistance observed at low current in the two-point measurements must be due to contact interface specific (metal/graphene) effects and is unrelated to the film. ${ }^{40}$ Thus, for two-point measurements, depending on the power applied

\footnotetext{
${ }^{40}$ Although the lead wires and related connections are ohmic and theoretically self-heat (resulting in an increase of resistance in response to the temperature rise) the power dissipated by the setup at the low current setpoints is miniscule and all auxiliary components have a comparatively very low resistances, so they likely do not contribute to the response at this temperature (other than a small, additive, static series resistance offset included in all two-point measurements).
} 
to the device (current and voltage), either the interface effects or film effects may dominate. This leads to the various behaviors shown in Figure 5.33. In four-point measurements, only Joule heating effects on the film impact the behavior. Joule heating in the low current regime is not sufficient to affect the film resistance, and thus the resistance remains constant after startup. For higher current setpoints $(>10 \mathrm{~mA})$, the decrease in resistivity is consistent with a rise in temperature due to Joule heating. The leveling off in the resistance value with time is due to the establishment of an equilibration temperature where Joule heating and gas cooling balance.

The mechanism of the resistance decrease for both the film and the interface is straightforward. It is temperature related in both cases. At $\mathrm{t}=0$ a voltage is applied to the device, current begins to flow and, due to charge scattering events, self-heating begins. This rises the temperature of the device. Because most of the voltage drop occurs across the contact interface, the majority of the heat is dissipated there. The decrease in resistance is thus due to thermal promotion of charge carriers to slightly higher energy levels where the Schottky barrier effects are reduced (see Section $\$ 5.2$ for an in-depth discussion of this effect). Although much smaller, there is also a voltage drop across the film, between the source and drain contacts. This too leads to a temperature increase of the film and reduction in film resistance. The resistance decrease in this case is, however, due to thermal promotion of charge carriers which increases the population in the conduction band of the film (recall conductivity is proportional to carrier density, $\mathrm{N}_{\mathrm{d}}$, and carrier mobility, $\mu$ ).

It is interesting to note that the decrease in film resistance (four-point measurements) is approximately an order of magnitude less that the decrease in the combined graphene/metal interface and film resistance change (two-point measurements). Although more film is captured in the two-point measurements, this would account for a reduction of two- to three-fold at most. Consequently, the data suggest that both the resistance decrease (at high current setpoints) and resistance increase (at low current setpoints) is dominated by the resistance change of the metal/graphene interface.

The mechanism causing the increase in device resistance (two-point measurement) when the device is powered at low setpoints, is unclear. As discussed earlier, the effect is isolated to the contact/film interface and is observable only at low current/voltage setpoints. However, as seen in Figure 5.33, there is evidence of this mechanism at play for setpoints as high as $50 \mathrm{~mA}$ (as seen by the rise in resistance observable after the fall over the initial 8 seconds of operation). It is unclear 
whether this mechanism is at play at setpoints higher than this or not. Considering this data only, it is unclear if the effect is time dependent or temperature dependent because, due to the nature of self-heating, the temperature increases with time. However, as will be discussed in Section \$5.3.3, further testing, where the gas flow rate is modulated, indicates the increase in resistance after startup for low setpoints is independent of temperature. Even so, it is difficult to confidently assign a mechanism to this particular behavior. However, two potential phenomena are discussed next which may explain the behavior; each amount to an electronic equilibration of the metal/graphene interface.

Current crowding within the charge trapping pathways may contribute to the reduction in current flow with time (increasing resistance) observed in the two-point measurements collected at low setpoints. As the pathways become saturated with carriers of like charge, charges may become trapped. When a carrier becomes surround by other carriers of the same charge, the propensity for this carrier to move is reduced, as it is repelled by the coulomb field of the neighboring carriers. This effect is more readily apparent at low setpoints because the driving potential (voltage) across the interface is small and the thermally-induced increase in mobility through the hopping pathways is also small. Stated another way, the charge trapping pathways may only accommodate a small number of carriers before carrier crowding occurs in the pathway at low voltages. As the pathways become crowded with time, this is reflected in the observed increase in resistance.

Alternatively, capacitive effects at the contact/film interface (i.e. junction capacitance) may be responsible for the increase in resistance. The very low conductivity across the interface at low voltages may make the interface appear as a pseudo-parallel plate capacitor (where the metal contact acting as one plate and the film as the other, with the Schottky barrier between). This would hinder the DC flow of carriers, particularly at low voltage. If this is the case, the rise in resistance is effectively recording the charge accumulation (analogous to the charging of a capacitor plate) on one side of the interface. In fact, the very recent report by Levi, et al. which details a similarly constructed graphene Schottky diode, showed their device to act as a varactor diode. ${ }^{41(663)}$ Their device was shown to have a capacitance-voltage curve which peaked at $0 \mathrm{~V}$ bias and decreased

\footnotetext{
${ }^{41}$ Varactor (a device with a variable reactance) diodes are devices which show a voltage dependent capacitance across their junction (i.e. a voltage-dependent change in capacitive reactance, hence the name, variable + reactance $=$ varactor). (746)
} 
with increasing voltage. Assuming a similar scenario is occurring here would support this hypothesis and would fully explain the increase in resistance with time at low biases. Collecting $\mathrm{C}-\mathrm{V}$ (capacitance vs voltage) curves, which the current setup is not capable of, on these devices will go a long way to determining if this mechanism is responsible.

A final note on this behavior is that the specific setpoint at which each effect dominates varies between samples and device designs. Furthermore, because the four-point measurements can only be taken using a room temperature setup, it is unknown exactly how the two effects change as the device is externally heated. However, the added insight that the four-point measurements of Figure 5.34 provides to the interpretation of the two-point measurements of Figure 5.33 can be extended to the two-point high temperature gas test measurements discussed later in this section.

In summary, while the startup effects are complicated and present an opportunity for an interesting scientific investigation, in regard to gas sensing, the results of this study show that these startup behaviors can be ignored. That is to say, as long as a steady state has been reached by the device, the complex swings in device resistance at startup do not need to be considered when differentiating between the thermal effects and chemical effects of the gas environment on the device response. This simplifies the primary topic of discussion in Section $\$$ 5.3.4.

\section{§5.3.3 Inert Gas Exposure: Effect of Flow Rate, Physical Properties and Establishment of the Thermal Effect}

Before the results of gas sensing can be discussed, various exogenous influences must be identified and their effects on the device response examined. This section will discuss these effects, which are purely physical in origin (i.e. not chemically derived). Specifically, the physical effect of gas composition and flow rate on device response are investigated here. These effects influence the rate of heat removal ( $\dot{Q}$ via Equation 5-6) from the device which, when powered, always generates heat by Joule heating. The amount of heat energy generated is proportional to power via Equation 5-5 and is thus depended on the device setpoint; lower setpoint generate less heat and vice versa.

In Equation 5-6, it may be recalled the quantity $\left(\frac{k A}{l}\right)$ represents the effective heat transfer coefficient. While the area (A) of the device remains constant in a given experiment, the thermal conductivity (k) will depend on the gas composition. The effective thermal boundary layer thickness $(l)$ will be determined by the flowrate and also, to some extent, the viscosity and density 
of the gas, which is composition dependent. In general, however, the flowrate will be the dominate factor controlling the boundary layer thickness which will decrease with increasing flow rate and vice versa. Therefore, the device response due to any combination of these mechanisms is referred to here as a 'thermal response' or 'thermal effect'. These are effects which are derived from the thermal interactions of the device with its environment and thus, are independent of the device's chemical environment. Table 5-2 lists the thermally relevant physical properties of the gases used in this work, the specific heat capacity $\left(\mathrm{C}_{\mathrm{P}}\right)$ and thermal conductivity $(\mathrm{k})$, of which thermal conductivity is the most important.

Table 5-2: Specific heat $\left(\mathrm{C}_{\mathrm{P}}\right)$ and thermal conductivity $(\mathrm{k})$ of the gases used in this study (data collected at $\sim 300 \mathrm{~K}){ }^{(664)}$

\begin{tabular}{|c|c|c|}
\hline & $\mathbf{C}_{\mathbf{P}}$ & $\mathbf{k}$ \\
\cline { 2 - 3 } & $\left(\frac{J}{g \cdot K}\right)$ & $\left(\frac{W}{m \cdot K}\right)$ \\
\hline Hydrogen $\left(\mathrm{H}_{2}\right)$ & 14.267 & 0.182 \\
\hline Helium $(\mathrm{He})$ & 5.300 & 0.149 \\
\hline Methane $\left(\mathrm{CH}_{4}\right)$ & 2.260 & 0.035 \\
\hline Carbon Monoxide $(\mathrm{CO})$ & 0.104 & 0.025 \\
\hline Argon $(\mathrm{Ar})$ & 0.523 & 0.017 \\
\hline
\end{tabular}

To illustrate the effect of gas composition on the device thermal response, consider the experimental data shown in Figure 5.35. This test was carried out in the small test cell, using the MK0* device package and no external heating (the setup was at room temperature). The device was operated in a constant current mode with a $60 \mathrm{~mA}$ setpoint. Argon is used as the carrier gas and helium is used as the pulsed analyte gas. Given the inert nature of both argon and helium, it is assumed that both gases do not chemically interact with the device and the only response is due to the differences in thermal conductivity of $\operatorname{Ar}\left(0.017\left(\frac{W}{m \cdot K}\right)\right)$ and $\mathrm{He}\left(0.149\left(\frac{W}{m \cdot K}\right)\right)$ since the flow rates are kept constant (thus, $l$ is unchanged). The gas flow is initially composed of $100 \%$ Ar. After 410 seconds of equilibration time, the gas flow is switched stepwise to $100 \%$ He for 200 seconds and then switched back to Ar. This process is repeated several times as seen in the figure. By cycling between the two gases, the device response to the physical properties of its gas environment can be observed. The data indicated by the black circles $(0)$ is the two-point response and shows the response of the device (film + contacts). The four-point reflects the film response alone and is 
indicated by the blue diamonds $(\diamond)$. Figure $5.35(A, B)$ plots the device response as the absolute change in resistance and a percent relative change in resistance, respectively.
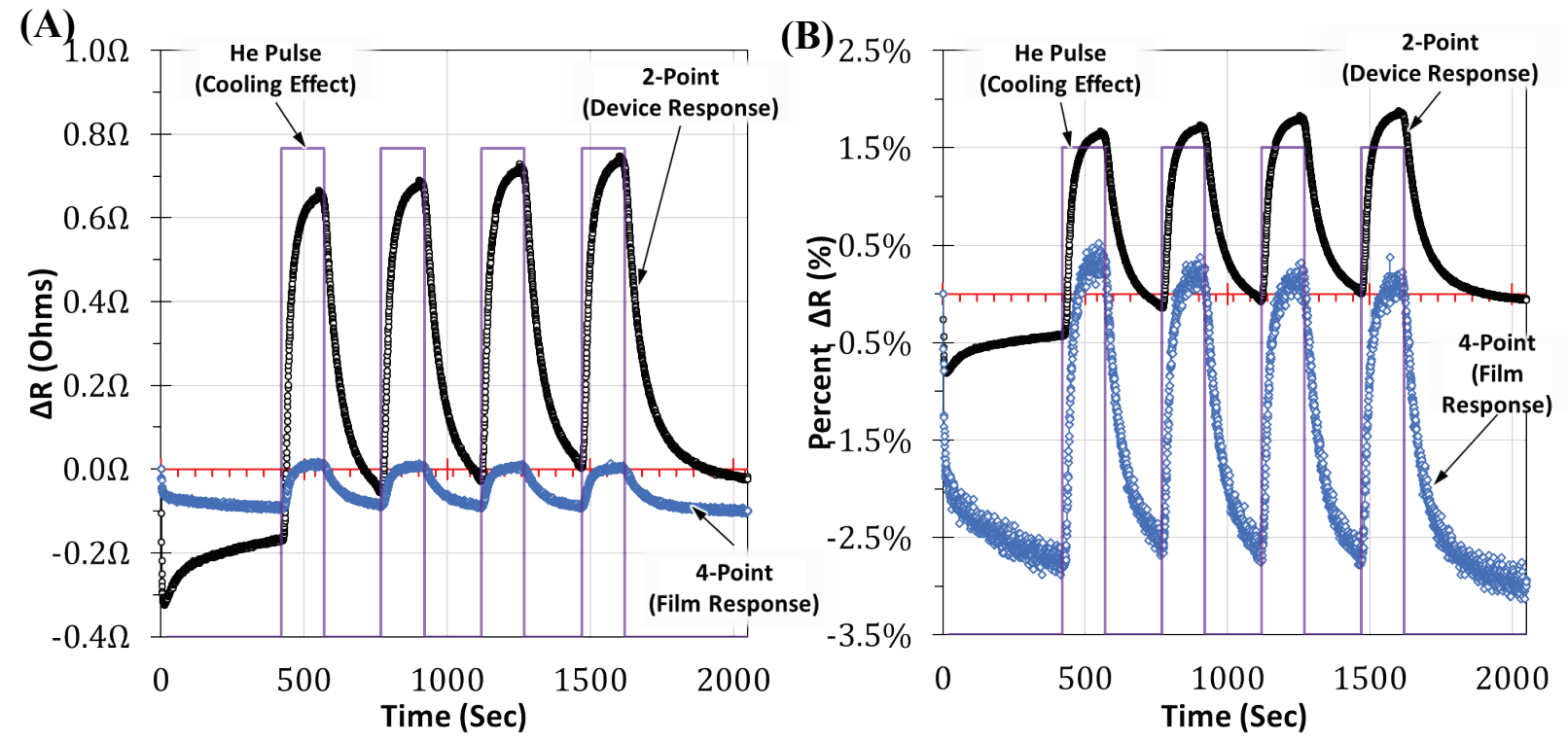

Figure 5.35: The device behavior in response to cycling between two inert gases, argon and helium, with different physical properties. (A) The response taken as the change in resistance and (B) the same response calculated as a percentage change in resistance. The setpoint was $60 \mathrm{~mA}$.

As seen in Figure 5.35 the device response initially assumes a value consistent with the level of Joule heating at the operational setpoint and the heat transfer rate resulting from the gas flow rate and composition of the gas stream at that time. When the flow is switched to He (at the same flowrate) the primary change is an increase in the thermal conductivity of the gas stream. From the data shown in Table 5-2, it can be seen that the thermal conductivity of helium is a factor of $9 \mathrm{x}$ higher than argon. The physical consequence of this is a higher effective heat transfer coefficient and a higher rate of heat transfer from the sample to the gas stream. Overall, this shifts the balance between the heat removal rate and heat generation rate towards heat removal, resulting in a lower equilibrium temperature for the device at the given operation setpoint. For both twoand four-point measurements an increase in resistance is observed in response to reducing the device temperature. This is consistent with all previously discussed results.

Figure 5.36 illustrates a similar thermal response. However, in this case, the response is due to changes in the volumetric/mass flowrate $(\dot{V}$ or $\dot{m})$. This influences the effective heat transfer coefficient by changing the boundary layer thickness. In Figure 5.36 the device response to a stepwise increase in flowrate from 10 SCCM to 700 SCCM and back to 10 SCCM of argon is shown (small test cell, room temperature, TLM contact pattern, MK0* package design with a 
$100 \mathrm{~mA}$ current setpoint). The data shown in Figure 5.36 was collected from the same device which produced the data shown in Figure 5.32, Figure 5.33, Figure 5.34, and Figure 5.35.
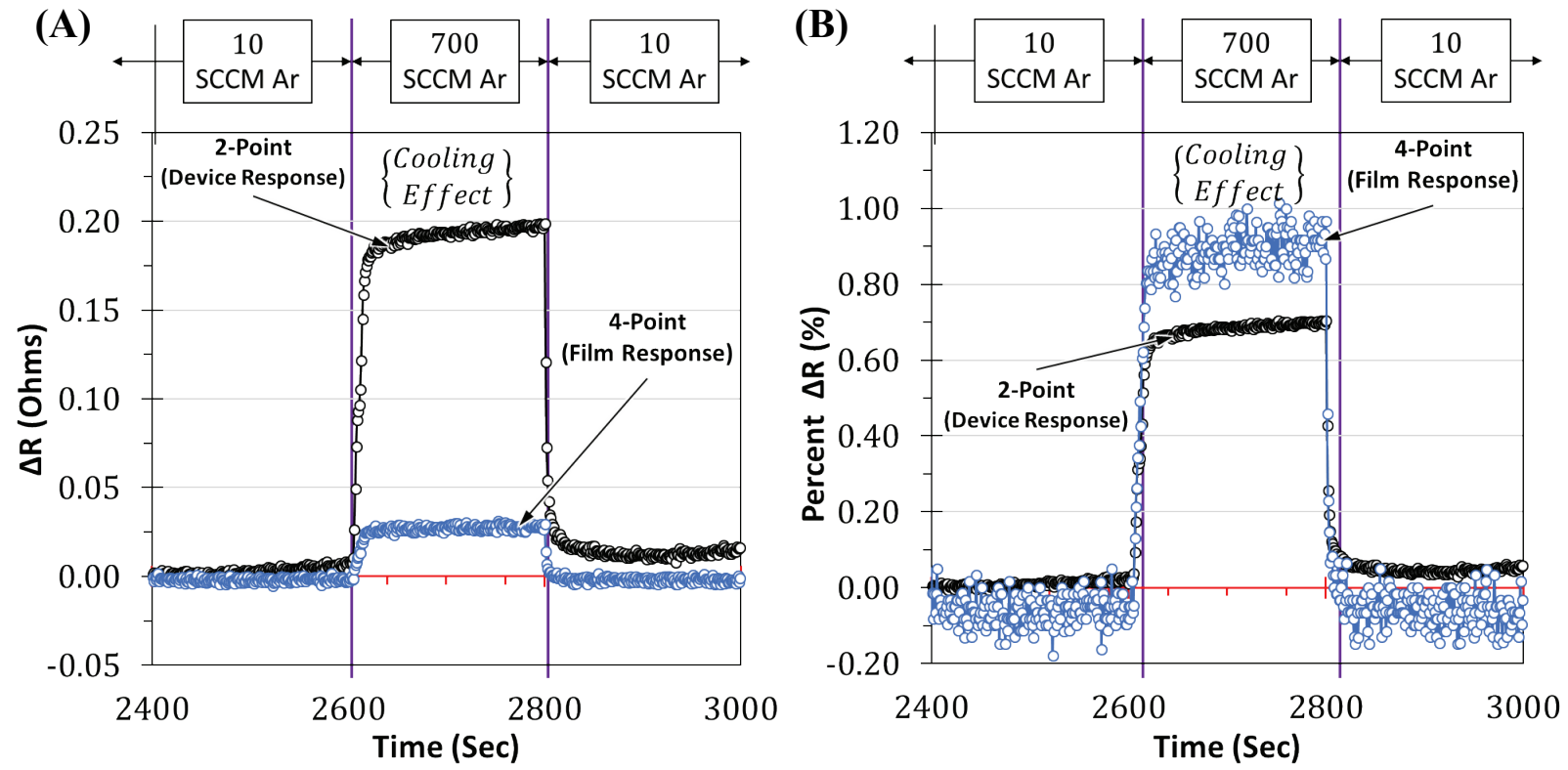

Figure 5.36: The device response due to changes in gas flowrate. The device was allowed to come to a full thermal equilibrium under a flow of 10 SCCM of argon. The flow was then stepwise increased to 700 SCCM for 200 seconds, then reduced back to 10 SCCM. The device response to a purely thermal (cooling) effect is thus illustrated. (A) The response as an absolute change in resistance and (B) a percentage change in resistance. The current setpoint was 100mA. The setpoint was 100mA.

In this test, it can be seen once again in both the two- and four-point measurements, that increasing the heat transfer coefficient, and consequently lowering the device temperature, results in an increase in device resistance. It is clear from these results that the film contributes the smallest absolute change in resistance but undergoes the largest percentage change.

Figure 5.36 illustrates the effect of cooling the device by increasing the gas flowrate. Figure 5.37 presents the companion data to this, where the effect of heating the device by reducing the gas flowrate is recorded. Figure 5.37 shows the results of rising the device temperature by reducing the Ar flowrate in a stepwise manner. This has the effect of increasing the boundary layer thickness and, thus, reducing the effective heat transfer rate. As a result, the temperature of the device increases to a new (higher) temperature, for the duration of the pulse. This test was carried out in the small test cell, using a $2.5 \mathrm{~mm}$ x $2.5 \mathrm{~mm}$ device having the interdigitated sensor contact pattern, was mounted using the MK0 package design and operated in constant voltage mode. Initially, the flowrate was set to $500 \mathrm{SCCM}$ of argon. At $\mathrm{t}=0$ a bias was applied to the device. After some time, the gas flow was switched off (0 SCCM) for 100 seconds and then back on to 
500 SCCM. This was done twice in each test. This test was carried out at six different voltage setpoints: $0.100 \mathrm{~V}, 0.500 \mathrm{~V}, 1.00 \mathrm{~V}, 1.50 \mathrm{~V}, 2.00 \mathrm{~V}$ and $2.30 \mathrm{~V}$.

(A)

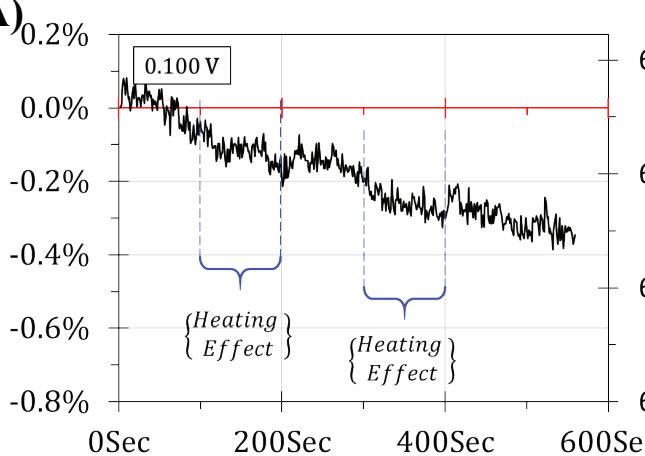

(B)
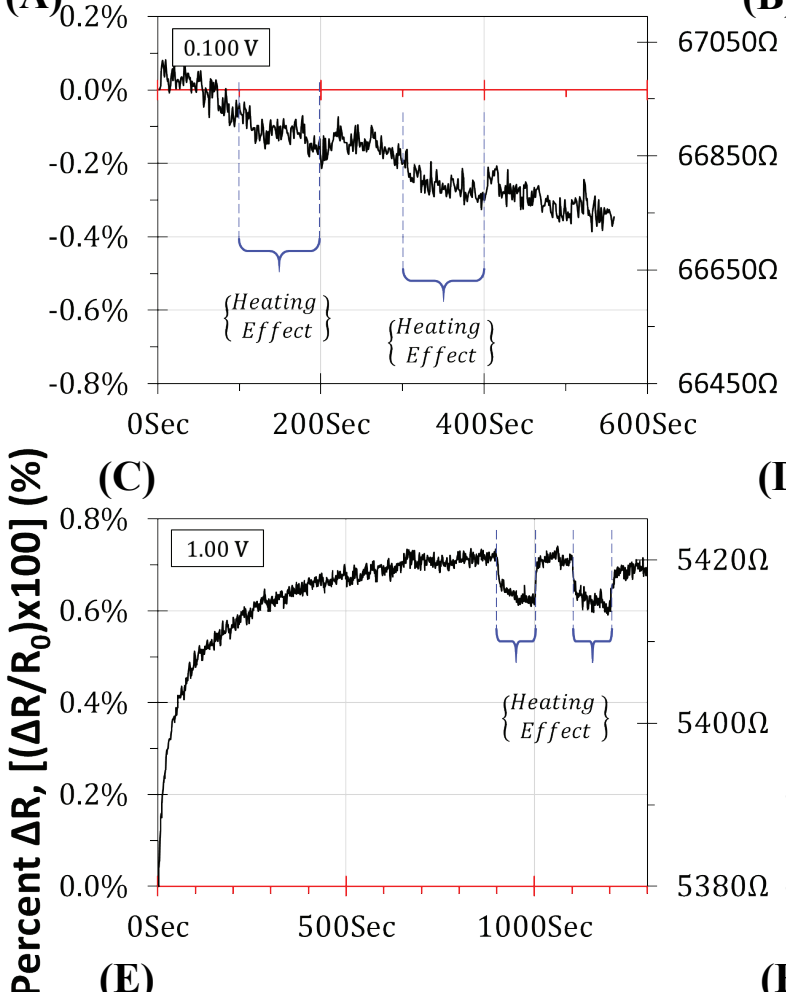

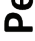

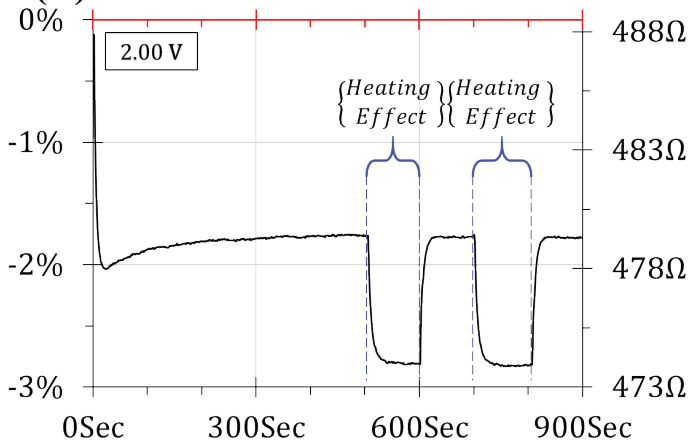

(F)
(D)
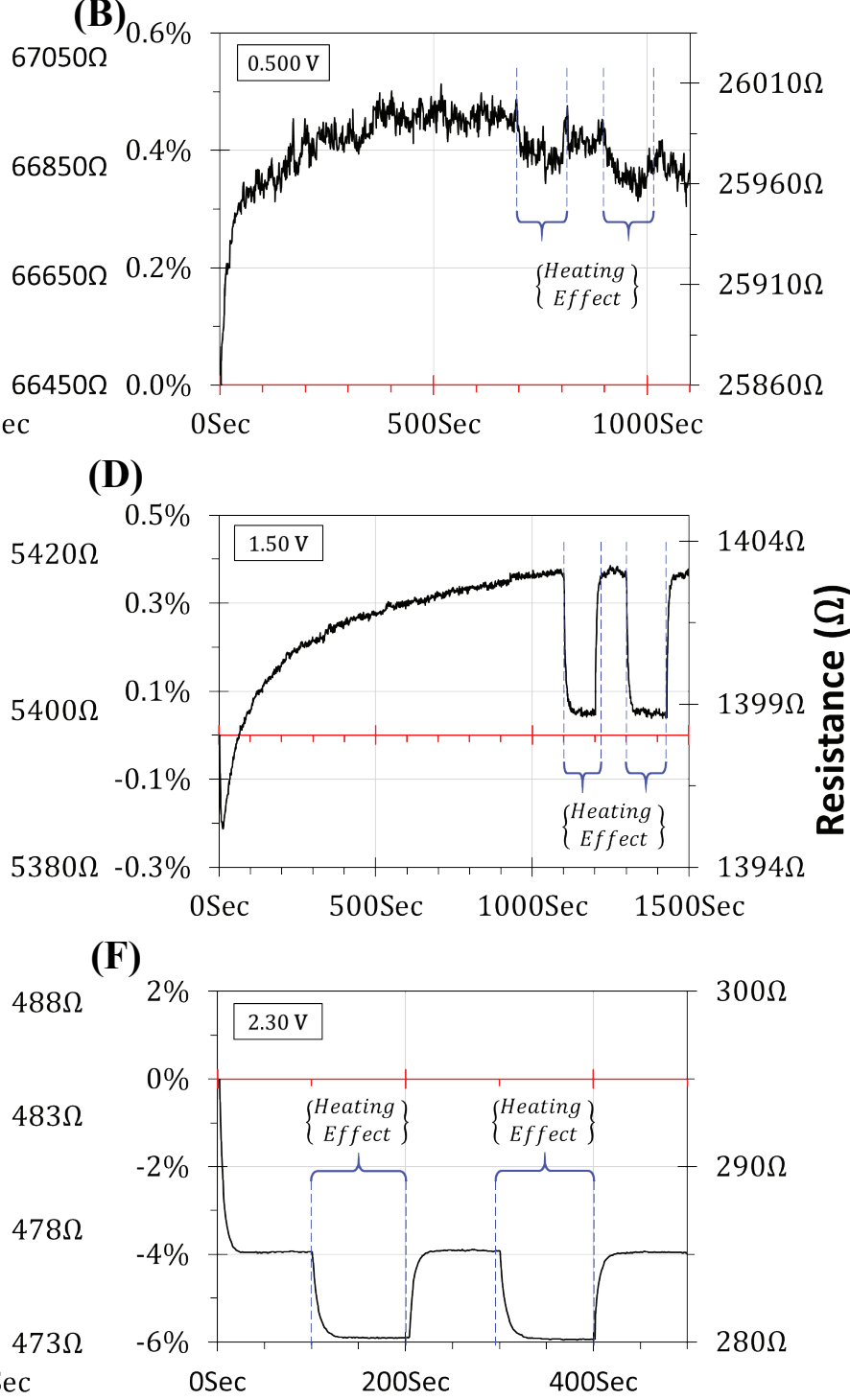

Time (Sec)

Figure 5.37: The startup behavior and subsequent response to reducing the Ar gas flowrate from 500 SCCM to o SCCM, effectively heating the device, performed at six different constant voltage setpoints. (A) $0.100 \mathrm{~V}$, (B) $0.500 \mathrm{~V}$, (C) $1.00 \mathrm{~V}$, (D) $1.50 \mathrm{O},(\mathrm{E}) 2.00 \mathrm{~V},(\mathrm{~F}) 2.30 \mathrm{~V}$.

A crucial point is revealed in Figure 5.37; at room temperature and for setpoints, heating the device reduces the resistance of the device. The implications of this observation are twofold. First, in regard to the startup behavior as discussed in Section \$5.3.2, this observation indicates that the rise in resistance observed at low setpoints at device startup is a time dependent phenomena and not temperature related. For instance, consider Figure 5.37(B,C,D). After a voltage is applied 
at $\mathrm{t}=0$, the device resistance increases. This initial rise in resistance is not the result of self-heating effects because, when the temperature of the device is raised further by a reduction in the gas flow rate, a reduction in resistance is observed. Therefore, the initial resistance increase cannot be due to heating. Stated another way, both the initial rise in resistance (seen at low setpoints) and the fall in resistance seen due to stopping the flow of gas cannot both be due to device heating. This establishes that the initial rise in resistance in not temperature related. This gives insight into the possible mechanisms responsible for the initial rise in resistance, which are discussed at the end of Section \$5.3.2. The second implication of this observation involves the interpretation of chemical versus thermal gas effects and will be discussed in-depth in the next section.

A defining characteristic of the thermal response illustrated in Figure 5.36 and Figure 5.37 is the rapid response rate. The high thermal conductivity of both the film and the SiC substrate mean that the temperature of the device rapidly changes and tracks with the environmentally modulated self-heating effects. The device temperature swings are rapidly reflected in the data. In fact, at the flowrates used in these experiments (10-700 SCCM), there appears to be no lag between the composition of the gas mixture within the chamber and the thermal response (i.e. the thermal response rate is faster than the rate of change in the concentration in the test chamber).

That is, the thermal response is reflective of the instantaneous composition of the gas mixture in the chamber and tracks with the actual shape of the gas concentration pulse. This can be concluded by comparing the observed thermal response to the theoretically calculated concentration of the analyte gas within the test chamber. Using elementary principles of chemical reactor design engineering, the system can be modeled as a plug flow reactor (PFR) in series with a poorly mixed continuously stirred tank reactor (CSTR). (665) Using this approach, the concentration in the test chamber can be calculated as function. This is the so-called 'C-curve', commonly used to calculate the pulse shape, or residence time distribution, and evaluate flow characteristics of a feed stream though a reactor system. This is qualitatively shown in Figure 5.38. 


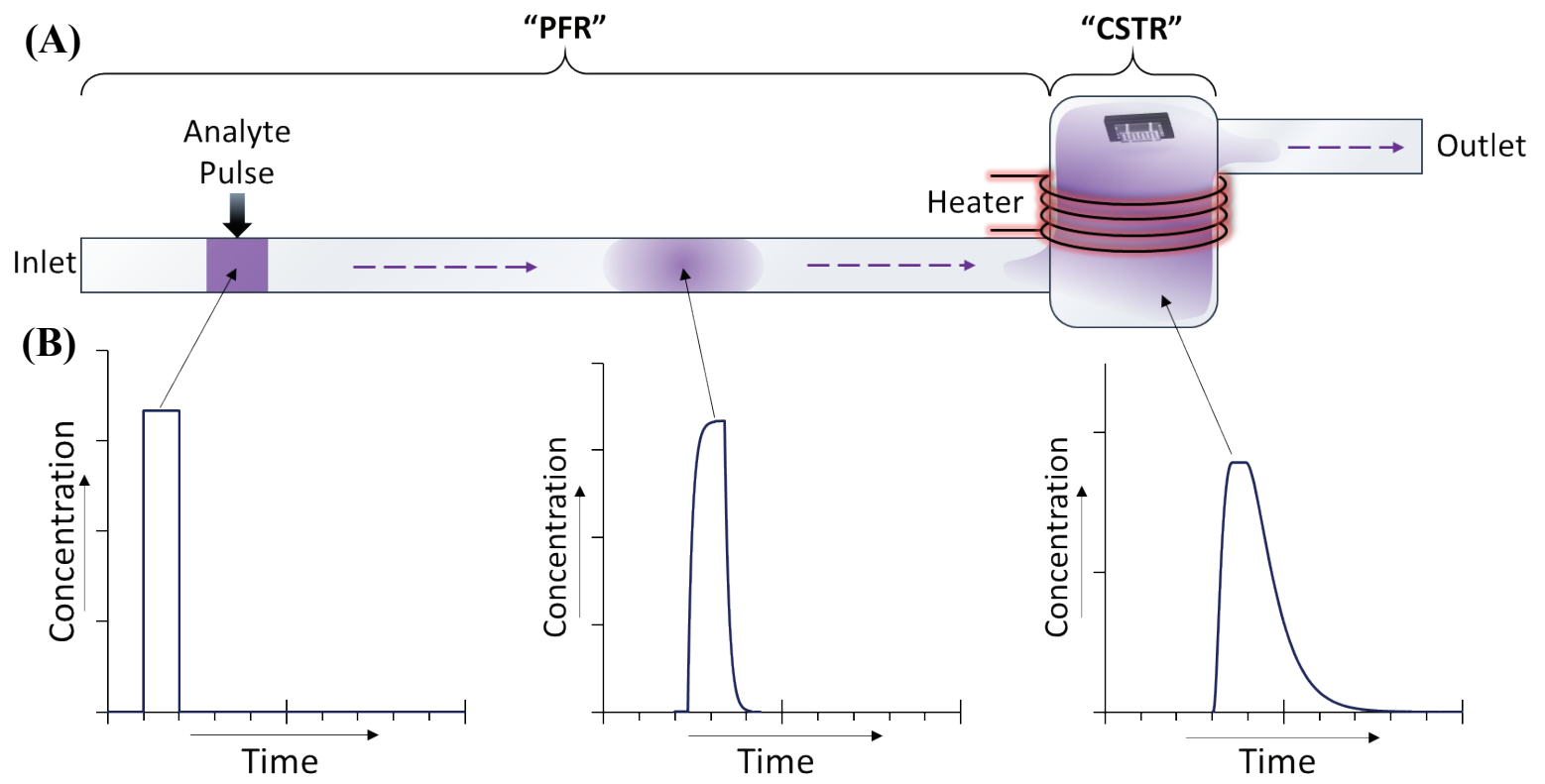

Figure 5.38: By modeling the test setup as a PFR in series with a CSTR, the concentration in the test chamber can be qualitatively calculated. (A) A generic schematic of the testing system. (B) The gas pulse shape at various points in the test system. The mixing is enhanced at higher temperatures.

The pulse of analyte gas moved linearly down the system as a plug. Diffusion of the analyte pulse into the carrier stream at the plug faces blurs out the leading and trailing edge of the pulse. Mixing within the test chamber broadens out the pulse response further. At higher temperatures, the increased molecular diffusion increases the gas mixing within the test chamber and the $\mathrm{C}$-curve broadens slightly (the flow within the chamber becomes less laminar-like and more turbulent-like). Thus, although the input function of the analyte is a pure square pulsed impulse, due to the diffusion and mixing which occurs, the concentration in the test chamber is broadened in the way shown in Figure 5.38(B). This sets an upper limit to the response of the devices. The thermal effect matches qualitatively very well with this model, indicating the thermal response is very rapid and is reflective of the composition in the chamber. On the other hand, the chemical response, discussed in Section \$5.3.4, is observed to be much slower. The rapid response of the thermal effect can therefore be used to help differentiate between thermal and chemical effects.

In summary, the results presented and discussed in this section show two important results. First, the physical environment, and by extension its influence on the device temperature, must be considered when analyzing the response of these devices. Second, increasing the device temperature always reduces the resistance of the device, regardless of the voltage or current setpoint. Conversely, reducing the temperature of the device always increases the device resistance. Although all results discussed in this section were collected at room temperature, 
similar effects were observed at all low to moderately-high temperatures tested. An important caveat to this is device operation at high temperatures where the device has surpassed its point of minimum conductivity. Above this point, the temperature effects reverse. Further heating of the device reduces conductivity and vice versa. This was discussed in Section $\$ 5.2 .1$. Additionally, through four-point and two-point measurements, the environmental effects were shown to affect both the film and the contact interface in much the same way as discussed in Section $§ 5.3 .2$.

\section{§5.3.4 Restometric Sensing of Hydrogen Gas: Thermal and Chemical Response}

This section presents and discusses the results of SOP devices functioning as gas sensors. A large number of devices $(>40)$ were exposed to various gas compositions across a wide range of temperatures and device setpoints. In almost all experiments $(>500)$, the devices show a clear response to a change in the composition of gas environment for all gases tested $\left(\mathrm{CO}, \mathrm{Ar}, \mathrm{H}_{2}, \mathrm{CH}_{4}\right.$, $\mathrm{He})$. In the vast majority of testing conditions, thermal effects dominate the response. The thermal response and related effects are discussed in Sections $\$$ 5.3.1 through Section $\$$ 5.3.3. Under certain conditions, however, a chemical response can be observed.

Figure 5.39(A) shows the IV curve of a device collected at three different temperatures $\left(25^{\circ} \mathrm{C}, 230^{\circ} \mathrm{C}, 410^{\circ} \mathrm{C}\right)$ collected under a steady state flow of $20 \mathrm{SCCM}$ of UHP argon. This test was carried out in the large test bed, using the MK III sensor package design. Figure 5.39(B) shows the positive quadrant of this data over a reduced voltage range. Six points of device operation are indicated by the colored arrows. Figure 5.39(C) plots the resistance of the device, calculated at a $0.100 \mathrm{~V}$ bias, as it is heated from room temperature to $810^{\circ} \mathrm{C}$ (similar data was discussed in Section \$5.2.1). The back circles ( $($ ) are the data points. The red dashed line indicates a polynomial best fit line described by, $\mathrm{R}=-4 \times 10^{-13} \mathrm{~T}^{5}+1 \times 10^{-9} \mathrm{~T}^{4}-1 \times 10^{-6} \mathrm{~T}^{3}+0.0005 \mathrm{~T}^{2}-0.1178 \mathrm{~T}+$ 21.02 , where $\mathrm{R}$ is the device resistance $(\Omega)$ and $\mathrm{T}$ is the temperature $\left({ }^{\circ} \mathrm{C}\right)$. The important fact to note here is the transition or turning point occurring at $296^{\circ} \mathrm{C}$. This is the temperature where the device response to a temperature change reverses. For temperatures less than this (referred to as the 'low temperature regime') the conductivity is dominated by thermionic emission and an increase in temperature reduces the effective resistance of the device. For temperatures above this 
(referred to as the 'high temperature regime') scattering effects begin to dominate and an increase in temperature increases the device resistance.

(A)

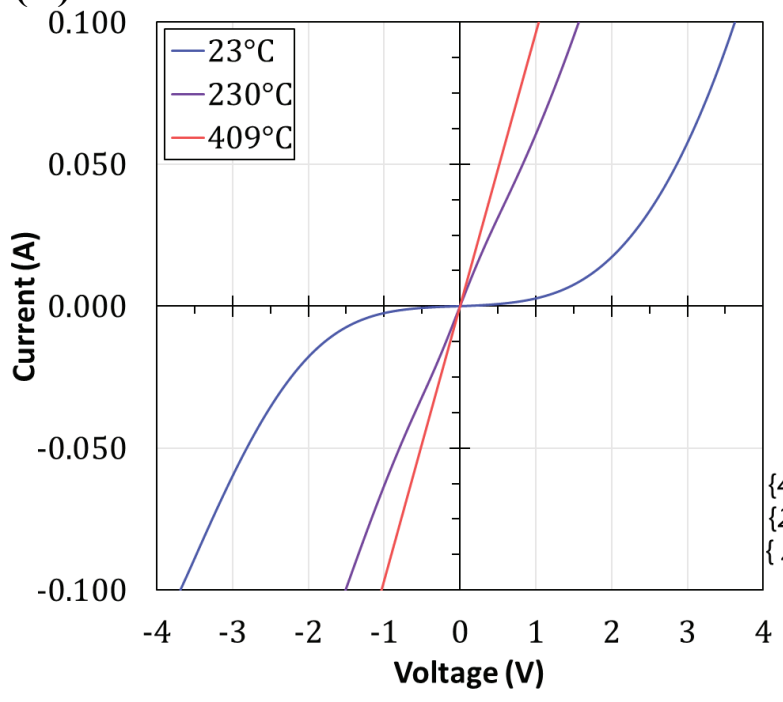

(B)

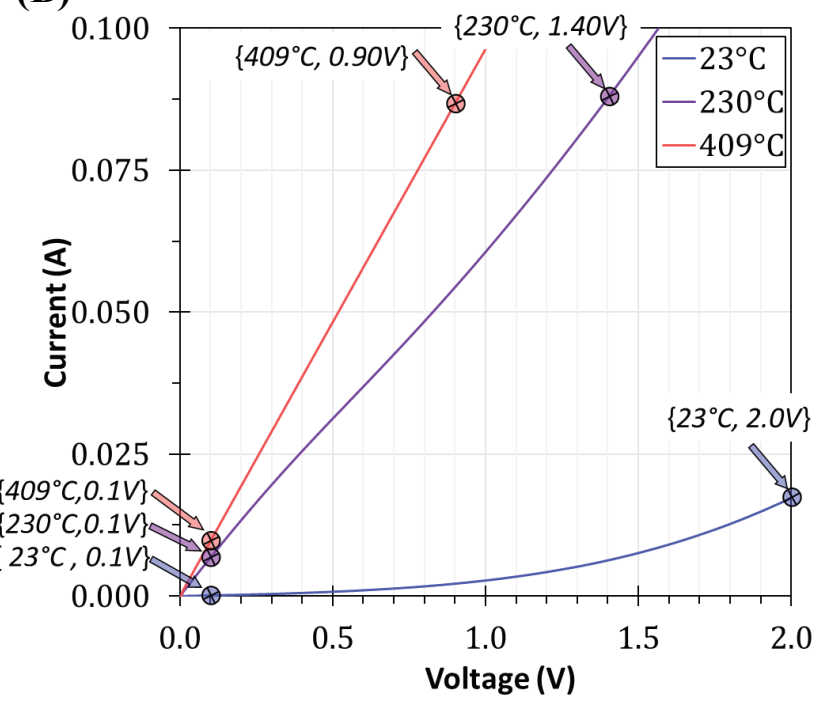

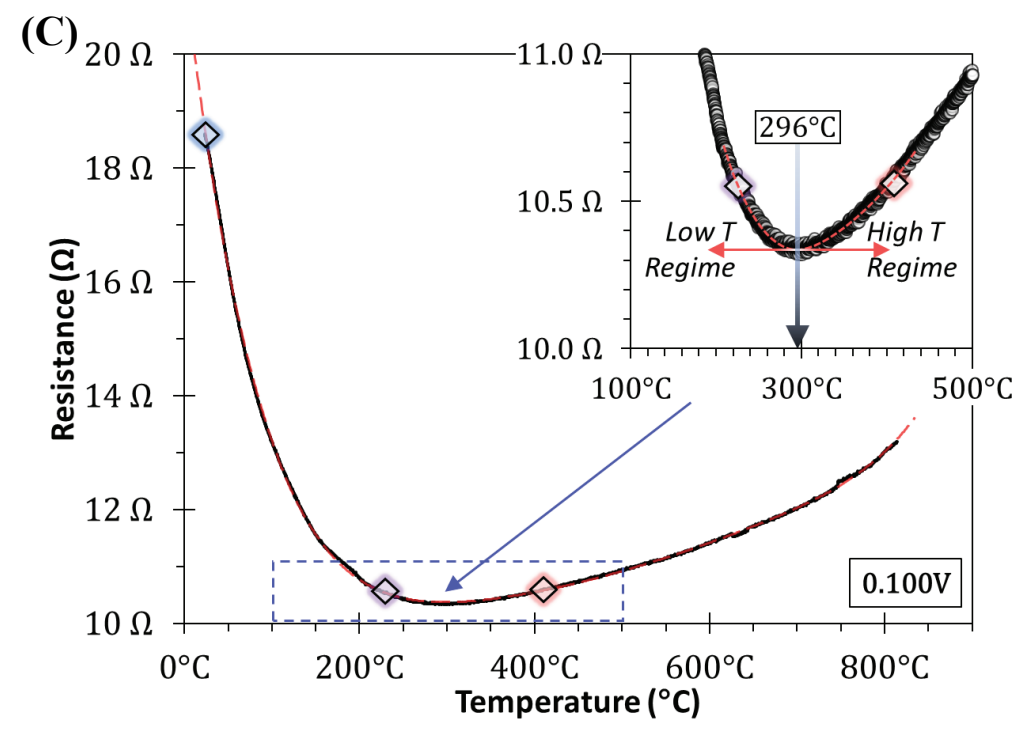

Figure 5.39: (A) IV curve of the device under test. As expected, the device transitions from back-to-back Schottky behavior at room temperature to apparent Ohmic behavior at high temperature. (B) The positive quadrant, spanning a reduced voltage range. Six different operation points are indicated by the crossed circles ( $\square$ ). (C) The two-point resistance measured at $0.100 \mathrm{~V}$ as the device is heated from room temperature to $810^{\circ} \mathrm{C}$. The three test temperatures are indicated by the diamonds $(\diamond)$. Inset: an expanded view of the plot around the resistance reversal point. Note the transition occurs at $296^{\circ} \mathrm{C}$.

The operation points shown in Figure 5.39 $(A, B)$ are generically classified as being at either high- or low- setpoints and are uniquely described by their coordinates in temperature/voltage parameter space. The low setpoints are: $\left\{23^{\circ} \mathrm{C}, 0.100 \mathrm{~V}\right\},\left\{230^{\circ} \mathrm{C}, 0.100 \mathrm{~V}\right\},\left\{409^{\circ} \mathrm{C}, 0.100 \mathrm{~V}\right\}$. The high setpoints are: $\left\{23^{\circ} \mathrm{C}, 2.00 \mathrm{~V}\right\},\left\{230^{\circ} \mathrm{C}, 1.40 \mathrm{~V}\right\},\left\{409^{\circ} \mathrm{C}, 0.90 \mathrm{~V}\right\}$. What follows is a discussion of tests conducted at each setpoint (indicated in Figure 5.39(B)) using argon as the carrier gas and 
pulses of hydrogen gas as the analyte gas. At all points in the tests, the gas flow was either 100\% argon or $100 \%$ hydrogen gas at a constant volumetric flowrate of 20 SCCM.

Figure 5.40 shows the results of two hydrogen pulse tests, both collected at low temperature $\left(23^{\circ} \mathrm{C}\right)$ with the device operating in constant voltage mode. The left vertical axis is the relative percentage change in the two-point resistance of the device and the right vertical axis is the corresponding absolute resistance (calculated from the measured current flow and the voltage setpoint using Ohms' law). In both tests, after 800 seconds of equilibration time, the device was exposed to an extended pulse of pure hydrogen gas for 2,000 seconds. Figure 5.40(A) shows the response of the device at a low setpoint of $0.100 \mathrm{~V}$ and Figure $5.40(B)$ shows the same test carried out at a high setpoint of $2.00 \mathrm{~V}$.

(A)

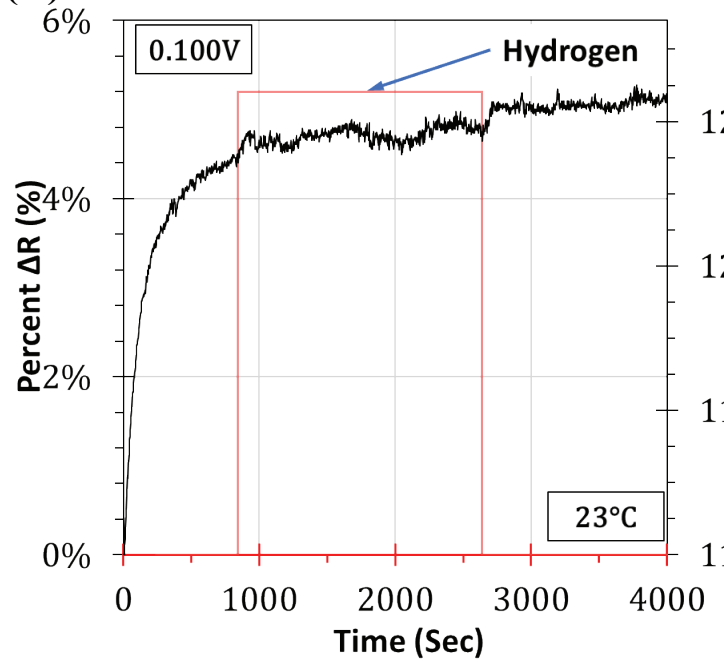

(B)

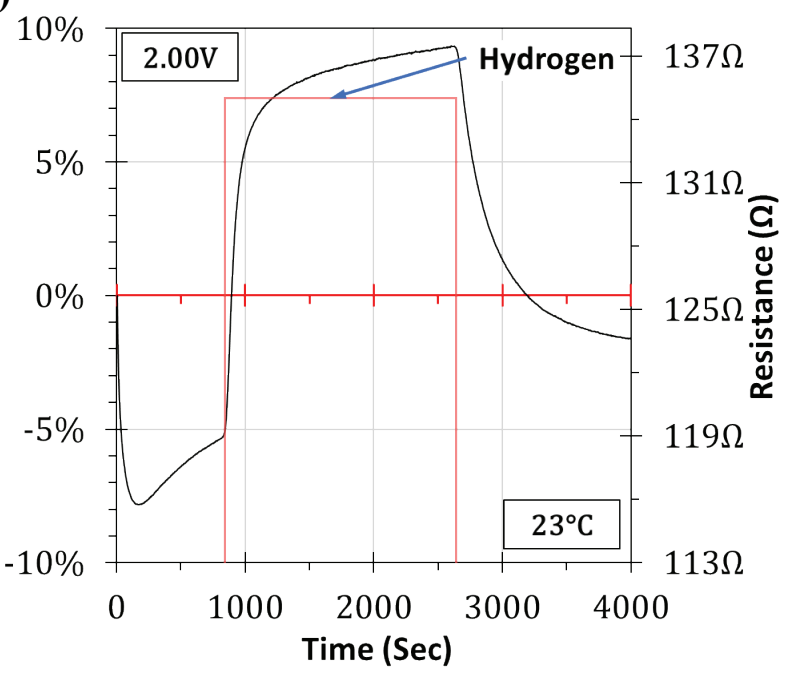

Figure 5.40: The results of a hydrogen pulse test from the same device at (A) 0.100V and (B) 2.0oV. Both tests were carried out at $23^{\circ} \mathrm{C}$. The red line indicted the applied hydrogen pulse.

In Figure 5.40(A), the anomalous rise in resistance, characteristic of the low setpoint operation, is seen at startup (this was discussed in Section \$5.3.2). There is effectively no change in resistance in response to the hydrogen gas pulse. The fact that no thermal response to hydrogen is seen at this low setpoint is due to two reasons. First, the amount of heat generated at the low set point is very small and thus, because the device is only slightly above the ambient temperature, the cooling effect of hydrogen is minimal. ${ }^{42}$ Secondly, by examination of Figure 5.39(B), it is clear

\footnotetext{
${ }^{42}$ From the values shown in Figure 5.40(A), at a setpoint voltage of $0.100000 \mathrm{~V}$, the device resistance is approximately $\sim 1220 \Omega$. Therefore, via $\mathrm{P}=\mathrm{I}^{2} \mathrm{R}$, only $0.000012 \mathrm{~J} / \mathrm{sec}$ of energy is consumed by the device to drive the $0.000080 \mathrm{~A}$ of current. This heat generation rate is comparatively much slower than the heat transfer rate capacity, and thus, the sample is only marginally about its ambient temperature.
} 
that this setpoint is far removed from the onset of the thermionic conduction region. Therefore, a small temperature change at this voltage has only a very small effect on the device conductivity in this region (i.e. a very large temperature change would be required to affect the injection of carriers at this reduced voltage).

In Figure 5.40(B), on startup the resistance of the device decreases; this is a hallmark of the device self-heating effects (discussed in Section \$5.3.1). The gradual rise prior to the onset of the pulse is again indicative of the anomalous rise in resistance (discussed in Section \$5.3.2). On exposure to hydrogen gas, the device resistance increases. This is a result of the cooling effect hydrogen has on the device due to the increased transfer of waste heat from the device into the gas stream (discussed in Section $\$$ 5.3.3). In this temperature regime, cooling the device increases the device resistance (as established in Section \$5.2.1). Therefore, these results are internally consistent with a thermally driven response. This thermal response is very rapid and is reflective of the real-time concentration of the gas in the chamber (recall the square input pulse is spread out in the time domain, due to diffusion within the system as discussed in Section§5.3.3).

Figure 5.41 shows the results of a similar test, collected at $230^{\circ} \mathrm{C}$. The results of the low $0.100 \mathrm{~V}$ setpoint test are shown in Figure 5.41(A) and the high $1.40 \mathrm{~V}$ setpoint results are shown in Figure $5.41(B)$. Similar to the testing at $23^{\circ} \mathrm{C}$, Figure $5.41(B)$ shows a thermally driven response to hydrogen exposure. On startup and during the 7,000 second equilibration period the resistance decreases as the device experiences self-heating. On exposure to the cooling effect of the hydrogen

(A)

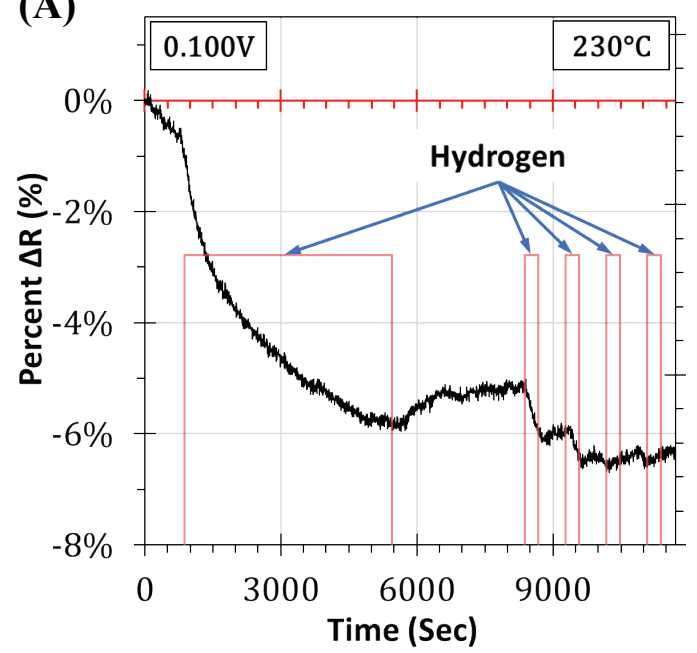

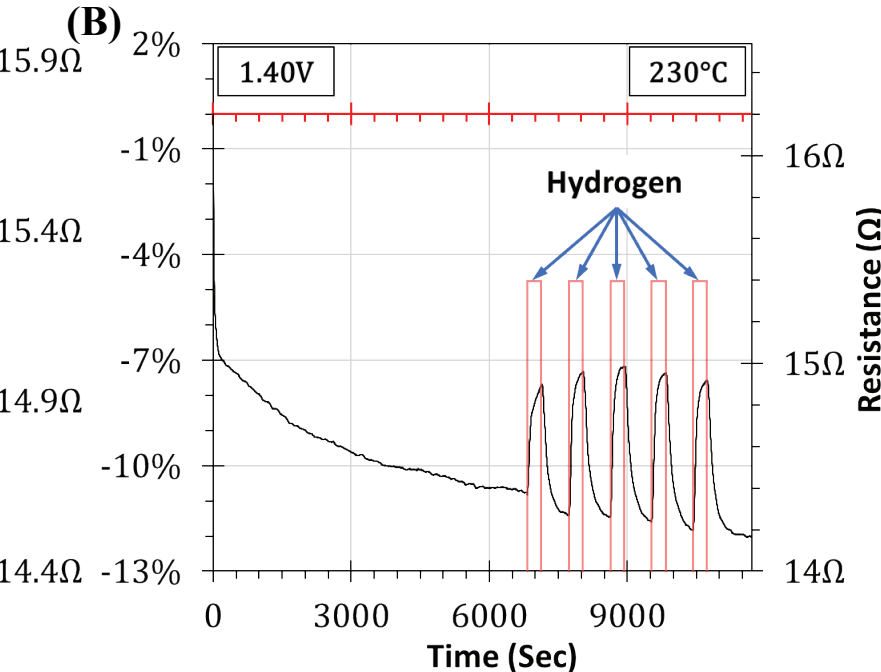

Figure 5.41: The results of a hydrogen pulse test from the same device at $0.100 \mathrm{~V}$ and $0.900 \mathrm{~V}$. Both tests were carried out at $230^{\circ} \mathrm{C}$. (A) Contrary to what would be expected for a thermal effect, the resistance decreases on hydrogen exposure, indicating this is a chemically derived effect when the device is operated at a low setpoint. (B) During high setpoint operation, only a thermal effect is observable. 
gas, the resistance increases. Again, this is consistent with a thermally driven effect. The opposite effect is observed in Figure 5.41(A); the device resistance decreases on exposure to hydrogen gas. It is argued here that this is a result of a chemical effect.

In Figure 5.41(A), after $\sim 800$ seconds of equilibration time, the device is hit with a 4,560second-long pulse of hydrogen gas. In response, the resistance decreases. This is in stark contrast to what would be expected for a thermal response at this temperature. This behavior is repeated later in the test when the device is exposed to four 300-second-long pulses of hydrogen. Recall that, in this temperature regime, reducing the device temperature increases the device resistance (more broadly discussed in Section \$5.2 and firmly established for this device in Figure 5.39(C)). Furthermore, as established in Section $\$$ 5.3.3, heating the device reduces the resistance regardless of the setpoint. Clearly in this case (Figure 5.41(A)), exposing the device to hydrogen gas, which was shown to have a cooling effect due to its physical properties (established in Section \$5.3.3) reduces the resistance of the device - the opposite of what would be expected of a thermal effect. Therefore, as this does not follow the established behavior of the thermal effect, this is likely a chemically derived effect.

In support of this conclusion, consider the overall nature of the response. After the initial hydrogen pulse, the gas flow is switched back to a pure argon flow for $\sim 3,000$ seconds. This is many times over what is required to fully purge the test chamber (for comparison, the companion test shown in Figure 5.41(B) shows a complete evacuation of the test chamber in the $\sim 600$ seconds between pulses, consistent with purge time calculations using the flowrate and chamber volume). The device response only partially recovers, even though at this point the device has been under a pure argon environment for over 2,000 seconds. Were this a thermal response, the signal would have tracked with the composition of the gas environment and returned to approximately the same level as before the hydrogen pulse (as seen for most other testes).

Furthermore, consider the leading edge of the device response signal to each hydrogen pulse in Figure 5.41(A). The response to each pulse asymptotically approaches the same value in all cases. This is indicative of a Type I Langmuir isotherm adsorption model with a low sticking probability and a low desorption rate. This, and other adsorption models, are discussed in Section $\$ 2.2 .1$, along with a general discussion of physi- and chemi- sorption theory. The Type I Langmuir adsorption model is one of the simplest descriptions of adsorption and is applicable to a wide variety of cases. Classically it is described as modeling the adsorption of a monolayer of molecules 
onto a flat surface. More specifically, it models the accumulation of molecules onto a finite number of adsorption sites, all equally accessible energetically, temporally and physically (i.e. the energy of adsorption is the same for all sites, the time required to access and interact with each site is the same and the sites are all distributed equidistance from each other in real space).

Further insight is gained by examining the response of the device as a function of gas concentration. Figure 5.42 shows the response to five different concentrations of hydrogen gas in argon. After 800 seconds of equilibration time under a flow of 20 SCCM of pure argon, the gas feed was switched to a hydrogen/argon mixture and fed at a rate of 20 SCCM for 300 seconds. This was followed by a purge using pure argon gas feed at 20 SCCM for 600 seconds. This was performed using a 5\%, 25\%,50\%,75\% and 100\% hydrogen in argon mixture. In Figure 5.42(A) this test was conducted on the device when powered at a constant voltage setpoint of $0.200 \mathrm{~V}$. This is a setpoint where a chemical response to hydrogen is observable. Figure 5.42(A) shows the same test conducted at a constant voltage setpoint of $1.40 \mathrm{~V}$ where only the thermal response is observable. The setpoint used in Figure 5.42(A) is a slightly higher (0.200V vs. $0.100 \mathrm{~V})$ setpoint than what was used in Figure 5.41(A) but still clearly leading to a chemical response.

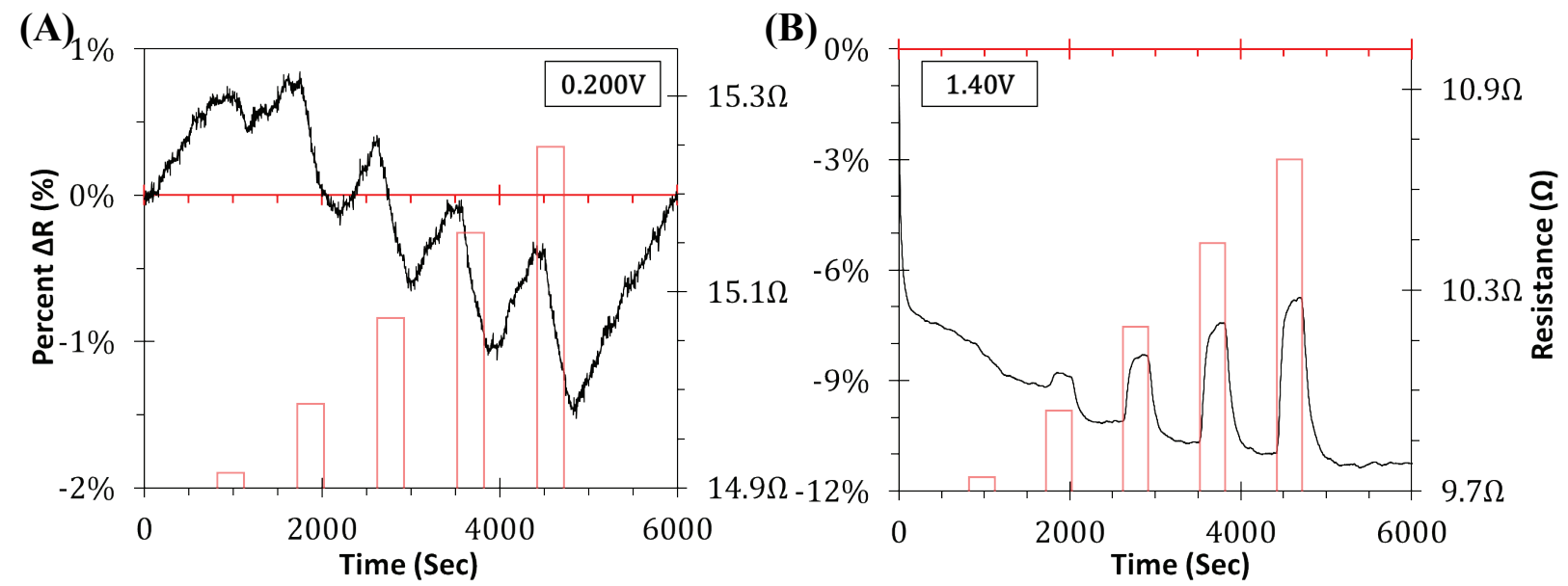

Figure 5.42: The results of a hydrogen concentration study for a device operated in the chemically responsive regime and thermally responsive regime. (A) When operating the device at $0.200 \mathrm{~V}$, a chemical response is seen. (B) When operated at $1.40 \mathrm{~V}$, a thermal response is seen. The device is at the same temperature, $230^{\circ} \mathrm{C}$, in both cases. The hydrogen/argon pulse mixtures are indicated by the red bars and correspond to a $5 \%, 25 \%, 50 \%, 75 \%$ and $100 \%$ hydrogen concentrations.

In Figure 5.42(A), it is observed that due to the slow desorption process (as evidenced by the results shown in Figure 5.41(A)), the device response does not return to its starting value before it is exposed to the next hydrogen pulse. The apparent downward drift in response seen in Figure $5.42(A)$ is thus due to an accumulation of gas on the device. It is interesting to note that, when the 
drift in the signal baseline is taken into account, the magnitude of each response is approximately the same for all gas concentrations above $25 \%$. This indicates that the device-gas interaction rate is saturated at a concentration somewhere between $5-25 \%$ hydrogen. Therefore, the gas sensing mechanism is a reaction rate limited process rather than mass transport limited (increasing the number of hydrogen molecules available to the device surface does not increase the response rate).

From these tests, it is unclear if the chemical effect is present at high voltages. It is possible the chemical effect is still present at the high setpoint, but the much larger thermal effect masks the response. Figure 5.43(A) plots the two tests shown in Figure 5.42 on the same graph for comparison. Here it is illustrated why the chemical response is not seen at high voltage, even if it is occurring. Not only does the thermal response drive the signal in the opposite direction of any potential chemical response, but the thermal response is much larger in magnitude- more than sufficient to eclipse any chemical response.
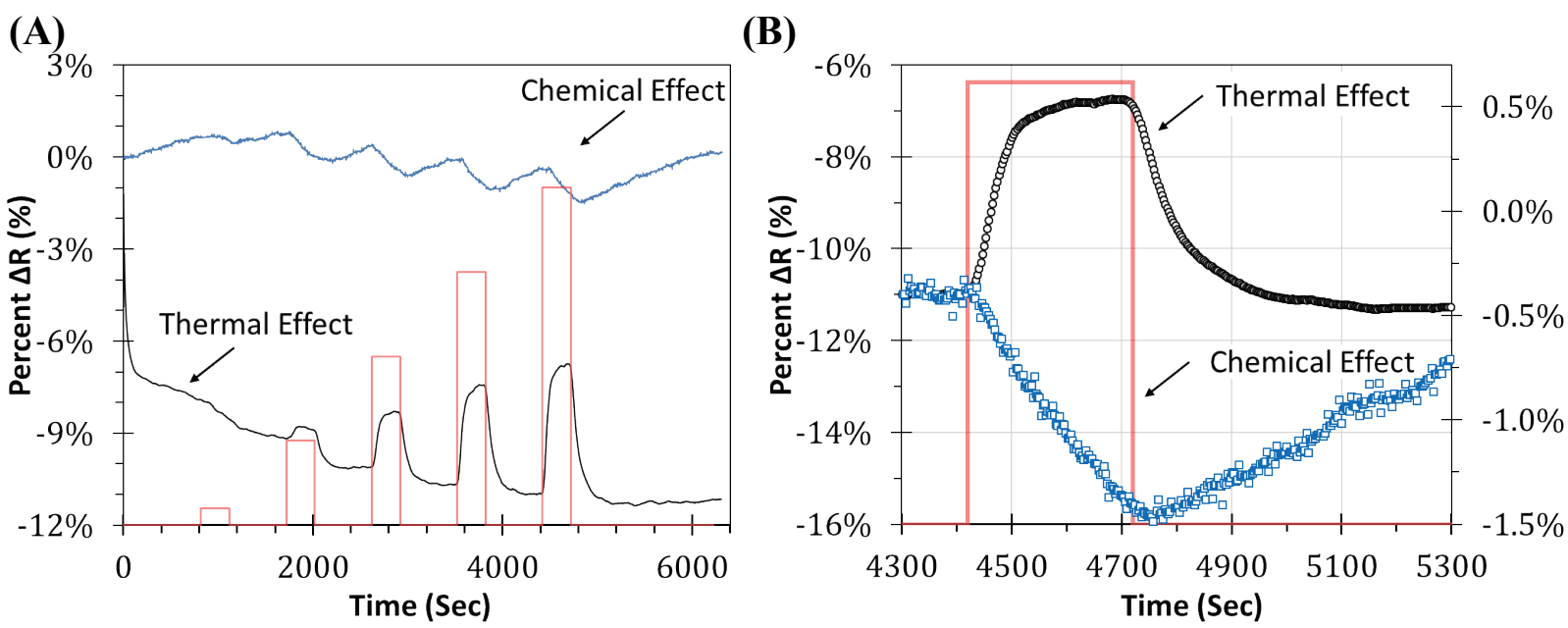

Figure 5.43: (A) The results of the hydrogen concentration study showing the thermal and chemical response on the same scale. The red line indicates the hydrogen mix pulses. Sequentially, they are $5 \%, 25 \%$, $50 \%, 75 \%$ and $100 \%$ hydrogen in argon. (B) A comparison of the device response curves to a 300 sec pulse of $100 \% \mathrm{H}_{2}$ when the device is at a low setpoint (chemical effects dominant) and a high setpoint (thermal effects dominate).

Figure 5.43(B) compares the thermal and chemical response to the pulse of $100 \%$ hydrogen gas, taken from Figure 5.43(A). Here, the different response and recovery rates between the two effects are illustrated. The thermal response tracks closely with the composition of the chamber environment. The chemical effect on the other hand, is slow to respond. This is again consistent with a surface interaction limited process. 
Shown in Figure 5.44 are the results of a similar test, conducted at a high temperature $\left(409^{\circ} \mathrm{C}\right)$, at two different constant voltage setpoints. In both tests, after 800 seconds of equilibration time, the device was exposed to five pulses of hydrogen gas. The hydrogen pulses were 300 seconds in duration, interspersed with 600 seconds of an argon purge at the same volumetric flowrate (20 SCCM). Figure 5.44(A) presents the results obtained at a low voltage setpoint of $0.100 \mathrm{~V}$ and Figure $5.44(B)$ presents the results obtained at a high voltage setpoint of $0.900 \mathrm{~V}$.
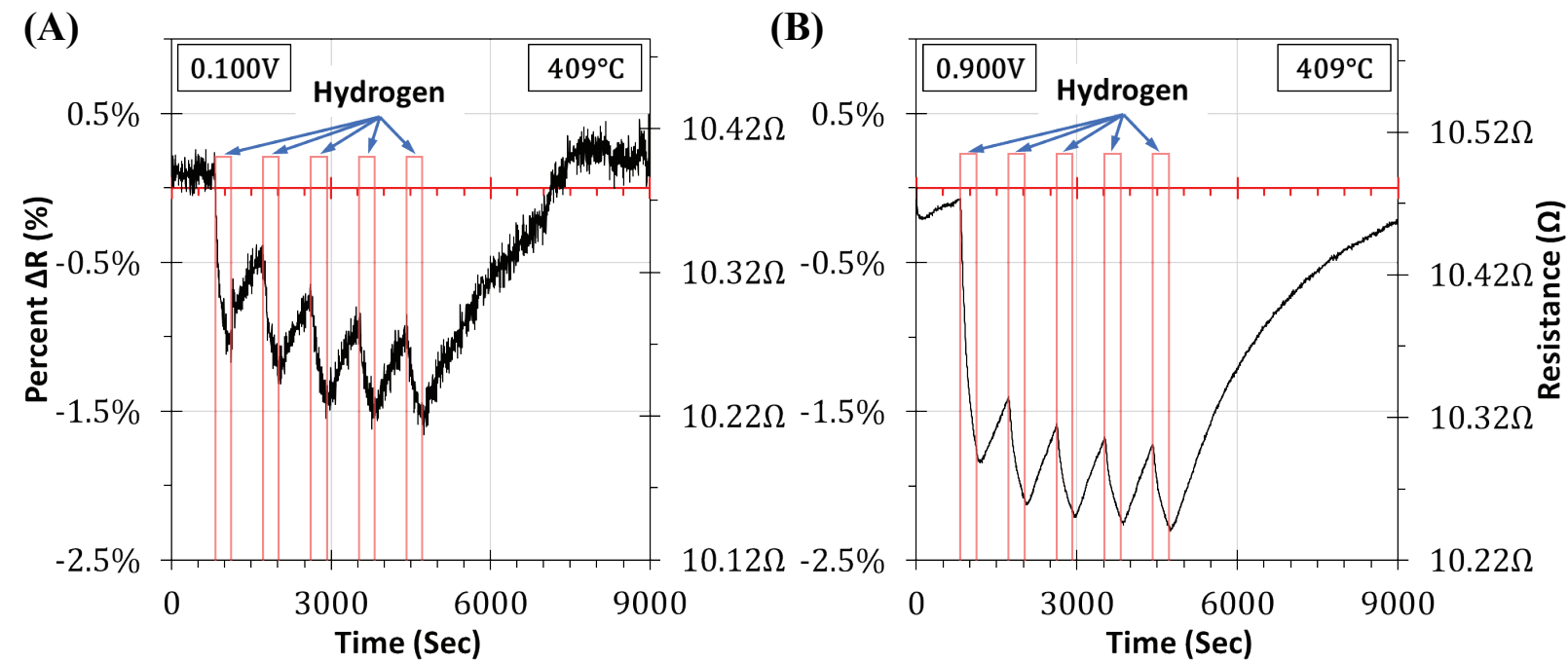

Figure 5.44: The results of a hydrogen pulse test $\left(100 \% \mathrm{H}_{2}\right.$ for $300 \mathrm{sec}, 100 \%$ Ar for $\left.600 \mathrm{sec}\right)$ from the same device at (A) $0.100 \mathrm{~V}$ and (B) $0.900 \mathrm{~V}$. Both tests were carried out at $409^{\circ} \mathrm{C}$.

In both cases the resistance of the device decreases on exposure to hydrogen. Recall from Figure $5.39(\mathrm{C})$ that this temperature, $409^{\circ} \mathrm{C}$, is above the resistance turnaround point. This means that the sample has transitioned into the high temperature regime where the thermal effects have flipped. Due to various scattering effects (Section \$5.2.2), which now dominate over thermionic emission, heating the device reduces the conductivity and cooling the device increases the conductivity in this regime. Considering these facts, it is difficult to assess if these results are thermally or chemically driven (both effects now move the device signal in the same direction: decreasing resistance).

An aspect of the response shown in Figure 5.44(A) not accounted for by a thermal effect is the slow device response time. In fact, the trailing edge of the response curves matches qualitatively with the chemical recovery curve seen in Figure 5.43(B). Furthermore, the magnitude of the response is also similar. Although the enhanced mixing within the chamber at high temperature may contribute this slower recovery (see the PFR/CSTR discussion in Section §5.3.3). 
These two facts argue in favor of assigning at least a portion of the response of Figure 5.44 $(A, B)$ to be chemical in nature. Figure 5.44(B) shares the same similarities as well, indicating that both a chemical and thermal effect may be occurring at these conditions.

\section{§5.3.5 Restometric Sensing of Methane and Carbon Monoxide Gas: Thermal Response}

The SOP device response was also tested using methane $\left(\mathrm{CH}_{4}\right)$ and carbon monoxide $(\mathrm{CO})$ gas. Due to a chemical reaction which occurred at high temperature between $\mathrm{CO}$ and the stainlesssteel walls of the small test cell, $\mathrm{CO}$ testing was limited to room temperature. This is discussed in detail in Appendix $\S C$.1.

The devices show a clear thermal response to both $\mathrm{CO}$ and $\mathrm{CH}_{4}$ gases across a range of concentrations. No chemical response to either gas was observed, however $\mathrm{CH}_{4}$ and $\mathrm{CO}$ gases were not tested as thoroughly as $\mathrm{H}_{2}$ was. Figure 5.45 shows the results of four pulses of pure $\mathrm{CH}_{4}$ gas, collected at a low and high setpoint $(10 \mathrm{~mA}$ and $100 \mathrm{~mA})$ at room temperature. Both responses are thermal in origin.
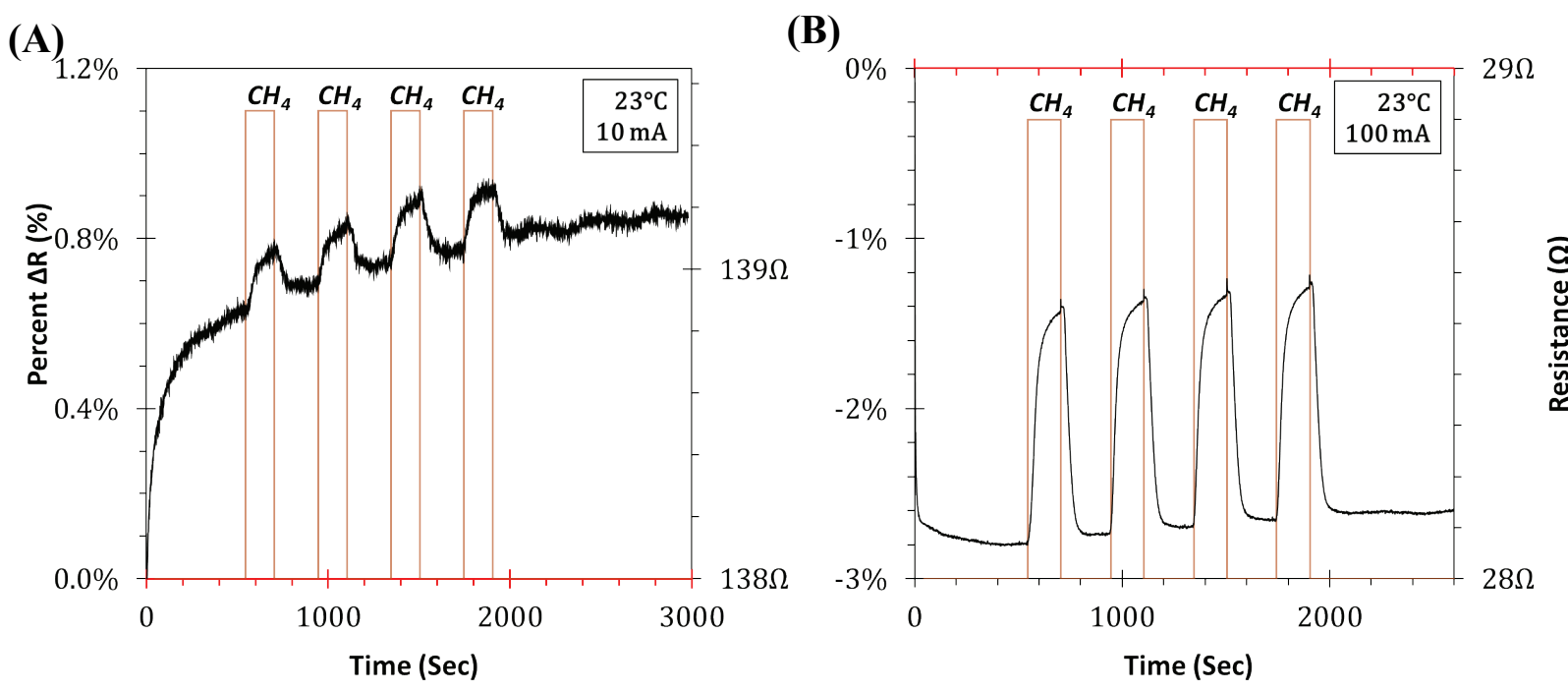

Figure 5.45: Four 300-second-long pulses of pure $\mathrm{CH}_{4}$ gas in a carrier stream of pure Ar. (A) Device response at $10 \mathrm{~mA}$ and at (B) $100 \mathrm{~mA}$. Both tests were conducted at room temperature in the small test nnl1 
Figure 5.46 shows the results of pulses pure $\mathrm{H}_{2}$ and $\mathrm{CO}$ gas. The difference in signal between $\mathrm{H}_{2}$ and $\mathrm{CO}$ is clear. The gases are differentiable by the magnitude of the response. This difference in magnitude reflects the difference in heat removal rates afforded to the device by the different gas environments. The heat is more readily removed from the device in a pure $\mathrm{H}_{2}$ environment than in a pure $\mathrm{CO}$ environment.
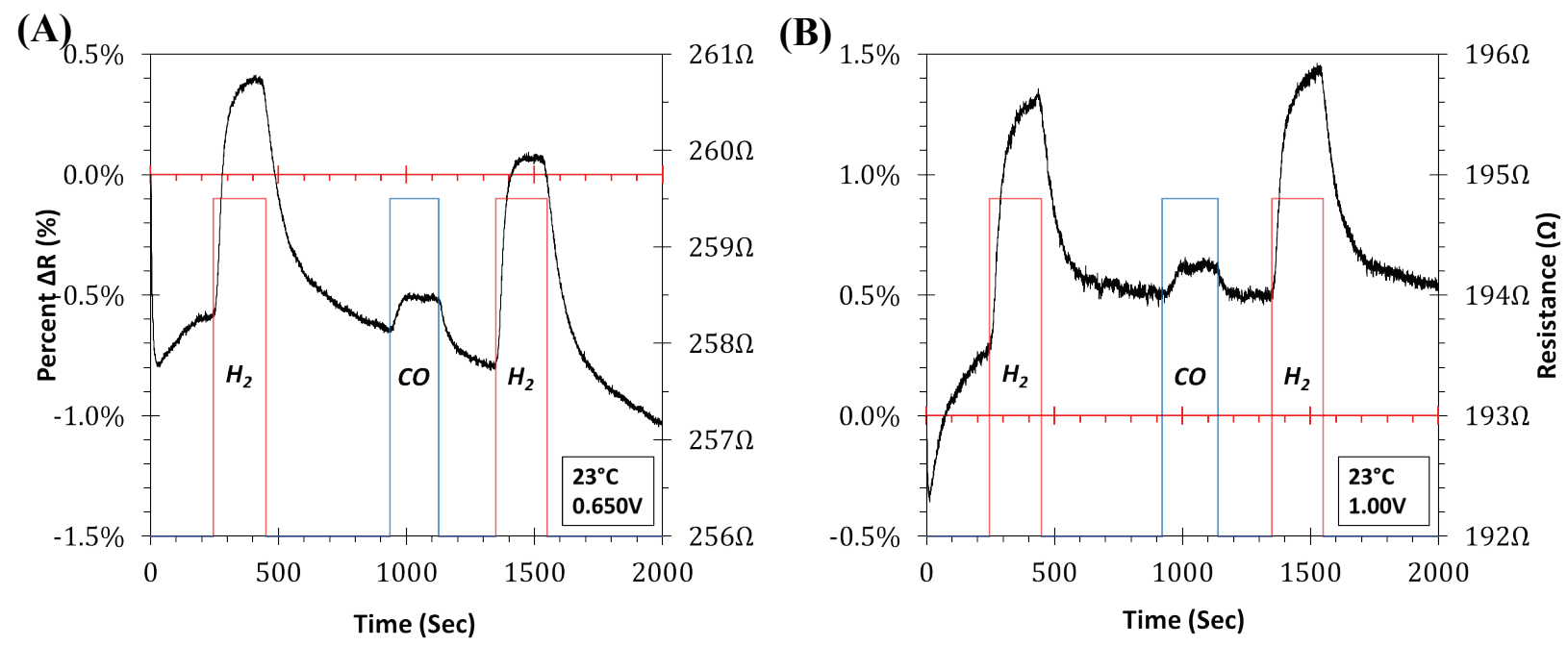

Figure 5.46: The device response to $\mathrm{H}_{2}$ and $\mathrm{CO}$ is shown at both a low set point (A) $0.650 \mathrm{O}$ and at a higher setpoint (B) 1.ooV. Both tests were conducted at room temperature in the small gas test cell.

\section{§5.3.6 Film Decoupling by Hydrogen Testing}

The device thermal response to hydrogen gas is fully and completely reversible at all conditions (setpoints and temperatures). After the analyte pulse, device response returns to the prepulse signal level (disregarding the global shifts in the signal baseline due to the larger timescale thermal equilibration/drift of the device/testing apparatus; the pulse responses essentially 'ride' on this baseline). The thermal response tracks with the composition of the gas environment. The rise and fall rate of the pulse response matches with a simple exponential growth and decay function as the test chamber first fills with analyte gas and then is purged with inert gas (see the PFR/CSTR discussion in Section \$5.3.3). This is consistent with the proposed mechanism of the thermal response. The devices chemical response is also largely reversable but does require longer for the response to return to the initial value. 
There are, however, conditions where the device response does not recover. This was observed during testing with hydrogen gas at very high temperatures $\left(\geq 500^{\circ} \mathrm{C}\right)$. The result was a permanent change in the device behavior. An example of this is shown in Figure 5.47. The device is at $500^{\circ} \mathrm{C}$ and operated in a constant voltage mode at $0.250 \mathrm{~V}$. Note the large change in resistance, much larger than any thermal or chemical response ever observed. After the initial hydrogen exposure (at $\sim 1300$ seconds), the device response is permanently changed and an additional hydrogen pulse (at $\sim 2750$ seconds) during the same test had little effect. It was found that by annealing the device, in a pure argon environment at a higher temperature (several hundred degrees higher), the response could be made to partially recover (the response returned to within $\sim 50 \%$ of the pre-hydrogen value). However, after extensive high temperature exposure to hydrogen gas (many thermal cycles and long durations under hydrogen gas at high temperature), the device response irrevocably changed in most cases.

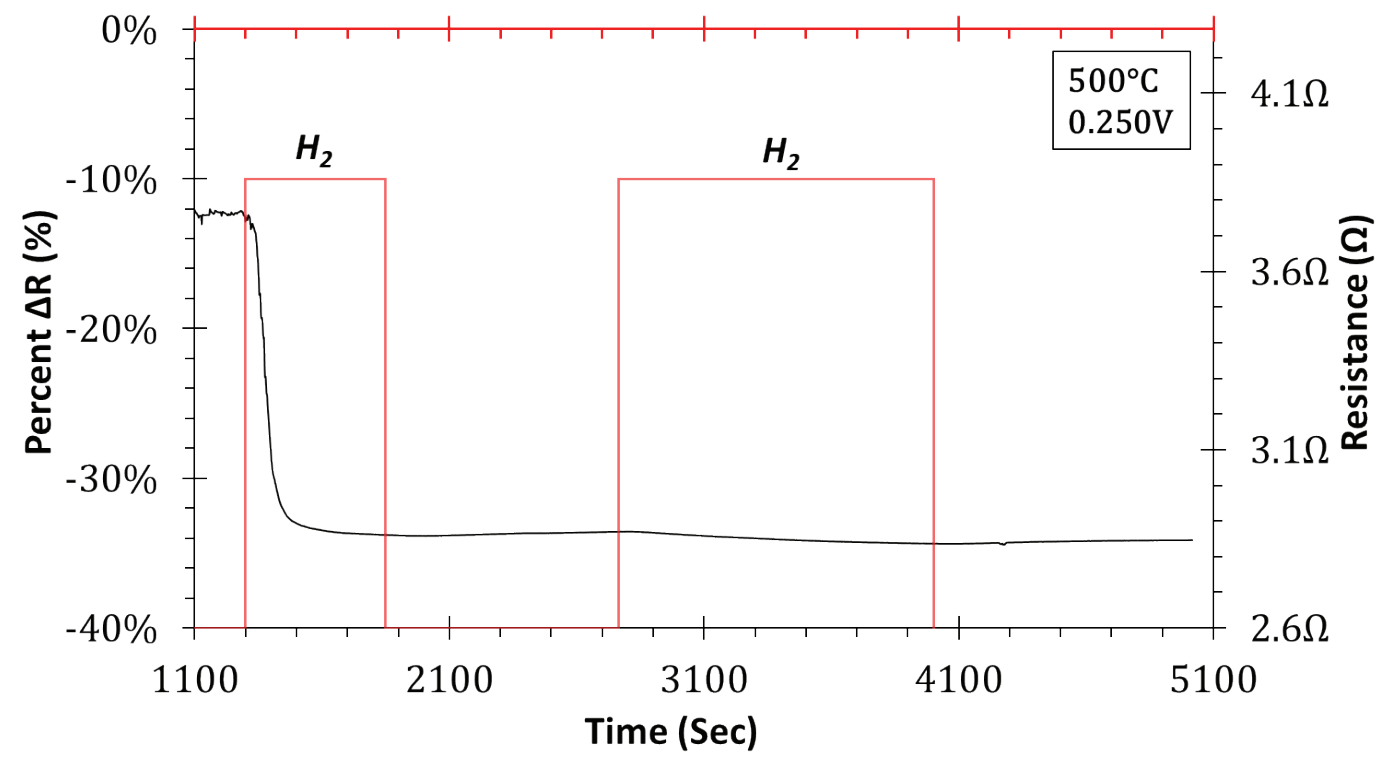

Figure 5.47: An example of the irreversible and large change in device resistance occasionally observed in devices exposed to hydrogen at high temperatures.

This permanent resistance change in devices exposed to hydrogen at very high temperatures is apparent at all temperatures, indicating a fundamental change of the device. Given what is known about the effects of high temperature hydrogen on the buffer layer, the most likely explanation is that this permanent change is the result of partial buffer layer decoupling. Recall the primary effect of high temperature hydrogen annealing (Section $\$ 4.3$ ) was a partial decoupling of the buffer layer. 
Figure 5.48 plots two IV curves from the same device, collected before $(\diamond)$ and after $(\circ)$ high temperature hydrogen gas testing. Both data sets were collected at room temperature and under a flow of 20 SCCM of argon. In the interim between the two curves, the device was exposed to pulses of hydrogen/argon gas mixtures at $23^{\circ} \mathrm{C}, 370^{\circ} \mathrm{C}$ and $786^{\circ} \mathrm{C}$. This particular device was exposed to a total of twenty-three, 300-second-long pulses of hydrogen/argon mixtures, which ranged from $5 \%-100 \%$ hydrogen. Not all devices show a permanent change and some devices underwent more extensive hydrogen exposures before a similar change is observed.

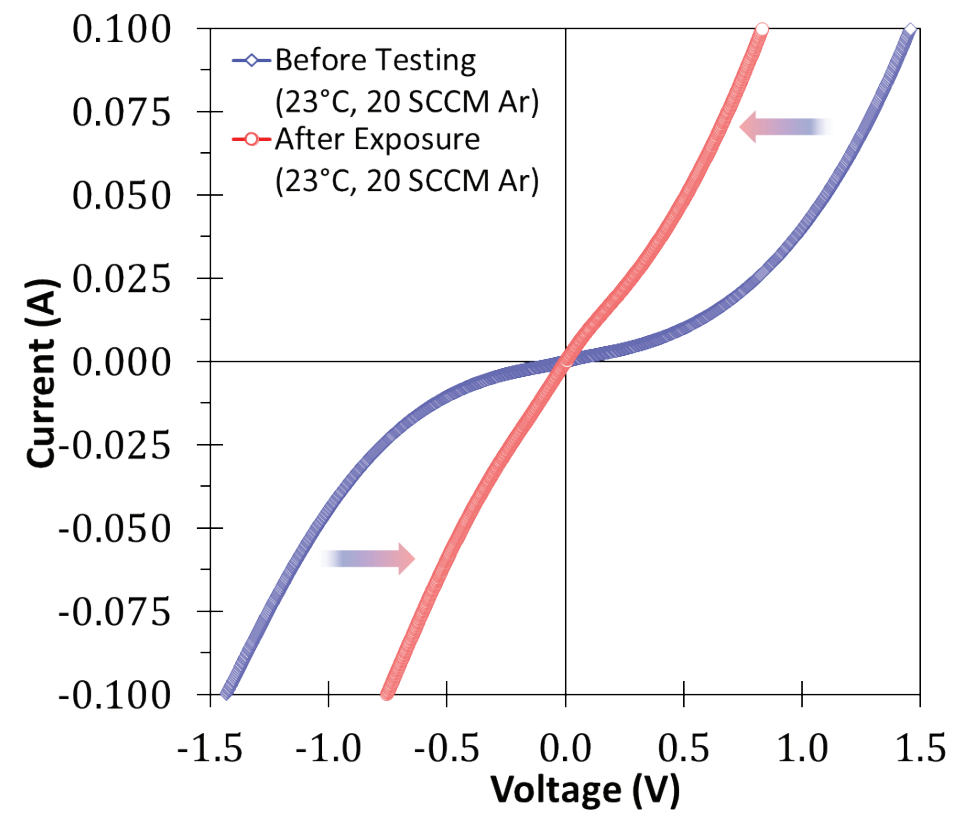

Figure 5.48: The IV curve collected before $(\diamond)$ and after $(\circ)$ high temperature gas testing with hydrogen. Both curves were collected under the same conditions: $23^{\circ} \mathrm{C}$ and 20 SCCM of UHP argon.

These IV results are qualitatively consistent with what is expected for the decoupling of the buffer layer. Goler, et al., show a similar transition in IV curve on buffer layer freeing. (234) Table 5-3 lists the device parameters as calculated using the modified Richardson-Dushman analysis (disused in Section \$5.1.3). Because this device utilizes the sensor pattern, some device parameters are not known, and specific values cannot be accurately calculated. However, because it is the same device in both cases, the relative changes can be calculated. By calculating the relative change in device resistance, as calculated from the linear (high voltage) portion of the IV curves (which is reflective of the resistance of the film, following the procedure detailed in Section \$5.1.2), the change in carrier mobility can be calculated. Following this approach, it is clear that exposure to high temperature hydrogen improves all electrical parameters. Even though the film 
is in the early stages of decoupling, there is a vast improvement in film properties. These results add motivation for the goal of decoupling the buffer layer in future work.

Table 5-3: Change in device parameters after high temperature hydrogen exposure.

\begin{tabular}{|l|c|}
\hline \multicolumn{1}{|c|}{$\left(\right.$ After $\left.\mathbf{H}_{2}\right)-\left(\right.$ Before $\left.\mathbf{H}_{2}\right)$} & $\% \Delta$ \\
\hline Change in Carrier Concentration $\left(\Delta \mathrm{N}_{\mathrm{D}}\right)$ & $-55.7 \%$ \\
\hline Change in Schottky Barrier Height $\left(\Delta \Phi_{\mathrm{B}}\right)$ & $-22.9 \%$ \\
\hline Change in Film Resistivity $(\Delta \rho)$ & $-10.8 \%$ \\
\hline Change in Carrier Mobility $(\Delta \mu)$ & $+60.5 \%$ \\
\hline
\end{tabular}

Following this electrical analysis, the sample was removed from the device packaging and analyzed using XPS and Raman spectroscopy. Figure 5.49 compares the XPS spectrum collected from the sample just after fabrication (before any gas testing) to the XPS spectrum collected after gas testing. The spectrum collected after gas testing is similar to those spectra observed during the experimentation discussed in Section $\$ 4.3$ and supports the hypothesis that buffer layer decoupling is occurring.

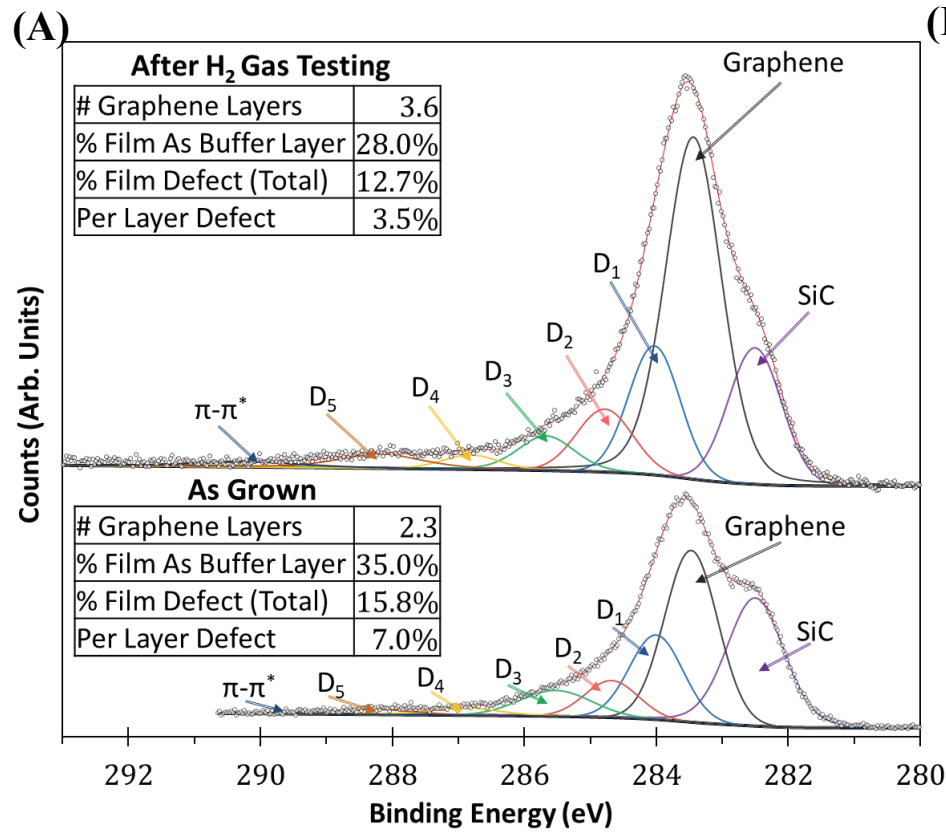

(B)

\begin{tabular}{|c|c|c|c|}
\hline \multicolumn{5}{|c|}{ After $\mathrm{H}_{2}$ Gas Testing } \\
\hline Peak ID & Position (eV) & Separation (eV) & \%Area \\
\hline SiC & 282.5 & $\varnothing$ & 16.7 \\
\hline Graphene & 283.4 & 0.9 & 48.0 \\
\hline D1: S1 & 284.0 & 1.5 & 15.2 \\
\hline D2: S2 & 284.8 & 2.3 & 8.1 \\
\hline D3 & 285.7 & 3.2 & 4.9 \\
\hline D4 & 286.8 & 4.3 & 2.1 \\
\hline D5 & 288.2 & 5.7 & 3.5 \\
\hline$\pi-\pi *$ & 290.2 & 7.7 & 1.6 \\
\hline \hline \multicolumn{5}{|c|}{ As Grown } \\
\hline Peak ID & Position (eV) & Separation (eV) & $\%$ Area \\
\hline SiC & 282.5 & $\emptyset$ & 29.7 \\
\hline Graphene & 283.5 & 1.0 & 34.6 \\
\hline D1: S1 & 284.0 & 1.5 & 17.1 \\
\hline D2: S2 & 284.7 & 2.2 & 7.5 \\
\hline D3 & 285.5 & 3.0 & 7.2 \\
\hline D4 & 286.9 & 4.4 & 2.4 \\
\hline D5 & 288.3 & 5.8 & 1.6 \\
\hline$\pi-\pi^{*}$ & 290.1 & 7.6 & 0.1 \\
\hline
\end{tabular}

Figure 5.49: (A) Top, the C1s spectra collected from the film after high temperature hydrogen gas testing. Bottom, the C1s spectra before gas testing. Inset are the calculated film parameters from the deconvolution of the data. (B) The corresponding deconvolution results.

In Figure 5.49, the peak positions remain remarkably unchanged $( \pm 0.1 \mathrm{eV})$ indicating no change in chemical state has occurred. The most apparent change is the large growth in the 
graphene peak. This leads to an apparent increase in the number of graphene layers (from 2.3 to 3.6), as there is no reason to expect additional carbon on the surface, as discussed in Section $\$ 4.3$, the increase in the graphene peak is likely due to a densification of the graphene overlayer. This causes an increased attenuation effect of the $\mathrm{SiC}$ signal from the substrate resulting in a thicker layer calculation (APPENDIX D).

Overall, the film parameters are improved after high temperature hydrogen exposure. The fraction of the carbon in the film associated with the buffer layer is reduced (from $35 \%$ to $28 \%$ ) and the total oxygen-based defect level is reduced (15.8\% to $12.7 \%)$. Some of these improvements are due to the way in which the values are calculated (as percentages) and the effect of the increase in the graphene peak. However, the general improvements in the film quality is undeniable.

The fact that the graphene peak has not substantially shifted indicates the decoupling process is in the very early stages. Based on the discussion in Section $\$ 4.3$, the decoupling process reveals itself first as a growth in the graphene peak intensity and then, as the process continues, the graphene peak shifts to higher binding energies. Considering this, it is interesting to note the large change in the electrical properties of this device, even though the buffer layer is only partially beginning to be decoupled at this point. The Raman spectra, shown in Figure 5.50, supports this. In fact, the spectrum matches well with data collected from the hydrogen decoupling experiments

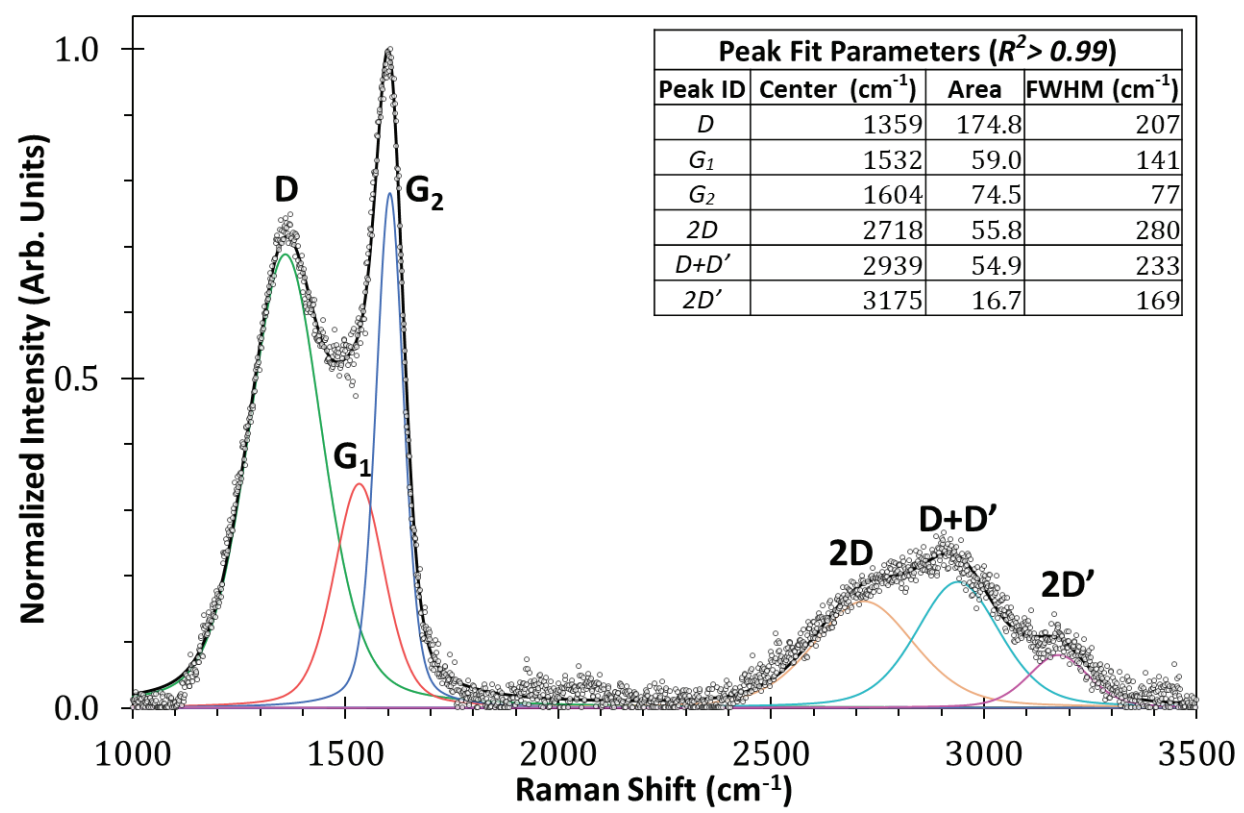

Figure 5.50: Raman spectra collected from the film after high temperature hydrogen gas testing. The $\mathrm{D} / \mathrm{G}$ ratio ( 1.31), narrowing of all peaks and slight growth of the two-phonon peaks are all indicative of the buffer layer decoupling process. 
carried out at $700^{\circ} \mathrm{C}-800^{\circ} \mathrm{C}$. This is not surprising because the highest temperature this device was exposed to hydrogen during gas testing was $786^{\circ} \mathrm{C}$.

\section{§5.3.7 Gas Sensing Mechanism and Future Directions}

The two device responses to gas are the thermal response and the chemical response. The thermal response is straightforward and is explained in Sections $\$ 5.3 .1$ and $\$ 5.3 .3$. In short, the thermal response is simply due to a shift of the balance between the self-heating effects and heat removal rate afforded to the device by the gas environment. The swings in device response observed immediately following startup, discussed in Section \$5.3.2, are due to a thermal equilibration and an electronic equilibration; these can be ignored in regard to investigating the gas sensing mechanism. A comprehensive explanation for the chemical effect is more complicated, however. A discussion of the chemical effect due to hydrogen gas follows, based on the observations detailed earlier in this section.

The following discussion assumes, for the moment, that the interaction process which produces the chemical effect is based on a simple adsorption process (as opposed to $\mathrm{H}_{2}$ intercalation into the film stack, diffusion into the contact-film interface, or other obscure effects). Examination of the chemical response shows the process to be surprisingly slow. Using the kinetic theory of gases, the flux $(F)$ of hydrogen molecules onto the device surface can be calculated using the following equations:

$$
F=\frac{P}{\sqrt{2 \pi m k_{B} T}}
$$

Where $\mathrm{P}$ and $\mathrm{T}$ are the gas pressure and the temperature, respectively, $\mathrm{m}$ is the mass of the gas molecule and $\mathrm{k}_{\mathrm{B}}$ is the Boltzmann constant.

Using Equation 5-7 and taking the properties of hydrogen gas and the temperature $\left(230^{\circ} \mathrm{C}\right)$ and pressure (1 atm) where a clear chemical response is observed, the device surface experiences

a flux of hydrogen molecules at a rate of $8.4 \times 10^{23} \frac{\mathrm{H}_{2} \text { Impacts }}{\mathrm{cm}^{2} \cdot \mathrm{Sec}}$. Given an areal density of $\sim 3.8 \times 10^{15} \mathrm{~cm}^{-1}$ for graphene, it is clear that the surface interaction that leads to the chemical response is not limited by the availability of $\mathrm{H}_{2}$ molecules. Considering the slow device response and recovery rates, the reaction probability must be very low and, once an adsorption event has occurred, the desorption probably is even lower. Analysis of chemical response curves shapes indicates that the desorption process occurs at about $1 / 3$ to $1 / 5$ the rate of adsorption. 
A similar chemical response to what has been discussed here has be observed in several other SOP devices tested. The conditions under which the chemical response can be observed varies somewhat from sensor-to-sensor and in some cases the chemical/thermal responses are more difficult to deconvolute. In order to speculate on the mechanism of the chemical response, and based on the results previously discussed, the following directly observable facts are listed:

1) In the temperature range where chemical effects are observed, the effect of the chemically derived response to hydrogen is to increase the device conductivity.

2) The chemical response is not seen at low temperatures.

3) The chemical response is most clearly observable at low voltages (although it may be present at high voltages as well, obscured by self-heating effects).

4) The response rate of the chemical effect is very slow.

5) The recovery rate of the chemical response is even slower at only $\sim 20-30 \%$ the forward rate.

6) The rate and magnitude of the response saturate at a hydrogen concentration between $5-25 \%$.

7) From \#6 and applying the kinetic theory of gases, the chemical effect is reaction rate limited and not mass transport limited.

8) The rate of the chemical response increases with temperature.

9) The magnitude of the chemical response increases with temperature.

A final observation: recall from the results discussed in Section $\$ \mathbf{5 . 2}$ that, with higher temperatures, the amount of current injection due to charge trap hopping increases. Additionally, due to the thermal promotion of charge carriers, the suppression of current injection due to the Schottky barrier is reduced. It is therefore interesting to note that the regions of device operation where a chemical effect is observed (high temperatures, low voltages) correspond to the regions where charge trap hoping carrier injection dominate. This may simply be due to the fact that selfheating is reduced under theses conditions (the device is much more conductive at these high temperatures and the low voltages both work to produce less waste heat) and the thermal effect no longer swamps out the chemical effect. Or, there may be some relationship between charge trap hoping current injection and the chemical effect. However, exactly how hydrogen adsorption would affect charge trap hopping is unclear.

There are several mechanisms which could explain the chemical effect. Given their complexities and absent more complex testing, it is difficult assign with certainty any one 
particular mechanism. No one observation completely precludes any single explanation and no combination of observations conclusively point to a particular explanation. What follows is a discussion of potential mechanisms which may account for the chemical response, using the nine observations previously listed as a guide. This discussion is based on classic adsorption theory, a general discussion of which has been given in Section $\$ 2.2 .1$.

The explanation routinely given for gas sensing for sensors of the type used here is that of charge transfer. Although there are many variations of this theory, generically the charge transfer theory considers the adsorption of a gas molecule onto the sensing material of the device (the fundamentals of gas adsorption are discussed in Section \$2.2.1). The electrodynamics of the adsorption events are such that a charge (either a hole or electron) transfer event occurs, and a charge is transferred from the adsorbed molecule into the sensing material. In this way gas adsorption effectively dopes the material, adding either holes or electrons and changes the carrier concentration. Depending on the type of the sensing material (n-type or p-type), and whether the adsorbed molecule acts as an electron donor or acceptor, the device conductivity may be increased or decreased. This adsorption and charge transfer may be mediated by defect sites. Structural defects (like a carbon vacancy or a crystallite edge site) or chemical defects (like an oxygen functional group attachment) may act as preferential adsorption sites or mediate the charge transfer. For this mechanism to be a practical means of gas sensing, the analyte gas must readily transfer charges and the number of charge carriers in the sensing material must be low. This is so that the overall fractional change in the carrier concertation is detectable (via a change in device conductivity).

A charge transfer mechanism fits some of the observations recorded here. For example, there are already a large number of carries natively present in the film, particularly at elevated temperature (see Section \$5.2.2). A charge transfer from the gas (if it is occurring) would result in a relatively small change in the total carrier concentration. This may explain the small chemical response. Additionally, there is broad consensus in the simulation community that chemisorption and physisorption of hydrogen onto graphene is not a chemically or thermodynamically favorable process. ${ }^{(666)(238)(667)(239)}$ First principle studies show that the physisorption of hydrogen onto graphene is highly unlikely and in fact, hydrogen is more likely to tunnel through the hexagonal ring structure than adsorb onto it. ${ }^{(238)}$ The same study found that chemisorption of hydrogen onto 
a graphene sheet bends the sheet out of plane by $0.47 \AA$. This matches with work by Boukhavalov, et al. who calculated a similar value of $0.40 \AA$ for the out of plane bending and a total radius of distortion in the graphene sheet surrounding the adsorption site to be $3.8 \AA .{ }^{(668)}$ Given the high degree of tethering and interactions with the buffer layer (discussed in Section $\$ 4.1$ ) the rigidity of the films used here (and the fact that they are under tensile stress) may reduce these interactions and explain the low response and slow rate. In support of this supposition, are the results of work done by Casolo, et al. , which states that for the chemisorption of hydrogen to occur, the film must be relaxed to allow for the required surface reconstruction. ${ }^{(667)}$ The fact that there is a substantial energy barrier to hydrogen chemisorption may explain why there is no chemical response at low temperature. $^{43}$

Although these reports are on the study of hydrogen adsorption, the general idea can be applied to any gas. Specifically, the adsorption of all gases should be enhanced if the graphene sheet is relaxed and free to bend and reconstruct to accommodate the adsorption of gas. This is yet another motivator for the decoupling of the films produced in this work from their $\mathrm{SiC}$ substrates.

As discussed in Section \$2.2.2 it has been widely reported that the addition of metallic nanoparticles onto a gas sensing surface will enhance the interaction with the analyte gas. Therefore, the addition of metallic nanoparticles is another avenue by which the chemical sensitivity of these devices may be enhanced. In pursuit of this, the preliminary results of four different metallic nanoparticle nucleation/decoration methods are presented and discussed in APPENDIX B. For hydrogen detection specifically, noble metal nanoparticles may catalytically facilitate the surface decomposition of $\mathrm{H}_{2}$, which may be a more easily detectable process than simple adsorption.

An alternate explanation is the chemical response may involve interactions with chemical defects in the film which act as scattering sites to charge carriers. In this scenario, hydrogen reacts (chemically) with defect sites to rehybridize their electron orbitals or otherwise perturb the charge distribution in such a way as to reduce their effectiveness as charge scattering sites. The increase

\footnotetext{
${ }^{43}$ For this reason, the performance of most graphene-based gas sensing devices has focused on the detecting of complex molecules like $\mathrm{NO}_{2}, \mathrm{NH}_{3}, \mathrm{CO}_{2}$ or $\mathrm{H}_{2} \mathrm{~S}$ which result in greater interactions with the film and a correspondingly larger charge transfer. These gases have been shown to be detectable in the parts per million (PPM) or even parts per billion (PPB) range. On the other hand, the lowest lower limit of detection for $\mathrm{H}_{2}$ is in the low parts per hundred (\%) range and requires the addition of sensitizers like platinum metal. (358) (747)
} 
in conductivity observed would thus be due to a reduction in carrier scattering. This would explain why no chemical effect is observed at low temperature (where scattering effects are not a dominant contributor to device behavior). The slow response time may also be explained by this; a reaction and reordering of a defect site, being a complex process, would have slower kinetics than a simple adsorption and charge transfer process. Both the faster response rate and the larger response magnitude observed in testing at $409^{\circ} \mathrm{C}$ (Figure 5.44) fit this explanation as well. Higher temperatures lead to a higher reaction rate and higher temperatures make more sites chemically activate. Both would lead to a larger response at higher temperature. Furthermore, scattering becomes the dominant mechanism at high temperature, increasing the prominence of this chemical response.

Another mechanism which may explain the chemical response, is diffusion of hydrogen into the layered film. At very high temperature, this is almost certainly the case (this idea is the basis for the hydrogen-induced decoupling of the buffer layer experiments discussed in Section $\S 4.3)$. Hydrogen testing at very high temperatures $\left(>500^{\circ} \mathrm{C}\right)$, where hydrogen diffusion into the film is clearly occurring, is discussed in Section $\$$ 5.3.6. If a similar process is occurring at these moderately high temperatures $\left(\sim 200^{\circ} \mathrm{C}-400^{\circ} \mathrm{C}\right)$, then the chemical response may simply be the results of a partial film decoupling or $\mathrm{H}_{2}$ intercalation between layers as reflected in the conductivity of the device. Being that this would be a severely diffusion limited process, this would explain the slow response rate, the temperature dependence and the fact that the response rate is saturated at a low hydrogen concentration. As discussed in Section $\$ 2.1 .3$, others have shown hydrogen intercalation to be a reversable process but doing so requires elevating the device temperature to drive desorption. Therefore, counter to this explanation is the fact that the device signal does recover, even though the temperature of the device is relativity constant (excluding the self-heating effects, which have been discussed extensively).

A somewhat more insidious explanation for the chemical response may be a function of the photosensitivity of the devices (device photosensitivity will be discussed in Section \$5.4). In short, the device may be reacting to irradiation from the heating elements of the large furnace used in the large test bed. Although this explanation would seem to be the least likely, it cannot be rejected entirely. Thus, for the sake of thoroughness, a brief discussion of how the photosensitivity could explain the observed behavior has been included in the Appendix $\$$ A.4.1. 
In addition to the results and mechanisms previously discussed, there may also be separate and unrelated interactions at the metal-film interface with hydrogen. This could impact the Schottky barrier height or influence tunneling effects by effecting the charge depletion region. In support of this, an interesting effect is observed in the devices under a zero-bias condition. This effect is illustrated in Figure 5.51. Figure 5.51 examines the zero-crossing of a series of IV plots recorded from the same device and collected from room temperature up to $550^{\circ} \mathrm{C}$, in $50^{\circ} \mathrm{C}$ increment, under a pure argon (Figure 5.51(A)) and under a pure hydrogen (Figure 5.51(B)) atmosphere.
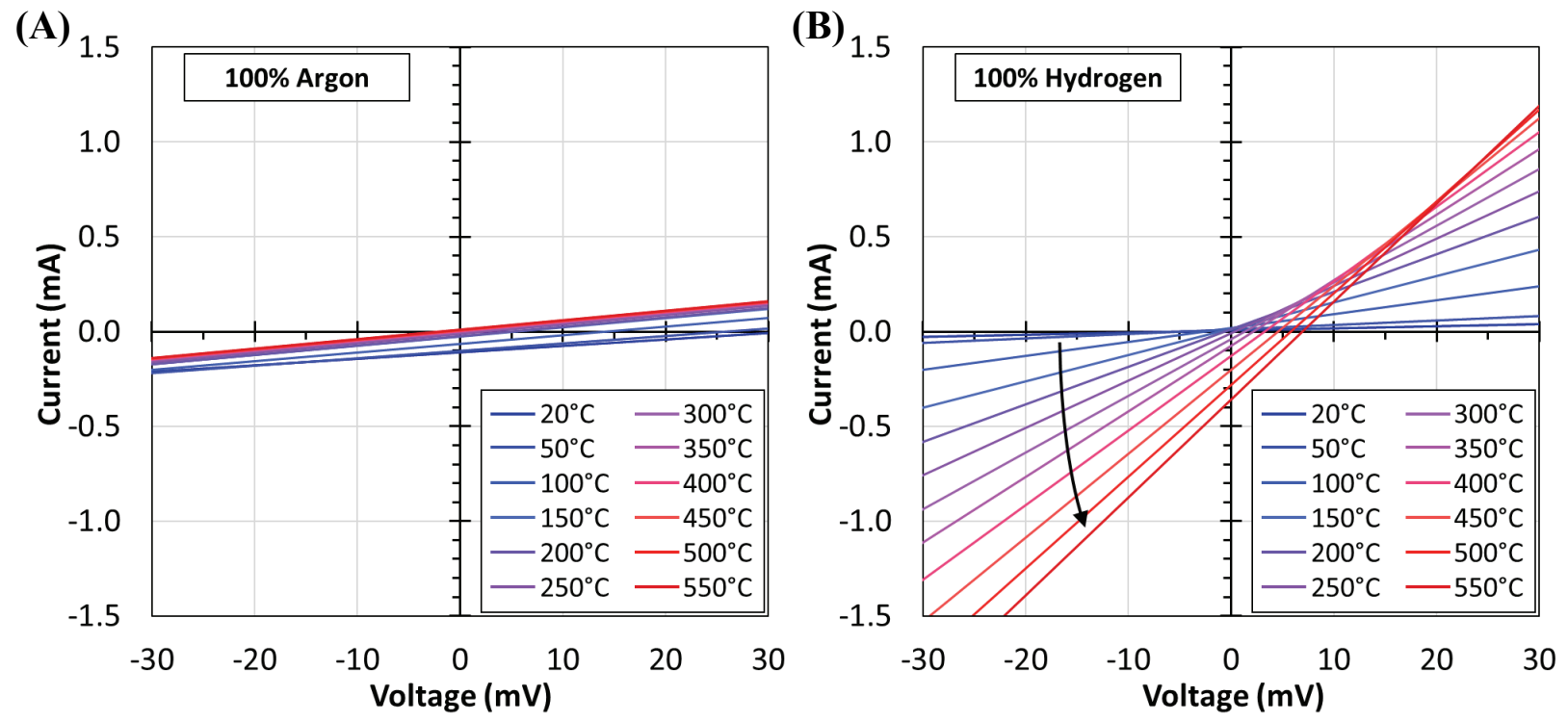

Figure 5.51: The IV plots collected at various temperatures in (A) a pure argon environment and in (B) a pure hydrogen environment. A substantial splash current is seen in the pure hydrogen environment.

The important difference between the plots of Figure 5.51 is the substantial splash current seen when the device is under a pure hydrogen environment. The splash current phenome is discussed in Section \$5.2.1 and is the term given to a current flow due to the thermal excitations of electrons out of the film and into the contacts (note that this is different from the thermoelectric effect and is most analogous to the photoelectric effect). Essentially, electrons are thermallypumped out of the film. A splash current is seen under argon (Figure 5.16), but comparable current flows are only seen when the device is substantially hotter $\left(800^{\circ} \mathrm{C}\right.$ under argon vs. $550^{\circ} \mathrm{C}$ under hydrogen for $\sim 0.4 \mathrm{~mA}$ ).

From Figure 5.51(B) it can be seen that the amount of splash current scales linearly with the increase in temperature. It is not clear what the specific process is which leads to the larger splash current under hydrogen as compared to argon. The hydrogen atmosphere may lower the 
Schottky barrier height, which would increase the amount of splash current. However, for there to be a balance of current flow in one direction, the Schottky barriers at the source and drain contact would have to be lowered asymmetrically (one barrier lowered more than the other). Alternatively, a surface reaction may be producing an electrochemical current. This is an area of the parameter space ( $0 \mathrm{~V}$ bias condition) has not been thoroughly investigated and may be an area of interest for future work.

\section{\$5.3.8 Summary of Device Performance in Gas Sensing Applications}

In summary, SOP devices successfully detect changes in the gas composition of their environment. There is a thermally derived and a chemically derived component to the device response. In the majority of cases, the thermal effects dominate the device signal. A thermal detection scheme has long been a reliable method for gas sensing. For example, the widely used Explosimeter ${ }^{\circledR}$ used in mine safety applications utilizes a Wheatstone bridge (resistor network) where the atmospheric composition is determined by temperature changes of a resistive element (via exothermic catalytic gas decomposition on the sensing surface). The common laboratory mass flow controller (MFC) and mass air flow (MAF) sensors in automotive engine applications use a similar principle (temperature-induced changes of resistance) for determining gas flowrates. ${ }^{44}$ With further development, the devices studied here could be used in similar applications on the wafer level; they may be particularly well suited for high temperature applications and the low power requirements make them intrinsically safe. The fact that such a device would be entirely solid state and planar, mean that small, inexpensive and extremely mechanically rugged devices could be produced, suitable for harsh environments, such as in space faring applications.

There are conditions where only a chemical effect is observed. In theory, chemical sensing would have many advantages over thermal sensing (limits of detection, response speed, selectivity, etc.). However, these benefits have not yet be realized in the devices tested here for reasons related to the buffer layer, discussed shortly. Nonetheless, a purely chemical effect is observed in response to hydrogen gas exposure at moderately high temperatures $\left(\sim 230^{\circ} \mathrm{C}\right)$ and low device setpoints $(<10 \mathrm{~mA}$ or $<0.20 \mathrm{~V})$. However, these bonds where a chemical effect were observed are not absolute and were found to vary from sample to sample.

\footnotetext{
${ }^{44}$ More information on the Wheatstone bridge (752), Explosimeter (758), mass flow controller operation (743) (755) (757) and other thermally derived gas sensing techniques (748) (749) (742) may be found in the references and in the Section $§$ 2.2.2 discussion.
} 
Section $\$ 5.3 .1$ establishes the self-heating phenomena observed in the devices and the resulting effect on the device response. Section $\$$ 5.3.2 shows that the film and the contact interface, which behave differently, combine to produce the two-point device response. Section $\$ 5.3 .2$ also shows that the decrease in device resistance with time is due to self-heating effects. The anonymous increase in resistance with time at low voltages, however, is most likely due to capacitive effects of the metal-energy barrier-graphene structure formed at the contact/film interface. Section $\$$ 5.3.3 show that both gas flowrate and gas composition influence the temperature of the device and thus, the device response. Section $\$ \mathbf{5 . 3 . 4}$ explores the conditions where a transition from a thermal to a chemical effect can be observed. It is unclear if the chemical effect is present at high voltages due to the onset of the thermal response, which is much larger in magnitude and masks any potential chemical effect which may occur. No chemical effect is seen at low temperature. At high temperature, the thermal effects flips and increasing temperature now increases the device resistance. This means that the thermal effect and the potential chemical effect both result in a reduction in device resistance. Therefore, it is difficult to differentiate between these effects when the devices are at high temperature. However, the overall shape of the response curve indicates a chemical effect. Section $\$ 5.3 .5$ show the devices to be sensitive to both CO and $\mathrm{CH}_{4}$ gas, although this sensitivity is derived from a purely thermal mechanism. This section concludes with a discussion of the results of very high temperature testing with hydrogen. This resulted in a partial decoupling of the film and a marked improvement in the electrical properties of the device.

There are two reasons for the small chemical response observed in these devices. First, the primary gas tests, hydrogen, has been shown to have a very low level of interaction with graphene. This makes $\mathrm{H}_{2}$ one of the more challenging gases to detect. Secondly, the tight coupling of the buffer layer to the substrate and the resulting coupling of the graphene overlayers to the substrate through the buffer layer, effectively desensitize the graphene film. This happens for a variety of reasons, which are summarized next.

First, consider the larger picture. As discussed in Chapter 2, the primary advantage of using nanomaterials (low-D materials) in gas sensing applications is that they have no internal volume. Thus, their behavior is governed entirely by the surface interactions. However, by binding tightly to the substrate, the carbon film produced here loses this advantage. The coupling of the electronic and vibrational states of the surface film with the substrate mean that the film is only 
pseudo-freestanding. As discussed in the electronic characterization section, this has led to excessive doping from the substrate to the film, which has increased the number of charge carriers. This leads to the second point. Considering the larger number of carriers already present in the film, a change in the carrier population, due to interacts with adsorbed gas molecules, is fractionally a very small change, resulting in a correspondingly small change in the signal (conductivity value) in response to an analyte gas. Recall that one of the motivations outlined in Section $\$ 2.2 .2$ for utilizing graphene as a gas sensing material is that graphene has a low number of native charge carriers; however, this is no longer the case when the films experience substantial doping, such as is the case here.

The third point is that the electrical communication from the film to the substrate may effectively 'short-out' the film response. The coupling (physically and electronically) between the film and the substrate can serve as a carrier source/sink, such that even if there is a large number of charge transfer events, the film electronically re-equilibrates to the pre-adsorbed carrier concentration by exchanging excess carriers with the bulk. This is a consequence of the first point.

The fourth point is that the mechanical coupling of the film to the substrate prevents the subtle conformational changes in the graphene film required for chemisorption of gas molecules. This decreases the strong surface interaction required for gas detection. Furthermore, by bonding with the substrate, the film may be become more chemically inert through the passivation of many of the dangling $\pi$-bonds of the graphene sheet. The electron clouds of the $\pi$-bonds are involved with bonding to the substrate and are therefore unavailable to interact with the environment, which further reduces sensitivity to gas (see Section $\$ 1.1$ for an atomistic description of the electron orbitals in graphene).

Finally, from the decoupling discussed in Section \$5.3.6, it is clear that it is the buffer layer that is responsible for the Schottky nature of the films (illustrated by the transition to Ohmic behavior observed after partial buffer layer decoupling). The Schottky characteristic of the devices make them highly temperature sensitivity, which is the fundamental basis for the thermal response. For these reasons, it is concluded that all aspects (sensitivity, response speed, etc.) of the chemically derived gas sensing may be improved by a complete decoupling of the films from the substrate. However, as will be discussed in the following section, this may not be advisable for photodetection applications. 


\section{§5.4 Response to Light Irradiation}

This chapter concludes with a discussion of the sensor response to visible light irradiation. In these tests, the SOP devices functioned as light (photo) detectors. The experimental setup is described in detail in Section $\mathbf{\$ 3 . 5}$. Of all device testing modes, light sensing was the most limited; however, initial results are promising. Further study and a refinement of the experimental setup will be required to further explore the photo response of these devices.

All photodetection experiments were performed in the open-air environment of the laboratory, using the MK0* package design. Approximately 25 SOP devices were tested. The devices used either the interdigitated sensor or parallel contact pattern designs and were $2.5 \mathrm{~mm} \mathrm{x}$ $2.5 \mathrm{~mm}$ in dimension. The devices were irradiated with either a red $(650 \mathrm{~nm} \pm 10, \sim 1.9 \mathrm{eV})$, green $(532 \mathrm{~nm} \pm 10, \sim 2.3 \mathrm{eV})$ or purple $(405 \mathrm{~nm} \pm 10, \sim 3.1 \mathrm{eV})$ low power $(<5 \mathrm{~mW})$ diode laser. ${ }^{45}$ Using the Keithley 4200 electrical characterization equipment, the photo response was then recorded. The devices were operated in either a powered mode (constant voltage bias) or unpowered mode (constant current mode at $0 \mathrm{~A}$ or constant voltage mode at $0 \mathrm{~V}$ ).

\section{§5.4.1 Positional Sensitivity}

The first step to evaluate the photo response of the $\mathrm{SiC} /$ Graphene devices is to determine which part of the device is photosensitive. For these tests, the Keithley 4200 was set to operate in constant voltage mode, with an applied voltage of $0.0 \mathrm{~V}$. The current through the device was recorded as a function of time. A red laser was rastered across the surface of the SOP devices to determine the position on the sample which resulted in the largest photocurrent. In all cases, the largest response was produced when the laser spot irradiated the area of the device around the $\mathrm{Ti} / \mathrm{Au}$ contact to which electrical contact to the Keithley 4200 was made. Irradiation of $\mathrm{Ti} / \mathrm{Au}$ contacts which were not electrically connected to the test setup did not result in a large photo response. This was true for both the sensor contact and parallel contact designs (both designs are described in technical detail in Section \$3.3.1). However, this effect is most clearly illustrated when using the parallel contact designs. Recall that the parallel contact design presents five sets of six parallel noncontiguous contacts, whereas the interdigitated sensor design provided only two circuitous interlocking contacts. Therefore, all positional sensitivity testing data presented here

\footnotetext{
${ }^{45}$ The fundamental relationship between frequency, wavelength and energy are pertinent to the discussion contained within this section; recall that higher frequencies correspond to shorter wavelengths and higher photon energies. This is described analytically by the Plank-Einstein relation, detailed in Appendix §A.5.1.
} 
were carried out on devices using parallel contact exclusively (even though the effect is observable in both designs).

To further investigate this positional sensitivity (i.e. device response as a function of the position of the laser spot on the sample) the laser spot was rastered across a rectangular contact. In this case, the primary contact irradiated $(100 \mu \mathrm{m} \times 400 \mu \mathrm{m}$ is size $)$ was the first contact in a series of six parallel contacts. This set of six was located at the physical edge of the sample. A red laser was scanned across both the short and long dimensions of the contact, as illustrated schematically in Figure 5.52. This was accomplished by manually moving the X-Y translational sample stages of the laser testing apparatus by hand in a stepwise fashion. This moved the laser spot across the sample.

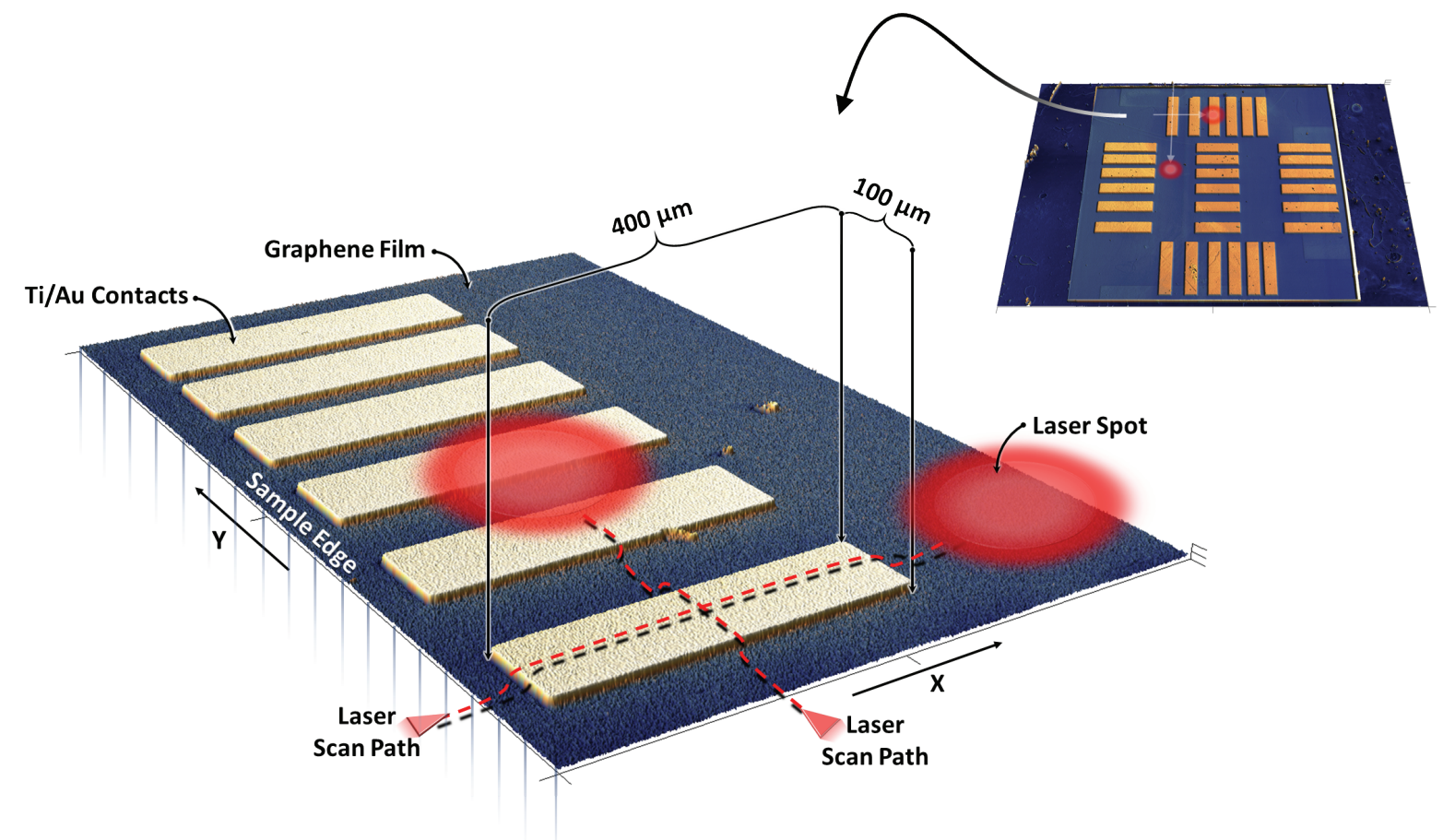

Figure 5.52: Illustration of the two laser scan paths. Shown is a false color, $3 \mathrm{D}$ projection of a SEM image of a $2.5 \mathrm{~mm}^{2}$ sample with the parallel contact pattern design. The path of the laser spot across the sample is shown. The approximant laser spot size is shown to scale.

Electrical contact was made between the first and last contacts in the parallel array, with each contact connected to an independent SMU. In this way, the current flow at each contact was independently measured. The resulting photocurrent measured at the irradiated contact was then recorded as a function of laser position. Figure 5.53(A) plots the photocurrent recorded as the laser is scanned in the $\mathrm{X}$ - direction (across the long dimension of the Ti/Au contact) and Figure 5.53(B) plots the corresponding data collected as the laser spot is scanned in the Y-direction (across the short dimension of the contact). The $\mathrm{x}$-axis of both plots is arbitrary distance. The jitter and 
clumping of data points is due to the manual stepwise motion of the sample stage and the comparatively high sampling rate of the data stream.
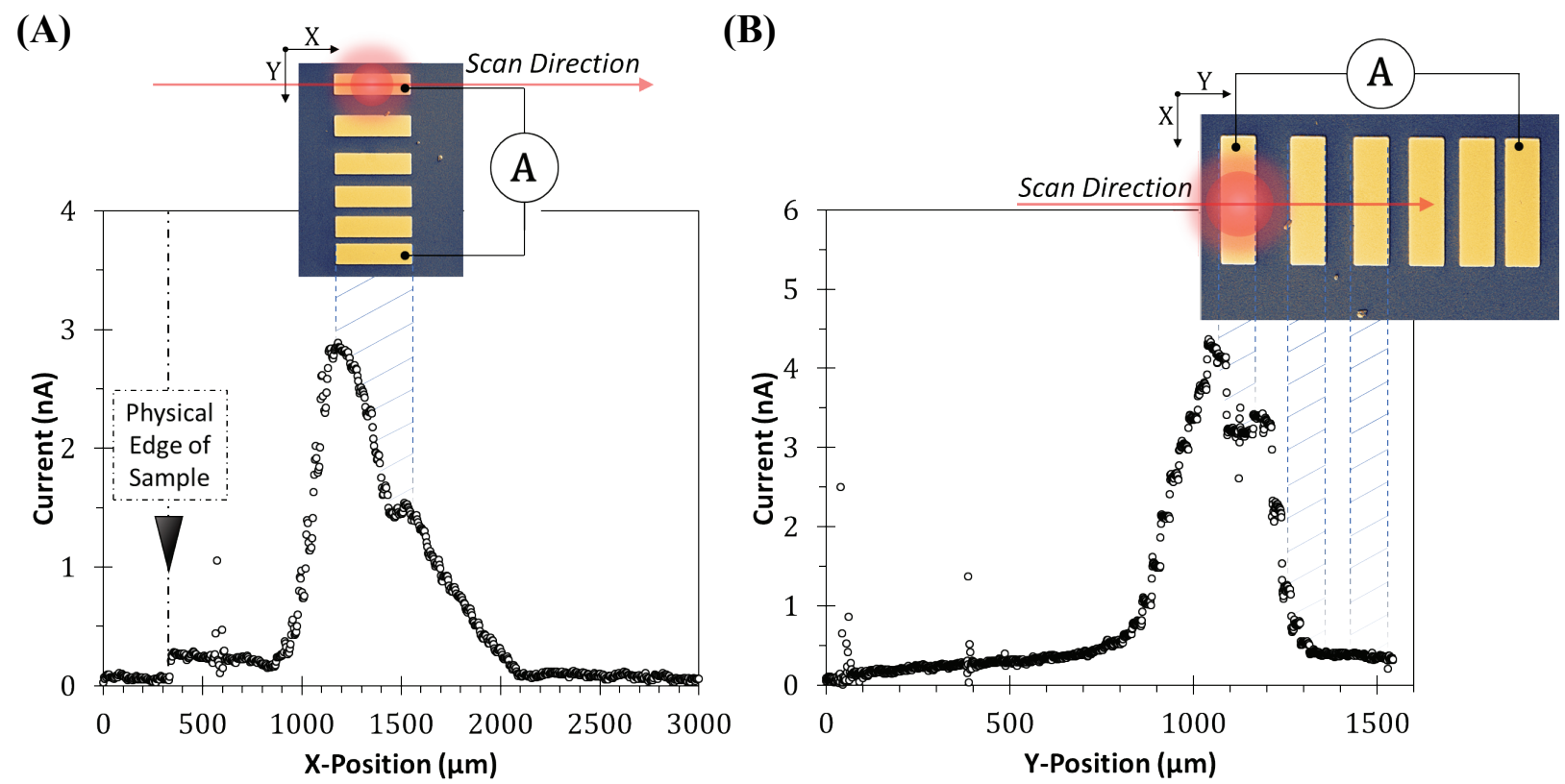

Figure 5.53: Photocurrent generated as a function of position by irradiation with red laser light. (A) Photocurrent as the laser is first scanned onto the sample and then across the length of a Ti/Au contact. (B) Photocurrent as the laser is scanned across the width of the same contact. The relative position is correlated with the photo response, as indicated by the false color images and the dashed drop lines. The laser spot size is drawn to relative scale. Note that the inset contact schematic has been rotated $90^{\circ}$ from (A) to (B) for clarity.

Several factors limit the spatial resolution of the photo response plots. These include the relatively large laser spot size, diffusion at the edges of the laser spot, the slight nonsymmetrical geometry of the laser spot and a nonuniform laser intensity within the spot size. Regardless of these limiting factors, it is clear that the largest photocurrent is generated when the electrical contact is irradiated. Specifically, irradiation of the interface between the metal contact and the graphene film (i.e. at the contact edges) produces the largest response. Areas more remote from the metal contact produce little photocurrent and irradiation of contacts not directly in the circuit do not produce a photocurrent (see Figure 5.53(B)).

That data shown in Figure 5.53 is the current recorded at the irradiated contact. During the same test, the current flow at the other contact was also recorded. Both electrical contacts produced an identical response, but the sign of the current flow is reversed. This data is provided in Appendix $\boldsymbol{\$}$ A.5.2. This effect is simply reflective of the electrical circuit formed. The photoinduced carrier flow direction is into the contact being irradiated, with makeup electrons pulled from the other electrical connected contact. This explains why irradiation of the metal contacts not 
directly contacted to the measurement circuitry do not produce a response. Charge carriers are pumped from the film into the contact and, being that there is no path to ground, are trapped and cannot contribute to the signal.

These observations begin to give insight into the photo response mechanism of the devices. Clearly, the contact/film interface, and the related phenomena which arises there, play a key role; as opposed to a purely film or film/substrate derived effect. Film effects are not responsible here because irradiation of the film far from the contacts does not produce a large photo response. There have been several reports on graphene devices which show a similar photo sensitivity at the metal/contact interface. ${ }^{(669)}$ Additionally, Echtermeyer, et al. show that the corners of a square contact produce a larger photo response as compared to the straight contact edge. (670) They attribute this to a concentration of electric field lines at these type of corner locations (a "lightningrod" effect). Given the likely mechanism of the photo response observed here (discussed in Section \$5.4.5), this explains the small humps in the data shown in Figure 5.53(A,B), which correspond to strong irradiation of the contact corners.

Figure 5.54 summarizes the positional sensitivity results observed here for irradiation of the device at three different positions. Irradiating at position 1 generates photocarriers which travel to the contact, then through the measurement circuitry where they are recorded and ultimately, to ground. This shifts the electronic equilibrium of the device (a positive charge, proportional to the amount of lost electrons, is developed) and makeup electrons are pulled from the other contact connected to the circuity, labeled 3 in Figure 5.54. Similarly, irradiation at position 3 causes the

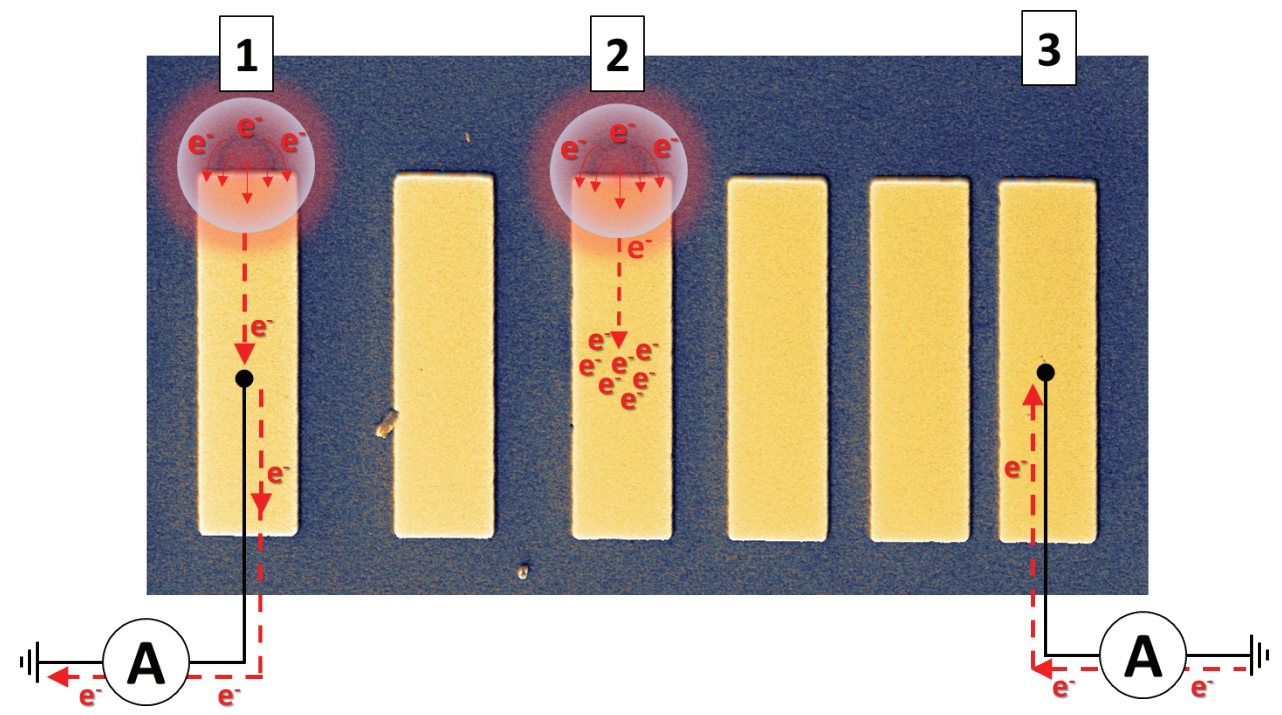

Figure 5.54: The photocurrent circuit produced by irradiating at two different positions. 
same effect, but the current flow is in the opposite direction. Irradiation at position 2, however, does not produce a recordable photocurrent, even though photocarriers are generated. The carriers produced fall into the metal contact and trapped, bounded by the energetic Schottky barrier 'walls' which surround the contact.

In summary, irradiation of the film/contact interface generates high energy photocarriers in the film (which are charge carriers photonically promoted to a high energy level, proportional to the energy of the incident light). These photocarriers, under the influence of the built-in electric field of the depletion region, fall to the lower energy levels available within the metal contacts. This is the same process which generates the depletion region in the first place. In this case, however, the depletion region is temporally populated with photocarriers, which then exit the depletion region under the same mechanism by which the original free carriers left (i.e. by falling into the metal contact). The metal contacts are effectively energetic wells surrounded by the Schottky barrier. Thus, if the irradiated contact is not electrically connected to the circuit (excluding the film, which is inaccessible due to the Schottky barrier), the photocarriers become trapped and cannot contribute to the photo signal. If the contact is connected to a ground, however, the photocarriers can lower their energy even further by traveling through the measurement circuitry to the electrical ground. In this way, a measurable photocurrent is generated; but only when a contact edge is irradiated, which is also directly connected to the circuitry. Finally, the fact that irradiation of the film far from a contact does not produce a large response indicates that the contact/film interface plays a key role in the response mechanism. Further discussion of this mechanism is given in Section $\$$ 5.4.5.

\section{\$5.4.2 Pulse Response}

To evaluate the unpowered photo response of the devices, the electronic monitoring equipment was programed to operate in a constant voltage mode, with a setpoint of $0.0 \mathrm{~V}$ (to measure the short-circuit current) or in a constant current mode, with a setpoint of $0.0 \mathrm{~A}$ (to measure the open circuit voltage). Both illustrate self-powered photo response of the devices (i.e. the devices were not externally powered). For these tests, the red laser ( $<5 \mathrm{~mW}, 650 \mathrm{~nm} \pm 10, \sim 1.9 \mathrm{eV}$ ) was used. Using the focusing optics of the testing apparatus, the on-sample laser spot size was minimized $\sim 200 \mu \mathrm{m} \pm 25 \%$ in diameter. The laser spot was then rastered across the Ti/Au contact in a grid like pattern and the photo response was recorded as a function of position. The laser spot 
was then repositioned to the coordinate which resulted in the largest photo response. The laser was then pulsed on and off to produce the pulse responses shown in Figure 5.55.
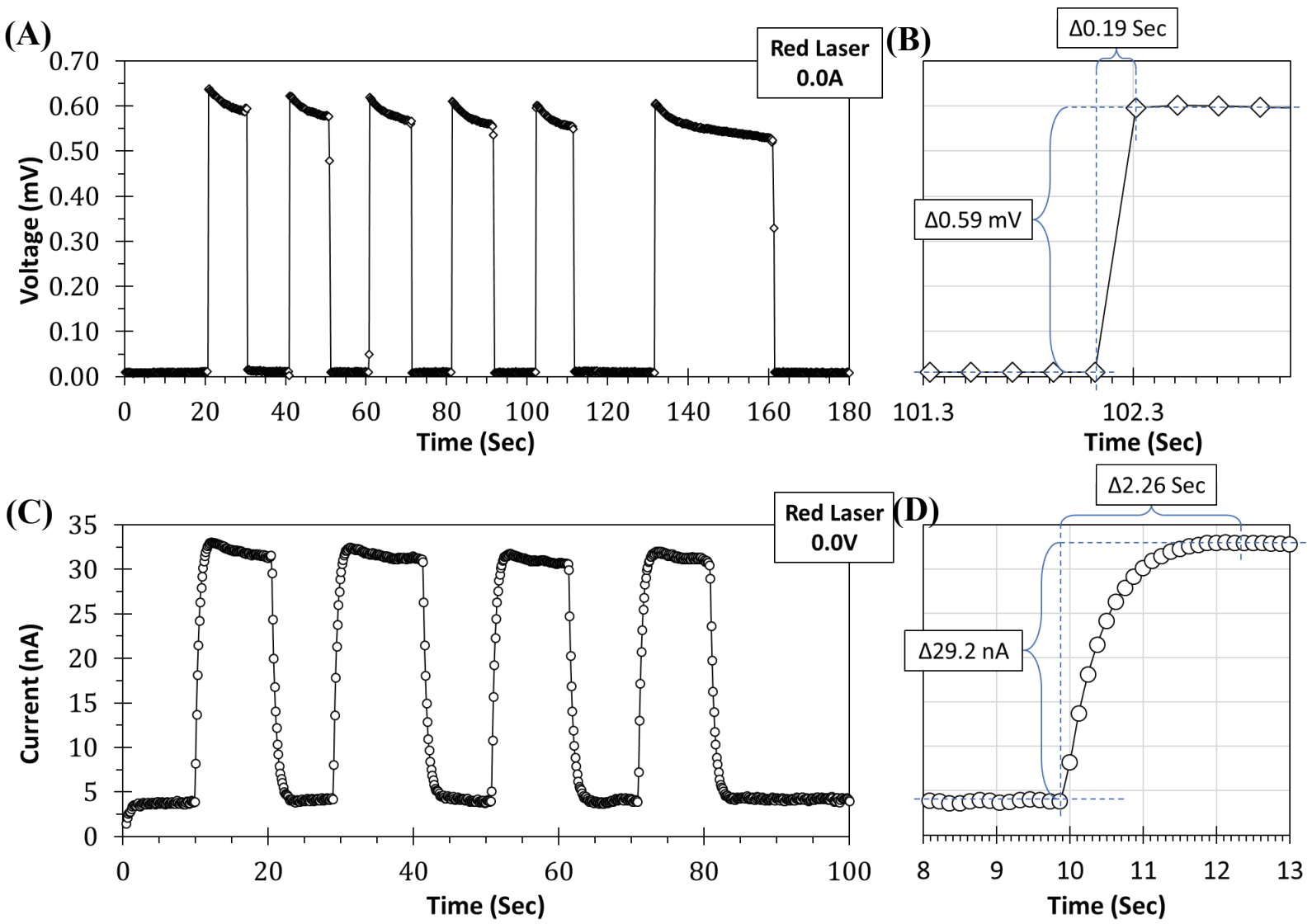

Figure 5.55: (A) Photovoltage due to five pulses of red laser light. (B) Higher resolution plot of one pulse, illustrating the fast response time. (C) Photocurrent due to four pulses of red laser light. (D) Higher resolution plot of one pulse, illustrating the, comparatively, slower response time.

The photovoltage produced from five pulses of red laser light, for a device operated in constant current mode (0.0A), are shown in Figure 5.55(A,B). A higher resolution plot of a single pulse is shown in Figure 5.55(B). The results collected from irradiating a different device with four pulses of red laser light in constant voltage mode $(0.0 \mathrm{~V})$ are shown in Figure 5.55(C,D). A higher resolution plot of a single pulse is shown in Figure 5.55(D).

The results show that both a photocurrent and a photovoltage are produced. There appears to be no decrease in response between successive pulses (i.e. no bleaching effect, etc.). The slight drop in response during a single pulse (most notable in Figure 5.55(A)) is most likely due to the fact that the laser is powered on and off to generate the pulses. The drop in the device response may reflect a small decrease in conversion efficiency of the laser diode as it heats up, and the beam 
power decreases and stabilizes. Subsequent tests using a shuttering setup, where the laser was continuously powered and the beam was intermittently blocked, show this is the case.

Setting aside the finer details, not only is the photovoltage response larger than the photocurrent, the response rate is also much faster. This is evident by comparing Figure 5.55(B and D), even though the sampling rates are different. The photovoltage response takes, at most, 0.19 seconds to reach the full response (the data sampling rate is slower than the response rate). Analogously, the photocurrent response takes $\sim 2.26$ seconds. This gives insight into the response mechanism, discussed in Section $\$$ 5.4.5.

\section{§5.4.3 Frequency Response}

Using the same testing methodology as the pulse tests described in Section \$5.4.2, the device frequency response was evaluated. This was done by exposing the same device to pulses of three different laser frequencies: red $(650 \mathrm{~nm} \pm 10$ or $\sim 461 \mathrm{THz})$, green $(532 \mathrm{~nm} \pm 10$ or $\sim 564 \mathrm{THz})$ and purple ${ }^{46}(405 \mathrm{~nm} \pm 10$ or $\sim 740 \mathrm{THz}) .{ }^{47}$ Each laser was rated at $<5 \mathrm{~mW} .{ }^{48}$ For each frequency test, the same spot on the sample was irradiated.

Figure 5.56 shows the photocurrent produced in response to five pulses of laser light at the three different frequencies. The relative magnitude of the device response is illustrated in Figure 5.56(A). The leading edge of a pulse is shown to the right and illustrates the different in device response rise time for each frequency. The first two pulses of each test are shown in greater detail in Figure 5.56(B,C,D). Shorter wavelengths lead to both a larger and a faster response.

\footnotetext{
${ }^{46}$ Although commonly referred to as 'violet' light, this frequency is referred to here as 'purple light' so as not to be confused with ultra-violet light.

${ }^{47}$ A new, higher quality red laser was used in this set of experiments than in the previous set; even though both lasers were rated to have the same output power (" $<5 \mathrm{~mW}$ "), this may account for the larger photocurrent recorded for red light in this set of experiments. Sample-to-sample variations and variations in irradiation location on the sample contribute as well.

${ }^{48}$ The actual laser power outputs were not measured; therefore, there may also be a difference in the beam intensity of each laser. However, the larger trends observed here are beyond what would reasonably be expected due to slight power variations between lasers.
} 

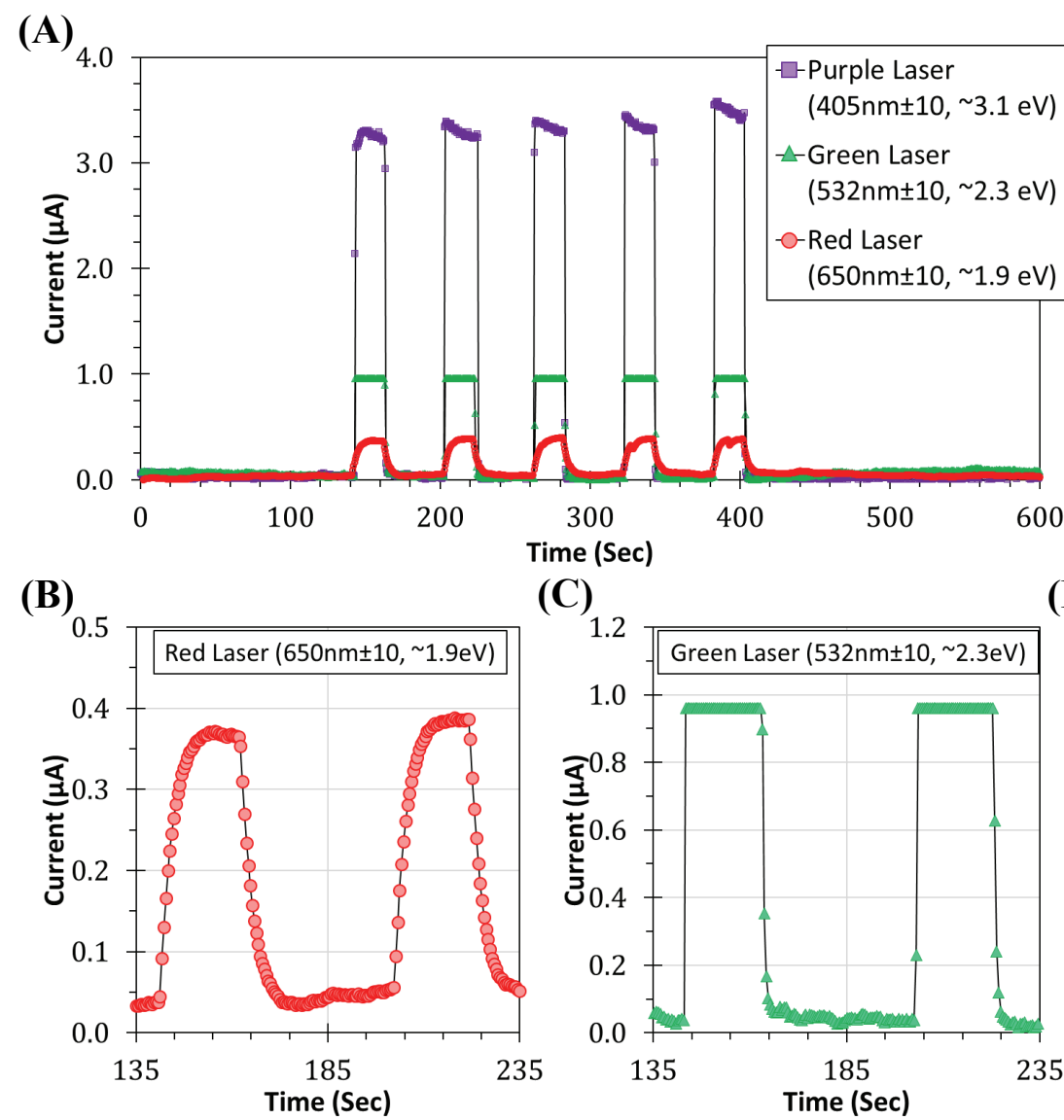

(C)
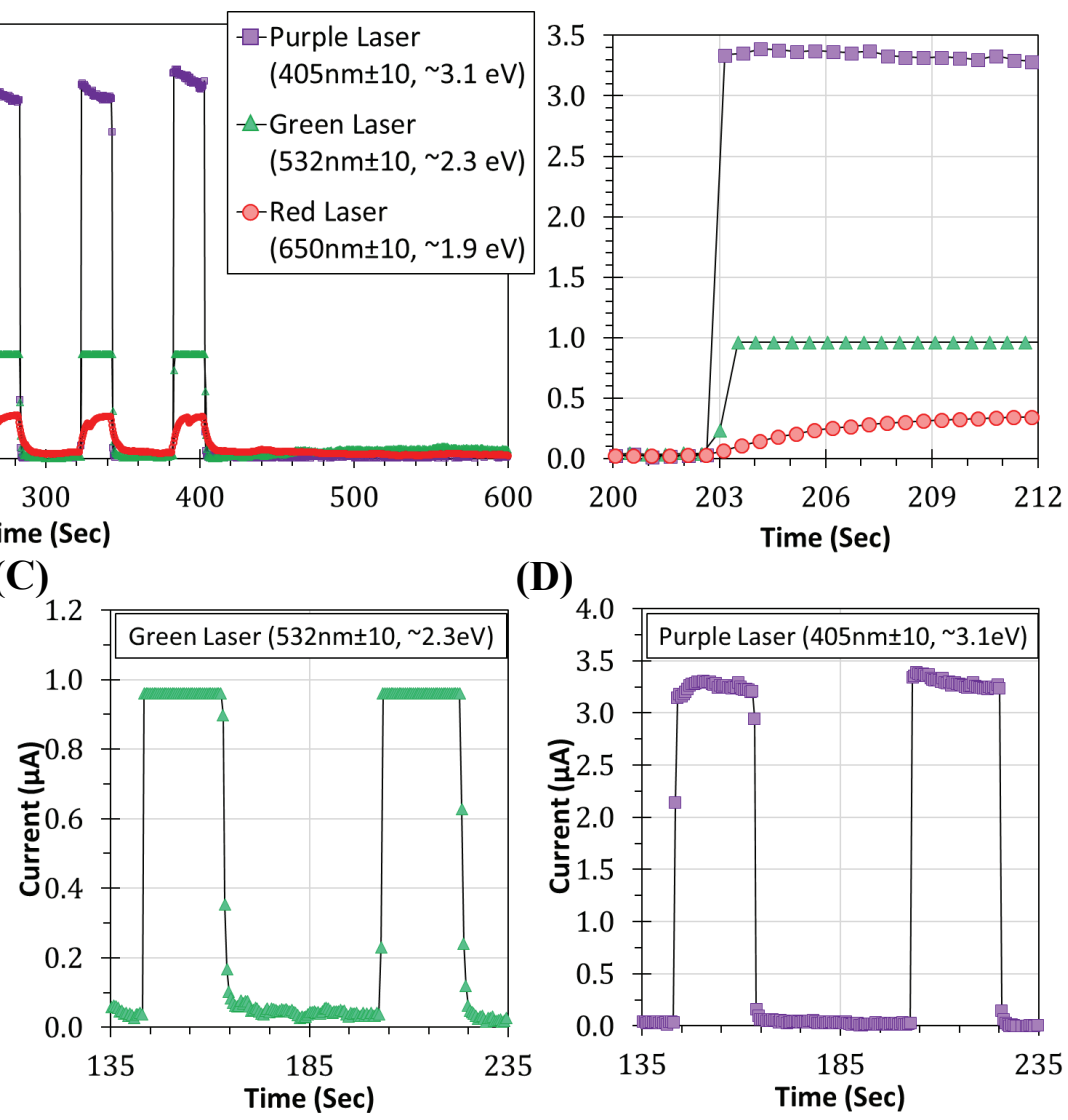

(D)

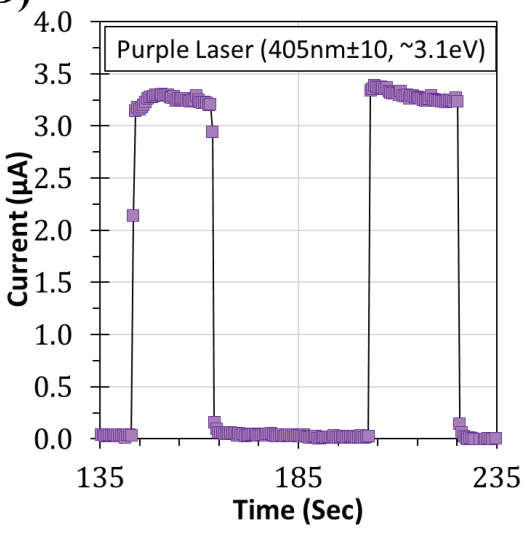

Figure 5.56: (A) The photocurrent generated in response to five pulses of laser light. Three different laser wavelengths were tested. The inspection box to the right shows leading edge of a pulse, highlighting the difference in rise time. Higher resolution plots of the first two pulse are shown in (B) red laser light, (C) green laser light and (D) purple laser light.

The average photocurrent generated as a function of the wavelength and energy of the irradiating light, is plotted in Figure 5.57. The Y-error bars are calculated from the variation in maximum response and the x-error bars are calculated from the manufacturer's specification of a $\pm 10 \mathrm{~nm}$ variation in laser wavelength for each laser used. The data match exceptionally well to a best-fit exponential curve, indicating there is an exponential relationship between the device signal response and the irradiation energy. 
(A)

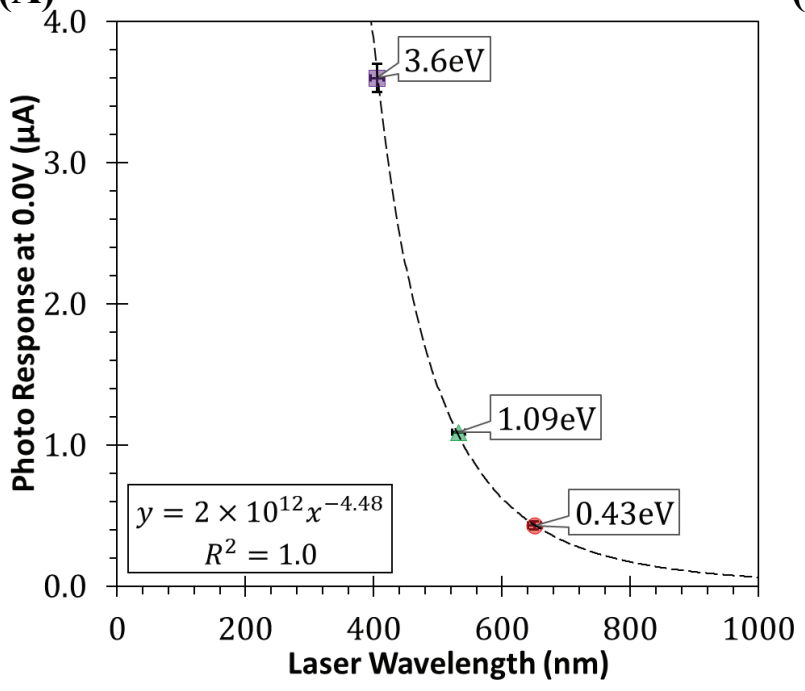

(B)

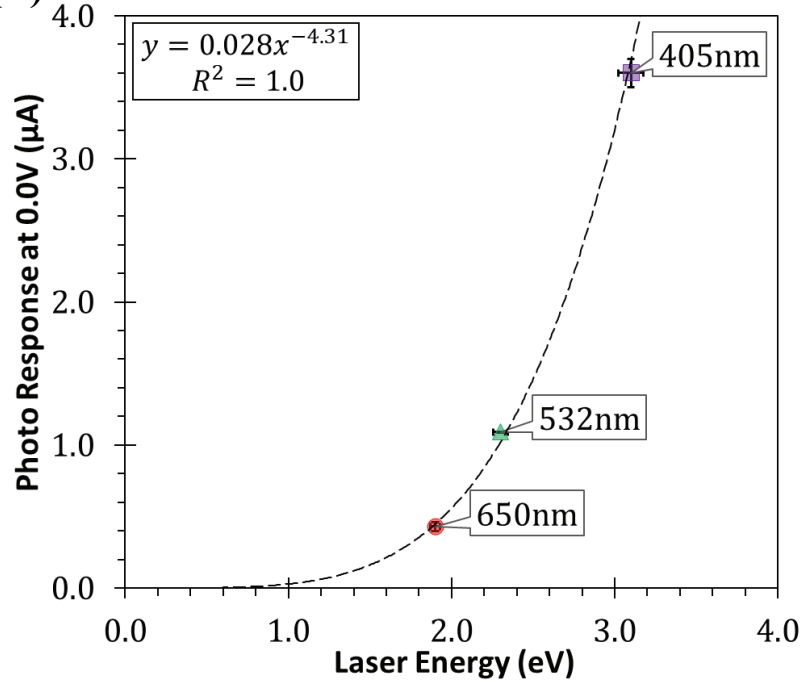

Figure 5.57: (A) Photocurrent as a function of laser wavelength and (B) as a function of laser energy. The dashed lines correspond to the best fit power law line for each dataset.

Given that the adsorption spectrum is notoriously flat for graphene (this is especially true in this spectral region) the exponential increase in response with increasing frequency is not due to spectral adsorption differences. ${ }^{(371)}$ Rather, however, this behavior is best explained by a carrier multiplication (CM) effect. The CM effect was first predicted to occur in graphene by Winzer, et $a l$. and was later experimentally verified by Plötzing, et al. ${ }^{(671)(672)}$ Alternative to, or working synergistically with the $\mathrm{CM}$ effect, is participation of the $\mathrm{SiC}$ substrate in the photocarrier generation process. The nominal band gap for pristine $6 \mathrm{H}-\mathrm{SiC}$ is $3.3 \mathrm{eV}$. This means that purple photons $(3.6 \mathrm{eV}, 405 \mathrm{~nm})$ contain enough energy to promote charge carriers across the band gap in $6 \mathrm{H}-\mathrm{SiC}$. Both possibilities are discussed in Section \$5.4.5. Although it is worth noting that the modified $6 \mathrm{H}-\mathrm{SiC}$ interface presents here (beneath the carbon film) may have a substantially different electronic band configuration and possess a variety of inter-gap states from which carriers may be promoted, thus making the material photosensitive to longer wavelength (lower energy) radiation than it otherwise would be.

A direct comparison of the device performance to literature reports is difficult without knowing the precise power of the lasers used here (specified only as " $<5 \mathrm{~mW}$ "). However, a preliminary analysis indicates the devices studied here perform comparably to similar devices reported. Huang, et al., for example, report on the UV response of a very similar device to what is used here. ${ }^{(410)}$ They use the selective sublimation method to grow epitaxial graphene on $\mathrm{SiC}$ (polymorph unclear) on which they construct a two terminal (Ti/ $\mathrm{Au}, 5 \mathrm{~nm} / 60 \mathrm{~nm})$ device. Although 
they tested only UV wavelengths, by using the best fit equation shown in Figure 5.57, the predicted results of these devices can be estimated. Allowing for the uncertainty in laser powers (and intensity due to beam spot size), the photo results presented here are within $30 \%$ of their reported values, which they claim are four orders of magnitude higher than previous reports.

\section{\$5.4.4 Powered Response}

Consistent with the previously described approach, the device photo repose was measured for a device under power. When powered, the devices display a similar positional sensitivity as discussed in Section $\$ \mathbf{5 . 4}$.1 in that a response was only seen when the device was irradiated near a contact. In this test, a constant voltage of $0.500 \mathrm{~V}$ was applied across the device (interdigitated sensor contact pattern, MK0* package design, open air) and the current was measured as a function of time. Again, the self-heating effect (described in detail in Section \$5.3.1) is observable. After 380 second of equilibration time, the sample was irradiated with five red laser pulses. The results are shown in Figure 5.58, where the current is recorded as a function of time for a constant voltage of $0.500 \mathrm{~V}$ applied across the device. Several interesting aspects of the device are apparent and are annotated in the figure.

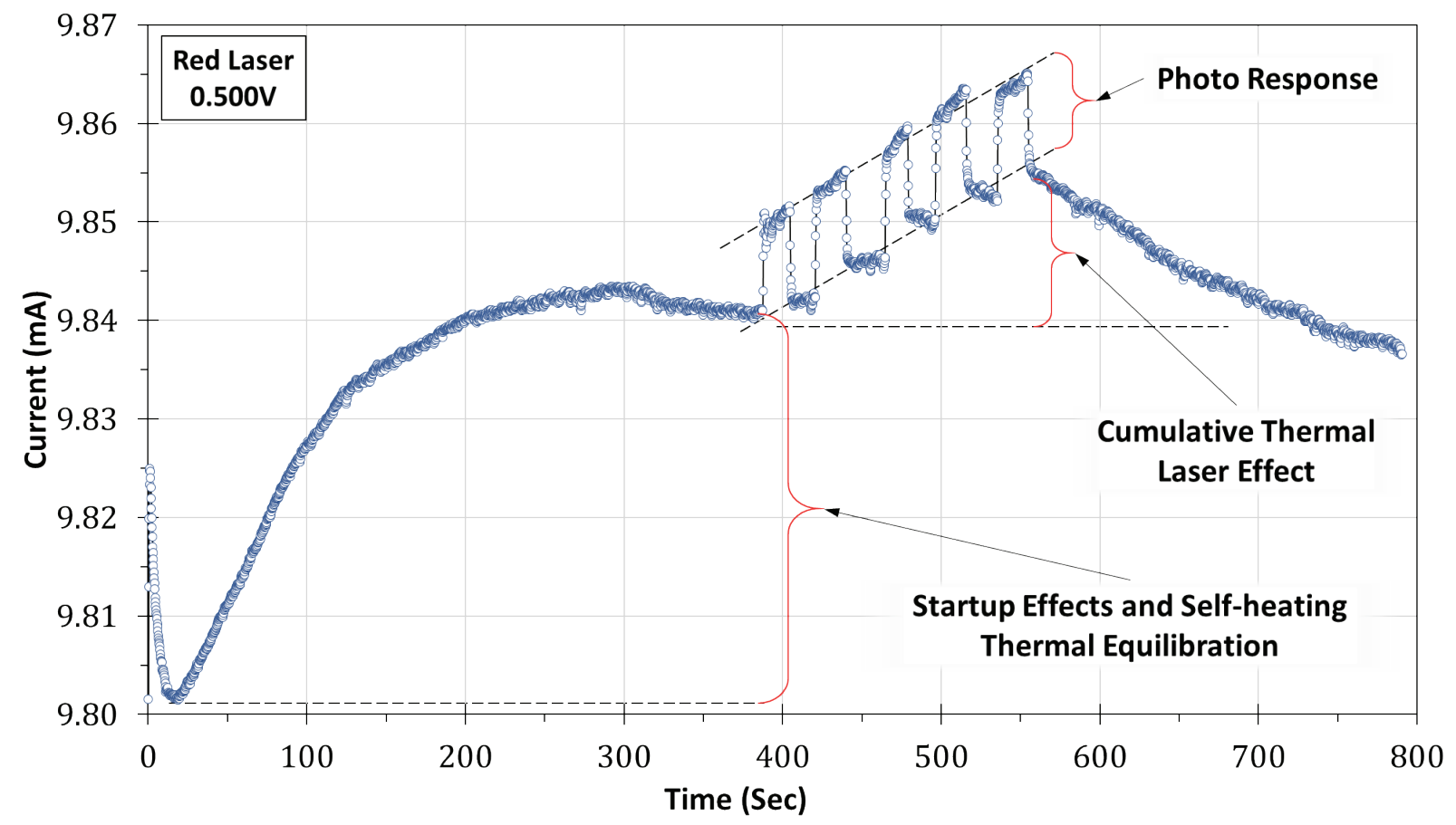

Figure 5.58: The powered photo response. Both thermal effects and photo effects can be seen.

As always, the initial startup and device thermal equilibration is observable. In response to five $\sim 20$ second long pulses of red laser light, the photo response is clearly indicated by the step 
wise increase in current. This response is, however, superimposed onto the laser-induced heating of the device. This heating reduces the device resistance and more current is passed through the device at the constant voltage setpoint. The considerably different time constant of the photo response and the laser induced thermal response make them easily differentiable.

A similar test was conducted for an applied voltage of $1.0 \mathrm{~V}$ across the device. These results show an almost $4 \mathrm{X}$ increase in photocurrent. However, by applying a voltage, a substantial dark current is generated; the device conducts current when a voltage is applied, regardless whether the device is illuminated or not. Increased device voltage also leads to the device self-heating thermal effects (self-heating effects are discussed in detail in Section \$5.3.1). These effects combine to cloud the device response and thus, the photo response is not as clearly revealed in the data. Figure 5.59 plots the average photo response to the same red laser on the same device operated at three different voltage levels; $0.0 \mathrm{~V}, 0.5 \mathrm{~V}$ and $1.0 \mathrm{~V}$. Also plotted on the secondary axis is the on/off ratio.

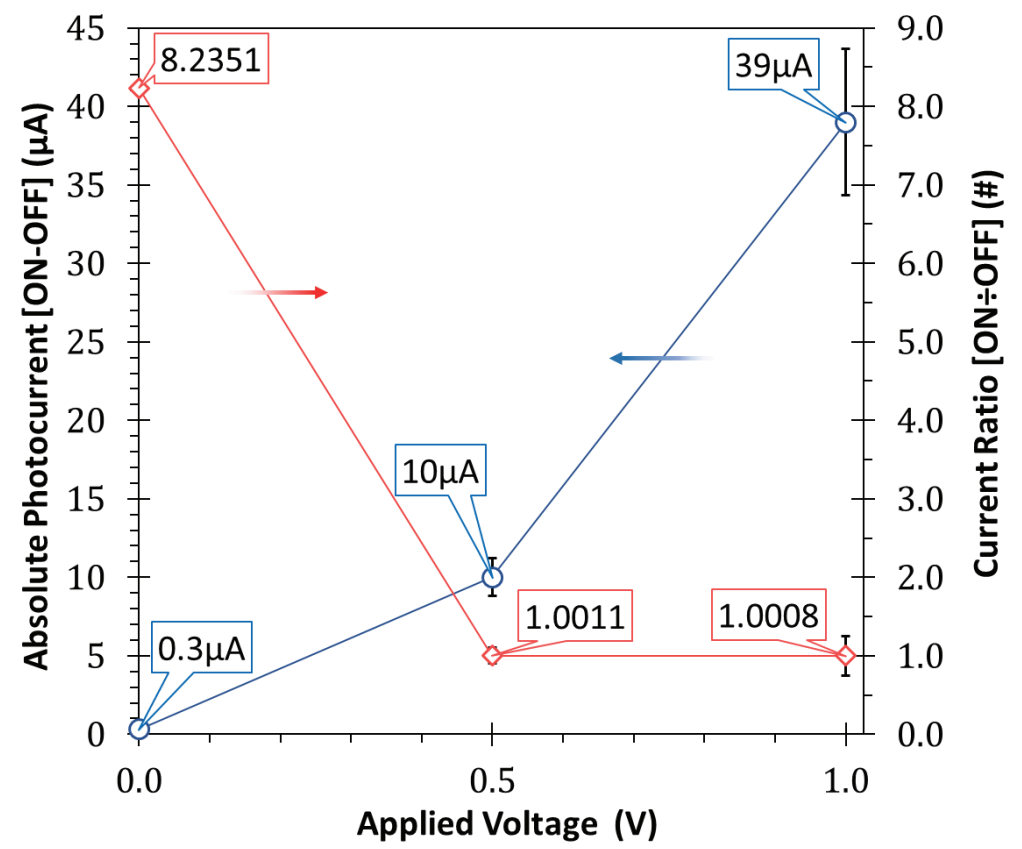

Figure 5.59: Photocurrent recorded due to irradiation with red laser light as a function of applied voltage across the device. Error bars are calculated from the minimum and maximum amplitude of the photo response to five pulses of laser light in each case.

The higher photocurrents observed for the powered devices is likely due to the enhancement of the built-in electric filed of the Schottky contact by the externally applied bias. A higher applied device voltage results in a larger photo response, but this response rides on the dark current signal floor, which is then further offset by photo-induced sample heating. Therefore, 
although a larger response is observed for a powered device, the very low dark current through the device at a $0.0 \mathrm{~V}$ bias condition makes device operation in a self-powered mode more attractive.

\section{§5.4.5 Sensing Mechanism and Future Directions}

A critical analysis of the results presented in each section give insight into the photo response mechanism. What follows is a discusses of the observational results presented in this section. The potential photo response mechanisms which explain these observatories are evaluated.

There are four mechanisms commonly discussed in the literature used to explain the photo response in graphene devices. The following review articles provide a thorough overview of these mechanisms. ${ }^{(673)(382)(674)(675)}$ Following the categorization given by Koppens, et al. in their review article, they are:

1) The photothermoelectric effect; where a light induced temperature gradient in a device results in the thermoelectric effect- a voltage is produced due to a photo-induced temperature differential and does not require the device to be powered. In the literature, the 'photothermoelectric effect' is used colloquially to refer to the photon-induced heating and resulting current generated by the Seebeck effect; i.e. the photothermoelectric effect is simply the thermoelectric effect as a result of heating by light irradiation and is not a separate effect. $\mathrm{Xu}$, et al. cite this effect to explain the photo response of their graphene device (exfoliated graphene on $\mathrm{SiO}_{2} / \mathrm{Si}$ wafer). ${ }^{(611)}$

2) Plasmonic wave effects (sometimes referred to as Dyakonov-Shur operation, named after the authors who first proposed it) ${ }^{(676)}$; where a complex plasma resonance is generated in the graphene device due to electromagnetic radiation. In these cases, a standing plasma wave arises at a contact, resulting in a voltage. Wang, et al. cite this effect for their device. ${ }^{(677)}$

3) Bolometric detection and the photogating effect; both mechanisms are based on the relationship between the temperature of the device and its conductivity-photo induced heating changes the resistance of the device, which is detectable by a change in the current through the device. Bolometric detection relies on changes in the carrier mobility $(\mu)$ due to thermally induced scattering effects where photogating relies on changes in carrier concentration $\left(\mathrm{N}_{D}\right)$ due to charge injection into the film. In the latter, Koppens, et al. cite nanoparticles, molecules or charge traps as carrier sources. In the device studied here, thermal promotion of carriers out of charge traps or over the Schottky barrier and into the film would be potential carrier sources. Importantly, however, both bolometric and photogating require the device to be powered. Riaz, 
et al. cite bolometric detection to explain photosensitivity in their nanocrystalline graphenebased devices. ${ }^{(657)}$

4) Photovoltaic effects. This is the most commonly cited mechanism-particularly for graphene devices having Schottky contacts. In this case, electron-hole (e-h) pairs are generated by the photoelectric effect. Under the influence of the built-in electric field in the depletion region of the Schottky contacts, these pairs are accelerated away from each other before they have an opportunity to recombine.

Although the devices studied here are temperature sensitive, there has been no evidence of the thermoelectric effect occurring in these devices. Similarly, for bolometric and photogating detection, the fact that there is a substantial photocurrent at $0.0 \mathrm{~V}$ bias indicates that a bolometric based detection is not the primary mechanism. However, like the thermal effect discussed extensively in gas sensing (Section \$5.3.1) when the device is powered, the photoinduced heating of the film does affect the device conductivity (see Section \$5.4.4). And in this sense, there is a component of the photo response which can be attributable to a photogating effect (thermal promotion of charge carriers into the film from traps or the metal contacts). The time scale that this occurs on is, however, much longer and there is a clear difference between it and the primary mechanism. Bolometric based detection, on the other hand, would only occur when scattering mechanisms dominate, which as shown in Section $\$ 5.2 .2$, occurs when the device is above $100^{\circ} \mathrm{C}$, which is not the case here (all testing was performed at room temperature).

As for plasmonic wave effects, the device design used here makes this mechanism unlikely. The mechanism proposed by Dyakonov and Shur typically involve a transistor device design. Additionally, in the devices studied here, there is no cavity or other structure in which a plasmonic resonance could occur. Furthermore, the plasma wave resonance requires long range order of the 2D gas of electrons, which the devices studied here most likely do not have due to the dispersed oxygen defects (which break up the distributed cloud of $\pi$ electrons) and, more importantly, the interactions with the buffer layer.

Of these potential mechanisms, the photovoltage effect is most likely. This effect explains the observations of Section $\$ 5.4 .1$ where a photo response is only achieved when the contact/film interface was illuminated; presumably this corresponded to illumination of the Schottky contact depletion region. Illumination of the film generates charge carriers through the photoelectric effect. Photocarriers generated in the depletion region are accelerated away from each other by the built- 
in field of the depletion region before they recombine (the electric field and band diagram of the Schottky contact are discussed theoretically in Section $\$ 2.2 .3$ and practically for these devices in Section $\$ 5.1 .1$.

Due to the low number of charger carriers and small dimensions of the depletion region, both carrier mobility and the carrier mean free path are higher in this region. The result is that an appreciable fraction of the photocarriers generated in this region may transit the contact-film interface and contribute to the photocurrent. When the metal contact is provided with an electrical path to a lower potential (i.e. earth ground in the $0.0 \mathrm{~V}$ condition), the carriers transit the interface and follow this potential gradient, through the measurement equipment, and a photocurrent is recorded. The larger absolute photocurrent observed when the device is powered is likely due to the external enhancement of the electric field of the depletion region. This results in a larger fraction of generated photocarriers surviving (i.e. not recombining with holes) that transit to the contact, resulting in a larger photocurrent. When the contact is isolated by measurement circuitry and no current is allowed to flow (i.e. the $0.0 \mathrm{~A}$ condition) a voltage builds as photocarriers fall into the lower energy states available in the metal contact where they are preserved (there are few holes to combine with in the metal), and the electrical potential of the irradiated contact is elevated.

Irradiation of the film outside the depletion region likely also generates e-h pairs but, as there is no electric field to separate the charges, the charges quickly recombine, and the energy of recombination is thermalized into lattice vibrations of the film. When under an external bias (the device is powered), there is an electric field across the entire device. However, the carrier concentration $\left(\mathrm{ND}_{\mathrm{D}}\right)$ is high and the mobility is low $(\mu)$ in the bulk expanse of the film, resulting in a small carrier mean free path. Photocarrier scattering with acoustic phonons has been shown to syphon the carrier energy into thermal lattice vibrations, in a way 'shorting out' the photocurrent. ${ }^{(671)}$ Photocarriers generated in the bulk film likely encounter many scattering events as they move under the influence of the externally applied bias. Thus, they have a lower drift velocity which 
presents them with many opportunities to recombine with holes before reaching a contact. These processes are illustrated in the electronic band diagrams in Figure 5.60.

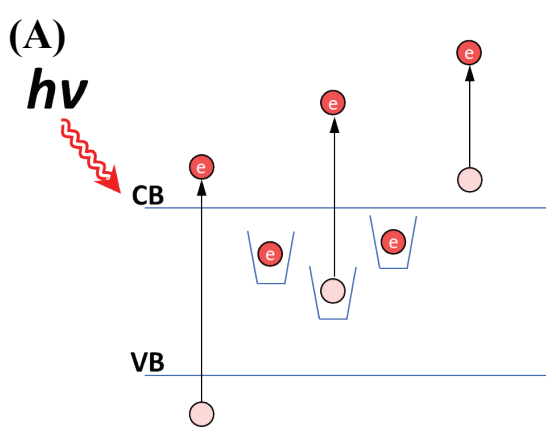

(B)

(C)

(D)

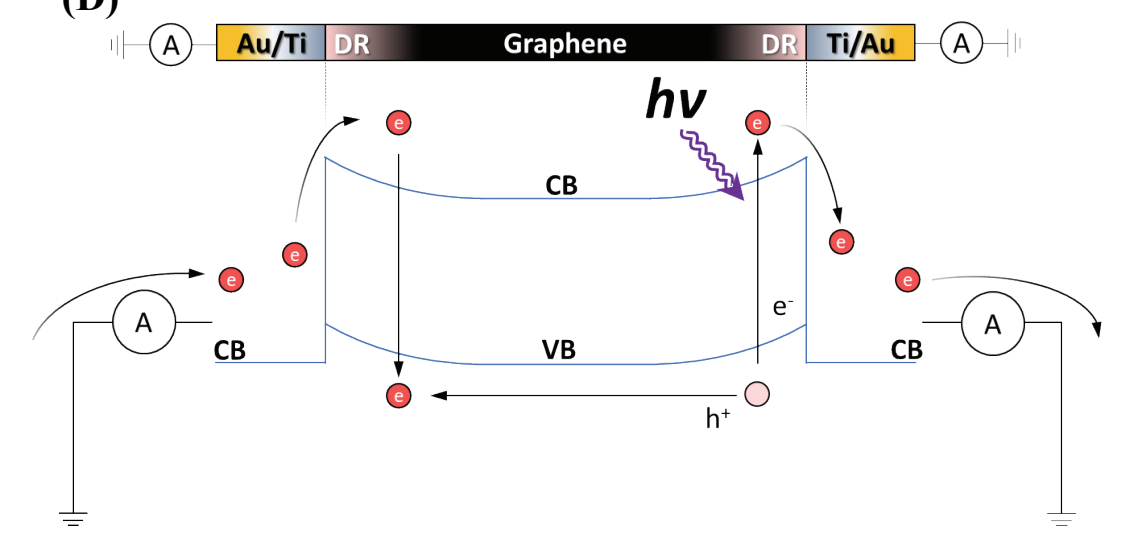

Figure 5.60: Various charge carrier processes which occur when the device is irradiated. (A) Irradiation can lead to promotion of charge carriers from the valence band (VB) or from charge traps into the conduction band (CB); or carriers already in the VB may be promoted to even higher levels. (B) The carrier recombination and energy thermalization process. (C) The carrier multiplication process mediated by carrier-carrier interactions; in this case, rather than energy going into thermalization process, the energy causes additional e-h pairs to be generated. (D) The overall electron flow in a device due to the injection of photonic energy. The direction of charge pumping can reverse, if the opposite contact is irradiated.

This explains the positional sensitivity of the response, why no photo response was seen when the bulk film expanse is irradiated even when the device is powered, the photocurrent and photovoltage pulse response and the powered response. Figure 5.60(D) shows the overall electron balance and illustrates why an equal and opposite current is recorded at the opposing contact, which is not irradiated (see Appendix $\$ \boldsymbol{A}$.5.2). Election-hole (e-h) pairs are photonically generated within the depletion region. These high energy mobile electrons, under the influence of the builtin field, migrate and fall into the available empty states in the metal contact. This is analogous to forward biasing the diode junction. The accumulation of holes in the film at the point of irradiation (the corresponding electrons fall into the conduction band of the metal contact), creates a gradient and pulls make up electrons from the rest of the film. Ultimately, these make-up electrons come 
from the other contact, and creates a current flow into the film. This make-up current is equal (and in the opposite direction) to the current recorded at the illuminated contact. The overall system essentially uses photonic energy to pump electrons around the circuit. The explanation for the frequency response (higher frequencies leading to an exponentially larger photo response) in the devices studied here is explained by a carrier multiplication (CM) process (this process is occasionally referred to as an avalanche multiplication process).

The CM process is predicated on the idea that higher energy photons produce higher energy e-h pairs. When the high energy electron relaxes to a lower quantized energy state, the energy differential may be given up by several different mechanisms. If the energy quantum is large, a photon may be produced (i.e. radiative e-h recombination). ${ }^{(678)}$ This process is rare in these types of experiments, although it has been observed in plasma-oxidized graphene when excited with a $530 \mathrm{~nm}$ light source. ${ }^{(679)}$

Alternativity, if there are both lower level electrons and corresponding higher energy vacant states available, multiple lower energy e-h pairs may be produced by the energy released by the initial electron relaxation. ${ }^{(671)}$ The high energy relaxation is a complex process, mediated by electron-electron scattering events (Coulomb interactions). ${ }^{(678)}$

Lastly, if the energy quantum is too small to be released by either process, or if there are not states available for electron promotion, the energy differential is simply thermalized into lattice vibrations and the film heats up slightly. The second relaxation process, which produces addition e-h pairs, is the CM process. Carrier multiplication has been experimentally observed in graphene devices before. ${ }^{(672)(680)(681)(682)}$

Considering this, the CM process may explain the increase in signal with increasing photon energy. However, the CM process may not completely explain this signal-energy relationship. Results reported by Chen, et la., show a linear relationship between photo response and photon energy, where in this work, an exponential relationship is seen. ${ }^{(681)}$ This discrepancy may simply be due to incorrectly interpreting the relationship (there is a large degree of spread in Chen, et la. data and the data set of this work is small). Alternatively, the e-h generation/relaxation process occurring here may be more complex, potentially involving the substrate and buffer layer in a synergistic fashion. Others have suggested that the $\mathrm{SiC}$ substrate in $\mathrm{SiC} /$ Graphene devices does play a role in carrier transport routes. ${ }^{(683)}$ 
It is noted that bulk $6 \mathrm{H}-\mathrm{SiC}$ (the substrates used in the SOP devices tested here) is cited as having a bandgap of $\sim 3.03 \mathrm{eV}$ (more detailed information on $\mathrm{SiC}$ can be found in Section 3.1.1). This is smaller than the excitation energy of the highest frequency light used (purple light $/ 405 \mathrm{~nm} / 3.1 \mathrm{eV}$ ). This means that purple photons contain enough energy to promote carriers across the gap. Furthermore, the $\mathrm{SiC}$ layers directly tethered to the buffer layer likely has a different bandgap than the bulk $6 \mathrm{H}-\mathrm{SiC}$ material. However, it is unclear whether these terminal layers will have a higher or lower bandgap, as compared to the bulk. If lower, then photocarriers excited across the band gap of the substrate may play an amplifying role for the higher energy irradiations. Ultimately, the CM process is complex and not completely understood by the larger scientific community at this time. The related references provide a more in-depth discussion of the process. (671) (673) (678) (684) (685)

Similar to gas detection applications, light detection applications of these devices would also stand to benefit from the addition of metallic nanoparticles, albeit due to a completely different mechanism. APPENDIX B presents and discusses the preliminary results of four different metallic nanoparticle nucleation/decoration methods. The underlying theory for how metallic nanoparticles could improve the photodetection performance of graphene device is covered in Section $\$ 2.2 .3$. In short, by coupling with the plasmonic shells which surround the particles, the light-device interactions can be increased, increasing device performance. In this way, nanoparticles would effectively act as an impedance matching device for the graphene film to the incident light.

As previously stated, graphene is largely transparent to broad spectrum light; incident light waves do not interact strongly with the distributed electron gas of graphene. Nanoparticles, however, present a dense electron free cloud on their surface. When exposed to electromagnetic waves with a wavelength similar to the particles physical dimension, the exterior electron shell may be driven into a collective and coherent oscillation. Under exposure to the sinusoidal nature of the electromagnetic incident waves, the electron shell is displaced from its equilibrium position around the positively charged core. This results in a nanosized oscillating dipole, which roughly oscillates in time with the frequency of the incident radiation. These coherent oscillations are called surface plasmons, a type of quasiparticle.

The nanoparticle plasmon excitations on the nanoparticle surface may then transfer their energy to surface plasmons on the graphene sheet on which they sit. These graphene surface plasmon may transform the energy (i.e. when the surface plasmon wave collapses) into a detectable 
form (i.e. the generation of electron-hole pairs). In this way, a random array of nanoparticles could enhance device performance.

A further refinement of this idea involves the deposition of nanoparticles in such a way as to produce large scale order. If properly arranged, light antennas may be constructed on the graphene surface. By placing nanoparticles in close enough proximity to each other, such that their plasmon shells may constructively interact, the larger nanoparticle structure would exhibit a resonance response at a particular excitation frequency. The resonance frequency would be a function of the particle size and spacing. Figure 5.61 illustrates this basic idea.
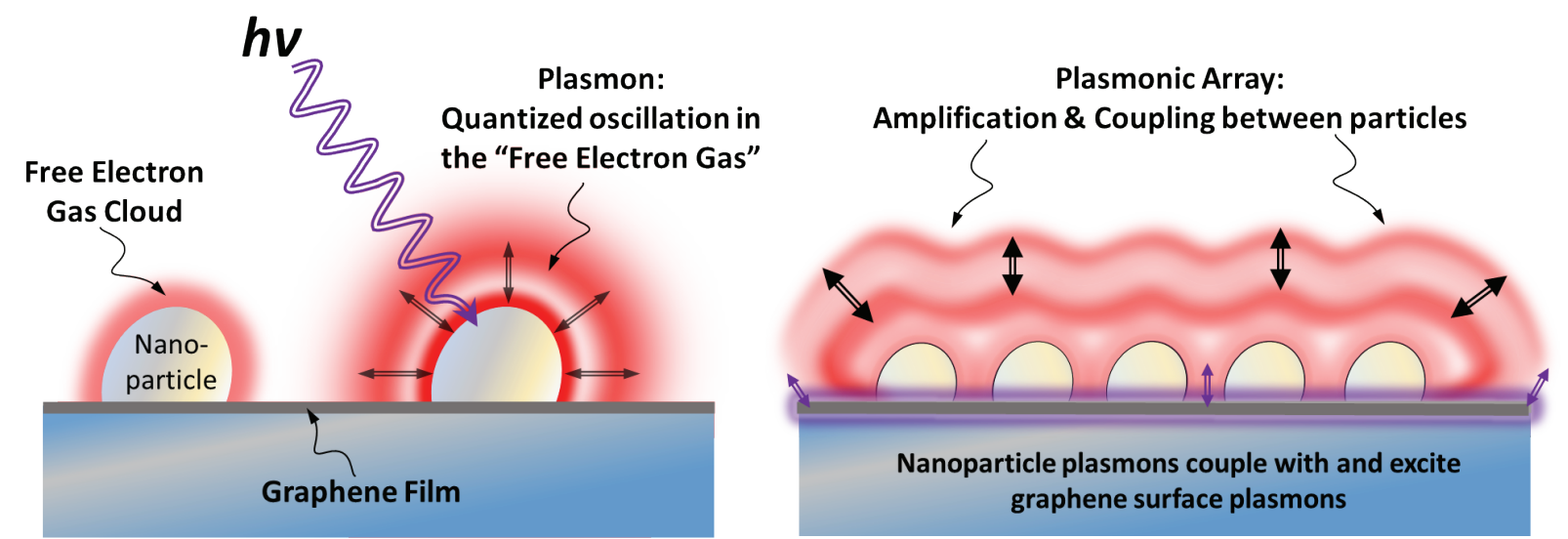

Figure 5.61: The basic idea behind a light antenna constructed from nanoparticles. High frequency electromagnetic radiation drives the plasmon shell surrounding a nanoparticle into oscillation. A highdensity array of nanoparticles can lead to a constructive interaction between theses oscillations of neighboring particles, leading to plasmonic resonance.

This idea is analogous to a simple Yagi style TV or radio antenna. In this analogy, each nanoparticle may act as an antenna element (dipole). Each element resonates (electromagnetically) and constructively interferes with its neighbor elements. More elements lead to a higher signal gain (the resonance magnitude of the larger structure is enhanced in a linear fashion with a logarithmic increase in the number of elements) and a narrower bandwidth sensitivity (the resonance frequency of the larger structure is narrowed). The nanoparticle layout scheme and potential antenna design are enormous. Whether through this mechanism or another, there have been several literature reports which have shown significant enhancement in the photo response, as well as response tunability, due to the addition of plasmonic structures on the graphene surface. (381) (406) (399) (400)

The optoelectronic applications for the devices studied here are wide ranging and rich, which these tests only scratch the surface of. Further testing and a refinement of the testing 
apparatus is required to further quantify the photo detection performance of these devices. Testing with a wide frequency range of well-characterized illumination sources are an obvious first step. Without knowing the precise power of each excitation beam, many of the common figures of merit cannot be precisely determined. Typical figures of merit, as cited by Shin, et al. in their recent review of graphene photodetectors, include quantities like the external quantum efficiency (EQE, photon conversion efficiency of the device), noise-equivalent power (NEP, a measurement of the combined inherent noise of the detector and is used to determine the lowest detectable signal threshold), linear dynamic range (LDR, the range of incidence illumination powers that scale the device response linearly, i.e. doubling the power doubles the signal etc., and is related to response saturation or 'sensor blinding') and response speed (which requires a higher data sampling rate and much higher $\mathrm{A} / \mathrm{D}$ conversion speed than what the current testing setup is capable of). ${ }^{\left({ }^{3} 2\right)}$

Considering the temperature stability of these devices, characterization of the high temperature photodetection performance may provide further insight into the device operation. Furthermore, unique device applications may present themselves and could fill important niche use cases in military and spacefaring applications. And as it relates to gas sensing (Section §5.3), combining the gas testing with device illumination may provide another avenue to achieve gas sensitivity. Xhu, et al. have combined gas and light detection with their graphene devices successfully. ${ }^{(310)}$

Testing with decoupled films will also be important to future work. There are reports in the literature which have shown hydrogen decoupling to both help and harm the photo detection of $\mathrm{SiC} /$ Graphene devices. ${ }^{(686)(687)}$ Although it is suspected that decoupling the film will reduce the Schottky barrier, which would reduce the depletion region and the photocurrent, Malic, et al. show that doping of the graphene film reduces the CM effect. ${ }^{(684)}$ Thus, by decoupling (or only partially decoupling) the film, which would reduce the substrate-induced doping, the internal gain caused by the CM effect may be enhanced. Whether an improvement is achieved or not is likely dependent on the specific photo-detection mechanism. Thus, testing with decoupled films will likely provide further insight into these devices. Once a comprehensive mechanism is determined, future devices may be intelligently designed with this in mind.

The design of graphene devices used for photodetection reported in the literature vary widely, however, based on the recent review by Shin, et al. on graphene-based semiconductor heterostructures for photodetection, the photodetection performance of the relatively simple 
$\mathrm{SiC} /$ Graphene devices tested in this work compare well with other, more complicated designs. ${ }^{(382)}$ A refinement of the testing apparatus will be required before rigorous quantitative comparisons can be made. Furthermore, it is worth noting that the device design used in this work has not been optimized for photo detection applications.

For example, the Schottky barrier (and thus the depletion region and resulting built in electric field) could be tuned by using metals with a different work function. Recall the contacts used here are comprised of $300 \mathrm{~nm}$ of Au on top of 30nm of Ti. By using a metal other than titanium as the interface between the gold and graphene film, the interface energies may be engineered. Figure 5.62 plots the work function of several metals as a function of their atomic number (the values given are for the pure material, and represent the average value calculated from the various allotropes and crystal faces measured). ${ }^{(688)}$

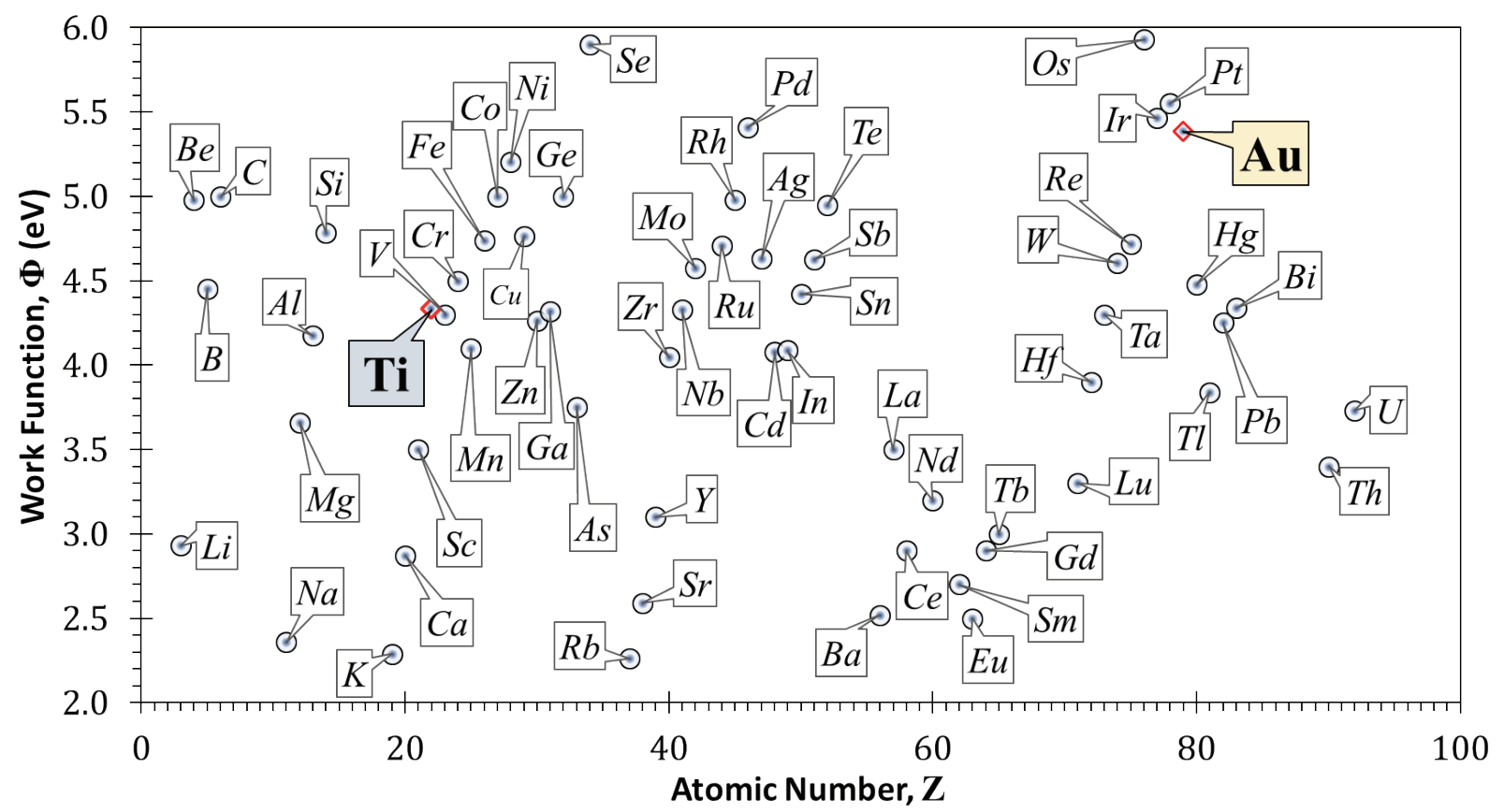

Figure 5.62: The nominal metal work function as a function of atomic number. The metals used for the electrical contacts for the devices studied here are highlighted. These data are averaged from the tabulated data found in the most recently available edition of the CRC Handbook of Chemistry and Physics. (688)

When interfaced with the film, each metal will form a Schottky contact with different parameters. Thus, the depletion region and intensity of the built-in field can be engineered by choosing the appropriate metal. Of course, not all the metals shown in Figure 5.62 are practical, but clearly there is a wide array from which to choose.

The device performance may be further enhanced by simply modifying the contact geometry. Because the detectivity is derived from the depletion region formed at the metal/film 
interface, a redesign of the geometry of the contact layout, such that the depletion region is extended or made to overlap with itself, would increase the photo sensitive area of the devices. Rather than the rectangular contacts used here, contacts with complex and irregular boundaries
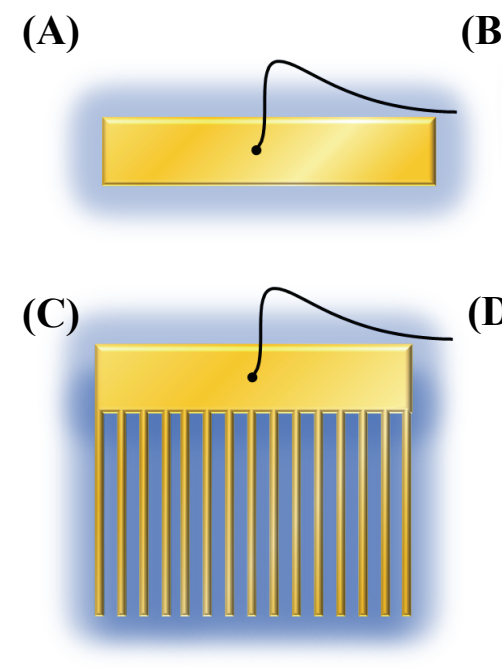

(D)

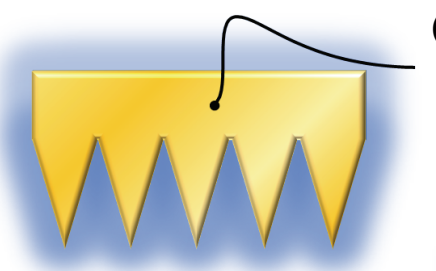

(E)
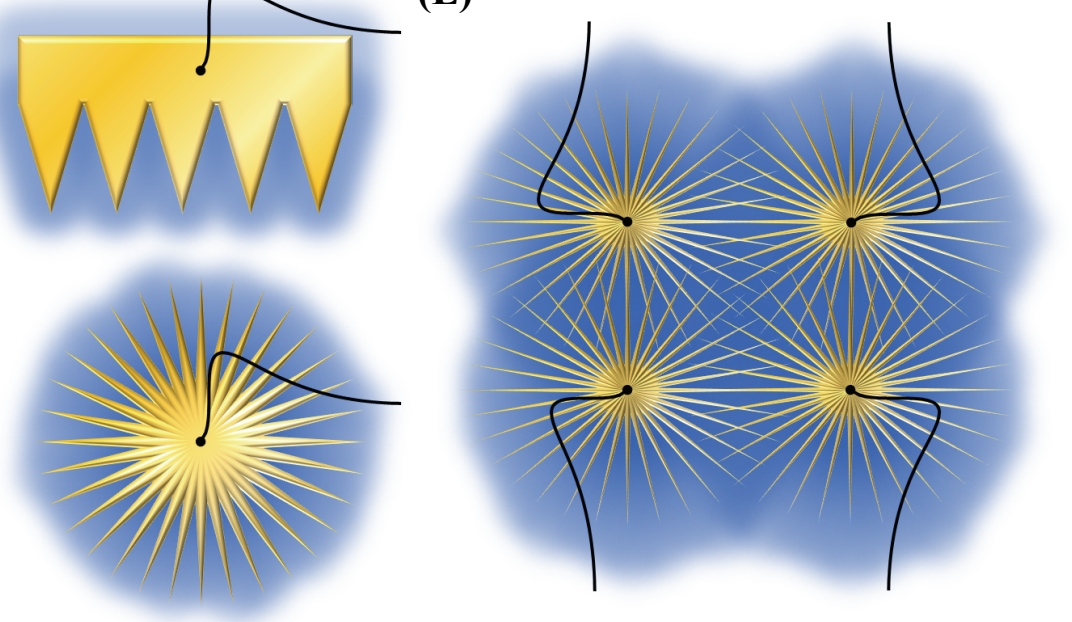

(F)

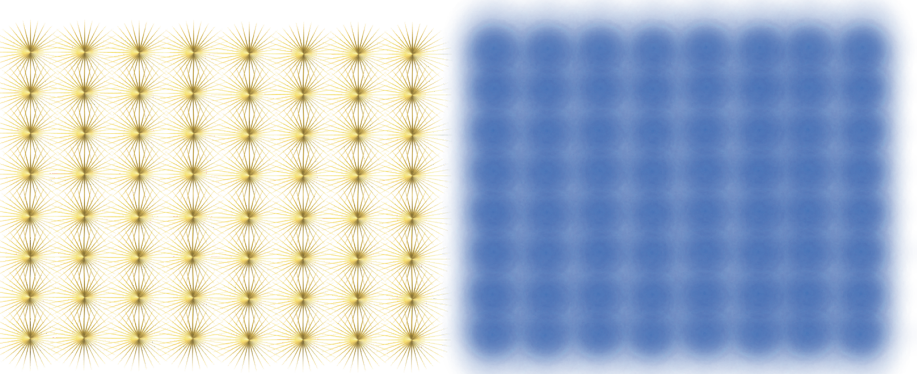

Figure 5.63: Different geometrical designs for the metal contacts and the resulting depletion region. (A) The contact design used in this work. (B) A toothed design. (C) A comb design. (D) Starburst design. (E) An array of starbursts. (F) A larger array of the starburst contact design and the corresponding depletion region.

would enhance the film depletion region due to the complex field interactions. Figure 5.63 schematically presents this idea.

The blue shaded area represents the depletion region in the film. The more complex designs (like the starburst design shown in Figure 5.63(D-F)) could capitalize on the 'lighting-rod' effect discussed in Section $\$$ 5.4.1 and may produce film areas with very high sensitivities. Gridded arrays of these types of contacts could be easily fabricated using the same shadow making procedures used in this work (detailed in Section \$3.3.1). Each element (pixel) in the grid may be electrically connected to obtain a high sensitivity or each may be independently electrically monitored to map the illumination coverage over the device. From here, it is easy to see how a high resolution, high sensitivity detector could be fabricated. 
This general concept could be extended to the nanoscale. The contact 'tentacles' could be made to narrow down to where plasmon effects may arise and further enhance device performance. Appendix $\$ B$. 2 presents the experimental results of several nanoparticle decoration methods. In particular, the electronic growth method, where nanoscale dendric and bladed structures were grown outwards from the electrical contacts present an intriguing possibility. Not to mention the fractal-like growth may also make for a good light antenna design. Again, this presents yet another fascinating area of future work.

\section{\$5.4.6 Summary of Device Performance in Photo Detection Applications}

Although many Schottky junction devices show a photo detection capability (they operate under the same principle as previously described), graphene-based devices have been speculated to have superior performance for all the same reasons which make graphene attractive for use in electronic devices in general. ${ }^{(675)}$ In addition, graphene's largely transparent and antireflective nature make it well suited as a starting material from which to build more complicated and decorated devices.

Despite both the simple device design and simple testing apparatus, the $\mathrm{SiC} / \mathrm{Graphene}$ devices studied here show a clear photo response. This takes the form as either a photocurrent or photovoltage (Section \$5.4.2) and is observable when the device is both powered and unpowered (Section \$5.4.4). Using three different wavelengths ( $\mathrm{red} / 650 \mathrm{~nm} / 0.43 \mathrm{eV}$, green $/ 532 \mathrm{~nm} / 1.09 \mathrm{eV}$ and purple $/ 405 \mathrm{~nm} / 3.6 \mathrm{eV}$ ), the devices showed an exponential increase in photocurrent with a linear increase in frequency/energy (Section \$5.4.3). The most likely explanation for this is a carrier multiplication process, which acts as an internal gain mechanism, as a result of the high energy electrons produced due to irradiation with high energy photons (Section \$5.4.5). The devices show a positional sensitivity to the location of the excitation beam on the device surface (Section \$5.4.1). The largest photo response was observed when the contact edges were directly illuminated. This indicates that the Schottky contact plays a critical role. When all the observations are considered, the most probable mechanism of the photo response is a simple photovoltage (photoelectric) effect (Section \$5.4.5). Overall, the performance of these unoptimized devices compares well with other reports in the literature. 


\section{CHAPTER 6: SUMMARY}

This chapter delivers a synopsis of the work detailed throughout this document. Specifically, it provides an overview of the information presented in each chapter, highlighting the motivations and key takeaway points. Included are the section headings where the material summarized may be found in the proceeding chapters, where they are discussed in much greater detail.

It total, over 300 graphene/ $\mathrm{SiC}$ samples were fabricated, from which more than 2,000 XPS and Raman spectra were collected and individually analyzed, as well over 1,000 AFM images. More than 180 graphene/SiC electronic devices were constructed, from which more than 5,000 individual electrical measurements were collected. Over 280 gas sensing and photo detection testes conducted on these samples.

\section{\$6.1 Chapter 1: Introduction}

Chapter 1 introduces graphene as a material and makes the general case for graphene research. Beginning with an atomistic description of graphene, the generic structure is described (Section \$1.1). Next, the mechanical and electrical properties are explained in the context of the electron orbital arrangements. Expanding on this view slightly, graphene's larger structure is described in a crystallographic context and some relevant properties are discussed.

Following this introduction to graphene fundamentals, graphene's position on the world stage is detailed (Section $\$ \mathbf{1 . 2}$ ). Simply put, 16 years after its discovery, graphene continues to attract worldwide interest, with billions of research dollars spent each year. This interest comes from government entities, academic institutions, and increasingly, the privet sector.

The worldwide research attention (and money) directed towards graphene is indicative of the global consensus that the promises of graphene are too great to ignore. In fact, the ever-growing increase in government funding from all major nations point towards a burgeoning graphene 'arms race'. Despite the billions of dollars spent each year in support of graphene development, graphene has yet to experience a break-out moment.

This point leads to the grand challenge facing graphene, which is fundamentally one of production and manufacturability (Section \$1.3). This is particularly true for electronic 
applications where high-quality graphene is required. Further, considering the scale and precision essential in most electronics, both synthesis and processing must be done in a scalable, repeatable way. Moreover, it is highly desirable that this be done within the current semiconductor processing ecosystem, which has matured over the past several decades. A graphene processing technology which uses techniques already deployed in the industry today would be highly advantages as it would minimis the disruption cost (technological and financial) of graphene integration and accelerate its adoption. To this end, the plasma assisted growth method described in this work has been investigated.

\section{§6.2 Chapter 2: Brief Review of the Relevant Literature and Statement of Research Goals}

Following the identification of the grand challenge facing graphene, as highlighted in the previous chapter, Chapter 2 begins with a brief literature survey and discussion of the common methods of graphene fabrication (Section \$2.1). Ignoring the finer details, all methods essentially produce either large quantities of poor-quality graphene or small quantiles of high-quality graphene. Although poor-quality graphene has many uses, in order to take advantage of the benefits inherent to graphene, electronic applications require comparatively high-quality graphene. However, the incredibly small production yields of high-quality graphene mean that graphene produce by these methods (like mechanical exfoliation) will forever be confined to one-off research devices or are too laborious (like chemical vapor deposition) and do not lend themselves to easy manufacturability of electronic devices.

One method of graphene production, however, stands out. Potentially straddling both challenges of quality and quantity, and being particularly well suited for electronic applications, is graphene growth directly from a carbon-containing semiconductor. Principally, this method takes the form of selective sublimation (thermal decomposition) of silicon carbide (SiC) (Section \$2.1.3). Therefore, Chapter 2 pays particular attention to this method. Due to its relevance to this work, the buffer-layer produced as a result of the sublimation process is also detailed extensively.

Following the discussion of the selective sublimation method, the method utilized by this work, the plasma assisted growth method, is introduced and the motivations for pursuing this method are discussed (Section $\$ \mathbf{2 . 1 . 5}$ ). Sharing many similarly with the selective sublimation method, the plasma assisted method provides an alternative route for growing graphene from a 
$\mathrm{SiC}$ substrate, while also retaining all the benefits of the selective sublimation method. The plasma assisted growth process is potentially a more flexible and controllable process. Greater control is gained by the inherently different method by which carbon is removed from the substrate. In the sublimation method, thermodynamics govern the carbon removal process, which is modulated by temperature and pressure. In contrast, the plasma assisted method uses plasma chemistry to control the removal process. The plasma chemistry and plasma kinetics, which both influence the graphene growth process, can be finely controlled though a variety of methods (explored in Chapter 4).

Furthermore, as compared to selective sublimation, the plasma assisted process is a comparatively more manufacturable process. In addition to the milder processing conditions, the plasma method uses only techniques and tools currently deployed at scale in the semiconductor industry today. Also, in addition to the associated with achieving uniform sublimation and thus uniform film growth on the wafer scale, is the fact that the thermodynamics of sublimation process inherently limits the manufacturing process. For instance, the very high temperatures required for sublimation $\left(<1,500^{\circ} \mathrm{C}\right.$ for high quality film growth) would destroy any device features present on the wafer. Thus, graphene growth by this method must always be the first device fabrication step and can only be done once. This limits the flexibility of any process which uses the sublimation method.

In contrast, the plasma assisted method use reactive ion etching, which is a process already used in concert with a variety of techniques (i.e. is already integrated in production lines) and can be utilized at many stages of a process. Additionally, the plasma assisted graphene growth can be limited to specific wafer areas using simple lithographic masking techniques. Patterned graphene growth allows for the potential of much more complex device designs. However, even considering these advantages, this is not to ignore the challenges and limitations of the plasma assisted method. This research begins to address some of these potential issues.

The sublimation method of graphene production has been in development since 2004. Due to its attractiveness for electronic applicants, it has received much research attention from across the globe. The scientific communities understanding of the process has matured and the technique has been refined since its inception. However, the sublimation method still faces significant fundamental challenges. On the other hand, the plasma assisted method has been in development since only 2012 solely by the Stinespring laboratory at West Virginia University, Morgantown 
WV. Thus, the primary goal of this research is to enhance the understanding of the plasma assisted graphene growth method and improve upon its implementation.

To further improve upon the manufacturability of the plasma process a key step in the process, the rapid thermal annealing step (RTA), was been modified from an ultra-high vacuum rapid thermal anneal (UHV-RTA) to be instead carried out at atmospheric pressure (an atmospheric-pressure rapid thermal anneal, AP-RTA). Using this modification to the plasma process as the new standard, the graphene produced by this method has been characterized. Following the characterization of the standard process, the effects of various processing parameters were investigated. Together, these studies have enhanced our understanding of the plasma assisted process as a whole and identified the challenges that the process faces.

Chapter 2 concludes with a brief literature survey of graphene-based electronic sensing devices (Section $\$ 2.2$ ). The fundamentals of gas adsorption in general are given (Section $\$ 2.2 .1$ ), followed by reports on graphene-based gas sensing devices (Section $\$ 2.2 .2)$. Subsequent to this is a survey of recent literature reports regarding graphene used in light sensing (photo detection) applications (Section $\$ \mathbf{2 . 2 . 3}$ ) is given. This provides context for the secondary goal of this research, which is application development of simple electronic devices constructed using graphene produced using the plasma assisted growth method. In addition to the obvious benefits of creating functioning devices, application development provided an opportunity to study the practical challenges of implementing graphene into electronic devices, as well as provide a unique prospective into the films produced by the plasma assisted process.

In summary, the grand challenge of graphene has been identified as being production and manufacturing related. The novel plasma assisted method of graphene grown from $\mathrm{SiC}$ has been identified as a step towards surmounting this challenge. However, given the novel nature of this method, much remains to be understood about the process. This work primary aims to develop a more thorough understanding of the films produced by the process, as well as the process itself. Furthermore, a considerable amount of insight can be gained by applying the films produced to real-world device applications. To these ends, Chapter 2 concludes with an explicit statement of the goals of this research (Section $\$ 2.3)$. Simply put, they are:

Goal 1) Development of the novel plasma assisted graphene growth method. 


\section{Goal 2) Development of simple electronic devices fabricated from graphene produced by the plasma assisted method.}

The results collected in pursuit of Goal 1 are detailed primarily in Chapter 4 and the results pertaining to Goal 2 are detailed in Chapter 5.

\section{\$6.3 Chapter 3: Experimental Designs and Characterization Setups}

By describing the experimental and characterization setups, details how the goals layed out in the previous chapter were to be tackled. Chapter 3 beginnings with a technical description of the materials and processing tools used in the plasma assisted graphene growth method (Section \$3.1). Following this, the film analysis techniques employed in service of Goal 1 are described (Section \$3.2). Both the theoretical basis and principles of operation are discussed. Specifically, as they relate to the interpretation of the data provided in later chapters. This work relies heavily on several material characterization methods and many of the concussions reached in later chapters rely on a thorough understanding of the fundamental processes by which the data are collected. Therefore, an in-depth discussion of each technique is given to add meaning and context to the data presented in the results chapters. Pertinent to Goal 2, the chapter concludes with a description of the methods utilized in device fabrication (Section $\$ 3.3$ ). Finally, the experimental apparatuses used for gas and light testing of the graphene devices are described in technical detail (Section \$3.4).

\section{§6.4 Chapter 4: Results and Discussion - Graphene Synthesis, Parametric Investigation and Hydrogen Treatment}

In pursuit of Goal 1, Chapter 4 begins with a detailed characterization of the standard plasma assisted method (termed SOP), which now utilizes the atmospheric-pressure rapid thermal annealing step (Section \$4.1). To investigate the SOP films, the material was analyzed at each step in the process. AFM analysis (Section $\$$ 4.1.1) shows the $\mathrm{SiC}$ substrate to be planarized by the plasma etch step, removing all traces of the atomic steps and crystal defects. XPS analysis shows that after the etch, the SiC surface becomes substantially enriched in carbon, with a substantial fluorinated component, originating from the etchant gas. Analysis of the C1s region in the XPS spectra show that the surface carbon exists in a wide verity of chemical states. Raman analysis indicates the carbon film to be in a largely amorphous configuration, although some order is retained. 
After the AP-RTA step, however, the film crystallinity increases, the fluorine species are removed, and the film densifies. Although the oxygen defect component is increased by the APRTA, the graphene films produced are of remarkably high quality, particularly when compared to reduce graphene oxide films. Further XPS analysis (Section \$4.1.2) shows that in addition to the expected $\mathrm{sp}^{2}$ hybridized carbon (indicative of a graphene film) and the oxygenated carbon species, there is a substantial portion of the $\mathrm{C} 1 \mathrm{~s}$ spectra which is unassigned. Comparison with the literature reports on graphene produced by sublimation indicated these peaks to be buffer-layer related. The buffer-layer is a graphene-like layer, which is partial covalently bound to dangling silicon bonds in the substrate interface. This bonding partially rehybridizes the $\mathrm{sp}^{2}$ carbon into a $\mathrm{sp}^{3}$-like conformation and, due to the lattice mismatch between the native graphene and $\mathrm{SiC}$ structures, the carbon film is under tensile stress.

Contrary to the high quality indicated by XPS, initial Raman analysis (Section \$4.1.3) indicated the produced films are highly defective. This discrepancy is reconciled, however, when the buffer-layer hypothesis is considered. A coupling of the vibrational density of states of the film with the substrate, and the resulting tensile stress experienced by the film, explain the Raman spectra (the splitting of the G peak, the larger FWHM values and the suppression of the 2D peak). This is again consistent with literature reports.

The existence of the buffer-layer is one of the major revelations uncovered in pursuit of Goal 1. Both the amount of buffer-layer material detected by XPS and the degree of coupling revealed by Raman analysis, indicate that the buffer-layer produced using the plasma assisted process is extensively bound to the substrate, as compared to the buffer-layer produced by the sublimation method. Furthermore, the specific nature of the covalent boding may be different as well. As will be discussed, the device performance detailed in Chapter 5 support the existence of this buffer-layer.

The next section, Section $\$ 4.2$, details the parametric investigation of the plasma assisted process. Using the SOP process detailed in Section $\$ 4.1$ as a base-case, the effect of substrate morphology (Section \$4.2.1), ICP and RIE powers (Section \$4.2.2), plasma chamber pressure (Section \$4.2.3), the maximum annealing temperature (Section \$4.2.4), heating (Section \$4.2.5) and cooling (Section \$4.2.6) rates were investigated. The overall concludes of the parametric investigation are stated in Section \$4.2.7. This extensive study provided insight into what the most 
important controllable processing parameters were. Although each parameter effects of the final product in various ways, it was found that the most important parameters were the ICP and RIE powers. Although there is a high degree of synergy between the two parameters, by independently varying the ICP power (which sets the reactivity of the plasma) and RIE power (which sets the DC bias and thus, the kinetic energy of the plasma ions), the rate and selectivity of the etch could be controlled. The rate and selectivity of the etch thus control the surface damage and the balance of excess carbon on the surface, which ultimately determines the quality and number of graphene layers produced. However, in all parameter combinations tested, both XPS and Raman analysis indicate a buffer-layer is always present.

The recognition of the existence of a buffer-layer provided the motivation for the final part of Chapter 4, high temperature hydrogen treatment (Section \$4.3). Literature reports on the sublimation method have shown that high temperature hydrogen treatment can lead to passivation of the bonds between the buffer-layer and substrate, resulting in a decoupling of the film and a transformation into quasi-freestanding sheets of graphene. This section details the effect of treating both etched-only samples and etch + AP-RTA'ed samples in a pure hydrogen environment, at various temperatures (Section \$4.3.1) and for various durations (Section \$4.3.2). Higher temperatures and longer durations lead to an improvement (reduction) in the degree of tethering between the buffer-layer and SiC substrate, as determined by XPS and Raman analysis. The largest reduction in film tethering was found for samples thermally cycled from low to high temperature many times under hydrogen (Section $\$$ 4.3.3). The overall conclusions on the hydrogen treatment are given in Section $\$ 4.3 .4$.

Analysis indicate that high temperature treatment resulted in a partial decoupling of the buffer-layer. However, in no cases was full decoupling achieved. The fact that similar conditions had led to a full decoupling in graphene produced by sublimation add credibility to the earlier hypothesis that the buffer-layers produced by the plasma assisted method are fundamentally different from those produced by sublimation. Specifically, that the tethering/coupling is more extensive in these films and the specifics of the bonding interface may be more complex. 


\section{\$6.5 Chapter 5: Results and Discussion - Electrical Characterization, Temperature Behavior, Gas Testing and Light Testing}

Chapter 5 details the results as they relate to Goal 2. It is also noted that the results collected in this chapter also inform the conceptual understanding of the material produced by the plasma assisted method, which is the subject of Goal 1. Thus, the results presented in Chapter 4 and Chapter 5 work in tandem to accomplish Goal 1 and have produced an internally consistent picture of the plasma assisted process and bolster the conclusions of Chapter 4.

Chapter 5 starts with room temperature, open air electrical characterization of the graphene devices produced using the SOP plasma assisted method (Section \$5.1). Simple two-point analysis show the devices exhibited a back-to-back Schottky behavior (Section \$5.1.1). The Schottky behavior is due to the formation of a charge depletion layer within the graphene film, as a result of the bending of electronic energy bands at the interface between the semiconducting film and the metal contacts. Given that pristine freestanding graphene displays metallic behavior, the semiconducting nature of these films (as evidenced by the formation of the Schottky barrier) is likely a result of doping due to film coupling to the substrate via the buffer-layer. Various diode parameters are calculated.

Using a more complex four-point analysis and an application of the transmission line model (TLM), the graphene film conductivity was calculated (Section \$5.1.2). Next, using a modified Richardson-Dushman Analysis, the Schottky barrier height $\left(\Phi_{\mathrm{B}}\right)$, carrier concentration $\left(\mathrm{N}_{\mathrm{d}}\right)$ and carrier mobility $(\mu)$ of the film were calculated (Section \$5.1.3). Overall, the per-layer conductivity, carrier concentration and carrier mobility are all slightly higher than expected. The higher carrier concentration is explained by doping from the substrate via the buffer-layer. The higher conductivity is explained by various buffer-layer related effects. Most notably are the additional conduction paths provided due to the electrical communication between the film and substrate via the buffer-layer. This effects the TLM-derived calculations, leading to a slight inaccuracy in the conductivity value which in turn, leads to a artificially higher calculated carrier mobility. Essentially, all these calculations fundamentally assume a freestanding (electronically isolated) film. The existence of the buffer-layer prevents the film from being truly freestanding, thus explaining the slight difference in expected values. 
Analysis of select non-SOP samples show that lower annealing temperatures produce devices with higher Schottky barriers (Section \$5.1.4). Recalling that lower annealing temperatures produced films with more carbon in the film participating in the buffer layer indices that the hypothesis that the Schottky behavior is derived, in part, by effects caused by the buffer layer is supported. The electrical characterization of non-SOP samples contributes to Goal 1 . The section concludes with a brief summary of the electrical characterization results and discussion (Section §5.1.5).

Following the room temperature electrical characterization, the device response to an explicit temperature change was studied (Section $\$ 5.2$ ). By analyzing the two-point measurements collected as a function of temperature, it was determined that two charge charrier injection mechanisms are principally at play in the devices (Section \$5.2.1). They are, thermionic emission and charge trap hopping. The practical effect of these process was to increase the effective conductivity of the devices with increasing temperature (the film itself also increases in conductivity, but for different reasons, related to intrinsic carrier promotion, discussed later).

Increasing the temperature increased charge injection via thermionic emission by the thermal promotion of charge carriers over the Schottky barrier that exists between the metal contacts and the graphene film. Additionally, the promotion of charge carriers to higher energy levels, where a greater number of charge traps are present, also increases the carrier injection via charge trap hopping conduction through the barrier. Analysis of the onset of trap hopping conduction as a function of temperature, indicated that there are change trap states at the metalfilm interface, which are homogenously distributed in space but exponentially distributed across energy levels. These realizations contribute to Goal 1.

Again, utilizing the modified Richardson-Dushman analysis, the Schottky barrier height $\left(\Phi_{\mathrm{B}}\right)$, carrier concentration $\left(\mathrm{N}_{\mathrm{d}}\right)$ and carrier mobility $(\mu)$ of the film are calculated as a function of temperature (Section \$5.2.2). The observation that the Schottky barrier height increases with temperature, indicates there is a degree of Schottky barrier height inhomogeneity at the interface. This is most likely due to surface defects. This includes the oxygen defects as discussed in Section \$4.1.2. Starting at a relatively low temperature, there is a direct correlation observed between the increase in carrier concentration to the reduction in carrier mobility. This indicates that carriercarrier interactions contribute to the scattering mechanism. Comparison of the carrier mobility data 
to literature repoints suggest that, in addition to this, five other scattering mechanisms contribute to varying degrees across the temperature range tested.

Section $\$ 5.2$ closes with a brief analysis of the thermal behavior of several non-SOP samples, which reveal unique behavior not observable at room temperature (Section \$5.2.3). Most notable are the non-SOP samples which used the $4 \mathrm{H}-\mathrm{SiC}$ polymorph as the substrate and exhibited fascinating electrical behaviors. In addition to the Schottky characteristics typical of the SOP devices, substantially more charge trapping conduction is observed in these non-SOP devices. Furthermore, an unusual electrical behavior was displayed by these 4H-SiC non-SOP samples; a negative differential resistance (NDR) was clearly observed during device operation at elevated temperature. NDR occurs when an incase in voltage decreases the current through the device (i.e. the differential change in resistance is negative). This is a purely quantum mechanical phenomenon, made possible by resonance of the electron wavefunction with charge traps at the Schottky barrier. In this way, the charge traps provide a shortcut through the Schottky barrier, but only for electrons at specific energy level. The impact of this is a voltage band which leads to high conduction through the device (corresponding to the electron resonance), followed by a reduction in current flow at higher voltages (the electrons are no longer in resonance with the traps and the shortcut is closed). A detailed discussion of the phenomena is given at the end of Section \$5.2.3. The section concludes with a brief summary of the device temperature-related results and discussion (Section \$5.2.4).

Section $\$ 5.3$ presents the electrical response of SOP devices in different gas environments across a wide temperature range. In these testes, the devices were made to function as gas sensors. Because of the temperature sensitivity of the devices, the section begins with a discussion of the various implications of the self-heating effects observed and are discussed in the context of how they impact the device gas sensing data (Section \$5.3.1). Next, the disparate influences of the contact interface and the film to device equilibration after startup are examined (Section $\$ 5$.3.2). Following this, by varying the gas flowrate and using two inert gases with different thermal properties, the device response to non-chemical effects are further established (Section \$5.3.3).

The device response to hydrogen gas is discussed next (Section \$5.3.4). Using the established behaviors of the devices discussed in the proceeding sections (Section $\$ 5.3 .1$, Section \$5.3.2 and Section $\$$ 5.3.3) the thermal and chemical response of the devices are separated and 
discussed. It was concluded that under the vast majority of conditions, the thermal response of the device to hydrogen eclipse any chemical effect which may occur. However, at high temperature and low voltages, a chemical response is observed. These conditions correspond to situations where current injection by charge trap hopping is the dominant mechanism. Next, the device response to methane gas and carbon monoxide gas are given (Section \$5.3.5). For these gases, only a thermal response is observable. However, these gases were not tested across as wide a range of conditions as hydrogen was and were conducting primary at room temperature. At low temperature, the charge traps are not accessible and therefore, the current flow is limited by thermionic emission.

Devices which were repeatedly exposed to very high temperature $\left(\geq 500^{\circ} \mathrm{C}\right)$ under a hydrogen atmosphere during gas testing were observed to undergo a permanent change in their electrical properties. Analysis of these devices is discussed in Section §5.3.6. Gas testing at these conditions effectively mimic the hydrogen decoupling experiments described in Section $\$ 4.3$. Although the gas tests of Section $\$$ 5.3.6 were performed chronologically before the decupling experiments of Section $\$ 4.3$, analysis of the device gas response data provided a unique opportunity to electronically observe what, in hindsight, was most surely the partial decoupling process of the film. An analysis of the room temperature data shows a transition from Schottky behavior to Ohmic behavior. A substantial improvement in all measured device parameters was also observed. This included a reduction in the carrier concentration, reduction in the Schottky barrier height, a decrease in the film resistivity and an increase in carrier mobility. XPS and Raman analysis of these films after testing support the hypothesis that these changes are derived from a partial film decoupling process.

Section $\$ 5.3$ concludes with a discussion of the possible origins of the chemical effect and future directions for gas sensing (Section \$5.3.7). There are many possible mechanisms which may drive the observed behavior including, charge transfer, reaction with and rehybridization of defect sites or diffusion of gas molecules into the film layers. However, regardless of the mechanism, concentration experiments indicate that the process is reaction rate limited.

Considering the fundamental theory of operation for graphene-based gas detectors, it is somewhat surprising that a more substantial chemical effect is not observed. However, if the coupling of the film to the substrate via the buffer layer is considered, then this behavior is 
explained fully. The fact that the films are not completely free standing, and thus are under tensile stress, may prevent the film from exhibiting the atomic scale flexibility require to accommodate the adsorption of gas molecules, onto the otherwise featureless graphene surface and explain the low sticking coefficient and slow chemical response speed. Additionally, the large charge carrier concentration in the films, a result of doping from the substrate via the buffer-layer, also works to reduce the device chemical sensitivity. This means that if the gas sensing mechanism is charge transfer related, the relatively small change (addition or reduction) of charge carriers in the film due to the adsorbed gas pales in comparison to the number of carriers already present in the film. Recall that one of the fundamental ideas behind graphene gas sensing is to take advantage of an electrically isolated graphene sheet with a low number of highly mobile charge carriers. Thus, these observations provide further motivation for film decoupling for the future directions of gas sensor development. The section concludes with a brief summary of the gas sensing results and discussion (Section $\$ 5.3 .8$ ).

Section $\$ 5.4$ discusses the results of the device response to visible laser irradiation. Essentially, the devices are made to act as light (photo) sensors. Section $\$ \mathbf{5 . 4 . 1}$ describes the position dependence of device response and establishes that the largest photo response was achieved when the interface between the metal contacts and the film was irritated. Illuminating this interface location, the devices were irradiated in a pulsed on/off manner which showed a photovoltage or photocurrent could be repeatably produced when the device was unpowered (Section \$5.4.2).

Section $§ 5.4 .3$ details the device response as a function of the wavelength of the irradiating light. An exponential relationship was observed between the light wavelength and the produced photocurrent with shorter wavelengths (high frequencies, higher photon energies) producing larger photocurrents. Next, the photocurrent produced by irradiating the device under an external bias was detailed (Section \$5.4.4). Both the thermal effect of laser irradiation and the photo effect were clearly observable and easily deconvoluted. Increasing the applied bias increased the absolute photo response, at the expense of increasing the dark current and noise floor. If all aspects considered, the best sensor performance was achieved when the device was self-powered (no external bias). 
Finally, Section $\$ \mathbf{5 . 4 . 5}$ discusses the potential mechanism and future direction for photodetection using these devices was discussed. The most likely explanation is the simple photoelectric effect. By irradiating the device at the interface between the film and contact, the charge depletion layer is illumined. Under the influence of the same forces which extracted the charges from the depletion region on its initial formation (electronic equilibrium by alignment of the Fermi levels between the film and metal), the photocarriers generated in this region are quickly extracted into the contacts, where they are observed as a current (or voltage, depending on the setup of the test). The low carrier density and high mobilities in this region allow the photogenerated change carriers to be separated before they recombine and thermalize their energies. In this way, a photo response is produced. Considering the exponential relationship between the irradiation wavelength and photo response, a carrier multiplication process is likely also a play. Carrier multiplication (or carrier avalanche) results from a recombination of a high energy e-h pair producing multiple lower energy e-h pairs.

Future work for light sensing applications involve an optimization of the device structure. The metal used in the contacts can be selected to tune the Schottky barrier and thus the depletion region. The geometry of the contacts can be changed to enhance the depletion region formed in the film and enhance the photo-response. Additionally, while the photo response is dependent on the Schottky contact formation, testing of partially decoupled films may also be fruitful. If all other things remain constant, partially decoupled films will still produce Schottky contacts (although with lower barrier heights). However, the higher mobility and lower carrier concentration may result in longer carrier half-life's and thus, a larger photo-response. The section concludes with a brief summary on the photodetection results and discussion (Section $\$ 5.4 .6$ ). 


\section{CHAPTER 7: Conclusions ANd Future Directions}

Chapter 7 concludes this work by presenting the overarching conclusions and places these within the context of the larger body of scientific work in the field. In Section $\$$ 7.1.1, the conclusions reached from work relating to Goal 1 (process development) are presented. In Section $\$$ 7.1.1, the conclusions regarding the work conducted in pursuit of Goal 2 (application development) are presented. The chapter ends with Section $\$ \mathbf{7 . 2}$, where a technological roadmap for the further development of the plasma assisted growth method is given. Suggested future research avenues and directions are provided.

\section{\$7.1 Conclusions}

Graphene faces a grand challenge of production and manufacturability limitations. This is especially true for electronic applications of graphene. To tackle this problem, many methods to produced graphene are being developed. For electronic applicants, two of these methods aim to produced wafer-scale graphene films. They are chemical vapor deposition (CVD) of carbon onto a catalytically active substrate and growth from $\mathrm{SiC}$ by selective sublimation (thermal decomposition). However, neither method easily lends itself to practical, scalable manufacturing. Although the CVD process can produce good-quality large area graphene sheets, to construct an electronic device requires a transfer of the atomically thin film to the electronic device structurea process which is laborious and is difficult to translate into a scalable process. The selective sublimation method grows graphene directly onto a high-performance semiconductor (SiC). However, the film growth is not uniform across the wafer (step edge growth and pit formation) and the harsh thermal environment required for silicon sublimation severely limits manufacturability.

The plasma assisted graphene growth method utilized in this work attempts to tackle graphene's grand challenge and provide an alternative to the sublimation growth method. In support of this are Goal 1 and Goal 2 of this work. Section $\$ 7.1 .1$ discusses Goal 1 and summaries the relating conclusions. Section $\$$ 7.1.2 discusses Goal 2 and summaries the relating conclusions. Recall the general problem statement is given in Section $\$ \mathbf{1 . 3}$ and the specific motivation for these goals is discussed in Section $\$ 2.3$. 


\section{\$7.1.1 Goal 1: Process Development Conclusions}

Compared to selective sublimation, the novel plasma assisted method presents an alternative route towards wafer-scale graphene growth from $\mathrm{SiC}$, while being a fundamentally more scalable and manufacturable process. The plasma assisted graphene growth method is a twostep process, consisting of a halogen-based plasma etch, followed by a rapid thermal anneal. However, unlike the selective synthesis method which has been in development by a number of research groups across the globe since 2004, many fundamental aspects of the novel plasma assisted growth method remain a mystery. As such, questions regarding many synthesis parameters and structural aspects of the resulting material had yet to be answered at the start of this work. This work aimed to elucidate the process as a whole and fill in some of the knowledge gaps. This led to Goal 1 and its three objectives, summarized in Table 7-1:

Table 7-1: Goal 1 and the three related objectives.

\section{Goal 1: Development of the novel plasma assisted graphene growth method.}

Objective 1A: Characterize the SOP process.

Objective 1B: Investigate the impact of processing parameters on the produced films and improve the understanding of the fundamental science.

Objective 1C: Decouple the SOP films by hydrogen intercalation.

The work towards Goal 1 is primarily detailed in Chapter 4. This work can be further broken down into three objectives, the results of which are detailed in the three main sections of Chapter 4. Objective 1A (Section \$4.1) focused on characterization of films produced using a refined iteration of the plasma assisted growth method using standard processing parameters (referred to as the SOP process). The modification to the method was to exchange the ultra-high vacuum $\left(\sim 10^{-9}\right.$ torr) rapid thermal anneal (UHV-RTA) step with an atmospheric pressure $(\sim 760$ torr) rapid thermal anneal (AP-RTA) step. This greatly simplifies the process and further improves the processability and implementation of the plasma assisted growth technique. Objective $1 B$ (Section $\$ 4.2$ ) was to determine the impact of the various processing parameters on the films produced, thereby improving the understanding of the fundamental science. To these ends, a detailed parametric study was conducted. Objective 1C (Section $\$ 4.3$ ), motivated by conclusions reached in the first section, was to decouple the SOP films from the substrate. Motivated by 
literature reports, the effect of high temperature hydrogen exposure on the films was investigated. The broad conclusions reached related to each objective are summarized in Table 7-2.

Table 7-2: Goal 1 objectives and resulting conclusions.

\begin{tabular}{|c|c|}
\hline \multicolumn{2}{|r|}{ GOAL 1 Conclusions } \\
\hline $\begin{array}{l}\text { Objective 1A: } \\
\text { SOP process } \\
\text { characterization } \\
(\text { Section } \$ 4.1)\end{array}$ & $\begin{array}{l}\text { - The UHV-RTA step can be replaced with an AP-RTA step, with minimal impact } \\
\text { on the film. } \\
\text { - The SOP process produces good-quality films. } \\
\text { - The SOP process produces films with a highly tethered buffer-layer. }\end{array}$ \\
\hline $\begin{array}{l}\text { Objective 1B: } \\
\text { Parametric } \\
\text { investigation } \\
(\text { Section } \$ 4.2)\end{array}$ & $\begin{array}{l}\text { - The most important parameter controlling the film thicknesses are the ICP and } \\
\text { RIE powers. } \\
\text { - AP-RTA parameters impact the chemical defect concentration; longer times and } \\
\text { higher temperatures increase the amount of oxygen defects. }\end{array}$ \\
\hline $\begin{array}{l}\text { Objective 1C: } \\
\text { Film decoupling } \\
\text { via. } \mathrm{H}_{2} \text { treatment } \\
(\text { Section } \$ 4.3)\end{array}$ & $\begin{array}{l}\text { - High temperature exposure to hydrogen gas only partially decouples the buffer } \\
\text { layer. } \\
\text { - High temperatures and longer durations improve film decoupling. } \\
\text { - The most complete decoupling results from cyclic thermal cycles under } \\
\text { hydrogen. }\end{array}$ \\
\hline
\end{tabular}

The conclusions of Objective 1A (SOP process characterization, Section $\$ 4.1$ ) are that the UHV-RTA step could be replaced with an AP-RTA step, with minimal impact on the film. This new process produced good-quality films. Comparisons of the characterization results to literature reports indicate that the SOP films are tightly coupled to the substrate by way of a buffer-layer.

The conclusions relating to Objective 1B (parametric investigation, Section \$4.2) found that, while each processing parameter effect the films is various ways, the most profound impact on the films was due to the specific ICP and RIE powers used. Extended annealing times were found to be largely detrimental to the film quality. In general, the best quality films were produced from the most rapid temperature anneals.

The conclusions relating to Objective 1C (film decoupling via. $\mathrm{H}_{2}$ treatment, Section $\$ 4.3$ ) determined that high temperature exposure to hydrogen gas improves film quality by partially decoupling the films. However, complete film decoupling was not achieved. Qualitatively, more extensive decoupling was observed to correlated with higher temperatures and longer durations of hydrogen exposure. However, the most complete film decoupling was found to result when films were thermally cycled to high temperatures while under hydrogen gas. 


\section{\$7.1.2 Goal 2: Application Development Conclusions}

To further investigate the films produced by the SOP process and test the utility of the $\mathrm{SiC} / \mathrm{Graphene}$ composites produced by the plasma assisted method, simple electronic devices were constructed. This allowed for the electrical characterizations of the films. Additionally, the device performance provided an alternant window into the films produced. Thus, the application development was also used to inform the conclusions drawn in regard to Goal 1 and produced an internally consistent picture of this research. The field of sensors was chosen as the class of electronic device to be fabricated due to the simplicity of construction, as well as being a particularly well-suited application of graphene and to meet the industrial demand. A planar, multiterminal device consisting of metal contacts, the graphene film and the $\mathrm{SiC}$ substrate were constructed. Gas sensing and light sensing (photo detection) were selected as the sensing modes for the devices. Goal 2 had four objectives, summarized in Table 7-3:

Table 7-3: Goal 2 and the four related objectives.

\section{Goal 2: Development of simple electronic devices using graphene produced by the plasma assisted method.}

Objective 2A: Electrically characterizes the simple electronic devices.

Objective 2B: Investigate the temperature dependence of the device electrical properties.

Objective 2C: Investigate the gas sensing performance of the devices.

Objective 2D: Investigate the photo diction capability of the devices.

The objectives of Goal 2 are detailed in the main sections of Chapter 5. Objective $2 A$ (Section \$5.1) was to electrically characterize the fabricated devices at room temperature. Objective 2B (Section \$5.2) was to investigate how the electrical properties of the devices changed with temperature. This was done to gain a more detailed understanding of the electronic nature of the devices (and films) and inform the sensing results. Objective 2B (Section \$5.3) was to investigate the gas sensing performance of the devices. Hydrogen was chosen for the primary gas due to the industry demand for a sensor capable of detecting hydrogen gas, in the percentage concentration range, at high temperature $\left(>200^{\circ} \mathrm{C}\right)$. Objective $2 B$ (Section $\left.\$ 5.4\right)$ investigated the photo detection capability of the devices. The broad concludes reached from each objective are summarized in Table 7-4. 
Table 7-4: Goal 2 objectives and resulting conclusions.

\begin{tabular}{|c|c|}
\hline \multicolumn{2}{|r|}{ GOAL 2 Conclusions } \\
\hline $\begin{array}{l}\text { Objective 2A: } \\
\text { Electrical } \\
\text { device } \\
\text { characterization } \\
(\text { Section } \$ 5.1)\end{array}$ & $\begin{array}{l}\text { - Devices display back-to-back Schottky behavior, indicating the formation of a } \\
\text { charge depletion region at the contact interface. } \\
\text { - Film conductivity, carrier concentration and carrier mobility are high, indicating } \\
\text { substantial substrate induced doping and a coupling of the electronic and } \\
\text { vibrational states of the film to the substrate via the buffer-layer. }\end{array}$ \\
\hline $\begin{array}{l}\text { Objective 2B: } \\
\text { Response to } \\
\text { temperature } \\
\text { (Section } \$ 5.2)\end{array}$ & $\begin{array}{l}\text { - Devices are highly temperature sensitive. } \\
\text { - Due to thermally mediated change injection, device conductivity increases up to } \\
\text { modestly high temperatures, after which thermally induced carrier scattering } \\
\text { mechanism dominate carrier flow. } \\
\text { - Thermionic conduction is the primary carrier injection mechanism with charge } \\
\text { trap hopping also contributing, but to a lesser extent. } \\
\text { - Charge trap hopping conduction increase exponentially with temperature, } \\
\text { indicative of trap distribution in energy-space. } \\
\text { - The effective Schottky barrier height increases with temperature, indicative of } \\
\text { structure interfacial inhomogeneities. } \\
\text { - Non-SOP samples show significantly different behavior, which merit further } \\
\text { investigation; most notably are } 4 \mathrm{H}-\mathrm{SiC} \text { samples which display a negative } \\
\text { differential resistance regime. }\end{array}$ \\
\hline $\begin{array}{l}\text { Objective 2C: } \\
\text { Gas sensing } \\
\text { (Section } \$ 5.3 \text { ) }\end{array}$ & $\begin{array}{l}\text { - Devices are sensitive to the environmental gas composition across all } \\
\text { temperatures tested. } \\
\text { - Gas sensitivity is primarily thermally derived. } \\
\text { - A chemically derived sensitivity is observable at high temperature and low } \\
\text { voltage. }\end{array}$ \\
\hline $\begin{array}{l}\text { Objective 2D: } \\
\text { Photo detection } \\
\text { (Section } \$ 5.4 \text { ) }\end{array}$ & $\begin{array}{l}\text { - Devices show a response to light irradiation when the device is in both a powered } \\
\text { and unpowered state. } \\
\text { - Device response takes the form of a photovoltage or photocurrent, produced by } \\
\text { the photoelectric effect. } \\
\text { - The greatest sensitivity is at the metal/film interface, indicating the depletion } \\
\text { layer and built-in field assists in the e-h separation process. } \\
\text { - There is an exponential relationship between irradiation frequency and photo } \\
\text { response magnitude, indicative of an internal carrier multiplication (avalanche) } \\
\text { process. }\end{array}$ \\
\hline
\end{tabular}

The conclusions of Objective 2A (electrical device characterization, Section $\$ 5.1$ ) were that the simple devices constructed displayed back-to-back Schottky behavior, indicating the formation of a charge depletion region at the contact interface. The film conductivity, carrier concentration and carrier mobility were found to be high, indicating substantial substrate induced doping and a coupling of the electronic and vibrational states of the film to those of the substrate, via the buffer-layer. 
The conclusion of Objective 2B (Response to temperature, Section \$5.2) are that the devices are highly temperature sensitive. Due to thermally mediated charge injection, device conductivity increases up to modestly high temperatures, after which thermally induced carrier scattering mechanisms dominate carrier flow. Thermionic conduction is the primary carrier injection mechanism. Charge trap hopping also contributes, but to a lesser extent. Charge trap hopping conduction increase exponentially with temperature, indicative of the trap distribution in energy-space. The effective Schottky barrier height increases with temperature, indicative of structure interfacial inhomogeneities. Non-SOP samples show significantly different behavior, which merit further investigation; most notably are films grown from a $4 \mathrm{H}-\mathrm{SiC}$ substrate, which display a negative differential resistance regime due to the resonant tunneling of charge carriers into the film.

The conclusion of Objective 2C (Gas sensing, Section \$5.3) are that devices are sensitive to the environmental gas composition across all temperatures tested. Gas sensitivity is primarily thermally derived; however, a chemically derived sensitivity is observable at high temperature and low voltage.

The conclusion of Objective 2D (Photo detection, Section \$5.4) are that devices show a response to visible light irradiation. Device are photo sensitive in both a powered and unpowered state. Device response takes the form of a photovoltage or photocurrent, resulting from the photoelectric effect. The greatest response is observed when the metal/film interface is irradiated, indicating the depletion layer and built-in field assists in the separation of the e-h pairs generated. There is an exponential relationship between irradiation frequency and photo response magnitude, indicative of an internal carrier multiplication (avalanche) process.

\section{\$7.2 Future Directions and Recommendations}

The work detailed in the preceding chapters touches on many aspects of the plasma assisted process and its application. This has exposed a wide array of directions future work can proceed into. Many of the experiments detailed in Chapter 4 and Chapter 5 can be improved and extended to extract more information in future work. These improvements are expanded on throughout the results and discussion sections of those chapters. For device applications, the future directions as they related to gas sensing are given in Section $\$ 5.3 .7$ and, as they relate to light sensing, are given in Section \$5.4.5. Additionally, based on the promising results detailed in Section $\$ 5.1 .4$, the 
performance of non-SOP devices should be investigated as well. And this should not be limited to just the applications studied here. Given the unique electrical properties of some non-SOP devices, there is good reason to believe that these devices may perform radically different from the SOP devices. This final section, however, will focus on the future directions of the work more broadly and look towards the overarching goal of this work, which is to address the grand challenge facing graphene. To this end, the future directions look towards a further refinement of the plasma assisted growth process.

The recommendations for future improvements to the synthesis process are be broken down into two basic blocks, based on their respective timeframes. First, is an improvement on the implementation of the process as it stands today. It is recommended that the immediate future work continue to focus on hydrogen treatment of the films, with the goal being to fully decouple the films from their substrates thereby improving film quality and device performance. The second recommendation, which looks towards more distant future work, is a continuation of the larger trend of improving on the plasma assisted process, with the goal being improved manufacturability and increased film quality.

\section{§7.2.1 Near-term Future Work}

In regard to the more immediate future work relating to the high temperature hydrogen exposure experiments, extending the parameter space covered (Section $\$ 4.3$ ) is a logical first step. If the trends observed in this work were to continue, future experiments should focus on increasing the time, temperature, and most importantly, the number of thermal cycles. The next, more aggressive step would be to move from molecular hydrogen $\left(\mathrm{H}_{2}\right)$ exposure to atomic hydrogen $(\mathrm{H})$ exposure. This smaller, more reactive form of hydrogen would potentially more readily penetrate the film and react with the interface covalent bonds. Others have reported on the use of atomic hydrogen to decouple graphene films from their $\mathrm{SiC}$ substrates. However, the short half-life of atomic hydrogen, particularly at atmospheric pressure, necessitates the source be located physically very close to the sample to provide the necessary flux to the surface. An atmospheric pressure catalytically assisted thermal cracker may prove challenging to implement in the current AP-RTA system but could be done using commercially available systems.

If full film decoupling is achieved, based on the film characterization results detailed in Section $\$ 4.3$ and the observed improvements in electrical properties discussed Section $\$$ 5.3.6, 
than it is expected that a substantial improvement can be realized in many electronic device applications. It is noted, however, that full film decoupling may not be desirable for all applications. For example, photo detection applications derive their functionality from the presence of Schottky contacts, which are themselves formed due to complex buffer layer interactions. Therefore, device performance in select applications may be improved by tuning the degree of film coupling. Thus, rather than a simply full-coupled or fully-decoupled film, establishing a method by which this parameter can be controlled with a high degree of granularity is highly desirable. This may be achieved by the aforementioned hydrogen treatment route.

\section{\$7.2.2 Long-term Future Work}

The long-term goals of this work are to develop the plasma assisted process in a way which improves the manufacturability of the process as well as improve the film quality. Therefore, in the same why that this work improved the manufacturability of the process by exchanging the UHV-RTA step for an AP-RTA step, it is recommended that future work examine the potential of combining the annealing step with the etch step. Looking future into future work, continuing the track of improved film quality by hydrogen treatment, the addition of hydrogen to the combination step should be investigated. To provide an overview of this developmental path, a technological roadmap showing the past, current and future work is given in Figure 7.2, where dashed lines represent the proposed future work.

\section{Technological Roadmap: \\ Plasma Assisted Graphene Growth}

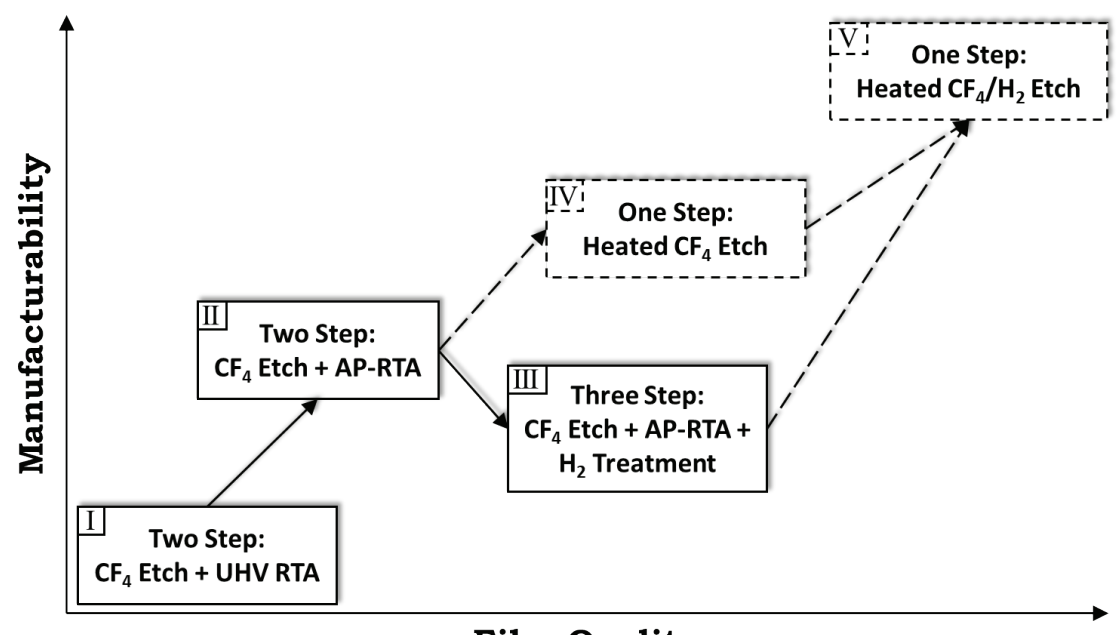

Film Quality

Figure 7.1: Suggested technological roadmap for the plasma assisted graphene growth process. Dashed lines represent future work. 
The technological step labeled I in Figure 7.2 represents the state of the art for the plasma assisted growth process at the start of this work. Steps labeled II and III represent the improvements to this process detailed in this work. Steps labeled IV and V represent future work. These steps are discussed next.

Step IV involves the combination of the etching step with the annealing step. Recall that in its first iteration, the plasma assisted process used a halogen etch to generate the carbon rich surface and then provided the energy required to crystalize the surface during an ultra-high vacuum annealing step. This work investigated the films produced when this step was replaced with an atmospheric pressure anneal and shows that this was a viable alternative. By preforming the anneal at atmospheric pressure rather than under UHV, the equipment and procedures are vastly simplified. Combing the etch step with the annealing step could continue this trend. This could be done by utilizing a heated sample chuck in the ICP/RIE chamber. This would be a significant departure from the current method and may present many challenges. However, by combining these steps, not only would the process and equipment setup be simplified, the films may be improved.

There are two basic iterations of this combination of steps. In the first, the etch would be performed to completion and then the annealing step would immediately follow. This case is relatively straightforward and would likely produce films of similar quality to the films of this work, but with lower oxygen defects. By preforming both steps within a single piece of equipment the processing is simplified, and the sample production time is reduced. Furthermore, the fact that the sample would not be exposed to air between the etch and anneal, as well as the reduce sample handling by human hands, would both undoubtedly improve film quality. However, the most compelling reason to combine these steps would be the ability to annealing the substrate during the etching process. This second iteration of the combination of steps, would result in a thermally enhanced plasma etch.

Applying heat during the etch could impact the process in several ways. The etch rate would almost certainly be enhanced. The enhanced kinetics of the various chemical etching processes may result in an enhanced selectivity. The greater volatilization rates and removal of the etch products may also improve the etch by quickly desorbing the Si- reaction products. Additionally, the enhanced mobility afforded to the surface atoms by the higher temperature may 
reduce the propensity of the film to form the covalent tethering bonds to the substrate (which, as shown in Appendix $\boldsymbol{\$ A}$.1.6, are formed during the etch). All of these effects may work to produce higher quality films. Note that there is evidence uncovered during this work which supports this basic idea. In Section $\$ 4.1$ it was noted that films produced where no helium flow was used to cool the ICP/RIE sample chuck produced higher quality films then those films which were produced using a He cooling flow. The analysis of films produced both with and without this He cooling flow are included in Appendix $\$ \mathbf{A}$.1.3.

Because the surface would already be at an elevated energetic state during the etch (due to ongoing surface plasma chemistry and because the film would be under intense ion bombardment), the maximum temperature required for recrystallization may also be reduced (for example, the sample may only need to be heated to a few hundred of ${ }^{\circ} \mathrm{C}$ ). This would further improve processability. The synergy between the plasma surface chemistry and thermal-derived enhancement would provide a fascinating study. Because the interplay between the ICP and RIE powers on the etching process would undoubtedly be dramatically affected, the trends reported here would not hold true for these elevated temperatures. Thus, the effects of the ICP/RIE powers on the films would need to be reinvestigated. If heating the substrate during the etch still produced coupled films, then the next technological step shown in should be Figure 7.2 considered, discussed next.

The fact that $\mathrm{H}_{2}$ and $\mathrm{CF}_{4}$ plasmas are compatible allow for a further combination of processing steps. Thus, the next technological iteration of the process, labeled V in Figure 7.2, would be to conduct the hydrogen treatment simultaneously with the combined $\mathrm{CF}_{4}$ etching + annealing steps. An investigation of the effect of adding a fractional background of $\mathrm{H}_{2}$ during or after the etch step would be informative. Forming a hydrogen plasma would provide a source of atomic hydrogen and hydrogen ions (protons). There small size, high reactivity, and directional kinetic energy would provide a method to hydrogen treat the films in a way which has yet to be done. These highly reactive hydrogen plasma species may more readily cleave the bonding between the substrate and surface film, resulting in a more through decoupling. Alternatively, the hydrogen plasma treatment may be conducted after the etch + annealing steps. The fact that the hydrogen plasma would be highly tunable would also add a degree of controllability not available to current hydrogen-based film decoupling methods. Clearly there are many unknows regarding 
these proposed modifications to the plasma process (for example, the competing chemistry between $\mathrm{CF}_{4}$ and $\mathrm{H}_{2}$ plasmas), but they will only reveal themselves through aggressive experimentation.

In conclusion, the plasma assisted growth method is in the early stages of development and as a result, there are a vast array of development avenues available at this time (only some of which have been mentioned here). As previously stated, this method is a unique process that is fundamentally different from all other graphene synthesis methods. One facet of this is that, unlike other growth methods, there are many aspects of the plasma process that can be independently controlled and modified. Furthermore, the basic process provides an agile paradigm which can be extensively modified, while simultaneously serving as a robust framework on which other process may be built. Overall, the plasma assisted growth method is a highly tunable graphene synthesis process which is well suited for electronic device manufacturing using current semiconductor processes. The work detailed here lays only the most basic groundwork for a process which has great potential. 


\section{WORKS CITED}

1. The Band Theory of Graphite. Wallace, PR. 9, 1947, Physical Review, Vol. 71, pp. 622-634.

2. Electric field effect in Atomically Thin Carbon Films. Novoselov, KS, et al. 5696, 2004, Science, Vol. 306, pp. 666-669.

3. Impermeability of Graphene and Its Applications. Berry, V. 2013, Carbon, Vol. 62, pp. 1-10.

4. The Electronic Properties of Graphene. Castro Neto, AH, et al. 1, 2009, Vol. 81, pp. 109-162.

5. Interplay of Wrinkles, Strain, and Lattice Parameter in Graphene on Iridium. Hattab, H, et al. 2, 2012, Nano Letters, Vol. 12, pp. 678-682.

6. Thermal Properties of Graphene: Fundamentals and Applications. E, Pop, C, Varshney and A, Roy. 2012, Materials Reserach Society Bulletin, Vol. 37, pp. 1273-1274.

7. Van der Waals Radii of Elements. Batsanov, SS. 9, 2001, Inorganic Materials, Vol. 37, pp. 871-885.

8. Measurement of the Elastic Properties and Intrinsic Strength of Monolayer Graphene. Lee, C, et al. 5887, 2008, Science, Vol. 321, pp. 385-388.

9. Extraordinary Physical Properties of Functionalized Graphene. Wei, Weili and Qu, Xiaogang. 14, 2012, Small, Vol. 8, pp. 2138-2148.

10. Wear Resistance Limited by Step Edge Failure: The Rise and Fall of Graphene as an Atomically Thin Lubricating Material. Qi, YZ, et al. 1, 2017, ACS Applied Materials \& Interfaces, Vol. 9, pp. 1099-1106.

11. Thermal Properties of Graphene and Nanostructured Carbon Materials. Balandin, AA. 8, 2011, Nature Materials, Vol. 10, pp. 569-581.

12. Superior Thermal Conductivity of Single-Layer Graphene. Balandin, AA Ghosh, S, et al. 3, 2008, Nano Letters, Vol. 8, pp. 902-907.

13. Unusual Enhancement in Intrinsic Thermal Conductivity of Multi layer Graphene by Tensile Strains. Kuang, YD, Lindsay, L and Huang, BL. 8, 2015, Nano Letters, Vol. 15, pp. 6121-6127.

14. Two-Dimensional Phonon Transport in Graphene. Nika, DL and Balandin, AA. 23, 2012, Journal of Physics-Condensed Matter, Vol. 24.

15. Thermal Conductivity of Graphene and Graphite: Collective Excitations and Mean Free Paths. Fugallo, G, et al. 11, 2014, Nano Letters, Vol. 14, pp. 6109-6114.

16. Properties of graphene: a theoretical perspective. D.S.L. Abergel, V. Apalkov, J. Berashevich , K. Ziegler, Tapash Chakraborty. 4, s.1. : Advances in Physics, 2010, Vol. 59, pp. 261-482.

17. Two-dimensional Carbon Nanostructures: Fundamental Properties, Synthesis, Characterization, and Potential Applications. Wu, YH, Yu, T and Shen, ZX. 7, 2010, Journal Of Applied Physics, Vol. 108.

18. Giant Intrinsic Carrier Mobilities in Graphene and Its Bilayer. Morozov, SV, Novoselov, KS and Katsnelson, MI. 1, 2008, Vol. 100.

19. Effect of Charge Puddles and Ripples on the Chemical Reactivity of Single Layer Graphene Supported by SiO2/Si Substrate. Fan, XY, Nouchi, R and Tanigaki, K. 115, 2011, Journal Of Physical Chemistry C, Vol. 26, pp. 12960-12964. 
20. Molecular Dynamics Study of Ripples in Graphene Nanoribbons on 6H-SiC(0001): Temperature and Size Effects. Tang, C, et al. 11, 2008, Journal Of Applied Physics, Vol. 104.

21. Effects of Thermal Rippling On the Frictional Properties of Free Standing Graphene. Smolyanitsky, A. 37, 2015, RSC Advances, Vol. 5, pp. 29179-29184.

22. Coulomb Interaction, Ripples, and the Minimal Conductivity of Graphene. Herbut, IF, Juricic, V and Vafek, O. 4, 2008, Physical Review Letters, Vol. 100.

23. Approaching Ballistic Transport in Suspended Graphene. Du, X, et al. 8, 2008, Nature Nanotechnology, Vol. 3, pp. 491-495.

24. Micrometer-Scale Ballistic Transport in Encapsulated Graphene at Room Temperature. Mayorov, AS, et al. 6, 2011, Nano Letters, Vol. 11, pp. 2396-2399.

25. New effects in graphene with high carrier mobility. Morozov, S.C. 4, 2012, Uspekhi Fizicheskikh Nauk, Russian Academy of Sciences, Vol. 55, pp. 408-412.

26. Ultrahigh Electron Mobility in Suspended Graphene. Bolotin, KI, et al. 9-10, 2008, Solid State Communications, Vol. 146, pp. 351-355.

27. Approaching the Dirac Point in High-Mobility Multilayer Epitaxial Graphene. Orlita, M, Faugeras, C and Plochocka, P. 26, 2008, Vol. 101.

28. Magnetoconductance Oscillations and Evidence for Fractional Quantum Hall States in Suspended Bilayer and Trilayer Graphene. Bao, WZ, et al. 24, 2010, Physical Review Letters, Vol. 105.

29. Interaction-Driven Spectrum Reconstruction in Bilayer Graphene. Mayorov, AS, et al. 6044, 2011, Science, Vol. 333, pp. 860-863.

30. Evidence for a Spontaneous Gapped State in Ultraclean Bilayer Graphene. Bao, WZ, et al. 2012. Proceedings Of The National Academy Of Sciences Of The United States Of America. Vol. 109, pp. 1080210805.

31. Opening a Band Gap Without Breaking Lattice Symmetry: A New Route Toward Robust Graphenebased Nanoelectronics. Kou, LZ, et al. 13, 2014, Nanoscale, Vol. 6, pp. 7474-7479.

32. Molecular Doping and Band-Gap Opening of Bilayer Graphene. Samuels, AJ and Carey, JD. 3, 2013, ACS NANO, Vol. 7, pp. 2790-2799.

33. Charge Neutrality and Band-gap Tuning of Epitaxial Graphene on SiC by Molecular Doping. Coletti, C, et al. 23, 2010, Physical Review B, Vol. 81.

34. Bandgap Tuning of Mono- and Bilayer Graphene Doped With Group IV Elements. Kaplan, D, et al. 18, 2013, Journal Of Applied Physics, Vol. 113.

35. Properties of suspended graphene membranes. Lau, CN, Bao, WZ and Velasco, J. 6, 2012, Materials Today, Vol. 15, pp. 238-245.

36. Stacking-dependent Band Gap and Quantum Transport in Trilayer Graphene. Bao, W, et al. 12, 2011, Nature Physics, Vol. 7, pp. 948-952.

37. Band Gap and Correlated Phenomena in Bilayer and Trilayer Graphene. Lee, Y, et al. 2013. Proceedings of SPIE. Vol. 8725.

38. $n$ Situ Transport Measurements and Band Gap Formation of Fluorinated Graphene. Zhao, R, et al. 34, 2015, Journal Of Physical Chemistry C, Vol. 119, pp. 20150-20155. 
39. Temperature Dependent Transfer Characteristics of Graphene Field Effect Transistors Fabricated Using Photolithography. Venugopal, G and Kim, SJ. 3, 2011, Current Applied Physics, Vol. 11, pp. S381S384.

40. Dirac Point and Transconductance of Top-gated Graphene Field-effect Transistors Operating at Elevated Temperature. Hopf, T, et al. 15, 2014, Journal Of Applied Physics, Vol. 116.

41. Temperature and Gate Voltage Dependent Electrical Properties of Graphene Field-effect Transistors. Feng, TT, et al. 2014, Carbon, Vol. 78, pp. 250-256.

42. Observation of an electrically tunable band gap in trilayer graphene. Lui, Chun Hung, et al. 2011, Nature Physics, Vol. 7, pp. 94-947.

43. Electrical Observation of a Tunable Band Gap in Bilayer Graphene Nanoribbons at Room Temperature. Szafranek, BN, et al. 11, 2010, Applied Physics Letters, Vol. 96.

44. Graphene Field-Effect Transistors with High On/Off Current Ratio and Large Transport Band Gap at Room Temperature. Xia, FN, et al. 2, 2010, Nano Letters, Vol. 10, pp. 715-718.

45. Improvement of mechanical properties of graphene oxide/poly(allylamine) composites by chemical crosslinking. Satti, A, Larpent, P and Gun'ko, Y. 12, 2010, Carbon, Vol. 48, pp. 3376-3381.

46. Enhancement of the Electrical Properties of Graphene Grown by Chemical Vapor Deposition via Controlling the Effects of Polymer Residue. Suk, JW, et al. 4, 2013, Nano Letters, Vol. 13, pp. 1462-1467.

47. Manipulating the electronic and chemical properties of graphene via molecular functionalization. Mao, HY, et al. 2, 2013, Progress in Surface Science, Vol. 88, pp. 132-159.

48. First principles calculations of the electronic and chemical properties of graphene, graphane, and graphene oxide. Rosas, JJH, et al. 5, 2011, Journal of Molecular Modeling, Vol. 17, pp. 1133-1139.

49. How Do the Electrical Properties of Graphene Change with its Functionalization? Sreeprasad, T.S. and Berry, Vikas. 3, 2013, Small, Vol. 9, pp. 341-350.

50. Substrate-induced Bandgap Opening in Epitaxial Graphene. Zhou, SY, et al. 10, 2007, Nature Materials, Vol. 6, pp. 770-775.

51. Controlling the Electronic Structure of Bilayer Graphene. Taisuke, Ohta, et al. 5789, 2006, Science, Vol. 313, pp. 951-954.

52. n-Type Behavior of Graphene Supported on Si/SiO2 Substrates. Hugo, Romero, et al. 2008, ACS Nano, pp. 2037-2044.

53. Effective Doping of Single-layer Graphene From Underlying SiO2 Substrates. Yumeng, Shi, et al. 11, 2009, Phys. Rev. B, Vol. 79.

54. Two-Dimensional Phonon Transport in Supported Graphene. Seol, JH, et al. 5975, 2010, Science, Vol. 328, pp. 213-216.

55. Challenges and Opportunities in Graphene Commercialization. Zurutuza, A and Marinelli, C. 10, 2014, Nature Nanotechnology, Vol. 9, pp. 730-734.

56. Mertens, Ron and Peleg, Roni. www.graphene-info.com. [Online] Graphene Info: The Graphene Experts, 2018.

57. Smart Reinvention of the Contact Lens with Graphene. Choi, K and Park, HG. 6, 2017, ACS Nano, Vol. 11, pp. 5223-5226. 
58. Quirky Graphene. Patel, P. 6, 2018, Scientific American, Vol. 318, pp. 20-20.

59. Towards the Artsutanov's Dream of the Space Elevator: The Ultimate Design of a 35 GPa Strong Tether Thanks to Graphene. Pugno, NM. 2, 2013, ACTA Astronautica, Vol. 82, pp. 221-224.

60. Web of Science. [Online] Clarivate Analytics, 2019. wcs.webofknowledge.com.

61. About Graphene Flagship. [Online] 2019. https://graphene-flagship.eu/project/Pages/About-GrapheneFlagship.aspx.

62. National Graphene Institute (NGI): About. [Online] The University of Manchester. [Cited: 9 15, 2019.] https://www.graphene.manchester.ac.uk/about/ngi/.

63. Graphene Reserch Centre (GRC): The National University of Singapore Centre for Advanced 2D Materials. [Online] The National Universtity of Singapore, 2019. https://graphene.nus.edu.sg/about-us/.

64. New graphene industrial park nears completion. [Online] Xinhua Silk Road: Information Service; China Economic Information Service, Xinhua News Agency, 2019. https://en.imsilkroad.com/p/97198.html.

65. Beijing Graphene Institue of Graphene Reserach. [Online] [Cited: 8 24, 2019.] http://bgigraphene.com/.

66. Congressional Hearing before the U.S. China Economic and Security Review Commission 116th Congress First Session on 6/7/2019: Hearing on Technology, Trade, and Military-Civil Fusion: China's Pursuit of Artificial Intelligence, New Materials and New Energy. Washington, DC: Transcript: https://www.uscc.gov/sites/default/files/transcripts/June\%207\%2C\%202019\%20Hearing\%20Transcript.p df Video: https://www.senate.gov/isvp/?type=live\&comm=uscc\&filename=uscc060719, 2019.

67. Tour, James M. Testimony before the Subcommittee on Digital Commerce and Consumer Protection, United States House of Representatives: 'Graphene and Establishing U.S. Preeminence In the Field' 3/15/2017. Washington, DC: Written Submisson: https://docs.house.gov/meetings/IF/IF17/20170315/105701/HHRG-115-IF17-Wstate-TourJ20170315.pdf Video: https://youtu.be/QwPtKmQm08s, 3 15, 2017.

68. Washington, Lindsey and Duckworth, Caroline. Project on International Peace and Security (PIPS) White Paper: Cornering the Graphene Market- Countering Beijing's Strategy for Industry Domination . [Online] The College of William \& Mary, 2019. [Cited: 8 26, 2019.] https://www.wm.edu/offices/globalresearch/projects/pips/white_papers/pips.2018-

2019.whitepaper.11.6.executivesummary.lindsey.washington.pdf.

69. Sokolnikov, Andre U. Graphene for Defense and Security. s.1. : CRC Press: Taylor \& Francis Group, 2017. ISBN: 978-1-4987-2762-4.

70. EDA Study to Assess Graphene Potential for Defence. [Online] European Defence Agency, 1211 , 2017. [Cited: 8 26, 2019.] https://www.eda.europa.eu/info-hub/press-centre/latest-news/2017/12/11/edastudy-to-assess-graphene-potential-for-defence.

71. Brussels, BE: Work starts on 'Graphene Roadmap in Defence'. eda.europa.eu. [Online] European Defence Agency, May 29, 2018. https://www.eda.europa.eu/info-hub/press-centre/latestnews/2018/05/29/work-starts-on-graphene-roadmap-in-defence.

72. European Defence Agency: Final Meeting of the OB Study 'Graphene in Defence'. [Online] European Defence Agency. [Cited: 8 26, 2019.] https://eda.europa.eu/docs/default-source/documents/final-meetingof-the-ob-study-graphene-in-defence.pdf. 
73. Graphene Landscape IP Report, August 2018. [Online] Derwent: Powering IP Innovation, Aug 2018. http://clarivate.com/derwent/wp-content/uploads/sites/3/dlm_uploads/2019/07/ANALYTICS_LandscapeGraphene-August-2018.pdf.

74. www.graphene-info.com/companies. [Online] 2019. https://www.graphene-info.com/companies.

75. Neufeld, Dorothy. Graphene Investing News. www.investingnews.com. [Online] July 31, 2019. https://investingnews.com/daily/tech-investing/nanoscience-investing/graphene-investing/investing-ingraphene-companies/.

76. Obtaining a Sustainable Competitive Advantage from Patent Information: A Patent Analysis of the Graphene Industry. Yang, X, Yu, X and Liu, X. 12, 2018, Sustainability, Vol. 10.

77. Trends Analysis of Graphene Research. Zou, LX, et al. 1, 2018, Journal of Data and Information Science, Vol. 3, pp. 82-100.

78. Perforene: Graphene Membrane. www.LockheadMartin.com. [Online] 2019.

79. Sharon, Madhuri Sharon and Maheshwar. Graphene: An Introduction to the Fundamentals and Industrial Applicaions. s.1. : John Wiley \& Sons, 2015. ISBN: 978-1-118-84256-0.

80. A Study on Technology Competition of Graphene Biomedical Technology Based on Patent Analysis. Yang, X, Liu, X and Song, J. 13, 2019, Applied Sciences-Basel, Vol. 9.

81. Pan, Siwen. Graphene Market Global Overview - Daydream - Dynovel - May 2019. [Online] Daydream: 5 to 5 Market Access, May 28, 2019. https://www.daydream.eu/graphene-market-globaloverview-daydream-dynovel-may-2019/.

82. Science and Technology Roadmap for Graphene, Related Two-Dimensional Crystals, and Hybrid Systems. Ferrari, AC, et al. 11, 2015, Nanoscale, Vol. 7, pp. 4598-4810.

83. A Roadmap for Graphene. Novoselov, KS, et al. 7419, 2012, Nature, Vol. 490, pp. 192-200.

84. A Decade of Graphene Research: Production, Applications and Outlook. Randviir, EP, Brownson, DAC and Banks, CE. 9, 2014, Materials Today, Vol. 19, pp. 426-432.

85. Graphene-Based Composite Materials. Stankovich, S, et al. 7100, 2006, Nature, Vol. 442, pp. 282-286.

86. Graphene-based Materials and Their Composites: A Review on Production, Applications and Product Limitations. Mohan, VB, et al. 2018, Composites Part B- Engineering, Vol. 142, pp. 200-220.

87. Aligned-Graphene Composites: A Review. Wang, F, Wang, HY and Mao, J. 1, 2019, Journal of Materials Science, Vol. 54, pp. 36-61.

88. Graphene: Corrosion-Inhibiting Coating. Prasai, D, et al. 2, 2012, ACS Nano, Vol. 6, pp. 1102-1108.

89. Impermeable Barrier Films and Protective Coatings Based on Reduced Graphene Oxide. Su, Y, et al. 10.1038/ncomms5843, 2014, Nature Communications, Vol. 5.

90. Impermeable Atomic Membranes from Graphene Sheets. Bunch, JS, et al. 8, 2008, Nano Letters, Vol. 8, pp. 2458-2462.

91. Graphene-Based Membranes. Liu, GP, Jin, WQ and Xu, NP. 15, 2015, Chemical Society Reviews, Vol. 44, pp. 5016-5030.

92. Recent Developments in Graphene-Based Membranes: Structure, Mass-Transport Mechanism and Potential Applications. Sun, PZ, Wang, KL and Zhu, HW. 12, 2016, Advanced Materials, Vol. 28, pp. 2287-2310. 
93. Light-Responsive Actuators Based on Graphene. Gao, YY, et al. 2019, Frontiers in Chemistry, Vol. 7.

94. Graphene-based Composite for Dielectric Elastomer Actuator: A Comprehensive Review. PanahiSarmad, M, Zahiri, B and Noroozi, M. 2019, Sensors and Actuators A-Physical, Vol. 293, pp. 222-241.

95. Graphene-Based Actuators. Park, S, et al. 2, 2010, Small, Vol. 6, pp. 210-212.

96. Graphene-Based Aptasensors: From Molecule-Interface Interactions to Sensor Design and Biomedical Diagnostics. Wang, L, Wu, AG and Wei, G. 7, 2018, Analyst, Vol. 143, pp. 1526-1543.

97. Research Progress of Gas Sensor Based on Graphene and Its Derivatives: A Review. Tian, WC, Liu, XH and Yu, WB. 7, 2018, Applied Sciences-Basel, Vol. 8.

98. Graphene and Its Sensor-based Applications: A Review. Nag, A, Mitra, A and Mukhopadhyay, SC. 2018, Sensors and Actuators A-Physical, Vol. 270, pp. 177-194.

99. Graphene-Based Light Sensing: Fabrication, Characterisation, Physical Properties and Performance. De Sanctis, A, et al. 9, 2018, Materials, Vol. 11.

100. Graphene Photodetectors for High-Speed Optical Communications. Mueller, T, Xia, FNA and Avouris, P. 5, 2010, Nature Photonics, Vol. 4, pp. 297-301.

101. Carbon Nanotube- and Graphene-Based Nanomaterials and Applications in High-Voltage Supercapacitor: A Review. Yang, ZF, et al. 2019, Carbon, Vol. 141, pp. 467-480.

102. An Overview of the Applications of Graphene-Based Materials in Supercapacitors. Huang, Y, Liang, JJ and Chen, YS. 12, 2012, Small, Vol. 8, pp. 1805-1834.

103. Recent Advances of Graphene-Based Materials for High-Performance and New-Concept Supercapacitors. Shi, XY, et al. 1, 2018, Journal of Energy Chemistry, Vol. 27, pp. 25-42.

104. High-Speed Graphene Transistors With a Self-Aligned Nanowire Gate. Liao, L, et al. 7313, 2010, Nature, Vol. 467, pp. 305-308.

105. Graphene-based Plasmonic Nano-Antenna for Terahertz Band Communication in Nanonetworks. Jornet, JM and Akyildiz, IF. 12, 2013, IEEE Journal on Selected Areas in Communications, Vol. 31, pp. 685-694.

106. Broad Electrical Tuning of Graphene-Loaded Plasmonic Antennas. Yao, Y, et al. 3, 2013, Nano Letters, Vol. 13, pp. 1257-1264.

107. Reconfigurable Terahertz Plasmonic Antenna Concept Using a Graphene Stack. Tamagnone, M, et al. 21, 2012, Applied Physics Letters, Vol. 101.

108. Application of Graphene-Based Flexible Antennas in Consumer Electronic Devices. Scida, A, et al. 3 , 2018, Materials Today, Vol. 21, pp. 223-230.

109. Large-Scale Pattern Growth of Graphene Films for Stretchable Transparent Electrodes. Kim, KS, et al. 7230, 2009, Nature, Vol. 457, pp. 706-710.

110. Graphene-Based Transparent Conductive Films: Material Systems, Preparation and Applications. Ma, YJ and Zhi, LJ. 1, 2019, Small Methods, Vol. 3.

111. A Review of Graphene Based Transparent Conducting Films for Use in Solar Photovoltaic Applications. Rosli, NN, et al. 2019, Renewable \& Sustainable Energy Reviews, Vol. 99, pp. 83-99.

112. Graphene/Si Schottky Solar Cells: A Review of Recent Advances and Prospects. Kong, XY, et al. 2, 2019, RSC Advances, Vol. 9, pp. 863-877. 
113. Graphene Materials and Their Use in Dye-Sensitized Solar Cells. Roy-Mayhew, JD and Aksay, IA. 12, 2014, Chemical Reviews, Vol. 114, pp. 6323-6348.

114. Graphene Oxide: A Promising Membrane Material for Fuel Cells. Farooqui, UR, Ahmad, AL and Hamid, NA. 2018, Renewable \& Sustainable Energy Reviews, Vol. 82, pp. 714-733.

115. Graphene and Graphene-Based Materials for Energy Storage Applications. Zhu, JX, et al. 17, 2014, Small, Vol. 10, pp. 3480-3498.

116. An Overview of graphene in energy production and storage applications. Brownson, DAC, Kampouris, DK and Banks, CE. 11, 2011, Journal of Power Sources, Vol. 196, pp. 4873-4885.

117. Graphene Hybridization for Energy Storage Applications. Li, XL and Zhi, LJ. 9, 2018, Chemical Society Reviews, Vol. 47, pp. 3189-3216.

118. Graphene/Semiconductor Hybrid Heterostructures for Optoelectronic Device Applications. Xie, C, et al. 2018, Nano Today, Vol. 19, pp. 41-83.

119. Graphene Photonics and Optoelectronics. Bonaccorso, F, et al. 9, 2010, Nature Photonics, Vol. 4, pp. 611-622.

120. Graphene Photonics, Plasmonics, and Broadband Optoelectronic Devices. Bao, QL and Loh, KP. 5 , 2012, ACS Nano, Vol. 6, pp. 3677-3694.

121. Graphene Oxide Photonics. Ghofraniha, N and Conti, C. 5, 2019, Journal of Optics, Vol. 21.

122. Graphene as Biomedical Sensing Element: State of Art Review and Potential Engineering Applications. Kumar, R, et al. 2018, Composites Part B-Engineering, Vol. 134, pp. 193-206.

123. Biomedical Applications of Graphene and Graphene Oxide. Chung, C, et al. 10, 2013, Accounts of Chemical Research, Vol. 46, pp. 2211-2224.

124. Biomedical Applications of Graphene. Shen, H, et al. 3, 2012, Theranostics, Vol. 2, pp. 283-294.

125. Recent Advances in Three-Dimensional Graphene Based Materials for Catalysis Applicaions. Qiu, BC, Xing, MY and Zhang, JL. 6, 2018, Chemcial Society Reviews, Vol. 47.

126. Graphene-based Materials for Catalysis. Machado, BF and Serp, P. 1, 2012, Catalysis Science \& Technology, Vol. 2, pp. 54-75.

127. Chemistry of Graphene Derivatives: Synthesis, Applications, and Perspectives. Sturala, J, et al. 23, 2018, Chemistry-A European Journal, Vol. 24, pp. 5992-6006.

128. Assembly of Graphene-Aligned Polymer Composites for Thermal Conductive Applications. Zhang, ZX, et al. 2018, Composite Communications, Vol. 9, pp. 33-41.

129. Review of Electrical Properties of Graphene Conductive Composites. Alemour, B, et al. 4, 2018, International Journal of Nanoelectronics and Materials, Vol. 11, pp. 371-398.

130. Ahmed, Khaled and Schuegraf, Klaus. Transistor Wars: Rival Architectures Face Off In a Bid to Keep Moore's Law Alive. IEEE Spectrum. Oct 28, 2011.

131. Intel Ivy Bridge Unveiled - The First Commercial Tri-gate, High-k, Metal-gate CPU. James, D. San Jose : s.n., 2012. 2012 IEEE Custom Integrated Circuits Conference (CICC).

132. 3D ICs in the Real World. James, D. s.1. : IEEE, 2014. 25th Annual Semi Advanced Semiconductor Manufacturing Conference and Workshop-Proceedings. pp. 113-119. 
133. Three-Dimensional Integration of Nanotechnologies for Computing and Data Storage On a Single Chip. Shulaker, MM, et al. 7661, 2017, Nature, Vol. 547, pp. 74-93.

134. Ye, P, Ernst, T and Khare, M. The Last Silicon Transister: Nanosheet Devices Could Be The Final Evolutionary Step For Moore's Law. IEEE Spectrum. Jul 2019, pp. 31-35.

135. Celasco, Edvige and Chaika, Alexander, [ed.]. Handbook of Graphene: Volumes 1-8. Hoboken : John Wiley \& Sons, Inc., 2019. ISBN: 978-1-119-46861-5.

136. Morgan, Hywel, Rout, Chandra and Late, Dattatray, [ed.]. Fundamentals and Sensing Applications of 2D Materials, 1st Ed. Duxford: Woodhead Publishing of Elsevier, 2019. ISBN:978-0-08-102577-2.

137. Production, Properties and Potential of Graphene. Soldano, C, Mahmood, A and Dujardin, E. 8, 2010, Carbon, Vol. 48, pp. 2127-2150.

138. Graphene and Graphene Oxide: Synthesis, Properties, and Applications. Zhu, YW, et al. 35, 2010, Advanced Materials, Vol. 22, pp. 3906-3924.

139. Energetics of Interplanar Binding in Graphite. Schabel, MC and Martins, JL. 11, 1992, Physical Review B, Vol. 46, pp. 7185-7188.

140. A Review on Mechanical Exfoliation for the Scalable Production of Graphene. Yi, M and Shen, ZG. 22, 2015, Journal of Materials Chemistry A, Vol. 3, pp. 11700-11715.

141. Size Controlled Synthesis of Graphene Oxide Sheets on a Large Scale Using Chemical Exfoliation. Zhang, L, et al. 14, 2009, Carbon, Vol. 47, pp. 3365-3368.

142. Controlled Synthesis of Few-layered Graphene Sheets on a Large Scale Using Chemical Exfoliation. Zhang, L, et al. 8, 2010, Carbon, Vol. 48, pp. 2367-2371.

143. Exfoliation and Chemical Modification Using Microwave Irradiation Affording Highly Functionalized Graphene. Economopoulos, SP, et al. 2, 2010, Acs Nano, Vol. 4, pp. 7499-7507.

144. The Exfoliation of Graphene in Liquids by Electrochemical, Chemical, and Sonication-Assisted Techniques: A Nanoscale Study. Xia, ZY, et al. 37, 2013, Advanced Functional Materials, Vol. 23, pp. 4684-4693.

145. The chemistry of Graphene Oxide. Dreyer, DR, et al. 1, 2010, Chemical Society Reviews, Vol. 39, pp. 228-240.

146. Chemically Derived Graphene Oxide: Towards Large-Area Thin-Film Electronics and Optoelectronics. Eda, G and Chhowalla, M. 22, 2010, Advanced Materials, Vol. 22, pp. 2392-2415.

147. Structural Defects in Graphene. Banhart, F, Kotakoski, J and Krasheninnikov, AV. 1, 2011, ACS Nano, Vol. 5, pp. 26-41.

148. Graphene Oxide, Highly Reduced Graphene Oxide, and Graphene: Versatile Building Blocks for Carbon-Based Materials. Compton, OC and Nguyen, ST. 6, 2010, Small, Vol. 6, pp. 711-723.

149. Functional Composite Materials Based on Chemically Converted Graphene. Bai, H, Li, C and Shi, GQ. 9, 2011, Advanced Materials, Vol. 23, pp. 1089-1115.

150. High-yield Production of Graphene by Liquid-phase Exfoliation of Graphite. Hernandez, Y, et al. 9, 2008, Nature Nanotechnology, Vol. 3, pp. 563-568.

151. Direct Ink Writing of Graphene-Based Solutions for Gas Sensing. Loh, HA, et al. 7, 2019, ACS Applied Nano Materials, Vol. 2, pp. 4104-4112. 
152. Synthesis of Graphene-Based Nanosheets via Chemical Reduction of Exfoliated Graphite Oxide. Stankovich, S, et al. 7, 2007, Carbon, Vol. 45, pp. 1558-1565.

153. Designed CVD Growth of Graphene via Process Engineering. Yan, K, et al. 10, 2013, Accounts of Chemical Research, Vol. 46, pp. 2263-2274.

154. Review of CVD Synthesis of Graphene. Munoz, R and Gomez-Aleixandre, C. 10-12, 2013, Chemical Vapor Depositon, Vol. 19, pp. 297-322.

155. The Chemistry of CVD Graphene. Plutnar, J, Pumera, M and Sofer, Z. 23, 2018, Journal of Materials Chemistry C, Vol. 6, pp. 6082-6101.

156. Recent Trends in Graphene Materials Synthesized by CVD with Various Carbon Precursors. Kairi, MI, et al. 2, 2018, Journal of Materials Science, Vol. 53, pp. 851-879.

157. Review of Chemical Vapor Deposition of Graphene and Related Applications. Zhang, Y, Zhang, LY and Zhou, CW. 10, 2013, Accounts Of Chemical Research, Vol. 46, pp. 2329-2339.

158. Kinetic Control of Catalytic CVD for High-Quality Graphene at Low Temperatures. Weatherup, RS, Dlubak, B and Hofmann, S. 11, 2012, ACS Nano, Vol. 6, pp. 9996-10003.

159. Influence of Copper Crystal Surface on the CVD Growth of Large Area Monolayer Graphene. Zhao, L, et al. 7, 2011, Solid State Communications, Vol. 151, pp. 509-513.

160. Epitaxial growth mechanisms of graphene and effects of substrates. Ozcelik, VO, Cahangirov, S and Ciraci, S. 23, 2012, Physical Review B, Vol. 85.

161. The Surface Science of Graphene: Metal Interfaces, CVD Synthesis, Nanoribbons, Chemical Modifications, and Defects. Batzill, M. 3-4, 2012, Surface Science Reports, Vol. 67, pp. 83-115.

162. Electrochemical Delamination of CVD-Grown Graphene Film: Toward the Recyclable Use of Copper Catalyst. Wang, Y, et al. 12, 2011, ACS Nano, Vol. 5, pp. 9927-9933.

163. Epitaxial Graphene on Cu(111). Gao, L, Guest, JR and Guisinger, NP. 9, 2010, Vol. 10, pp. 35123516.

164. Large-Area Graphene Single Crystals Grown by Low-Pressure Chemical Vapor Deposition of Methane on Copper. Li, XS, et al. 9, 2011, Journal of the American Chemical Society, Vol. 133, pp. 28162819.

165. A Review of Chemical Vapour Deposition of Graphene on Copper. Mattevi, C, Kim, H and Chhowalla, M. 10, 2011, Journal Of Materials Chemistry, Vol. 21, pp. 3324-3334.

166. Soulubility of Carbon in Solid Gold, Copper and Silver. McLellan, RB. 6, 1969, Scripta Metallurgica, Vol. 3, p. 389.

167. Transfer of CVD-Grown Monolayer Graphene onto Arbitrary Substrates. Suk, JW, et al. 9, 2011, ACS Nano, Vol. 5, pp. 6916-6924.

168. Large Area, Few-Layer Graphene Films on Arbitrary Substrates by Chemical Vapor Deposition. Reina, A, et al. 1, 2009, Nano Letters, Vol. 9, pp. 30-35.

169. Doping Graphene With Metal Contacts. Giovannetti, G, et al. 2, 2008, Physical Review Letters, Vol. 101.

170. Cohesion Energetics of Carbon Allotropes: Quantum Monte Carlo Study. Shin, H, et al. 11, 2014, Journal Of Chemical Physics, Vol. 140. 
171. Large-Scale Synthesis of Few-Layered Graphene using CVD. Wang, XB, et al. 1-3, 2009, Chemical Vapor Deposition, Vol. 15, pp. 53-56.

172. Ultrathin Epitaxial Graphite: 2D Electron Gas Properties and a Route Toward Graphene-based Nanoelectronics. Berger, C, et al. 52, 2004, Journal of Physical Chemistry B, Vol. 108, pp. 19912-19916.

173. Graphene Growth on Silicon Carbide: A Review. Mishra, N, et al. 9, 2016, Physica Status Solidi AApplications and Materials Science, Vol. 213, pp. 2277-2289.

174. Combining Graphene With Silicon Carbide: Synthesis and Properties - A Review. Shtepliuk, I, Khranovskyy, V and Yakimova, R. 11, 2016, Semiconductor Science and Technology, Vol. 31.

175. Graphene on Cubic SiC. Chaika, AN, Aristov, VY and Molodtsova, OV. 2017, Progress in Materials Science, Vol. 89, pp. 1-30.

176. Epitaxial Graphene on SiC: A Review of Growth and Characterization. Yazdi, GR, Iakimov, T and Yakimova, R. 5, 2016, Crystals, Vol. 6.

177. Large Area and Structured Epitaxial Graphene Produced by Confinement Controlled Sublimation of Silicon Carbide. de Heer, WA, et al. 41, 2011, Proceedings Of The National Academy Of Sciences Of The United States Of America, Vol. 108, pp. 16900-16905.

178. Recent Progress in Synthesis, Properties and Potential Applications of SiC Nanomaterials. Wu, RB, et al. 2015, Progress in Materials Science, Vol. 72, pp. 1-60.

179. Feng, Zhe Chuan, [ed.]. SiC Power Materials: Devices and Applications. s.l. : Springer, 2004.

180. Fraley, Bill. Silicon Carbide Devices and Technology. s.1. : NY Research Press, 2015.

181. Choyke, W.J. and Matsunami, H. Silicon Carbide Recent Major Advances. [ed.] G. Pensl. s.1. : Springer, 2004.

182. Replacing Si to SiC: Opportunities and Challenges. Nawaz, M and Ilves, K. Lausanne, Switzerland: IEEE, 2016. 2016 46TH European Soli-State Device Research Conference (ESSDERC). Vols. Proceedings of the European Solid-State Device Research Conference, pp. 472-475.

183. Status of Silicon Carbide (SiC) As a Wide-bandgap Semiconductor for High-temperature Applications: A Review. Casady, JB and Johnson, RW. 10, 1996, Solid-State Electronics, Vol. 39, pp. 14091422.

184. Baliga, B. Jayant. Silicon Carbide Power Devices. s.1. : World Scientific Publishing Co. Pte. Ltd., 2005.

185. Tsunenobu, Kimoto and James, Cooper. Fundamentals of Silicon Carbide Technology: Growth, Characterization, Devices, and Applications. s.1. : John Wiley \& Sons, 2014.

186. Device Applications of Epitaxial Graphene on Silicon carbide. Beshkova, M, Hultman, L and Yakimova, R. 2016, Vacuum, Vol. 128, pp. 186-197.

187. Initial Stages of the Graphite-SiC(0001) Interface Formation Studied by Photoelectron Spectroscopy. Emtsev, KV, et al. Newcastle upon Tyne, ENGLAND : s.n., 2007. 6th European Conference on Silicon Carbide and Related Materials. Vols. 556-557, pp. 525-528.

188. Study of the crystal and electronic structure of graphene films grown on 6H-SiC (0001). Davydov, VY, et al. 2017, Semiconductors, pp. 1072-1080. 
189. Formation Mechanism of Graphene Buffer Layer on SiC(0001). Strupinski, W, et al. 2015, Carbon, Vol. 81, pp. 63-72.

190. The transition from 3 C SiC(111) to graphene captured by Ultra High Vacuum Scanning Tunneling Microscopy. B. Gupta, E. Placidi, C. Hogan, N. Mishra, F. Iacopi, N. Motta. 2015, Carbon, Vol. 91, pp. 378-385.

191. Why graphene growth is very different on the C face than on the Si face of SiC: Insights from surface equilibria and the (3 $x 3$ 3)-3C-SiC((1)over-bar(1)over-bar(1)over-bar) reconstruction. Nemec, L, et al. 16, 2015, Physical Review B, Vol. 91.

192. Tip induced mechanical deformation of epitaxial graphene grown on reconstructed $6 \mathrm{H}-\mathrm{SiC}(0001)$ surface during scanning tunneling and atomic force microscopy studies. Meza, JAM, et al. 25, 2015, Nanotechnology, Vol. 26.

193. Effects of substrate orientation on the structural and electronic properties of epitaxial graphene on SiC(0001). Robinson, JA, et al. 22, 2011, Applied Physics Letters, Vol. 98.

194. Effect of SiC Wafer Miscut Angle on the Morphology and Hall Mobility of Epitaxially Grown Graphene. Dimitrakopoulos, C, et al. 22, 2011, Applied Physics Letters, Vol. 98.

195. Step-edge Instability During Epitaxial Growth of Graphene From SiC(0001). Borovikov, V and Zangwill, A. 12, 2009, Physical Review B, Vol. 80.

196. Microscopic Mechanisms of Initial Formation Process of Graphene on SiC(0001) Surfaces: Selective Si Desorption from Step Edges. Imoto, F, et al. 9, 2017, Journal of Physical Chemistry C, Vol. 121, pp. 5041-5049.

197. Pit Formation During Graphene Synthesis on SiC(0001): In Situ Electron Microscopy. Hannon, JB and Tromp, RM. 24, 2008, Physical Review B, Vol. 77.

198. Formation Process of Graphene on SiC (0001). Norimatsu, W and Kusunoki, M. 4, 2010, Physica ELow-Dimensional Systems \& Nanostructures, Vol. 42, pp. 691-694.

199. A Morphology Study on the Epitaxial Growth of Graphene and Its Buffer Layer. Kruskopf, M, et al. 2018, Thin Solid Flims, Vol. 659, pp. 7-15.

200. Sequential Control of Step-Bunching During Graphene Growth on SiC (0001). Bao, JF, et al. 8, 2016, Applied Physics Letters, Vol. 109.

201. Epitaxial Graphene: How Silicon Leaves the Scene. Sutter, P. 3, 2009, Nature Materials, Vol. 8.

202. Towards Wafer-Size Graphene Layers by Atmospheric Pressure Graphitization of Silicon Carbide. Emtsev, KV, et al. 3, 2009, Nature Materials, Vol. 8, pp. 203-207.

203. Interface Structure and Mechanics Between Graphene and Metal Substrates: A First-Principles Study. Xu, ZP and Buehler, MJ. 48, 2010, Journal Of Physics-Condensed Matter, Vol. 22.

204. Strain Superlattices and Macroscale Suspension of Graphene Induced by Corrugated Substrates. Reserbat-Plantey, A, et al. 9, 2014, Nano Letters, Vol. 14, pp. 5044-5051.

205. Raman Spectroscopy of Epitaxial Graphene on a SiC Substrate. Ni, ZH, et al. 11, 2008, Physical Review B, Vol. 77.

206. Effects of Mismatch Strain and Substrate Surface Corrugation on Morphology of Supported Monolayer Graphene. Aitken, ZH and Huang, R. 12, 2010, Journal Of Applied Physics, Vol. 107. 
207. Modification of Electrical Properties of Graphene by Substrate-Induced Nanomodulation. Lee, JK, et al. 8, 2013, Nano Letters, Vol. 13, pp. 3494-3500.

208. Carrier Transport in Two-Dimensional Graphene Layers. Hwang, EH, Adam, S and Das Sarma, S. 18, 2007, Physical Review Letters, Vol. 98.

209. Electronic Structure of Epitaxial Graphene Layers on sic: Effect of the Substrate. Varchon, F, et al. 12, 2007, Physical Review Letters, Vol. 99.

210. Understanding and Controlling the Substrate Effect on Graphene Electron-Transfer Chemistry via Reactivity Imprint Lithography. Wang, QH, et al. 9, 2012, Nature Chemistry, Vol. 4, pp. 724-732.

211. Atomic Surface Structure of Graphene and Its Buffer Layer on SiC(0001): A Chemical-specific Photoelectron Diffraction Approach. de Lima, LH, et al. 8, 2013, Physical Review B, Vol. 87.

212. Contribution of the Buffer Layer to the Raman Spectrum of Epitaxial Graphene on SiC(0001). Fromm, F, et al. 2013, New Journal of Physics, Vol. 15.

213. Theoretical Study on Epitaxial Graphene Growth by Si Sublimation from SiC(0001) Surface. Kageshima, H, et al. 9, 2011, Japanese Journal of Applied Physics, Vol. 50.

214. Carbon Rehybridization at the Graphene/SiC(0001) Interface: Effect on Stability and Atomic-Scale Corrugation. Sclauzero, G and Pasquarello, A. 16, 2012, Physical Review B, Vol. 85. 10.1103/PhysRevB.85.161405.

215. Formation of a Buffer Layer for Graphene on C-Face SiC\{0001\}. He, GW, Srivastava, N and Feenstra, RM. 4, 2014, Journal of Electronic Materials, Vol. 43, pp. 819-827.

216. Graphene Buffer Layer on Si-terminated SiC Studied With an Empirical Interatomic Potential. Lampin, E, et al. 10, 2010, Journal of Applied Physics, Vol. 107.

217. Band Gap Opening Induced by the Structural Periodicity in Epitaxial Graphene Buffer Layer. Nair, MN, et al. 4, 2017, Nano Letters, Vol. 17, pp. 2681-2689.

218. Structure and Evolution of Semiconducting Buffer Graphene Grown on SiC(0001). Conrad, M, et al. 19, 2017, Vol. 96.

219. Structural Determination of Bilayer Graphene On SiC(0001) Using Synchrotron Radiation Photoelectron Diffraction. Razado-Colambo, I, et al. 8, 2018, Scientific Reports.

220. Effect of Buffer Layer Coupling on the Lattice Parameter of Epitaxial Graphene on SiC(0001). Schumann, T, et al. 4, 2014, Physical Review B, Vol. 90.

221. On The Lattice Parameters of Silicon Carbide. Stockmeier, M, et al. 3, 2009, Jorunal of Applied Physics, Vol. 105.

222. Interaction, Growth, and Ordering of Epitaxial Graphene on SiC\{0001\} Surfaces: A Comparative Photoelectron Spectroscopy Study. Emtsev, KV, et al. 15, 2008, Physical Review B, Vol. 77.

223. Synthesis of Freestanding Graphene on SiC by a Rapid-Cooling Technique. Bao, JF, et al. 20, 2016, Physical Review Letters, Vol. 117.

224. Decoupling the Graphene Buffer Layer from SiC(0001) via Interface Oxidation. Ostler, M, et al. [ed.] RP Devaty, et al. 2012. Silicon Carbide and Related Materials . Vols. 717-720.

225. Synergistic Effect of $\mathrm{H} 2 \mathrm{O}$ and $\mathrm{O}-2$ On the Decoupling of Epitaxial Monolayer Graphene From SiC(0001) via Thermal Treatments. Bom, NM, et al. 2014, Carbon, Vol. 78, pp. 298-304. 
226. Formation of High-Quality Quasi-Free-Standing Bilayer Graphene On SiC(0001) By Oxygen Intercalation Upon Annealing In Air. Oliveira, MH, et al. 2013, Carbon, Vol. 52, pp. 83-89.

227. Decoupling Graphene From SiC(0001) via Oxidation. Oida, S, et al. 4, 2010, Physical Review B, Vol. 82.

228. Decoupling of Epitaxial Graphene via Gold Intercalation Probed By Dispersive Raman Spectroscopy. Pillai, PB, et al. 18, 2015, Journal of Applied Physics, Vol. 117.

229. Electronic Properties of Zero-Layer Graphene on 6H-SiC(0001) Substrate Decoupled By Silicon Intercalation. Silly, MG, Li, G and Dappe, YJ. 12-13, 2014, Surface and Interface Analysis, Vol. 46, pp. 1273-1277.

230. Buffer Layer Free Large Area Bi-layer Graphene on SiC(0 0 O 1). Virojanadara, C, et al. 2, 2010, Surface Science, Vol. 604, pp. L4-L7.

231. Hydrogenation of the Buffer-Layer Graphene on 6H-SiC (0001): A Possible Route for the Engineering of Graphene-Based Devices. Lin, YP, Ksari, Y and Themlin, JM. 3, 2015, Nano Research, Vol. 8, pp. 839850.

232. Control of Epitaxial Graphene Thickness on 4H-SiC(0001) and Buffer Layer Removal Through Hydrogen Intercalation. Hassan, J, et al. [ed.] RP Devaty, et al. 2012. Materials Science Forum. Vols. 717720 .

233. First-principles Study of Preferential Sites of Hydrogen Incorporated in Epitaxial Graphene on 6HSiC(0001). Lee, B, Han, S and Kim, YS. 7, 2010, Physical Review B, Vol. 81.

234. Revealing the atomic structure of the buffer layer between SiC(0001) and epitaxial graphene. Goler, S, et al. 2013, Carbon, Vol. 51, pp. 249-254.

235. Epitaxial Graphene Transistors: Enhancing Performance via Hydrogen Intercalation. Robinson, JA, et al. 9, 2011, Nano Letters, Vol. 11, pp. 3875-3880.

236. Hydrogen Transport Within Graphene Mutilayers by Means of Flexural Phonons. Camiola, VD, et al. 1, 2015, 2D Materials, Vol. 2.

237. Graphene: A Perfect Nanoballoon. Leenaerts, O, Partoens, B and Peeters, FM. 19, 2008, Applied Physics Letters, Vol. 93.

238. First Principles Study of the Permeability of Graphene to Hydrogen Atoms. Miao, M, et al. 8, 2013, Physical Chemistry Chemical Physics, Vol. 15, pp. 16132-16137.

239. Rapid Communication: Permeability of Two-Dimensional Graphene and Hexagonal-Boron Nitride to Hydrogen Atom. Gupta, V, Kumar, A and Ray, N. 5, 2018, Pramana-Journal of Physics, Vol. 91.

240. Large Homogeneous Mono-/bi-layer Graphene on 6H-SiC(0001) and Buffer Layer Elimination. Virojanadara, C, et al. 37, 2010, Journal of Physics D: Applied Physics, Vol. 43.

241. High Electron Mobility in Epitaxial Graphene on 4H-SiC(0001) via Post-Growth Annealing Under Hydrogen. Pallecchi, E, et al. 2014, Scientific Reports, Vol. 4.

242. Structural and Electronic Properties of Epitaxial Graphene on SiC(0 00 1): A Review of Growth, Characterization, Transfer Doping and Hydrogen Intercalation. Riedl, C, Coletti, C and Starke, U. 37, 2010, Journal of Physics D: Applied Physics, Vol. 43.

243. Enhancement of Band Gap and Evolution of In-gap States in Hydrogen-adsorbed Monolayer Graphene on SiC(0001). Sugawara, K, et al. 2017, Carbon, Vol. 124, pp. 584-587. 
244. Hydrogen Intercalation of Epitaxial Graphene and Buffer Layer Probed by Mid-infrared Absorption and Raman Spectroscopy. Kunc, J, Rejhon, M and Hlidek, P. 4, 2018, AIP Advances, Vol. 8.

245. Tuning a Schottky Barrier of Epitaxial Graphene/4H-SiC (0001) by Hydrogen Intercalation. Dharmaraj, P, Jesuraj, PJ and Jeganathan, K. 5, 2016, Applied Physics Letters, Vol. 108.

246. Uniform Coverage of Wuasi-free Standing Monolayer Graphene on SiC By Hydrogen Intercalation. Yu, CC, et al. 4, 2017, Journal of Materials Science- Materials in Electronics, Vol. 28, pp. 3884-3890.

247. Effects of hydrogen Intercalation On Transport Properties of Quasi-Free-Standing Monolayer Graphene. Tanabe, S, et al. 4, 2014, Japanese Journal of Applied Physics, Vol. 53.

248. Exposure of Epitaxial Graphene on SiC(0001) to Atomic Hydrogen. Guisinger, NP, et al. 4, 2009, Nano Letters, Vol. 9, pp. 1462-1466.

249. Structural consequences of hydrogen intercalation of epitaxial graphene on SiC(0001). Emery, JD, et al. 16, 2014, Applied Physics Letters, Vol. 105.

250. Thesis: Epitaxial Graphene on Silicon Carbide Surfaces: Growth, Characterization, Doping and Hydrogen Intercalation. Riedl, Christian. 2010.

251. Hydrogen intercalation of graphene grown on $6 H-S i C(0001)$. Watcharinyanon, $\mathrm{S}$, et al. 17-18, 2011, Surface Science, Vol. 605, pp. 1662-1668.

252. Core-level Photoelectron Spectroscopy Study of Interface Structure of Hydrogen-Intercalated Graphene On n-type 4H-SiC(0001). Maeda, F, et al. 8, 2013, Physical Revicew B, Vol. 88.

253. Bandstructure Manipulation of Epitaxial Graphene on SiC(0001) by Molecular Doping and Hydrogen Intercalation. Starke, U. MRS Spring Meeting. Vol. 1246.

254. Electrical Characterization of Bilayer Graphene Formed by Hydrogen Intercalation of Monolayer Graphene on SiC(0001). Tanabe, S, et al. 2, 2012, Japanse Journal of Applied Physics, Vol. 15.

255. Direct Experimental Evidence For the Reversal Of Carrier Type Upon Hydrogen Intercalation In Epitaxial Graphene/SiC(0001). Rajput, S, Li, YY and Li, L. 4, 2014, Applied Physics Letters, Vol. 104.

256. Quasi-Free-Standing Epitaxial Graphene on SiC Obtained by Hydrogen Intercalation. Riedl, C, et al. 24, 2009, Physical Review Letters, Vol. 103.

257. Raman Probing of Hydrogen-Intercalated Graphene on Si-face 4H-SiC. Shtepliuk, I, et al. 2019, Materials Science in Semiconductor Processing, Vol. 96, pp. 145-152.

258. A new direct growth method of graphene on Si-face of 6H-SiC by synergy of the inner and external carbon sources. Yang, ZY, et al. 2018, Applied Surface Science, Vol. 436, pp. 511-518.

259. Synthesis of high-quality monolayer graphene by low-power plasma. Hong, HK, et al. 1, 2019, Current Applied Physics, Vol. 19, pp. 44-49.

260. Induction Plasma Synthesis of Graphene Nano-flakes with In Situ Investigation of Ar-H-2-CH4 Plasma by Optical Emission Spectroscopy. Mohanta, A, Lanfant, B and Leparoux, M. 5, 2019, Plasma Chemistry and Plasma Processing, Vol. 39.

261. Effect of Hydrogen Concentration on Graphene Synthesis Using Microwave-Driven Plasma-Mediated Methane Cracking. Singh, M, et al. 2019, Vol. 143, pp. 802-813.

262. Novel Surface Chemical Synthesis Route for Large Area Graphene-on-Insulator Films. Raghavan, S, et al. 3, 2012, Journal of Vacuum Science \& Technology B, Vol. 30. 
263. Characterization of Graphene-On-Insulator Films Formed Using Plasma Based Surface Chemistry. Raghavan, S, et al. 2016, Carbon, Vol. 99, pp. 212-221.

264. Chaudhari, Saurabh. Development of Graphene and Graphene-Nanoparticle Composites for Sensor Applications. s.1., Morgantown, WV : West Virginia University, 2015.

265. Graphene Based Electrochemical Sensors and Biosensors: A Review. Shao, YY, et al. 10, 2000, Electroanalysis, Vol. 22, pp. 1027-1036.

266. Biological and Chemical Sensors Based on Graphene Materials. Liu, YX, Dong, XC and Chen, P. 6, 2012, Chemical Society Reviews, Vol. 41, pp. 2283-2307.

267. Graphene-based Electronic Sensors. He, QY, et al. 6, 2012, Chemical Science, Vol. 3, pp. 1764-1772.

268. Graphene Based Sensors and Biosensors. Justino, CIL, et al. 2017, Trac-Trends in Analytical Chemistry, Vol. 91, pp. 53-66.

269. Reduced Graphene Oxide Molecular Sensors. Robinson, JT, et al. 10, 2008, Nano Letters, Vol. 8, pp. 3137-3140.

270. Graphene-based Gas Sensors. Yuan, WJ and Shi, GQ. 35, 2013, Journal of Materials Chemistory A, Vol. 1, pp. 10078-10091.

271. Recent Advances in Graphene Based Gas Sensors. Varghese, SS, et al. 2015, Sensors and Actuators B-Chemical, Vol. 218, pp. 160-183.

272. Graphene in Light: Design, Synthesis and Applications of Photo-active Graphene and Graphene-Like Materials. Wang, HX, et al. 8, 2013, Small, Vol. 9, pp. 1266-1283.

273. Kreuzer, H.J. and Gortel, Z.W. Physisorption Kinetics . [ed.] P Toennies, G Ertl and R Gromer. s.1. : Springer-Verlag, 1986.

274. Nobel Lectures: Chemistry 1922-1941. s.1. : Elsevier Publishing Company, 1966.

275. On the Determination of Molecular Fields - II From the Equation of State of a Gas. Jones, JE. 738, 1924, Proceedings of the Royal Society of London Series A- Containing Papers of a Mathematical and Physical Character, Vol. 106, pp. 463-477.

276. Insights Into the Modeling of Adsorption Isotherm Systems. Foo, KY and Hameed, BH. 1, 2010, Chemcial Engingeering Journal, Vol. 156, pp. 2-10.

277. Selective Adsorption Computations With Digital Computers. Rose, A, Lombardo, RJ and Williams, TJ. 11, 1951, Industrial and Engieering Chemistry, Vol. 43, pp. 2454-2459.

278. A Review of Theoretical-Models of Adsorption, Diffusion, Desorption, and Reaction of Gases on Metal-Surfaces. Lombardo, SJ and Bell, AT. 1-2, 1991, Surface Science Reports, Vol. 13, pp. 1-72.

279. Molecular Modeling of Adsorption and Ordering At Solid Interfaces. Hentschke, R. 2, 1997, Macromolecular Theory and Simulations, Vol. 6, pp. 287-316.

280. Atomistic Modeling of Gas Adsorption in Nanocarbons. Zollo, G and Gala, F. 2012, Journal of Nanomaterials.

281. Ab Initio Investigation Into the Physisorption of Noble Gases On Graphene. Shepard, R, Shepard, S and Smeu, M. 2019, Surface Science, Vol. 682, pp. 38-42.

282. Artificial Neural Network Modelling of Amido Black Dye Sorption On Iron Composite Nano Material: Kinetics and Thermodynamics Studies. Ali, I, et al. 2018, Journal of Molecular Liquids, Vol. 250, pp. 1-8. 
283. Use of Artificial Neural Networks and Genetic Algorithms for Prediction of Sorption of An Azo-Metal Complex Dye Onto Lentil Straw. Celekli, A, Bozkurt, H and Geyik, F. 2013, Bioresource Technology, Vol. 129, pp. 396-401.

284. Machine-Learning-Augmented Chemisorption Model for CO2 Electroreduction Catalyst Screening. Ma, XF, et al. 18, 2015, Journal of Physical Chemistry Letters, Vol. 6, pp. 3528-3533.

285. Adsorption of CO on Low-Energy, Low-Symmetry Pt Nanoparticles: Energy Decomposition Analysis and Prediction via Machine-Learning Models. Gasper, R, Shi, HB and Ramasubramaniam, A. 10, 2017, Journal of Physical Chemistry C, Vol. 121, pp. 5612-5619.

286. Robust Machine Learning Models for Predicting High CO2 Working Capacity and CO2/H-2 Selectivity of Gas Adsorption in Metal Organic Frameworks for Precombustion Carbon Capture. Dureckova, H, et al. 7, 2019, Journal of Physical Chemistry C, Vol. 123, pp. 4133-4139.

287. Ibach, Harald. Physics of Surfaces and Interfaces. New York: Springer-Verlag Berlin Heidelberg, 2006. ISBN: 3-540-34709-7.

288. Masel, Richard I. Principles of Adsorption and Reaction on Solid Surfaces. s.1. : Wiley-Interscience Publication, 1996. ISBN: 0-471-30392-5.

289. Kolasinski, Kurt W. Surface Science: Foundations of Catalysis and Nanoscience. 3ed. s.1. : Wiley, 2012. p. 120. ISBN: 978-0-470-66556-5.

290. Site Hopping of Single Chemisorbed Oxygen Molecules on Si(111)-(7x7) Surfaces. Hwang, IS, Lo, RL and Tsong, TT. 25, 1997, Physical Review Letters, Vol. 78, pp. 4797-4800.

291. Diffusion of Single Hydrogen Atoms on Si(111)-(7x7) Surfaces. Lo, RL, et al. 25, 1998, Physical Review Letters, Vol. 80, pp. 5584-5587.

292. Real-time Observation of Molecular Motion On A Surface. Backus, EHG, et al. 5755, 2005, Science, Vol. 310, pp. 1790-1793.

293. Thommes, M, et al. Physisorption of Gases, With Special Reference To The Evaluation of Surface Area and Pore Size Distribution (IUPAC Technical Report). IUPAC (International Union of Pure and Applied Chemistry). s.1. : Jurnal of Pure and Applied Chemistry, 2015. pp. 1051-1069.

294. Rouquerol, Françoise, Rouquerol, Jean and Sing, Kenneth. Adsorption by Powders and Porous Solids: Principles, Methodology and Applications. s.1. : Elsevier, 1999. ISBN: 978-0-12-598920-6.

295. Nanomaterial-Based Gas Sensors: A Review. Xu, K, et al. 2, 2018, Instrumentation Science and Technology, Vol. 46, pp. 115-145.

296. Recent Developments in 2D Nanomaterials for Chemiresistive-Type Gas Sensors. Choi, SJ and Kim, ID. 3, 2018, Electronic Materials Letters, Vol. 14, pp. 221-260.

297. Two-Dimensional Transition Metal Dichalcogenides and Metal Oxide Hybrids for Gas Sensing. Lee, E, Yoon, YS and Kim, DJ. 10, 2018, ACS Sensors, Vol. 3, pp. 2045-2060.

298. Hydrogen-gas Sensors Based on Graphene Functionalized Palladium Nanoparticles: Impedance Response As A Valuable Sensor. Martinez-Orozco, RD, Antano-Lopez, R and Rodriguez-Gonzalez, V. 10, 2015, New Journal of Chemistry, Vol. 39, pp. 8044-8054.

299. Impedance Spectroscopic Analysis of Nanoparticle Functionalized Graphene/p-Si Schottky Diode Sensors. Uddin, MA, et al. 11, 2016, Japanese Journal of Applied Physics, Vol. 55. 
300. Gas Concentration Effect on Channel Capacitance in Graphene Based Sensors. Akbari, E, et al. 10, 2013, Journal of Computational and Theoretical Nanoscience, Vol. 10, pp. 2449-2452.

301. Tunable Reverse-Biased Graphene/Silicon Heterojunction Schottky Diode Sensor. Singh, A, et al. 8, 2014, Small, Vol. 10, pp. 1555-1565.

302. High-Performance Schottky Diode Gas Sensor Based on the Heterojunction of Three-Dimensional Nanohybrids of Reduced Graphene Oxide-Vertical ZnO Nanorods on an AIGaN/GaN Layer. Triet, NM, et al. 6, 2017, ACS Applied Materials \& Interfaces, Vol. 9, pp. 30722-30732.

303. Bias- and Gate-Tunable Gas Sensor Response Originating from Modulation in the Schottky Barrier Height of a Graphene/MoS2 van der Waals Heterojunction. Tabata, H, et al. 44, 2018, ACS Applied Materials \& Interfaces, Vol. 10, pp. 38387-38393.

304. Solid State Gas Sensors. Moseley, PT. 3, 1997, Measurement Science and Technology, Vol. 8, pp. 223-237.

305. Solid State Gas Sensors: State of the Art and Future Activites. Capone, S., et al. 5, 2003, Journal of Optoelectronics and Advanced Materials, Vol. 5, pp. 1335 - 1348.

306. Low-Frequency Fluctuations in Solids: 1/f Noise. Dutta, P and Horn, PM. 3, 1981, Review of Modern Physics, Vol. 53, pp. 497-516.

307. Two-dimensional Gas of Massless Dirac Fermions in Graphene. Novoselov, KS, et al. 7065, 2005, Nature, Vol. 438, pp. 197-200.

308. The Necessity of Structural Irregularities for the Chemical Applications of Graphene. Eftekhari, A and Garcia, H. 2017, Materials Today Chemistry, Vol. 4, pp. 1-16.

309. Gas Detection Using Low-temperature Reduced Graphene Oxide Sheets. Lu, GH, Ocola, LE and Chen, JH. 8, 2009, Applied Physics Letters, Vol. 94.

310. Photo-induced Selective Gas Detection Based On Reduced Graphene Oxide/Si Schottky Diode. Zhu, M, et al. 2015, Carbon, Vol. 84, pp. 138-145.

311. Selective detection of Carbon Monoxide (CO) Gas by Reduced Graphene Oxide (rGO) at Room Temperature. Panda, D, et al. 53, 2016, RSC Advances, Vol. 6, pp. 47337-47348.

312. Graphene Oxide for Gas Detection Under Standard Humidity Conditions. Donarelli, M, et al. 3, 2015, 2D Materials, Vol. 2.

313. Electrochemical Sensing and Biosensing Platform Based on Chemically Reduced Graphene Oxide. Zhou, M, Zhai, YM and Dong, SJ. 14, 2009, Analytical Chemistry, Vol. 81, pp. 5603-5613.

314. A Comprehensive Review of Gas Sensors Useing Carbon Materials. Kim, MI and Lee, YS. 5, 2016, Journal of Nanoscience and Nanotechnology, Vol. 16, pp. 4310-4319.

315. Practical Chemical Sensors from Chemically Derived Graphene. Fowler, JD, et al. 2, 2009, ACS Nano, Vol. 3, pp. 301-306.

316. Effects of charge transfer interaction of graphene with electron donor and acceptor molecules examined using Raman spectroscopy and cognate techniques. Rakesh, Voggu, et al. 47, 2008, Journal of Physics: Condensed Matter, Vol. 20.

317. Adsorption of $H(2) O, N H(3), C O, N O(2)$, and NO on graphene: A first-principles study. Leenaerts, $\mathrm{O}$, Partoens, B and Peeters, FM. 12, 2008, Physical Review B, Vol. 77. 
318. Metal to Insulator Transition in Epitaxial Graphene Induced by Molecular Doping. Zhou, SY, et al. 8, 2008, Vol. 101.

319. The Transfer Doping of Graphite and Graphene. Sque, SJ, Jones, R and Briddon, PR. 9, 2007, Physica Status Solidi A-Applications And Materials Science, Vol. 204, pp. 3078-3084.

320. Tuning the Electronic Structure of Graphene by an Organic Molecule. Lu, YH, et al. 1, 2009, Vol. 113 , pp. $2-5$.

321. A Highly Sensitive Hydrogen Sensor with Gas Selectivity Using a PMMA Membrane-Coated Pd Nanoparticle/Single-Layer Graphene Hybrid. Hong, J, et al. 6, 2015, ACS Applie Materials and Interfaces, Vol. 7, pp. 3554-3561.

322. Dielectrophoretic assembly of Pt nanoparticle-reduced graphene oxide nanohybrid for highlysensitive multiple gas sensor. Wang, JW, et al. 2015, Sensors and Actuators B- Chemcials, Vol. 220, pp. 755-761.

323. Hybrid Graphene-metal Nanoparticle Systems: Electronic Properties and Gas Interaction. Tjoa, V, et al. 39, 2011, Journal of Materials Chemistry, Vol. 21, pp. 15593-15599.

324. Prospects for Graphene-Nanoparticle-Based Hybrid Sensors. Yin, PT, et al. 31, 2013, Physical Chemistry Chemical Physics, Vol. 15, pp. 12785-12799.

325. Uniform decoration of silver nanoparticle on exfoliated graphene oxide sheets and its ammonia gas detection. Kavinkumar, T and Manivannan, S. 1, 2016, Ceramics International, Vol. 42, pp. 1769-1776.

326. Platinum Nanoparticle Ensemble-on-Graphene Hybrid Nanosheet: One-Pot, Rapid Synthesis, and Used as New Electrode Material for Electrochemical Sensing. Guo, SJ, et al. 7, 2010, ACS Nano, Vol. 4, pp. 3959-3968.

327. Graphene Oxide Coupled with Gold Nanoparticels for Localized Surface Plasmon Resonance Based Gas Sensor. Michela, Cittadini' Marco, Bersani, Francesco, Perrozzi and Luca, Ottaviano. 2014, Carbon, Vol. 69, pp. 452-459.

328. Hydrogen-gas Sensors Based on Graphene Functionalized Palladium Nanoparticles: Impedance Response as a Valuable Sensor. 2015, New J. Chem., Vol. 39, pp. 8044-8054.

329. Low Temperature Acetylene Gas Sensor Based on Ag Nanoparticles-loaded ZnO-reduced Graphene Oxide Hybrid. Uddin, A.S.M. Iftekhar, Phan, Duy-Thach and Chung, Gwiy-Sang. 2015, Sensors and Actuators B, Vol. 207, pp. 362-369.

330. Synthesies of Graphene Supported Pd Nanoparticles for Amperometric NO Gas Sensors. Wang, Huan. 2015. International Conference on Chemical, Material and Food Engineering. pp. 355-358.

331. Flexible Hydrogen Sensors Using Graphene With Palladium Nanoparticle Decoration. Chung, MG, et al. 2012, Sensors and Actuators B-Chemcial, Vol. 169, pp. 387-392.

332. High-Sensitivity and Fast-Response Hydrogen Sensor for Safety Application Using Pt NanoparticleDecorated 3D Graphene. Phan, DT, Youn, JS and Jeon, KJ. 2019, Renewable Energy, Vol. 144, pp. 167174.

333. Enhancement of Hydrogen Physisorption on Graphene and Carbon Nanotubes by Li Doping. Cabria, I, Lopez, MJ and Alonso, JA. 20, 2005, Journal of Chemical Physics, Vol. 123.

334. Attracted by Long-Range Electron Correlation: Adenine on Graphite. Ortmann, F., Schmidt, W. G. and Bechstedt1, F. 18, 2005, Phys. Rev. Lett., Vol. 95. 
335. Hierarchical Interactions and Their Influence upon the Adsorption of Organic Molecules on Graphene Film. Roos, Michael, et al. 24, 2011, Journal of the American Chemical Society, Vol. 133, pp. 9208-9211.

336. Symmetry Breaking of Graphene Monolayers by Molecular Decoration. Dong, XC, et al. 13, 2009, Physical Review Letters, Vol. 102.

337. Magnetic Properties and Diffusion of Adatoms On a Graphene Sheet. Lehtinen, PO, et al. 1, 2003, Vol. 91.

338. Electrical Probing and Tuning of Molecular Physisorption on Graphene. Kulkarni, G., et al. 1, 2016, Nano Letters, Vol. 16, pp. 695-700.

339. Anomalously Large Reactivity of Single Graphene Layers and Edges toward Electron Transfer Chemistries. Sharma, R, et al. 2, 2010, Nano Letters, Vol. 10, pp. 398-405.

340. Preferential Binding of Peptides to Graphene Edges and Planes. Kim, SN, et al. 37, 2011, Journal of the American Chemical Society, Vol. 133, pp. 14480-14483.

341. Adsorption Capacity of H2O, NH3, CO, and NO2 On The Pristine Graphene. Lin, XQ, Ni, J and Fang, C. 3, 2013, Journal of Applied Physics, Vol. 113.

342. Nature of Graphene Edges: A Review. Acik, M and Chabal, YJ. 7, 2011, Japanese Journal of Applied Physics, Vol. 50.

343. Preferential Functionalization on Zigzag Graphene Nanoribbons: First-principles Calculations. Lee, H. 35, 2010, Jouna of Physics-Condensed Matter, Vol. 22.

344. Unique Chemical Reactivity of a Graphene Nanoribbon's Zigzag Edge. Jiang, DE, Sumpter, BG and Dai, S. 13, 2007, Journal of Chemical Physics, Vol. 126.

345. Quantum Transport in Graphene Nanoribbons: Effects of Edge Reconstruction and Chemical Reactivity. Dubois, SMM, et al. 4, 2010, ACS Nano, Vol. 4, pp. 1971-1976.

346. Doping Single-Layer Graphene with Aromatic Molecules. Xiaochen, Dong, et al. 12, 2009, Small, Vol. 5.

347. Elisabetta, Comini, Guido, Faglia and Giorgio, Sberveglieri. Solid State Gas Sensing. s.1. : Springer, 2009.

348. Detection of individual gas molecules adsorbed on graphene. Schedin, F, et al. 9, 2007, Nature Materials, Vol. 6, pp. 652-655.

349. Improving Gas Sensing Properties of Graphene by Introducing Dopant and Defect: A First-Principles Study. Zhang, YH, et al. 18, 2009, Nanotechnology, Vol. 20.

350. CO Chemisorption at Vacancies of Supported Graphene Films: A Candidate for a Sensor? Celasco, E, et al. 23, 2016, Physical Chemistry Chemical Physics, Vol. 18, pp. 18692-18696.

351. Improving SO2 Gas Sensing Properties of Graphene by Introducing Dopant and Defect: A FirstPrinciples Study. Liu, XY, et al. 2014, Applied Surface Science, Vol. 313, pp. 405-410.

352. Atomic Chemisorption on Graphene with Stone-Thrower-Wales Defects. Chen, L, et al. 10, 2011, Carbon, Vol. 49, pp. 3356-3361.

353. Atomic nitrogen chemisorption on graphene with extended line defects. Li, Y, et al. 39, 2012, Journal of Materials Chemistry, Vol. 22, pp. 21167-21172. 
354. Curvature Effect on the Barrier from the Physisorption to the Chemisorption of H2 on Graphene. Kang, B, et al. 3, 2011, Bulletion of the Korean Chemical Society, Vol. 32, pp. 934-938.

355. Room Temperature Detection of Individual Molecular Physisorption Using Suspended Bilayer Graphene. Sun, J, Muruganathan, M and H, Mizuta. 4, 2016, Science Advances, Vol. 2.

356. Intrensic Responce of Graphene Vapor Sensors. Dan, YP, et al. 4, 2009, Nano Letters, Vol. 9, pp. 1472-1475.

357. Gas Sensor Based on p-phenylenediamine Reduced Graphene Oxide. Hu, Nantao, et al. 1, 2012, Sensors and Actuators B-Chemcial, Vol. 163, pp. 107-114.

358. A Review on Graphene-Based Gas/Vapor Sensors with Unique Properties and Potential Applications. Wang, T, et al. 2, 2016, Nano-Micro Letters, Vol. 8, pp. 95-119.

359. Intrinsic Response of Graphene Vapor Sensors. Yaping Dan, Ye Lu, Nicholas J. Kybert, Zhengtang Luo,. 4, 2009, Nano Letters, Vol. 9, pp. 1472-1475.

360. Ab Initio Study of Adsorption Properties of Hazardous Organic. Junsu Lee, Kyung-Ah Min, Suklyun Hong, Gunn Kim. 618, 2015, Chemical Physical Letters, pp. 57-62.

361. Carbon Nanotubes and Graphene-based Chemical Sensors. Misra, Abha. 3, 2014, Current Science Special Section: Carbon Technology, Vol. 107, pp. 419-429.

362. Chemical Vapor Sensing Properties of Graphene Based on Geometrical Evaluation. Hwang, Sukju, et al. 4, 2012, Current Applied Phyiscs, Vol. 12, pp. 1017-1022.

363. Adsorption of Small Organic Molecules on Graphene. Lazar, Petr, et al. 16, 2013, Journal of The American Chemical Society, Vol. 135, pp. 6372-6377.

364. Enhanced Gas Sensing Properties to Acetone Vapor Achieved by Alpha-Fe2O3 Particles Ameliorated with Reduced Graphene Oxide Sheets. Zhang, B, et al. 2017, Sensors and Actuators B-Chemcial, Vol. 241, pp. 904-914.

365. Gas Adsorption on Graphene Doped with B, N, Al, and S: A Theoretical Study. Dai, JY, Yuan, JM and Giannozzi, P. 23, 2009, Applied Physics Letters, Vol. 95.

366. A DFT Study of Volatile Organic Compounds Adsorption on Transition Metal Deposited Graphene. Kunaseth, Manaschai, et al. 2017, Applied Surface Science, Vol. 396, pp. 1712-1718.

367. Adsorption of Organic Molecules to van der Waals Materials: Comparison of Fluorographene and Fluorographite with Graphene and Graphite. Karlicky, F, et al. 3, 2017, Journal of Chemical Theory and Computiation, Vol. 13.

368. The Nature of High Surface Energy Sites in Graphene and Graphite. Lazar, Petr, et al. 2014, Carbon, Vol. 73, pp. 448-453.

369. Interplay betwen Ethanol Adsorption to High Energy Sites and Clustering on Grahene and Graphite Alters the Measured Isosteric Adsorption Enthalpies. Karlicky, Frantisek, et al. 35, 2015, Journal of Physical Chemistry C, Vol. 119.

370. High-Temperature Ceramic Gas Sensors: A Review. Akbar, S, Dutta, P and Lee, CH. 4, 2006, International Journal of Applied Ceramic Technology, Vol. 3, pp. 302-311.

371. Fine Structure Constant Defines Visual Transparency of Graphene. Nair, RR, et al. 5881, 2008, Science, Vol. 320. 
372. Graphene Plasmonics: Challenges and Opportunities. Abajo, Garcia de and Javier, F. 3, 2014, ACS Photonics, Vol. 1, pp. 135-152.

373. Advances in Graphene-based Optoelectronics, Plasmonics and Photonics. Nguyen, Bich Ha and Nguyen, Van Hieu. 1, 2016, Advances in Natural Sciences-Nanoscience and Nanotechnology, Vol. 7.

374. Graphene Plasmonics: A platfrom for Strong Light-Matter Interactions. Frank, Koppens, Darrick, Chang and and Javier, García de Abajo. 11, 2011, Nano Letters, pp. 3370-3377.

375. Graphene-Silicon Schottky Diodes. Chen, CC, et al. 5, 2011, Nano Letters, Vol. 11, pp. 1863-1867.

376. Graphite Based Schottky Diodes Formed on Si, GaAs, and 4H-SiC Substrates. Tongay, S, Schumann, T and Hebard, AF. 22, 2009, Applied Physics Letters, Vol. 95.

377. The Graphene-Semiconductor Schottky Junction. Li, XM and Zhu, HW. 2016, Physics Today, Vol. 69 , pp. 47-51.

378. Ning, Tak H. and Taur, Yuan. Fundamentals of Modern VLSI Devices. 2nd. s.1. : Cambridge University PRess, 2009. pp. 108-120.

379. Hu, Chenming Calvin. Modern Semiconductor Devices for Integrated Circuits. s.1. : Wiley, 2010. pp. 133-148.

380. Graphene Schottky Diodes: An Experimental Review of the Rectifying Graphene/Semiconductor Heterojunction. Di Bartolomeo, A. 2016, Physics Reports-Review Section of Phyisics Letters, Vol. 606, pp. 1-58.

381. Synergistic Effects of Plasmonics and Electron Trapping in Graphene Short-Wave Infrared Photodetectors with Ultrahigh Responsivity. Chen, ZF, et al. 1, ACS Nano, Vol. 11, pp. 430-437.

382. Graphene-Based Semiconductor Heterostructures for Photodetectors. Shin, DH and Choi, SH. 7, 2018, Micromachines, Vol. 9.

383. On-Chip Integrated, Silicon-Graphene Plasmonic Schottky Photodetector with High Responsivity and Avalanche Photogain. Goykhman, I, et al. 5, 2016, Nano Letters, Vol. 16, pp. 3005-3013.

384. Junction Investigation of Graphene/silicon Schottky Diodes. Mohammed, M, et al. 2012, Nanoscale Research Letters, Vol. 7, pp. 1-6.

385. Graphene/silicon Schottky Solar Cells: Technical Strategies for Performance Optimization. Ju, SH, et al. 2018, Optical Communications, Vol. 428, pp. 258-268.

386. Ultrafast Graphene Photodetector. Xia, FN, et al. 2, 2009, Nature Nanotechnology, Vol. 4, pp. 839843.

387. Tunable Graphene-Silicon Heterojunctions for Ultrasensitive Photodetection. An, XH, et al. 3, 2013, Nano Letters, Vol. 13, pp. 909-916.

388. Enhanced light-matter interaction of graphene-gold nanoparticle hybrid films for high-performance SERS detection. Du, YX, et al. 23, 2014, Journal of Materials Chemistry C, Vol. 2, pp. 4683-4691.

389. Synthesis of Visible-Light Responsive Graphene Oxide/TiO2 Composites with $p / n$ Heterojunction. Chen, C, et al. 2010, ACS Nano, pp. 6425-6432.

390. Graphene Sheets Grafted Ag@AgCl Hybrid with Enhanced Plasmonic Photocatalytic Activity under Visible Light. Zhang, H, et al. 13, 2011, Enviromental Science \& Technology, Vol. 45, pp. 5731-5736. 
391. Plasmon-Induced Photon Manipulation by Ag Nanoparticle-Coupled Graphene Thin-Film: Light Trapping for Photovoltaics. Kanade, P, et al. 1, Plasmonics, Vol. 10, pp. 157-164.

392. Enhanced Efficiency of Graphene-silicon Schottky Junction Solar Cells by Doping with Au nanoparticles. Liu, X, et al. 18, 2014, Applied Physics Letters, Vol. 105.

393. Dual-plasmonic Au/graphene/Au-enhanced Ultrafast, Broadband, Self-driven Silicon Schottky Photodetector. Wang, L, et al. 50, 2018, Nanotechnology, Vol. 29.

394. The Rich Photonic World of Plasmonic Nanoparticle Arrays. Wang, WJ, et al. 3, 2018, Materials Today, Vol. 21, pp. 303-314.

395. Henzie, J, et al. Nanofabrication of Plasmonic Structures. s.1. : Annual Review of Physical Chemistry, 2009. pp. 147-165. Vol. 60.

396. Evidence of Plasmonic Coupling in Gallium Nanoparticles/Graphene/SiC. Yi, CW, et al. 17, 2012, Small, Vol. 8, pp. 2721-2730.

397. Plasmonic Coupling of Silver Nanoparticles Covered by Hydrogen-Terminated Graphene for Surfaceenhanced Raman Spectroscopy. Liu, CY, et al. 18, 2011, Optics Express, Vol. 19, pp. 17092-17098.

398. Graphene Photo-detector Enhanced by Plasmonic Coupling. Maleki, A, et al. Singapore : IEEE, 2017. 2017 Conference On Lasers and Electro-Optics Pacific Rim.

399. Strong Plasmonic Enhancement of Photovoltage in Graphene. Echtermeyer, TJ, et al. 2011, Nature Communications, Vol. 2.

400. Significantly Enhanced Infrared Absorption of Graphene Photodetector Under Surface-Plasmonic Coupling and Polariton Interference. Zhang, Y, et al. 23, 2018, Optics Express, Vol. 26, pp. 30862-30872.

401. Extremely narrow plasmon resonances based on diffraction coupling of localized plasmons in arrays of metallic nanoparticles. Kravets, VG, Schedin, F and Grigorenko, AN. 8, 2008, Physical Review Letters, Vol. 101.

402. Plasmonics of Coupled Graphene Micro-structures. Yan, HG, et al. 2012, New Journal of Physics, Vol. 14.

403. Graphene-Antenna Sandwich Photodetector. Fang, ZY, et al. 7, 2012, Nano Letters, Vol. 12, pp. 38083813.

404. The optical Properties of Metal Nanoparticles: The Influence of Size, Shape, and Dielectric Environment. Kelly, KL, et al. 3, 2003, Journal of Physical Chemictry B, Vol. 107, pp. 668-677.

405. Chemistry and Properties of Nanocrystals of Different Shapes. Burda, C, et al. 4, 2005, Chemical Reviews, Vol. 105, pp. 1025-1102.

406. Plasmon Resonance Enhanced Multicolour Photodetection by Graphene. Liu, Y, et al. 2011, Nature Communications, Vol. 2.

407. Plasmon-Induced Doping of Graphene. Fang, Zheyu, et al. 11, 2012, ACS Nano, Vol. 6, pp. 1022210228.

408. Controlling Graphene Plasmons with Resonant Metal Antennas and Spatial Conductivity Patterns. Alonso-Gonzalez, P, et al. 6190, 2014, Science, Vol. 344, pp. 1369-1373.

409. Measurement of the Optical Conductivity of Graphene. Mak, KF, et al. 19, 2008, Physical Review Letters, Vol. 101. 
410. A Self-powered Sensitive Ultraviolet Photodetector Dased on Epitaxial Graphene on Silicon Carbide. Huang, J, et al. 6, 2016, Chinese Physics B, Vol. 25.

411. Interfacial Amplification for Graphene-based Position-Sensitive-Detectors. Wang, WH, et al. 2017, Light-Science \& Applications, Vol. 6.

412. The History of the Development of Silicon Carbide Refractories. Geiger, CF. 1, 1923, Journal of The American Ceramic Society, Vol. 6, pp. 301-306.

413. About the Rectifier and Photoelectric Effect on the Silicon Carbide Detector. Specht, P. 3-4, 1934, Zetschrift Fur Physik, Vol. 90, pp. 145-165.

414. The Silicon Carbide Electrode. Hume, DN and Kolthoff, IM. 1941, Journal of The American Chemcial Society, Vol. 63, pp. 2805-2806.

415. Silicon Carbide Non-ohmic Resistors. Anonymous. 1946, Nature, Vol. 158, p. 742.

416. Review of SiC Crystal Growth Technology. Wellmann, PJ. 10, 2018, Semiconductor Science and Technology, Vol. 33.

417. History \& Milestones. [Online] Cree, 2019. www.cree.com/about/history-and-milestones.

418. Neudeck, Philip G. Silicon Carbide Technology. The VLSI Handbook. s.1. : CRC Press LLC., 2006, pp. 5-1 5-33.

419. Kittel, Charles. Introduction to Solid State Physics. [ed.] Stuart Johnson. 8th. s.1. : John Wiley \& Sons, Inc., 2005. p. 190. ISBN: 0-471-41526-X.

420. High Performance Silicon Carbide Power PackagingPast Trends, Present Practices, and Future Directions. Seal, S and Mantooth, HA. 3, 2017, Energies, Vol. 10.

421. Silicon Carbide and Diamond for High Temperature Device Applications. Willander, M, et al. 1, 2006, Journal of Materials Scienes- Materials in Electronics, Vol. 17, pp. 1-25.

422. Review of Silicon Carbide Power Devices and Their Applicaions. She, X, et al. 10, 2017, IEEE Transactions on Industrial Electronics, Vol. 64, pp. 8193-8205.

423. Micro- and Nanomechanical Structures for Silicon Carbide MEMS and NEMS. Zorman, CA and Parro, RJ. 7, 2008, Physica Status Solidi B- Basic Solid State Physics, Vol. 245, pp. 1404-1424.

424. Silicon Carbide for Microelectromechanical Systems. Mehregany, M, et al. 3, 2000, International Materials Reviews, Vol. 45, pp. 85-108.

425. Advances in Silicon Carbide Science and Technology At The Micro- and Nanoscales. Maboudian, R, et al. 5, 2013, Journal of Vacuume Sciences \& Technology A, Vol. 31.

426. Impact of silicon carbide semiconductor technology in Photovoltaic Energy System. Pushpakaran, BN, et al. 2016, Renewable \& Sustainable Energy Revies, Vol. 55, pp. 971-989.

427. Technological Breakthroughs In Growth Control of Silicon Carbide for High Power Electronic Devices. Matsunami, H. 10, 2004, Japanese Journal of Applied Physics Part 1- Regular Papers Nrief Communications \& Review Papers, Vol. 43, pp. 6835-6847.

428. Kimoto, Tsunenobu and Copper, James A. Fundamentals of Silicon Carbide Technology. Solaris South Tower, Singapore : John Wiley \& Sons Singapore Pte. Ltd., 2014. 
429. Electron Diffraction Methods For The Analysis Of Silicon Carbide Surfaces and The Controlled Growth Of Polytype Heterostructures. Winkelmann, A (Winkelmann, A), Schroter, B (Schroter, B) and Richter, W. 17, 2004, Journal of Physics- Condensed Matter, Vol. 16, pp. A1555-S1578.

430. Roewer, G, et al. Structure and Bonding; Silicon Carbide: A Survey of Synthetic Approaches, Properties and Applications. [ed.] DMP Mingos. New York: Springer-Verlag Verline Heidelberg, 2002, Vol. 101, pp. 95-135.

431. STM Profilometry of Low-Load Vickers Indentations in a Silicon Single Crystal. Walls, MG, Chaudhri, MM and Tang, TB. 3, 1992, Journal of Physics D Applied Physics, Vol. 25.

432. Lide, David R. CRC Handbook of Chemistry and Physics. Boca Raton, FL : CRC Press, 2005.

433. A Perspective On Non-stoichiometry in Silicon Carbide. Majid, A. 2, 2018, Ceramics International, Vol. 44, pp. 1277-1283.

434. Towards a Unified View of Polytypism in Silicon-Carbide. Fisher, GR and Barnes, P. 2, 1990, Philosophical Magazine B-Physics of Condensed Matter Statistical Mechanics Electronic Optical and Magnetic Properties, Vol. 61, pp. 217-236.

435. Silicon Carbide Substrates and Epitaxy Data Sheet: Cree, Inc. [Online] https://www.wolfspeed.com/materials/products.

436. HPSI - Semi-insulating SiC Substrates. [Online] Cree, Inc. (Wolfspeed), 2019. www.wolfspeed.com/materials/products/semi-insulating-sic-substrates.

437. CVD Growth and Properties Of On-Axis Vanadium Doped Semi-Insulating 4H-SiC Epilayers. Karhu, R, et al. 4, 2019, Vol. 125. 10.1063/1.5057389.

438. Deep Defect Centers In Silicon Carbide Monitored With Deep Level Transient Spectroscopy. Dalibor, T, et al. 1, 1997, Physica Status Solidi A- Applied Reserach, Vol. 162, pp. 199-225.

439. Growth of SiC Substrates. Powell, A, et al. 3, 2006, International Journal of High Speed Electronics and Systems, Vol. 16, pp. 751-777.

440. High-Purity Semi-Insulating 4H-SiC for Microwave Device Applications. Jenny, JR, et al. 5, 2003, Journal of Electronic Materials, Vol. 32, pp. 432-436.

441. High Purity Semi-Insulating 4H-SiC Epitaxial Layers by Defect-Competition Epitaxy: Controlling Si Vacancies. Chandrashekhar, MVS, et al. 2, 2012, Applied Physics Express, Vol. 5.

442. Optical Characterization of Silicon Carbide Polytypes. Devaty, RP and Choyke, WJ. 1, 1997, Physica Status Solidi A- Applications and Materials Science, Vol. 162, pp. 5-38.

443. Comparison Of 3C-SiC, 6H-SiC and 4H-SiC MESFETs Performances. Codreanu, C, et al. 1-2, 2000, Materials Science in Semiconductor Processing, Vol. 3, pp. 137-142.

444. Silicon Carbide of Ni/6H-SiC and Ti/4H-SiC Type Schottky Diode Current-Voltage Characteristics Modelling. Panchenko, PV, et al. 2017, Journal of Physics: Conference Series.

445. Comparison of Graphene Formation on C-face and Si-face SiC \{0001\} Surfaces. Luxmi, et al. 23, 2010, Physical Review B, Vol. 82.

446. Nojiri, K. Dry Etching Technology for Semiconductors. s.1. : Springer, 2015.

447. Lieberman, M. A. and Lichtenberg, A. J. Principles of Plasma Discharges and Materials Processing. 2nd. New Jersey : Wiley-Interscience, 2005. pp. 387 - 483. 
448. Ion Assisted and Electron Assisted Gas Surface Chemistry Importatn Effect in Plasma Etching. JW, Coburn and HF, Winters. 5, 1979, Journal of Applied Physics, Vol. 50, pp. 3189-3196.

449. http://www.triontech.com/minilock_phantom.htm. www.triontech.com. [Online] Trion Technology.

450. Detection of Chamber Conditioning by CF4 Plasmas In An Inductively Coupled Plasma Reactor. Cruden, BA, et al. 2002, Journal of Vaccum Science \& Technology B, pp. 353-363.

451. Technology, Trion. Minilock-Phantom III Operator \& Maintenance Manual. Tempe, AZ : s.n., 2007.

452. Introduction to Gas Discharges. Braithwaite, NSJ. 4, 2000, Plasma Sources Sci. Technol., Vol. 9, pp. 517-527.

453. Henry, Michael David. ICP Etching of Silicon: For Micro and Nanoscale Devices . s.1. : California Insititute of Technology, 2010.

454. Complete plasma physics, plasma chemistry, and surface chemistry simulation of SiO2 and Si etching in CF4 plasmas. Gogolides, E, et al. Athens, Greece: Elsevier Science Bv, Po Box 211, 1000 Ae Amsterdam, Netherlands, 1998. Vol. 42, pp. 391-394.

455. On the Role of Radicals in Kinetics of Plasma Etchers in Ar/CF4 Mixtures. Nikitovic, Z, Stojanovic, V and Petrovic, ZL. Herceg Novi, Montenegro : Polish Acad Sciences Inst Physics, Al Lotnikow 32-46, PL-02-668 WARSAW, POLAND, 2009. Vol. 115, pp. 765-767.

456. Radical kinetics in an inductively-coupled plasma in CF4. Booth, JP, et al. Tara Natl Pk, Yuggoslavia : s.n., 2004. Vol. 740, pp. 252-267. 22nd Summer School and International Symposium on the Physics of Ionized Gases.

457. Nojiri, Kazuo. Dry Etching Technology for Semiconductors. Japan : Lam Research Co., Ltd., 2012. pp. 11-30.

458. Lieberman, M. A. and Lichtenberg, A. J. Principles Of Plasma Discharges And Materials Processing. 2nd. New Jersey : Wiley-Interscience, 2005. pp. 586 - 596.

459. Reactive ion etching of $6 \mathrm{H}-\mathrm{SiC}$ in $\mathrm{SF} 6 / \mathrm{O}-2$ and $\mathrm{CF} 4 / \mathrm{O}-2$ with $\mathrm{N}-2$ additive for device fabrication. $\mathrm{R}$, Wolf and R, Helbig. 3, 1996, Journal of the Electrochemical Society, Vol. 143, pp. 1037-1042.

460. Progress in quantitative Surface Analysis by X-ray Photoelectron Spectroscopy: Current Status and Perspectives. Powell, CJ and Jablonski, A. Special Issue, 2010, Journal of Electron Spectroscopy and Related Phenomena, Vol. 178, pp. 331-346.

461. X-ray Photoelectron Spectroscopy: Progress and Perspectives. Fadley, CS. Special Issue, 2010, Journal of Electron and Spectroscopy and Related Phenomena, Vol. 178, pp. 2-32.

462. The Nobel Prize in Physics 1981. https://www.nobelprize.org/prizes/physics/1981/summary/. [Online] Nobel Media AB.

463. Siegbahn, Kal. Nobel Lecture: Electron Spectroscopy for Atoms, Molecules and Condensed Matter. s.1., Sweden : Institute of Physics, University of Uppsala, December 8, 1981.

464. Brundle, C.R. and Baker, A.D, [ed.]. Electron Spectroscopy: Theory, Techniques and Applications. s.1. : Academic Press, 1978. Vol. 2.

465. Alford, Terry L., Feldman, L.C. and Mayer, James W. Fundamentals of Nanoscale Film Analysis. New York : Springer Science \& Business Media, 2007. ISBN: 0-387-29260-8. 
466. Moulder, J.,F., et al. Handbook of X-ray photoelectron spectroscopy. s.1. : Physical Electronics,Inc, 1995.

467. Oswald, Steffen. X-Ray Photoelectron Spectroscopy in Analysis of Surfaces. Encyclopedia of Analytical Chemistry: Applications, Theory and Instrumentation. s.1. : John Wiley \& Sons, Ltd., 2013.

468. Alexander V. Naumkin, Anna Kraut-Vass, Stephen W. Gaarenstroom, and Cedric J. Powell. National Institute of Standards and Technology (NIST) X-ray Photoelectron Spectroscopy Database: NIST Standard Reference Database 20, Version 4.1. [Online] U.S. Secretary of Commerce on behalf of the United States of America, 2012. https://srdata.nist.gov/xps/.

469. Comparison Of Intrinsic Zero-Energy Loss and Shirley-Type Background Corrected Profiles of XPS Spectra for Quantitative Surface Analysis: Study of Cr, Mn and Fe Oxides. Oku, M, et al. 16, 2008, Applied Surface Science, Vol. 254, pp. 5141-5148.

470. The Gaussian-Lorentzian Sum, Product, and Convolution (Voigt) Functions In The Context Of Peak Fitting X-ray Photoelectron Spectroscopy (XPS) Narrow Scans. Jain, V Biesinger, MC and Linford, MR. 548-553, 2018, Applied Surface Science, Vol. 447, pp. 548-553.

471. Casa Software Ltd. CasaXPS Manual 2.3.15: Processing Software for XPS Spectra. 1.3. s.1. : Casa Software Ltd., 2009.

472. Vickerman, J. C. Surface analysis: The principal techniques. s.1. : John Wiley \& Sons, 2011.

473. Surface Studies Relevant to Silicon-Carbide Chemical Vapor-Depostion. Stinespring, CD and Wormhoudt, JC. 4, 1989, Journal of Applied Physics, Vol. 65, pp. 1733-1742.

474. The Nobel Prize in Physics 1986. NobelPrize.org. [Online] Nobel Media AB 2018. $<$ https://www.nobelprize.org/prizes/physics/1986/summary/>.

475. 9 Scientific Pioneers to Receive the 2016 Kavli Prizes . [Online] 6 01, 2016. $<$ http://kavliprize.org/events-and-features/9-scientific-pioneers-receive-2016-kavli-prizes $>$.

476. Atomic Force Microscope. Binning, G. and Quate, C. F. 9, 1986, Physical Review Letters, Vol. 56, pp. 930-933.

477. Force-distance Curves by Atomic Force Microscopy. Cappella, B and Dietler, G. 1-3, 1999, Surface Science Reports, Vol. 34, pp. 1-103.

478. High-Resolution Imaging of Antibodies by Tapping-Mode Atomic Force Microscopy: Attractive and Repulsive Tip-Sample Interaction Regimes. San Paulo, A and Garcia, R. 3, 2000, Biophysical Journal, Vol. 78, pp. 1599-1605.

479. Dynamic Atomic Foce Microscopy Methods. Garcia, R and Perez, R. 6-8, 2002, Surface Science Reports, Vol. 47, pp. 197-3001.

480. Advances in Atomic Force Microscopy. Giessibl, FJ. 3, 2003, Reviews of Modern Physics, Vol. 75, pp. $949-983$.

481. A Review of Atomic Force Microscopy Imaging Systems: Application to Molecular Metrology and Biological Sciences. Jalili, N and Laxminarayana, K. 8, 2004, Mechatronics, Vol. 14, pp. 907-945.

482. Gwyddion: An Open-Source Software For SPM Data Analysis. Necas, D and Klapetek, P. 1, 2012, Central European Journal of Physics, Vol. 10, pp. 181-188.

483. A New Type of Seconday Radiation. Raman, CV and Krishnan, KS. 1928, Nature, Vol. 121, pp. 501502. 
484. A New Radiation. Raman, CV. 1928, Indian Jurnal of Physics, Vol. 2, pp. 368-376.

485. The Nobel Prize in Physics 1930. [Online] Nobel Media AB. https://www.nobelprize.org/prizes/physics/1930/summary/.

486. Harris, Daniel C. and Bertolucci, Michael D. Symmetry and Spectroscopy: An Introduction to Vibrational and Electronic Spectroscopy . New York : Dover Publications, Inc., 1978. ISBN: 0-486-66144$\mathrm{X}$.

487. Chimenti, Robert V. Spectrometers: Excitation source parameters dictate Raman spectroscopy outcomes. [Online] Endeavor Business Media, LLC., 1 24, 2017. https://www.laserfocusworld.com/testmeasurement/spectroscopy/article/16548136/spectrometers-excitation-source-parameters-dictate-ramanspectroscopy-outcomes.

488. Renishaw, PLC. Raman Spectroscopy Explained. Gloucestershire, UK, United Kingdom : s.n., 2018. 489. Socrates, George. Infarared and Raman Characteristic Group Frequencies. New York : John Wiley \& Sones, LTD, 2004.

490. Raman Spectroscopy of Carbon Materials: Structural Basis of Observed Spectra. Wang, Y, Alsmeyer, DC and McCreery, RL. 5, 1990, Chemistry of Materials, Vol. 2, pp. 557-563.

491. Raman spectroscopy of graphene and Graphite: Disorder, Electron-phonon Coupling, Doping and Nonadiabatic Effects. Farrari, AC. 1-2, 2007, Solid State Communications, Vol. 143, pp. 47-57.

492. Raman Spectrum of Graphene and Graphene Layers. Ferrari, AC, et al. 18, 2006, Physical Review Letters, Vol. 97.

493. Raman Spectroscopy as a Versatile Tool for Studying the Properties of Graphene. Ferrari, AC and Basko, DM. 4, 2013, Nature Nanotechnology, Vol. 8, pp. 235-246.

494. Quantifying Defects in Graphene via Raman Spectroscopy at Different Excitation Energies. Cancado, LG, et al. 8, 2011, Nano Letters, Vol. 11, pp. 3190-3196.

495. Raman Spectroscopy in Graphene. Malard, LM, et al. 5-6, 2009, Physics Reports-Review Section of Physics Letters, Vol. 473, pp. 51-87.

496. Basic Concepts and Recent Advances of Crystallographic Orientation Determination of Graphene by Raman Spectroscopy. Lan, YC, et al. 10, 2018, Crystals, Vol. 8.

497. A Guide to and Review of the Use of Multiwavelength Raman Spectroscopy for Characterizing Defective Aromatic Carbon Solids: from Graphene to Amorphous Carbons. Merlen, A, Buijnsters, JG and Pardanaud, C. 10, 2017, Coatings, Vol. 7.

498. Growth and Raman Spectroscopy of Thickness-controlled Rotationally Faulted Multilayer Graphene. Kato, H, Itagaki, N and Im, HJ. 2019, Carbon, Vol. 141, pp. 76-82.

499. Quantifying Ion-induced Defects and Raman Relaxation Length in Graphene. Lucchese, MM, et al. 5, 2010, Carbon, Vol. 48, pp. 1592-1597.

500. Interpretation of Raman Spectra of Disordered and Amorphous Carbon. Ferrari, AC and Robertson, J. 20, 2000, Physical Review B, Vol. 62, pp. 14095-14107.

501. Raman Scattering Characterization on SiC. Harima, H. 1, 2006, Microelectronic Engieering, Vol. 83, pp. 126-129. 
502. Effect of Doping on the Raman Scattering of 6H-SiC Crystals. Li, XB, Chen, ZZ and Shi, EW. 10, 2010, Physica B-Condensed Matter, Vol. 405, pp. 2423-2426.

503. Raman Spectra of Epitaxial Graphene on SiC(0001). Rohrl, J, et al. 20, 2008, Applied Physics Letters, Vol. 92.

504. Selective Epitaxial Growth of Graphene on SiC. Camara, N, et al. 12, 2008, Applied Physics Letters, Vol. 93.

505. First- and Second-order Raman Scattering from Semi-insulating 4H-SiC. Burton, JC, Sun, L and Long, FH. 11, 1999, Physical Review B, Vol. 59, pp. 7282-7284.

506. An Empirical Study on Raman Peak Fitting and Its Application to Raman Quantitative Research. Yuan, XY and Mayanovic, RA. 10, 2017, Applied Spectroscopy, Vol. 71, pp. 2325-2338.

507. Raman Spectroscopy of Graphene-based Materials and Its Applications In Related Devices. Wu, JB, et al. 5, 2018, Chemical Society Reviews, Vol. 47, pp. 1822-1873.

508. Fityk: A General-Purpose Peak Fitting Program. Wojdyr, M. 2010, Applied Crystallography, Vol. 43, pp. 1126-1128.

509. Scanning Electron Microscopy 1928-1965. McMullan, D. 3, 1995, The Journal of Scanning Microscopies, Vol. 17, pp. 175-185.

510. Media, Nobel. The Nobel Prize in Physics 1986. https://www.nobelprize.org/prizes/physics/1986/press-release/. [Online]

511. JEOL Ltd. SEM: Scanning Electron Microscope A to Z Basic Knowledge For Using The SEM. Tokyo, Akishima, Japan : s.n. pp. 1 - 30. No.1101B992C(Ks).

512. Seonday Electron Emission in the Scanning Electron Microscope. Seiler, H. 11, 1983, Journal of Applied Physics, Vol. 54, pp. R1-R18.

513. JEOL Ltd. Invitation to the SEM World. Tokyo, Akishima, Japan : s.n. pp. 1-59. No.118C496(Ks).

514. Ohm's Law Survives to the Atomic Scale. Weber, B, et al. 6064, 2012, Science, Vol. 335, pp. 64-67.

515. Schroder, Dieter K. Semiconductor Material and Device Characterization, 3ed Edition. s.1. : IEEE Press, Wiley-Interscience, John Wiley \& Sons Inc., 2006. ISBN: 0-471-73906-5.

516. Shockley, W. Research and Investigation of Inverse Epitaxial UHF Power Transistors. WrightPatterson Air Foce Base, Ohio. 1964. Technical Documentary Report. Report No. A1-TOR-64-207.

517. Obtaining the Specific Contact Resistance from Transmission Line Model Measurements. K., Reeves G. and B., Harrison H. 5, 1982, IEEE: Electron Device Letters, Vol. 3, pp. 111-113.

518. Contact Resistance Measurements in GaAs MESFET's and MODFET's by Magneto-TLM Technique. Look, D.C. 8, 1988, J. Electrochem. Soc.: Solid-State Science and Technology, Vol. 135, pp. 2054-2058.

519. Contact resistivity and Current Flow Path At Metal/graphene Contact. Nagashio, K, et al. 14, 2010, Applied Physics Letters, Vol. 97.

520. Models for Contacts to Planar Devices. BERGER, HH. 2, 1972, Solid-State Electronics, Vol. 15, p. 145.

521. Effects of Electron Confinement on Thermionic Emission Current in a Modulation Doped Heterostructure. Anwar, A, et al. 5, 1999, Journal of Applied Physics, Vol. 85, pp. 2663-2666. 
522. Epitaxial-Graphene/Graphene-Oxide Junction: An Essential Step Towards Epitaxial Graphene Electronics. Wu, XS, et al. 2, 2008, Physical Review Letters, Vol. 101.

523. Temperature-Dependent Electrical Property Transition of Graphene Oxide Paper. Huang, XY, et al. 45, 2012, Nanotechnology, Vol. 23.

524. Gold Wire and Ribbbon Product Data Sheet. Ametek Electronic Components and Packaging. [Online] https://www.ametek-ecp.com/products/wire-and-ribbon/gold-wire-and-ribbon.

525. Transient Joule Heating of Graphene, Nanowires and Filaments: Analytical Model for CurrentInduced Temperature Evolution Including Substrate and End Effects. Chandran, KSR. 2015, International Journal of Heat and Mass Transfer, Vol. 88, pp. 14-19.

526. Extended study of the atomic step-terrace structure on hexagonal SiC (0001) by chemical-mechanical planarization. Shi, XL, et al. 2013, Applied Surface Science, Vol. 284, pp. 195-206.

527. Special Limits of Error: Type K Thermocouple Wire. http://assets.omega.com/resources/z218-220.pdf . [Online] Omega Engineering, 2019.

528. Initial Oxidation of Si-Terminated 6H-SiC(0001). Amy, F, et al. 2000, Materials Science Forum, Vols. 338-3, pp. 395-398.

529. Reconstructed Oxide Structures Stable in Air: Silicate Monolayers on Hexagonal SiC Surfaces. Starke, U, et al. 4, 1999, Journal of Vacuum Science \& Technology A: Vacuum Surfaces and Films, Vol. 17, pp. 1688-1692.

530. Graphitization Process of SiC(0001) Studied By Electron Energy Loss Spectroscopy. Langer, T, et al. 11, 2009, Applied Physics Letters, Vol. 94.

531. Photoemission Studies of Graphene on SiC: Growth, Interface, and Electronic Structure. Bostwick, A, et al. 2008, Advances in Solid State Physics, Vol. 47, pp. 159-170.

532. Band-Bending at the Graphene-SiC Interfaces: Effect of the Substrate. Chen, W, et al. 1, 2010, Japanese Journal of Applied Physics, Vol. 49.

533. Complementary Schottky Diode Formation With Carbon Buffer and p-Doped Single Layer Graphene on Intrinsic SiC via Fluorine Intercalation. Lee, SY, et al. 2019, Carbon, Vol. 142, pp. 254-260.

534. High Quality Graphene Grown by Sublimation on 4H-SiC (0001). Lebedev, AA, et al. 4, 2018, Semiconductors, Vol. 52, pp. 1882-1885.

535. Highly n-Doped Graphene Generated Through Intercalated Terbium Atoms. Daukiya, L, et al. 3, 2018, Physical Review B, Vol. 97.

536. Quasi Free-Standing Epitaxial Graphene Fabrication On 3C-SiC/Si(111). Amjadipour, M, et al. 14, 2018, Nanotechnology, Vol. 29.

537. A Table-top Formation of Bilayer Quasi-Free-Standing Epitaxial-Graphene on SiC(0001) By Microwave Annealing in Air. Kim, KS, et al. 2018, Carbon, Vol. 130, pp. $792-798$.

538. The Atomic Structure of a Bare Buffer Layer on SiC(0001) Chemically Resolved. de Lima, LH, et al. 8, 2014, Chemical Communications, Vol. 50, pp. 13571-13574.

539. Structural Investigation of nanocrystalline Graphene Grown on (6 root $3 \times 6$ root 3)R30 DegreesReconstructed SiC Surfaces by Molecular Beam Epitaxy. Schumann, T, et al. 2013, New Journal of Physics, Vol. 15. 
540. Graphene Investigated by Synchrotron Radiation. Hicks, J and Conrad, EH. 12, 2012, MRS Bulletin, Vol. 37, pp. 1203-1213.

541. Probing the Thermal Deoxygenation of Graphene Oxide Using High-Resolution In Situ X-ray-Based Spectroscopies. Ganguly, A, et al. 34, 2011, Journal of Phusical Chemistry C, Vol. 115, pp. 17009-17019.

542. The Route to Functional Graphene Oxide. Haubner, K, et al. Chemphyschem, Vol. 11, pp. 2131-2139.

543. Chemical Analysis of Graphene Oxide Films After Heat and Chemical Treatments by X-ray Photoelectron and Micro-Raman Spectroscopy. Yang, D, et al. 1, 2009, Carbon, Vol. 47, pp. 145-152.

544. Insights Into Lew-Fayer Epitaxial Graphene Growth on 4H-SiC(000(1)over-bar) Substrates From STM Studies. Biedermann, LB, et al. 12, 2009, Physical Review B, Vol. 79.

545. Studies of SiC Formation on Si(100) by Chemical Vapor Depostion. Bozso, F, et al. 8, 1985, Journal of Applied Physics, Vol. 57, pp. 2771-2778.

546. Graphene Growth by Molecular Beam Epitaxy Using a Solid Carbon Source. Moreau, E, et al. 2, 2010, Phys. Status Dolidi A, Vol. 207, pp. 300-303.

547. Surface Morphology and Characterization of Thin Graphene Films on SiC Vicinal Substrate. Penuelas, J, et al. 3, 2009, Physical Review B, Vol. 79.

548. Structure of Few-layer Epitaxial Graphene on 6H-SiC (0001) at Atomic Resolution. Weng, XJ, et al. 20, 2010, Applied Physics Letters, Vol. 97.

549. Raman Characterization of Defects and Dopants in Graphene. Beams, R, Cancado, LG and Novotny, L. 8, 2015, Vol. 27.

550. Raman Spectroscopy of Graphene and Bilayer under Biaxial Strain: Bubbles and Balloons. Zabel, J, et al. 2, 2012, Nano Letters, Vol. 12, pp. 617-621.

551. Evolution of the Raman Spectra From Single-, Few-, and Many-Layer Graphene With Increasing Disorder. Ferreira, EHM, et al. 12, 2010, Physical Review B, Vol. 82.

552. High-throughput Solution Processing of Large-scale Graphene. Tung, VC, et al. 1, 2009, Nature Nanotechnology, Vol. 4, pp. 25-29.

553. Patterning of Two-Dimensional Graphene Oxide on Silicon Substrates. Ishikawa, R, et al. 6, 2010, Japanese Journal of Applied Physics, Vol. 49.

554. Towards Wafer-size Graphene Layers By Atmospheric Pressure Graphitization of Silicon Carbide. Emtsev, KV, et al. 3, 2009, Nature Materials, Vol. 8, pp. 203-207.

555. Nucleation of Epitaxial Graphene on SiC(0001). Robinson, J, et al. 1, 2010, ACS Nano, Vol. 4, pp. 153-158.

556. General Equation for the Determination of the Crystallite Size L-a of Nanographite by Raman Spectroscopy. Cancado, LG, et al. 16, 2006, Applied Physics Letters, Vol. 88.

557. Comparison of Epitaxial Graphene Growth on Polar and Nonpolar 6H-SiC Faces: On the Growth of Multilayer Films. Daas, BK, et al. 7, 2012, Crystal Growth \& Design, Vol. 12, pp. 3379-3387.

558. Schottky Contacts on CF4/H2 Reactive Ion Etch b-SiC. G, Constantinidis, et al. 2, 1998, Solid-State Electronics, Vol. 42, pp. 253-256.

559. New X-ray Insight Into Oxygen Intercalation in Epitaxial Graphene Grown On 4H-SiC(0001). Kowalski, G, et al. 10, 2015, Journal of Applied Physics, Vol. 117. 
560. Effect of Substrate Polishing on the Growth of Graphene on 3C-SiC(111)/Si(111) by High Temperature Annealing. Gupta, B, et al. 18, 2016, Nanotechnology, Vol. 27.

561. The Growth and Morphology of Epitaxial Multilayer. Hass, J, de Heer, WA and Conrad, EH. 32, 2008, Journal of Phusics-Condensed Matter, Vol. 20.

562. Resonant Raman Spectroscopy of Disordered, Amorphous, and Diamond-Like Carbon. Ferrari, AC and Robertson, J. 7, 2001, Physical Review B, Vol. 64.

563. Probe Enhanced, Nano-Raman Spectroscopy (PERS); A Sensitive Technique for Vibrational Surface Spectroscopy. Spizzirri, P.G. 2010, Microscopy: Science, Technology, Applications and Education.

564. Nanoscale Chemical Imaging of Single-Layer Graphene. Stadler, J, Schmid, T and Zenobi, R. 10, 2011, ACS Nano, Vol. 5, pp. 8442-8448.

565. Narrow Plasmon Resonances Enabled by Quasi-Freestanding Bilayer Epitaxial. Daniels, KM, et al. 2, 2017, 2D Materials, Vol. 4.

566. Graphane: A Two-Dimensional Hydrocarbon. Sofo, JO, Chaudhari, AS and Barber, GD. 15, 2007, Physical Review B, Vol. 75.

567. Back-to-back Schottky Diodes: The Generalization of the Diode Theory in Analysis and Extraction of Electrical Parameters of Nanodevices. Chiquito, AJ, et al. 22, 2012, Journal of Physics: Condensed Matter, Vol. 24.

568. A Review on Conduction Mechanisms in Dielectric Films. Chiu, FC. 2014, Advances in Materials Science and Engieering.

569. Electron-Transport of Inhomogeneous Schottky Barriers. Tung, RT. 24, 1991, Applied Physics Letter, Vol. 58, pp. 2821-2823.

570. Electron-Transport at Metal-Semiconductor Interfaces - General Theory. Tung, RT. 23, 1992, Vol. 45, pp. 13509-13523.

571. Alshaeer, Fadwa. PhD Thesis (sic): A Study of Current Transport in Schottky Diodes Based on AlInSb/InSb-QW Heterostructures. School of Physics and Astronomy, Cardiff University. 2018.

572. Schottky Diodes With High Series Resistance- Limitations of Forward I-V Methods. Aubry, V and Meyer, F. 12, 1994, Journal of Applied Physics, Vol. 76, pp. 7973-7984.

573. An improved Forward IV Method For Nonideal Schottky Diodes With High Series Resistance. 10, 1984, IEEE Transactions On Electron Devices, Vol. 31.

574. Forward IV Plot For Nonideal Schottky Diodees With High Series Resistance. Cibils, RM and Buitrago, RH. 2, 1985, Journal of Applied Physics, Vol. 58, pp. 1075-1077.

575. Origin of Voltage-Dependent High Ideality Factors in Graphene-Silicon Diodes. Park, HK and Choi, J. 1, 2018, Advanced Electronic Materials, Vol. 4.

576. Current-voltage Characteristics and Parameter Retrieval of Semiconducting Nanowires. Zhang, ZY, et al. 7, 2006, Applied Physics Letters, Vol. 88.

577. Extraction of Graphene-Titanium Contact Resistances useing Transfer Length Measurement and a Curve-Fit Method. Anteroinen, J., et al. 8, 2012, World Academy of Science, Engineering and Technology International Journal of Materials and Metallurgical Engineering, Vol. 6, pp. 801-804. 
578. Contact Resistance in Few and Multilayer Graphene Devices. Venugopal, A, Colombo, L and Vogel, EM. 1, 2010, Applied Physics Letters, Vol. 96.

579. Metal/graphene Contact As a Performance Killer of Ultra-high Mobility Graphene - Analysis of Intrinsic Mobility and Contact Resistance. Nagashio, K, et al. Baltimore, MD : s.n., 2009, 2009 IEEE International Electron Devices Meeting, pp. 527-530.

580. Contacting Graphene. Robinson, JA, et al. 5, 2011, Applied Physics Letters, Vol. 98.

581. Three-dimensional Stacked Multilayer Graphene Interconnects. Yu, TH, et al. 8, 2011, IEEE Electron Device Letters, Vol. 32, pp. 1110-1112.

582. Resistivity of Graphene Nanoribbon Interconnects. Murali, R, et al. 6, 2009, IEEE Electron Device Letters, Vol. 30, pp. 611-613.

583. Measurements of the Sheet Resistance and Conductivity of Thin Epitaxial Graphene and SiC Films. Krupka, J and Strupinski, W. 8, 2010, Applied Physics Letters, Vol. 96.

584. Dirac Particles in Epitaxial Graphene Films Grown on SiC. Berger, C, et al. 2008, Advances in Solid State Physics, Vol. 47.

585. Improving the Electrical Properties of Graphene Layers By Chemical Doping. Khan, MF, et al. 5, 2014, Science and Technology of Advanced Materials, Vol. 15.

586. Effect of a High-kappa Environment on Charge Carrier Mobility in Graphene. Ponomarenko, LA, et al. 20, 2009, Physical Review Letters, Vol. 102.

587. Determination of Work Function of Graphene under a Metal Electrode and Its Role in Contact Resistance. Song, SM, et al. 8, 2012, Nano Letters, Vol. 12, pp. 3887-3892.

588. Electronic Transport Properties of Individual Chemically Reduced Graphene Oxide Sheets. GomezNavarro, C, et al. 11, 2007, Nano Letters, Vol. 7, pp. 3499-3503.

589. Contact Resistance in Graphene-Based Devices. Russo, S, et al. 4, 2010, Physica E-Low-Dimensional Systems \& Nanostructures, Vol. 42, pp. 677-679.

590. Reducing Contact Resistance in Graphene Devices through Contact Area Patterning. Smith, JT, et al. s.1. : 7, 2013, ACS Nano, Vol. 4, pp. 3661-3667.

591. Electrical Properties of the Graphene/4H-SiC (0001) Interface Probed By Scanning Current Spectroscopy. Sonde, S, et al. 24, 2009, Physical Review B, Vol. 80.

592. Resistivity Anisotropy Measured Using Four Probes in Epitaxial Graphene On Silicon Carbide. Kobayashi, K, et al. 3, 2015, Applied Physics Express, Vol. 8.

593. Magnetophonon Spectroscopy of Dirac Fermion Scattering By Transverse and Longitudinal Acoustic Phonons in Graphene. Greenaway, MT, et al. 15, 2019, Physical Review B, Vol. 100.

594. Generalized Two-Temperature Model for Coupled Phonons in Nanosized Graphene. An, M, et al. 9, 2017, Nano Letters, Vol. 17, pp. 5805-5810.

595. Temperature-Dependent Resistivity in Bilayer Graphene Due to Flexural Phonons. Ochoa, H, et al. 23, 2011, Physical Review B, Vol. 83.

596. Interaction of Massless Dirac Electrons With Acoustic Phonons in Graphene at Low Temperatures. Kubakaddi, SS. 7, 2009, Physical Review B, Vol. 79. 
597. Impact of Electron-Electron Coulomb Interaction On the High Harmonic Generation Process in Graphene. Avetissian, HK and Mkrtchian, GF. 11, 2018, Physical Review B, Vol. 97.

598. Electron-Electron and Electron-Phonon Interactions in Graphene. Davydov, S. Yu. 3, 2018, Semiconductors, Vol. 52, pp. 353-358.

599. Modified Monte Carlo Method for Study of Electron Transport in Degenerate Electron Gas in the Presence of Electron-Electron Interactions, Application to Graphene. Borowik, P, Thobel, JL and Adamowicz, L. 2017, Journal of Computational Physics, Vol. 341, pp. 397-405.

600. Charge Trapping and Scattering in Epitaxial Graphene. Farmer, DB, et al. 20, 2011, Physical Review B, Vol. 84.

601. Charge Trapping in Monolayer and Multilayer Epitaxial Graphene. Liu, CI, et al. 2016, Journal of Nanomaterials.

602. Transmission Through Barriers and Resonant Tunneling In an Interacting One-dimensional Electron Gas. Kane, CL and Fisher, MPA. 23, 1992, Physical Review B, Vol. 46, pp. 15233-15262.

603. Theory of Electron Tunneling in Semiconductor Junctions. Fredkin, DR and Wannier, GH. 5, 1962, Physical Review, Vol. 128.

604. Theory of Electron Tunneling in Semiconductors (Direct Tunneling Across $p$ - $n$ Junctions). Y, Takeuti and H, Funada. 10, 1965, Journal of the Physical Society of Japan, Vol. 20, pp. 1854-1865.

605. Afanas, Valery V. Analysis of the Charge Trapping Kinetics: Fundamentals and Recent Advances. [book auth.] Valery V. Afanas'ev. Internal Photoemission Spectroscopy, 2nd Ed. s.1. : Elsevier Ltd., 2014, pp. 213-254.

606. Space Charge Limited Conduction With Exponential Trap Distribution in Reduced Graphene Oxide Sheets. Joung, D, et al. 9, 2010, Applied Physics Letters, Vol. 97.

607. Electronic Conduction Mechanisms in Insulators. Chiang, TH and Wager, JF. 1, 2018, IEEE Transactions on Electron Devices, Vol. 65, pp. 223-230.

608. High-Temperature Characteristics of AlxGa1-xN/GaN Schottky Diodes. Xiaoling, Z, et al. 3, 2009, Journal of Semiconductors, Vol. 30.

609. Sharma, Bijay Kumar. Solid State Physics and Devices-the Harbinger of Third Wave of Civilization. 2011.

610. Valve Technology- A Practical Guide. Dixey, Graham. s.1. : MIEE via Maplin Magazine, 1993.

611. Photo-Thermoelectric Effect at a Graphene Interface Junction. Xu, XD, et al. 2, 2010, Nano Letters, Vol. 10, pp. 562-566.

612. Giant Thermoelectric Effect in Graphene. Dragoman, D and Dragoman, M. 20, 2007, Vol. 91.

613. Giant Magneto-Photoelectric Effect in Suspended Graphene. Sonntag, J, et al. 2017, New Journal of Physics, Vol. 19.

614. What Does Annealing Do to Metal-Graphene Contacts? Leong, WS, Nai, CT and Thong, JTL. 7, 2014, Nano Letters, Vol. 14, pp. 3840-3847.

615. Rapid Thermal Annealing of Graphene-Metal Contact. Balci, O and Kocabas, C. 24, 2012, Applied Physics Letters, Vol. 101. 
616. Annealing Effect on Schottky Barrier Inhomogeneity of Graphene/n-type Si Schooky Diodes. Lin, YJ and Lin, JH. 2014, Applied Surface Science, Vol. 311, pp. 224-229.

617. Temperature Dependent Transport Characteristics of Graphene/n-Si Diodes. Parui, S, et al. 24, 2015, Journal of Applied Physics, Vol. 116.

618. A study of Temperature-Related Non-Linearity At The Metal-Silicon Interface. Gammon, PM, et al. 11, 2013, Journal of Applied Physics , Vol. 112.

619. Electron-Transport of Inhomogeneous Schottky Barriers- A Numerical Study. Sullivan, JP, et al. 2, 1991, Journal of Applied Physics, Vol. 70, pp. 7403-7424.

620. The Physics and Chemistry of the Schottky Barrier Height. Tung, RT. 1, 2014, Applied Physics Reviews, Vol. 1.

621. Yu, Peter Y. and Cardona, Manuel. Graduate Texts In Physics: Fundamentals of SemiconductorsPhysics and Materials Properties 4th Ed. New York: Springer, 2010. pp. 203-236. ISBN: 978-3-64200709-5.

622. Chauhan, Jyotsna. Nanomaterial, Vol IV. s.1. : Educreation Publishing, 2019. p. 116. ISBN: 978-935373-011-6.

623. Electric Properties of n-GaN: Effect of Different Contacts on the Electronic Conduction. Abdalla, S, et al. 2, 2013, International Journal of Physics, Vol. 1.

624. Lundstrom, Mark. Fundamentals of Carrier Transport, 2nd Ed. New York : Camberidge University Press, 2009. pp. 54-80. ISBN: 978-0-521-63134-1.

625. Hall Coefficient Factor For Polar Mode Scattering in N-Type GaAs. Stillman, GE, Wolfe, CM and Dimmock, JO. 6, 1970, Journal of Physics and Chemistry of Solids, Vol. 31.

626. An Exact Solution of the Linearized Boltzmann Equation With Applications to the Hall Mobility and Hall Factor of n-GaAs. Fletcher, K and Butcher, PN. 2, 1972, Journal of Physics C: Solid State Physics, Vol. 5.

627. Resonant Tunneling Diodes: Models and Properties. Sun, JP, et al. 4, 1998, Proceedings of the IEEE, Vol. 86, pp. 641-661.

628. Croke, ET and Schulman, JN. Encyclopedia of Materials: Science and Technology (2nd Ed): Resonant Tunneling Diodes. 2001. pp. 8185-8192.

629. Theoretical Study of a Molecular Resonant Tunneling Diode. Seminario, JM, Zacarias, AG and Tour, JM. 13, 2000, Journal of the American Chemical Society, Vol. 122, pp. 3015-3020.

630. Frequency Limitations of Resonant-Tunnelling Diodes in Sub-THz and THz Oscillators and Detectors. Feiginov, M. 4, 2019, Journal of Infrared Millimeter and Terahertz Waves, Vol. 40, pp. 365-394.

631. Resonant Tunneling Diodes for Sub-Terahertz and Terahertz Oscillators. Asada, M, Suzuki, S and Kishimoto, N. 6, 2008, Japanese Journal of Applied Physics, Vol. 47, pp. 4375-4384.

632. Miura, N. Comprehensive Semiconductor Science and Technology: Volume 2 Magneto-Spectroscopy of Semiconductors. [ed.] P. Bhattacharya, R. Fornari and H. Kamimura. s.1. : Elsevier, 2011. pp. 256-342. ISBN: 978-0-444-53143-8.

633. Robinett, Richard W. Quantum Mechancis: Classical Results, Modern Systems, and Visualized Examples (2nd Ed.). New York : Oxford University Press, 2006. ISBN-10: 0-19-853097-8. 
634. Messiah, Albert. Quantum Mechanics (Dover Edition). Paris : Dover Publications, 2014. pp. 77-114. ISBN: 978-0-486-78455-7.

635. Computer-Generated Motion Pictures of 1-Dimensional Quantum-Mechanical Transmission and Reflection Phenomena. Goldberg, A, Schemy, HM and Schwartz, JL. 3, 1967, American Journal of Physics, Vol. 35, pp. 177-186.

636. Zur Quantenmechanik der Stoßvorgänge (On the Quantum Mechanics of Collision Processes, Translated by D. H. Delphenich). Born, Max. 1926, Zeit. Phys., Vol. 37, pp. 863-867.

637. Kramers, H.A. Quantum Mechanics. s.1. : Dover, 1957. ISBN: 978-0-486-66772-0.

638. Novel AlInAsSb/InGaAs Double-Barrier Resonant Tunneling Diode with High Peak-to-Valley Current Ratio at Room Temperature. Su, YK, et al. 4, 2000, IEEE Electron Device Letters, Vol. 21, pp. 146-148.

639. Single-well Resonant-Tunneling Diode Heterostructures Based on In0.53Ga0.47As/AlAs/InP With the Peak-To-Valley Current Ratio of 22:1 At Room Temperature. Syzranov, VS, et al. 8, 2013, Bulletiin of the Lebedev Physics Institute, Vol. 40.

640. Experimental Studies On the NDR of Si1-xGx/Si Triple Barrier Resonant Tunneling Diodes. Xu, DX, et al. 5, 1912, Solid-State Electroncis, Vol. 35, pp. 611-614.

641. Repeatable Room Temperature Negative Differential Resistance in AlN/GaN Resonant Tunneling Diodes Grown on Sapphire. Wang, D, et al. 2, 2019, Advanced Electronic Materials, Vol. 5.

642. Measurements of Temperature Characteristics and Estimation of Terahertz Negative Differential Conductance in Resonant-Tunneling-Diode Oscillators. Asada, M, Suzuki, S and Fukuma, T. 11, 2017, AIP Advances, Vol. 7.

643. Degradation Study of AlAs/GaAs Resonant Tunneling Diode IV Curves Under Influence of High Temperatures. Makeev, MO, Meshkov, SA and Sinyakin, VY. St. Petersburg : s.n., 2017. 4th International School and Conference On Optoelectronics, Photonics, Engineering and Nanostructures. Vol. 917.

644. Study of Degradation Processes Kinetics in Ohmic Contacts of Resonant Tunneling Diodes Based on Nanoscale AlAs/GaAs Heterostructures under Influence of Temperature. Makeev, MO and Meshkov, SA. 2017. 2017 2nd International Conference On Smart Materials Technologies (ICSMT 2017).

645. Resonant Tunnelling Diodes Based On Graphene/h-BN Heterostructure. Nguyen, VH, et al. 32, 2012, Journal of Physics D-Applied Physics, Vol. 45.

646. Negative Differential Resistances in graphene Double Barrier Resonant Tunneling Diodes. Song, Y, Wu, HC and Guo, Y. 9, 2013, Applied Physics Letters, Vol. 102.

647. Shape Effects in Graphene Nanoribbon Resonant Tunneling Diodes: A Computational Study. Teong, H, et al. 8, 2009, Journal of Applied Physics, Vol. 105.

648. Advanced Micro- and Nano-Gas Sensor Technology: A Review. Nazemi, H, et al. 6, 2019, Sensors, Vol. 19.

649. Johnson-Nyquist Noise for a 2D Electron-Gas In a Narrow Channel. Bulashenko, OM and Kochelap, VA. 39, 1993, Journal of Physics-Condensed Matter, Vol. 5, pp. L469-L474.

650. Plasmonic Mass and Johnson-Nyquist Noise. Chee, J, et al. 35, 2015, Nanotechnology, Vol. 26.

651. In-Situ Temperature Measurements of Joule-Heated Graphene Using Near-Infrared CCD Imaging System. Saito, T, Suda, R and Shirakashi, JI. 2013. 2013 13th IEEE Conference on Nanotechnology (IEEENANO). pp. 717-721. 
652. Infrared Microscopy of Joule Heating in Graphene Field Effect Transistors. Bae, MH, et al. 2009. 2009 9th IEEE Conference on Nanotechnology (IEEE-NANO). pp. 818-821.

653. Kozlowska, A, et al. Graphene Joule Heating Measurements in Environmental Chamber. Progress in Automation, Robotics and Measuring Techniques, Vol 3: Measuring Techniques and Systems. 2015, pp. 129-135.

654. Scaling of High-Field Transport and Localized Heating in Graphene Transistors. Bae, MH, et al. 10, 2011, ACS Nano, Vol. 5, pp. 7936-7944.

655. Role of Joule Heating on Current Saturation and Transient Behavior of Graphene Transistors. Islam, S, et al. 2, 2013, IEEE Electron Device Letters, Vol. 34, pp. 166-168.

656. Self-Heating and Failure in Scalable Graphene Devices. Beechem, TE, et al. 2016, Scientific Reports, Vol. 6.

657. Light Emission, Light Detection and Strain Sensing With Nanocrystalline Graphene. Riaz, A, et al. 32, 2015, Nanotechnology, Vol. 26.

658. Self-heating and Nonlinear Current-Voltage Characteristics In Bilayer Graphene. Viljas, JK, et al. 20, 2011, Physical Review B, Vol. 83.

659. Joule-Heating Induced Thermal Voltages in Graphene Three-Terminal Nanojunctions. Butti, P, et al. 13, 2018, Applied Physics Letters, Vol. 112.

660. Nanoscale Joule Heating, Peltier Cooling and Current Crowding at Graphene-Metal Contacts. Grosse, KL, et al. 5, 2011, Nature Nanotechnology, Vol. 6, pp. 287-290.

661. Graphene Electrode Diagnostic with IR Imaging of Joule Heat Emission. Gawlik, G, et al. 2019, Inferared Physics \& Technology, Vol. 97, pp. 48-53.

662. Probing Charge Trapping and Joule Heating In Graphene Field-Effect Transistors By Transient Pulsing. Ramamoorthy, H, et al. 8, 2017, Semiconductor Science and Technology, Vol. 32.

663. Graphene Schottky Varactor Diodes for High-Performance Photodetection. Levi, A, et al. 8, 2019, ACS Photonics, Vol. 6, pp. 1910-1915.

664. Green, Don, [ed.]. Perry's Chemical Engineerings' Handbook 7th Ed. s.1. : McGraw-Hill, 1997. ISBN: ISBN 0-07-049841-5.

665. Fogler, H. Scott. Elements of Chemical Reaction Engieering. 5th. s.1. : Prentice Hall, 2016. ISBN: 9780-13-388751-8.

666. First-principles Study Of the Structural and Energetic Properties of H Atoms on a Graphite (0001) Surface. Sha, XW and Jackson, B. 3, 2002, Surface Science, Vol. 496, pp. 318-330.

667. Understanding Adsorption of Hydrogen Atoms on Graphene. Casolo, S, et al. 5, 2009, Journal of Chemical Physics, Vol. 130.

668. Hydrogen on Graphene: Electronic structure, Total Energy, Structural Distortions and Magnetism From First-Principles Calculations. Boukhvalov, DW, Katsnelson, MI and Lichtenstein, AI. 3, 2008, Physical Review B, Vol. 77.

669. Role of Contacts in Graphene Transistors: A Scanning Photocurrent Study. Mueller, T, et al. 24, 2009, Vol. 79. 
670. Photothermoelectric and Photoelectric Contributions to Light Detection in Metal-Graphene-Metal Photodetectors. Echtermeyer, TJ, et al. 7, 2014, Nano Letters, Vol. 14, pp. 3733-3742.

671. Carrier Multiplication in Graphene. Winzer, T, Knorr, A and Malic, E. 12, 2010, Nano Letters, Vol. 10, pp. 4839-4843.

672. Experimental Verification of Carrier Multiplication in Graphene. Plotzing, T, et al. 9, 2014, Nano Letters, Vol. 14, pp. 5371-5375.

673. Photodetectors Based on Graphene and Other Two-Dimensional Materials and Hybrid Systems. Koppens, FHL, et al. 10, 2014, Nature Nanotechnology, Vol. 9, pp. 780-793.

674. Graphene and Graphene-like Two-Dimensional Materials in Photodetection: Mechanisms and Methodology. Sun, ZH and Chang, HX. 5, 2014, ACS Nano, Vol. 8, pp. 4133-4156.

675. Graphene-Based Materials In The Infrared and Terahertz Detector Families: A Tutorial. Rogalski, A. 2, 2019, Advances In Optics and Photonics, Vol. 11, pp. 314-379.

676. Detection, Mixing, and Frequency Multiplication of Terahertz Radiation By Two-Dimensional Electronic Fluid. Dyakonov, M and Shur, M. 3, 1996, IEEE Transactions on Electron Devices, Vol. 43, pp. 380-387.

677. Intrinsic photo-conductance triggered by the plasmonic effect in graphene for terahertz detection. Wang, L, Chen, XS and Lu, W. 3, 2016, Nanotechnology, Vol. 27.

678. Ultrafast Collinear Scattering and Carrier Multiplication in Graphene. Brida, D, et al. 2013, Nature Communications, Vol. 4.

679. Making Graphene Luminescent by Oxygen Plasma Treatment. Gokus, T, et al. 12, 2009, ACS Nano, Vol. 3, pp. 3963-3968.

680. Tunable Carrier Multiplication and Cooling in Graphene. Johannsen, JC, et al. 1, 2015, Nano Letters, Vol. 15, pp. 326-331.

681. Photovoltaic Effect and Evidence of Carrier Multiplication in Graphene Vertical Homojunctions with Asymmetrical Metal Contacts. Chen, JJ, et al. 9, 2015, ACS Nano, Vol. 9, pp. 8851-8858.

682. High-Performance Graphene/ $\beta$-Ga2O3 Heterojunction Deep Ultraviolet Photodetector with HotElectron Excited Carrier. Lin, RC, et al. 26, 2018, ACS Applied Materials \& Interfaces, Vol. 10, pp. 2241922426.

683. Rectified Photocurrent In a Planar ITO/Graphene/ITO Photodetector On SiC By Local Irradiation of Ultraviolet Light. Yang, JW, et al. 40, 2017, Journal of Physics D- Applied Physics, Vol. 50.

684. Review On Carrier Multiplication In Graphene. Malic, E, et al. 12, 2016, Physica Status Solidi BBasic Solid State Physics, Vol. 253, pp. 2303-2310.

685. Current Enhancement Due to Field-Induced Dark Carrier Multiplication In Graphene. Jago, R, Wendler, F and Malic, E. 2, 2017, 2D Materals, Vol. 4.

686. Enhanced Performance of a Visible Light Detector Made with Quasi-Free-Standing Graphene on SiC. Li, XM, et al. 19, 2019, Materials, Vol. 12.

687. Epitaxial Graphene (EG)/SiC Based Schottky Emitter Bipolar Phototransistors or UV Detection and Effect of Hydrogen Intercalation On Device I-V Characteristics. V. S. N. Chava, M. V. S. Chandrashekhar, K. M. Daniels, B. G. Barker and A. B. Greytak. Orlando, FL : s.n., 2016. 15th IEEE Sensors Conference. pp. 1-3. 
688. Haynes, W. M., [ed.]. CRC Handbook of Chemistry and Physics, 97th Ed. s.1. : CRC Press, Taylor \& Francis Group, 2017. p. 12.123. ISB: 978-1-4987-5429-3.

689. CFx Radical Production and Loss in a CF4 Reactive Ion Etching Plasma: Fluorine Rich Conditions. Booth, JP, et al. 6, 1999, Vol. 85, pp. 3097-3107.

690. Effect of CHF3 Addition on Reactive Ion Etching of Aluminum Using Inductively Coupled Plasma. Saito, S, Sugita, K and Tonotani, J. 5A, 2005, Japanese Journal of Applied Physics Part 1: Regular Papers Brief Communicatinos \& Review Papers, Vol. 44, pp. 2971-2975.

691. A Simple Method to Synthesize Silver Nanoparticles By Photo-reduction. Courrol, LC, Silva, FRDO and Gomes, L. 1-3, 2007, Colloides and Surface A, Vol. 305, pp. 54-57.

692. TiO2-graphene Nanocomposites: UV-assisted Photocatalytic Reduction Of Graphene Oxide. Williams, G, Seger, B and Kamat, PV. 7, 2008, ACS Nano, Vol. 2, pp. 1487-1491.

693. Anchoring Semiconductor and Metal Nanoparticles on a Two-Dimensional Catalyst Mat. Storing and Shuttling Electrons with Reduced Graphene Oxide. Lightcap, IV, Kosel, TH and Kamat, PV. 2, 2010, Nano Letters, Vol. 10, pp. 577-583.

694. Facile Photochemical Synthesis of Graphene-Pt Nanoparticle Composite for Counter Electrode in Dye Sensitized Solar Cell. Tjoa, V, et al. 7, 2012, ACS Applied Materals \& Interfaces, Vol. 4, pp. 34473452.

695. Sodium Borohydride Digest. www.dow.com. [Online] 2003. [Cited: 7 29, 2017.] http://www.dow.com/assets/attachments/industry/pharma_medical/chemical_reagents/reducing_agents/so dium_borohydride_digest.pdf.

696. Real-time Tracking of Metal Nucleation via Local Perturbation of Hydration Layers. Harniman, RL, et al. s.1. : Nature Communications, 2017, Vol. 8. 10.1038/s41467-017-01087-1.

697. Absolute Electronegativity and Hardness- Applications to Inorganic Chemistroy. Pearson, RG. 4, 1988, Inorganic Chemistry, Vol. 27, pp. 734-740.

698. Silver Doped Reduced Graphene Oxide as a Promising Plasmonic Photocatalyst for Oxidative Coupling of Benzylamines Under Visible Light Irradiation. Kumar, A, Sadanandhan, AM and Jain, SL. 23, 2019, New Journal of Chemistry, Vol. 43, pp. 9116-9122.

699. www.qsil.com. [Online] QSIL, 8 17, 2019. https://www.qsil.com/en/material/about-fused-quartz/.

700. Solid-state graphene formation via a nickel carbide intermediate phase. Xiong, W, et al. 120, 2015, RSC Advances, Vol. 5, pp. 99037-99043.

701. Graphene Growth by a Metal-Catalyzed Solid-State Transformation of Amorphous Carbon. Rodriguez-Manzo, JA, Pham-Huu, C and Banhart, F. 2, 2011, ACS Nano, Vol. 5, pp. 1529-1534.

702. Formation of Monolayer Graphene by Annealing Sacrificial Nickel Thin Films. Pollard, AJ, et al. 38, 2009, Journal of Physical Chemistry C, Vol. 113, pp. 16565-16567.

703. In Situ X-ray Diffraction Study of Graphitic Carbon Formed During Heating and Cooling of Amorphous-C/Ni Bilayers. Saenger, KL, et al. 15, 2010, Applied Physics Letters, Vol. 96.

704. Synthesis of Large-Area Graphene Layers on Poly-Nickel Substrate by Chemical Vapor Deposition: Wrinkle Formation. Chae, SJ, et al. 22, 2009, Advanced Materials, Vol. 21. 
705. Inouye, H. High-Temperature Reactions of Type 304 Stainless Steel in Low Concentrations of Carbon Dioxide and Carbon Monoxide. Oak Ridge National Laboratory . Oak Ridge, Tennessee : U.S. Atomic Energy Commission, 1962. pp. 1-49.

706. Initiation and Growth of Iron Metal Dusting in CO-H-2-H2O Gas Mixtures. Zhang, JQ, Schneider, A and Inden, G. 4, 2008, CORROSION SCIENCE , Vol. 50, pp. 1020-1034.

707. Oxidation, Carburisation and Metal Dusting of 304 Stainless Steel in $\mathrm{CO} / \mathrm{CO} 2$ and $\mathrm{CO} / \mathrm{H}-2 / \mathrm{H} 2 \mathrm{O}$ Gas Mixtures . Zhang, J, Boddington, K and Young, DJ. 11, 2008, CORROSION SCIENCE, Vol. 50, pp. 3107 3115.

708. Cox, Arthur Reeves. The High Temperature Reactions of Carbon Monoxide with Iron, Nickel, and Austentic Stainless Steel. s.l.: Georgia Institute of Technology, 1962. Master Thesis of Science in Metallurgy.

709. Wang L, Liu J, Wang F, Yu S. Preparation of carbonyl nickel powder involves decomposing mixture of carbonyl nickel steam and carbon monoxide and controlling the disproportionation reaction of carbon monoxide at high temp through heating. CN102070205-A China, May 25, 2010.

710. Heterogeneous Catalyst Deactivation and Regeneration: A Review. Argyle, MD and Bartholomew, CH. 1, 2015, Catalysts, Vol. 5, pp. 145-269.

711. Acute Nickel Carbonyl Poisoning. Kurta, DL, Bean, BS and Krenzelock, EP. 1, 1993, Amearican Journal of Emergency Medicine, Vol. 11, pp. 64-66.

712. Chemical Bonding and Electronic Structures of the Al2SiO5 Polymorphs, Andalusite, Sillimanite, and Kyanite: X-ray Photoelectron- and Electron Energy Loss Spectroscopy Studies. Ohuchi, FS, et al. 5-6, 2006, American Mineralogist, Vol. 91, pp. 740-746.

713. Suppression of Aluminum Contamination in Polysilicon Reactive Ion Etching Using Highly Purified Chlorine Gas. Sato, M and Arita, Y. 7, 1997, Journal of the Electrochemical Society, Vol. 144, pp. 2512547.

714. Reactive Ion Etching of Aluminum and Aluminum-Alloys in an RF Plasma Contating Halogen Species. Schaible, P. M., Metzger, W. C. and Anderson, J. P. 2, 1978, Journal of Vacuum Science \& Technology, Vol. 15, pp. 334-337.

715. Transient Enhanced Diffusion of Aluminum in SiC During High Temperature Ion Implantation. Usov, IO, et al. 11, 1999, Journal of Applied Physics, Vol. 86, pp. 6039-6042.

716. Ion Implantation and Annealing Effects in Silicon Carbide. Heera, V and Skorupa, W. 1997. Materials Modification and Synthesis by Ion Beam Processing. Vol. 438, pp. 241-252.

717. Site-Competition Eptiaxy for Superior Silicon-Carbide Electronics. Larkin, DJ, et al. 13, 1994, Applied Physics Letters, Vol. 65, pp. 1659-1661.

718. Rao, Shailaja P. Dissertation: Implant Annealing of Al Dopants in Silicon Carbide Useing Silane Overpressure. s.l. : University of South Florida, 2005.

719. Ratner, DB and Castner, DG. Surface Analysis- The Principal Techniques, 2nd Edition: Electron Spectroscopy for Chemical Analysis. [ed.] J Vickerman and I Gilmore. s.1. : Wiley and Sons, 2009.

720. The 6H-SiC Structure Model: Further Refinement From SCXRD Data From a Terrestrial Moissanite. Capitani, GC, Di Pierro, S and Tempesta, G. 2-3, 2007, American Mineralogist, Vol. 92, pp. 403-407.

721. NIST electron inelastic-mean-free-path database. Powell, CJ and Jablonski, A. 2000, U.S. Department of Commerce, Technology Administration Standard Reference Data Program. 
722. Atomic and Electronic Structure of Few-Layer Graphene On SiC(0001) Studied With Scanning Tunneling Microscopy and Spectroscopy. Lauffer, P, et al. 15, 2008, Physical Review B, Vol. 77.

723. Kenny, Ryan and Watt, Jeff. White Paper: The Breakthrough Advantage for FPGAs with Tri-Gate Technology. [Online] [Cited: 2727 2019, https:/www.intel.com/content/dam/www/programmable/us/en/pdfs/literature/wp/wp-01201-fpga-tri-gatetechnology.pdf.

724. Hruska, Joel. GlobalFoundries Announces 14nm Validation With AMD Zen Silicon. [Online] ExtreamTech, 11 6, 2015. https://www.extremetech.com/computing/217664-globalfoundries-announces$14 \mathrm{~nm}$-validation-with-amd-silicon.

725. www.AMD.com. [Online] 2019. https://www.amd.com/en/products/cpu/amd-ryzen-threadripper3970x.

$\begin{array}{lllll}\text { 726. } & \text { 7nm } & \text { Technology. } & \text { www.TSMC.com. } & \text { [Online] }\end{array}$ https://www.tsmc.com/english/dedicatedFoundry/technology/7nm.htm.

727. The Nobel Prize in Chemistry 1932. s.1. : Nobel Media, 2019.

728. Transparent Conductive Electrodes Based on Graphene-Related Materials. Woo, YS. 1, 2019, Micromachines, Vol. 10.

729. Transparent, Conductive Graphene Electrodes for Dye-Sensitized Solar Cells. Wang, X, Zhi, LJ and Mullen, K. 1, 2008, Nano Letters, Vol. 8, pp. 323-327.

730. ISO 18118:2015 Surface chemical analysis -- Auger electron spectroscopy and X-ray photoelectron spectroscopy -- Guide to the use of experimentally determined relative sensitivity factors for the quantitative analysis of homogeneous materials. 22015.

731. Quantitative Electron Spectroscopy of Surfaces: A Standard Data Base for Electron Inelastic Mean Free Paths in Solids. Seah, M.P. and Dench, W.A. 1, 1979, Surface and Interface Analysis, Vol. 1.

732. Nicholson, Dwight R. Introduction to Plasma Theory. New York : John Wiley \& Sons, Inc., 1983. 0471-09045-X.

733. Brown, Sanbrorn C. Introduction to Electrical Discharges in Gases . s.1. : Massachusetts Institute of Technology, 1966.

734. Federal Communications Commission. Title 47 Telecommunications Federal Regulations: Chapter 1, Subchapter A, Part 2, Subpart B: §2.106 Table of Frequency Allocations. 1 22, 2019.

735. International Telecommunication Union. Radio Regulatoins Articles. 2016.

736. Why Can't We See Hydrogen in X-ray Photoelectron Spectroscopy? Stojilovic, N. 10, 2012, Journal of Chemical Education, Vol. 89, pp. 1331-1332.

737. Origin of the D Peak in the Raman Spectrum of Microcrystalline Graphite. Pocsik, I, et al. 1998, Journal of Non-Crystalline Solids, Vol. 227, pp. 1083-1086.

738. Observation of Raman Band Shifting With Excitation Wavelength for Carbons and Graphites. Vidano, RP, et al. 2, 1981, Solid State Communications, Vol. 39, pp. 341-344.

739. America, U.S. Secretary of Commerce on behalf of the United States of. National Institute of Standards and Technology: Chemistry WebBook, SRD 69. [Online] U.S. Department of Commerce, 2019. https://webbook.nist.gov/cgi/cbook.cgi?ID=C7783611\&Mask=4. 
740. On the Runge Example. Epperson, JF. 4, 1987, American Mathematical Monthly, Vol. 94, pp. 329341.

741. Über empirische Funktionen und die Interpolation zwischen äquidistanten Ordinaten (About Empirical Functions and the Interpolation Between Equidistant Ordinates). Runge, C. 1901, Zeitschrift für Mathematik und Physik (Journal of Mathematics and Physics), Vol. 46, pp. 224-243.

742. Gas Sensors: A Review. Yunusa, Z., et al. 4, 2014, Sensors and Transducers, Vol. 168, pp. 61-75.

743. A Critical Evaluation of Thermal Mass Flow Meters. Tison, SA. 4, 1996, Journal of Vacuum Science and Technology A- Vacuum Surfaces and Films, Vol. 14, pp. 2582-2591.

744. Rhoderick, E.H. and Williams, R. Metal-semiconductor Contacts, 2nd. s.1. : Clarendon Press Oxford, 1988. ISBN: 0198593368.

745. Comments on the Conduction Mechanism in Schottky Diodes. Rhoderick, E.H. 10, 1972, Journal of Physics D: Applied Physics, Vol. 5, pp. 1920-1929.

746. Mortenson, Kenneth E. Variable Capacitance Diodes: The Operation and Characterization of Varactor, Charge Storage, and PIN Diodes for RF and Microwave Applications. s.1. : Artech, 1974. ISBN: 0890060150.

747. Gas Sensors Using Carbon Nanomaterials: A Review. Llobet, E. SI, 2013, Sensors and Actuators BChemcial, Vol. 179, pp. 32-45.

748. Low Concentration Response Hydrogen Sensors Based on Wheatstone Bridge. Jiang, HC, et al. 5, 2019, Sensors, Vol. 19.

749. Miniaturized Single Chip Arrangement of a Wheatstone Bridge Based Calorimetric Gas Sensor. Illyaskutty, N, et al. 2, 2018, Chemosensors, Vol. 6.

750. Dynamic Transmission and Reflection Phenomena For A Time-Dependent Rectangular Potential. Haavig, DL and Reifenberger, R. 12, 1982, Physical Review B, Vol. 26, pp. 6408-6420.

751. Extraction of Schottky Diode Parameters Including Parallel Conductance Using a Vertical Optimization Method. Ferhat-Hamida, A, et al. 5, 2002, Solid-State Electronics, Vol. 46, pp. 615-619.

752. The Genesis of the Wheatstone Bridge. Ekelof, S. 1, s.1.: IET, 2001, Engineering Science and Education Journal, Vol. 10, pp. 37-40.

753. Wave Packet Scattering From Time-Varying Potential Barriers in One Dimension. Dimeo, RM. 2, 2014, American Journal of Physics , Vol. 82, pp. 142-152.

754. Substation Installation. 11-474, US Government: War Department Technical Manual TM. June 30, 1944, p. 46. LC Call \#: U408.3.A13 TM 11-474 Jun. 1944.

755. Thermal Mass Flow Sensor For Gases: Bypass Principle. www.bronkhorst.com. [Online] Bronkhorst High-Tech B.V. https://www.bronkhorst.com/int/service-support/technologies/thermal-mass-flow-sensorfor-gases-bypass-principl/.

756. The Nobel Prize in Physics 1954. www.NobelPrize.org. [Online] Nobel Media AB 2019. https://www.nobelprize.org/prizes/physics/1954/summary/.

757. Mass Air Sensors (MAF) - Automotive. Sensorland.com. [Online] https://www.sensorland.com/HowPage060.html. 
758. Explosimeter Combustible Gas Indicators: Data Sheet 03-00-03. [Online] MSA. http://site.jjstech.com/pdf/MSA/explosimeter-combustible-gas-indicator_datasheet.pdf.

759. DOE Handbook: Electrical Safety . Washington, D.C. : U.S. Department of Energy, 2013. DOEHDBK-1092-2013 . 


\section{APPENDIX A: SUPPORTING INFORMATION}

This appendix provides supporting information and ancillary data to the results presented in Chapter 4 and Chapter 5. Primary, these data are given to support the conclusions made in the main body of the text. Also included in this appendix are data which, while not strictly germane to the main discussion, provide the data-driven motivations for various process modifications and approaches to the data analysis.

\section{§A.1 Supporting Information for Section $\$ 4.1$}

This section includes supporting information for Section $\$ 4.1$ in the main text. Recall, Section $\$ 4.1$ discusses the AFM, XPS and Raman characterization of the films produced by the SOP process.

\section{§A.1.1 Additional Characterization of As-Received 6H-SiC Wafer by AFM}

Figure A.1 illustrates the basic manufacturing steps which lead to the off-axis nature of $\mathrm{SiC}$ wafers and the origin of the atomically stepped surfaces. Growth begins from a seed crystal in a highly controlled environment. After growth is completed a single large, high quality crystal ingot is produced. The ingot is then diced into sections which are then chemo-mechanically polished into flat wafers. Although ideal crystal growth occurs purely epitaxially, real growth occurs slightly off axis. The dicing and polishing steps also contribute to the off-axis nature of the wafers, in that the cuts and polished surfaces are not purely perpendicular to the growth direction. During the polishing step, the wafers are uniformly flattened on the wafer scale. This exposes the atomic steps, which protrude out of the wafer surface. By measuring the angle between the step terraces and an imaginary $\mathrm{x}$-axis (horizontal direction) the effective degree of off-axis can be determined. There are two ways this can be conceptually view, as shown in Figure A.1(C). The first orientation (referred to as 'surface-leveled'), is such that the entire wafer surface is leveled (the wafer is lain flat), resulting in the atomic steps angling out from the surface. This orientation presents the surface such that the edges of each crystal step are all at the same height (and thus, the surface, taken as a whole, is leveled). The second orientation (referred to as 'facet-leveled'), tilts the entire wafer such that all the parallel step terraces are horizontally aligned. In this case, 
the atomic terraces are parallel to the horizontal direction, producing a stair-stepped surface. All equivalent angles are shown in Figure A.1(C).

(A)
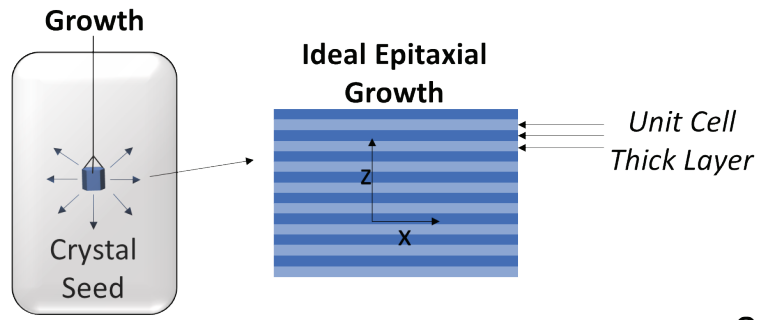

(B)
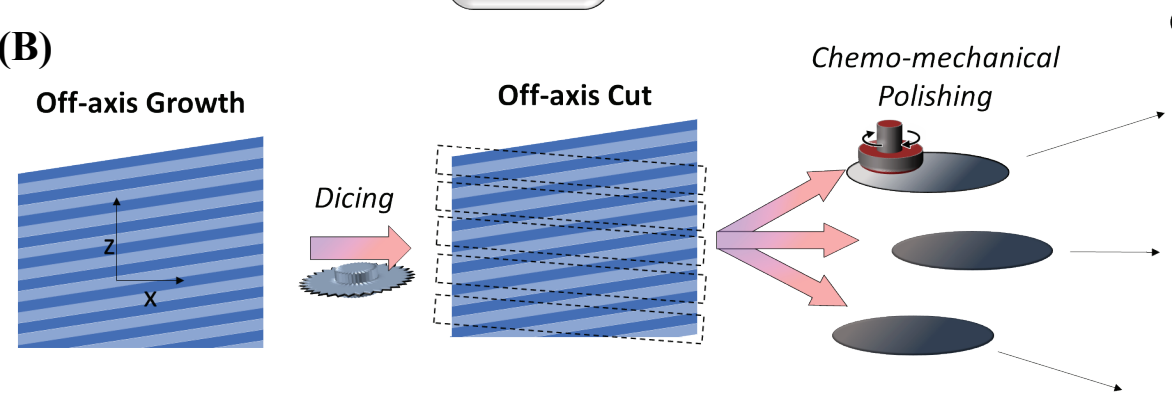

Off-axis Growth + Off-axis Cut

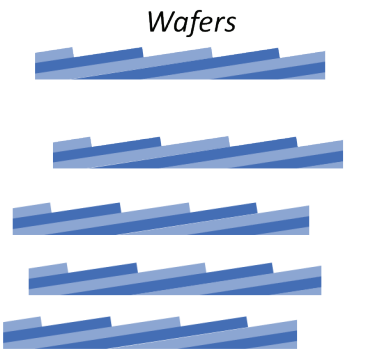

(C)
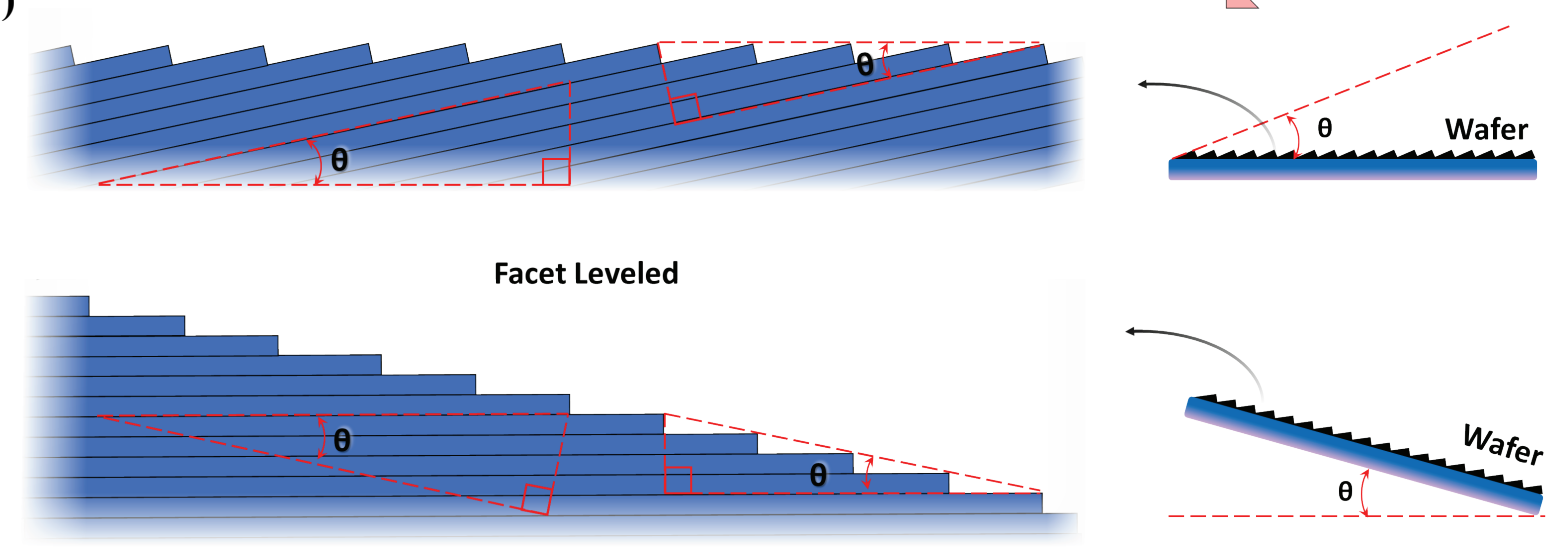

Figure A.1: The wafer manufacturing process and the origin of the atomically stepped surface. (A) Ideal fully epitaxial crystal growth. (B) Actual growth occurs slightly off axis. The wafer dicing from the master ingot is also slightly off axis. The chemo-mechanical polishing step flattens the wafers such they the wafer as a whole is geometrically uniform. (C) Measurements of the wafer miss cut angle. All angles marked $\theta$ are equivalent.

The as-received $6 \mathrm{H}-\mathrm{SiC}$ wafers used in this work were characterized using AFM. Figure A.2 shows AFM images collated from six randomly chosen locations across the surface of a $1 \mathrm{~cm}^{2}$ diced, uncleaned $6 \mathrm{H}-\mathrm{SiC}$. The atomic steps are clearly seen, as well as debris remaining from the dicing process. The step height and growth direction vary across the surface. These images have been surface-level; this allows for the entire surface to be within the range of the color bar and is a more practical view of the surface. This allows all surface feature to be revealed within a narrow 
color bit depth window. However, this view can be more conceptually difficult to interpret in a crystallographic context.
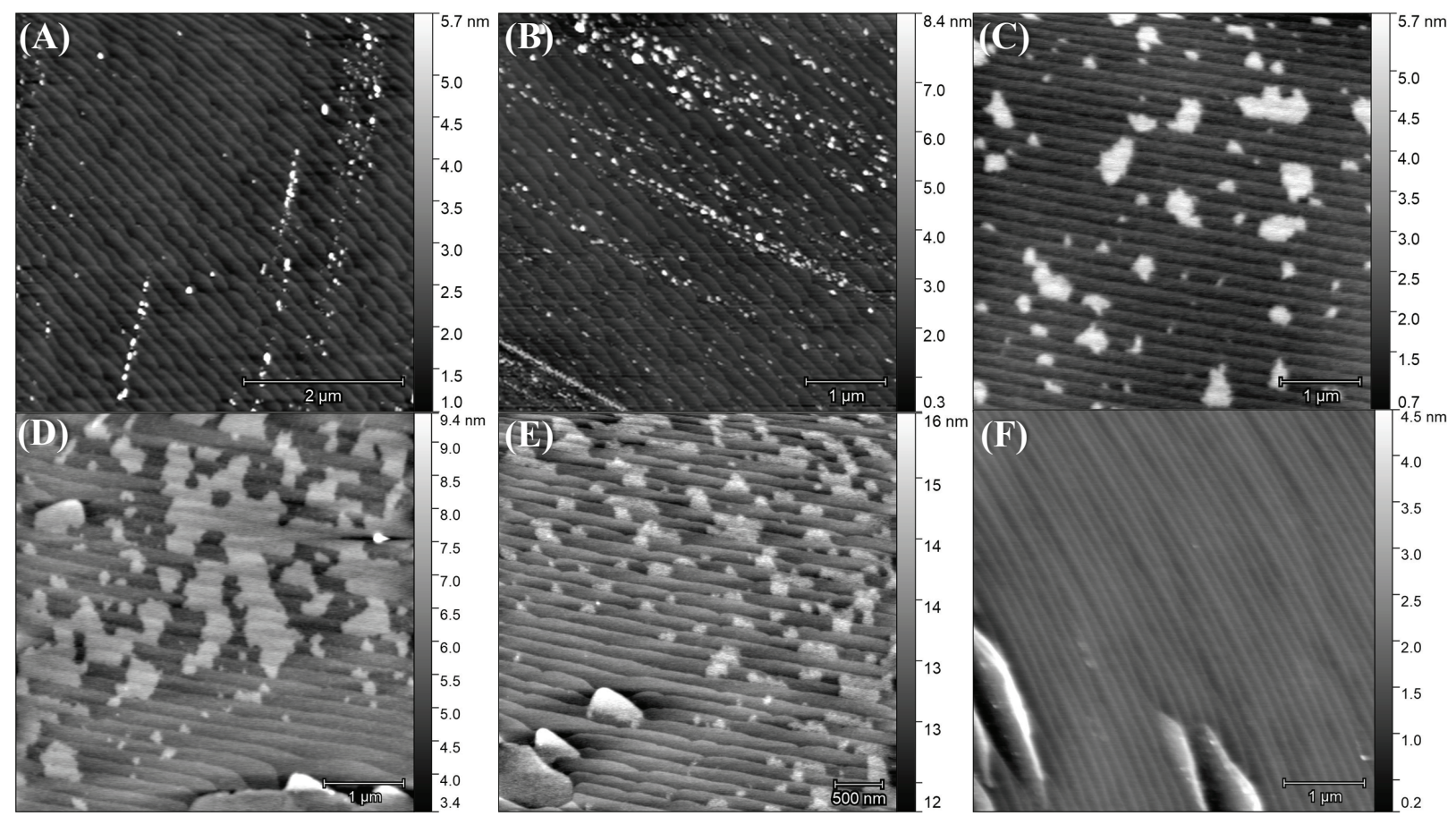

Figure A.2: AFM images collected from six randomly chosen locations across the wafer surface. The atomic steps are clearly seen, as well as polishing debris.

Figure A.3 show AFM images collected over an area containing a high concentration of crystal edge defects. These areas are not typical of the average surface; however, they are present on all wafers analyzed. The arrows in Figure A.3(C) indicate pores present in the blade-like defects. These defects are notable because they appear to be completely removed during the ICP/RIE etch and do not affect the graphitization process. This is presumably due the aggressive

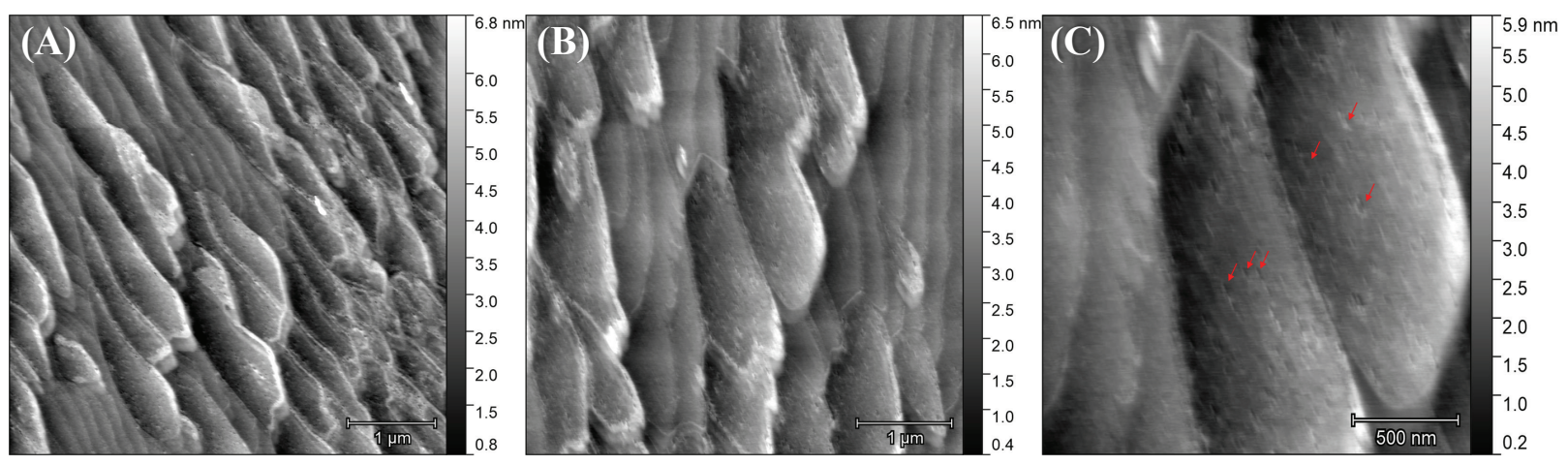

Figure A.3: AFM images showing an area containing a high concentration of crystal edge defects. As indicated by the red arrows, the blades contain pores at least $0.4-0.6 \mathrm{~nm}$ deep. However, these are not fully resolved by the $10 \mathrm{~nm}$ AFM tip. 
nature of the etch and the large amount of material removed. Additional step analysis is shown in Figure A.4. The step height indicates a small degree of step bunching.
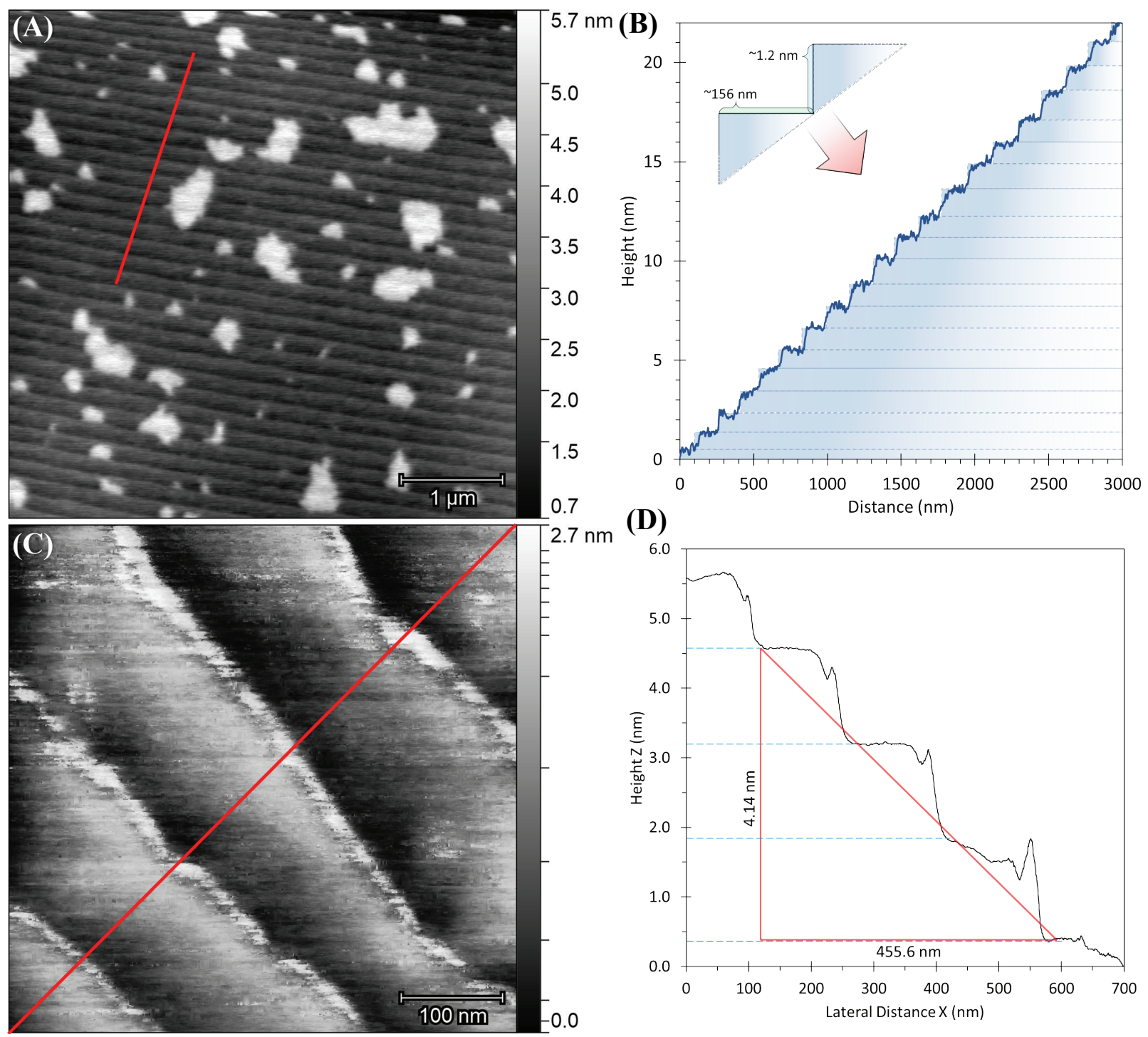

Figure A.4: AFM showing the atomically stepped surface. (B) Line profile corresponding to the red line in (A). (C) High magnification AFM image of the surface. (D) Line profile corresponding to the red line in (C). 


\section{§A.1.2 Motivation for including a rest step during the ICP/RIE step in the standard synthase process:}

The XPS data collected from a sample produced using the standard process but without a rest step during the ICP/RIE etch is shown in Figure A.5. The effect of not including the rest step appear to not be statistically significant when compared against the results in Figure 4.11.

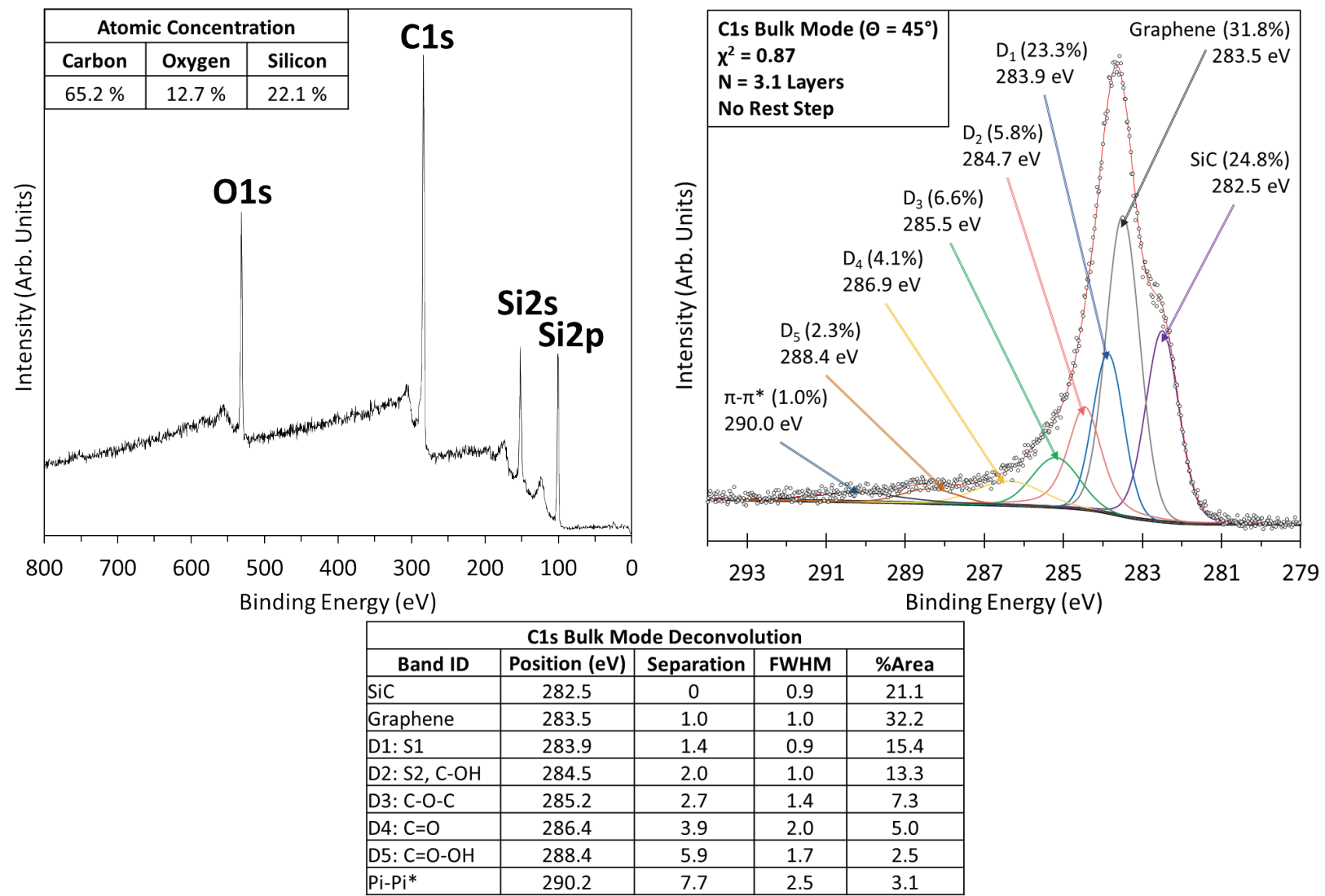

Figure A.5: XPS spectra from a sample produced using the standard process but with no rest step during the ICP/RIE. This yielded good films, but not significantly better than films which incorporated a rest step during the etch.

\section{\$A.1.3 Motivation for not using helium cooling flow during the ICP/RIE step in the standard synthase process:}

Two samples were produced using the identical standard synthesis process except for the use of a helium backing flow used during the ICP/RIE etch to cool the carrier wafer. A comparison of the C1s XPS results are shown in Figure A.6. 


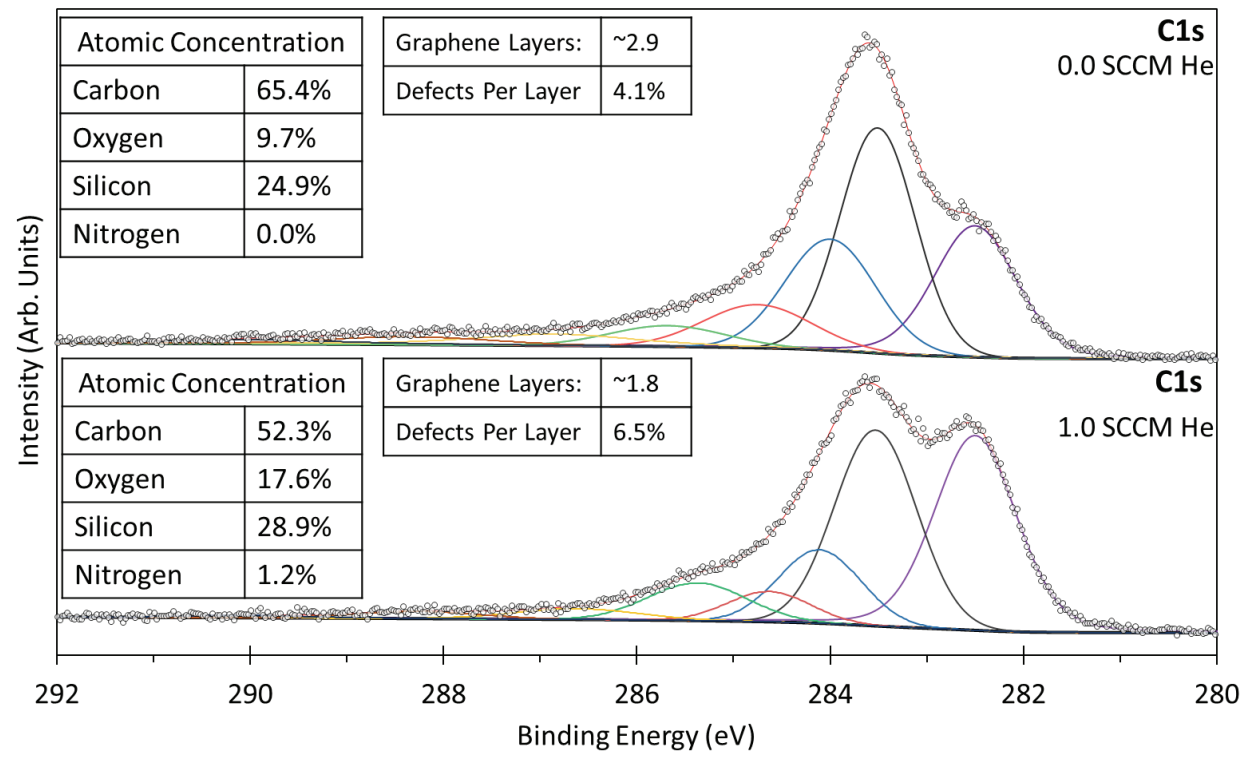

Figure A.6: The C1s region of a sample where no helium flow was used (top) and a sample where the standard helium flow of 1.o SCCM was used. Running the etch process without helium flow resulted in more carbon layers, will lower defect less and less nitrogen contamination. For simplicity, the blue curve combines the $\mathrm{S} 1$ and $\mathrm{S} 2$ peaks.

As can be seen, slightly higher quality films (more graphene, less defects per layer and lower $\mathrm{N}_{2}$ contamination) are produced when no helium flow is used. The thicker carbon films are likely a result of an increased etching effect due to a more reactive plasma being generated. By not leaking He into the chamber to cool the carrier wafer, and thereby not diluting the chemically active component of the plasma, a more energetic $\mathrm{CF}_{4}$ plasma is formed. The He gas line, which is plumbed though a separate manifold and supply line from the process gases, is eliminated as a potential source of contaminants as well. Furthermore, the gas itself provides an additional source of trace contaminants, which is eliminated by forgoing the He cooling flow. Additionally, by not running with a cooling He flow, the samples undergoing the etch reach much higher temperatures than they otherwise would. Exactly how much hotter the samples get is not known. However, sample temperatures reach high enough to bake the temperature resistant boron nitride CoolGrease (CGR7016), used to anchor the samples to the carrier wafer, into a brown powder. A final note is that the higher sample temperature reached by not using a cooling gas may be beneficial to the process. The increased substrate temperature would increase the chemical reactivity of the surface during the chemical etch, as well as increase the removal rate of volitional etchant species and result in an initial annealing of the film. This may explain the improvement in the XPS spectra shown in Figure A.6. 


\section{§A.1.4 Motivation for the preconditioning of the etch chamber before the ICP/RIE step in standard synthase process:}

A series of six $6 \mathrm{H}-\mathrm{SiC}$ samples were sequentially run though the ICP/RIE etch and APRTA steps. The samples were then analyzed by XPS to determine the effect of ICP/RIE chamber aging on the synthesized samples, as shown in Figure A.7. Repeated etching using $\mathrm{CF}_{4}$ leaded to a decrease in the total number of carbon layers produced and an increase in the per-layer defect concentration, simultaneously. Ultimately, it was determined that material build up on the chamber walls provide a source of condemnation (contributing to the increase in defect concentration) and detunes the electrodynamics of the chamber (decreasing the number of carbon layers produced).

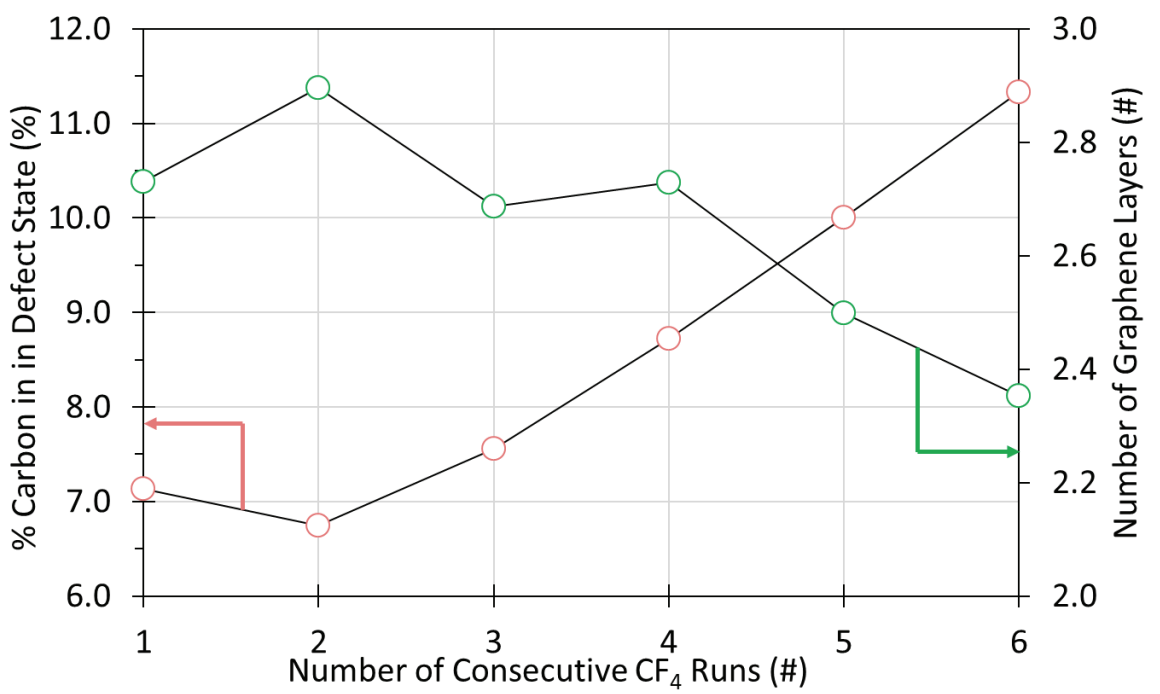

Figure A.7: The effect of consecutive ICP/RIE etch runs (no $\mathrm{O}_{2}$ cleaning or $\mathrm{CF}_{4}$ conditioning steps between runs) on the amount of defective carbon and the number of graphene layers, as determined by XPS analysis of the C1s region. A clear trend is seen in that more defects and less graphene is formed as the number of successive runs increases. Including an $\mathrm{O}_{2}$ clean and one $\mathrm{CF}_{4}$ run (with not sample) was determined to be the best balance between a steady DC bias and chamber performance. This corresponds to operating the chamber with a sample loaded at the run \#2 point in the figure, which yielded the most layers of defect free graphene.

Visual inspection of the ICP region through the main chamber view port reviled arcing between the main plasma cloud and the chamber walls. The arcing frequency was observed to increase with additional $\mathrm{CF}_{4}$ runs. The material which is deposited on the chamber walls is insulative (determined by charging during XPS analysis). The arcing results from electrical charge buildup in the plasma cloud, which is no longer neutral. This is because free electrons cannot be as easily blead to ground through the walls, which are coated with an insulating film. More importantly, because the plasma cloud is no longer neutral, the potential developed between the 
plasma and the sample is reduced. Thus, the -DC bias developed on the sample is decreased, resulting in less RIE action and few carbon layers produced.

This conclusion is supported by the correlation between the number of $\mathrm{CF}_{4}$ runs and the amount of material build up inside the chamber, observed during the routine manual cleaning of the chamber. Based on the results shown in Figure A.7, it was determined that one $\mathrm{O}_{2}$ clean and one $\mathrm{CF}_{4}$ conditioning step (standard process, but with no sample) were to be performed before each ICP/RIE run in which a SiC sample was to be processed. The chamber detuning can be observed by a gradual drop in the -DC bias from run-to-run (as well as over the duration of a single run) as material begins to build up. The $\mathrm{O}_{2}$ clean between runs removes some of this material, but not all of it (hence the required manual cleanings).

XPS analysis of the material which builds up on the ICP/RIE chamber walls, shown in Figure A.8, show the presence of fluorene. Combined with the observed correlation between the number of etch runs and the amount of material, this indicates the material build up to be a result of fluorine etching. This material is most likely a combination of a fluorocarbon polymer film and aluminum fluoride polymer film, which both are known to be products of fluorine-based plasmas. ${ }^{(689)(690)}$ Furthermore, it has been shown also that fluorocarbon polymer film forms preferentially on the grounded components within the etching chamber, consistent with these observations. ${ }^{(689)}$ Therefore, while additional $\mathrm{CF}_{4}$ conditioning runs stabilize the -DC bias somewhat, only a single $\mathrm{CF}_{4}$ run was used to condition the chamber. 

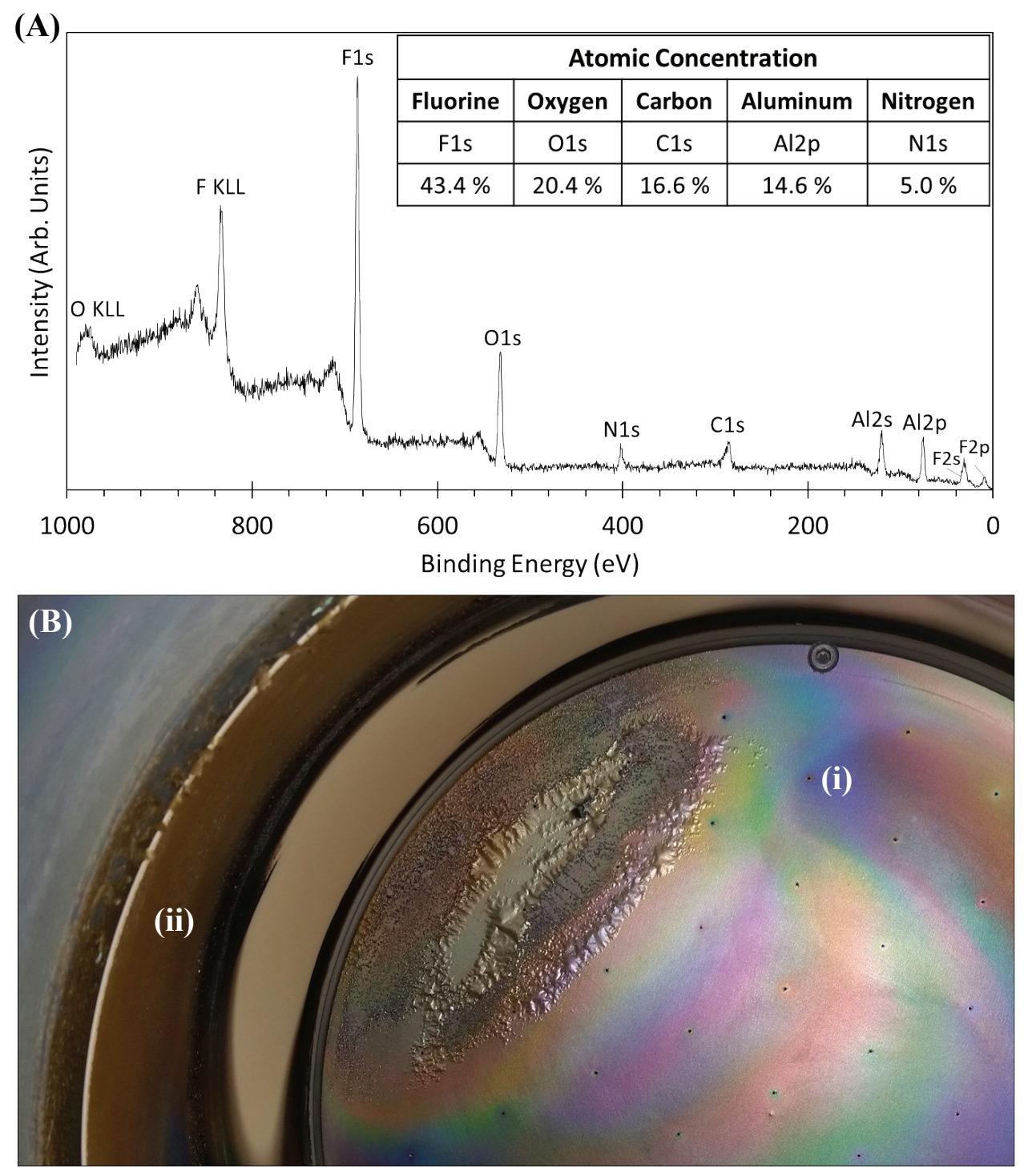

Figure A.8: (A) XPS data collected from the material which builds up on the ICP/RIE chamber walls. The presence of fluorine indicated the material buildup is related to the $\mathrm{CF}_{4}$ etching. (B) Picture of the upper lid of the process chamber showing the showerhead (i) and upper chamber walls (ii). The material buildup is clear. This material was removed and analyzed give the spectra shown in (A). XPS results and literature reports indicated the material is most likely a combination of a fluorocarbon polymer film and aluminum fluoride polymer film. (689) (690) 


\section{§A.1.5 Motivation for the two peak $G$ fit of Raman spectra:}

Using a single $G$ peak to fit the Raman spectra from sample produced using the standard process results in a poor fit, as shown in Figure A.9. Thus, a bimodal G peak is required for a good fit of the spectrum.

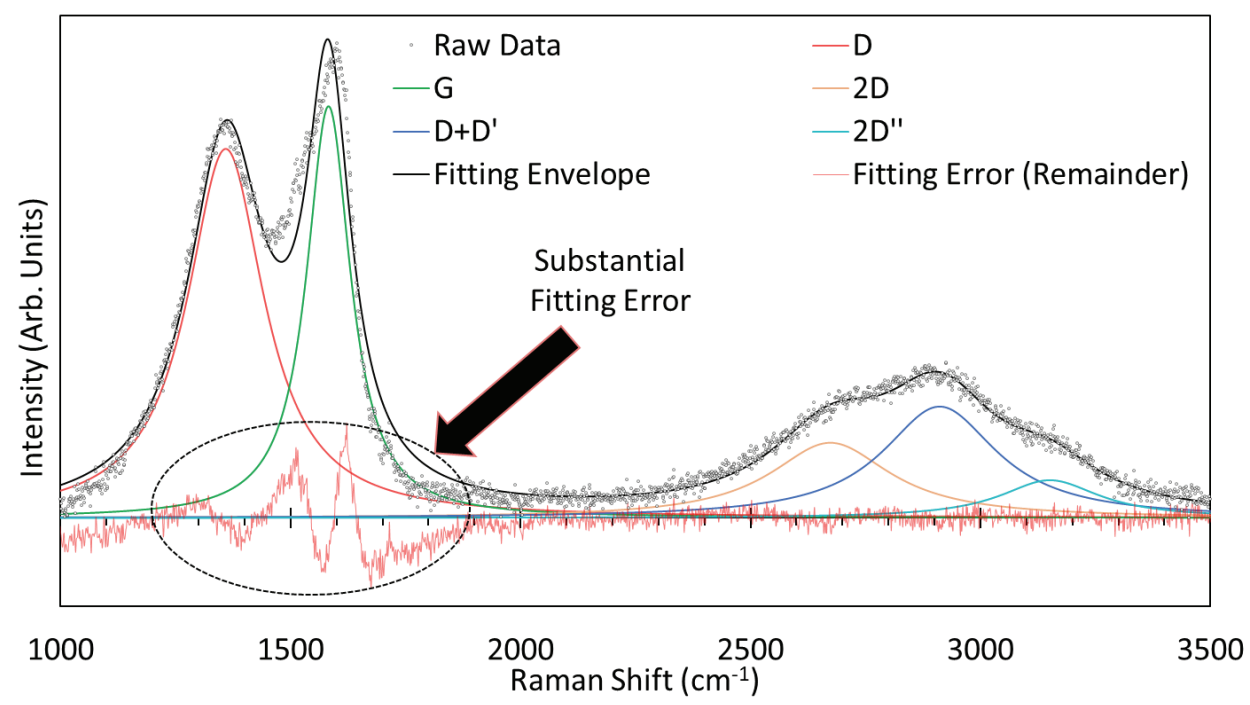

Figure A.9: The fitting results using a single G peak fit. Substantial errors result and a poor fit is achieved.

\section{§A.1.6 Raman spectra of an etched only sample (no AP-RTA):}

The Raman spectrum taken from a sample which has undergone only the standard etching process (no thermal annealing of any kind) is shown in Figure A.10. The spectrum is consistent with a largely amorphous surface. However, the films must have a semblance of order to enter the coherent vibrational states required to produce a Raman signal.

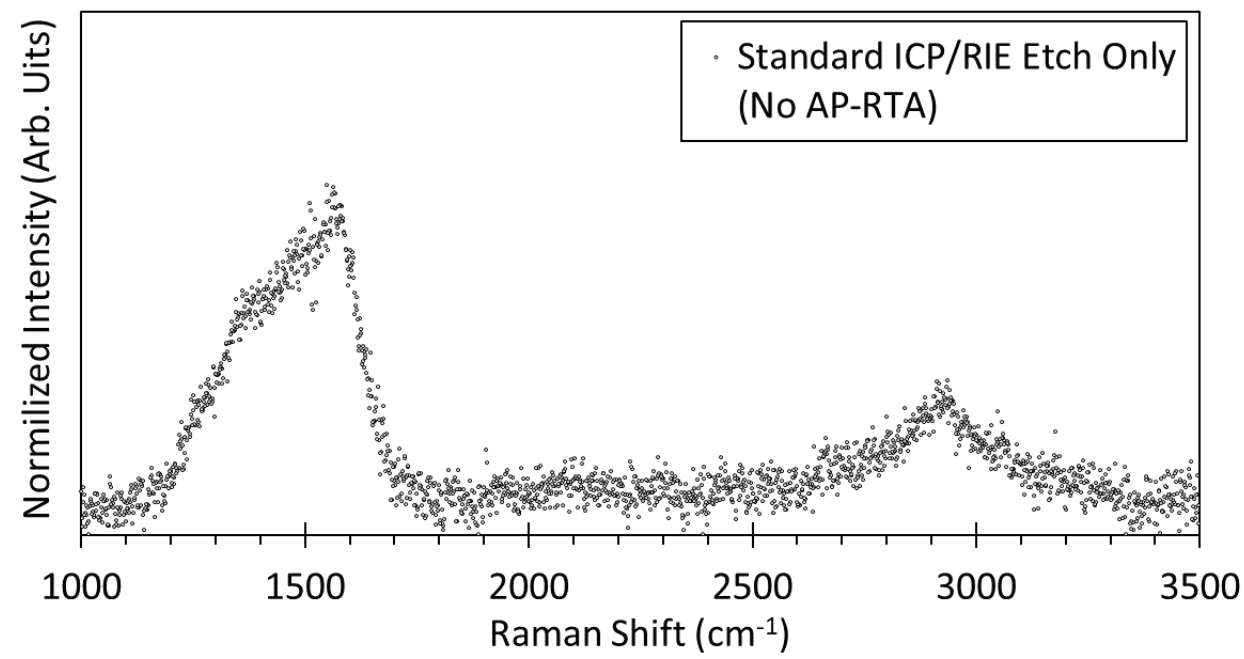

Figure A.10: Raman spectra collected for a sample which has been etched using the standard process but has not undergone the AP-RTA process 
This spectrum leads to two possible interpretations regarding the buffer layer. First, this spectrum may be reflective of semi-ordered, tethered, interfacial carbons of the film. These carbons may be the precursor to the buffer layer, which then becomes more fully formed during the anneal. This interpretation would mean that the tethering bonds of the buffer layer are formed during the annealing step.

Alternatively, this spectrum may be the result of a very highly tethered film, whose vibrational states are highly suppressed, resulting in this weak spectrum. Then, during the anneal, both the amorphous component and highly tethered carbons crystallinity increase, resulting in the Raman spectra typical of the SOP samples. This second interpretation would essentially mean that the films exit the etch already highly tethered. In both cases, the order imparted to these carbons would be derived from the periodicity of the tethering sites, which would be the silicon terminus sites of the substrate.

More study is required to fully elucidate the specifics of this transitional (post-etched and pre-annealed) film. As mentioned in Section $\$$ 7.2.2, heating during the etching process may provide valuable insight.

\section{§A.1.7 $\mathrm{CF}_{4}$ and $\mathrm{Cl}_{2}$ SOP Etch on Silicon}

Figure A.11 shows XPS survey spectra for three samples. First, the as-received silicon wafer. Second, a silicon wafer processed using the SOP process, but using $\mathrm{Cl}_{2}$ gas in the ICP-RIE step. Third, a silicon wafer fully processed using the full SOP, using $\mathrm{CF}_{4}$ plasma. The important note here is that approximately the same amount of carbon is present in all three cases. This carbon is likely atmospheric in origin (adventitious carbon) and is thus present on all samples. This shows that the carbon films produced during the SOP process must originate from the carbon in the substrate and not the etching gas; otherwise the third sample would have a carbon content consistent with the $\mathrm{SiC}$ samples processed using the $\mathrm{SOP}$. The $\mathrm{Cl}_{2}$ data is included because, in 
previous work, $\mathrm{Cl}_{2}$ was used as the etchant gas to produce graphene films on $\mathrm{SiC}$. Therefore, these data demonstrably show that in the halogen etch, the carbon source is always from the substrate.
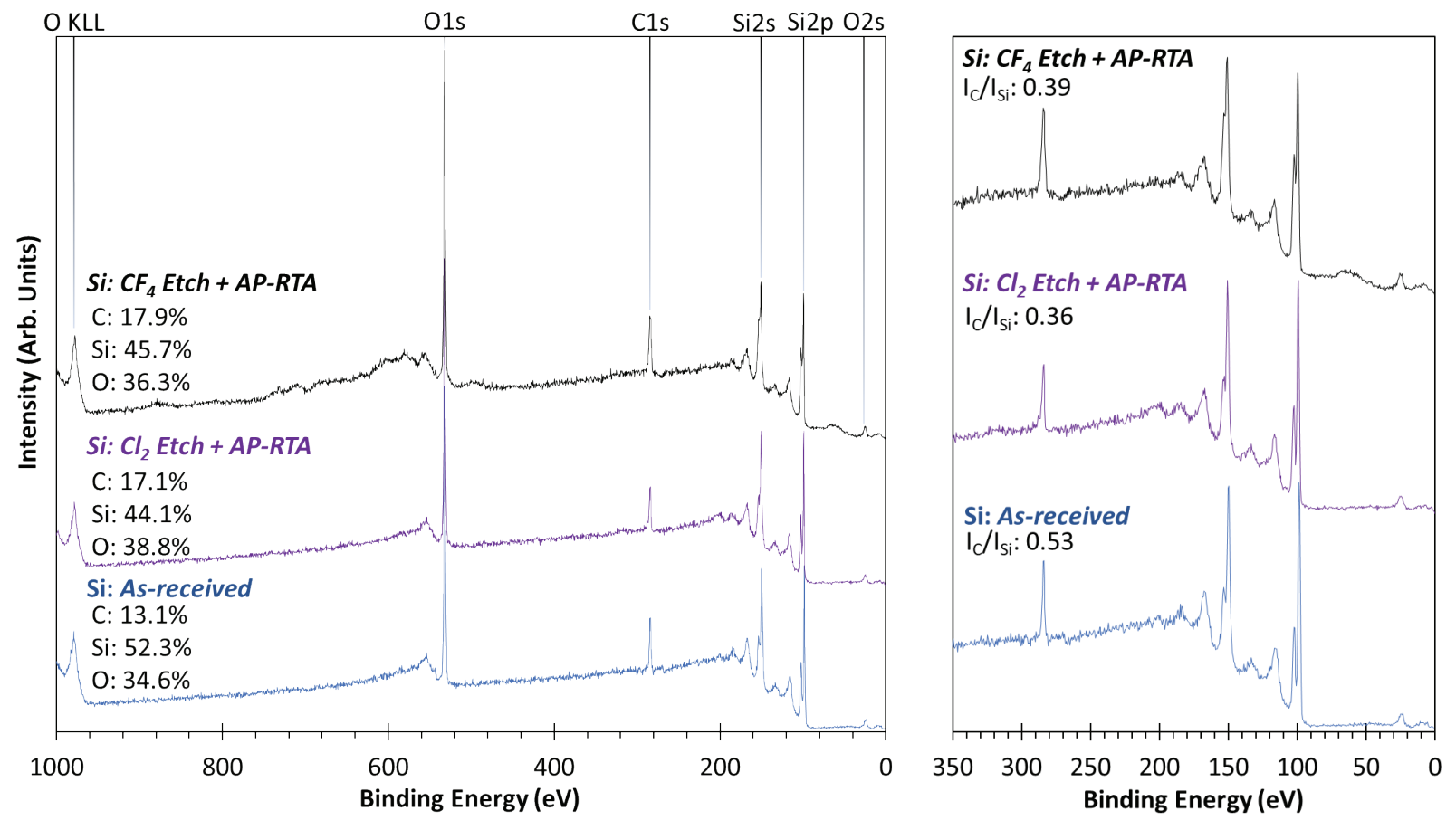

Figure A.11: XPS spectra of three different silicon test samples. They are: the as-received silicon, silicon which has undergone the SOP process using $\mathrm{Cl}_{2}$ gas as the halogen plasma source and the standard SOP process using $\mathrm{CF}_{4}$ gas as the halogen plasma source. The relative atomic concentrations are annotated. Also shown is a more detailed view of the same data. The ratio between the C1s and Si2p peaks are annotated.

\section{§A.2 Supporting Information for Section $\$ 4.2$}

This section includes supporting information for Section $\$ 4.2$ in the main text. Recall,

Section $\$ 4.2$ discusses the results of the parametric investigation into the graphene synthesis process.

\section{§A.2.1 DC bias as a function of etch time, at 600W ICP and various RIE powers}

The variation of the -DC bias can be seen a function of etch time. Figure A.12 plots this data for eight different RIE power setpoints. The variability in the DC bias (indicated by the error bars, as well as the overall trends) is reflective of the stability of the plasma at each setpoint. For an ICP power of $600 \mathrm{~W}$, higher RIE powers lead to larger fluctuations in -DC bias. 


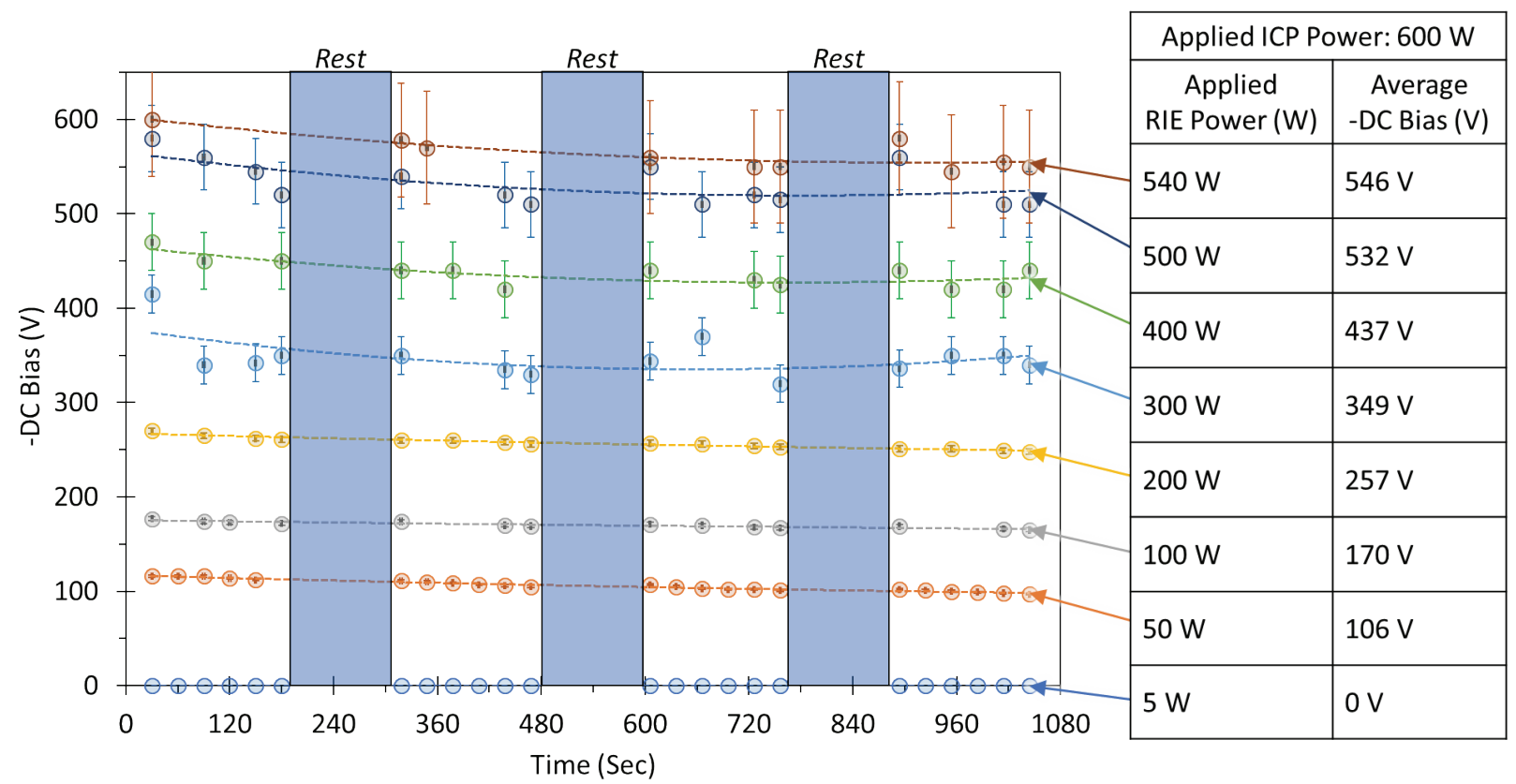

Figure A.12: The variation of the -DC bias can be seen a function of etch time.

\section{§A.2.2 Heat maps of the number Carbon Layers and percent Buffer Layer taken as a function of ICP and RIE Powers.}

Figure A.13 maps the ICP power against the RIE power (as opposed to DC bias, as is shown in the main text) as a function of the number of carbon layers and as the percent carbon as buffer layer in the film. Although the RIE power is the controllable parameter, the DC bias is the more meaning full parameter. Furthermore, depending on the conditions of the chamber, multiple
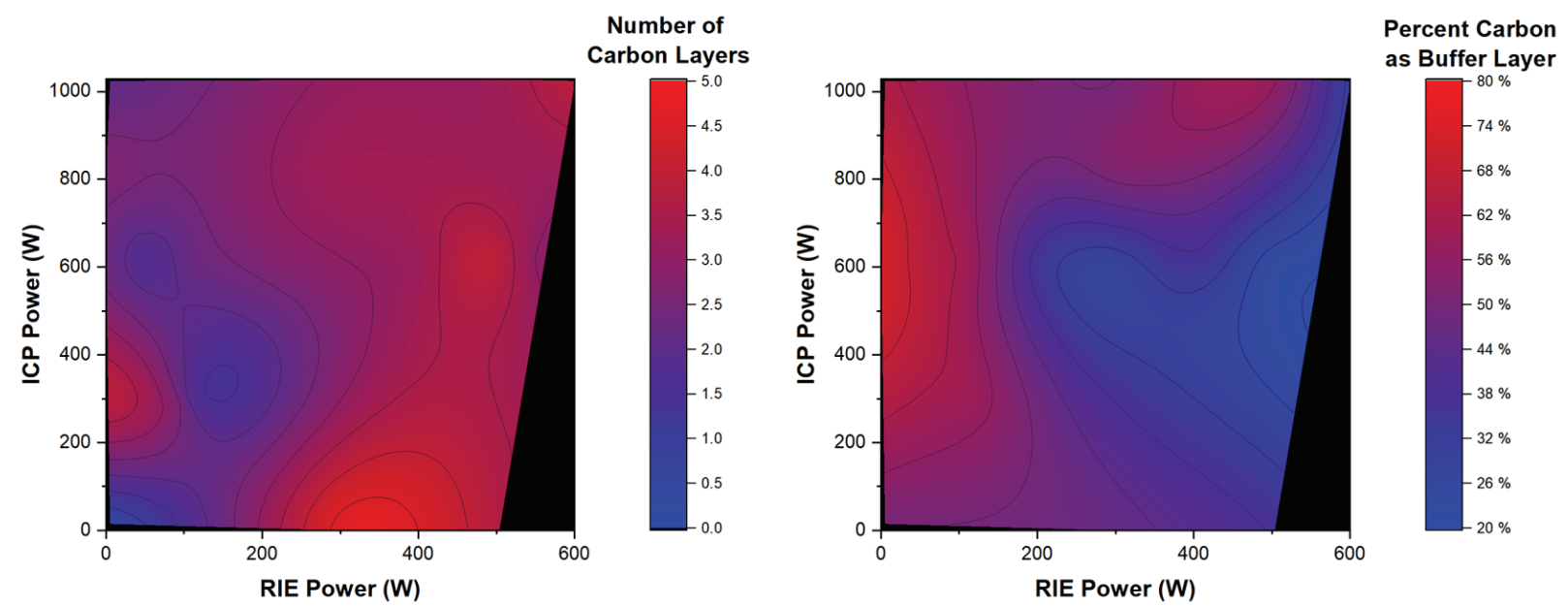

Figure A.13: Heat maps of the number of carbon layers and the percent carbon which is participating in the buffer layer across the rang of ICP and RIE powers. 
RIE powers will produce the same DC bias, or, depending on chamber age, the same RIE power will not always result in the same DC bias. Thus, the DC bias is the more reliable parameter from which to draw correlations from. Furthermore, it is the DC bias which set the ion energy, which determines many aspects of the etch and the RIE power sets the DC bias (i.e. the DC bias is further 'downstream' and has a more direct effect on the film than the RIE power form which it is derived).

\section{§A.2.3 Raman and XPS of SOP samples: D/G ratio and number of carbon layers}

The analysis shown in Figure A.14 indicated that there is no correlation between the D/G ratio (Raman analysis), amount of buffer layer carbon (XPS analysis), the Raman D peak center (Raman analysis) or D peak FWHM (Raman analysis) and the number of carbon layers (XPS analysis).
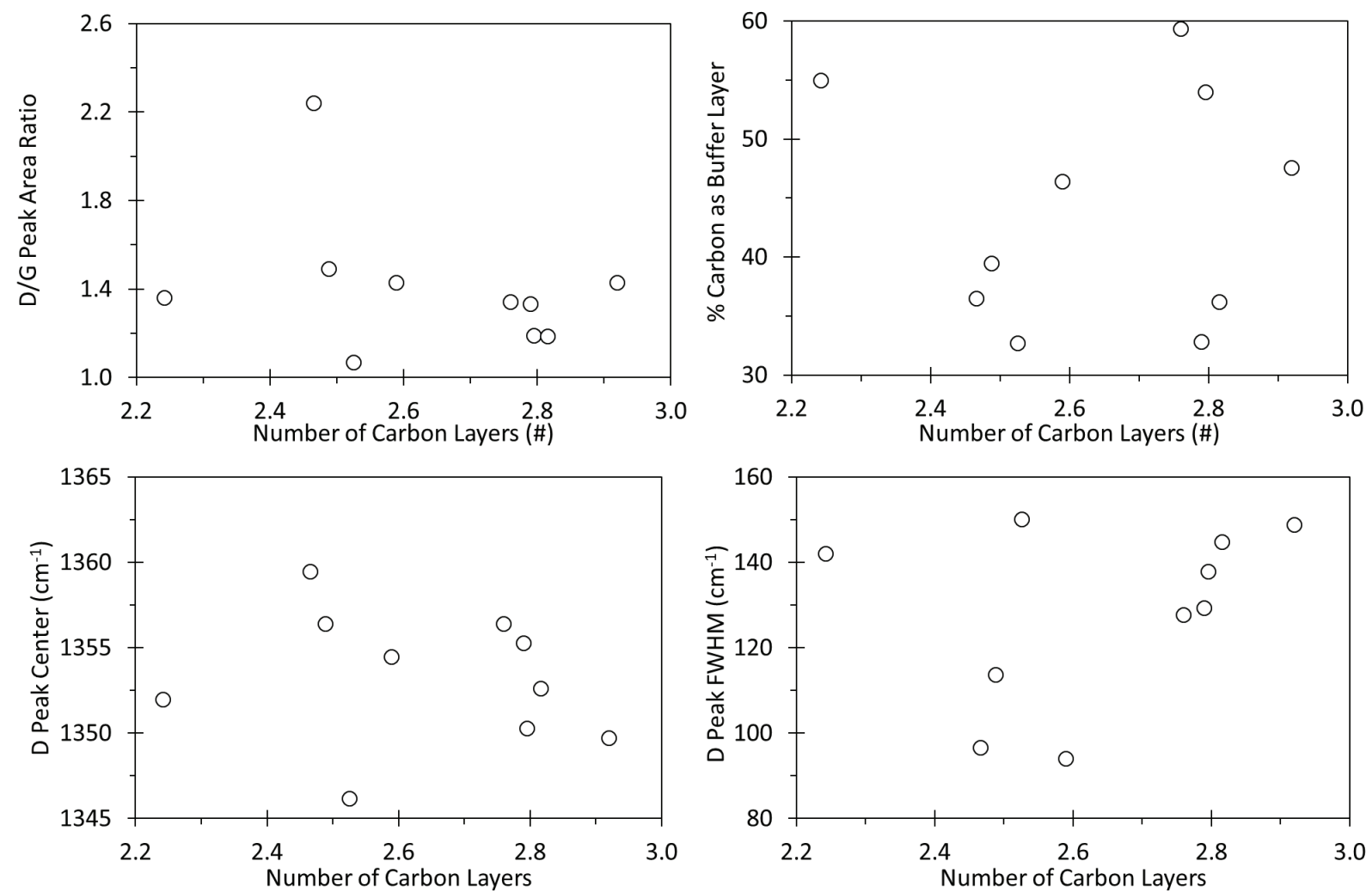

Figure A.14: Plots of the D/G peak area ratio, \% carbon as buffer layer, D peak position and D peak FWHM plotted as a function of the number of carbon layers. No correlation was found.

\section{§A.2.4 Motivation for taking the chemical defect concentration on a per layer basis}

Figure A.15 shows the per layer defect density plotted as a function of the number of layers.

No trend is seen, which indicates that the defect density is not tied to the number of layers. Thus, 
the defect density of the films produced must be taken on a per layer basis before a meaningful comparison can be made between samples which do not have the same number of layers. Otherwise, the film with more layers will always show a higher amount of defects by XPS analysis. Furthermore, for any study which shows a trend involving a changing chemical defect concentration, it can be concluded that the trend does not simply track with the number of carbon

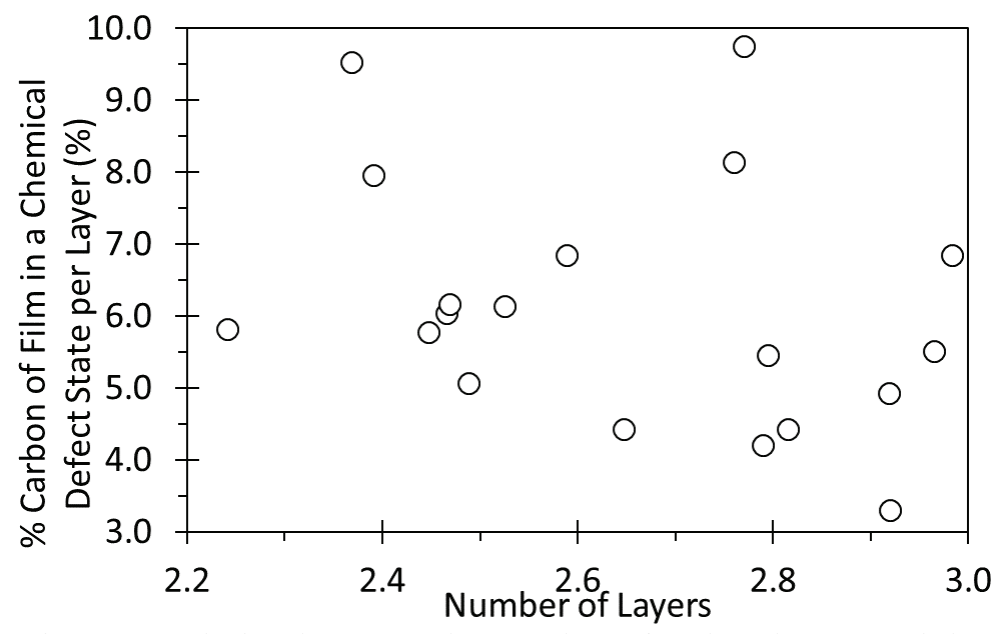

Figure A.15: There is no correlation between the number of carbon layers and the chemical defect density of each layer. This indicates that all layers are equally defective (i.e. the $2^{\text {nd }}$ layer has as many chemical defects as the zed layer).

layers produced and thus must be a function of another parameter.

\section{\$A.2.5 AP-RTA max temperatures vs. time at temperature and taking the buffer layer on a per layer basis}

Although a rigorous attempt was made to limit the number of dependent variables, by the natural fact that the heating and cooling rates are constant between samples, samples exposed to higher temperatures also experienced more time at elevated temperature. Simply taking the maximum temperature as the sole influencing parameter does not capture the amount of time the samples spend at temperature and their effects on the film. This leads to the natural question whether the observed trends are a function of the high temperature exposure or on the extend time at elevated temperature. In order to capture these effects, the area under the annealing curves are integrated. While this does lead to a somewhat clumsy dimensional analysis (the units of the integrated RTA curve area are ${ }^{\circ} \mathrm{C} \cdot \mathrm{Sec}$ ), general trends in film properties are found when they are plotted against the integrated area. Conducing the analysis in this way captures both temperature effects and time effects but weights them equally (increasing the time by $1 \mathrm{sec}$ is indistinguishable from increasing the temperature $1^{\circ} \mathrm{C}$ ). 
The comparison between the maximum temperature encountered during the AP-RTA and the corresponding area under the annealing curve is shown in Figure A.16, for all samples used in the AP-RTA temperature study. The area under the curve was calculated using a simple trapezoidal integration on the measured data. The curves were integration from the time when the sample reached $200^{\circ} \mathrm{C}$ during heat up to the time the samples passed $200^{\circ} \mathrm{C}$ during cool down. The slight variation in heating and cooling rates account for the slight difference in area for the similar temperature.

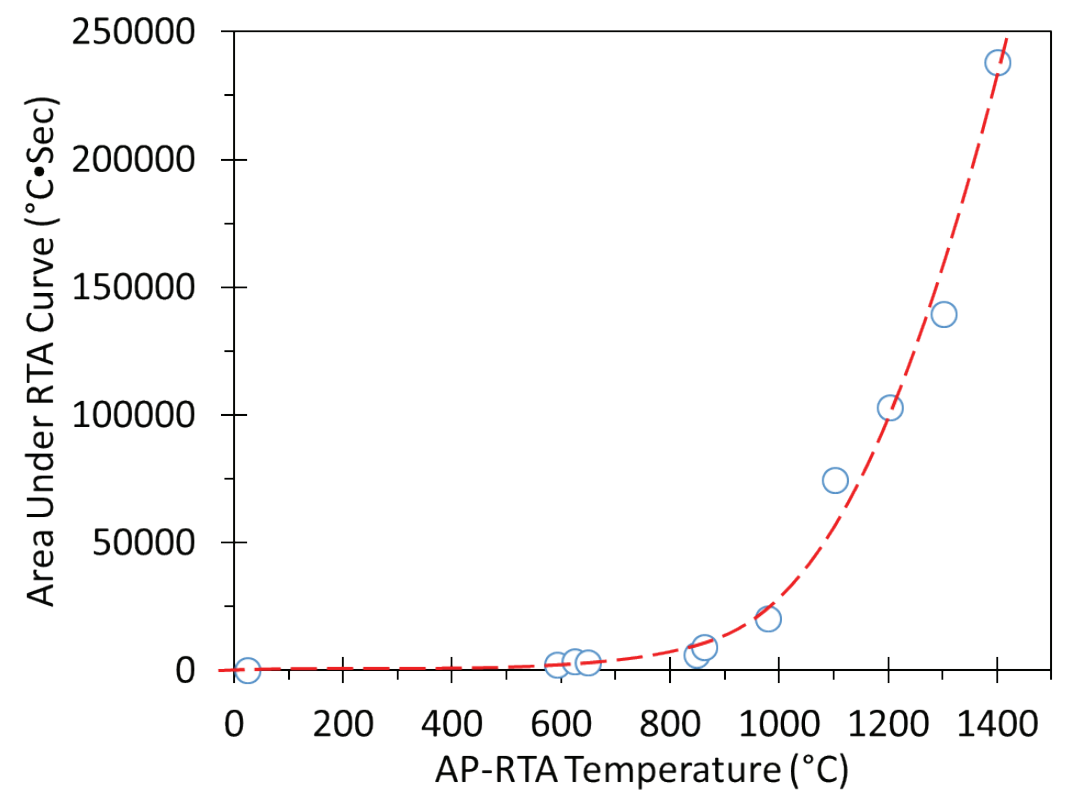

\begin{tabular}{|c|c|}
\hline $\begin{array}{c}\text { Max AP-RTA } \\
\text { Temperature }\left({ }^{\circ} \mathbf{C}\right)\end{array}$ & $\begin{array}{c}\text { Area Under } \\
\text { RTA Curve }\left({ }^{\circ} \mathbf{C} \cdot \mathbf{S e c}\right)\end{array}$ \\
\hline 25 & 0 \\
\hline 592 & 2350 \\
\hline 626 & 3622 \\
\hline 649 & 3016 \\
\hline 848 & 6078 \\
\hline 862 & 9200 \\
\hline 979 & 20325 \\
\hline 1076 & 64665 \\
\hline 1102 & 54064 \\
\hline 1106 & 74572 \\
\hline 1202 & 124524 \\
\hline 1203 & 102774 \\
\hline 1302 & 143108 \\
\hline 1303 & 139494 \\
\hline 1400 & 237917 \\
\hline 1400 & 322087 \\
\hline
\end{tabular}

Figure A.16: Tabulated and plotted area under the curve for each temperature. Obviously, but importantly, a linear incase in the maximum temperature leads to an exponential increase in the time at a sample is exposed to elevated temperature.

While the amount of time at temperature does have an effect, the trends are most clear when plotted against the maximum RTA temperature. Thus, it is concluded that it is the temperature (and correspondingly the thermal energy provided) that is the primary driving forces for the observed trends (and not as much the time at elevated temperature). Figure A.17 show plots of various parameters as a function of the area under the RTA curve. 
(A)

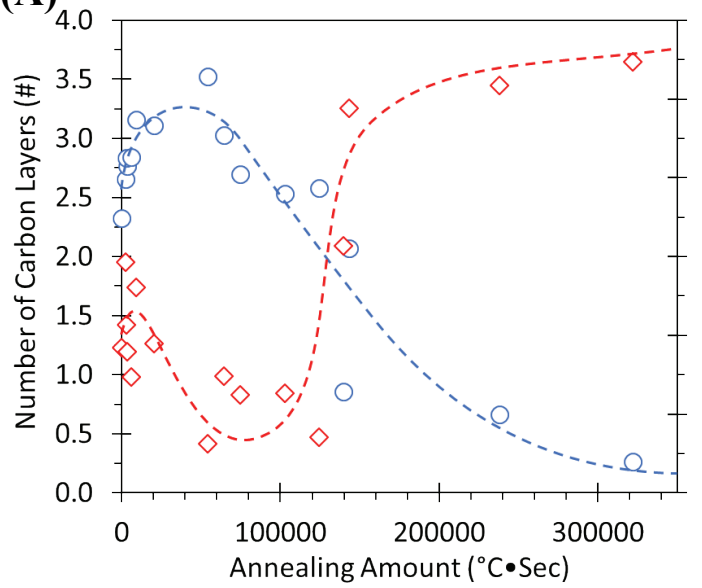

$75 \%$

$65 \%$

$55 \%$

$45 \%$

$35 \%$

$25 \%$

$15 \%$
(B)

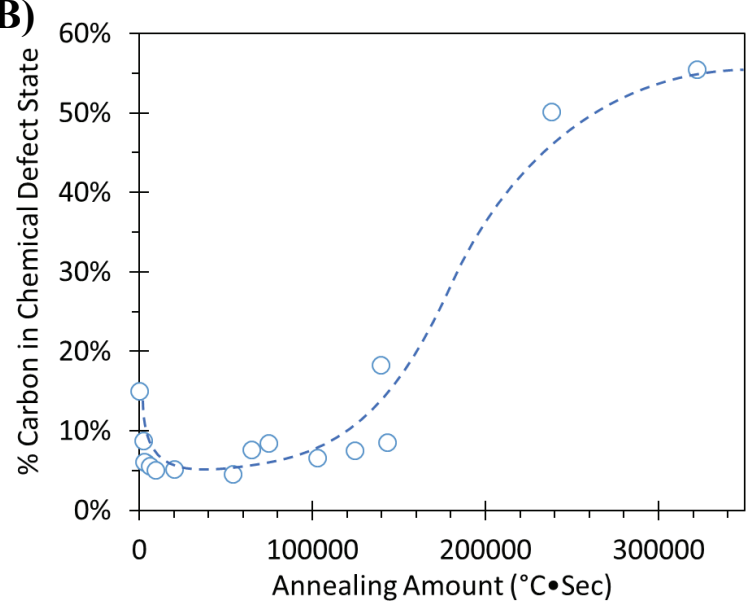

Figure A.17: Plots of various film parameters, taken as a function of the annealing amount (area under the RTA curve).

However, the ratio of buffer layer carbons to film carbons is one parameter which trends with the time-at-temperature more so than the maximum temperature. Figure A.18 plots the amount of buffer layer carbon divided by the amount of graphene layer basis (i.e. the ratio of buffer layer atoms to graphene layer atoms). Two important conclusions can be arrived at. First, the

(A)

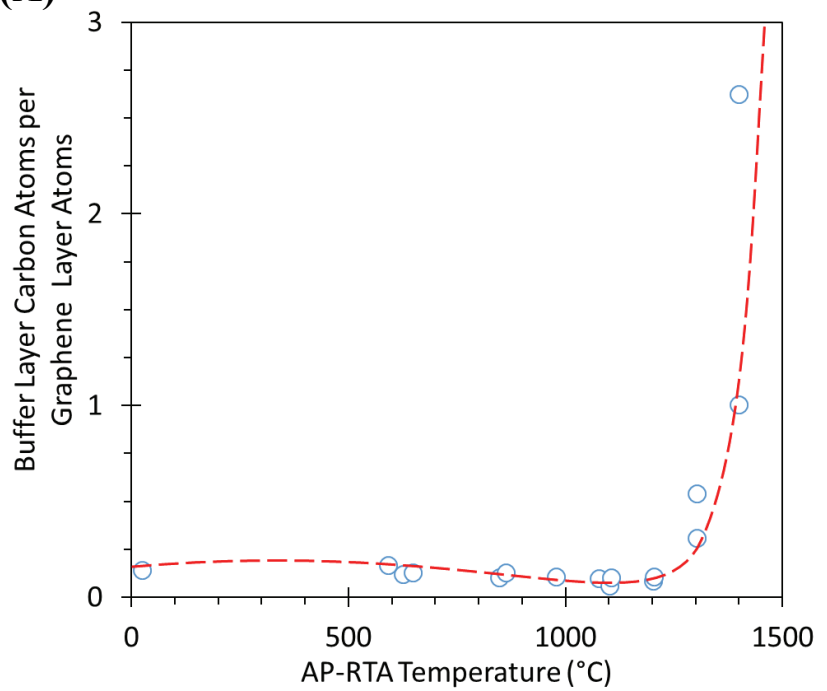

(B)

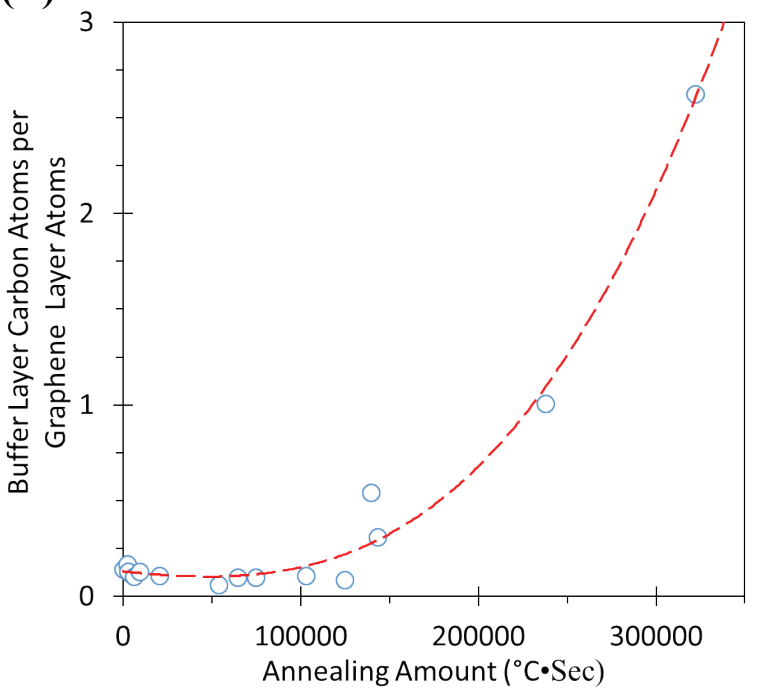

Figure A.18: (A) The number of buffer layer carbon atoms per graphene carbon layer atoms as a function of AP-RTA temperature (ratio of buffer carbon to graphene carbon atoms). It is clear that for the majority of temperatures, the buffer layer is largely independent of the number of layers. Given the nature of the buffer layer, this observation agrees with intuition. At high temperatures $\left(>1100^{\circ} \mathrm{C}\right)$ the carbon in the uppermost graphene layers are consumed by trace oxygen. Leaving films which are mostly buffer layer. (B) The same data, but plotted against the amount of annealing the samples experience (the integrated are under the annealing curve). It is unclear whether the large uptick in buffer layer (indicating the graphene layers has been consumed or burnt off) is due to the high temperature (A) or the extended time spent at temperature (B). Results from the dwell time studies indicated that it may be the latter. 
amount of buffer layer is largely independent of the number of graphene layers. Given that the buffer layer is simply an interfacial layer between the graphene layers and the SiC substrate, one would expect this to be the case. Second, at temperatures about $1100^{\circ} \mathrm{C}$, as the graphene layers are burnt off, only the buffer layer remains. Therefore, these films are made up of mostly buffer-layer carbon.

\section{§A.2.6 Atomic Concentrations during the AP-RTA process}

The elemental atomic concentrations, shown as a function of annealing temperature, can be found in Figure A.19. It is important to note that the atomic concentration is not as influenced by the densification of the overlayer film in the same why that the film thickness is. Given that the drop in fluorine concentration coincides with the increase in the carbon concentration, the absolute amount of carbon on the samples is largely constant up to $1100^{\circ} \mathrm{C}$, as would be expected. After $1100^{\circ} \mathrm{C}$ however, the amount of carbon decrease sharply. This coincides with an uptick in oxygen and silicon. This is reflective of the film 'burring' off the surface, leaving behind oxidizes carbon (from the film) and silicon oxide (from the substrate).

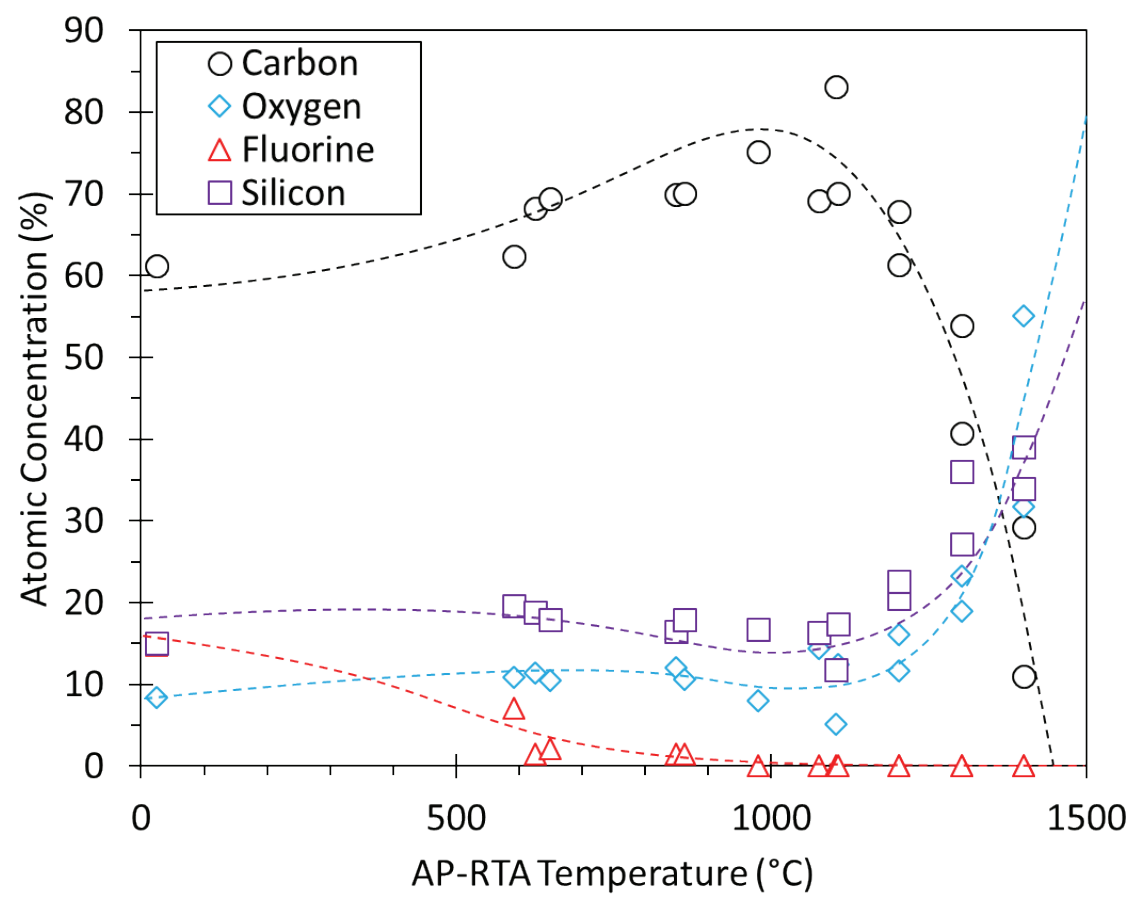

Figure A.19: The elemental atomic concentration across various AP-RTA temperatures. The increase in carbon concentration is due to the removal of fluorine species, as well as a densification of the carbon film leading to an attenuation of the silicon signal from the bulk. At temperatures above $1000^{\circ} \mathrm{C}$ as sharp increase in oxygen content is observed, coinciding with a decrease in carbon, indicating the carbon film is being consumed by oxygen. 


\section{§A.2.7 AP-RTA Cycling $\left(1200^{\circ} \mathrm{C}-500^{\circ} \mathrm{C} \mathrm{X} \mathrm{5)}\right.$}

With the heater set to $1400^{\circ} \mathrm{C}$, a sample was thermally cycled between $1200^{\circ} \mathrm{C}$ and $500^{\circ} \mathrm{C}$ by repeatedly withdrawing and inserting the RTA tube. The RTA profile and the XPS and Raman characterization results are shown in Figure A.20. All other synthesis parameters are identical to the SOP process.

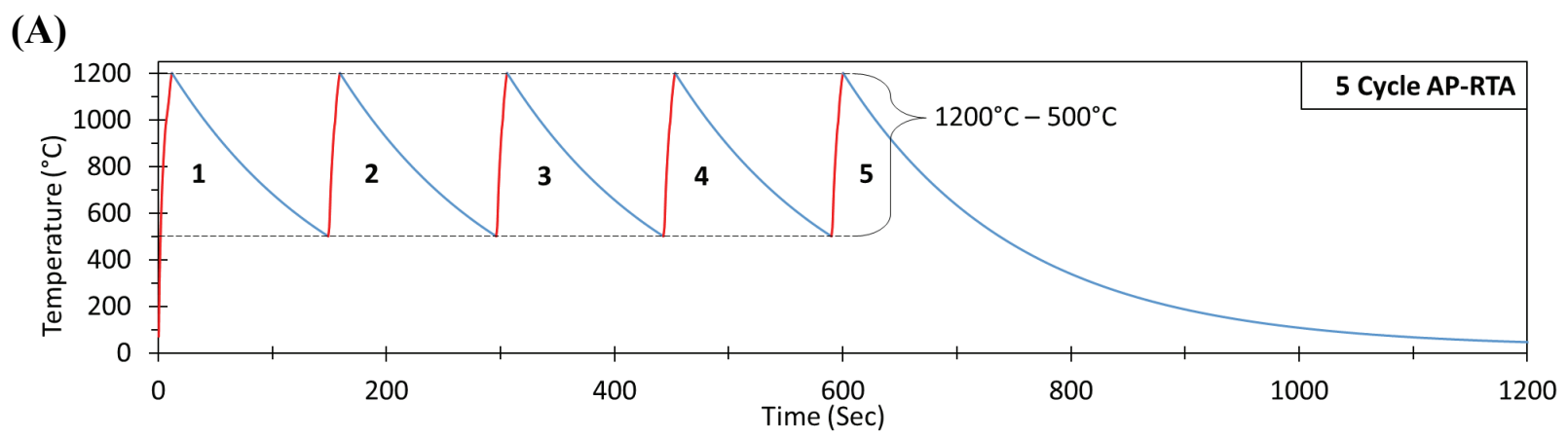

(B)

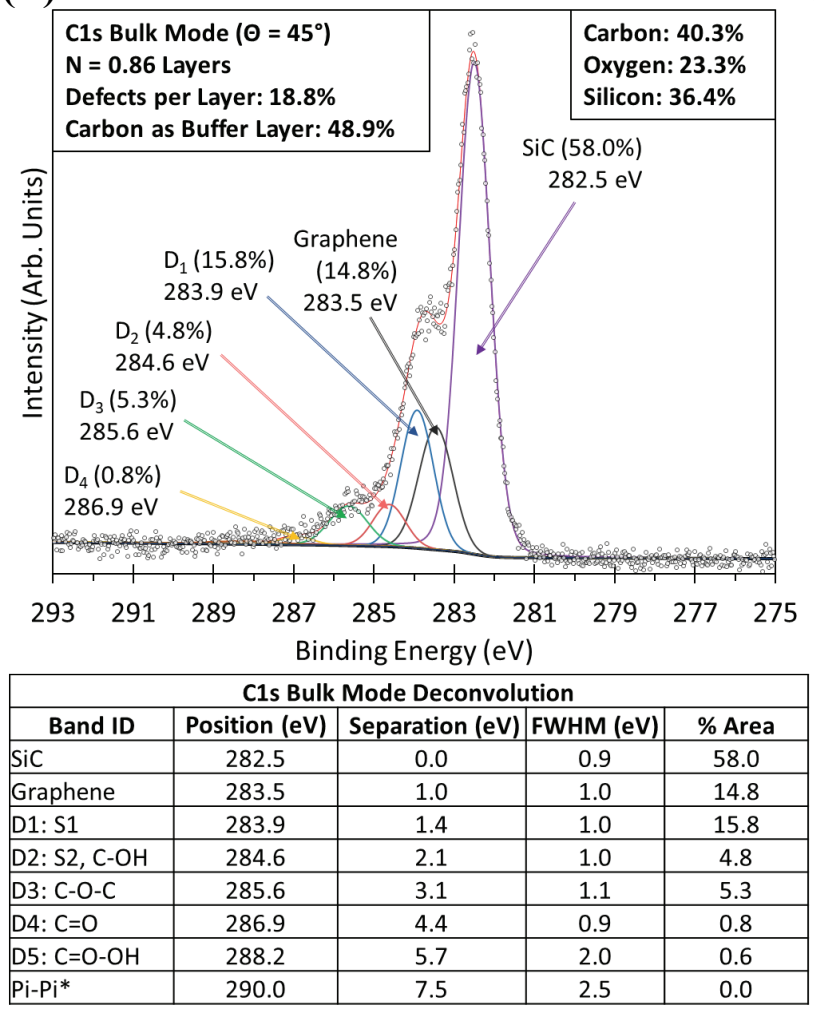

(C)

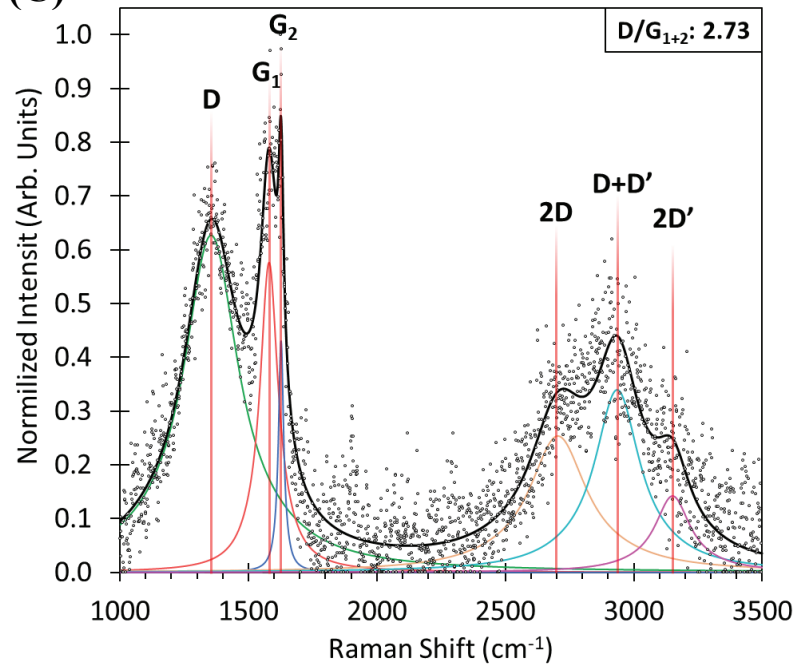

\begin{tabular}{|l|c|c|c|c|}
\hline \multicolumn{5}{|c|}{ Raman Deconvolution } \\
\hline Peak ID & $\begin{array}{c}\text { Center } \\
\left(\mathbf{c m}^{-1}\right)\end{array}$ & $\begin{array}{c}\text { Normalized } \\
\text { Height }\end{array}$ & $\begin{array}{c}\text { Area } \\
\text { (Arb. Units) }\end{array}$ & $\begin{array}{c}\text { FWHM } \\
\left(\mathbf{c m}^{-1}\right)\end{array}$ \\
\hline D & 1355 & 0.63 & 276.0 & 269.5 \\
\hline$G 1$ & 1580 & 0.58 & 83.3 & 88.5 \\
\hline$G 2$ & 1627 & 0.43 & 17.5 & 24.9 \\
\hline 2D & 2704 & 0.25 & 116.9 & 282.2 \\
\hline$D+D^{\prime}$ & 2935 & 0.34 & 122.5 & 220.9 \\
\hline 2D' & 3151 & 0.14 & 40.3 & 173.6 \\
\hline
\end{tabular}

Figure A.20: (A) The approximant heating and cooling profile for the thermally cycled sample. (B) XPS analysis of the C1s region. The dominant peaks are labeled, and the peak parameters are tabulated below the figure. Inset also are the atomic concentration data collected from higher resolution scans of each element. (C) Raman analysis of the same sample. The primary peaks are labeled, and the peak parameters are tabulated below the figure.

From the results shown in Figure A.20(B,C), it can be concluded that the effect of thermally cycling is similar to an extended annealing time. However, there are some differences. Some 
aspects of the Raman spectra indicate improvements in the crystallinity, namely growth in the 2phonon region (2D, D+D' and 2D') and the narrowing of the G peak. The G peak has been fitted with two curves, however given the low signal level, coupled with the noise from the SiC substrate which clouds the signal in around $1600 \mathrm{~cm}^{-1}$, the G peak may be mono-modal in this case. The D peak remains large, resulting in a large $\mathrm{D} / \mathrm{G}_{1+2}$. The XPS spectra show most of the carbon has been removed from the surface, yet there remains an appreciable fraction of non-buffer layer graphene. What this may be indicating is that, although the majority of the carbon has been removed through the oxidation process, that which remains as unbound graphene is of relatively good quality. The thermal 'exercising' of the interfacing bonds may have helped to decouple the film that remained.

\section{§A.2.8 Dwell Time}

To investigate the effect of an extended annealing time, a sample was allowed to rest at temperature under a flow of 100 SCCM of ultra-high purity (UHP) argon $(>99.9999 \%$ research plus purity) for approximately 15 minutes following the SOP heating rate. The sample was then withdrawn from the furnace and allowed to rapidly and naturally cool down following the Standard Synthesis cooling rate. The AP-RTA profile and film characterization an analysis can be found in Figure A.21. All other synthesis parameters are identical to the Standard Process. 


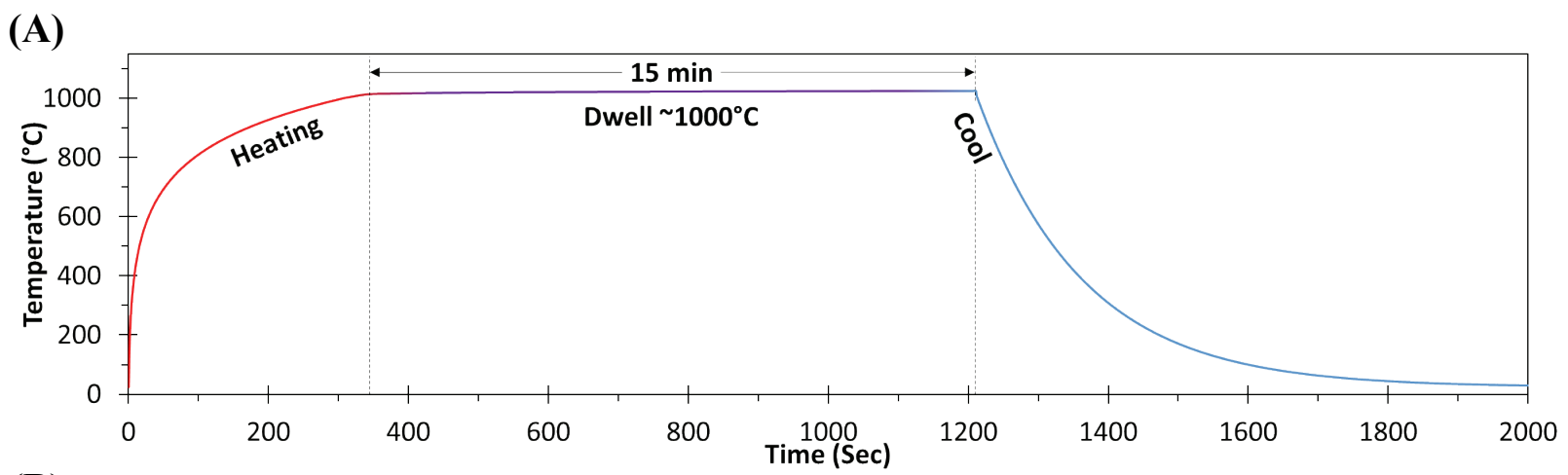

(B)

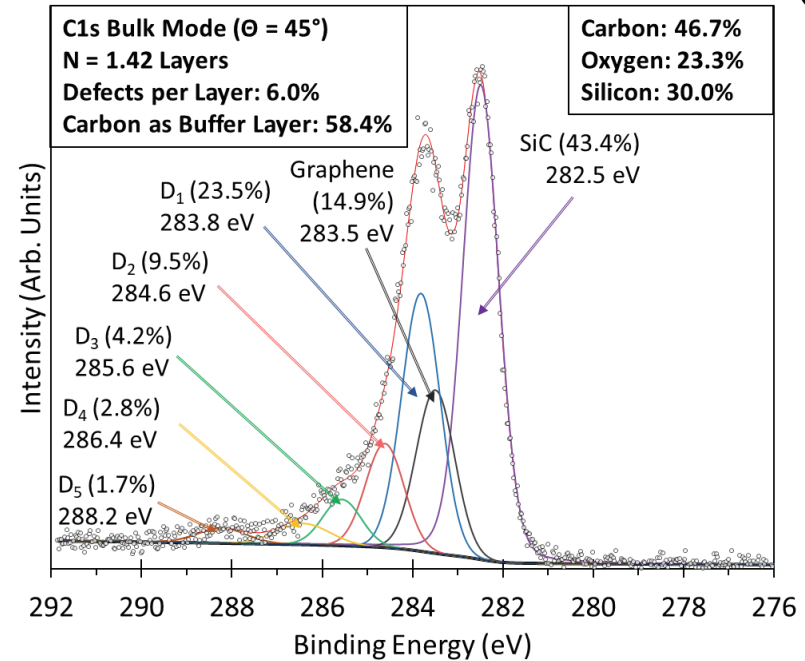

\begin{tabular}{|l|c|c|c|c|}
\hline \multicolumn{5}{|c|}{ C1s Bulk Mode Deconvolution } \\
\hline \multicolumn{1}{|c|}{ Band ID } & Position (eV) & Separation (eV) & FWHM (eV) & \% Area \\
\hline SiC & 282.5 & 0.0 & 0.9 & 43.4 \\
\hline Graphene & 283.5 & 1.0 & 1.0 & 14.9 \\
\hline D1: S1 & 283.8 & 1.3 & 1.0 & 23.5 \\
\hline D2: S2, C-OH & 284.6 & 2.1 & 1.0 & 9.5 \\
\hline D3: C-O-C & 285.6 & 3.1 & 1.0 & 4.2 \\
\hline D4: C=O & 286.4 & 3.9 & 1.4 & 2.8 \\
\hline D5: C=O-OH & 288.2 & 5.7 & 1.3 & 1.7 \\
\hline Pi-Pi* & 290.2 & 7.7 & 2.5 & 0.0 \\
\hline
\end{tabular}

(C)

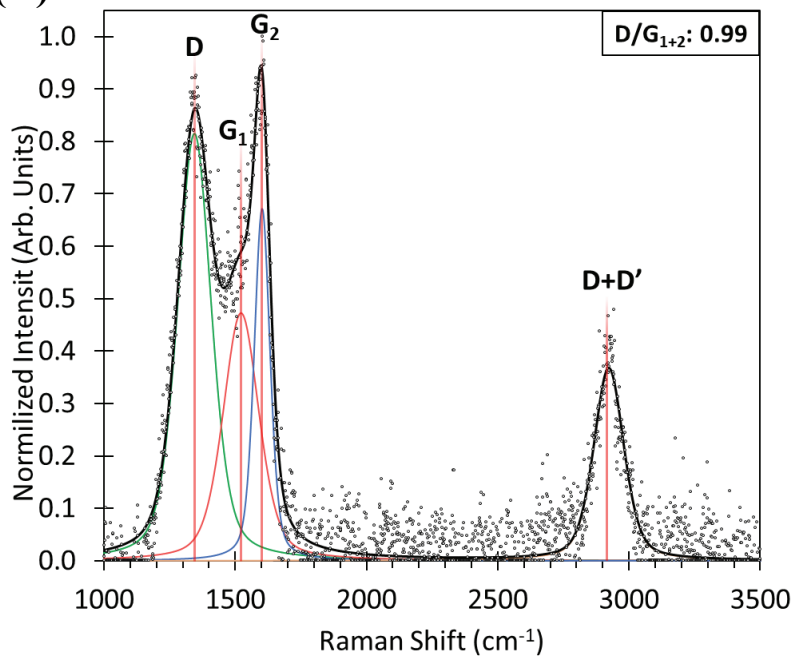

\begin{tabular}{|l|c|c|c|c|}
\hline \multicolumn{5}{|c|}{ Raman Deconvolution } \\
\hline Peak ID & $\begin{array}{c}\text { Center } \\
\left(\mathbf{c m}^{-1}\right)\end{array}$ & $\begin{array}{c}\text { Normalized } \\
\text { Height }\end{array}$ & $\begin{array}{c}\text { Area } \\
(\text { Arb. Units) }\end{array}$ & $\begin{array}{c}\text { FWHM } \\
\left(\mathbf{c m}^{-1}\right)\end{array}$ \\
\hline D & 1344 & 0.81 & 156.66 & 151.90 \\
\hline G1 & 1522 & 0.47 & 96.81 & 161.85 \\
\hline G2 & 1602 & 0.67 & 60.58 & 71.27 \\
\hline 2D & - & - & - & - \\
\hline D+D' & 2923 & 0.37 & 62.61 & 135.10 \\
\hline 2D' & - & - & - & - \\
\hline
\end{tabular}

Figure A.21: (A) The approximant heating and cooling profile showing the extended anneal dwell time. (B) XPS analysis of the C1s region. The dominant peaks are labeled, and the peak parameters are tabulated below the figure. Inset also are the atomic concentration data collected from higher resolution scans of each element. (C) Raman analysis of the same sample. The primary peaks are labeled, and the peak parameters are tabulated below the figure.

The film has a combined buffer layer and graphene thickness of $0.5 \pm 0.1 \mathrm{~nm}$ or approximately 1.5 carbon layers. This is essentially half the thickness of the film produced by the standard anneal. From Figure A.21(B) it is clear that extending the anneal time leads to an increase in the SiC-C component and a reduction in the graphene component. As comparted films which are annealed using the standard procedure (detailed in Chapter 3.1), the buffer layer species ( $\mathrm{D}_{1}$ and $\mathrm{D}_{2}$ ) are enhanced slightly overall, while $\mathrm{D}_{3}$ remains the same and $\mathrm{D}_{5}$ decreases. This suggests 
that the graphene component is gasified (oxidized) by low level oxygen-based impurities $(\sim 0.5$ $\mathrm{ppm}$ ) in the Ar purge gas. This is consistent with numerous observations on the effect of extending the annealing time. Not surprisingly, the total atomic oxygen content for the extended anneal is which is significantly higher $(23.3 \%)$ than that for the standard anneal $(\sim 15.1 \%)$. The overall conclusion is that extended anneal times longer than standard anneal leads to deleterious effects on the graphene film.

\section{§A.2.9 The $\mathrm{D} / \mathrm{G}$ ratio}

Figure A.22 plots the D/G ratio as a function of increasing AP-RTA temperature.

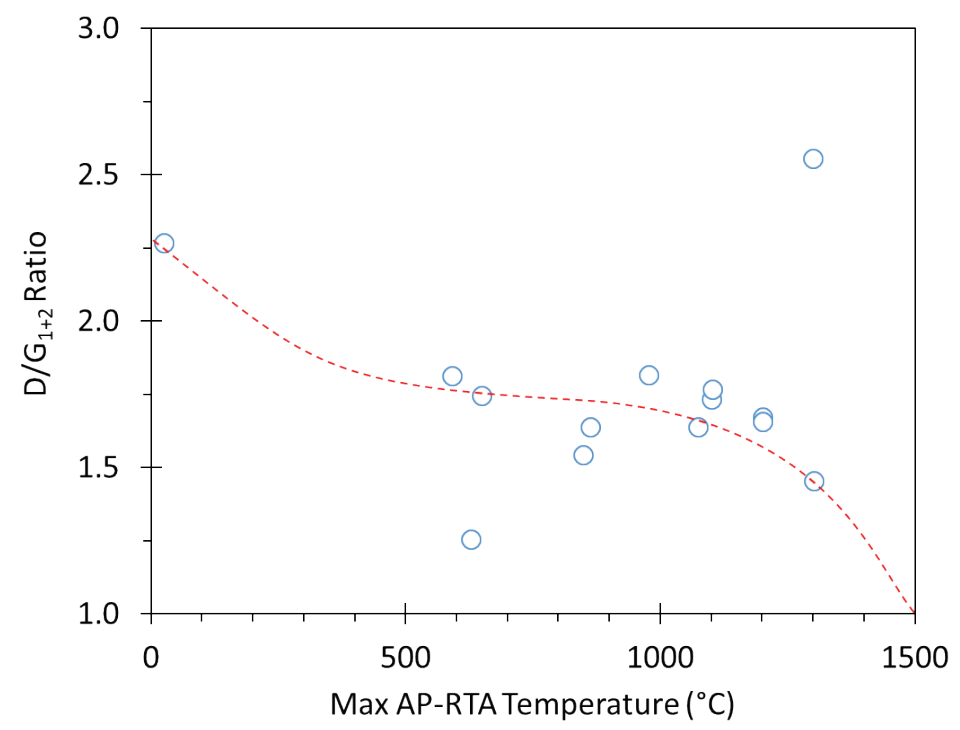

Figure A.22: The D/G ratio as a function of increasing AP-RTA temperature.

\section{§A.3 Supporting Information for Section $§ 4.3$}

This section includes supporting information for Section $\$ 4.3$ in the main text.

\section{§A.3.1 Literature Reports on Graphene XPS Peak Shifting}

Table A-1 summarizes the literature reports on the evolution of the C1s region of the XPS spectra resulting from decoupling of the buffer layer. The processes attributed by each report to explain these shifts have been quoted. 
Table A-1: Literature explanations for the observed Graphene-SiC peak separation after buffer layer decoupling.

\begin{tabular}{|c|c|c|c|c|c|}
\hline \multirow[t]{2}{*}{ Ref } & \multirow{2}{*}{$\begin{array}{c}\text { Explanation for Peak Separation Increase on } \\
\text { Decoupling }\end{array}$} & \multicolumn{3}{|c|}{\begin{tabular}{|c|} 
SiC-Graphene \\
Separation $(e V)$ \\
\end{tabular}} & \multirow{2}{*}{$\begin{array}{l}\text { Decoupling } \\
\text { Agent }\end{array}$} \\
\hline & & Before & After & $\Delta$ & \\
\hline (537) & $\begin{array}{l}\text { "These shifts toward lower binding energies indicate increase } \\
\text { of the upward bending of the SiC bands." }\end{array}$ & 1.04 & 1.42 & 0.38 & $\begin{array}{c}\text { Oxygen } \\
\text { Intercalation } \\
\end{array}$ \\
\hline (565) & $\begin{array}{l}\text { "...from the change in EG Fermi energy owing to the decoupled } \\
\text { nature of QFS BLG from the substrate..." }\end{array}$ & 1.00 & 1.80 & 0.80 & Hydrogen \\
\hline (559) & $\begin{array}{l}\text { "The position of } \mathrm{SiC} \text { components is characteristic, just like in } \\
\text { the case of the graphene layer decoupled, as a } \\
\text { result of hydrogen } 21 \text { and oxygen intercalation." }\end{array}$ & 1.00 & 1.77 & 0.77 & $\begin{array}{l}\text { Oxygen } \\
\text { Intercalation }\end{array}$ \\
\hline (241) & $\begin{array}{l}\text { "However, the } \mathrm{SiC} \text { bulk component in the } \mathrm{C} 1 \mathrm{~s} \text { core level } \\
\text { spectra shifts } 0.2 \mathrm{eV} \text { towards lower binding energy. This } \\
\text { variation in the band bending in the sample } \mathrm{BH} 2 \text {, confirms the } \\
\text { partial saturation of } \mathrm{Si} \text { dangling bond and reveals that the } \\
\text { graphene/SiC interfacial region is modified after } \\
\text { hydrogenation." }\end{array}$ & 0.90 & 1.10 & 0.20 & Hydrogen \\
\hline \multirow[t]{2}{*}{ (225) } & \multirow{2}{*}{$\begin{array}{l}\text { "Moreover, the binding energy difference between graphene } \\
\text { and bulk } \mathrm{SiC} \text { components is increased after annealings (in } 0.77 \\
\mathrm{eV} \text { for (b) and in } 0.68 \mathrm{eV} \text { for (c)). This observation is related to } \\
\mathrm{SiC} \text { band bending, due to the electronic decoupling of graphene } \\
\text { as result of the intercalation." }\end{array}$} & 0.70 & 1.47 & 0.77 & $\begin{array}{l}\text { Oxygen } \\
\text { Intercalation } \\
+\mathrm{H}_{2} \mathrm{O} \\
\end{array}$ \\
\hline & & 0.70 & 1.38 & 0.68 & \begin{tabular}{|c|} 
Oxygen \\
Intercalation
\end{tabular} \\
\hline (226) & $\begin{array}{l}\text { "The shift of } 0.4 \mathrm{eV} \text { in binding energy between the graphene } \\
\text { components of the } \mathrm{C} 1 \mathrm{~s} \text { spectra corresponds to a difference in } \\
\text { doping level between the pristine monolayer graphene (Fig. 1a) } \\
\text { and the decoupled bilayer graphene obtained upon annealing in } \\
\text { air (Fig. 1b). Also the SiC bulk component in the C1s (Fig. 1) } \\
\text { and Si2p (Fig. 2) core level spectra shifts } 0.7 \mathrm{eV} \text { towards lower } \\
\text { binding energy. This reveals that the BL/SiC interfacial region } \\
\text { is modified after annealing, causing a different band bending." }\end{array}$ & 0.94 & 1.26 & 0.40 & $\begin{array}{c}\text { Oxygen } \\
\text { Intercalation }\end{array}$ \\
\hline (256) & $\begin{array}{l}\text { "The difference in binding energy location between the } \\
\text { monolayer and decoupled bilayer components is about } 0.4 \mathrm{eV} \text {, } \\
\text { which perfectly agrees with the shift of the Fermi level } \\
\text { measured via ARPES. The total shift of the SiC component is } 1 \\
\mathrm{eV} \text {, which confirms that on the SiC surface hydrogen bonds are } \\
\text { present which cause a respective band bending." }\end{array}$ & 1.00 & 1.61 & 0.61 & Hydrogen \\
\hline
\end{tabular}

\section{§A.4 Supporting Information for Section \$5.3}

This section includes supporting information for Section $§ 5.3$. Recall, Section $§ 5.3$ deals with the device response to gas exposure. 


\section{§A.4.1 Plank's Law Calculation for Heater Element Spectral Emission and a Photonic Explanation for the Chemical Effect Observed During $\mathrm{H}_{2}$ Gas Sensing}

Plank's Law for blackbody radiation is given by,

$$
B_{(\lambda, T)}=\frac{2 h c^{2}}{\lambda^{5}\left(e^{\left(\frac{h c}{\lambda k_{B} T}\right)}-1\right)}
$$

where the radiance, $B_{(\lambda, T)}$, is a function of the wavelength $(\lambda)$ emitted and the temperature $(\mathrm{T})$ of the body in units of $\frac{W}{s r \cdot m^{3}}$; that is, the energy emitted per unit area, through a square radian (solid angle), a given wavelength (if Equation A-1 is solved in terms of frequency, the units become $\left.\frac{W}{s r \cdot m^{2} \cdot H z}\right) ; \mathrm{h}$ is the Planck constant, $\mathrm{c}$ is the speed of light and $\mathrm{k}_{\mathrm{B}}$ is the Boltzmann constant.

Figure A.23, plots $B_{(\lambda)}$ for three different temperatures, $23^{\circ} \mathrm{C}, 230^{\circ} \mathrm{C}, 409^{\circ} \mathrm{C}$, and are the heater setpoints discussed in Section \$5.3.4 in regard hydrogen gas sensing. However, the actual temperature of the heating element itself is much higher. It is unknow what the exact temperature of the element is, but for a heater temperature of $\sim 400^{\circ} \mathrm{C}$ a conservative estimate of $1000^{\circ} \mathrm{C}$ is considered here. 
(A)

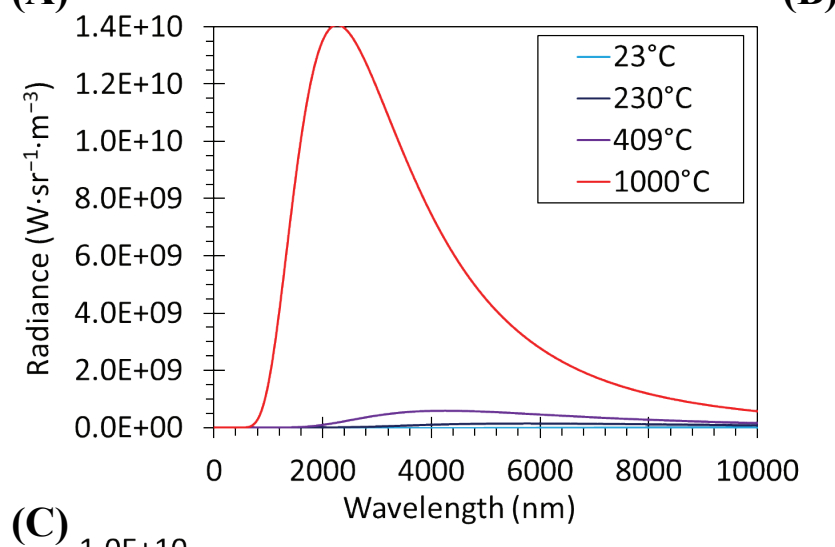

(B)

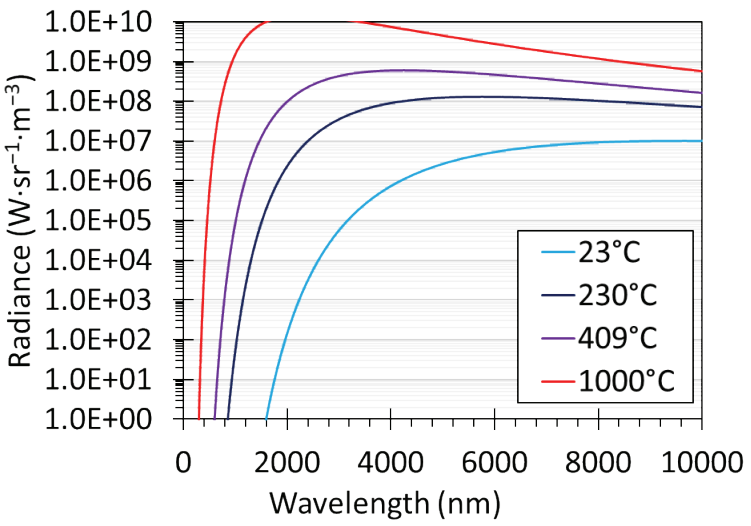

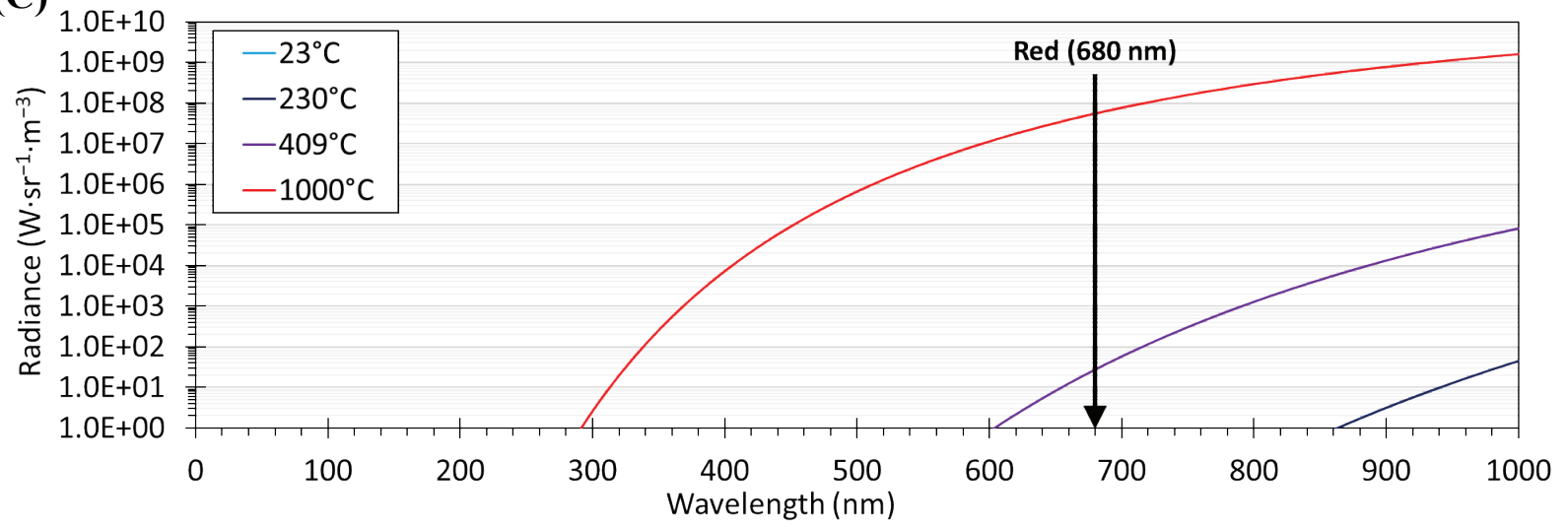

Figure A.23: (A) The spectral radiance calculated at for different temperatures. (B) The same data on a simi- $\log _{10}$ scale. (C) The visible spectral region of the same data. The arrow indicates the wavelength of light the devices have shown photosensitivity to.

The following discussion explores a photonically derived explanation for the observed decrease in the device resistance (see as an increase in the current flow) when a pulse of hydrogen gas is fed through the large test bed. Recall that small photocurrent is produced when the devices are exposed to visible light irradiation. Also recall that the in the large test bed setup, the device is housed within a quartz tube inside the body cavity of the heater. There is a direct line-of-site from the heating elements to the device. When hydrogen is fed into the gas testing system, the temperature of the heater drops slightly. This is due to the cooling effect of the hydrogen stream and is why the temperature of the device is reduced when exposed to hydrogen, as discussed in the main text. In response to this temperature drop, the heater's PID control increases the electrical power supplied to the heating elements in order to maintain the constant temperature setpoint. The elements, which are now hotter, emit more energy across a broader range of wavelengths, including emission in the visible spectrum. This is observable by the naked eye when heating elements are viewed and is confirmed by the heaters built in ammeter. Therefore, the increased 
current flow observed when the device is exposed to hydrogen could be due to an increase in the photocurrent generated in the device due to illumination by the heating elements.

Given that the actual heating elements are substantially hotter than the setpoint of the heater, the amount of radiated energy is not trivial. However, for the temperature range over which the chemical effect is observed, the peak emission is well into the IR region- a low energy region which would not lead to a large photo response. However, as discussed in Section $\$ 5.4 .3$, the devices have not been photo tested with a wide range of light energies. So, it is unknown what the device photosensitivity is across the spectrum emitted by the heating elements. It is possible that one of the frequency bands emitted by the heater elements is generating a photocurrent. Furthermore, the intensity of irradiation over the frequency region which was observed to produce a photo response is low; this is further reduced by the étendue property (spreading out) of light as it travels from the heating element to the device. Although it cannot be entirely ruled out, this explanation for the chemical effect seems unlikely and is considered here for completeness.

\section{§A.5 Supporting Information for Section $\$ 5.4$}

This section includes supporting information for Section $\$ 5.4$ in the main text.

\section{§A.5.1 The Planck-Einstein Relation}

The Planck-Einstein relation gives the relationship between a photon's energy and frequency, which is proportional through Planck's constant. The relationship is thus,

$$
E=h v \quad \text { Equation A-2 }
$$

where $\mathrm{E}$ is the photo energy $(\mathrm{eV}), \mathrm{h}$ is Planck's constant $\left(4.135667696 \times 10^{-15} \mathrm{eV} \cdot \mathrm{Sec}\right)$ and $v$ is the frequency (cycles/Sec). In terms of wavelength, the Planck-Einstein relation is given by,

$$
E=\frac{h c}{\lambda}
$$

Equation A-3 
where $\mathrm{c}$ is the speed of light in a vacuum $(299,792,458 \mathrm{~m} / \mathrm{sec})$ and $\lambda$ is the wavelength $(\mathrm{m})$. Figure A.24 plots this relationship. The colors are only approximations; due to differences in perception, the relationship between photon wavelength and apparent color is, quite literally, in the eye of the beholder.

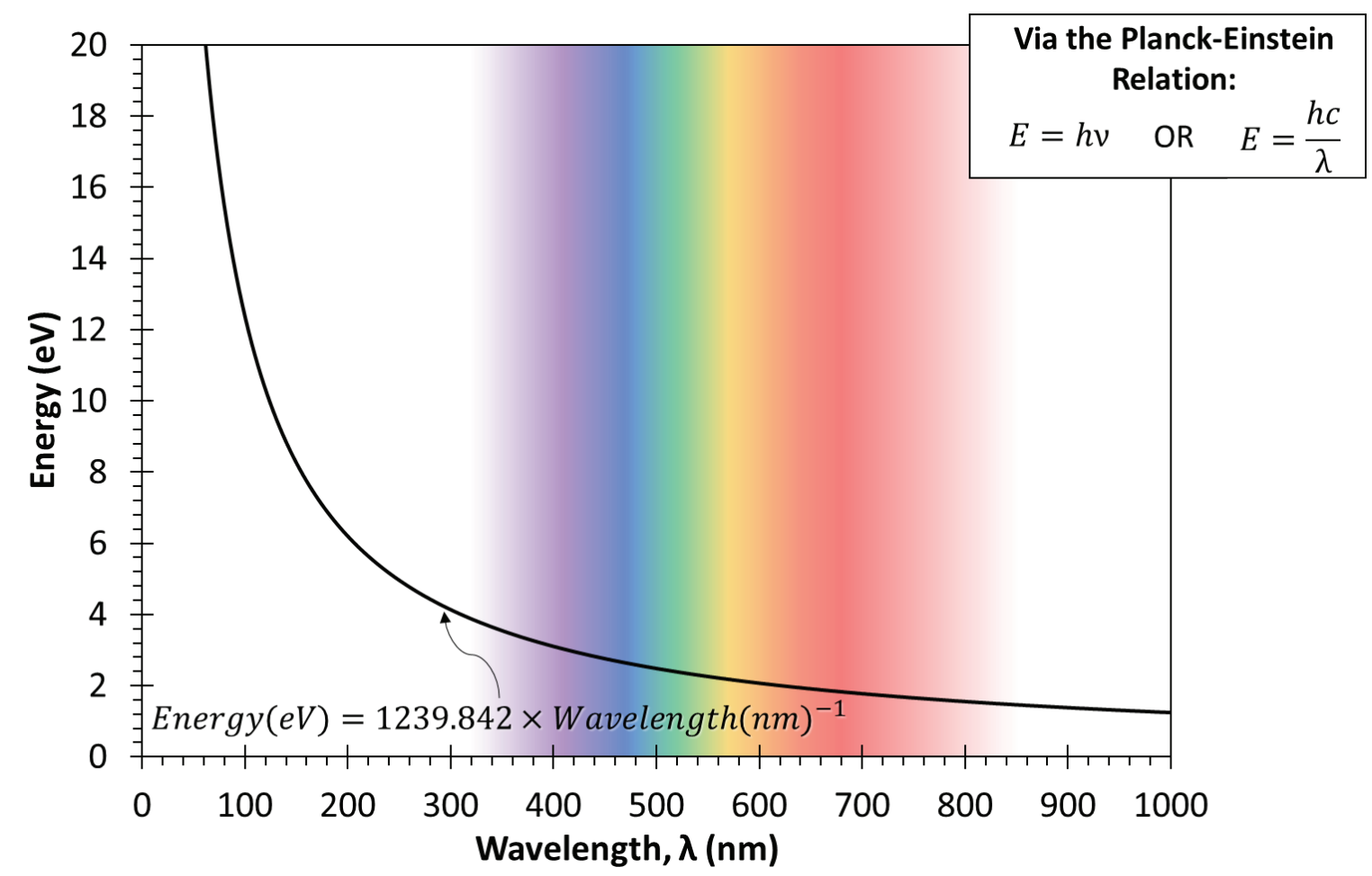

Figure A.24: The relationship between a photons energy and its wavelength, as given by the PlanckEinstein relation. 


\section{§A.5.2 Laser Irradiation}

By monitoring two electrical contacts independently, the current flow through each may be recorded separately. Figure A.25(A) records the current flow through both contacts as one contact is directly illuminated with a purple laser in a pulsed fashion. Figure A.25(B) plots the current recorded from both contacts as a red laser is scanned across one of the metal contacts. An equal and opposite current is recorded in all cases.

(A)

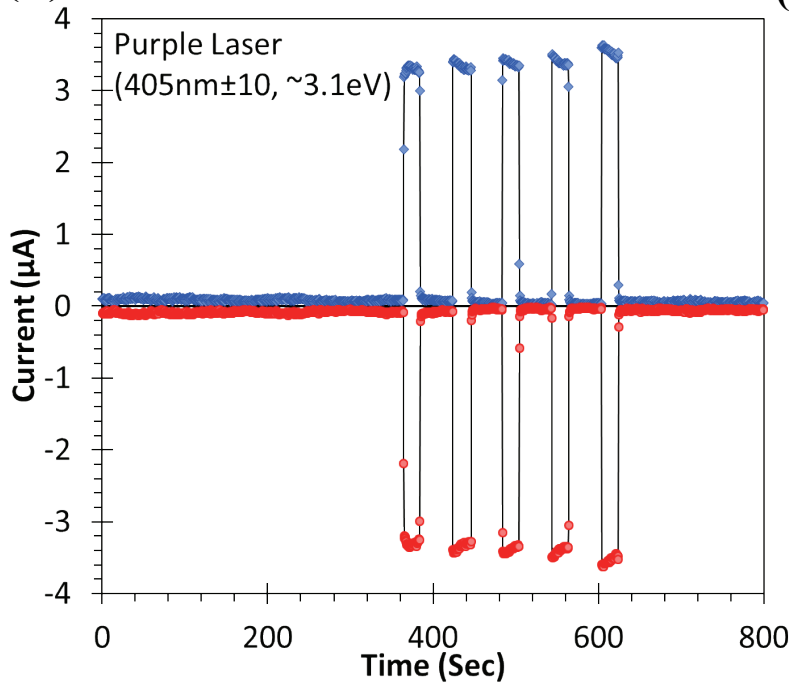

(B)

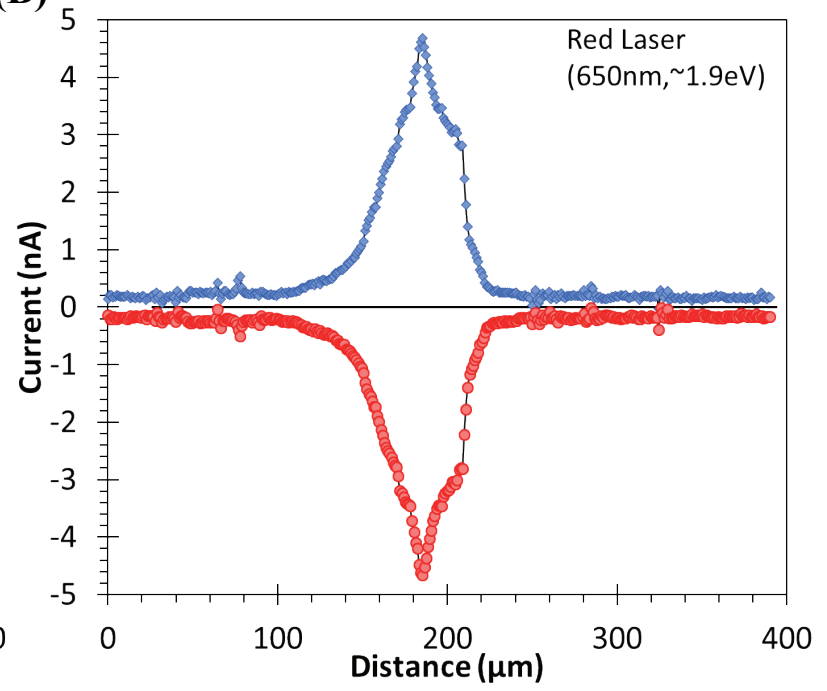

Figure A.25: (A) The current recorded from two contacts as one is irradiated with a purple laser. (B) Similar data collected when a red laser is scanned across one of the contacts. 


\section{APPENDIX B: PRELIMINARY STUdies INTO METALliC NANOPARTICLE DECORATION METHODS}

APPENDIX $B$ details the experimental setup, results and discussion of the preliminary investigation into decorating the $\mathrm{SiC} /$ Graphene devices with metallic nanoparticles. Four different methods are detailed; a chemical, electrical, photonic and evaporative route. The nucleation experiments are documenting here to provide a starting point for future work. As described in Section $\$ 2.2$, theory suggests that both gas sensing and photo detection platforms would stand to benefit from the addition of metallic nanoparticles. For gas sensing, it has been suggested that nanoparticles potentially serve as additional gas adsorption sites, as well as aid in change transfer events. This would manifest itself as an enhanced sensitivity and selective in a decorated gas sensor. For photo detection, plasmonic structures (arising from metallic nanoparticles) can enhance light-matter interactions and enhance the sensitivity of a photo detector and a means by which to tune the sensitivity bandwidth.

To these ends, several exploratory studies were conducted to investigate the post-syntheses decoration of the produced films with metallic nanoparticles. Four distinct methods were tested; chemical nucleation, photo-nucleation, electro-nucleation and e-beam deposition. Each method was qualitatively evaluated based on the ease of execution, repeatability and the resulting nanoparticle character (size, coverage, morphology, etc.). The results of these preliminary studies are detailed in this section.

\section{§B.1 Nanoparticle Decoration: Experimental Setup}

A variety of methods were explored to decorate the graphene surfaces. They included a chemical route, a photonic route, an electrical route and an evaporative route. Described here is the experimental setup and the underpinning theory for each.

\section{§B.1.1 Chemical Route for Metallic Nanoparticle Decoration}

The chemical route for nanoparticle decoration was an extension of previously reported efforts to deposit metallic nanoparticles onto graphene produced by the plasma assisted growth method. ${ }^{(264)}$ Those experiments involved a dilute aqueous $\mathrm{NaBH}_{4}$ solution combined with an aqueous metal salt solution in the presence of a graphenated $\mathrm{SiC}$ sample. The work detailed here is a modification of these experiments and an extension to samples which had electrical contacts, 
with the specific aim for use in sensing applications. The precise experimental setup was one of the focuses of this study and more detail is given in the results section. A generic description, along with what the setup which yielded the best results, is described here.

The experimental setup used here can be seen in Figure B.1. The volume and concentration of $\mathrm{NaBH}_{4}$ ranged between $1-50 \mathrm{~mL}$ and $2.71-12.95 \mathrm{mM}$. The specific salt used depended on the desired metal to be nucleated; for silver $\mathrm{AgNO}_{3}$ was used, for iridium $\mathrm{H}_{2} \mathrm{Cl}_{6} \mathrm{Ir}$ was used, for platinum $\mathrm{H}_{2} \mathrm{PtCl}_{6}$ was used and for gold as $\mathrm{HAuCl}_{4} \bullet 3 \mathrm{H}_{2} \mathrm{O}$ was used. The metal salt was dissolved into 1 - $50 \mathrm{~mL}$ of water at a concentration of between 0.213 - $126.8 \mathrm{mM}$. The burette in Figure B.1 was filled with either the $\mathrm{NaBH}_{4}$ or an aqueous metal salt solution. The stopcock was set to a very slow drip rate such that several hours were required to empty the burette.

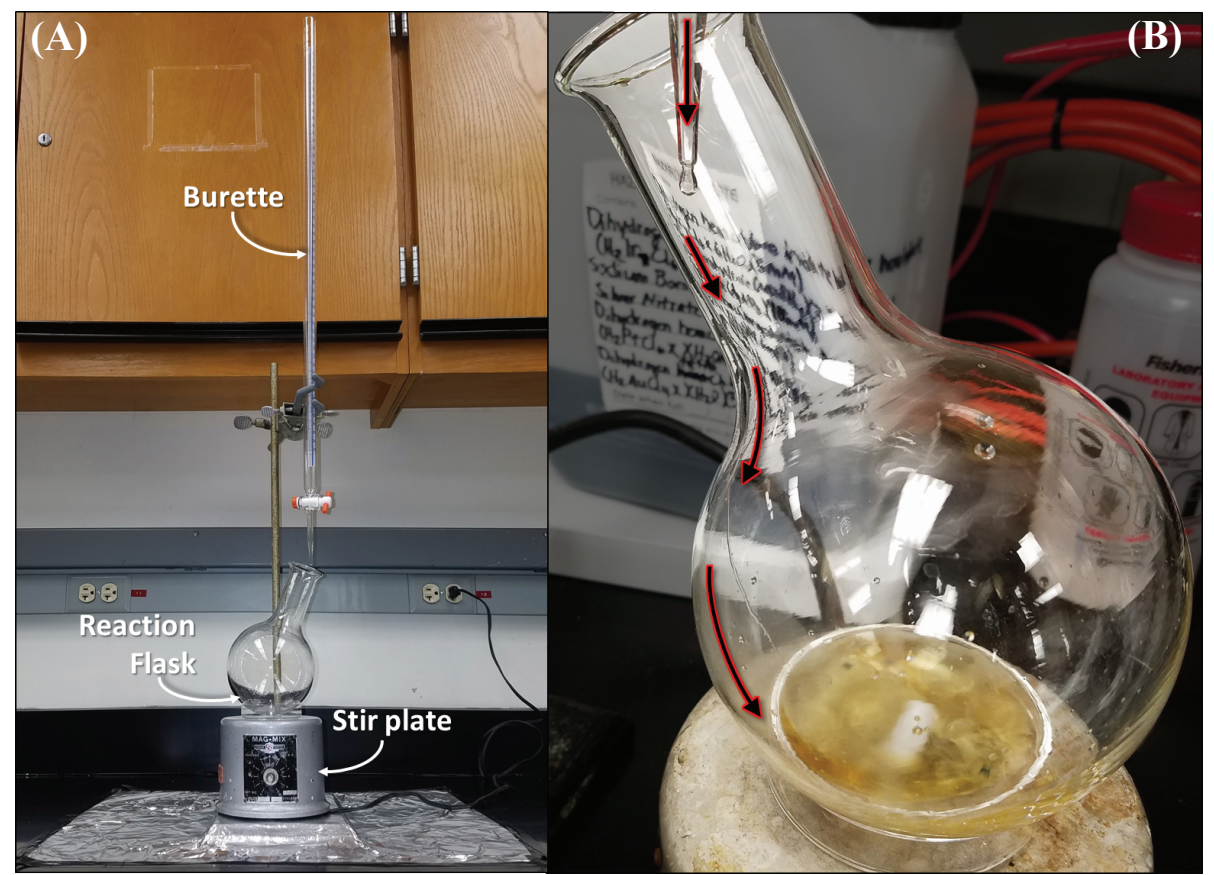

Figure B.1: (A) The equipment setup for nucleation experiments using $\mathrm{NaBH}_{4}$. (B) Close up view illustrating the drip path of the burette solution into the flask solution with stir bar.

A graphenated $\mathrm{SiC}$ sample was placed in a round bottom flask along with $50 \mathrm{~mL}$ of either the $\mathrm{NaBH}_{4}$ or the metal salt solution. A magnetic stir plate was used with a Teflon coated magnetic stir bar placed inside the round bottom flask. The stir bar rotation was set at a constant speed for each experiment. The speed was set such that either a standing vortex would form within the flask, or to a very low speed of less than 60 RPM. The setup was arranged such that the liquid droplet from the burette fell into the neck of the flask, spread across the flask wall and ran down the side into the liquid. The vortex rim chased along the interior wall of the flask and sampled the film of 
precursor liquid running down the sidewall and quickly integrated it into the main body of the liquid. The geometry of this setup was found to be an important parameter.

After the entirety of the burette solution was added, the sample was allowed to incubate in the resulting mixture in the round bottom flask for a total time of 12 hours. The timing clock was started at the addition of the first drop of precursor solution from the burette. The solution was stirred throughout the entire duration of the experiment, including both during the dropwise addition of the burette solution and subsequent incubation. After the incubation period, samples were gently rinsed with DI water for 15 seconds and allowed to air dry. Samples were then analyzed with XPS and AFM.

\section{§B.1.2 Photonic Route for Metallic Nanoparticle Decoration}

This method for nanoparticle nucleation utilized ultraviolet light to photonically reduce a substrate surface in the presence of a metal salt solution. This method was based on work published by others on graphene oxide. ${ }^{(691)(692)(693)(694)}$ The underlying theory is as follows; in a liquid phase solution, consisting of a metal salt precursor in a water/methanol mixture, the surface of a semiconductor is irradiated with photons. If the photons are of a sufficient energy, electrons are promoted across the energy band gap into the conduction band. It has been reported by others that the role of methanol as a hole scavenger is an important component in the process. ${ }^{(693)(694)}$ The process is continuous. The combination of electron promotion and hole scavenging leads to a net negative charge (surface reduction) on the semiconductor surface. This negative charge acts on the positive metal ions in solution, driving nucleation events, which lead to nanoparticle formation and their subsequent growth on the semiconductor surface.

A schematic of this process is shown in Figure B.3. It is noted that others have reported that, due to the surface reduction effects, this process also chemically reduces the graphene sheet (i.e. removes functional groups) leading to a partial return of the $\pi$ - $\pi$ graphene network. ${ }^{(693)}$ 


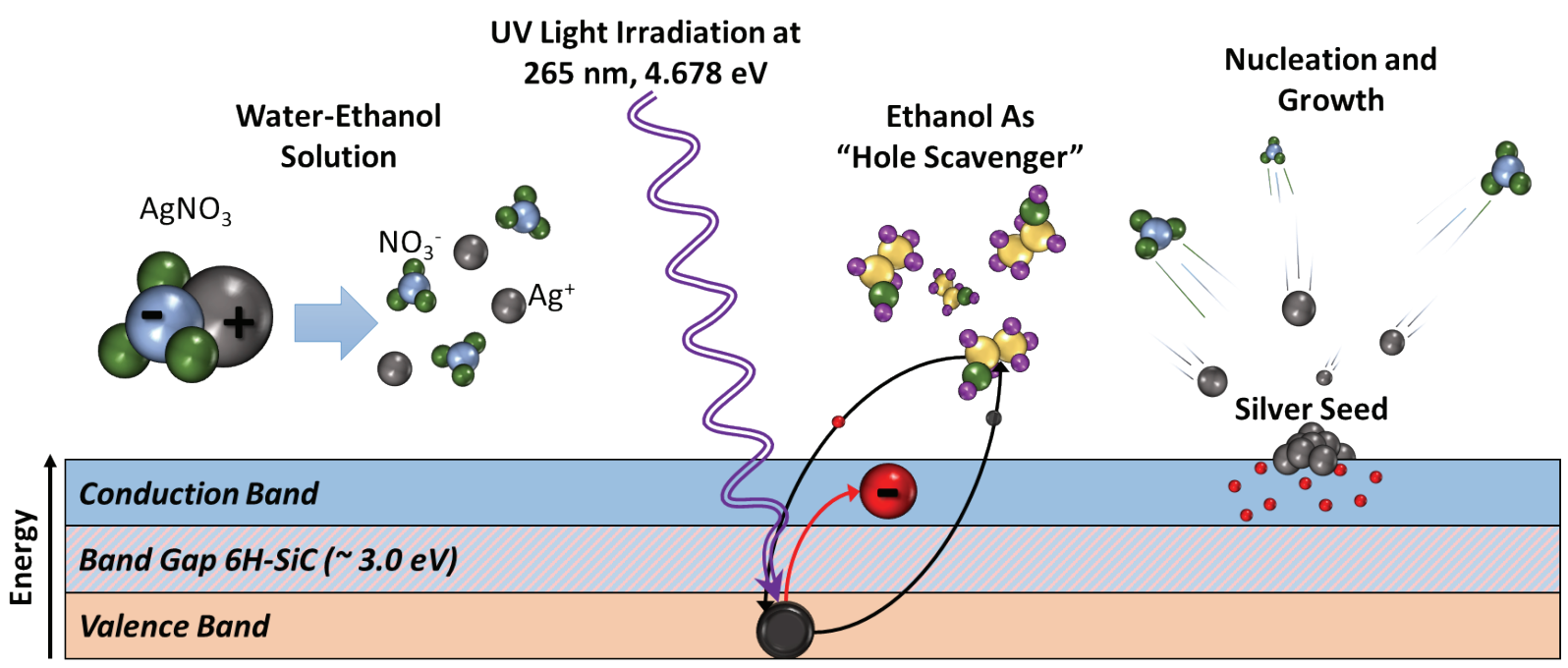

Figure B.2: A schematic of the photo-nucleation process. Depicted is the proposed mechanism for silver nucleation. Light irradiation of the semiconductor surface promotes electrons into the conduction band while ethanol scavenges holes left in the valence band, leading to a net negative charge accumulation on the surface. This charge accumulation acts on ions within the solution and drives nanoparticle growth.

The photo-nucleation experiments were conducted by Michael Matthews, a member of the Stinespring lab group. Graphenated samples were placed inside either a quartz or borosilicate beaker. The beaker also contained $50 \mathrm{~mL}$ of an $\mathrm{AgNO}_{3}$ salt solution of between $3-100 \mathrm{mM}$ in a water-ethanol mixture. The water-ethanol mixture concentration ranged between $0-100 \%$ ethanol. The beaker was placed in a XL-1500 UV Spectrolinker crosslinker obtained from Spectronics Corp. The crosslinker was turned on and the sample was irradiated with $265 \mathrm{~nm}$ light at a density of $120 \mathrm{~mJ} / \mathrm{cm}^{2}$ for between 10 - 30 mins. An image of the crosslinker used and a sample under irradiation can be seen in Figure B.2. After the incubation period, samples were gently rinsed with DI water for 15 seconds and allowed to air dry. Samples were then analyzed with XPS and AFM.

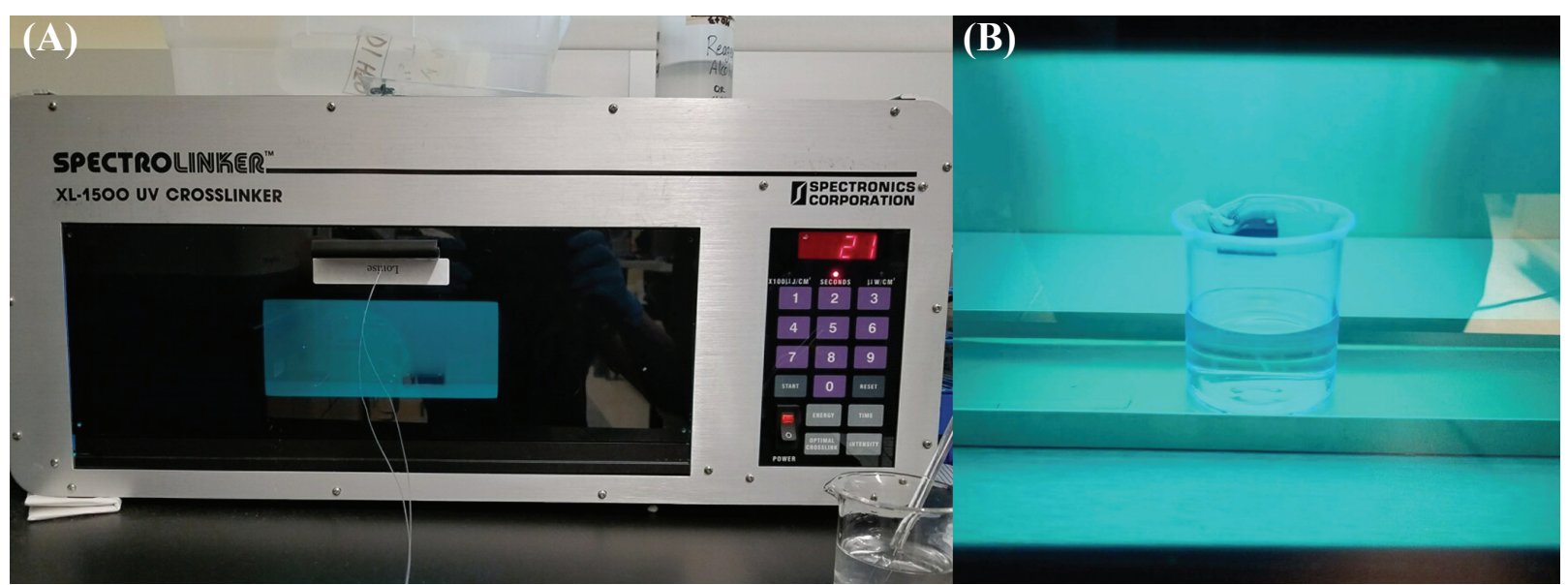

Figure B.3: (A) The XL-1500 UV crosslinker used to irradate the graphenenated samples. (B) A beaker of $\mathrm{AgNO}_{3}$ in a water-ethanol mixture with a graphenated sample under UV light. 


\section{§B.1.3 Electrical Route for Metallic Nanoparticle Decoration}

The electrical route was broken into three subsets of experiments. All three utilized the same principle, but on different size scales. They are termed here generically as the macro, micro and nano experiments. The experiments were all conducted in the liquid phase. The sample was immersed fully in an aqueous salt solution. The specific salt solution depended on the desired metal to be nucleated; for silver $\mathrm{AgNO}_{3}$ was used, for iridium $\mathrm{H}_{2} \mathrm{Cl}_{6} \mathrm{Ir}$ was used, for platinum $\mathrm{H}_{2} \mathrm{PtCl}_{6}$ was used and for gold as $\mathrm{HAuCl}_{4} \bullet 3 \mathrm{H}_{2} \mathrm{O}$ was used. Electrical contact was made to the sample and the sample was biased negative. A second probe was biased positively and brought in close proximity to the sample surface. The goal was to electrically reduce the sample surface to drive nucleation events drawing on the free positive metallic ions in solution. After the incubation period, all samples were gently rinsed with DI water for 15 seconds and allowed to air dry. Samples were then analyzed with XPS and AFM.

For the macro set of experiments, the sample was mounted to a glass slide substrate by double-sided Kapton tape. Large Ti/Au contact pads were evaporated onto the glass substrate using e-beam evaporation as described in Section $\$ 3.3 .1$. Gold wire bonds were made between the large contact pads on the glass to the Ti/Au pattern evaporated onto the sample. Electrical contact was made between the large contact pads of the glass slide (now electrically connected to the sample via wire bonds) to the negative terminal of a variable voltage power supply by means of alligator clip style electrodes. The teeth of the electrodes were wrapped in aluminum foil to increase the contact area between the flat pads and the alligator clips. A small aliquot of salt solution was dropped onto the surface of the sample, forming a small ball of liquid.

The power source was a GW Instek GPS-3030DD laboratory DC power supply purchased from Good Will Instrument Co., Ltd. The positive terminal of the power supply was connected to a blunt electrode. This electrode was mounted in a ring stand such that the tip of the electrode was suspended directly above the sample; but not touching either the sample or the liquid droplet. This setup can be seen in Figure B.4. The concentration of the salt solution was $10 \mathrm{mM}$ in water. The power supply was set to a programmed voltage and powered on. Voltages were between $1 \mathrm{~V}-10 \mathrm{~V}$.

The experiment was allowed to run for a pre-determined about of time. Typically, this was between 5 to 30 seconds. After the allotted time, the power supply was turned off and the sample was then gently rinsed with DI water. The sample was then analyzed via SEM. 

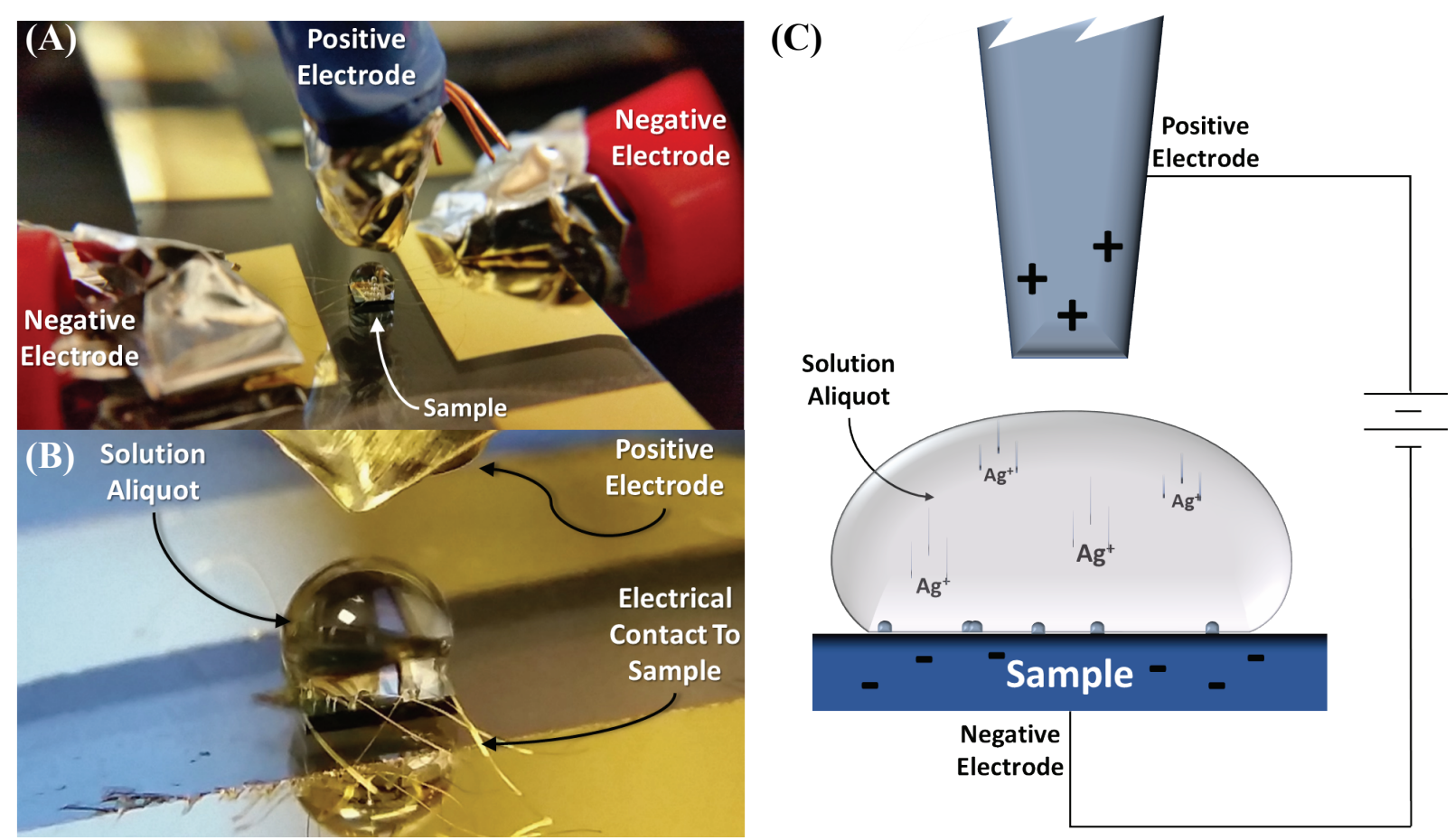

Figure B.4: (A) Magnified view of the macro set of elecro-nucleation experimental setup. (B) Higher magnification view of the experimental setup showing the positive electrode, solution aliquot and the ultra-sonic wire bonds used to make electrical contact with the sample. (C) Simple conceptual schematic of the experimental setup.

The micro set of experiments was a refinement of the macro set of experiments and utilized a miniaturized positive electrode to further confine the electric filed. This was done to drive nucleation to a small area of the sample, rather than over the whole sample as in the macro experiments. For these tests, a silicon substrate was used for the test substrate. Again, a small aliquot of salt solution was deposited onto the sample. However, for the micro set of experiments, the tip of the positive electrode was submerged into the droplet of liquid but was not in contact with the sample surface.

The sample was mounted to a glass slide with double-sided copper tape. Contact to the negative terminal of the power supply was achieved via an alligator style clip connected to the copper tape. A high precision mechanical manipulator (purchased from Signatone Corp. Model S725-PLM Micropositioner) was used to precisely position the positive electrode. The positive electrode was an ultra-sharp tungsten electrical probe tip (purchased from Signatone Corp. Model $\mathrm{SE}-\mathrm{TB}, 1.25$-inch in length with a tip diameter of $25 \mu \mathrm{m})$. The same power supply was used in this 
set of micro experiments as was used in the macro set of experiments. Between 1 and $10 \mathrm{~V}$ were applied for between 30 - 120 seconds. The experimental setup is shown in Figure B.5.

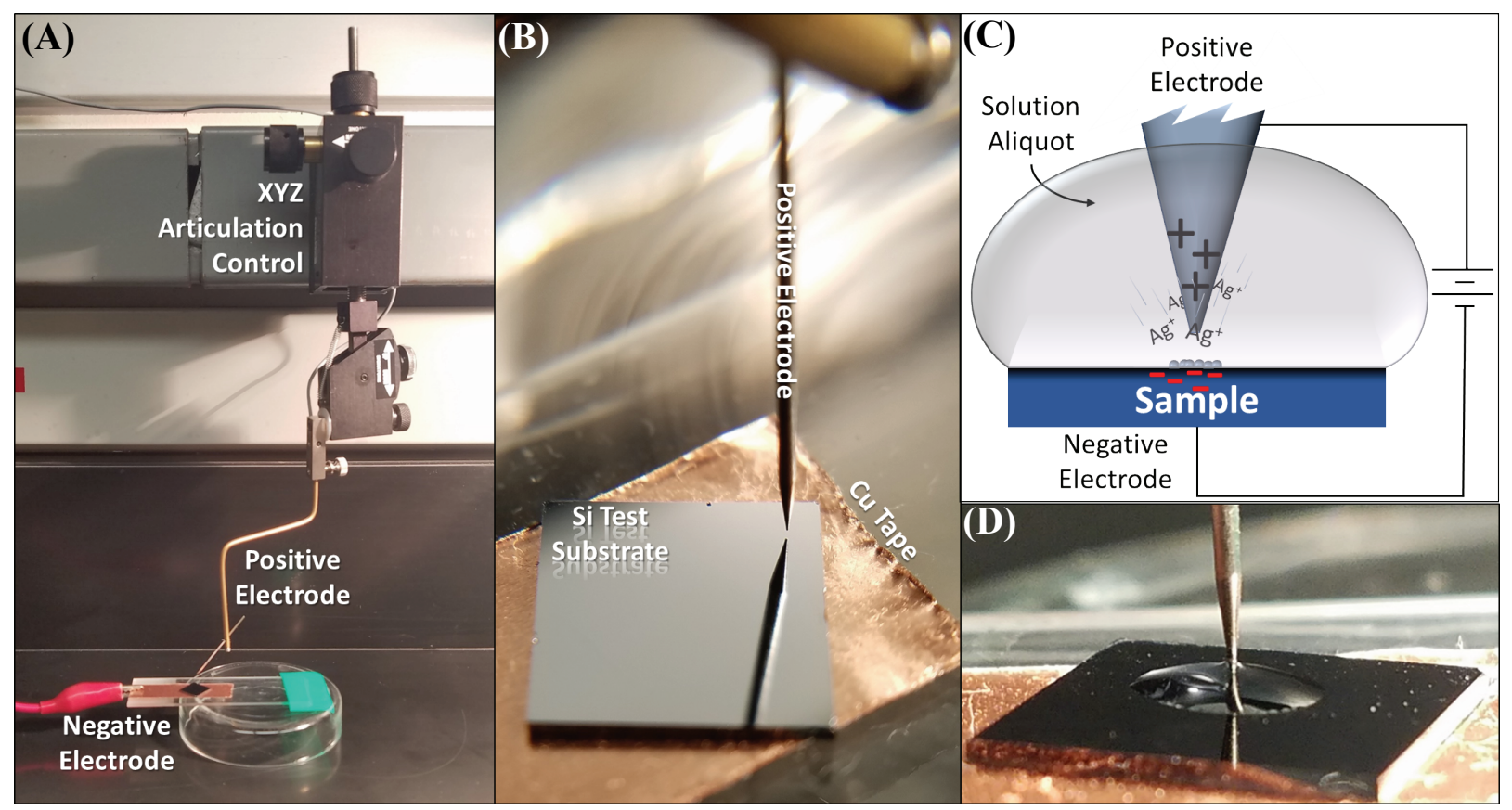

Figure B.5: (A) The experimental setup for the micro set of elecro-nucleation experiments. (B) Magnified view showing the positioning of the positive electrode to the substrate. (C) Simple conceptual schematic of the experimental setup. (D) Magnified view of the positive electrode and substrate with aliquot of precursor solution. Note that in this set of experiments, the positive electrode was submerged in the liquid.

The nano set of experiments was the final set of experiments investigating the feasibility of electrically induced nucleation of metallic nanoparticles. The goal was to achieve a tight confinement and direction of the electric field to drive the nucleation of individual nanoparticles at specific locations and in a precise arrangement. This set of experiments utilized an Agilent 5500 SPM AFM (described in detail in Section $\$ 3.2 .5)$.

The built-in power supply of the AFM was used to develop the voltage to drive nucleation. A $1 \mathrm{~cm}^{2}$ silicon sample was mounted onto a sample stage equipped with an electrochemical test cell. The test cell contained a $1 \mathrm{mM}$ aqueous $\mathrm{AgNO}_{3}$ solution for all experiments. Electrical contact was made from the AFM power supply to the sample via the electrochemical cell electrode and a platinum wire. The sample was mounted in the liquid cell with double-sided copper tape. The end of the platinum wire was coiled beneath the copper tape and the insulating electrochemical cell bottom such that electrical contact was made from the platinum wire, through the copper tape and to the sample. Because the metal head of AFM scanner is electrically grounded and makes contact with the liquid within the cell, the scanner head was insulated with Teflon tape. This was done to 
prevent its participation in the electrochemical circuit. The tip was submerged into the liquid and brought into near contact with the sample surface resulting in a tip-sample separation estimated to be on the order of 10's of nanometers. This was done by operating the AFM in tapping mode and approaching the sample until the tip oscillation was reduced to $90 \%$ of its free oscillation in the liquid. The sample was grounded, and the tip was biased positively. Voltages used were between $1-10 \mathrm{~V}$ and the duration of the applied voltages between $10-60$ seconds. The experimental setup can be seen in Figure B.6.

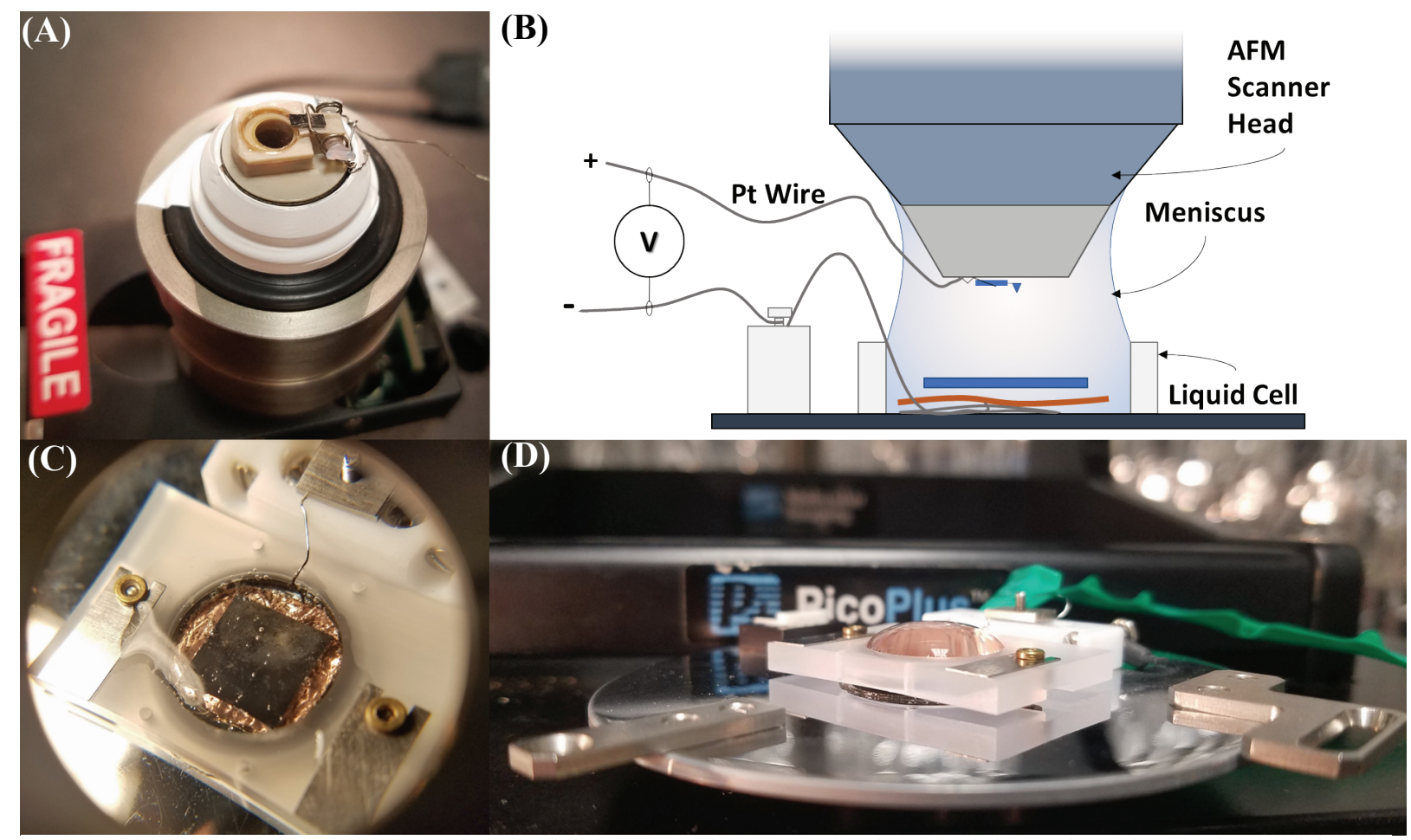

Figure B.6: (A) The AFM scanner head with insulated scanner body, AFM tip and Pt electrode. (B) Simple conceptual schematic of the experimental setup. (C) Magnified view of the liquid cell holding a test substrate and a liquid salt solution. (D) View of the sample stage with liquid test cell installed. The surface meniscus of the liquid salt solution within the cell can be seen as well.

\section{§B.1.4 Evaporative Route for Metallic Nanoparticle Decoration}

The goal of this set of experiments was to determine if it was feasible to deposit nanoparticles, evenly and over a large area, in a manner which had few process constraints and was easily scalable and repeatable. To do this, a series of experiments were devised which used an e-beam evaporator to evaporate a fractional monolayer of metal onto a sample surface which was to coalesce into individual nanoparticles. The same custom Kurt J. Lesker e-Beam Evaporator system (described in greater detail in Section \$3.3.1) was used here as was used to deposit electrical contacts onto the graphene samples for electrical testing. 
This series of experiments tested the feasibility of nickel and gold metal. Clean $1 \mathrm{~cm}^{2}$ silicon substrates were used. The sample preparation and equipment operation were largely similar to the electrical contact deposition step discussed in Section \$3.3.1. However, in these tests only one metal was deposited per experiment and the deposition parameters were heavily modified. The Kurt J. Lesker e-Beam Evaporator used a computer interface which allowed for the modification of a wide array of system parameters. These were used to tune the deposition. A picture of the main process chamber is shown in Figure B.7(B).
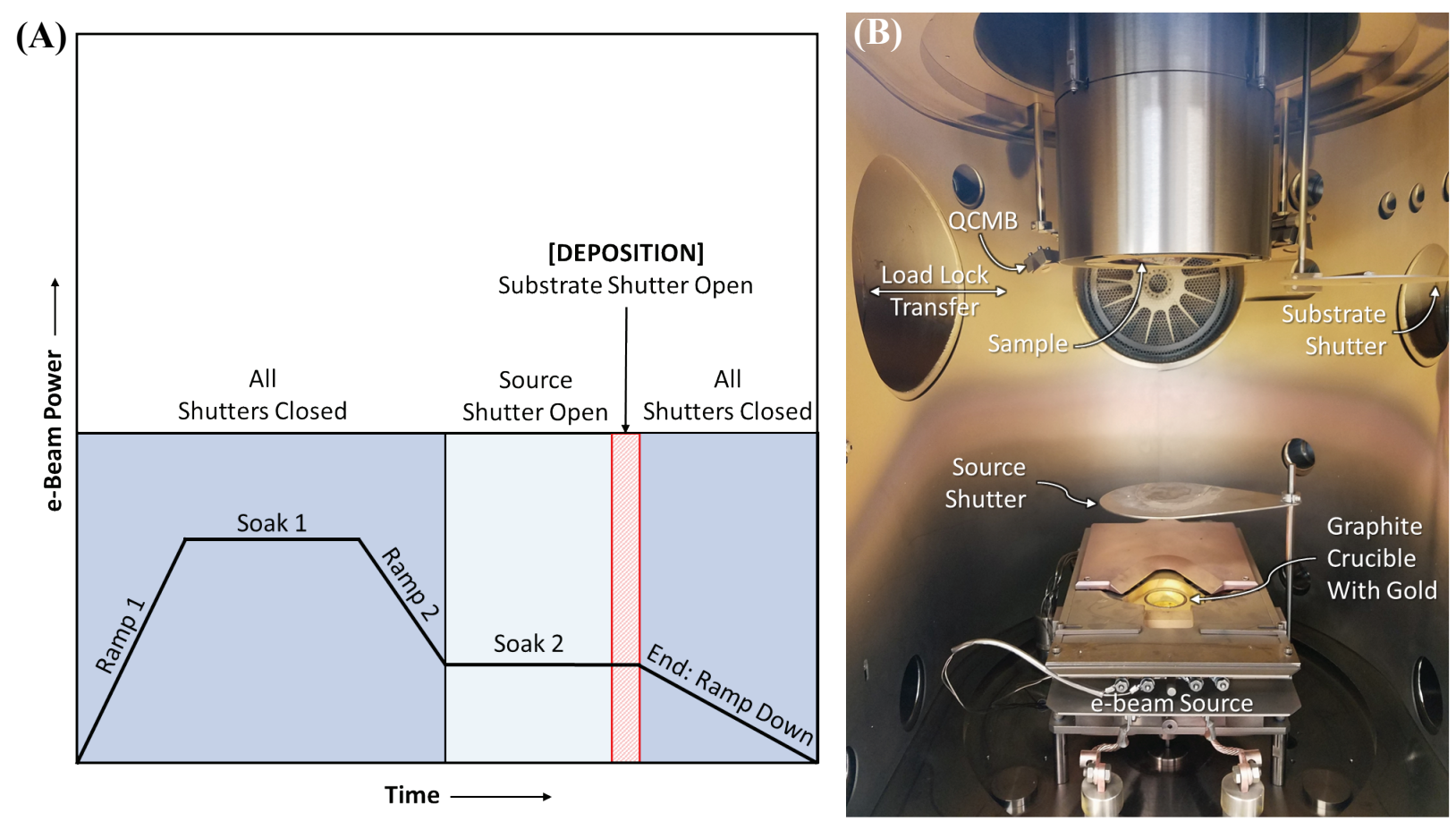

Figure B.7: (A) Generic example of the nanoparticle deposition power profile with each phase labeled. (B) Real world picture of the interior of the process chamber. The pertinent components are label. Note that the substrate shutter is shown in the OPEN position and the source shutter is shown in the CLOSED position.

Once the samples were loaded into the main chamber and the pressure had reached the $10^{-}$ ${ }^{8}$ Torr range, the deposition recipe was started. The deposition recipe was divided into a multistep process as can be seen in Figure B.7(A). For the Ramp 1, Soak 1 and Ramp 2 phases both the source and substrate shutters were closed. The Ramp 1 phase ramped the e-beam emission current to the Soak 1 power set point so as not to thermally shock the components in the system. The purpose of the Soak 1 phase was to desorb any volatile contaminants on the surface of the metal and to fully melt the metal within the crucible. This step ensured that the crucible was adequately conditioned and cleaned before the Soak 2 step- which for these experiments was at an unusually 
low power as compared to the normal operation of this tool. The Soak 2 phase was operated with the source shutter open and the power set to the final deposition power. This was done so that entire chamber was exposed to the evaporative flux at the deposition set point to bring the chamber into a steady state condition and thermal equilibrium. The deposition step consisted solely of opening the substrate shutter to expose the sample surface to the flux of metal atoms emanating from the molten metal in the crucible. After the end point had been reached, the substrate shutter was closed, and the e-beam power ramped to zero emission current. The sample was then transferred to the load lock and then removed for XPS and AFM analysis.

\section{§B.2 Nanoparticle Decoration: Preliminary Results and Discussion}

Each nanoparticle decoration method tested are summarized in this section. All methods were successful in depositing metallic nanoparticles. XPS and AFM analysis were the primary tools used to characterize these methods. Overall, the evaporative route for metallic nanoparticle decoration (Section $\$$ B.2.4) shows the most promise. The most attractive aspect to this method is the ease of deposition and potentially high degree of reproducibility.

\section{§B.2.1 Chemical Route for Metallic Nanoparticle Decoration}

Past work has been conducted by the Stinespring lab group on liquid phase nucleation of metallic nanoparticles on to a graphene surface where a detailed parametric study was conducted on the effect of nucleation time and precursor concentration. ${ }^{(264)}$ That work was continued here with an aim to determine the feasibility of the chemically mediated route for nanoparticle decoration on the as-fabricated $\mathrm{SiC} /$ graphene devices produced in this work. The conclusions of this study are based on the decoration and characterization of over 60 samples nucleated using this chemical route. Silver, gold, platinum, and iridium were all successfully nucleation from the liquid phase reduction reaction with $\mathrm{NaBH}_{4}$ and their respective salt precursors $\left(\mathrm{AgNO}_{3}, \mathrm{HAuCl}_{4} \cdot 3 \mathrm{H}_{2} \mathrm{O}\right.$, $\mathrm{H}_{2} \mathrm{PtCl}_{6}$ and $\mathrm{H}_{2} \mathrm{Cl}_{6} \mathrm{Ir}$, respectably). As determined from the past work, the optimum deposition parameters (time and concentrations) were used here to decorate the graphinated sensors with nanoparticles. Discussed here are general observations and the effects of the modifications made to the previously published procedures.

In short summary, graphinated $\mathrm{SiC}$ samples were placed in a small beaker containing $47 \mathrm{~mL}$ of $2.71 \mathrm{mM} \mathrm{NaBH}_{4}$, to which then $1 \mathrm{~mL}$ of $10 \mathrm{mM}$ metallic salt of choice was added to induce 
nucleation. The samples were allowed to incubate in this solution for $12 \mathrm{hrs}$. $\mathrm{NaBH}_{4}$ acted as a reducing agent which caused particle nucleation from metal ions. The underlying concept is that the chemically reduced surface (more electronegative) attracts positive metal ions from the solution and promote nanoparticle nucleation on the surface. However, it was observed that nucleation occurred both in the liquid phase and on the surface of the device. Solution phase nucleation was obvious from the observed formation of stable colloidal solutions or from precipitation of particles form the liquid visible to the naked eye. Surface nucleation was determined from XPS (used for elemental detection) and AFM (partial characterization).

While this chemical route was shown to produce nanoparticles, it was found to have several drawbacks. These included poor repeatability, poor nanoparticle area coverage and sized distribution, degradation of the deposited electrical contact and detrimental chemical modification of the device surface. Over the course of this work several small modifications were made to the procedure in an effort to address these issues. However, these issues were never completely resolved, and so other method of nanoparticle nucleation were investigated (detailed in the following subsections).

The primary issue of this nucleation method were the lack of reliability and inconstancy in producing nanoparticle decorated surfaces. Additionally, for experiments which did yield nanoparticles, the surface coverage was irregular with large areas completely undecorated. Both issues are suspected to have stemmed from the decomposition of $\mathrm{NaBH}_{4}$ by way of the Equation $B-1$ with water and with the sodium replacement reaction with metallic salt by Equation $B-2$ to Equation B-5, depending on the metal salt used. ${ }^{(695)}$

$$
\begin{array}{cc}
\mathrm{NaBH}_{4}+4 \mathrm{H}_{2} \mathrm{O} \rightarrow 4 \mathrm{H}_{2}+\mathrm{NaBH}(\mathrm{OH})_{4} & \text { Equation B-1 } \\
2 \mathrm{AgNO}_{3}+2 \mathrm{NaBH}_{4} \rightarrow 2 \mathrm{Ag}+\mathrm{H}_{2}+\mathrm{B}_{2} \mathrm{H}_{6}+2 \mathrm{NaNO}_{3} & \text { Equation B-2 } \\
2 \mathrm{HAuCl}_{4}+3 \mathrm{NaBH}_{4} \rightarrow 2 \mathrm{Au}+3 \mathrm{BH}_{4}+5 \mathrm{HCl}+3 \mathrm{NaCl} & \text { Equation B-3 } \\
2 \mathrm{H}_{2} \mathrm{PtCl}_{6}+3 \mathrm{NaBH}_{4} \rightarrow 2 \mathrm{Pt}+8 \mathrm{H}_{2}+3 \mathrm{BCl}_{3}+3 \mathrm{NaCl} & \text { Equation B-4 } \\
2 \mathrm{H}_{2} \mathrm{IrCl}_{6}+3 \mathrm{NaBH}_{4} \rightarrow 2 \mathrm{Ir}+8 \mathrm{H}_{2}+3 \mathrm{BCl}_{3}+3 \mathrm{NaCl} & \text { Equation B-5 }
\end{array}
$$


All reactions generate hydrogen gas and occur in the solution and at the sample surface. This is evident by the gradual appearance of gas bubbles on the sample. The gas evolution over time is shown in Figure B.8.

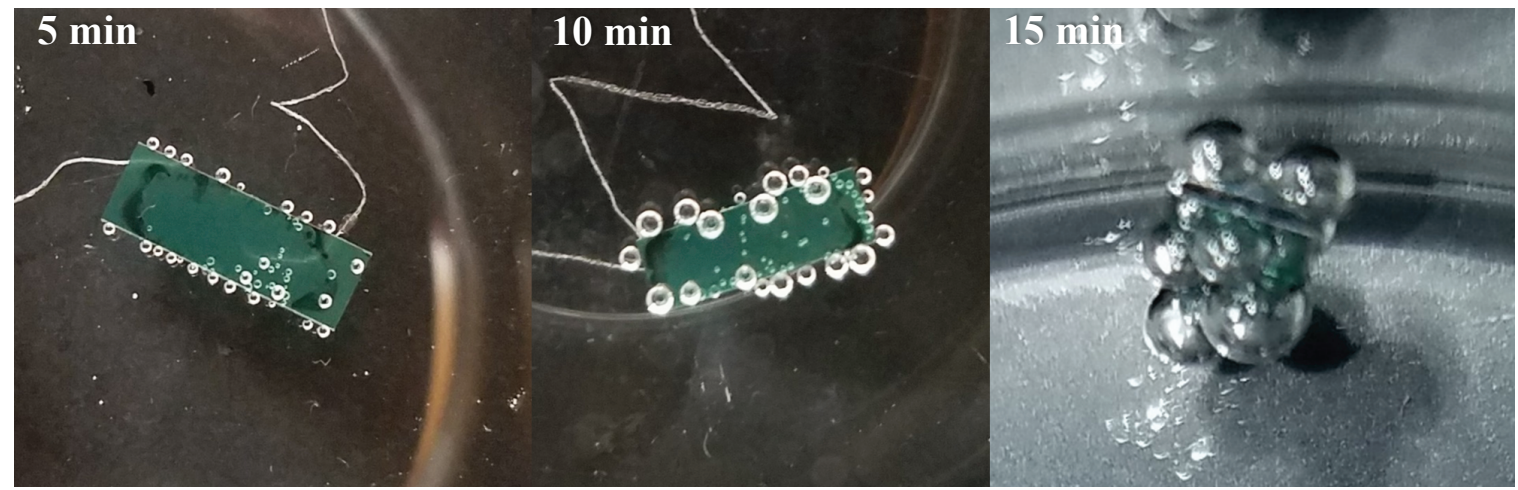

Figure B.8: Bubble formation on a graphinated sample at 5, 10 and 15 minutes in a solution of $2.71 \mathrm{mM}$. $\mathrm{NaBH}_{4}$. At 15 min the sample has begone to become buoyant and has stood up on end. Shortly thereafter the sample floated to the surface.

Gas bubble formation lead to two problems. First, the bubbles shield the area at the bubble attachment site from the nanoparticle precursors in the solution, effectively stopping and preventing nucleation from occurring at those sites. Second, the samples eventually float to the surface and unsubmerge caused by the buoyancy of the attached gas bubbles. This gas generation is believed to be the reason for both the inconsistency between trials and the irregular surface coverage. This impact of this detrimental gas formation was lessened but, never fully eliminated, by the addition of a spinning Teflon stir bar to the beaker. The turbulence caused by the spinning stir bar continually agitated the sample and sweep the sample surface of gas bubbles.

Silver nitrate $\left(\mathrm{AgNO}_{3}\right)$ was chosen as the prototypical metal salt for which to test various modifications to the nucleation procedure. It was found that the nucleation method was sensitive to both the manner in which, and the mixing rate of, the precursor solution. Slow addition of an initially dilute salt solution to an initially concentrated $\mathrm{NaBH}_{4}$ solution was found to yield the best nucleation outcome, in terms of the amount of nucleated material and the morphology of the nucleated particles. The dependence of the nucleation on the mixing method was apparent from the observed color change of the reaction solution.

The effect of the precursor mixing rate can be seen in Figure B.9. The composition of mixtures is the same in both; $47 \mathrm{~mL}$ of $2.25 \mathrm{mM} \mathrm{NaBH}_{4}$ and $10 \mathrm{~mL}$ of $12.95 \mathrm{mM} \mathrm{AgNO}_{3}$. In Figure 
B.9(A) the $\mathrm{NaBH}_{4}$ was added dropwise while the in Figure B.9(B) were simply poured together. Clearly, by mixing the precures slowly, a stable colloidal solution is formed in Figure B.9(A).

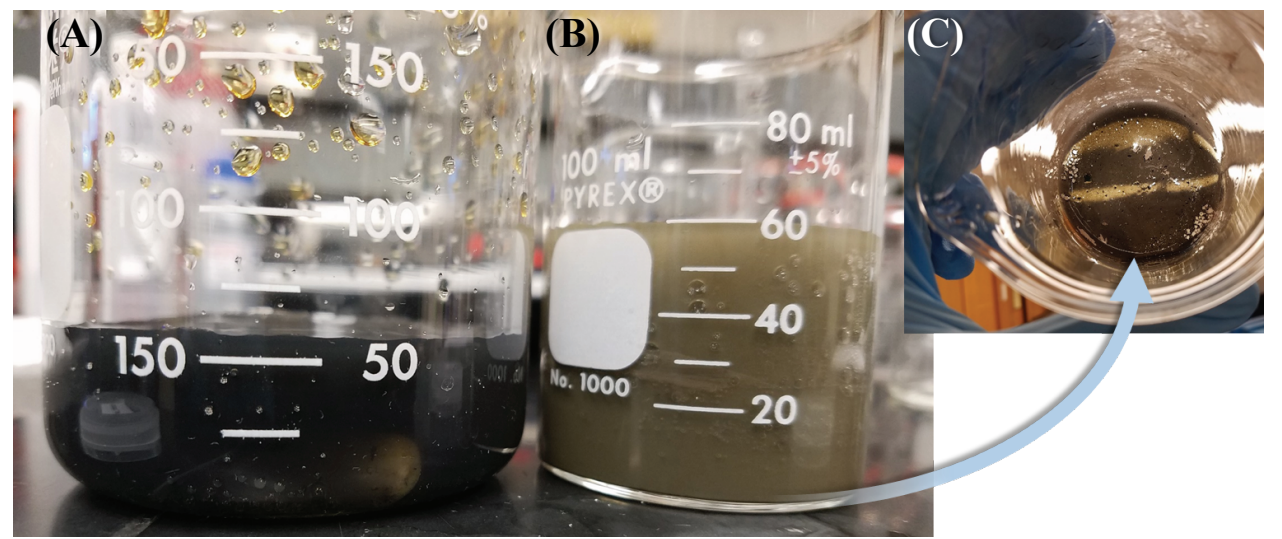

Figure B.9: Both beakers contain the same mixture: $47 \mathrm{~mL}$ of $2.25 \mathrm{mM} \mathrm{AgNO}_{3}$ and $10 \mathrm{~mL}$ of $12.95 \mathrm{mM}$ $\mathrm{NaBH}_{4}$. (A) A stable colloidal solution of silver particles was formed by using dropwise mixing. (B, C) Large particles have formed which precipitate out of the liquid as a result of the pour mixing.

Additionally, it was found that the slow mixing of a relatively dilute salt solution into a relatively concentrated $\mathrm{NaBH}_{4}$ solution lead to better nucleation than did the slow mixing of a relatively dilute $\mathrm{NaBH}_{4}$ solution into a relatively concentrated salt solution, even though the final concentrations in both were identical. Again, this is clear through the observation of the final solution, as shown in Figure B.10. In Figure B.10(A), where dilute $\mathrm{NaBH}_{4}$ was added to concentrated $\mathrm{AgNO}_{3}$, large particles formed and precipitate out where in Figure B.10(B),

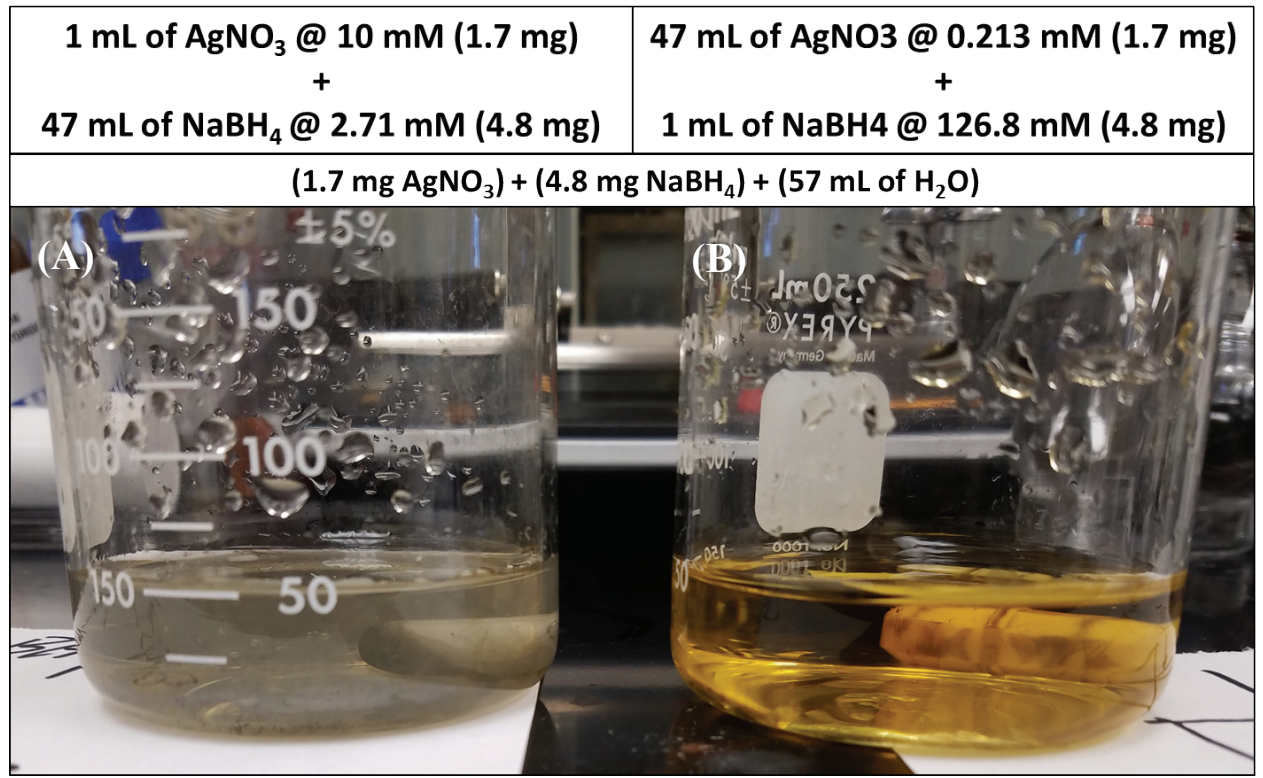

Figure B.10:(A) The result of dropwise mixing of dilute $\mathrm{NaBH}_{4}$ into a concentrated $\mathrm{AgNO}_{3}$ salt solution. Large particles have formed and have begun to precepted out of solution. (B) The result of dropwise mixing of dilute $\mathrm{AgNO}_{3}$ salt solution into concentrated $\mathrm{NaBH}_{4}$; a stable colloidal solution has formed. 
concentrated $\mathrm{AgNO}_{3}$ was added to dilute $\mathrm{NaBH}_{4}$ and a stable colloidal solution was formed. Note that the final concentrations of both components are the same in both Figure B.10(A) and (B).

It is clear that the slow addition of the metal salt leads to smaller particle formation, where faster mixing leads to large particle growth. Given that the total amount of metal atoms is the same in both cases, it follows that the growth rate and therefore the size, are limited only by the availability of reactant atoms. By controlling the rate of available atoms, the nucleation process can be slowed down. This limits the reaction rate by starving nucleation sites of reactive atoms which ultimately leads to smaller particle formation. This prevents nucleation runaway events which occur when there is an abundance of nucleation atoms at a single nucleation site, leading to large particle formation at these sites.

This hypothesis is supported by AFM analysis of the nucleated surfaces prepared by mixing a dilute $\mathrm{NaBH}_{4}$ solution into a concentrated salt solution. Shown in Figure B.11 are AFM images from three different samples showing varying degrees of nucleation runaway. In Figure B.11(A), a high number of large nucleation events have proceeded to a much greater extent than is desirable. Figure B.11(B) is dominated by a smaller number of sites where it is clear that rapid runaway nunation has occurred. In Figure B.11(C) many desirable nanoparticles are seen (i.e. small, uniform and well distributed), with only one instance of a runway nucleation event. The fact that each sample was produced in an identical fashion further illustrates the variations in repeatability of the liquid nucleation process. 


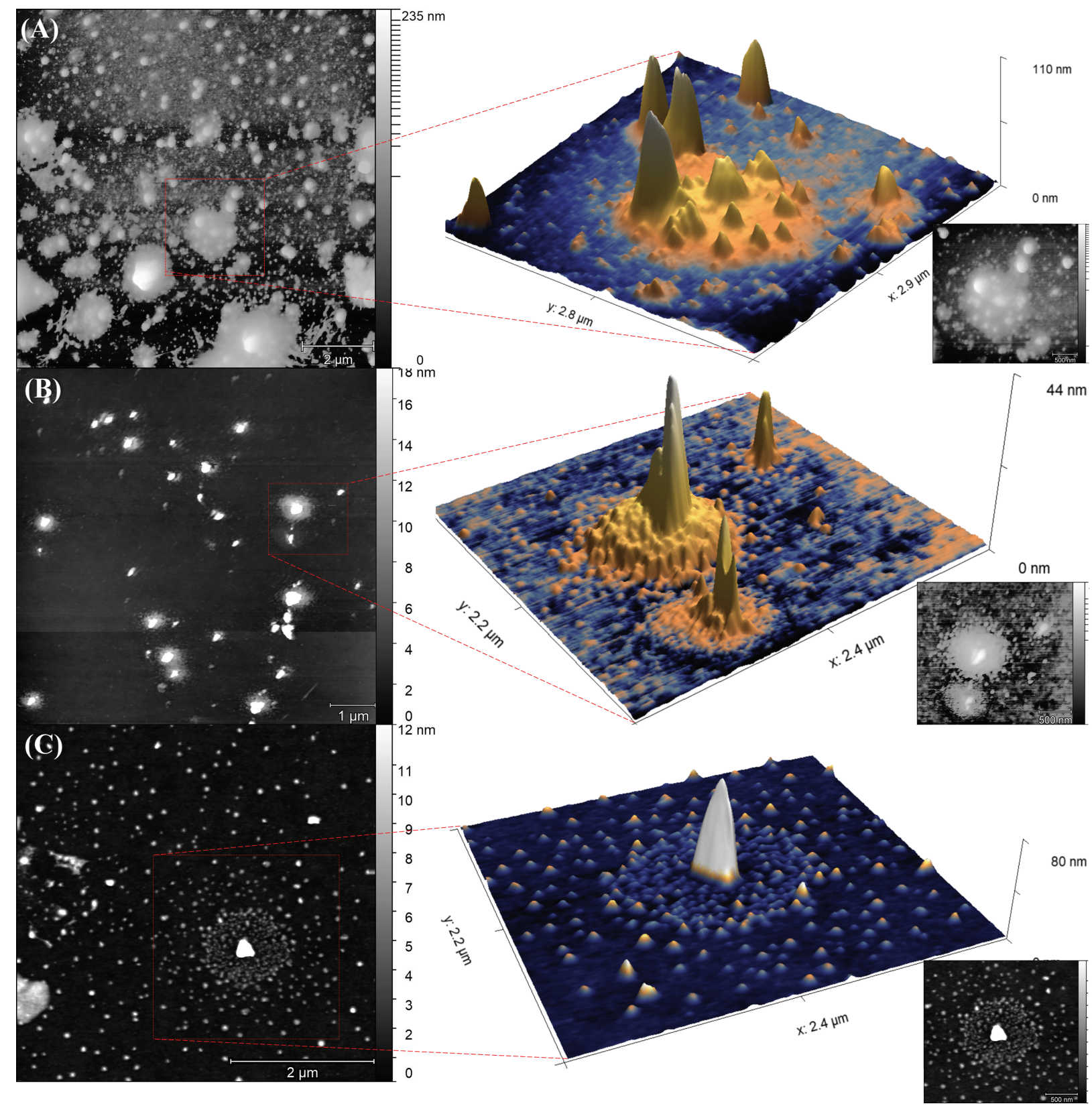

Figure B.11: Evidence of nucleation runaway based on the observed structures. Two samples, treated under the same conditions, showing varying degrees of nucleation runaway. The key features in both are the large particles surrounded by a growing nest of smaller nanoparticles. The false color bar ranges have been truncated to more fully resolve the feature of both images. Also shown are false colored 3D renders of select nucleation sites at different stages of runaway.

While these conclusions are not profound, they do indicate that the rate of reaction, governed by the availability of atoms from which each nucleation site may draw from, has a large impact on the end result. Based on these conclusions, the experimental setup was modified to what is shown in Figure B.12. A round bottom flask was used in conjunction with a magnetic stir bar to 
generate a standing vortex within the liquid in the flask. The burette setup was arranged such that the liquid droplet from the burette tip fell into the neck of the flask, spread across the flask wall and ran down the side into the liquid. The vortex rim chased along the interior wall of the flask and sampled the film of precursor liquid running down the sidewall and quickly integrated it into the main body of the liquid. This rapid integration of the dilute salt solution ensures thorough mixing of the nucleation precures. This mixing method ensures a better matchup between nucleation sites and precursor atoms due to the slow and thorough mixing which occurs. While this setup did not complete eliminate runaway nucleation events, it did reduce the frequency of their occurrence, as determined from subsequent AFM measurements.

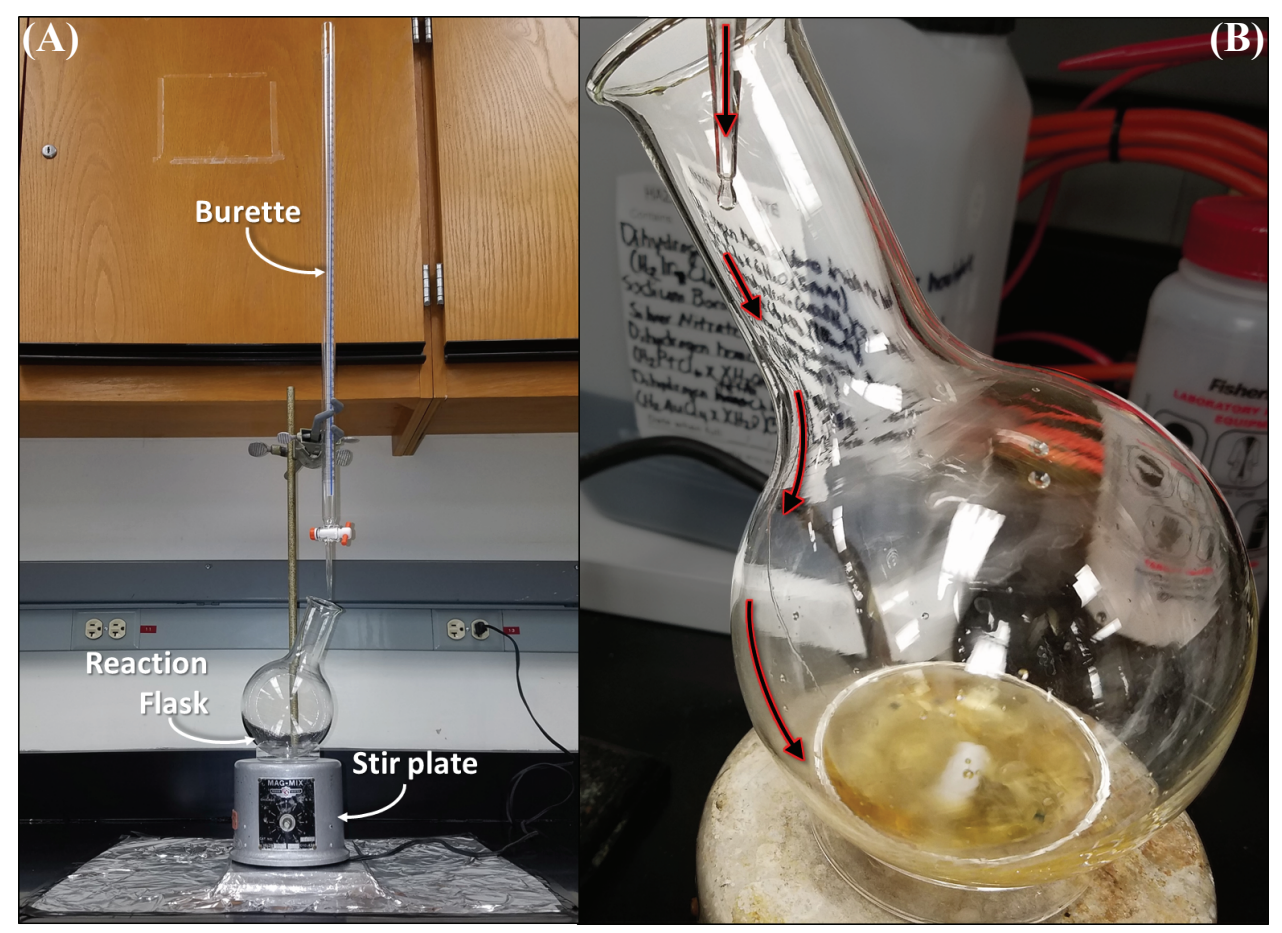

Figure B.12: (A) The modified equipment setup for nucleation experiments using $\mathrm{NaBH}_{4}$. (B) Close up view illustrating the drip path of the burette solution into the flask solution with stir bar.

While the chemistry is very similar for each metal, it is not unreasonable to suspect that in practice the resulting nucleation may be very different, even under identical conditions. However, a detailed parametric study was not conducted here. Rather, a simple exploratory study was preformed, using the optimum parameters and experimental setup devised for Ag nucleation, to determine if other metals could be made to nucleate onto the graphinated surfaces.

The following results represent the 'best case' scenario for each metal, in terms of partial coverage, morphology and distribution. However, as previously stated, even using the optimum 
parameters and the improved setup shown in Figure B.12, this nucleation method perpetually suffered from poor repeatability. AFM and XPS analysis are shown for each; note that the area coverage from AFM analysis does not match up with the elemental concentration as detected by XPS analysis. This is not surprising. There are several reasons for this, most of which stem from the inherently different detection methods and fundamental difference in the two quantities. This is particular case the discrepancy is most likely due to large difference in analysis area (25-100 $\mathrm{um}^{2}$ for AFM and $7850 \mathrm{um}^{2}$ for XPS). The larger XPS analysis region captures areas which have little or no nucleated material which. When these bare areas are convoluted with the signal from the covered areas, the recorded concentration is reduced. This is reflective of the 'patchiness' of the coverage inherent to the liquid nucleation process and is more representative of the samples coverage as a whole. Whereas analysis of AFM images is only conduced on scans which show particle coverage and is, by its nature, more selective of decorated areas with higher coverage. 
Figure B.13 shows the analysis of a graphenated sample which showed good nucleation of Ag nanoparticles.

(A)
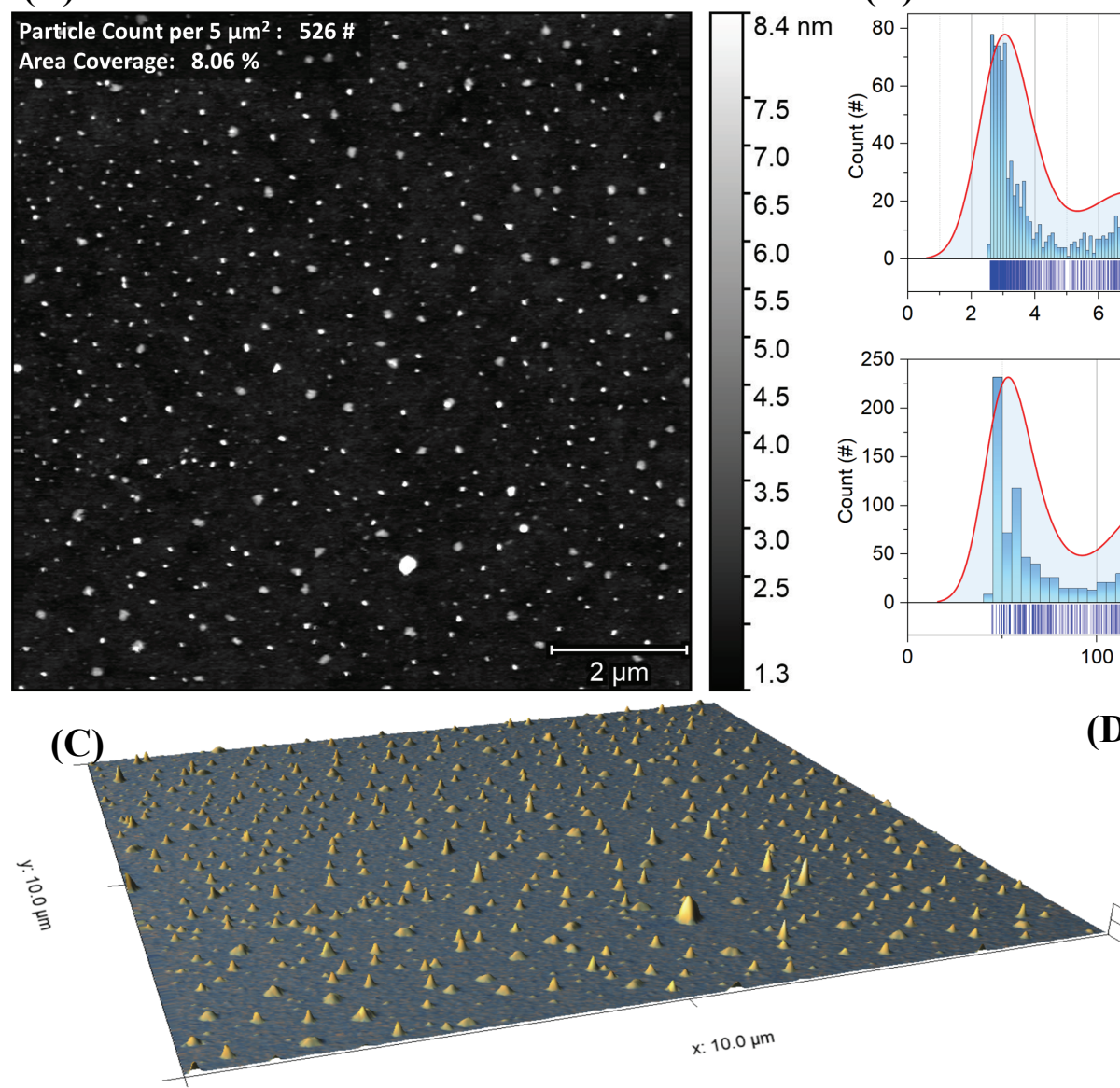

(B)
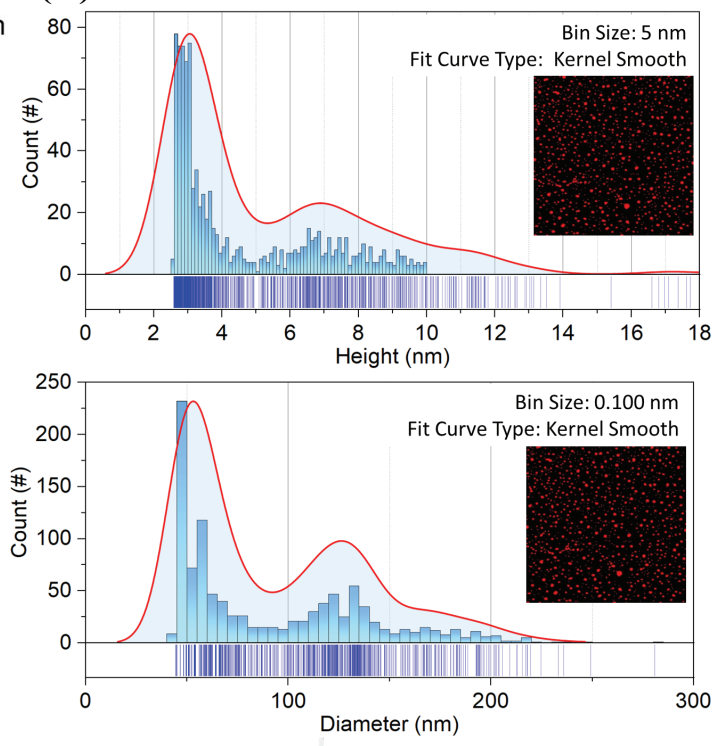

(D)

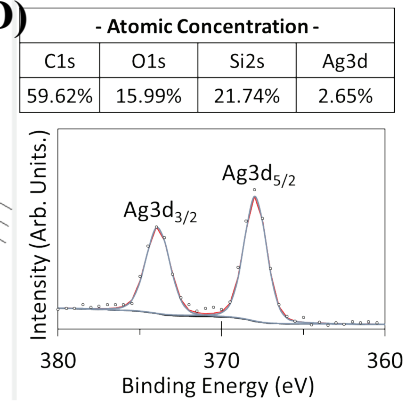

Figure B.13: (A) AFM image showing nanoparticles. (B) Histogram analysis showing the height and diameter distribution of the nanoparticles in (A), using a combination height and curvature thresholding detection method; inset in (B) are the detection maps. (C) 3-D false colored image of (A). (D) XPS results showing the elemental distribution and a section of the survey scan showing the presence of silver. 
Figure B.14 shows the analysis of a graphenated sample which showed good nucleation of Au nanoparticles.

(A)

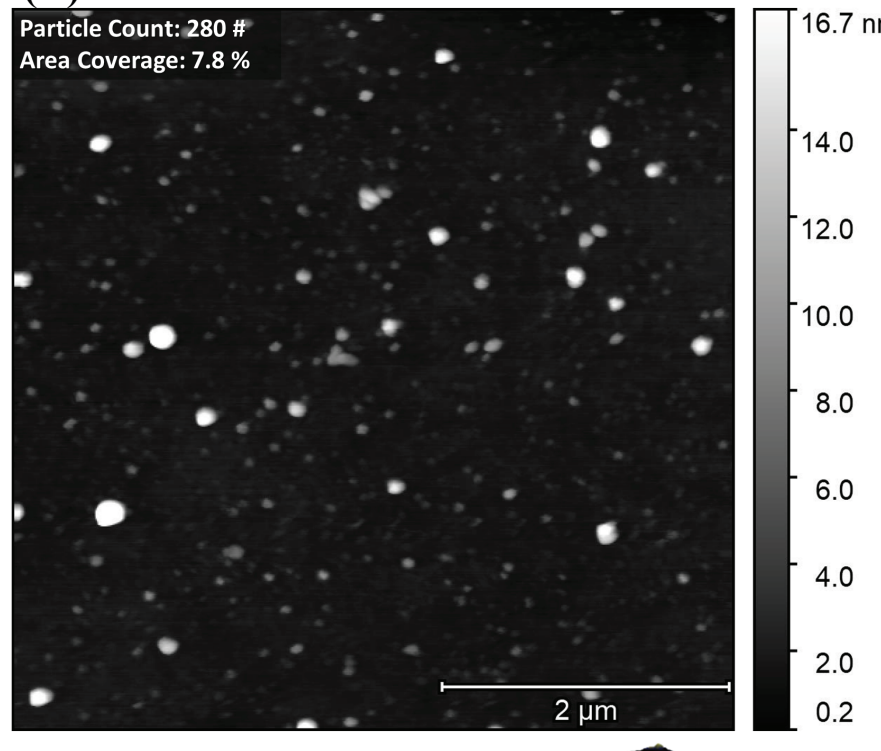

(B)
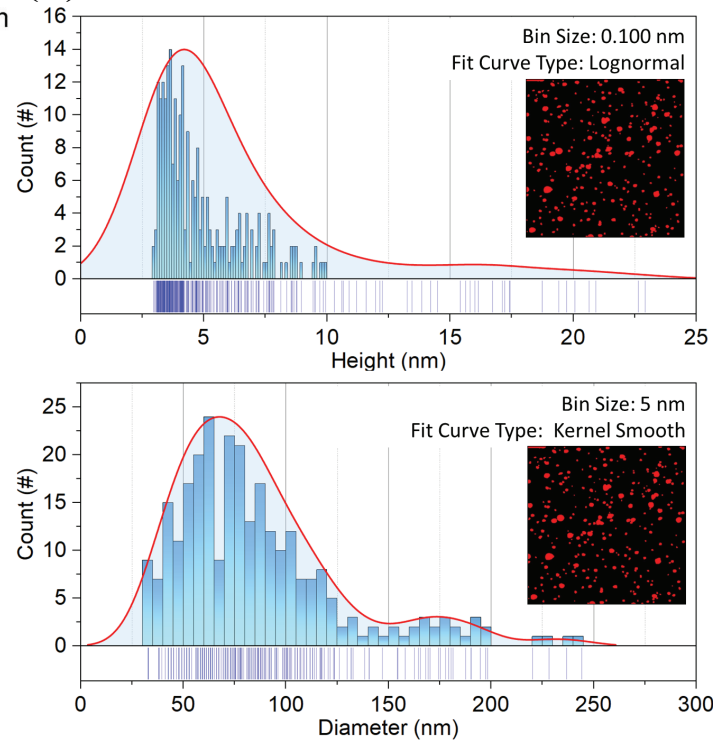

\begin{tabular}{|c|c|c|c|c|}
\hline \multicolumn{5}{|c|}{ - Atomic Concentration - } \\
\hline C1s & N1s & O1s & Si2s & Au4f \\
\hline $60.59 \%$ & $0.94 \%$ & $20.07 \%$ & $18.15 \%$ & $0.25 \%$ \\
\hline
\end{tabular}

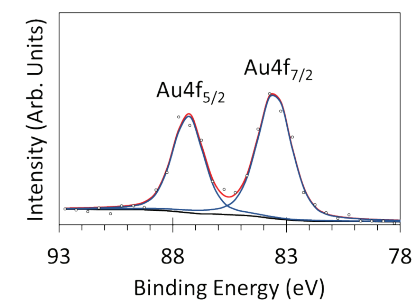

Figure B.14: (A) AFM image showing nanoparticles. (B) Histogram analysis showing the height and diameter distribution of the nanoparticles in (A), using a combination height and curvature thresholding detection method; inset in (B) are the detection maps. (C) 3-D false colored image of (A). (D) XPS results showing the elemental distribution and a section of the survey scan showing the presence of gold. 
Figure B.15 shows the analysis of a graphenated sample which showed good nucleation of Pt nanoparticles.

(A)
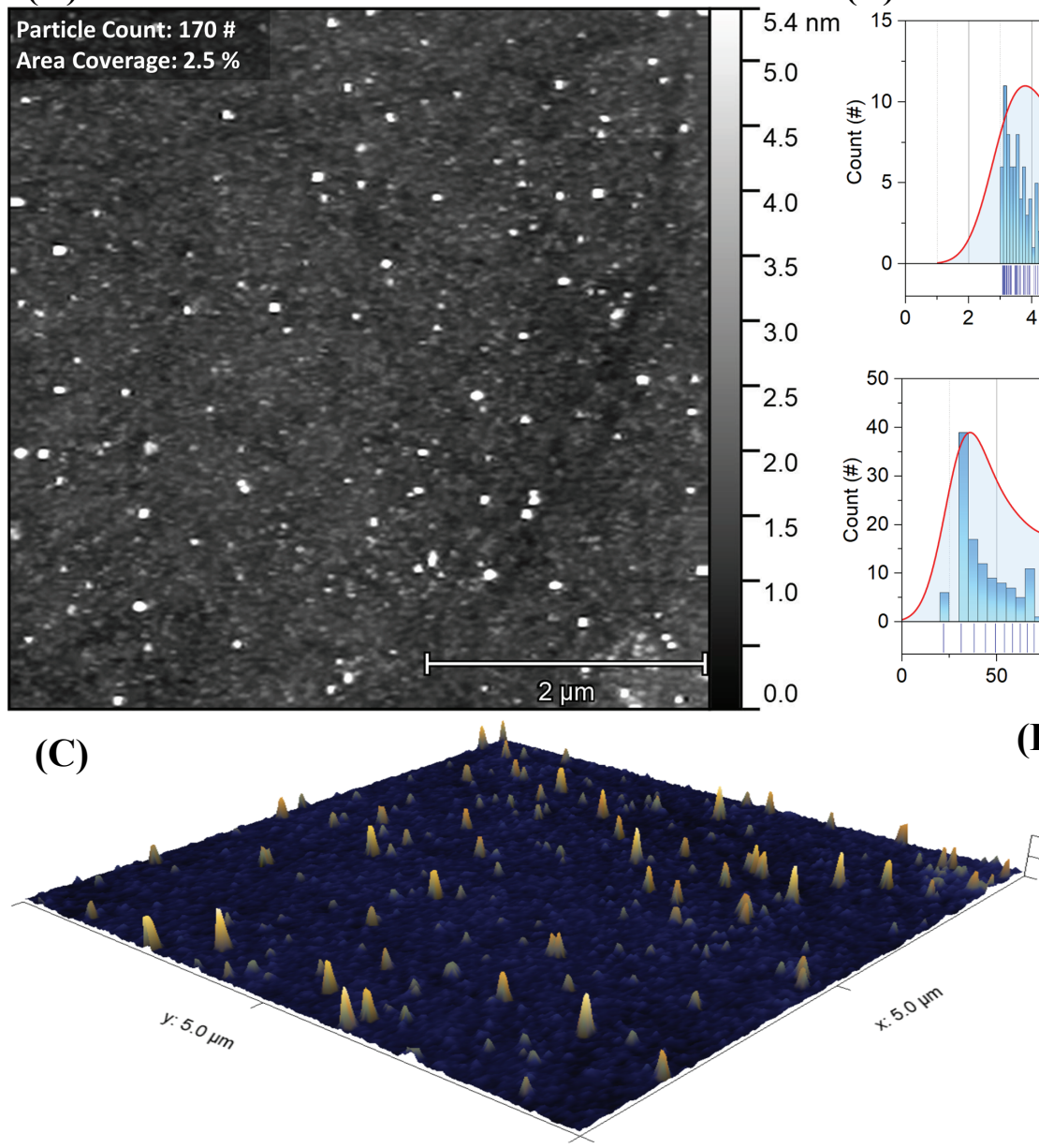

(B)
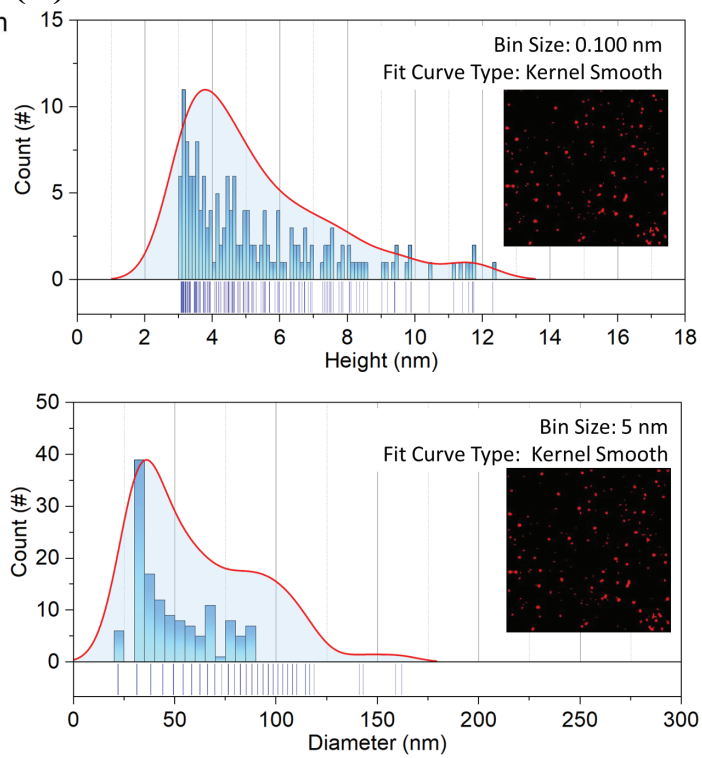

(D)
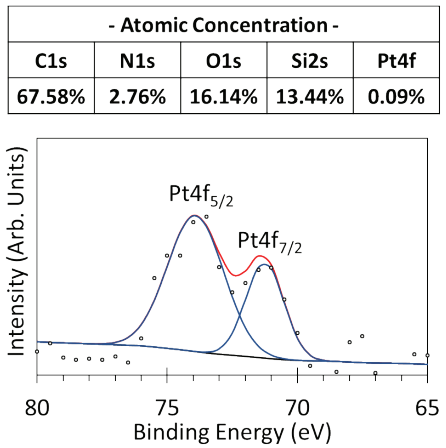

Figure B.15: (A) AFM image showing nanoparticles. (B) Histogram analysis showing the height and diameter distribution of the nanoparticles in (A), using a combination height and curvature thresholding detection method; inset in (B) are the detection maps. (C) 3-D false colored image of (A). (D) XPS results showing the elemental distribution and a section of the survey scan showing the presence of platinum. 
Nucleation of iridium were the most difficult to reproduce. Figure B.16 shows the analysis of a graphenated sample which showed nucleation of Ir nanoparticles. The XPS Ir peaks shown in

(A)

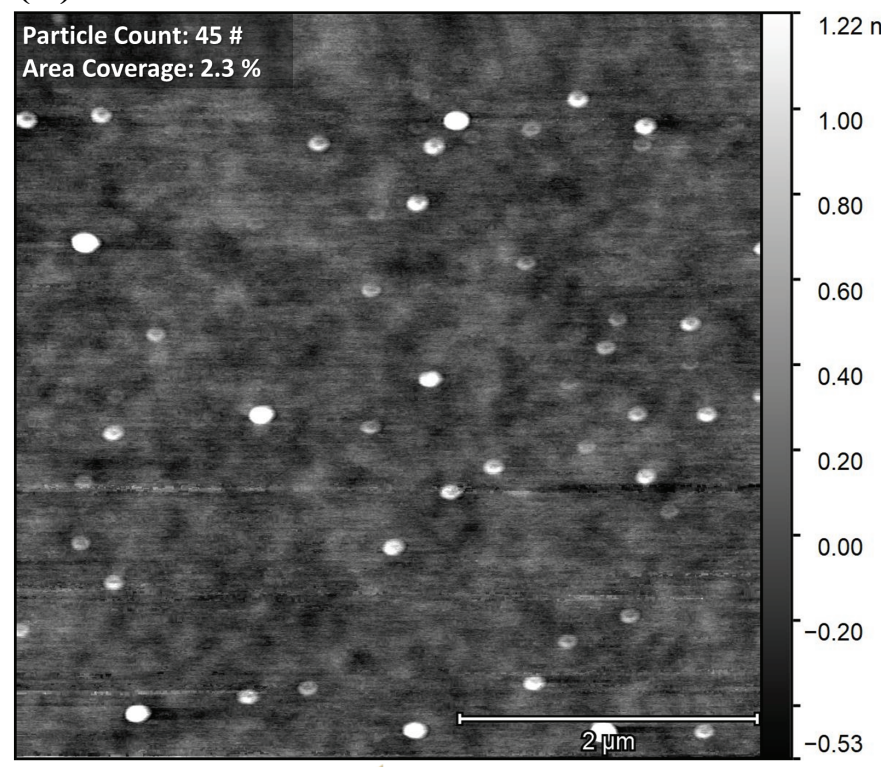

(C)
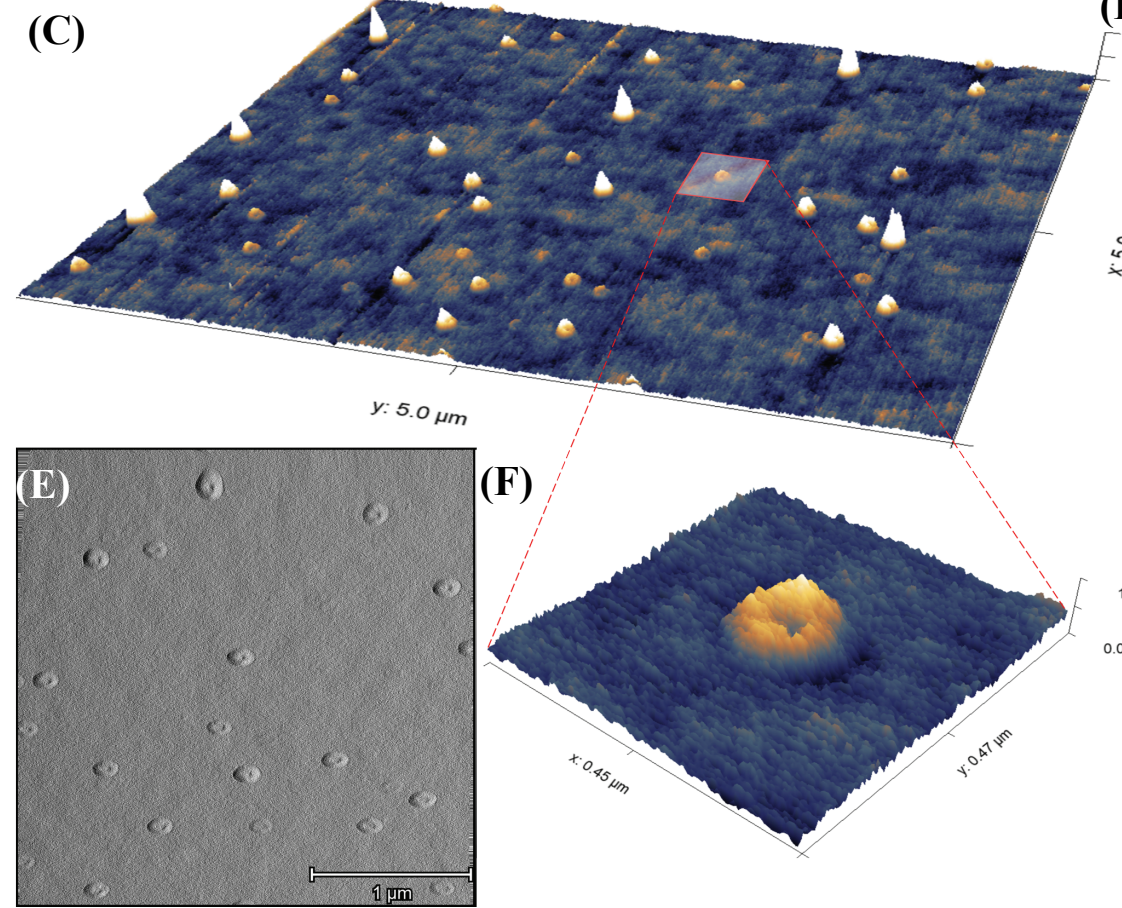

(F)

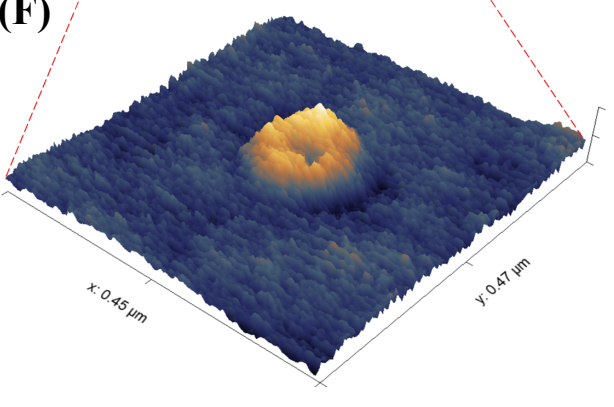

(B)
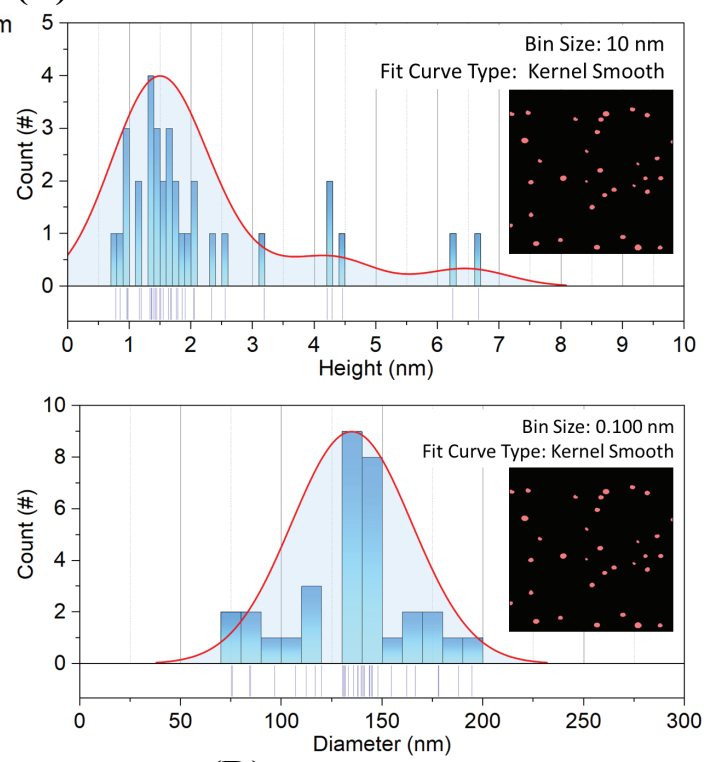

(D) - Atomic Concentration \begin{tabular}{|c|c|c|c|c|}
\hline C1s & N1s & O1s & Si2s & Ir4f \\
\hline $56.24 \%$ & $0.72 \%$ & $25.16 \%$ & $17.81 \%$ & $0.08 \%$ \\
\hline
\end{tabular}

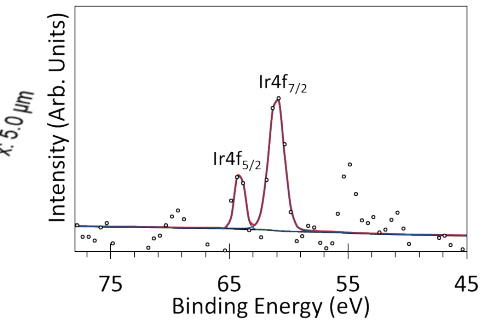

Figure B.16: (A) AFM image showing nanoparticles. (B) Histogram analysis showing the height and diameter distribution of the nanoparticles in (A), using a combination height and curvature thresholding detection method; inset in (B) are the detection maps. (C) 3-D false colored image of (A). (D) XPS results showing the elemental distribution and a section of the survey scan covering the Ir4f emission range. (E) Phase image on the same sample, more clearly revealing the donut-like structure of the nanoparticles. (F) Zoomed in region of (C) showing the donut-like structure. (G) Phase image of (F).

Figure B.16(D) are almost lost in the signal noise floor. This is due to two reasons. First, evidently 
there is very little Ir on the surface. Second, is the high relative sensitivity factor (RSF) of Ir. The Ir4f emission line has an RSF of 5.484 and a corrected RSF of 742.302 (the corrected RSF value takes into account the RSF as well as parameters specific to the particular instrument). For comparison, the $\mathrm{C} 1 \mathrm{~s}$ emission line RSF is 0.314 and the corrected RSF is 6.682; put another way, iridium is $111 \mathrm{x}$ harder to detect than carbon using this specific XPS instrument.

Clearly, Ir is present at levels around the detection limit of XPS. However, the low amount of Ir notwithstanding, these results are intriguing due to the unique morphology of the nanoparticles observed in the AFM analysis. Rather than the hemispherical or platelet-like structures seen for the other metals tested, donut-like structures are seen for Ir. While these structures can be explained by tip-damage induced artifacts, the fact that these donut structures have only been observed with Ir experiments indicate they are exclusive to Ir. Further study is required to determine the degree of reproducibility of these structures. These unique structures produced here are particular interesting, specifically, the nano-cavity within each particle. These particles could find interesting applications as photonic structures in light detection applications, for example.

In conclusion, the chemically mediated method for nanoparticle decoration of the graphenated samples, while intermittently successful, was found to be too unreliable. Both in terms of the number of trials which lead to successful nucleation and, for the trials which were successful, poor overall surface coverage of the whole sample. Additionally, this nucleation method was found to be highly sensitive to the concentration of reactants at the sample surface, and thus the specific method and rate of mixing were identified as key parameters. Recent real time direct observation of the nucleation of metal nanoparticles using similar chemistry show the nucleation of each particle to be a chaotic and dynamic process. ${ }^{(696)}$ Thus, the size distribution, unique morphologies and inconsistent results are not surprising.

Furthermore, the graphenated surfaces may not have a purely homogeneous atomic composition. Because this nucleation method relies on the chemistry occurring between the precursor solution and the surface, the heterogeneous nature of the surface may influence the frequency and extent of nucleation events. Variations in atomic composition across the sample surface, and from sample to sample, may also have contributed to the otherwise puzzling surface coverages and variations between samples. For example, small variations in the trace amounts of 
fluorine left in the film from the etching process (albeit at levels below the detection limit of XPS) may contribute to the large variations observed between nucleation samples. Given its extreme electronegativity, small amounts of fluorine may lead to large effects on nucleation (fluorine is the most electronegative element with an absolute electronegativity of $10.41 \mathrm{eV}$ and a 3.98 on the relative Pauling scale). ${ }^{(697)}$ Oxygen defects may play a similar role.

Two more observations lead to the conclusion of the chemically mediated route for nanoparticles; chemical modification of the graphene by $\mathrm{NaBH}_{4}$ and degradation of the $\mathrm{Ti} / \mathrm{Au}$ electrical contacts. The detrimental effects this process had to the adhesion and mechanical integrity of the Ti/Au electrical contacts can be seen in the SEM images shown in Figure B.17. Contact degradation may have been due to both the corrosive nature of the $\mathrm{NaBH}_{4}\left(\mathrm{NaBH}_{4}\right.$ is strongly basic with a $\mathrm{pH}$ of $\sim 10$ at the concentration used here, $10 \mathrm{mM}$ in water) as well as the decomposition on the sample surface (forming $\mathrm{H}_{2}$ gas) which may have worked to mechanically exfoliating the contacts. Undoubtedly, the mechanical agitation of the solution and sample (required to prevent excessive bubble attachment on the sample) compounded the contact degradation issue. This could be avoided by preforming the nucleation step before the contact deposition step; however, doing so would require an extensive redesign of the device fabrication pipeline.

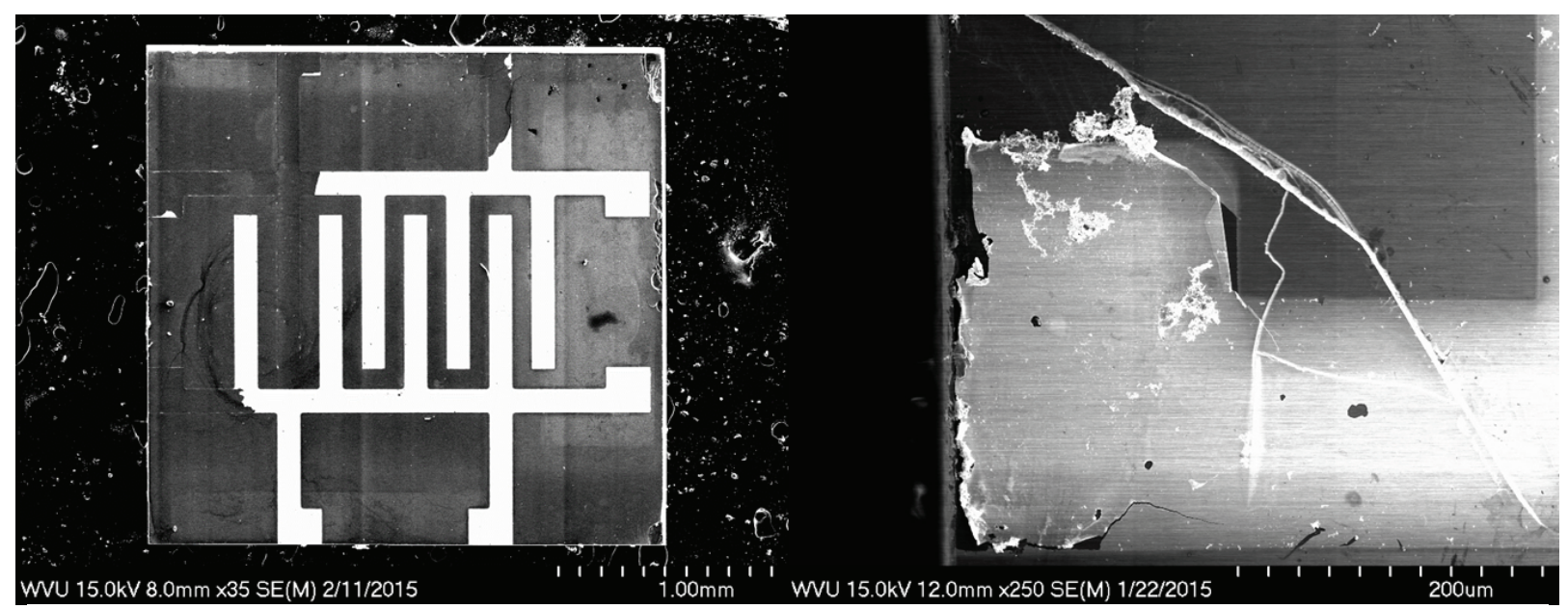

Figure B.17: SEM images of a $2.5 \mathrm{~mm}$ square SiC-Graphene sample after Au nucleation using $\mathrm{NaBH}_{4}$. Note the degradation of the Ti/Au contacts (bright areas of the image). 


\section{§B.2.2 Electrical Route for Metallic Nanoparticle Decoration}

This section details the investigation of electrically reducing the sample surface to drive nucleation. This was accomplished by either directly electrifying the sample or capacitively (electrostatically) coupling with the sample while it was in an aqueous precursor solution. By electrically reducing the surface (as opposed to chemically), nucleation could be achieved but without the need for caustic $\mathrm{NaBH}_{4}$. Advantages of nucleating without $\mathrm{NaBH}_{4}$ are the reduction of damage to the surface film and limiting the liquid phase nucleation which was observed to occur in precursor solution (bulk phase nucleation). Furthermore, this method allows for precise control over the voltage and duration which gives greater control over the nucleation process.

As a proof of concept, a simple series of experiments were designed to determine the feasibility of the electro nucleation method. They have been generically grouped into three subsets of experiments, termed here as the macro, micro and nano experiments. The experimental setup for each is described in Appendix $\mathbf{\$ B . 1 . 3 . ~ I n ~ m o s t ~ c a s e s , ~ t h e ~ s a m p l e ~ w a s ~ b i a s e d ~ b y ~ e l e c t r o s t a t i c ~}$ coupling (capacitive coupling). The samples were connected to the negative terminal of a power supply. A probe, connected to an earth ground or the positive side of the power supply, was brought into close proximity to the sample. The specifies of how this was done are what constitute the macro, micro and nano scale experiments. As was the case for the chemically mediated route, silver (via $\mathrm{AgNO}_{3}$ ) was used as the prototypical metal used for this exploratory study. Although $\mathrm{Au}, \mathrm{Pt}$ and Ir were all successfully nucleated using this method (via the macro experimental design).

To test on the macro level, the graphenated samples were electrified in two different ways. The first, where current was passed directly through the device, termed here as the DC method. The second, by electrostatic coupling, as described earlier and termed here as the EC method. In both cases a small aliquot $(\sim 10 \mu \mathrm{L})$ of a $10 \mathrm{mM} \mathrm{AgNO}_{3}$ aqueous solution was pipetted on the graphenated sample. The samples were electrified for a predetermined about to time. Then the setup was deenergized and the samples were removed, gently washed with water and then characterized with SEM.

The results of the macro DC experiments with a graphenated sample with contacts and a $\mathrm{AgNO}_{3}$ salt solution are given in Figure B.18. For 600 seconds a bias of $1.0 \mathrm{~V}$ was applied across the sample by the source measurement unit of the Keithley 4200 SCS system used elsewhere in 
this work. By using this system, the current could be recorded during the duration of the deposition process (current vs. time, It). This effectively allowed for the real time monitoring of the deposition process. Additionally, current vs. voltage (IV) curves were also recorded at three different stages of the experiment. First, in open air before any treatment, second after the addition of the salt solution (no bias) and third at the end of the experiment, after the sample was washed with water. SEM images of the sample are also shown in Figure B.18(D-E). 

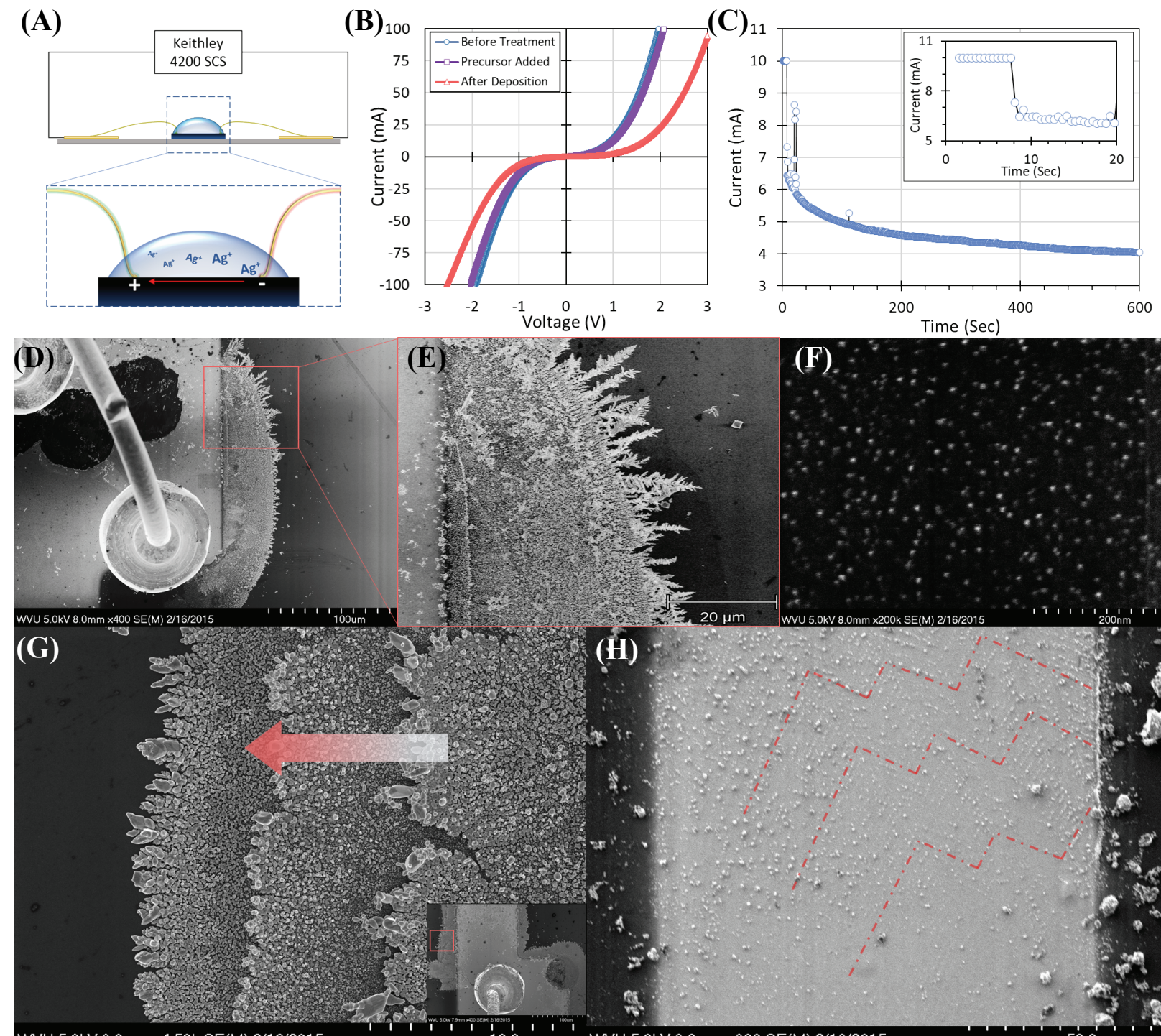

WVU 5.0kV 8.0mm $\times 4.50 \mathrm{k}$ SE(M) 2/16/2015

Figure B.18: (A) Schematic of the DC method. (B) IV plot showing the slight change in electrical character of the device after deposition. (C) The current flow resulting from a $1.0 \mathrm{~V}$ bias applied across the device during the 600 seconds of deposition. Inset within is the first 20 seconds of deposition; the large drop indicates that the majority of the deposition occurs within the first 10 seconds. (D) SEM of a ball wire bond made to a deposited Ti/Au contact on the device. (E) Magnified view of the inspection box shown in (D), showing the tree-like growth, emanating out from the current injection site. (F) SEM image take approximately at the midpoint between two contacts, revealing the presence of nanoparticles. (G) SEM taken adjacent to another current injection site (shown in the inset) illustrating a layer by layer growth. $(\mathrm{H})$ SEM of a Ti/Au contact with particles deposited on-top. Note the particle alignment, as illustrated by the dotted lines. 
Four observations are noted. First, is the somewhat unexpected increase in resistance of the device after Ag deposition. Inspection of Figure B.18(B) shows that after deposition, the device retains its Schottky behavior and a slight rectification character has appeared. This is most likely due to additional band bending resulting from the asymmetrical $\mathrm{Ag}$ deposition at the current injection sites. Second, based on Figure B.18(C), after an $\sim 8$ second delay, the majority of deposition appears to occur very quickly over $\sim 2-3$ seconds. Thirdly, is the observed preferential growth around the current injection sites. Interestingly, rather a single sheet of material growing from the injection electrode across the surface to then terminate at the ground electrode, layer growth proceeds out a finite amount before second- and third-layer growth begins. This confined growth may be influenced by the charge depletion region which is responsible for the Schottky behavior of the device. Lastly, is the observed particle alignment seen in Figure B.18(H). This could be due to complex interactions in electric field alignment along the surface, directed $\mathrm{Ag}$ deposition. This opens up the intriguing possibility for pattered partial deposition by manipulating the applied electric field.

To deposit material more evenly across the surface, the EC was used. For the EC setup, the current was not be monitored; however, IV data was taken in open air before treatment, after the addition of the precursor solution and after the deposition and water wash. The setup can be seen 
in Figure B.19(A,B). Using a laboratory power supply, a bias of $30 \mathrm{~V}$ was applied between the sample and the probe for 360 seconds. These results are shown in Figure B.19(C-F).

(A)

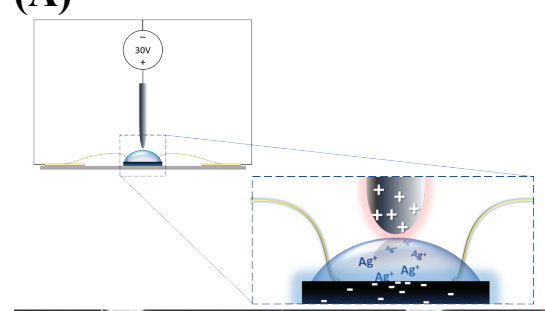

(B)

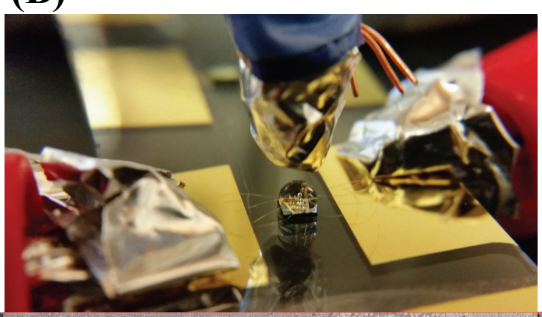

(C)

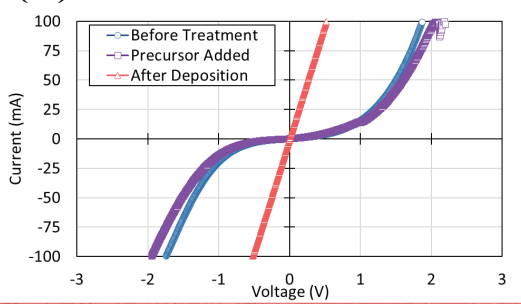

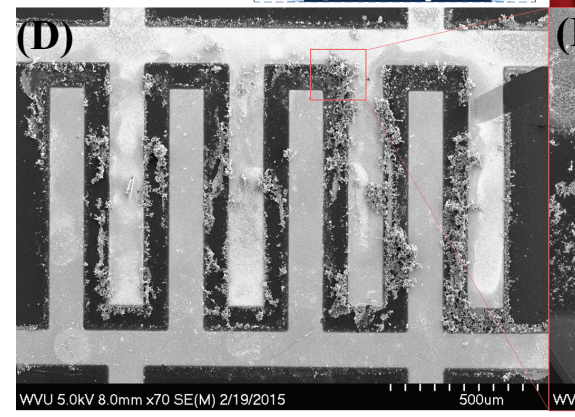

(E)

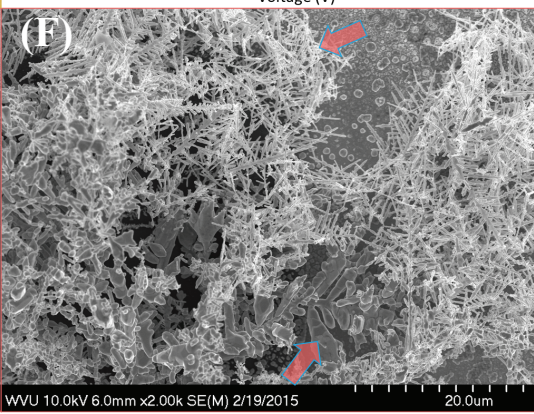

Figure B.19: (A) Schematic of the EC setup. (B) Real-world picture of the setup. The spacing between the probe and sample was estimated to be $\sim 5 \mathrm{~mm}$. (C) IV data collected from the sample before, after precursor addition and after deposition. (D-E) SEM images of the sample showing the extensive needle-like Ag growth. The arrows in (F) show the two types of Ag structures: thin, young needles and old, girthy needles.

Three observations are noted. First, material growth occurred to a much greater extent as compared to the DC method; to the point where the contacts have been shorted by the growth of $\mathrm{Ag}$ needles. This is evident by the ohmic behavior shown in Figure B.19(C) and the SEM image in of $(D)$. This is most likely due the much higher voltage used, as well as the fact that the whole device acted as an electrode rather than only once set of contacts, as was the case in the DC experimental setup. Second, as can be seen in Figure B.19(E, F), growth appears to occur in two stages; first as tree-like needle growth and second as needle thickening (this indicates that the needle length growth is self-limiting in some way). Lastly, the structure growth observed in the EC setup appears to occur 3-dimenitally as opposed to the more 2-dimentianl sheet-like growth seen in the DC experiment. This indicates that growth occurs parallel to the bias orientation and perpendicular to the field gradient; in the DC setup the field gradient decreased across the sample surface from one electrode to the other and in the EC setup, from the sample upward (in the Zdirection) towards the suspended probe.

During the course of the experiments (both DC and EC), it was observed that the precursor droplet evaporated, although not completely. This was due to the small amount of liquid and 
relatively large surface area of the droplet. This effectively changed the concentration of the solution (drastically increasing it) during the process, leading to both crystallization and drying effects as seen in Figure B.20.

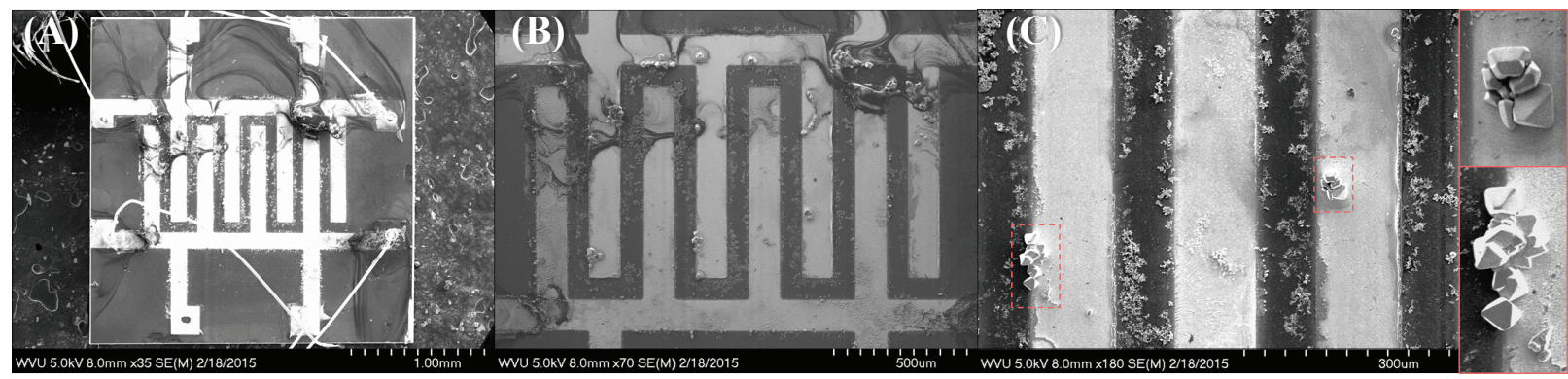

Figure B.20: (A, B) Drying effects, as a result of a decreasing droplet size, can be seen. (C) Ag crystals, as a result of the concentration of $\mathrm{AgNO}_{3}$ increasing to beyond the solubility point, crashing out of solution and crystalizing (magnified insets).

Therefore, the setup was modified to what is shown schematically in Figure B.21. The sample was mounted to a substrate (a TO header) and suspended upside-down in a beaker of precursor solution. A foil plate was placed under the beaker and electrically connected to the positive terminal of a laboratory power supply. The negative terminal was connected to the sample and a $30 \mathrm{~V}$ bias was applied to the sample. The spacing between the sample and the foil electrode was estimated to be $\sim 3 \mathrm{~cm}$. This setup increased the reservoir from which nucleation atoms could be pulled from and a constant concertation was maintained in the solution.

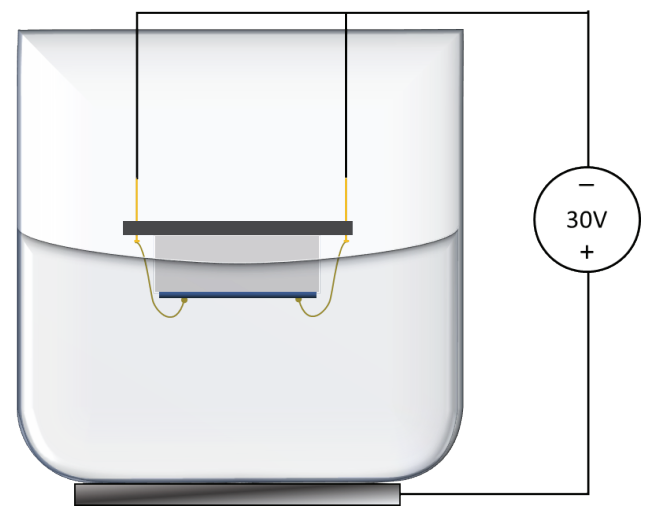

Figure B.21: Simple schematic showing the modified EC deposition setup.

This setup was used for the nucleation of $\mathrm{Ag}, \mathrm{Au}, \mathrm{Pt}$ and $\mathrm{Ir}$ using the EC method. All experiments used a $30 \mathrm{~V}$ bias and the experiment was allowed to run for 330 seconds. Material was successfully nucleated for each metal salt used. Complex, intricate structures were grown from Ag and Pt salts. Material was deposited using Au and Ir salts; structures were small and globular. 
SEM results for Ag deposition using this new EC setup are shown in Figure B.22. Growth occurred over the entire surface, largely independent of contact location, as can be seen in Figure B.22(A-C). The more even deposition observed here may be the result of a weaker and more uniform filed generated across the sample surface, due the electrical field spread caused by the larger probe spacing and attenuation due to the interfacial quarts bottom of the beaker. The fundamental feature is shown in Figure B.22(F). Similar to the needle like tree growth observed in Figure B.19(F), but with a more 3-dimentinoal and fractal like growth seen in. However, additional growth has manifested itself here as additional needle-on-needle growth resulting in a more fractal-like pattern.

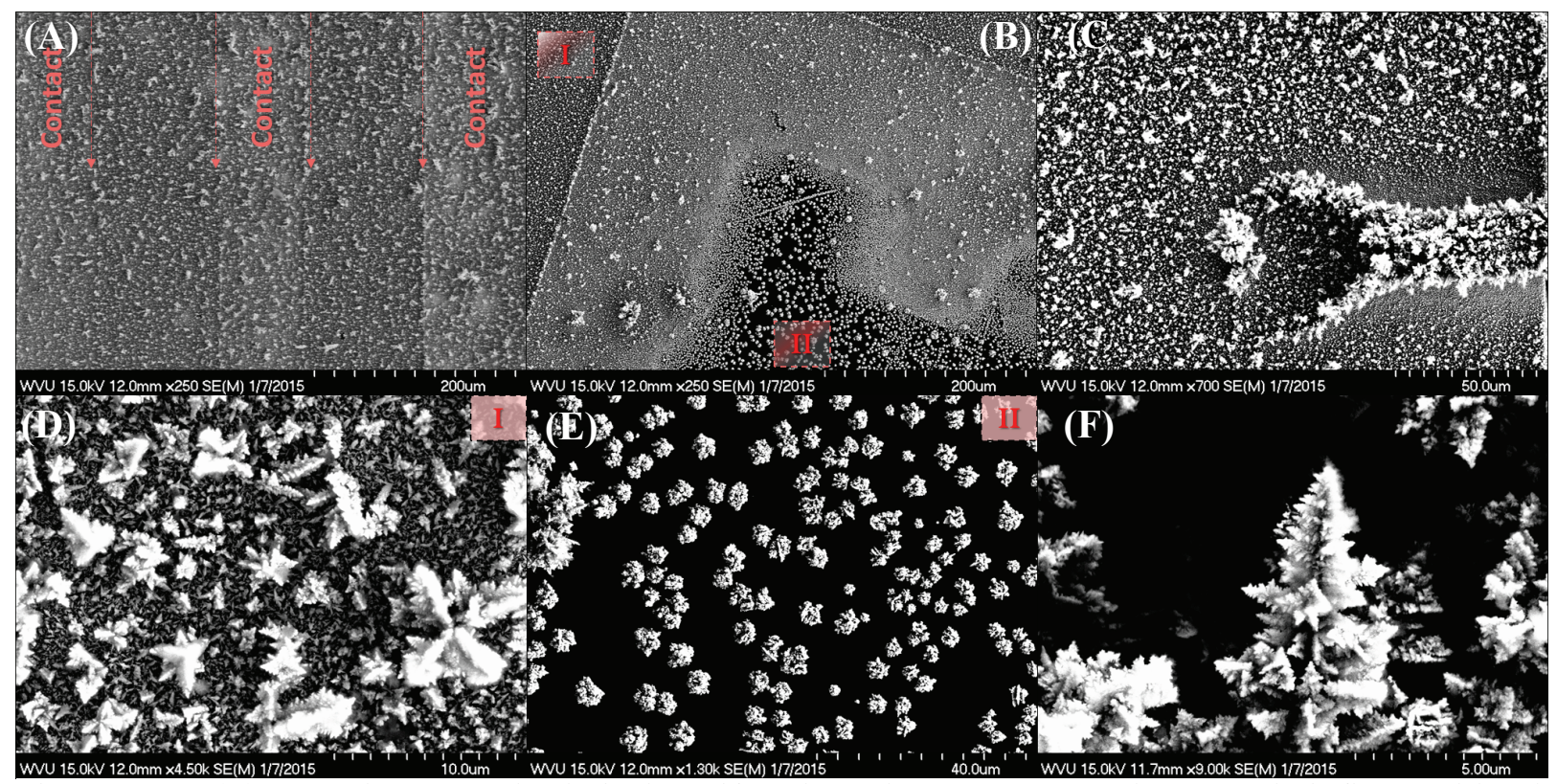

Figure B.22: (A) Growth occurred relatively uniformly and appears not to be strongly influenced by the $\mathrm{Ti} / \mathrm{Au}$ contacts. The location of contacts has been annotated. (B) There are two regions of growth: a dense filed of growth, annotated "I" and a sparse field of growth, annotated "II". (C) SEM showing indiscriminate growth on the graphenated surface, $\mathrm{Ti} / \mathrm{Au}$ contact and on an Au wire wedge bond. (D) Magnified view of the area 'I' annotated in (B). (E) Magnified view of the area 'II' annotated in (B). (F) Close up view of the primary growth feature. 
SEM results for Au using this new EC setup are shown in Figure B.23. Here, no intricate features are observed, only simple globular structures.

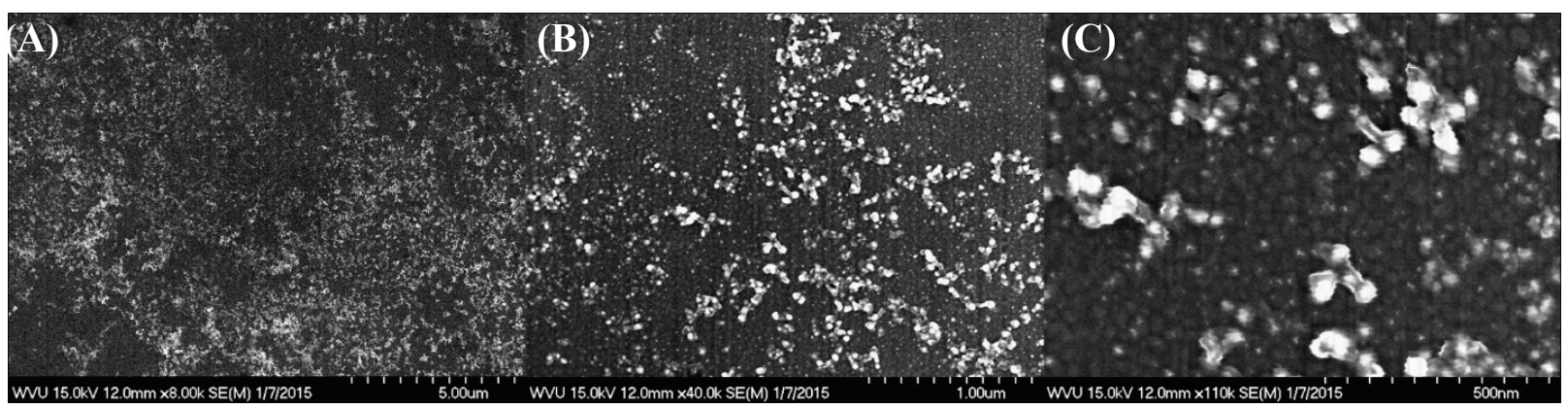

Figure B.23: Large area Au deposition of globular like structures.

SEM results for Ir using this new EC setup are shown in Figure B.24. Similar to the Au deposition seen in Figure B.23, deposited material consists solely of globular structures.

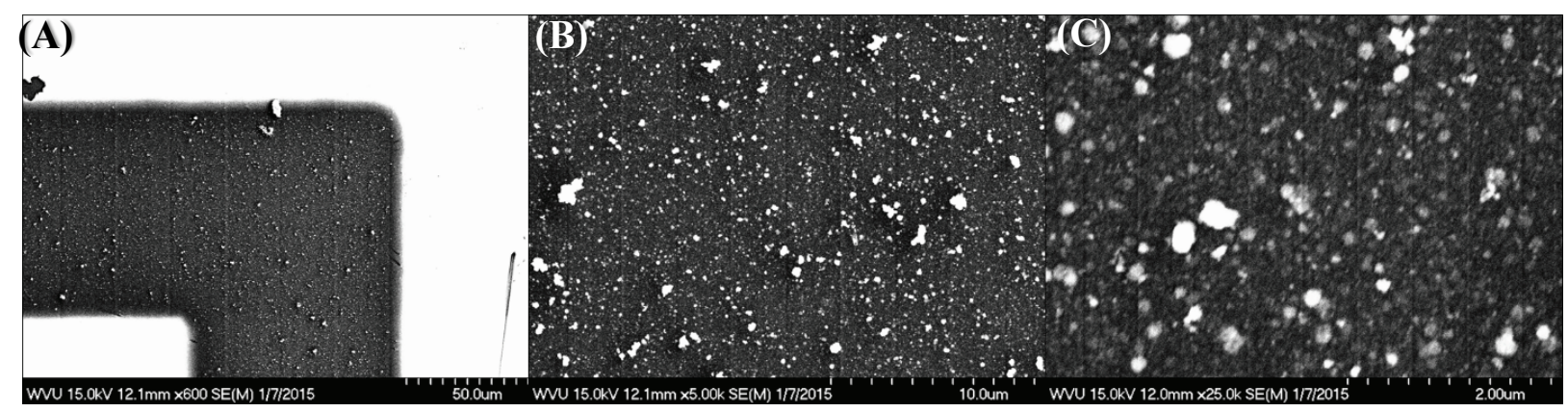

Figure B.24: Large area Ir deposition of globular like structures.

SEM results for Pt using this new EC setup are shown in Figure B.25. Complex, large area growth has occurred. Growth was characterized by large, interconnected blade structures creating sheets of material which occasional delaminated and curled up from the substrate. From these sheets, nodules of secondary growth appear to have occurred as well. 


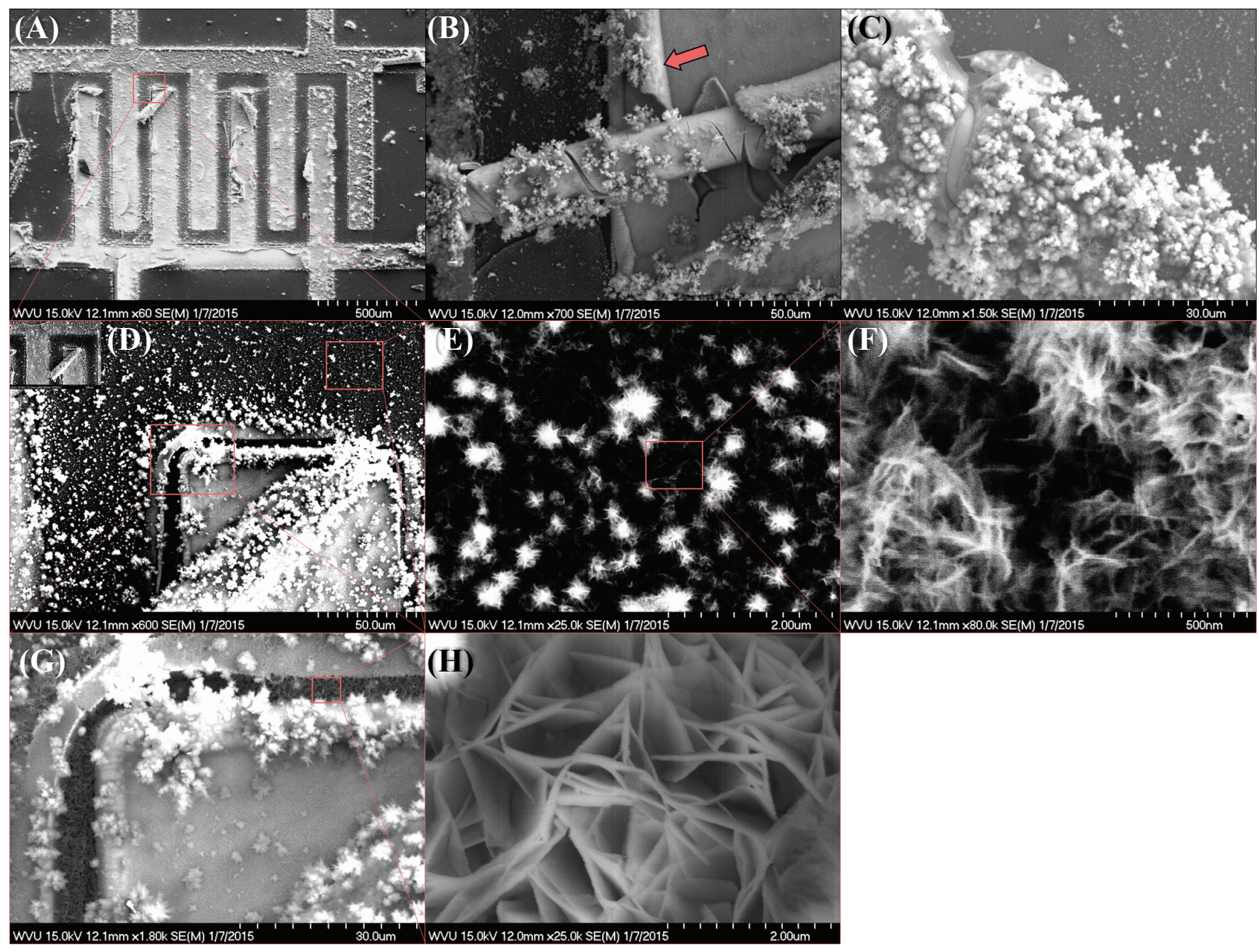

Figure B.25: SEM analysis revealing the growth structures. (A) The large area deposition can be seen. (B) The delaminated sheets of material; also visible is growth on a gold wire bond wire. (C) Magnified view of growth on a wire bond wire; the nodule growth is easily seen. (A,D,G,H) show successive magnified views revealing the interconnected bladed-like structure of the underlying sheets. (D,E,F) show successive magnified views showing a slightly different morphology to the underlying sheets.

The macro set of experiments were intriguing and were the proof of concept for the electro nucleation method, proving that metal cations can be driven to the graphene surface by an electric field and self-assemble into complex structures, which cover the entire surface. However, the macro setup lead to a much greater amount of nucleation that was desirable; as illustrated by the micron size nucleated features and the shorting of the device in some cases. Clearly, this method calls for a parametric study investigating the effects of the applied voltage, solution concertation and, in the case of the EC method, the distance between electrodes.

However, for the light detection and gas sensing applications, noncontiguous and discrete structures are desired. A new setup was devised to determine if, by decreasing the probe-sample 
spacing and confining the electric fields, discrete structures could be formed. This was the micro series of experiments. The experimental setup is described in Appendix $\$ B$.1.3. The primary difference between the micro and macro experiments were the decrease in probe tip size $(25 \mu \mathrm{m}$ vs. $\sim 10 \mathrm{~mm})$ and probe spacing of the positive electrode $(\sim 150 \mu \mathrm{m}$ vs. $\sim 5 \mathrm{~mm})$ and the fact that the positive electrode was in contact with the precursor solution. For all micro experiments a 1 $\mathrm{mM}$ aqueous $\mathrm{AgNO}_{3}$ solution was used as the precursor and $1 \mathrm{~cm}^{2}$ silicon substrates was used. The results of applying a $10 \mathrm{~V}$ bias between the sample and probe for 120 seconds can be seen in Figure B.26. Growth was observed to originated from the substrate and grow up to and meet the probe. Growth then extended radially, and the resulting tree-like structure was formed.

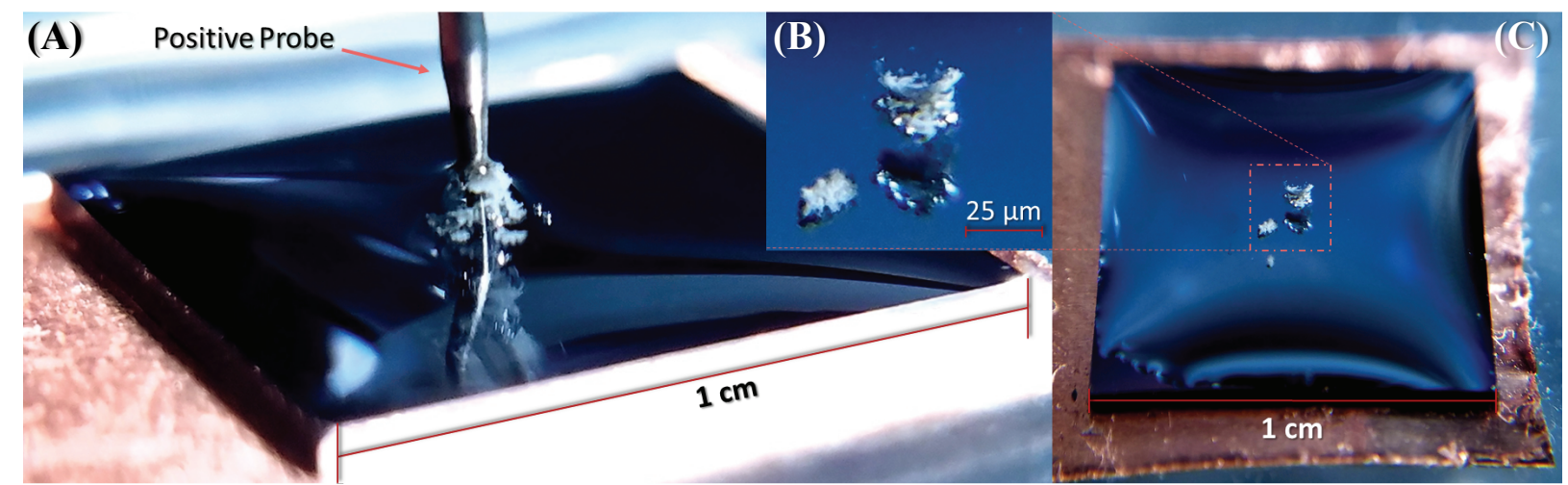

Figure B.26: (A) The resulting Ag structure grown between the sample and the probe after the application of a $10 \mathrm{~V}$ bias for 120 seconds. (B) Magnified view of (C) showing the Ag structure after the probe has been removed. The structure is still attached to the substrate.

By sequence reducing the applied voltage and the duration, successively smaller structures could be formed. At an applied bias to $5 \mathrm{~V}$ and duration of 30 seconds, nano-scale structures could be directed to grown beneath the biasing probe. AFM analysis of this area reveal many dendritic structures formed; typically, these were several micrometers across and 100-200 nm tall. Surrounding these larger structures were scattered spherical nanoparticles. An example of the structures formed can be seen in the AFM image in Figure B.27. 


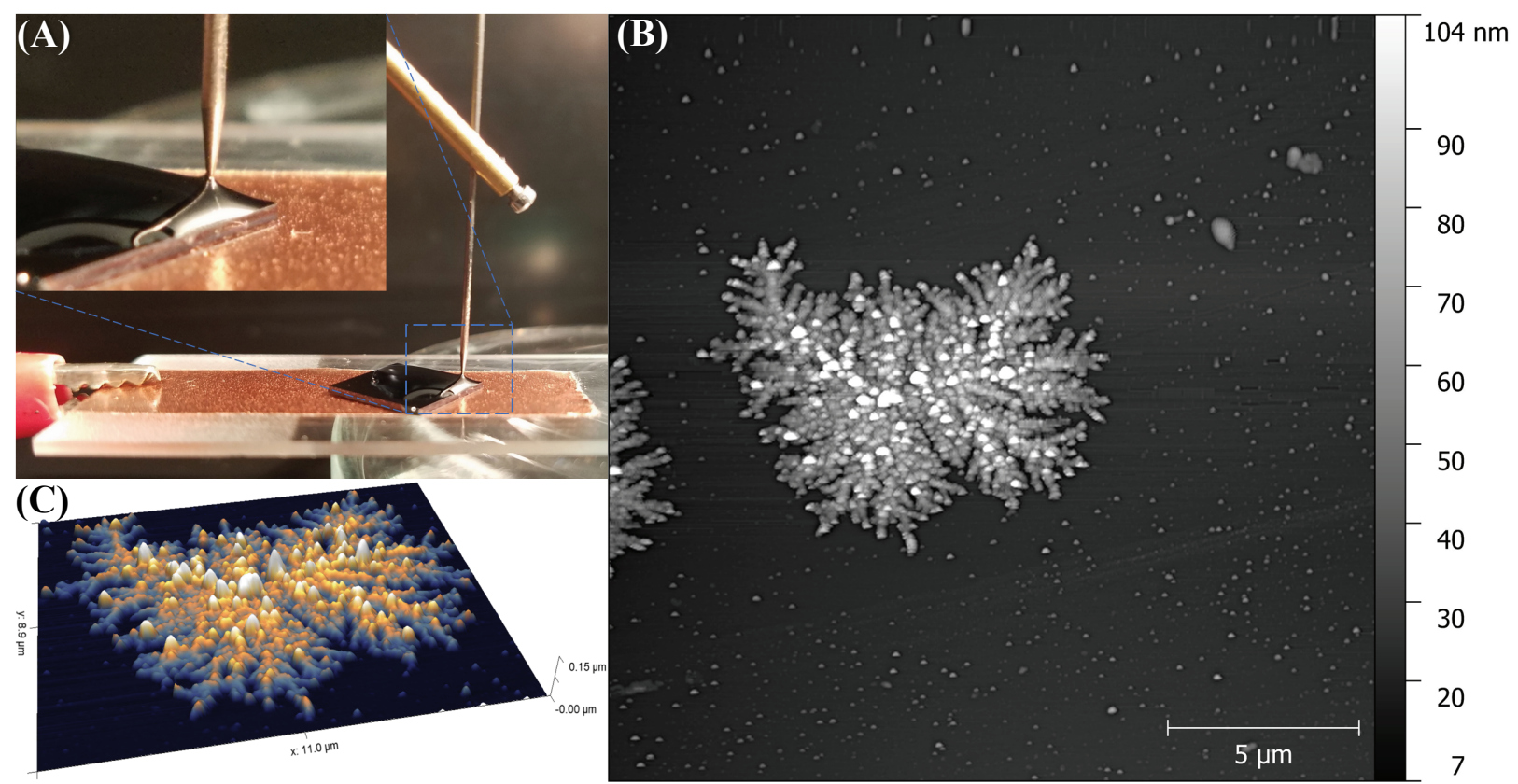

Figure B.27: (A) The probe and substrate after 30 seconds of $5 \mathrm{~V}$ biasing. No large structures are observed. (B) AFM image of the nanoscale dendritic structures formed beneath the biasing probe. (C) False colored $3 \mathrm{D}$ image of the large structure seen in (B).

The micro set of experiments were successful and demonstrated two key points. First, material deposition could be confined to discrete areas of the substrate by using a small biasing probe and thereby confining the electric fields (field spread over the surface of the substrate could be limited). Second, having the biasing probe in contact with the conductive precursor solution did not prevent nucleation (no detrimental shorting effects).

The final extension of the border electro nucleation experiments was the nano set of experiments. The goal was to successfully nucleate a single nanoparticle by using an ultrasharp AFM tip as the biasing probe. For these experiments an Agilent 5500 AFM was used. The AFM was equipped with an electrochemical liquid test cell and a built-in power supply which was used for sample biasing. The experimental setup is described in Section 2.2.2. Consistent with the general experimental design for the previous electro nucleation tests, the AFM tip was grounded, and the sample was biased negatively. An AFM stage equipped with a modified liquid containment cell was used to house the sample and precursor solution.

The challenges encountered with the nano set of experiments were practical in nature and were related to the operation of the AFM in a liquid environment. Variations of the refractive index of the precursor solution due to composition changes and temperature fluctuations during 
experimentation lead to poor laser alignment on the AFM tip. Additionally, maintaining a consistent tip-sample separation was difficult to achieve in liquid. Not surprisingly, this was observed to be an important parameter for nucleation. As was the case for the standard AFM operation used in this work, the tip-sample separation was set by the dampening of the tip oscillation. The absolute separation for a given dampening factor is a function of the tip geometry, resonance frequency and specific fluid parameters of the imagining medium. However, a rough estimated for the tip-sample spacing can be determined after the fact by switching into contact mode and driving the tip in the sample surface and then calculating the distance travelled from the dampened setpoint to the hard contact. It this way, it was discovered that otherwise identical approach setpoints resulted in very different absolute tip-sample separations. This was due to a noisy feedback signal, as a result of operating in a liquid environment, resulted in premature false tip engagement. Perturbations in the feedback signal 'trick' the PI controller into registering that the setpoint has been reached before it should. This resulted in vastly different absolute tip-sample spacings for the same dampening setpoint and was ultimately responsible for the variability between identical experiments. While this method proved to be challenging, it was ultimately successful in the nucleation of a single nanoparticle. 
In Figure B.28 the results of a successful nucleation can be seen. Figure B.28(A,B) show the area before and after the nucleation. A $5 \mathrm{um}^{2}$ area was imaged (Figure B.28(A)), then the tip was moved to the center of the scan window and a $10 \mathrm{~V}$ bias was applied for 60 seconds. The area was then immediately rescanned revealing the successful nucleation of a nanoparticle (Figure B.28(B)).
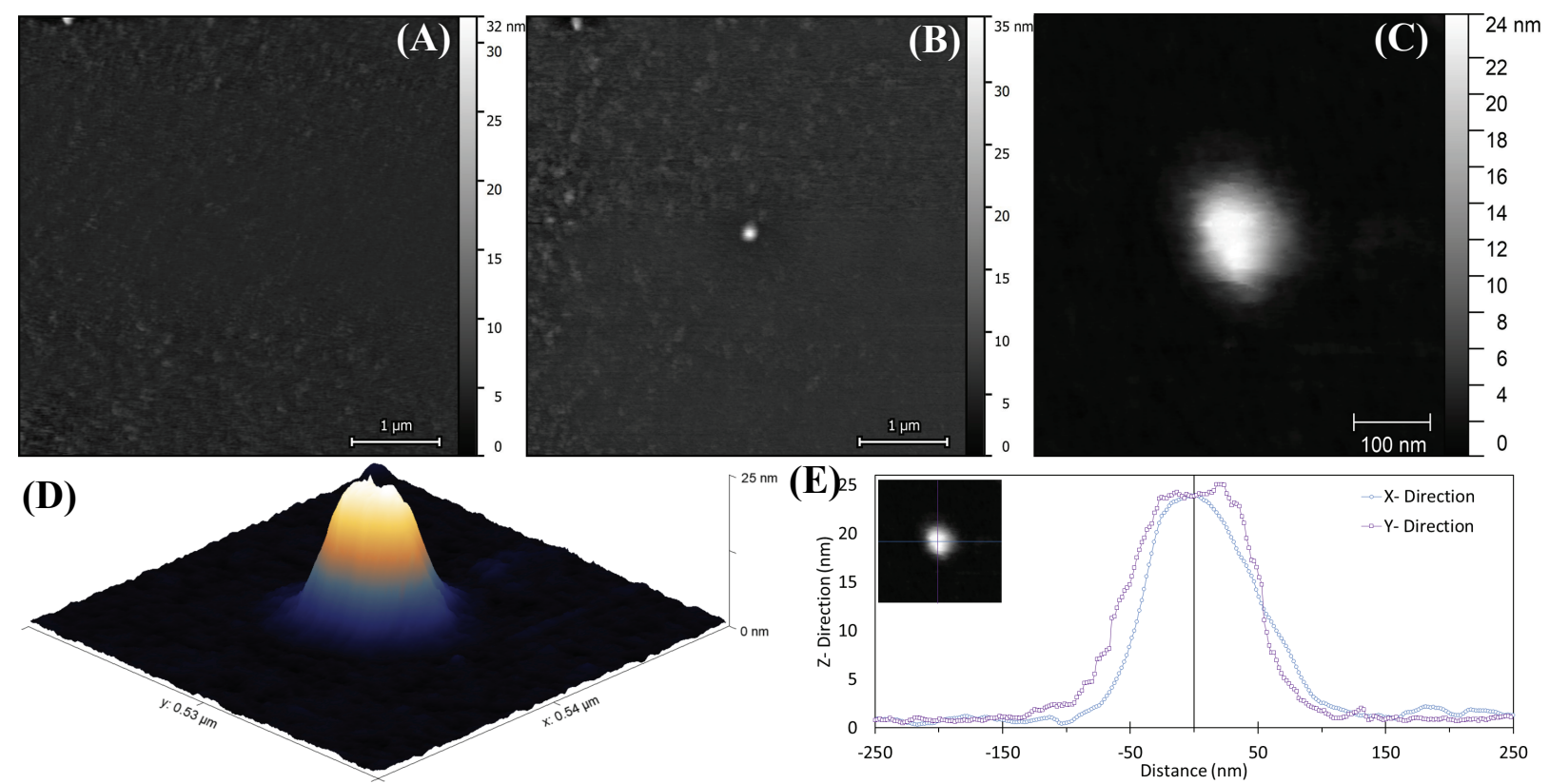

Figure B.28:(A) AFM of the substrate in precursor liquid before the applied voltage. (B) AFM of the same area immediately after the nucleation experiment showing the growth of a single spherical particle exactly where the tip had been during the experiment. (C) Additional higher magnification of the same particle. (D) False colored $3 \mathrm{D}$ representation of the particle. (E) Perpendicular line profiles of the particle showing a rough symmetry, a particle height of $\sim 24 \mathrm{~nm}$ and radius of $\sim 50 \mathrm{~nm}$. 
It was discovered that if growth were allowed to continue until the particle grew until it made contact with the AFM tip, or if the tip were in physical contact with the substrate when the bias was applied, dendritic structures would grow. Examples of this can be seen in Figure B.29. In Figure B.29(A,B) grow proceeded until a Ag wire bridged the gap between the substrate and the tip. After which growth bloomed from the Ag wire attachment point out across the sample surface. This was observed in real time through the optical microscope attachment of the AFM. Figure B.29(C,D) show the resulting growth when a bias is applied when the AFM tip is in contact with the surface. This is similar to the macro set of experiments where growth was instigated by passing current across the sample surface and similar structures were formed.

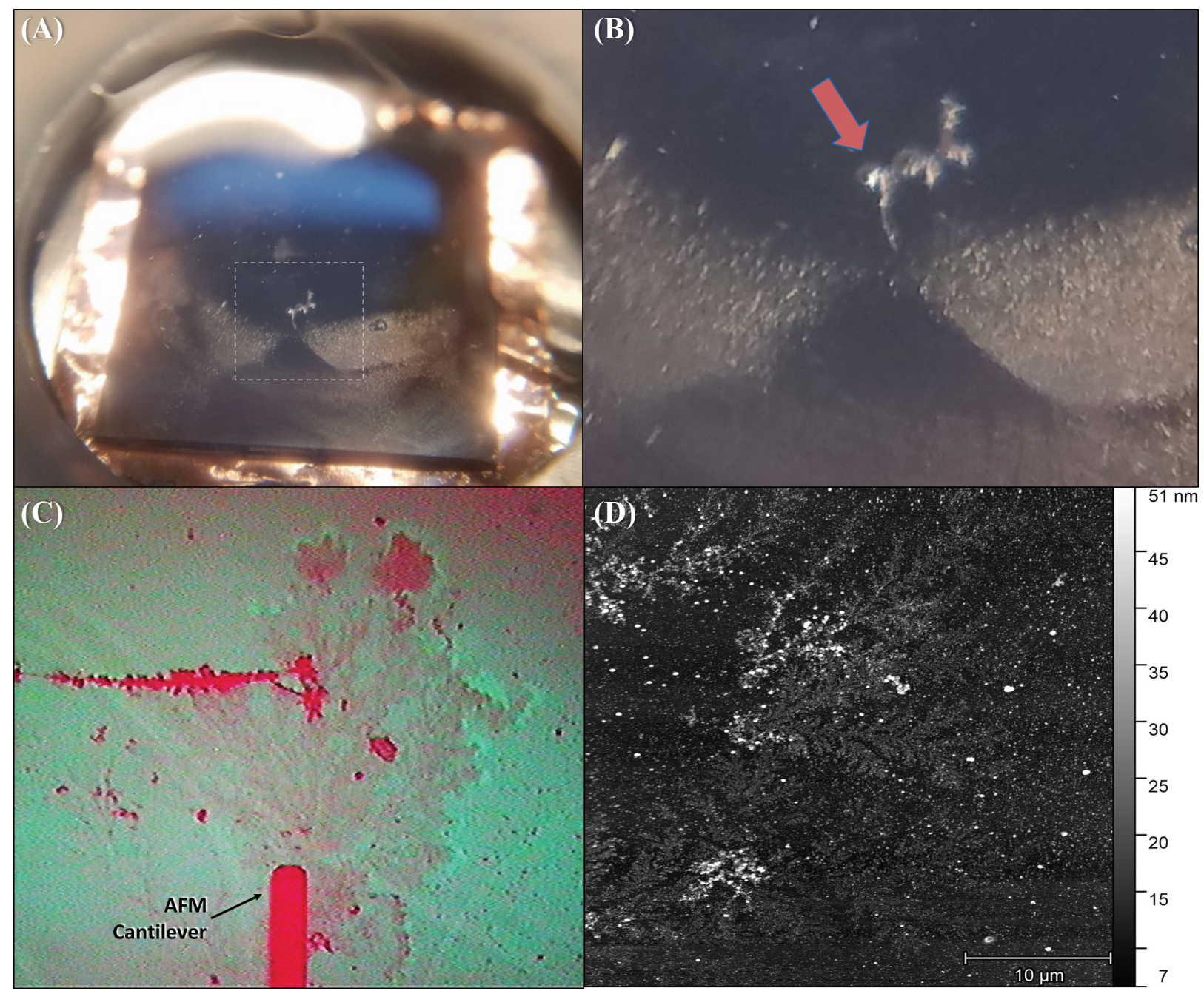

Figure B.29: The resulting growth when electrical contact being made between the AFM tip and the sample. (A) A view of the electrochemical test cell with sample. Electrical contact was made when an Ag wire grew between the AFM tip and sample. (B) Magnified view of (A) with arrow indicating the Ag wire. (C) A view through the built-in optical microscope of the AFM showing the dendritic growth which occurs when the AFM tip is in physical contact and a bias is applied. (D) AFM image of a different sample showing a similar effect. 
Note that in all cases the actual tip-sample spacing was unlikely to be the same. For example, clearly the length of the $\mathrm{Ag}$ wire in Figure B.29(A) indicate that the spacing was much larger than the experiment which grew the particle seen in Figure B.28(B), even though both used the same dampening setpoint.

In summary, all electro deposition experiment groups (macro, micro and nano) were successful in the nucleation of metallic particles. These results are encouraging and there are clearly many areas which have yet to be optimized that can lead to an improvement in the deposition results in each case. Furthermore, the preliminary results indicated that, by slight modification of the experimental setups, may intriguing possibilities are presented.

For example, in the macro and micro set of experiments, there remains a vast parameter space yet to be optimized. And although too densely packed to be particularly useful in the current form, the wide variety of complex structures which formed (metallic needles, blades, blooms, etc.) is particularly intriguing. Their high aspect ratio, sharp angles and fractal-like growth patterns undoubtedly present a complex distribution of surface plasmons which may result in interesting electromagnetic field interactions, as comparted to the typical symmetrical spherical nanoparticle. Plasmonic antennas constructed from such structures is an interesting avenue of potential future research for light applications.

For the nano experiments, an enticing prospect would be the full computerization and automation of the deposition. The nature of this process could allow for the precise control over the location and size of the particles. This could allow for the generation of extremely complex geometric or fractal patterns. In conjunction with multi-tipped AFM chips, the process could be massively scaled up.

\section{§B.2.3 Photonic Route for Metallic Nanoparticle Decoration}

This section details the investigation of the nucleation of metallic nanoparticles driven by the photonic reduction of standardly synthesized samples by UV light irradiation in a metallic salt precursor solution. This work is motivated by literature reports for photonically induced nanoparticle formation. ${ }^{(691)(693)}$ This method has been applied to dispersed sheets of graphene oxide in solution. ${ }^{(694)(692)(698)}$ However, it application to graphene grown on $\mathrm{SiC}$ has drawn little attention in the research community. Michael Matthews preformed many of the nucleation 
experiments in this study. He has spearheaded the photo-nucleation investigation and provided valuable insight into the process.

Over 30 individual photo nucleation experiments were conducted. As with many of the other nucleation studies, silver (via $\mathrm{AgNO}_{3}$ salt) was used as the prototypical nucleation metal. The conditions which produced the most optimum nucleation in terms of coverage, uniformity and particle morphology are given in Table B-1.

Table B-1: Optimum conditions found for the photo-nucleation of silver nanoparticles.

\begin{tabular}{|l|l|}
\hline Light Source & $\begin{array}{l}\text { Deep UV @ 265 nm } \\
(4.68 \mathrm{eV}, 1131 \mathrm{THz})\end{array}$ \\
\hline Irradiation Time & $30 \mathrm{~min}$ \\
\hline Solvent & $100 \%$ Anhydrous Ethanol \\
\hline Salt Concentration & $3 \mathrm{mM} \mathrm{AgNO}$ \\
\hline Container Material & Quartz \\
\hline Total Volume & $50 \mathrm{~mL}$ \\
\hline
\end{tabular}

The analysis of a sample produced using the optimum conditions is shown in Figure $B .30(A)$. The substrate used (the same for all in this study) was a $\mathrm{SiC}$ sample graphinated using the Standard Synthesis procedure. A particle coverage of $\sim 9.9 \%$ was achieved, with a particle 
density of $\sim 119$ particles per $\mu \mathrm{m}^{2}$. The average particle size was found to be $26.6 \mathrm{~nm}$ in diameter and a height of $1.4 \mathrm{~nm}$.

(A)

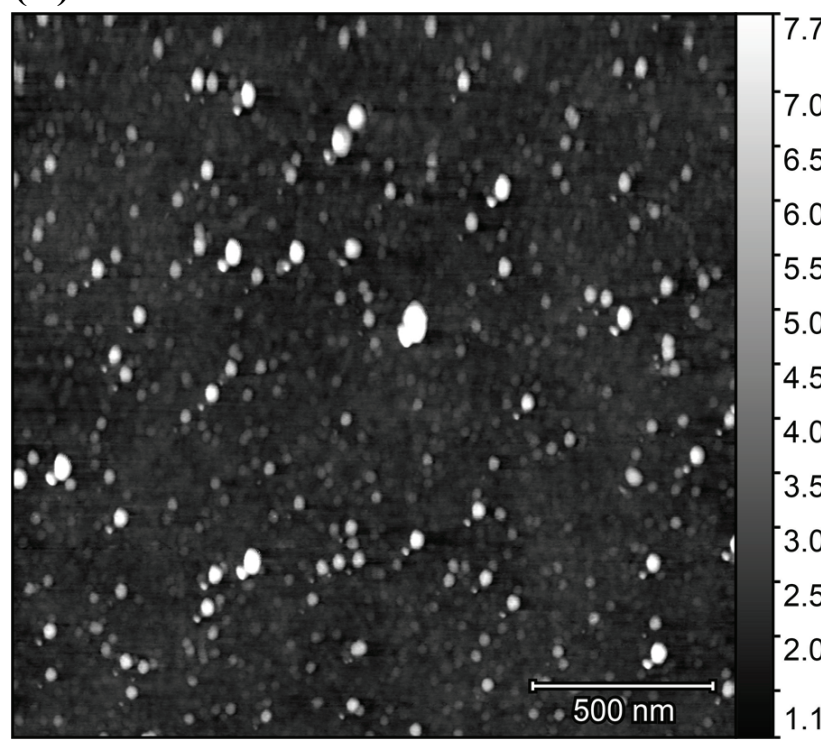

\begin{tabular}{|c|c|c|c|}
\hline \multicolumn{4}{|c|}{ - Atomic Concentration - } \\
\hline C1s & 01s & Si2s & Ag3d \\
\hline $70.5 \%$ & $15.4 \%$ & $13.8 \%$ & $0.3 \%$ \\
\hline
\end{tabular}

(B)
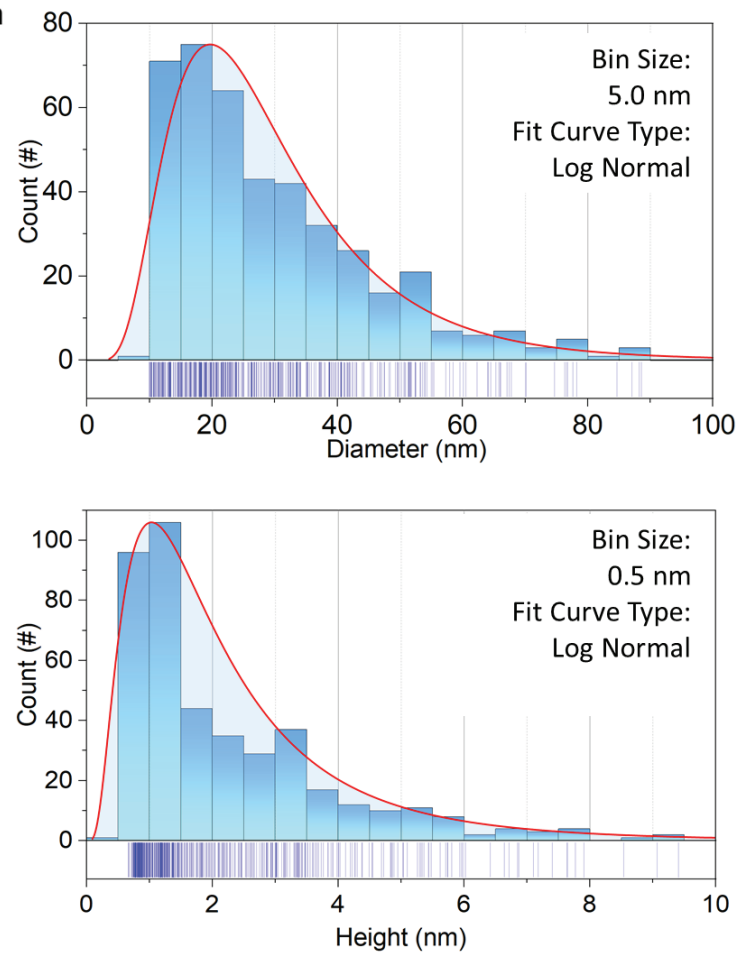

Figure B.30: (A) $5 \mu \mathrm{m} \times 5 \mu \mathrm{m}$ AFM image of a photo nucleated sample produced under the optimum conditions described in Table B-1. The XPS derived atomic concentrations and particle statistics are included below. (B) Histogram plots for particle diameter and height extracted from the analysis of (A).

The XPS analysis will always undermeasures the silver concentration as compared to the AFM calculated coverage due to fundamental differences in how these values are calculated between the two methods. In this instance, this is most likely due to the presence of a physiosorbed atmospheric oxide layer (increasing the oxygen fraction) and, particularly for samples with vary short particles, an oversampling of the substrate (increasing the carbon and silicon fractions) which occurs when photoelectrons escape from the substrate beneath a short particle.

Irradiation times of 10, 15 and 30 minutes were studied; ratios ranging from $100 \%$ water to $100 \%$ ethanol were studied; two container materials were used, quartz and borosilicate; various total volumes were studied. The effects of salt concentration were not studied. The primary observations were as follows:

I) Longer irradiation times resulted in higher silver concentrations as determined by XPS analysis. 
II) The grater the fraction of ethanol in the precursor solution, the greater the extent of nucleation as determined by XPS and AFM analysis.

III) A quartz reactor always resulted in greater nucleation, as compared to experiments performed in a borosilicate reactor, as determined by both XPS and AFM analysis.

IV) All other things being equal, smaller total liquid volumes resulted in greater nucleation, as determined by XPS analysis.

V) Nucleation events occur preferentially on the film areas which are more oxidized, as determined by AFM and XPS analysis.

VI) Nucleation is highly preferential to a metalized surface.

While observation I is intuitive, it remains to be seen what the exact relationship between irradiation time and particle coverage is (e.g. linear, exponential, diminishing log, etc.) and to what extent increasing the irradiation time continues to increase nucleation.

Observation II is explained by the difference in ionization energy of water vs. methanol. The light, at this frequency, passing through one meter of this material, decrees by this many e factors (e-foldings).

The amount of silver detected by XPS was found to increase as the fraction of water in the precursor solution decreased. A solution of $100 \%$ water produced few particles and with a silver concentration of $<0.2 \%$ (via XPS). This is as compared to experiments using solutions of $100 \%$ ethanol, which showed a silver concentration of $\sim 1.3 \%$ (via XPS). Furthermore, many samples using high concentrations of water routinely resulted in no detectable silver on the graphene surfaces. The enhanced nucleation activity for the high ethanol concentrations may be explained by the hole scavenging role that ethanol plays in the charge transfer mechanics as described in literature reports. ${ }^{(693)}\left({ }^{694)}\right.$ Given the higher absorbance coefficient of water over ethanol, light attenuation may also play a factor. The reduction in UV intensity delivered to sample surface, which sets at the bottom of the beaker during UV exposure, decreases the negative charge build up on the surface and results in less nucleation. This is similar to observations III and IV.

Observation III is explained be the higher transmittances of quartz over borosilicate in the UV rage. ${ }^{(699)}$ Conducting the experiments in a quartz container results in a higher energy density delivered to the sample surface. Although the XL-1500 Crosslinker is illuminated from the ceiling of the chamber, all the internal surfaces are treated with a highly reflective coating. Because the reaction is carried out in a beaker placed in the center of the chamber, the beaker is illuminated in 
three dimensions. Therefore, by using a container transparent to UV, the energy delivered to the sample in increased. Observation IV is also reflective of the importance of illimitation intensity at the sample surface. A larger volume of liquid results in more diffusion and attenuation of the light. Therefore, a small volume result in a small column of liquid though which to transmit light. Although observations II, III and IV are not profound they point towards the importance of irradiation intensity on the nanoparticles produced. This shows that light intensity, and easily controllable parameter, can be used to fine tune the extent of nucleation in future studies.

Observation $\mathrm{V}$ is explained by the high electronegatively of the oxygen. Oxidized areas of the film hold a higher negative charge, as compared to the surrounding film and are thus seen as preferential sites for nucleation events. Evidence of this is shown in Figure B.31, where two different areas on the same sample are compared. This sample began as a Standard Sample. The sample was then physically shadow masked and exposed to a light ICP/RIE oxygen plasma. This resulted in a highly oxidized graphene surface in the exposed areas and in the protected areas,

(A)

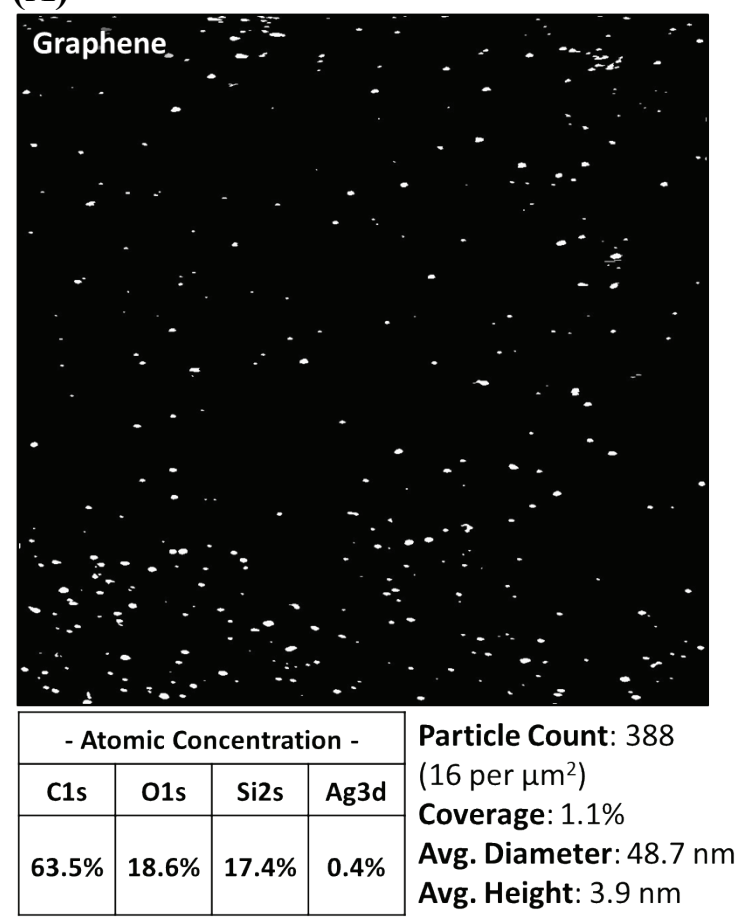

(B)

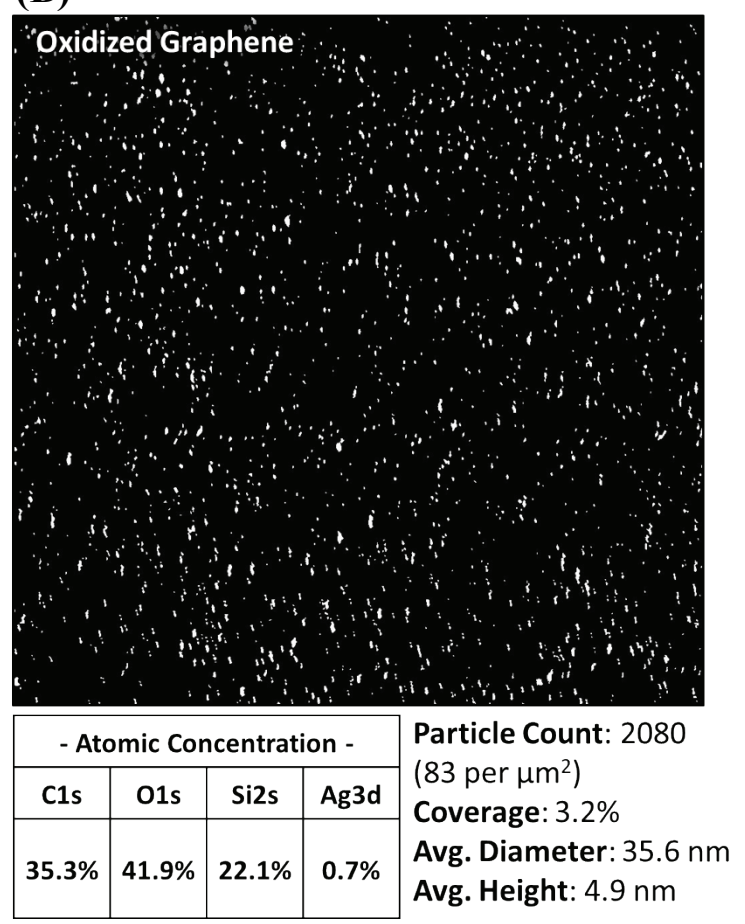

Figure B.31: Particle detection maps from two $5 \mu \mathrm{m}$ X $5 \mu \mathrm{m}$ AFM scans on the same sample. The effect of surface oxide on nucleation is illustrated. (A) The plain graphene surface sees moderate particle coverage. (B) An area of the same sample but exposed to a light oxygen plasm. A higher surface oxide concentration results in more nucleation as evident from the higher particle cover and count. The nucleation reaction was carried out in a quartz beaker containing $50 \mathrm{~mL}$ of a $3 \mathrm{mM} \mathrm{AgNO}_{3}$ aqueous solution of $8 \%$ ethanol and was irradiated with $265 \mathrm{~nm}$ light for 30 minutes. 
normal unadulterated graphene. The sample then underwent the UV nucleation process and the two different areas were analyzed. The oxidized areas show a higher frequency of nucleation, confirmed both by AFM and XPS analysis

The particle analysis supports the conclusion that oxygen defect sites in the graphene surface serve as nucleation centers. The oxidized surface is covered with many more ( $>5 \mathrm{x}$ as many) and slightly smaller (27\% smaller in diameter) particles. Given that the precursor concentration and nucleation time were identical in both cases, both observations are indicative of a surface with many more sites available for nucleation.

Observation IV was discovered when comparing samples which had $\mathrm{Ti} / \mathrm{Au}$ contacts deposited and those which did not. The samples were processed identically. However, analysis of the graphene surfaces of both showed very little silver on the graphene surface of the sample which had $\mathrm{Ti} / \mathrm{Au}$ contacts. On further analysis, it was discovered that the samples with $\mathrm{Ti} / \mathrm{Au}$ did experience nucleation, but that it was almost entirely confined the contacts. Furthermore, the nucleation had proceeded to such a large extent that the surrounding graphene surface was effectively starved of silver cations for nucleation. This explain why, under conditions which normally produced higher particle coverages, little nucleation was seen on the graphene surface of samples which had Ti/Au contacts. This is illustrated in Figure B.32, which shows the secondary 
X-ray emission intensity (SXI) map of a sample with Ti/Au contacts over two different locations and their corresponding XPS spectrum covering the Ag3d region.

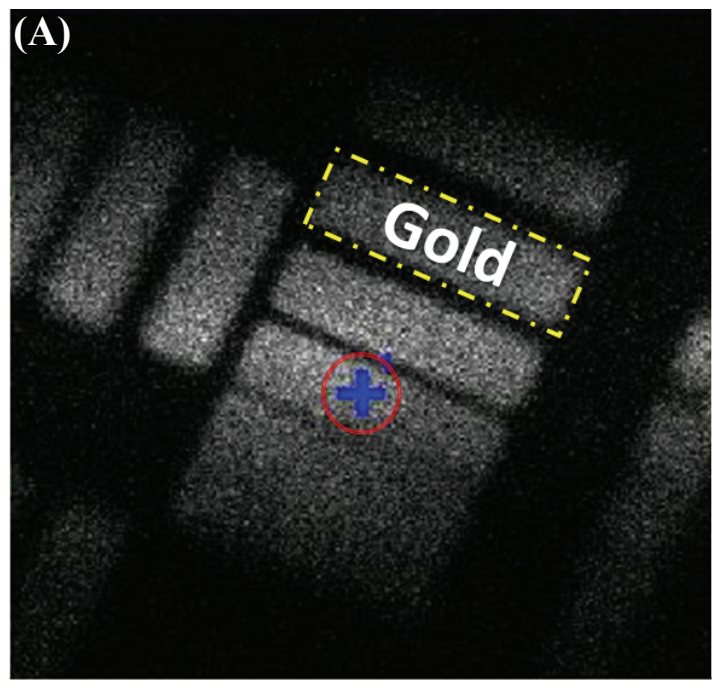

(B)

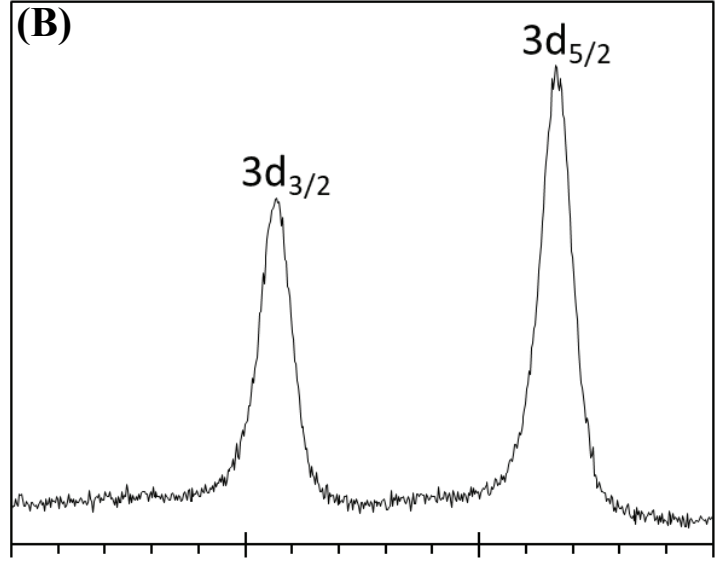

380
375
370

Binding Energy (eV)

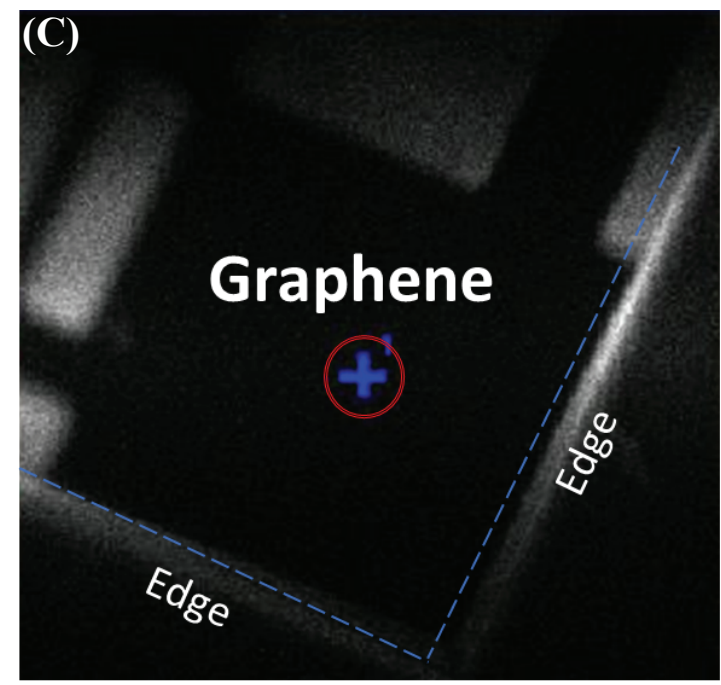

(D)
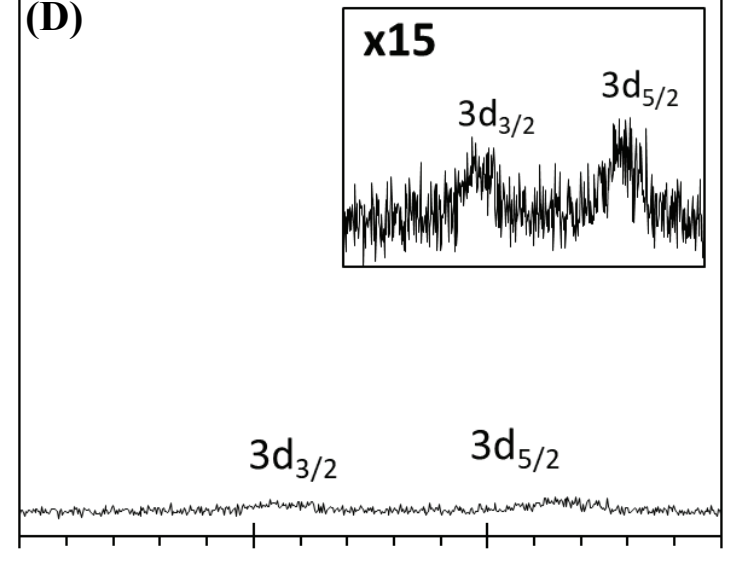

380

365
370

Binding Energy (eV)

Figure B.32: Secondary X-ray emission intensity (SXI) map of two different areas of the same sample. The $\mathrm{Ti} / \mathrm{Au}$ show up as bright areas in the image and correspond to areas of high electron emission. The blue plus marks the center of the collection area for the spectra shown in each panel. The red line represents the $\sim 100 \mu \mathrm{m}$ spot size of the XPS. (A) The SXI map showing the area sampled for the spectrum in (B). (B) The Ag3d region collected from a Ti/Au metal contact pad. Very high levels of silver are detected. (C) Corresponding SXI map and spectrum for the graphene surface of the same sample. The sample edges are also marked. Very low levels of silver are detected. This show the preferential silver deposition onto the metal contacts.

There are many possible explanations for why the Ti/Au contacts show high levels of nucleation. They range from morphological differences (surface roughness, etc.) to strong noble metal interactions (noble metals are known to interaction with one another, in particular gold and silver) to electronically derived mechanics (the metal contacts may serve as de facto charge collection sites during the photo reduction process). ${ }^{(210)}$ Regardless of the mechanism, the results 
are clear. This can be avoided by conducing the nucleation process before the contact deposition step.

Future studies can investigate applying this method to samples in between the ICP/RIE and the AP-RAT steps. Samples at this stage in the process, which have not yet undergone the APRTA process, have a large fraction of fluorine (which is highly electronegative) embedded in the carbon film. These films may provide a fascinating surface on which to nucleate nanoparticles. The particle interaction with the carbon reordering process would be very interesting. Particularly if nickel were used, which has many interesting interactions with carbon. More pertinently, nickel has been shown to assist in the transformation of amorphous carbon films into graphene. ${ }^{(700)(701)}$ (702) (703) (704)

In summary, the photo-nucleation method for the decoration of graphenated samples was shown to be successful. However, there is room for further refinement of the nucleation parameters to fine tune the process and increase the reproducibility of the process. Given the propensity for nucleation to occur preferentially on oxidized surfaces, this feature could be used utilized to direct nucleation to purposefully oxidized areas to template the nucleation.

\section{§B.2.4 Evaporative Route for Metallic Nanoparticle Decoration}

Using the custom Kurt J. Lesker e-Beam Evaporator system (described in greater detail in Section $§ 3.3 .1$ ) metallic nanoparticle decoration of silicon test wafers segments was achieved by e-beam evaporation of both gold and nickel. This study focuses primarily on gold deposition. Decoration was achieved by running at low e-beam powers (given as a percentage of delivered power from the $5 \mathrm{~kW}$ e-beam power supply) for short deposition times (less than 1 minute). The resulted in the deposition of a fractional monolayer of metal which took the form or nano-islands or nanoparticles. Deposition of metal was confirmed by XPS analysis and the nanoparticle nature of the deposited material was directly observed by AFM. Details on the specifics of the

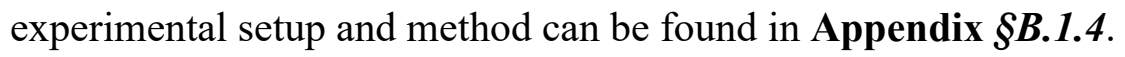


AFM analysis of a silicon test wafer decorated with gold nanoparticles using the evaporative method is shown in Figure B.33. These particles were produced using a deposition power of 5\% for a duration of 17 seconds and excellent coverage and distribution is demonstrate. Particle analysis of the $5 \mu \mathrm{m} \times 5 \mu \mathrm{m}$ AFM scan shown in Figure B.33 reveals 1168 discreet particles, for a density of $\sim 47$ particles per $\mu \mathrm{m}^{2}$. Histogram distributions of the diameter and height are shown as well. The average particle height is $2.7 \mathrm{~nm}$ and the average particle diameter is $44.2 \mathrm{~nm}$ (without tip induced broadening compensation). This gives a projected area coverage of $\sim 9.7 \%$. XPS analysis show the gold atomic concentration to be $\sim 2.2 \%$. The XPS analysis underestimates the coverage in this case for the same reasons as discussed in the previous section; the presence of an atmospherically derived oxide layer and an over sampling of the substrate from beneath short particles.
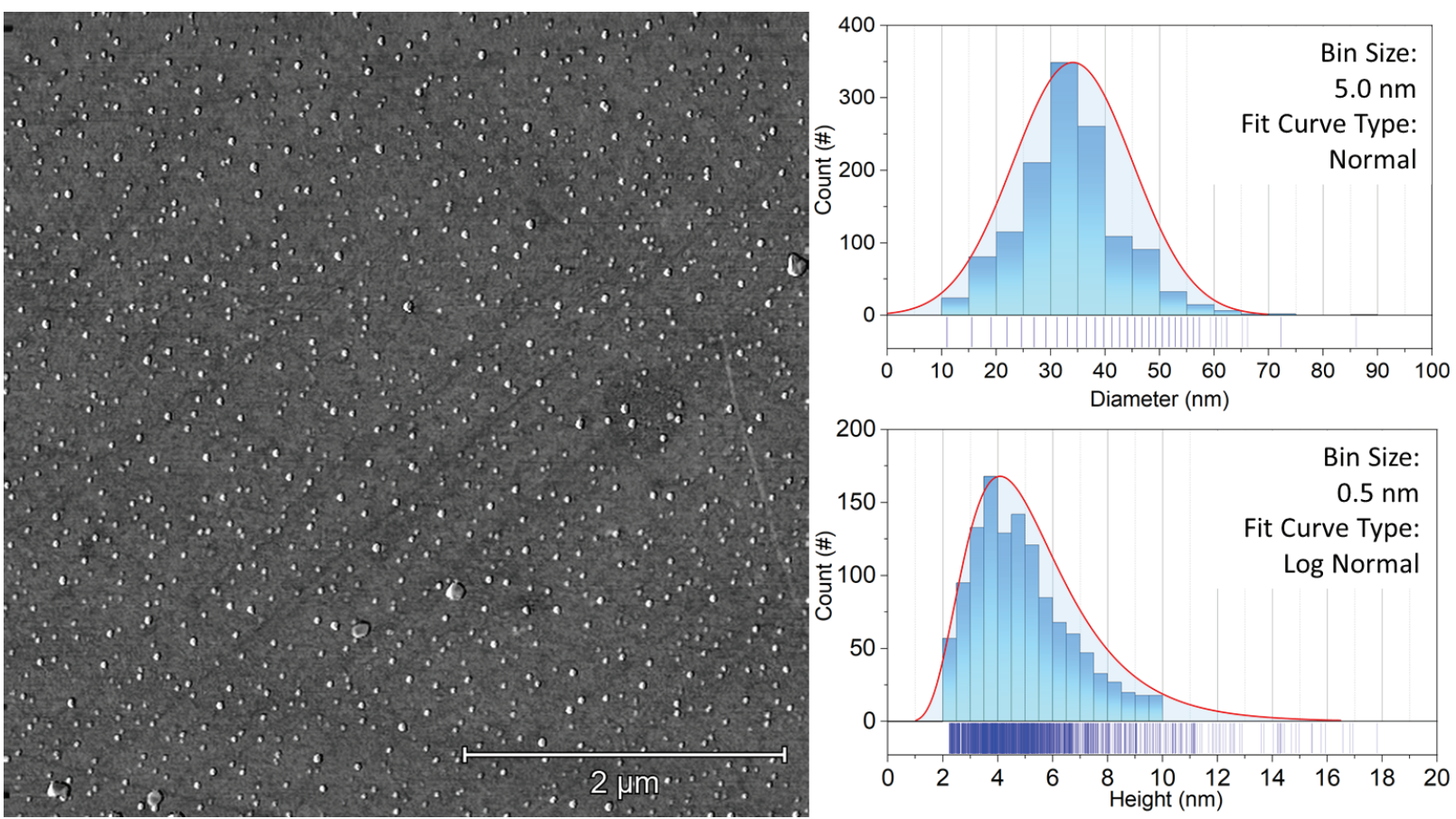

Figure B.33: Phase image of a silicon wafer decorated with gold nanoparticles. Excellent coverage and distribution are demonstrated.

A series of experiments were preformed to determine the effect of the e-beam deposition power and the deposition duration. The results are tabulated in Table B-2. No strong correlation was found between either the deposition power or duration and particle statistics. Samples $\mathrm{C}, \mathrm{K}$ and $\mathrm{L}$, for example, used identical deposition parameters but this resulted in three vastly different particle coverages $(4.7 \%, 1.2 \%$ and $0 \%)$. Because the operational parameters are around the evaporating threshold, the recent thermal history of crucible influences subsequent depositions. 
For example, samples J, K and L were processed in series. A power of 10\% (Sample J) is evidently above the evaporation threshold, while a power of 5\% (Sample L) is below. Sample K was process using a power which was also below the evaporation threshold. However, due to the residual heat retained by the crucible from the decoration of Sample J, this resulted in nanoparticle deposition.

Table B-2: Partial sample list, arranged by nanoparticle coverage area.

\begin{tabular}{|c|c|c|c|c|c|c|c|}
\hline Sample & $\begin{array}{c}\text { e-Beam } \\
\text { Power } \\
(\%)\end{array}$ & $\begin{array}{c}\text { Duration } \\
\text { (Sec) }\end{array}$ & $\begin{array}{c}\text { Particle } \\
\text { Count } \\
\left(\text { per } 25 \mu \mathrm{m}^{2}\right)\end{array}$ & $\begin{array}{l}\text { Coverage: } \\
\text { Projected } \\
\text { Area (\%) }\end{array}$ & $\begin{array}{c}\text { Average } \\
\text { Particle } \\
\text { Diameter } \\
(\mathbf{n m}) \\
\end{array}$ & $\begin{array}{c}\text { Average } \\
\text { Particle } \\
\text { Height } \\
\text { (nm) }\end{array}$ & $\begin{array}{c}\text { Au Atomic } \\
\text { Concentration } \\
\text { (Via. XPS) }\end{array}$ \\
\hline $\mathbf{A}$ & $5 \%$ & $40 \mathrm{Sec}$ & 1229 & $11.0 \%$ & $41.9 \mathrm{~nm}$ & $3.6 \mathrm{~nm}$ & $3.3 \%$ \\
\hline B & $5 \%$ & $17 \mathrm{Sec}$ & 1306 & $5.2 \%$ & $30.2 \mathrm{~nm}$ & $3.8 \mathrm{~nm}$ & $2.2 \%$ \\
\hline C & $5 \%$ & $45 \mathrm{Sec}$ & 1063 & $4.7 \%$ & $6.1 \mathrm{~nm}$ & $4.8 \mathrm{~nm}$ & $0.8 \%$ \\
\hline D & $5 \%$ & $25 \mathrm{Sec}$ & 2595 & $4.5 \%$ & $15.3 \mathrm{~nm}$ & $0.7 \mathrm{~nm}$ & \\
\hline $\mathbf{E}$ & $5 \%$ & $8 \mathrm{Sec}$ & 274 & $4.5 \%$ & $52.9 \mathrm{~nm}$ & $5.9 \mathrm{~nm}$ & \\
\hline $\mathbf{F}$ & $5 \%$ & $31 \mathrm{Sec}$ & 2237 & $3.7 \%$ & $17.0 \mathrm{~nm}$ & $1.0 \mathrm{~nm}$ & \\
\hline G & $5 \%$ & $23 \mathrm{Sec}$ & 765 & $3.6 \%$ & $32.6 \mathrm{~nm}$ & $5.4 \mathrm{~nm}$ & \\
\hline $\mathbf{H}$ & $8 \%$ & $17 \mathrm{Sec}$ & 326 & $2.6 \%$ & $43.0 \mathrm{~nm}$ & $3.1 \mathrm{~nm}$ & \\
\hline I & $8 \%$ & $9 \mathrm{Sec}$ & 804 & $2.1 \%$ & $23.9 \mathrm{~nm}$ & $6.7 \mathrm{~nm}$ & \\
\hline $\mathbf{J}$ & $10 \%$ & $2 \mathrm{Sec}$ & 1350 & $2.0 \%$ & $16.1 \mathrm{~nm}$ & $0.5 \mathrm{~nm}$ & \\
\hline $\mathbf{K}$ & $5 \%$ & $45 \mathrm{Sec}$ & 1504 & $1.2 \%$ & $11.6 \mathrm{~nm}$ & $3.2 \mathrm{~nm}$ & \\
\hline $\mathbf{L}$ & $5 \%$ & $45 \mathrm{Sec}$ & 0 & $0.0 \%$ & - & - & \\
\hline
\end{tabular}

Although there is a clear need for the refinement of the deposition protocol, this process consistently produced well dispersed sheets of nanoparticles. Figure B.34 shows the particle detection maps from the samples listed in Table B-2. The detection map, labeled B' $\left(90^{2} \mu \mathrm{m}\right)$, was collected from Sample B and covered an area of $1800 \mu \mathrm{m}$. This map illustrates the large and consistent coverage inherent to this method of nanoparticle decoration. 


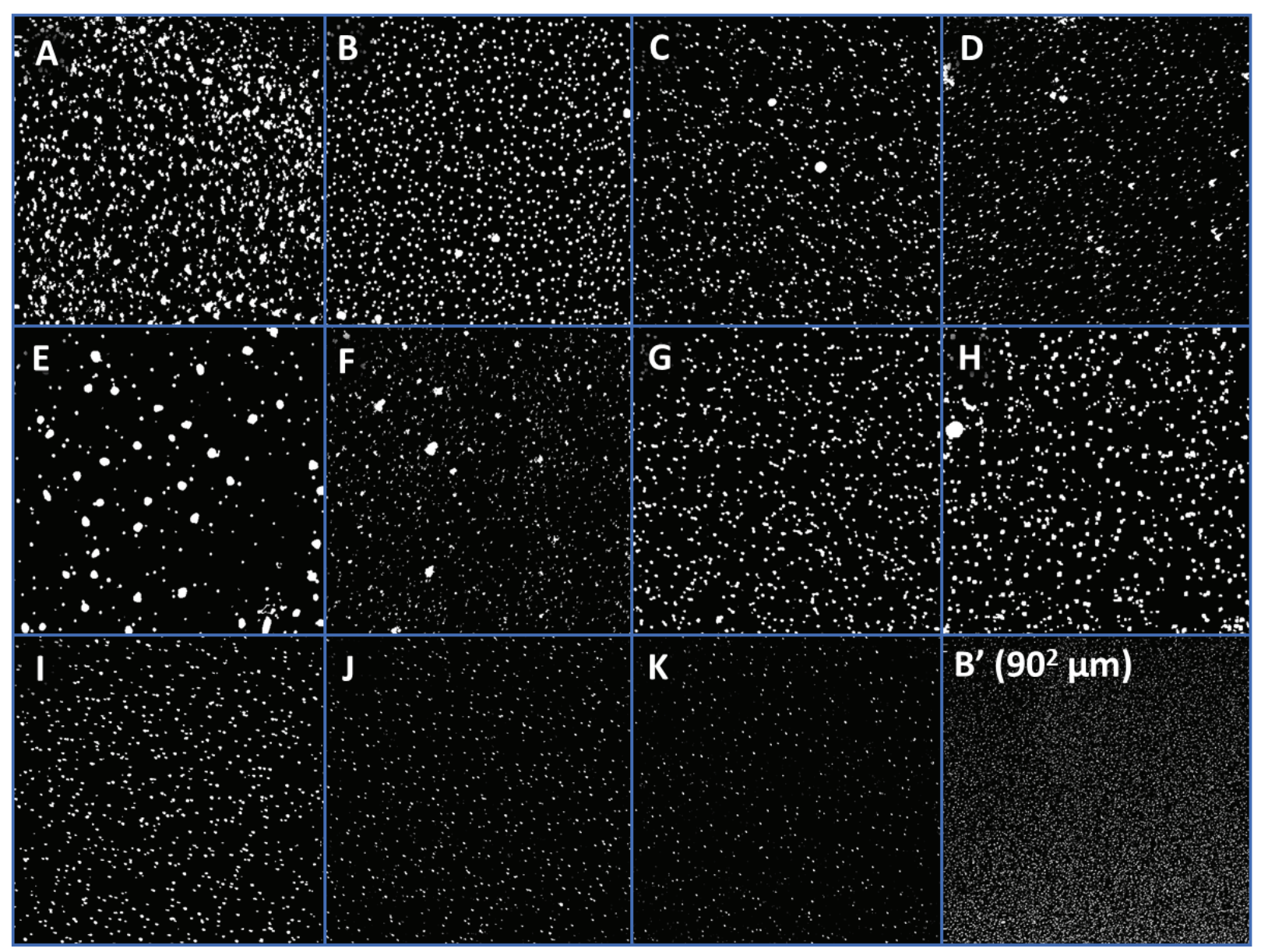

Figure B.34: Particle detection maps corresponding top the samples listed in Table B-2, excluding sample $\mathrm{L}$ which did not have nanoparticles. All maps correspond to a $5 \mu \mathrm{m}$ x $5 \mu \mathrm{m}\left(25 \mu \mathrm{m}^{2}\right)$ area, excluding B' which corresponds to a $90 \mathrm{X} 90 \mu \mathrm{m}\left(1800 \mu \mathrm{m}^{2}\right)$ area.

This leads to the two key conclusions of this study. Frist, special attention needs to be paid to the chamber condoning step and the time between depositions as it relates to the starting crucible temperature. Second, this process is a very promising route for the deposition of metallic nanoparticles. E-beam evaporation is a mature technology and the process is largely automated. This shows the decoration of samples could become both very reproducible and, given the fine control over the power and deposition time, highly tunable.

In summary, gold nanoparticles were successfully deposited onto a silicon wafer via ebeam evaporation. Excellent coverage and uniformity were demonstrated. It was fond that, due to the low e-beam powers used, the preconditioning of the chamber and crucible are important and substantially effect deposition. Further refinement of the preconditioning steps will most likely lead a more direct correlation between deposition parameters (i.e. power and time) to the resulting 
nanoparticle metrics (size, distribution, coverage, etc.). With further parameter tuning, this method shows great promise.

Future studies will focus on extending this process to the graphenated $\mathrm{SiC}$ samples and on the refinement of the deposition parameters. By terminating the deposition process in the very early stages of film formation a fractional monolayer of material is deposited which takes the form of isolated islands of material. The interactions each island has with the underlying substrate influences the geometry of the parties (a balance between metal spreading on the surface with the tendency for the metal to 'ball-up' on itself). Heating of the substrate, either during or after deposition, may prove to be an important step. Adding thermal energy to the sample may make the deposited material more mobile and allow the interactions between the metal and substrate to dominate the morphology of the nanoparticles. Depending on the propensity for wetting of the substrate by the metal, substrate heating may lead to either a coalescence (making the particles more spherical) or spreading (more island-like) of the deposited metal. Therefore, a heating step may provide an additional means of morphological control over the particles.

Lastly, this deposition method is particularly well suited for the investigation of the interaction between nickel and the partially amorphous carbon surface following the ICP/RIE step. The deposition of a fractional nickel layer before the AP-RTA step may provide a means by which the reordering process of film into graphene sheets may be enhance. Literature reports show that the presence of nickel films greatly assist in the formation of graphene from amorphous carbon films. ${ }^{(700)(701)(702)(703)(704)}$ This will be explored in future work. 


\section{APPENDIX C: CONTAMINATION INVESTIGATIONS}

Appendix $\mathrm{C}$ details the investigation on the origins of two metallic contamination phenomena referenced in the main text, involving gas sensing and separately, graphene synthesis. Both investigations represent a significant time investment. In gas testing, a nickel contamination was observed as a result of a chemical transport phenomenon mediated by chemistry occurring between the $\mathrm{CO}$ test gas and the stainless-steel walls of the small test cell during high temperature tests. During graphene synthesis, an aluminum contamination was observed and found to be due, ultimately, to sputtering of components within the plasma chamber. Although problematic at the time, both processes, particularly the in-situ plasma sputtering and metal implantation during etching, present some novel metal doping/decoration opportunities.

\section{§C.1 Nickel Contamination During Gas Testing}

When testing at temperature above $400^{\circ} \mathrm{C}$ with carbon monoxide gas in the small test cell, the devices were observed to unexpectantly cease to function. Visual inspection of the sample package revealed a white film deposited onto the hot components of the test package. This can be seen in Figure C.1 which shows the sensor before and after exposure to $\mathrm{CO}$ at elevated temperatures. This film was conductive and caused an electrical short across the device contacts. After confirming with XPS, the material was discovered to consist of primarily nickel, iron, and carbon (with trace amounts of other compounds), presumably scavenged by the $\mathrm{CO}$ from the stainless-steel walls (316 SS) of the test chamber. Although the chamber walls are not heated

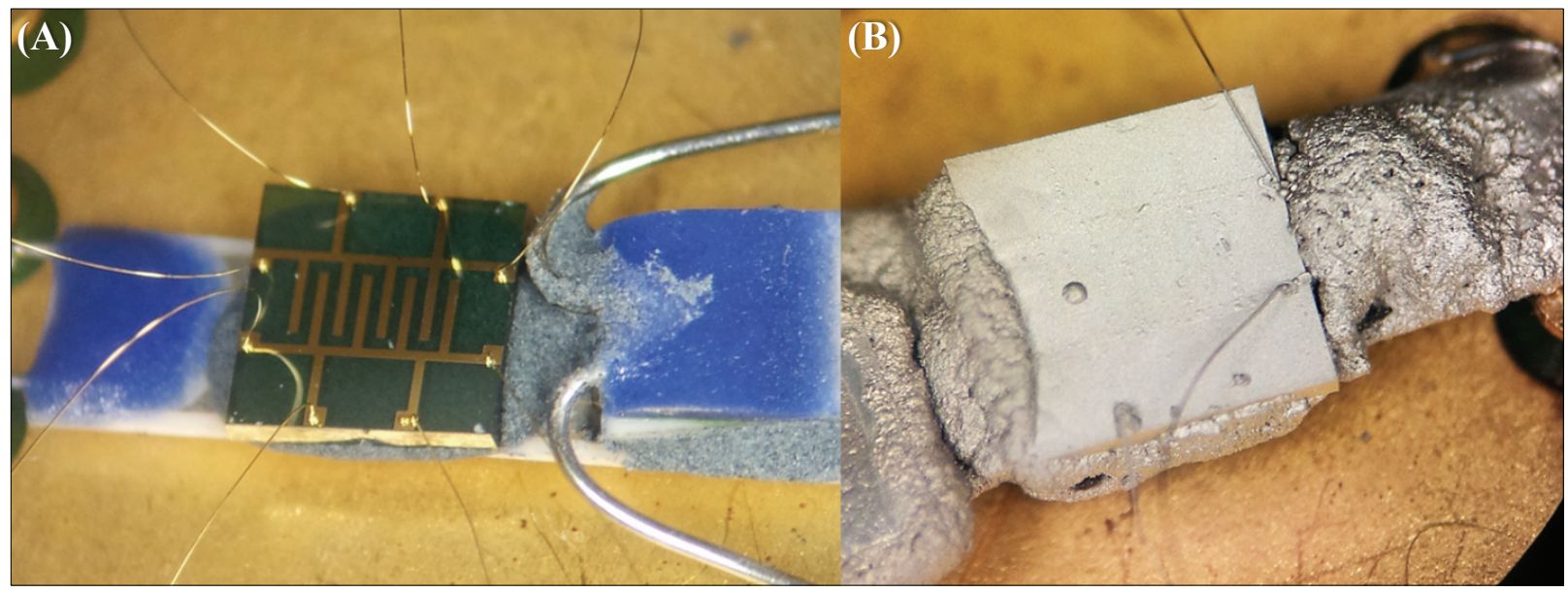

Figure C.1: (A) An example of a device for reference before testing (B) After high temperature testing with carbon monoxide. The light-colored nickel film is clearly seen. The compostion of the film was determinond by XPS. 
directly in the small test cell setup they undoubtably experience a large temperature increase through radiative and conductive heat transfer from the sample heater which is above $400^{\circ} \mathrm{C}$ and located only mm away from the test cell walls. This conclusion is supported by reports in the literature. (705) (706) (707) (708) (709) Based on various reports in catalysis literature, the film is most likely some form of nickel carbonyl. ${ }^{(710)}$ Nickel tetracarbonyl is highly toxic. ${ }^{(711)}$ For this reason, $\mathrm{CO}$ was removed from the gas testing suite.

\section{§C.2 Aluminum Contamination During Sample Synthesis}

This section will detail the investigation into the source of aluminum contamination in the graphenated samples. While not directly pertinent to this work, this investigation represents a large time investment and has important implications. Occasionally during routine XPS analysis of films

produced using the standard synthesis process, trace amounts of aluminum would be detected (on approximately $10 \%$ of samples). This was not unprecedented as historically with the plasma assisted process an intermittent aluminum contamination had been observed, but the source had not been determined.

During the course of this work, there was abrupt and massive (orders of magnitude) increase in the levels of aluminum contamination and $100 \%$ of samples produced were contaminated. Additionally, after the initial abrupt appearance of these high levels of contamination, the aluminum levels were observed to rise with each subsequent sample produced. None of these highly contaminated samples were used in this work. The first step in tracking down 
the source of contamination was to conducted a thorough analysis of the contaminated samples. These results are shown in Figure C.2.

(A)

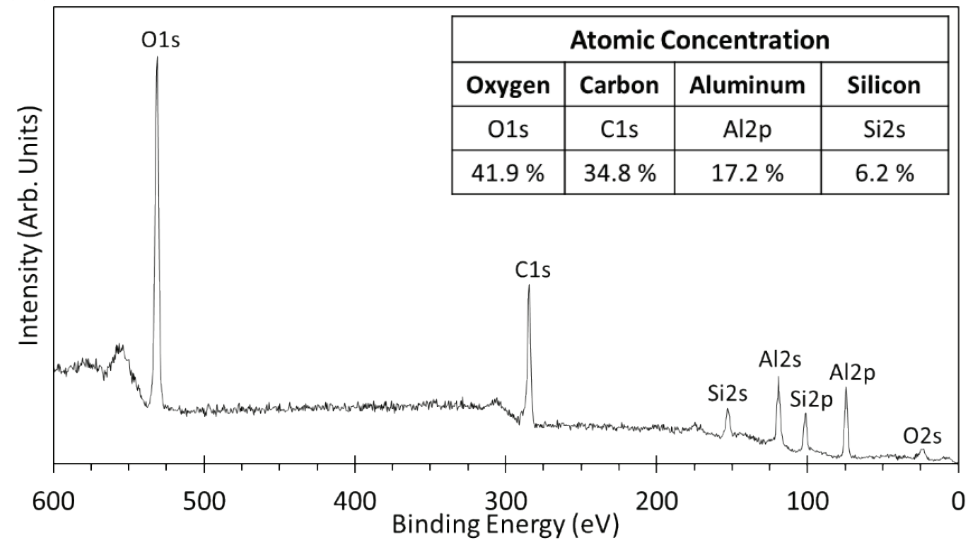

(B)

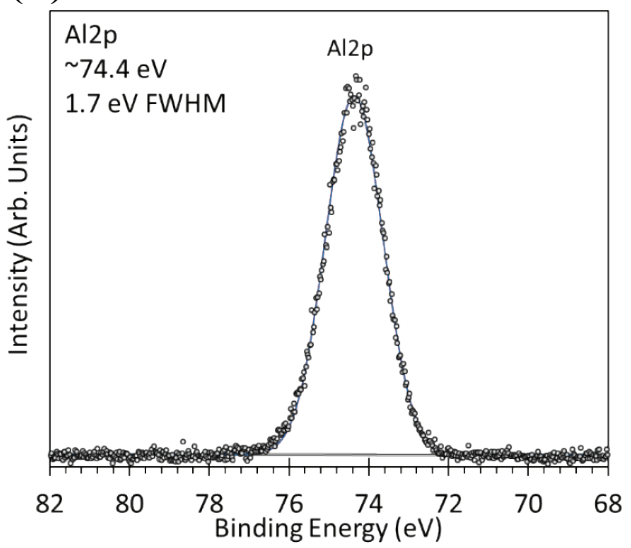

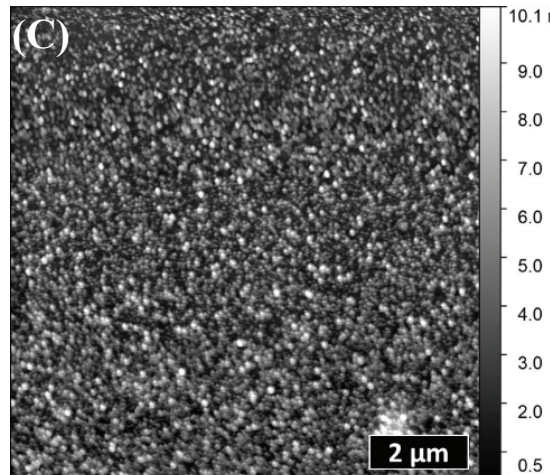
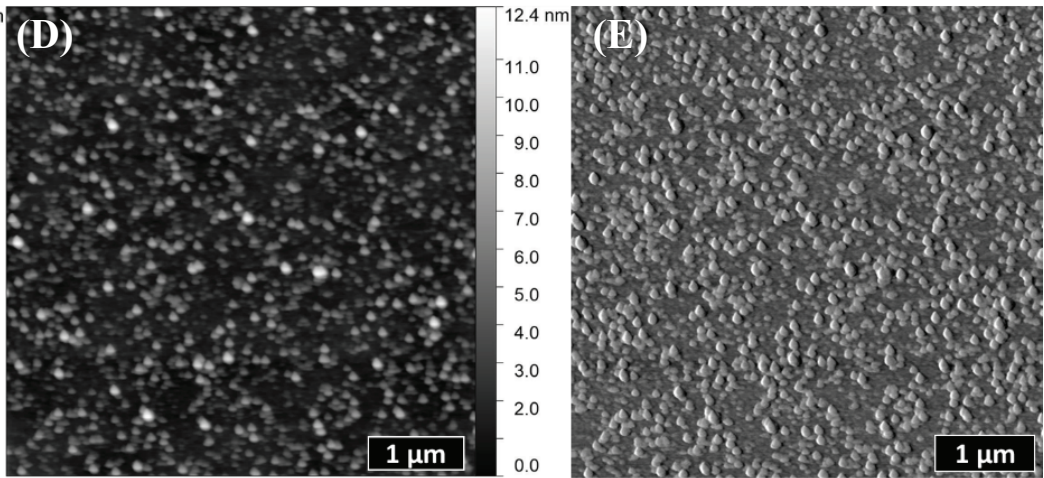

Figure C.2: (A) XPS survey data from a contaminated sample. Inset are the atomic concentrations. The presence of aluminum always correlates to high levels of oxygen. (B) The Al2p region showing the unambiguous presence of aluminum. (C) Representative AFM image collected from a contaminated sample. The surfaces are textured and covered with what appear to be nanoparticles. (D) Higher magnification AFM image from a different contaminated sample. (E) Phase image of (D). The phase shift indicates the particles to be of a different material than the background.

The XPS spectra have been shifted using the $\mathrm{SiC}-\mathrm{C}$ peak at $282.5 \mathrm{eV}$ as a charging reference consistent with the rest of this work. XPS results reveal that the presence of aluminum always correlates with the observation of high levels of oxygen; $41.9 \%$ in the case shown in Figure C.2. For reference, standardly synthesized samples have nominal oxygen concentrations of $\sim 15 \%$. The XPS results indicate the aluminum contamination to most likely be present in the form of an aluminum suboxide $\left(\mathrm{Al}_{2} \mathrm{O}_{3-\mathrm{x}}\right)$ and aluminosilicate $\left(\mathrm{Al}_{2} \mathrm{SiO}_{5}\right)$. The atomic concentrations $(\mathrm{Al}: 17.2 \%$, Si: $6.2 \%, \mathrm{O}: 41.9 \% \rightarrow 2.8: 1: 6.8)$ match reasonably well with the sociometric ratio of aluminosilicate $\left(\mathrm{Al}_{2} \mathrm{SiO}_{5} \rightarrow\right.$ 2:1:5) with balance of aluminum and oxygen contributing from the suboxides of aluminum and substrate silicon. Literature reports support this assignment of the XPS 
peaks. ${ }^{(712)}$ AFM analysis of the contaminated samples (Figure C.2(C-E)), which had a very high aluminum concentrations, showed textured surface with nanoparticle-like features.

Due to the insidious nature of the contamination, the intricacies of the process and the observed bi-conditional requirements for contamination (discussed later), determining the source was a tour de force over several months. Ultimately, it was determined that the contamination was coming primarily from anodized aluminum components from within the ICP/RIE chamber (the centering ring and electrostatic chuck specifically). A description of how this was determined and the mechanism by which aluminum was transported to the samples follows. Figure C.3 is a simplified logic flow diagram. The diagram is arranged so as to preserve the logic flow and loosely corresponds to the order in which the testing was carried out.

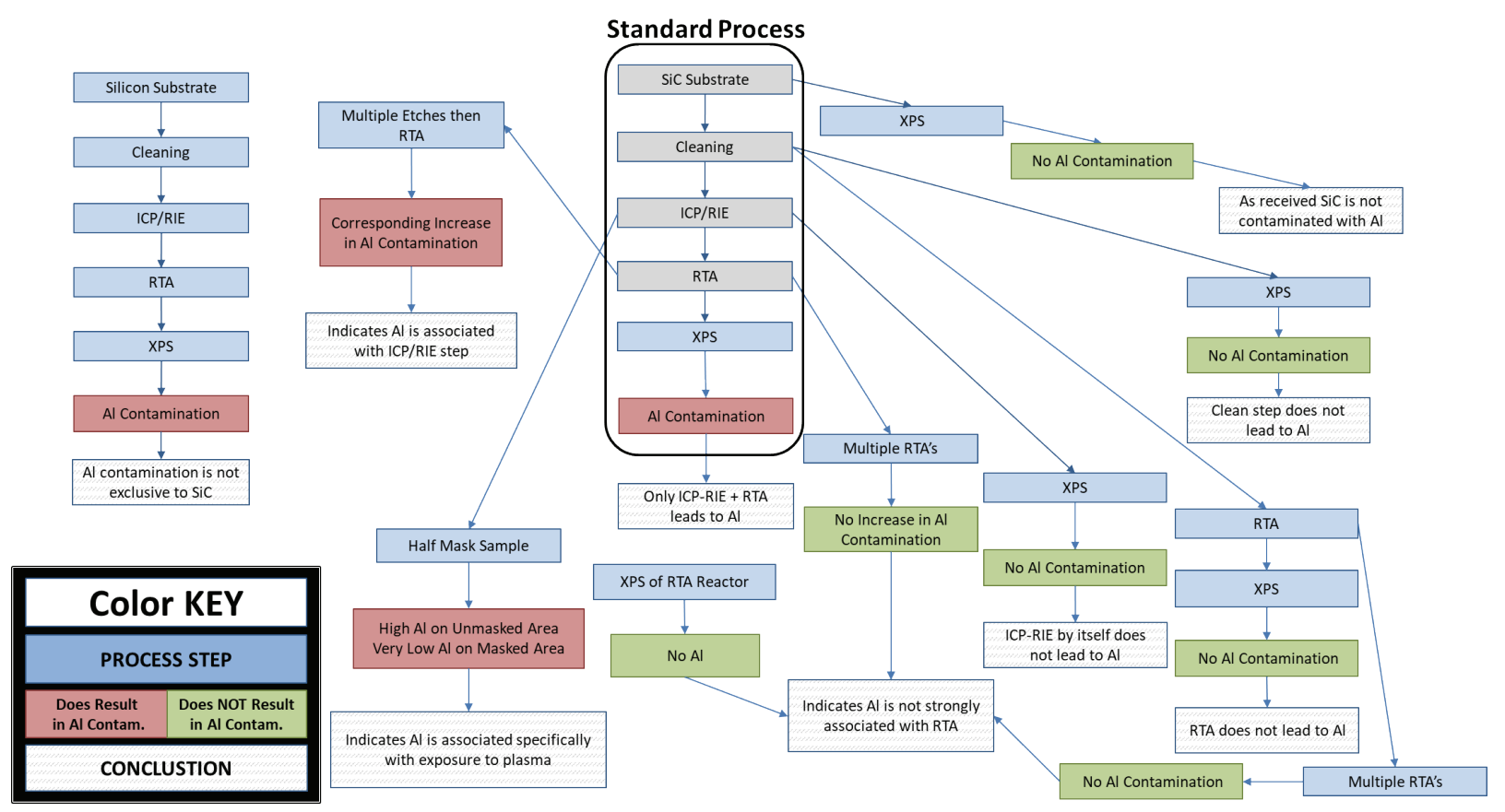

Figure C.3: Flow diagram summarizing the major experiments conducted to determine the source of aluminum contamination.

XPS analysis was performed on the samples after each step of the graphitization process. A simple logic test was applied to the results, which indicated the contamination was related to the ICP/RIE and/or the AP-RTA step. However, neither step on its own resulted in detectable aluminum by XPS. Experiments were conducted to further probe the ICP/RIE and AP-RTA steps. First, the AP-RTA step was investigated. The RTA reactors are made of quartz and aluminum is known to be a possible contaminate of natural quartz; however, XPS analysis showed no aluminum in the quartz reactor. A virgin $\mathrm{SiC}$ sample was put through multiple AP-RTA cycles and analyzed 
after each cycle; no aluminum was detected. A SiC sample was etched and then analyzed; no aluminum was detected. A SiC sample was etched and then AP-RTA'ed; aluminum was detected. Thus, it was determined that no single step in the process lead to detectable aluminum; only the combination of both an etch and AP-RTA resulted in aluminum contamination. A sample was then taken through multiple AP-RTA cycles. After the first AP-RTA cycle, aluminum was detected. Subsequent AP-RTA cycles had no effect on aluminum levels. The ICP-RIE step was then investigated. A series of samples were put through a range of ICP/RIE cycles; some samples experiencing few etch cycles, others many etch cycles. Each sample was then AP-RTA'ed and analyzed. It was found that the aluminum contamination levels tracked precisely with the number of ICP/RIE cycles, with samples that experienced many cycles showing the most aluminum. A sample was masked off (thereby protecting it from the etching plasma) and put though a single etch cycle and then a single AP-RTA cycle. XPS analysis showed very high levels of aluminum on the exposed area and only trace amounts on the masked off area. Therefore, it was concluded that the aluminum was not related to the AP-RTA but to the ICP-RIE (specifically to exposure to the $\mathrm{CF}_{4}$ plasma) but that the AP-RTA was required for the contamination to be detectable.

Visual inspection of the interior of the ICP/RIE chamber revealed etching had occurred to the hard-anodized aluminum centering ring (Figure C.4 $(A, B)$ ). The ring was replaced with a new, freshly anodized aluminum ring. This led to a drastic reduction in aluminum contamination in newly etched samples. However, after $\sim 6$ etch runs, aluminum levels had risen to the previous levels. Visual inspection of the new ring showed the hard anodization had been broken though by the etching process. The ring was then replaced with a pure ceramic ring, which is much more resistant to the plasma. This component substitution almost eliminated the aluminum contamination. It was later discovered that the initial appearance of the aluminum coincided with the replacement the original ceramic centering ring with the hard-anodized aluminum ring. Further investigations revealed a secondary source of contamination originating from the anodized electrostatic chuck (Figure C.4(C,D)). This was the source of the original trace aluminum contamination seen in samples produced before this work. The electrostatic chuck is protected by the carrier wafer and is only slowly etched by plasma which undercuts the wafer. This does lead to the occasional, and unavoidable, contamination of samples. This is mitigated by periodic replacement of the components before a complete breakthrough of the protective anodization occurs. It is worth noting that there are literature reports that the addition of oxygen to the chamber 
during halogen-based plasma etching can suppress the etching of anodized aluminum. ${ }^{(713)}$ This may provide a useful method to suppress aluminum contamination of the samples. However, the effect of including oxygen during the etch step on the oxygen related defect states in the carbon films would require investigation.
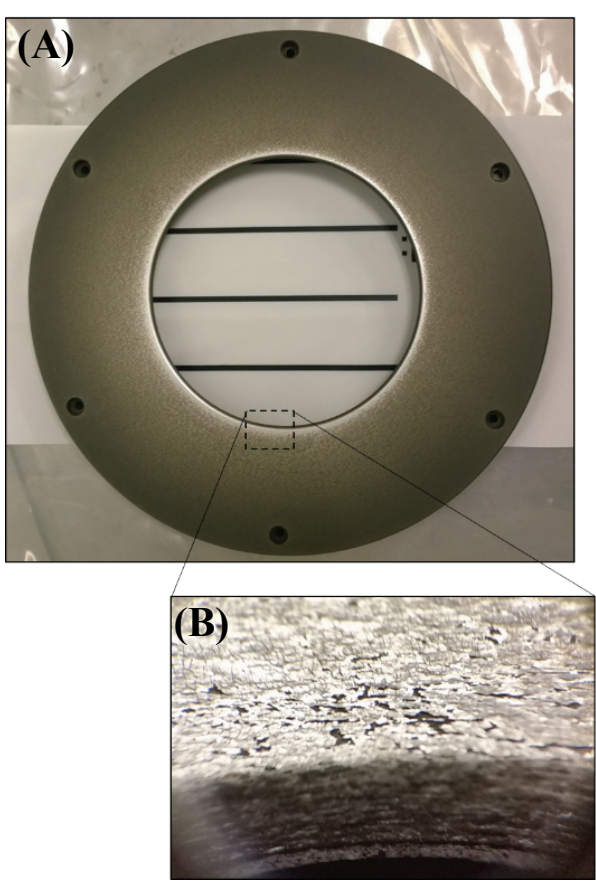
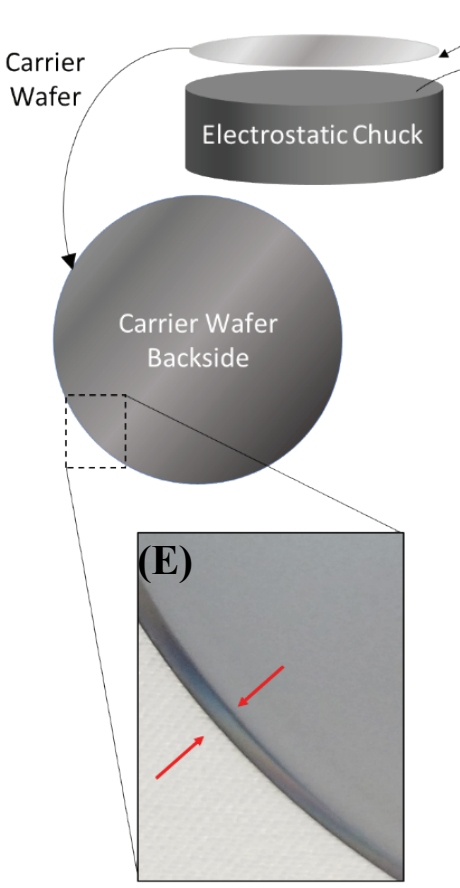

Plasma

Undercut
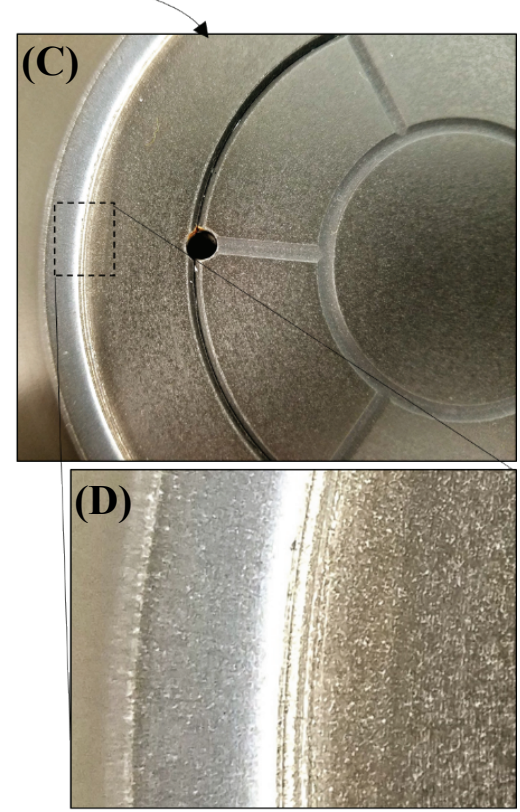

Figure C.4: The primary contamination source: (A) The anodized aluminum centering ring. This was found to be the primary source of contamination. The speckling of lighter color is where the anodization has broken through and the bare aluminum is exposed. (B) A magnified view of the inner portion of the ring showing excessive etching of the unprotected aluminum has occurred, producing pitting. The secondary contamination source: (C) The electrostatic chuck. (D) Zoomed in view of the edge of the electrostatic chuck, the light speckled area along the edge is bare aluminum exposed where the anodization has broken through. This is due to plasma undercutting occurring between the carrier wafer and the chuck. (E) The backside of a used carrier wafer, showing discoloration, indicating plasma undercutting has occurred.

The following theory explains the aluminum contamination: $\mathrm{CF}_{\mathrm{x}}$ ions, accelerated under the RIE field, impact not only the sample but also other components at the base of the ICP/RIE chamber, including the centering ring. While the anodization is somewhat resistive to the plasma it is quickly etched through by the plasma. Anodized aluminum has been shown to be etched by halogen plasmas. ${ }^{(713)}$ Once breakthrough occurs, the bare aluminum is exposed to the highly reactive $\mathrm{CF}_{4}$ plasma products and is aggressively etched. $\mathrm{RF}$ based halogen plasmas have been shown to etch aggressively etch aluminum. ${ }^{(714)}$ Aluminum is either sputtered into the plasma region or transported as products of an aluminum and $\mathrm{CF}_{\mathrm{x}}$ reaction. Once in the main plasma region, and subject to the ICP/RIE RF fields, aluminum species are accelerated back towards the 
bottom of the chamber into the centering ring and $\mathrm{SiC}$ sample being processed. A simple schematic of this process is depicted in Figure C.5.

(A)

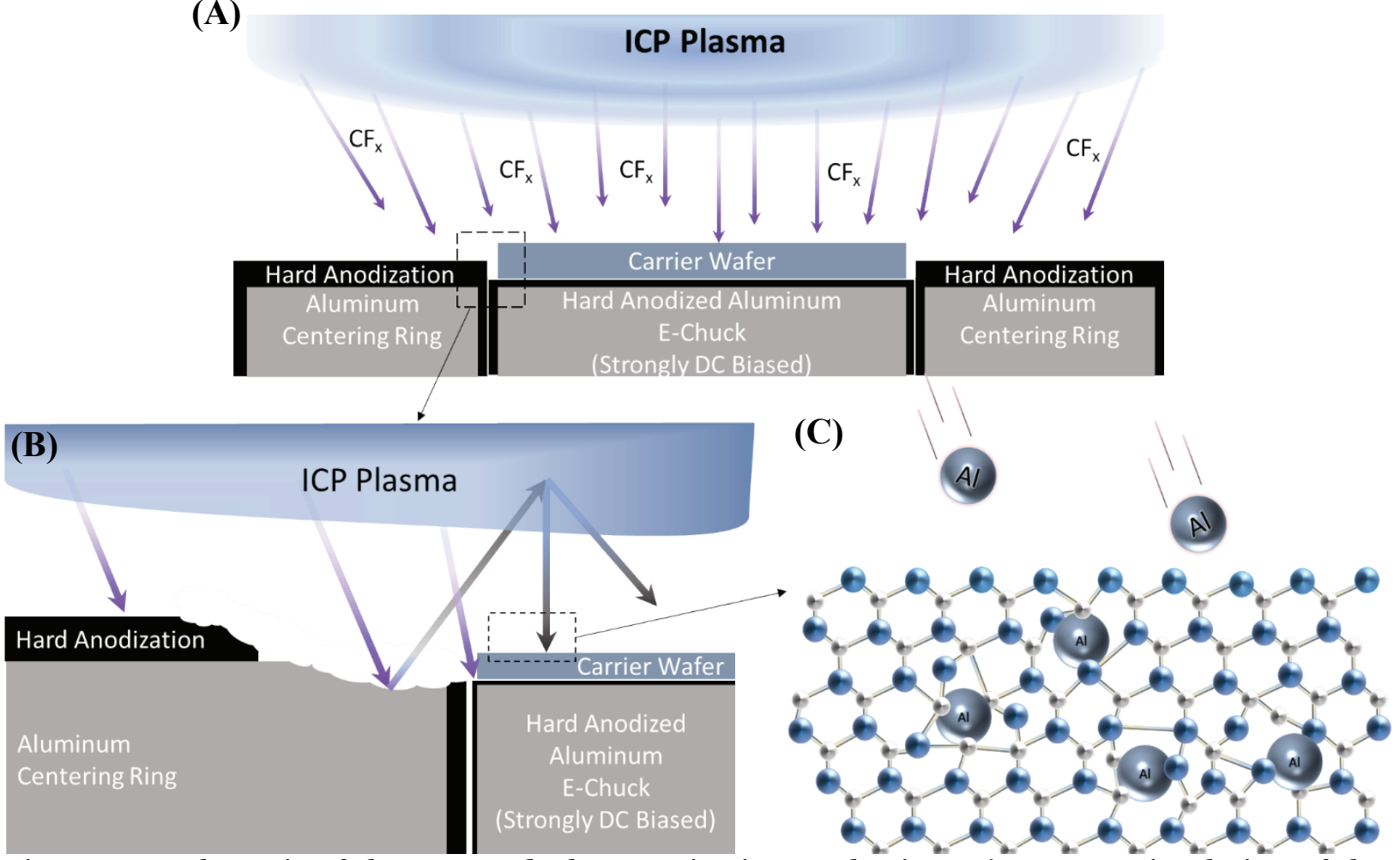

Figure C.5: Schematic of the proposed Al contamination mechanism. A) Cross-sectional view of the chamber interior with key components labeled. B) Depiction of the etching and sputtering process which damages the anodization and exposes the underlying bare aluminum. The arrows indicating the path of the $\mathrm{CF}_{\mathrm{x}}$ and aluminum. $\mathrm{C}$ ) Depiction showing the impregnation of the $\mathrm{SiC}$ by the aluminum. The size discrepancy between silicon atoms and aluminum has been exaggerated for clarity.

This results in dispersed aluminum implanted over a range of depths within the $\mathrm{SiC}$. Because of this, the aluminum concentrations within the sampling depth are below the detection limit of XPS. This explains why samples which have undergone an etched only (and thus are contaminated) do not show detectable aluminum. During the AP-RTA, sufficient thermal energy is imparted to the system for the implanted aluminum to become mobile. Aluminum atoms selfsegregate to the grain boundaries or defect site. In this case, the reconstructed surface acts as a sink for interstitials (aluminum) and precipitates out of the $\mathrm{SiC}$ matric. It then aggregates and, at high levels, forms the textured and particulated surface seen in Figure C.2(C-E). The thermally driven migration of aluminum to defect sites within a $\mathrm{SiC}$ matrix has been previously reported. ${ }^{(381)}(715)$ 
Once aluminum has become concentrated at the surface by this process, it is detectable by XPS analysis. This explains the bi-conditional requirement that a sample must be processed through both the ICP/RIE and AP-RTA steps before aluminum is detected. The generic concentration profiles shown Figure C.6 illiterate this point. Channeling effects, which are known to occur with aluminum implantation of hexagonal $\mathrm{SiC}$, can lead to deeper ion implantation ranges than would otherwise be expected. ${ }^{(716)}$

Aluminum Depth Concentration Profile

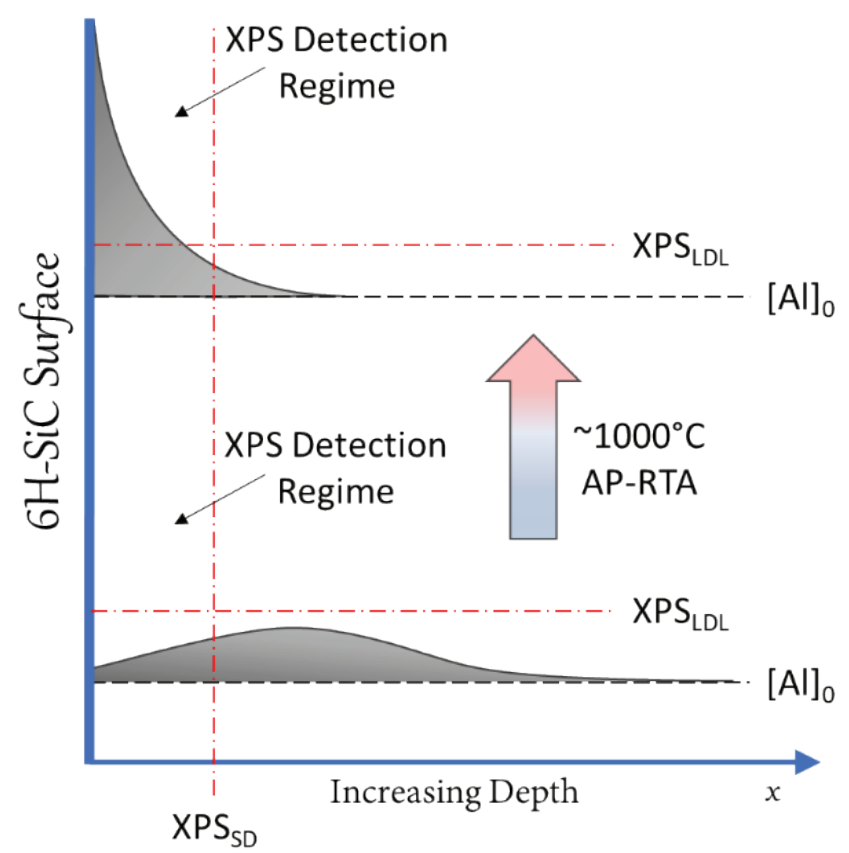

Figure C.6: Proposed aluminum concentration depth profiles which explain the bi-conditional process requirement for detectable aluminum contamination. XPSSD is the sampling depth of the XPS. XPS LDL $_{\text {is }}$ the lower detection limit of XPS and [Al $]_{\mathrm{o}}$ corresponds to the zero aluminum. For XPS analysis to detect aluminum, it must be at concentrations about the XPS $S_{L D L}$ and within the XPSSD. A graphical representation of when these conditions are satisfied is labeled as the XPS detection regime.

In reality, the process is probably much more complex. For example, given that aluminum implantation is occurring simultaneously with selective silicon remove from the $\mathrm{SiC}$, and that aluminum ions preferentially reside at silicon lattice sites in $\mathrm{SiC}$, the situation becomes increasing complex. ${ }^{(717)(718)}$ Furthermore, the co-implantation of $\mathrm{CF}_{\mathrm{x}}$ ions and their reaction with the oxygen and aluminum present further complicate the picture. Nonetheless, this basic theory as stated fits well with the observations and the aluminum contamination problem has been largely solved by this investigation. 
After the primary aluminum containment issue had been solve, it was observed that the occasional appearance of aluminum (resulting from plasma undercutting of the carrier wafer and into the electrostatic chuck) was found to be a function of ICP and RIE powers. Figure C.7 shows the atomic concentration of aluminum and oxygen as a function of ICP and RIE powers and as a function of ICP power and DC bias. This makes drawing conclusions related to the carbon films produced at these parametric locations difficult, as the presence of aluminum changes the fundamental nature of the films.
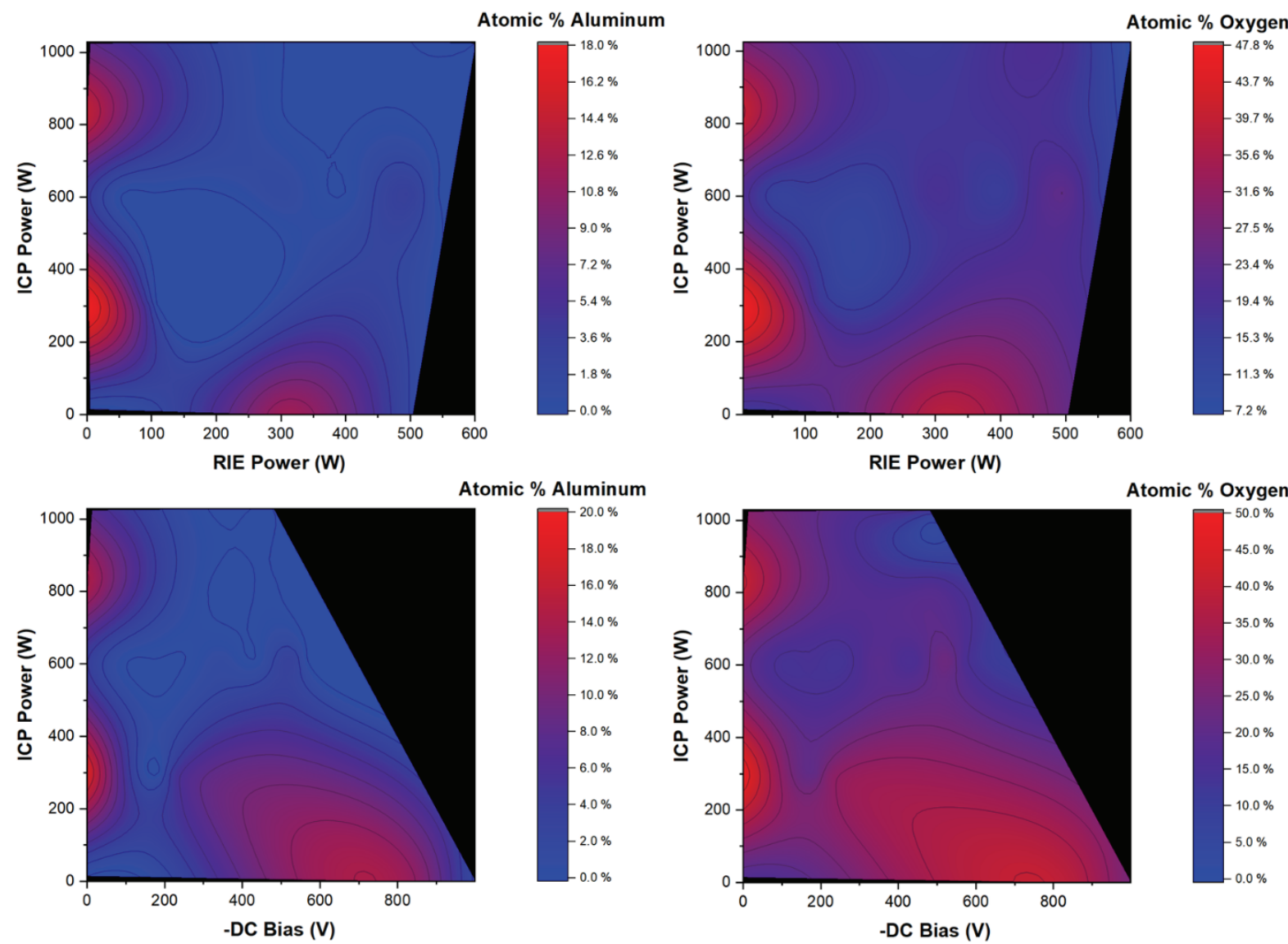

Figure C.7: Atomic concentrations of aluminum and oxygen across the parameter space investigated during the ICP/RIE power study.

Although these contaminated surfaces present a fascinating system, resulting from an equally fascinating formation mechanism, the resulting films produced are too dissimilar to the primary films under study in this work. Thus, their detailed study has been relegated to future work. The prospect of the purposeful decoration or doping of SiC/graphene during the etching process is intriguing. Metals other than aluminum could be introduced to the ICP/RIE chamber resulting in a similar 'cross-contamination' during the etch. The interplay between the etching 
removal process and the deposition addition process may present a unique situation at the sample surface. Such a scenario may represent a unique method of add-material addition. 


\section{APPENDIX D: GRAPHENE LAYER CALCULATION BY XPS ANALYSIS}

Appendix D details the methodology behind the calculation for the number of graphene layers as determined by XPS analysis. The mathematics of the attenuation calculation are detailed. Also, the conceptual interpretation of the system and the motivation for this method are given.

The thickness of the graphene films formed in this work were estimated using a simple layer model and Beer's Law. Beer's Law states that if $\mathrm{I}^{\circ}$ is the intensity of photoelectrons emitted from a layer of atoms buried at a depth of $d$ in the substrate, then the detected intensity emitted from the surface is given by

$$
I=I^{o} e^{-\left(\frac{d}{\lambda \sin \theta}\right)} \quad \text { Equation D-1 }
$$

where $\lambda$ is the electron inelastic mean free path for photoelectrons of interest and $\theta$ is the takeoff angle for the photoelectrons measured relative to the surface. ${ }^{(719)}$ The exponential term accounts for attenuation of the photoelectrons due to the overlying material. The value for Io depends on a number of factors associated with the sample including the areal density of atoms producing the photoelectrons, the photon absorption cross-section for the atomic orbital of interest, the probability of producing a photoelectron, the inelastic mean free path of the photoelectrons as well as instrumental factors including the photon flux and the detection efficiency. ${ }^{(719)}$

Based on Equation D-1, it is clear that for electrons emitted normal to the surface $\sim 95 \%$ of the detected electrons come from a depth $\mathrm{d}<3 \lambda$. This is frequently referred to as the escape depth. For electrons emitted with low take off angles (e.g., $\left.5^{\circ}\right)$, the effective inelastic mean free path $(\lambda$ $\sin \theta$ ) is very small. Analyses based on such electrons are said to be obtained in the "surface analysis mode," while analyses at larger angles (e.g. $45^{\circ}$ ) are said to be in the "bulk analysis mode." 
To use Equation D-1 to determine the thickness of the graphene layers produced in these studies, a simplified mode of the graphene-SiC substrate system was proposed. This is illustrated in Figure D.1 which shows a graphene film consisting of three equally spaced layers on the $\mathrm{SiC}$ surface. Only a portion of the $6 \mathrm{H}-\mathrm{SiC}$ unit cell is shown. Here the spacing (d) is taken as $0.346 \mathrm{~nm}$ based on the measurements of Weng, et al. ${ }^{(548)}$ The deepest layer of graphene is referred to as the buffer layer and it is bonded to the topmost Si layer in the SiC substrate. The spacing between the topmost $\mathrm{Si}$ and the buffer layer (do) should nominally be $0.189 \mathrm{~nm}$ based on bulk Si-C spacings. Thus, the value of $0.200 \mathrm{~nm}$ represents a slightly relaxed value used by Asensio and coworkers. ${ }^{(219)}$ The value of $D_{1}$ is taken as $0.063 .{ }^{(219)}$ The spacing between the adjacent carbon layers $\left(D_{2}\right)$ in $6 \mathrm{H}-\mathrm{SiC}$ is taken as one-sixth the unit cell dimension or $0.252 \mathrm{~nm} .{ }^{(720)}$

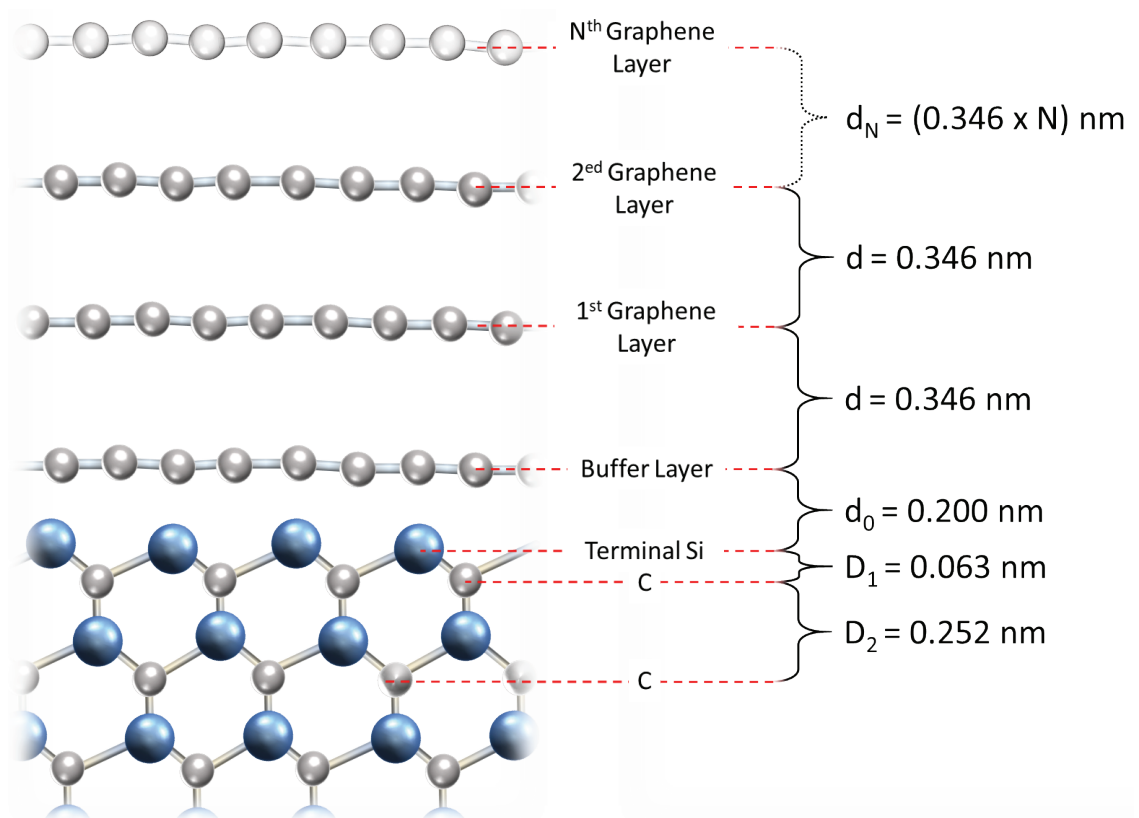

Figure D.1: Schematic of a three-layer (two graphene + one buffer) film on a 6H-SiC (ooo1) substrate surface. This is an idealized model and does not attempt to account for complex interactions between the buffer layer and substrate.

Following Yates and coworkers, if $I_{G}^{o}$ represents the intensity of the C1s peak for a single layer of graphene, then for $\mathrm{N}$ equally spaced graphene layers

$$
I_{G}=I_{G}^{o}\left(1+e^{-\left(\frac{d}{\lambda \sin \theta}\right)}+e^{-\left(\frac{2 d}{\lambda \sin \theta}\right)}+\cdots \ldots+e^{-\left(\frac{(N-1) d}{\lambda \sin \theta}\right)}\right) \quad \text { Equation D-2 }
$$




$$
I_{G}=I_{G}^{o} \sum_{n=0}^{N-1} e^{-\left(\frac{n d}{\lambda \sin \theta}\right)}
$$

Equation D-3

where $\lambda$ is the electron inelastic mean free path for $\mathrm{C} 1 \mathrm{~s}$ photoelectrons and $\theta$ as noted above is the takeoff angle (relative to the surface) for the photoelectrons. ${ }^{(545)}$ The inelastic mean free path for $\mathrm{C} 1 \mathrm{~s}$ photoelectrons was taken as $1.5 \mathrm{~nm} .{ }^{(721)} \mathrm{In}$ a similar manner, the intensity of the C1s peak from $\mathrm{SiC}$ can be calculated as

$$
I_{S i C}^{\infty}=I_{\text {SiC }}^{o} e^{-\left(\frac{D_{1}}{\lambda \sin \theta}\right)} \sum_{n=0}^{\infty} e^{-\left(\frac{n D_{2}}{\lambda \sin \theta}\right)} \quad \text { Equation D-4 }
$$

were $I_{\text {SiC }}^{o}$ is the intensity of the C $1 \mathrm{~s}$ XPS peak for a layer of carbon in $6 \mathrm{H}-\mathrm{SiC}$. The factor of $e^{-\left(\frac{D_{1}}{\lambda \sin \theta}\right)}$ accounts for the attenuation of the carbon signal by the overlying Si layer associated with the buffer layer assumed to always be present on the (0001) surface which interfaces with the graphene layer(s). Based on these considerations, the measured ratio of $\mathrm{I}_{\mathrm{G}} / \mathrm{Isic}_{\mathrm{SiC}}$ should thus be given by

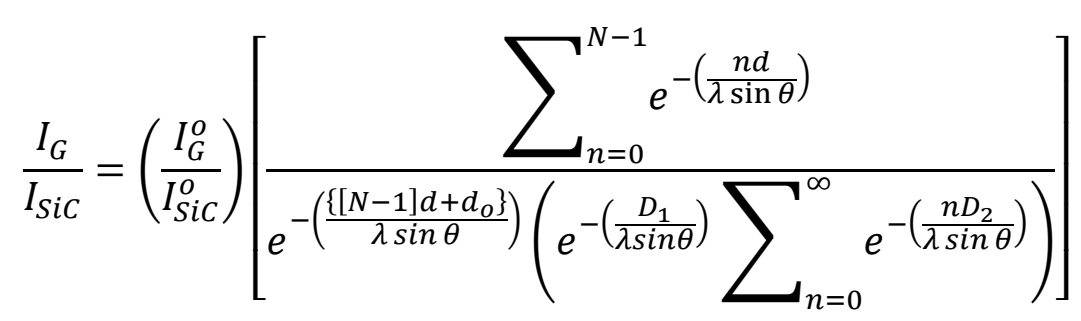

Here the exponential factor, $e^{-\left(\frac{\left[[N-1] d+d_{0}\right\}}{\lambda s i n \theta}\right)}$, takes into account of the attenuation of the $\mathrm{SiC}$ signal by the overlaying graphene layer.

Since the photoelectron peaks considered here at all $\mathrm{C} 1 \mathrm{~s}$ peaks, all material related factors with the exception of the areal density of carbon atoms should be the same for both graphene and $\mathrm{SiC}$. Moreover, since the $\mathrm{C} 1 \mathrm{~s}$ peaks for both graphene and $\mathrm{SiC}$ are measured at the same time under the same conditions, all instrumental factors (including take off angle) should also be the same. Consequently, the ratio the intensity ratio for the carbon atoms in a single graphene and $\mathrm{SiC}$ layer should be given by 


$$
\frac{I_{G}^{o}}{I_{S i C}^{o}}=\frac{A_{G}}{A_{S i C}} \quad \text { Equation D-6 }
$$

where, $A_{\mathrm{Sic}} \sim 1.22 \times 10^{15} / \mathrm{cm}^{2}$ and $\mathrm{AG} \sim 3.82 \times 10^{15} / \mathrm{cm} .{ }^{(722)}$ Using Equation D-5 and Equation D-6, calculations for the intensity ratio for different number of graphene layers can easily be performed using MS Excel or similar program. Figure D.2 shows a plot of number of graphene layers $(\mathrm{N})$ as a function of intensity ratio $\left(\mathrm{IG}_{\mathrm{G}} / \mathrm{Isic}_{\mathrm{Sic}}\right)$. Note that this count includes the carbon atoms in the buffer layer. This plot was used to determine number of layers corresponding to a given ratio of intensities.

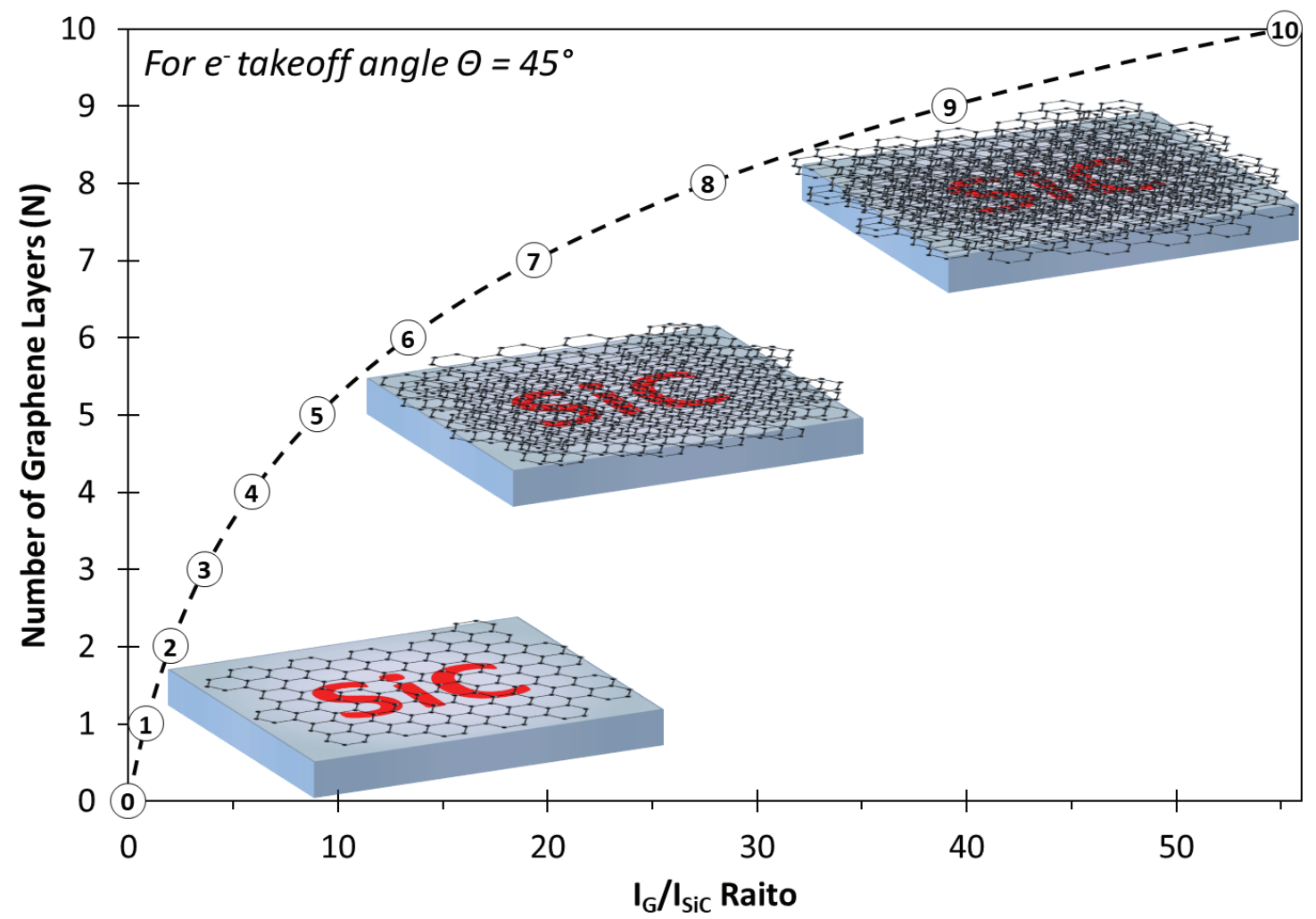

Figure D.2: Plot of number of graphene layers $(\mathrm{N})$ as a function of intensity ratio $\left(\mathrm{I}_{\mathrm{G}} / \mathrm{I}_{\mathrm{Sic}}\right)$. The dashed line corresponds to the pseudo-spline interpretation used to estimate the fractional coverage.

Recall that the data generated from any XPS measurement is the weighted confluence of the photoelectron signal produced from all material within the analysis spot size $(\sim 100 \mu \mathrm{m}$ diameter in this work). Thus, the parameters calculated from the resulting data are nominal values. As such, fractional graphene coverages are routinely calculated. For example, the number of calculated layers may be $1 / 2$. It is worth noting that the proper interoperation of this is not that a 
graphene layer is one-half the normal thickness has been produced, but rather that one-half of the analysis region is covered with graphene, nominally (i.e. factional coverage). ${ }^{49}$

To determine the fractional coverage (i.e. intensity rations which fall between data points), a local $\mathrm{n}^{\text {th }}$ degree polynomial was fitted to capture the surrounding data points (resulting in a pseudo-spline interpolation over the whole data set). This was done to achieve a greater granularity in the interpolation between points and avoid Runge's phenomenon for polynomial interpolation. ${ }^{50}$

While quite simple, both mathematically and computationally, this model could readily be expanded to include a variety of complexities. These include differences in the inelastic mean free path for $\mathrm{C} 1 \mathrm{~s}$ electron in graphene layers and $\mathrm{SiC}$, differences in areal density for each graphene layer, differences in graphene layer spacing, and similar variations in the near surface layers of the $\mathrm{SiC}$ substrate. Although this information is important as a part of understanding the physics and chemistry of the buffer layer, the numerical differences turn out to be relatively small and do not significantly change the calculated thickness of the graphene film. In this vein, however, we refer to the calculated thicknesses as estimates.

\footnotetext{
${ }^{49}$ Although this is just one interpretation; any aerial coverage which would average to a $1 / 2$ layer would be valid. For example, $1 / 4$ of the surface may have two layers and the remaining $3 / 4$ having no graphene (island stacking). There is no way to differentiate based solely on the XPS data. However, given the nature of the growth and the self-attenuation which would occur for an isolated, many-layer graphene stack, coverage scenarios which yield the fewest number of total layers are the most likely.

${ }^{50}$ Briefly state, Runge's phenomenon is a mathematical situation in which no $\mathrm{n}^{\text {th }}$ order polynomial exists which adequality fits the data, across the entire data range. ${ }^{(741)(740)}$ For example, in this work, a polynomial which accurately captures the data space between 1 layer and 2 layers does not accurately fit the data space between 9 layers and 10 layers. Therefore, polynomials were fitted to only the few neighboring data points thereby simulating a spline interpolation.
} 